\title{
Glacier inventory and atlas of
}

West Greenland

$G E \cup S$

Report file no.

\section{5}

Anker Weidick, Carl Egede Bøggild and Niels Tvis Knudsen

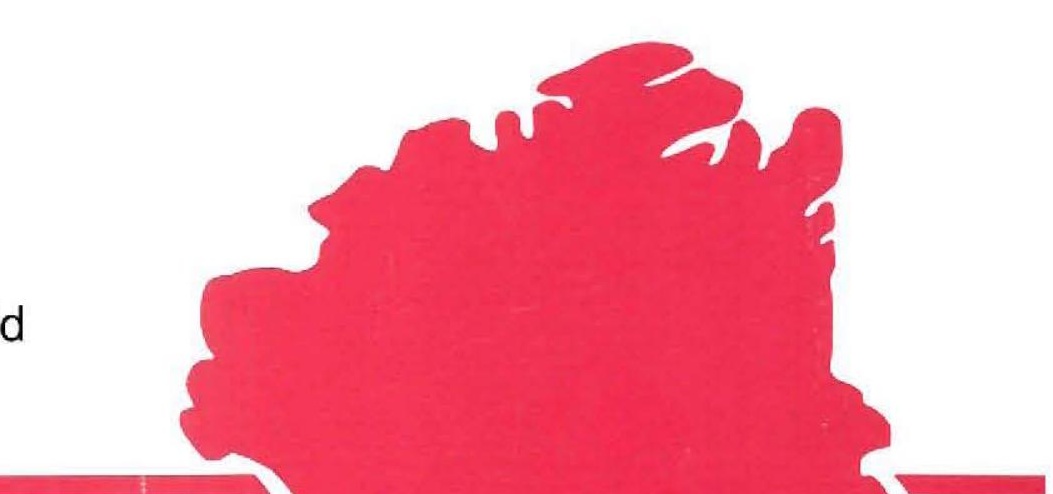


GRøNLANDS GEOLOGISKE UNDERS $\emptyset$ GELSE RAPPORT 158

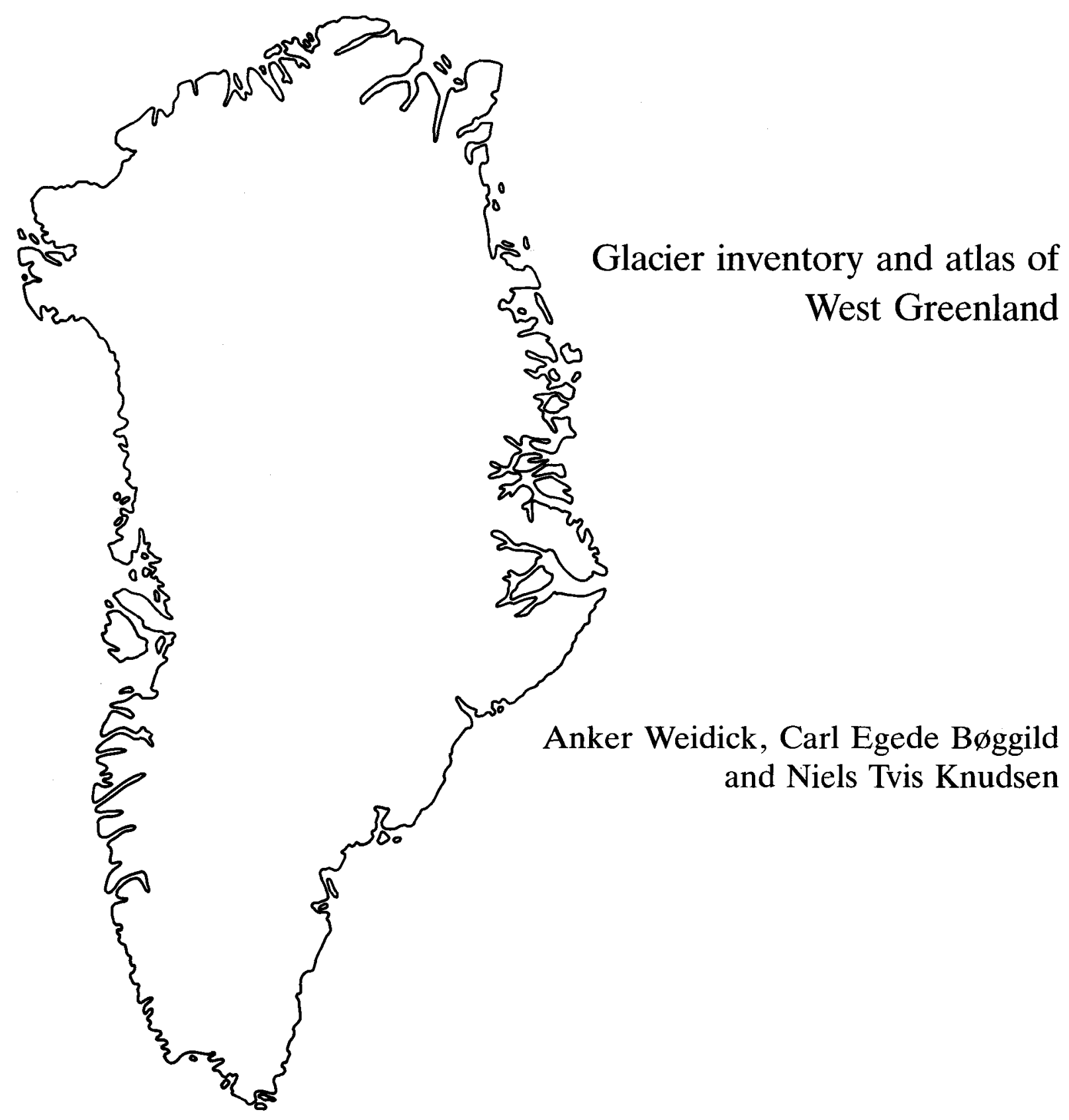


Weidick, A., Bøggild, C. E. \& Knudsen, N. T. 1992: Glacier inventory and atlas of West Greenland. Rapp. Grønlands geol. Unders. 158, pp.

The glacier inventory and atlas provides the localisation and description of over 5000 glacier units in western Greenland between $59^{\circ} 30^{\prime}$ and $71^{\circ} 00^{\prime} \mathrm{N}$. Registration is based on natural hydrological basins, in accordance with the recommendations of the International Commission on Snow and Ice (ICSI) but modified and simplified to meet Greenland conditions. The work is divided into three parts: a description of the procedure used in compilation of the glacier inventory and the glaciological conditions, a tabulated presentation of the information and description of the individual glacier units, and a 1:300 000 map presentation showing the position and delineation of the individual glacier units.

\section{Authors' addresses:}

A. W., Geological Survey of Greenland, Øster Voldgade 10, DK-1350 Copenhagen K, Denmark.

C. E. B., Geological Survey of Greenland, Øster Voldgade 10, DK-1350 Copenhagen K, Denmark. Present address: Alfred-Wegener-Institut für Polar- und Meeresforschung, Postfach 120161, Columbusstrasse, D-2850 Bremerhaven, Germany.

N. T. K., Geological Institute, University of Aarhus, Langelandsgade, bygn. 521, DK-8000 Århus C, Denmark.

\section{Dansk sammendrag}

Registrering og atlas dækker lokalisering og beskrivelse af over 5000 gletscherenheder i det vestlige Grønland mellem $59^{\circ} 30^{\prime}$ og $71^{\circ} 00^{\prime} \mathrm{N}$. Registreringen er udført efter et system foreslået af International Commission on Snow and Ice (ICSI) og er baseret på de naturligt forekommende hydrologiske bassiner, men modificeret og simplificeret med henblik på de specielle grønlandske forhold. Atlasset er delt i tre afsnit: En beskrivelse af arbejdsprocedurerne for udarbejdelse af gletscherregistret og de generelle glaciologiske forhold $i$ området, en tabellarisk fremstilling af information og beskrivelse af de enkelte gletscherenheder, samt et atlas i 1:300 000 angivende de enkelte gletscherenheders position og afgrænsning.

\section{Imaqarnersiuineq}

Qinerlerfissiami nunallu assingini Kitaani sermeqarfiit 5000-t avannarpasissutsip $59^{\circ} 30^{\prime}$ aamma $71^{\circ} 00$ akornanniittut sumiissuserneqarlutillu allaaserineqarsimapput. Nalunaarsuineq International Commission on Snow and Ice (ICSI)-mit nalunaarsueriaaseq siunnersuutigineqarsimasoq malillugu ingerlanneqarsimavoq, tassanilu nunami imminik erngup katersuuffigisartagai tunngaviusimallutik, kisiannili Kalaallit Nunaanni immikkut pissutsit peqqutigalugit allanngortiterneqarsimalluni pisariillisarneqarsimallunilu. Nunap assingi im- mikkoortunut pingasunut immikkoortiterneqarsimapput: Siullermi sermit nalunaarsorneqarnerini suleriaaseq nunamilu immikkoortumi ataatsimut isigalugu sermeqarfiit pissusaat allaaserineqarsimavoq, aappaanilu sermeqarfinnut ataasiakkaanut paasissutissat tabelinngorlugit allaaserineqarsimapput, kiisalu pingajuani nunap assingani 1:300 000 uuttuuteqartumi sermeqarfiit ataasiakkaat sumiiffii killingilu nalunaarsorneqarsimallutik.

\section{Grønlands Geologiske Undersøgelse Ujarassiortut Kalaallit Nunaanni Misissuisoqarfiat Geological Survey of Greenland}

The Geological Survey of Greenland (GGU) is a research institute affiliated to the Mineral Resources Administration for Greenland (MRA) within the Danish Ministry of Energy. As with all other activities involving the non-living resources in Greenland, GGU's investigations are carried out within the framework of the policies decided jointly by the Greenland Home Rule Authority and the Danish State. 


\section{Contents}

Introduction. $\ldots \ldots \ldots \ldots \ldots \ldots \ldots \ldots \ldots \ldots \ldots \ldots, \quad 5$

Background ......................... 5

Main sources ....................... 5

Aim of the atlas $\ldots \ldots \ldots \ldots \ldots \ldots \ldots \ldots \ldots \ldots$

Restriction of information $\ldots \ldots \ldots \ldots \ldots \ldots \ldots \quad 7$

Glacier identification $\ldots \ldots \ldots \ldots \ldots \ldots \ldots \ldots \ldots \ldots, 7$

Identification code ..................... 7

International reference $\ldots \ldots \ldots \ldots \ldots \ldots \ldots \ldots, 7$

District and fjord devision in Greenland ........ 7

Hydrological basins and the individual glacier units 7

Comments on the West Greenland hydrological basins 9

Identification ..................... 9

Local glaciers and the Inland Ice margin........ 9

Local glaciers $\ldots \ldots \ldots \ldots \ldots \ldots \ldots \ldots \ldots \ldots, 9$

The Inland Ice and its total size compared to larger local glaciers $\ldots \ldots \ldots \ldots \ldots \ldots \ldots \ldots \ldots \ldots$

Inland Ice delineation $\ldots \ldots \ldots \ldots \ldots \ldots \ldots$

Surface of the Inland Ice

Sector delineation and subsurface topography of the Inland Ice. . . . . . . . . . . . . . . . . . . . . .

Hydrological and dynamic sectors............

Glacier change and identification code $\ldots \ldots \ldots \ldots$.

Information registered on the West Greenland glaciers

Glacier identification (code)................. 12

Glacier name........................ 12

Geographical coordinates and orientation of glaciers . 12

Highest and lowest glacier elevation........... 12

Surface areas ..................... 12

Morphology code .................... 13

Remarks on the map and morphology code ....... 13

Work description $\ldots \ldots \ldots \ldots \ldots \ldots \ldots \ldots \ldots \ldots \ldots . \ldots \ldots$

Geographical coordinates ................ 14

Area determination ................... 14

Height determination. .................. 14

Storage of information $\ldots \ldots \ldots \ldots \ldots \ldots \ldots \ldots . \ldots \ldots$

Inventory atlas sheets $\ldots \ldots \ldots \ldots \ldots \ldots \ldots \ldots \ldots$

Morphology and outline of Holocene glacier history ... 15

Coastal terrain ........................ 15

Subsurface of the south-western slope of the Inland Ice 15

Outline of the Holocene glaciation............. 16

Present climate and glacial conditions ........... 16

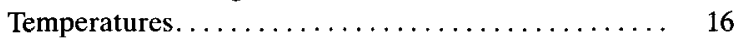

Coastal stations..................... 16

The Inland Ice $\ldots \ldots \ldots \ldots \ldots \ldots \ldots \ldots \ldots$

Precipitation and accumulation $\ldots \ldots \ldots \ldots \ldots \ldots, 17$
Precipitation over the coastland $\ldots \ldots \ldots \ldots \ldots \ldots, 17$

Accumulation over the Inland Ice........... 18

Glaciation and snow lines............... 18

Comments on minor local glaciers (glacierets, mountain glaciers and valley glaciers) $\ldots \ldots \ldots \ldots \ldots \ldots \ldots$

Glacierets and snow fields $\ldots \ldots \ldots \ldots \ldots \ldots \ldots \ldots$

Size, extent and volume $\ldots \ldots \ldots \ldots \ldots \ldots \ldots . \ldots 19$

Changes .................................. 19

Mountain glaciers...................... 19

Size, extent and volume $\ldots \ldots \ldots \ldots \ldots \ldots \ldots .20$

Changes ......................... 21

Mass-balance measurements. ............. 21

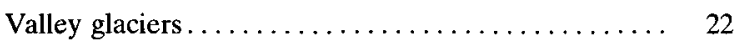

Size, extent and volume $\ldots \ldots \ldots \ldots \ldots \ldots \ldots \ldots, 22$

Changes ................................ 22

Mass-balance measurements............. 23

Large, local glaciers (ice caps and ice fields) ....... 23

Definitions....................... 23

Composite and solitary ice caps and ice fields ..... 23

Distribution and limitations ............... 23

Julianehåb ice cap ................... 25

Size and extent of major ice caps and ice fields.... 25

Volume............................... 26

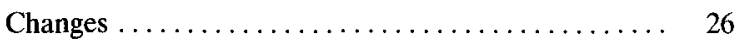

Mass-balance measurements................. 27

Calf ice production from ice caps . . . . . . . . . 28

Ice domes............................ 28

The south-western slope of the Inland Ice ........ 28

Limitations....................... 28

Sector division of the Inland Ice - calf ice producing and quiet sectors of the ice sheet ............ 29

Calf ice producing sectors............... 30

Quiet sectors ...................... 30

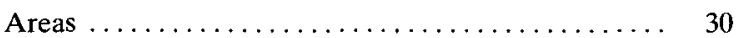

General trends in recent marginal change ........ 30

Thickness change of the marginal parts of the ice sheet 34

Local change of the ice margin ............. 35

Modelling of Inland Ice margin response ........ 36

Mass balance measurements.................. 37

Acknowledgements . . . . . . . . . . . . . . 37

References......................... 40

Glacier names in West Greenland c. $60^{\circ}-71^{\circ} \ldots \ldots \ldots .43$

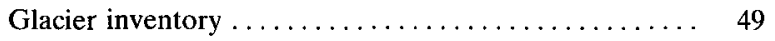

Glacier atlas .......................... 95 


\section{Introduction}

\section{Background}

During the International Hydrological Decade 1965-74, the International Commission on Snow and Ice (ICSI) recommended registration of all perennial snow and ice masses of the world (Müller et al., 1977). The work thus set in progress is coordinated by the World Glacier Monitoring Service (WGMS, earlier: 'Temporary Technical Secretariat (TTS) for the World Glacier Inventory', ETH, Zürich).

The main work on glacier registration during the International Hydrological Decade was undertaken in the 'classical' glacier countries (e.g. the Alps, Scandinavia) with possibilities for detailed work on individual glaciers. Work along the same outline for polar ice sheets such as those of Antarctica or Greenland, which make up respectively $c .90 \%$ and $c .9 \%$ of the present total amount of glacier ice in the world $\left(=29\right.$ mill. $\mathrm{km}^{3}$ water), is not feasible and these areas were not considered during the early phase of the proposed registration.

For the Greenland area, two lines of development have promoted a move towards an attempt at a glacier inventory. The first appearance of the Landsat satellite images following the launching of ERTS-1 on July 23rd 1972 (ERTS = Earth Resources Technology Satellite: Williams \& Carter, 1976) has provided possibilities for differentiating glaciers in remote regions. Next, the onset of the energy crisis in 1973 invoked an interest in the development of hydropower in West Greenland.

Since the major part of potential water resources in Greenland is stored as a solid phase, a natural first step to the utilization of this resource must therefore be to survey it.

Ninety-two per cent of Greenland's total population of about 55000 inhabitants are located in West Greenland between latitudes $60^{\circ}$ and $70^{\circ} \mathrm{N}$, in towns and settlements generally sited on the coastal parts of the broad 'ice-free' land area between Davis Strait and the Inland Ice. This broad strip of land (for which the term 'coastland' is used for convenience in this report) is $100-200 \mathrm{~km}$ wide in places, and has been given the highest priority in resource mapping; the present work is devoted to this area.

Mapping of all the hydrological basins in West Greenland, including a first attempt of sector division of the south- western part of the Inland Ice, was therefore necessary in order to make a resource estimate for this sector of the Inland Ice as well as all major local glaciers (Weidick \& Olesen, 1980). At this stage the base maps used were the Geological Survey of Greenland (GGU) map sheets at 1:500 000 with $200 \mathrm{~m}$ contour interval.

\section{Main sources}

The basin division of the inventory is based on a simplified version of the instructions by the Temporary Technical Secretariat of the World Glacier Inventory (Müller et al., 1977) and the present work has facilitated from experience gained in TTS.

At the same time valuable help was supplied from the EROS office of the U.S. Geological Survey in Reston, Va., U.S.A., and its project for a Satellite Image Atlas of Glaciers (edited by R. Williams \& J. Ferrigno). A selection of good Landsat images of Greenland from this office greatly facilitated a first order division of the ice sheet and the larger local ice caps.

The most important base for the work are the topographic maps of West Greenland by Kort- og Matrikelstyrelsen, Denmark (National Survey and Cadastre, formerly the Geodetic Institute). Nearly the whole of Greenland is covered by map sheets at 1:250 000 with at least $100 \mathrm{~m}$ contour interval, and the same institute's collection of aerial photographs has provided the primary base for the location of individual glaciers.

\section{Aim of the atlas}

The aim of the atlas is a procurement of a natural reference system for information on Greenland glaciers, i.e. the establishment of an address for the individual glacier units. Considerable information on these glaciers occurs as scattered references in published scientific literature, and the lack of proper specifications of glacier localities has inevitably led to the loss of important glacier information.

The reluctance to establish a comprehensive reference system is due to the overwhelming number of glacier units involved. Minor glaciers are often lacking on official maps, and only very few glaciers of the total number have a name.

The first step therefore was to modify the system 


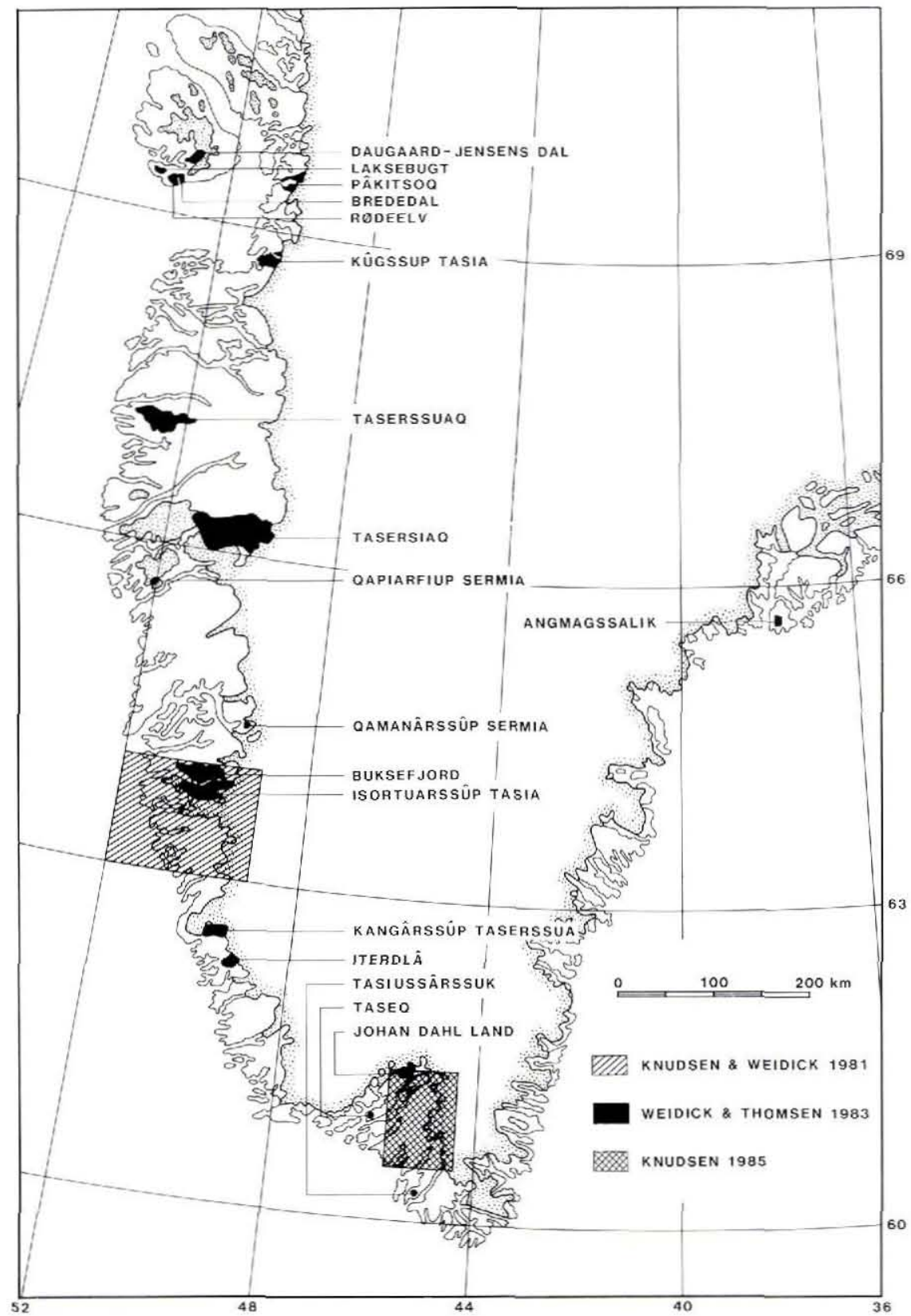

Fig. 1. Areas covered by former inventory work on glacicrs.

suggested by the TTS so that it could satisfy the Greenland conditions of one major, continental ice sheet (the Inland Ice) and a large number of relatively minor local glaciers on the up to $200 \mathrm{~km}$ wide strip of coastland (cf. Weidick, 1980). Location and delineation (identification) of the individual glacier units were also made at this stage. The background for the identification code is given in the next chapter.

The next step was collection and registration of basic data for the morphology code of each of over 5000 glacier units of the area, a process described in the chapter on Information registered on the West Greenland glaciers. For selected hydropower localities, the immediate need of an inventory resulted in the inventory atlas being preceded by two earlier works covering specific areas, namely those by Knudsen \& Weidick (1981) and by Weidick \& Thomsen (1983). A third area in South Greenland was compiled by Knudsen, but never published as a separate report. The areas covered by these three compilations are included in the atlas and the localities are shown on the map of Fig. 1.

In addition to the desire for the establishment of a 
natural registration system, the perspective for this first attempt at a glacier inventory for a large part of Greenland is to provide a basis for the planning of future glaciological investigations, for instance to define suitable key areas for monitoring future glacier changes (e.g. in connection with the supposed 'greenhouse effect'). In this context it should be mentioned that between 10 and $20 \%$ of the world's local glaciers are situated in Greenland, a fact often forgotten due to the existence and overwhelming dominance of the Inland Ice (Weidick, 1990b). Local glaciers will respond faster to global warming and are therefore important for the evaluation of any initial rise of sea level (Meier, 1985).

\section{Restriction of information}

Since coding of the individual glacier is an a prior requirement for filing of glacier information, and was urgently required at the same time at many different potential hydropower sites, part of the work was carried out under a certain time constraint. This, together with the restrictions arising from the quality of the 1:250 000 base map and the varying quality of aerial photographs requires the authors to emphasize that the general information of the atlas has been worked out at a scale of 1:250 000 and is not suitable for studies at greater detail.

Only a few of the Greenland glaciers are covered by detailed maps at scales greater than 1:250 000. With more precise surveying the surface areas given for the individual glacier units may be expected to change significantly.

\section{Glacier identification}

\section{Identification code \\ International reference}

As an example of a complete glacier code, Jakobshavn Isbræ is chosen here. This is a major outlet of the Inland Ice, and drains a large part of it (Atlas sheet $6900-05)$. Its identification code is:

\section{GL 2U 1GC 06002}

where the first part of the code (GL 2U) is given by the TTS World Glacier Inventory indicating the administrative unit Greenland (Greenland = GL) situated in North America (2U). This first part of the code (GL $2 U)$ has been omitted from all references in this inventory, since all data in this description refer to localities in Greenland.

\section{District and fjord division in Greenland}

The next combination of digit and letters (in the example 1GC) refer to the district and fjord division of the Greenland coastland. As shown in Fig. 2, the main division is into West, North and East Greenland, denoted by the digits 1,2 and 3 respectively. These main areas are then subdivided into districts (in the example $\mathbf{G}=$ inner part of Disko Bugt), which within each main area is denoted by a letter, beginning with South Greenland and moving clockwise around Greenland.
The position of the first two places in the national code (in the example 1G) is shown on the map of Fig. 2 covering the whole of Greenland. The subsequent subdivision down to individual glaciers has only been made for the West Greenland area covered by this inventory.

Each district is a compromise between a natural division along main watersheds and a need for approximate equal-sized areas of the coastland. The subsequent letter thus denotes the fjords, counted clockwise around Greenland, i.e. in western Greenland beginning from the south. In the example $\mathrm{C}=$ Jakobshavn Isfjord (see Atlas sheet 6900-04).

\section{Hydrological basins and the individual glacier units}

The succeeding two digits (in the example 06) indicate the number of the natural hydrological basin in the fjord, counted clockwise from the entrance (see Atlas sheet 6900.04-05). In the original version (Weidick \& Olesen, 1980) the basins were closed units (in order to calculate their discharge). This division left some of the coastal areas outside the system, and since glaciers occur in these localities, the coastal areas have been included in the nearest hydrological basin so that the division refers to a 'basin area' rather than to a natural hydrological basin.

The final three digits (in the example 002) refer to the number of the glacier unit in the basin area. A glacier 


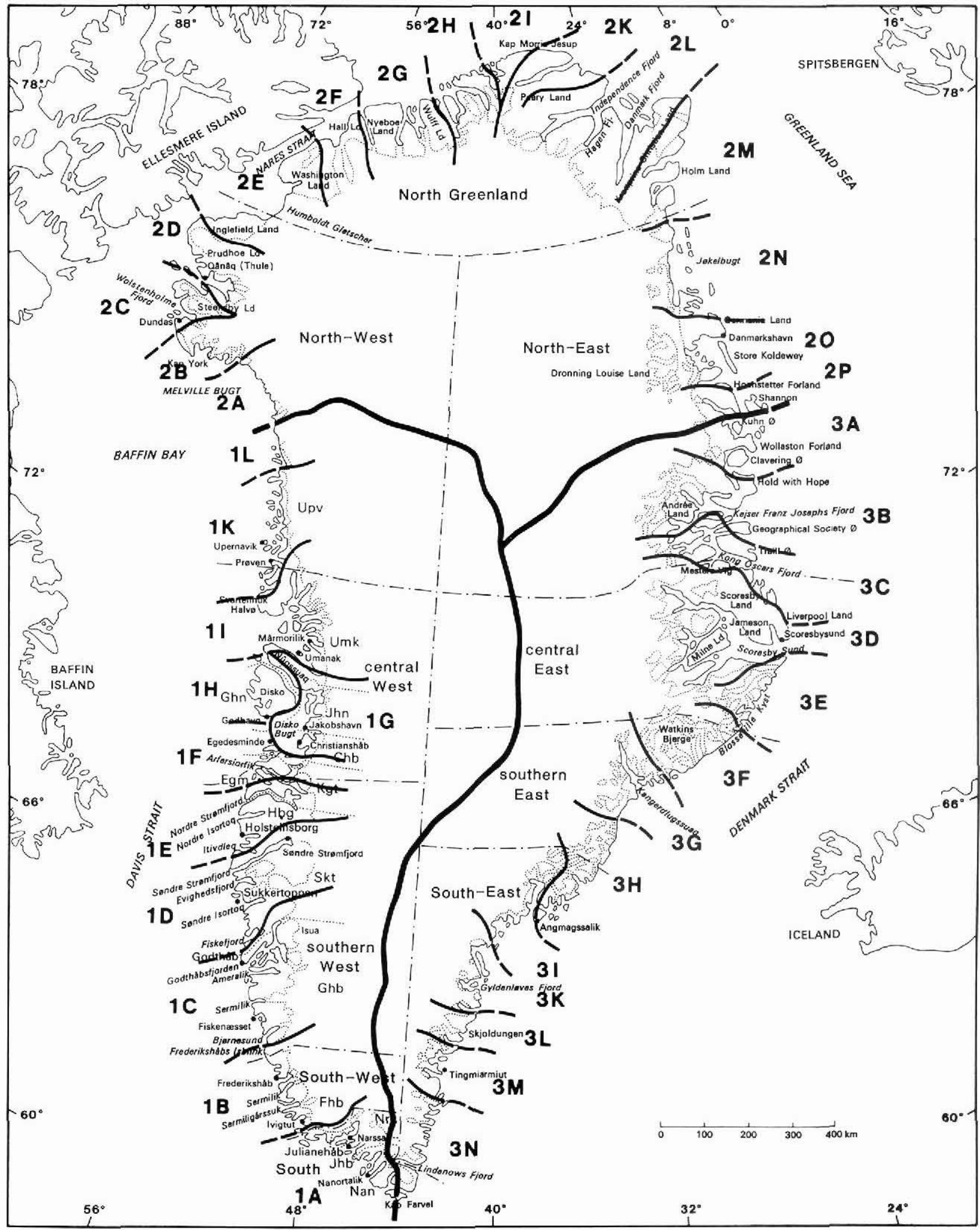

Fig. 2. Glacier hydrological main division of Greenland into districts. 
unit is in this context defined as a single minor glacier or a sector of a larger glacier (i.e. an ice cap or the Inland Ice). They are numbered clockwise in the basin (see Atlas sheet 6900-05).

A few inconsistencies can be encountered in the atlas due to discoveries of minor glaciers in a basin, subsequent to the original numbering, e.g. as better photographs became available. In such cases it has been decided to retain the original numbering of the original glaciers rather than change the entire system for a strict clockwise numeric order. In any case, the location of the glacier is determined by its listed geographical coordinates.

\section{Comments on the West Greenland hydrological basins}

In a few places watersheds pass over flat plateaus or across lowlands of the coastal shore flat, and here determination of the exact position of the watershed (and thus the basin boundary) is often difficult.

In the case of the Egedesminde district $(1 \mathrm{~F})$, this problem produced insurmountable difficulties, and a proper basin division has been omitted for parts of this area (1FD). Since there are no glaciers in the district this has had no consequences for filing glacier information.

\section{Identification}

Glaciers are located and their areas determined on the basis of the aerial photograph collection maintained by Kort- og Matrikelstyrelsen, Denmark (KMS). Most of the photographs in this collection were taken between 1948 and 1968 but in places older photographs have been utilized. The photographs are of variable quality, some showing the terrain partly covered by new snow. The extent of some glacier areas listed is thus questionable since in reality they may be semi-perennial snow patches.

A new series of aerial photographs taken in 1985 totally covers West Greenland. This series provides a benchmark for future glacier inventories, and as expected, several glaciers show considerable deviation in form from that given on the maps, and in some cases have been shown to be semi-perennial snow patches.

By 1986 the glacier atlas presented here had reached a stage where any thorough revision and updating required more resources than were available. Since the most important goal of the inventory, to locate and code the glacier units, had been achieved, the status of glaciers are maintained on the basis of the pre-1985 in- formation, and the limitations of these data on the atlas must be kept in mind.

\section{Local glaciers and the Inland Ice margin}

The presence of one superglacier (the Inland Ice) and about 5000 local glaciers in the West Greenland coastland implies certain limitations on homogenization of the information on the glaciers. It is necessary therefore to stress the distinction between local glaciers and sectors of the Inland Ice.

\section{Local glaciers}

The expression 'local glaciers' covers all glacier types with the exception of the Inland Ice. A common feature for all the different glacier types is their small size in comparison to the Inland Ice, and hence generally the possibilities of better delineation on the basis of surface maps, on determination of mass balance and on determination of the total change in area and volume of the glacier body.

Ice caps and ice fields are usually divided into sectors by flow lines, radiating out from a centre or a divide, and the sectors are determined from a right angle to the height contours of the map.

The total area of any individual glacier was originally believed to be a minimum requirement of data information which could be based on the current topographic maps at 1:250 000. However, re-measurement of some of the glaciers on the basis of better, more detailed maps may change the measured area by up to several square kilometres.

Maximum and minimum altitude have usually been taken from the best current topographic maps.

For determination of mass balance a conventional procedure is used with measurements of both accumulation and ablation and giving the resulting annual net gain or loss covering the entire body. It can be empirically related to climatic series in order to simulate longer periods of run-off.

\section{The Inland Ice and its total size compared to larger local glaciers}

According to most definitions, the main feature of the Inland Ice is a form which, apart from a narrow marginal zone, is independent of the subglacial topography. To the above definition can be included its mere size, i.e. a total area (according to Holtzscherer \& Bauer, 1954) estimated at $1726400 \mathrm{~km}^{2}$. This figure can be compared 
10

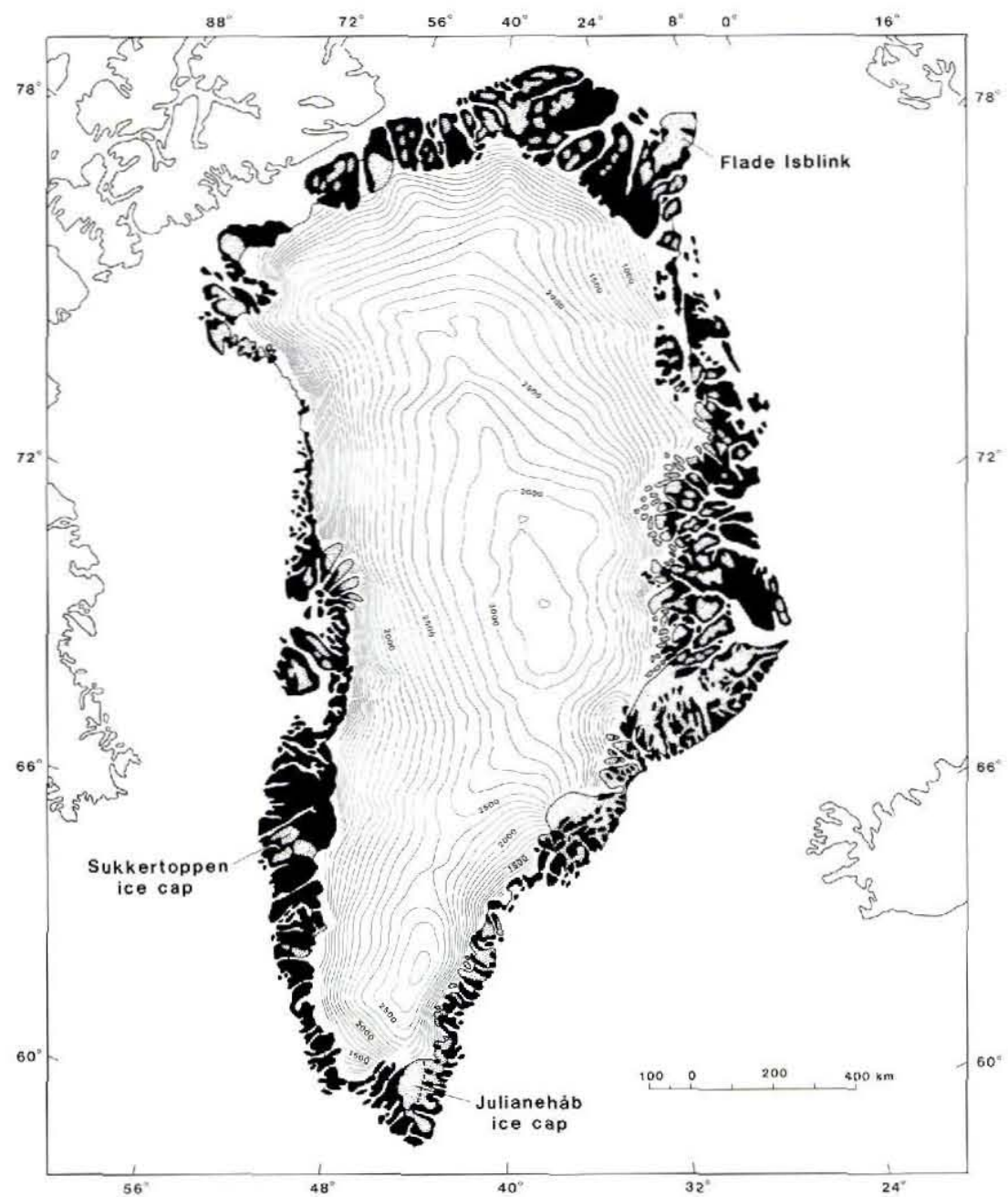

Fig. 3. Major local glaciers and ice domes of Greenland (dotted areas). Determination based on Landsat images. Contours compiled from various sources, especially from the Electromagnetics Institute, Technical University of Denmark.

with those for the largest local glaciers on the Greenland coastland, e.g.:

Flade Isblink (North-East Greenland) c. 9000 $\mathrm{km}^{2}$

Julianchăb ice cap (South Greenland) c. 6500 $\mathrm{km}^{2}$

Sukkertoppen ice cap (West Greenland) c. 2000 $\mathrm{km}^{2}$.

\section{Inland Ice delineation}

The form and extent of the Inland Ice is indicated on the map of Fig. 3. There may be doubt whether some marginal ice domes, especially in North and East Greenland, should be included in the total area of the Inland Ice. These areas are shown dotted in Fig. 3. Those connected with the ice sheet have been included in the area determination of the Inland Ice by Holtzscherer \& Bauer (1954). Even disregarding these areas of the Inland Ice, the ice sheet is still unique in its large size.

\section{Surface of the Inland Ice}

The standard 1:250 000 GI (KMS) map sheets cover only the coastland of Greenland. For the Inland Ice the best general topographic maps are those produced by the International Civil Aviation Organization (ICAO). However, the present ICAO maps at 1:1 million contain errors in elevation of over $100 \mathrm{~m}$ and in the trend of contour lines from one map sheet to another. Areal determinations based on these sheets must therefore be considered unreliable.

Radar altimetry has improved determination of altitudes of the interior parts of the ice sheet (Bindschadler et al., 1989; Fig. 17). Unfortunately the method is dependent on the slope of the surface which means that the altitude of the marginal areas below $1500-1800 \mathrm{~m}$ 
a.s.l. is still poorly determined, as the marginal slopes are uncertain.

\section{Sector delineation and subsurface topography of the Inland Ice}

The major water resources for future hydropower in Greenland are connected with special 'quiet' sectors (cf. section on Quiet sectors) of the Inland Ice and a solution to the problem of dividing the ice sheet into sectors is therefore important. An approach for improving correct sector division in the ablation area is made by mapping surface flow features by IDIMS (Interactive Digital Image Manipulation System) enhancement of Landsat images (Thomsen, 1983) to determine superficial flow lines of the ice.

Further improvement of the sector division is dependent on knowledge of surface and subsurface topography of the ice sheet marginal areas, which is usually lacking today. In this context progress has been made, since Landsat images of the ice sheet margin seen under low sun angle reveals patterns of the subglacial topography even where there is an ice cover of more than $1000 \mathrm{~m}$ (Thomsen, 1983).

Detailed maps of the subsurface below the ice margin have only been made for minor areas around Isukasia near Godthåbsfjord (glacier code 1CH02013; Colbeck, 1974), areas around Jakobshavn Isbræ at Alangordliup and Sarqardliup sermia (glacier codes 1GB06001 and 1GB07001; Thorning \& Hansen, 1987) and at Pâkitsoq (glacier codes 1GC04003 to 1GE04001; Thomsen et al., 1988).

\section{Hydrological and dynamic sectors}

Even a sector division based on ice flow can be misleading when applied to glacier hydrological problems. This is due to the fact that englacial and subglacial water drainage of the ice margin does not necessarily follow the surface ice flow patterns.

All investigated sectors of the Inland Ice margin in West Greenland exhibit a difference between the topographic upland (the 'topographic ablation area') and the 'hydrological ablation area', as originally pointed out by Clement (1983).

The topographic ablation area for a given basin (watershed) in the ice-free coastland is determined by flow lines (of ice) constructed from the ice surface contour lines and should only be taken as a first approximation for defining the hydrological ablation area. This approximation is, for instance, applied in technical feasibility reports for hydropower as the Inland Ice contribution to a given hydrological basin (Weidick, 1990a). A determination of the hydrological ablation area requires detailed investigation of mass balance, surface and subsurface topography and of the hydraulic conditions in the ice margin. So far investigations have only been undertaken on a large scale at Pâkitsup ilordlia (Pâkitsoq) near Jakobshavn (sectors 1GE04001-02 and 1GE05002-04), and a review of this 'Pâkitsoq case story' is given by Thomsen et al. (1988). At this locality subsequent control of the calculated hydraulic conditions was made by drilling (Thomsen \& Olesen, 1990).

In Table 1 , estimates of the hydrological ablation areas for some of the sites shown in Fig. 1 are given. They are essentially based on comparison between measured runoff and calculated ablation. The difference in areas is essentially related to the fact that the Inland Ice margin does not, as in the case of most local glaciers, rest in a topographic terrain. It hides a terrain where the drainage pattern of the subglacial valley systems cannot be expected to coincide with the direction of surface drainage of the ice margin.

From the above it follows that mass balance measurements can mainly be related to single lines of the ablation areas in certain sectors. They are not a direct expression for a net gain or loss of this sector, and they can only, for instance, through a degree-day concept, be linked to meteorological records in order to produce discharge series.

\section{Glacier change and identification code}

Codes for sectors of the Inland Ice and for larger ice caps or ice fields are connected to the snout or outlet and not, as generally for local glaciers, to the central parts of the glacier (cf. section on Geographical coordinates).

The above is a general rule. Local glaciers can split up or merge, as also can outlets from the margin of the Inland Ice or an ice cap, and thus change the drainage. For these relatively few cases it has been decided to maintain the original identification codes, leading to minor inconsequences, rather than to re-arrange the whole system each time such events occur. 
Table 1. Ablation investigations on the Inland Ice margin

\begin{tabular}{lccccc}
\hline Name & $\begin{array}{c}\text { Topographic } \\
\text { ablation } \\
\text { area km }\end{array}$ & $\begin{array}{c}\text { Hydrological } \\
\text { ablation } \\
\text { area km }\end{array}$ & $\begin{array}{c}\text { ELA } \\
\mathrm{m} \\
\text { a.s.l. }\end{array}$ & $\begin{array}{c}\text { Slope } \\
\text { at } \\
\text { ELA }\end{array}$ & $\begin{array}{c}\text { Identification } \\
\text { codes }\end{array}$ \\
\hline Nordbogletscher & 89 & $57-35$ & $1400 \pm 100$ & $4.5 \%$ & 1 AG05001 \\
Isortuarssûp sermia & 347 & $310-260$ & $1380 \pm 100 ?$ & $1.1 \%$ & 1 CE05025 \\
Tilníngnilik & 561 & $391-260$ & $1175 \pm 100$ & $1.0 \%$ & 1 GB07002-04 \\
Pâkitsup ilordlia & $380-157$ & $150-118$ & $1175 \pm 100$ & $0.9 \%$ & $\left\{\begin{array}{l}1 \text { GE04001-02 } \\
1 G E 07001-02\end{array}\right.$ \\
\hline
\end{tabular}

\author{
ELA = Equilibrium line altitude \\ Reference Nordbogletscher: Clement $(1983,1984)$ \\ - Isortuarssûp sermia: Braithwaite (1989) \\ - Tiníngnilik: Braithwaite \& Thomsen (1984) \\ - Pâkitsup ilordlia (Pâkitsoq): Braithwaite \& Thomsen (1984), Thomsen (1984).
}

\title{
Information registered on the West Greenland glaciers
}

The description of the information registered given below is taken, with a few additions and minor alterations, from that of Knudsen \& Weidick (1981) and Weidick \& Thomsen (1983).

\section{Glacier identification (code)}

The glacier code is based on the division of the Greenland coastland into hydrological basins as described in a previous chapter.

\section{Glacier name}

Only rarely do official names for glaciers exist, and where they are found (most described glaciers) spelling of the names or even the names may vary. Due to the descriptive character of Greenland place names, these are often repeated from area to area. The place names are not tabulated with the main data but are listed after the text; here the old Greenlandic spelling is retained since this version still appears on the majority of published topographic map sheets.

\section{Geographical coordinates and orientation of glaciers}

The location of each glacier is given in geographical coordinates. The location is defined as a point situated on the central line of movement in the ablation area.
If no ice is visible on the glacier or the snow field, the coordinate point is placed in the central part of the glacier area. For the sectors or outlets of the Inland Ice the coordinate point is placed in the ablation zone close to the margin so that redefinition of the total sector area will not influence the coordinates given (cf. section on Glacier change and identification code).

Orientation refers to the ablation area in the down glacier direction and is given according to the cardinal points (N, NE, E, SE, S, SW, W, NW).

\section{Highest and lowest glacier elevation}

Elevations are given for local glaciers, but for many areas accurate values cannot be given; altitudes extracted from topographic maps should in most cases be considered as little better than estimates.

For the Inland Ice values for the highest glacier elevation are omitted, and $1800 \mathrm{~m}$ a.s.l. is assumed as an upper limit of the measured glacier area.

\section{Surface areas}

The surface area given for local glaciers comprises the total area of the glacier within the marked delineation. Areas have been measured by digitizing using a high resolution digitizer. Each area was measured three times and averaged. Areas quoted for the Inland Ice sectors only cover the marginal areas below $1800 \mathrm{~m}$ a.s.l. 


\section{Morphology code}

Each glacier is classified with a 6-digit code according to the classification system as given in Table 2. A description of the classification system in the version applied here is given in Table 2 .

It should be noted that digit 6 (activity of tongue or ice margin) relates to the width of neoglacial recession determined in case of retreat, and lack of trimline zone, form of glacier lobe and documentation in cases of advance; it is therefore a very generalized and subjective estimate of the net change of the glacier over the last $100-200$ years.

\section{Remarks on the map and morphology code}

The presence of prominent moraines (neoglacial extent of the glacier lobe), proglacial and glacier dammed lakes and nunataks are given on the map sheets, but is simplified to general outlines.

Rock glaciers occur frequently in South Greenland and north of latitude $69^{\circ} \mathrm{N}$, but are not included in the inventory since they are not considered as a part of the permanent snow and ice cover.

\section{Work description}

An enlargement of all relevant 1:250 000 Geodetic Institute map sheets were made to a scale of 1:100 000, and on this base the present map division was determined (Fig. 4). All glaciers were localized and coded according to the basins, and their boundaries drawn from the best available aerial photographs.

Table 2. Explanation of the morphology code of the glaciers

\begin{tabular}{|c|c|c|c|c|c|c|}
\hline & & & Digit 3 & Digit 4 & Digit 5 & Digit 6 \\
\hline & $\begin{array}{c}\text { Primary } \\
\text { Classification }\end{array}$ & Form & $\begin{array}{c}\text { Frontal } \\
\text { Characteristics }\end{array}$ & $\begin{array}{l}\text { Longitudinal } \\
\text { profile }\end{array}$ & $\begin{array}{l}\text { Major source } \\
\text { of nourishment }\end{array}$ & $\begin{array}{l}\text { Activity } \\
\text { of tongue }\end{array}$ \\
\hline 0 & $\begin{array}{l}\text { uncertain } \\
\text { or misc. }\end{array}$ & $\begin{array}{l}\text { uncertain } \\
\text { or misc. }\end{array}$ & $\begin{array}{l}\text { normal } \\
\text { or misc. }\end{array}$ & $\begin{array}{l}\text { uncertain } \\
\text { or misc. }\end{array}$ & $\begin{array}{l}\text { uncertain } \\
\text { or misc. }\end{array}$ & uncertain \\
\hline 1 & $\begin{array}{l}\text { continental } \\
\text { ice sheet }\end{array}$ & $\begin{array}{l}\text { compound } \\
\text { basin }\end{array}$ & Piedmont & $\begin{array}{l}\text { even } \\
\text { regular }\end{array}$ & $\begin{array}{l}\text { snow and/or } \\
\text { drift snow }\end{array}$ & $\begin{array}{c}\text { marked } \\
>1.5 \mathrm{~km} \text { retreat }\end{array}$ \\
\hline 2 & ice field & $\begin{array}{l}\text { compound } \\
\text { basin }\end{array}$ & $\begin{array}{l}\text { expanded } \\
\text { foot }\end{array}$ & hanging & $\begin{array}{l}\text { avalanche ice } \\
\text { and/or snow }\end{array}$ & $\begin{array}{c}\text { slight } \\
<1.5 \mathrm{~km} \text { retreat }\end{array}$ \\
\hline 3 & ice cap & simple basin & lobed & cascading & superimposed ice & stationary \\
\hline 4 & & cirque & $\begin{array}{l}\text { calving in sea } \\
\text { considerable calf ice }\end{array}$ & ice-fall & & $\begin{array}{c}\text { slight } \\
\text { advance }\end{array}$ \\
\hline 5 & valley glacier & niche & $\begin{array}{l}\text { calving in sea } \\
\text { grounded }\end{array}$ & interrupted & & $\begin{array}{l}\text { marked } \\
\text { advance }\end{array}$ \\
\hline 6 & mountain glacier & outlet & calving in lake & & & possible surge \\
\hline 7 & $\begin{array}{l}\text { glacieret and } \\
\text { snow field }\end{array}$ & ice apron & confluent & & & known surge \\
\hline 8 & ice shelf & group & & & & oscillating \\
\hline 9 & rock glacier & remnant & & & & \\
\hline
\end{tabular}




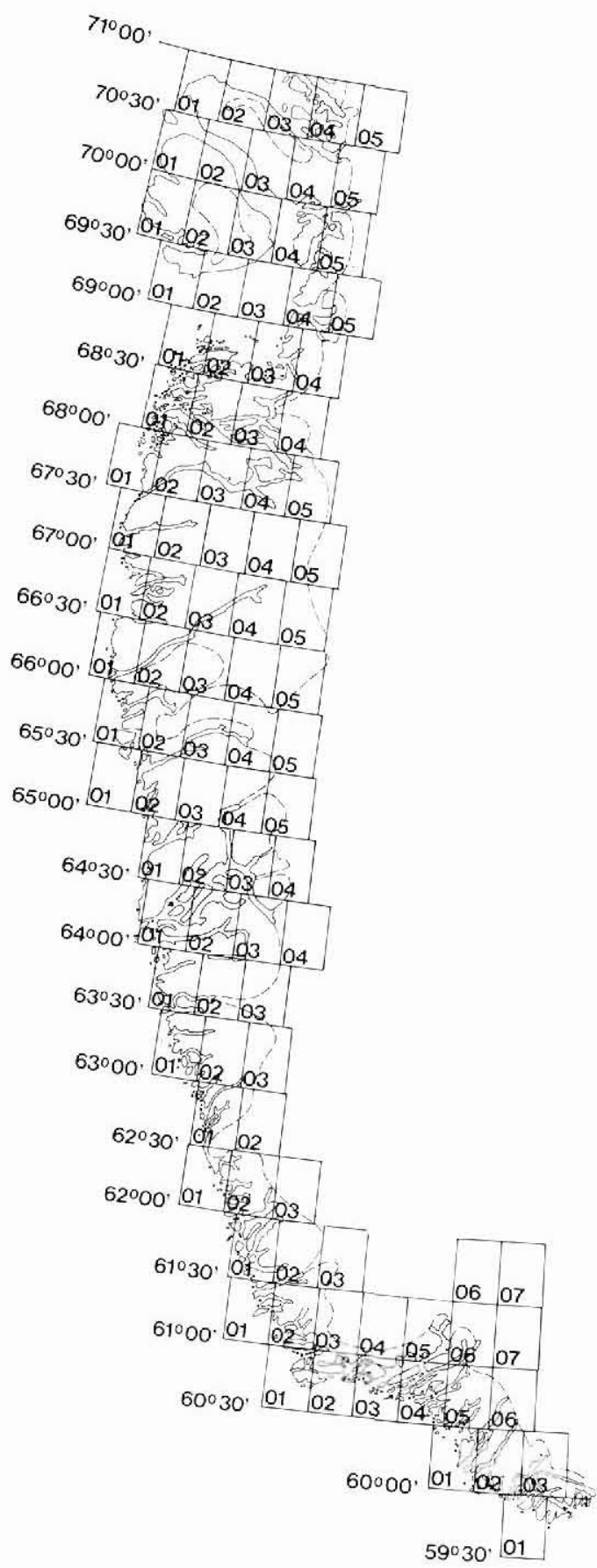

Fig. 4. Map division of the glacier atlas.

Trimline zones, which are the basis for digit 6 of the morphology code, were mapped on the 1:100 000 work sheets. 'Marked retreat' indicates a recession of more than $1.5 \mathrm{~km}$ from neoglacial moraines, 'slight retreat' less than this distance. 'Slight advance' here implies the occurrence of a narrow trimline (often restricted to small parts of the surroundings of the lobe) whereas 'marked advance' involves formation of push moraines.
In general the numerals 3-5 in the morphology code's digit 6 (Table 2) can only be documented to a small degree. In the few cases where it is possible, however, this subjective description seems verified. The classification is especially useful for the ice sheet margin, since it clearly distinguishes groups of markedly retreating sectors from those that are advancing (Weidick, 1991a, b).

Other, essential elements of the work were the determination of: (a) geographical coordinates; (b) areas; (c) heights Comments in respect of these determinations are given below.

\section{Geographical coordinates}

The coordinates were measured on photocopies of the original glacier work sheets (enlargements of 1:250 000 GI maps to $1: 100000$ ).

Location are given in whole minutes of longitude and latitude. However the average area between the coordinate intersection (minute $\times$ minute) is only $0.46 \mathrm{~km}^{2}$ and $57 \%$ of the glacier units encountered are below this value; the coordinates given for a specific glacier unit can thus be situated outside the drawn boundaries of the glacier unit.

\section{Area determination}

All areas were determined on the Tetronix digitizer of the Geological Survey of Greenland. In the early works with Tetronix, the area determinations were separate from storage of the results; later these functions were coupled.

\section{Height determination}

For determination of minimum and maximum altitudes, a linear interpolation between the $100 \mathrm{~m}$ contour lines has been applied in the cases where the limits occurred between contours. The determination is given to the nearest $50 \mathrm{~m}$, but the varying quality of maps and the original scale $(1: 250000)$ of the map sheets used places limits on the accuracy of this determination.

For valley glaciers an uncertainty in the maximum altitude arises essentially due to the narrow spacing between the contour lines. On ice caps the maximum altitudes may be uncertain due to the difficulties of determining exact contour trends in flat, extensive firn areas. In the tabulation, heights are given in tens of metres (in the example given below: $99=990 \mathrm{~m}, 74=$ $740 \mathrm{~m})$. 


\begin{tabular}{llllllllll}
\hline & & & & & & & MO \\
CODE & & LAT & LONG & ORI & HIGH & LOW & AREA & CODE & MAP \\
IAA & 01001 & $60^{\circ} 03^{\prime} \mathrm{N}$ & $44^{\circ} 45^{\prime} \mathrm{W}$ & $\mathrm{NW}$ & 99 & 74 & 0012.00 & 740110 & $6000-02$
\end{tabular}

\section{Storage of information}

Data were originally stored as lists, but in 1984 a dicision was made to place them in the database DATATRIEVE. Registration and recording has been time consuming due to the size of the enterprise and the limited resources, concurrent with changes of hardware and software in GGU. The structure of the storage in DATATRIEVE can be considered as a matrix with 9 vertical columns and 5606 lines:

Each line represents a glacier unit or a basin without glaciers (number of glacier units $=0$ ). In total there are 5297 glacier units. Each column contains one type of information for all glacier units.

\section{Inventory atlas sheets}

In the atlas the 1:100 000 work sheets have been reduced to a publication scale of 1:300 000 after the final drawing; an index map of all atlas sheets is shown in Fig. 4. The sheets are grouped in strips each covering half a degree of latitude, and each strip is divided into sheets. Each sheet is denoted by the southernmost latitude of the sheet and a number in the strip (e.g. 593001).

All atlas sheets adjoin but glacier numbers and basin numbers covering two sheets or more are repeated on the individual sheets.

\section{Morphology and outline of Holocene glacier history}

\section{Coastal terrain}

The coastal parts of West Greenland are underlain mainly by Archaean rock formations. Only to the north, on the island of Disko and the outer parts of the peninsula of Nûgssuaq, are these formations covered by younger deposits of Mesozoic and Tertiary age, dominantly basalts.

Apart from a partially developed strandflat with hills up to $300 \mathrm{~m}$, most of the broad coastal land areas consist of uplands and highlands with hilly plateau surfaces or ridges, and with heights from 500 to $1500 \mathrm{~m}$ a.s.l. Higher elevations are encountered in two main regions, namely South Greenland $\left(60^{\circ}-61^{\circ} \mathrm{N}\right)$ and Sukkertoppen (Manîtsoq; $65^{\circ} \mathrm{N}$ ), where altitudes above $2000 \mathrm{~m}$ and alpine topography is seen. Minor areas of appreciable height are also found at Sermilik in Frederikshåb (Pâmiut; $63^{\circ} \mathrm{N}$ ) and around Sermilik in the area of Godthåb (Nûk; $64^{\circ} \mathrm{N}$ ).

On Disko and western Nûgssuaq $\left(69^{\circ}-70^{\circ} \mathrm{N}\right)$ the terrain also reaches high altitudes, here mainly as plateaus; the plateau surfaces increase in height from above 700 $\mathrm{m}$ on the west coast to above $2000 \mathrm{~m}$ in the interior. The plateau surfaces are well preserved on Disko, whereas on Nûgssuaq they are generally dissected into minor units or even alpine peaks.

The coastal land areas are penetrated by fjords and major valleys, whose main trends run east-west or north-east/south-west. This direction trend means that the location of the glaciation limit is strongly influenced by the concentration of occurrences of minor glaciers on shady (north facing) slopes of the valleys and fjords.

\section{Subsurface of the south-western slope of the Inland Ice}

In general the subsurface of the marginal areas of the Inland Ice in West Greenland is a continuation of the terrain of the ice-free coastland, but the general altitude decreases eastwards below the ice sheet margin. A major depression under Jakobshavn Isfjord seems to ramify eastward under the ice sheet from its main centre at the front of Jakobshavn Isbræ (1GC06002).

Jacobshavn Isbræ is at present a major drainage channel for a large part of the centre of the Inland Ice, and the position of the outlet may have been inherited from a major Tertiary river drainage system of central Greenland.

As mentioned earlier, detailed mapping of the absolute altitudinal conditions of the ice margin subsurface is scarce, whereas the relative configuration of the subsurface can be detected from Landsat images. 


\section{Outline of the Holocene glaciation}

The long-term glacier history of the coastland is closely related to variations in the extent of the Inland Ice.

Little is known about the pre-Wisconsinan extent of the main ice sheet, but there is strong evidence that the margin of the ice sheet during the last ice age (Wisconsin) was situated close to the present outer coast (e.g. Kelly, 1985; Weidick, 1985).

Between 10000 and 6000 years ago a strong recession of the Inland Ice margin took place (Fig. 5) so that by 6000 B.P. the margin was everywhere at or beyond the present, and this recession culminated about 4000 years B.P. with the Inland Ice position up to $10-20 \mathrm{~km}$ behind the present (Weidick et al., 1990).

The colder climate of the past $2000-3000$ years has caused readvances leading to the present position, but with many local modifications in intensity and time. Best known are the changes after the maximum readvance at the end of the Little Ice Age (A.D. 1500-1850) whose outlines will be referred to in the following chapters.

Post-glacial rise of the snow line occurred prior to the retreat of the Inland Ice from the coastland at most localities. Evidence of an older extent of local glaciers greater than that of the Little Ice Age are therefore scarce. The main evidence for this greater extent is reported from South Greenland around the corrie glaciers of $1 \mathrm{AC} 16$ and $1 \mathrm{AC} 17$ near Nanortalik (Weidick, $1963)$ and glaciers of the same type (1EF05003-05) at the entrance to the Nordre Strømfjord area (Kelly, 1975).

\section{Present climate and glacial conditions}

The coastland of West Greenland is fairly well covered by meteorological stations placed in the towns. Some of these have climatic records going back more than 100 years (although with some interruptions), though the observation periods of individual stations varies greatly (e.g. Frydendahl, 1989).

\section{Temperatures}

It is necessary to discern between the climatic measurements of the coastland and the climatic records of the ice sheet (the Inland Ice).

\section{Coastal stations}

A review of January, July and mean annual temperatures of some West Greenland stations is shown in Table 3. In addition to the decreasing trend from south to north, the higher continentality of the inland stations of Narssarssuaq and Søndre Strømfjord are reflected by their higher annual temperature amplitudes. However, the summer temperatures especially show little latitudinal variation over the coastal area, as is shown by the compilation of temperature distribution over Greenland as a whole by Ohmura (1987), which is based on the period 1951-60 as reference decade. The distribution of July temperatures in Fig. 6 is based on Ohmura's work and illustrates the uniformity of summer temperatures over large stretches along the Inland Ice margin.
Long term observation series all indicate a trend of increasing temperatures throughout the last century, which culminated in the 1940s-1950s, and was followed by a decrease during subsequent decades. An example from Nûk / Godthåb is shown in Fig. 20. With regard to seasonal changes during the period, the greatest change occurs in the winter, least in the summer months (Lysgaard, 1949; Frydendahl, 1989). As a comment to the curve of change in mean annual temperature in Nûk / Godthåb shown in Fig. 20, it can be added that while the decadal annual mean temperature in the period $1881 / 90$ to $1971 / 80$ rose by $1.3^{\circ} \mathrm{C}$ (from $-2.6^{\circ}$ to $-1.3^{\circ}$ ), the July temperature for the same period only rose $0.2^{\circ} \mathrm{C}$ (from $6.5^{\circ}$ to $6.7^{\circ}$ ), while that of January rose by as much as $3.0^{\circ} \mathrm{C}$ (from $-10.3^{\circ}$ to $-7.3^{\circ}$ ). For Jakobshavn about $500 \mathrm{~km}$ further to the north the temperature change curve followed the same trend: the decadal annual mean here rose $2.0^{\circ} \mathrm{C}$ (from $-6.3^{\circ}$ to $-4.3^{\circ}$ ), whereas the increase for July only rose $0.9^{\circ} \mathrm{C}$ (from $7.5^{\circ}$ to $8.4^{\circ}$ ) and that for January $5.5^{\circ} \mathrm{C}$ (from -18.3 to $\left.-12.8^{\circ}\right)$.

\section{The Inland Ice}

The compilation of Ohmura (1987) shows that for the south-western slope of the ice sheet the August mean temperature varies from $0^{\circ} \mathrm{C}$ at about $1000-1200 \mathrm{~m}$ a.s.l. to $-10^{\circ} \mathrm{C}$ in the highest parts (the South Dome) of the ice sheet. This implies that all of the southern part of the 


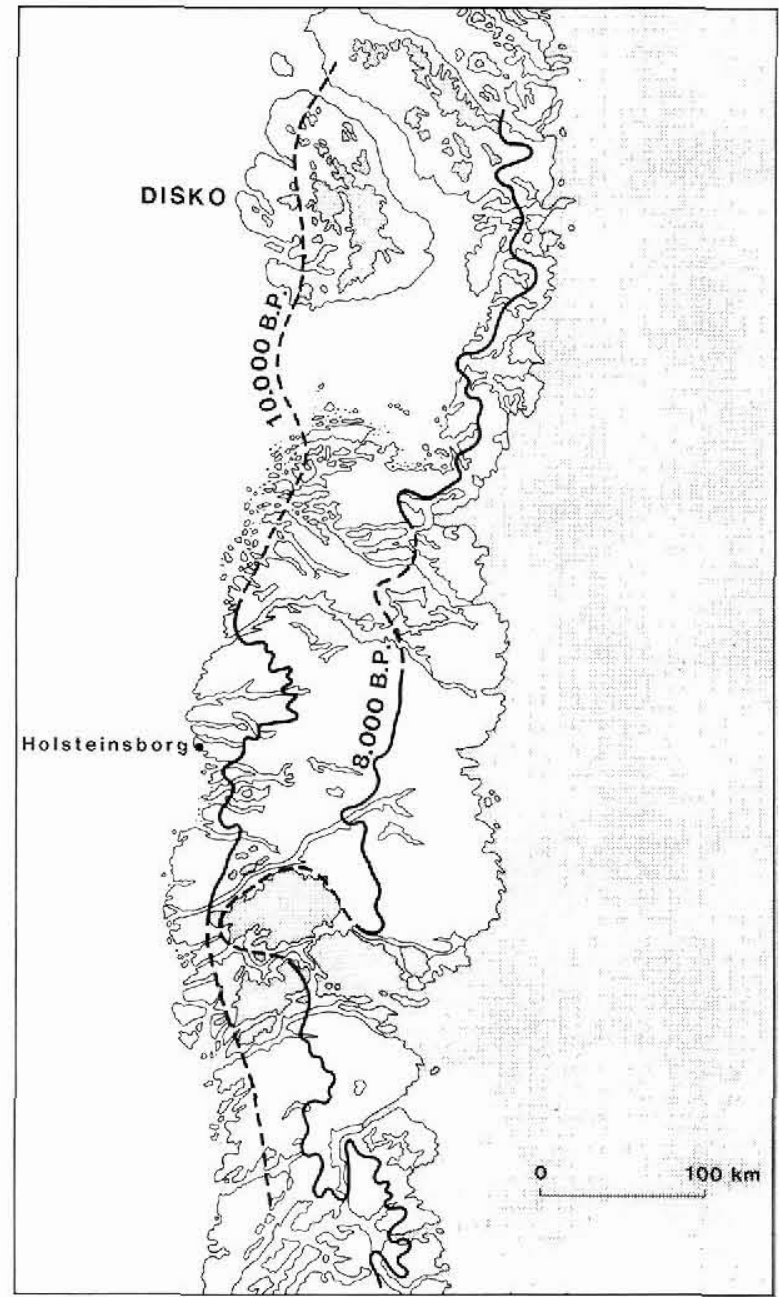

Fig. 5. Approximate extension of the Inland Ice in West Greenland between $64^{\circ} \mathrm{N}$ and $70^{\circ} \mathrm{N}$ at about 10000 and 8000 B.P. according to Weidick (1985).
Inland Ice in the present day situation must be situated inside or below the percolation facies of Benson \& Motyka (1979).

Trends of temperature change for the last century revealed by the oxygen isotope variations recorded in ice cores, indicate the same development as registered from the coastal stations; i.e. a gradual increase of temperature with a culmination in the 1940s or 1950) (e.g. Dansgaard et al., 1973; cf. Fig. 20).

\section{Precipitation and accumulation Precipitation over the coastland}

A geographical plot of values of precipitation on the basis of the meteorological records of the coastal stations is shown in Fig. 6. These stations are all situated close to sea level, and variations in precipitation due to exposure of the individual basins and other topographic factors are little known. Generally precipitation increases with altitude. Thus, for the Buksefjord basin near Godthăb / Nûk, Thomsen \& Jørgensen (1984) give a mean precipitation increase of $7 \%$ per $100 \mathrm{~m}$.

Although the precipitation records of the meteorological stations do not cover the same period, the plot furnishes the trends of decreasing precipitation towards inland areas and towards the north. The values decrease from above $100 \mathrm{~cm}$ at the southern and south-western outer coasts to below $13-20 \mathrm{~cm}$ in the inland areas around Søndre Strømfjord.

Extremely low precipitation in some inland areas compared to coastal areas is due to the rain shadow effect of the highlands around Sukkertoppen and in the north, the highlands of Disko island and the Nûgssuaq peninsula.

Table 3. Average temperatures, January, July and annual precipitation at selected stations in West Greenland. Data refer to 1961-70 averages from the Danish Meteorological Institute, Copenhagen

\begin{tabular}{|c|c|c|c|c|c|c|}
\hline \multirow[t]{2}{*}{ Station } & \multicolumn{2}{|c|}{ Location } & \multicolumn{3}{|c|}{ Temperature ${ }^{\circ} \mathrm{C}$} & \multirow{2}{*}{$\begin{array}{l}\text { Precipitation } \mathrm{mm} \mathrm{a}^{-1} \\
\text { Annual average }\end{array}$} \\
\hline & Lat. ${ }^{\circ} \mathrm{N}$ & Long. ${ }^{\circ} \mathrm{W}$ & January & July & average & \\
\hline Julianehåb & 61 & 46 & -3.3 & +7.2 & +1.4 & 860 \\
\hline Narssarssuaq & 61 & 45 & -4.2 & +10.2 & +1.7 & 606 \\
\hline Godthåb & 64 & 52 & -5.8 & +6.6 & -0.8 & 839 \\
\hline Søndre Strømfjord & 67 & 51 & -20.9 & +10.5 & -6.3 & $145^{\circ}$ \\
\hline Holsteinsborg & 67 & 54 & -10.7 & +6.2 & -3.1 & 350 \\
\hline Jakobshavn & 69 & 51 & -11.8 & +8.0 & -3.6 & 244 \\
\hline Upernavik & 73 & 56 & -16.6 & +4.9 & -6.9 & 267 \\
\hline
\end{tabular}

\footnotetext{
* Measurements 1981-90.
} 


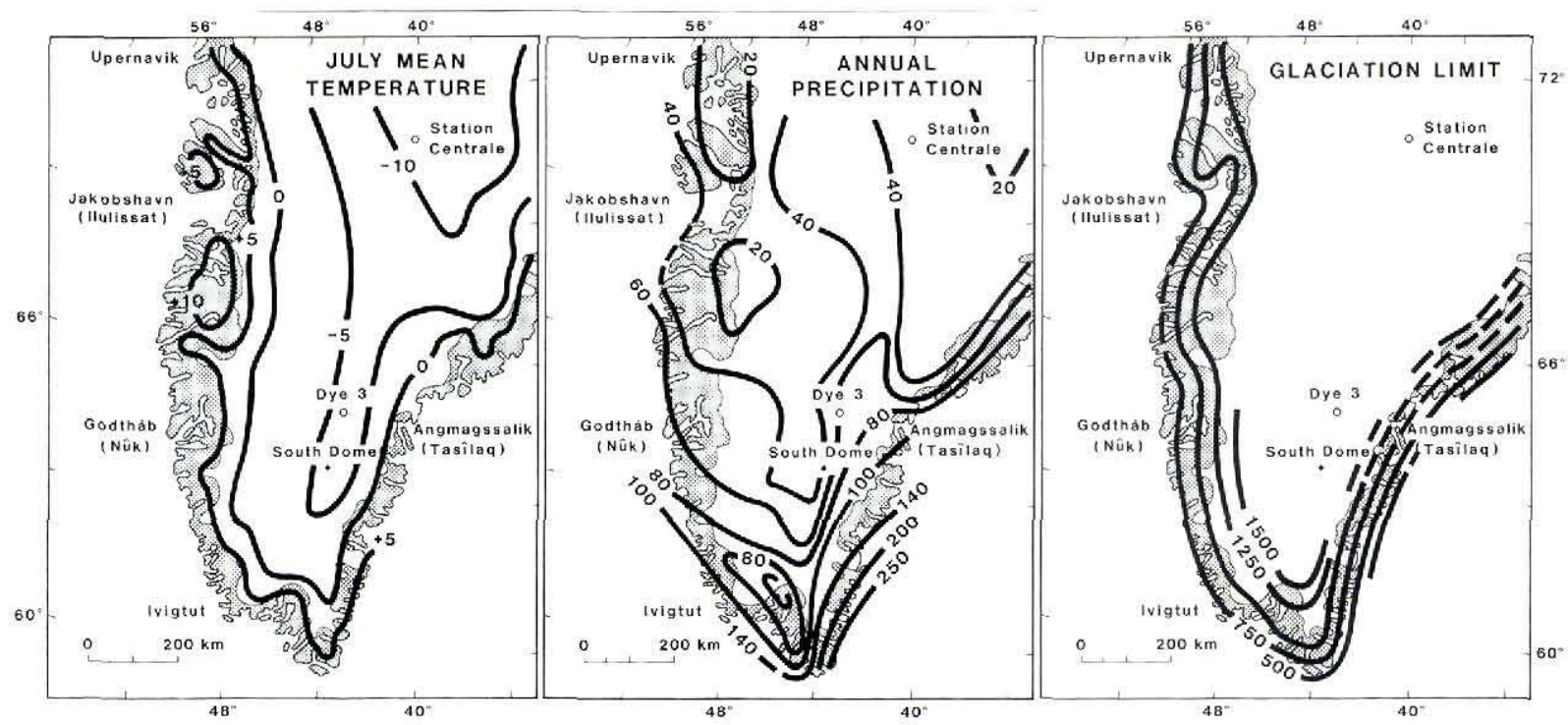

Fig. 6. Left: July mean surface temperature reduced to 1951-1960 according to Ohmura (1987). Middle: Annual precipitation on the coastland and accumulation on the Inland Ice, mainly according to Reeh (1989). Figures indicate centimetres a ${ }^{-1}$. Right: Glaciation limit over South and South-West Greenland. Figures indicate metres a.s.I.

\section{Accumulation over the Inland Ice}

The accumulation pattern over the Inland Ice is illustrated in Fig. 6 (middle). The distribution is based on the compilation of Reeh (1989) which relies mainly on the data of Benson (1962) and Ragle \& Davies (1962). The area south of $c .69^{\circ} \mathrm{N}$ is covered by a few measurements on the western slope. The rain shadow effect of the coastland influences not only inland areas, but also the pattern of accumulation on the lower part of the ice sheet slope.

The accumulation area of the ice sheet is characterized by a marked decrease of accumulation towards the north, and by a decrease with altitude. The studies of Diamond (1956), Gerdel (1961) and Langway (1962) all indicate that an accumulation or precipitation maximum occurred around 1920 at the Inland Ice station of Eismitte (Station Centrale), and at Upernavik town just north of the region covered by the atlas. However, at Ivigtût (a coastal station at $62^{\circ}$ ) this same period is one of minimum precipitation. Up to the present no clear trends in the fluctuation of precipitation can be seen, nor does the available data support any zonal shift of precipitation or enable any accumulation maximum or minimum to be traced.

\section{Glaciation and snow lines}

The general trend of the glaciation limit is shown in Fig. 6 (right). The altitude of the glaciation limit on the Inland Ice margin increases from about $1300 \mathrm{~m}$ in cen- tral West Greenland to $1500-1600 \mathrm{~m}$ a.s.l. in South Greenland. The altitude also increases towards inland areas. Along the major fjord systems, i.e. Godthåbsfjord at around $64^{\circ} \mathrm{N}$, a relative lowering of this limit can be seen. A more detailed positioning of glaciation limit has been made by Humlum (1985), and shows the same trends as in the map presented by Weidick \& Olesen (1980); however, comparison of values between places can differ by up to c. $200 \mathrm{~m}$. For both maps it should be stressed that local topography (cf. coastal terrain) implies considerable local deviation from the general trends given in Fig. 6.

In connection with mass balance measurements, the equilibrium line altitude (ELA) has been determined at Nordbogletscher (1AG05001, 61 $31^{\circ} \mathrm{N}$ ), Qamanârssûp sermia $\left(1 \mathrm{CH} 21002,64^{\circ} 30^{\prime} \mathrm{N}\right)$ and at Pâkitsoq $\left(1 \mathrm{GE} 04002-04\right.$ and $\left.1 \mathrm{GE} 07001-02,69^{\circ} 30^{\prime} \mathrm{N}\right)$ such as shown in Table 1. The ELA shows a general decrease from $c .1400$ to $1170 \mathrm{~m}$ a.s.l. over 8 degrees of latitude.

The figures quoted above for the ELA are an average under present conditions, and are estimated to vary by up to $100 \mathrm{~m}$ in altitude from year to year. Nevertheless, the measured ELA is somewhat below the glaciation limit, a characteristic which also holds true for local glaciers in the coastland where mass balance has been measured (Weidick \& Thomsen, 1986).

On the Inland Ice, oxygen isotope mapping has facilitated a procedure of rapid determination of an 'average equilibrium line' (Reeh \& Thomsen, 1986; Reeh et al., 1987). This line is determined by a linear increase of $\delta^{18} \mathrm{O}$ values from the ice margin towards the interior at 
the ELA (due to the age of the ice), and a decrease of the same values from the ELA to the top of the ice sheet (due to the height/temperature conditions of newly fallen snow). The limit determined in this way at Pâkitsoq $\left(69^{\circ} 45^{\prime} \mathrm{N}\right)$ is at $1100 \mathrm{~m}$ a.s.l., i.e. almost identical to the average ELA measured by mass-balance measurements at this locality (cf. Table 1).

\section{Comments on minor local glaciers (glacierets, mountain glaciers and valley glaciers)}

\section{Glacierets and snow fields}

According to Müller et al. (1977, p. 13), "A glacieret is a small ice mass of indefinite shape in hollows, river beds and on protected slopes developed from snow drifting, avalanching and/or especially heavy accumulation in certain years; usually no marked flow patterns are visible and, therefore, no clear distinction from snowfield is possible. Exists for at least two summers".

From this description it seems that a snow field is covered partly by the same definition as glacieret, but without the same degree of firnification.

\section{Size, extent and volume}

In total 2076 units of glaciers and snow fields have been recorded with a total area of $553 \mathrm{~km}^{2}$. Their individual size ranges from $0.01 \mathrm{~km}^{2}$ to $4.97 \mathrm{~km}^{2}$.

Thicknesses have not been measured on any of these features but it is estimated that in general it rarely exceeds $10 \mathrm{~m}$.

\section{Changes}

Glacierets and snow fields are generally extremely sensitive to minor climatic changes although direct information on this transitional type to seasonal snow cover is very restricted.

Pitman (1973) has described the change of the height of a 'perennial snow line' since A.D. 1680 on the basis of snow patches around Frederikshåb Isblink $\left(62^{\circ} 30^{\prime} \mathrm{N}\right)$. A pronounced reduction of snow patches in the area essentially took place in this century. On this basis Pitman determined the change in the annual mean temperature of the region since 1680 .

The strong reduction of snow patches is also revealed by the presence of pronounced widespread vegetationbare plateaus just below the present snow line on aerial photographs taken since the 1930s. Good examples can be found in the regions of Godthabsfjord $\left(64^{\circ} 30^{\prime} \mathrm{N}\right)$ and Disko-Nûgssuaq $\left(69^{\circ}-70^{\circ} \mathrm{N}\right)$.

In an archaeological description of cairns, erected in connection with reindeer hunting, some cairns close to the present snowline are recorded to have melted out of semiperennial snow cover on the plateaus within the last decade (Rosing, 1988). Dating of moss from the cairns indicates erection between A.D. 1290 and 1400 (J. Rosing, personal communication). The cairns are located in Godthåbsfjord in the areas of 1CH35-1CD38.

Another well-known example is a small snowpatch on the mountain Qaqarssuaq at the town of Narssaq (1AG01), situated in a hollow c. $425 \mathrm{~m}$ a.s.l. The snowpatch is perennial over some summers.

Examples of the opposite trend (i.e. apparent permanence of glacierets and snow fields) are also known from West Greenland, as well as elsewhere, especially in the shadow slopes of steep mountains. An example from West Greenland (Kobbefjord near Godthåb / Nûk) is shown in Fig. 7. Here the snow and ice cover of the north-eastern flanks of the $1188 \mathrm{~m}$ high mountain of Hjortetakken (Kingigtorssuaq) can be seen to have altered little between the years of 1853 and 1984. Although not fully documented, the climatic conditions around 1850 must, in terms of temperature, be comparable with those of the coldest measured spells between 1875 and 1910 (cf. Fig. 20).

Of the examples given above, only that of Hjortetakken is included in the registration since under present climatic conditions the referred location of snow and ice must be considered as perennial. The other examples indicate the great variability of this feature. This also implies that registration of features less than about 0.1 to $0.2 \mathrm{~km}^{2}$ must be occasional.

\section{Mountain glaciers}

Mountain glaciers are defined as glaciers "of any shape, sometimes similar to a valley glacier but much 


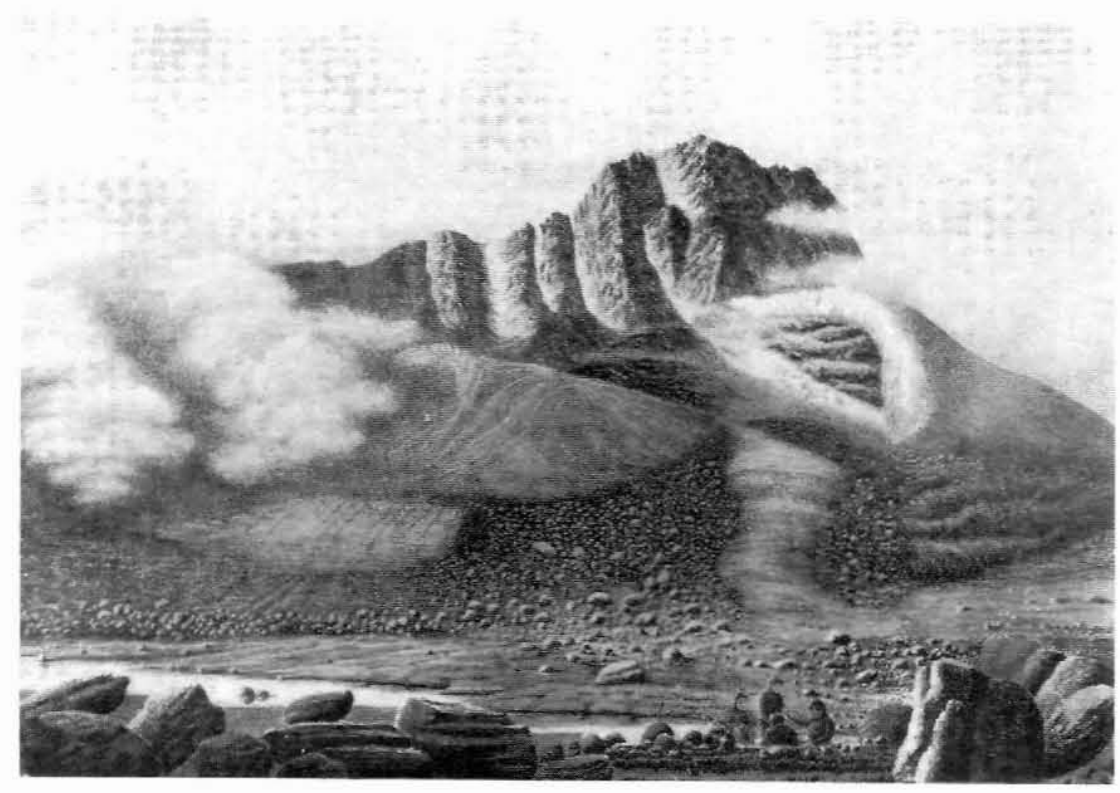

Fig. 7. Glacier 1CG01022 near Godthăb / Nûk. The small cirque glacier must be considered a transitional form to glacieret and snow/ice field. The pictures of 1853 (H. Rink) and 1984 (C. Egede Bøggild) indicate little change of the glacier thickness.

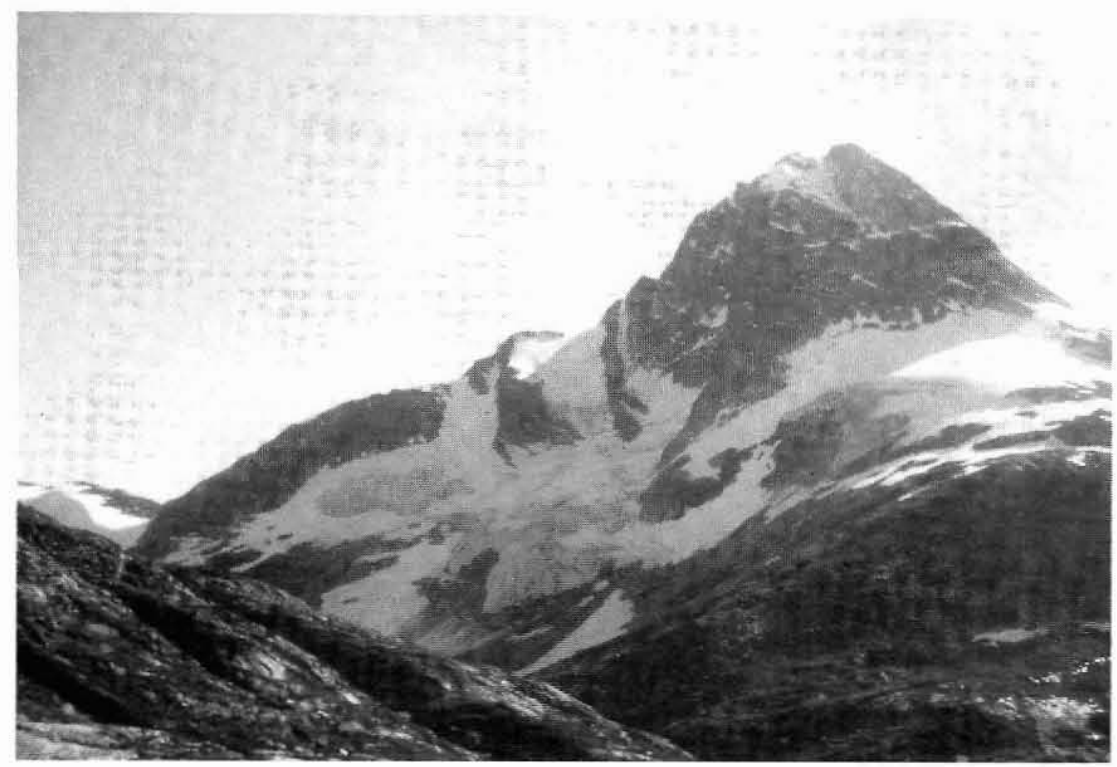

smaller; frequently located in a cirque or niche" (Müller et al., 1977, p. 13).

\section{Size, extent and volume}

Mountain glaciers have a widespread distribution, and they occur most commonly as cirque glaciers.

Within the region covered by the atlas a total number of 1473 mountain glaciers have been registered; they occur throughout West Greenland, especially in the outer parts of the coastal stretch. The have a total area of $1178 \mathrm{~km}^{2}$, and vary in size from $0.02 \mathrm{~km}^{2}$ to $10 \mathrm{~km}^{2}$.

No measurements of thickness seem to have been recorded. Most of the cirque glaciers have an area of about $1 \mathrm{~km}^{2}$. Volume/area plots of Scandinavian glaciers (Østrem et al., 1988) for this glacier size imply an average volume of under $0.1 \mathrm{~km}^{3}$ and an average thickness of about $50 \mathrm{~m}$. However, according to Müller \& Scherler (1980) the relationship of mean thickness $(D$, in metres) to glacier area $\left(A\right.$, in $\left.\mathrm{km}^{2}\right)$ of alpine glaciers is:

$$
D=5.2+15.4 \sqrt{A}
$$

which will give an average thickness of $20 \mathrm{~m}$ for a glacier of this size. 
Fig. 8. Narssaq Bræ, South Greenland (1AG01001) seen from the west. In the foreground on the northern flank of the glacier is a large marginal moraine, indicating the maximum position of the glacier in historical time. As late as around 1900 the glacier still had an extension as indicated by the moraine.

Kort- og Matrikelstyrelsen, Denmark, serics 501 C-N no. 35595 , 15th September 1947. Published with permission A.200/87.

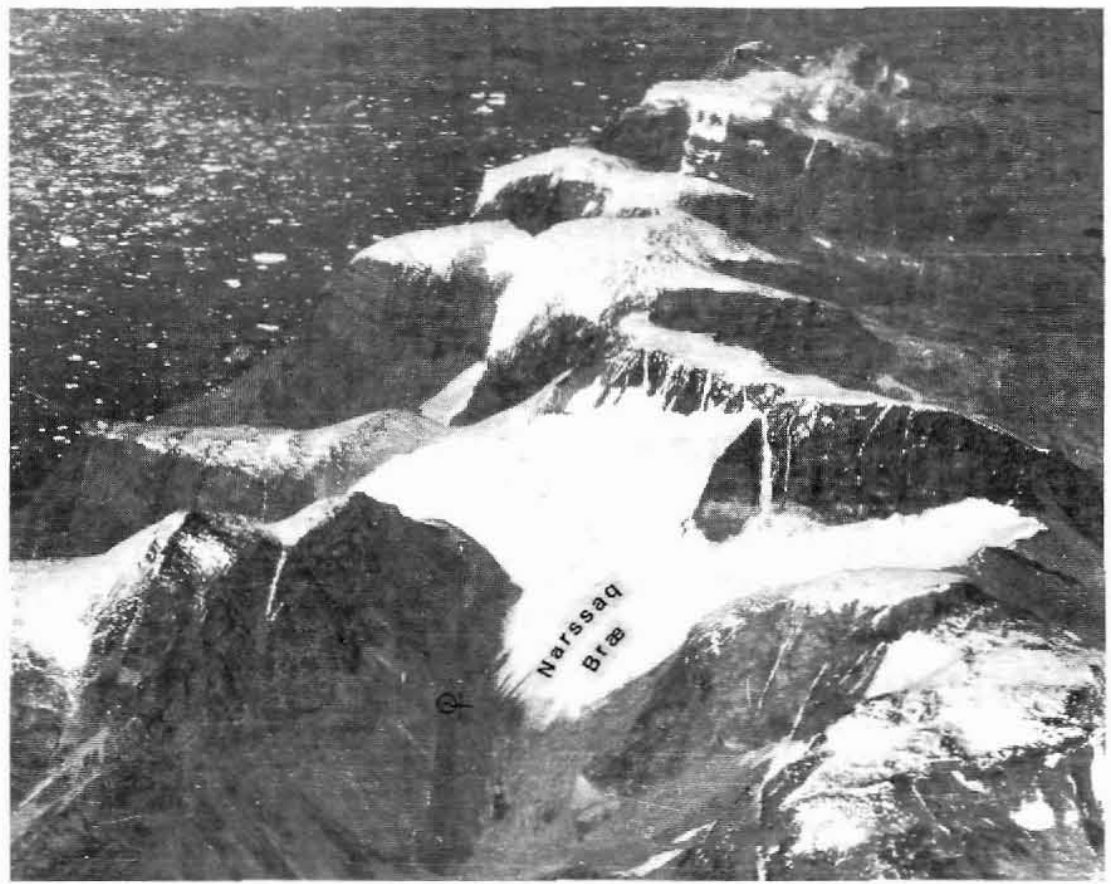

\section{Changes}

Mountain glaciers are often extremely sensitive to climatic change, and a reduction in area of $20-40 \%$ in this century can often be documented. Outstanding examples are shown on the atlas sheets where the recession from a neoglacial maximum is shown with a dotted signature. As an illustration Narssaq Bre is shown in Fig. 8 .

Many listed glacierets (first digits 78_-) appear to be remnants of original cirque glaciers, and their reduction in size is believed to have taken place essentially during this century.

Considering the large number of glaciers of this type, relatively few have been investigated for frontal fluctuations over the past century or more; the examples which have been studied show a rather uniform trend, as indicated in Fig. 9.

A common feature of the glaciers investigated is that most of them exhibit well-defined neoglacial moraines suitable for lichenometric dating, which are easily recognizable for documentation on photographs. With some deviations they all show the latest neoglacial maximum to have taken place about $1850-1900$, with subsequently a recession which, after a short standstill around 1920, accelerated over the period between 1920 and 1960 .

Over the past two decades glaciers of this type in the southern part of Greenland have continued their reces- sion and thinning. In contrast more northern examples of glacier change, e.g. in the Sukkertoppen area, indicate an initial readvance (Fig. 9).

Surging behaviour has been documented for a single cirque glacier in the Disko and Nûgssuaq area (1HD06009), and others in the area show signs of earlier surging activity (1HD06005, 1HE09094). The cause of this concentration of surge activity in basalt terrain is unknown (Weidick, 1984, 1988).

Another feature of basalt terrain is the frequent occurrence of mudflows, especially on the north coast of Disko. They often originate from perennial snow patches (especially ice aprons), remnants of cirque glaciers or even existing cirque glaciers. Their flow seem to be initiated only as isolated events (Weidick, 1968).

\section{Mass-balance measurements}

Mass-balance measurements have been undertaken on the following mountain glaciers:

1AG01001 (Narssaq Bræ) during the periods 1970/71 and 1980/83 (Larsen, 1973; Clement, 1982, 1984).

1AG05008/09 (Valhaltindegletscher) during the period 1978/83 (Clement, 1984).

1 CG14033 ('Glacier 33') from 1982 (Braithwaite, 1989).

Extrapolation of the mass balance of Narssaq Bræ 


\section{CIRQUE GLACIERS}

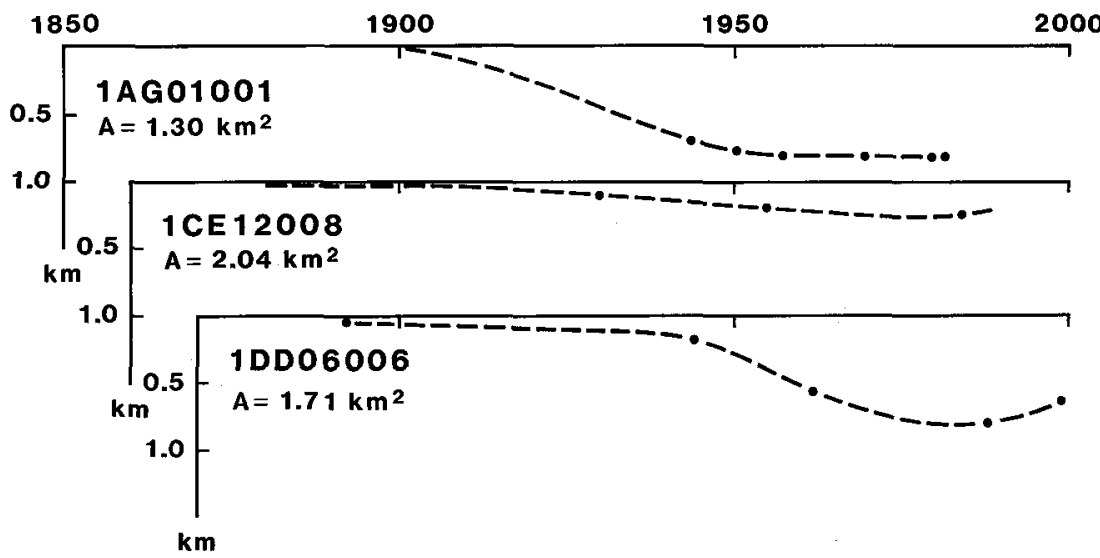

Fig. 9. Examples of change in frontal position of cirque and valley glaciers in West Greenland throughout the last century. Although simplified due to scattered observations and datings, the general trend of recession of glacier tongues throughout this century is apparent. Area (A) about 1950 is indicated.

\section{VALLEY GLACIERS}

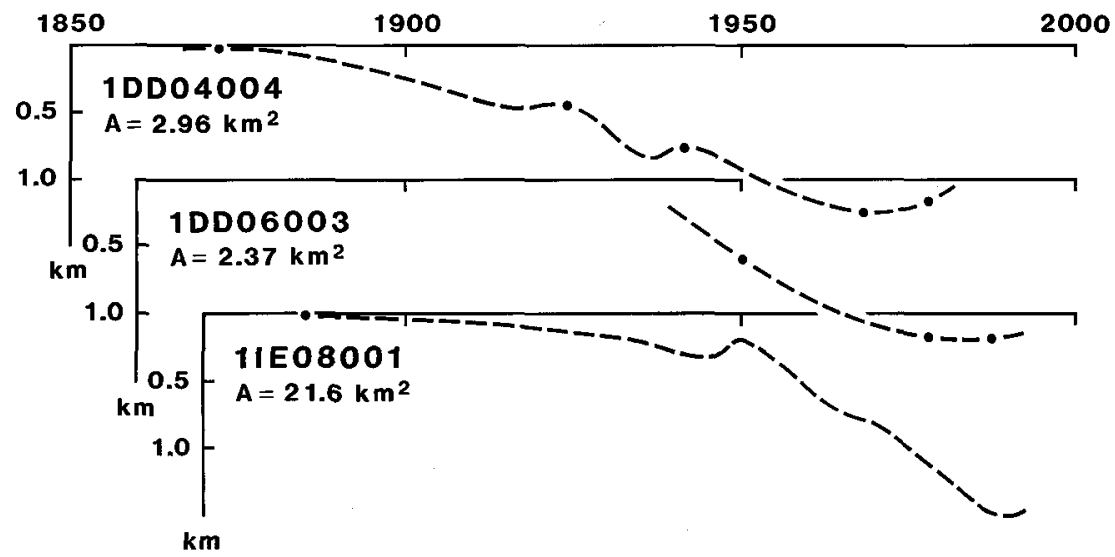

and Valhaltindegletscher back to the early 1960 s has been made for both glaciers on the basis of meteorological records of temperature and precipitation, measured at nearby stations in Narssaq and Narssarssuaq, respectively. Both results indicate a nearly continuous loss of mass from the glaciers, and the same might be concluded from the results of studies of 'Glacier 33' (Braithwaite, 1989).

\section{Valley glaciers}

The definition of a valley glacier by Müller et al. (1977, p. 13) that it "flows down a valley, the catchment in most cases well defined" is followed here, although with a stronger emphasis on the requirement that the glacier tongue partly or wholly fills a valley.

\section{Size, extent and volume}

A total of 310 valley glaciers are listed in the inventory, with a total area of $1370 \mathrm{~km}^{2}$ and sizes ranging from $0.03 \mathrm{~km}^{2}$ to $39.1 \mathrm{~km}^{2}$.

Thickness has not been measured on any of these features, but since transitional types to cirque glaciers frequently occur, the approach of relating area to mean thickness discussed under cirque glaciers may also be applied to valley glaciers.

\section{Changes}

Valley glaciers show the same high sensitivity to climatic changes as cirque glaciers. The size of the frontal change however, often exceeds that of cirque glaciers. 
Some examples of the change of frontal positions of valley glaciers are shown in Fig. 9, which shows that their behaviour is close to that of cirque glaciers. The examples shown also include 1IE08001, just north of the region described in the atlas, which has been chosen due to its relatively good information coverage (i.e. Gribbon, 1970). However, it should be noted that the investigated number of both valley and mountain glaciers is so few that any generalization on behavioural trends should be treated with caution.

For West Greenland glaciers in general, Beschel (1961) concluded there had been an early neoglacial maximum about A.D. 1600-1750 near the outer coast and a later neoglacial advance about A.D. 1850-1880 in the inland areas. However, this picture may be somewhat simplified, since mass-balance conditions can change the response situation for different areas and glacier types. Gordon (1981) concluded that investigations on mountain and valley glaciers in the Sukkertoppen area showed that for the last century: "In general, fluctuations of the glaciers have been in sympathy with prevailing climatic trends and show a relatively rapid response following temperature changes and a lagged response of at least 9 years following precipitation changes. Fluctuations of larger valley and ice-field outlet glaciers are out of phase with the others which may reflect a greater time lag of $20-30$ years in their response to precipitation changes".

Surging behaviour is also characteristic in the case of valley glaciers in the basalt areas of Disko and Nûgssuaq. The glaciers 1HD06026, 1HD06030, 1HE09095 and 1IA02034 all show signs of former surging or pulsing activity.

\section{Mass-balance measurements}

For valley glaciers the only short-term investigation of mass balance is that reported by E. v. Drygalski (1897) on the 'Kome Gletscher' (1IB29004).

\section{Large, local glaciers (ice caps and ice fields)}

\section{Definitions}

An ice cap is defined as a dome-shaped ice body with radial flow, whereas an ice field is defined as a more or less horizontal mass of sheet or blanket type of a thickness insufficient to obscure the subsurface topography.

Both types vary in size from features just larger than glacierets to those with a size of thousands of square kilometres. Common for the two types is their ability to cover the terrain whereas cirque and valley glaciers are limited in size and are largely controlled by the terrain.

It can be difficult to discern between the two forms. Even such a well-accepted feature such as Sukkertoppen Iskappe (Figs $3 \& 10$ ) has no simple radial outflow, and is not completely dome shaped. Transitional forms often occur, and the use of the prefix 3- (ice caps) and 2- (ice fields) in the tables is sometimes arbitrary.

\section{Composite and solitary ice caps and ice fields}

Since ice caps and ice fields according to definition (radial outflow) can cover watersheds of several hydrological basins they are mainly split up into sectors, each related to a tongue or part of the ice margin related to a specific frontal drainage area. The sectors are tabulated according to these hydrological basins.

In the following these units are re-arranged to define the individual ice cap or ice field, and each of these units are labelled by either their name (a few cases) or, where lacking a proper name (most cases), by the nearest place name or by the spot height of the nearest mountain peak. All the larger and most complex ice caps are indicated in Fig. 10 and tabulated by their area in Table 4.

Minor ice caps and ice fields (only a few square kilometres) can often be conceived as a unit, draining essentially to one basin. They are tabulated in the same way as other glacier units in the inventory.

\section{Distribution and limitations}

In western Greenland the major ice caps and ice fields are concentrated essentially in certain areas around latitudes $61^{\circ}$ and $63^{\circ}-64^{\circ} \mathrm{N}$, around $66^{\circ} \mathrm{N}$, and around $69^{\circ}-71^{\circ} \mathrm{N}$ (Disko, Nûgssuaq, Umanak area).

For some of these features a delineation problem exists. This may be due to attached perennial snow patches (e.g. Isortuarssuk ice cap) or to shrinkage dur- 


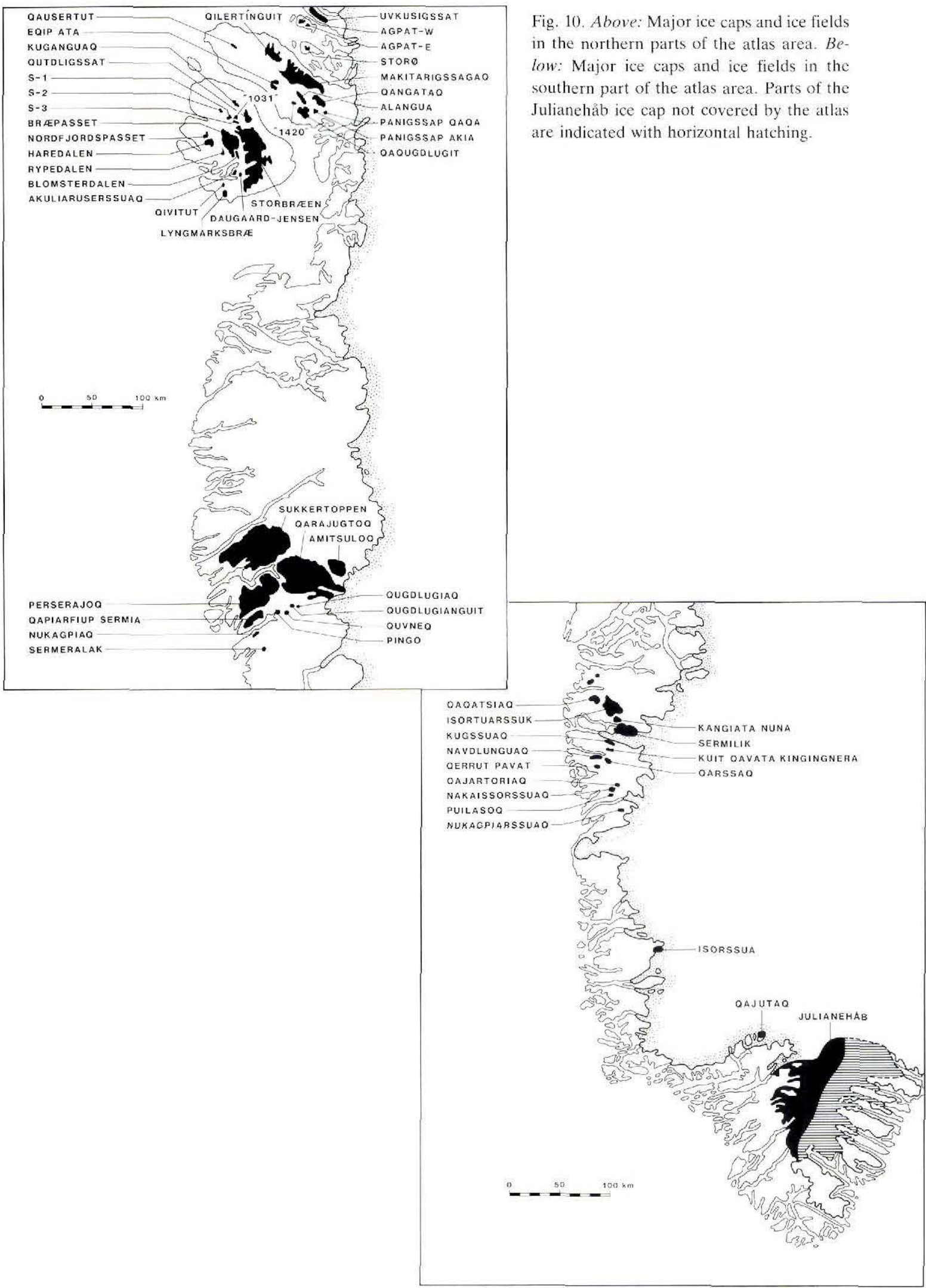


Table 4. Areas of ice caps and ice fields in $\mathrm{km}^{2}$

\begin{tabular}{|c|c|c|c|c|c|c|c|}
\hline \multicolumn{2}{|l|}{ Sydgrønland } & \multicolumn{2}{|c|}{ West Grønland } & \multicolumn{2}{|l|}{ Disko } & \multicolumn{2}{|c|}{ Nûgssuaq and Ũmánaq } \\
\hline Julianehåb & 6500 & Qarajugtoq & 2010 & Storbræen & 908 & Makitarigssagaq & 379 \\
\hline Isortuarssuk & 177 & Sukkertoppen & 1983 & Bræpasset & 254 & Qilertínguit & 116 \\
\hline Sermilik & 117 & Perserajoq & 692 & Qutdligssat & 65 & Qaqugdlugit & 81 \\
\hline Isorssua & 40 & Amitsulôq & 193 & Haredalen & 40 & Uvkusigssat & 79 \\
\hline Navdlúnguaq & 34 & Qapiarfiup sermia & 116 & Lyngmarksbræ & 32 & Qangataq & 65 \\
\hline Kûgssuaq & 33 & Nukagpiaq & 32 & Kûganguaq & 21 & Alángua & 63 \\
\hline Qaqatsiaq & 30 & Qugdlugianguit & 15 & Blomsterdalen & 18 & Eqip ata & 61 \\
\hline Nakaissorssuaq & 29 & Sermeralak & 13 & Akuliaruserssuaq & 17 & Agpat-W & 28 \\
\hline Kuit qavata & 24 & Quvneq & 7 & S-1 & 17 & Panigssap akia & 22 \\
\hline Qajutaq & 20 & Qugdlugiaq & 7 & $S-3$ & 11 & ' 1420 ' & 13 \\
\hline Puilassoq & 19 & & & Nordfjordspas. & 8 & Agpat-E & 10 \\
\hline Kangiata nuna & 15 & & & '1031' & 7 & Panigssap qaqa & 10 \\
\hline Qajartoriaq & 9 & & & $S-2$ & 7 & Qausertut & 10 \\
\hline \multirow{3}{*}{ Qarssaq } & 9 & & & Qivitut & 7 & Storø & 8 \\
\hline & & & & Rypedalen & 6 & & \\
\hline & & & & Daugaard-Jensen & al 6 & & \\
\hline
\end{tabular}

ing this century to such a degree that the ice caps and ice fields today are close to disintegrating into minor, local forms (especially frequent on Disko).

Another delineation problem occurs at places where an ice cap merges with the Inland Ice (e.g. Qarajugtoq). However, in most cases in western Greenland the delineation between two ice bodies can be determined rather precisely, and only the Julianehåb ice cap requires specific comment.

\section{Julianehåb ice cap}

The northern limit of this ice cap or ice field is drawn from the west through the depression filled by the ice stream of Qôrqup sermia (1AG08001 = the Inland Ice part and 1AG08002 = the Julianehåb ice cap part) and from here north-east towards the head of Anoritup kangerdlua on the east coast of Greenland.

There is a gradual transition of the surface from the southern slope of the Inland Ice to the Julianehåb ice cap in the south. The suggested border line between the two ice bodies is rather arbitrary. The main criterion is that the ice cap surface to the south is influenced essentially by the subsurface and by nunataks protruding from this surface, whereas these features disappear at the southern slope of the Inland Ice proper.

Julianehåb ice cap consists of several ice centres merging with complex outflow patterns. To the west this complex is clearly discernible from the coastland, which is only covered by minor glaciers. To the east the delimitation of the Julianehåb ice cap from the local ice domes between the East Greenland fjords (Fig. 11) can only be clearly detected on Landsat images.

The total area of the Julianehåb ice cap is estimated to be $6500 \mathrm{~km}^{2}$, of which only the western parts amounting to $c .2970 \mathrm{~km}^{2}$ are covered by the glacier atlas.

\section{Size and extent of major ice caps and ice fields}

The Julianehåb ice cap is by far the largest of the ice fields and ice caps of western Greenland, and is the major body in a cluster of ice caps and ice fields. The other members of this cluster are situated partly to the south-east, and especially to the east which is outside the area covered by the atlas (Fig. 11).

Other clusters of large ice caps and ice fields are located on Fig. 10. In Table 4 the individual ice caps and ice fields are arranged according to size within each of the major areas, down to areas of $c .6 \mathrm{~km}^{2}$. The large difference between the Julianehåb ice cap $\left(6500 \mathrm{~km}^{2}\right)$ and the adjacent ice cap Qarajugtoq $\left(2010 \mathrm{~km}^{2}\right)$ is apparent, and fits into a hyperbolic form of the relationship between areas and numbers of ice caps and ice sheets as given in Fig. 12. The total area covered by ice caps and ice fields is $11473 \mathrm{~km}^{2}$ (if the eastern part of the Julianehåb ice cap, not covered by the atlas, is included the total area would be $15000 \mathrm{~km}^{2}$ ). The total number of units of this type in the atlas area is 1171 . 


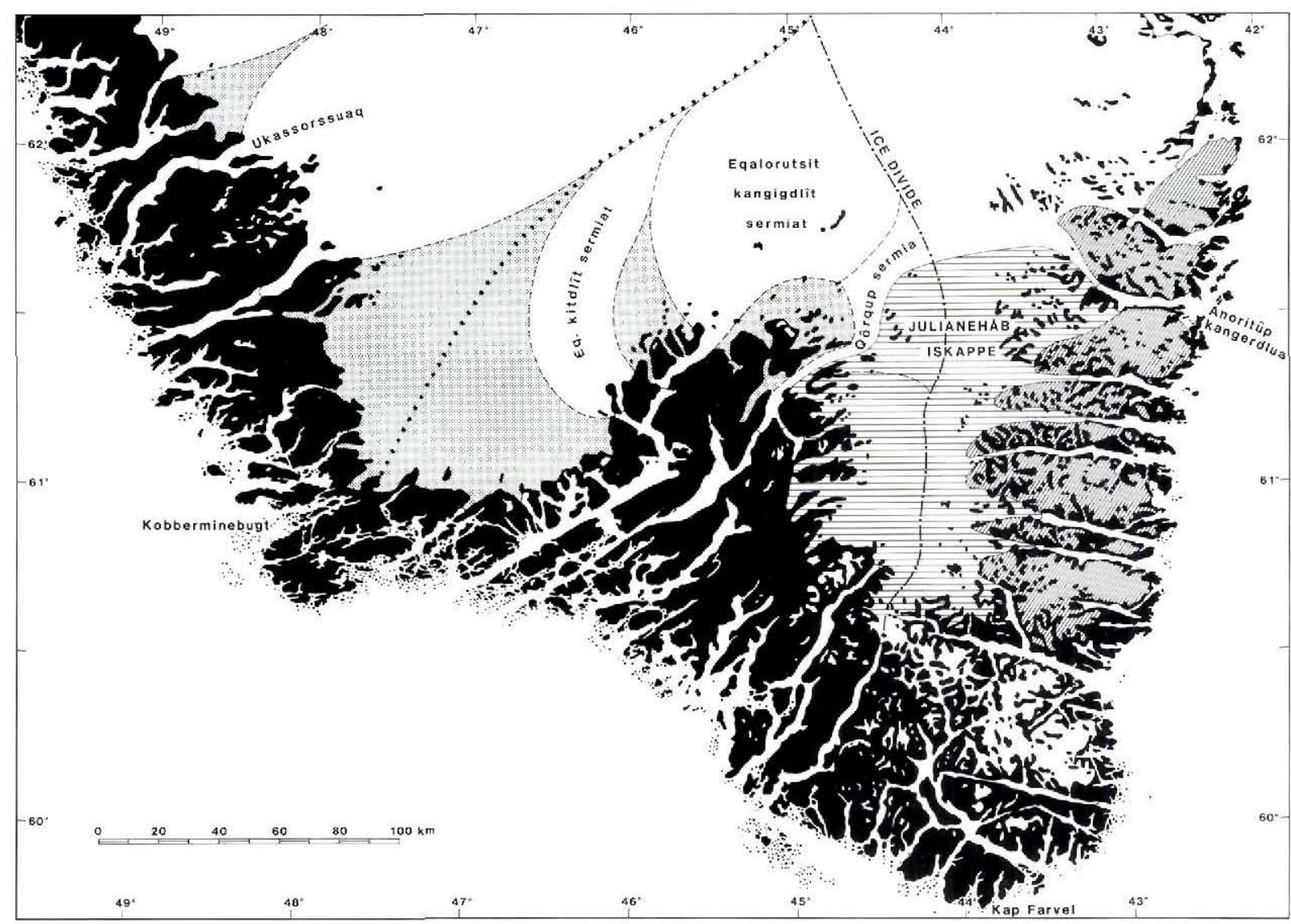

Fig. 11. Julianehab ice cap (horizontal hatching) and the neighbouring ice caps to the east (oblique hatching). To the north the south slope of the Inland Ice is delimited by the ice stream of Qôrqup sermia. From Weidick 1988.

\section{Volume}

Soundings of ice thickness have been undertaken by radar on the Julianehåb ice cap (S. Overgaard, Electromagnetics Institute. Technical University, Denmark) and by gravity on Sukkertoppen Iskappe $\left(1983 \mathrm{~km}^{2}\right)$ (Bull, 1963).

On the Julianeháb ice cap four lines were measured from which an average thickness of about $450 \mathrm{~m}$ was estimated; however, the scarcity of reflections and the alpine configuration of the subsurface places limits on the reliability of this figure. The same can be said of an estimate of an average thickness of about $300 \mathrm{~m}$ of Sukkertoppen Iskappe, as this is based on only a single traverse.

Both estimates given above are higher than the average thicknesses for ice covers with areas of between 2000 and $8000 \mathrm{~km}^{2}$. Figures given by Cailleux \& Lagarec (1977) and based on measurements elsewhere in the world, suggest an average of about $150-200 \mathrm{~m}$.

\section{Changes}

Fluctuations of many outlets from ice caps and ice fields are known from the literature (e.g. Weidick. 1968). Often the last major advance of the outlets was initiated in the first half of the last century, culminated during the 1850 s, and was followed by a gradual retreat that has accelerated throughout this century. The reduction of the length of the glacier outlets on land is most often a matter of 1-2 km, but with considerable deviation from this mean.

Greatest interest has been attached to outlets with exorbitantly large fluctuations, but these examples are usually those which, at least during their culmination, terminated with a tongue floating in a fjord. Thus the glacier of Sermeq (1AC07020) of the Julianehảb ice cap is supposed to have advanced $12 \mathrm{~km}$ down the fjord in the first half of the 19th century. It maintained this advanced position until near the end of the 1960s, when this $10 \mathrm{~km}$ extension disintegrated over a few years presumably as a consequence of a previous long period of thinning of the outlet surface (Weidick, 1988a). 


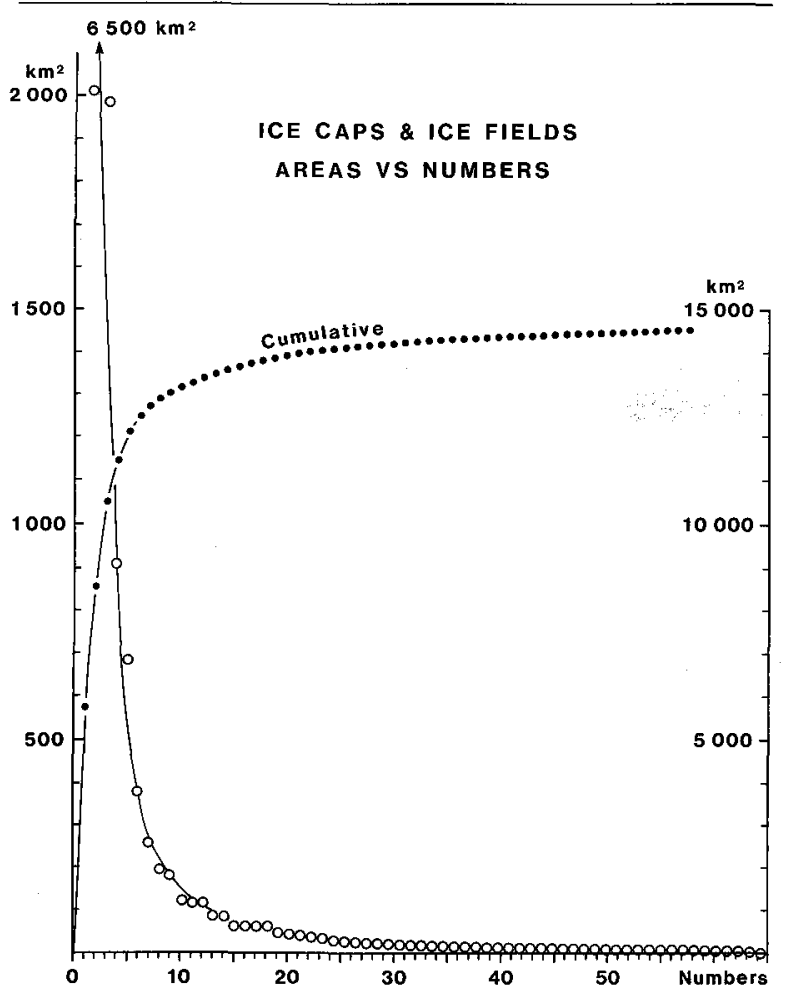

Fig. 12. Ice caps and ice fields in the atlas area arranged according to their size and numbers.

Recent thinning of comparable magnitude has been reported from 1DF20002 (Sukkertoppen Iskappe) at the head of Evighedsfjord whose glacier termination was earlier partly floating but today rests on land, i.e. on raised marine deposits with an age of 6510 radiocarbon years B.P. (Sugden, 1972).

On Disko several examples are known of land based glaciers which had a larger extension in historical time (e.g. 1HB15029, 1HB10027, 1HB10036, 1HB15039 and 1HE01024). These glacier fluctuations on Disko may possibly be related to surge behaviour referred to in Table 5 (Weidick, 1988b). Such surges have been especially concentrated on the island of Disko, and it seems that the glaciers here during the Little Ice Age and until the early part of this century passed through a phase of surge activity, which in most cases is now extinct.

Several glacier lobes in Disko island and the Nûgssuaq peninsula reveal today large quantities of dead ice at their fronts; this could be a relict of former surge activity. However, the appearance of the dead ice often detached remnants at the foot of the firn-carrying plateaus - might just as well be an indication of the strong sensitivity of ice caps and ice fields to climatic change, since the plateau surfaces are mainly situated close to the present snow line.
The rise of the snow line in this century here implies a strong thinning of the ice cover, and the cut-off outlets are often left as extensive dead ice areas in the valleys. Examples described from Nûgssuaq are 1IB29004 ('Kome- Gletscher') and 1IB30003 ('SarfarfikGletscher') (e.g. Drygalski, 1897).

\section{Mass-balance measurements}

Investigation of mass balance of ice caps and ice fields in western Greenland has been concentrated in the area around latitude $67^{\circ} \mathrm{N}$, where work on energy balance and accumulation on Sukkertoppen Iskappe goes back to 1938 (Etienne, 1940). The work was continued in 1962-64 with investigation of thickness, accumulation and ablation in the central and eastern parts of the area (Goldthwait, 1964; Rundle, 1965).

Subsequent mass-balance work on the ice caps of the region has been carried out by the Geological Survey of Greenland in connection with the planning for hydropower in Greenland; this work was therefore essentially devoted to establishing time series of mass-balance measurements. The GGU work has covered the following ice caps, or parts of ice caps and ice fields:

\section{Table 5. Surging glaciers in West Greenland}

Glacier Solitary $\quad$ Sector of greater
ice cap or ice field

$\begin{array}{ll}1 \text { AC 07020? } & \\ 1 \text { HB } 10036 & \\ 1 \text { HB } 11029 & \\ 1 \text { HB } 15012 & \\ 1 \text { HB } 15013 & \\ 1 \text { HB } 15018 & \\ 1 \text { HB } 15029 & \\ 1 \text { HB } 15031 & \\ 1 \text { HB } 15039 & \\ 1 \text { HC } 05004 & \text { ice field } \\ 1 \text { HD } 06005 & \text { cirque glacier } \\ 1 \text { HD } 06009 & \text { cirque glacier } \\ 1 \text { HD } 06026 & \text { valley glacier } \\ 1 \text { HD } 06030 & \text { valley glacier }\end{array}$

$1 \mathrm{HE} 01011$

1 HE 01015

1 HE 01024

$1 \mathrm{HE} 09051$

$1 \mathrm{HE} 09090$

$1 \mathrm{HE} 09094$

1 HE 09095

1 IA 02034

1 IB 26003
Julianehåb ice cap Bræpasset ice field Brepasset ice field Bræpasset ice field Bræpasset ice field Storbræen (ice cap) Storbræen Storbræen Storbræen

Storbræen Storbræen

ice field Storbræen

ice field cirque glacier valley glacier valley glacier 
Sukkertoppen Iskappe, 1982-89 (O. B. Olesen, personal communication). Measurements covered only the lobe of 1DG16250 ('Glacier A' of Rundle, 1965), i.e. 'Tasersiaq Gletscher', on the 1:250 000 topographic map sheet.

Qapiarfiup sermia, 1980-89 (Olesen, 1986) covering the entire ice cap.

Amitsulôq, with measurements from 1981 covering the entire ice cap (Olesen, 1986; cf. Fig. 13).

In areas further north, early measurements on ablation of the lobes of Agssakait sermiat (1IB26003) and Sermiarssuit sermikavsâ (1IB27003) were made by Drygalski (1897).

\section{Calf ice production from ice caps}

Several lobes of the larger ice caps terminate in the West Greenland fjords, and have a small calf-ice production. Measurements on the rate of movement have been reported from Sermeq in Tasermiut (1AB06001, a lobe from the Julianehåb ice cap) which moves at a maximum rate of $3.7 \mathrm{~m} / 24 \mathrm{~h}$ (Steenstrup, 1881), whereas Mott (in Etienne, 1940) for the outlet of Tâterat sermiat from Sukkertoppen Iskappe (1DF11005) reported a maximum movement of $60 \mathrm{~cm} / 24 \mathrm{~h}$ at the front of the glacier.

\section{Ice domes}

Ice domes are defined as dome-shaped surface features in the marginal area of the Inland Ice sheet with their own firn area and almost radial movement.

Ice domes are partly surrounded by outlets from the ice sheet but only contribute to a limited degree to the nourishment of the ice sheet. This is due to their comparatively negligible size. The ice domes can be considered as transitional areas between the Inland Ice sheet proper and the local glaciers described earlier. Whether they influence growth or decay of the ice sheet margin proper is not clearly demonstrated, but is feasible.

Ice domes occur frequently in the northern parts of the Umanak district, i.e. just north of the area dealt with in this atlas. In most of western Greenland they only occur in minor, uncharacteristic forms.

The codified descriptions do not contain possibilities for distinction of a special category, and ice domes are here classified as ice caps.

Of the composite larger ice caps of the atlas area, that coming closest to the definition of an ice dome is Isorssua. Isorssua is divided into the 4 part sectors of 1BF03015 and 1BF03032-34, and its position is shown in Fig. 10. Its area is $40 \mathrm{~km}^{2}$ (Table 4).

\section{The south-western slope of the Inland Ice}

A definition and general information on the Inland Ice has been given in the chapter on Glacier identification. The following remarks concern only the western slope of the Inland Ice covered by the atlas between $61^{\circ} \mathrm{N}$ and $71^{\circ} \mathrm{N}$.

\section{Limitations}

The extent of the Inland Ice treated in the atlas reaches from fjord $1 \mathrm{AG}$ (Tunugdliarfik) at $61^{\circ} \mathrm{N}$ to $1 \mathrm{IC}$ (Itivdliarssûp kangerdlua) at $71^{\circ} \mathrm{N}$.

The ice sheet is generally well defined towards the coastland in western Greenland. Confluence with local glaciers is restricted to three areas:

1. South Greenland, where the southern edge (slope) merges with the Julianehåb ice cap between 1AG08001 and 1AG08002 (Qôrqup sermia);

2. South-West Greenland with partial confluence to local ice domes at sector 1BF03035 between Sermiligârssuk and Sermilik fjords;

3. West Greenland at Sukkertoppen / Manitsoq where the Qarajugtoq ice cap merges with the Inland Ice along a short stretch. 
Only in the case of the Julianehåb ice cap is the delimitation of the Inland Ice somewhat arbitrary, due to the lack of a detailed survey of the upper zone of confluence with the Julianchảb ice cap (Qôrqup sermia). The total area of Qôrqup sermia is given in Table 6 as $1406 \mathrm{~km}^{2}$; of which the total Inland Ice part is estimated at $646 \mathrm{~km}^{2}$ and the area below $1800 \mathrm{~m}$ to 158 $\mathrm{km}^{2}$.

\section{Sector division of the Inland Ice - calf ice producing and quiet sectors of the ice sheet}

The problem of defining sectors of the ice sheet related to the hydrological conditions of the ice margin was treated in the section on local glaciers, and in the following the division presented must be conceived as a first approach towards a description of the dynamic conditions (flow lines) of the ice margin. The essential content of the sector division presented is its value for the registration of glacial events and the approximate division of the ice sheet margin into calf-ice producing sectors and quiet sectors (Fig. 14).

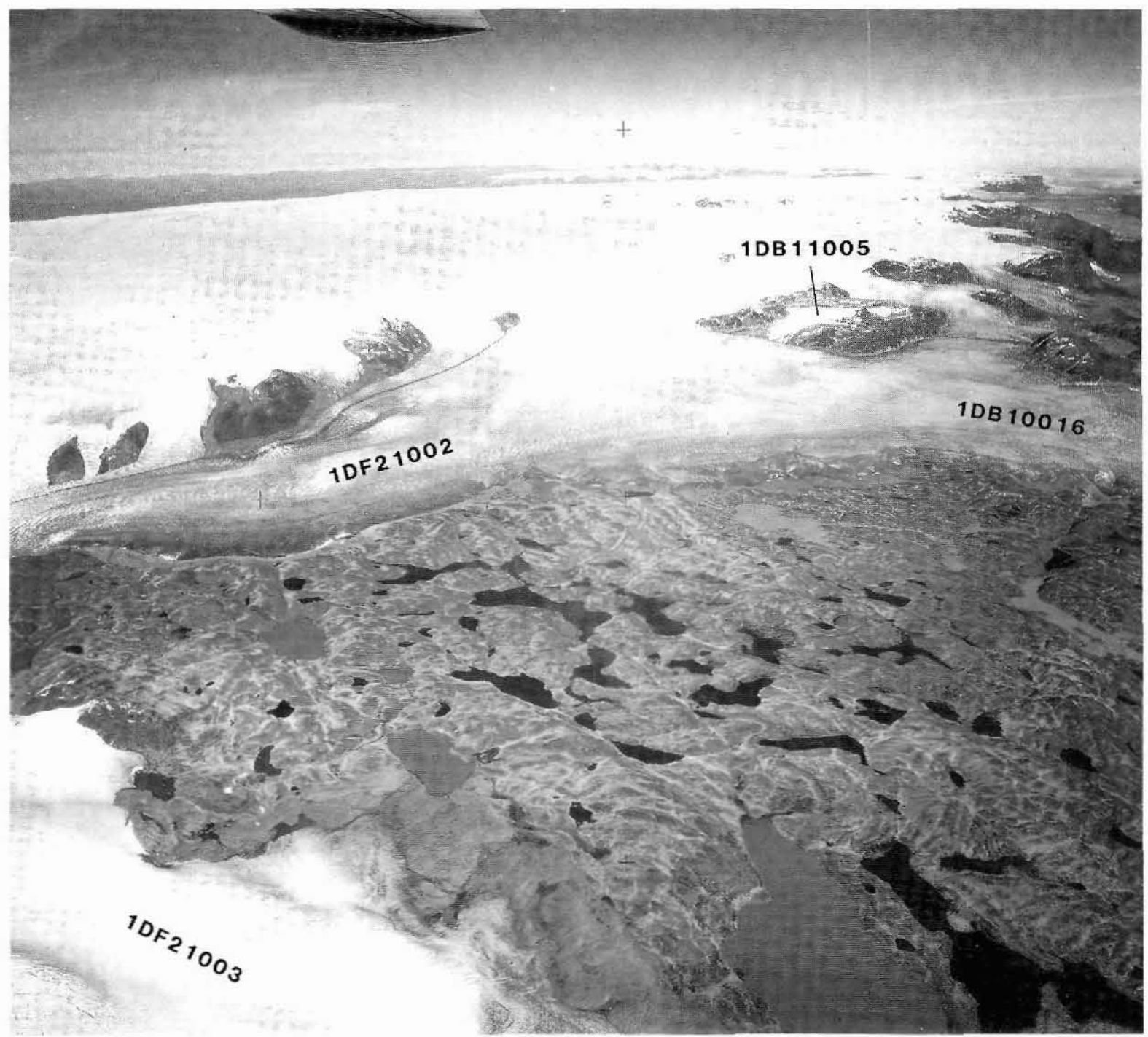

Fig. 13. Ice caps in the Sukkertoppen/Manîtsoq region, seen from the west. Foreground: Qarajugtôq ice cap. Background: Amitsulôq ice cap and Inland Ice. Kort- og Matrikelstyrelsen, Denmark, series 505 D- $\varnothing$ no. 4721 . 20th August 1948. Published with permission A.200/87. 
Table 6. Estimated area of Qôrqup sermia

\begin{tabular}{lccr}
\hline Area (in $\mathrm{km}^{2}$ ) & $\begin{array}{c}\text { 1 AG 08001 } \\
\text { (Inland Ice part) }\end{array}$ & $\begin{array}{c}\text { 1 AG 08002 } \\
\text { (ice cap part) }\end{array}$ & Total \\
\hline below $1800 \mathrm{~m}$ a.s.l. & $\underline{158}$ & 175 & 333 \\
above $1800 \mathrm{~m}$ a.s.l. & 488 & 585 & 1073 \\
total Qôrqup sermia & 646 & $\underline{760}$ & 1406 \\
\hline
\end{tabular}

Underlined figures are those tabulated.

The characteristics of the two types of sectors are given below.

\section{Calf ice producing sectors}

Calf ice producing sectors are confined to the termination of outlets at the heads of deep fjords and inlets, i.e. to very restricted areas of the total ice margin (Fig. $15)$.

The expression 'tidewater glaciers' is often applied to these localities, stressing their connection to the marine environment. A distinction is made between polar and subpolar glaciers mainly having floating termini, and temperate glaciers which are grounded (Meier \& Post, 1987). Within the atlas area all transitions and varieties seem to occur.

Major calf-ice production is confined to fjords with deep proglacial fjords continuing into and under the ice sheet margin. These drainage channels for the ice act as large sinks for large parts of the Inland Ice and as foci for convergent flow of the ice margin, and they may influence the nearby quiet sectors. They can be defined as fast tidal glaciers, characterized by permanent surges (Paterson, 1968).

The best known (and largest) example of these fast tidal glaciers is Jakobshavn Isbræ (1GC06002), where frontal movement is up to $23 \mathrm{~m} / \mathrm{d}$ (Lingle et al., 1981; Hughes, 1986), and calf-ice production has been estimated at between $26 \mathrm{~km}^{3} \mathrm{a}^{-1}$ (Bauer, 1968a) and c. 40 $\mathrm{km}^{3} \mathrm{a}^{-1}$ (Bindschadler, 1984).

Other tidal glaciers have essentially lower rates of movement and calf-ice production (Bauer, 1968a; Carbonnell \& Bauer, 1968; Weidick \& Olesen, 1980). The amount of calf-ice production can only be determined very approximately so that the presentation of the distribution of calf-ice production in Fig. 15 gives only an impression of the relative magnitude. Apart from Jakobshavn Isbræ, which is unique both in size of calfice production and rate of movement (cf. Fig. 16), a distinction can be made among the other outlets of Fig.
15 between a few large producers (the 'ice streams' according to the original definition by Rink (1857) whose rate of movement at the front usually is more than $1 \mathrm{~km} \mathrm{a}^{-1}$; and the calf-ice production more than about $2 \mathrm{~km}^{3}$ ice $\mathrm{a}^{-1}$ ) and a large number of minor outlets with lower production and movement.

Some of the outlets change character with time. Thus in 1870 Nordenskiöld Gletscher (1FC19001) at the visit of A. E. Nordenskiöld (Nordenskiöld, 1886) was producing calf ice in appreciable amounts; subsequently sedimentation seems to have developed a tidal flat along large parts of the front, so that the reported productivity in 1968 (about $1 \mathrm{~km}^{3}$ ice $\mathrm{a}^{-1}$, Bauer, 1968a) may well now be an overestimate.

\section{Quiet sectors}

Quiet sectors are marginal areas of the Inland Ice which rest and end on land, in lakes or in shallow fjords with no appreciable calf-ice production, and whose main marginal loss is in the form of meltwater. As in the case of Nordenskiöld Gletscher, transitional forms to tidewater glaciers can be found. The extent of quiet sectors of the Inland Ice margin could most simply be defined as the parts of the ice margin between the ice streams. Hydropower planning is usually connected to suitable localities at this form of ice margin.

In a dynamic sense the marginal movement of these parts of the ice margin is divergent. The rate of movement generally decreases from horizontal velocities of about $100-200 \mathrm{~m} \mathrm{a}^{-1}$ at the equilibrium line (1200-1600 $\mathrm{m}$ a.s.l.) to nil at the margin where the ice margin is totally landbased. More or less floating parts of the ice margin in lakes can have movements of $100 \mathrm{~m} \mathrm{a}^{-1}$ or more.

\section{Areas}

Determination of total areas of individual sectors of the Inland Ice is given only for those parts of the sectors below $1800 \mathrm{~m}$ a.s.l. The area of the tabulated ice streams amounts to $31 \%$ of the total sector area of 101 $000 \mathrm{~km}^{2}$ covered by the atlas, whereas the intervening 242 quiet sectors form $69 \%$ of the total area. The total area of these sectors of the Inland Ice projected up to the ice divide is estimated to cover $c .400000 \mathrm{~km}^{2}$ (Weidick \& Olesen, 1980).

\section{General trends in recent marginal change}

The plot of changes of the ice margin in Fig. 17 is based on digit 6 of the morphology code given in Table 


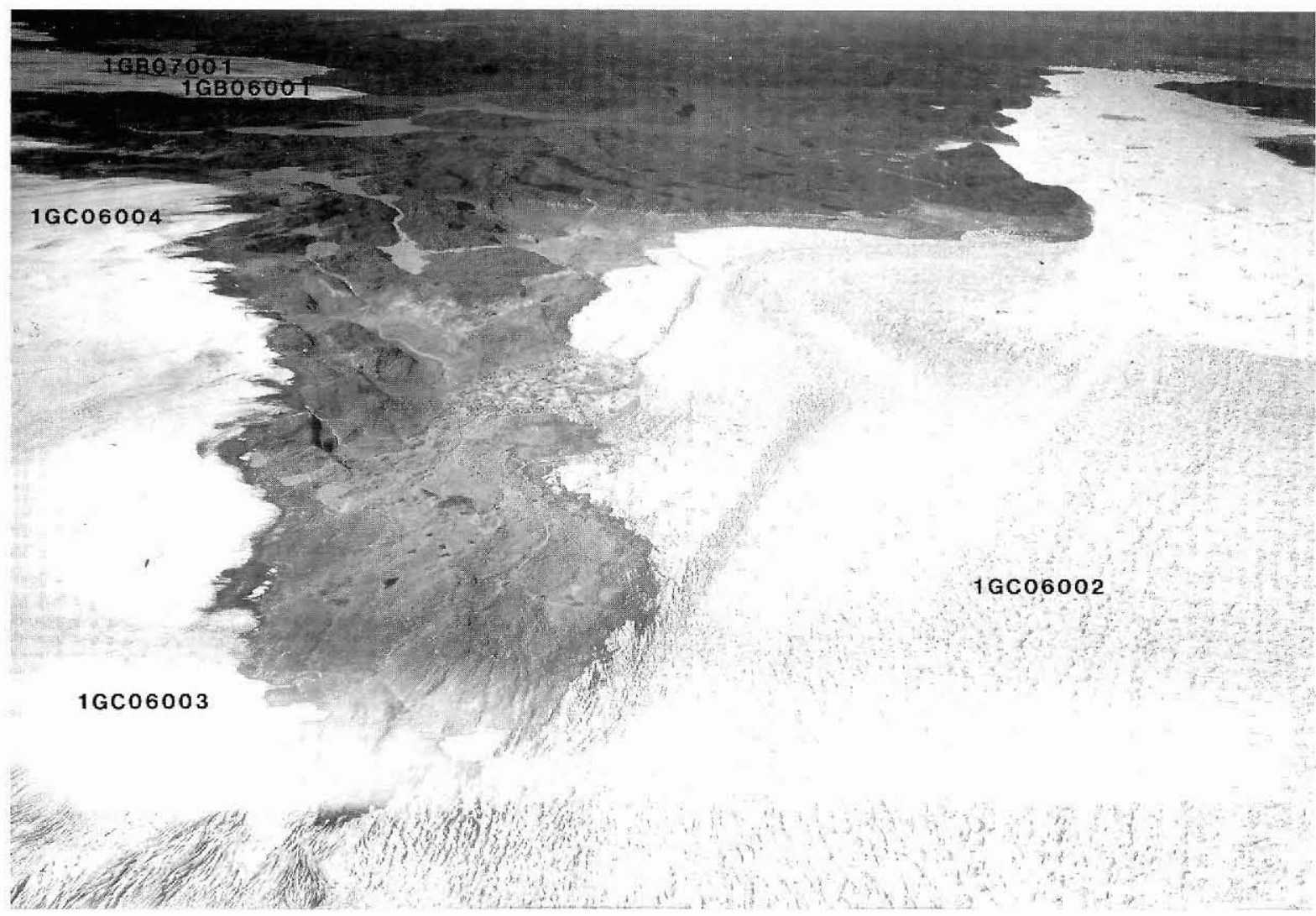

Fig. 14. Quiet sectors and ice stream of the Inland Ice, here seen from the cast. Foreground left: Quiet sectors of 1GC060(03-04. Foreground right: The ice stream of Jakobshavn Isbræ (1GC06002). Background: Tidewater glaciers of Alángordliup sermia (1GB06001) and Sarqardliup sermia (1GB07001). Kort-og Matrikelstyrelsen, Denmark, series 518 D-V no. 8025, 22 nd July 1949. Published with permission A.200/87.

2. The plot therefore indicates the net change of the ice margin within a period of up to 2 centuries (c. 1750? to 1950) without any consideration of short time oscillations of the ice margin within this period.

It can be seen from Fig. 17 that the most pronounced areas of net marginal retreat are confined to low areas of the ice sheet margin situated relatively close to the coast. Thus in the south these areas are concentrated around the glacier aprons of Qagssimiut and Frederikshåb Isblink. Both areas approach in form and their sensitivity to climatic change that of piedmont glaciers.

Further to the north, recessional areas are also related to a relatively close proximity to the sea, chiefly around the fjord complexes of Godthåbsfjord, Disko Bugt and Umanaq Fjord where several ice streams and other tidal glaciers debouch into the fjords.

Minor recessions and more frequent occurrences of stationary or even advancing glacier margins are to be found at higher altitudes of the ice margin (e.g. the area between Godthảbsfjord and Søndre Strømfjord). This may be explained by a decreasing (and faster) thickness response at greater elevations of the ice margin, as demonstrated by Reeh (1983) in modelling of the response of the essentially land based sectors 1GC04003-1GF06002 (Pâkitsoq locality in Disko Bugt, Fig. 21) for the period A.D. 600 to 1950.

In general it is difficult on the basis of present information to document anything else than a 'general advance' in most recent time for the advancing sectors, since the geological evidence is buried beneath the present ice and the documentary evidence is sparse. Only for the area around Eqalorutsit kangigdlit sermiat is there sufficient evidence that the ice margin throughout the last century (or possible more) has been steadily advancing (Weidick, 1988b). In this area major parts of the ice margin are obstructed by or abut high mountains. This is also the case in the area north-east of Narssarssuaq where general recession has been noticed throughout the same period. Here as elsewhere, changes in accumulation pattern of the interior and topographic conditions of the ice margin must influence the overall general trend of recent recession. 
11G- RINK ISBRE (KANGIGDLIUP SERMIA)

1KG - UPERNAVIK ISSTRøM (SERMEOI

IINGIA ISERAE ISAGOLIARUTSIP SEFMIAI $11 \mathrm{H}-\mathrm{Umi}$

IF- KanGERdLUGSSÚp SERMERSSUA

IIE - KANGEFDLUARSSÂP SERMA R

$\mathrm{km}^{3}$ ice/year

40

30

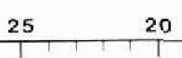

20

100

1 GH17001 SERMEO AVANGNAROLEO

KUJATOLEO

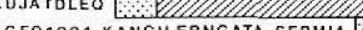

1GFOSO01 EQIP SERMIA

$16 C 06002$ JAKOQSHAVN ISBAAE 1

11020001 PERDLERFIUP SERMIA 11B03001 SERMILIK

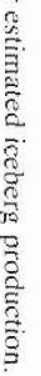

1 B B06001 ALANGORDLIUP SERMIA

$1 G B 07001$ SARQARDLIUP SERMIA

1FC 19001 NOHDENSKIÖLD GLETSCHER

1FC 30001 USUGDLUP SERMIA

TCH 17002 NARSSAP SERMIA

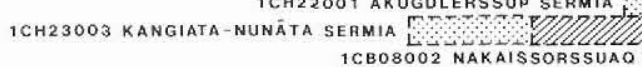
1 CBOBOO2 NAKAISSORSSUAO

1BG03002 AVANGNARDLEQ BRE

$18 F 03002$ UKÁSSERSSUAD

18006001 SERMILIGÁASSUK BRE WU $18 B 05001$ ARSUK BRAE

AHO2001 OALERAGDLIT SERMIA

TAHO6001/O3 EOALERUTSIT XITDLAT SERMIAT

1AHO8O22 EQALORUTSIT KANGIGOLIT SERMIAT
1AGOBO01/O2 QOORQUP SERMIA

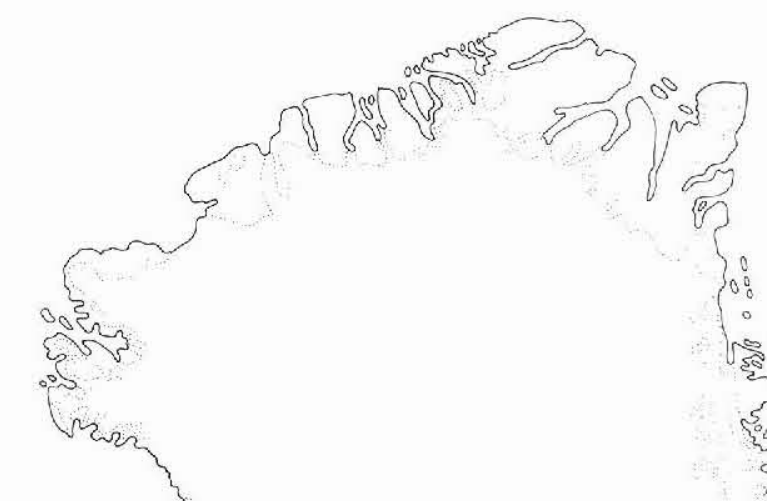

$\omega$

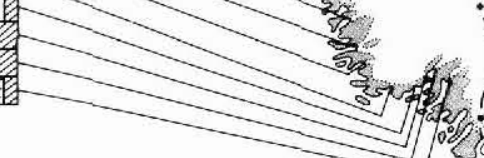




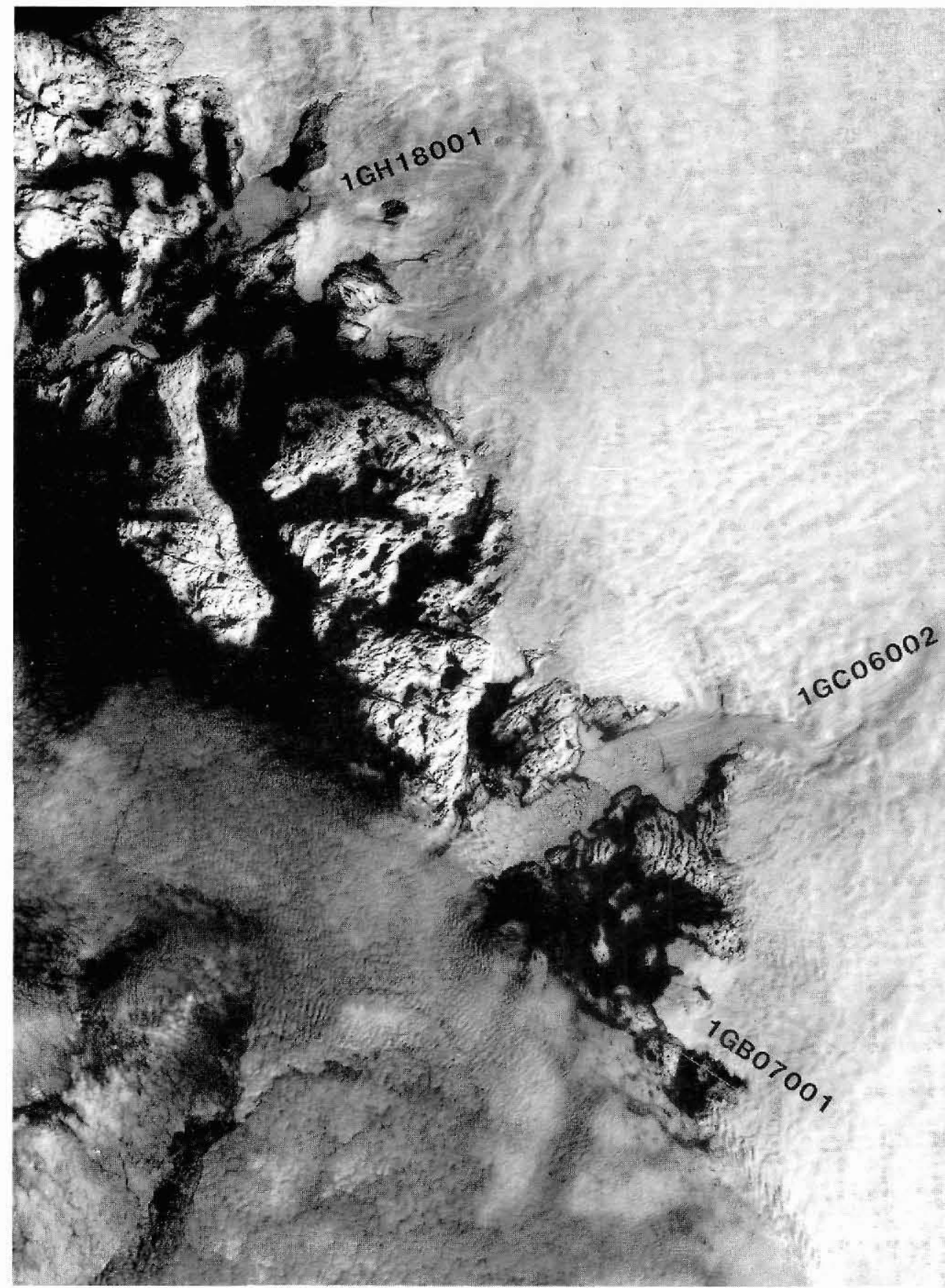

Fig. 16. Central West Greenland around Disko Bugt. Inland Ice margin from Sarqardliup sermia (1GB07001) in the south to Sermeq kujatdleq (1GH18001) in the north. The lineaments on the ice margin indicate trends of the calf ice producing ice streams (fast tidewater glaciers) in the fjords. The patterns on the ice surface in the intervening quiet sectors are a reflection of the subsurface topography of the ice margin. Landsat scene 11/11, 27th September 1979.

Updating of the atlas with results from the 1980s has only been made on the basis of local observations during GGU field investigations in the region of Narssarssuaq to Qagssimiut, the Frederikshåb Isblink area, Godthåbsfjord and the Søndre Strømfjord to Disko
Bugt area; reports from other expeditions or field work (e.g. report by the ACG/Øresund in Isukasia area in the 1980s, Scholz (1987) in the Søndre Strømfjord area) have also been used supplemented with a perusal of the extensive aerial photograph coverage of 1985 . The gen- 


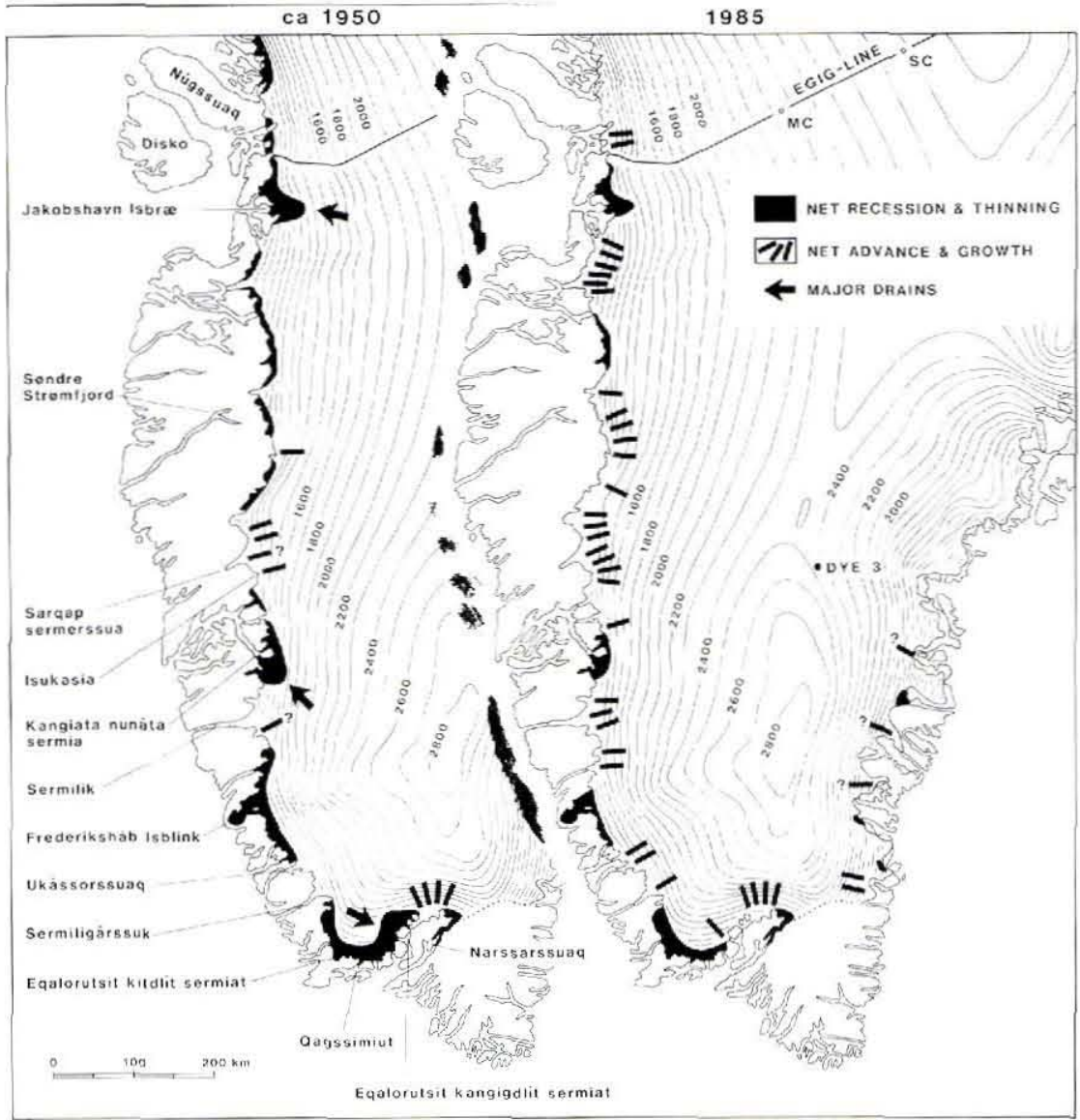

Fig. 17. Marginal activity in 1950 and 1985. Width of the black zone indicates the estimates of net thinning and recession. The western part of the EGIG-line is shown with the stations of Milcent (MC) and Station Centrale (SC). The position of the Dye 3 station is also indicated. Height contour lines according to Bindschadler et al. (1989).

eral impression (cf. Fig. 17) is that the general recession of the past century or two has been succeeded by an initial advance ('turn of the tide). mainly in the relatively high lying parts of the ice margin (Weidick. 1991) from which it may spread in the future to lowland areas.

\section{Thickness change of the marginal parts of the ice sheet}

Data concerning the change in thickness of the ice margin, determined by repeated photogrammetry, is only available around three localities:

1. In South Greenland at the ice margin in sector 1AG05001 (Nordbogletscher) and at IAG07008 (Nordgletscher; Knudsen, 1986). Nordbogletscher terminates in a lake $660 \mathrm{~m}$ a.s.l. and the repeated surveys for 1953 and 1981 covered an area of $17 \mathrm{~km}^{2}$ between the glacier front and an altitude of $950 \mathrm{~m}$ a.s.I. An average total increase in thickness of about $30 \mathrm{~m}$ could be demonstrated during this period of 28 years, related to a frontal advance of $300-700 \mathrm{~m}$ for the same period.
Nordgletscher terminates on land at about $650 \mathrm{~m}$ a.s.l. Repeated surveys for the period 1952-81 covered an area of $21 \mathrm{~km}^{2}$, from the glacier front to an altitude of $900 \mathrm{~m}$ a.s.l. A total increase in glacier thickness for this period varied between $120 \mathrm{~m}$ (at the front) to $30 \mathrm{~m}$ (at $900 \mathrm{~m}$ a.s.l.). The increase is connected to a frontal advance of $750 \mathrm{~m}$.

2. In the Godthåbsfjord area at the margins of sectors ICH21001 (no name) and 1CH21002 (Qamanârssûp sermia; Knudsen \& Moller, 1982; Fig. 18).

$1 \mathrm{CH} 21001$ terminates partly in a frontal lake at about $440 \mathrm{~m}$ a.s.l. Repeated surveying 1968-1980 covered an area of $3 \mathrm{~km}^{2}$. i.e. from the glacier front to about $600 \mathrm{~m}$ a.s.l. An average total increase in thickness of $20 \mathrm{~m}$ was measured for the 12 year period related to a frontal advance of $50-150 \mathrm{~m}$. Qamanârssûp sermia terminates partly on land and partly in lakes at about $70 \mathrm{~m}$ a.s.l. and a survey for the period 1968-80 covered an area of $15 \mathrm{~km}^{2}$, i.e. from the glacier front to $c .900 \mathrm{~m}$ a.s.l. The glacier at some parts is thickening, at others thinning, with a nearly stable front throughout the period (Knudsen. 
1983).

Both glaciers are surrounded by a well-developed trimline zone indicating frontal recessions of about 2 $\mathrm{km}$. The general recession up to the 1950 s has reversed to the present phase of re-advance at $1 \mathrm{CH} 21001$.

3. Pâkitsoq area in Disko Bugt, covering a wide spectrum of sectors between 1 GC04003 and 1GE03004 of which most terminate partly in frontal lakes and partly on land (Fig. 19).

A single sector is a tidewater glacier (1GC04001 Sermeq avangnardleq) with a relatively low rate of movement of $500 \mathrm{~m} \mathrm{a}^{-1}$ and an estimated calf-ice production of $0.2 \mathrm{~km}^{3}$ ice $\mathrm{a}^{-1}$ (Bauer, 1968a).

A repeat survey was made by Thomsen (Thomsen $e t$ al., 1988) on the basis of aerial photographs from 1959 and 1985, and covered an area (c. $140 \mathrm{~km}^{2}$ ) from sea level to about $500 \mathrm{~m}$ a.s.l.; a mean total thinning of $14 \mathrm{~m}$ for the 26 years was determined. At the ice margin the thinning was related to an increase in superficial moraine, but rarely by distinctive recession of the ice margins.

The detailed studies referred to above fit into the pattern of development indicated by Fig. 17 and opens up the question of a general trend of recent and thickening expansion of the Inland Ice surface.

A geodetic profile across the Inland Ice from the Disko Bugt area of West Greenland to the Kong Oscar Fjord area of East Greenland has been measured. The lower parts of the western profile up to $1200 \mathrm{~m}$ a.s.l. were measured in the period 1948-59 (Bauer, 1968b), and the whole profile during the period 1959-68 (Seckel, 1977). For the ablation area a thinning of the

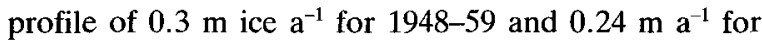
$1959-68$ was determined whereas in the accumulation area the altitude increased by an average of $0.085 \mathrm{~m}$ snow $\mathrm{a}^{-1}$.

Zwally (1989), on the basis of satellite altimetry, found an average increase of the elevation of the Inland Ice surface of $0.2-0.3 \mathrm{~m} \mathrm{a}^{-1}$ for the period 1978-86, which he ascribed to increased accumulation due to warmer climate. Braithwaite (1990) explained this increase as a consequence of a decrease in mean temperatures (and of ablation) in the region over the same decade. Zwally's results have been questioned by Douglas et al. (1990). The statement of a general increase in accumulation over the last 300 years at Crête at $71^{\circ} 07^{\prime} \mathrm{N}, 37^{\circ} 19^{\prime} \mathrm{W}$ by Clausen et al. (1988) and the earlier trends in accumulation determined at the stations of Milcent at $70^{\circ} 18^{\prime} \mathrm{N}, 44^{\circ} 45^{\prime} \mathrm{W}$ and Dye 3 at $65^{\circ} 11^{\prime} \mathrm{N}, 43^{\circ} 49^{\prime} \mathrm{W}$ by Reeh et al. (1978) show only in part homogeneous patterns. The reports of an increas- ing number of sectors turning from recession to advance during the period 1950 to 1985 may be the consequence of a slight increase in accumulation in the centuries after the Little Ice Age, combined with the stabilization of temperature trends after 1950 (Weidick, 1991).

The initiation of this general thickening, its total value in water equivalent and its spreading rate to the lower reaches of the ice margin, where local frontal responses are modified by the surrounding topography, is still not known in detail.

\section{Local change of the ice margin}

The generalized trends of marginal change in Fig. 17 do not furnish details of the local 'noise' caused by specific morphological features (or by local climate). It is only in rare cases that glacier changes over the last few centuries can be established from historical and geological records. These can be used as 'case stories' elucidating the influence of specific local features.

The location of recessions greater than $1.5 \mathrm{~km} \mathrm{(=}$ 'marked retreat' in Table 2) can be plotted from the data list and are not specified in detail here. These are confined to lower ocean-near parts of the ice sheet (Qagssimiut area and Frederikshăb Isblink as shown in Fig. 17) or to large calf ice producing ice streams (Eqalorutsit kitdlit sermiat 1AH06001-03, Kangiata nunâta sermia $1 \mathrm{CH} 23003$, Jakobshavn Isbræ $1 \mathrm{GC} 06002$ and, north of the atlas area, Upernavik Isstrøm at $72^{\circ} \mathrm{N}$ ).

It is the ice streams which exhibit the greatest retreat over the last two centuries known in western Greenland with, as shown in Fig. 20, recessions of from 10-26 km. However, it should be noted that the tentative curves of frontal change of these ice streams are based on a few isolated dated events, and that the curves therefore mark trends rather than furnishing exact descriptions of frontal change.

For example, a short re-advance at Eqalorutsit kitdlit sermiat occurred around 1944, which moved the front of the glacier $4 \mathrm{~km}$ down the fjord for a few years only (Weidick, 1988b). Such short time pulsations may have occurred at many or all outlets, but they can usually not be documented from the scattered evidence at individual localities. Monitoring of Landsat imagery may reveal the frequency of future secondary pulsations of this type. Studies of the most recent decade of Landsat imagery at Jakobshavn Isbræ (Stove et al., 1984) has revealed tertiary annual pulsations of this glacier front, with winter positions about $2 \mathrm{~km}$ further out in the fjord than summer positions.

Apart from their great amplitude the curves of Fig. 20 also indicate the variety of trends that also occur at lowland outlets with minor changes, namely that some 


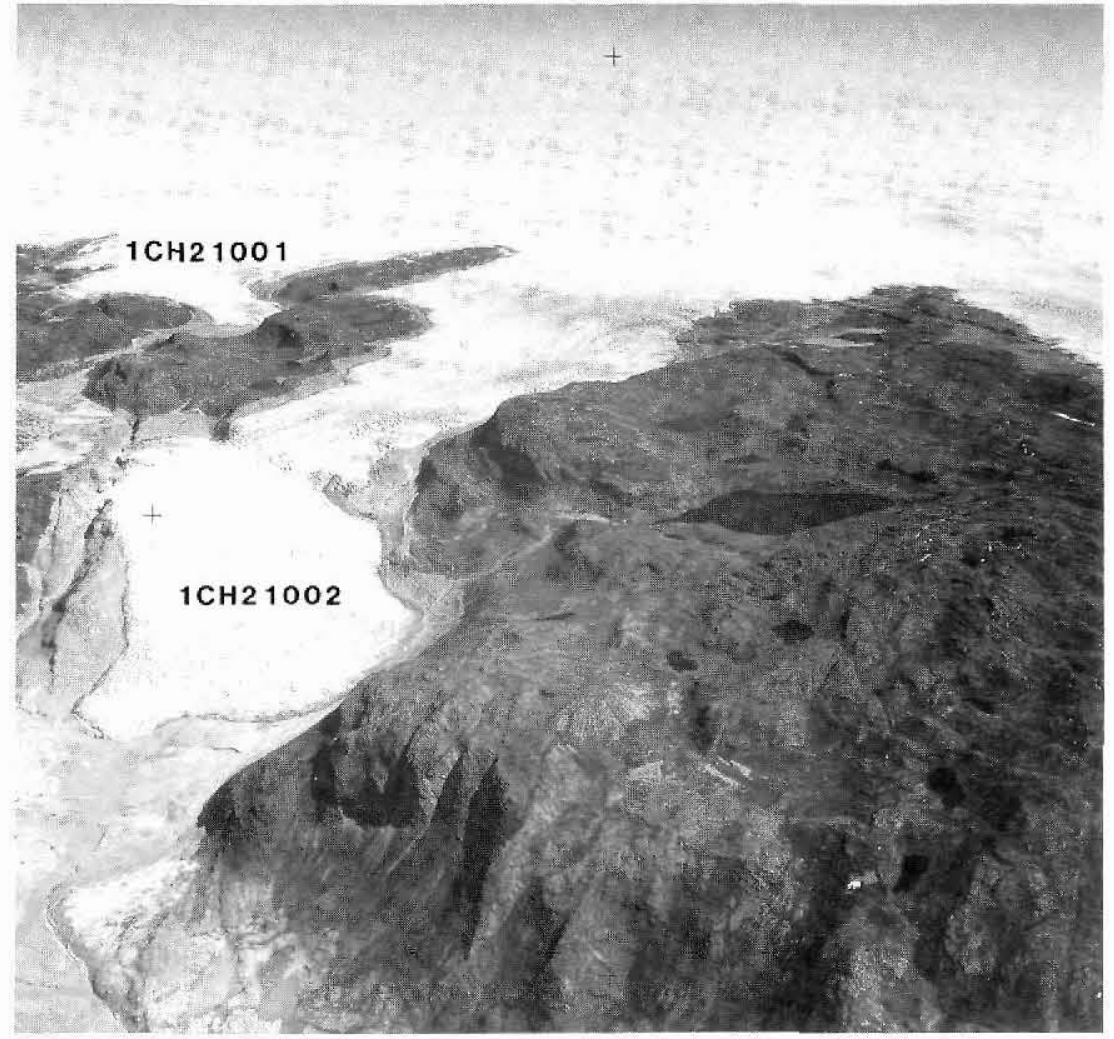

Fig. 18. Outlets $1 \mathrm{CH} 21001$ and 1CH21002 (Qamanârssûp sermia) seen from the west. The outlets can be seen surrounded by a wide trimline zone indicating the maximum extent in the 18 th century (cf. Fig. 21). The recession of $1 \mathrm{CH} 21001$ is reversed to an advance around 1960. Kort- og Matrikelstyrelsen. Denmark, series 505 E- $\varnothing$ no. 7419 . August 1948. Published with permission A.200/87.

glaciers attained their maximum position already in the 18 th century whereas most others first reached their maximum extent in the 19th century (Weidick, 1968). For all the outlets shown, the 20th century is remarkable for a general shrinkage until the 1950 s, followed by an initial recent re-advance traced at some of the more sensitive glaciers.

Few data are available on the bathymetry of the main ice producing fjords, on the annual changes of rate of movement or position of glacier fronts of the ice streams, and of the thickness of the ice streams themselves. It is therefore difficult to establish the role and importance of tidewater glaciers in the total mass balance.

The topographic influence on change of ice flux from one sector to another can be illustrated in the changes of Jakobshavn Isbra (Fig. 20). A pronounced thinning of Jakobshavn Isbræe after 1850 led to protrusion of subglacial thresholds at the southern margin of Jakobshavn Isbra. This caused a change in direction of some of the ice flow from Jakobshavn Isbræ to the region around Alángordliup sermia (1GB06001) and Sarqardliup sermia (1GB07001), sufficient to cause apparent stability or re-advance of the ice margin in these areas in spite of the increased ablation in the middle of this century (Weidick, 1968).

\section{Modelling of inland ice margin response}

Theoretical calculations of the change of the ice margin position from A.D. 600-1980 (Reeh, 1983) are based on current data on the topography of the ice margin surface and subsurface, the measured climatic record of Jakobshavn since 1873, the ice margin mass balance since 1982, and ice core records of changes in accumulation and temperature during the past 1400 years in central Greenland (Milcent and Crete stations).

The calculations include the Pâkitsoq area north of Jakobshavn Isbra (sectors 1GC04003 to 1GE04003), and the results from two of the sectors (1GE08001 and GC04003) are shown in Fig. 21. Mode and amplitude for the past few centuries fit in well with the historically documented trends, with one minor discrepancy - that the advance of 1880 at GE08001 reached a maximum, whereas on the calculated curve 1750 showed a maximum. However, the discrepancy is only a matter of 100-200 metres and, as was emphasized by Reeh (1983), the basal data for the calculations are still insufficient for such detail. 


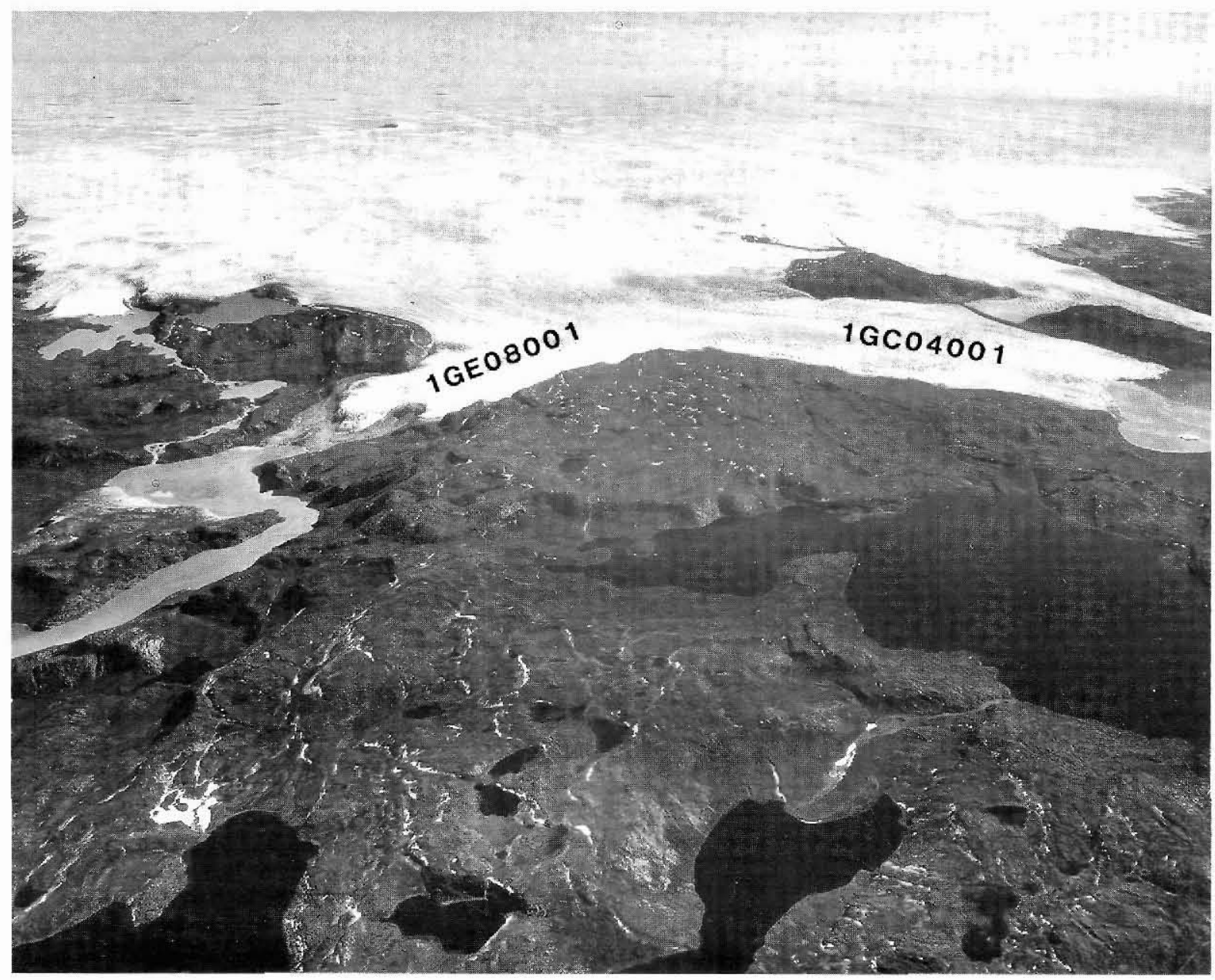

Fig. 19. Outlets 1GE08001 and 1GC04001 seen from the west. The sectors are surrounded by trimline zones indicating the recession since around 1880. Kort- og Matrikelstyrelsen, Denmark, series 518 A- $\varnothing$ no. 1474, 18th July 1949. Published with permission A.200/87.

\section{Mass balance measurements}

Mass balance measurements have been made on the following outlets of the Inland Ice:

Nordbogletscher (1AG05001) at latitude $61^{\circ} \mathrm{N}$ during the period 1978-1983 (Olesen, 1978; Clement, 1983).

Isortuarssup sermia (1CE05025) at $63^{\circ} 50^{\prime} \mathrm{N}$ during the period 1984-1988 (Braithwaite, 1989).

Qamanârssûp sermia (1CH21002) at $64^{\circ} 30^{\prime} \mathrm{N}$ during the period 1979-88 (Braithwaite, 1989).

Jakobshavn Isbræ $(1 \mathrm{GCO} 6002)$ at $69^{\circ} 15^{\prime}$ during the period of 1984-1988 (Eckelmeyer \& Harrison, 1990).

Pâkitsoq area (1GE04001-1GE07002) at $69^{\circ} 30^{\prime} \mathrm{N}$ since 1982 (Thomsen et al., 1988).

At most localities the measurements extend from the ice margin to the equilibrium line along a single traverse. Exceptions are Isortuarssup sermia and Jakobshavn Isbra where only a more restricted area was covered.
The height of the equilibrium line altitude can be estimated at about $1200 \mathrm{~m}$ a.s.l. in the Jakobshavn Isbræ area (Braithwaite \& Thomsen, 1984a. b; cf. section on Glaciation and snow lines). However, the $\delta^{18} \mathrm{O}-$ line (Reeh \& Thomsen, 1986), which also has a relation to the average ELA altitude points rather to an average of $1100 \mathrm{~m}$ for this locality (cf. Table 1).

Acknowledgements. Thanks are due to Grethe Fuglsang Hansen for the patient drawing of the 100 atlas map sheets (and for many suggested improvements during compilation) and to Peter Svendsen for typing and corrections. Editorial comments and corrections by colleagues W. S. Watt, A. K. Higgins and F. Kalsbeek of GGU are greatly appreciated.

The scientific content has benefitted from many discussions with our colleagues Niels Reeh, Henrik Højmark Thomsen, Roger Braithwaite and Ole B. Olesen.

Last but not least, thanks are due to the Geological Survey of Greenland for making the work and its publication possible. 
ACCUMULATION DYE -3
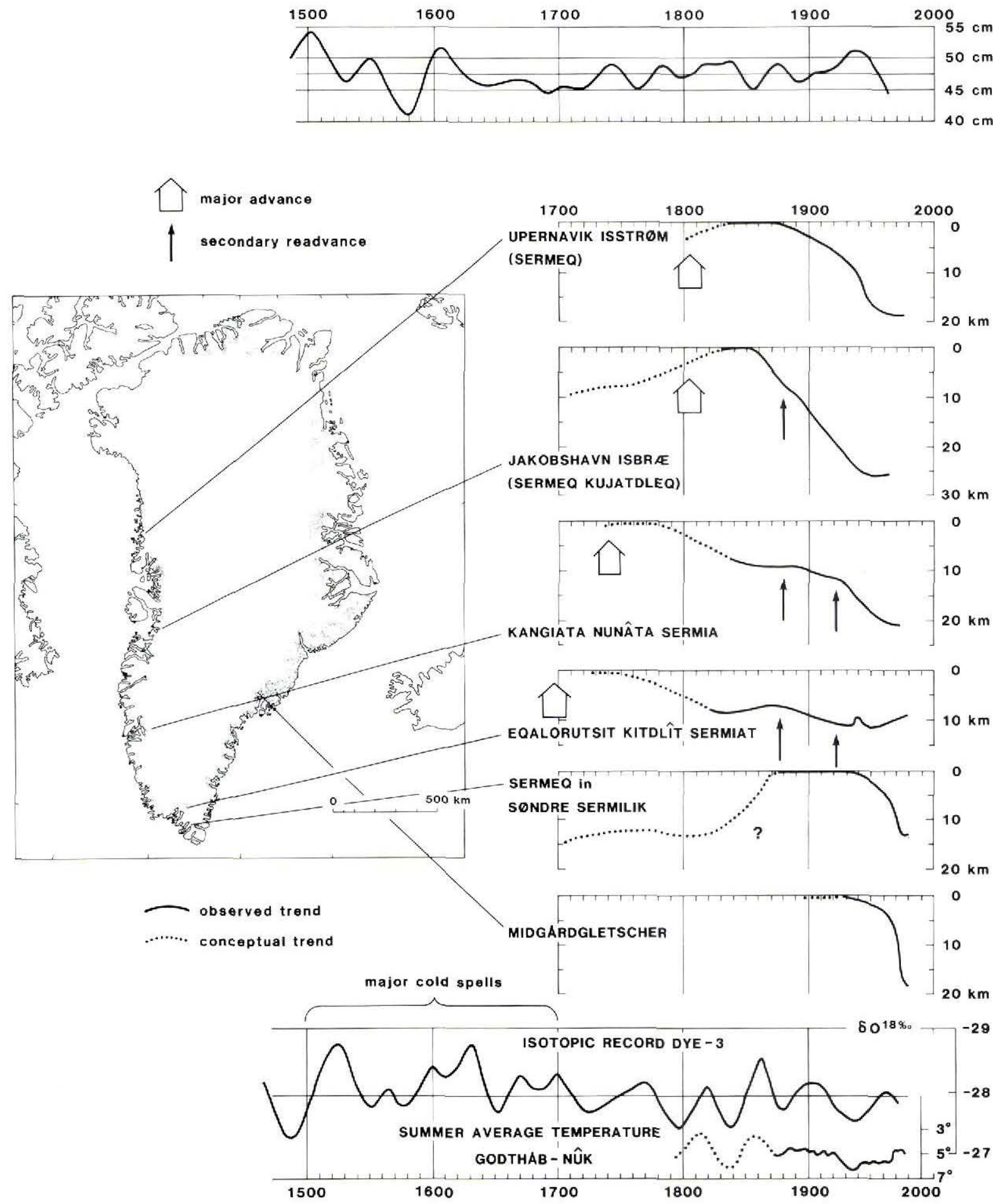

Fig. 20. Top: Variation of the annual accumulation (precipitation) at Dye 3 ice core record for the period A.D. 1500 to 1970 (after Dansgaard et al., 1973). Middle section: Response curves of some major calf-ice producing outlets from the Inland Ice (Upernavik Isstrøm, Jakobshavn Isbre, Kangiata nunata sermia and Eqalorutsit kitdlit sermiat) and from other extensive ice covers (Sermeq in Søndre Sermilik and Midgårdgletscher near Angmagssalik). Bottom: $\delta^{18} \mathrm{O}$ record from the Dye 3 ice core (Dansgaard $e t$ al., 1973 ) and summer temperatures in Godthåb (compiled from various sources; here from Weidick, 1982). 

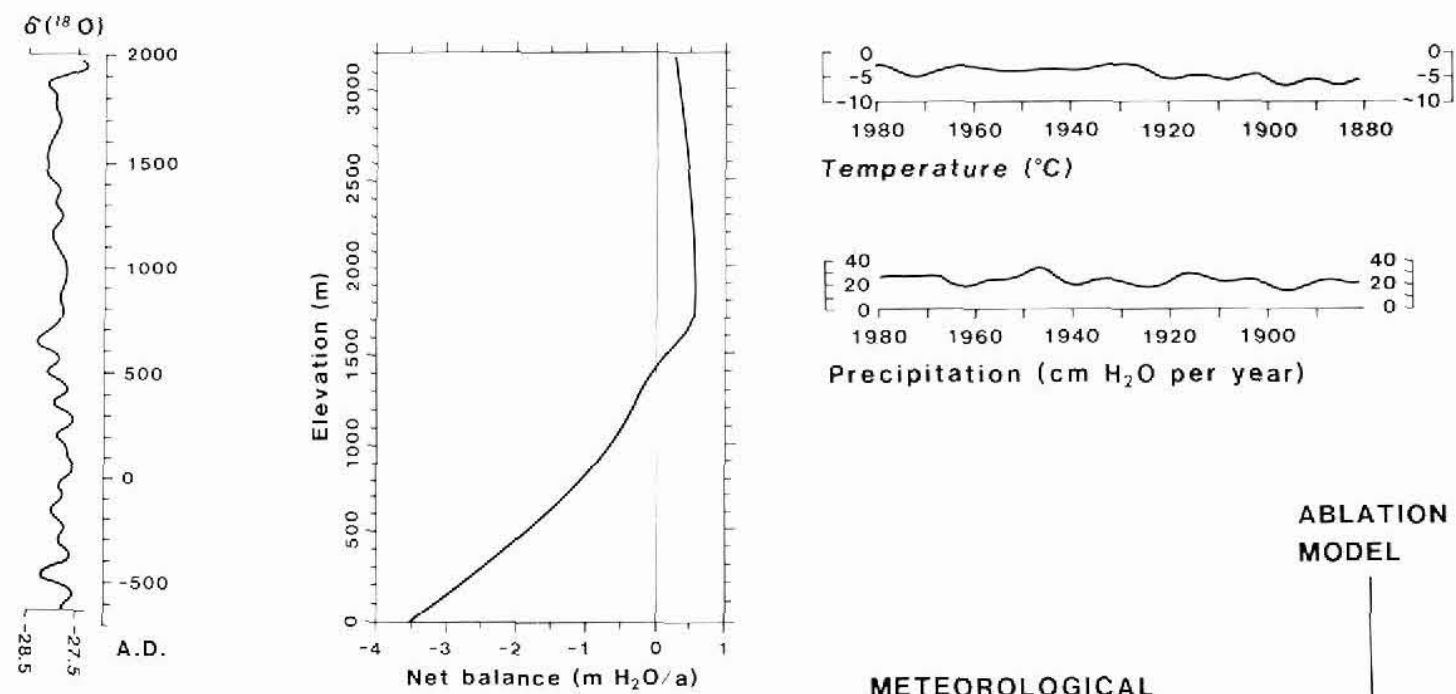

ICE CORE
INFORMATION

MASS BALANCE VS ALTITUDE
ABLATION MODEL

METEOROLOGICAL OBSERVATIONS JAKOBSHAVN SINCE 1873

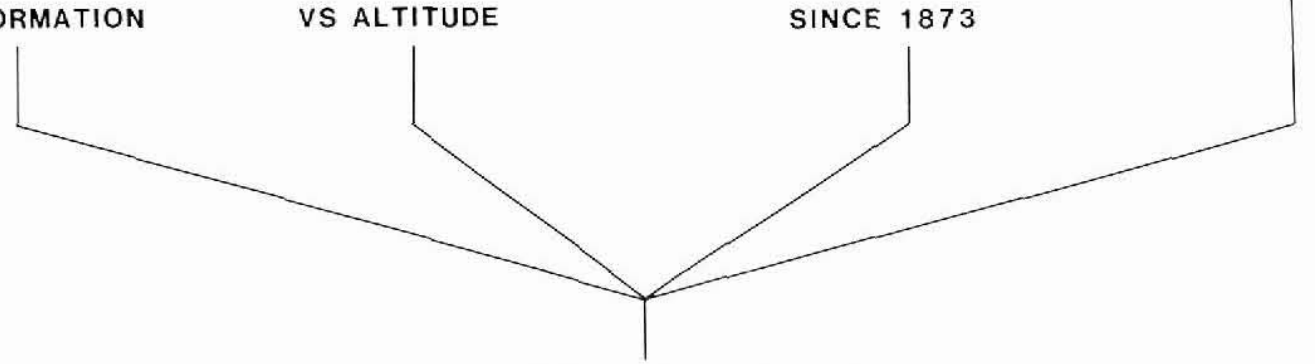

INPUT FOR CALCULATION OF 2600 YEARS OF MASS BALANCE HISTORY

ICE DYNAMICAL MODEL
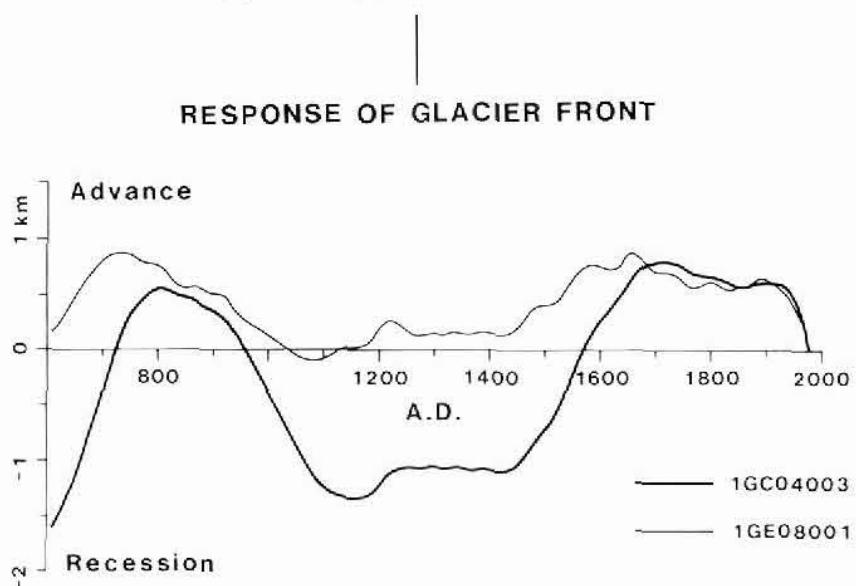

Fig. 21. Sketch of data and principle for simulation of the Inland Ice response to climatic change A.D. 600 to A.D. 1980 aceording to Rech (1983). 


\section{References}

ACG/Øresund 1981: Isukasia ore mine field investigations 1981 - report on movement of glaciers and ice surface elevation. Unpublished report by Arctic Consultant Gp., 4 pp.

Bauer, A. 1968a: Missions aériennes de reconnaissance au Groenland 1957-68. Meddr Gronland 173(3), $116 \mathrm{pp}$.

Bauer, A. 1968b: Mouvement et variation d'altitude de la zone d'ablation ouest de l'Indlandsis du Groenland entre 1948 et 1959. Meddr Gronland 174(1), 79 pp.

Belknap, R. L. 1941: Physiographic studies in the Holsteinsborg District of southern Greenland. Univ. Michigan Stud. Scient. Ser. 6, 205-255.

Benson, C. S. 1962: Stratigraphic studies in the snow and firn of the Greenland ice sheet. Res. Rep. Snow Ice Permfrost Res. Establ. 70, 93 pp.

Benson, C. S. \& Motyka, R. J. 1979: Glacier-volcano interactions on Mt. Wrangell, Alaska. In Annual Report of the Geophysical Institute, Univ. Alaska, 1977-78, 1-25.

Beschel, R. E. 1961: Dating rock surfaces by lichen growth and its application to glaciology and physiography (lichenometry). In Raasch, G. O. (ed.) Geology of the Arctic 2, 10441062.

Bindschadler, R. A. 1984: Jakobshavn glacier drainage basin: a balance assessment. J. geophys. Res. 89, C2, 2066-2072.

Bindschadler, R. A., Zwally, H. J., Major, J. A. \& Brenner, A. C. 1989: Surface topography of the Greenland ice sheet from satellite radar altimetry. Natn. Aeronaut. Space Admin. Spec. Publ. 503, 105 pp.

Braithwaite, R. J. 1989: Glaciers and hydropower for Nuuk / Godthåb, West Greenland. Open File Ser. Grønlands geol. Unders. 89/2, 49 pp.

Braithwaite, R. J. 1990: Recent thickening of the Greenland ice sheet was only transient. Unpubl. intern. GGU rep., 12 pp.

Braithwaite, R. J. \& Thomsen, H. H. 1984a: Runoff conditions at Kuussuup Tasia, Christianshåb, estimated by modelling. Grønlands geol. Unders. Gletscher-hydrol. Meddr 84/2, $23 \mathrm{pp}$.

Braithwaite, R. J. \& Thomsen, H. H. 1984b: Runoff conditions at Paakitsup Akuliaruserssua, Jakobshavn, estimated by modelling. Grønlands geol. Unders. Gletscher-hydrol. Meddr 84/3, 19 pp.

Bull, C. 1963: Glaciological reconnaissance of the Sukkertoppen Ice Cap, south-west Greenland. J. Glaciol. 4, 813-816.

Cailleux, A. \& Lagarec, D. 1977: Nombre, surface et volume des glaciers du globe. Studia Geologia Polonica 52, 83-96.

Carbonell, M. \& Bauer, A. 1968: Exploitation des couvertures photographiques aeriennes répétées du front des glaciers vêlant dans Disko Bugt et Umanak Fjord Juin-Juillet 1964. Meddr Grønland 173(5), 78 pp.

Chamberlin, T. C. 1894: Glacial studies in Greenland. II. The glaciers of Disko Island. J. Geol. 2, 768-788.

Clausen, H. B., Gundestrup, N. S. \& Johnsen, S. J. 1988: Glaciological investigations in the Crête area, central Greenland. Ann. Glaciol. 10, 10-15.

Clement, P. 1982: Glaciologi på Narssaq Bræ - massebalancen
1981 og 1982. Grønlands geol. Unders. Gletscher-hydrol. Meddr 82/5, 27 pp.

Clement, P. 1983: Glacial-hydrologiske forhold i Nordbos $\emptyset$ bassinet, Johan Dahl Land. Grønlands geol. Unders. Gletscher-hydrol. Meddr 83/9, 54 pp.

Clement, P. 1984: Glaciological activities in the Johan Dahl Land area, South Greenland, as a basis for mapping hydropower potential. Rapp. Grønlands geol. Unders. 120, 113121.

Colbeck, S. C. 1974: A study of glacier flow for an open-pit mine: an exercise in applied glaciology. J. Glaciol. 13, 401414.

Dansgaard, W., Johnsen, S. J., Clausen, H. B. \& Gundestrup, N. 1973: Stable isotope glaciology. Meddr Grønland 197(2), $53 \mathrm{pp}$.

Diamond, M. 1956: Precipitation trends in Greenland during the past 30 years. Res. Rep. Cold, Reg. Res. Engng Lab. 22, 4 pp.

Douglas, B. C., Cheney, R. E., Miller, L., Agreen, R. W., Carter, W. E. \& Robertson, D. S. 1990: Greenland Ice Sheet: is it growing or shrinking? Science $\mathbf{2 4 8}, 288$ only.

Drygalski, E. v. 1897: Grönland-Expedition der Gesellschaft für Erdkunde zu Berlin, 1891-1893. 1, 555 pp. Berlin: Kühl Publ. House.

Eckelmeier, K. \& Harrison, W. D. 1990: Jakobshavn Isbræ, West Greenland: seasonal variations in velocity - or lack thereof. J. Glaciol. 36, 82-88.

Etienne, E. 1940: Expeditionsbericht der Grönland-Expedition der Universität Oxford 1938. Veröff. Geophys. Inst. Univers. Leipzig (2), 13, 227 pp.

Fenger, J. \& Laut, P. 1989: Drivhuseffekten - global luftforurening og klimaaendringer, 186 pp. Fiskers Forlag.

Froda, F. 1925: Some observations made in North-Greenland 1923. Meddr Gronland 59(4), 39-54.

Frydendahl, K. 1989: Global og regional temperaturudvikling siden 1850. Danish Meteorological Institute, Scientific Report 89-6, 113 pp.

Gerdel, R. W. 1961: A climatological study of the Greenland ice sheet. Folia Geogr. Danica 9, 84-106.

Giesecke, K. L. 1910: Mineralogisches Reisejournal über Grönland 1806-13. 2te vollständige Ausgabe. Meddr Grønland 35, $532 \mathrm{pp}$.

Goldthwait, R. P. 1964: Tasersiaq area - Sukkertoppen Ice Cap studies. Ohio State University Research Foundation. Report 1701-Final, 65 pp.

Gordon, J. E. 1981: Glacier margin fluctuations during the 19th and 20th centuries in the Íkamiut kangerdluarssuat area, West Greenland. Arct. Alp. Res. 13, 47-62.

Gribbon, P. W. F. 1970: Frontal recession of Sermikavsak, West Greenland. J. Glaciol. 9, 279-282.

Holtzscherer, J.-J. \& Bauer, A. 1954: Contribution à la eonnaissance de l'Inlandsis du Groenland. Publs Ass. int. Hydrol. scient. 39, 244-296.

Hughes, T. 1986: The Jakobshavn effect. Geophys. Res. Letter 13, $46-48$. 
Humlum, O. 1985: The glaciation level in West Greenland. Arct. Alp. Res. 17, 311-319.

Jahn, A. 1938: Dyluwialne i postdyluwialne ruchy pionowe Grenlandii Zachodniej w świetle teras nadbrzeżnych fiordu Arfersiorfik. Czas. Geogr. 16, 307-324.

Jespersen, E. 1911: Nyopdagede Nordo-Ruiner JulianehaabDistrikt. Meddr Grønland 50(2), 97-104.

Kelly, M. 1975: A summary of the Quaternary geology of the Nordre Strømfjord area. Unpubl. intern. GGU rep., 13 pp.

Kelly, M. 1985: A review of the Quaternary geology of western Greenland. In Andrews, J. T. (ed.) Quaternary environments eastern Canadian Arctic, Baffin Bay and western Greenland, 461-501. Boston: Allen \& Unwin.

Knudsen, N. T. 1983: Photogrammetric investigations at glaciers in West Greenland. Rapp. Gronlands geol. Unders. 115, 115-117.

Knudsen, N. T. 1986: Changes of Nordbogletscher and Nordgletscher. Ann. Glaciol. 8, 106-110.

Knudsen, N. T. \& Møller, J. T. 1982: Photogrammetric survey of Qamanârssûp sermia. In Andreasen, J.-O., Knudsen, N. T. \& Møller, J. T. Glaciological investigations at Qamanârssûp sermia. Field Report 1980. Grønlands geol. Unders. Gletscher-hydrol. Meddr 82/4, 1-26.

Knudsen, N. T. \& Weidick, A. 1981: Glacier inventory, West Greenland $60^{\circ}-64^{\circ} \mathrm{N}$. Gronlands geol. Unders. Gletscher-hydrol. Meddr 81/4, 14 pp. + maps and tables.

Koch, J. P. \& Wegener, A. 1930: Wissenschaftliche Ergebnisse der dänischen Expedition nach Dronning Louises-Land und quer über das Inlandeis von Nordgrönland 1912-13. Unter Leitung von Hauptmann J. P. Koch. Meddr Grønland 75, 676 pp.

Langway, C. C. 1962: Some physical and chemical investigations of a 411 meter deep Greenland ice core and their relationship to accumulation. Publs Ass. int. Hydrol. scient. 58, 101-118.

Larsen, L. B. 1973: Water balance investigations in the Narssaq River Basin, South Greenland. A contribution to the International Hydrological Decade. 159. Unpublished dissertation, Univ. Copenhagen.

Lingle, C. S., Hughes, T. J. \& Kollmeyer, R. C. 1981: Tidal flexure of Jakobshavns Glacier, West Greenland. J. geo phys. Res. 86, B5, 3960-3968.

Lysgaard, L. 1949: Recent climatic fluctuations. Folia Geogr. Danica 5, part 1 (text) 85 pp., part 2 (tables) 94 pp., part 3 (curves) 35 pp.

Meier, M. F. 1985: Mass balance of the glacier and the small ice caps of the world. In Lingle, C. S. (ed.) Glaciers, ice sheets and sea level: effects of a $\mathrm{CO}_{2}$-induced climate change. Report on workshop held in Seattle, Washington, Sept. 13-15, 1984, 139-144.

Meier, M. \& Post, A. 1987: Fast tidewater glaciers. J. geophys. Res. 92, B9, 9051-9058.

Müller, F., Caflisch, T. \& Müller, G. 1977: Instructions for compilation and assemblage of data for a World Glacier Inventory. Temporary Technical Secretariat for World Glacier Inventory, International Commission on Snow and Ice, Department of Geography, Swiss Federal Institute of Technology (ETH), Zürich, 28 pp. + appendix.
Müller, F. \& Scherler, K. 1980: Some comments on the Swiss glacier inventory. Publs Ass. int. Hydrol. scient. 126, 185192.

Nordenskiöld, A. E. 1886: Grönland, seine Eiswüsten im Inneren und seine Ostküste. Schilderungen der Zweiten Dickson'schen Expedition ausgeführt im J. 1883, 505 pp. Leipzig: Brockhaus.

Ohmura, A. 1987: New temperature distribution maps for Greenland. Z. Gletscherkunde Glazialgeol. 23, 1, 1-45.

Olesen, O. B. 1978: Glaciological investigations in Johan Dahl Land, South Greenland, as a basis for hydroelectric planning. Rapp. Gronlands geol. Unders. 90, 84-86.

Olesen, O. B. 1986: Fourth year of glaciological field work at Tasersiaq and Qapiarfiup sermia, West Greenland. Rapp. Grønlands geol. Unders. 130, 121-126.

Østrem, G., Selvig, K. D. \& Tăndberg, K. 1988: Atlas over Breer i Sør-Norge. Revidert utgave utarbeidet på grunnlag af flybilder tatt i perioden 1969-1986. Meddelelse $\mathrm{nr} .61 \mathrm{fra}$ Hydrologisk Avdeling, Norges Vassdrags- og Energiverk, Vassdragsdirektoratet. Text: $54 \mathrm{pp}$., maps and tables, $180 \mathrm{pp}$.

Paterson, S. 1968: The physics of glaciers, $380 \mathrm{pp}$. 2nd edition 1981. Pergamon Press.

Petersen, F. 1898: Opmaalingsexpditionen til EgedesmindeDistrikt 1897. Beretning om Rejsen. Meddr Grønland 14(3), 263-399.

Petersen, H. C. 1979: Et kajakfund fra Kûkulûp tasia. Grønland 1979, 257-263.

Pitman, G. T. K. 1973: A lichenometrical study of snow patch variation in the Frederikshåb district, South-West Greenland, and its implications for studies of climatic and glacial fluctuations. Bull. Grønlands geol. Unders. 104 (also Meddr Grønland 192,7), $31 \mathrm{pp}$.

Ragle, R. H. \& Davies, T. C. 1962: South Greenland traverses. J. Glaciol. 4, 129-131.

Reeh, N. 1983: Ikke-stationær beregningsmodel for Indlandsisens randzone. Grønlands geol. Unders. Gletscher-hydrol. Meddr 83/7, $81 \mathrm{pp}$.

Reeh, N. 1989: Dynamic and climatic history of the Greenland Ice Sheet. In Fulton, R. J. (ed.) Quaternary geology of Canada and Greenland. Geology of Canada 1, Chapter 14 (also The geology of North America K-1. 793-822. Boulder: Geol. Soc. America).

Reeh, N. \& Thomsen, H. H. 1986: Model for simulering af ilt-isotop variation i smeltevandsafstrømningen fra Indlandsisens rand ved Pâkitsup akuliarusersua, Jakobshavn. Grønlands geol. Unders. Gletscher-hydrol. Meddr 86/1, 34 pp.

Reeh, N., Clausen, H. B., Dansgaard, W., Gundestrup, N., Hammer, C. U. \& Johnsen, S. J. 1978: Secular trends of accumulation rates of three Greenland stations. I. Glaciol. 20, 27-30.

Reeh, H., Hammer, C. U., Thomsen, H. H. \& Fisher, D. A. 1987: Use of tracer constituents to test flow models for ice sheets and ice caps. In The physical basis of ice sheet modelling. Publis Ass. int. Hydrol. scient. 170, 299-310.

Rink, H. 1857: Grønland, geographisk og statistisk beskrevet. Bd. 1, Det Nordre Inspectorat, 206 pp. København: A. Høst og Søn.

Rosing, J. 1988: Rensdyrjægere i Itinnera. In Møbjerg, T., 
Grønnow, B. \& Schulz-Lorentzen, H. (ed.) Paloeoeskimoisk forskning $i$ Grønland, 69-79. Aarhus Universitetsforlag.

Rundle, A. S. 1965: Glaciological investigations on Sukkertoppen Ice Cap, southwest Greenland, summer 1964. Rep. Inst. Polar Studies 14, $11 \mathrm{pp.}$

Scholz, H. 1987: Expeditionsbericht Westgrönland 1986/87. Beiträge zur Geologie und Botanik der eisrandnahe Gebiete in der Umgebung des Örkendals westlich von Söndre Strömfjord, Westgrönland, $87 \mathrm{pp}$. Unpublished report.

Seckel, H. 1977: Höhenänderungen in Grönländischen Inlandeis zwischen 1959 und 1968. Meddr Grønland 187(4), 58 pp.

Steenstrup, K. J. V. 1881: Bemærkninger til et geognostisk Oversigtskaart over en Del af Julianehaabs Distrikt. Meddr Gronland 2(2), 27-41.

Steenstrup, K. J. V. 1901: Beretning om en Undersøgelsesrejse til Øen Disko i Sommeren 1898. Meddr Grønland 24(3), 249-306.

Stove, G. C., Green, K., Birnie, R. V., Davison, G., Bagot, K., Palmer, M., Kearn, G., Ritchie, P. F. S. \& Sugden, D. E. 1984(?): The use of Landsat data to monitor iceberg production. Monitoring ice production from West Greenland tidewater glaciers using Landsat data: Results from the AGRISPINE experiment for the Jakobshavn Isbra, Macaulay Institute for Soil Research, Craigiebuckler, Aberdeen, Scotland, and the National Remote Sensing Centre, Space and new Concepts Department, Royal Aircraft Establishment, Farnborough, Hampshire, England, 32 pp.

Sugden, D. 1972: Deglaciation and isostasy in the Sukkertoppen Ice Cap area, West Greenland. Arct. Alp. Res. 4, 97117.

Thomsen, H. H. 1983: Satellitdata - et redskab til studier af Indlandsisens randzone i forbindelse med vandkraftunders $\emptyset$ gelser. Grønlands geol. Unders. Gletscher-hydrol. Meddr 83/8, 24 pp.

Thomsen, H. H. 1984: Glaciologiske undersøgelser i Disko Bugt området 1983. Grønlands geol. Unders. Gletscher-hydrol. Meddr 84/1, 31 pp.

Thomsen, H. H. \& Olesen, O. B. 1990: Continued glaciological investigations with respect to hydropower and ice-climate relationships, at Pâkitsoq, Jakobshavn, West Greenland. Rapp. Grønlands geol. Unders. 148, 83-86.

Thomsen, H. H., Thorning, L. \& Braithwaite, R. J. 1988; Glacier-hydrological conditions on the Inland Ice north-east of Jakobshavn / Ilulissat, West Greenland. Rapp. Grønlands geol. Unders. 138, 1 map sheet.

Thomsen, Th. \& Jørgensen, G. H. 1984: Hydrological datamodel work in Greenland. Nordic Hydrol. 15, 39-56.

Thorning, L. \& Hansen, E. 1987: Electromagnetic reflection survey 1986 at the Inland Ice margin of the Pâkitsoq basin, central West Greenland. Rapp. Grønlands geol. Unders. 135, 87-95.

Weidick, A. 1963: Ice margin features in the Julianehåb district, South Greenland. Bull. Grønlands geol. Unders. 35 (also Meddr Grønland 165,3), 133 pp.

Weidick, A. 1968: Observations on some Holocene glacier fluctuations in West Greenland. Bull. Gronlands geol. Unders. 73 (also Meddr Grønland 165,6), 202 pp.

Weidick, A. 1980: Hydrological basins in West Greenland and their relationship to a preliminary sectorial division of the Inland Ice sheet. Publs Ass. int. Hydrol. scient. 126, 49-55.

Weidick, A. 1985: Review of glacier changes in West Greenland. Z. Gletscherk. Glazialgeol. 21, 301-309.

Weidick, A. 1988a: Gletschere i Sydgrønland. Geologi i Grønland 2, $80 \mathrm{pp}$.

Weidick, A. 1988b: Surging glaciers in Greenland - a status. Rapp. Grønlands geol. Unders. 140, 106-110.

Weidick, A. 1990a: Glaciers and the hydropower planning for Greenland. 'Polartech', Proceedings of the International conference on development and commercial utilization of technologies in polar regions, 14-16 August 1990, Copenhagen, 654-663.

Weidick, A. 1990b: Human activities and the ice cover of Greenland. In Kotlyakov, V. N. \& Sokolov, V. E. (ed.) Arctic research. Advances and prospects 2, 23-34. Moscow: Nauka.

Weidick, A. 1991: Present-day expansion of the southern part of the Greenland Inland Ice. Rapp. Gronlands geol. Unders. 152, 73-79.

Weidick, A. \& Olesen, O. B. 1980: Hydrological basins in West Greenland. Rapp. Grønlands geol. Unders. 94, 51 pp.

Weidick, A. \& Thomsen, H. H. 1983: Lokalgletschere og Indlandsisens rand $\mathrm{i}$ forbindelse med udnyttelse af vandkraft i bynære bassiner. Grønlands geol. Unders. Gletscher-hydrol. Meddr 83/2, 129 pp.

Weidick, A. \& Thomsen, H. H. 1986: A decade of glacier investigations for utilisation of Greenland hydropower. Rapp. Grønlands geol. Unders. 128, 157-169.

Weidick, A., Oerter, H., Reeh, N., Thomsen, H. H. \& Thorning, L. 1990: The recession of the Inland Ice margin during the Holocene climatic optimum of the Jakobshavn Isfjord area of West Greenland. Palaeogeogr. Palaeoclimat. Palaeoecol. (Global planet. Change Sect.) 82, 389-399.

Williams, R. S. \& Carter, W. D. 1976: ERTS-1 a new window on our planet, 362 pp. Washington: US Government Print Office.

Zwally, H. J. 1989: Growth of Greenland ice sheet: interpretation. Science 246, 1589-1591. 


\section{Glacier names in West Greenland $c .60^{\circ}-71^{\circ}$}

The geographical names of the listed glaciers are taken from the 1:250 000 map sheets published by Kort- og Matrikelstyrelsen, Danmark (KMS: formerly the Geodetic Institute) or those found in descriptions of the glaciers.

The Greenland Place Names Committee was formerly the authority responsible for approval of old and new geographical names in Greenland. Responsibility for authorization of new place names was transferred to the Greenland Home Rule authorities in Nûk / Godthåb in 1984. The list below was prepared by Anker Weidick, with the assistance of E. Hansen (place name office, KMS); control of translation and corrections of localities was undertaken by H. C. Petersen (Nunat Aqqinik Aalajangiisartut - Grønlands Stednavnsnævn Greenland Place Names Authority).

Many glaciers have both Danish (in the list designated 'Dan.') and Greenlandic place names. Some Danish place names have a hybrid construction in the sense that a nearby Greenlandic place name is used in connec- tion with the designation for a glacier, i.e. 'Gletscher' (German) or 'Bræ' (Norwegian); e.g. 1 BB 05004 'Arsuk Bræ'. Some glaciers were named after persons (e.g. 1 FC 19001 'Nordenskiöld Gletscher') or for a country (e.g. 1 FC 30001 'Polonia Gletscher'). Names of USA origin are found around Søndre Strømfjord (designated by 'Am.'); e.g. 1 DG 02002 'Russell Gletscher'.

Place names of Greenlandic origin have a descriptive character, as is evident from the translations given below. The spelling employed is that of the old Greenland orthography, that still found on nearly all published maps sheets. A new orthography, decreed in 1973 by the Greenland authorities, is gradually replacing the old, and the new spellings immediately follow the original spellings in brackets; English translations are given in inverted commas. Where several sectors are covered by the same glacier name the identification codes are grouped together.

Locations of the place names given below are shown in Fig. 22 by their identification codes. 


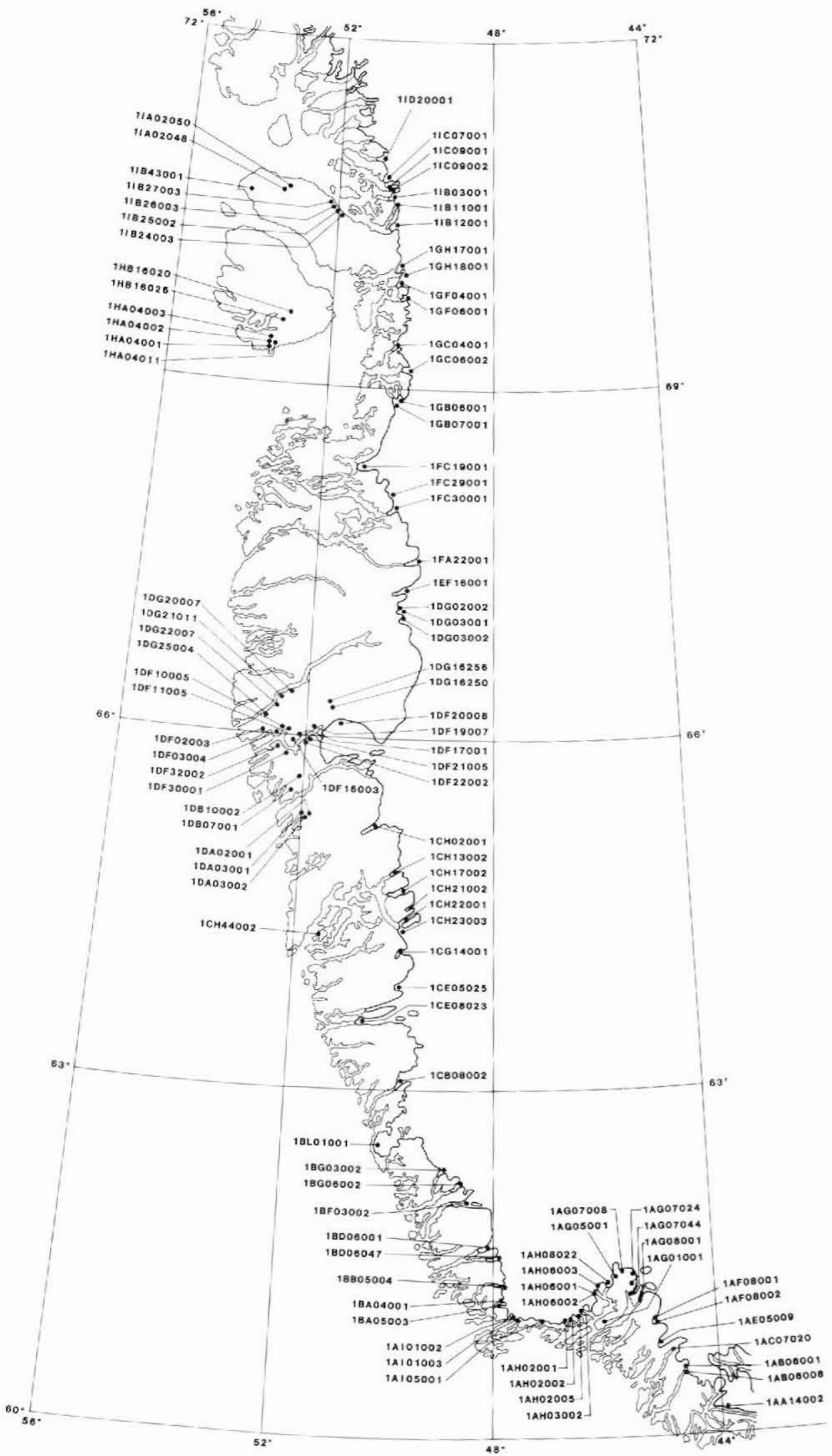

Fig. 22. Location of glacier names given in translation. 
1 AA 14002 Sermêrúnerit (Sermeerunnerit): 'Where the glacier is melted away'.

1 AB 06001 Sermeq (Sermeq): 'The glacier'.

$1 \mathrm{AB} 06008$ Sermitsiaq (Sermitsiaq): 'The rather large glacier'.

1 AC 07020 Sermeq (Sermeq): 'The glacier'.

1 AE 05009 Sioragdlip sermia (Siorallip Sermia): 'The glacier of the sand plain'.

1 AF 08001 Jespersen Bræ (Dan.). For the Rev. E. Jespersen, Julianehåb / Qaqortoq, who described the area and its glaciers (Jespersen, 1911).

1 AF 08002 Sermeq kangigdleq (Sermeq Kangilleq): 'The easternmost (and/or innermost) glacier'.

1 AG 01001 Narssaq Bræ (Dan.). After the town of Narssaq (Narsaq): 'The plain'.

1 AG 05001 Nordbogletscher (Dan.). Nordbo = Norseman. Kûkulûp sermia (Kuukuluup Sermia): 'The glacier of the poor (or mediocre) river'.

1 AG 07008 Nordgletscher (Dan.). Nord = north.

1 AG 07024 Østgletscher (Dan.). Øst = cast.

1 AG 07044 Sydgletscher (Dan.). Syd:= south.

1 AG 07044 Kiagtût sermiat (Kiattuut Sermiat): 'The glacier of the warm places'.

1 AG 08001 Qôrqup sermia (Qooqqup Sermia): 'The glacier in the valley'.

1 AH 02001 Qaleragdlit sermia (Qalerallit Sermia): 'The glacier of the halibut'

1 AH 02002 Naujat sermiat (Naajat Sermiat): 'The glacier of the sea- gulls'.

1 AH 02005 Ilordlit sermiat (Ilorlliit Sermiat): 'The glacier of the innermost parts' (of the fjord?).

1 AH 03002 Sermínguaq (Serminnguaq): 'The small glacier'.

$1 \mathrm{AH} \mathrm{06001,} 1 \mathrm{AH} 06002$ and

1 AH 06003 Eqalorutsit kitdlit sermiat (Eqalorutsit Killiit Sermiat): 'The western glacier of the poor salmon place'. Comment: Eqalorutsit (the poor salmon place) is situated near this glacier. It is possible that the place name in fact only relates to the western parts of this glacier (1 AH 06001 and $1 \mathrm{AH} \mathrm{06002)}$ whereas $1 \mathrm{AH} 06003$ should be designated as the eastern branch of the glacier (Eqalorutsit kangigdlît sermiat, see below).

1 AH 08022 Eqalorutsit kangigdît sermiat (Eqalorutsit Kangilliit Sermiat): 'The eastern glacier of the poor salmon place'. It is possible that the place name in fact covers $1 \mathrm{AH} 06003$ (see above) since the local population uses Qajütap sermia for glacier $1 \mathrm{AH} 08022$.

1 AH 08022 Qajũtap sermia (Qajuuttap Sermia): 'Qajutaq's glacier'. Qajútaq (Qajuuttaq): 'The ladle' was the name of a hunter who lived at the present town of Narssaq in the beginning of the 19th century (Giesecke, 1910, p. 220). This glacier name was documented by H. C. Petersen (1979).

1 AI 01002 Nordre Qipisarqo Bræ (Dan.). Qipisarqo (Qipisaqqo): 'The river that bends and winds'. Nordre $=$ northern.

1 AI 01003 Søndre Qipisarqo Bræ (Dan.). Søndre = south- ern.

1 AI 05001 Sermilik Bræ / Sermitsialik Bræ (Dan.). Sermilik (Sermilik): 'Place with glacier(s)' or 'Icefjord'. In older publications the name is found as: Sermitsialik (Sermitsialik): 'The place with the rather large glacier'.

1 BA 04001 Nordre Qôrnoq Bra (Dan.). Qôrnoq (Qoornoq): 'Narrow sound between two mountain tracts'. Nordre $=$ northern.

1 BA 05003 Søndre Qôrnoq Bræ (Dan.). Søndre = southern. 1 BB 05004 Arsuk Bræ (Dan.). Arsuk (Arsuk): 'The favourite place'. Sermeq (Sermeq): 'The glacier' (e.g. $1 \mathrm{AB} 06001,1 \mathrm{AC} 07020$ ).

1 BD 06001 Sermiligârssuk Bræ (Dan.). Sermiligârssuk (Sermiligaarsuk): 'The inconspicuous (fjord) which is provided with glaciers'.

1 BD 06047 Sioralik Bra (Dan.). Sioralik (Sioralik): 'The place with a sandy plain'.

1 BF 03002 Sermilik Bra (Dan.). Sermilik (Sermilik): 'Place with glacier(s)'. Ukâssorssuaq (Ukaasorsuaq): 'The great glacier which usually calves frequently' (calving by falling down: ukarpoq).

1 BG 03002 Avangnardleq Bræ (Dan.). Avangardleq (Avannarleq): 'The northernmost'.

1 BG 06002 Nigerdlikasik Bræ (Dan.). Nigerdlikasik (Nigerlikasik): 'The insignificant place situated farthest out towards the south-west wind'.

1 BL 01001 Frederikshåb Isblink (Dan.). After the town of Frederikshåb / Pâmiut (Paamiut). 'Isblink' originally an expression for the glare over the Inland Ice which can be observed from far out to sea. Here the meaning has come to denote a large lobe from the Inland Ice.

1 CB 08002 Nákâssorssuaq (Nakkaasorsuaq): 'The great calving glacier' (from nakaiva: drops it, i.e. calving by falling down) ef. 1 DF 19007 . Spelt Nakaissorssuaq on 1:250 000 KMS map.

1 CE 05025 Isortuarssûp sermia (Isortuarsuup Sermia): 'The glacier of the very muddy locality'.

1 CE 08023 Sermilik (Sermilik): 'The ice fjord'. Comment: The name refers to the fjord in front of the glacier (misplaced on 1:250 000 KMS map). Glacier name: Sermeq (cf. 1 AB 06001, 1 AC 07020).

1 CG 14001 Kangaussarssûp sermia (Kangaassarsuup Sermia): 'The glacier at the large headland-like area'.

1 CH 02001 Sarqap sermerssua (Saqqap Sermersua): 'The great glacier of the sunny side'.

$1 \mathrm{CH} 13002$ Kangilínguata sermia (Kangilinnguata Sermia): 'The glacier of the small country to the east'.

$1 \mathrm{CH} 17002$ Narssap sermia (Narsap Sermia): 'The glacier of the plain'.

1 CH 21002 Qamanârssûp sermia (Qamanaarsuup Sermia): 'The glacier at the place where one lies in wait (for game)'.

1 CH 22001 Akugdlerssûp sermia (Akullersuup Sermia): 'The glacier of the large area in the middle'.

1 CH 23003 Kangiata nunâta sermia (Kangiata Nunaata Sermia): 'The glacier of the country to the east'. 
1 CH 44002 Sermitsiaq (Sermitsiaq): 'The rather great glacier'.

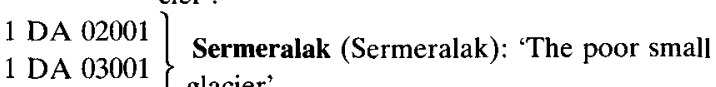

1 DA 03002 'glacier'.

1 DB 07001 Apússuit (Apussuit): 'The great amounts of snow'.

1 DB 10002 Qapiarfiup sermia (Qapiarfiup Sermia): 'The scraping bench (name of a mountain) glacier'.

1 DF 02003 Sermeralak (Sermeralak): 'The poor little glacier'.

1 DF 03004 Sermitsiaq (Sermitsiaq): 'The rather large glacier'.

1 DF 10005 Avangnardliup sermia (Avannarliup Sermia): 'The glacier of the northernmost branch (of a fjord)'.

1 DF 11005 Tâterât sermiat (Taateraat Sermiat): 'The glacier of the kittiwakes (mountain)'.

1. DF 16003 Tâterâtsiait sermiat (Taateraatsiaat Sermiat): 'The glacier of the rather large kittiwakes (mountain)'.

1 DF 17001 Tâterâtsiait sermiat (Taateraatsiaat Sermiat). As above.

1 DF 19007 Nákarajôq (Nakkarajooq): 'The glacier that calves frequently' (nákarpoq: fall down).

1 DF 20008 Quantum Gletscher (Dan.). Origin of name unknown.

1 DF 21005 Aulasâma (Aalasaama): 'The glacier that brings fish up (to the surface)'. Comment: When the ice drops into the fjord (during calving) fish are thrown up to the surface.

1 DF 22002 Tapa (Tapa): 'The dancer'. The name relates to avalanches from the steep mountain slopes at the glacier, sounding like the stamping feet of a dancer. Greenlandic dancing involves stamping on the ground.

1 DF 23004 Inorersut (Inorersut): 'The killer'. This hanging glacier has killed passing kayakers by its calving.

1 DF 30001 Umingmak (Umimmak): 'The musk ox'. Comment: The musk ox was first introduced into West Greenland in the 1960s whereas the place name is older. It is therefore unsure whether the derivation from the root of the word (umik: beard, whiskers of a seal, curtain of a tent) should be translated in this way.

1 DF 32002 Sârdloq (Saarloq): 'The bald'. The locality is situated at a low, level and bare foreland.

1 DG 02002

1 DG 03001 Russell Glacier (Am.). Name found on map of the 1927-30 University of Michigan Expeditions to the Søndre Strømfjord area (Belknap, 1941).

1 DG 03002 Leveret Glacier (Am.). As above.

1 DG 16250 Tasersiaq Gletscher (Dan.), cf. below. Tasersiaq: 'The lake added over and above, or additional lake'.

1 DG 16256 Sarfartôq Gletscher (Dan.). Sarfartôq (Sarfartooq): 'The place with a strong current'. Miligaersartoq (Miligaarsartoq): 'The glacier that blocks it (i.e. the river Tasersiaq) but will usually be broken up'. On the 1:250 000 KMS maps the name Miligaersartoq, possibly by mistake, is attached to 1 DG 16250.

1 DG 20007 Sermínguaq (Serminnguaq): 'The small glacier'.

1 DG 21011 Vimmelskaftet (Dan.). For a Copenhagen street with a winding (sinuous) form. Name refers to the winding form of the glacier lobe.

1 DG 22007 Lyngbræ (Dan.). Lyng = heather. Might refer to the surroundings of the glacier front.

1 DG 25004 Fagerbræ (Dan.). Fager = an old lyric word for beautiful, bræ is glacier.

1 EF 16001 Isúnguata sermia (Isunnguata Sermia): 'The glacier at the small terminal area' e.g. small land incission in the ice margin.

1 FA 22001 Inugpait quatat (Inuppaat Quuat): 'Urinating place for many people' (might refer to the strange odour of the vegetation on the mountain slopes). According to information from the local population (H. C. Petersen, personal communication), this place name should refer to a river about 10 $\mathrm{km}$ north of the glacier outlet, draining sectors 1 FA 21001 and 1 FA 21002. The name of 1 FA 22001 should be Avangnardliup sermia.

1 FA 22001 Avangnardliup sermia (Avannarliup Sermia); 'The glacier of the northern area'.

1 FC 19001 Nordenskiöld Gletscher (Dan.). For A. E. Nordenskiöld, Swedish geologist and explorer who in 1870 and 1883 investigated and described the glacier (Nordenskiöld, 1886). Akuliarutsip sermerssua (Akuliarutsip Sermersua): 'The large glacier between the mountains'.

1 FC 29001 J. P. Koch Gletscher (Dan.). For colonel J. P. Koch, Danish explorer and surveyor who crossed the Inland Ice in 1912-13 (Koch \& Wegener, 1930).

1 FC 30001 Polonia Gletscher (Dan.). The Danish name originates from a Polish expedition that investigated the outlet and its surroundings in 1937 ( $\mathrm{Jahn}$, 1938). Usugdlûp sermia (Usulluup Sermia): 'The glacier at the bad penis'. The place name (Usugdluk) refers to a narrow headland in the fjord in front of the glacier.

1 GB 06001 Alángordliup sermia (Alanngorliup Sermia): 'The glacier of the fjord on the most shadowy side'.

1 GB 07001 Sarqardliup sermia (Saqqarliup Sermia): 'The glacier of the fjord on the most sunny side'.

1 GC 04001 Sermeq avangnardleq (Sermeq Avannarleq): 'The northernmost glacier'.

1 GC 06002 Jakobshavn Isbræ (Dan.). After the nearby town of Jakobshavn / Ilulíssat (Ilulissat). Sermeq kujatdleq (Sermeq Kujalleq): 'The southernmost glacier'.

1 GF 04001 Kangilerngata sermia (Kangilerngata Sermia): 'The easternmost glacier'.

1 GF 06001 Eqip sermia (Eqip Sermia): 'The glacier at the inlet'.

1 GH 17001 Sermeq avangnardleq (Sermeq Avannarleq): The 
northernmost glacier'.

1 GH 18001 Sermeq kujatdleq (Sermeq Kujalleq): 'The southernmost glacier'.

1 HA 04001 Lyngmarksbra (Dan.). The glacier at the heather field.

1 HA 04002 Petersen Gletscher (Dan.). For lieutenant F. Petersen (Froda) who investigated the glacier in 1897 and 1923 (Petersen (Froda), 1898; Froda, 1925).

1 HA 04003 Chamberlin Gletscher (Dan.). For T. C. Chamberlin, American geologist who described the glacier in 1893 and 1894 (Chamberlin, 1894).

1 HA 04011 Pjetursson Gletscher (Dan.). After H. Pjetursson, Icelandic geologist who worked in the area in 1897 together with Froda (cf. 1 HA 04002 above).

1 HB 16020 Steenstrup Gletscher (Dan.). For the Danish geologist K. J. V. Steenstrup who described the glaciers on Disko during visits in the 1870 s and in 1888 (Steenstrup, 1901).

1 HB 16026 C. A. Nielsen Bræ (Dan.). Origin of name uncertain, possibly for C. A. Nielsen, manager 1901-04 at Godhavn / Qeqertarssuaq (Qeqertarsuaq).

1 IA 02048 Auvfarssûp sermia (Aaffarsuup Sermia): 'The glacier at the great reindeer hunting area'.

1 IA 02050 Qiterdlikavsaup sermia (Qiterlikasaap Sermia): 'The poor middle glacier (glacier in the poor middle places)'.

1 IB 24003 Sorqaup sermia (Soqqaap Sermia): 'The baleen glacier'. Comment: sorqaup = baleen (genitive), i.e. the place (mountain or glacier) which by its formation suggests the mouth of a baleen whale.

1 IB 25002 Umiartorfiup sermia (Umiartorfiup Sermia): 'The glacier of Umiartorfik'. (Umiartorfik: the place where (or to which place) people travel by umiaq.)

1 IB 26003 Agssakait sermiat (Assakaat Sermiat): 'The glacier at the place of downfalling (agssakâq: that which has been turned upside down). The name might refer to landslides from the surrounding mountain slopes.

1 IB 27003 Sermiarssuit sermikavsât (Sermiarsuit Sermikassaat): 'The insignificant glacier of the rather large glaciers'.
1 IB 03001 Sermilik (Sermilik): 'Ice fjord' or 'place with glaciers'.

1 IB 11001 Lille Gletscher (Dan.). Lille = small.

1 IB 12001 Store Gletscher (Dan.). Store = large.

1 IB 29004 'Kome Gletscher'. Unauthorized name used in several older descriptions of the glacier (e.g. Drygalski, 1897). Name derived from locative case of Kûk (Kuuk), older spelling Kook: 'The river'. The name could therefore be translated to: 'The glacier at the river'. No proper name of the glacier is known.

1 IB 30003 'Sarfarfik-Gletscher' or 'Sarfarfik Bræ'. Unauthorized names used in several old descriptions of the glacier (e.g. Drygalski, 1887). Name derived from the river in front of the glacier. Earlier referred to as 'Sarfarfik', authorized form Sarfâgfîp kugssinerssua (Sarfaarfiip Kuusinersua): 'The place of the strong current'.

1 IB 43001 Sermersalik (Sermersalik): 'The place with the glaciated area'.

1 IC 07001 Sermeq silardleq (Sermeq Silarleq): 'The outermost glacier'. Comment: Sila 'that which is outside'. Some map sheets have the name Sisôrtartoq (Sisoortartoq): 'what usually slides down (calves)'.

1 IC 09001 Silardleq (Silarleq): 'The outermost'. Comment: Authorized to Sisôrtartukavsak (Sisoortartukassak). 'What insignificantly usually slides down (calves)'.

1 IC 09002 Kangigdleq (Kangilleq): 'The most easterly' (or innermost), cf. Sermeq silardleq above in the same fjord.

1 ID 20001 Ingnerit (Innerit): 'The fire'. Comment: The name refers to a coastal locality on the northern side of the fjord (Ingnerit fjord on older maps, now: Perdlerfiup kangerdlua). The name Ingnerit occurs on the 1939 Geodetic Institute map of this area.

1 ID 20001 Perdlerfiup sermia (Perlerfiup Sermia): 'The glacier at the place where people died from starvation'. Comment: The name is used on recent maps. The locality of Perdlerfik (Perlerfik) is situated on the south side of the fjord Perdlerfiup kangerdlua (Perlerfiup Kangerlua) about $6 \mathrm{~km}$ from the present glacier front. 


\section{Main data of glaciers}

CODE Identification code

LAT Latitude

LONG Longitude

ORI Orientation of lower part of glacier

HIGH At local glaciers: highest elevation given on the current maps.

At Inland Ice sectors: an upper area limitation is set at $1800 \mathrm{~m}$ according to current maps

LOW Lowest glacier elevation

AREA Total area of local glaciers (in square kilometres)

MO CODE Morphology code

MAP Reference to map sheet in atlas.

Table 7. Total area coverage of glaciers in the atlas

\begin{tabular}{lcc}
\hline Type & Area $\mathbf{k m}^{2}$ & Number of units \\
\hline Local glaciers & & \\
Glacierets & 553 & 2076 \\
Mountain glaciers & 1178 & 1473 \\
Valley glaciers & 1370 & 310 \\
Ice caps & 10215 & $\mathbf{1 2 5 8}$ \\
Ice fields & $\underline{11473}$ & $\underline{334}$ \\
Local glaciers total & 14574 & 5030 \\
Inland Ice margin & & \\
below 1800 m a.s.1. & $\underline{100729}$ & $\underline{567}$ \\
Grand total & 115303 & 5297 \\
\hline
\end{tabular}




\begin{tabular}{|c|c|c|c|c|c|c|c|c|c|c|c|c|c|c|c|c|c|}
\hline CODE & LAT & LONG & ORI & HIGH & Low & AREA & $\begin{array}{c}\text { MO } \\
\text { CODE }\end{array}$ & MAP & CODE & LAT & LONG & ORI & HIGH & LOW & AREA & $\begin{array}{c}\text { MO } \\
\text { CODE }\end{array}$ & MAP \\
\hline 1AA01001 & $60^{\circ} 03^{\prime} \mathrm{N}$ & $44^{\circ} 45^{\prime} \mathrm{W}$ & NW & 99 & 74 & 0000.01 & 740110 & $6000-02$ & $1 \mathrm{AA} 05012$ & $60^{\circ} 05^{\prime} \mathrm{N}$ & $44^{\circ} 32^{\prime} \mathrm{W}$ & $w$ & 123 & 100 & 0000.06 & 780010 & $6000-02$ \\
\hline $1 \mathrm{AA} 01002$ & $60^{\circ} 04^{\prime} \mathrm{N}$ & $44^{\circ} 46^{\prime} \mathrm{W}$ & $\mathrm{NW}$ & 100 & 63 & 0000.18 & 720110 & $6000-02$ & 1 AA05013 & $60^{\circ} 06^{\prime} \mathrm{N}$ & $44^{\circ} 32^{\prime} \mathrm{W}$ & E & 90 & 70 & 0000.04 & 780010 & $00-02$ \\
\hline $1 \mathrm{AA} 01003$ & $60^{\circ} 04^{\prime} \mathrm{N}$ & $44^{\circ} 47^{\prime} \mathrm{W}$ & NE & 84 & 74 & 0000.07 & 740110 & $6000-02$ & $1 \mathrm{AA05014}$ & $60^{\circ} 07 \cdot \mathrm{N}$ & $44^{\circ} 31^{\prime} \mathrm{w}$ & $\mathbf{E}$ & 153 & 49 & 0000.18 & 643012 & $6000-02$ \\
\hline $1 \mathrm{AA} 01004$ & $60^{\circ} 05^{\prime} \mathrm{N}$ & $44^{\circ} 48^{\prime} \mathrm{W}$ & $\mathbf{N}$ & 101 & 73 & 0000.88 & 523112 & $6000-02$ & $1 \mathrm{AA0} 0015$ & $60^{\circ} 07^{\prime} \mathrm{N}$ & $44^{\circ} 31^{\prime} \mathrm{v}$ & E & 130 & 50 & 0000.41 & 623312 & $6000-02$ \\
\hline $1 \mathrm{AA} 02001$ & $60^{\circ} 05^{\prime} \mathrm{N}$ & $44^{\circ} 49^{\prime} \mathrm{V}$ & N & 105 & 70 & 0000.10 & 730110 & $6000-02$ & $1 \mathrm{AA0} 0016$ & $60^{\circ} 09^{\prime} \mathrm{N}$ & $44^{\circ} 30^{\prime} \mathrm{v}$ & NE & 152 & 12 & 0001.46 & 623312 & $6000-02$ \\
\hline $1 \mathrm{AA0} 2002$ & $60^{\circ} 05^{\prime} \mathrm{N}$ & $44^{\circ} 47^{\prime} \mathrm{W}$ & $\mathrm{E}$ & 105 & 90 & 0000.03 & 780110 & $6000-02$ & $1 \mathrm{AA} 05017$ & $60^{\circ} 09^{\prime} \mathrm{N}$ & $44^{\circ} 31^{\prime} \mathrm{v}$ & E & 91 & 60 & 0000.08 & 750010 & $6000-02$ \\
\hline $1 \mathrm{AA0} 2003$ & $60^{\circ} 05^{\prime} \mathrm{N}$ & $44^{\circ} 49^{\prime} \mathrm{W}$ & u & 98 & 60 & 0000.11 & 640110 & $6000-02$ & $1 \mathrm{AA} 05018$ & $60^{\circ} 09, \mathrm{~N}$ & $44^{\circ} 31^{\prime} \mathrm{v}$ & $\mathrm{N}$ & 96 & 72 & 0000.06 & 750010 & $6000-02$ \\
\hline $1 \mathrm{AA0} 2004$ & $60^{\circ} 04^{\prime} \mathrm{N}$ & $44^{\circ} 46^{\prime} \mathrm{V}$ & NW & 121 & 59 & 0000.18 & 740110 & $6000-02$ & 1AA05019 & $60^{\circ} 09^{\prime} \mathrm{N}$ & $44^{\circ} 32^{\prime} \mathrm{w}$ & N & 85 & 10 & 0000.87 & 620010 & $6000-02$ \\
\hline 1 AA02005 & $60^{\circ} 04^{\prime} \mathrm{N}$ & $44^{\circ} 47^{\prime} \mathrm{W}$ & NW & 120 & 59 & 0000.15 & 640110 & $6000-02$ & $1 \mathrm{AA} 05020$ & $60^{\circ} 09^{\prime} \mathrm{N}$ & $44^{\circ} 34^{\prime} \mathrm{W}$ & $\mathrm{N}$ & 89 & 49 & 0000.28 & 640012 & $6000-02$ \\
\hline $1 \mathrm{AA0} 2006$ & $60^{\circ} 03^{\prime} \mathrm{N}$ & $44^{\circ} 48^{\prime} \mathrm{W}$ & $\mathrm{SE}$ & 90 & 80 & 0000.43 & 780010 & $6000-02$ & $1 \mathrm{AA} 05021$ & $60^{\circ} 09^{\prime} \mathrm{N}$ & $44^{\circ} 35^{\prime} \mathrm{W}$ & $\mathrm{N}$ & 130 & 39 & $0 b 00.69$ & 623212 & $6000-02$ \\
\hline 1AA02007 & $60^{\circ} 04^{\prime} \mathrm{N}$ & $44^{\circ} 45^{\prime} \mathrm{W}$ & $\mathrm{SE}$ & & & 0000.06 & 750010 & $6000-02$ & $1 \mathrm{AA0} 05022$ & $60^{\circ} 11 \cdot \mathrm{N}$ & $44^{\circ} 31^{\prime}$, & SW & 169 & 65 & 0000.93 & 643010 & $6000-02$ \\
\hline 1 AA03001 & $60^{\circ} 05^{\prime} \mathrm{N}$ & $44^{\circ} 44^{\prime} \mathrm{W}$ & NE & 90 & 80 & 0000.09 & 740010 & $6000-02$ & IAA06001 & $60^{\circ} 11 \cdot \mathrm{N}$ & $44^{\circ} 30^{\prime} \mathrm{v}$ & $\mathrm{E}$ & 140 & 120 & 0000.04 & 780010 & $6000-02$ \\
\hline 1 AA03002 & $60^{\circ} 06^{\prime} \mathrm{N}$ & $44^{\circ} 45^{\prime} \mathrm{W}$ & $\mathrm{N}$ & 120 & 98 & 0000.12 & 740110 & $6000-02$ & 1 AA06002 & $60^{\circ} 11$, N & $44^{\circ} 30^{\prime} \mathrm{W}$ & SE & 130 & 110 & 0000.07 & 750010 & $\begin{array}{l}6000-02 \\
6000-02\end{array}$ \\
\hline $1 \mathrm{AA} 03003$ & $60^{\circ} 06^{\prime} \mathrm{N}$ & $33^{\circ} 45^{\prime} \mathrm{W}$ & $\mathrm{NW}$ & 78 & 60 & 0000.10 & 740110 & $6000-02$ & $1 \mathrm{AA06003}$ & $60^{\circ} 12^{\prime} \mathrm{N}$ & $44^{\circ} 28^{\prime}$ w & $\mathbf{s}$ & 140 & 33 & 0004.12 & 523111 & $6000-02$ \\
\hline IAA03004 & $60^{\circ} 06^{\prime} \mathrm{N}$ & $44^{\circ} 46^{\prime} \mathrm{W}$ & NW & 110 & 70 & 0000.08 & 700110 & $6000-02$ & $1 \mathrm{AA06004}$ & $60^{\circ} 10^{\prime} \mathrm{N}$ & $44^{\circ} 23^{\prime} \mathrm{W}$ & SW & 131 & 59 & 0001.51 & 523312 & $6000-03$ \\
\hline $1 \mathrm{AA0} 0005$ & $60^{\circ} 07^{\prime} \mathrm{N}$ & $44^{\circ} 45^{\prime} \mathrm{W}$ & $\mathrm{N}$ & 97 & 85 & 0000.05 & 730110 & $6000-02$ & 1AA06005 & $60^{\circ} 10^{\prime} \mathrm{N}$ & $44^{\circ} 22^{\prime} \mathrm{V}$ & SW & 113 & 110 & 0000.14 & 770212 & $6000-03$ \\
\hline $1 \mathrm{AA} 03006$ & $60^{\circ} 08^{\prime} \mathrm{N}$ & $44^{\circ} 41^{\prime} \mathrm{W}$ & SW & 110 & 90 & 0000.03 & 700010 & $6000-02$ & $1 \mathrm{AA} 06006$ & $60^{\circ} 10^{\prime} \mathrm{N}$ & $44^{\circ} 22^{\prime} \mathrm{v}$ & $\mathrm{V}$ & 105 & 95 & 0000.16 & 770112 & $6000-03$ \\
\hline $1 \mathrm{AA} 03007$ & $60^{\circ} 07 / \mathrm{N}$ & $44^{\circ} 41, \mathrm{~W}$ & $\mathrm{SW}$ & 120 & 105 & 0000.05 & 630010 & $6000-02$ & 1AA06007 & $60^{\circ} 11^{\prime} \mathrm{N}$ & $44^{\circ} 21^{\prime}$, & SE & 147 & 45 & 0001.17 & 670212 & $6000-03$ \\
\hline $1 \mathrm{AA0} 03008$ & $60^{\circ} 09^{\prime} \mathrm{N}$ & $44^{\circ} 38^{\prime} \mathrm{W}$ & SE & 101 & 93 & 0000.04 & 770010 & $6000-02$ & $1 \mathrm{AA} 06008$ & $60^{\circ} 10^{\prime} \mathrm{N}$ & $44^{\circ} 20^{\prime} \mathrm{W}$ & SW & 80 & 58 & 0000.26 & 790010 & $6000-03$ \\
\hline $1 \mathrm{AA} 03009$ & $60^{\circ} 09^{\prime} \mathrm{N}$ & $44^{\circ} 36^{\prime}$ & sw & 140 & 80 & 0000.40 & 780010 & $6000-02$ & $1 \mathrm{AA} 06009$ & $60^{\circ} 10^{\prime} \mathrm{N}$ & $44^{\circ} 20^{\prime} \mathrm{W}$ & W & 81 & 60 & 0000.18 & 790012 & $6000-03$ \\
\hline 1 AA03010 & $60^{\circ} 09^{\prime} \mathrm{N}$ & $44^{\circ} 36^{\prime}$, & $\mathrm{v}$ & 141 & 121 & 0000.07 & 780010 & $6000-02$ & 1 AA06010 & $60^{\circ} 10^{\prime} \mathrm{N}$ & $44^{\circ} 19^{\prime} \mathrm{W}$ & V & $\begin{array}{l}81 \\
103\end{array}$ & 90 & 0000.07 & 790012 & $0-03$ \\
\hline 1AA03011 & $60^{\circ} 08^{\prime} \mathrm{N}$ & $44^{\circ} 37^{\prime} \mathrm{W}$ & $\mathrm{v}$ & 119 & 77 & 0000.45 & 640010 & $6000-02$ & 1 AA07001 & $60^{\circ} 10^{\prime} \mathrm{N}$ & $44^{\circ} 19^{\prime}$ W & $\mathrm{SE}$ & 103 & 90 & 0000.13 & 780010 & $6000-03$ \\
\hline $1 \mathrm{AA} 03012$ & $60^{\circ} 08^{\prime} \mathrm{N}$ & $44^{\circ} 37^{\prime} \mathrm{W}$ & NW & 100 & 66 & 0000.73 & 643010 & $6000-02$ & $1 \mathrm{AA0} 0002$ & $60^{\circ} 11^{\prime} \mathrm{N}$ & $44^{\circ} 19^{\prime}$ W & $\mathrm{N}$ & 95 & 30 & 0000.89 & 643212 & $6000-03$ \\
\hline $1 \mathrm{AA} 03013$ & $60^{\circ} 12^{\prime} \mathrm{N}$ & $44^{\circ} 38^{\prime} \mathrm{W}$ & NW & 102 & 70 & 0000.19 & 643012 & $6000-02$ & $1 \mathrm{AA} 07003$ & $60^{\circ} 11 \cdot \mathrm{N}$ & $44^{\circ} 19^{\prime} \mathrm{V}$ & NE & 95 & 30 & 0000.04 & 750012 & $6000-03$ \\
\hline $1 \mathrm{AA} 03014$ & $60^{\circ} 07^{\prime} \mathrm{N}$ & $44^{\circ} 38^{\prime} \mathrm{W}$ & NW & 107 & 90 & 0000.04 & 780010 & $6000-02$ & $1 \mathrm{AA} 07004$ & $60^{\circ} 11^{\prime} \mathrm{N}$ & $44^{\circ} 20^{\prime} \mathrm{W}$ & N & 110 & 90 & 0000.09 & 780010 & $6000-03$ \\
\hline $1 \mathrm{AA} 03015$ & $60^{\circ} 08^{\prime} \mathrm{N}$ & $44^{\circ} 36^{\prime} \mathrm{V}$ & $\mathrm{s}$ & 100 & 76 & 0000.06 & 750010 & $6000-02$ & 1AA07005 & $60^{\circ} 11 \cdot \mathrm{N}$ & $44^{\circ} 20^{\prime}$ & $\mathrm{N}$ & 110 & 90 & 0000.14 & 643112 & $6000-03$ \\
\hline 1AA03016 & $60^{\circ} 08^{\prime} \mathrm{N}$ & $44^{\circ} 33^{\prime} \mathrm{W}$ & w & 159 & 100 & 0001.14 & 270110 & $6000-0$ & 1AA07006 & $60^{\circ} 11^{\prime} \mathrm{N}$ & $44^{\circ} 21^{\prime \prime}$ & $\mathbf{N}$ & 105 & 35 & 0000.77 & 623212 & $6000-03$ \\
\hline 1 AA03017 & $60^{\circ} 08^{\prime} \mathrm{N}$ & $44^{\circ} 34^{\prime}$ ' & 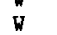 & 76 & 56 & 0000.15 & 750110 & $6000-02$ & 1AA07007 & $60^{\circ} 12^{\prime} \mathrm{N}$ & $44^{\circ} 23^{\prime} \mathrm{W}$ & $\mathrm{N}$ & 143 & 50 & 0000.17 & 643212 & $\begin{array}{l}6000-03 \\
6000-03\end{array}$ \\
\hline $1 \mathrm{AA} 03018$ & $60^{\circ} 08^{\prime} \mathrm{N}$ & $44^{\circ} 33^{\prime} \mathrm{w}$ & SW & 140 & 80 & 0000.41 & 640112 & $6000-02$ & $1 \mathrm{AA} 07008$ & $60^{\circ} 11 \cdot \mathrm{N}$ & $44^{\circ} 23^{\prime}$, & $\mathrm{NW}$ & 156 & 65 & 0000.36 & 640112 & $6000-03$ \\
\hline $1 \mathrm{AA} 03019$ & $60^{\circ} 08^{\prime} \mathrm{N}$ & $44^{\circ} 32^{\prime} \mathrm{V}$ & SW & 188 & 63 & 0001.17 & 543112 & $6000-02$ & $1 \mathrm{AA} 07009$ & $60^{\circ} 11 \cdot \mathrm{N}$ & $44^{\circ} 25^{\prime} \mathrm{v}$ & $\mathrm{NE}$ & 80 & 49 & 0000.72 & 640112 & $6000-03$ \\
\hline 1 AA03020 & $60^{\circ} 07^{\circ} \mathrm{N}$ & $44^{\circ} 32, \mathrm{~V}$ & $\mathrm{~W}$ & $\begin{array}{l}156 \\
156\end{array}$ & 63 & 0002.18 & 543110 & $6000-0$ & 1 AA07010 & $60^{\circ} 12, \mathrm{~N}$ & $44^{\circ} 25^{\prime}$ & E & $\begin{array}{r}80 \\
142\end{array}$ & $\begin{array}{r}49 \\
119\end{array}$ & 0000.45 & $\begin{array}{l}640112 \\
643112\end{array}$ & $6000-03$ \\
\hline 1 AA03021 & $60^{\circ} 07^{\prime} \mathrm{N}$ & $44^{\circ} 34^{\prime}, \mathrm{v}$ & NW & 90 & 72 & 0000.18 & 770010 & $6000-0$ & 1AA08001 & $60^{\circ} 13^{\prime} \mathrm{N}$ & $44^{\circ} 19^{\prime}$ W & $\mathbf{N}$ & 87 & 85 & 0000.06 & 780010 & $6000-03$ \\
\hline 1 AA03022 & $60^{\circ} 06^{\prime} \mathrm{N}$ & $44^{\circ} 34^{\prime} \mathrm{V}$ & NW & 315 & 71 & 0000.45 & 643012 & $6000-02$ & 1AA08002 & $60^{\circ} 12^{\prime} \mathrm{N}$ & $49^{\circ} 20^{\prime} \mathrm{W}$ & $\mathrm{NE}$ & 80 & 80 & 0000.07 & 790012 & $6000-03$ \\
\hline $1 \mathrm{AA0} 3023$ & $60^{\circ} 06^{\prime} \mathrm{N}$ & $44^{\circ} 35^{\prime} \mathrm{W}$ & $\mathrm{N}$ & 88 & 76 & 0000.26 & 650012 & $6000-0$ & $1 \mathrm{AA08003}$ & $60^{\circ} 13^{\prime} \mathrm{N}$ & $44^{\circ} 21^{\prime}$ W & $\mathrm{N}$ & 101 & 79 & 0000.09 & 643112 & $6000-03$ \\
\hline $1 \mathrm{AA} 03024$ & $60^{\circ} 05^{\prime} \mathrm{N}$ & $44^{\circ} 37^{\prime} \mathrm{V}$ & NW & 95 & 63 & 0000.34 & 640010 & $6000-02$ & $1 \mathrm{AA08004}$ & $60^{\circ} 13^{\prime} \mathrm{N}$ & $44^{\circ} 22^{\prime} \mathrm{W}$ & $\mathrm{N}$ & 100 & 78 & 0000.13 & 643112 & $0-03$ \\
\hline $1 \mathrm{AA} 03025$ & $60^{\circ} 06^{\prime} \mathrm{N}$ & $44^{\circ} 37^{\prime} \mathrm{V}$ & N & 102 & 61 & 0000.22 & 640010 & $6000-0$ & $1 \mathrm{AA} 08005$ & $60^{\circ} 13^{\prime} \mathrm{N}$ & $44^{\circ} 22^{\prime} \mathrm{W}$ & $\mathrm{N}$ & 100 & 80 & 0000.11 & 643212 & $6000-03$ \\
\hline $1 \mathrm{AAO} 3026$ & $60^{\circ} 05^{\prime N}$ & $44^{\circ} 38^{\prime} \mathrm{V}$ & $\mathrm{v}$ & 101 & 88 & 0000.08 & 740010 & $6000-0$ & 1 AA08006 & $60^{\circ} 13 \cdot \mathrm{N}$ & $44^{\circ} 25^{\prime}$ & N & 110 & 90 & 0000.52 & 653212 & $6000-03$ \\
\hline $1 \mathrm{AA0} 03027$ & $60^{\circ} 05^{\prime} \mathrm{N}$ & $44^{\circ} 38^{\prime \prime} \mathrm{W}$ & NW & 100 & 70 & 0000.07 & 740010 & $6000-0$ & $1 \mathrm{AA} 08007$ & $60^{\circ} 13^{\prime} \mathrm{N}$ & $44^{\circ} 26^{\prime} \mathrm{H}$ & E & 132 & 105 & 0000.02 & 750010 & $6000-02$ \\
\hline $1 \mathrm{AA0} 3028$ & $60^{\circ} 05^{\prime} \mathrm{N}$ & $44^{\circ} 37^{\prime \prime} \mathrm{W}$ & $\mathrm{s}$ & 85 & 70 & 0000.04 & 700010 & $6000-$ & $1 \mathrm{AA} 08008$ & $60^{\circ} 13^{\prime} \mathrm{N}$ & $44^{\circ} 27^{\prime} \mathrm{W}$ & $\mathbf{E}$ & 100 & 80 & 0000.53 & 620012 & $6000-02$ \\
\hline 1 AA03029 & $60^{\circ} 05^{\prime} \mathrm{N}$ & $44^{\circ} 37^{\circ} \mathrm{W}$ & W & 90 & 55 & 0000.10 & 780010 & $6000-02$ & 1AA08009 & $60^{\circ} 14^{\prime} \mathrm{N}$ & $44^{\circ} 27^{\prime \prime}$ & $\mathrm{NE}$ & 123 & 100 & 0000.10 & 780010 & $6000-02$ \\
\hline 1 AA03030 & $60^{\circ} 04^{\prime} \mathrm{N}$ & $44^{\circ} 38^{\prime} \mathrm{W}$ & $\mathrm{NW}$ & 92 & 80 & 0000.06 & 780010 & $6000-02$ & 1 AA08010 & $60^{\circ} 16, \mathrm{~N}$ & $44^{\circ} 25^{\prime}$ W & $\mathrm{SE}$ & 150 & $\begin{array}{r}100 \\
42\end{array}$ & 0003.82 & 623112 & $\begin{array}{l}0-02 \\
0-03\end{array}$ \\
\hline $1 A A 04001$ & $60^{\circ} 04^{\prime} \mathrm{N}$ & $44^{\circ} 39^{\prime} \mathrm{V}$ & SW & 93 & 81 & 0000.04 & 780010 & $6000-0$ & 1AA08011 & $60^{\circ} 15^{\prime} \mathrm{N}$ & $44^{\circ} 24^{\prime} \mathrm{W}$ & s & 110 & 95 & 0000.04 & 670112 & $6000-03$ \\
\hline $1 \mathrm{AAO} 04002$ & $60^{\circ} 03^{\prime} \mathrm{N}$ & $44^{\circ} 38^{\prime}$ W & E & 103 & $\begin{array}{l}01 \\
100\end{array}$ & 0000.03 & 780010 & $6000-$ & $1 \mathrm{AA08012}$ & $60^{\circ} 17, \mathrm{~N}$ & $44^{\circ} 22^{\prime} \mathrm{W}$ & s & $\begin{array}{l}110 \\
160\end{array}$ & 90 & 0007.44 & 523110 & $6000-03$ \\
\hline 1 AA04003 & $60^{\circ} 04^{\prime} \mathrm{N}$ & $44^{\circ} 36^{\prime} \mathrm{W}$ & SW & 99 & 94 & 0000.04 & 780010 & $6000-($ & $1 \mathrm{AA} 08013$ & $60^{\circ} 16^{\prime} \mathrm{N}$ & $44^{\circ} 21^{\prime} \mathrm{W}$ & s & & 90 & 0002.76 & 200110 & $6000-03$ \\
\hline $1 \mathrm{AA0} 0004$ & $60^{\circ} 05^{\prime} \mathrm{N}$ & $44^{\circ} 36^{\prime} \mathrm{W}$ & s" & 131 & 96 & 0000.32 & 673112 & $6000-02$ & $1 \mathrm{AA08014}$ & $60^{\circ} 17 \cdot \mathrm{N}$ & $44^{\circ} 20^{\prime} \mathrm{W}$ & S & 162 & 35 & 0012.11 & 523311 & $6000-03$ \\
\hline $1 \mathrm{AA} 04005$ & $60^{\circ} 05^{\prime} \mathrm{N}$ & $44^{\circ} 35^{\prime} \mathrm{W}$ & $\mathrm{s}$ & 120 & $\begin{array}{l}70 \\
58\end{array}$ & 0000.34 & 780010 & $6000-02$ & $1 \mathrm{AA} 08015$ & $60^{\circ} 15^{\prime N} \mathrm{~N}$ & $44^{\circ} 17^{\prime} \mathrm{W}$ & sw & 150 & 35 & 0001.24 & 623112 & $6000-03$ \\
\hline 1 AA04006 & $60^{\circ} 03^{\prime} \mathrm{N}$ & $44^{\circ} 35^{\prime} \mathrm{V}$ & s & $\begin{array}{r}120 \\
99\end{array}$ & 78 & 0000.04 & 790010 & $6000-02$ & $1 \mathrm{AA} 08016$ & $60^{\circ} 15^{\prime N} \mathrm{~N}$ & $44^{\circ} 17^{\prime} \mathrm{W}$ & NW & 140 & 55 & 0000.38 & 643112 & $6000-03$ \\
\hline $1 \mathrm{AAO} 4007$ & $60^{\circ} 03^{\prime} \mathrm{N}$ & $44^{\circ} 35^{\prime} \mathrm{v}$ & W & 132 & 58 & 0001.13 & 673112 & & $1 \mathrm{AA} 08017$ & $60^{\circ} 14^{\prime} \mathrm{N}$ & $44^{\circ} 16^{\prime \prime}$ & $\mathrm{s}$ & 140 & 130 & 0000.16 & 740110 & $6000-03$ \\
\hline $1 \mathrm{AA} 04008$ & $60^{\circ} 02^{\prime} \mathrm{N}$ & $44^{\circ} 35^{\prime} \mathrm{V}$ & U & 90 & & 0000.04 & 780010 & $6000-02$ & $1 \mathrm{AA09001}$ & $60^{\circ} 14^{\prime} \mathrm{N}$ & $44^{\circ} 14^{\prime} \mathrm{W}$ & $\mathrm{SE}$ & 140 & & 0000.26 & 650112 & $6000-03$ \\
\hline 1AA04009 & $60^{\circ} 01 / \mathrm{N}$ & $44^{\circ} 35^{\prime} \mathrm{V}$ & NW & 92 & 79 & 0000.05 & 790010 & $6000-02$ & $1 \mathrm{AA} 09002$ & $60^{\circ} 15, \mathrm{~N}$ & $44^{\circ} 14^{\prime} \mathrm{W}$ & $\begin{array}{l}S E \\
E\end{array}$ & $\begin{array}{l}140 \\
113\end{array}$ & 82 & 0000.39 & 750010 & 60 \\
\hline 1AA05001 & $60^{\circ} 01, \mathrm{~N}$ & $44^{\circ} 34^{\prime} \mathrm{W}$ & $\mathrm{s}$ & 101 & 88 & 0000.07 & 700010 & $6000-02$ & $1 \mathrm{AA} 09003$ & $60^{\circ} 15^{\prime N}$ & $44^{\circ} 14^{\prime \prime} \mathrm{W}$ & E & 101 & 90 & 0000.17 & 650010 & $6000-03$ \\
\hline $1 \mathrm{AA}$ & $60^{\circ} 02, \mathrm{~N}$ & $44^{\circ} 34^{\prime \prime} \mathrm{W}$ & $\mathrm{SE}$ & 100 & 89 & 0000.04 & 750010 & & 1AA09004 & $60^{\circ} 15^{\prime N}$ & $40^{\circ} 15, \mathrm{~W}$ & $\mathrm{NE}$ & 140 & 30 & 0002.47 & 633112 & $6000-03$ \\
\hline $1 \mathrm{AAO}$ & $60^{\circ} 02^{\prime} \mathrm{N}$ & $44^{\circ} 33^{\prime} \mathrm{W}$ & $\mathrm{s}$ & 80 & 69 & 0000.06 & 780010 & $6000-02$ & $1 \mathrm{AA} 09005$ & $60^{\circ} 15^{\prime} \mathrm{N}$ & $44^{\circ} 16^{\prime}, \mathrm{W}$ & $\mathrm{N}$ & 120 & 120 & 0000.08 & 770010 & $6000-03$ \\
\hline $1 \mathrm{AA0} 5004$ & $60^{\circ} 03^{\prime} \mathrm{N}$ & $44^{\circ} 33^{\prime} \mathrm{W}$ & $\mathrm{N}$ & 85 & 71 & 0000.08 & 653412 & $6000-02$ & $1 \mathrm{AA} 09006$ & $60^{\circ} 16^{\prime} \mathrm{N}$ & $44^{\circ} 16^{\prime} \mathrm{b}$ & $\mathrm{NE}$ & 130 & & 0001.15 & 670012 & $6000-03$ \\
\hline 1 AA05005 & $60^{\circ} 05^{\prime} \mathrm{N}$ & $44^{\circ} 34^{\prime} \mathrm{W}$ & s & 91 & 79 & 0000.16 & 780010 & $6000-02$ & $1 \mathrm{AA09007}$ & $60^{\circ} 17{ }^{\prime N}$ & $44^{\circ} 17^{\prime}$ W & SE & 60 & & 0000.37 & 670010 & $6000-03$ \\
\hline $1 \mathrm{AA} 05006$ & $60^{\circ} 05^{\prime} \mathrm{N}$ & $44^{\circ} 34^{\prime}$ v & s & 90 & 75 & 0000.18 & 780010 & $6000-02$ & $1 \mathrm{AA} 09008$ & $60^{\circ} 177^{\prime} \mathrm{N}$ & $44^{\circ} 16^{\prime} \mathrm{W}$ & NE & 110 & 70 & 0000.14 & 770010 & $6000-03$ \\
\hline $1 \mathrm{AA} 0$ & $60^{\circ} 04^{\prime} \mathrm{N}$ & $44^{\circ} 33^{\prime} \mathrm{v}$ & $\mathrm{s}$ & 116 & 100 & 0000.06 & 780010 & $6000-02$ & 1AA09009 & $60^{\circ} 18^{\prime} \mathrm{N}$ & $44^{\circ} 17^{\prime} \mathrm{W}$ & $\mathrm{E}$ & 150 & 50 & 0000.97 & 633312 & $6000-03$ \\
\hline $1 \mathrm{AA} 05008$ & $60^{\circ} 04^{\prime} \mathrm{N}$ & $44^{\circ} 33^{\prime} \mathrm{W}$ & $\mathrm{NE}$ & 130 & 120 & 0000.05 & 780010 & $6000-02$ & IAA09010 & $60^{\circ} 19^{\prime} \mathrm{N}$ & $44^{\circ} 18^{\prime}, \mathrm{W}$ & $\mathrm{N}$ & 150 & 32 & 0003.02 & 623112 & $6000-03$ \\
\hline 1 AA05009 & $60^{\circ} 05^{\prime} \mathrm{N}$ & $44^{\circ} 33^{\prime} \mathrm{W}$ & NE & 102 & 88 & 0000.17 & 643112 & $6000-02$ & $1 \mathrm{AA} 09011$ & $60^{\circ} 19^{\prime} \mathrm{N}$ & $44^{\circ} 19^{\prime} \mathrm{W}$ & $\mathbf{N}$ & 160 & 160 & 0000.06 & 780010 & -03 \\
\hline 1 AA05010 & $60^{\circ} 05^{\prime} \mathrm{N}$ & $44^{\circ} 34^{\prime} \mathrm{V}$ & $\mathrm{E}$ & 120 & 70 & 0001.5 & 646112 & 6000 & $1 \mathrm{AA} 09012$ & $60^{\circ} 20^{\prime} \mathrm{N}$ & $44^{\circ} 21^{\prime} \mathrm{W}$ & $\mathrm{NE}$ & 160 & 120 & 0000.10 & 780010 & $6000-03$ \\
\hline $1 \mathrm{AA05011}$ & $60^{\circ} 06^{\prime} \mathrm{N}$ & $44^{\circ} 32^{\prime} \mathrm{W}$ & SW & 120 & 90 & 0000.06 & 780010 & $6000-02$ & 1AA09013 & $60^{\circ} 20^{\prime} \mathrm{N}$ & $44^{\circ} 22^{\prime} \mathrm{W}$ & E & 140 & 50 & 0002.32 & 633312 & $6000-03$ \\
\hline
\end{tabular}




\begin{tabular}{|c|c|c|c|c|c|c|c|}
\hline LOW & AREA & $\begin{array}{l}\text { MO. } \\
\text { CODE }\end{array}$ & MAP & CODE & LAT & LONG & OR \\
\hline 120 & $\begin{array}{l}0000.05 \\
0000.15\end{array}$ & $\begin{array}{l}780010 \\
633312\end{array}$ & $\begin{array}{l}6000-03 \\
6000-03\end{array}$ & $\begin{array}{l}\text { 1AA12009 } \\
\text { 1AA12010 }\end{array}$ & $\begin{array}{l}60^{\circ} 15^{\prime} \mathrm{N} \\
60^{\circ} 15^{\prime} \mathrm{N}\end{array}$ & $\begin{array}{l}44^{\circ} 01^{\prime} \mathrm{W} \\
44^{\circ} 02^{\prime} \mathrm{W}\end{array}$ & $\mathrm{Nh}$ \\
\hline 75 & 0000.08 & 680310 & $6000-03$ & 1AA12011 & $60^{\circ} 15^{\prime N}$ & $44^{\circ} 03^{\prime} W$ & NW \\
\hline 105 & 0002.10 & 623312 & $6000-03$ & $1 \mathrm{AA} 12012$ & $60^{\circ} 14^{\prime} \mathrm{N}$ & $44^{\circ} 05^{\prime} \mathrm{W}$ & \\
\hline 65 & 0001.08 & 680312 & $6000-03$ & 1AA12013 & $60^{\circ} 13^{\prime} \mathrm{N}$ & $44^{\circ} 06^{\prime} \mathrm{W}$ & NW \\
\hline 90 & 0000.04 & 780010 & $6000-03$ & $1 \mathrm{AA12014}$ & $60^{\circ} 13^{\prime} \mathrm{N}$ & $44^{\circ} 08^{\prime} \mathrm{W}$ & \\
\hline 13 & 0000.05 & 780010 & $6000-03$ & 1AA12015 & $60^{\circ} 11^{\prime} \mathrm{N}$ & $44^{\circ} 08^{\prime} \mathrm{W}$ & \\
\hline 41 & 0000.61 & 640112 & $6000-03$ & 1AA13001 & $60^{\circ} 12^{\prime} \mathrm{N}$ & $44^{\circ} 06^{\prime} \mathrm{W}$ & \\
\hline & 0000.20 & 750010 & 6000-03 & 1AA13002 & $60^{\circ} 13^{\prime} \mathrm{N}$ & $49^{\circ} 05^{\prime}$ W & \\
\hline 125 & 0003.01 & 203112 & $6000-03$ & $1 \mathrm{AA} 13003$ & $60^{\circ} 13^{\prime} \mathrm{N}$ & $44^{\circ} 04^{\prime} \mathrm{W}$ & \\
\hline 150 & 0000.07 & 750010 & $6000-03$ & 1AA13004 & $60^{\circ} 14^{\prime} \mathrm{N}$ & $44^{\circ} 03^{\prime} \mathrm{V}$ & \\
\hline 150 & 0000.06 & 750110 & $6000-03$ & 1AA13005 & $60^{\circ} 16^{\prime} \mathrm{N}$ & $44^{\circ} 00^{\prime} \mathrm{V}$ & \\
\hline 12 & 0009.69 & 523112 & $6000-03$ & 1 AA13006 & $60^{\circ} 13^{\prime} \mathrm{N}$ & $44^{\circ} 02^{\prime} \mathrm{V}$ & $\mathrm{W}$ \\
\hline & & 750010 & $6000-03$ & $1 \mathrm{AA} 13007$ & $60^{\circ} 12^{\prime} \mathrm{N}$ & $44^{\circ} 00^{\prime} \mathrm{W}$ & \\
\hline 40 & 0000.39 & 750010 & $6000-03$ & $1 \mathrm{AA13008}$ & $60^{\circ} 12^{\prime} \mathrm{N}$ & $44^{\circ} 01^{\prime} \mathrm{W}$ & \\
\hline 55 & 0000.08 & 780010 & $6000-03$ & 1AA13009 & $60^{\circ} 11^{\prime} \mathrm{N}$ & $44^{\circ} 00^{\prime} \mathrm{W}$ & \\
\hline & 0003.96 & 523112 & $6000-03$ & $1 \mathrm{AA} 13010$ & $60^{\circ} 11^{\prime} \mathrm{N}$ & $43^{\circ} 58^{\prime} \mathrm{W}$ & \\
\hline 65 & 0000.74 & 740112 & $6000-03$ & $1 \mathrm{AA} 14001$ & $60^{\circ} 11^{\prime} \mathrm{N}$ & $43^{\circ} 57^{\prime} \mathrm{W}$ & \\
\hline 140 & 0000.07 & 770010 & $6000-03$ & 1AA14002 & $60^{\circ} 13^{\prime} \mathrm{N}$ & $43^{\circ} 54^{\prime} \mathrm{W}$ & \\
\hline 70 & 0002.05 & 543212 & & $1 \mathrm{AA}$ & $60^{\circ} 10^{\prime} \mathrm{N}$ & $43^{\circ} 52^{\prime} \mathrm{W}$ & \\
\hline 17 & 0002.93 & 533212 & $6000-03$ & $1 \mathrm{AA} 15001$ & $60^{\circ} 08^{\prime} \mathrm{N}$ & $43^{\circ} 52^{\prime} \mathrm{W}$ & \\
\hline 110 & 0000.75 & 270010 & $6000-03$ & 1AA15002 & $60^{\circ} 08^{\prime} \mathrm{N}$ & $43^{\circ} 52^{\prime} \mathrm{W}$ & N \\
\hline 20 & 0000.10 & 780010 & $6000-03$ & 1AA15003 & $60^{\circ} 08^{\prime} \mathrm{N}$ & $43^{\circ} 53^{\prime} \mathrm{W}$ & \\
\hline & 0003.35 & 523112 & $6000-03$ & $1 \mathrm{AA} 1$ & $60^{\circ} 08^{\prime} \mathrm{N}$ & $43^{\circ} 55^{\prime} \mathrm{W}$ & \\
\hline & & 640110 & & $1 \mathrm{AA}$ & $60^{\circ} 08^{\prime} \mathrm{N}$ & $43^{\circ} 57^{\prime} \mathrm{V}$ & NW \\
\hline & 0000 & 653212 & 600 & $1 \mathrm{AA} 15006$ & $60^{\circ} 08^{\prime} \mathrm{N}$ & $43^{\circ} 59^{\prime} \mathrm{W}$ & \\
\hline & 0000.72 & 670112 & $6000-03$ & $1 \mathrm{AA} 15007$ & $60^{\circ} 10^{\prime} \mathrm{N}$ & $43^{\circ} 58^{\prime} \mathrm{V}$ & \\
\hline 80 & 0001.27 & 670212 & $6000-03$ & $1 \mathrm{AA1} 15008$ & $60^{\circ} 08^{\prime} \mathrm{N}$ & $43^{\circ} 59^{\prime} \mathrm{W}$ & \\
\hline 110 & 0000.32 & 603512 & 600 & & & $3 . \mathrm{W}$ & \\
\hline 50 & & & & 1AA15010 & $60^{\circ} 09^{\prime} \mathrm{N}$ & $44^{\circ} 04^{\prime} \mathrm{W}$ & $\mathrm{N}$ \\
\hline & & & & 1AA15011 & $60^{\circ} 09^{\prime} \mathrm{N}$ & $44^{\circ} 05^{\prime} \mathrm{V}$ & \\
\hline 40 & 0001.47 & 633412 & $6000-03$ & 1AA16001 & $60^{\circ} 09^{\prime} \mathrm{N}$ & $44^{\circ} 05^{\prime} \mathrm{V}$ & \\
\hline 40 & 0001.31 & 633312 & $6000-03$ & 1AA16002 & $60^{\circ} 08^{\prime} \mathrm{N}$ & $44^{\circ} 05^{\prime} \mathrm{W}$ & \\
\hline & 0001.66 & 623412 & 600 & & & & \\
\hline & & 683310 & & $1 \mathrm{AA}$ & $60^{\circ} 07^{\prime} \mathrm{N}$ & $44^{\circ} \mathrm{O} 2^{\prime} \mathrm{V}$ & \\
\hline 20 & 0002.72 & 623412 & 600 & $1 \mathrm{AA} 17001$ & $60^{\circ} 06^{\prime} \mathrm{N}$ & $44^{\circ} 02^{\prime} \mathrm{V}$ & \\
\hline & 0000.13 & 780010 & & $1 \mathrm{AA}$ & $60^{\circ} 07^{\prime} \mathrm{N}$ & $44^{\circ} 01 \cdot \mathrm{V}$ & \\
\hline 20 & 0002.00 & 633512 & & $1 \mathrm{AA}$ & $5 \cdot \mathrm{N}$ & $43^{\circ} 59^{\prime} \mathrm{V}$ & \\
\hline & 0121 & 224111 & & & & $44^{\circ} 00^{\prime} \mathrm{V}$ & 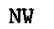 \\
\hline 40 & & 750010 & & $1 \mathrm{AA} 18001$ & $60^{\circ} 05^{\prime} \mathrm{N}$ & $43^{\circ} 59^{\prime} \mathrm{W}$ & \\
\hline 40 & 000 & 643012 & 60 & 1AA1 & $60^{\circ} 08^{\prime} \mathrm{N}$ & $43^{\circ} 59^{\prime} \mathrm{W}$ & \\
\hline & 0004.06 & 523112 & 60 & $1 \mathrm{AA}$ & $60^{\circ} 07^{\prime} \mathrm{N}$ & $43^{\circ} 57^{\prime} \mathrm{V}$ & \\
\hline 70 & 0000.66 & 643112 & 60 & 1AA18 & $60^{\circ} 06^{\prime} \mathrm{N}$ & $43^{\circ} 55^{\prime} \mathrm{v}$ & \\
\hline 140 & 0000.08 & 750010 & 60 & $1 \mathrm{AA} 18$ & $60^{\circ}$ & $43^{\circ} 44^{\prime} \mathrm{V}$ & \\
\hline 90 & 000 & 650010 & & $1 \mathrm{AA} 18006$ & $60^{\circ}$ & $43^{\circ} 57^{\prime} \mathrm{W}$ & \\
\hline 10 & 0009 & 523 & 6 & $1 \mathrm{AA}$ & & $56 \cdot \mathrm{W}$ & \\
\hline 40 & 0000 & 633 & & $1 \mathrm{AA} 18008$ & & $43^{\circ} 56^{\prime} \mathrm{W}$ & \\
\hline 14 & 0003.19 & 633112 & 60 & $1 \mathrm{AA} 19001$ & & $43^{\circ} 56^{\circ} \mathrm{W}$ & \\
\hline 90 & 0000.56 & 780010 & & $1 \mathrm{AA}$ & $59^{\circ}$ & $43^{\circ} 57^{\prime} \mathrm{W}$ & \\
\hline 42 & 0000.21 & 780010 & & $1 \mathrm{AA}$ & $\stackrel{N}{N}$ & $8, \mathrm{~V}$ & \\
\hline 52 & & 653012 & & $1 \mathrm{AAI}$ & $59^{\circ} 55^{\prime} \mathrm{N}$ & $43^{\circ} 59^{\prime} \mathrm{W}$ & \\
\hline & 000 & 563 & & 1 AA20001 & $59^{\circ} 55^{\prime} \mathrm{N}$ & $43^{\circ} 58^{\prime} \mathrm{W}$ & \\
\hline 80 & 30 & 750010 & $6000-03$ & $1 \mathrm{AA} 20002$ & $59^{\circ} 55^{\prime} \mathrm{N}$ & $43^{\circ} 57 \cdot \mathrm{W}$ & \\
\hline & & 780010 & & 1 AA20003 & $59054^{\prime} \mathrm{N}$ & $44^{\circ} 00^{\prime} \mathrm{W}$ & \\
\hline & 00 & 7800 & & $1 \mathrm{AA} 20$ & $59^{\circ} 54^{\prime} \mathrm{N}$ & $44^{\circ} 01^{\prime} \mathrm{W}$ & \\
\hline 60 & 0000 . & 770010 & $6000-03$ & 1AA20005 & $59^{\circ} 53^{\prime} \mathrm{N}$ & $44^{\circ} 02^{\prime} \mathrm{W}$ & \\
\hline 110 & 0000.36 & 770010 & $6000-03$ & 1AA20006 & $59^{\circ} 53^{\prime} \mathrm{N}$ & $44^{\circ} 03^{\prime} \mathrm{W}$ & NW \\
\hline & & & & 1AA21001 & $59^{\circ} 53^{\prime} \mathrm{N}$ & $44^{\circ} 03^{\prime} \mathrm{v}$ & \\
\hline & & 623012 & $6000-03$ & 1AA21002 & $59^{\circ} 52^{\prime} \mathrm{N}$ & $44^{\circ} 03^{\prime} \mathrm{W}$ & \\
\hline 75 & 0001.21 & 630212 & $6000-03$ & $1 \mathrm{AA} 21003$ & $59^{\circ} 52^{\prime} \mathrm{N}$ & $44^{\circ} 02^{\prime} \mathrm{w}$ & \\
\hline 30 & 0003.52 & 531212 & $6000-03$ & $1 \mathrm{AA} 21004$ & $59^{\circ} 51^{\prime} \mathrm{N}$ & $44^{\circ} 02^{\prime} \mathrm{v}$ & \\
\hline
\end{tabular}

HIGH
113
130
100
110
105
120
118
100
128
120
120
147
140
130
130
122
130
140
159
136
110
110
130
130
110
120
80
130
120
105
90
90
103
110
130
110
119
110
120
80
109
90
140
150
100
100
79
70
110
122
110
110
70
80
90
91
90
80
109




\begin{tabular}{|c|c|c|c|c|c|c|c|c|}
\hline CODE & LAT & LONG & ORI & HIGH & LOW & AREA & $\begin{array}{c}\text { MO } \\
\text { CODE }\end{array}$ & MAP \\
\hline 1AA22001 & $60^{\circ} 00^{\prime} \mathrm{N}$ & $44^{\circ} 08^{\prime} \mathrm{W}$ & SW & 72 & 68 & 0000.05 & 780010 & $6000-03$ \\
\hline $1 \mathrm{AA2} 2002$ & $59^{\circ} 59^{\prime} \mathrm{N}$ & $44^{\circ} 07^{\prime} \mathrm{W}$ & SW & 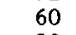 & 60 & 0000.03 & 780010 & $5930-01$ \\
\hline 1AA22003 & $59 \circ 57 / \mathrm{N}$ & $44^{\circ} 07^{\prime} \mathrm{W}$ & W & 80 & 40 & 0000.13 & 750010 & $30-01$ \\
\hline 1AA22004 & $59^{\circ} 57^{\prime} \mathrm{N}$ & $44^{\circ} 08^{\prime} \mathrm{W}$ & $\mathbf{N}$ & 110 & 71 & 0000.30 & 643112 & $5930-01$ \\
\hline 1AA22005 & $59^{\circ} 57^{\prime} \mathrm{N}$ & $44^{\circ} 08^{\prime} \mathrm{W}$ & $\mathrm{s}$ & 70 & 60 & 0000.13 & 790010 & 5930-01 \\
\hline $1 \mathrm{AA} 22006$ & $59^{\circ} 57^{\prime} \mathrm{N}$ & $44^{\circ} 06^{\prime} \mathrm{W}$ & $\mathrm{s}$ & 80 & 50 & 0001.11 & 220110 & $5930-01$ \\
\hline 1AA22007 & $59^{\circ} 57^{\circ} \mathrm{N}$ & $44^{\circ} 05^{\prime} \mathrm{v}$ & 5 & & 51 & 0001.13 & 223110 & $5930-01$ \\
\hline 1AA22008 & $59^{\circ} 58^{\prime} \mathrm{N}$ & $44^{\circ} 04^{\prime} \mathrm{V}$ & $\mathrm{N}$ & 100 & 100 & 0000.18 & 200110 & $5930-01$ \\
\hline 1 AA22009 & $59^{\circ} 58^{\prime} \mathrm{N}$ & $44^{\circ} 03^{\prime} \mathrm{V}$ & E & 70 & 40 & 0000.06 & 643112 & $5930-01$ \\
\hline 1 AA22010 & $60^{\circ} 00^{\prime} \mathrm{N}$ & $44^{\circ} 02^{\prime} \mathrm{W}$ & E & 70 & 53 & 0000.11 & 770112 & $6000-03$ \\
\hline 1AA22011 & $59^{\circ} 59^{\prime} \mathrm{N}$ & $44^{\circ} 03^{\prime} \mathrm{W}$ & $\mathrm{NE}$ & 81 & 78 & 0000.06 & 653132 & 5930-01 \\
\hline 1AA22012 & $60^{\circ} 00^{\prime} \mathrm{N}$ & $44^{\circ} 04^{\prime} \mathrm{W}$ & $\mathrm{N}$ & 70 & 30 & 0001.31 & 643112 & $6000-03$ \\
\hline $1 \mathrm{AA2} 2013$ & $60^{\circ} 00^{\prime} \mathrm{N}$ & $44^{\circ} 05^{\prime} \mathrm{W}$ & N & 80 & 45 & 0000.25 & 643212 & $6000-03$ \\
\hline $1 \mathrm{AA} 22014$ & $59^{\circ} 59^{\prime} \mathrm{N}$ & $44^{\circ} 05^{\prime} \mathrm{W}$ & $v$ & 80 & 42 & 0000.22 & 633212 & $5930-01$ \\
\hline 1AA22015 & $59^{\circ} 59^{\prime} \mathrm{N}$ & $44^{\circ} 04^{\prime} \mathrm{W}$ & NW & 112 & 30 & 0001.81 & 533112 & $5930-01$ \\
\hline 1 AA22016 & $59^{\circ} 58^{\prime} \mathrm{N}$ & $44^{\circ} 05^{\prime} \mathrm{W}$ & $\mathbf{N}$ & 100 & 18 & 0004.13 & 526412 & $5930-01$ \\
\hline 1AA22017 & $60^{\circ} 00^{\prime} \mathrm{N}$ & $44^{\circ} 08^{\prime} \mathrm{W}$ & $\mathrm{NW}$ & & & 0000.19 & 643012 & $6000-03$ \\
\hline 1AA23001 & $59^{\circ} 55^{\prime} \mathrm{N}$ & $44^{\circ} 15^{\prime} \mathrm{W}$ & $v$ & 100 & 80 & & 640010 & $5930-01$ \\
\hline $1 \mathrm{AA} 23002$ & $59^{\circ} 55^{\prime} \mathrm{N}$ & $44^{\circ} 14^{\prime} \mathrm{W}$ & SE & 100 & 80 & 0000 . & 780010 & $5930-01$ \\
\hline 1 AA23003 & $59^{\circ} 55^{\prime} \mathrm{N}$ & $44^{\circ} 13^{\prime} \mathrm{W}$ & E & 90 & 50 & 0000.63 & 646112 & $5930-01$ \\
\hline 1 AA23004 & $59^{\circ} 56^{\prime} \mathrm{N}$ & $44^{\circ} 13^{\prime} \mathrm{W}$ & $\mathrm{SE}$ & 100 & 82 & 0000.22 & 640112 & $5930-01$ \\
\hline 1AA23005 & $59^{\circ} 57^{\prime} \mathrm{N}$ & $44^{\circ} 11^{\prime} \mathrm{W}$ & $\mathrm{SW}$ & 80 & 70 & 0000.27 & 643112 & $5930-01$ \\
\hline $1 \mathrm{AA} 2$ & & $44^{\circ} 10^{\prime} \mathrm{W}$ & $\mathrm{NE}$ & 120 & 90 & & & \\
\hline 1AA23007 & $59^{\circ} 59^{\prime} \mathrm{N}$ & $44^{\circ} 10^{\prime} \mathrm{W}$ & $\mathbf{N}$ & 90 & 60 & 00 & 770010 & -01 \\
\hline $1 \mathrm{AA} 25001$ & $59^{\circ} 57^{\prime} \mathrm{N}$ & $44^{\circ} 15^{\prime} \mathrm{W}$ & $\mathrm{w}$ & 90 & 70 & 0000.15 & 780010 & $5930-01$ \\
\hline 1 AA25002 & Nי & $44^{\circ} 14^{\prime} \mathrm{W}$ & s & 100 & 85 & 00 & 780010 & $5930-01$ \\
\hline IAA2: & $59^{\circ} 56^{\prime} \mathrm{N}$ & $44^{\circ} 13^{\prime} \mathrm{W}$ & NW & 100 & 67 & & 643112 & -01 \\
\hline $1 \mathrm{AA} 2$ & & $4 \cdot \mathrm{W}$ & $\mathrm{v}$ & 82 & 69 & & & \\
\hline $1 \mathrm{AA} 2$ & $59^{\circ} 55^{\prime} \mathrm{N}$ & $44^{\circ} 14^{\prime} \mathrm{W}$ & $\mathrm{NV}$ & 90 & 49 & 00 & 750010 & 593 \\
\hline 1 AA25006 & $59^{\circ} 55^{\prime} \mathrm{N}$ & $44^{\circ} 15^{\prime} \mathrm{W}$ & NW & 80 & 68 & 0000.08 & 750010 & $5930-01$ \\
\hline 1 AA26001 & $59^{\circ} 58^{\prime} \mathrm{N}$ & $44^{\circ} 11^{\prime} \mathrm{W}$ & $v$ & 100 & 35 & 0000.72 & 633112 & 5930-01 \\
\hline $1 \mathrm{AA} 2$ & $59^{\circ} 57^{\prime} \mathrm{N}$ & $44^{\circ} 12^{\prime} \mathrm{W}$ & SW & 80 & 50 & 0001.37 & 620112 & $5930-01$ \\
\hline $1 \mathrm{AA2}$ & & & N & 90 & 50 & & & \\
\hline $1 \mathrm{AA} 26004$ & $59^{\circ} 57^{\prime} \mathrm{N}$ & $44^{\circ} 14^{\prime} \mathrm{W}$ & $\mathrm{NW}$ & 90 & 60 & 00 & 640010 & -01 \\
\hline 1AA27001 & $60^{\circ} 01^{\prime} \mathrm{N}$ & $44^{\circ} 23^{\prime} \mathrm{W}$ & SE & 126 & 100 & & 10 & -03 \\
\hline 1 AA27002 & $60^{\circ}$ & $44^{\circ} 22^{\prime} \mathrm{W}$ & N & 90 & 80 & & 10 & .03 \\
\hline $1 \mathrm{AA} 2$ & $60^{\circ}$ & $44^{\circ} 22^{\prime} \mathrm{W}$ & SE & 135 & 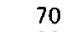 & & & \\
\hline 1 AA2 & & & SE & 130 & 90 & & & \\
\hline 1AA27005 & $60^{\circ} 03^{\prime} \mathrm{N}$ & $44^{\circ} 19^{\prime} \mathrm{W}$ & $\mathbf{E}$ & 90 & 80 & 000 & 750110 & \\
\hline 1AA27006 & $60^{\circ} 03^{\prime} \mathrm{N}$ & $44^{\circ} 19^{\prime} \mathrm{W}$ & E & 100 & 80 & 000 & 10 & -03 \\
\hline 1 AA2 & & $44^{\circ} 18^{\prime} \mathrm{W}$ & 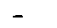 & 100 & J & & & \\
\hline $1 A A$ & & $44^{\circ} 20^{\prime} \mathrm{V}$ & $\mathbf{N}$ & 118 & 60 & 000 & & .03 \\
\hline $1 \mathrm{AA}$ & & $44^{\circ} 20^{\prime} \mathrm{W}$ & $\mathbf{N}$ & 100 & 70 & & & \\
\hline 1AA27010 & 60 & $44^{\circ} 21^{\prime} \mathrm{W}$ & $\mathrm{N}$ & 100 & 60 & & & -03 \\
\hline $1 \mathrm{AA}$ & & $44^{\circ} 2$ & $\mathrm{SE}$ & 8 & 60 & & & \\
\hline $1 \mathrm{AA} 2$ & & ' W & $\mathrm{E}$ & 12 & 90 & & & \\
\hline 1AA27013 & $60^{\circ}$ & $44^{\circ} 19^{\prime} \mathrm{W}$ & $\mathrm{N}$ & 120 & 105 & 00 & 623 & -03 \\
\hline $1 \mathrm{AA} 28$ & & & $\mathrm{~N}$ & 98 & 75 & & & \\
\hline 1 AA28002 & $60^{\circ} 06^{\prime} \mathrm{N}$ & $44^{\circ} 26^{\prime} \mathrm{W}$ & $\mathrm{s}$ & $12 C$ & 60 & .67 & 673112 & -02 \\
\hline 1AA28003 & & $44^{\circ} 24^{\prime} \mathrm{W}$ & SE & 60 & 60 & & & \\
\hline & $60^{\circ} 05^{\prime} \mathrm{N}$ & $44^{\circ} 23^{\prime} \mathrm{W}$ & $\mathrm{N}$ & 102 & 96 & 0000.07 & 780010 & 10-03 \\
\hline $1 \mathrm{AA2}$ & & $44^{\circ} 23^{\prime} \mathrm{W}$ & $\mathrm{SE}$ & 120 & 100 & 000 & 780010 & $6000-03$ \\
\hline & & & $\mathbf{N}$ & 100 & 50 & & 620112 & \\
\hline & $60^{\circ} 09$ & $44^{\circ}$ & SW & 90 & 70 & 00 & & \\
\hline 1AA28008 & $60^{\circ} 04^{\prime} \mathrm{N}$ & $44^{\circ} 22^{\prime} \mathrm{W}$ & SW & 100 & 75 & & & \\
\hline & $60^{\circ} 03^{\prime} \mathrm{N}$ & $44^{\circ} 22^{\prime \prime} \mathrm{V}$ & $\mathbf{N N}$ & 110 & 90 & 0000 & 790 & $6000-03$ \\
\hline 010 & $60^{\circ} 03^{\prime} \mathrm{N}$ & $44^{\circ} 23^{\prime} \mathrm{K}$ & $\mathbf{N H}$ & 110 & & & 780010 & $6000-03$ \\
\hline 1AA & $60^{\circ} 07^{\prime} \mathrm{N}$ & $44^{\circ} 23^{\prime} \mathrm{V}$ & NW & 96 & 8 & 00 & 780010 & $6000-03$ \\
\hline & $60^{\circ} 07^{\prime} \mathrm{N}$ & $44^{\circ} 24^{\prime} \mathrm{W}$ & NW & & 70 & 0000.33 & 670212 & $6000-03$ \\
\hline 9003 & $60^{\circ} 07^{\prime} \mathrm{N}$ & $44^{\circ} 25^{\prime} \mathrm{W}$ & $\mathbf{N}$ & 105 & 60 & 0000.70 & 623312 & $6000-03$ \\
\hline 0 & $60^{\circ} 07^{\prime} \mathrm{N}$ & $44^{\circ} 25^{\prime} \mathrm{W}$ & $\mathrm{NE}$ & & 促 & 0000.64 & 770112 & $6000-c$ \\
\hline
\end{tabular}

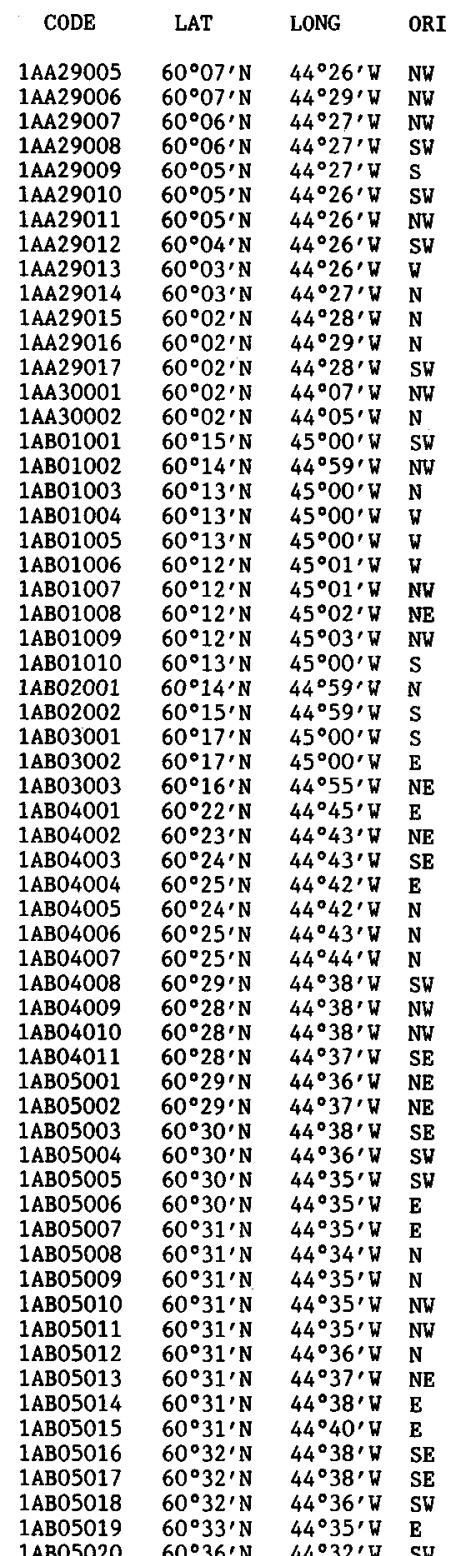

$\begin{array}{rrc}\text { HIGH } & \text { LOW } & \text { AREA } \\ 121 & 90 & 0000.38 \\ 90 & 60 & 0000.05 \\ 100 & 80 & 0000.60 \\ 80 & 70 & 0000.13 \\ 90 & 80 & 0000.16 \\ 100 & 80 & 0000.08 \\ 100 & 80 & 0000.21 \\ 100 & 70 & 0000.11 \\ 90 & 68 & 0000.10 \\ 80 & 60 & 0000.10 \\ 110 & 90 & 0000.03 \\ 90 & 80 & 0000.05 \\ 100 & 90 & 000.07 \\ 80 & 75 & 0000.01 \\ 85 & 80 & 0000.01 \\ 90 & 81 & 0000.04 \\ 91 & 88 & 0000.03 \\ 95 & 65 & 0000.08 \\ 105 & 87 & 0000.05 \\ 105 & 86 & 0000.06 \\ 105 & 80 & 0000.20 \\ 102 & 60 & 0000.45 \\ 82 & 72 & 0000.04 \\ 92 & 79 & 0000.01 \\ 110 & 93 & 0000.02 \\ 80 & 70 & 0000.09 \\ 105 & 95 & 0000.04 \\ 85 & 85 & 0000.01 \\ 100 & 72 & 0000.06 \\ 90 & 67 & 0000.15 \\ 120 & 110 & 0000.07 \\ 117 & 93 & 0000.54 \\ 131 & 90 & 0000.56 \\ 100 & 84 & 0000.18 \\ 120 & 90 & 0000.19 \\ 110 & 73 & 0000.48 \\ 120 & 94 & 0000.06 \\ 137 & 69 & 0002.54 \\ 118 & 93 & 0000.02 \\ 130 & 114 & 0000.06 \\ 92 & 79 & 0000.11 \\ 110 & 87 & 0000.18 \\ 95 & 85 & 0000.07 \\ 110 & 85 & 0000.43 \\ 120 & 90 & 0000.13 \\ 145 & 120 & 0000.10 \\ 110 & 92 & 0000.31 \\ 120 & 10 & 0000.30 \\ 125 & 60 & 0000.08 \\ 110 & 80 & 0000.09 \\ 120 & 100 & 0000.05 \\ 120 & 90 & 0000.06 \\ 110 & 80 & 0000.43 \\ 110 & 87 & 0000.19 \\ 120 & 98 & 0000.20 \\ 105 & 95 & 0000.81 \\ 120 & 82 & 0000.25 \\ 105 & 70 & 0000.75 \\ 125 & 102 & 0000.06 \\ 145 & 140 & 0000.06 \\ 165 & 180 & 0007.30 \\ & & \end{array}$

$\stackrel{\text { MO }}{\text { CODE }}$

MAP N

$673212 \quad 6000-02$

$750010 \quad 6000-02$

$643212 \quad 6000-02$

$750010 \quad 6000-02$

$780010 \quad 6000-02$

$780010 \quad 6000-02$

$780010 \quad 6000-02$

$770010 \quad 6000-02$

$780010 \quad 6000-02$

$780010 \quad 6000-02$

$780010 \quad 6000-02$

$790010 \quad 6000-03$

$790010 \quad 6000-03$

$750010 \quad 6000-02$

$750010 \quad 6000-02$

$700012 \quad 6000-02$

$640111 \quad 6000-02$

$700012 \quad 6000-02$

$780010 \quad 6000-02$

$780010 \quad 6000-02$

$790012 \quad 6000-02$

$780010 \quad 6000-02$

$780010 \quad 6000-02$

$790012 \quad 6000-02$

$790012 \quad 6000-02$

$643110 \quad 6000-02$

$640010 \quad 6000-02$

$6401126000-02$

$627312 \quad 6000-02$

$643112 \quad 6000-02$

$5231126000-02$

$740110 \quad 6000-02$

$790110 \quad 6000-02$

$670112 \quad 6030-06$

$753012 \quad 6030-06$

$740012 \quad 6030-06$

$643212 \quad 6030-06$

$643312 \quad 6030-06$

$740010 \quad 6030-06$

$750212 \quad 6030-06$

$750112 \quad 6030-06$

$643112 \quad 6030-06$

$6131126030-06$

633112 6030-06

$6431126030-06$

$770010-6030-06$

$790012 \quad 6030-06$ 


\begin{tabular}{|c|c|c|c|c|}
\hline CODE & LAT & LONG & ORI & HIGH \\
\hline & $\begin{array}{l}60^{\circ} 35^{\prime} \mathrm{N} \\
60^{\circ} 35^{\prime} \mathrm{N}\end{array}$ & $44^{\circ} 31^{\prime} \mathrm{V}$ & $\mathrm{SW}$ & 92 \\
\hline $\begin{array}{l}1 \mathrm{AB} 05022 \\
1 \mathrm{AB} 05023\end{array}$ & $\begin{array}{l}60^{\circ} 35^{\prime} \mathrm{N} \\
60^{\circ} 35^{\prime} \mathrm{N}\end{array}$ & $\begin{array}{l}44^{\circ} 30^{\prime} \mathrm{V} \\
44^{\circ} 30^{\prime} \mathrm{V}\end{array}$ & SW & $\begin{array}{l}80 \\
80\end{array}$ \\
\hline $1 \mathrm{AB} 05024$ & $60^{\circ} 34^{\prime} \mathrm{N}$ & $44^{\circ} 30^{\prime} \mathrm{V}$ & W & 90 \\
\hline $1 \mathrm{AB} 05025$ & $60^{\circ} 34^{i} \mathrm{~N}$ & $44^{\circ} 31^{\prime} \mathrm{W}$ & SW & 120 \\
\hline BO5026 & $60^{\circ} 33^{\prime} \mathrm{N}$ & $44^{\circ} 31^{\prime} \mathrm{W}$ & NW & 110 \\
\hline $1 \mathrm{AB} 06001$ & $60^{\circ} 35^{\prime} \mathrm{N}$ & $44^{\circ} 27^{\prime} \mathrm{W}$ & $\mathrm{SW}$ & 150 \\
\hline $1 \mathrm{AB06002}$ & $60^{\circ} 34^{\prime} \mathrm{N}$ & $44^{\circ} 26^{\prime} \mathrm{W}$ & W & 90 \\
\hline $1 \mathrm{AB} 06003$ & $60^{\circ} 33^{\prime} \mathrm{N}$ & $44^{\circ} 25^{\prime} \mathrm{V}$ & $\mathrm{N}$ & 135 \\
\hline $1 \mathrm{AB} 06004$ & $60^{\circ} 33^{\prime} \mathrm{N}$ & $44^{\circ} 26^{\prime} \mathrm{W}$ & W & 120 \\
\hline $\mathrm{BB} 06005$ & $60^{\circ} 33^{\prime} \mathrm{N}$ & $44^{\circ} 25^{\prime} \mathrm{W}$ & s & 119 \\
\hline $1 \mathrm{AB} 06006$ & $60^{\circ} 33^{\prime} \mathrm{N}$ & $44^{\circ} 24^{\prime} \mathrm{W}$ & $\mathrm{s}$ & 150 \\
\hline $1 \mathrm{AB} 06007$ & $60^{\circ} 32^{\prime} \mathrm{N}$ & $44^{\circ} 21^{\prime} \mathrm{W}$ & $\mathrm{s}$ & 145 \\
\hline $1 \mathrm{AB} 06008$ & $60^{\circ} 32^{\prime} \mathrm{N}$ & $44^{\circ} 23^{\prime} \mathrm{W}$ & W & 150 \\
\hline AB07001 & $60^{\circ} 31^{\prime} \mathrm{N}$ & $44^{\circ} 21^{\prime} \mathrm{W}$ & W & 110 \\
\hline $1 \mathrm{AB}$ & $60^{\circ} 30^{\prime} \mathrm{N}$ & $44^{\circ} 20^{\prime \prime} \mathrm{V}$ & s & 160 \\
\hline 003 & $60^{\circ} 30^{\prime} \mathrm{N}$ & $44^{\circ} 19^{\prime} \mathrm{W}$ & $\mathrm{s}$ & 165 \\
\hline B07004 & $60^{\circ} 30^{\prime} \mathrm{N}$ & $44^{\circ} 19^{\prime} W$ & $\mathrm{~s}$ & 165 \\
\hline $1 \mathrm{AB} 07005$ & $60^{\circ} 30^{\prime} \mathrm{N}$ & $44^{\circ} 21^{\prime} \mathrm{W}$ & W & 140 \\
\hline $1 \mathrm{AB} 07006$ & $60^{\circ} 29^{\prime} \mathrm{N}$ & $44^{\circ} 22^{\prime} \mathrm{W}$ & NW & 140 \\
\hline & & $44^{\circ} 24^{\prime} \mathrm{W}$ & N & 140 \\
\hline $1 \mathrm{ABC}$ & $60^{\circ} 30^{\prime} \mathrm{N}$ & $44^{\circ} 25^{\prime} \mathrm{W}$ & $\mathbf{N}$ & 110 \\
\hline $1 \mathrm{ABO}$ & $60^{\circ} 29^{\prime} \mathrm{N}$ & $44^{\circ} 26^{\prime} \mathrm{W}$ & NW & 90 \\
\hline $1 \mathrm{AB} 07010$ & $60^{\circ} 30^{\prime} \mathrm{N}$ & $44^{\circ} 26^{\circ} \mathrm{v}$ & $\mathrm{NW}$ & 140 \\
\hline $1 \mathrm{ABO}$ & $60^{\circ} 29^{\prime} \mathrm{N}$ & $44^{\circ} 27^{\prime} \mathrm{W}$ & NW & 150 \\
\hline & 60 & $44^{\circ} 23^{\prime} \mathrm{W}$ & $\mathrm{s}$ & 120 \\
\hline $1 \mathrm{ABC}$ & $60^{\circ} 28^{\prime} \mathrm{N}$ & $44^{\circ} 21^{\prime} \mathrm{v}$ & U & 164 \\
\hline $1 \mathrm{AB} 08003$ & $60^{\circ} 27^{\prime} \mathrm{N}$ & $44^{\circ} 26^{\prime} \mathrm{W}$ & $\mathrm{N}$ & 160 \\
\hline $1 \mathrm{ABC}$ & $60^{\circ} 27^{\prime} \mathrm{N}$ & $44^{\circ} 27^{\prime} \mathrm{W}$ & N & 90 \\
\hline $1 \mathrm{AB}$ & $60^{\circ} 27^{\prime} \mathrm{N}$ & $44^{\circ} 26^{\prime} \mathrm{W}$ & SW & 155 \\
\hline & & $44^{\circ} 27^{\prime} \mathrm{V}$ & $\mathrm{NW}$ & 165 \\
\hline 003 & $60^{\circ} 25^{\prime} \mathrm{N}$ & $44^{\circ} 25^{\prime} \mathrm{W}$ & $\mathrm{W}$ & 140 \\
\hline $1 \mathrm{AB}$ & $60^{\circ} 2$ & $44^{\circ} 25^{\prime} \mathrm{V}$ & $W$ & 155 \\
\hline & $60^{\circ} 2$ & $44^{\circ} 26^{\prime} \mathrm{V}$ & SW & 160 \\
\hline & & $44^{\circ} 24^{\prime} \mathrm{W}$ & $\mathrm{W}$ & 170 \\
\hline & 6 & $44^{\circ} 24^{\prime} \mathrm{W}$ & $\mathrm{W}$ & 150 \\
\hline $1 \mathrm{AB}$ & $60^{\circ} 22^{\prime} \mathrm{N}$ & $44^{\circ} 25^{\prime} \mathrm{W}$ & $\mathbf{N}$ & 100 \\
\hline & 6 & $44^{\circ}$ & $\mathrm{NE}$ & 160 \\
\hline & & $44^{\circ}$ & $\mathrm{NE}$ & 150 \\
\hline & & & $\mathrm{E}$ & 110 \\
\hline & 6 & $44^{\circ} 29^{\prime} \mathrm{W}$ & E & 110 \\
\hline $1 \mathrm{AB}$ & $60^{\circ} 2$ & $44^{\circ} 30^{\prime} \mathrm{N}$ & E & 163 \\
\hline & 6 & $\mathrm{w}$ & $\mathbf{N}$ & 80 \\
\hline & & $44^{\circ}$ & H & 120 \\
\hline & & $44^{\circ} 33^{\prime} \mathrm{V}$ & 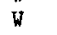 & 102 \\
\hline & 6 & $44^{\circ}$ & $\mathrm{W}$ & 122 \\
\hline & & & $\mathrm{v}$ & 100 \\
\hline & & $44^{\circ} 29^{\prime} \mathrm{V}$ & W & 130 \\
\hline & & $44^{\circ} 30^{\prime} \mathrm{W}$ & W & 180 \\
\hline & $60^{\circ} 23$ & $44^{\circ} 32^{\prime} \mathrm{W}$ & NW & 180 \\
\hline & & & W & 120 \\
\hline & 6 & $44^{\circ} 32^{\prime} \mathrm{W}$ & SW & 150 \\
\hline & & $44^{\circ}$ & $\mathrm{W}$ & 140 \\
\hline & & & W & \\
\hline & & & $\mathrm{N}$ & 120 \\
\hline & 60 & $44^{\circ} 27^{\prime} \mathrm{W}$ & $\mathrm{V}$ & 160 \\
\hline & 60 & $44^{\circ} 28^{\prime}, \mathrm{w}$ & W & 100 \\
\hline & & 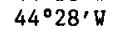 & NW & \\
\hline & & $44^{\circ} 29^{\prime} \mathrm{v}$ & $\mathrm{N}$ & 75 \\
\hline $1 \mathrm{AB}$ & $60^{\circ} 21^{\prime} \mathrm{N}$ & $44^{\circ} 30^{\prime} \mathrm{V}$ & $\mathrm{N}$ & 95 \\
\hline $1 \mathrm{AB} 11011$ & $60^{\circ} 20^{\prime} \mathrm{N}$ & $44^{\circ} 29^{\prime} \mathrm{W}$ & NW & 130 \\
\hline
\end{tabular}

\begin{tabular}{rccc|} 
LOW & AREA & MO & \\
& CODE & MAP \\
79 & 0000.31 & 640212 & $6030-06$ \\
78 & 0000.05 & 750010 & $6030-06$ \\
75 & 0000.05 & 75010 & $6030-06$ \\
80 & 0000.05 & 780010 & $6030-06$ \\
90 & 0000.13 & 750010 & $6030-06$ \\
80 & 0000.05 & 750010 & $6030-06$ \\
& 0025.00 & 365311 & $6030-06$ \\
30 & 0001.13 & 533112 & $6030-06$ \\
120 & 0000.16 & 770010 & $6030-06$ \\
90 & 0000.13 & 753212 & $6030-06$ \\
110 & 0000.06 & 770012 & $6030-06$ \\
47 & 0004.10 & 363110 & $6030-06$ \\
100 & 0000.30 & 27010 & $6030-06$ \\
5 & 0023.80 & 363112 & $6030-06$ \\
62 & 0002.43 & 633112 & $6030-06$ \\
130 & 0000.06 & 750010 & $6030-06$ \\
155 & 0000.03 & 75010 & $6030-06$ \\
155 & 0000.05 & 750010 & $6030-06$ \\
50 & 0002.13 & 533112 & $6030-06$ \\
125 & 0000.50 & 770112 & $6000-03$ \\
125 & 0000.40 & 653112 & $6000-03$ \\
95 & 0000.16 & 670010 & $6030-06$ \\
60 & 0000.93 & 633112 & $6000-02$ \\
140 & 0000.05 & 680212 & $6030-06$ \\
& 0001.63 & 623212 & $6000-02$ \\
120 & 0000.05 & 770112 & $6000-03$ \\
26 & 0019.08 & 513112 & $6000-03$ \\
140 & 0000.06 & 780010 & $6000-02$ \\
50 & 0000.07 & 780010 & $6000-02$ \\
125 & 0000.02 & 780010 & $6000-02$ \\
140 & 0000.07 & 78010 & $6000-02$ \\
105 & 0000.05 & 790012 & $6000-03$ \\
130 & 0000.03 & 750012 & $6000-03$ \\
80 & 0000.51 & 653312 & $6000-02$ \\
90 & 0000.50 & 653312 & $6000-03$ \\
85 & 0000.49 & 210112 & $6000-03$ \\
45 & 0005.19 & 523112 & $6000-03$ \\
130 & 0000.39 & 643412 & $6000-02$ \\
80 & 0000.23 & 643412 & $6000-02$ \\
70 & 0000.16 & 640212 & $6000-02$ \\
45 & 0000.44 & 643112 & $6000-02$ \\
50 & 0000.74 & 630512 & $6000-02$ \\
57 & 0000.24 & 630112 & $6000-02$ \\
70 & 0000.55 & 640112 & $6000-02$ \\
80 & 0000.06 & 750112 & $6000-02$ \\
100 & 0000.31 & 770112 & $6000-02$ \\
40 & 0001.48 & 533212 & $6000-02$ \\
90 & 0000.15 & 680010 & $6000-02$ \\
73 & 0002.89 & 533112 & $6000-02$ \\
60 & 0001.37 & 643112 & $6000-02$ \\
90 & 0000.09 & 780110 & $6000-02$ \\
120 & 0000.17 & 790010 & $6000-02$ \\
110 & 0000.05 & 790010 & $6000-02$ \\
80 & 0000.12 & 770010 & $6000-02$ \\
110 & 0000.27 & 780010 & $6000-02$ \\
65 & 0001.51 & 633112 & $6000-02$ \\
80 & 0000.32 & 673112 & $6000-02$ \\
68 & 0000.24 & 743012 & $6000-02$ \\
10 & 0000.20 & 743012 & $6000-02$ \\
70 & 0000.15 & 753110 & $6000-02$ \\
70 & 0001.20 & 643112 & $6000-02$ \\
& & &
\end{tabular}

\begin{tabular}{|c|c|c|c|c|}
\hline CODE & LAT & LONG & ORI & HIGH \\
\hline $1 \mathrm{AB} 11012$ & $60^{\circ} 20^{\prime} \mathrm{N}$ & $44^{\circ} 30^{\prime} \mathrm{W}$ & 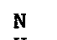 & 120 \\
\hline $1 \mathrm{AB} 11013$ & $60^{\circ} 20^{\prime} \mathrm{N}$ & $44^{\circ} 31^{\prime} \mathrm{W}$ & w & \\
\hline $1 \mathrm{AB} 11014$ & $60^{\circ} 19^{\prime} \mathrm{N}$ & $44^{\circ} 32^{\prime} \mathrm{W}$ & $\mathrm{N}$ & 130 \\
\hline $1 \mathrm{AB} 12001$ & $60^{\circ} 21^{\prime} \mathrm{N}$ & $44^{\circ} 32^{\prime}$ W & NW & 100 \\
\hline$A B B 12002$ & $60^{\circ} 21^{\prime} \mathrm{N}$ & $44^{\circ} 31^{\prime} \mathrm{W}$ & su & 120 \\
\hline $1 \mathrm{AB12003}$ & $60^{\circ} 20^{\prime} \mathrm{N}$ & $44^{\circ} 35^{\prime} \mathrm{W}$ & NV & 139 \\
\hline $1 \mathrm{AB} 12004$ & $60^{\circ} 19^{\prime} \mathrm{N}$ & $44^{\circ} 35^{\prime} \mathrm{W}$ & NG & 115 \\
\hline $1 \mathrm{AB} 12005$ & $60^{\circ} 19^{\prime} \mathrm{N}$ & $44^{\circ} 36^{\prime} \mathrm{W}$ & N & 110 \\
\hline $1 \mathrm{AB} 12006$ & $60^{\circ} 19^{\prime} \mathrm{N}$ & $44^{\circ} 38^{\circ}, \mathrm{w}$ & NE & 130 \\
\hline $1 \mathrm{AB} 12007$ & $60^{\circ} 20^{\prime} \mathrm{N}$ & $44^{\circ} 39^{\prime} \mathrm{W}$ & $\mathrm{NW}$ & 100 \\
\hline $1 \mathrm{AB} 12008$ & $60^{\circ} 20^{\prime} \mathrm{N}$ & $44^{\circ} 39^{\prime} \mathrm{W}$ & NV & 120 \\
\hline $1 \mathrm{AB} 12009$ & $60^{\circ} 20^{\prime} \mathrm{N}$ & $44^{\circ} 39^{\prime} \mathrm{W}$ & $\mathrm{v}$ & 90 \\
\hline $1 \mathrm{AB} 12010$ & $60^{\circ} 19^{\prime} \mathrm{N}$ & $44^{\circ} 40^{\prime} \mathrm{W}$ & W & 110 \\
\hline $1 \mathrm{AB} 12011$ & $60^{\circ} 19^{\prime} \mathrm{N}$ & $44^{\circ} 41^{\prime} \mathrm{W}$ & $\mathrm{N}$ & 100 \\
\hline $1 \mathrm{AB} 13001$ & $60^{\circ} 18^{\prime} \mathrm{N}$ & $44^{\circ} 41^{\prime} \mathrm{W}$ & NW & 110 \\
\hline $1 \mathrm{AB} 13002$ & $60^{\circ} 18^{\prime} \mathrm{N}$ & $44^{\circ} 41^{\prime} \mathrm{W}$ & W & 60 \\
\hline $1 \mathrm{AB} 13003$ & $60^{\circ} 19^{\prime} \mathrm{N}$ & $44^{\circ} 38^{\prime} \mathrm{W}$ & $\mathbf{S}$ & 135 \\
\hline $1 \mathrm{AB} 13004$ & $60^{\circ} 18^{\prime} \mathrm{N}$ & $44^{\circ} 35^{\prime} \mathrm{W}$ & SW & 120 \\
\hline $1 \mathrm{AB} 13005$ & $60^{\circ} 17^{\prime} \mathrm{N}$ & $44^{\circ} 35^{\prime} \mathrm{W}$ & SW & 120 \\
\hline $1 \mathrm{AB} 13006$ & $60^{\circ} 19^{\prime} \mathrm{N}$ & $44^{\circ} 30^{\prime} \mathrm{W}$ & $\mathrm{s}$ & 140 \\
\hline $1 \mathrm{AB} 13007$ & $60^{\circ} 19^{\prime} \mathrm{N}$ & $44^{\circ} 29^{\prime} \mathrm{W}$ & $\mathrm{s}$ & 170 \\
\hline $1 \mathrm{AB} 13008$ & $60^{\circ} 20^{\prime} \mathrm{N}$ & $44^{\circ} 27^{\prime} \mathrm{W}$ & $\mathrm{SE}$ & 105 \\
\hline $1 \mathrm{AB} 13009$ & $60^{\circ} 20^{\prime} \mathrm{N}$ & $44^{\circ} 27^{\circ}, \mathrm{W}$ & $\mathbf{E}$ & 115 \\
\hline $1 \mathrm{AB} 13010$ & $60^{\circ} 20^{\prime} \mathrm{N}$ & $44^{\circ} 27^{\prime} \mathrm{W}$ & $\mathbf{E}$ & 115 \\
\hline $1 \mathrm{AB} 13011$ & $60^{\circ} 21^{\prime} \mathrm{N}$ & $44^{\circ} 28^{\prime} \mathrm{W}$ & $\mathbf{E}$ & 140 \\
\hline $1 \mathrm{AB} 13012$ & $60^{\circ} 21^{\prime} \mathrm{N}$ & $44^{\circ} 27^{\prime} \mathrm{W}$ & $\mathbf{S}$ & 150 \\
\hline $1 \mathrm{AB} 13013$ & $60^{\circ} 21^{\prime} \mathrm{N}$ & $44^{\circ} 27^{\prime} \mathrm{W}$ & SE & 170 \\
\hline $1 \mathrm{AB} 13014$ & $60^{\circ} 22^{\prime} \mathrm{N}$ & $44^{\circ} 27^{\prime} \mathrm{W}$ & NE & 160 \\
\hline $1 \mathrm{AB} 13015$ & $60^{\circ} 22^{\prime} \mathrm{N}$ & $44^{\circ} 22^{\prime} \mathrm{W}$ & $\mathrm{s}$ & 182 \\
\hline $1 \mathrm{AB} 13016$ & $60^{\circ} 23^{\prime} \mathrm{N}$ & $44^{\circ} 21^{\prime} \mathrm{W}$ & $\mathrm{s}$ & 160 \\
\hline $1 \mathrm{AB} 13017$ & $60^{\circ} 21^{\prime} \mathrm{N}$ & $44^{\circ} 20^{\prime} \mathrm{W}$ & $\bar{w}$ & 150 \\
\hline $1 \mathrm{AB} 13018$ & $60^{\circ} 20^{\prime} \mathrm{N}$ & $44^{\circ} 24^{\prime} \mathrm{W}$ & NW & 90 \\
\hline $1 \mathrm{AB} 13019$ & $60^{\circ} 20^{\prime} \mathrm{N}$ & $44^{\circ} 24^{\prime} \mathrm{w}$ & $\eta$ & 100 \\
\hline $1 \mathrm{AB} 13020$ & $60^{\circ} 19^{\prime} \mathrm{N}$ & $44^{\circ} 24^{\prime} \mathrm{W}$ & $\mathrm{w}$ & 120 \\
\hline $1 \mathrm{AB} 13021$ & $60^{\circ} 19^{\prime} \mathrm{N}$ & $44^{\circ} 24^{\prime} \mathrm{W}$ & W & 110 \\
\hline $1 \mathrm{AB} 13022$ & $60^{\circ} 18^{\prime} \mathrm{N}$ & $44^{\circ} 24^{\prime} \mathrm{W}$ & NW & 110 \\
\hline $1 \mathrm{AB} 13023$ & $60^{\circ} 17^{\prime} \mathrm{N}$ & $44^{\circ} 27^{\prime} \mathrm{W}$ & $\mathrm{N}$ & 170 \\
\hline $1 \mathrm{AB} 13024$ & $60^{\circ} 18^{\prime} \mathrm{N}$ & $44^{\circ} 27^{\prime} \mathrm{W}$ & $\mathrm{N}$ & 170 \\
\hline $1 \mathrm{AB} 13025$ & $60^{\circ} 17^{\prime} \mathrm{N}$ & $44^{\circ} 28^{\circ} \mathrm{W}$ & v & 135 \\
\hline $1 \mathrm{AB} 13026$ & $60^{\circ} 17^{\prime N} \mathrm{~N}$ & $44^{\circ} 28^{\circ} \mathrm{k}$ & W & 150 \\
\hline $1 \mathrm{AB} 13027$ & $60^{\circ} 16^{\prime} \mathrm{N}$ & $44^{\circ} 28^{\prime} \mathrm{W}$ & $\mathrm{NW}$ & 162 \\
\hline $1 \mathrm{AB} 13028$ & $60^{\circ} 16^{\prime} \mathrm{N}$ & $44^{\circ} 30^{\prime} \mathrm{W}$ & NW & 100 \\
\hline $1 \mathrm{AB} 13029$ & $60^{\circ} 16^{\prime} \mathrm{N}$ & $44^{\circ} 30^{\prime} \mathrm{W}$ & $\mathrm{w}$ & 120 \\
\hline $1 \mathrm{AB} 13030$ & $60^{\circ} 16^{\prime} \mathrm{N}$ & $44^{\circ} 30^{\prime} \mathrm{w}$ & SW & 110 \\
\hline $1 \mathrm{AB} 13031$ & $60^{\circ} 16^{\prime} \mathrm{N}$ & $44^{\circ} 29^{\prime} \mathrm{W}$ & $\mathbf{s}$ & 150 \\
\hline $1 \mathrm{AB} 13032$ & $60^{\circ} 16^{\prime} \mathrm{N}$ & $44^{\circ} 28^{\prime} \mathrm{W}$ & SW & 120 \\
\hline $1 \mathrm{AB} 13033$ & $60^{\circ} 14^{\prime} \mathrm{N}$ & $44^{\circ} 27^{\prime \prime} \mathrm{W}$ & $\mathrm{SW}$ & 120 \\
\hline $1 \mathrm{AB} 13034$ & $60^{\circ} 13^{\prime} \mathrm{N}$ & $44^{\circ} 28^{\prime} \mathrm{V}$ & $\mathrm{N}$ & 160 \\
\hline $1 \mathrm{AB} 13035$ & $60^{\circ} 14^{\prime} \mathrm{N}$ & $44^{\circ} 30^{\prime} \mathrm{v}$ & $\mathrm{N}$ & 100 \\
\hline $1 \mathrm{AB} 1303$ & $60^{\circ} 14^{\prime} \mathrm{N}$ & $44^{\circ} 31^{\prime} \mathrm{k}$ & $\mathrm{NE}$ & 144 \\
\hline $1 \mathrm{AB} 13037$ & $60^{\circ} 14^{\prime} \mathrm{N}$ & $44^{\circ} 32^{\prime} \mathrm{H}$ & $\mathrm{NE}$ & 110 \\
\hline $1 \mathrm{AB} 13038$ & $60^{\circ} 13^{\prime} \mathrm{N}$ & $44^{\circ} 34^{\prime} \mathrm{W}$ & NW & 110 \\
\hline $1 \mathrm{AB}$ & $60^{\circ} 13^{\prime} \mathrm{N}$ & $44^{\circ} 34^{\prime} \mathrm{V}$ & NW & 130 \\
\hline $1 \mathrm{AB} 130$ & $60^{\circ} 14^{\prime} \mathrm{N}$ & $44^{\circ} 36^{\prime} \mathrm{W}$ & NE & 120 \\
\hline $1 \mathrm{AB} 13041$ & $60^{\circ} 15^{\prime} \mathrm{N}$ & $44^{\circ} 35^{\prime} \mathrm{W}$ & $\mathrm{N}$ & 100 \\
\hline $1 \mathrm{AB} 13042$ & $60^{\circ} 14^{\prime} \mathrm{N}$ & $44^{\circ} 36^{\prime} \mathrm{W}$ & $\mathrm{NE}$ & 130 \\
\hline $1 A B 13 \cap 4$ & $60^{\circ} 15^{\prime} \mathrm{N}$ & $44^{\circ} 38^{\prime} \mathrm{w}$ & $\mathrm{N}$ & 110 \\
\hline $1 \mathrm{AB} 13044$ & $60^{\circ} 14^{\prime} \mathrm{N}$ & $44^{\circ} 39^{\prime} \mathrm{W}$ & $\mathrm{N}$ & 120 \\
\hline $1 \mathrm{AB} 13045$ & $60^{\circ} 14^{\prime} \mathrm{N}$ & $44^{\circ} 40^{\prime} \mathrm{W}$ & NW & 100 \\
\hline$n$ & $0^{\circ} 14^{\prime} \mathrm{N}$ & $44^{\circ} 41^{\prime} \mathrm{W}$ & NW & 125 \\
\hline 10 & $60^{\circ} 13^{\prime} \mathrm{N}$ & $44^{\circ} 41^{\prime} \mathrm{w}$ & SW & 130 \\
\hline
\end{tabular}

$\begin{array}{rc}\text { LOW } & \text { AREA } \\ 67 & 0000.82 \\ 60 & 0000.12 \\ 20 & 0004.32 \\ 65 & 0000.17 \\ 105 & 0000.30 \\ 39 & 0003.36 \\ 90 & 0000.14 \\ 70 & 0001.03 \\ 80 & 0000.61 \\ 90 & 0000.14 \\ 87 & 0000.08 \\ 77 & 0000.13 \\ 77 & 0000.91 \\ 78 & 0000.13 \\ 85 & 0000.17 \\ 50 & 0000.07 \\ 87 & 0000.74 \\ 70 & 0001.04 \\ 90 & 0000.41 \\ 120 & 0000.86 \\ 130 & 0000.23 \\ 80 & 0000.07 \\ 87 & 0000.08 \\ 65 & 0000.28 \\ 90 & 0000.41 \\ 90 & 0000.73 \\ 40 & 0003.54 \\ 130 & 0000.25 \\ 35 & 0003.32 \\ 100 & 0000.09 \\ 70 & 0000.10 \\ 70 & 0000.07 \\ 70 & 0000.17 \\ 75 & 0000.16 \\ 40 & 0000.67 \\ 38 & 0001.14 \\ 50 & 0002.24 \\ 40 & 0000.71 \\ 80 & 0000.47 \\ 70 & 0000.89 \\ 60 & 0002.35 \\ 85 & 0000.07 \\ 100 & 0000.06 \\ 100 & 0000.47 \\ 130 & 0000.06 \\ 120 & 0000.09 \\ 80 & 0000.11 \\ 45 & 0004.08 \\ 50 & 0000.35 \\ 30 & 0001.19 \\ 60 & 0000.24 \\ 95 & 0000.07 \\ 60 & 0000.56 \\ 78 & 0000.34 \\ 60 & 0000.05 \\ 40 & 0001.24 \\ 60 & 0000.36 \\ 60 & 0002.26 \\ 58 & 0000.09 \\ 90 & 0000.18 \\ 115 & 0000.05 \\ & \end{array}$

$\begin{array}{cc}\text { M0 } & \\ \text { CODE } & \text { MAP } \\ & \\ 643112 & 6000-02 \\ 623212 & 6000-02 \\ 553112 & 6000-02 \\ 740110 & 6000-02 \\ 780010 & 6000-02 \\ 523012 & 6000-02 \\ 640110 & 6000-02 \\ 623110 & 6000-02 \\ 643112 & 6000-02 \\ 780010 & 6000-02 \\ 780010 & 6000-02 \\ 64012 & 6000-02 \\ 643112 & 6000-02 \\ 750010 & 6000-02 \\ 753010 & 6000-02 \\ 780010 & 6000-02 \\ 670012 & 6000-02 \\ 623212 & 6000-02 \\ 750010 & 6000-02 \\ 633210 & 6000-02 \\ 623312 & 6000-02 \\ 673012 & 6000-02 \\ 770010 & 6000-02 \\ 643212 & 6000-02 \\ 670212 & 6000-02 \\ 640112 & 6000-02 \\ 623412 & 6000-02 \\ 770010 & 6000-02 \\ 523112 & 6000-03 \\ 640112 & 6000-03 \\ 780010 & 6000-03 \\ 650112 & 6000-03 \\ 640112 & 6000-03 \\ 650112 & 6000-03 \\ 623212 & 6000-03 \\ 623312 & 6000-03 \\ 623212 & 6000-02 \\ 643312 & 6000-02 \\ 643312 & 6000-02 \\ 643312 & 6000-02 \\ 623412 & 6000-02 \\ 740210 & 6000-02 \\ 750010 & 6000-02 \\ 750010 & 6000-02 \\ 780010 & 6000-02 \\ 780010 & 6000-02 \\ 770110 & 6000-02 \\ 623112 & 6000-02 \\ 643012 & 6000-02 \\ 623112 & 6000-02 \\ 643112 & 6000-02 \\ 780110 & 6000-02 \\ 620110 & 6000-02 \\ 643112 & 6000-02 \\ 750112 & 6000-02 \\ 543112 & 6000-02 \\ 780110 & 6000-02 \\ 623112 & 6000-02 \\ 690010 & 6000-02 \\ 673112 & 6000-02 \\ 750010 & 6000-02 \\ & \end{array}$




\begin{tabular}{|c|c|c|c|c|}
\hline CODE & LAT & LONG & ORI & HIGH \\
\hline 4002 & $60^{\circ} 13^{\prime} \mathrm{N}$ & $44^{\circ} 41^{\prime} \mathrm{W}$ & $w$ & 120 \\
\hline $1 \mathrm{AB} 14003$ & $60^{\circ} 13^{\prime} \mathrm{N}$ & $44^{\circ} 39^{\prime} \mathrm{W}$ & $\mathrm{V}$ & 130 \\
\hline $1 \mathrm{AB} 14004$ & $60^{\circ} 13^{\prime} \mathrm{N}$ & $44^{\circ} 39^{\prime} \mathrm{W}$ & W & 115 \\
\hline $1 \mathrm{AB} 14005$ & $60^{\circ} 13^{\prime} \mathrm{N}$ & $44^{\circ} 39^{\prime} \mathrm{W}$ & v & 130 \\
\hline $1 \mathrm{AB} 14006$ & $60^{\circ} 14^{\prime} \mathrm{N}$ & $44^{\circ} 37^{\prime} \mathrm{W}$ & w & 140 \\
\hline $1 \mathrm{AB} 14007$ & $60^{\circ} 13^{\prime} \mathrm{N}$ & $44^{\circ} 37^{\prime} \mathrm{V}$ & W & 140 \\
\hline $1 \mathrm{AB} 14008$ & $60^{\circ} 13^{\prime} \mathrm{N}$ & $44^{\circ} 35 \%$ & NW & 140 \\
\hline $1 \mathrm{AB} 14009$ & $60^{\circ} 12^{\prime} \mathrm{N}$ & $44^{\circ} 35^{\prime} \mathrm{W}$ & NW & 130 \\
\hline $1 \mathrm{AB} 14010$ & $60^{\circ} 13^{\prime} \mathrm{N}$ & $44^{\circ} 31^{\prime} \mathrm{W}$ & W & 120 \\
\hline $1 \mathrm{ABI} 4011$ & $60^{\circ} 12^{\prime} \mathrm{N}$ & $44^{\circ} 31^{\prime} \mathrm{V}$ & NW & 140 \\
\hline $1 \mathrm{AB} 14012$ & $60^{\circ} 12^{\prime} \mathrm{N}$ & $44^{\circ} 32, \mathrm{~W}$ & $\mathrm{NW}$ & 140 \\
\hline $1 \mathrm{AB} 14013$ & $60^{\circ} 09^{\prime} \mathrm{N}$ & $44^{\circ} 36^{\prime} \mathrm{W}$ & $\mathrm{N}$ & 130 \\
\hline $1 \mathrm{AB} 14014$ & $60^{\circ} 09^{\prime} \mathrm{N}$ & $44^{\circ} 37^{\prime} \mathrm{W}$ & $\mathbf{N}$ & 129 \\
\hline $1 \mathrm{AB} 14015$ & $60^{\circ} 09^{\prime} \mathrm{N}$ & $44^{\circ} 38^{\prime} \mathrm{W}$ & $\mathbf{N}$ & 100 \\
\hline $1 \mathrm{AB} 14016$ & $60^{\circ} 09^{\prime} \mathrm{N}$ & $44^{\circ} 39^{\prime} \mathrm{W}$ & $\mathbf{N}$ & 160 \\
\hline $1 \mathrm{AB} 14017$ & $60^{\circ} 10^{\prime} \mathrm{N}$ & $44^{\circ} 40^{\prime} \mathrm{W}$ & N & 120 \\
\hline $1 \mathrm{AB} 14018$ & $60^{\circ} 09^{\prime} \mathrm{N}$ & $44^{\circ} 10^{\prime} \mathrm{W}$ & $\mathrm{E}$ & 120 \\
\hline $1 \mathrm{AB1} 4019$ & $60^{\circ} 09^{\prime} \mathrm{N}$ & $44^{\circ} 10^{\prime} \mathrm{W}$ & NW & 110 \\
\hline $1 \mathrm{AB} 15001$ & $60^{\circ} 09^{\prime} \mathrm{N}$ & $44^{\circ} 39^{\prime} \mathrm{W}$ & SW & 110 \\
\hline $1 \mathrm{AB} 15002$ & $60^{\circ} 08^{\prime} \mathrm{N}$ & $44^{\circ} 40^{\prime} \mathrm{W}$ & NW & 110 \\
\hline $1 \mathrm{AB} 1$ & $60^{\circ} 08^{\prime} \mathrm{N}$ & $44^{\circ} 40^{\prime} \mathrm{W}$ & NW & 125 \\
\hline $1 A B$ & $60^{\circ} 08^{\circ} \mathrm{N}$ & $44^{\circ} 41^{\prime} \mathrm{V}$ & $\mathbf{N}$ & 127 \\
\hline $1 \mathrm{AB} 15005$ & $60^{\circ} 08^{\prime} \mathrm{N}$ & $44^{\circ} 42^{\prime} \mathrm{W}$ & $\mathbf{N}$ & 100 \\
\hline $1 \mathrm{AB} 15006$ & $60^{\circ} 08^{\prime} \mathrm{N}$ & $44^{\circ} 43^{\prime} \mathrm{W}$ & NW & 100 \\
\hline $1 \mathrm{ACO} 01001$ & $60^{\circ} 23^{\prime} \mathrm{N}$ & $45^{\circ} 05^{\prime} \mathrm{W}$ & NW & 100 \\
\hline 110 & $60^{\circ} 23^{\prime} \mathrm{N}$ & $45^{\circ} 04^{\prime} \mathrm{V}$ & $\mathrm{N}$ & 95 \\
\hline $1 \mathrm{ACC}$ & & $45^{\circ} 03^{\prime} \mathrm{v}$ & $\mathrm{N}$ & 105 \\
\hline $1 \mathrm{ACO} 004$ & $60^{\circ} 23^{\prime} \mathrm{N}$ & $45^{\circ} 02^{\prime} \mathrm{W}$ & NW & 95 \\
\hline $1 \mathrm{ACO} 01005$ & $60^{\circ} 25^{\prime} \mathrm{N}$ & $45^{\circ} 02^{\prime}$ W & W & 110 \\
\hline $1 \mathrm{AC} 01006$ & $60^{\circ} 25^{\prime} \mathrm{N}$ & $45^{\circ} 02^{\prime} \mathrm{W}$ & SE & 105 \\
\hline & $' \mathrm{~N}$ & $45^{\circ} 07^{\prime}$ & & 105 \\
\hline $1 \mathrm{ACC}$ & N & $45^{\circ} 06^{\prime}$ W & SW & 110 \\
\hline $1 \mathrm{ACO} 2003$ & $60^{\circ} 29^{\prime} \mathrm{N}$ & $45^{\circ} 04^{\prime} \mathrm{V}$ & SW & 120 \\
\hline $1 \mathrm{AC} 02004$ & $60^{\circ} 29^{\prime} \mathrm{N}$ & $45^{\circ} 04^{\prime} \mathrm{W}$ & SW & 117 \\
\hline $1 \mathrm{ACO} 3001$ & $60^{\circ} 33^{\prime} \mathrm{N}$ & $44^{\circ} 56^{\prime} \mathrm{W}$ & W & 140 \\
\hline & $36^{\prime} \mathrm{N}$ & $44^{\circ} 54^{\prime} \mathrm{W}$ & NE & 100 \\
\hline $1 \mathrm{ACC}$ & & 44052,6 & $\mathrm{~N}$ & 100 \\
\hline $1 \mathrm{AC} 03004$ & $60^{\circ} 37^{\prime} \mathrm{N}$ & $44^{\circ} 52^{\prime} \mathrm{W}$ & NW & 122 \\
\hline $1 \mathrm{ACO} 3005$ & $60^{\circ} 37 / \mathrm{N}$ & $44^{\circ} 53^{\prime} \mathrm{W}$ & $\mathbf{N}$ & 110 \\
\hline $1 \mathrm{AC03006}$ & $60^{\circ} 36^{\prime} \mathrm{N}$ & $44^{\circ} 54^{\prime} \mathrm{W}$ & N & 115 \\
\hline $1 \mathrm{ACO}$ & $60^{\circ} 37 \cdot \mathrm{N}$ & $44^{\circ} 55 \%$ & $\mathrm{NE}$ & 120 \\
\hline $1 \mathrm{ACO}$ & & 44051 ' W & $\mathrm{N}$ & 90 \\
\hline 1AC04002 & $60^{\circ} 40^{\prime} \mathrm{N}$ & $44^{\circ} 52^{\prime} \mathrm{W}$ & $\mathrm{NE}$ & 100 \\
\hline $1 \mathrm{ACO} 4003$ & $60^{\circ} 40^{\prime} \mathrm{N}$ & $44^{\circ} 53^{\prime} \mathrm{W}$ & $\mathrm{NE}$ & 100 \\
\hline $1 \mathrm{ACO} 4$ & $60^{\circ}$ & $44^{\circ} 54^{\prime} \mathrm{W}$ & $\mathrm{NE}$ & 160 \\
\hline $1 \mathrm{ACO} 4$ & $60^{\circ} 41^{\prime} \mathrm{N}$ & $44^{\circ} 54^{\prime} \mathrm{W}$ & SE & 140 \\
\hline $1 \mathrm{ACO} 4006$ & & $44^{\circ} 51$, & $\mathrm{s}$ & 130 \\
\hline $1 \mathrm{ACO}$ & $2, \mathrm{~N}$ & $44^{\circ} 51^{\prime} \mathrm{W}$ & NE & 100 \\
\hline & $60^{\circ} 42^{\prime} \mathrm{N}$ & $44^{\circ} 52^{\prime} \mathrm{W}$ & SE & 140 \\
\hline 10 & $60^{\circ} 43^{\prime} \mathrm{N}$ & $44^{\circ} 50^{\prime} \mathrm{W}$ & $\mathrm{NE}$ & 110 \\
\hline & $60^{\circ} 43^{\prime} \mathrm{N}$ & $44^{\circ} 51^{\prime} \mathrm{W}$ & NE & 130 \\
\hline $1 \mathrm{AC}$ & & & $\mathrm{NE}$ & 140 \\
\hline $1 \mathrm{AC} 05006$ & $60^{\circ} 43^{\prime} \mathrm{N}$ & $44^{\circ} 53^{\prime} \mathrm{W}$ & E & 140 \\
\hline $1 \mathrm{AC} 05007$ & $60^{\circ} 44^{\prime} \mathrm{N}$ & $44^{\circ} 52^{\prime} \mathrm{N}$ & $\mathrm{N}$ & 110 \\
\hline $\mathrm{AC05008}$ & $60^{\circ} 45^{\prime} \mathrm{N}$ & $44^{\circ} 52^{\prime} \mathrm{W}$ & SE & 120 \\
\hline & & $44^{\circ} 51^{\prime} \mathrm{V}$ & sv & 160 \\
\hline $1 \mathrm{AC} 05010$ & $60^{\circ} 45^{\prime} \mathrm{N}$ & $44^{\circ} 50^{\prime} \mathrm{W}$ & W & 150 \\
\hline $1 \mathrm{AC} 05011$ & $60^{\circ} 44^{\prime} \mathrm{N}$ & $44^{\circ} 49^{\prime} \mathrm{W}$ & SW & 170 \\
\hline $1 \mathrm{AC} 0501$ & $60^{\circ} 44^{\prime} \mathrm{N}$ & $44^{\circ} 47^{\prime} \mathrm{W}$ & SW & 140 \\
\hline & & & & $=0$ \\
\hline $1 \mathrm{AC} 05014$ & $43^{\prime} \mathrm{N}$ & $46^{\prime} \mathrm{W}$ & SW & 16 \\
\hline
\end{tabular}

$\begin{array}{rccc}\text { LOW } & \text { AREA } & \text { MODE } & \text { MAP } \\ & & & \\ 90 & 0000.11 & 750010 & 6000-02 \\ 105 & 0000.51 & 670112 & 6000-02 \\ 100 & 0000.05 & 780010 & 6000-02 \\ 110 & 0000.06 & 750010 & 6000-02 \\ 120 & 0000.39 & 780010 & 6000-02 \\ 110 & 0000.13 & 670010 & 6000-02 \\ 100 & 0000.37 & 670010 & 6000-02 \\ 100 & 0000.10 & 750010 & 6000-02 \\ 90 & 0000.40 & 780010 & 6000-02 \\ 50 & 0000.95 & 770010 & 6000-02 \\ 70 & 0000.99 & 673012 & 6000-02 \\ 90 & 0000.27 & 780010 & 6000-02 \\ 90 & 0000.17 & 780010 & 6000-02 \\ 90 & 0000.02 & 780010 & 6000-02 \\ 90 & 0000.31 & 780010 & 6000-02 \\ 90 & 0000.06 & 780010 & 6000-02 \\ 100 & 0000.19 & 780010 & 6000-03 \\ 90 & 0000.09 & 650010 & 6000-03 \\ 98 & 0000.23 & 780010 & 6000-02 \\ 100 & 0000.04 & 780010 & 6000-02 \\ 90 & 0000.03 & 750010 & 6000-02 \\ 70 & 0000.24 & 74010 & 6000-02 \\ 80 & 0000.04 & 780010 & 6000-02 \\ 70 & 0000.11 & 750010 & 6000-02 \\ 80 & 0000.26 & 640112 & 6000-02 \\ 70 & 0000.36 & 64312 & 6000-02 \\ 85 & 0000.47 & 640112 & 6000-02 \\ 80 & 0000.06 & 780010 & 6000-02 \\ 95 & 0000.04 & 780010 & 6000-02 \\ 90 & 0000.04 & 780010 & 6000-02 \\ 100 & 0000.05 & 78010 & 6000-02 \\ 95 & 0000.05 & 780010 & 6000-02 \\ 100 & 0000.04 & 780010 & 6000-02 \\ 110 & 0000.14 & 770010 & 6000-02 \\ 120 & 0000.05 & 78010 & 6030-06 \\ 90 & 0000.12 & 640112 & 6030-06 \\ 70 & 0000.09 & 750110 & 6030-06 \\ 120 & 0000.08 & 750110 & 6030-06 \\ 80 & 0000.25 & 643112 & 6030-06 \\ 75 & 0000.63 & 643112 & 6030-06 \\ 80 & 0000.80 & 740002 & 6030-06 \\ 80 & 0000.05 & 750010 & 6030-06 \\ 82 & 0000.67 & 643112 & 6030-06 \\ 75 & 0000.55 & 643112 & 6030-06 \\ 72 & 0002.20 & 623112 & 6030-06 \\ 130 & 0000.30 & 740010 & 6030-06 \\ 85 & 0000.53 & 780112 & 6030-06 \\ 80 & 0000.15 & 750112 & 6030-06 \\ 98 & 0001.25 & 623212 & 6030-06 \\ 90 & 0000.23 & 643012 & 6030-06 \\ 70 & 0000.97 & 643212 & 6030-06 \\ 80 & 0001.01 & 643312 & 6030-06 \\ 105 & 0000.44 & 670112 & 6030-06 \\ 110 & 0000.48 & 630112 & 6030-06 \\ 110 & 0000.22 & 770112 & 6030-06 \\ 105 & 0001.96 & 623312 & 6030-06 \\ 120 & 0000.43 & 653312 & 6030-06 \\ 110 & 0000.21 & 643012 & 6030-06 \\ 105 & 0001.02 & 643212 & 6030-06 \\ 105 & 0000.94 & 643212 & 6030-06 \\ 120 & 0000.08 & 643212 & 6030-06 \\ & & & \end{array}$

\begin{tabular}{|c|c|c|c|}
\hline CODE & LAT & LONG & ORI \\
\hline $\begin{array}{l}\text { 1AC05015 } \\
1 \text { ACO5016 }\end{array}$ & $\begin{array}{l}60^{\circ} 43^{\prime} \mathrm{N} \\
60^{\circ} 42^{\prime} \mathrm{N}\end{array}$ & $\begin{array}{l}44^{\circ} 46^{\prime} \mathrm{W} \\
44^{\circ} 47^{\prime} \mathrm{W}\end{array}$ & $\mathrm{SW}_{\mathrm{W}}$ \\
\hline $1 \mathrm{AC} 06001$ & $60^{\circ} 44^{\prime} \mathrm{N}$ & $44^{\circ} 45^{\prime} \mathrm{W}$ & SE \\
\hline $1 \mathrm{AC} 06002$ & $60^{\circ} 44^{\prime} \mathrm{N}$ & $44^{\circ} 45^{\prime} \mathrm{W}$ & $\mathrm{SE}$ \\
\hline 1AC06003 & $60^{\circ} 44^{\prime} \mathrm{N}$ & $44^{\circ} 42^{\prime} \mathrm{W}$ & $\mathrm{NE}$ \\
\hline $1 \mathrm{ACO6004}$ & $60^{\circ} 45^{\prime} \mathrm{N}$ & $44^{\circ} 42^{\prime} \mathrm{W}$ & \\
\hline $1 \mathrm{AC} 06005$ & $60^{\circ} 45^{\prime} \mathrm{N}$ & $44^{\circ} 41^{\prime} \mathrm{W}$ & $\mathrm{SE}$ \\
\hline 1AC06006 & $60^{\circ} 45^{\circ} \mathrm{N}$ & $44^{\circ} 42^{\prime} \mathrm{W}$ & $\mathrm{E}$ \\
\hline $1 \mathrm{AC} 06007$ & $60^{\circ} 46^{\prime} \mathrm{N}$ & $44^{\circ} 40^{\prime} \mathrm{V}$ & SQ \\
\hline $1 A C 07001$ & $60^{\circ} 45^{\prime} \mathrm{N}$ & $44^{\circ} 38^{\prime} \mathrm{W}$ & \\
\hline $1 \mathrm{AC} 07002$ & $60^{\circ} 46^{\prime} \mathrm{N}$ & $44^{\circ} 38^{\prime} \mathrm{W}$ & $\mathbf{E}$ \\
\hline $1 \mathrm{AC} 07003$ & $60^{\circ} 46^{\prime} \mathrm{N}$ & $44^{\circ} 38^{\prime} \mathrm{V}$ & E \\
\hline $1 \mathrm{AC} 07004$ & $60^{\circ} 46^{\prime} \mathrm{N}$ & $44^{\circ} 37^{\prime \prime}$ & B \\
\hline$A C$ & $60^{\circ} 46^{\prime} \mathrm{N}$ & $44^{\circ} 38^{\prime}, \mathrm{W}$ & $E$ \\
\hline $1 \mathrm{AC} 07006$ & $60^{\circ} 47 \cdot \mathrm{N}$ & $44^{\circ} 37^{\prime} \mathrm{W}$ & \\
\hline $1 \mathrm{AC} 07007$ & $60^{\circ} 47^{\prime} \mathrm{N}$ & $44^{\circ} 38^{\prime} \mathrm{W}$ & $\bar{E}$ \\
\hline $1 \mathrm{AC} 07008$ & $60^{\circ} 48^{\prime} \mathrm{N}$ & $44^{\circ} 37^{\prime} \mathrm{W}$ & $\bar{s}$ \\
\hline 1 AC07009 & $60^{\circ} 477^{\prime N}$ & $44^{\circ} 35^{\prime} \mathrm{W}$ & s \\
\hline $1 \mathrm{ACO} 07010$ & $60^{\circ} 47 \cdot \mathrm{N}$ & $44^{\circ} 35^{\prime} \mathrm{W}$ & W \\
\hline $1 \mathrm{AC} 07011$ & $60^{\circ} 48^{\prime} \mathrm{N}$ & $44^{\circ} 36^{\prime} \mathrm{W}$ & v \\
\hline $1 \mathrm{AC} 07012$ & $60^{\circ} 48^{\prime} \mathrm{N}$ & $44^{\circ} 35^{\prime} \mathrm{W}$ & NE \\
\hline $1 A C 07013$ & $60^{\circ} 48^{\prime} N$ & $44^{\circ} 36^{\prime} \mathrm{W}$ & SE \\
\hline $1 \mathrm{AC} 07014$ & $60^{\circ} 48^{\prime} \mathrm{N}$ & $44^{\circ} 36^{\prime} \mathrm{W}$ & $\mathrm{E}$ \\
\hline $1 \mathrm{AC} 07015$ & $60^{\circ} 48^{\prime} \mathrm{N}$ & $44^{\circ} 34^{\prime \prime} \mathrm{W}$ & $\mathrm{s}$ \\
\hline $1 \mathrm{AC} 07016$ & $60^{\circ} 48^{\prime} \mathrm{N}$ & $44^{\circ} 32^{\prime} \mathrm{W}$ & SW \\
\hline $1 \mathrm{AC} 07017$ & $60^{\circ} 49^{\prime} \mathrm{N}$ & $44^{\circ} 32^{\prime} \mathrm{W}$ & $\pi$ \\
\hline $1 \mathrm{AC} 07018$ & $60^{\circ} 49^{\prime} \mathrm{N}$ & $44^{\circ} 33^{\prime} \mathrm{W}$ & $\mathrm{E}$ \\
\hline $1 \mathrm{AC} 07019$ & $60^{\circ} 51^{\prime} \mathrm{N}$ & $44^{\circ} 31^{\prime} \mathrm{W}$ & $\mathrm{s}$ \\
\hline $1 \mathrm{AC} 07020$ & $60^{\circ} 49^{\prime} \mathrm{N}$ & $44^{\circ} 26^{\prime} \mathrm{V}$ & SW \\
\hline $1 \mathrm{AC} 07021$ & $60^{\circ} 47 \times N$ & $44^{\circ} 27^{\prime}$ & $\mathrm{W}$ \\
\hline $1 \mathrm{AC} 07022$ & $60^{\circ} 46^{\prime} \mathrm{N}$ & $44^{\circ} 27^{\prime} \mathrm{W}$ & \\
\hline $1 \mathrm{AC} 07023$ & $60^{\circ} 46^{\prime} \mathrm{N}$ & $44^{\circ} 28^{\prime} \mathrm{W}$ & $\mathbf{N}$ \\
\hline $1 A C 07024$ & $60^{\circ} 46^{\prime} \mathrm{N}$ & $44^{\circ} 28^{\prime} \mathrm{W}$ & N \\
\hline $1 \mathrm{AC} 07025$ & $60^{\circ} 46^{\prime} \mathrm{N}$ & $44^{\circ} 29^{\prime} \mathrm{W}$ & NW \\
\hline & $60^{\circ} 46^{\prime} \mathrm{N}$ & $44^{\circ} 29^{\prime} \mathrm{W}$ & $\mathrm{W}$ \\
\hline $1 \mathrm{AC} 07027$ & $60^{\circ} 44^{\prime} \mathrm{N}$ & $44^{\circ} 27^{\prime} \mathrm{W}$ & NW \\
\hline $1 \mathrm{ACO} 07028$ & $60^{\circ} 44^{\prime} \mathrm{N}$ & $44^{\circ} 30^{\prime} \mathrm{W}$ & \\
\hline 1 AC07029 & $60^{\circ} 43^{\prime} \mathrm{N}$ & $44^{\circ} 32^{\prime} \mathrm{W}$ & $\mathrm{w}$ \\
\hline $1 A C 07030$ & $60^{\circ} 42 \cdot \mathrm{N}$ & $44^{\circ} 31^{\prime}, W$ & $\mathrm{NW}$ \\
\hline & $60^{\circ} 41^{\prime} \mathrm{N}$ & $44^{\circ} 32^{\prime} \mathrm{W}$ & $\mathrm{NE}$ \\
\hline $1 \mathrm{AC} 07032$ & $60^{\circ} 41^{\prime} \mathrm{N}$ & $44^{\circ} 34^{\prime} \mathrm{W}$ & $\mathrm{NE}$ \\
\hline $1 A C 07033$ & $60^{\circ} 42^{\prime} \mathrm{N}$ & $44^{\circ} 34^{\prime} W$ & \\
\hline $1 \mathrm{AC} 07034$ & $60^{\circ} 43^{\prime} \mathrm{N}$ & $44^{\circ} 35^{\prime} \mathrm{W}$ & \\
\hline $1 \mathrm{AC}$ & $60^{\circ} 42^{\prime} \mathrm{N}$ & $44^{\circ} 37^{\prime W}$ & NW \\
\hline & $60^{\circ} 41^{\prime} \mathrm{N}$ & $44^{\circ} 35^{\prime} \mathrm{W}$ & SW \\
\hline $1 \mathrm{AC} 08003$ & $60^{\circ} 40^{\prime} \mathrm{N}$ & $44^{\circ} 33^{\prime \prime}$ & SW \\
\hline $1 \mathrm{AC} 08004$ & $60^{\circ} 40^{\prime} \mathrm{N}$ & $44^{\circ} 32^{\prime} \mathrm{W}$ & SW \\
\hline $1 \mathrm{AC} 08005$ & $60^{\circ} 40^{\prime} \mathrm{N}$ & $44^{\circ} 35^{\prime} \mathrm{W}$ & $\mathrm{W}$ \\
\hline $1 \mathrm{AC}$ & $60^{\circ} 39^{\prime} \mathrm{N}$ & $44^{\circ} 34^{\prime} \mathrm{V}$ & $\mathrm{NE}$ \\
\hline $1 \mathrm{AC} 08007$ & $60^{\circ} 39^{\prime} \mathrm{N}$ & $44^{\circ} 36^{\prime} \mathrm{W}$ & $\mathbf{N}$ \\
\hline $1 \mathrm{AC} 08008$ & $60^{\circ} 39^{\prime} \mathrm{N}$ & $44^{\circ} 37^{\prime} \mathrm{W}$ & N \\
\hline 0 & $60^{\circ} 40^{\prime} \mathrm{N}$ & $44^{\circ} 38^{\prime} \mathrm{W}$ & $\mathrm{NE}$ \\
\hline $1 \mathrm{AC} 08010$ & $60^{\circ} 39^{\prime} \mathrm{N}$ & $44^{\circ} 39^{\prime} \mathrm{W}$ & $\mathrm{s}$ \\
\hline $1 \mathrm{AC} 08011$ & $60^{\circ} 39^{\prime} \mathrm{N}$ & $44^{\circ} 39^{\prime} \mathrm{W}$ & SW \\
\hline $1 \mathrm{ACO} 08012$ & $60^{\circ} 38^{\prime} \mathrm{N}$ & $44^{\circ} 39^{\prime} \mathrm{W}$ & v \\
\hline $1 \mathrm{AC} 08013$ & $60^{\circ} 38^{\prime} \mathrm{N}$ & $44^{\circ} 40^{\prime} \mathrm{W}$ & W \\
\hline $1 \mathrm{AC} 08014$ & $60^{\circ} 38^{\prime} \mathrm{N}$ & $44^{\circ} 40^{\prime} \mathrm{W}$ & $\mathrm{NW}$ \\
\hline $1 \mathrm{AC} 08015$ & $60^{\circ} 38^{\prime} \mathrm{N}$ & $44^{\circ} 41^{\prime} \mathrm{W}$ & 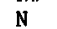 \\
\hline $\mathrm{ACO} 0001$ & . & $44^{\circ} 4^{\circ} 1^{\prime} \mathrm{V}$ & v \\
\hline $1 \mathrm{AC} 09002$ & $60^{\circ} 38^{\prime} \mathrm{N}$ & $44^{\circ} 40^{\prime} \mathrm{W}$ & SW \\
\hline
\end{tabular}

HIGH
120
100
120
150
115
140
115
135
150
140
130
130
130
130
120
150
160
130
130
110
140
130
170
110
100
160
140
170
210
210
185
180
140
130
130
190
150
169
140
140
120
140
100
150
150
150
110
140
150
110
110
110
100
110
120
110
110
95
100
120
120
1

$\begin{array}{rc}\text { LOW } & \text { AREA } \\ & \\ 95 & 0000.64 \\ 90 & 0000.07 \\ 80 & 0000.70 \\ 80 & 0001.10 \\ 75 & 0000.30 \\ 80 & 0001.70 \\ 100 & 0000.30 \\ 100 & 0000.50 \\ 70 & 0008.80 \\ 120 & 0000.20 \\ 120 & 0000.20 \\ 130 & 0000.10 \\ 110 & 0000.30 \\ 90 & 0000.20 \\ 90 & 0000.40 \\ 80 & 0002.00 \\ 90 & 0001.30 \\ 120 & 0000.40 \\ 130 & 0000.10 \\ 110 & 0000.10 \\ 110 & 0000.30 \\ 130 & 0000.20 \\ 150 & 0000.50 \\ 80 & 0000.50 \\ 80 & 0000.20 \\ 110 & 0000.40 \\ 130 & 0000.20 \\ 100 & 0002.72 \\ & 0094.90 \\ 185 & 0086.10 \\ 145 & 0000.10 \\ 110 & 0000.40 \\ 80 & 0000.10 \\ 90 & 0000.20 \\ 190 & 0017.00 \\ 100 & 0000.80 \\ 150 & 0000.56 \\ 90 & 0045.40 \\ 70 & 0001.31 \\ 90 & 0001.95 \\ 70 & 0000.30 \\ 60 & 0000.81 \\ 60 & 0000.69 \\ 130 & 0000.50 \\ 130 & 0000.10 \\ 90 & 0000.30 \\ 30 & 0009.10 \\ 100 & 0000.60 \\ 80 & 0000.50 \\ 85 & 0000.39 \\ 95 & 0000.19 \\ 90 & 0000.06 \\ 80 & 0000.43 \\ 95 & 0000.15 \\ 80 & 0000.24 \\ 90 & 0000.08 \\ 70 & 0000.31 \\ 80 & 0000.10 \\ 95 & 0000.18 \\ 105 & 0000.09 \\ & \end{array}$

CODE

MAP 


\begin{tabular}{|c|c|c|c|c|c|c|c|c|c|c|c|c|c|c|c|c|c|}
\hline CODE & LAT & LONG & ORI & HIGH & LOW & AREA & $\begin{array}{c}\text { MO } \\
\text { CODE }\end{array}$ & MAP & CODE & LAT & LONG & ORI & HIGH & LOW & AREA & $\begin{array}{c}\text { MO } \\
\text { CODE }\end{array}$ & MAP \\
\hline $1 \mathrm{AC} 09004$ & $60^{\circ} 37^{\prime} \mathrm{N}$ & $44^{\circ} 38^{\prime} \mathrm{W}$ & SW & 120 & 90 & 0000.24 & 643112 & $6030-06$ & $1 \mathrm{AC} 12014$ & $60^{\circ} 32^{\prime} \mathrm{N}$ & $44^{\circ} 40^{\prime} \mathrm{W}$ & NH & 110 & 80 & 0000.19 & 643212 & $6030-06$ \\
\hline $1 \mathrm{AC} 09005$ & $60^{\circ} 37^{\prime} \mathrm{N}$ & $44^{\circ} 38^{\prime} \mathrm{W}$ & $\mathrm{SE}$ & 130 & 120 & 0000.07 & 780010 & $6030-06$ & $1 \mathrm{AC12015}$ & $60^{\circ} 31, \mathrm{~N}$ & $44^{\circ} 41^{\prime}$ W & $\mathrm{NU}$ & 120 & 95 & 0000.26 & 643112 & $6030-06$ \\
\hline $1 \mathrm{AC} 09006$ & $60^{\circ} 38^{\prime} \mathrm{N}$ & $44^{\circ} 38^{\prime} \mathrm{W}$ & $N E$ & 110 & 90 & 0000.27 & 643212 & $6030-06$ & $1 \mathrm{AC12016}$ & $60^{\circ} 31 \cdot \mathrm{N}$ & $44^{\circ} 41^{\prime}$ W & $N$ & 120 & 90 & 0000.26 & 640112 & $6030-06$ \\
\hline $1 \mathrm{AC} 09007$ & $60^{\circ} 39^{\prime} \mathrm{N}$ & $44^{\circ} 35^{\prime} \mathrm{W}$ & SW & 130 & 110 & 0000.61 & 633212 & $6030-06$ & $1 \mathrm{AC} 12017$ & $60^{\circ} 31, \mathrm{~N}$ & $44^{\circ} 41^{\prime} \mathrm{W}$ & W & 120 & 100 & 0000.14 & 643112 & $6030-06$ \\
\hline $1 \mathrm{AC} 09008$ & $60^{\circ} 38^{\prime} \mathrm{N}$ & $44^{\circ} 34^{\prime} \mathrm{W}$ & SW & 140 & 100 & 0000.09 & 653212 & $6030-06$ & $1 \mathrm{AC} 12018$ & $60^{\circ} 30^{\prime} \mathrm{N}$ & $44^{\circ} 41^{\prime} \mathrm{W}$ & sw & 100 & 90 & 0000.09 & 690112 & $6030-06$ \\
\hline $1 \mathrm{AC} 09009$ & $60^{\circ} 39^{\prime} \mathrm{N}$ & $44^{\circ} 32^{\prime} \mathrm{W}$ & $\mathrm{s}$ & 170 & 100 & 0000.93 & 623312 & $6030-06$ & $1 \mathrm{AC} 12019$ & $60^{\circ} 30^{\prime} \mathrm{N}$ & $44^{\circ} 41^{\prime} \mathrm{W}$ & $\mathrm{s}$ & 120 & 100 & 0000.06 & 750010 & $6030-06$ \\
\hline 1 AC09010 & $60^{\circ} 38^{\circ} \mathrm{N}$ & $44^{\circ} 31^{\prime}$ W & W & 160 & 39 & 0002.97 & 363212 & $6030-06$ & 1 AC12020 & $60^{\circ} 30^{\prime} \mathrm{N}$ & $44^{\circ} 39^{\prime \prime} \mathrm{W}$ & $\mathrm{sH}$ & 110 & 90 & 0000.08 & 780010 & $6030-06$ \\
\hline $1 \mathrm{AC} 09011$ & $60^{\circ} 38^{\prime} \mathrm{N}$ & $44^{\circ} 31^{\prime} \mathrm{W}$ & W & 160 & 80 & 0011.60 & 363212 & $6030-06$ & $1 \mathrm{ACl} 2021$ & $60^{\circ} 30^{\prime} \mathrm{N}$ & $44^{\circ} 38^{\prime \prime} \mathrm{W}$ & $\mathrm{W}$ & 110 & 80 & 0000.17 & 643212 & $6030-06$ \\
\hline $1 \mathrm{AC} 09012$ & $60^{\circ} 37 \cdot \mathrm{N}$ & $44^{\circ} 33^{\prime} \mathrm{W}$ & $\mathrm{NW}$ & 140 & 100 & 0000.21 & 653312 & $6030-06$ & $1 \mathrm{AC1} 2022$ & $60^{\circ} 29^{\prime} \mathrm{N}$ & $44^{\circ} 39^{\prime} \mathrm{W}$ & NW & 110 & 75 & 0000.28 & 643112 & $6000-02$ \\
\hline $1 \mathrm{AC} 09013$ & $60^{\circ} 37^{\prime} \mathrm{N}$ & $44^{\circ} 34^{\prime} \mathrm{W}$ & $\mathrm{NW}$ & 120 & 70 & 0000.08 & 680210 & $6030-06$ & $1 \mathrm{AC} 12023$ & $60^{\circ} 29^{\prime} \mathrm{N}$ & $44^{\circ} 39^{\circ} \mathrm{W}$ & W & 80 & 60 & 0000.19 & 640112 & $6000-02$ \\
\hline $1 \mathrm{AC} 09014$ & $60^{\circ} 36^{\prime} \mathrm{N}$ & $44^{\circ} 35^{\prime}, W$ & NW & 120 & 110 & 0000.12 & 750210 & $6030-06$ & $1 \mathrm{ACl} 2024$ & $60^{\circ} 29^{\prime} \mathrm{N}$ & $44^{\circ} 40^{\prime} \mathrm{W}$ & NW & 100 & 70 & 0000.26 & 640112 & $6000-02$ \\
\hline $1 \mathrm{ACO} 0015$ & $60^{\circ} 36^{\prime} \mathrm{N}$ & $44^{\circ} 34^{\prime} \mathrm{W}$ & SW & 90 & 70 & 0000.07 & 643112 & $6030-06$ & 1AC12025 & $60^{\circ} 28^{\prime} \mathrm{N}$ & $44^{\circ} 40^{\prime} \mathrm{v}$ & $\mathrm{V}$ & 110 & 90 & 0000.18 & 640112 & $6000-02$ \\
\hline $1 \mathrm{ACO} 9016$ & $60^{\circ} 33^{\prime} \mathrm{N}$ & $44^{\circ} 36^{\prime} \mathrm{W}$ & NW & 100 & 70 & 0000.06 & 680310 & $6030-06$ & $1 \mathrm{ACl} 2026$ & $60^{\circ} 28^{\prime} \mathrm{N}$ & $44^{\circ} 41^{\prime} \mathrm{W}$ & NT & 100 & 80 & 0000.39 & 680112 & $6000-02$ \\
\hline $1 \mathrm{ACO} 0017$ & $60^{\circ} 33^{\prime} \mathrm{N}$ & $44^{\circ} 37^{\prime} \mathrm{W}$ & $\mathrm{N}$ & 120 & 70 & 0000.83 & 643312 & $6030-06$ & $1 \mathrm{AC} 12027$ & $60^{\circ} 27^{\prime} \mathrm{N}$ & $44^{\circ} 42^{\prime} \mathrm{W}$ & $\mathrm{NU}$ & 95 & 70 & 0000.39 & 680112 & $6000-02$ \\
\hline $1 \mathrm{AC} 09018$ & $60^{\circ} 33^{\prime} \mathrm{N}$ & $44^{\circ} 38^{\prime \prime}$ & NE & 120 & 70 & 0000.86 & 643212 & $6030-06$ & $1 \mathrm{AC12028}$ & $60^{\circ} 27 \times \mathrm{N}$ & $44^{\circ} 43^{\prime} \mathrm{W}$ & $\mathrm{NV}$ & 115 & 70 & 0000.20 & 680112 & $6000-02$ \\
\hline $1 \mathrm{AC} 09019$ & $60^{\circ} 34^{\prime} \mathrm{N}$ & $44^{\circ} 40^{\prime} \mathrm{V}$ & $\mathrm{NE}$ & 181 & 85 & 0002.76 & 623112 & $6030-06$ & $1 \mathrm{AC} 12029$ & $60^{\circ} 26^{\prime} \mathrm{N}$ & $44^{\circ} 44^{\prime} \mathrm{W}$ & $\mathbf{N}$ & 100 & 70 & 0000.39 & 640112 & $6000-02$ \\
\hline 1AC09020 & $60^{\circ} 34^{\prime} \mathrm{N}$ & $44^{\circ} 40^{\prime} \mathrm{W}$ & NE & 150 & 90 & 0000.61 & 633212 & $6030-06$ & $1 \mathrm{AC} 12030$ & $60^{\circ} 27^{\prime} \mathrm{N}$ & $44^{\circ} 45^{\prime}, W$ & NE & 109 & 90 & 0000.08 & 780012 & $6000-02$ \\
\hline $1 \mathrm{AC} 09021$ & $60^{\circ} 34^{\prime} \mathrm{N}$ & $44^{\circ} 41^{\prime} \mathrm{W}$ & $\mathrm{E}$ & 120 & 85 & 0000.96 & 623112 & $6030-06$ & $1 \mathrm{AC1} 2031$ & $60^{\circ} 26^{\prime} \mathrm{N}$ & $44^{\circ} 44^{\prime} \mathrm{W}$ & NW & 125 & 105 & 0000.05 & 780012 & $6000-02$ \\
\hline $1 \mathrm{ACO} 0022$ & $60^{\circ} 35^{\prime} \mathrm{N}$ & $44^{\circ} 42^{\prime} \mathrm{W}$ & NE & 140 & 110 & 0000.15 & 770010 & $6030-06$ & $1 \mathrm{ACl} 2032$ & $60^{\circ} 25^{\prime} \mathrm{N}$ & $44^{\circ} 46^{\prime} \mathrm{W}$ & $\mathrm{NV}$ & 80 & 45 & 0001.07 & 643212 & $6000-02$ \\
\hline 1 ACO9023 & $60^{\circ} 35^{\prime} \mathrm{N}$ & $44^{\circ} 42^{\prime} \mathrm{W}$ & SE & 160 & 150 & 0000.14 & 770010 & $6030-06$ & $1 \mathrm{AC1} 2033$ & $60^{\circ} 25 \times \mathrm{N}$ & $44^{\circ} 47^{\circ}, 6$ & $\mathrm{NE}$ & 100 & 60 & 0000.34 & 643212 & $6000-02$ \\
\hline $1 \mathrm{AC} 09024$ & $60^{\circ} 35^{\circ} \mathrm{N}$ & $44^{\circ} 42^{\prime}$ & $\mathrm{N}$ & 110 & 80 & 0000.03 & 790012 & $6030-06$ & $1 \mathrm{ACl} 2034$ & $60^{\circ} 26^{\prime} \mathrm{N}$ & $44^{\circ} 47^{\circ} \mathrm{V}$ & E & 100 & 75 & 0000.15 & 780010 & $6000-02$ \\
\hline $1 \mathrm{ACO} 9025$ & $60^{\circ} 36^{\prime} \mathrm{N}$ & $44^{\circ} 42^{\prime} \mathrm{W}$ & $\mathrm{N}$ & 90 & 75 & 0000.03 & 790012 & $6030-06$ & $1 \mathrm{AC} 12035$ & $60^{\circ} 26^{\prime} \mathrm{N}$ & $44^{\circ} 47^{\prime} \mathrm{W}$ & E & 100 & 80 & 0000.16 & 780010 & $6000-02$ \\
\hline $1 \mathrm{AC} 09026$ & $60^{\circ} 36^{\prime} \mathrm{N}$ & $44^{\circ} 43^{\prime} \mathrm{W}$ & N & 100 & 80 & 0000.04 & 790012 & $6030-06$ & 1 AC1 2036 & $60^{\circ} 27^{\prime} \mathrm{N}$ & $44^{\circ} 48^{\prime} \mathrm{W}$ & W & 100 & 90 & 0000.03 & 750010 & $6000-02$ \\
\hline $1 \mathrm{ACl} 0001$ & $60^{\circ} 35^{\prime} \mathrm{N}$ & $44^{\circ} 44^{\prime} W$ & $\forall$ & 100 & 80 & 0000.08 & 783112 & $6030-06$ & $1 \mathrm{AC1} 2037$ & $60^{\circ} 26^{\prime} \mathrm{N}$ & $44^{\circ} 48^{\prime} \mathrm{W}$ & W & 90 & 80 & 0000.41 & 780010 & $6000-02$ \\
\hline $1 \mathrm{ACl} 0002$ & $60^{\circ} 35^{\prime} \mathrm{N}$ & $44^{\circ} 43^{\prime} \mathrm{W}$ & SW & 120 & 100 & 0000.08 & 750010 & $6030-06$ & $1 \mathrm{AC} 12038$ & $60^{\circ} 26^{\prime} \mathrm{N}$ & $44^{\circ} 48^{\prime \prime} \mathrm{W}$ & $\mathrm{N}$ & 90 & 75 & 0000.42 & 643112 & $6000-02$ \\
\hline $1 \mathrm{ACl} 10003$ & $60^{\circ} 34^{\prime} \mathrm{N}$ & $44^{\circ} 43^{\prime} \mathrm{W}$ & $W$ & 110 & 80 & 0000.26 & 743012 & $6030-06$ & $1 \mathrm{AC} 13001$ & $60^{\circ} 26^{\prime} \mathrm{N}$ & $44^{\circ} 50^{\prime} \mathrm{W}$ & SW & 100 & 75 & 0000.07 & 780010 & $6000-02$ \\
\hline $1 \mathrm{AC} 10004$ & $60^{\circ} 34^{\prime} \mathrm{N}$ & $44^{\circ} 43^{\prime} \mathrm{W}$ & w & 150 & 120 & 0000.09 & 753010 & $6030-06$ & $1 \mathrm{AC} 13002$ & $60^{\circ} 25^{\prime} \mathrm{N}$ & $44^{\circ} 47^{\prime} \mathrm{W}$ & $\mathrm{W}$ & 115 & 100 & 0000.15 & 643112 & 6000-02 \\
\hline $1 \mathrm{AC} 10005$ & $60^{\circ} 34^{\prime} \mathrm{N}$ & $44^{\circ} 44^{\prime} \mathrm{w}$ & W & 110 & 90 & 0000.16 & 640012 & $6030-06$ & $1 \mathrm{ACl} 13003$ & $60^{\circ} 24^{\prime} \mathrm{N}$ & $44^{\circ} 47^{\prime} \mathrm{W}$ & W & 120 & 100 & 0000.53 & 623112 & $6000-02$ \\
\hline $1 \mathrm{ACl} 10006$ & $60^{\circ} 33^{\prime} \mathrm{N}$ & $44^{\circ} 42, \mathrm{~V}$ & W & 120 & 70 & 0000.30 & 643212 & $6030-06$ & $1 \mathrm{ACl} 13004$ & $60^{\circ} 24^{\prime} \mathrm{N}$ & $44^{\circ} 48^{\prime} \mathrm{W}$ & $\mathrm{w}$ & 100 & 80 & 0000.13 & 770010 & $6000-02$ \\
\hline $1 \mathrm{AC} 10007$ & $60^{\circ} 33^{\prime} \mathrm{N}$ & $44^{\circ} 43^{\prime} \mathrm{W}$ & 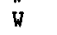 & 90 & 75 & 0000.08 & 770012 & $6030-06$ & $1 \mathrm{ACl} 3005$ & $60^{\circ} 24^{\prime} \mathrm{N}$ & $44^{\circ} 48^{\prime} \mathrm{W}$ & NV & 100 & 85 & 0000.11 & 780010 & $6000-02$ \\
\hline $1 \mathrm{AC} 10008$ & $60^{\circ} 33^{\prime} \mathrm{N}$ & $44^{\circ} 43^{\prime} \mathrm{W}$ & $\mathrm{NW}$ & 90 & 75 & 0000.08 & 770012 & $6030-06$ & $1 \mathrm{ACl} 3006$ & $60^{\circ} 24^{\prime} \mathrm{N}$ & $44^{\circ} 50^{\prime} \mathrm{W}$ & $\mathrm{N}$ & 95 & 75 & 0000.04 & 780010 & $6000-02$ \\
\hline $1 \mathrm{AC} 10009$ & $60^{\circ} 32^{\prime} \mathrm{N}$ & $44^{\circ} 45^{\prime} \mathrm{W}$ & $\mathrm{N}$ & 110 & 95 & 0000.06 & 700010 & $6030-06$ & $1 \mathrm{AC} 13007$ & $60^{\circ} 24^{\prime} \mathrm{N}$ & $44^{\circ} 50^{\prime} \mathrm{V}$ & NV & 95 & 85 & 0000.05 & 690012 & $6000-02$ \\
\hline $1 \mathrm{AC} 10010$ & $60^{\circ} 32^{\prime} \mathrm{N}$ & $44^{\circ} 45^{\prime} \mathrm{W}$ & W & 110 & 90 & 0000.16 & 600010 & $6030-06$ & 1AC13008 & $60^{\circ} 23, \mathrm{~N}$ & $44^{\circ} 51^{\prime} \mathrm{W}$ & $\mathrm{NV}$ & 90 & 80 & 0000.05 & 780010 & $6000-02$ \\
\hline $1 \mathrm{AC} 10011$ & $60^{\circ} 32^{\prime} \mathrm{N}$ & $44^{\circ} 46^{\prime} \mathrm{W}$ & $\mathrm{NW}$ & 110 & 90 & 0000.16 & 770012 & $6030-06$ & $1 \mathrm{AC} 13009$ & $60^{\circ} 23, \mathrm{~N}$ & $44^{\circ} 52^{\prime}, \overline{1}$ & $\mathrm{w}$ & 95 & 80 & 0000.17 & 640012 & $6000-02$ \\
\hline 1AC10012 & $60^{\circ} 32^{\prime} \mathrm{N}$ & $44^{\circ} 47^{\prime} \mathrm{W}$ & E & 100 & 100 & 0000.03 & 750012 & & $1 \mathrm{ACl} 3010$ & $60^{\circ} 23^{\prime} \mathrm{N}$ & $44^{\circ} 52^{\prime}, \mathrm{W}$ & $\mathrm{sw}$ & 80 & 60 & 0000.06 & 690012 & $6000-02$ \\
\hline $1 \mathrm{ACl} 10013$ & $60^{\circ} 33^{\prime} \mathrm{N}$ & $44^{\circ} 47, \mathrm{~W}$ & $\mathrm{NW}$ & 80 & 60 & 0000.11 & 750012 & $6030-06$ & $1 \mathrm{AC} 13011$ & $60^{\circ} 22^{\prime} \mathrm{N}$ & $44^{\circ} 54^{\prime} \mathrm{W}$ & $\mathrm{W}$ & 100 & 80 & 0000.03 & 790010 & $6000-02$ \\
\hline $1 \mathrm{ACl} 10014$ & $60^{\circ} 32^{\prime} \mathrm{N}$ & $44^{\circ} 48^{\prime} \mathrm{W}$ & $\mathrm{NV}$ & 90 & 60 & 0000.15 & 670012 & $6030-06$ & $1 \mathrm{ACl} 3012$ & $60^{\circ} 2$ & $44^{\circ} 55^{\prime \prime}$ & W & 110 & 80 & 000 & 670112 & 600 \\
\hline $1 \mathrm{AC} 10015$ & $60^{\circ} 31^{\prime} \mathrm{N}$ & $44^{\circ} 49$, W & $\mathrm{NW}$ & 80 & 60 & 0000.59 & 643212 & $6030-06$ & $1 \mathrm{ACl} 13013$ & $60^{\circ} 21, \mathrm{~N}$ & $44^{\circ} 56^{\prime} \mathrm{W}$ & NW & $\begin{array}{r}110 \\
90\end{array}$ & 80 & 0000.04 & 640112 & $6000-02$ \\
\hline $1 \mathrm{AC} 10016$ & $60^{\circ} 30^{\prime} \mathrm{N}$ & $44^{\circ} 51^{\prime} \mathrm{w}$ & $\mathrm{NW}$ & 110 & 60 & 0001.63 & 670112 & $6030-06$ & $1 \mathrm{AC} 14001$ & $60^{\circ} 21^{\prime} \mathrm{N}$ & $44^{\circ} 56^{\prime} \mathrm{W}$ & $\mathrm{s}$ & 110 & 90 & 0000.02 & 780010 & $6000-02$ \\
\hline $1 \mathrm{AC} 10017$ & $60^{\circ} 30^{\prime} \mathrm{N}$ & $44^{\circ} 52^{\prime} \mathrm{W}$ & $v$ & 110 & 90 & 0000.25 & 670112 & & 1 AC14002 & $60^{\circ} 22^{\prime} \mathrm{N}$ & $44^{\circ} 51^{\prime} \mathrm{W}$ & $\mathbf{E}$ & 110 & 90 & 0000.05 & 780010 & 60 \\
\hline $1 \mathrm{AC} 10018$ & $60^{\circ} 30^{\prime} \mathrm{N}$ & $44^{\circ} 53^{\prime}, \mathrm{W}$ & NW & 110 & 90 & 0000.11 & 670112 & 6030 & $1 \mathrm{ACl} 14003$ & $60^{\circ} 25^{\prime} \mathrm{N}$ & $44^{\circ} 44^{\prime} \mathrm{W}$ & $\mathrm{sw}$ & 11 & 85 & 00 & 780010 & 60 \\
\hline $1 \mathrm{AC} 10019$ & $60^{\circ} 29^{\prime} \mathrm{N}$ & $44^{\circ} 54^{\prime} \mathrm{W}$ & $\mathrm{NW}$ & 110 & 80 & 0000.23 & 643112 & 6000 & 1 AC14 & $60^{\circ} 24^{\prime} \mathrm{N}$ & $44^{\circ} 45^{\prime} \mathrm{W}$ & W & 100 & 70 & 0000.43 & 670012 & $6000-02$ \\
\hline 1AC11001 & $60^{\circ} 32^{\prime} \mathrm{N}$ & $44^{\circ} 47^{\prime} \mathrm{W}$ & $\mathrm{SE}$ & 140 & 130 & 0000.06 & 780010 & 6030 & $1 \mathrm{AC1} 4005$ & $60^{\circ} 23^{\prime} \mathrm{N}$ & $44^{\circ} 44^{\prime} \mathrm{W}$ & W & 95 & 80 & 0000.06 & 780010 & $6000-02$ \\
\hline $1 \mathrm{AC} 11002$ & $60^{\circ} 32^{\prime} \mathrm{N}$ & $44^{\circ} 46^{\prime} \mathrm{W}$ & $\mathrm{v}$ & 110 & 95 & 0000.15 & 770012 & 6030 & $1 \mathrm{AC1} 4006$ & $60^{\circ} 23^{\prime} \mathrm{N}$ & $44^{\circ} 45^{\prime} \mathrm{W}$ & NW & 100 & 70 & 0000.30 & 770012 & $6000-02$ \\
\hline $1 \mathrm{AC} 11003$ & $60^{\circ} 31 \cdot \mathrm{N}$ & $44^{\circ} 46^{\prime} \mathrm{W}$ & SW & 110 & 9 & 0000.40 & 640012 & & $1 \mathrm{AC} 14007$ & $60^{\circ} 23^{\prime} \mathrm{N}$ & $44^{\circ} 47^{\prime} \mathrm{W}$ & $\mathbf{N}$ & 100 & 60 & 0000.10 & 780010 & $6000-02$ \\
\hline $1 \mathrm{AC} 11004$ & $60^{\circ} 30^{\prime} \mathrm{N}$ & $44^{\circ} 47^{\prime} \mathrm{W}$ & v & 120 & 90 & 0000.24 & 640012 & $6030-06$ & $1 \mathrm{AC} 14008$ & $60^{\circ} 2$ & $44^{\circ} 47^{\prime} \mathrm{W}$ & $\mathrm{sW}$ & 10 & 95 & 00 & 780010 & 600 \\
\hline 1AC11005 & $60^{\circ} 30^{\prime} \mathrm{N}$ & $44^{\circ} 47^{\prime} \mathrm{W}$ & W & 120 & 10 & 0000.08 & 780010 & 6030 & $1 \mathrm{AC}$ & $60^{\circ} 22^{\prime} \mathrm{N}$ & $44^{\circ} 46^{\prime} \mathrm{W}$ & $\mathrm{W}$ & 120 & 115 & .08 & 778 & 60 \\
\hline $1 \mathrm{AC} 11006$ & $60^{\circ} 29^{\prime} \mathrm{N}$ & $44^{\circ} 46^{\prime}$ W & NW & 120 & 95 & 0000.14 & 780010 & & $1 \mathrm{AC}$ & $60^{\circ} 22^{\prime} \mathrm{N}$ & $44^{\circ} 46^{\prime} \mathrm{V}$ & NW & 110 & 90 & 0000.30 & 643112 & $6000-02$ \\
\hline $1 \mathrm{AC1} 2001$ & $60^{\circ} 29^{\prime} \mathrm{N}$ & $44^{\circ} 46^{\prime} \mathrm{W}$ & $\mathrm{s}$ & 120 & 100 & 0000.03 & 780010 & 600 & 1AC14011 & $60^{\circ} 21$, N & $44^{\circ} 47^{\prime \prime} \mathrm{W}$ & $\mathrm{W}$ & 90 & 80 & 0000.05 & 770012 & $6000-02$ \\
\hline $1 \mathrm{AC} 12002$ & $60^{\circ} 30^{\prime} \mathrm{N}$ & $44^{\circ} 45^{\prime} \mathrm{W}$ & SE & 120 & 90 & 0000.26 & 780010 & $6030-06$ & 1AC14012 & $60^{\circ} 21 \cdot \mathrm{N}$ & $44^{\circ} 48^{\prime} \mathrm{V}$ & $\hat{\mathrm{W}}$ & 110 & 95 & 0000.03 & 770012 & $6000-02$ \\
\hline $1 \mathrm{AC} 12003$ & $60^{\circ} 30^{\prime} \mathrm{N}$ & $44^{\circ} 45^{\prime} \mathrm{w}$ & $\mathrm{E}$ & 115 & 100 & 0000.10 & 740010 & $6030-06$ & $1 \mathrm{AC} 14013$ & $60^{\circ} 1$ & $44^{\circ} 48^{\prime}$ & $\mathrm{NW}$ & 100 & 80 & 000 & 780010 & 600 \\
\hline $1 \mathrm{AC} 12004$ & $60^{\circ} 30^{\prime} \mathrm{N}$ & $44^{\circ} 45^{\prime} \dot{W}$ & $\mathrm{E}$ & 110 & 100 & 0000.92 & 623312 & 6030 & $1 \mathrm{AC} 14$ & $60^{\circ} 19^{\prime} \mathrm{N}$ & $44^{\circ} 49^{\prime}$ V & $\mathrm{NV}$ & 90 & 80 & 0000.09 & 640112 & 60 \\
\hline $1 \mathrm{ACl} 2005$ & $60^{\circ} 31^{\prime} \mathrm{N}$ & $44^{\circ} 45^{\prime \prime}$ & $\mathrm{s}$ & & & 0000.07 & 780010 & 60 & 1AC14015 & $60^{\circ} 19, \mathrm{~N}$ & $44^{\circ} 49^{\prime} \mathrm{V}$ & $\mathrm{N}$ & 90 & 70 & 0000.11 & 670112 & $\begin{array}{l}-06 \\
-02\end{array}$ \\
\hline $1 \mathrm{AC} 12006$ & $60^{\circ} 31^{\prime} \mathrm{N}$ & $44^{\circ} 45^{\prime} \mathrm{W}$ & NE & 120 & 95 & 0000.08 & 780010 & 6030 & $1 \mathrm{AC} 14016$ & $60^{\circ} 19^{\prime} \mathrm{N}$ & $44^{\circ} 50^{\prime}$ W & $\mathrm{NW}$ & 90 & 85 & 0000.05 & 770112 & $6000-02$ \\
\hline $1 \mathrm{AC} 12007$ & $60^{\circ} 31 \cdot \mathrm{N}$ & $44^{\circ} 45^{\prime} \mathrm{V}$ & $\mathrm{E}$ & 130 & 110 & 0000.04 & 780010 & $6030-06$ & $1 \mathrm{AC} 14017$ & $60^{\circ} 18^{\prime} \mathrm{N}$ & $44^{\circ} 50^{\prime} \mathrm{W}$ & $\mathrm{NW}$ & 80 & 70 & 0000.08 & 670112 & $6000-02$ \\
\hline $1 \mathrm{AC} 12008$ & $60^{\circ} 32^{\prime} \mathrm{N}$ & $44^{\circ} 45^{\prime} \mathrm{W}$ & $\mathrm{E}$ & 140 & 90 & 0000.17 & 780010 & $6030-06$ & 1AC14018 & $60^{\circ} 18^{\prime} \mathrm{N}$ & $44^{\circ} 50^{\prime} \mathrm{W}$ & $\mathrm{N}$ & 90 & 60 & 0000.14 & 770110 & $6000-02$ \\
\hline $1 \mathrm{AC1} 2009$ & $60^{\circ} 33^{\prime} \mathrm{N}$ & $44^{\circ} 42^{\prime} \mathrm{V}$ & SW & 100 & 80 & 0000.04 & 770012 & $6030-06$ & 1 AC14019 & $60^{\circ} 18^{\prime} \mathrm{N}$ & $44^{\circ} 51^{\prime}$ & $\mathrm{N}$ & 80 & 65 & 0000.08 & 643112 & $6000-02$ \\
\hline $1 \mathrm{AC} 12010$ & $60^{\circ} 33^{\prime} \mathrm{N}$ & $44^{\circ} 41^{\prime} \mathrm{W}$ & SW & 100 & 80 & 0000.61 & 640112 & 6030 & $1 \mathrm{AC1} 4020$ & $60^{\circ} 18^{\prime} \mathrm{N}$ & $44^{\circ} 52^{\prime \prime}$ & NW & 80 & 60 & 0000.05 & 670112 & $6000-02$ \\
\hline $1 \mathrm{AC} 12011$ & $60^{\circ} 32^{\prime} \mathrm{N}$ & $44^{\circ} 41^{\prime} \mathrm{W}$ & W & 110 & 80 & 0000.17 & 690112 & 6030 & $1 \mathrm{AC1} 5001$ & $60^{\circ} 18^{\prime} \mathrm{N}$ & $44^{\circ} 52^{\prime} \mathrm{W}$ & W & 90 & 70 & 0000.03 & 750010 & $6000-02$ \\
\hline 1AC12012 & $60^{\circ} 32^{\prime} \mathrm{N}$ & $44^{\circ} 40^{\prime} \mathrm{W}$ & SW & 90 & 70 & 0001.42 & 643112 & $6030-06$ & $1 \mathrm{AC} 15002$ & $60^{\circ} 16^{\prime} \mathrm{N}$ & $44^{\circ} 56^{\prime} \mathrm{W}$ & $\mathbf{N}$ & 90 & 75 & 0000.16 & 640012 & $6000-02$ \\
\hline $1 \mathrm{ACl} 2013$ & $60^{\circ} 32^{\prime} \mathrm{N}$ & $44^{\circ} 41^{\prime} \mathrm{W}$ & $\mathrm{SW}$ & 100 & 80 & 0000.09 & 770010 & $6030-06$ & $1 \mathrm{AC} 15003$ & $60^{\circ} 17 \cdot \mathrm{N}$ & $44^{\circ} 56^{\prime} \mathrm{W}$ & $\mathrm{NE}$ & 95 & 70 & 0000.10 & 670112 & $6000-02$ \\
\hline
\end{tabular}




\begin{tabular}{|c|c|c|c|}
\hline ODE & LAT & & $R 7$ \\
\hline 407500 & 6 & & \\
\hline 001 & $60^{\circ} 17^{\prime} \mathrm{N}$ & $44^{\circ} 59^{\prime} \mathrm{W}$ & \\
\hline & $60^{\circ} 16^{\prime} \mathrm{N}$ & $44^{\circ} 59^{\prime} \mathrm{W}$ & \\
\hline 16003 & $60^{\circ} 16^{\prime} \mathrm{N}$ & $44^{\circ} 59^{\prime} \mathrm{W}$ & \\
\hline 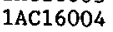 & $60^{\circ} 15^{\prime} \mathrm{N}$ & $44^{\circ} 59^{\prime} \mathrm{W}$ & \\
\hline 005 & $60^{\circ} 15^{\prime} \mathrm{N}$ & $45^{\circ} 00^{\prime} \mathrm{W}$ & \\
\hline 10 & $\mathrm{~N}$ & $45^{\circ} 02 \cdot W$ & \\
\hline C16007 & $60^{\circ} 15^{\prime} \mathrm{N}$ & $45^{\circ} 02^{\prime} \mathrm{W}$ & \\
\hline C16008 & $60^{\circ} 15^{\prime} \mathrm{N}$ & $45^{\circ} 03^{\prime} \mathrm{W}$ & $\mathrm{NW}$ \\
\hline & ${ }^{\circ} 14 \cdot \mathrm{N}$ & & \\
\hline & $\mathrm{N}$ & W & NW \\
\hline 1 & 6 & $45^{\circ} 11^{\prime} \mathrm{W}$ & \\
\hline $1 \mathrm{ACl}$ & $60^{\circ}$ & $5^{\circ} 10^{\prime} \mathrm{W}$ & $\mathrm{NE}$ \\
\hline $1 \mathrm{ACl} 17$ & $60^{\circ} 1$ & $45^{\circ} 16^{\prime} \mathrm{W}$ & \\
\hline $1 \mathrm{AC} 17004$ & $\mathrm{~N}$ & $45^{\circ} 15^{\circ} \mathrm{W}$ & \\
\hline & & & $\mathrm{NE}$ \\
\hline & & $45^{\circ} 15^{\prime} \mathrm{V}$ & \\
\hline $1 \mathrm{ACl}$ & & $45^{\circ} 14^{\prime} W$ & $\mathbf{N}$ \\
\hline $1 \mathrm{ACl} 7$ & $7 \cdot \mathrm{N}$ & $45^{\circ} 14^{\prime} \mathrm{W}$ & $\mathrm{N}$ \\
\hline & & & \\
\hline & & & \\
\hline & & & \\
\hline $1 \mathrm{ACl}$ & & $17 \mathrm{~W}$ & $\mathrm{~N}$ \\
\hline $1 \mathrm{ACl}$ & & $18^{\prime \prime}$ & \\
\hline & & & D \\
\hline & & & \\
\hline & & 19,6 & N \\
\hline $1 \mathrm{AC} 1$ & & 20,0 & \\
\hline & & $18, \mathrm{~W}$ & vi \\
\hline & & & NW \\
\hline & & $\mathrm{W}$ & $\mathrm{NW}$ \\
\hline & N & $19, \mathrm{~W}$ & NW \\
\hline $1 \mathrm{AC}$ & & $18, \mathrm{~W}$ & \\
\hline & & & \\
\hline & & & \\
\hline & & & \\
\hline & & $45^{\circ} 17^{\prime} \cdot \mathrm{V}$ & NW \\
\hline & & $45^{\circ} 19^{\prime}$ & \\
\hline & & $9, \mathrm{~W}$ & \\
\hline & & $\mathrm{v}$ & \\
\hline & & & $\mathrm{Nh}$ \\
\hline & & $45^{\circ} 12, v$ & 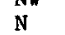 \\
\hline & & 11, W & \\
\hline & & $1, \mathrm{~W}$ & \\
\hline & & & \\
\hline & & & NW \\
\hline & & $3, \mathrm{v}$ & NW \\
\hline & & & NW \\
\hline & & & \\
\hline & & & \\
\hline & & & S \\
\hline & & & $\mathrm{s}$ \\
\hline & & & E \\
\hline & & $44^{\circ} 52^{\prime \prime} \mathrm{W}$ & 2 \\
\hline & & & NW \\
\hline & & & \\
\hline & & & \\
\hline & 6 & W & $\mathrm{NE}$ \\
\hline & & $44^{\circ} 5$ & \\
\hline & & & \\
\hline & & & \\
\hline
\end{tabular}

$\begin{array}{rrccc}\text { HIGH } & \text { LOW } & \text { AREA } & \text { MODE } & \text { MAP } \\ 80 & 55 & 0000.10 & 780010 & 6000-02 \\ 90 & 75 & 0000.04 & 780010 & 6000-02 \\ 100 & 60 & 0000.18 & 640112 & 6000-02 \\ 100 & 80 & 0000.07 & 670112 & 6000-02 \\ 100 & 90 & 0000.21 & 64312 & 6000-02 \\ 70 & 60 & 0000.10 & 690010 & 6000-02 \\ 75 & 45 & 0000.10 & 780010 & 6000-02 \\ 80 & 75 & 0000.15 & 670312 & 6000-02 \\ 100 & 75 & 0000.32 & 640512 & 6000-02 \\ 85 & 60 & 0000.13 & 780010 & 6000-02 \\ 85 & 75 & 0000.08 & 780010 & 6000-02 \\ 80 & 65 & 0000.06 & 780010 & 6000-01 \\ 85 & 65 & 0000.08 & 780010 & 6000-01 \\ 100 & 65 & 0000.56 & 643312 & 6000-01 \\ 85 & 65 & 0000.05 & 750010 & 6000-01 \\ 120 & 55 & 0000.54 & 673012 & 6000-01 \\ 65 & 50 & 0000.06 & 780010 & 6000-01 \\ 90 & 70 & 0000.33 & 670112 & 6000-01 \\ 90 & 80 & 0000.34 & 670112 & 6000-01 \\ 90 & 80 & 0000.03 & 790010 & 6000-01 \\ 90 & 70 & 0000.03 & 790012 & 6000-01 \\ 90 & 70 & 0000.04 & 780010 & 6000-01 \\ 105 & 50 & 0001.97 & 536122 & 6000-01 \\ 85 & 75 & 0000.11 & 650210 & 6000-01 \\ 95 & 80 & 0000.02 & 780010 & 6000-01 \\ 60 & 45 & 0000.07 & 740112 & 6000-01 \\ 110 & 45 & 0000.73 & 643212 & 6000-01 \\ 70 & 50 & 0000.34 & 640112 & 6000-01 \\ 75 & 60 & 0000.17 & 780010 & 6000-01 \\ 75 & 55 & 0000.05 & 750112 & 6000-01 \\ 80 & 70 & 0000.12 & 750112 & 6000-01 \\ 65 & 50 & 0000.14 & 750112 & 6000-01 \\ 100 & 80 & 0000.09 & 780012 & 6000-01 \\ 100 & 85 & 0000.08 & 753112 & 6000-01 \\ 75 & 60 & 0000.07 & 750010 & 6000-01 \\ 105 & 90 & 0000.12 & 750010 & 6000-01 \\ 80 & 70 & 0000.03 & 750010 & 6000-01 \\ 65 & 55 & 0000.03 & 770112 & 6000-01 \\ 90 & 75 & 0000.08 & 770112 & 6000-01 \\ 70 & 50 & 0000.07 & 780010 & 6000-01 \\ 90 & 70 & 0000.04 & 750010 & 6000-01 \\ 85 & 65 & 0000.06 & 730212 & 6000-01 \\ 75 & 70 & 0000.04 & 780010 & 6000-01 \\ 100 & 85 & 0000.05 & 780012 & 6000-01 \\ 80 & 65 & 0000.34 & 670112 & 6000-01 \\ 80 & 65 & 0000.12 & 670112 & 6000-01 \\ 80 & 70 & 0000.13 & 673112 & 6000-01 \\ 70 & 65 & 0000.05 & 780010 & 6000-01 \\ 90 & 80 & 0000.11 & 643212 & 6000-01 \\ 110 & 95 & 0000.04 & 750012 & 6030-05 \\ 115 & 100 & 0000.10 & 750010 & 6030-05 \\ 115 & 95 & 0000.12 & 700010 & 6030-05 \\ 115 & 95 & 0000.20 & 700010 & 6030-05 \\ 130 & 105 & 0000.15 & 670112 & 6030-06 \\ 120 & 110 & 0000.11 & 670112 & 6030-06 \\ 120 & 105 & 0000.21 & 740002 & 6030-06 \\ 130 & 100 & 0000.26 & 740002 & 6030-06 \\ 130 & 100 & 0000.18 & 740002 & 6030-06 \\ 140 & 100 & 0000.51 & 640110 & 6030-06 \\ 150 & 130 & 0000.31 & 643112 & 6030-06 \\ 150 & 110 & 0000.64 & 620111 & 6030-06\end{array}$

CODE

LAT

$44^{\circ} 53$

IADO1013 60 $60^{\circ} 42^{\prime} \mathrm{N} \quad 44^{\circ} 53^{\prime} \mathrm{W}$

$\begin{array}{llll}1 \text { ADD } 1014 & 60^{\circ} 41 ' \mathrm{~N} & 44^{\circ} 53^{\prime} \mathrm{V} & \mathrm{NW} \\ 1 \mathrm{AD} 01015 & 60^{\circ} 411^{\prime} \mathrm{N} & 44^{\circ} 54^{\prime} \mathrm{N} & \mathrm{NE}\end{array}$

$1 \mathrm{AD01016} 60^{\circ} 41$ ' N $44^{\circ} 54^{\prime} \mathrm{W} \mathrm{NE}$

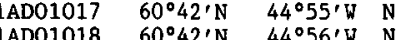

$1 \mathrm{AD01019} \quad 60^{\circ} 42^{\prime} \mathrm{N} \quad 44^{\circ} 566^{\prime} \mathrm{W} \quad \mathrm{NW}$

$1 \mathrm{AD01020} \quad 60^{\circ} 42^{\prime} \mathrm{N} \quad 44^{\circ} 57^{\prime} \mathrm{W} \mathrm{W}$

1 AD01022 $60^{\circ} 41^{\prime} \mathrm{N} \quad 44^{\circ} 56^{\prime} \mathrm{N}$ SW

$\begin{array}{lll}1 \mathrm{AD} 01023 & 60^{\circ} 40^{\prime} \mathrm{N} & 44^{\circ} 56^{\prime} \mathrm{V} \\ 1 \mathrm{AD} 01024 & 60^{\circ} 40^{\prime} \mathrm{N} & 44^{\circ} 56^{\prime} \mathrm{V}\end{array}$

$\begin{array}{lll}1 \mathrm{AD} 01024 & 60^{\circ} 40^{\prime} \mathrm{N} & 44^{\circ} 56^{\prime} \mathrm{V} \\ 1 \mathrm{ADO} 01025 & 60^{\circ} 39^{\prime} \mathrm{N} & 44^{\circ} 58^{\prime} \mathrm{V}\end{array}$

$\begin{array}{llll}1 \mathrm{ADO1026} & 60^{\circ} 39^{\prime} \mathrm{N} & 44^{\circ} 58^{\prime} \mathrm{N} & \mathrm{NE} \\ 1 \mathrm{AD} 01027 & 60^{\circ} 40^{\prime} \mathrm{N} & 45^{\circ} 00^{\prime} \mathrm{N} & \mathrm{NNE}\end{array}$

$\begin{array}{llll}1 \text { ADD01027 } & 60^{\circ} 40^{\prime} \mathrm{N} & 45^{\circ} 00 \cdot \mathrm{N} & \mathrm{NW} \\ 1 \mathrm{ADD01028} & 60^{\circ} 40^{\prime} \mathrm{N} & 45^{\circ} 00^{\prime} \mathrm{W} & \mathrm{SW}\end{array}$

$1 A D 0102960^{\circ} 40^{\prime} \mathrm{N} \quad 45^{\circ} 00$, N

1 AD01031 $60^{\circ} 39^{\prime} \mathrm{N} \quad 45^{\circ} 01$ 'N NW

$1 \mathrm{ADO} 103260^{\circ} 39^{\prime} \mathrm{N} \quad 5^{\circ} 02, \mathrm{~N}$ NH

$\begin{array}{llll}1 \mathrm{ADO} 01033 & 60^{\circ} 39^{\prime} \mathrm{N} & 45^{\circ} 02 \cdot \mathrm{W} & \mathrm{S} \\ 1 \mathrm{AD01034} & 60^{\circ} 38^{\prime} \mathrm{N} & 45^{\circ} 02, \mathrm{~W} & \mathrm{~V}\end{array}$

$\begin{array}{llll}1 \mathrm{AD01035} & 60^{\circ} 37^{\prime} \mathrm{N} & 45^{\circ} 02, \mathrm{~N} & \mathrm{NW} \\ 1 \mathrm{AD01036} & 60^{\circ} 38^{\prime} \mathrm{N} & 45^{\circ} 05^{\prime} \mathrm{V} & \mathrm{N}\end{array}$

$\begin{array}{llll}1 \mathrm{AD} 01036 & 60^{\circ} 38^{\prime} \mathrm{N} & 45^{\circ} 05^{\prime} \mathrm{N} & \mathrm{N} \\ 1 \mathrm{AD} 01037 & 60^{\circ} 377^{\prime} \mathrm{N} & 45^{\circ} 05^{\prime} \mathrm{V} & \mathrm{N}\end{array}$

$\begin{array}{llll}1 \mathrm{AD01037} & 60^{\circ} 37^{\prime} \mathrm{N} & 45^{\circ} 05^{\prime} \mathrm{W} & \mathrm{N} \\ 1 \mathrm{ADO1038} & 60^{\circ} 37^{\prime} \mathrm{N} & 45^{\circ} 06^{\prime} \mathrm{W} & \mathrm{NW}\end{array}$

$\begin{array}{llll}1 \mathrm{ADO} 01038 & 60^{\circ} 377^{\prime} \mathrm{N} & 45^{\circ} 06^{\prime} \mathrm{W} & \mathrm{NW} \\ 1 \mathrm{ADO} 2001 & 60^{\circ} 40^{\prime} \mathrm{N} & 44^{\circ} 56^{\prime} \mathrm{W} & \mathrm{E}\end{array}$

$1 \mathrm{ADO} 200260^{\circ} 40^{\prime} \mathrm{N} \quad 44^{\circ} 56^{\prime} \mathrm{V}$ SE

$1 \mathrm{ADO} 2004 \quad 60^{\circ} 40^{\prime} \mathrm{N} \quad 44^{\circ} 55^{\prime} \mathrm{W}$ SV

$1 \mathrm{ADO} 200560^{\circ} 39^{\prime} \mathrm{N} \quad 44^{\circ} 53^{\prime} \mathrm{W}$

$1 \mathrm{ADO2006} \quad 60^{\circ} 39^{\prime} \mathrm{N} \quad 44^{\circ} 53^{\prime} \mathrm{W}$

$1 \mathrm{ADO} 2007 \quad 60^{\circ} 38^{\prime} \mathrm{N} \quad 44^{\circ} 55^{\prime} \mathrm{W}$

IAD02009 $60^{\circ} 37^{\prime} \mathrm{N} \quad 44^{\circ} 56^{\prime} \mathrm{W}$

$\begin{array}{llll}1 \mathrm{ADO} 2010 & 60^{\circ} 37^{\prime} \mathrm{N} & 44^{\circ} 56^{\prime} \mathrm{V} & \mathrm{NW} \\ 1 \mathrm{ADO} 2011 & 60^{\circ} 36^{\prime} \mathrm{N} & 44^{\circ} 56^{\prime} \mathrm{V} & \mathrm{NH}\end{array}$

$\begin{array}{llll}1 \mathrm{ADO} 2011 & 60^{\circ} 366^{\prime} \mathrm{N} & 44^{\circ} 56^{\prime} \mathrm{V} & \mathrm{N} \\ 1 \mathrm{ADO} 02012 & 60^{\circ} 35^{\prime} \mathrm{N} & 44^{\circ} 54^{\prime} \mathrm{V} & \mathrm{V}\end{array}$

$1 \mathrm{ADO} 2012$

$\begin{array}{ll}60^{\circ} 35^{\prime} \mathrm{N} & 44^{\circ} 54^{\prime}, \mathrm{W} \\ 60^{\circ} 35^{\prime} \mathrm{N} & 44^{\circ} 55^{\prime} \mathrm{W}\end{array}$

ADO2014 $60^{\circ} 35^{\prime} \mathrm{N} \quad 44^{\circ} 55^{\prime} \mathrm{N}$

$1 \mathrm{AD02016} 60^{\circ} 34^{\prime} \mathrm{N} \quad 44^{\circ} 577^{\prime} \mathrm{N}$ N

$1 \mathrm{AD0} 2017 \quad 60^{\circ} 34^{\prime} \mathrm{N} \quad 44^{\circ} 59^{\prime} \mathrm{V} \quad \mathrm{NW}$

$1 \mathrm{ADO2018} \quad 60^{\circ} 34^{\prime} \mathrm{N} \quad 55^{\circ} 58^{\prime} \mathrm{N} N$

$\begin{array}{lllll}1 \text { ADO2019 } & 60^{\circ} 34^{\prime} \mathrm{N} & 44^{\circ} 49^{\prime} \mathrm{W} & \mathrm{N} \\ 1 \mathrm{ADO} 2020 & 60^{\circ} 34^{\prime} \mathrm{N} & 45^{\circ} 01^{\prime} \mathrm{W} & \mathrm{N}\end{array}$

$1 \mathrm{ADO} 2021 \quad 60^{\circ} 34^{\prime} \mathrm{N} \quad 45^{\circ} 02^{\prime} \mathrm{W} \quad \mathrm{N}$

1AD02022 $60^{\circ} 34^{\prime} \mathrm{N} \quad 45^{\circ} 03^{\prime} \mathrm{W} N$

$\begin{array}{llll}1 \mathrm{ADO} 2023 & 60^{\circ} 34^{\prime} \mathrm{N} & 45^{\circ} 04^{\prime} \mathrm{V} & \mathrm{N} \\ 1 \mathrm{ADO} 2024 & 60^{\circ} 34^{\prime} \mathrm{N} & 45^{\circ} 04^{\prime} \mathrm{V} & \mathrm{V}\end{array}$

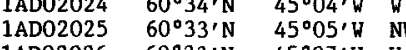

IADO2027 $60^{\circ} 38^{\prime} \mathrm{N} \quad 44^{\circ} 59^{\prime} \mathrm{W}$

$1 \mathrm{AD0} 300160^{\circ} 32^{\prime} \mathrm{N} \quad 45^{\circ} 07^{\prime} \mathrm{V}$

1 AD03002 $60^{\circ} 33^{\prime} \mathrm{N} \quad 45^{\circ} 03^{\prime} \mathrm{W}$

$1 \mathrm{AD} 03003 \quad 60^{\circ} 33^{\prime} \mathrm{N} \quad 45^{\circ} 02, \mathrm{~V}$

$1 \mathrm{AD03004} 60^{\circ} 33^{\prime} \mathrm{N} \quad 45^{\circ} 00^{\prime} \mathrm{W}$

$1 \mathrm{AD03005} 60^{\circ} 322^{\prime} \mathrm{N} \quad 44^{\circ} 59^{\prime} \mathrm{W} W$

$1 \mathrm{AD03006} 60^{\circ} 31^{\prime} \mathrm{N} \quad 45^{\circ} 00^{\prime} \mathrm{N} N$

$\begin{array}{llll}1 \mathrm{AD0} 03007 & 60^{\circ} 31^{\prime} \mathrm{N} & 45^{\circ} 01^{\prime} \mathrm{W} & \mathrm{N} \\ 1 \mathrm{AD0} 000 & 60^{\circ} 31, \mathrm{~N} & 45^{\circ} 02, \mathrm{~W} & \mathrm{~N}\end{array}$ $-06$ -06
-06 06 06 06 06 -06
-05
-05 -1
-05 05 -05
-06 06 $-06$ 06 06 $-06$

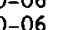
$-06$ $-06$ $-05$ -05
0

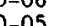
$-05$ $-06$ $-05$ 


\begin{tabular}{|c|c|c|c|c|c|c|c|c|c|c|c|c|c|c|c|c|c|}
\hline CODE & LAT & LONG & ORI & HIGH & LOW & AREA & $\begin{array}{l}\text { MO, } \\
\text { CODE }\end{array}$ & MAP & CODE & LAT & LONG & ORI & HIGH & LOW & AREA & $\begin{array}{c}\text { MO } \\
\text { CODE }\end{array}$ & MAP \\
\hline 1AD03009 & $60^{\circ} 31^{\prime} \mathrm{N}$ & $45^{\circ} 03^{\prime \prime}$ & $W$ & 100 & & 0000.10 & 653010 & $6030-05$ & $1 \mathrm{AE} 05017$ & $60^{\circ} 49^{\prime} \mathrm{N}$ & $44^{\circ} 36^{\prime} \mathrm{W}$ & $\mathrm{N}$ & 195 & 105 & 0005.10 & 526110 & $6030-06$ \\
\hline $1 \mathrm{AD03010}$ & $60^{\circ} 31^{\prime N}$ & $45^{\circ} 02, \mathrm{~W}$ & $\mathrm{NW}$ & 100 & 75 & 0000.33 & 643112 & $6030-05$ & 1AE05018 & $60^{\circ} 50^{\prime} \mathrm{N}$ & $44^{\circ} 39^{\prime} \mathrm{W}$ & $\mathrm{N}$ & 180 & 160 & 0000.10 & 700010 & $6030-06$ \\
\hline 1AD03011 & $60^{\circ} 31^{\prime} \mathrm{N}$ & $45^{\circ} 04^{\prime} \mathrm{W}$ & NW & 95 & 85 & 0000.04 & 780010 & $6030-05$ & $1 \mathrm{AE} 05019$ & $60^{\circ} 50^{\prime} \mathrm{N}$ & $44^{\circ} 39^{\prime} \mathrm{W}$ & $\mathrm{N}$ & 160 & 140 & 0000.30 & 700010 & $6030-06$ \\
\hline 1 AD03012 & $60^{\circ} 30^{\prime} \mathrm{N}$ & $45^{\circ} 09^{\prime} \mathrm{W}$ & NW & 110 & 105 & 0000.06 & 780010 & $6030-05$ & $1 \mathrm{AE} 05020$ & $60^{\circ} 49^{\prime} \mathrm{N}$ & $44^{\circ} 40^{\prime} \mathrm{W}$ & $\mathrm{W}$ & 190 & 90 & 0011.20 & 530110 & $6030-06$ \\
\hline 1 AD03013 & $60^{\circ} 30^{\prime} \mathrm{N}$ & $45^{\circ} 04^{\prime} \mathrm{W}$ & NW & 105 & 85 & 0000.21 & 643112 & $6030-05$ & $1 \mathrm{AB} 05021$ & $60^{\circ} 49^{\prime} \mathrm{N}$ & $44^{\circ} 44^{\prime} \mathrm{W}$ & $\mathrm{N}$ & 200 & 100 & 0003.70 & 523110 & $6030-06$ \\
\hline $1 \mathrm{AD} 03014$ & $60^{\circ} 30^{\prime} \mathrm{N}$ & $45^{\circ} 04^{\prime} \mathrm{W}$ & W & 110 & 100 & 0000.08 & 750012 & $6030-05$ & $1 \mathrm{AE} 05022$ & $60^{\circ} 46^{\prime} \mathrm{N}$ & $44^{\circ} 48^{\prime} \mathrm{W}$ & NW & 190 & 100 & 0015.30 & 523110 & $6030-06$ \\
\hline 1AD03015 & $60^{\circ} 30^{\prime} \mathrm{N}$ & $45^{\circ} 05^{\prime} \mathrm{V}$ & $\mathrm{NE}$ & 105 & 80 & 0000.19 & 643112 & $6030-05$ & $1 \mathrm{AEO} 05023$ & $60^{\circ} 48^{\prime} \mathrm{N}$ & $44^{\circ} 46^{\prime} \mathrm{W}$ & NW & 186 & 160 & 0000.60 & 770010 & $6030-06$ \\
\hline 1AD03016 & $60^{\circ} 30^{\prime} \mathrm{N}$ & $45^{\circ} 06^{\prime} \mathrm{V}$ & $\mathrm{N}$ & 85 & 75 & 0000.04 & 750012 & $6030-05$ & $1 \mathrm{AEO} 5024$ & $60^{\circ} 48^{\prime} \mathrm{N}$ & $44^{\circ} 46^{\prime} \mathrm{V}$ & NW & 150 & 105 & 0000.30 & 633110 & $6030-06$ \\
\hline 1AD03017 & $60^{\circ} 30^{\prime} \mathrm{N}$ & $45^{\circ} 06^{\prime} \mathrm{W}$ & NW & 110 & 85 & 0000.14 & 643112 & $6000-02$ & 1AE05025 & $60^{\circ} 48^{\prime} \mathrm{N}$ & $44^{\circ} 46^{\prime} \mathrm{V}$ & NH & 160 & 110 & 0000.90 & 533310 & $6030-06$ \\
\hline 1AD03018 & $60^{\circ} 30^{\prime} \mathrm{N}$ & $45^{\circ} 07^{\prime} \mathrm{W}$ & $\mathbf{N}$ & 95 & 60 & 0000.44 & 643112 & $6000-02$ & $1 \mathrm{AEO} 0026$ & $60^{\circ} 47 \cdot \mathrm{N}$ & $44^{\circ} 45^{\prime} \mathrm{W}$ & NW & 192 & 180 & 0000.50 & 700010 & $6030-06$ \\
\hline $1 \mathrm{AD} 03019$ & $60^{\circ} 30^{\prime} \mathrm{N}$ & $45^{\circ} 08^{\prime} \mathrm{W}$ & $\mathrm{N}$ & 95 & 85 & 0000.05 & 753112 & $6030-05$ & $1 \mathrm{AE} 05027$ & $60^{\circ} 47 \cdot \mathrm{N}$ & $44^{\circ} 47^{\prime} \mathrm{V}$ & NW & 170 & 95 & 0000.80 & 533410 & $6030-06$ \\
\hline $1 \mathrm{AD} 03020$ & $60^{\circ} 30^{\prime} \mathrm{N}$ & $45^{\circ} 18^{\prime} \mathrm{W}$ & $\mathrm{N}$ & 110 & 95 & 0000.15 & 643112 & $6000-02$ & 1 AE05028 & $60^{\circ} 47^{\prime} \mathrm{N}$ & $44^{\circ} 48^{\prime} W$ & SU & 175 & 115 & 0000.50 & 770010 & $6030-06$ \\
\hline 1 AD03021 & $60^{\circ} 30^{\prime} \mathrm{N}$ & $45^{\circ} 19^{\prime W}$ & $\mathrm{~N}$ & 90 & 80 & 0000.21 & 643112 & $6000-02$ & 1AE05029 & $60^{\circ} 477^{\prime} \mathrm{N}$ & $44^{\circ} 48^{\prime} \mathrm{W}$ & Nu & 130 & 120 & 0000.10 & 750010 & $6030-06$ \\
\hline $1 \mathrm{ADO} 3022$ & $60^{\circ} 30^{\prime} \mathrm{N}$ & $45^{\circ} 20^{\prime} \mathrm{W}$ & $\mathrm{N}$ & 95 & 85 & 0000.06 & 743112 & $6000-02$ & $1 \mathrm{AE} 05030$ & $60^{\circ} 49^{\prime} \mathrm{N}$ & $44^{\circ} 50^{\prime} \mathrm{W}$ & $\mathrm{NV}$ & 140 & 140 & 0000.20 & 750010 & $6030-06$ \\
\hline $1 \mathrm{AE} 04001$ & $60^{\circ} 56^{\prime} \mathrm{N}$ & $44^{\circ} 54^{\prime} W$ & $\mathrm{v}$ & 110 & 95 & 0000.80 & 632110 & $6030-06$ & $1 \mathrm{AE} 05031$ & $60^{\circ} 49^{\prime} \mathrm{N}$ & $44^{\circ} 49^{\prime} \mathrm{W}$ & $\mathbf{N}$ & 150 & 140 & 0000.80 & 770010 & $6030-06$ \\
\hline $1 \mathrm{AE} 04002$ & $60^{\circ} 55^{\prime} \mathrm{N}$ & $44^{\circ} 53^{\prime} \mathrm{W}$ & NW & 170 & 150 & 0000.19 & 600010 & $6030-06$ & $1 \mathrm{AE} 05032$ & $60^{\circ} 50^{\prime} \mathrm{N}$ & $44^{\circ} 52^{\prime} \mathrm{W}$ & W & 160 & 110 & 0004.80 & 530110 & $6030-06$ \\
\hline $1 \mathrm{AE} 04003$ & $60^{\circ} 55^{\prime} \mathrm{N}$ & $44^{\circ} 54^{\prime} \mathrm{W}$ & $\mathrm{SW}$ & 160 & 100 & 0001.82 & 670010 & $6030-06$ & $1 \mathrm{AE} 05033$ & $60^{\circ} 49^{\prime} \mathrm{N}$ & $44^{\circ} 53^{\prime} \mathrm{W}$ & N & 170 & 90 & 0005.00 & 523110 & $6030-06$ \\
\hline $1 \mathrm{AE} 04004$ & $60^{\circ} 54^{\prime} \mathrm{N}$ & $44^{\circ} 55^{\prime} \mathrm{W}$ & $\mathrm{N}$ & 140 & 110 & 0000.18 & 600010 & $6030-06$ & $1 \mathrm{AE} 05034$ & $60^{\circ} 49 \cdot \mathrm{N}$ & $44^{\circ} 55^{\prime} \mathrm{W}$ & N & 150 & 90 & 0001.70 & 533110 & $6030-06$ \\
\hline 1AE04005 & $60^{\circ} 54^{\prime} \mathrm{N}$ & $44^{\circ} 55^{\prime} \mathrm{W}$ & W & 130 & 130 & 0000.10 & 750110 & $6030-06$ & 1AE05035 & $60^{\circ} 49^{\prime} \mathrm{N}$ & $44^{\circ} 56^{\prime} \mathrm{W}$ & $\mathrm{NE}$ & 130 & 110 & 0000.13 & 750010 & $6030-06$ \\
\hline $1 \mathrm{AE} 04006$ & $60^{\circ} 54^{\prime} \mathrm{N}$ & $44^{\circ} 55^{\prime} \mathrm{W}$ & NW & 130 & 100 & 0000.50 & 600110 & $6030-06$ & 1 AE05036 & $60^{\circ} 48^{\prime} \mathrm{N}$ & $44^{\circ} 54^{\prime} \mathrm{W}$ & $\mathrm{SW}$ & 150 & 140 & 0000.10 & 750010 & $6030-06$ \\
\hline $1 \mathrm{AE} 04007$ & $60^{\circ} 53 \cdot \mathrm{N}$ & $44^{\circ} 56^{\prime} \mathrm{W}$ & NW & 135 & 95 & 0001.20 & 623110 & $6030-06$ & 1 AE05037 & $60^{\circ} 48^{\prime} \mathrm{N}$ & $44^{\circ} 54^{\prime} \mathrm{W}$ & SW & 140 & 140 & 0000.10 & 750110 & $6030-06$ \\
\hline $1 \mathrm{AE} 04008$ & $60^{\circ} 53^{\prime} \mathrm{N}$ & $44^{\circ} 57^{\prime} \mathrm{V}$ & $\mathrm{w}$ & 130 & 130 & 0000.10 & 750110 & 6030-06 & 1 AE05038 & $60^{\circ} 46^{\prime} \mathrm{N}$ & $44^{\circ} 50^{\prime} \mathrm{W}$ & $w$ & 110 & 110 & 0000.10 & 753110 & $6030-06$ \\
\hline 1AE04009 & $60^{\circ} 52, \mathrm{~N}$ & $44^{\circ} 58^{\prime} \mathrm{W}$ & $\mathbf{N}$ & 115 & 105 & 0000.20 & 580010 & 6030-06 & $1 \mathrm{AE} 05039$ & $60^{\circ} 46^{\prime} \mathrm{N}$ & $44^{\circ} 50^{\prime} \mathrm{W}$ & NE & 182 & 110 & 0002.40 & 523110 & $6030-06$ \\
\hline $1 \mathrm{AE} 04010$ & $60^{\circ} 52^{\prime} \mathrm{N}$ & $45^{\circ} 00^{\prime} \mathrm{V}$ & NE & 115 & 100 & 0000.19 & 570010 & $6030-06$ & 1 AE 05040 & $60^{\circ} 46^{\prime} \mathrm{N}$ & $44^{\circ} 51^{\prime} \mathrm{W}$ & $\mathrm{N}$ & 120 & 100 & 0000.25 & 753110 & -06 \\
\hline $1 \mathrm{AE} 04011$ & $60^{\circ} 50^{\prime} \mathrm{N}$ & $45^{\circ} 02^{\prime} \mathrm{W}$ & $\mathbf{N}$ & 135 & 120 & 0000.27 & 670010 & $6030-05$ & 1AE05041 & $60^{\circ} 43^{\prime} \mathrm{N}$ & $45^{\circ} 03^{\prime} \mathrm{W}$ & $\mathrm{N}$ & 120 & 120 & 0000.10 & 700010 & $6030-05$ \\
\hline $1 \mathrm{AE} 04012$ & $60^{\circ} 49^{\prime} \mathrm{N}$ & $45^{\circ} 03^{\prime} \mathrm{W}$ & $\mathrm{sw}$ & 115 & 115 & 0000.11 & 500010 & $6030-05$ & $1 \mathrm{AE} 05042$ & $60^{\circ} 43^{\prime} \mathrm{N}$ & $45^{\circ} 03^{\prime} \mathrm{W}$ & W & 120 & 120 & 0000.10 & 700010 & $6030-05$ \\
\hline $1 \mathrm{AE} 04013$ & $60^{\circ} 48^{\prime} \mathrm{N}$ & $45^{\circ} 04^{\prime} \mathrm{W}$ & $\mathrm{N}$ & 120 & 110 & 0000.14 & 570010 & 6030 & 1 AE05043 & $60^{\circ} 43^{\prime} \mathrm{N}$ & $45^{\circ} 03^{\prime} \mathrm{W}$ & NW & 120 & 115 & 0000.10 & 700010 & $6030-05$ \\
\hline $1 \mathrm{AE} 04014$ & $60^{\circ} 48^{\prime} \mathrm{N}$ & $45^{\circ} 05^{\prime} \mathrm{W}$ & NW & 120 & 105 & 0000.08 & 550010 & $6030-05$ & 1 AE05044 & $60^{\circ} 43^{\prime} \mathrm{N}$ & $45^{\circ} 04^{\prime} \mathrm{W}$ & NW & 115 & 105 & 0000.10 & 700010 & $6030-05$ \\
\hline $1 \mathrm{AE} 04015$ & $60^{\circ} 48^{\prime} \mathrm{N}$ & $45^{\circ} 05^{\prime} \mathrm{W}$ & NV & 140 & 100 & 0000.65 & 643112 & $6030-05$ & $1 \mathrm{AE} 05045$ & $60^{\circ} 43^{\prime} \mathrm{N}$ & $45^{\circ} 04^{\prime} \mathrm{W}$ & $\mathrm{N}$ & 115 & 100 & & & \\
\hline 1AE04016 & $60^{\circ} 47^{\prime} \mathrm{N}$ & $45^{\circ} 07^{\prime} \mathrm{W}$ & NW & 150 & 85 & 0001.60 & 633110 & $6030-05$ & 1AE05046 & $60^{\circ} 43^{\prime} \mathrm{N}$ & $45^{\circ} 04^{\prime} \mathrm{W}$ & N & 110 & 95 & 0000.10 & 753010 & $6030-05$ \\
\hline $1 \mathrm{AE} 04017$ & $60^{\circ} 47^{\prime} \mathrm{N}$ & $45^{\circ} 07^{\prime} \mathrm{W}$ & NW & 160 & 110 & 0000.30 & 633110 & $6030-05$ & $1 \mathrm{AEO} 05047$ & $60^{\circ} 43^{\prime} \mathrm{N}$ & $45^{\circ} 02^{\prime} \mathrm{W}$ & sw & 125 & 125 & 0000.18 & 700010 & $6030-05$ \\
\hline 1AE04018 & $60^{\circ} 46^{\prime} \mathrm{N}$ & $45^{\circ} 08^{\prime} \mathrm{W}$ & W & 160 & 95 & 0001.80 & 633110 & 6030-05 & 1AE05048 & $60^{\circ} 42^{\prime} \mathrm{N}$ & $45^{\circ} 03^{\prime} \mathrm{W}$ & $\mathrm{N}$ & 110 & 110 & 0000.20 & 750010 & $6030-05$ \\
\hline 1AE04019 & $60^{\circ} 46^{\prime} \mathrm{N}$ & $45^{\circ} 09^{\prime} \mathrm{W}$ & $w$ & 120 & 100 & 0000.34 & 653110 & 6030-05 & $1 \mathrm{AE} 05049$ & $60^{\circ} 42, \mathrm{~N}$ & $45^{\circ} 03^{\prime} \mathrm{W}$ & $\mathrm{N}$ & 120 & 120 & 0000.10 & 700010 & $6030-05$ \\
\hline $1 \mathrm{AE} 04020$ & $60^{\circ} 46^{\prime} \mathrm{N}$ & $45^{\circ} 09^{\prime} \mathrm{W}$ & NW & 80 & 70 & 0000.05 & 700010 & 6030-05 & $1 \mathrm{AE} 05050$ & $60^{\circ} 42^{\prime} \mathrm{N}$ & $45^{\circ} 04^{\prime} \mathrm{W}$ & $\mathrm{N}$ & 130 & 90 & 00 & 653010 & $6030-05$ \\
\hline 1 AE04021 & $60^{\circ} 46^{\prime} \mathrm{N}$ & $45^{\circ} 09^{\prime} \mathrm{W}$ & NW & 750 & 650 & 0000.10 & 700010 & $6030-05$ & $1 \mathrm{AE} 05051$ & $60^{\circ} 41^{\prime} \mathrm{N}$ & $45^{\circ} 05^{\prime} \mathrm{W}$ & NV & 115 & 90 & & 590012 & $6030-05$ \\
\hline 1AE04022 & $60^{\circ} 43^{\prime} \mathrm{N}$ & $45^{\circ} 12^{\prime} \mathrm{W}$ & $\mathbf{N}$ & 120 & 85 & 0000.06 & 590112 & 6030-05 & $1 \mathrm{AE} 05052$ & $60^{\circ} 41, \mathrm{~N}$ & $45^{\circ} 06^{\prime} \mathrm{W}$ & NW & 130 & 120 & 0000.08 & 590010 & $6030-05$ \\
\hline $1 \mathrm{AE} 04023$ & $60^{\circ} 43^{\prime} \mathrm{N}$ & $45^{\circ} 13^{\prime} \mathrm{W}$ & $\mathrm{N}$ & 120 & 90 & 0000.30 & 633110 & & 1 AE05053 & $60^{\circ} 41^{\prime} \mathrm{N}$ & $45^{\circ} 07 \cdot \mathrm{W}$ & NW & 110 & 90 & 11 & 010 & -05 \\
\hline 1AE04024 & $60^{\circ} 43^{\prime} \mathrm{N}$ & $45^{\circ} 13^{\prime} \mathrm{w}$ & $\mathrm{N}$ & 120 & 90 & 0000.23 & 643110 & 603 & 1AE05054 & $60^{\circ} 40^{\prime} \mathrm{N}$ & $45^{\circ} 08^{\prime} \mathrm{W}$ & $\mathrm{W}$ & 115 & 95 & 0000.10 & 753110 & $\begin{array}{l}-05 \\
-05\end{array}$ \\
\hline $1 \mathrm{AE} 04025$ & $60^{\circ} 43^{\prime} \mathrm{N}$ & $45^{\circ} 14^{\prime} \mathrm{W}$ & NW & 130 & 100 & 0001.00 & 623110 & 60 & 1AE05055 & $60^{\circ} 40^{\prime} \mathrm{N}$ & $45^{\circ} 08^{\prime} \mathrm{W}$ & $\mathrm{W}$ & 120 & 90 & 0000.30 & 630110 & $6030-05$ \\
\hline 1AE04026 & $60^{\circ} 42^{\prime} \mathrm{N}$ & $45^{\circ} 14^{\prime} \mathrm{W}$ & NW & 120 & 75 & 0000.30 & 650110 & 60 & $1 \mathrm{AE} 05056$ & $60^{\circ} 43^{\prime} \mathrm{N}$ & $45^{\circ} 11 \cdot \mathrm{W}$ & E & 120 & 100 & 0000.16 & 750012 & $6030-05$ \\
\hline $1 \mathrm{AE} 04027$ & $60^{\circ} 42 \cdot \mathrm{N}$ & $45^{\circ} 15^{\prime} \mathrm{W}$ & NW & 95 & 80 & 0000.07 & 590010 & -05 & 1 AEO5057 & $60^{\circ} 37 / \mathrm{N}$ & $45^{\circ} 16^{\prime} \mathrm{W}$ & 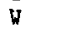 & 95 & 75 & 0000.03 & 790012 & $6030-05$ \\
\hline $1 \mathrm{AE} 04028$ & $60^{\circ} 42^{\prime} \mathrm{N}$ & $45^{\circ} 14^{\prime} \mathrm{W}$ & SW & 130 & 95 & 0000.17 & 623110 & $6030-05$ & $1 \mathrm{AFO} 4001$ & $61^{\circ} 01^{\prime} \mathrm{N}$ & $45^{\circ} 18^{\prime} \mathrm{W}$ & w & 130 & 90 & 0000.40 & 750010 & -06 \\
\hline $1 \mathrm{AE} 04029$ & $60^{\circ} 41^{\prime} \mathrm{N}$ & $45^{\circ} 14^{\prime} \mathrm{W}$ & NW & 120 & 90 & 0000.26 & 633110 & $6030-05$ & 1AF05001 & $61^{\circ} 02^{\prime} \mathrm{N}$ & $45^{\circ} 17^{\prime} \mathrm{W}$ & s & 130 & 115 & 0000.70 & 363110 & $6100-06$ \\
\hline $1 \mathrm{AE} 04030$ & $60^{\circ} 41^{\prime} \mathrm{N}$ & $45^{\circ} 15^{\prime} \mathrm{W}$ & NW & 120 & 95 & 0000.20 & 643110 & 60 & $1 \mathrm{AF} 05002$ & $61^{\circ} 01^{\prime} \mathrm{N}$ & $45^{\circ} 11^{\prime} \mathrm{W}$ & SW & 180 & 105 & 0001.70 & 530110 & $6100-06$ \\
\hline 1 AE 04031 & $60^{\circ} 41^{\prime} \mathrm{N}$ & $45^{\circ} 15^{\prime} \mathrm{W}$ & v & 120 & 105 & 0000.03 & 590010 & 60 & $1 \mathrm{APO} 5003$ & $99^{\prime} \mathrm{N}$ & $45^{\circ} 12^{\prime} \mathrm{W}$ & $\mathrm{N}$ & 14 & 120 & 0000.12 & 770112 & $6030-05$ \\
\hline $1 \mathrm{AE} 05001$ & $60^{\circ} 42^{\prime} \mathrm{N}$ & $45^{\circ} 12^{\prime} \mathrm{W}$ & $\mathrm{SE}$ & 110 & 85 & 0000.25 & 640110 & $6030-05$ & 1 AF0 5004 & $99^{\prime} \mathrm{N}$ & $45^{\circ} 13^{\prime} \mathrm{W}$ & $\mathrm{N}$ & 140 & 120 & 0000.08 & 770112 & $6030-05$ \\
\hline 1AE05002 & $60^{\circ} 42^{\prime} \mathrm{N}$ & $45^{\circ} 12^{\prime} \mathrm{W}$ & $\mathrm{NE}$ & 135 & 115 & 0000.60 & 630110 & $6030-05$ & $1 \mathrm{AF} 07001$ & $60^{\circ} 58^{\prime} \mathrm{N}$ & $45^{\circ} 12^{\prime} \mathrm{W}$ & SW & 120 & 110 & 0000.08 & 790010 & $6030-05$ \\
\hline 1AE05003 & $60^{\circ} 46^{\prime} \mathrm{N}$ & $45^{\circ} 08^{\prime} \mathrm{W}$ & SW & 160 & 100 & 0000.60 & 633110 & 6030 & $1 \mathrm{AF} 08001$ & & $45^{\circ} 01^{\prime} \mathrm{W}$ & $s$ & 210 & 5 & 0098.70 & 363112 & $6030-05$ \\
\hline 1AE05004 & $60^{\circ} 46^{\prime} \mathrm{N}$ & $45^{\circ} 06^{\prime} \mathrm{W}$ & $\mathrm{SE}$ & 150 & 150 & & 750010 & & 1AF08002 & $60^{\circ} 57^{\prime} \mathrm{N}$ & $44^{\circ} 57^{\prime} \mathrm{W}$ & $\mathrm{SW}$ & 210 & 20 & 0320.60 & 363112 & $6030-06$ \\
\hline 1 AE05005 & $60^{\circ} 46^{\prime} \mathrm{N}$ & $45^{\circ} 05^{\prime} \mathrm{W}$ & $\mathrm{E}$ & 130 & 120 & 0000.10 & 750110 & $6030-05$ & $1 \mathrm{AFO} 8003$ & $60^{\circ} 56^{\prime} \mathrm{N}$ & $44^{\circ} 48^{\prime} \mathrm{W}$ & $\mathrm{v}$ & 160 & 102 & 0062.90 & 363012 & $6030-06$ \\
\hline 1AE05006 & $60^{\circ} 46^{\prime} \mathrm{N}$ & $45^{\circ} 05^{\prime} \mathrm{W}$ & E & 120 & 100 & 0000.10 & 753110 & 6030 & 1AF08004 & $60^{\circ} 56^{\prime} \mathrm{N}$ & $44^{\circ} 51^{\prime} \mathrm{W}$ & $\mathrm{NE}$ & 130 & 110 & 0000.40 & 750010 & $6030-06$ \\
\hline $1 \mathrm{AE} 05007$ & $60^{\circ} 47^{\prime} \mathrm{N}$ & $45^{\circ} 05^{\prime} \mathrm{W}$ & E & 120 & 110 & 0000.10 & 750110 & 6030 & $1 \mathrm{AFOB}$ & $5 / N$ & $44^{\circ} 52^{\prime} \mathrm{W}$ & $\mathrm{E}$ & 150 & 140 & 0000.20 & 750010 & $6030-06$ \\
\hline $1 \mathrm{AE} 05008$ & $60^{\circ} 47^{\prime} \mathrm{N}$ & $45^{\circ} 06^{\prime} \mathrm{W}$ & $\mathbf{s}$ & 140 & 140 & 0000.10 & 770010 & 6030 & $1 \mathrm{AF} 08006$ & $60^{\circ} 56^{\prime} \mathrm{N}$ & $44^{\circ} 50^{\prime} \mathrm{W}$ & $\mathbf{N}$ & 110 & 102 & 0002.00 & 536110 & $6030-06$ \\
\hline 1AE05009 & $60^{\circ} 51^{\prime} \mathrm{N}$ & $44^{\circ} 53^{\prime} \mathrm{W}$ & SW & 210 & 45 & 0124.30 & 363110 & $6030-06$ & 1AG01001 & $60^{\circ} 15^{\prime} \mathrm{N}$ & $45^{\circ} 55^{\prime} \mathrm{W}$ & sw & 12 & 90 & 0001.30 & 620112 & $6030-04$ \\
\hline $1 \mathrm{AE} 05010$ & $60^{\circ} 53^{\prime} \mathrm{N}$ & $44^{\circ} 46^{\prime} \mathrm{W}$ & $\mathrm{SW}$ & 118 & 110 & 0000.20 & 736110 & & $1 \mathrm{AGO}$ & $61^{\circ} 25^{\prime} \mathrm{N}$ & $45^{\circ} 23^{\prime} \mathrm{W}$ & $\mathrm{s}$ & 180 & 66 & 0166.08 & 166114 & $6100-06$ \\
\hline 1AE05011 & $60^{\circ} 50^{\prime} \mathrm{N}$ & $44^{\circ} 45^{\prime} \mathrm{W}$ & SW & 135 & 110 & 0004.20 & 363110 & 6030 & $1 \mathrm{AG} 05002$ & $61^{\circ} 28^{\prime} \mathrm{N}$ & $45^{\circ} 17^{\prime} \mathrm{W}$ & W & 140 & 115 & 0000.48 & 600112 & $6100-06$ \\
\hline 1AE05012 & $60^{\circ} 52^{\prime} \mathrm{N}$ & $44^{\circ} 45^{\prime} \mathrm{W}$ & 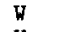 & 115 & 115 & 0000.20 & 756110 & $6030-$ & 1 AG05003 & $61^{\circ} 28^{\prime} \mathrm{N}$ & $45^{\circ} 17^{\prime} \mathrm{W}$ & SW & 150 & 150 & 0000.14 & 640111 & $6100-06$ \\
\hline $1 \mathrm{AE} 05013$ & $60^{\circ} 52^{\prime} \mathrm{N}$ & $44^{\circ} 45^{\prime} \mathrm{W}$ & $w$ & 120 & 120 & 0000.10 & 750110 & 6030 & $1 \mathrm{AG} 05004$ & $61^{\circ} 28^{\prime} \mathrm{N}$ & $45^{\circ} 17^{\prime} \mathrm{W}$ & $\mathrm{v}$ & 140 & 115 & 0000.10 & 640111 & $6100-06$ \\
\hline 1AE05 & $60^{\circ} 51 \cdot \mathrm{N}$ & $44^{\circ} 45^{\prime} \mathrm{W}$ & $v$ & 120 & 120 & 0000.10 & 636110 & $6030-06$ & 1 AG05005 & $61^{\circ} 27^{\prime} \mathrm{N}$ & $45^{\circ} 16^{\prime} \mathrm{W}$ & s & 150 & 135 & 0000.19 & 640112 & $6100-06$ \\
\hline 1AE05015 & $60^{\circ} 52^{\prime} \mathrm{N}$ & $44^{\circ} 41^{\prime} \mathrm{W}$ & SW & 210 & 120 & 0052.30 & 366110 & $6030-06$ & $1 \mathrm{AG} 05006$ & $61^{\circ} 26^{\prime} \mathrm{N}$ & $45^{\circ} 17^{\prime} \mathrm{W}$ & $\mathrm{V}$ & 120 & 114 & 0000.15 & 770012 & $6100-06$ \\
\hline 1AE05016 & $60^{\circ} 51^{\prime} \mathrm{N}$ & $44^{\circ} 38^{\prime} \mathrm{W}$ & SW & 210 & 120 & 0042.90 & 360110 & $6030-06$ & 1AG05007 & $61^{\circ} 26^{\prime} \mathrm{N}$ & $45^{\circ} 18^{\prime} \mathrm{W}$ & NE & 144 & 112 & 0000.66 & 640112 & $6100-06$ \\
\hline
\end{tabular}




\begin{tabular}{|c|c|c|c|c|c|c|c|c|c|c|c|c|c|c|c|c|c|}
\hline CODE & LAT & LONG & ORI & HIGH & LOW & AREA & $\begin{array}{c}\text { MO } \\
\text { CODE }\end{array}$ & MAP & CODE & LAT & LONG & ORI & HIGH & LOW & AREA & $\begin{array}{c}\text { MO } \\
\text { CODE }\end{array}$ & MAP \\
\hline 05008 & $61{ }^{\circ} 2$ & 45 & $\mathbf{N}$ & 164 & 124 & 00 & 6 & 61 & 1 & N & 4 & NE & 150 & 85 & 0 & 533110 & 610 \\
\hline 1AG05009 & $61^{\circ} 26^{\prime} \mathrm{N}$ & $45^{\circ} 20^{\prime} \mathrm{w}$ & $\mathbf{N}$ & 160 & 108 & 0000.93 & 640112 & $6100-06$ & 1 AG10006 & $61^{\circ} 09^{\prime} \mathrm{N}$ & $45^{\circ} 02^{\prime}$ W & $\mathrm{N}$ & 150 & 90 & 0001.60 & 533110 & $6100-06$ \\
\hline 1AG05010 & $61^{\circ} 25^{\prime} \mathrm{N}$ & $45^{\circ} 20^{\prime} \mathrm{W}$ & $\mathbf{s}$ & 130 & 111 & 0000.14 & 650112 & $6100-06$ & 1 AG10007 & $61^{\circ} 14^{\prime} \mathrm{N}$ & $45^{\circ} 06^{\prime} \mathrm{W}$ & SW & & 13 & 0182.30 & 363012 & $6100-06$ \\
\hline $1 \mathrm{AG} 05011$ & $61^{\circ} 19^{\prime} \mathrm{N}$ & $45^{\circ} 19^{\prime} \mathrm{W}$ & $\mathrm{w}$ & 110 & 93 & 0000.31 & 770012 & $6100-06$ & $1 \mathrm{AG} 10008$ & $61^{\circ} 20^{\prime} \mathrm{N}$ & $44^{\circ} 49^{\prime} \mathrm{V}$ & W & 170 & 100 & 0018.70 & 300010 & 6100-07 \\
\hline $1 \mathrm{AG} 05012$ & $61^{\circ} 18^{\prime} \mathrm{N}$ & $45^{\circ} 21^{\prime} \mathrm{w}$ & NE & 130 & 110 & 0000.18 & 780012 & $6100-06$ & 1AG10009 & $61^{\circ} 22 \cdot \mathrm{N}$ & $44^{\circ} 46^{\prime} \mathrm{W}$ & N & 135 & 125 & 0000.30 & 756110 & 6100-07 \\
\hline $1 \mathrm{AG} 05013$ & $61^{\circ} 16^{\prime} \mathrm{N}$ & $45^{\circ} 25^{\prime} \mathrm{W}$ & NW & 120 & 110 & 0000.20 & 770010 & $6100-06$ & 1 AG10010 & $61^{\circ} 20^{\prime} \mathrm{N}$ & $44043 \cdot 6$ & SW & 150 & 140 & 0000.70 & 630110 & 6100-07 \\
\hline 1AG05014 & $61^{\circ} 26^{\prime} \mathrm{N}$ & $45^{\circ} 19^{\prime} \mathrm{W}$ & E & 110 & 100 & 0000.07 & 770012 & $6100-06$ & IAG10011 & $61^{\circ} 18^{\prime} \mathrm{N}$ & $44^{\circ} 54^{\prime} \mathrm{V}$ & $W$ & & 70 & 0200.40 & 367112 & $6100-07$ \\
\hline 1AG06001 & $61^{\circ} 17^{\prime} \mathrm{N}$ & $45^{\circ} 23^{\prime} \mathrm{W}$ & W & 135 & 115 & 0000.37 & 670012 & $6100-06$ & 1AG10012 & $61^{\circ} 17^{\prime} \mathrm{N}$ & $44^{\circ} 52^{\prime} \mathrm{W}$ & SW & 120 & 120 & 0000.80 & 636110 & $6100-07$ \\
\hline $1 \mathrm{AG} 06002$ & $61^{\circ} 16^{\prime} \mathrm{N}$ & $45^{\circ} 22^{\prime} \mathrm{W}$ & NW & 120 & 105 & 0000.09 & 770012 & $6100-06$ & $1 \mathrm{AG} 10013$ & $61^{\circ} 16^{\prime} \mathrm{N}$ & $44^{\circ} 52^{\prime} \mathrm{V}$ & NW & 150 & 120 & 0001.40 & 750010 & 6100-07 \\
\hline $1 \mathrm{AG} 07001$ & $61^{\circ} 17^{\prime} \mathrm{N}$ & $45^{\circ} 20^{\circ} \mathrm{w}$ & $\mathrm{s}$ & 120 & 115 & 0000.08 & 780010 & $6100-06$ & 1 AG10014 & $61^{\circ} 15^{\prime} \mathrm{N}$ & $44^{\circ} 52, \mathrm{~W}$ & W & 150 & 140 & 0000.80 & 653110 & $6100-07$ \\
\hline $1 \mathrm{AG} 07002$ & $61^{\circ} 18^{\prime} \mathrm{N}$ & $45^{\circ} 18^{\prime} \mathrm{W}$ & SE & 135 & 130 & 0000.18 & 780010 & $6100-06$ & 1AG10015 & $61^{\circ} 15^{\prime} \mathrm{N}$ & $44^{\circ} 53^{\prime} \mathrm{W}$ & $\mathrm{NW}$ & 155 & 120 & 0002.70 & 630110 & 6100-07 \\
\hline 1AG07003 & $61^{\circ} 26^{\prime} \mathrm{N}$ & $45^{\circ} 16^{\prime} \mathrm{W}$ & $\mathrm{NE}$ & 130 & 130 & 0000.07 & 780010 & $6100-06$ & $1 \mathrm{AG} 10016$ & $61^{\circ} 13^{\prime} \mathrm{N}$ & $44^{\circ} 57 \cdot \mathrm{W}$ & $\mathbf{s}$ & 130 & 120 & 0001.30 & 600112 & 6100-07 \\
\hline 1 AG07004 & $61^{\circ} 28^{\prime} \mathrm{N}$ & $45^{\circ} 14^{\prime} \mathrm{V}$ & SE & 125 & 120 & 0000.12 & 770012 & $6100-06$ & 1 AG10017 & $61^{\circ} 13^{\prime} \mathrm{N}$ & $44^{\circ} 59, \mathrm{v}$ & SW & 140 & 120 & 0000.50 & 653110 & $6100-07$ \\
\hline $1 \mathrm{AG07005}$ & $61^{\circ} 28^{\prime} \mathrm{N}$ & $45^{\circ} 15^{\prime} \mathrm{w}$ & $\mathrm{s}$ & 140 & 135 & 0000.24 & 670012 & $6100-06$ & 1AG10018 & $61^{\circ} 12^{\prime} \mathrm{N}$ & $44^{\circ} 58, \mathrm{~V}$ & $N W$ & 130 & 100 & 0000.10 & 750110 & $6100-07$ \\
\hline 1 AG07006 & $61^{\circ} 28^{\prime} \mathrm{N}$ & $45^{\circ} 16^{\prime} \mathrm{W}$ & $\mathrm{NE}$ & 150 & 120 & 0000.27 & 670012 & $6100-06$ & 1AG10019 & $61^{\circ} 12^{\prime} \mathrm{N}$ & $44^{\circ} 57^{\prime \prime}$ & $\mathrm{N}$ & 140 & 110 & 0000.40 & 650110 & 6100-07 \\
\hline 1AG07007 & $61^{\circ} 28^{\prime} \mathrm{N}$ & $45^{\circ} 17^{\prime} \mathrm{W}$ & N & 140 & 115 & 0000.56 & 780010 & $6100-06$ & 1 AG10020 & $61^{\circ} 13^{\prime} \mathrm{N}$ & $44 \circ 55,6$ & SW & 135 & 100 & 0000.40 & 750110 & $6100-07$ \\
\hline $1 \mathrm{AG} 07008$ & $61^{\circ} 27^{\prime} \mathrm{N}$ & $45^{\circ} 08^{\prime} \mathrm{W}$ & $S E$ & 180 & 65 & 0100.49 & 163114 & $6100-06$ & 1AG10021 & $61^{\circ} 14^{\prime} \mathrm{N}$ & $44^{\circ} 52, v$ & $\mathrm{~s}$ & 156 & 110 & 0003.80 & 633110 & $6100-07$ \\
\hline 1AG07009 & $61^{\circ} 31^{\prime} \mathrm{N}$ & $45^{\circ} 05^{\prime} \mathrm{W}$ & W & 175 & 135 & 0003.11 & 623112 & $6130-06$ & 1 AG10022 & $61^{\circ} 13^{\prime} \mathrm{N}$ & $44^{\circ} 48^{\prime}$ & SW & 170 & 110 & 0013.10 & 363112 & $6100-07$ \\
\hline $1 \mathrm{AG} 07010$ & $61^{\circ} 30^{\prime} \mathrm{N}$ & $45^{\circ} 05^{\prime} \mathrm{W}$ & $\mathrm{NW}$ & 180 & 150 & 0000.48 & 670112 & $6130-06$ & 1AG10023 & $61^{\circ} 10^{\prime} \mathrm{N}$ & $44^{\circ} 51, \mathrm{v}$ & w & 210 & 13 & 0179.80 & 366112 & $6100-07$ \\
\hline $1 \mathrm{AG07011}$ & $61^{\circ} 30^{\prime} \mathrm{N}$ & $45^{\circ} 06^{\prime} \mathrm{W}$ & $\mathrm{NW}$ & 155 & 130 & 0000.37 & 770112 & $6130-06$ & 1AG10024 & $61^{\circ} 12^{\prime} \mathrm{N}$ & $44^{\circ} 42,1$ & s & 130 & 120 & 0000.80 & 636110 & $6100-07$ \\
\hline $1 \mathrm{AG} 07012$ & $61^{\circ} 30^{\prime} \mathrm{N}$ & $45^{\circ} 07^{\prime} \mathrm{w}$ & $\mathrm{W}$ & 145 & 120 & 0000.13 & 780112 & $6130-06$ & 1 AG10025 & $61^{\circ} 11^{\prime} \mathrm{N}$ & $44^{\circ} 42^{\prime}$ W & $\mathrm{w}$ & 110 & 110 & 0000.20 & 633110 & $6100-07$ \\
\hline $1 \mathrm{AG} 07013$ & $61^{\circ} 29^{\prime} \mathrm{N}$ & $45^{\circ} 07, \mathrm{~W}$ & SW & 120 & 115 & 0000.31 & 770112 & $6100-06$ & 1 AG10026 & $61^{\circ} 11^{\prime} \mathrm{N}$ & $44^{\circ} 41$, w & $\mathrm{W}$ & 135 & 115 & 0000.50 & 633110 & $6100-07$ \\
\hline 1AG07014 & $61^{\circ} 30^{\prime} \mathrm{N}$ & $45^{\circ} 03^{\prime} \mathrm{W}$ & $\mathrm{v}$ & 160 & 155 & 0000.15 & 670112 & $6130-06$ & 1AG10027 & $61^{\circ} 10^{\prime} \mathrm{N}$ & $44^{\circ} 40^{\prime} \mathrm{W}$ & SW & 130 & 110 & 0003.30 & 531110 & $6100-07$ \\
\hline 1AG07015 & $61^{\circ} 29^{\prime} \mathrm{N}$ & $45^{\circ} 04^{\prime} \mathrm{W}$ & SW & 135 & 120 & 0000.12 & 770112 & $6100-06$ & $1 \mathrm{AG} 10028$ & $61^{\circ} 10^{\prime} \mathrm{N}$ & $44^{\circ} 49^{\prime \prime}$ & W & 170 & 80 & 0001.50 & 523410 & 6100-07 \\
\hline $1 \mathrm{AG} 07016$ & $61^{\circ} 30^{\prime} \mathrm{N}$ & $45^{\circ} 02 \cdot \mathrm{W}$ & SW & 170 & 120 & 0002.94 & 220110 & $6130-06$ & 1AG10029 & $61^{\circ} 09 \cdot \mathrm{N}$ & $44^{\circ} 50^{\prime}$, & NW & 180 & 60 & 0001.10 & 533410 & $6100-07$ \\
\hline 1 AG07017 & $61^{\circ} 29^{\prime} \mathrm{N}$ & $45^{\circ} 01 \cdot \mathrm{W}$ & $\mathrm{NW}$ & & 135 & 0001.06 & 670112 & $6100-06$ & 1 AG10030 & $61^{\circ} 09 \cdot \mathrm{N}$ & $44^{\circ} 52,6$ & SW & 150 & 150 & 0001.30 & 230110 & 6100-07 \\
\hline $1 \mathrm{AG} 07018$ & $61^{\circ} 28^{\prime} \mathrm{N}$ & $45^{\circ} 03^{\prime} \mathrm{w}$ & W & 125 & 110 & 0000.16 & 780010 & $6100-06$ & 1AG10031 & $61^{\circ} 09^{\prime} \mathrm{N}$ & $44^{\circ} 54^{\prime}$, & 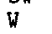 & 150 & 140 & 0000.50 & 633110 & $6100-07$ \\
\hline $1 \mathrm{AG} 07019$ & $61^{\circ} 28^{\prime} \mathrm{N}$ & $45^{\circ} 02^{\prime} \mathrm{W}$ & $\mathrm{W}$ & 120 & 110 & 0000.14 & 780010 & $6100-06$ & $1 \mathrm{AG} 10032$ & $61^{\circ} 08^{\prime} \mathrm{N}$ & $44^{\circ} 54^{\prime}$ & W & 125 & 125 & 0000.10 & 700010 & 6100-07 \\
\hline $1 \mathrm{AGO} 07020$ & $61^{\circ} 28^{\prime} \mathrm{N}$ & $45^{\circ} 01 \cdot \mathrm{V}$ & SW & 150 & 140 & 0000.52 & 780010 & $6100-06$ & 1AG10033 & $61^{\circ} 09 \cdot \mathrm{N}$ & $44^{\circ} 50^{\prime}$ W & SW & 160 & 125 & 0002.10 & 533110 & 6100-07 \\
\hline $1 \mathrm{AG} 07021$ & $61^{\circ} 28^{\prime} \mathrm{N}$ & $45^{\circ} 00^{\prime} \mathrm{w}$ & SW & 175 & 160 & 0000.50 & 670110 & $6100-07$ & 1 AG10034 & $61^{\circ} 09, \mathrm{~N}$ & $44^{\circ} 47^{\prime}$ ' & SW & 170 & 90 & 0002.80 & 533110 & $6100-07$ \\
\hline $1 \mathrm{AG} 07022$ & $61^{\circ} 29^{\prime} \mathrm{N}$ & $44^{\circ} 59^{\prime} \mathrm{W}$ & $\mathrm{SE}$ & 175 & 160 & 0001.25 & 640010 & 6100 & IAG10035 & $61^{\circ} 05, \mathrm{~N}$ & $44^{\circ} 59 \mathrm{~V}$ & $\mathrm{~W}$ & 205 & 20 & 0299.30 & 367112 & $6100-07$ \\
\hline $1 \mathrm{AG} 07023$ & $61^{\circ} 28^{\prime} \mathrm{N}$ & $44^{\circ} 58^{\prime} \mathrm{V}$ & $\mathrm{SW}$ & 175 & 155 & 0000.22 & 780012 & $6100-07$ & 1AG10036 & $61^{\circ} 04^{\prime} \mathrm{N}$ & $44^{\circ} 41^{\prime \prime}$ & SW & 130 & 110 & 0001.00 & 623110 & $6100-07$ \\
\hline $1 \mathrm{AG} 07024$ & $61^{\circ} 27^{\prime} \mathrm{N}$ & $44^{\circ} 59^{\prime} \mathrm{W}$ & SW & 180 & 70 & 0063.58 & 163112 & $6100-07$ & 1AG10037 & $61^{\circ} 03^{\prime} \mathrm{N}$ & $44^{\circ} 59^{\prime} \mathrm{V}$ & W & 195 & 200 & 0280.70 & 367112 & $6100-07$ \\
\hline $1 \mathrm{AG} 07025$ & $61^{\circ} 31^{\prime} \mathrm{N}$ & $44^{\circ} 55^{\prime} \mathrm{w}$ & $\mathrm{s}$ & 205 & 195 & 0000.34 & 770110 & 6130 & $1 \mathrm{AG} 10038$ & $61^{\circ} 03^{\prime} \mathrm{N}$ & $45^{\circ} 08^{\prime}$ & $\hat{W}$ & 135 & 100 & 0001.01 & 643112 & $6100-06$ \\
\hline $1 \mathrm{AG} 07026$ & $61^{\circ} 31^{\prime N} \mathrm{~N}$ & $44^{\circ} 54^{\prime} \mathrm{w}$ & s & 210 & 185 & 0000.62 & 670112 & 6130 & 1AG10039 & $61^{\circ} 03^{\prime} \mathrm{N}$ & $45^{\circ} 09^{\prime \prime}$ & $\mathrm{N}$ & 145 & 95 & 0001.40 & 523110 & $6100-06$ \\
\hline $1 \mathrm{AG} 07027$ & $61^{\circ} 28^{\prime} \mathrm{N}$ & $44^{\circ} 54^{\prime} \mathrm{W}$ & s & 180 & 115 & 0059.81 & 167112 & 6100 & $1 \mathrm{AG} 10040$ & $61^{\circ} 02 \cdot \mathrm{N}$ & $45^{\circ} 10^{\prime \prime}$ & NW & 160 & 125 & 0000.13 & 753310 & $6100-06$ \\
\hline 1 AG07028 & $61^{\circ} 30^{\prime} \mathrm{N}$ & $44^{\circ} 50^{\prime} \mathrm{W}$ & $\mathrm{SE}$ & 170 & 170 & 0000.13 & 770112 & $6130-$ & $1 \mathrm{AG} 10041$ & $61^{\circ} 02 \cdot \mathrm{N}$ & $45^{\circ} 12, \mathrm{v}$ & NW & 160 & 150 & 0000.31 & 633110 & $6100-06$ \\
\hline $1 \mathrm{AG} 07029$ & $61^{\circ} 27^{\prime} \mathrm{N}$ & $44^{\circ} 51^{\prime} \mathrm{W}$ & s & 140 & 135 & 0000.32 & 670112 & 6100 & 1 AG10042 & $61^{\circ} 03^{\prime} \mathrm{N}$ & $45^{\circ} 15^{\prime}$, & $\mathbf{N}$ & 150 & 90 & 0000.10 & 753310 & $6100-06$ \\
\hline $1 \mathrm{AG} 07030$ & $61^{\circ} 31^{\prime} \mathrm{N}$ & $44^{\circ} 45^{\prime} \mathrm{w}$ & $\mathrm{W}$ & 200 & 190 & 0000.19 & 700110 & 6130 & $1 \mathrm{AG} 10043$ & $61^{\circ} 03^{\prime} \mathrm{N}$ & $45^{\circ} 15^{\prime}$ & $\mathrm{N}$ & 150 & 110 & 0000.30 & 643110 & 6100-06 \\
\hline $1 \mathrm{AG} 07031$ & $61^{\circ} 26^{\prime} \mathrm{N}$ & $44^{\circ} 52, \mathrm{w}$ & $\mathrm{W}$ & 175 & 170 & 0000.14 & 780010 & 6100 & 1AG10044 & $61^{\circ} 03, \mathrm{~N}$ & $45^{\circ} 16^{\prime}$, & NE & 140 & 120 & 0000.20 & 633110 & $6100-06$ \\
\hline $1 \mathrm{AG} 07032$ & $61^{\circ} 26^{\prime} \mathrm{N}$ & $44^{\circ} 53^{\prime} \mathrm{W}$ & $\mathrm{N}$ & 165 & 165 & 0000.08 & 780010 & 6100 & 1AG10045 & $61^{\circ} 03^{\prime} \mathrm{N}$ & $45^{\circ} 18^{\prime}$, & $\mathrm{W}$ & 150 & 115 & 00 & 533110 & $0-06$ \\
\hline $1 \mathrm{AG} 07033$ & $61^{\circ} 26^{\prime} \mathrm{N}$ & $44^{\circ} 55^{\prime} \mathrm{W}$ & $\mathrm{NW}$ & 155 & 135 & 0000.71 & 670110 & $6100-07$ & 1AG10046 & $61^{\circ} 02^{\prime} \mathrm{N}$ & $45^{\circ} 17^{\prime \prime}$ & E & 135 & 125 & 0000.35 & 360110 & $6100-06$ \\
\hline $1 \mathrm{AG} 07034$ & $61^{\circ} 26^{\prime} \mathrm{N}$ & $44^{\circ} 56^{\prime} \mathrm{W}$ & NW & 150 & 145 & 0000.15 & 780112 & 6100 & 1AG10047 & $61^{\circ} 02^{\prime} \mathrm{N}$ & $45^{\circ} 18^{\prime} \mathrm{V}$ & W & 135 & 115 & 0000.10 & 360110 & $6100-06$ \\
\hline $1 \mathrm{AG} 07035$ & $61^{\circ} 26^{\prime} \mathrm{N}$ & $44^{\circ} 57 \cdot \mathrm{W}$ & w & 145 & 140 & 0000.48 & 770112 & 6100 & $1 \mathrm{AG} 10048$ & $61^{\circ} 04^{\prime} \mathrm{N}$ & $45^{\circ} 17^{\prime \prime V}$ & $\mathrm{NE}$ & 150 & 120 & 0000.18 & 633110 & $6100-06$ \\
\hline $1 \mathrm{AG} 07036$ & $61^{\circ} 25^{\prime} \mathrm{N}$ & $44^{\circ} 59^{\prime} \mathrm{W}$ & - & 130 & 125 & 0000.33 & 780010 & 6100 & IAG10049 & $61^{\circ} 04^{\prime} \mathrm{N}$ & $45^{\circ} 18^{\prime} \mathrm{V}$ & N & 150 & 120 & 0000.10 & 633110 & $6100-06$ \\
\hline $1 \mathrm{AG} 07037$ & $61^{\circ} 25^{\prime} \mathrm{N}$ & $44^{\circ} 55^{\prime} \mathrm{W}$ & $\mathrm{s}$ & 150 & 140 & 0000.24 & 780012 & 6100 & 1AHO2OO1 & $61^{\circ} 01^{\prime} \mathrm{N}$ & $46^{\circ} 40^{\prime \prime}$ W & S & 180 & & 04 & 164111 & $6100-04$ \\
\hline $1 \mathrm{AG} 07038$ & $61^{\circ} 26^{\prime} \mathrm{N}$ & $44^{\circ} 54^{\prime} \mathrm{W}$ & $\mathrm{s}$ & 155 & 150 & 0000.14 & 770012 & $6100-07$ & $1 \mathrm{AHO} 2002$ & $61^{\circ} 02, \mathrm{~N}$ & $46^{\circ} 34^{\prime \prime}$ W & $\mathrm{s}$ & 180 & & 0211.81 & 165211 & $6100-04$ \\
\hline 1 AG07039 & $61^{\circ} 26^{\prime} \mathrm{N}$ & $44^{\circ} 53^{\prime} \mathrm{W}$ & $\mathrm{SE}$ & 140 & 140 & & 780010 & 610 & $1 \mathrm{AHO} 2$ & $61^{\circ} 04^{\prime} \mathrm{N}$ & $46^{\circ}$ & $\mathrm{s}$ & 180 & & 0155.39 & 165211 & 6100-05 \\
\hline $1 \mathrm{AG} 07040$ & $61^{\circ} 26^{\prime} \mathrm{N}$ & $44^{\circ} 49^{\prime} \mathrm{W}$ & $\mathrm{s}$ & 180 & 55 & 0061.47 & 167112 & $6100-07$ & $1 \mathrm{AHO} 2004$ & $61^{\circ} 05^{\prime N}$ & $46^{\circ} 26^{\prime} \mathrm{V}$ & $\mathrm{s}$ & 180 & & 0051.62 & 163211 & $6100-05$ \\
\hline 1 AG07041 & $61^{\circ} 28^{\prime} \mathrm{N}$ & $44^{\circ} 44^{\prime} \mathrm{W}$ & sW & 157 & 130 & 0000.62 & 670110 & $6100-07$ & $1 \mathrm{AHO} 2005$ & $61^{\circ} 06^{\prime} \dot{\mathrm{N}}$ & $46^{\circ} 23^{\prime}$ & $\mathrm{s}$ & 180 & & 0108.37 & 162112 & 6100-05 \\
\hline $1 \mathrm{Ag} 07042$ & $61^{\circ} 28^{\prime} \mathrm{N}$ & $44^{\circ} 42^{\prime} \mathrm{W}$ & SW & 176 & 120 & 0004.71 & 263112 & 6100 & $1 \mathrm{AHO} 03001$ & $61 \cdot 06 \cdot \mathrm{N}$ & $46^{\circ} 20^{\prime \prime}$ & $s$ & 180 & 10 & .61 & 163111 & 6100-05 \\
\hline 1 AG07043 & $61^{\circ} 27^{\prime} \mathrm{N}$ & $44^{\circ} 41, \mathrm{~W}$ & s & 180 & 140 & 0 & 77 & & $1 \mathrm{AHO}$ & $61^{\circ} 06^{\prime} \mathrm{N}$ & $8^{\prime \prime} \mathrm{W}$ & s & 180 & 15 & 0065.55 & 163112 & $6100-05$ \\
\hline $1 \mathrm{AG07044}$ & $61^{\circ} 14^{\prime} \mathrm{N}$ & $45^{\circ} 18^{\prime} \mathrm{W}$ & SW & 180 & 10 & 0150.25 & 163112 & 6100 & $1 \mathrm{AH} 04001$ & $61^{\circ} 09^{\prime} \mathrm{N}$ & $46^{\circ} 15^{\prime} \mathrm{W}$ & SE & 180 & 45 & 0096.70 & 166111 & 6100-05 \\
\hline $1 \mathrm{AG} 08001$ & $61^{\circ} 12^{\prime} \mathrm{N}$ & $45^{\circ} 12^{\prime} \mathrm{W}$ & SW & 180 & & 0157.75 & 164111 & $6100-06$ & $1 \mathrm{AH} 04002$ & $61^{\circ} 08^{\prime} \mathrm{N}$ & $46^{\circ} 08^{\prime} \mathrm{V}$ & SE & 180 & 10 & 0086.22 & 166111 & $6100-05$ \\
\hline 1 AG08002 & $61^{\circ} 12^{\prime} \mathrm{N}$ & $45^{\circ} 11^{\prime} \mathrm{W}$ & SW & 240 & & 0759.75 & 364111 & $6100-06$ & $1 \mathrm{AHO} 0003$ & $61^{\circ} 11^{\prime} \mathrm{N}$ & $46^{\circ} 09^{\prime \prime}$ & $\mathrm{SE}$ & 180 & 15 & 010 & 163111 & $6100-05$ \\
\hline $1 \mathrm{AG} 09001$ & $61^{\circ} 09^{\prime} \mathrm{N}$ & $45^{\circ} 06^{\prime} \mathrm{W}$ & $\mathrm{N}$ & 165 & 85 & 0004.50 & 533010 & 6100 & $1 \mathrm{AH} 06001$ & $61^{\circ} 14^{\prime} \mathrm{N}$ & $46^{\circ} 11^{\prime n}$ & $\mathrm{SE}$ & 180 & 20 & 0116.37 & 164111 & $6100-05$ \\
\hline $1 \mathrm{AG}$ & $61^{\circ} 09^{\prime} \mathrm{N}$ & $17 \% \mathrm{~W}$ & $\mathrm{~N}$ & 145 & 100 & 000 & 650000 & & $1 \mathrm{AH} 06002$ & $61^{\circ} 15^{\prime N}$ & $46^{\circ} 08^{\prime} \mathrm{K}$ & $\mathrm{SE}$ & 180 & & 0641.20 & 164111 & $6100-05$ \\
\hline $1 \mathrm{AG} 10001$ & $61^{\circ} 08^{\prime} \mathrm{N}$ & $45^{\circ} 08^{\prime}$ W & $\mathrm{W}$ & 160 & 130 & 0000.43 & 633110 & $6100-06$ & $1 \mathrm{AH} 06003$ & $61^{\circ} 16^{\prime} \mathrm{N}$ & $46^{\circ} 04^{\prime \prime}$ & $\mathrm{S}$ & 180 & & 0383.77 & 164111 & $6100-05$ \\
\hline $1 \mathrm{AG} 10002$ & $61^{\circ} 08^{\prime} \mathrm{N}$ & $45^{\circ} 09^{\prime} \mathrm{W}$ & SW & 140 & 115 & 0000.84 & 523110 & $6100-06$ & $1 \mathrm{AHO} 0001$ & $61^{\circ} 23^{\prime} \mathrm{N}$ & $46^{\circ} 00^{\prime}$ & $\mathrm{s}$ & 180 & 80 & 0066.27 & 163112 & 6100-05 \\
\hline $1 \mathrm{AG} 10003$ & $61^{\circ} 07^{\prime} \mathrm{N}$ & $45^{\circ} 07^{\prime} \mathrm{W}$ & $\mathrm{v}$ & 125 & 100 & 0000.20 & 670110 & $6100-06$ & $1 \mathrm{AH} 08002$ & $61^{\circ} 24^{\prime} \mathrm{N}$ & $45^{\circ} 54^{\prime}$ W & 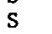 & 180 & 10 & 0219.27 & 167112 & 6100-05 \\
\hline $1 \mathrm{AG} 10004$ & $61^{\circ} 07^{\prime} \mathrm{N}$ & $45^{\circ} 05^{\prime} \mathrm{W}$ & SW & 165 & 125 & 0002.20 & 533110 & $6100-06$ & $1 \mathrm{AH} 08003$ & $61^{\circ} 27, \mathrm{~N}$ & $45051^{\prime \prime V}$ & SW & 145 & 10 & 0001.72 & 533112 & $6100-05$ \\
\hline
\end{tabular}




\begin{tabular}{|c|c|c|c|}
\hline CODE & LAT & LONG & ORI \\
\hline 14 & 61 & $45^{\circ} 50^{\circ} \mathrm{V}$ & $\mathrm{N}$ \\
\hline 1AH08005 & $61^{\circ} 27^{\prime} \mathrm{N}$ & $45^{\circ} 49^{\prime} \mathrm{W}$ & E \\
\hline 1 AH08006 & $61^{\circ} 28^{\prime} \mathrm{N}$ & $45^{\circ} 50^{\prime} \mathrm{W}$ & $E$ \\
\hline 1AH08007 & $61^{\circ} 27^{\prime} \mathrm{N}$ & $45^{\circ} 48^{\prime} \mathrm{W}$ & SE \\
\hline 1AH08008 & $61^{\circ} 28^{\prime} \mathrm{N}$ & $45^{\circ} 46^{\prime} \mathrm{W}$ & $\mathrm{SW}$ \\
\hline $1 \mathrm{AH} 08009$ & $61^{\circ} 28^{\prime} \mathrm{N}$ & $45^{\circ} 45^{\prime} \mathrm{W}$ & SW \\
\hline $1 \mathrm{AHO} 08010$ & $61^{\circ} 28^{\prime} \mathrm{N}$ & $45^{\circ} 45^{\prime} \mathrm{W}$ & \\
\hline 1АHOBO11 & $61^{\circ} 27^{\prime} \mathrm{N}$ & $45^{\circ} 44^{\prime} \mathrm{W}$ & NW \\
\hline $1 \mathrm{AHOBO12}$ & $61^{\circ} 26^{\prime} \mathrm{N}$ & $45^{\circ} 43^{\prime} \mathrm{W}$ & sW \\
\hline $1 \mathrm{AH} 08013$ & $61^{\circ} 26^{\prime} \mathrm{N}$ & $45^{\circ} 29^{\prime} \mathrm{W}$ & \\
\hline AHOBO14 & $61^{\circ} 29^{\prime} \mathrm{N}$ & $45^{\circ} 45^{\prime} \mathrm{W}$ & $\mathrm{NE}$ \\
\hline 1AH08015 & $61^{\circ} 29^{\prime} \mathrm{N}$ & $45^{\circ} 45^{\prime} \mathrm{W}$ & $\mathrm{NE}$ \\
\hline $1 \mathrm{AH} 08016$ & $61^{\circ} 29^{\prime} \mathrm{N}$ & $45^{\circ} 44^{\prime} \mathrm{W}$ & $\mathrm{s}$ \\
\hline 1AH08017 & $61^{\circ} 30^{\prime} \mathrm{N}$ & $45^{\circ} 43^{\prime} \mathrm{W}$ & sw \\
\hline 1AH08018 & $61^{\circ} 28^{\prime} \mathrm{N}$ & $45^{\circ} 40^{\prime} \mathrm{W}$ & $\mathrm{SW}$ \\
\hline 1АH08019 & $61^{\circ} 28^{\prime} \mathrm{N}$ & $45^{\circ} 38^{\prime} \mathrm{W}$ & 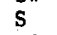 \\
\hline 1АН08020 & $61^{\circ} 29^{\prime} \mathrm{N}$ & $45^{\circ} 39^{\prime} \mathrm{W}$ & \\
\hline $1 \mathrm{AH} 08021$ & $61^{\circ} 31^{\prime} \mathrm{N}$ & $45^{\circ} 37^{\prime} \mathrm{W}$ & \\
\hline $1 \mathrm{AH} 08022$ & $61^{\circ} 20^{\prime} \mathrm{N}$ & $45^{\circ} 48^{\prime} \mathrm{W}$ & SW \\
\hline $1 \mathrm{AH} 10001$ & $61^{\circ} 16^{\prime} \mathrm{N}$ & $45^{\circ} 41^{\prime} \mathrm{V}$ & NW \\
\hline $1 \mathrm{AH} 11001$ & $61^{\circ} 14^{\prime} \mathrm{N}$ & $45^{\circ} 41^{\prime} \mathrm{V}$ & $\cdots$ \\
\hline 1AH11002 & $61^{\circ} 13^{\prime} \mathrm{N}$ & $45^{\circ} 41^{\prime} \mathrm{W}$ & NW \\
\hline 1AH13001 & $61^{\circ} 01^{\prime} \mathrm{N}$ & $45^{\circ} 52^{\prime} \mathrm{W}$ & NW \\
\hline $1 \mathrm{AH} 13002$ & $61^{\circ} 00^{\prime} \mathrm{N}$ & $45^{\circ} 56^{\prime} \mathrm{W}$ & $\mathrm{N}$ \\
\hline $1 \mathrm{AH} 13003$ & $61^{\circ} 00^{\prime} \mathrm{N}$ & $45^{\circ} 55^{\prime} \mathrm{V}$ & $\mathrm{N}$ \\
\hline 1AI01001 & $61^{\circ} 07^{\prime} \mathrm{N}$ & $47^{\circ} 36^{\prime} \mathrm{W}$ & \\
\hline $1 \mathrm{AI} 01002$ & $61^{\circ} 06^{\prime} \mathrm{N}$ & $47^{\circ} 35^{\prime} \mathrm{W}$ & SW \\
\hline 1AI01003 & $61^{\circ} 04^{\prime} \mathrm{N}$ & $47^{\circ} 30^{\prime} \mathrm{W}$ & $\mathrm{S}$ \\
\hline $1 \mathrm{AI} 02001$ & $61^{\circ} 05^{\prime} \mathrm{N}$ & $47^{\circ} 26^{\prime} \mathrm{V}$ & $\mathrm{s}$ \\
\hline $1 \mathrm{AI} 02002$ & $61^{\circ} 05^{\prime} \mathrm{N}$ & $47^{\circ} 18^{\prime}$ & \\
\hline $1 \mathrm{AI} 03001$ & $61^{\circ} 05^{\prime} \mathrm{N}$ & $47^{\circ} 12^{\prime} \mathrm{W}$ & s \\
\hline 1AI04001 & $61^{\circ} 04^{\prime} \mathrm{N}$ & $47^{\circ} 04^{\prime} \mathrm{V}$ & $\mathbf{s}$ \\
\hline 1AI04002 & $61^{\circ} 01{ }^{\prime N}$ & $47^{\circ} 01^{\prime} \mathrm{W}$ & $\mathrm{s}$ \\
\hline IAI05001 & $60^{\circ} 59^{\prime} \mathrm{N}$ & $47^{\circ} 00^{\prime} \mathrm{W}$ & SW \\
\hline $1 \mathrm{AI} 06001$ & $61^{\circ} 00^{\prime} \mathrm{N}$ & $46^{\circ} 53^{\prime} \mathrm{W}$ & \\
\hline $1 \mathrm{BA0} 01001$ & $61^{\circ} 18^{\prime} \mathrm{N}$ & $47^{\circ} 44^{\prime} \mathrm{W}$ & \\
\hline $1 \mathrm{BAO} 01002$ & $61^{\circ} 15^{\prime} \mathrm{N}$ & $47^{\circ} 50^{\prime} \mathrm{v}$ & $\mathrm{N}$ \\
\hline $1 \mathrm{BA01003}$ & $61^{\circ} 15^{\prime} \mathrm{N}$ & $47^{\circ} 51^{\prime} \mathrm{W}$ & NW \\
\hline $1 \mathrm{BA01004}$ & $61^{\circ} 15^{\prime} \mathrm{N}$ & $47^{\circ} 52^{\prime} \mathrm{W}$ & $\mathbf{N}$ \\
\hline $1 B A 01005$ & $61^{\circ} 14^{\prime} \mathrm{N}$ & $47^{\circ} 51^{\prime} \mathrm{W}$ & \\
\hline 1BA01006 & $61^{\circ} 14^{\prime} \mathrm{N}$ & $47^{\circ} 52 . \mathrm{V}$ & \\
\hline $1 \mathrm{BAO} 1007$ & $61^{\circ} 13^{\prime} \mathrm{N}$ & $47^{\circ} 51, v$ & s \\
\hline 1BA01008 & $61^{\circ} 13^{\prime} \mathrm{N}$ & $47^{\circ} 52^{\prime} \mathrm{W}$ & $\mathbf{N}$ \\
\hline $1 \mathrm{BAO} 1009$ & $61^{\circ} 13^{\prime} \mathrm{N}$ & $47^{\circ} 56^{\prime} \mathrm{W}$ & $\mathbf{E}$ \\
\hline $1 \mathrm{BA01010}$ & $61^{\circ} 12^{\prime} \mathrm{N}$ & $47^{\circ} 56^{\prime} \mathrm{W}$ & \\
\hline 1BA01011 & $61^{\circ} 12, \mathrm{~N}$ & $47^{\circ} 55^{\prime} \mathrm{W}$ & SW \\
\hline $1 \mathrm{BAO} 1012$ & $61^{\circ} 15 \cdot \mathrm{N}$ & $47^{\circ} 57^{\prime} \mathrm{v}$ & E \\
\hline 1BAO3001 & $61^{\circ} 17^{\prime} \mathrm{N}$ & $47^{\circ} 42, \mathrm{~W}$ & SW \\
\hline 1BA03002 & $61^{\circ} 17^{\prime} \mathrm{N}$ & $47^{\circ} 44^{\prime} \mathrm{W}$ & NW \\
\hline $1 \mathrm{BA0} 3003$ & $61^{\circ} 18^{\prime} \mathrm{N}$ & $47^{\circ} 40^{\prime} \mathrm{W}$ & SW \\
\hline $1 \mathrm{BAO}$ & $61^{\circ} 15^{\prime} \mathrm{N}$ & $47^{\circ} 39^{\prime} \mathrm{W}$ & SW \\
\hline 1BA03005 & $61^{\circ} 12^{\prime} \mathrm{N}$ & $47^{\circ} 51^{\prime} \mathrm{W}$ & W \\
\hline 1BA03006 & $61^{\circ} 12^{\prime} \mathrm{N}$ & $47^{\circ} 52^{\prime} \mathrm{W}$ & \\
\hline 1BA04001 & $61^{\circ} 12^{\prime} \mathrm{N}$ & $47^{\circ} 44^{\prime} \mathrm{W}$ & SW \\
\hline 1BA05001 & $61^{\circ} 10^{\prime} \mathrm{N}$ & $47^{\circ} 39^{\prime} \mathrm{W}$ & SW \\
\hline $1 \mathrm{BAO}$ & $61^{\circ} 10^{\prime} \mathrm{N}$ & $47^{\circ} 43^{\prime} \mathrm{V}$ & $\mathrm{NV}$ \\
\hline 1BA05003 & $61^{\circ} 09^{\prime} \mathrm{N}$ & $47^{\circ} 40^{\prime} \mathrm{W}$ & sv \\
\hline 1BA05004 & $61^{\circ} 08^{\prime} \mathrm{N}$ & $47^{\circ} 42^{\prime} \mathrm{W}$ & W \\
\hline 1BA05005 & $61^{\circ} 08^{\prime} \mathrm{N}$ & $47^{\circ} 48^{\prime} \mathrm{W}$ & $\mathrm{NW}$ \\
\hline & $61^{\circ} 07^{\prime} \mathrm{N}$ & $47^{\circ} 4$ & SW \\
\hline 1BA08001 & $61^{\circ} 04^{\prime} \mathrm{N}$ & $48^{\circ} 13^{\prime} \mathrm{W}$ & NW \\
\hline
\end{tabular}

\begin{tabular}{|c|c|c|c|c|}
\hline HIGH & LOW & AREA & $\begin{array}{l}\text { MO } \\
\text { CODE }\end{array}$ & MAP \\
\hline & 150 & 0000.11 & 700110 & 6100-05 \\
\hline 167 & 140 & 0001.41 & 543210 & $6100-05$ \\
\hline 140 & 110 & 0000.14 & 770012 & $6100-05$ \\
\hline 180 & 40 & 0085.25 & 167112 & $6100-05$ \\
\hline 140 & 90 & 0001.01 & 643112 & $6100-05$ \\
\hline 155 & 140 & 0000.25 & 770012 & $6100-05$ \\
\hline 135 & 120 & 0000.11 & 770012 & $6100-05$ \\
\hline 130 & 105 & 0000.24 & 670112 & 6100-05 \\
\hline 124 & 115 & 0000.04 & 780010 & $6100-06$ \\
\hline 170 & 150 & 0000.22 & 700110 & $6100-06$ \\
\hline 150 & 110 & 0000.09 & 770010 & $6100-05$ \\
\hline 170 & 160 & 0000.11 & 700010 & $6100-05$ \\
\hline 180 & 45 & 0081.35 & 167112 & $6100-05$ \\
\hline 170 & 100 & 0001.30 & 533210 & $6100-06$ \\
\hline 125 & 95 & 0000.42 & 533112 & $6100-06$ \\
\hline 125 & 100 & 0000.82 & 276110 & $6100-06$ \\
\hline & 125 & 0018.90 & 203110 & $6100-06$ \\
\hline 180 & 65 & 0026.49 & 167112 & $6130-06$ \\
\hline 180 & & 0872.99 & 164114 & $6100-05$ \\
\hline 105 & 100 & 0000.10 & 780010 & $6100-06$ \\
\hline 110 & 95 & 0000.15 & 780010 & $6100-06$ \\
\hline 110 & 100 & 0000.05 & 780010 & $6100-06$ \\
\hline 90 & 60 & 0000.14 & 643312 & $6100-05$ \\
\hline 110 & 75 & 0000.36 & 643312 & $6100-05$ \\
\hline 120 & 90 & 0000.29 & 643212 & $6100-05$ \\
\hline 180 & 30 & 0045.70 & 163112 & $6100-03$ \\
\hline 180 & 40 & 0213.00 & 163112 & $6100-03$ \\
\hline 180 & 10 & 0141.60 & 163111 & $6100-03$ \\
\hline 180 & 10 & 0271.75 & 163112 & $6100-03$ \\
\hline 180 & 10 & 0171.79 & 163111 & $6100-03$ \\
\hline 180 & 5 & 0269.91 & 163111 & $6100-04$ \\
\hline 180 & 20 & 0208.63 & 163112 & $6100-04$ \\
\hline 180 & 25 & 0056.87 & 163112 & $6100-04$ \\
\hline 180 & & 0227.27 & 164112 & $6030-03$ \\
\hline 180 & 15 & 0211.30 & 163112 & $6100-04$ \\
\hline 180 & 55 & 0061.57 & 163112 & $6100-03$ \\
\hline 80 & 75 & 0000.06 & 780010 & $6100-03$ \\
\hline 90 & 75 & 0000.05 & 770010 & $6100-03$ \\
\hline 95 & 70 & 0000.07 & 770010 & $6100-03$ \\
\hline 77 & 72 & 0000.04 & 780010 & $6100-03$ \\
\hline 70 & 55 & 0000.05 & 770010 & $6100-03$ \\
\hline 90 & 85 & 0000.07 & 780010 & $6100-03$ \\
\hline 92 & 70 & 0000.28 & 643112 & $6100-03$ \\
\hline 70 & 60 & 0000.02 & 770010 & $6100-03$ \\
\hline 70 & 60 & 0000.04 & 770010 & $6100-03$ \\
\hline 80 & 74 & 0000.04 & 770010 & $6100-03$ \\
\hline 100 & 90 & 0000.02 & 770010 & $6100-03$ \\
\hline 180 & 59 & 0042.88 & 163112 & $6100-03$ \\
\hline 100 & 90 & 0000.10 & 770010 & $6100-03$ \\
\hline 180 & 90 & 0065.72 & 163112 & $6100-03$ \\
\hline 180 & 55 & 0045.65 & 163112 & $6100-03$ \\
\hline 85 & 80 & 0000.17 & 770010 & $6100-03$ \\
\hline 80 & 75 & 0000.03 & 780010 & $6100-03$ \\
\hline 180 & & 0168.88 & 165111 & $6100-03$ \\
\hline 180 & 30 & 0050.42 & 163112 & $6100-03$ \\
\hline 75 & 55 & 0000.06 & 780110 & $6100-03$ \\
\hline 180 & & 0067.67 & 163111 & $6100-03$ \\
\hline 60 & 50 & 0000.13 & 780110 & $6100-03$ \\
\hline 75 & 65 & 0000.03 & 770110 & $6100-03$ \\
\hline 180 & 20 & 0065.14 & 163112 & $6100-03$ \\
\hline 45 & 30 & 0001.32 & 780110 & $6100-02$ \\
\hline
\end{tabular}

CODE

LAT

LONG

HIGH

MAP

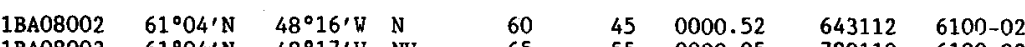

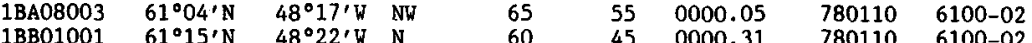

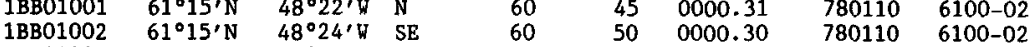

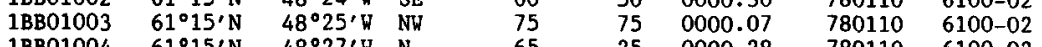

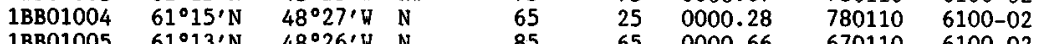

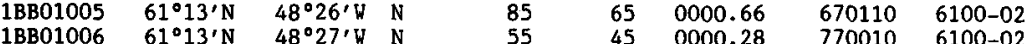

$\begin{array}{lllllllll}1 \mathrm{BB} 01007 & 61^{\circ} 14^{\prime} \mathrm{N} & 48^{\circ} 28^{\prime} \mathrm{W} & \mathrm{NE} & 120 & 100 & 0000.19 & 770010 & 6100-02\end{array}$

$1 \mathrm{BB} 0100861^{\circ} 13^{\prime} \mathrm{N} \quad 48^{\circ} 29^{\prime} \mathrm{W}$ W

$1 \mathrm{BB} 0100961^{\circ} 13^{\prime} \mathrm{N} \quad 48^{\circ} 27^{\prime} \mathrm{W}$

$\begin{array}{llll}1 \mathrm{BB} 01010 & 61^{\circ} 13^{\prime} \mathrm{N} & 48^{\circ} 26^{\prime} \mathrm{W} & \mathrm{SE} \\ 1 \mathrm{BBO} 0001 & 61^{\circ} 28^{\prime} \mathrm{N} & 48^{\circ} 02^{\prime} \mathrm{W} & \mathrm{SE}\end{array}$

$48^{\circ} 02$, W $\mathrm{NW}$

1 BB0 4003

$61^{\circ} 27^{\prime} \mathrm{N} \quad 47^{\circ} 57^{\prime} \mathrm{W}$ SW

$1 \mathrm{BB} 04005$

$61^{\circ} 25^{\prime} \mathrm{N}$

$47^{\circ} 57, \mathrm{~W}$ NE

$1 \mathrm{BB} 04007$

6.

$1 \mathrm{BB} 04008$

$61^{\circ} 22$ '

$47^{\circ} 59^{\prime} \mathrm{W}$ N

$1 \mathrm{BB} 05002$

(

$47^{\circ} 50^{\prime} \mathrm{N} N$

$1 \mathrm{BB} 0500$

$1^{\circ} 20^{\prime}$

$47^{\circ} 47$ ' W SW

1 BB05005

.18.N

$47^{\circ} 54 . \mathrm{V}$

$1 \mathrm{BB} 07001$

$47^{\circ} 55^{\prime} \mathrm{W} \mathrm{N}$

$1 B B 07002$

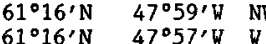

-1
-1

$61^{\circ} 06^{\prime} \mathrm{N} \quad 48^{\circ} 57^{\circ} \mathrm{W}$

$1 \mathrm{BC} 02001$

$1026^{\prime} \mathrm{N} \quad 48^{\circ} 21^{\circ} \mathrm{W} \quad \mathrm{NW}$

$\begin{array}{llll}1 \mathrm{BC} 02002 & 61^{\circ} 26^{\prime} \mathrm{N} & 48^{\circ} 17^{\prime} \mathrm{W} & \mathrm{NW} \\ 1 \mathrm{BC} 03001 & 61^{\circ} 25^{\prime} \mathrm{N} & 48^{\circ} 16^{\prime} \mathrm{W} & \mathrm{W}\end{array}$

$1 \mathrm{BCO} 3002$

1BD06001

$610^{\circ} 35^{\prime}$

$48^{\circ} 15^{\prime} \mathrm{W}$ NW

(1)

$61^{\circ} 40^{\prime} \mathrm{N} \quad 47^{\circ} 37^{\prime} \mathrm{V}$
$61^{\circ} \mathrm{N}$

(1)

$61041, \mathrm{~N}$

$47^{\circ} 41^{\prime} \mathrm{W}$

$1 \mathrm{BD} 06006$

$61^{\circ} 40^{\prime}$

$47^{\circ} 43 \prime \mathrm{W}$
$47^{\circ} 43.6$

1 BD06008

$61^{\circ} 40^{\prime} \mathrm{N} \quad 47^{\circ} 46^{\prime} \mathrm{W}$

$61^{\circ} 40^{\prime} \mathrm{N} \quad 47^{\circ} 41^{\prime} \mathrm{W}$

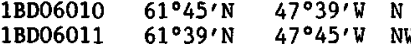

$1 \mathrm{BD} 06012$

$10^{\circ} 39^{\prime}$

$47^{\circ} 45^{\prime} \mathrm{N}$ NW

(1800014

$61^{\circ} 38^{\prime} \mathrm{N}$

$\begin{array}{lll}47^{\circ} 46^{\prime} \mathrm{W} & \mathrm{SW} \\ 47^{\circ} 47^{\prime} \mathrm{W} & \mathrm{NW}\end{array}$

1 BD06015

-5
$610^{\circ} 39^{\prime} \mathrm{N}$

$47^{\circ} 49 \cdot \mathrm{W}$

$61038^{\prime} \mathrm{N}$

$47^{\circ} 50^{\prime} \mathrm{W} N \mathrm{NN}$

61039'N $477^{\circ} 52$, N

$1 \mathrm{BD} 0601961^{\circ} 38^{\prime} \mathrm{N} \quad 47^{\circ} 52, \mathrm{~N} \quad \mathrm{NW}$

$\begin{array}{llll}\text { 1BD06020 } & 61^{\circ} 38^{\prime} \mathrm{N} & 47^{\circ} 54^{\prime} \mathrm{W} & \mathrm{NW} \\ 1 \mathrm{BDD06021} & 61^{\circ} 39^{\prime} \mathrm{N} & 47^{\circ} 55^{\prime} \mathrm{W} & \mathrm{E}\end{array}$

BD0602

$1 \mathrm{BD} 06023$

$61^{\circ} 39^{\prime} \mathrm{N} \quad 47^{\circ} 56^{\prime} \mathrm{W}$ SW

1 BD06025

$\begin{array}{lll}61^{\circ} 38^{\prime} \mathrm{N} & 477^{\circ} 57^{\prime} \mathrm{V} & \mathrm{W} \\ 61^{\circ} 38^{\prime} \mathrm{N} & 47055^{\prime} \mathrm{V} & \mathrm{NU}\end{array}$

1BD06026
$1 \mathrm{BD} 06027$

$61^{\circ} 38^{\prime} \mathrm{N} \quad 47^{\circ} 54^{\prime} \mathrm{W} \quad \mathrm{N}$

$\begin{array}{ll}65 & 0000.35\end{array}$

6100-02

$\begin{array}{ll}670112 & 6100-02 \\ 780010 & 6100-02\end{array}$

$780010 \quad 6100-02$

$780010 \quad 6100-02$

$163112 \quad 6100-03$

$780010 \quad 6100-03$

$750010 \quad 6100-03$

$780010 \quad 6100-03$

$\begin{array}{ll}780010 & 6100-03 \\ 770010 & 6100-03\end{array}$

163112 6100-03

163112 6100-03

$780010 \quad 6100-03$

6100-03

$770010 \quad 6100-03$

$780110 \quad 6100-02$

$\begin{array}{ll}643110 & 6100-02 \\ 643112 & 6100-02\end{array}$

643112 6100-02

$6701106100-02$

$230010 \quad 6130-03$

6130-03

$770010 \quad 6130-03$

$780010 \quad 6130-03$

$780010 \quad 6130-03$

$\begin{array}{ll}163112 & 6130-03 \\ 770110 & 6130-03\end{array}$

$770110 \quad 6130-03$

\begin{tabular}{ll}
753112 & $6130-03$ \\
\hline $5130-03$
\end{tabular}

$750110 \quad 6130-03$

$780010 \quad 6130-03$

$780010 \quad 6130-03$

$780010 \quad 6130-03$

$770110 \quad 6130-03$

$770110 \quad 6130-03$

6130-03

$770010 \quad 6130-03$

$\begin{array}{ll}780010 & 6130-03 \\ 770010 & 6130-03\end{array}$

$\begin{array}{ll}780010 & 6130-03 \\ 770010 & 6130-03\end{array}$ 


\begin{tabular}{|c|c|c|c|c|c|c|c|c|c|c|c|c|c|c|c|c|c|}
\hline CODE & LAT & LONG & ORI & HIGH & LOW & AREA & $\begin{array}{c}\text { MO } \\
\text { CODE }\end{array}$ & MAP & CODE & LAT & LONG & ORI & HIGH & LOW & AREA & $\begin{array}{c}\text { MO } \\
\text { CODE }\end{array}$ & MAP \\
\hline 1BD06028 & $61^{\circ} 36^{\prime} \mathrm{N}$ & $47^{\circ} 57^{\prime} \mathrm{W}$ & NW & 110 & 110 & 0000.07 & 780010 & $6130-03$ & 1BF03001 & $62^{\circ} 05^{\prime N}$ & $48^{\circ} 37^{\prime} \mathrm{W}$ & SW & 180 & 60 & 0224.69 & 163112 & $6200-03$ \\
\hline 1BD06029 & $61^{\circ} 36^{\prime} \mathrm{N}$ & $47^{\circ} 57^{\prime} \mathrm{W}$ & $v$ & 105 & 100 & 0000.04 & 780010 & $6130-03$ & $1 \mathrm{BF} 03002$ & $61.58, \mathrm{~N}$ & $48^{\circ} 44^{\prime} \mathrm{W}$ & $\mathrm{SW}$ & 180 & & 1556.80 & 164113 & $6130-02$ \\
\hline 1BD06030 & $61^{\circ} 34^{\prime} \mathrm{N}$ & $48^{\circ} 01^{\prime} \mathrm{W}$ & $\mathrm{N}$ & 125 & 100 & 0000.07 & 780010 & $6130-02$ & 1BF03003 & $61^{\circ} 59 \cdot \mathrm{N}$ & $48^{\circ} 19 \cdot k$ & W & 180 & 20 & 0120.97 & 166112 & $6130-02$ \\
\hline 18D06031 & $61^{\circ} 34^{\prime} \mathrm{N}$ & $48^{\circ} 03^{\prime} \mathrm{W}$ & $\mathbf{N}$ & 125 & 110 & 0000.06 & 780010 & $6130-02$ & $1 \mathrm{BF} 03004$ & $61^{\circ} 55^{\prime} \mathrm{N}$ & $48^{\circ} 22^{\prime} \mathrm{W}$ & SW & 115 & 105 & 0000.17 & 780010 & $6130-02$ \\
\hline 1BD06032 & $61^{\circ} 37^{\prime} \mathrm{N}$ & $47^{\circ} 54^{\prime} \mathrm{W}$ & SW & 130 & 115 & 0000.09 & 780010 & $6130-03$ & $1 \mathrm{BF} 03005$ & $61^{\circ} 55^{\prime} \mathrm{N}$ & $48^{\circ} 23^{\prime} \mathrm{W}$ & E & 110 & 105 & 0000.17 & 780010 & $6130-02$ \\
\hline $1 \mathrm{BD} 06033$ & $61^{\circ} 38^{\prime} \mathrm{N}$ & $47^{\circ} 52^{\prime} \mathrm{W}$ & w & 140 & 130 & 0000.05 & 780010 & $6130-03$ & 1 BF03006 & $61^{\circ} 55^{\prime N}$ & $48^{\circ} 25$, & $\mathrm{s}$ & 100 & 80 & 0000.26 & 790010 & $6130-02$ \\
\hline 1BD06034 & $61^{\circ} 38^{\prime} \mathrm{N}$ & $47^{\circ} 48^{\prime} \mathrm{W}$ & SW & 140 & 130 & 0000.04 & 780010 & $6130-03$ & 1BF03007 & $61^{\circ} 55, \mathrm{~N}$ & $48^{\circ} 24^{\prime} \mathrm{W}$ & W & 110 & 105 & 0000.11 & 770010 & $6130-02$ \\
\hline $1 \mathrm{BD} 06035$ & $61^{\circ} 38^{\prime} \mathrm{N}$ & $47^{\circ} 48^{\prime} \mathrm{W}$ & SW & 140 & 125 & 0000.05 & 780010 & $6130-03$ & 1BF03008 & $61^{\circ} 53^{\prime} \mathrm{N}$ & $48^{\circ} 20^{\prime} \mathrm{w}$ & NV & 105 & 105 & 0000.21 & 780010 & $6130-02$ \\
\hline $1 \mathrm{BD} 06036$ & $61^{\circ} 38^{\circ} \mathrm{N}$ & $47^{\circ} 49^{\prime} \mathrm{W}$ & $\mathrm{w}$ & 160 & 140 & 0000.13 & 770010 & $6130-03$ & $1 \mathrm{BF} 03009$ & $61^{\circ} 58^{\circ} \mathrm{N}$ & $48^{\circ} 08^{\prime} \mathrm{W}$ & SW & 180 & 75 & 0089.70 & 163112 & $6130-02$ \\
\hline $1 \mathrm{BD} 06037$ & $61^{\circ} 38^{\prime} \mathrm{N}$ & $47^{\circ} 46^{\prime} \mathrm{W}$ & W & 180 & 40 & 0067.96 & 163111 & $6130-03$ & $1 \mathrm{BF} 03010$ & $61^{\circ} 59^{\prime} \mathrm{N}$ & $47^{\circ} 57^{\prime} \mathrm{W}$ & $\mathrm{W}$ & 180 & 105 & 0081.71 & 167112 & $6130-03$ \\
\hline 1BD06038 & $61^{\circ} 37^{\prime} \mathrm{N}$ & $47^{\circ} 44^{\prime} \mathrm{W}$ & w & 180 & 75 & 0036.21 & 163111 & $6130-03$ & $1 \mathrm{BF} 03011$ & $61^{\circ} 57 \cdot \mathrm{N}$ & $48^{\circ} 02^{\prime} \mathrm{W}$ & sW & 160 & 125 & 0000.68 & 603112 & $6130-02$ \\
\hline 1BD06039 & $61^{\circ} 36^{\prime} \mathrm{N}$ & $47^{\circ} 40^{\prime} \mathrm{W}$ & $\mathrm{W}$ & 180 & 75 & 0080.75 & 167112 & $6130-03$ & 1BF03012 & $61^{\circ} 58^{\circ} \mathrm{N}$ & $48^{\circ} 02^{\prime \prime} \mathrm{W}$ & $\mathrm{N}$ & 155 & 110 & 0001.12 & 770010 & $\begin{array}{l}0130-02 \\
6130-02\end{array}$ \\
\hline 1BD06040 & $61^{\circ} 35^{\prime} \mathrm{N}$ & $47^{\circ} 41^{\prime} \mathrm{W}$ & NW & 170 & 140 & 0001.06 & 700010 & $6130-03$ & $1 \mathrm{BF} 03013$ & $61^{\circ} 58^{\prime} \mathrm{N}$ & $48^{\circ} 03^{\prime} \mathrm{W}$ & & 140 & 105 & 0000.42 & 770010 & $6130-02$ \\
\hline $1 \mathrm{BD06041}$ & $61^{\circ} 35^{\prime} \mathrm{N}$ & $47^{\circ} 44^{\prime} \mathrm{W}$ & $\mathrm{N}$ & 140 & 130 & 0000.15 & 780010 & $6130-03$ & $1 B F 03014$ & $61^{\circ} 58^{\prime} \mathrm{N}$ & $48^{\circ} 05^{\prime} \mathrm{W}$ & 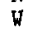 & 125 & 110 & 0000.20 & 780010 & $6130-02$ \\
\hline 1BD06042 & $61^{\circ} 35^{\prime} \mathrm{N}$ & $47^{\circ} 45^{\prime} \mathrm{W}$ & NW & 140 & 130 & 0000.07 & 770010 & $6130-03$ & 1BF03015 & $61^{\circ} 56^{\prime} \mathrm{N}$ & $48^{\circ} 05^{\prime} \mathrm{V}$ & W & 160 & 90 & 0025.44 & 226112 & $6130-02$ \\
\hline 1 BD06043 & $61^{\circ} 34^{\prime} \mathrm{N}$ & $47^{\circ} 45^{\prime} \mathrm{W}$ & s & 150 & 130 & 0000.19 & 780010 & $6130-03$ & 1BF03016 & $61.56, \mathrm{~N}$ & $48^{\circ} 07 \cdot \mathrm{V}$ & NW & 135 & 90 & 0001.32 & 633112 & $6130-02$ \\
\hline 1BD06044 & $61^{\circ} 35^{\prime} \mathrm{N}$ & $47^{\circ} 44^{\prime} \mathrm{W}$ & SE & 150 & 140 & 0000.07 & 780010 & $6130-03$ & 1BF03017 & $61^{\circ} 57^{\circ} \mathrm{N}$ & $48^{\circ} 08^{\prime} \mathrm{W}$ & SW & 120 & 115 & 0000.11 & 780010 & $6130-02$ \\
\hline 1BD06045 & $61^{\circ} 35^{\prime} \mathrm{N}$ & $47^{\circ} 41^{\prime} \mathrm{W}$ & E & 155 & 140 & 0000.10 & 780010 & $6130-03$ & $1 \mathrm{BF} 03018$ & $61^{\circ} 55^{\prime N}$ & $48^{\circ} 09^{\circ}$ & $\mathrm{N}$ & 120 & 105 & 0000.06 & 780010 & $6130-02$ \\
\hline 1BD06046 & $61^{\circ} 34^{\prime} \mathrm{N}$ & $47^{\circ} 42^{\prime} \mathrm{W}$ & SE & 160 & 150 & 0000.10 & 780010 & $6130-03$ & $1 \mathrm{BF} 03019$ & $61^{\circ} 55^{\prime N}$ & $48^{\circ} 10^{\prime} \mathrm{W}$ & $\mathrm{N}$ & 125 & 115 & 0000.23 & 770012 & $6130-02$ \\
\hline 1BD06047 & $61^{\circ} 37^{\prime} \mathrm{N}$ & $48^{\circ} 02^{\prime} \mathrm{W}$ & $\mathrm{W}$ & 180 & 4 & 0244.60 & 163111 & $6130-03$ & $1 \mathrm{BFO} 3020$ & $61 \circ 55^{\prime} \mathrm{N}$ & $48^{\circ} 11^{\prime} \mathrm{W}$ & NW & 115 & 115 & 0000.05 & 780010 & $6130-02$ \\
\hline 1BD06048 & $61^{\circ} 30^{\prime} \mathrm{N}$ & $47^{\circ} 54^{\prime} \mathrm{W}$ & NW & & 75 & 0000.26 & 750010 & $6130-03$ & $1 \mathrm{BF} 03021$ & $61.54 \mathrm{~N}$ & $48^{\circ} 12^{\prime} \mathrm{W}$ & $\mathrm{N}$ & 140 & 115 & 0001.02 & 780010 & $6130-02$ \\
\hline 1BD06049 & $61^{\circ} 29^{\prime} \mathrm{N}$ & $47^{\circ} 55^{\prime} \mathrm{W}$ & N & 80 & 75 & 0000.05 & 770010 & $6100-03$ & 1 BF03022 & $61054 \mathrm{~N}$ & $48^{\circ} 13^{1} \mathrm{~W}$ & NW & 130 & 105 & 0000.40 & 640012 & $\begin{array}{l}0130-02 \\
6130-02\end{array}$ \\
\hline 1BD06050 & $61^{\circ} 29^{\prime} \mathrm{N}$ & $47^{\circ} 54^{\prime} \mathrm{W}$ & NW & 95 & 85 & 0000.16 & 750010 & $6100-03$ & 1BF03023 & $61^{\circ} 53^{\prime N}$ & $48^{\circ} 15^{\prime \prime} \mathrm{W}$ & NW & 120 & 110 & 0000.12 & 700010 & $6130-02$ \\
\hline 1BD06051 & $61^{\circ} 28^{\prime} \mathrm{N}$ & $47^{\circ} 57^{\prime} \mathrm{W}$ & NW & 90 & 85 & 0000.06 & 750010 & $6100-03$ & $1 \mathrm{BF} 03024$ & $61^{\circ} 52^{\prime} \mathrm{N}$ & $48^{\circ} 16^{\prime} \mathrm{W}$ & $\mathrm{N}$ & 130 & 95 & 0001.41 & 673012 & $6130-02$ \\
\hline 1BD06052 & $61^{\circ} 28^{\prime} \mathrm{N}$ & $47^{\circ} 57^{\prime} \mathrm{W}$ & $\mathrm{N}$ & 90 & 85 & 0000.01 & 750010 & $6100-03$ & 1BF03025 & $61^{\circ} 52^{\prime} \mathrm{N}$ & $48^{\circ} 17^{\prime} \mathrm{W}$ & $\mathrm{N}$ & 120 & 80 & 0000.87 & 673112 & $6130-02$ \\
\hline 1BD06053 & $61^{\circ} 29^{\prime} \mathrm{N}$ & $48^{\circ} 01^{\prime} \mathrm{W}$ & $\mathrm{N}$ & 95 & 90 & 0000.04 & 650010 & $6100-02$ & 1BF03026 & $61052, \mathrm{~N}$ & $48^{\circ} 18^{\prime} \mathrm{W}$ & NW & 100 & 95 & 0000.06 & 780010 & $6130-02$ \\
\hline 1BD06054 & $61^{\circ} 28^{\prime} \mathrm{N}$ & $48^{\circ} 03^{\prime} \mathrm{W}$ & NW & 105 & 90 & 0000.07 & 750010 & $6100-02$ & 1BF03027 & $61.52, \mathrm{~N}$ & $48^{\circ} 19^{\prime} \mathrm{W}$ & $\mathrm{N}$ & 120 & 110 & 0000.05 & 780010 & $6130-02$ \\
\hline 1BD06055 & $61^{\circ} 28^{\prime} \mathrm{N}$ & $48^{\circ} 05^{\prime} \mathrm{W}$ & $\mathrm{NE}$ & 110 & 95 & 0000.03 & 780010 & $6100-02$ & 1BF03028 & $61^{\circ} 51$, N & $48^{\circ} 28^{\prime} \mathrm{W}$ & NW & 115 & 115 & 0000.27 & 770010 & $6130-02$ \\
\hline 1BD06056 & $61^{\circ} 29^{\prime} \mathrm{N}$ & $48^{\circ} 05^{\prime} \mathrm{W}$ & N & 95 & 90 & 0000.03 & 780010 & $6100-0$ & 1BF03029 & $61052 \cdot \mathrm{N}$ & $48^{\circ} 17^{\prime} \mathrm{W}$ & SW & 120 & 100 & 0000.81 & 700110 & $6130-02$ \\
\hline 1BD06057 & $61^{\circ} 30^{\prime} \mathrm{N}$ & $48^{\circ} 04^{\prime} \mathrm{W}$ & N & 85 & 75 & 0000.05 & 780010 & $6130-02$ & $1 \mathrm{BF} 03030$ & $61.53, \mathrm{~N}$ & $48^{\circ} 15^{\prime} \mathrm{W}$ & NE & 125 & 110 & 0000.33 & 770110 & $6130-02$ \\
\hline 1BD06058 & $61^{\circ} 30^{\prime} \mathrm{N}$ & $48^{\circ} 07^{\prime}$ W & NE & 90 & 80 & 0000.06 & 780010 & $6130-02$ & 1BF03031 & $61.54 \times \mathrm{N}$ & $48^{\circ} 12^{\circ} \mathrm{W}$ & E & 135 & 120 & 0000.15 & 780010 & $6130-02$ \\
\hline 1BD06059 & $61^{\circ} 30^{\prime} \mathrm{N}$ & $48^{\circ} 08^{\prime}$ W & W & 85 & 80 & 0000.11 & 780010 & $6130-02$ & $1 \mathrm{BFO} 3032$ & $61055 \mathrm{~N}$ & $48^{\circ} 07, \mathrm{~W}$ & ฟ & 135 & 90 & 0008.06 & 223112 & 6130-02 \\
\hline 1BE02001 & $61^{\circ} 41^{\prime} \mathrm{N}$ & $48^{\circ} 48^{\prime} \mathrm{W}$ & NW & 110 & 80 & 0000.35 & 603010 & $6130-02$ & 1BF03033 & $61^{\circ} 55 . \mathrm{N}$ & $48^{\circ} 04^{\prime} \mathrm{w}$ & SW & 135 & 110 & 0002.00 & 220110 & $6130-02$ \\
\hline $1 \mathrm{BE} 02002$ & $61^{\circ} 40^{\prime} \mathrm{N}$ & $48^{\circ} 48^{\prime} \mathrm{W}$ & W & 105 & 90 & 0000.28 & 700010 & $6130-02$ & $1 \mathrm{BF} 03034$ & $61^{\circ} 55^{\prime} \mathrm{N}$ & $48^{\circ} 02^{\prime} \mathrm{W}$ & $\mathrm{s}$ & 135 & 110 & 0004.44 & 220110 & $6130-02$ \\
\hline 1BE03001 & $61^{\circ} 41^{\prime} \mathrm{N}$ & $48^{\circ} 46^{\prime} \mathrm{W}$ & $\mathbf{N}$ & 120 & 75 & 0001.61 & 643112 & $6130-02$ & 1BF03035 & $61^{\circ} 57^{\prime} \mathrm{N}$ & $47^{\circ} 56^{\prime} \mathrm{W}$ & SW & 180 & 110 & 0092.19 & 167110 & $6130-03$ \\
\hline 1BE03002 & $61^{\circ} 41^{\prime} \mathrm{N}$ & $48^{\circ} 45^{\prime} \mathrm{Wl}$ & $\mathrm{NE}$ & 120 & 90 & 0000.22 & 770010 & $6130-02$ & $1 \mathrm{BF} 03036$ & $61^{\circ} 51^{\prime} \mathrm{N}$ & $48^{\circ} 12^{\prime} \mathrm{w}$ & $\mathrm{N}$ & 110 & 100 & 0000.07 & 780010 & $6130-02$ \\
\hline $1 \mathrm{BE} 03003$ & $61^{\circ} 40^{\prime} \mathrm{N}$ & $48^{\circ} 46^{\prime} \mathrm{W}$ & $\mathrm{s}$ & 120 & 95 & 0000.17 & 770010 & $6130-02$ & $1 B F 03037$ & $61051 \cdot \mathrm{N}$ & $48^{\circ} 13^{\prime} \mathrm{W}$ & NW & 120 & 90 & 0000.49 & 770112 & $6130-02$ \\
\hline 1BE03004 & $61^{\circ} 40^{\prime} \mathrm{N}$ & $48^{\circ} 46^{\prime} \mathrm{WI}$ & SW & 90 & 85 & 0000.10 & 780010 & $6130-02$ & 1BF03038 & $61.51, \mathrm{~N}$ & $48^{\circ} 14^{\prime} \mathrm{W}$ & NW & 100 & 85 & 0000.07 & 750110 & $6130-02$ \\
\hline $1 \mathrm{BE} 03005$ & $61^{\circ} 40^{\prime} \mathrm{N}$ & $48^{\circ} 45^{\prime} \mathrm{W}$ & E & 90 & 80 & 0000.16 & 780010 & $6130-02$ & $1 B F 03039$ & $61051, \mathrm{~N}$ & $48^{\circ} 02^{\prime} \mathrm{W}$ & sw & 180 & 74 & 0218.82 & 166112 & $6130-02$ \\
\hline $1 \mathrm{BE} 03006$ & $61^{\circ} 42^{\prime} \mathrm{N}$ & $48^{\circ} 35^{\prime} \mathrm{W}$ & s & 130 & 105 & 0000.24 & 790010 & $6130-02$ & 1BF03040 & $61^{\circ} 46^{\prime} \mathrm{N}$ & $48^{\circ} 11^{\prime} \mathrm{V}$ & NW & 115 & 90 & 0000.12 & 780010 & $6130-02$ \\
\hline 1BE03007 & $61^{\circ} 42^{\prime} \mathrm{N}$ & $48^{\circ} 24^{\prime} \mathrm{W}$ & $\mathrm{w}$ & 150 & 125 & 0000.06 & 770010 & $6130-02$ & $1 \mathrm{BF} 03041$ & $61^{\circ} 46^{\prime} \mathrm{N}$ & $48^{\circ} 13^{\prime} \mathrm{w}$ & $\mathrm{N}$ & 120 & 85 & 0003.72 & 270112 & $6130-02$ \\
\hline $1 \mathrm{BE} 03008$ & $61^{\circ} 41^{\prime} \mathrm{N}$ & $48^{\circ} 38^{\prime} \mathrm{W}$ & W & 110 & 65 & 0001.52 & 770112 & $6130-02$ & IBF03042 & $61^{\circ} 46^{\prime} \mathrm{N}$ & $48^{\circ} 15, W$ & $\mathrm{~N}$ & 120 & 100 & 0000.34 & 750012 & $\begin{array}{l}0130-02 \\
6130-02\end{array}$ \\
\hline 1BE03009 & $61^{\circ} 41^{\prime} \mathrm{N}$ & $48^{\circ} 33^{\prime} \mathrm{W}$ & NW & 95 & 70 & 0000.29 & 770112 & $6130-02$ & 1BF03043 & $61^{\circ} 48^{\prime} \mathrm{N}$ & $48^{\circ} 15^{\prime} \mathrm{v}$ & $\mathrm{N}$ & 125 & 95 & 0001.20 & 780012 & $6130-02$ \\
\hline $1 \mathrm{BEO}$ & $61^{\circ} 43^{\prime} \mathrm{N}$ & $48^{\circ} 32^{\prime} \mathrm{W}$ & $\mathrm{SE}$ & 120 & 100 & 0000.65 & 770010 & $6130-02$ & $1 \mathrm{BF} 03044$ & $61^{\circ} 48^{\prime} \mathrm{N}$ & $48^{\circ} 16^{\prime} \mathrm{V}$ & $\mathrm{NE}$ & 130 & 90 & 0000.95 & 643010 & $6130-02$ \\
\hline 1BE03011 & $61^{\circ} 43^{\prime} \mathrm{N}$ & $48^{\circ} 30^{\prime} W$ & $\mathrm{~s}$ & 140 & 110 & 0.27 & 780010 & $6130-02$ & 1BF03045 & $61^{\circ} 48^{\prime} \mathrm{N}$ & $48^{\circ} 17^{\prime \prime} \mathrm{v}$ & NW & 110 & 90 & 0000.23 & 780010 & $6130-02$ \\
\hline 1BE03012 & $61^{\circ} 43^{\prime} \mathrm{N}$ & $48^{\circ} 26^{\prime} \mathrm{W}$ & W & 120 & 100 & 0000.09 & 770010 & $6130-02$ & $1 \mathrm{BFO} 3$ & $61^{\circ} 48^{\prime} \mathrm{N}$ & $48^{\circ} 22^{\prime} \mathrm{V}$ & $\mathrm{N}$ & 110 & 95 & 0000.62 & 780010 & $6130-02$ \\
\hline 1BE04001 & $61^{\circ} 42^{\prime} \mathrm{N}$ & $48^{\circ} 26^{\prime} \mathrm{VI}$ & $s$ & 130 & 110 & 0000.11 & 780010 & $6130-02$ & 1BF03047 & $61^{\circ} 49 \cdot \mathrm{N}$ & $48^{\circ} 24^{\prime} \mathrm{K}$ & NW & 110 & 90 & 0000.97 & 780010 & $6130-02$ \\
\hline 1BE04002 & $61^{\circ} 43^{\prime} \mathrm{N}$ & $48^{\circ} 26^{\prime} \mathrm{W}$ & $\mathrm{s}$ & 110 & 105 & 0000.06 & 770010 & $6130-02$ & 1BF03048 & $61^{\circ} 49^{\prime} \mathrm{N}$ & $48^{\circ} 27^{\prime \prime}$ & NW & 105 & 72 & 0000.43 & 750010 & $6130-02$ \\
\hline 1BE04003 & $61^{\circ} 43^{\prime} \mathrm{N}$ & $48^{\circ} 21^{\prime} \mathrm{W}$ & $\mathrm{NE}$ & 110 & 95 & 0000.48 & 643112 & $6130-02$ & $1 \mathrm{BF} 03049$ & $61^{\circ} 48^{\prime} \mathrm{N}$ & $48^{\circ} 27^{\prime}$ & $\mathrm{s}$ & 135 & 120 & 0000.08 & 780010 & $6130-02$ \\
\hline 1BE04004 & $61^{\circ} 43^{\prime} \mathrm{N}$ & $48^{\circ} 22^{\prime} \mathrm{W}$ & $\mathrm{NU}$ & 105 & 85 & 0000.33 & 770010 & $6130-02$ & $1 \mathrm{BP} 03050$ & $61^{\circ} 48^{\prime} \mathrm{N}$ & $48^{\circ} 22^{\prime} \mathrm{V}$ & NW & 115 & 100 & 0000.27 & 780010 & $\begin{array}{l}0130-02 \\
6130-02\end{array}$ \\
\hline 1BE04005 & $61^{\circ} 42^{\prime} \mathrm{N}$ & $48^{\circ} 21^{\prime}$ ㅁ & SW & 90 & 70 & 0000.06 & 640010 & $6130-02$ & 1BF03051 & $61^{\circ} 47^{\prime} \mathrm{N}$ & $48^{\circ} 23^{\prime \prime 6}$ & $\mathrm{~N}$ & 105 & 100 & 0000.16 & 780010 & $6130-02$ \\
\hline 1BE04006 & $61^{\circ} 42^{\prime} \mathrm{N}$ & $48^{\circ} 20^{\prime}$ W & NE & 120 & 105 & 0000.13 & 780010 & $6130-02$ & 1BF03052 & $61^{\circ} 46^{\prime} \mathrm{N}$ & $48^{\circ} 24^{\prime} \mathrm{V}$ & N & 125 & 95 & 0000.29 & 780010 & $6130-02$ \\
\hline & $61^{\circ} 44^{\prime} \mathrm{N}$ & $48^{\circ} 18^{\prime} \mathrm{W}$ & $\mathrm{NV}$ & 125 & 95 & & 770010 & 0 & 1BF03053 & $61^{\circ} 47^{\prime} \mathrm{N}$ & $48^{\circ} 25^{\prime \prime}$ & $\mathrm{N}$ & 135 & 87 & 0001.75 & 276010 & $6130-02$ \\
\hline 1BE04008 & $61^{\circ} 44^{\prime} \mathrm{N}$ & $48^{\circ} 17^{\prime} \mathrm{W}$ & $\mathrm{W}$ & 125 & 100 & 0000.43 & 770010 & $6130-02$ & 1BF03054 & $61^{\circ} 47$, N & $48^{\circ} 27 \cdot \mathrm{V}$ & $\mathrm{N}$ & 105 & 87 & 0000.26 & 770010 & $6130-02$ \\
\hline 1BE04009 & $61^{\circ} 43^{\prime} \mathrm{N}$ & $48^{\circ} 16^{\prime} \mathrm{W}$ & su & 130 & 95 & 0000.52 & 770010 & $6130-02$ & 1BF03055 & $61^{\circ} 48^{\prime} \mathrm{N}$ & $48^{\circ} 30^{\circ} \mathrm{W}$ & $\mathrm{N}$ & 13 & 90 & 0000.57 & 750010 & $6130-02$ \\
\hline 1BE04010 & $61^{\circ} 43^{\prime} \mathrm{N}$ & $48^{\circ} 15^{\prime} \mathrm{W}$ & E & 120 & 105 & 0000.12 & 780010 & $6130-02$ & 1BF08001 & $61^{\circ} 51^{\prime} \mathrm{N}$ & $48^{\circ} 43^{\prime} \mathrm{V}$ & NW & 105 & 85 & 0000.28 & 780010 & $6130-02$ \\
\hline 1BE04011 & $61^{\circ} 44^{\prime} \mathrm{N}$ & $48^{\circ} 16^{\prime} \mathrm{W}$ & $\mathrm{NE}$ & 130 & 95 & & 270112 & $6130-02$ & $1 \mathrm{BF} 08002$ & $61^{\circ} 47 \cdot \mathrm{N}$ & $48^{\circ} 43^{\prime} \mathrm{W}$ & N & 110 & 95 & 0000.31 & 780010 & $6130-02$ \\
\hline $1 \mathrm{BE} 04012$ & $61^{\circ} 45^{\prime} \mathrm{N}$ & $48^{\circ} 15^{\prime} \mathrm{W}$ & $\mathrm{NE}$ & 130 & 115 & 0000.02 & 780010 & $6130-02$ & 1BF09001 & $61^{\circ} 47 \cdot \mathrm{N}$ & $48^{\circ} 38^{\prime} \mathrm{w}$ & NW & 120 & 85 & 0000.78 & 770010 & $6130-02$ \\
\hline 1BE04013 & $61^{\circ} 45^{\prime} \mathrm{N}$ & $48^{\circ} 15^{\prime} \mathrm{W}$ & $\mathrm{s}$ & 130 & 110 & 0000.08 & 780010 & $6130-02$ & 1BF09002 & $61^{\circ} 46^{\prime} \mathrm{N}$ & $48^{\circ} 39^{\prime} \mathrm{w}$ & NW & 110 & 100 & 0000.06 & 770010 & 6130-02 \\
\hline 1BE04014 & $61^{\circ} 46^{\prime} \mathrm{N}$ & $48^{\circ} 10^{\prime} \mathrm{W}$ & $\mathrm{NE}$ & 120 & 90 & 0000.45 & 770110 & $6130-02$ & 1BF09003 & $61^{\circ} 46^{\prime} \mathrm{N}$ & $48^{\circ} 39^{\prime \prime}$ & NW & 130 & 120 & 0000.12 & 780010 & $6130-02$ \\
\hline 1BE04015 & $61^{\circ} 48^{\prime} \mathrm{N}$ & $48^{\circ} 01^{\prime} \mathrm{W}$ & Su & 180 & 70 & 0137.33 & 160113 & $6130-02$ & 1BF09004 & $61^{\circ} 45^{\prime} \mathrm{N}$ & $48^{\circ} 39^{\prime} \mathrm{W}$ & $\because 7$ & 120 & 100 & 0000.30 & 750010 & $6130-02$ \\
\hline
\end{tabular}


LOW AREA CODE MAP

$\begin{array}{llll}120 & 0000.08 & 780010 & 6130-02\end{array}$
$120 \quad 0000.06$ $\begin{array}{ll}20 & 0000.02 \\ 95 & 0000.22\end{array}$ $100 \quad 0000.19$ $95 \quad 0000.47$

$100 \quad 0000.83$

$85 \quad 0000.02$ $105 \quad 0000.03$ $\begin{array}{ll}70 & 0002.69\end{array}$ $\begin{array}{ll}75 & 0000.26 \\ 80 & 0000.11\end{array}$ $\begin{array}{ll}100 & 0000.04 \\ 100 & 0000.48\end{array}$ $\begin{array}{ll}100 & 0000.48 \\ 105 & 0000.24\end{array}$ 1050000.24 0002.11 $\begin{array}{ll}75 & 0000.48 \\ 72 & 0000.94\end{array}$ $72 \quad 0001.43$ $\begin{array}{ll}72 & 0001.43 \\ 72 & 0001.15\end{array}$ 850000.50 $\begin{array}{ll}0000.33 \\ 95 & 0000.34\end{array}$ 0000.32
95 $90 \quad 0001.78$ $85 \quad 0004.47$ $85 \quad 0001.12$ $\begin{array}{ll}85 & 0000.68 \\ 75 & 0000.10\end{array}$ 0000.36
-5000.14 $\begin{array}{ll}85 & 0000.15 \\ 90 & 0000.14\end{array}$ $70 \quad 0001.10$ 850000.71 $85 \quad 0000.54$ 0000.63
70 $58 \quad 0200.95$ $\begin{array}{ll}0914.07 \\ 60 & 0293.04\end{array}$ $\begin{array}{ll}60 & 0293.04 \\ 60 & 0116.92\end{array}$ 0048.09
80 $\begin{array}{ll}80 & 0438.89 \\ 60 & 0197.21\end{array}$ \begin{tabular}{ll}
60 & 0197.21 \\
\hline 0 & 0469.49
\end{tabular} $95 \quad 0083.01$ $\begin{array}{ll}2 & 0104.70 \\ 60 & 0220.63\end{array}$

$\begin{array}{ll}65 & 0220.63 \\ 65 & 0000.32\end{array}$ 0030.18 0000.20 $\begin{array}{ll}60 & 0047.85 \\ 609.11\end{array}$ 50000.69 $\begin{array}{ll}90 & 0002.20 \\ 85 & 0001.23\end{array}$ $80 \quad 0000.54$
6130-02 $780010 \quad 6130-02$ $780010 \quad 6130-02$ $780010 \quad 6130-02$ $643312 \quad 6130-02$ 6130112 6130-02 $780010 \quad 6130-02$ $633112 \quad 6130-02$ 6130-02 $6130-02$
$6130-02$ $656110 \quad 6130-02$ $780010 \quad 6130-02$ $643112 \quad 6130-02$
$770110 \quad 6130-02$ $770112 \quad 6130-02$ $643212 \quad 6130-02$ 6130-02 $770010 \quad 6130-02$ $6130-02$
$643012 \quad 6130-02$ $643112 \quad 6130-02$ 643112 6130-02 $70010 \quad 6130-02$ $770010 \quad 6130-02$ $780010 \quad 6130-02$ $780010 \quad 6130-02$ $770010 \quad 6130-02$
770010 $770010 \quad 6130-02$ $630112 \quad 6130-02$ 163112 6200-02 164111 6200-02 163112 6200-03 $63112 \quad 6200-03$ $163112 \quad 6200-02$ $163112 \quad 6200-02$ 633112 6200-02 $67112 \quad 6230-02$ $200110 \quad 6230-02$ $\begin{array}{ll}167113 & 6230-02 \\ 260000 & 6230-03\end{array}$ $\begin{array}{ll}260000 & 6230-03 \\ 167113 & 6230-02\end{array}$ 6230-02 $540111 \quad 6230-02$ $540111 \quad 6230-02$ $167112 \quad 6230-02$
CODE

MAP

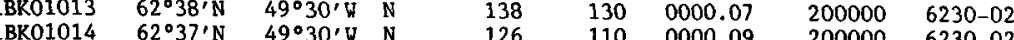
$\begin{array}{llllllll}1 \text { BRR01015 } 62^{\circ} 39 \cdot \mathrm{N} & 49^{\circ} 29^{\prime} \mathrm{W} & \mathrm{NE} & 130 & 105 & 0000.14 & 200000 & 6230-02\end{array}$

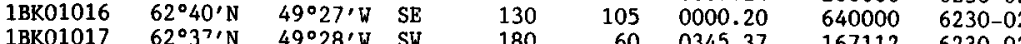

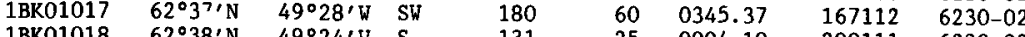

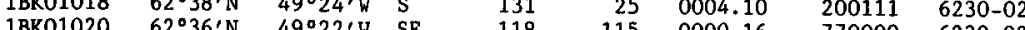
$\begin{array}{llllllll}1 \mathrm{BKO} 021 & 62^{\circ} 37^{\prime} \mathrm{N} & 49019, \mathrm{~V} & 118 & 115 & 0000.16 & 770000 & 6230-02\end{array}$

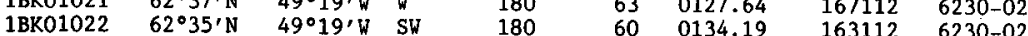

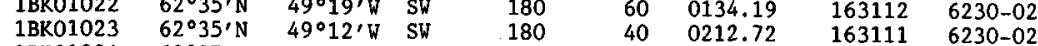

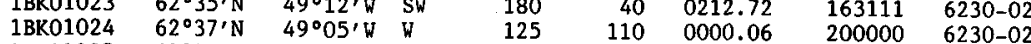

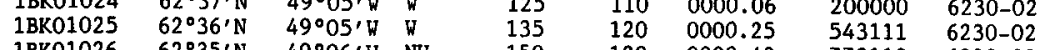

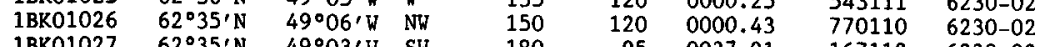

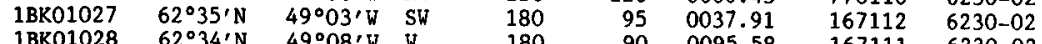
$\begin{array}{lllllllll}1 \mathrm{BKO} 01028 & 62^{\circ} 34^{\prime} \mathrm{N} & 49^{\circ} 08^{\prime} \mathrm{W} & \mathrm{W} & 180 & 90 & 0095.58 & 167111 & 6230-02 \\ 1 \mathrm{BKO} 01029 & 62^{\circ} 29^{\prime} \mathrm{N} & 49^{\circ} 13^{\prime} \mathrm{W} & \mathrm{W} & 180 & 21 & 0290.66 & 166111 & 6200-02\end{array}$

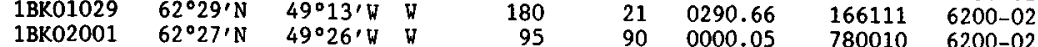

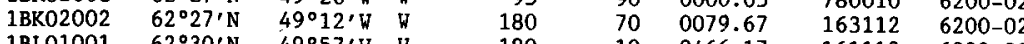

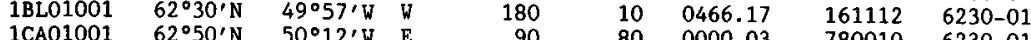

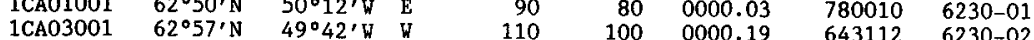

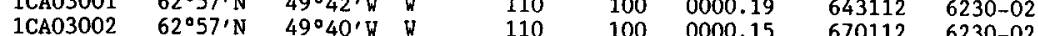

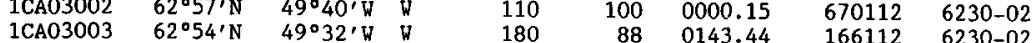

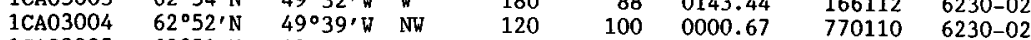
$\begin{array}{lllllllll}1 \mathrm{CA} 03005 & 62^{\circ} 51^{\prime} \mathrm{N} & 499^{\circ} 42, \mathrm{~W} & \mathrm{NW} & 115 & 90 & 0001.20 & 770110 & 6230-02\end{array}$

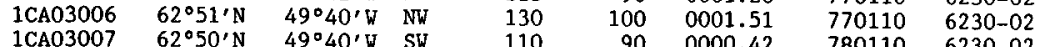

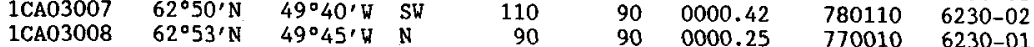

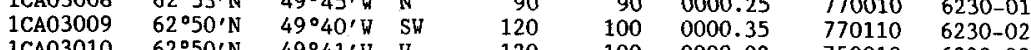

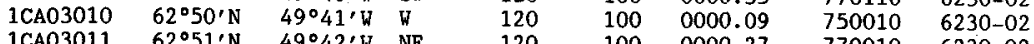

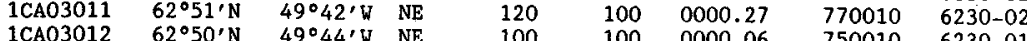

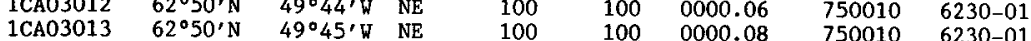

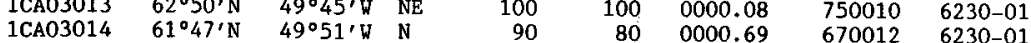

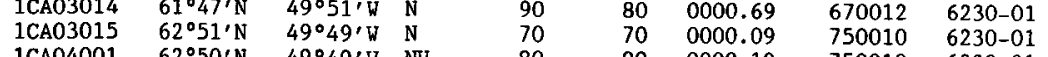

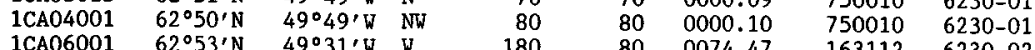

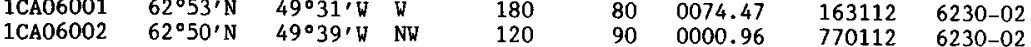

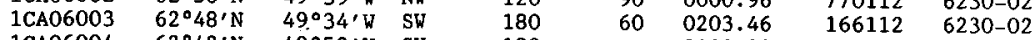
$\begin{array}{llllllll}1 \mathrm{CA} 06004 & 62^{\circ} 42^{\prime} \mathrm{N} & 49^{\circ} 52^{\prime} \mathrm{W} & \mathrm{SW} & 180 & 0102.92 & 162111 & 6230-01\end{array}$

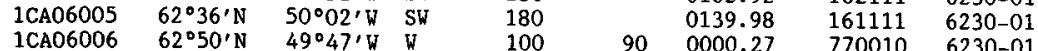

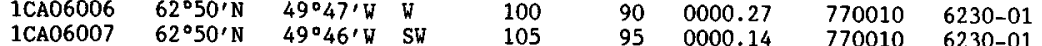
$\begin{array}{rrrrrrrrr}1 \mathrm{CA06007} & 62^{\circ} 50^{\prime} \mathrm{N} & 49^{\circ} 46^{\prime} \mathrm{W} & \mathrm{SW} & 105 & 95 & 0000.14 & 770010 & 6230-01 \\ 1 \mathrm{CA06008} & 62^{\circ} 49^{\prime} \mathrm{N} & 490^{\circ} 43^{\prime} \mathrm{W} & \text { SW } & 110 & 100 & 0000.10 & 750010 & 6230-02\end{array}$

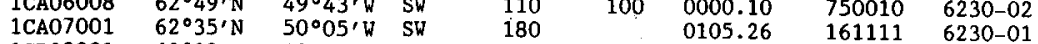

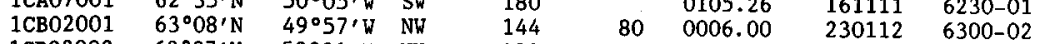

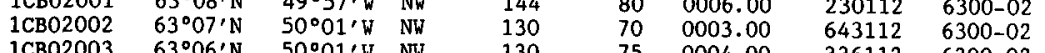

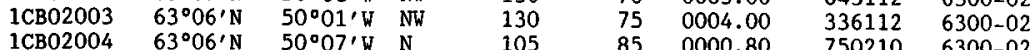

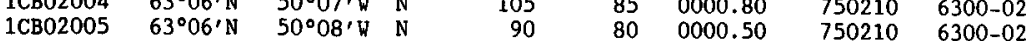

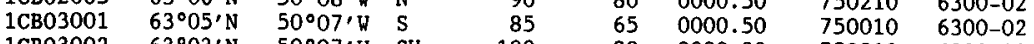
$\begin{array}{lllllllll}1 \mathrm{CB} 03002 & 63^{\circ} 03^{\prime} \mathrm{N} & 50^{\circ} 07^{\prime} \mathrm{W} & \mathrm{SW} & 100 & 80 & 0000.20 & 750210 & 6300-02\end{array}$ $\begin{array}{lllllllll}1 \mathrm{CB} 04001 & 63^{\circ} 03^{\prime} \mathrm{N} & 50^{\circ} 06^{\prime} \mathrm{W} & \mathrm{E} & 100 & 80 & 0001.00 & 233210 & 6300-02\end{array}$ \begin{tabular}{lllllllll}
$1 \mathrm{CBO} 4002$ & $63^{\circ} 04^{\prime} \mathrm{N}$ & $50^{\circ} 02^{\prime} \mathrm{W}$ & $\mathrm{SW}$ & 107 & 80 & 0009.00 & 360110 & $6300-02$ \\
\hline
\end{tabular}

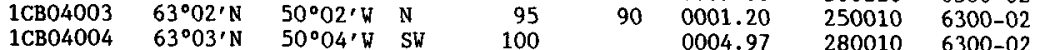
$\begin{array}{llllllll}1 \mathrm{CBO} 0004 & 63^{\circ} 03^{\prime} \mathrm{N} & 50^{\circ} 04^{\prime} \mathrm{W} & \mathrm{SW} & 100 & 0004.97 & 280010 & 6300-02 \\ 1 \mathrm{CBO} 4005 & 63^{\circ} 03^{\prime} \mathrm{N} & 50^{\circ} 03^{\prime} \mathrm{W} & \mathrm{NW} & & 0000.25 & 600010 & 6300-02\end{array}$ $\begin{array}{lllllllll}1 \mathrm{CB} 04005 & 63^{\circ} 03^{\prime} \mathrm{N} & 50^{\circ} 03^{\prime} \mathrm{W} & \mathrm{NW} & & & 0000.25 & 600010 & 6300-02 \\ 1 \mathrm{CB} 04006 & 63^{\circ} 00^{\prime} \mathrm{N} & 49^{\circ} 59^{\prime} \mathrm{W} & \mathrm{SW} & 100 & 80 & 0000.10 & 770010 & 6300-02\end{array}$ $\begin{array}{lllllllll}1 \mathrm{CB} 04006 & 63^{\circ} 00^{\prime} \mathrm{N} & 49^{\circ} 59^{\prime} \mathrm{W} & \mathrm{SW} & 100 & 80 & 0000.10 & 770010 & 6300-02 \\ 1 \mathrm{CB} 05001 & 63^{\circ} 02^{\prime} \mathrm{N} & 49^{\circ} 57^{\prime} \mathrm{W} & \mathrm{E} & 105 & 90 & 0003.90 & 233110 & 6300-02\end{array}$ $\begin{array}{lllllllll}1 \mathrm{CBO} 0002 & 63^{\circ} 01^{\prime} \mathrm{N} & 4^{\circ} 9^{\circ} 9^{\prime} \mathrm{W} & \mathrm{N} & 105 & 75 & 0008.90 & 233110 & 6300-02 \\ 1 \mathrm{CBB} 5003 & 63^{\circ} 04^{\prime} \mathrm{N} & 50^{\circ} 00^{\prime} \mathrm{W} & \mathrm{SE} & 115 & 85 & 0004.00 & 360110 & 6300-02\end{array}$ 
CODE LAT LONG ORI HIGH LOW AREA MO

\begin{tabular}{|c|c|c|c|c|c|c|c|c|}
\hline 1СB06001 & $63^{\circ} 05^{\prime} \mathrm{N}$ & $49^{\circ} 58^{\prime} \mathrm{W}$ & $\mathrm{SE}$ & 115 & 75 & 0010.00 & 363112 & $6300-02$ \\
\hline $1 \mathrm{CB} 07001$ & $63^{\circ} 06^{\prime} \mathrm{N}$ & $49^{\circ} 58^{\prime} \mathrm{w}$ & $\mathrm{NE}$ & 130 & 100 & 0002.00 & 366010 & $6300-02$ \\
\hline $1 \mathrm{CB} 07002$ & $63^{\circ} 07$ N & $49^{\circ} 48^{\prime} \mathrm{w}$ & $\mathrm{NW}$ & 120 & 105 & 0001.00 & 640010 & $6300-03$ \\
\hline $1 \mathrm{CB} 07003$ & $63^{\circ} 08^{\prime} \mathrm{N}$ & $49^{\circ} 47^{\prime} \mathrm{V}$ & SE & 141 & 105 & 0001.50 & 541110 & $6300-03$ \\
\hline 1 CB07004 & $63^{\circ} 07 \cdot \mathrm{N}$ & $49^{\circ} 47 \cdot \mathrm{V}$ & $\mathrm{NW}$ & 100 & 100 & 0000.50 & 753210 & $6300-03$ \\
\hline 1 CB07005 & $63^{\circ} 07 \cdot \mathrm{N}$ & $49^{\circ} 45, \mathrm{w}$ & $\mathrm{NW}$ & 105 & 100 & 0000.50 & 750210 & $6300-03$ \\
\hline $1 \mathrm{CB} 07006$ & $63^{\circ} 08^{\prime} \mathrm{N}$ & $49^{\circ} 43^{\prime} \mathrm{W}$ & N & 110 & 90 & 0000.50 & 750210 & $6300-03$ \\
\hline $1 \mathrm{CB} 07007$ & $63^{\circ} 08^{\prime} \mathrm{N}$ & $49^{\circ} 44^{\prime} \mathrm{W}$ & E & 130 & 100 & 0000.20 & 643110 & $6300-03$ \\
\hline 1CB07008 & $63^{\circ} 09^{\prime} \mathrm{N}$ & $49^{\circ} 43^{\prime} \mathrm{W}$ & N & 105 & 85 & 0000.50 & 750212 & $6300-03$ \\
\hline $1 \mathrm{CB} 07009$ & $63^{\circ} 09^{\prime} \mathrm{N}$ & $49^{\circ} 27^{\prime} \mathrm{W}$ & N & 168 & 100 & 0001.00 & 643112 & $6300-03$ \\
\hline $1 \mathrm{CB} 07010$ & $63^{\circ} 09^{\prime} \mathrm{N}$ & $49^{\circ} 30^{\prime} \mathrm{W}$ & $\mathrm{N}$ & 120 & 100 & 0000.50 & 643112 & $6300-03$ \\
\hline $1 \mathrm{CB} 07011$ & $63^{\circ} 07^{\prime} \mathrm{N}$ & $49^{\circ} 35^{\prime} \mathrm{W}$ & W & 180 & 110 & 0120.56 & 166112 & $6300-03$ \\
\hline $1 \mathrm{CB} 07012$ & $63^{\circ} 08^{\prime} \mathrm{N}$ & $49^{\circ} 30^{\prime} \mathrm{V}$ & NE & 155 & 110 & 0001.00 & 543110 & $6300-03$ \\
\hline 1CB07013 & $63^{\circ} 08^{\prime} \mathrm{N}$ & $49^{\circ} 32^{\prime} \mathrm{W}$ & NE & 150 & 105 & 0001.50 & 646112 & $6300-03$ \\
\hline $1 \mathrm{CB} 07014$ & $63^{\circ} 09^{\prime} \mathrm{N}$ & $49^{\circ} 34^{\prime} \mathrm{v}$ & w & 120 & 110 & 0000.40 & 750212 & $6300-03$ \\
\hline 1 CB07015 & $63^{\circ} 08^{\prime} \mathrm{N}$ & $49^{\circ} 34^{\prime} \mathrm{W}$ & $\mathrm{N}$ & 140 & 110 & 0000.70 & 750210 & $6300-03$ \\
\hline $1 \mathrm{CB} 08001$ & $63^{\circ} 06^{\prime} \mathrm{N}$ & $49^{\circ} 35^{\prime} \mathrm{W}$ & $w$ & 180 & 100 & 0055.36 & 163111 & $6300-03$ \\
\hline $1 \mathrm{CB} 08002$ & $63^{\circ} 04^{\prime} \mathrm{N}$ & $49^{\circ} 39^{\prime} \mathrm{W}$ & W & 180 & 50 & 0357.14 & 164211 & $6300-03$ \\
\hline $1 \mathrm{CB} 08003$ & $63^{\circ} 02^{\prime} \mathrm{N}$ & $49^{\circ} 33^{\prime} \mathrm{W}$ & $\mathbf{N}$ & 135 & 100 & 0000.90 & 633410 & $6300-03$ \\
\hline 1 СВ08004 & $63^{\circ} 02^{\prime} \mathrm{N}$ & $49^{\circ} 34^{\prime} \mathrm{V}$ & N & 160 & 100 & 0001.00 & 633410 & $6300-03$ \\
\hline 1 CB08005 & $63^{\circ} 02 \cdot \mathrm{N}$ & $49^{\circ} 36^{\prime}$ W & N & 160 & 100 & 0001.00 & 633410 & $6300-03$ \\
\hline 1CB09001 & $63^{\circ} 02 \cdot \mathrm{N}$ & $49^{\circ} 33^{\prime} \mathrm{W}$ & s & 155 & 145 & 0000.13 & 780010 & $6300-03$ \\
\hline $1 \mathrm{CB} 09002$ & $63^{\circ} 02, \mathrm{~N}$ & $49^{\circ} 32^{\prime} \mathrm{V}$ & $\mathrm{N}$ & 105 & 100 & 0000.12 & 780010 & $6300-03$ \\
\hline $1 \mathrm{CB} 09003$ & $63^{\circ} 02$, N & $49^{\circ} 30^{\prime} \mathrm{W}$ & E & 125 & 105 & 0000.15 & 750010 & $6300-03$ \\
\hline 1 CB09004 & $63^{\circ} 01 \cdot \mathrm{N}$ & $49^{\circ} 31 \cdot \mathrm{W}$ & $\mathrm{NW}$ & 120 & 105 & 0000.25 & 750010 & $6300-03$ \\
\hline 1 CBO9005 & $63^{\circ} 02, \mathrm{~N}$ & $49^{\circ} 29^{\prime}$, & N & 125 & 100 & 0000.22 & 640010 & $6300-03$ \\
\hline $1 \mathrm{CB} 09006$ & $63^{\circ} 03^{\prime} \mathrm{N}$ & $49^{\circ} 30^{\prime} \mathrm{W}$ & E & 120 & 110 & 0000.05 & 750010 & $6300-03$ \\
\hline $1 \mathrm{CB} 09007$ & $63^{\circ} 03^{\prime} \mathrm{N}$ & $49^{\circ} 27 \cdot \mathrm{W}$ & SW & 125 & 115 & 0000.12 & 770010 & $6300-03$ \\
\hline $1 \mathrm{CB} 09008$ & $63^{\circ} 02^{\prime} \mathrm{N}$ & $49^{\circ} 22^{\prime} \mathrm{W}$ & $\mathrm{W}$ & 180 & 105 & 0032.58 & 167112 & $6300-03$ \\
\hline 1 СВ09009 & $63^{\circ} 01 \cdot \mathrm{N}$ & $49^{\circ} 24^{\prime} \mathrm{W}$ & NW & 180 & 98 & 0098.55 & 166112 & $6300-03$ \\
\hline 1 CB09010 & $63^{\circ} 00^{\prime} \mathrm{N}$ & $49^{\circ} 24^{\prime} \mathrm{W}$ & n & 120 & 105 & 00.17 & 750012 & $6300-03$ \\
\hline 1 CBO9011 & $63^{\circ} 01$ ' N & $49^{\circ} 27^{2} \cdot \mathrm{W}$ & N & 115 & 110 & 0000.07 & 780010 & $6300-03$ \\
\hline $1 \mathrm{CB} 09012$ & $63^{\circ} 00^{\prime} \mathrm{N}$ & $49^{\circ} 22^{\prime} \mathrm{V}$ & W & 100 & 85 & 0000.19 & 750010 & $6300-03$ \\
\hline $1 \mathrm{CB} 09013$ & $62^{\circ} 58^{\prime} \mathrm{N}$ & $49^{\circ} 24^{\prime} \mathrm{W}$ & $\mathrm{V}$ & 180 & 71 & 0102.02 & 166112 & $6230-02$ \\
\hline $1 \mathrm{CB} 09014$ & $62^{\circ} 58^{\prime} \mathrm{N}$ & $49^{\circ} 30^{\prime} \mathrm{W}$ & NE & 85 & 65 & 0000.63 & 750110 & $6230-02$ \\
\hline 1CB09015 & $62^{\circ} 58$, N & $49^{\circ} 31^{\prime} \mathrm{W}$ & $\mathrm{N}$ & 100 & 95 & 0000.43 & 750112 & $6230-02$ \\
\hline $1 \mathrm{CB} 09016$ & $63^{\circ} 00^{\prime} \mathrm{N}$ & $49^{\circ} 43^{\prime} \mathrm{W}$ & $\mathrm{N}$ & 95 & 85 & 0000.74 & 750112 & $6300-03$ \\
\hline $1 \mathrm{CB} 09017$ & $62^{\circ} 59^{\prime} \mathrm{N}$ & $49^{\circ} 32^{\prime} \mathrm{V}$ & NW & 85 & 80 & 0000.27 & 750010 & $6230-02$ \\
\hline 1СВ09018 & $62.59 \cdot \mathrm{N}$ & $49^{\circ} 32^{\prime} \mathrm{v}$ & $\mathrm{NE}$ & 85 & 80 & 0000.42 & 780010 & $6230-02$ \\
\hline 1 CB09019 & $62^{\circ} 59$ ' N & $49^{\circ} 32^{\prime} \mathrm{w}$ & $\mathrm{NE}$ & 80 & 80 & 0000.07 & 780010 & $6230-02$ \\
\hline 1 CB10001 & $62^{\circ} 58^{\prime} \mathrm{N}$ & $49^{\circ} 36^{\prime} \mathrm{W}$ & N & 95 & 85 & 0003.25 & 270112 & $6230-02$ \\
\hline $1 \mathrm{CB} 10002$ & $62^{\circ} 58^{\prime} \mathrm{N}$ & $49^{\circ} 39^{\prime} \mathrm{W}$ & W & 105 & 95 & 0000.23 & 270112 & $6230-02$ \\
\hline $1 \mathrm{CB} 10003$ & $62^{\circ} 58^{\prime} \mathrm{N}$ & $49^{\circ} 36^{\prime} \mathrm{V}$ & $\mathrm{N}$ & 120 & 105 & 0000.08 & 780010 & $6230-02$ \\
\hline $1 \mathrm{CB} 10004$ & $62 \cdot 58 \cdot \mathrm{N}$ & $49^{\circ} 35, \mathrm{~W}$ & $\mathrm{~N}$ & 110 & 100 & 0000.40 & 780010 & $6230-02$ \\
\hline $1 \mathrm{CB} 10005$ & $62^{\circ} 59 \cdot \mathrm{N}$ & $49^{\circ} 34^{\prime} \mathrm{W}$ & s & 105 & 100 & 0000.05 & 780010 & $6230-02$ \\
\hline 1 CB10006 & $62^{\circ} 58, \mathrm{~N}$ & $49^{\circ} 32^{\prime} \mathrm{W}$ & $v$ & 100 & 95 & 0000.05 & 780010 & $6230-02$ \\
\hline 1CB10007 & $62^{\circ} 58$, N & $49^{\circ} 33^{\prime} \mathrm{V}$ & v & 100 & 90 & 0000.62 & 750112 & $6230-02$ \\
\hline $1 \mathrm{CB} 10008$ & $62^{\circ} 58^{\prime} \mathrm{N}$ & $49^{\circ} 31^{\prime} \mathrm{W}$ & W & 95 & 90 & 0000.09 & 770010 & $6230-02$ \\
\hline $1 \mathrm{CB} 10009$ & $62057 \cdot \mathrm{N}$ & $49^{\circ} 30^{\prime} \mathrm{v}$ & s & 105 & 95 & 0000.11 & 780010 & $6230-02$ \\
\hline $1 \mathrm{CB} 10010$ & $62^{\circ} 57^{\circ} \mathrm{N}$ & $49^{\circ} 31^{\prime} \mathrm{V}$ & $v$ & 95 & 85 & 0000.12 & 780010 & $6230-02$ \\
\hline 1CB10011 & $62.57, \mathrm{~N}$ & $49^{\circ} 31^{\prime}$ w & $\mathrm{N}$ & 80 & 80 & 0000.09 & 780010 & $6230-02$ \\
\hline $1 \mathrm{CB} 10012$ & $62^{\circ} 57^{\prime} \mathrm{N}$ & $49^{\circ} 30^{\prime} \mathrm{W}$ & SW & 95 & 80 & 0000.35 & 750010 & $6230-02$ \\
\hline $1 \mathrm{CB} 10013$ & $62^{\circ} 56^{\prime} \mathrm{N}$ & $49^{\circ} 28^{\prime} \mathrm{W}$ & W & 180 & 75 & 0105.72 & 166112 & $6230-02$ \\
\hline $1 \mathrm{CB} 10014$ & $62^{\circ} 57 / \mathrm{N}$ & $49^{\circ} 40^{\prime} \mathrm{W}$ & NE & 100 & 80 & 0000.36 & 640110 & $6230-02$ \\
\hline 1CB10015 & $62^{\circ} 57 \cdot \mathrm{N}$ & $49^{\circ} 42^{\prime} \mathrm{W}$ & $\mathrm{N}$ & 95 & 80 & 0000.89 & 643112 & $6230-02$ \\
\hline 1CB10016 & $63^{\circ} 00^{\prime} \mathrm{N}$ & $49^{\circ} 42$ ' W & NE & 90 & 9 & 0000.13 & 750010 & $6300-03$ \\
\hline $1 \mathrm{CB} 11001$ & $62^{\circ} 57^{\prime} \mathrm{N}$ & $49^{\circ} 55^{\prime} \mathrm{W}$ & N & 50 & 50 & 0000.16 & 780010 & $6230-01$ \\
\hline $1 \mathrm{CB} 11002$ & $62^{\circ} 54^{\prime} \mathrm{N}$ & $50^{\circ} 00^{\prime} \mathrm{W}$ & N & 50 & 50 & 0000.05 & 780010 & $6230-01$ \\
\hline $1 \mathrm{CB} 12001$ & $62 \cdot 52$, N & $50^{\circ} 05^{\circ} \mathrm{W}$ & N & 40 & 40 & 0000.07 & 750110 & $6230-01$ \\
\hline 1CB12002 & $62^{\circ} 52^{\prime} \mathrm{N}$ & $50^{\circ} 04^{\prime} \mathrm{W}$ & NE & 50 & 40 & 0000.18 & 640112 & $6230-01$ \\
\hline $1 \mathrm{CB} 12003$ & $62^{\circ} 51^{\prime} \mathrm{N}$ & $50^{\circ} 06^{\prime} \mathrm{V}$ & $N W$ & 50 & 40 & 0000.15 & 770012 & $6230-01$ \\
\hline
\end{tabular}

LAT

LONG

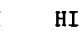

$\begin{array}{llll}1 \mathrm{CB} 12004 & 62^{\circ} 50^{\prime} \mathrm{N} & 50^{\circ} 10^{\prime} \mathrm{V} & \mathrm{NW} \\ 1 \mathrm{CB} 12005 & 62^{\circ} 50^{\prime} \mathrm{N} & 50^{\circ} 11^{\prime} \mathrm{W} & \mathrm{N}\end{array}$

$1 \mathrm{CC} 0300163^{\circ} 18^{\prime} \mathrm{N} \quad 50^{\circ} 45^{\prime} \mathrm{W}$

$\begin{array}{lll}1 \mathrm{CC} 03002 & 63^{\circ} 19 \cdot \mathrm{N} & 50^{\circ} 36 / \mathrm{W} \\ 1 \mathrm{CC} 03003 & 63^{\circ} 18^{\prime} \mathrm{N} & 50^{\circ} 38^{\prime} \mathrm{V}\end{array}$

$\begin{array}{llll}1 \mathrm{CC} 03004 & 63^{\circ} 18, \mathrm{~N} & 50^{\circ} 40^{\prime} \mathrm{N} & \mathrm{N}\end{array}$

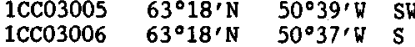

$\begin{array}{llll}1 \mathrm{CC} 03007 & 63^{\circ} 18^{\prime} \mathrm{N} & 50^{\circ} 35^{\prime} \mathrm{WI} & \mathrm{S} \\ 1 \mathrm{NE}\end{array}$

$1 \mathrm{CC} 0300863^{\circ} 17$ 'N $50^{\circ} 32^{\prime} \mathrm{W} N$

$1 \mathrm{CC} 0300963^{\circ} 17^{\prime} \mathrm{N} \quad 50^{\circ} 33^{\prime} \mathrm{W}, \mathrm{N}$

$1 \mathrm{CC} 03011$ 63.16, N $50^{\circ} 35^{\prime} \mathrm{W}$

$\begin{array}{llll}1 \mathrm{CC} 03012 & 63^{\circ} 16^{\prime} \mathrm{N} & 50^{\circ} 36^{\prime} \mathrm{N} N & \mathrm{~N}\end{array}$

$\begin{array}{llll}1 \mathrm{CC} 03013 & 63^{\circ} 15^{\prime} \mathrm{N} & 50^{\circ} 36^{\prime} \mathrm{V} & \mathrm{NW} \\ 1 \mathrm{CC} 03014 & 63^{\circ} 15 \cdot \mathrm{N} & 50^{\circ} 37^{\prime} \mathrm{N} & \mathrm{NW}\end{array}$

$50^{\circ} 37, \mathrm{NW}$

$\begin{array}{llll}1 \mathrm{CCO} 0001 & 63^{\circ} 14^{\prime} \mathrm{N} & 50^{\circ} 36^{\prime} \mathrm{V} & \mathrm{SE} \\ 1 \mathrm{CC} 05001 & 63^{\circ} 16^{\prime} \mathrm{N} & 50^{\circ} 32, \mathrm{~V} & \mathrm{~B}\end{array}$

$1 \mathrm{CC} 0500263^{\circ} 16^{\prime} \mathrm{N} \quad 50^{\circ} 33^{\prime} \mathrm{W} \mathrm{E}$

$1 \mathrm{CC} 05003 \quad 63^{\circ} 18^{\prime} \mathrm{N} \quad 50^{\circ} 30^{\prime} \mathrm{W} \quad \mathrm{NE}$

$\begin{array}{llll}1 \mathrm{CC} 05004 & 63^{\circ} 19^{\prime} \mathrm{N} & 50^{\circ} 28^{\prime} \mathrm{W} & \mathrm{E} \\ 1 \mathrm{CC} 05005 & 63^{\circ} 18^{\prime} \mathrm{N} & 50^{\circ} 27^{\prime} \mathrm{V} & \mathrm{NE}\end{array}$

$1 \mathrm{CC} 05006$ 63०19'N $50^{\circ} 25^{\prime} \mathrm{W} S$

$1 \mathrm{CC} 0500763^{\circ} 19^{\prime} \mathrm{N} \quad 50^{\circ} 25^{\prime} \mathrm{W}$ E

$\begin{array}{llll}1 \mathrm{CC} 05008 & 63^{\circ} 19^{\prime} \mathrm{N} & 50^{\circ} 24^{\prime} \mathrm{W} & \mathrm{S} \\ 1 \mathrm{CC} 05009 & 63^{\circ} 20^{\prime} \mathrm{N} & 50^{\circ} 23^{\prime} \mathrm{W} & \mathrm{S}\end{array}$

$\begin{array}{llll}1 \mathrm{CC} 05009 & 63^{\circ} 20^{\prime} \mathrm{N} & 50^{\circ} 23^{\prime} \mathrm{W} & \mathrm{S} \\ 1 \mathrm{CC} 05010 & 63^{\circ} 15^{\prime} \mathrm{N} & 50^{\circ} 26^{\prime} \mathrm{W} & \mathrm{W}\end{array}$

$\begin{array}{lll}1 \mathrm{CC} 07001 & 63^{\circ} 16^{\prime} \mathrm{N} & 50^{\circ} 24^{\prime} \mathrm{W} \\ 1 \mathrm{CC} 07002 & 63^{\circ} 21^{\prime} \mathrm{N} & 50^{\circ} 17^{\prime} \mathrm{W}\end{array}$

$1 \mathrm{CC} 0700363^{\circ} 21^{\prime} \mathrm{N} \quad 50^{\circ} 12^{\prime} \mathrm{W}$

$1 \mathrm{CC} 0800263^{\circ} 19 \cdot \mathrm{N} \quad 50^{\circ} 08^{\prime} \mathrm{W} \mathrm{SW}$

$1 \mathrm{CC} 0800363^{\circ} 18^{\prime} \mathrm{N} \quad 50^{\circ} 08^{\prime} \mathrm{W} \quad \mathrm{E}$

$1 \mathrm{CC} 0800463^{\circ} 17^{\prime} \mathrm{N} \quad 50^{\circ} 08^{\prime} \mathrm{W}$

$1 \mathrm{CC} 09001$ 63.19'N $49^{\circ} 16^{\prime} \mathrm{W}$ SE

$1 \mathrm{CC} 09002$

$63^{\circ} 19^{\prime} \mathrm{N} \quad 49^{\circ} 16^{\prime} \mathrm{W}$ NW

$1 \mathrm{CC} 09003$

$63^{\circ} 16^{\prime} \mathrm{N} \quad 49^{\circ} 25^{\prime} \mathrm{W}$

1 cC0900

$63^{\circ} 14^{\prime} \mathrm{N} \quad 49^{\circ} 17^{\prime} \mathrm{W}$

$1 \mathrm{cc09007}$

$63^{\circ} 12^{\prime} \mathrm{N} \quad 49^{\circ} 15^{\prime} \mathrm{W}$ NV

(cc0900

$\begin{array}{ll}63^{\circ} 12^{\prime} \mathrm{N} & 49^{\circ} 17^{\prime} \mathrm{W} \\ 63^{\circ} 11^{\prime} \mathrm{N} & 49^{\circ} 12^{\prime} \mathrm{N}\end{array}$

$\begin{array}{llll}1 \mathrm{C} C 09009 & 63^{\circ} 11^{\prime} \mathrm{N} & 49^{\circ} 18^{\prime} \mathrm{W} & \mathrm{NW}\end{array}$

1 CC09011 63010'N $49^{\circ} 19^{\prime} \mathrm{W}$

$1 \mathrm{CC} 0901263^{\circ} 10^{\prime} \mathrm{N} \quad 49^{\circ} 22^{\prime} \mathrm{W} N \mathrm{NW}$

$\begin{array}{llll}1 \mathrm{CC} 09013 & 63^{\circ} 09^{\prime} \mathrm{N} & 49^{\circ} 23^{\prime} \mathrm{W} & \mathrm{NW} \\ 1 \mathrm{CC} 09014 & 63^{\circ} 09^{\prime} \mathrm{N} & 49^{\circ} 26^{\prime} \mathrm{W} & \mathrm{NE}\end{array}$

$1 \mathrm{CC} 09014$

1CC09016 $63^{\circ} 10^{\prime} \mathrm{N} \quad 49^{\circ} 26^{\prime} \mathrm{W}$ E

$1 \mathrm{CC} 09017$

$63^{\circ} 10^{\prime} \mathrm{N} \quad 49^{\circ} 28^{\prime} \mathrm{W} N \mathrm{NW}$

$\begin{array}{lll}63^{\circ} 10^{\prime} \mathrm{N} & 49^{\circ} 43^{\prime} \mathrm{W} & \mathrm{N} \\ 63^{\circ} 10^{\prime} \mathrm{N} & 49^{\circ} 44^{\prime} \mathrm{W} & \mathrm{N} \\ 633^{\circ} 09^{\prime} \mathrm{N} & 49^{\circ} 46^{\prime} \mathrm{W} & \mathrm{N}\end{array}$

1 CC09019

$63^{\circ} 10^{\prime} \mathrm{N}$

1CC09020

$63^{\circ} 09^{\prime} \mathrm{N} \quad 49^{\circ} 47^{\prime} \mathrm{W}$

$1 C C 09022 \quad 63^{\circ} 09^{\prime} \mathrm{N} \quad 49^{\circ} 53^{\prime} \mathrm{W} N \mathrm{NW}$

1CC11001 $63^{\circ} 07^{\prime} \mathrm{N} \quad 50^{\circ} 15^{\prime} \mathrm{W}$ NE

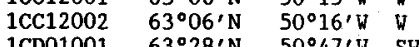

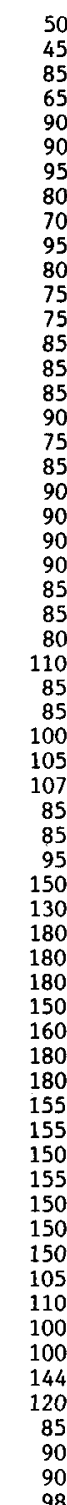

$780012 \quad 6230-01$

$\begin{array}{ll}270010 & 6300-01 \\ 750010 & 6300-01\end{array}$

$643110 \quad 6300-01$

$750110 \quad 6300-01$

$630010 \quad 6300-01$

$750110 \quad 6300-01$

$630010 \quad 6300-02$

$643010 \quad 6300-02$

$643010 \quad 6300-02$

633010
$6300-02$

$630010 \quad 6300-01$

$630010 \quad 6300-01$

$630010 \quad 6300-01$

$750110 \quad 6300-02$

6300-02

640110 6300-02

$750110 \quad 6300-02$

$233110 \quad 6300-02$

$233110 \quad 6300-02$

$233110 \quad 6300-02$

6300-02

$230110 \quad 6300-02$

263110 6300-02

233110 6300-02

$233110 \quad 6300-02$

$233110 \quad 6300-02$

$701010 \quad 6300-02$

$646110 \quad 6300-03$

$\begin{array}{ll}543110 & 6300-03 \\ 163112 & 6300-03\end{array}$

$163112 \quad 6300-03$

$6300-03$

$167113 \quad 6300-03$

$167112 \quad 6300-03$

$647112 \quad 6300-03$

$640112 \quad 6300-03$

$6431126300-03$

$643112 \quad 6300-03$

$643112 \quad 6300-03$

$643112 \quad 6300-03$

$640412 \quad 6300-03$

$6421126300-03$

642112 6300-03

$755210 \quad 6300-02$

$755210 \quad 6300-02$

$\begin{aligned} & 755210 \\ & 643110\end{aligned} 6300-01$ 


\begin{tabular}{|c|c|c|c|c|c|c|c|c|c|c|c|c|c|}
\hline CODE & LAT & LONG & ORI & HIGH & LOW & AREA & $\begin{array}{c}\text { MO } \\
\text { CODE }\end{array}$ & MAP & CODE & LAT & LONG & ORI & \\
\hline $1 \mathrm{CD} 03001$ & $63^{\circ} 28^{\prime} \mathrm{N}$ & $50^{\circ} 55^{\prime} \mathrm{W}$ & E & 98 & 55 & 0000.84 & 700010 & $6300-01$ & & & & $\mathbf{E}$ & \\
\hline $1 \mathrm{CD} 03002$ & $63^{\circ} 29^{\prime} \mathrm{N}$ & $50^{\circ} 55^{\prime} \mathrm{W}$ & SE & 102 & 48 & 0003.28 & 523111 & $6300-01$ & 1 CD04056 & Nי2 $63^{\circ} 27^{2}$ & $50^{\circ} 10^{\prime} \mathrm{W}$ & E & 12 \\
\hline 1CD03003 & $63^{\circ} 29^{\prime} \mathrm{N}$ & $50^{\circ} 54^{\prime} \mathrm{V}$ & V & 85 & 78 & 0000.20 & 700010 & $6300-01$ & $1 \mathrm{CD} 04057$ & $63^{\circ} 28^{\prime} \mathrm{N}$ & $50^{\circ} 12^{\prime} \mathrm{v}$ & $\mathrm{NE}$ & \\
\hline 1CD03004 & $63^{\circ} 29^{\prime} \mathrm{N}$ & $50^{\circ} 52^{\prime} \mathrm{W}$ & $\mathbf{S}$ & 85 & 52 & 0001.24 & 500112 & $6300-01$ & $1 \mathrm{CD} 04058$ & $63^{\circ} 28^{\prime} \mathrm{N}$ & $50^{\circ} 15^{\prime} \mathrm{W}$ & $\mathbf{N}$ & \\
\hline 1CD03005 & $63^{\circ} 29^{\prime} \mathrm{N}$ & $50^{\circ} 49^{\prime} \mathrm{W}$ & $\mathrm{NE}$ & 80 & 55 & 0000.70 & 653100 & $6300-01$ & $1 \mathrm{CD} 04059$ & $63^{\circ} 27 \cdot \mathrm{N}$ & $50^{\circ} 19^{\prime} \mathrm{W}$ & $\mathbf{N}$ & \\
\hline $1 \mathrm{CD} 03006$ & $63^{\circ} 28^{\prime} \cdot \mathrm{N}$ & $50^{\circ} 49, \mathrm{~W}$ & $\mathrm{~N}$ & 70 & 50 & 0000.60 & 650010 & $6300-01$ & 1 CD04060 & $63^{\circ} 27 \mathrm{~N}$ & $50^{\circ} 20^{\prime} \mathrm{W}$ & $\mathrm{N}$ & \\
\hline $1 \mathrm{CD} 03007$ & $63^{\circ} 27^{\prime} \mathrm{N}$ & $50^{\circ} 42 \cdot \mathrm{W}$ & $\mathrm{N}$ & 100 & 50 & 0000.70 & 650010 & $6300-01$ & 1CD04061 & $63^{\circ} 26^{\prime} \mathrm{N}$ & $50^{\circ} 23^{\prime} \mathrm{W}$ & $\mathbf{N}$ & \\
\hline 1CD04001 & $63^{\circ} 27^{\prime} \mathrm{N}$ & $50^{\circ} 48^{\prime} \mathrm{W}$ & SW & 85 & 15 & 0000.50 & 750110 & $6300-01$ & $1 \mathrm{CD} 04062$ & $63^{\circ} 26^{\prime} \mathrm{N}$ & $50^{\circ} 25^{\prime} \mathrm{W}$ & $\mathrm{N}$ & \\
\hline 1CD04002 & $63^{\circ} 28^{\prime} \mathrm{N}$ & $50^{\circ} 46^{\prime} \mathrm{W}$ & $\mathrm{NE}$ & 100 & 40 & 0002.60 & 530110 & $6300-01$ & $1 \mathrm{CD} 04063$ & $63^{\circ} 28^{\prime} \mathrm{N}$ & $50^{\circ} 26^{\circ} \mathrm{v}$ & N & \\
\hline $1 \mathrm{CD} 04003$ & $63^{\circ} 28^{\prime} \mathrm{N}$ & $50^{\circ} 47^{\prime} \mathrm{w}$ & E & 95 & 75 & 0000.30 & 750110 & $6300-01$ & $1 \mathrm{CD} 04064$ & $63^{\circ} 26^{\prime} \mathrm{N}$ & $50^{\circ} 28^{\prime}, \mathrm{W}$ & NW & \\
\hline 1CD04004 & $63^{\circ} 30^{\prime} \mathrm{N}$ & $50^{\circ} 50^{\prime} \mathrm{W}$ & NE & 90 & 55 & 0002.00 & 633110 & $6330-01$ & $1 \mathrm{CD} 04065$ & $63^{\circ} 26^{\prime} \mathrm{N}$ & $50^{\circ} 32^{\prime} \mathrm{V}$ & NW & \\
\hline $1 \mathrm{CD} 04005$ & $63^{\circ} 30^{\prime} \mathrm{N}$ & $50^{\circ} 36^{\prime} \mathrm{V}$ & SW & 120 & 95 & 0003.40 & 750110 & 6330-02 & $1 \mathrm{CD} 04066$ & $63^{\circ} 25^{\prime} \mathrm{N}$ & $50^{\circ} 31^{\prime} \mathrm{W}$ & SW & \\
\hline 1CD04006 & $63^{\circ} 31^{\prime} \mathrm{N}$ & $50^{\circ} 32^{\prime} \mathrm{W}$ & $\mathbf{s}$ & 140 & 60 & 0005.10 & 633112 & $6330-02$ & $1 \mathrm{CD04067}$ & $63^{\circ} 25^{\prime} \mathrm{N}$ & $50^{\circ} 32^{\prime} \mathrm{W}$ & SW & \\
\hline $1 \mathrm{CD} 04007$ & $63^{\circ} 30^{\prime} \mathrm{N}$ & $50^{\circ} 29^{\prime} \mathrm{W}$ & SW & 115 & 50 & 0002.10 & 643312 & $6330-02$ & ICDO4068 & $63^{\circ} 25^{\prime} \mathrm{N}$ & $50^{\circ} 33^{\prime \prime}$ & $\mathrm{NW}$ & \\
\hline $1 \mathrm{CD} 04008$ & $63^{\circ} 30^{\prime} \mathrm{N}$ & $50^{\circ} 27^{\prime} \mathrm{W}$ & SW & 110 & 80 & 0002.10 & 643312 & $6330-02$ & 1 CD04069 & $63^{\circ} 25^{\prime} \mathrm{N}$ & $50^{\circ} 35^{\prime} \mathrm{W}$ & NW & \\
\hline $1 \mathrm{CD} 04009$ & $63^{\circ} 30^{\prime} \mathrm{N}$ & $50^{\circ} 20^{\prime} \mathrm{V}$ & E & 125 & 50 & 0021.30 & 233112 & 6330 & $1 \mathrm{CD} 05001$ & $63^{\circ} 24^{\prime} \mathrm{N}$ & $50^{\circ} 34^{\prime} \mathrm{W}$ & $\mathbf{s}$ & \\
\hline $1 \mathrm{CD} 04010$ & $63^{\circ} 31^{\prime} \mathrm{N}$ & $50^{\circ} 17^{\prime} \mathrm{W}$ & E & 150 & 70 & 0011.20 & 223112 & $6330-02$ & $1 \mathrm{CD} 05002$ & $63^{\circ} 25^{\prime} \mathrm{N}$ & $50^{\circ} 27 \cdot \mathrm{W}$ & $\mathrm{W}$ & \\
\hline 1CD04011 & $63^{\circ} 31^{\prime} \mathrm{N}$ & $50^{\circ} 14^{\prime} \mathrm{W}$ & $\mathbf{s}$ & 150 & 140 & 0000.60 & 780010 & $6330-02$ & 1CD05003 & $63^{\circ} 25^{\prime} \mathrm{N}$ & $50^{\circ} 23, \mathrm{~V}$ & SE & \\
\hline 1CD04012 & $63^{\circ} 32^{\prime} \mathrm{N}$ & $50^{\circ} 10^{\prime} \mathrm{W}$ & s & 150 & 140 & 0001.30 & 760010 & $6330-02$ & $1 \mathrm{CD} 05004$ & $63^{\circ} 26^{\prime} \mathrm{N}$ & $50^{\circ} 22^{\prime \prime}$ & $\mathrm{SE}$ & 1 \\
\hline $1 \mathrm{CD} 04013$ & $63^{\circ} 31^{\prime} \mathrm{N}$ & $50^{\circ} 07^{\prime}$ & - & 100 & 90 & 0001.00 & 650112 & $6330-02$ & 1 CD05005 & $63^{\circ} 26^{\prime} \mathrm{N}$ & $50^{\circ} 20^{\prime} \mathrm{V}$ & SW & \\
\hline $1 \mathrm{CD} 04014$ & $63^{\circ} 32^{\prime} \mathrm{N}$ & $50^{\circ} 07^{\prime} \mathrm{W}$ & $\mathrm{s}$ & 110 & 100 & 0000.70 & 650012 & $6330-0$ & $1 \mathrm{CD} 05006$ & $63^{\circ} 25^{\prime} \mathrm{N}$ & $50^{\circ} 20^{\prime} \mathrm{W}$ & SW & \\
\hline $1 \mathrm{CD} 04015$ & $63^{\circ} 32^{\prime} \mathrm{N}$ & $50^{\circ} 07^{\prime} \mathrm{W}$ & $\mathbf{s}$ & 140 & 140 & 0000.60 & 780010 & $6330-02$ & 1CD06001 & $63^{\circ} 26^{\prime} \mathrm{N}$ & $50^{\circ} 18^{\prime} \mathrm{W}$ & $\mathrm{SW}$ & \\
\hline 1CD04016 & $63^{\circ} 32^{\prime} \mathrm{N}$ & $50^{\circ} 04^{\prime} \mathrm{W}$ & $\mathbf{s}$ & 145 & 105 & 0002.20 & 780010 & $6330-02$ & $1 \mathrm{CD} 06002$ & $63^{\circ} 26^{\prime} \mathrm{N}$ & $50^{\circ} 16^{\prime} \mathrm{W}$ & SE & \\
\hline 1CD04017 & $63^{\circ} 32^{\prime} \mathrm{N}$ & $50^{\circ} 02^{\prime} \mathrm{W}$ & NE & 110 & 100 & 0000.90 & 643112 & $6330-02$ & 1CD06003 & $63^{\circ} 26^{\prime} \mathrm{N}$ & $50^{\circ} 13^{\prime} \mathrm{V}$ & $\mathrm{SW}$ & 1 \\
\hline 1 CD 04018 & $63^{\circ} 33^{\prime} \mathrm{N}$ & $50^{\circ} 00^{\prime} \mathrm{W}$ & NE & 105 & 70 & 0000.60 & 753110 & $6330-02$ & $1 \mathrm{CD} 06004$ & $63^{\circ} 25^{\prime} \mathrm{N}$ & $50^{\circ} 15^{\prime} \mathrm{V}$ & NW & \\
\hline $1 \mathrm{CD} 04019$ & $63^{\circ} 34^{\prime} \mathrm{N}$ & $49^{\circ} 58^{\prime}$ & SW & 180 & 48 & 0295.98 & 166112 & 6330 & $1 \mathrm{CD} 07001$ & $63^{\circ} 24^{\prime} \mathrm{N}$ & $50^{\circ} 10^{\prime} \mathrm{W}$ & NW & \\
\hline $1 \mathrm{CD} 04020$ & $63^{\circ} 31^{\prime} \mathrm{N}$ & $49^{\circ} 56^{\prime} \mathrm{V}$ & W & 115 & 100 & 0000.50 & 700010 & $6330-03$ & $1 \mathrm{CD} 07002$ & $63^{\circ} 24^{\prime} \mathrm{N}$ & $50^{\circ} 11^{\prime} \mathrm{W}$ & NW & \\
\hline 1CD04021 & $63^{\circ} 32^{\prime} \mathrm{N}$ & $49^{\circ} 55^{\prime}$ & NW & 1.20 & 75 & 0001.90 & 653210 & $6330-03$ & $1 \mathrm{CD} 07003$ & $63^{\circ} 25^{\prime} \mathrm{N}$ & $50^{\circ} 13^{\prime} \mathrm{W}$ & E & \\
\hline 1CD04022 & $63^{\circ} 33^{\prime} \mathrm{N}$ & $49^{\circ} 54^{\prime} \mathrm{V}$ & $\mathrm{N}$ & 145 & 85 & 0003.50 & 633112 & $6330-03$ & $1 \mathrm{CD} 07004$ & $63^{\circ} 24^{\prime} \mathrm{N}$ & $50^{\circ} 02^{\prime} \mathrm{W}$ & s & 115 \\
\hline $1 \mathrm{CD} 04023$ & $63^{\circ} 33^{\prime} \mathrm{N}$ & $49^{\circ} 53^{\prime} \mathrm{W}$ & $\mathbf{N}$ & 120 & 95 & 0000.60 & 740010 & $6330-03$ & $1 \mathrm{CD} 07005$ & $63^{\circ} 24^{\prime} \mathrm{N}$ & $49 \circ 57$, है & E & \\
\hline 1CD04024 & $63^{\circ} 33^{\prime} \mathrm{N}$ & $49^{\circ} 52 ! \mathrm{V}$ & $\mathrm{N}$ & 145 & 70 & 0004 . & 643112 & & 1CD07006 & $63^{\circ} 26^{\prime} \mathrm{N}$ & $50^{\circ} 07 \cdot \mathrm{W}$ & $\mathrm{v}$ & \\
\hline $1 \mathrm{CD} 04025$ & $63^{\circ} 32^{\prime} \mathrm{N}$ & $49^{\circ} 52^{\prime} \mathrm{W}$ & SE & 145 & 70 & 0007.30 & 533110 & $6330-03$ & $1 \mathrm{CD} 08001$ & $63^{\circ} 19^{\prime} \mathrm{N}$ & $50^{\circ} 08^{\prime} \mathrm{W}$ & $\mathrm{NE}$ & \\
\hline 1CD04026 & $63^{\circ} 31^{\prime} \mathrm{N}$ & $49^{\circ} 53^{\prime} \mathrm{W}$ & E & 135 & 125 & 0000.36 & 750110 & $6330-03$ & $1 \mathrm{CDO} 0002$ & $63^{\circ} 20^{\prime} \mathrm{N}$ & $50^{\circ} 10^{\prime}$ & $\mathrm{N}$ & \\
\hline 1CD04027 & $63^{\circ} 33^{\prime} \mathrm{N}$ & $49^{\circ} 49^{\prime} \mathrm{W}$ & $\mathbf{N}$ & 110 & 70 & 0007.80 & 626112 & $6330-03$ & $1 \mathrm{CD} 08003$ & $63^{\circ} 20^{\prime} \mathrm{N}$ & $50^{\circ} 11^{\prime \prime}$ & $\mathrm{N}$ & 90 \\
\hline $1 \mathrm{CD} 04028$ & $63^{\circ} 34^{\prime} \mathrm{N}$ & $49^{\circ} 46^{\prime} \mathrm{W}$ & NE & 120 & 70 & 0001.80 & 645112 & $6330-03$ & $1 \mathrm{CD} 09001$ & $63^{\circ} 22^{\prime} \mathrm{N}$ & $50^{\circ} 17^{\prime} \mathrm{W}$ & W & \\
\hline $1 \mathrm{CD} 04029$ & $63^{\circ} 33^{\prime} \mathrm{N}$ & $49^{\circ} 45^{\prime} \mathrm{W}$ & $\mathrm{N}$ & 120 & 70 & 0002.60 & 645112 & & $1 \mathrm{CD} 09002$ & $63^{\circ} 21^{\prime} \mathrm{N}$ & $50^{\circ} 20^{\prime} \mathrm{W}$ & $\mathrm{N}$ & \\
\hline $1 \mathrm{CD} 04030$ & $63^{\circ} 35^{\prime} \mathrm{N}$ & $49^{\circ} 44^{\prime} \mathrm{W}$ & $\mathrm{N}$ & 125 & 100 & 0000.80 & 600110 & $6330-03$ & $1 \mathrm{CD} 09003$ & $63^{\circ} 21^{\prime} \mathrm{N}$ & $50^{\circ} 21^{\prime} \mathrm{W}$ & $\mathrm{N}$ & \\
\hline 1CD04031 & $63^{\circ} 34^{\prime} \mathrm{N}$ & $49^{\circ} 43^{\prime} \mathrm{W}$ & NE & 125 & 70 & 0001.20 & 603110 & $6330-03$ & $1 \mathrm{CD} 10001$ & $63^{\circ} 21^{\prime} \mathrm{N}$ & $50^{\circ} 25$, W & SW & \\
\hline 1CD04032 & $63^{\circ} 34^{\prime} \mathrm{N}$ & $49^{\circ} 42^{\prime} \mathrm{W}$ & $\mathrm{NE}$ & 120 & 105 & 0000.20 & 700010 & 633 & $1 \mathrm{CD} 1$ & $63^{\circ} 20^{\prime} \mathrm{N}$ & $50^{\circ} 26^{\prime}$ & $\mathrm{SW}$ & 110 \\
\hline $1 \mathrm{CD} 04033$ & $63^{\circ} 33^{\prime} \mathrm{N}$ & $49^{\circ} 40^{\prime} \mathrm{V}$ & E & 100 & 90 & 0000.30 & 700010 & $6330-03$ & $1 \mathrm{CD}$ & $63^{\circ} 19^{\prime} \mathrm{N}$ & $50^{\circ} 29^{\prime} \mathrm{W}$ & $\mathrm{N}$ & 9 \\
\hline $1 \mathrm{CD} 04034$ & $63^{\circ} 33^{\prime} \mathrm{N}$ & $49^{\circ} 41^{\prime} \mathrm{W}$ & $\mathrm{N}$ & 110 & & 0000.30 & 700010 & & 04 & $63^{\circ} 19^{\prime} \mathrm{N}$ & $50^{\circ} 32^{\prime} \mathrm{W}$ & NW & \\
\hline $1 \mathrm{CD} 04035$ & $63^{\circ} 33^{\prime} \mathrm{N}$ & $49^{\circ} 42^{\prime} \mathrm{W}$ & NE & 140 & 100 & 0001.40 & 600010 & 63 & 1CE01001 & $63^{\circ} 47^{\prime} \mathrm{N}$ & $51^{\circ} 04^{\prime} \mathrm{W}$ & w & \\
\hline 1CD04036 & $63^{\circ} 43^{\prime} \mathrm{N}$ & $49^{\circ} 12^{\prime}$ & 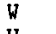 & 180 & 105 & 005 & 163 & & 1CE01002 & $63^{\circ} 46^{\prime} \mathrm{N}$ & $51^{\circ} 04^{\prime} \mathrm{W}$ & ט & 100 \\
\hline 1CD04037 & $63^{\circ} 35^{\prime} \mathrm{N}$ & $49^{\circ} 44^{\prime} \mathrm{W}$ & 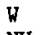 & 180 & 65 & 0212.71 & 167112 & $6330-03$ & 1CE01003 & $63^{\circ} 46^{\prime} \mathrm{N}$ & $51^{\circ} 03^{\prime \prime}$ & SW & 100 \\
\hline 1CD04038 & $63^{\circ} 29^{\prime} \mathrm{N}$ & $49^{\circ} 45^{\prime} \mathrm{W}$ & NW & 115 & 90 & 0001.10 & 700010 & $6300-03$ & 1CE01004 & $63^{\circ} 45^{\prime} \mathrm{N}$ & $51^{\circ} 03^{\prime} \mathrm{W}$ & $\mathbf{s}$ & \\
\hline $1 \mathrm{CD} 04039$ & $63^{\circ} 29^{\prime} \mathrm{N}$ & $49^{\circ} 47^{\prime}$ & $\mathrm{NW}$ & 100 & 100 & 0000 & 700010 & & $1 \mathrm{CE}$ & 'N & $51^{\circ} 01^{\prime} \mathrm{V}$ & NE & \\
\hline $1 \mathrm{CD} 04040$ & $63^{\circ} 28^{\prime} \mathrm{N}$ & $49^{\circ} 46^{\prime} \mathrm{W}$ & NW & 100 & 100 & 000 & 700 & & 1CE01006 & $63^{\circ} 46^{\prime} \mathrm{N}$ & $51 \circ 01$, & SE & \\
\hline 1CD04041 & $63^{\circ} 31^{\prime} \mathrm{N}$ & $49^{\circ} 35^{\prime} \mathrm{V}$ & SW & 180 & 42 & 0569.21 & & & 1CE01007 & $63^{\circ} 47^{\prime} \mathrm{N}$ & $50^{\circ} 59 \cdot \mathrm{v}$ & w & 110 \\
\hline 1CD04042 & $63^{\circ} 35^{\prime} \mathrm{N}$ & $49^{\circ} 20^{\prime} \mathrm{V}$ & Sw & 180 & 95 & 0250.43 & 163113 & $6330-03$ & 1CE01008 & $63^{\circ} 47 \cdot \mathrm{N}$ & $50^{\circ} 58^{\prime} \mathrm{V}$ & $\mathrm{s}$ & 110 \\
\hline $1 \mathrm{CD} 04043$ & $63^{\circ} 32^{\prime} \mathrm{N}$ & $49^{\circ} 17^{\prime} \mathrm{W}$ & $W$ & 180 & 100 & 0155.96 & 167113 & $6330-$ & 1CE01009 & $63^{\circ} 46^{\prime} \mathrm{N}$ & $50^{\circ} 56^{\prime} \mathrm{v}$ & SE & \\
\hline $1 \mathrm{CD} 04044$ & $63^{\circ} 29^{\prime} \mathrm{N}$ & $49^{\circ} 24^{\prime} \mathrm{W}$ & $\mathrm{V}$ & 180 & 65 & 0384.91 & 1671 & & 1CE01010 & $63^{\circ} 47 \cdot \mathrm{N}$ & $50^{\circ} 55^{\prime} \mathrm{W}$ & $\mathrm{s}$ & \\
\hline 1CD04045 & $63^{\circ} 25^{\prime} \mathrm{N}$ & $49^{\circ} 19^{\prime}$ W & NW & 18 & 65 & & & & 1CE01011 & $63^{\circ} 48^{\prime} \mathrm{N}$ & $50^{\circ} 54^{\prime} \mathrm{W}$ & E & \\
\hline $1 \mathrm{CD} 04046$ & $63^{\circ} 21^{\prime} \mathrm{N}$ & $49^{\circ} 10^{\prime} \mathrm{W}$ & $\mathrm{NW}$ & 180 & 110 & 0031.47 & 163113 & $6300-03$ & 1CE01012 & $63^{\circ} 49^{\prime} \mathrm{N}$ & $50^{\circ} 53^{\prime} \mathrm{W}$ & $\mathrm{SE}$ & 130 \\
\hline $1 \mathrm{CD} 04047$ & $63^{\circ} 20^{\prime} \mathrm{N}$ & $49^{\circ} 13^{\prime} \mathrm{W}$ & NW & 150 & 115 & 0001.80 & 633112 & $6300-03$ & 1CE01013 & $63^{\circ} 48^{\prime} \mathrm{N}$ & $50^{\circ} 51 \cdot \mathrm{W}$ & $\mathrm{NE}$ & \\
\hline $1 \mathrm{CD} 04048$ & $63^{\circ} 20^{\prime} \mathrm{N}$ & $49^{\circ} 14^{\prime} \mathrm{W}$ & NW & 150 & 125 & 0001 & 633112 & & 1CE01 & $63^{\circ} 48^{\prime} \mathrm{N}$ & $50^{\circ} 46^{\prime} \mathrm{W}$ & SW & 1 \\
\hline 1CD04049 & $63^{\circ} 20^{\prime} \mathrm{N}$ & $49^{\circ} 14^{\prime} \mathrm{W}$ & NW & 150 & 135 & & 640 & & 1CE01015 & $63^{\circ} 47^{\prime} \mathrm{N}$ & $50^{\circ} 47 \cdot \mathrm{v}$ & v & \\
\hline $1 \mathrm{CD} 04050$ & $63^{\circ} 25^{\prime} \mathrm{N}$ & $50^{\circ} 01^{\prime} \mathrm{W}$ & $\mathrm{N}$ & 140 & 65 & 0007.50 & 643112 & $6300-02$ & 1CE01016 & $63^{\circ} 46^{\prime} \mathrm{N}$ & $50^{\circ} 45, \mathrm{~W}$ & s & \\
\hline $1 \mathrm{CD} 04051$ & $63^{\circ} 25^{\prime} \mathrm{N}$ & $50^{\circ} 04^{\prime} \mathrm{W}$ & NE & 120 & 80 & 0001.30 & 640012 & $6300-02$ & $1 \mathrm{CE} 01$ & $63^{\circ} 45^{\prime} \mathrm{N}$ & $50^{\circ} 43^{\prime} \mathrm{v}$ & SW & \\
\hline $1 \mathrm{CD} 04052$ & $63^{\circ} 26^{\prime} \mathrm{N}$ & $50^{\circ} 03^{\prime}$ & E & 140 & 90 & 0000.70 & 640012 & $6300-$ & 1CE01018 & $63^{\circ} 45 \cdot \mathrm{N}$ & $50^{\circ} 42, \mathrm{v}$ & SW & \\
\hline $1 \mathrm{CD} 04053$ & $63^{\circ} 26^{\prime} \mathrm{N}$ & $50^{\circ} 03^{\prime} \mathrm{W}$ & $\mathbf{N}$ & 140 & 90 & 0000.80 & 640012 & $6300-02$ & 1CE01019 & $63^{\circ} 45^{\prime} \mathrm{N}$ & $50^{\circ} 41^{\prime} \mathrm{W}$ & $\mathrm{s}$ & 14 \\
\hline $1 \mathrm{CD} 04054$ & $63^{\circ} 26^{\prime} \mathrm{N}$ & $50^{\circ} 06^{\prime} \mathrm{W}$ & N & 157 & 50 & 0007.20 & 643112 & $6300-02$ & 1 CEO 1020 & $63^{\circ} 43^{\prime} \mathrm{N}$ & $50^{\circ} 37^{\prime} \mathrm{V}$ & SW & 13 \\
\hline
\end{tabular}

\begin{tabular}{rccc} 
& & MO & \\
LOW & AREA & CODE & MAP \\
75 & 0002.60 & 363112 & $6300-02$ \\
75 & 0002.10 & 363112 & $6300-02$ \\
50 & 0006.00 & 363112 & $6300-02$ \\
40 & 0008.50 & 363312 & $6300-02$ \\
60 & 0004.60 & 363511 & $6300-02$ \\
60 & 0002.20 & 533112 & $6300-02$ \\
80 & 0003.10 & 533110 & $6300-02$ \\
70 & 0000.50 & 633312 & $6300-02$ \\
85 & 0000.50 & 700110 & $6300-02$ \\
60 & 0001.20 & 633212 & $6300-02$ \\
55 & 0006.60 & 633112 & $6300-02$ \\
80 & 0002.20 & 633112 & $6300-02$ \\
80 & 0000.20 & 780010 & $6300-02$ \\
70 & 0000.50 & 750010 & $6300-02$ \\
50 & 0001.20 & 74310 & $6300-02$ \\
80 & 0000.20 & 650112 & $6300-02$ \\
70 & 0003.70 & 643112 & $6300-02$ \\
90 & 0000.20 & 643112 & $6300-02$ \\
90 & 0001.90 & 643112 & $6300-02$ \\
90 & 0000.40 & 643110 & $6300-02$ \\
90 & 0000.40 & 643112 & $6300-02$ \\
100 & 0002.80 & 673110 & $6300-02$ \\
85 & 0001.00 & 63310 & $6300-02$ \\
75 & 0003.50 & 643112 & $6300-02$ \\
75 & 0001.10 & 650010 & $6300-02$ \\
76 & 0000.20 & 746110 & $6300-02$ \\
76 & 0001.50 & 643112 & $6300-02$ \\
76 & 0000.30 & 77310 & $6300-02$ \\
95 & 0002.00 & 533112 & $6300-02$ \\
100 & 0000.70 & 250012 & $6300-02$ \\
95 & 0002.94 & 270012 & $6300-02$ \\
65 & 0002.20 & 263110 & $6300-02$ \\
70 & 0001.70 & 263110 & $6300-02$ \\
75 & 0000.70 & 263110 & $6300-02$ \\
75 & 0002.20 & 640210 & $6300-02$ \\
60 & 0003.60 & 203410 & $6300-02$ \\
70 & 0003.90 & 23410 & $6300-02$ \\
90 & 0000.70 & 750010 & $6300-02$ \\
55 & 0004.80 & 243112 & $6300-02$ \\
60 & 0003.10 & 243112 & $6300-02$ \\
45 & 0004.45 & 243112 & $6300-02$ \\
85 & 0000.70 & 200110 & $6330-01$ \\
90 & 0000.80 & 230110 & $6330-01$ \\
75 & 0000.70 & 233112 & $6330-01$ \\
80 & 0000.70 & 730010 & $6330-01$ \\
80 & 0000.80 & 230110 & $6330-01$ \\
80 & 0000.70 & 230110 & $6330-01$ \\
90 & 0000.80 & 240110 & $6330-01$ \\
85 & 0001.00 & 230110 & $6330-01$ \\
40 & 0002.50 & 233112 & $6330-01$ \\
85 & 0005.20 & 232112 & $6330-01$ \\
95 & 0000.40 & 230010 & $6330-01$ \\
110 & 0000.60 & 640110 & $6330-01$ \\
80 & 0004.00 & 236110 & $6330-01$ \\
90 & 0004.40 & 367110 & $6330-01$ \\
20 & 0013.10 & 363412 & $6330-01$ \\
95 & 0005.30 & 360110 & $6330-02$ \\
95 & 0005.00 & 360110 & $6330-02$ \\
60 & 0004.70 & 363112 & $6330-02$ \\
95 & 0008.80 & 360112 & $6330-02$ \\
80 & 0003.10 & 366112 & $6330-02$ \\
& & & \\
\hline 50
\end{tabular}




\begin{tabular}{|c|c|c|c|c|c|c|c|c|c|c|c|c|c|c|c|c|c|}
\hline CODE & LAT & LONG & ORI & HIGH & Low & AREA & $\begin{array}{c}\text { MO } \\
\text { CODE }\end{array}$ & MAP & CODE & LAT & LONG & ORI & HIGH & LOW & AREA & $\begin{array}{c}\text { MO } \\
\text { CODE }\end{array}$ & MAP \\
\hline 1CE01021 & $63^{\circ} 43^{\prime} \mathrm{N}$ & $50^{\circ} 37^{\prime} \mathrm{W}$ & $\mathrm{NW}$ & 110 & 80 & 0000.80 & 360110 & $6330-02$ & 1CE05034 & $63^{\circ} 47{ }^{\prime N}$ & $49^{\circ} 45^{\prime} \mathrm{W}$ & $\mathrm{N}$ & 115 & 85 & & & \\
\hline 1CE01022 & $63^{\circ} 43^{\prime} \mathrm{N}$ & $50^{\circ} 40^{\prime} \mathrm{W}$ & E & 100 & 80 & 0002.90 & 536110 & $6330-02$ & 1CE05035 & $63^{\circ} 47 \cdot \mathrm{N}$ & $49^{\circ} 45^{\prime} \mathrm{W}$ & N & 145 & 90 & 0001.10 & 642412 & $6330-03$ \\
\hline 1CE01023 & $63^{\circ} 45^{\prime} \mathrm{N}$ & $50^{\circ} 44^{\prime} \mathrm{W}$ & NW & 130 & 50 & 0005.20 & 533112 & $6330-02$ & 1 CE05036 & $63^{\circ} 46^{\prime} \mathrm{N}$ & 49044,6 & $\mathrm{~N}$ & 130 & 130 & 0000.10 & 780010 & $6330-03$ \\
\hline 1CE01024 & $63^{\circ} 42^{\prime} \mathrm{N}$ & $50^{\circ} 46^{\prime} \mathrm{W}$ & NW & 125 & 50 & 0005.30 & 533110 & $6330-01$ & 1 CE05037 & $63^{\circ} 46^{\prime} \mathrm{N}$ & $49^{\circ} 47^{\prime} \mathrm{W}$ & N & 130 & 110 & 0001.20 & 643112 & $6330-03$ \\
\hline 1CE01025 & $63^{\circ} 43^{\prime} \mathrm{N}$ & $50^{\circ} 48^{\prime} \mathrm{W}$ & NW & 85 & 80 & 0000.60 & 676110 & $6330-01$ & 1CE05038 & $63^{\circ} 46^{\prime} \mathrm{N}$ & $49^{\circ} 48^{\prime} \mathrm{W}$ & $\mathrm{N}$ & 120 & 85 & 0001.00 & 653112 & $6330-03$ \\
\hline 1CE01026 & $63^{\circ} 42^{\prime} \mathrm{N}$ & $50^{\circ} 48^{\prime} \mathrm{W}$ & $\mathrm{NW}$ & 115 & 70 & 0002.70 & 536110 & $6330-01$ & 1CE05039 & $63^{\circ} 46^{\prime} \mathrm{N}$ & $49^{\circ} 49^{\prime} \mathrm{W}$ & $\mathbf{N}$ & 110 & 90 & 0000.70 & 770012 & $6330-03$ \\
\hline 1CE01027 & $63^{\circ} 42^{\prime} \mathrm{N}$ & $50^{\circ} 53^{\prime} \mathrm{W}$ & $\mathrm{W}$ & 110 & 35 & 0005.00 & 530110 & 6330-01 & 1CE05040 & $63^{\circ} 45^{\prime} \mathrm{N}$ & $49^{\circ} 53^{\prime}$ & $N W$ & 130 & 85 & 0006.20 & 532412 & $6330-03$ \\
\hline 1CE01028 & $63^{\circ} 42^{\prime} \mathrm{N}$ & $50^{\circ} 53^{\prime} \mathrm{W}$ & $\mathbf{N}$ & 115 & 75 & 0000.80 & 647110 & $6330-01$ & 1CE05041 & $63^{\circ} 45^{\prime} \mathrm{N}$ & $49^{\circ} 54^{\prime} \mathrm{W}$ & NW & 145 & 95 & 0004.50 & 533112 & $6330-03$ \\
\hline 1СE01029 & $63^{\circ} 42^{\prime} \mathrm{N}$ & $50^{\circ} 52^{\prime} \mathrm{W}$ & $\mathbf{N}$ & 110 & 35 & 0000.80 & 647410 & $6330-01$ & 1CE05042 & $63^{\circ} 45^{\prime} \mathrm{N}$ & $49^{\circ} 56^{\prime} \mathrm{W}$ & $\mathrm{N}$ & 140 & 100 & 0001.30 & 536412 & $6330-03$ \\
\hline 1CE01030 & $63^{\circ} 43^{\prime} \mathrm{N}$ & $50^{\circ} 48^{\prime} \mathrm{W}$ & NE & 100 & 85 & 0000.90 & 530110 & $6330-01$ & 1CE05043 & $63^{\circ} 45^{\prime} \mathrm{N}$ & $49^{\circ} 59^{\prime} \mathrm{W}$ & $\mathbf{N}$ & 160 & 70 & 0035.10 & 531111 & $6330-02$ \\
\hline 1CE01031 & $63^{\circ} 43^{\prime} \mathrm{N}$ & $50^{\circ} 51^{\prime} \mathrm{W}$ & $\mathrm{W}$ & 105 & 70 & 0003.33 & 530110 & $6330-01$ & 1CE05044 & $63^{\circ} 45^{\prime} \mathrm{N}$ & $50^{\circ} 03^{\prime} \mathrm{W}$ & NW & 150 & 85 & 0003.40 & 532012 & $6330-02$ \\
\hline 1CE01032 & $63^{\circ} 44^{\prime} \mathrm{N}$ & $50^{\circ} 50^{\prime} \mathrm{W}$ & $\mathrm{N}$ & 85 & 70 & 0000.20 & 700010 & $6330-01$ & 1 CE05045 & $63^{\circ} 44^{\prime} \mathrm{N}$ & $50^{\circ} 05^{\prime} \mathrm{W}$ & $\mathrm{N}$ & 160 & 95 & 0004.90 & 532111 & $6330-02$ \\
\hline 1CE01033 & $63^{\circ} 44^{\prime} \mathrm{N}$ & $50^{\circ} 49^{\prime} \mathrm{W}$ & $\mathbf{N}$ & 90 & 70 & 0000.30 & 700010 & $6330-01$ & 1CE05046 & $63^{\circ} 44^{\prime} \mathrm{N}$ & $50^{\circ} 06^{\prime}$ U & NE & 150 & 95 & 0007.30 & 523111 & $6330-02$ \\
\hline 1CE01034 & $63^{\circ} 41^{\prime} \mathrm{N}$ & $51^{\circ} 00^{\prime} \mathrm{W}$ & $\mathrm{NW}$ & 95 & 65 & 0000.50 & 640110 & $6330-01$ & 1CE05047 & $63^{\circ} 45^{\circ} \mathrm{N}$ & $50^{\circ} 09^{\prime} \mathrm{W}$ & $\mathrm{NE}$ & 120 & 110 & 0000.40 & 650012 & $6330-02$ \\
\hline 1CE01035 & $63^{\circ} 42^{\prime} \mathrm{N}$ & $51^{\circ} 00^{\prime} \mathrm{H}$ & $\mathrm{N}$ & 85 & 75 & 0000.17 & 700010 & $6330-01$ & 1 CE05048 & $63^{\circ} 44^{\prime} \mathrm{N}$ & $50^{\circ} 09^{\prime} \mathrm{W}$ & $\mathrm{w}$ & 130 & 100 & 0000.80 & 770010 & $6330-02$ \\
\hline 1CE02001 & $63^{\circ} 41^{\prime} \mathrm{N}$ & $50^{\circ} 55^{\prime} \mathrm{W}$ & NW & 80 & 65 & 0000.40 & 640112 & $6330-01$ & 1CE05049 & $63^{\circ} 43^{\prime} \mathrm{N}$ & $50^{\circ} 11^{\prime} \mathrm{W}$ & NW & 140 & 65 & 0029.50 & 521112 & $6330-02$ \\
\hline 1CE02002 & $63^{\circ} 41^{\prime} \mathrm{N}$ & $50^{\circ} 54^{\prime} \mathrm{W}$ & SW & 100 & 75 & 0000.70 & 640112 & $6330-01$ & 1CE05050 & $63^{\circ} 43^{\prime} \mathrm{N}$ & $50^{\circ} 15^{\prime} \mathrm{V}$ & $\mathrm{NW}$ & 140 & 80 & 0002.10 & 533112 & $6330-02$ \\
\hline 1CE02003 & $63^{\circ} 40^{\prime} \mathrm{N}$ & $50^{\circ} 54^{\prime} \mathrm{W}$ & $W$ & 100 & 80 & 0001.10 & 533112 & $6330-01$ & 1CE05051 & $63^{\circ} 42, \mathrm{~N}$ & $50^{\circ} 18^{\prime}$ & NW & 150 & 70 & 0022.40 & 523112 & $6330-02$ \\
\hline 1CE02004 & $63^{\circ} 40^{\prime} \mathrm{N}$ & $50^{\circ} 57 . \mathrm{W}$ & NW & 100 & 75 & 0000.20 & 640112 & $6330-01$ & 1 CE05052 & $63^{\circ} 43^{\prime} \mathrm{N}$ & $50^{\circ} 22^{\prime \prime} \mathrm{W}$ & $\mathrm{N}$ & 100 & 65 & 0000.30 & 700010 & $6330-02$ \\
\hline 1CE02005 & $63^{\circ} 39^{\prime} \mathrm{N}$ & $50^{\circ} 57 \cdot \mathrm{V}$ & $\mathbf{N}$ & 100 & 80 & 0000.40 & 640112 & $6330-01$ & 1CE05053 & $63^{\circ} 43^{\prime} \mathrm{N}$ & $50^{\circ} 23^{\prime} \mathrm{W}$ & $\bar{v}$ & 120 & 85 & 0001.00 & 700010 & $6330-02$ \\
\hline 1CE02006 & $63^{\circ} 39^{\prime} \mathrm{N}$ & $50^{\circ} 56^{\prime} \mathrm{V}$ & SW & 90 & 80 & 0000.40 & 730112 & $6330-01$ & 1CE05054 & $63^{\circ} 42^{\prime} \mathrm{N}$ & $50^{\circ} 25^{\prime} \mathrm{V}$ & NW & 160 & 45 & 0016.10 & 523111 & $6330-02$ \\
\hline 1CE02007 & $63^{\circ} 39^{\prime} \mathrm{N}$ & $50^{\circ} 55^{\prime} \mathrm{W}$ & $\mathrm{E}$ & 100 & 85 & 0001.00 & 640112 & 6330-01 & 1CE05055 & $63^{\circ} 42, \mathrm{~N}$ & $50^{\circ} 28^{\prime} \mathrm{W}$ & $\mathrm{NW}$ & 95 & 60 & 0003.60 & 362010 & $6330-02$ \\
\hline 1CE03001 & $63^{\circ} 40^{\prime} \mathrm{N}$ & $50^{\circ} 52 . \mathrm{V}$ & $\mathrm{SE}$ & 100 & 80 & 0001.00 & 640112 & $6330-01$ & 1CE05056 & $63^{\circ} 43^{\prime} \mathrm{N}$ & $50^{\circ} 29^{\prime \prime}$ & NW & 90 & 70 & 0000.40 & 700010 & $6330-02$ \\
\hline 1CE03002 & $63^{\circ} 41^{\prime} \mathrm{N}$ & $50^{\circ} 52^{\prime} \mathrm{W}$ & $\mathrm{s}$ & 110 & 70 & 0003.20 & 640112 & $6330-01$ & 1 CE05057 & $63^{\circ} 41^{\prime} \mathrm{N}$ & $50^{\circ} 30^{\prime \prime}$ & 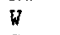 & 140 & 80 & 0002.20 & 363412 & $6330-02$ \\
\hline 1CE03003 & $63^{\circ} 40^{\prime} \mathrm{N}$ & $50^{\circ} 49^{\prime} \mathrm{W}$ & SW & 115 & 70 & 0002.40 & 532412 & $6330-01$ & 1CE05058 & $63^{\circ} 40^{\prime} \mathrm{N}$ & $50^{\circ} 29^{\prime} \mathrm{V}$ & $\mathrm{s}$ & 140 & 110 & 0000.80 & 573010 & $6330-02$ \\
\hline 1CE03004 & $63^{\circ} 40^{\prime} \mathrm{N}$ & $50^{\circ} 47 \cdot \mathrm{V}$ & $\mathbf{s}$ & 115 & 85 & 0002.00 & 532110 & $6330-01$ & 1CE05059 & $63^{\circ} 53^{\prime} \mathrm{N}$ & $50^{\circ} 10^{\prime} \mathrm{W}$ & $\mathrm{NE}$ & 130 & 120 & 0000.52 & 770010 & $6330-02$ \\
\hline 1CE04001 & $63^{\circ} 41^{\prime} \mathrm{N}$ & $50^{\circ} 44^{\prime} \mathrm{V}$ & $\mathrm{SE}$ & 110 & 75 & 0002.20 & 533112 & $6330-02$ & 1CE05060 & $63^{\circ} 53^{\prime} \mathrm{N}$ & $50^{\circ} 11^{\prime} \mathrm{V}$ & $E$ & 130 & 125 & 0000.23 & 770010 & $6330-02$ \\
\hline 1 CE04002 & $63^{\circ} 41^{\prime} \mathrm{N}$ & $50^{\circ} 43^{\prime} \mathrm{W}$ & SE & 115 & 65 & 0004.70 & 533112 & $6330-02$ & 1CE06001 & $63^{\circ} 40^{\prime} \mathrm{N}$ & $50^{\circ} 27 \cdot \mathrm{W}$ & $\mathrm{SW}$ & 100 & 85 & 0008.80 & 363112 & $6330-02$ \\
\hline 1CE05001 & $63^{\circ} 43^{\prime} \mathrm{N}$ & $50^{\circ} 35^{\prime} \mathrm{W}$ & NE & 110 & 60 & 0006.30 & 633111 & $6330-02$ & 1CE06002 & $63^{\circ} 39^{\prime} \mathrm{N}$ & $50^{\circ} 25^{\prime} \mathrm{W}$ & $\mathrm{W}$ & 160 & & 0026.50 & 364112 & $6330-02$ \\
\hline 1CE05002 & $63^{\circ} 45^{\prime} \mathrm{N}$ & $50^{\circ} 34^{\prime} \mathrm{V}$ & SE & 135 & 85 & 0005.40 & 363112 & $6330-02$ & 1CE06003 & $63^{\circ} 39^{\prime} \mathrm{N}$ & $50^{\circ} 24^{\prime} \mathrm{W}$ & $\mathrm{N}$ & 160 & 140 & 0001.60 & 740010 & $6330-02$ \\
\hline 1CE05003 & $63^{\circ} 45^{\prime} \mathrm{N}$ & $50^{\circ} 31^{\prime} \mathrm{W}$ & $\mathrm{s}$ & 120 & 70 & 0003.70 & 363112 & $6330-02$ & 1CE06004 & $63^{\circ} 38^{\prime} \mathrm{N}$ & $50^{\circ} 27^{\prime} \mathrm{V}$ & NW & 150 & 150 & 0000.50 & 740010 & $6330-02$ \\
\hline 1CE05004 & $63^{\circ} 45, \mathrm{~N}$ & $50^{\circ} 30^{\prime} \mathrm{W}$ & $\mathrm{s}$ & 110 & 90 & 0000.30 & 362112 & $6330-02$ & 1CE06005 & $63^{\circ} 39^{\prime} \mathrm{N}$ & $50^{\circ} 28^{\prime} \mathrm{W}$ & NW & 150 & 150 & 0000.10 & 740010 & $6330-02$ \\
\hline 1CE05005 & $63^{\circ} 40^{\prime} \mathrm{N}$ & $50^{\circ} 28^{\prime} \mathrm{W}$ & $\mathrm{s}$ & 130 & 80 & 0008.00 & 363112 & $6330-02$ & 1CE06006 & $63^{\circ} 39^{\prime} \mathrm{N}$ & $50^{\circ} 28^{\prime} \mathrm{W}$ & $\mathrm{N}$ & 75 & 65 & 0000.10 & 740010 & $6330-02$ \\
\hline 1 CE05006 & $63^{\circ} 46^{\prime} \mathrm{N}$ & $50^{\circ} 29^{\prime} \mathrm{V}$ & E & 135 & 125 & 0001.60 & 650112 & $6330-02$ & 1CE07001 & $63^{\circ} 37^{\prime N}$ & $50^{\circ} 29^{\prime} \mathrm{W}$ & NW & 160 & & 0015.70 & 523112 & $6330-02$ \\
\hline 1CE05007 & $63^{\circ} 46^{\prime} \mathrm{N}$ & $50^{\circ} 21^{\prime} W$ & NE & 115 & 95 & 0000.80 & 533110 & $6330-02$ & 1CE07002 & $63^{\circ} 36^{\prime} \mathrm{N}$ & $50^{\circ} 30^{\prime} \mathrm{W}$ & NW & 140 & 20 & 0002.80 & 530012 & $6330-02$ \\
\hline 1CE05008 & $63^{\circ} 46^{\prime} \mathrm{N}$ & $50^{\circ} 23^{\prime} \mathrm{W}$ & $\mathbf{N}$ & 110 & 95 & 0000.40 & 650010 & $6330-02$ & 1CE08001 & $63^{\circ} 35^{\prime} \mathrm{N}$ & $50^{\circ} 37^{\prime} \mathrm{W}$ & E & 70 & 70 & 0000.50 & 640010 & $6330-02$ \\
\hline 1CE05009 & $63^{\circ} 47^{\prime} \mathrm{N}$ & $50^{\circ} 28^{\prime} \mathrm{W}$ & E & 140 & 50 & 0052.00 & 333112 & $6330-02$ & 1CE08002 & $63^{\circ} 34^{\prime} \mathrm{N}$ & $50^{\circ} 30^{\prime} \mathrm{V}$ & $s$ & 115 & 100 & 000 & 700010 & $6330-02$ \\
\hline 1CE05010 & $63^{\circ} 49^{\prime} \mathrm{N}$ & $50^{\circ} 32^{\prime} \mathrm{V}$ & NE & 140 & 90 & 0020.30 & 360110 & $6330-02$ & 1CE08003 & $63^{\circ} 3$ & $50^{\circ} 28^{\prime} \mathrm{W}$ & $\mathrm{SE}$ & 145 & 85 & 0003.70 & 531210 & $6330-02$ \\
\hline 1CE0SO11 & $63^{\circ} 51^{\prime} \mathrm{N}$ & $50^{\circ} 30^{\prime} \mathrm{W}$ & E & 150 & 100 & 0004.60 & 603012 & $6330-$ & 1CE08004 & $63^{\circ} 35^{\prime} \mathrm{N}$ & $50^{\circ} 25^{\prime \prime}$ & SE & 150 & 105 & 0001.60 & 533110 & $6330-02$ \\
\hline 1CE05012 & $63^{\circ} 50^{\prime} \mathrm{N}$ & $50^{\circ} 28^{\prime}$ & $\mathrm{N}$ & 120 & 95 & 0001.6 & 650112 & $6330-02$ & 1CE08005 & $63^{\circ} 36^{\prime} \mathrm{N}$ & $50^{\circ} 18^{\prime} \mathrm{W}$ & SE & 165 & 70 & 0026.20 & 323110 & $6330-02$ \\
\hline 1CE05013 & $63^{\circ} 50^{\prime} \mathrm{N}$ & $50^{\circ} 26^{\prime} \mathrm{W}$ & N & 150 & 90 & 0002.50 & 633112 & $6330-02$ & 1CEOBO06 & $63^{\circ} 38^{\prime} \mathrm{N}$ & $50^{\circ} 06^{\prime} \mathrm{W}$ & SE & 140 & 85 & 0063.80 & 330110 & $6330-02$ \\
\hline 1CE05014 & $63^{\circ} 50^{\prime} \mathrm{N}$ & $50^{\circ} 25^{\prime} \mathrm{W}$ & NE & 105 & 85 & 0001.20 & 652412 & $6330-02$ & 1CE08007 & $63^{\circ} 40^{\prime} \mathrm{N}$ & $50^{\circ} 03^{\prime} \mathrm{W}$ & $\mathrm{s}$ & 145 & 105 & 00 & 533 & $6330-02$ \\
\hline 1CE05015 & $63^{\circ} 50^{\prime} \mathrm{N}$ & $50^{\circ} 24^{\prime} \mathrm{V}$ & NE & 135 & 85 & 0001.00 & 640412 & $6330-02$ & 1CE08008 & $63^{\circ} 41^{\prime} \mathrm{N}$ & $50^{\circ} 00^{\prime} \mathrm{V}$ & s & 135 & 90 & 0007.20 & 521110 & $6330-02$ \\
\hline 1CE05016 & $63^{\circ} 49^{\prime} \mathrm{N}$ & $50^{\circ} 23^{\prime} \mathrm{W}$ & E & 150 & 100 & 0000.80 & 653112 & $6330-02$ & 1CE08009 & $63^{\circ} 41^{\prime} \mathrm{N}$ & $49^{\circ} 54^{\prime} \mathrm{W}$ & $\mathrm{E}$ & 140 & 85 & 0.008 .70 & 530110 & $6330-03$ \\
\hline 1 CE05017 & $63^{\circ} 50^{\prime} \mathrm{N}$ & $50^{\circ} 22^{\prime} \mathrm{W}$ & $N$ & 125 & 90 & 0000.90 & 640012 & $6330-0$ & 1CE08010 & $63^{\circ} 42^{\prime} \mathrm{N}$ & $49^{\circ} 55^{\prime} \mathrm{W}$ & E & 150 & 95 & 0001.90 & 543112 & $6330-03$ \\
\hline 1CE05018 & $63^{\circ} 52^{\prime} \mathrm{N}$ & $50^{\circ} 26^{\prime} \mathrm{V}$ & NE & 150 & 105 & 0001.10 & 600010 & $6330-0$ & 1CE0B011 & $63^{\circ} 44^{\prime} \mathrm{N}$ & $49^{\circ} 55^{\prime} \mathrm{W}$ & $\mathrm{SE}$ & 140 & 120 & 0001.60 & 531110 & $6330-03$ \\
\hline 1CE05019 & $63^{\circ} 53^{\prime} \mathrm{N}$ & $50^{\circ} 03^{\prime} \mathrm{W}$ & NW & 105 & 95 & 0000.40 & 700010 & $6330-02$ & 1 CE 08012 & $63^{\circ} 43^{\prime} \mathrm{N}$ & $49^{\circ} 52^{\prime} \mathrm{W}$ & $\mathrm{SE}$ & 140 & 95 & 0005 & 533 & -03 \\
\hline 1CE05020 & $63^{\circ} 53^{\prime} \mathrm{N}$ & $50^{\circ} 04^{\prime} \mathrm{W}$ & $\mathbf{N}$ & 100 & 90 & 0000.10 & 700010 & $6330-02$ & 1CE08013 & $63^{\circ} 45^{\prime} \mathrm{N}$ & $49^{\circ} 49^{\prime} \mathrm{V}$ & $\mathrm{E}$ & 130 & 85 & 0001.00 & 266112 & $6330-03$ \\
\hline 1CE05021 & $63^{\circ} 53^{\prime} \mathrm{N}$ & $50^{\circ} 03^{\prime} W$ & NW & 105 & 95 & 0000.20 & 700010 & $6330-$ & 1CE08014 & $63^{\circ} 45^{\prime} \mathrm{N}$ & $49^{\circ} 42^{\prime} \mathrm{W}$ & NE & 130 & 100 & 0004.80 & 640010 & $6330-03$ \\
\hline 1CE05022 & $63^{\circ} 53^{\prime} \mathrm{N}$ & $50^{\circ} 03^{\prime} \mathrm{W}$ & $\mathrm{N}$ & 110 & 100 & 0000.10 & 700010 & $6330-($ & 1CE08015 & $63^{\circ} 42^{\prime} \mathrm{N}$ & $49^{\circ} 47^{\prime} \mathrm{W}$ & $\mathbf{N}$ & 110 & 100 & 0000.20 & 750010 & $6330-03$ \\
\hline 1CE05023 & $63^{\circ} 53^{\prime} \mathrm{N}$ & $50^{\circ} 02^{\prime} \mathrm{W}$ & $\mathbf{N}$ & 110 & 100 & 00 & 700010 & 633 & 1CE08016 & $63^{\circ} 42^{\prime} \mathrm{N}$ & $49^{\circ} 46^{\prime} \mathrm{W}$ & $\mathrm{NE}$ & 110 & 100 & 0000.20 & 750010 & $6330-03$ \\
\hline 1CE05024 & $63^{\circ} 52^{\prime} \mathrm{N}$ & $50^{\circ} 02, \mathrm{~V}$ & $\mathrm{~N}$ & 120 & 120 & 0000.20 & 700010 & $6330-02$ & 1CE08017 & $63^{\circ} 42^{\prime} \mathrm{N}$ & $49^{\circ} 47^{\prime} \mathrm{W}$ & $\mathrm{N}$ & 115 & 115 & 0000.30 & 750010 & $6330-03$ \\
\hline 1CE05025 & $63^{\circ} 50^{\prime} \mathrm{N}$ & $49^{\circ} 47^{\prime} \mathrm{K}$ & W & 180 & 45 & 0437.30 & 164112 & $6330-03$ & 1CE08018 & $63^{\circ} 42^{\prime} \mathrm{N}$ & $49^{\circ} 48^{\prime} \mathrm{W}$ & $\mathrm{NE}$ & 130 & 110 & 0000.30 & 600010 & $6330-03$ \\
\hline 1CE05026 & $63^{\circ} 48^{\prime} \mathrm{N}$ & $49^{\circ} 57^{\prime} \mathrm{W}$ & $\mathrm{N}$ & 115 & 105 & 0000.30 & 640112 & $6330-02$ & 1CE08019 & $63^{\circ} 42^{\prime} \mathrm{N}$ & $49^{\circ} 48^{\prime} \mathrm{W}$ & NE & 130 & 85 & 0002.80 & 640110 & $6330-03$ \\
\hline 1CE05027 & $63^{\circ} 48^{\prime} \mathrm{N}$ & $49^{\circ} 56^{\prime} \mathrm{W}$ & $\mathbf{N}$ & 135 & 120 & 0000.50 & 640012 & & 1CE08020 & $63^{\circ} 42^{\prime} \mathrm{N}$ & $49^{\circ} 50^{\prime} \mathrm{W}$ & $\mathbf{N}$ & 120 & 85 & 0001.10 & 600110 & $6330-03$ \\
\hline 1CE05028 & $63^{\circ} 48^{\prime} \mathrm{N}$ & $49^{\circ} 55^{\prime} \mathrm{W}$ & $\mathrm{N}$ & 130 & 115 & 0000.20 & 643112 & $6330-03$ & 1CE08021 & $63^{\circ} 40^{\prime} \mathrm{N}$ & $49^{\circ} 55^{\prime} \mathrm{W}$ & E & 145 & 95 & 0005.80 & 523 & $6330-03$ \\
\hline 1CE05029 & $63^{\circ} 47 \cdot \mathrm{N}$ & $49^{\circ} 56^{\prime} \mathrm{W}$ & $\mathrm{SW}$ & 130 & 130 & 0000.30 & 700010 & $6330-03$ & 1CE08022 & $63^{\circ} 46^{\prime} \mathrm{N}$ & $49^{\circ} 40^{\prime} \mathrm{W}$ & W & 180 & 110 & 0033.26 & 163112 & $6330-03$ \\
\hline 1CE05030 & $63^{\circ} 47^{\prime} \mathrm{N}$ & $49^{\circ} 55^{\prime} \mathrm{W}$ & $\mathrm{N}$ & 135 & 135 & 0000.30 & 700010 & $6330-03$ & 1CE08023 & $63^{\circ} 33^{\prime} \mathrm{N}$ & $50^{\circ} 32^{\prime} \mathrm{W}$ & $\mathrm{w}$ & 180 & & 0611.32 & 163112 & $6330-02$ \\
\hline 1CE05031 & $63^{\circ} 47^{\prime} \mathrm{N}$ & $49^{\circ} 53^{\prime} \mathrm{W}$ & $\mathrm{N}$ & 135 & 120 & 0000.60 & 643112 & $6330-$ & 1CE09001 & $63^{\circ} 36^{\prime} \mathrm{N}$ & $50^{\circ} 36^{\prime} \mathrm{W}$ & NE & 130 & 100 & 0000.80 & 640410 & $6330-02$ \\
\hline 1CE05032 & $63^{\circ} 477^{\prime N}$ & $49^{\circ} 54^{\prime} \mathrm{W}$ & s & 125 & 115 & 0000.20 & 700010 & $6330-03$ & 1CE09002 & $63^{\circ} 36^{\prime} \mathrm{N}$ & $50^{\circ} 40^{\prime} \mathrm{W}$ & NW & 130 & 15 & 0006.10 & 533410 & $6330-02$ \\
\hline 1CE05033 & $63^{\circ} 47^{\prime} \mathrm{N}$ & $49^{\circ} 47^{\prime} \mathrm{W}$ & $\mathbf{N}$ & 105 & 95 & 0000.10 & 750110 & $6330-03$ & 1CE09003 & $63^{\circ} 36^{\prime} \mathrm{N}$ & $50^{\circ} 36^{\prime} \mathrm{W}$ & & 130 & 90 & 0000.70 & 640010 & $6330-02$ \\
\hline
\end{tabular}


CODE

LAT

RI

\begin{tabular}{|c|c|c|c|c|}
\hline 1CE09004 & $63^{\circ} 37^{\prime} \mathrm{N}$ & $50^{\circ} 36^{\prime} \mathrm{W}$ & $\mathbf{E}$ & 100 \\
\hline 1CE09005 & $63^{\circ} 37^{\prime} \mathrm{N}$ & $50^{\circ} 35^{\prime} \mathrm{W}$ & E & 135 \\
\hline 1CE09006 & $63^{\circ} 37^{\prime} \mathrm{N}$ & $50^{\circ} 36^{\prime} \mathrm{W}$ & $\mathrm{N}$ & 140 \\
\hline 1 CE09007 & $63^{\circ} 37^{\prime} \mathrm{N}$ & $50^{\circ} 36^{\prime} \mathrm{W}$ & $\mathrm{N}$ & 140 \\
\hline 1CE09008 & $63^{\circ} 37^{\prime} \mathrm{N}$ & $50^{\circ} 37 \cdot \mathrm{W}$ & $\mathrm{N}$ & 150 \\
\hline 1CE09009 & $63^{\circ} 37^{\prime} \mathrm{N}$ & $50^{\circ} 38^{\prime} \mathrm{W}$ & NW & 140 \\
\hline 1CE09010 & $63^{\circ} 36^{\prime} \mathrm{N}$ & $50^{\circ} 43^{\prime} \mathrm{W}$ & $\mathbf{N}$ & 160 \\
\hline 1CE09011 & $63^{\circ} 37^{\prime} \mathrm{N}$ & $50^{\circ} 44^{\prime} \mathrm{W}$ & $\mathbf{N}$ & 105 \\
\hline 1CE09012 & $63^{\circ} 36^{\prime} \mathrm{N}$ & $50^{\circ} 46^{\prime} \mathrm{W}$ & NW & 100 \\
\hline 1CE09013 & $63^{\circ} 35^{\prime} \mathrm{N}$ & $50^{\circ} 41 ' \mathrm{~W}$ & & 100 \\
\hline 1CE09014 & $63^{\circ} 36^{\prime} \mathrm{N}$ & $50^{\circ} 41^{\prime} \mathrm{W}$ & $v$ & 90 \\
\hline 1CE10001 & $63^{\circ} 34^{\prime} \mathrm{N}$ & $50^{\circ} 54^{\prime} \mathrm{W}$ & NW & 135 \\
\hline 1CE10002 & $63^{\circ} 55^{\prime} \mathrm{N}$ & $50^{\circ} 54^{\prime} \mathrm{W}$ & $\mathrm{N}$ & 135 \\
\hline 1CE10003 & $63^{\circ} 35^{\prime} \mathrm{N}$ & $50^{\circ} 52, \mathrm{~W}$ & $\mathrm{~N}$ & 115 \\
\hline 1CE 10004 & $63^{\circ} 35^{\prime} \mathrm{N}$ & $50^{\circ} 50^{\circ} \mathrm{W}$ & NE & 95 \\
\hline 1CE10005 & $63^{\circ} 35^{\prime} \mathrm{N}$ & $50^{\circ} 49 \cdot \mathrm{W}$ & $\mathrm{NE}$ & 100 \\
\hline 1CE10006 & $63^{\circ} 34^{\prime} \mathrm{N}$ & $50^{\circ} 47^{\prime} \mathrm{W}$ & NW & 85 \\
\hline 1CE 10007 & $63^{\circ} 35^{\prime} \mathrm{N}$ & $50^{\circ} 47^{\prime} \mathrm{W}$ & $\mathrm{W}$ & 105 \\
\hline 1CE11001 & $63^{\circ} 34^{\prime} \mathrm{N}$ & $50^{\circ} 55^{\circ} \mathrm{W}$ & $\mathrm{v}$ & 130 \\
\hline $1 \mathrm{CE}$ & $63^{\circ} 35^{\prime} \mathrm{N}$ & $50^{\circ} 51, \mathrm{~V}$ & $\mathrm{SE}$ & 135 \\
\hline 1CE11003 & $63^{\circ} 34^{\prime} \mathrm{N}$ & $50^{\circ} 48^{\prime} \mathrm{W}$ & NW & 100 \\
\hline 1CE11004 & $63^{\circ} 33^{\prime} \mathrm{N}$ & $50^{\circ} 49^{\prime} \mathrm{W}$ & $\mathbf{w}$ & 100 \\
\hline 1CE11005 & $63^{\circ} 34^{\prime} \mathrm{N}$ & $50^{\circ} 46^{\prime} \mathrm{W}$ & SE & 110 \\
\hline & $63^{\circ}$ & $50^{\circ} 40^{\prime} \mathrm{W}$ & $\mathrm{sw}$ & 100 \\
\hline $1 \mathrm{CE}$ & $63^{\circ} 31^{\prime} \mathrm{N}$ & $50^{\circ} 40^{\prime} \mathrm{W}$ & NW & 80 \\
\hline 1CE 12002 & $63^{\circ} 31^{\prime} \mathrm{N}$ & $50^{\circ} 48^{\prime} \mathrm{W}$ & NE & \\
\hline 1CE 12003 & $63^{\circ} 31^{\prime} \mathrm{N}$ & $50^{\circ} 48^{\prime} \mathrm{W}$ & $\mathrm{N}$ & 120 \\
\hline 1 CE 12004 & $63^{\circ} 29^{\prime} \mathrm{N}$ & $50^{\circ} 51 \cdot \mathrm{W}$ & $\mathrm{N}$ & 75 \\
\hline 1CE12005 & $63^{\circ} 30^{\prime} \mathrm{N}$ & $50^{\circ} 54, \mathrm{~W}$ & NW & 77 \\
\hline $1 \mathrm{CE}$ & $63^{\circ} 30^{\prime} \mathrm{N}$ & $50^{\circ} 55^{\prime} \mathrm{W}$ & $\mathrm{N}$ & 92 \\
\hline 1CE12007 & $63^{\circ} 30^{\prime} \mathrm{N}$ & $50^{\circ} 57 \cdot \mathrm{W}$ & NW & 100 \\
\hline 1CE 12008 & $63^{\circ} 29^{\prime} \mathrm{N}$ & $50^{\circ} 58^{\prime} \mathrm{W}$ & $\mathbf{N}$ & 130 \\
\hline 1CE12009 & $63^{\circ} 29^{\prime} \mathrm{N}$ & $50^{\circ} 59^{\prime} \mathrm{W}$ & NV & 112 \\
\hline & $63^{\circ} 54^{\prime} \mathrm{N}$ & $51^{\circ} 10^{\prime} \mathrm{W}$ & SE & 90 \\
\hline $1 \mathrm{CF} 01002$ & $63^{\circ} 54^{\prime} \mathrm{N}$ & $51^{\circ} 10^{\circ} \mathrm{W}$ & $\mathrm{E}$ & 90 \\
\hline $1 \mathrm{CF} 01003$ & $63^{\circ} 56^{\prime} \mathrm{N}$ & $51^{\circ} 06^{\prime} \mathrm{W}$ & E & 90 \\
\hline 1 CF01004 & $63^{\circ} 57^{\prime} \mathrm{N}$ & $51^{\circ} 06^{\prime} \mathrm{W}$ & $\mathrm{SE}$ & 130 \\
\hline $1 \mathrm{CF}$ & $63^{\circ} 56^{\prime} \mathrm{N}$ & $51^{\circ} 03^{\prime} \mathrm{W}$ & $\mathrm{NE}$ & 95 \\
\hline 1 CFO & $63^{\circ} 57^{\prime} \mathrm{N}$ & $51^{\circ} 03^{\prime} \mathrm{W}$ & $\mathrm{N}$ & 95 \\
\hline 1 CF02003 & $63^{\circ} 58^{\prime} \mathrm{N}$ & $50^{\circ} 58^{\prime} \mathrm{W}$ & NE & 95 \\
\hline 1CF03001 & $63^{\circ} 58^{\prime} \mathrm{N}$ & $50^{\circ} 57^{\prime} \mathrm{W}$ & $\mathrm{E}$ & 110 \\
\hline 1CF03002 & $63^{\circ} 59^{\prime} \mathrm{N}$ & $50^{\circ} 54^{\prime} \mathrm{W}$ & sw & 120 \\
\hline 1CFO & $63^{\circ} 55^{\prime} \mathrm{N}$ & $50^{\circ} 47^{\prime} \mathrm{W}$ & $\mathbf{N}$ & 130 \\
\hline 1 CF04001 & $63^{\circ} 54^{\prime} \mathrm{N}$ & $50^{\circ} 44^{\prime} \mathrm{W}$ & $\mathbf{N}$ & 120 \\
\hline & $63^{\circ} 53^{\prime} \mathrm{N}$ & $50^{\circ} 27^{\prime} \mathrm{W}$ & $\mathrm{NE}$ & 125 \\
\hline $1 \mathrm{CF} 04$ & $63^{\circ} 53^{\prime} \mathrm{N}$ & $50^{\circ} 28^{\prime} \mathrm{W}$ & $\mathbf{N}$ & 125 \\
\hline 1CF04004 & $63^{\circ}$ & $50^{\circ} 29^{\prime} \mathrm{W}$ & $\mathbf{N}$ & 150 \\
\hline 1CFO & $63^{\circ} 53^{\prime} \mathrm{N}$ & $50^{\circ} 33^{\prime} \mathrm{W}$ & N & 125 \\
\hline 1CF04006 & $63^{\circ} 52^{\prime} \mathrm{N}$ & $50^{\circ} 34^{\prime} \mathrm{W}$ & W & 140 \\
\hline $1 \mathrm{CF}$ & $63^{\circ} 51^{\prime} \mathrm{N}$ & $50^{\circ} 36^{\prime} \mathrm{W}$ & $\hat{N}$ & 140 \\
\hline 1CF04008 & $63^{\circ} 50^{\prime} \mathrm{N}$ & $50^{\circ} 38^{\prime} \mathrm{W}$ & $\mathrm{N}$ & 140 \\
\hline 1CF04009 & $63^{\circ} 51^{\prime} \mathrm{N}$ & $50^{\circ} 39^{\prime} \mathrm{W}$ & $\mathbf{N}$ & 80 \\
\hline 1CF04 & $63^{\circ} 50^{\prime} \mathrm{N}$ & $50^{\circ} 41^{\prime} \mathrm{W}$ & $\mathrm{N}$ & 140 \\
\hline & $63^{\circ} 51^{\prime} \mathrm{N}$ & $50^{\circ} 43^{\prime} \mathrm{W}$ & $\mathrm{N}$ & 150 \\
\hline 1CF04012 & 63 & $50^{\circ} 47^{\prime \prime V}$ & NE & 140 \\
\hline 1CF04013 & $63^{\circ} 52^{\prime} \mathrm{N}$ & $50^{\circ} 50^{\prime} \mathrm{W}$ & NE & 115 \\
\hline 1CF04014 & $63^{\circ} 52^{\prime} \mathrm{N}$ & $50^{\circ} 53^{\prime} \mathrm{V}$ & NW & 130 \\
\hline & $63057, \mathrm{~N}$ & $50^{\circ} 51^{\prime} \mathrm{V}$ & $\mathbf{N}$ & 115 \\
\hline $1 \mathrm{CF} 04 \mathrm{C}$ & $63^{\circ} 52^{\prime} \mathrm{N}$ & $50^{\circ} 54^{\prime} \mathrm{W}$ & $\mathrm{N}$ & 95 \\
\hline (1) & $63^{\circ} 51$ 'N & $50^{\circ} 53^{\prime} \mathrm{V}$ & Nw & 110 \\
\hline
\end{tabular}

LOW

$45 \quad 0000.80$

CODE

LAT

LONG

ORI

CODE MAP

$\begin{array}{llll}110 & 0000.70 & 700012 & 6330-02 \\ 6330-02\end{array}$

135 0000.20 640012 6330-02

$\begin{array}{lll}0000.30 & 640010 & 6330-02\end{array}$

$\begin{array}{lll}0009.60 & 365412 & 6330-02\end{array}$

$\begin{array}{llll}70 & 0000.30 & 640410 & 6330-02 \\ 60 & 0000.50 & 640110 & 6330-01\end{array}$

$80 \quad 0000.70 \quad 700010 \quad 6330-02$

$\begin{array}{llll}90 & 0000.20 & 700010 & 6330-02 \\ 75 & 0001.00 & 653010 & 6330-01\end{array}$

$\begin{array}{llll}75 & 0001.00 & 653010 & 6330-01 \\ 75 & 0002.00 & 623410 & 6330-01\end{array}$

$\begin{array}{llll}60 & 0002.00 & 623410 & 6330-01 \\ 60 & 0001.40 & 630410 & 6330-01\end{array}$

$65 \quad 0000.80 \quad 640410 \quad 6330-01$

$\begin{array}{llll}50 & 0000.70 & 643110 & 6330-01\end{array}$

$\begin{array}{llll}45 & 0002.00 & 643110 & 6330-01\end{array}$

$\begin{array}{rrrr}75 & 0001.30 & 630010 & 6330-01 \\ 105 & 0002.50 & 530010 & 6330-0\end{array}$

$\begin{array}{llll}60 & 0001.70 & 530110 & 6330-01\end{array}$

$\begin{array}{llll}60 & 0000.30 & 700010 & 6330-01\end{array}$

$\begin{array}{llll}95 & 0001.10 & 500110 & 6330-01 \\ 55 & 0003.50 & 533112 & 6330-02\end{array}$

$\begin{array}{llll}55 & 0003.50 & 533112 & 6330-02 \\ 80 & 0000.01 & 700010 & 6330-01\end{array}$

0000.01

$\begin{array}{rr}110 & 0000.10 \\ 55 & 0000.03\end{array}$

0000.03
0000.32

$\begin{array}{ll}65 & 0000.07 \\ 60 & 0000.36\end{array}$

$100 \quad 0002.04$

$\begin{array}{ll}33 & 0000.28 \\ 75 & 0001.10\end{array}$

750001.10

$80 \quad 0000.30$

$\begin{array}{rr}125 & 0000.40 \\ 75 & 0000.70\end{array}$

$70 \quad 0000.50$

$\begin{array}{ll}90 \\ 105 & 0000.10\end{array}$

$100 \quad 0004.30$

0000.60

0000.20

$\begin{array}{ll}115 & 0001.00 \\ 110 & 0001.20\end{array}$

$\begin{array}{ll}110 & 0002.60 \\ 105 & 0001.00\end{array}$

$\begin{array}{ll}105 & 0001.00 \\ 100 & 0002.40\end{array}$

$\begin{array}{ll}90 & 0000.50 \\ 80 & 0009.40\end{array}$

0001.30

$\begin{array}{ll}80 & 0010.50 \\ 80 & 0001.00\end{array}$

$\begin{array}{ll}60 & 0010.00\end{array}$

$85 \quad 0001.00$

$\begin{array}{ll}80 & 0006.00 \\ 95 & 0001.70\end{array}$

$\begin{array}{ll}95 & 0001.70 \\ 75 & 0000.60\end{array}$

$\begin{array}{rr}105 & 0000.20 \\ 80 & 0000.60\end{array}$

$700010 \quad 6330-01$

$700010 \quad 6300-01$

$640010 \quad 6330-01$

$640010 \quad 6330-01$

$770010 \quad 6330-01$

$\begin{aligned} & 603312 \\ & 770412\end{aligned} 6300-01$

230112 6330-01

$230112 \quad 6330-01$

$360010 \quad 6330-01$

$700010 \quad 6330-01$

$730010 \quad 6330-01$

$640112 \quad 6330-01$

$5301126330-01$

630112 6330-01

$\begin{array}{ll}700010 & 6330-02 \\ 640112 & 6330-02\end{array}$

$640112 \quad 6330-02$

$\begin{array}{ll}640112 & 6330-02 \\ 730112 & 6330-02\end{array}$

$644112 \quad 6330-02$

643412 6330-02

$\begin{array}{ll}363112 & 6330-02 \\ 530112 & 6330-02\end{array}$

366112 6330-02

$6231126330-01$

$530112 \quad 6330-01$

$523112 \quad 6330-01$

$700012 \quad 6330-01$

$700012 \quad 6330-01$

1CF04019

$63^{\circ} 50^{\prime} \mathrm{N}$

$50^{\circ} 57^{\prime} \mathrm{W} \mathrm{N}$

1 Cr06002

$63^{\circ} 49 \prime \mathrm{N}$

$50^{\circ} 48^{\prime} \mathrm{W}$ W

$1 \mathrm{CFO6004}$

$63^{\circ} 49^{\prime} \mathrm{N}$

$50^{\circ} 50^{\prime} \mathrm{W} \quad \mathrm{W}$

1 CF06006

-1
0

$50^{\circ} 51, \mathrm{NW}$

(CF06007

$63^{\circ} 49^{\prime} \mathrm{N}$

每

$1 \mathrm{CF} 06009$

$63.49 \cdot \mathrm{N}$

$50^{\circ} 54, \mathrm{~W}$

1 CF06011

-

$\begin{array}{lll}50^{\circ} 55^{\prime}, \mathrm{W} & \mathrm{N} \\ 50^{\circ} 56^{\prime}, \mathrm{W} & \mathrm{N}\end{array}$

1CF06012

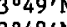

$50^{\circ} 59, \mathrm{~W} N$

1 CF06014

63.

$51^{\circ} 00^{\prime} \mathrm{W}$

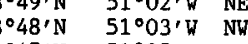

$1 \mathrm{CF} 0700$

$51^{\circ} 05$ ' W N

1 CF08001

$63^{\circ} 47$, N $510^{\circ} 02$, N

1 CG01001

51.06' W

CG01002

$64^{\circ} 11^{\prime} \mathrm{N} \quad 51^{\circ} 30^{\prime} \mathrm{W}$

1 CG01004

1CG01005

$51^{\circ} 26^{\prime} \mathrm{W}$

$64^{\circ} 10^{\prime} \mathrm{N} \quad 51^{\circ} 21^{\prime} \mathrm{W}$

$64^{\circ} 10^{\prime} \mathrm{N}$

$\begin{array}{lll}51^{\circ} 23^{\prime}, \mathrm{W} & \mathrm{N} \\ 51^{\circ} 22 & \mathrm{~W} & \mathrm{~S}\end{array}$

1 CG01007

. $51^{\circ} 20^{\prime} \mathrm{W}$

1 CG0100

$51^{\circ} 20^{\prime} \mathrm{W}$ S

1CG01011

$\begin{array}{lll}51^{\circ} 16^{\prime} \mathrm{W} & \mathrm{NW} \\ 51^{\circ} 21^{\prime} \mathrm{W} & \mathrm{NE}\end{array}$

1 CG01013

$51^{\circ} 20^{\prime} \mathrm{W}$

1 CG01014 $64^{\circ} 07^{\prime} \mathrm{N} \quad 51^{\circ} 26^{\prime} \mathrm{W}$ N

1 CG01016

$51^{\circ} 29^{\prime} \mathrm{W} N$

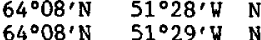

64.08 .

$51^{\circ} 29 \cdot \mathrm{W} N \mathrm{~N}$

(c01018

$64^{\circ} 09^{\circ}$

$51^{\circ} 33^{\prime} \mathrm{W}$

1 GG1020

$64^{\circ} 08^{\prime} \mathrm{N} \quad 51^{\circ} 34^{\prime} \mathrm{N} \quad \mathrm{NE}$

$64^{\circ} 08^{\prime} \mathrm{N} \quad 51^{\circ} 35^{\prime} \mathrm{W}$

$64^{\circ} 06^{\prime} \mathrm{N} \quad 51^{\circ} 20^{\prime} \mathrm{W} S \mathrm{~S}$

$64^{\circ} 08^{\prime} \mathrm{N}$

$51^{\circ} 11, \mathrm{~W}$ NE

$1 \mathrm{CG02003}$

$51^{\circ} 14^{\prime} \mathrm{W} E$

$\begin{array}{lll}64^{\circ} 08^{\prime} \mathrm{N} & 51^{\circ} 12^{\prime} \mathrm{W} & \mathrm{NE} \\ 64^{\circ} 10^{\prime} \mathrm{N} & 51^{\circ} 05^{\prime} \mathrm{W} & \mathrm{NB}\end{array}$

$64^{\circ} 09^{\prime} \mathrm{N} \quad 51^{\circ} 00^{\prime} \mathrm{W} N$

$\begin{array}{lll}64^{\circ} 17^{\prime} \mathrm{N} & 50^{\circ} 37^{\prime} \mathrm{W} & \mathrm{N} \\ 64^{\circ} 12^{\prime} \mathrm{N} & 49^{\circ} 31^{\prime} \mathrm{V} & \mathrm{N}\end{array}$

(c)

$\begin{array}{lll}64^{\circ} 12^{\prime} \mathrm{N} & 49^{\circ} 31^{\prime} \mathrm{W} & \mathrm{NW} \\ 64^{\circ} 10^{\prime} \mathrm{N} & 49^{\circ} 29^{\prime} \mathrm{W} & \mathrm{NW}\end{array}$

(cGi3002

$64^{\circ} 11^{\prime} \mathrm{N} \quad 49^{\circ} 42^{\prime} \mathrm{W}$ NW

$64^{\circ} 10^{\prime} \mathrm{N} \quad 49^{\circ} 46^{\prime} \mathrm{V} N \mathrm{NV}$

$\begin{array}{lll}64^{\circ} 05^{\prime} \mathrm{N} & 49^{\circ} 34^{\prime} \mathrm{N} & \mathrm{NW} \\ 63^{\circ} 58^{\prime} \mathrm{N} & 49^{\circ} 27, \mathrm{~W} & \mathrm{NW}\end{array}$

$63^{\circ} 56^{\prime} \mathrm{N} \quad 49^{\circ} 34^{\prime} \mathrm{W} \mathrm{N}$

$63^{\circ} 56^{\prime} \mathrm{N} \quad 49^{\circ} 56^{\prime} \mathrm{W}$ N

$\begin{array}{llll}1 \text { CG14006 } & 63^{\circ} 56^{\prime} \mathrm{N} & 49^{\circ} 56^{\prime}, \mathrm{W} & \mathrm{E} \\ 1 \mathrm{CG} 14007 & 63^{\circ} 56^{\prime} \mathrm{N} & 49^{\circ} 58^{\prime} \mathrm{W} & \mathrm{NW}\end{array}$

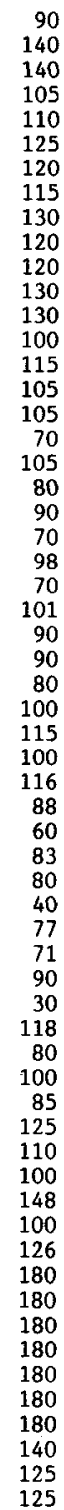

$\begin{array}{rrrr}70 & 0000.50 & 730010 & 6330-01 \\ 115 & 0000.60 & 330110 & 6330-01\end{array}$

$330110 \quad 6330-01$

$700110 \quad 6330-01$

640112 6330-01

$6431126330-01$

$640112 \quad 6330-01$

$643310 \quad 6330-01$

$640110 \quad 6330-01$

$537112 \quad 6330-01$

$240113 \quad 6330-01$

233112 6330-01

$630110 \quad 6330-01$

$640110 \quad 6330-01$

$700010 \quad 6330-01$

$780010 \quad 6400-01$

$780010 \quad 6400-01$

$780010 \quad 6400-01$

$\begin{array}{ll}780010 & 6400-01 \\ 770010 & 6400-01\end{array}$

$670112 \quad 6400-01$

$670112 \quad 6400-01$

$640112 \quad 6400-01$

$6701126400-01$

$670312 \quad 6400-01$

$770110 \quad 6400-01$

$270112 \quad 6400-01$

$643212 \quad 6400-01$

780010 6400-01

$780010 \quad 6400-01$

$780010 \quad 6400-01$

$640212 \quad 6400-01$

$750010 \quad 6400-01$

$790010 \quad 6400-01$

$643312 \quad 6400-02$

$770012 \quad 6400-02$

$643112 \quad 6400-02$

$\begin{array}{ll}780010 & 6400-02 \\ 750010 & 6400-02\end{array}$

$166111 \quad 6400-04$

163112 6400-04

$163111 \quad 6400-03$

163111 6400-03

163112 6330-03

$270110 \quad 6330-03$

$\begin{array}{ll}770110 & 6330-03 \\ 770110 & 6330-02\end{array}$

a 


\begin{tabular}{|c|c|c|c|c|c|c|c|c|c|c|c|c|c|c|c|c|c|}
\hline CODE & LAT & LONG & ORI & $\mathrm{HIGH}$ & LOW & AREA & $\begin{array}{c}\text { MO } \\
\text { CODE }\end{array}$ & MAP & CODE & LAT & LONG & ORI & $\mathrm{HIGH}$ & LOW & AREA & $\begin{array}{c}\text { MO } \\
\text { CODE }\end{array}$ & MAP \\
\hline 1CG14008 & $63^{\circ} 56^{\prime} \mathrm{N}$ & $\mathrm{W}$ & E & 135 & 125 & 00 & 710110 & 633 & $1 \mathrm{CG} 2$ & $64^{\circ} 03^{\prime} \mathrm{N}$ & $\mathrm{v}$ & $\mathbf{N}$ & 100 & 100 & 000 & 700010 & $6400-02$ \\
\hline ICG14009 & $63^{\circ} 58^{\prime} \mathrm{N}$ & $49^{\circ} 59^{\prime} \mathrm{W}$ & $N E$ & 115 & 105 & 0000.13 & 770110 & $6330-02$ & 1CG20004 & $64^{\circ} 02^{\prime} \mathrm{N}$ & $50^{\circ} 52 \% \mathrm{~V}$ & W & 130 & 100 & 0000.35 & 640112 & $6400-02$ \\
\hline 1CG14010 & $63^{\circ} 58^{\prime} \mathrm{N}$ & $50^{\circ} 01^{\prime} \mathrm{W}$ & $\mathrm{N}$ & 145 & 120 & 0000.43 & 770110 & $6330-02$ & $1 \mathrm{CG} 20005$ & $64^{\circ} 01^{\prime} \mathrm{N}$ & $50^{\circ} 52^{\prime} \mathrm{W}$ & w & 105 & 90 & 0000.10 & 700010 & $6400-02$ \\
\hline 1CG14011 & $63^{\circ} 58^{\prime} \mathrm{N}$ & $50^{\circ} 02^{\prime} \mathrm{W}$ & $\mathrm{N}$ & 150 & 125 & 0000.41 & 770112 & $6330-02$ & $1 \mathrm{CG} 20006$ & $64^{\circ} 00^{\prime} \mathrm{N}$ & $50^{\circ} 54^{\prime} \mathrm{W}$ & NW & 110 & 90 & 0002.90 & 533112 & $6400-02$ \\
\hline 1CG14012 & $63^{\circ} 57^{\prime} \mathrm{N}$ & $50^{\circ} 03^{\prime} \mathrm{W}$ & $\mathrm{NE}$ & 130 & 125 & 0000.54 & 770110 & $6330-02$ & $1 \mathrm{CG} 20007$ & $64^{\circ} 01^{\prime} \mathrm{N}$ & $50^{\circ} 55^{\prime}$ & NW & 110 & 100 & 0000.20 & 750012 & $6400-02$ \\
\hline 1CG14013 & $63057 / \mathrm{N}$ & $50^{\circ} 04^{\prime} \mathrm{W}$ & $\mathrm{NE}$ & 130 & 125 & 0000.07 & 770110 & $6330-02$ & 1 CG20008 & $64^{\circ} 01^{\prime} \mathrm{N}$ & $50^{\circ} 55^{\prime} \mathrm{V}$ & NW & 100 & 90 & 0000.10 & 750012 & $6400-02$ \\
\hline $1 \mathrm{CG} 14014$ & $63^{\circ} 57^{\prime} \mathrm{N}$ & $50^{\circ} 04^{\prime} \mathrm{W}$ & $\mathrm{NW}$ & 145 & 130 & 0000.62 & 740110 & $6330-02$ & ICG20009 & $64^{\circ} 00^{\prime} \mathrm{N}$ & $50^{\circ} 55, \mathrm{~W}$ & $\mathrm{Nw}$ & 120 & 90 & 0001.30 & 530112 & $6400-02$ \\
\hline 1CG14015 & $63^{\circ} 57^{\prime} \mathrm{N}$ & $50^{\circ} 05^{\prime} \mathrm{W}$ & $\mathrm{N}$ & 125 & 115 & 0000.12 & 770110 & $6330-02$ & 1CG20010 & $64^{\circ} 00^{\prime} \mathrm{N}$ & $50^{\circ} 55^{\prime} \mathrm{W}$ & NW & 120 & 90 & 0001.10 & 530112 & $6400-02$ \\
\hline $1 C G 14016$ & $63^{\circ} 56^{\prime} \mathrm{N}$ & $50^{\circ} 04^{\prime} \mathrm{W}$ & $\mathrm{N}$ & 150 & 140 & 0000.23 & 770110 & $6330-02$ & $1 C G 20011$ & $63^{\circ} 59^{\prime} \mathrm{N}$ & $50^{\circ} 54^{\prime} \mathrm{W}$ & $w$ & 125 & 105 & 0000.80 & 533112 & $6330-01$ \\
\hline 1CG14017 & $63^{\circ} 55^{\prime} \mathrm{N}$ & $50^{\circ} 07 \cdot \mathrm{W}$ & E & 125 & 120 & 0000.25 & 770110 & $6330-02$ & 1 CG20012 & $63^{\circ} 59^{\prime} \mathrm{N}$ & $50^{\circ} 56^{\prime} \mathrm{V}$ & $\mathrm{NE}$ & 120 & 75 & 0003.50 & 523112 & $6330-01$ \\
\hline 1CG14018 & $63^{\circ} 55^{\prime} \mathrm{N}$ & $50^{\circ} 07^{\prime} \mathrm{W}$ & $\mathrm{NE}$ & 120 & 120 & 0000.11 & 770110 & $6330-02$ & 1 CG20013 & $63^{\circ} 59 \cdot \mathrm{N}$ & $50^{\circ} 59^{\prime} \mathrm{W}$ & $\mathrm{N}$ & 120 & 70 & 0002.00 & 533112 & 6330-01 \\
\hline 1CG14019 & $63^{\circ} 55^{\prime} \mathrm{N}$ & $50^{\circ} 08^{\prime}, \widehat{W}$ & $v$ & 120 & 110 & 0000.34 & 770111 & $6330-02$ & 1CG21001 & $64^{\circ} 01^{\prime} \mathrm{N}$ & $51^{\circ} 06^{\prime}, \mathrm{V}$ & NW & 90 & 85 & 0000.12 & 670112 & $6400-02$ \\
\hline 1CG14020 & $63^{\circ} 55^{\prime} \mathrm{N}$ & $50^{\circ} 08^{\prime} \mathrm{W}$ & NW & 135 & 120 & 0000.75 & 770112 & $6330-02$ & 1CG22001 & $63^{\circ} 57 / \mathrm{N}$ & $51^{\circ} 04^{\prime} \mathrm{V}$ & $\mathrm{NE}$ & 115 & 95 & 0001.90 & 360112 & $6330-01$ \\
\hline 1CG14021 & $63^{\circ} 58^{\prime} \mathrm{N}$ & $50^{\circ} 10^{\prime} \mathrm{W}$ & - & 130 & 130 & 0000.06 & 700110 & $6330-02$ & $1 \mathrm{CG} 22002$ & $63^{\circ} 57^{\prime} \mathrm{N}$ & $51^{\circ} 06^{\prime}$ & $\mathrm{N}$ & 115 & 60 & 0001.40 & 363112 & $6330-01$ \\
\hline 1CG14022 & $63^{\circ} 54^{\prime} \mathrm{N}$ & $50^{\circ} 09^{\prime} \mathrm{W}$ & $\mathrm{NE}$ & 130 & 115 & 0000.15 & 740110 & $6330-02$ & $1 \mathrm{CG} 23001$ & $63^{\circ} 57^{\prime} \mathrm{N}$ & $51^{\circ} 07^{\prime} \mathrm{V}$ & NW & 115 & 90 & 0001.00 & 361112 & $6330-01$ \\
\hline 1 CG14023 & $63^{\circ} 54^{\prime} \mathrm{N}$ & $50^{\circ} 11 \cdot \mathrm{W}$ & - & 115 & 110 & 0000.06 & 750112 & $6330-02$ & 1 CG23002 & $63^{\circ} 56^{\prime} \mathrm{N}$ & $51^{\circ} 08^{\prime}$ & $\mathrm{W}$ & 90 & 75 & 0000.30 & 533112 & $6330-01$ \\
\hline 1CG14024 & $63^{\circ} 54^{\prime} \mathrm{N}$ & $50^{\circ} 13^{\prime} \mathrm{W}$ & - & 125 & 115 & 0000.05 & 770110 & $6330-02$ & 1CG23003 & $63^{\circ} 55^{\prime} \mathrm{N}$ & $51^{\circ} 09^{\prime}$ & NE & 95 & 90 & 0001.00 & 700010 & 6330-01 \\
\hline 1 CG14025 & $63^{\circ} 54^{\prime} \mathrm{N}$ & $50^{\circ} 14^{\prime} \mathrm{W}$ & NW & 135 & 125 & 0000.23 & 770110 & $6330-02$ & 1 CG23004 & $63^{\circ} 55^{\prime} \mathrm{N}$ & $51^{\circ} 10^{\prime} \mathrm{V}$ & NV & 95 & 80 & 0000.20 & 670112 & $6330-01$ \\
\hline $1 C G 14026$ & $63^{\circ} 54^{\prime} \mathrm{N}$ & $50^{\circ} 15^{\prime} \mathrm{W}$ & $\mathrm{N}$ & 125 & 125 & 0000.20 & 770110 & $6330-02$ & $1 \mathrm{CG} 24001$ & $63^{\circ} 55^{\prime} \mathrm{N}$ & $51^{\circ} 11^{\prime}$ & SW & 99 & 85 & 0002.60 & 230110 & $6330-01$ \\
\hline 1 CG14027 & $63^{\circ} 56^{\prime} \mathrm{N}$ & $50^{\circ} 25^{\prime} \mathrm{W}$ & NW & 150 & 110 & 0000.10 & 770110 & $6330-02$ & $1 \mathrm{CH} 02001$ & $65^{\circ} 15^{\prime} \mathrm{N}$ & $50^{\circ} 33,6$ & $\mathrm{SW}$ & 180 & 10 & 2047.81 & 163113 & $6500-04$ \\
\hline 1CG14028 & $63^{\circ} 56^{\prime} \mathrm{N}$ & $50^{\circ} 41 \cdot \mathrm{W}$ & $\mathrm{N}$ & 135 & 110 & 0000.27 & 770112 & $6330-02$ & $1 \mathrm{CH} 02002$ & $65^{\circ} 15^{\prime} \mathrm{N}$ & $49^{\circ} 53^{\prime} \mathrm{V}$ & $\mathrm{w}$ & 180 & 62 & 0334.30 & 163114 & $6500-05$ \\
\hline 1CG14029 & $63^{\circ} 56^{\prime} \mathrm{N}$ & $50^{\circ} 43^{\prime} \mathrm{W}$ & $\mathrm{N}$ & 135 & 100 & 0000.37 & 273110 & $6330-02$ & $1 \mathrm{CHO} 2003$ & $65^{\circ} 13^{\circ} \mathrm{N}$ & $49045, \mathrm{~W}$ & N & 124 & 110 & 0001.02 & 770010 & $6500-05$ \\
\hline 1CG14030 & $63^{\circ} 56^{\prime} \mathrm{N}$ & $50^{\circ} 44^{\prime} \mathrm{W}$ & $\mathrm{NW}$ & 135 & 90 & 0000.67 & 770110 & $6330-02$ & $1 \mathrm{CH} 02004$ & $65^{\circ} 08^{\prime} \mathrm{N}$ & $49^{\circ} 55^{\prime}$ & NW & 105 & 100 & 0000.58 & 780010 & $6500-05$ \\
\hline 1CG14031 & $63^{\circ} 59^{\prime} \mathrm{N}$ & $50^{\circ} 51^{\prime} \mathrm{V}$ & $\mathrm{NE}$ & 105 & 85 & 0003.18 & 533111 & $6330-01$ & $1 \mathrm{CH} 02005$ & $65^{\circ} 09^{\prime} \mathrm{N}$ & $49^{\circ} 56^{\prime} \mathrm{W}$ & NW & 110 & 95 & 0000.50 & 780010 & $6500-05$ \\
\hline $1 \mathrm{CG} 14032$ & $64^{\circ} 01^{\prime} \mathrm{N}$ & $50^{\circ} 45^{\prime} \mathrm{W}$ & SW & 115 & 110 & 0000.31 & 770110 & $6400-02$ & $1 \mathrm{CH} 02006$ & $65^{\circ} 10^{\prime} \mathrm{N}$ & $49 \circ 55^{\prime} \mathrm{V}$ & N & 110 & 100 & 0000.22 & 780010 & $6500-05$ \\
\hline 1CG14033 & $64^{\circ} 01^{\prime} \mathrm{N}$ & $50^{\circ} 39^{\prime} \mathrm{W}$ & $\mathrm{NE}$ & 145 & 110 & 0001.14 & 643111 & $6400-02$ & $1 \mathrm{CHO} 2007$ & $65^{\circ} 10^{\prime} \mathrm{N}$ & $49056, \mathrm{~W}$ & NW & 110 & 105 & 0000.52 & 780010 & $6500-05$ \\
\hline 1CG14034 & $64^{\circ} \mathrm{O} 2^{\prime} \mathrm{N}$ & $50^{\circ} 33^{\prime} \mathrm{W}$ & $\mathrm{N}$ & 115 & 100 & 0000.24 & 770110 & $6400-02$ & $1 \mathrm{CHO} 2008$ & $65^{\circ} 11^{\prime} \mathrm{N}$ & $49057, \mathrm{~V}$ & N & 110 & 110 & 0000.37 & 780010 & $6500-05$ \\
\hline 1CG16001 & $64^{\circ} 03^{\prime} \mathrm{N}$ & $50^{\circ} 34^{\prime} \mathrm{W}$ & N & 115 & 110 & 0000.09 & 790012 & $6400-02$ & 1 СHO2009 & $65^{\circ} 12^{\prime} \mathrm{N}$ & $49^{\circ} 57^{\prime} \mathrm{W}$ & N & 115 & 110 & 0000.73 & 780010 & $6500-05$ \\
\hline 1CG16002 & $64^{\circ} 02^{\prime} \mathrm{N}$ & $50^{\circ} 35^{\prime} \mathrm{W}$ & NW & 115 & 110 & 0000.07 & 790012 & $6400-02$ & $1 \mathrm{CHO} 2010$ & $65^{\circ} 11^{\prime} \mathrm{N}$ & $50^{\circ} 00^{\prime}$ W & No & 115 & 115 & 0000.36 & 780010 & $6500-05$ \\
\hline $1 \mathrm{CG} 16003$ & $64^{\circ} 02^{\prime} \mathrm{N}$ & $50^{\circ} 38^{\prime} \mathrm{W}$ & $\mathrm{N}$ & 120 & 110 & 0000.05 & 640112 & $6400-02$ & $1 \mathrm{CH} 02011$ & $65^{\circ} 10^{\prime} \mathrm{N}$ & $49^{\circ} 58^{\prime}$ W & NW & 115 & 110 & 0000.27 & 780010 & $6500-05$ \\
\hline 1CG16004 & $64^{\circ} 01^{\prime} \mathrm{N}$ & $50^{\circ} 40^{\prime} \mathrm{W}$ & $\mathrm{NE}$ & 140 & 85 & 0001.98 & 643112 & $6400-02$ & $1 \mathrm{CHO} 2012$ & $65^{\circ} 07^{\prime N}$ & $49^{\circ} 58^{\prime} \mathrm{W}$ & N & 110 & 100 & 0000.18 & 780010 & $6500-05$ \\
\hline 1CG16005 & $64^{\circ} 02^{\prime} \mathrm{N}$ & $50^{\circ} 41^{\prime}, \mathrm{W}$ & $\mathrm{N}$ & 110 & 100 & 0000.06 & 650112 & $6400-02$ & $1 \mathrm{CHO} 2013$ & $65^{\circ} 11^{\prime} \mathrm{N}$ & $49^{\circ} 44^{\prime} \mathrm{W}$ & w & 180 & 85 & 0188.75 & 163113 & $6500-05$ \\
\hline 1CG16006 & $64^{\circ} 02^{\prime} \mathrm{N}$ & $50^{\circ} 43^{\prime} \mathrm{W}$ & NE & 135 & 100 & 0005.82 & 273112 & $6400-02$ & $1 \mathrm{CHO} 2014$ & $65^{\circ} 09^{\prime} \mathrm{N}$ & $49^{\circ} 42^{\prime} \mathrm{W}$ & w & 180 & 80 & 0292.40 & 166113 & $6500-05$ \\
\hline 1 CG16007 & $64^{\circ} 04^{\prime} \mathrm{N}$ & $50^{\circ} 43^{\prime} \mathrm{W}$ & $\mathrm{SE}$ & 134 & 130 & 0000.13 & 780010 & $6400-02$ & $1 \mathrm{CH} 02015$ & $65^{\circ} 07 \times \mathrm{N}$ & $49044 \mathrm{r}$ & $\mathrm{W}$ & 180 & 85 & 0132.79 & 163113 & $6500-05$ \\
\hline 1CG16008 & $64^{\circ} 04^{\prime} \mathrm{N}$ & $50^{\circ} 39^{\prime} \mathrm{W}$ & E & 140 & 115 & 0000.17 & 780010 & $6400-02$ & $1 \mathrm{CH} 02016$ & $65^{\circ} 02^{\prime} \mathrm{N}$ & $49^{\circ} 50^{\prime} \mathrm{V}$ & $\mathrm{W}$ & 180 & 75 & 0526.41 & 166113 & $6500-05$ \\
\hline 1CG16009 & $64^{\circ} 05^{\prime} \mathrm{N}$ & $50^{\circ} 38^{\prime} \mathrm{W}$ & E & 130 & 120 & 0000.10 & 780010 & $6400-02$ & $1 \mathrm{CH} 08001$ & $64^{\circ} 58^{\prime} \mathrm{N}$ & $49^{\circ} 56^{\prime} \mathrm{V}$ & SW & 180 & 75 & 0056.60 & 166113 & $6430-03$ \\
\hline 1CG16010 & $64^{\circ} 05^{\prime} \mathrm{N}$ & $50^{\circ} 36^{\prime} \mathrm{W}$ & NW & 120 & 120 & 0000.09 & 750010 & $6400-02$ & $1 \mathrm{CH} 13001$ & $64^{\circ} 56^{\prime} \mathrm{N}$ & $49054^{\prime} \mathrm{W}$ & sw & 180 & 75 & 0089.74 & 163112 & $6430-03$ \\
\hline 1CG17001 & $64^{\circ} 07^{\prime} \mathrm{N}$ & $50^{\circ} 34^{\prime} \mathrm{V}$ & $\mathrm{NW}$ & 120 & 105 & 0000.53 & 780010 & $6400-02$ & $1 \mathrm{CH} 13002$ & $64^{\circ} 53^{\prime} \mathrm{N}$ & $49^{\circ} 53^{\prime} \mathrm{W}$ & SW & 180 & 5 & 0456.50 & 163112 & $6430-03$ \\
\hline 1CG17002 & $64^{\circ} 06^{\prime} \mathrm{N}$ & $50^{\circ} 37 \cdot \mathrm{W}$ & $N W$ & 140 & 85 & 0000.38 & 780010 & $6400-02$ & $1 \mathrm{CH} 13003$ & $64^{\circ} 53^{\prime} \mathrm{N}$ & $49042 \cdot \mathrm{W}$ & $\mathrm{w}$ & 180 & 75 & 0110.98 & 163112 & $6430-04$ \\
\hline 1 CG17003 & $64^{\circ} 05^{\prime} \mathrm{N}$ & $50^{\circ} 40^{\prime} \mathrm{W}$ & $\mathrm{N}$ & 130 & 105. & 0001.81 & 643112 & $6400-02$ & $1 \mathrm{CH} 13004$ & $64^{\circ} 50^{\prime} \mathrm{N}$ & $49^{\circ} 39^{\prime} \mathrm{W}$ & W & 180 & 75 & 0361.54 & 166112 & $6430-04$ \\
\hline ICG17004 & $64^{\circ} 05^{\prime} \mathrm{N}$ & $50^{\circ} 43^{\prime} \mathrm{W}$ & NE & 130 & 95 & 0000.32 & 643112 & $6400-02$ & 1 CH17001 & $64^{\circ} 49^{\prime} \mathrm{N}$ & 49033.6 & SW & 180 & 90 & 0037.78 & 163112 & $6430-04$ \\
\hline 1CG17005 & $64^{\circ} 05^{\prime} \mathrm{N}$ & $50^{\circ} 43^{\prime} \mathrm{W}$ & NE & 130 & 85 & 0000.39 & 643112 & $6400-02$ & $1 \mathrm{CH} 17002$ & $64^{\circ} 41^{\prime} \mathrm{N}$ & $49^{\circ} 48^{\prime}$, & $\mathrm{W}$ & 180 & & 1187.96 & 164113 & $6430-04$ \\
\hline 1 CG17006 & $64^{\circ} 07^{\prime} \mathrm{N}$ & $50^{\circ} 41 \cdot \mathrm{W}$ & $N E$ & 145 & 110 & 0000.72 & 643112 & 640 & $1 \mathrm{CH} 17003$ & $64^{\circ} 36^{\prime} \mathrm{N}$ & $49^{\circ} 26^{\prime} \mathrm{W}$ & W & 180 & 75 & 0075.84 & 163112 & $6430-04$ \\
\hline $1 \mathrm{CG} 18001$ & $64^{\circ} 08^{\prime} \mathrm{N}$ & $50^{\circ} 42 \cdot \mathrm{W}$ & $\mathbf{N}$ & 115 & 105 & 0000.06 & 780010 & $6400-02$ & $1 \mathrm{CH} 21001$ & $64^{\circ} 34^{\prime} \mathrm{N}$ & $49^{\circ} 27^{\prime} \mathrm{W}$ & SW & 180 & 40 & 0169.23 & 163111 & $6430-04$ \\
\hline 1CG18002 & $64^{\circ} 08^{\prime} \mathrm{N}$ & $50^{\circ} 43^{\prime} \mathrm{V}$ & NW & 145 & 135 & 0000.56 & 673212 & $6400-02$ & $1 \mathrm{CH} 21002$ & $64^{\circ} 29^{\prime} \mathrm{N}$ & $49^{\circ} 32^{\prime} \mathrm{V}$ & SW & 180 & 60 & 0327.20 & 163111 & 640 \\
\hline 1CG18003 & $64^{\circ} 07^{\prime} \mathrm{N}$ & $50^{\circ} 44^{\prime} \mathrm{W}$ & NW & 140 & 50 & 0001.49 & 613012 & $6400-02$ & $1 \mathrm{CH} 22001$ & $64^{\circ} 22^{\prime} \mathrm{N}$ & $49^{\circ} 37$, W & $\mathrm{sW}$ & 180 & & 0338.25 & 164111 & $6400-03$ \\
\hline ICG18004 & $64^{\circ} 07^{\prime} \mathrm{N}$ & $50^{\circ} 45^{\prime} \mathrm{W}$ & NW & 125 & 85 & 0000.43 & 670012 & $6400-02$ & $1 \mathrm{CH} 22002$ & $64^{\circ} 25^{\prime} \mathrm{N}$ & $49^{\circ} 14^{\prime} \mathrm{V}$ & $\mathrm{v}$ & 180 & 95 & 0193.39 & 163112 & $6400-04$ \\
\hline 1CG18005 & $64^{\circ} 07^{\prime} \mathrm{N}$ & $50^{\circ} 45^{\prime} \mathrm{W}$ & NW & 80 & 55 & 0000.49 & 643112 & & $1 \mathrm{CH} 23001$ & $64^{\circ} 22^{\prime} \mathrm{N}$ & $49^{\circ} 14^{\prime} \mathrm{W}$ & W & 180 & 95 & 0111.30 & 163112 & $6400-04$ \\
\hline $1 \mathrm{CG} 18006$ & $64^{\circ} 06^{\prime} \mathrm{N}$ & $50^{\circ} 46^{\prime} \mathrm{V}$ & NW & 130 & 90 & 0000.95 & 643112 & $6400-02$ & $1 \mathrm{CH} 23002$ & $64^{\circ} 20^{\prime} \mathrm{N}$ & $49^{\circ} 13^{\prime} \mathrm{W}$ & $\mathrm{W}$ & 180 & 90 & 0027.35 & 163112 & $6400-04$ \\
\hline 1CG18007 & $64^{\circ} 06^{\prime} \mathrm{N}$ & $50^{\circ} 45^{\prime} \mathrm{W}$ & $\mathrm{SE}$ & 80 & 45 & 0000.26 & 770010 & $6400-02$ & $1 \mathrm{CH} 23003$ & $64^{\circ} 15^{\prime} \mathrm{N}$ & $49^{\circ} 32, \mathrm{n}$ & $\mathrm{H}$ & 180 & & 1551.52 & 164111 & $6400-04$ \\
\hline 1CG18008 & $64^{\circ} 04^{\prime} \mathrm{N}$ & $50^{\circ} 45^{\prime} \mathrm{W}$ & W & 134 & 130 & 0000.38 & 780010 & $6400-02$ & 1CH 31001 & $64^{\circ} 28^{\prime} \mathrm{N}$ & $50^{\circ} 29^{\prime}$ & $\mathrm{NE}$ & 135 & 110 & 0000.26 & 643112 & $6400-02$ \\
\hline 1CG18009 & $64^{\circ} 04^{\prime} \mathrm{N}$ & $50^{\circ} 45^{\prime \prime} \mathrm{W}$ & N & 110 & 80 & & 643212 & & $1 \mathrm{CH} 31$ & $64^{\circ} 28^{\prime} \mathrm{N}$ & $50^{\circ} 30^{\prime}$ И & $\mathrm{N}$ & 150 & 110 & 0000.19 & 670112 & $6400-02$ \\
\hline 1CG18010 & $64^{\circ} 04^{\prime} \mathrm{N}$ & $50^{\circ} 46^{\prime} \mathrm{W}$ & $\mathrm{N}$ & 110 & 85 & 0000.16 & 643212 & $6400-02$ & $1 \mathrm{CH} 31003$ & $64^{\circ} 27^{\prime} \mathrm{N}$ & $50^{\circ} 31^{\prime}$, & $\mathrm{NE}$ & 160 & 120 & 0000.45 & 643112 & $6400-02$ \\
\hline 1CG19001 & $64^{\circ} 04^{\prime} \mathrm{N}$ & $50^{\circ} 44^{\prime} \mathrm{V}$ & SW & 134 & 125 & 0000.13 & 780010 & $6400-02$ & $1 \mathrm{CH} 31004$ & $64^{\circ} 28^{\prime} \mathrm{N}$ & $50^{\circ} 31$ ' & $\mathrm{NE}$ & 155 & 110 & 0000.55 & 633112 & $6400-02$ \\
\hline 1CG19002 & $64^{\circ} 02^{\prime} \mathrm{N}$ & $50^{\circ} 45^{\prime} \mathrm{W}$ & $\mathbf{N}$ & 120 & 105 & 0000.17 & 640112 & $6400-02$ & $1 \mathrm{CH} 31005$ & $64^{\circ} 28^{\prime} \mathrm{N}$ & $50^{\circ} 33^{\prime} \mathrm{W}$ & - & 155 & 155 & 0000.12 & 790010 & $6400-02$ \\
\hline $1 \mathrm{CG} 19003$ & $64^{\circ} 02^{\prime} \mathrm{N}$ & $50^{\circ} 47^{\prime} \mathrm{W}$ & N & 130 & 95 & 0000.92 & 613112 & 6400 & $1 \mathrm{CH}$ & $64^{\circ} 28^{\prime} \mathrm{N}$ & $50^{\circ} 33^{\prime} \mathrm{V}$ & $\mathrm{NE}$ & 145 & 110 & 0000.16 & 670112 & $6400-02$ \\
\hline 1CG19004 & $64^{\circ} 02^{\prime} \mathrm{N}$ & $50^{\circ} 49 \cdot \mathrm{W}$ & NW & 135 & 130 & 0000.24 & 780112 & 6400 & $1 \mathrm{CH} 31007$ & $64^{\circ} 29^{\circ} \mathrm{N}$ & $50^{\circ} 36^{\prime} \mathrm{W}$ & N & 115 & 105 & 0000.04 & 750010 & $6400-02$ \\
\hline 1CG19005 & $64^{\circ} 02^{\prime} \mathrm{N}$ & $50^{\circ} 50^{\prime} \mathrm{W}$ & NE & 140 & 100 & 0000.38 & 640112 & $6400-02$ & $1 \mathrm{CH} 32001$ & $64^{\circ} 28^{\prime} \mathrm{N}$ & $50^{\circ} 34^{\prime} \mathrm{W}$ & W & 155 & 145 & 0000.15 & 780010 & 6400-02 \\
\hline 1CG19006 & $64^{\circ} 03^{\prime} \mathrm{N}$ & $50^{\circ} 51^{\prime} \mathrm{W}$ & $\mathrm{NE}$ & 140 & 90 & 0001.06 & 643112 & $6400-02$ & $1 \mathrm{CH} 32002$ & $64^{\circ} 27^{\prime} \mathrm{N}$ & $50^{\circ} 33 \cdot \mathrm{k}$ & SW & 155 & 145 & 0000.15 & 780010 & $6400-02$ \\
\hline 1CG20001 & $64^{\circ} 03^{\prime} \mathrm{N}$ & $50^{\circ} 53^{\prime} \mathrm{W}$ & $\mathrm{N}$ & 135 & 90 & 0001.31 & 640112 & $6400-02$ & $1 \mathrm{CH} 32003$ & $64^{\circ} 27^{\prime} \mathrm{N}$ & $50^{\circ} 30^{\prime} \mathrm{V}$ & $\mathrm{SE}$ & 160 & 140 & 0000.22 & 780010 & $6400-02$ \\
\hline 1CG20002 & $64^{\circ} 03^{\prime} \mathrm{N}$ & $50^{\circ} 54^{\prime} \mathrm{W}$ & $\mathrm{N}$ & 135 & 90 & 0000.41 & 653112 & $6400-02$ & $1 \mathrm{CH} 32004$ & $64^{\circ} 27^{\prime} \mathrm{N}$ & $50^{\circ} 27 \cdot \mathrm{V}$ & 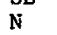 & 110 & $\begin{array}{r}140 \\
90\end{array}$ & 0000.10 & 750012 & $6400-02$ \\
\hline
\end{tabular}


$\begin{array}{llll}1 \mathrm{CH} 45003 & 64^{\circ} 12^{\prime} \mathrm{N} & 51^{\circ} 26^{\prime} \mathrm{N} & \mathrm{N} \\ 1 \mathrm{CH} 45004 & 64^{\circ} 12^{\prime} \mathrm{N} & 51^{\circ} 27 . \mathrm{H} & \mathrm{N}\end{array}$

$\begin{array}{lll}\text { 1CH45004 } & 64^{\circ} 12^{\prime} \mathrm{N} & 51^{\circ} 27 \cdot \mathrm{N} \\ 1 \mathrm{DA01001} & 65^{\circ} 21^{\prime} \mathrm{N} & 51^{\circ} 55 / \mathrm{N}\end{array}$

1 DA02001 65\%19'N $51^{\circ} 50^{\circ} \mathrm{W}$ NE

$1 \mathrm{DA03001} \quad 65^{\circ} 18^{\prime} \mathrm{N} \quad 51^{\circ} 51^{\prime} \mathrm{W}$

$\begin{array}{llll}\text { IDA03002 } & 65^{\circ} 18^{\prime} \mathrm{N} & 51^{\circ} 49^{\prime} \mathrm{W} & \mathrm{NE} \\ \text { IDA03003 } & 65^{\circ} 17^{\prime} \mathrm{N} & 51^{\circ} 45^{\prime} \mathrm{W} & \mathrm{NW}\end{array}$

$1 \mathrm{DA03004} 65^{\circ} 17^{\prime} \mathrm{N} \quad 51^{\circ} 47^{\prime} \mathrm{W} \quad \mathrm{NW}$

1DA04001 $65^{\circ} 16^{\prime} \mathrm{N} \quad 51^{\circ} 47^{\prime} \mathrm{W}$ SE

1DB01002 $65^{\circ} 34^{\prime} \mathrm{N} \quad 52^{\circ} 27^{\prime} \mathrm{W}$ E

1DBO1003

DBO1005

1DB01006

1DB01008

1DB01009

1DB01011

1DB02001

$1 \mathrm{DBO} 2003$

1DB03001
1DB03002

DB04001

1DB05001

1DB06001

$1 \mathrm{DB06003}$

1DB07001
$1 \mathrm{DB} 07002$

DB07003

$1 \mathrm{DB} 07004$
$1 \mathrm{DB} 07005$

1DB07006

1DB07008

1DB09001

$1 \mathrm{DB} 09003$

1DB09004

1DB10001

1DB10003
$1 \mathrm{DB1} 10004$

$1 \mathrm{DB} 10004$
$1 \mathrm{DB1} 10005$

$1 \mathrm{DB} 10006$
$1 \mathrm{DB} 10007$

1DB10007

$1 \mathrm{DB} 10010$

$\begin{array}{lll}65^{\circ} 34^{\prime} \mathrm{N} & 52^{\circ} 27^{\prime} \mathrm{W} & \mathrm{E} \\ 65^{\circ} 35^{\prime} \mathrm{N} & 52^{\circ} 25^{\prime} \mathrm{W} & \mathrm{E}\end{array}$

$65^{\circ} 37^{\prime N} \quad 52^{\circ} 23 \cdot \mathrm{W}$ SE

$65^{\circ} 377^{\prime} \mathrm{N} \quad 52^{\circ} 22^{\prime} \mathrm{W} \mathrm{S}$

$\begin{array}{ll}65^{\circ} 38^{\prime} \mathrm{N} & 52^{\circ} 21^{\circ} \mathrm{W} \\ & \end{array}$

$\begin{array}{lll}65^{\circ} 38^{\prime} \mathrm{N} & 52^{\circ} 19^{\prime} \mathrm{W} & \mathrm{S} \\ 65^{\circ} 39^{\prime} \mathrm{N} & 52^{\circ} 15^{\prime} \mathrm{W} & \mathrm{S}\end{array}$

$65^{\circ} 38^{\prime} \mathrm{N} \quad 52^{\circ} 16^{\prime} \mathrm{W}$ SW

$65^{\circ} 34^{\prime} \mathrm{N} \quad 52^{\circ} 16^{\prime} \mathrm{W} N$

$65^{\circ} 32, \mathrm{~N} \quad 52^{\circ} 20^{\prime} \mathrm{W}$

$65^{\circ} 31^{\prime} \mathrm{N} \quad 52^{\circ} 23^{\prime} \mathrm{W} \mathrm{W}$

$65^{\circ} 29^{\prime} \mathrm{N} \quad 52^{\circ} 21^{\prime} \mathrm{W} \quad \mathrm{SW}$

$65^{\circ} 29^{\prime} \mathrm{N} 52^{\circ} 17^{\prime} \mathrm{N}$

$65^{\circ} 30^{\prime} \mathrm{N} \quad 52^{\circ} 19^{\prime} \mathrm{V}$

$\begin{array}{lll}65^{\circ} 30^{\prime} \mathrm{N} & 52^{\circ} 17^{\prime} \mathrm{W} & \mathrm{NE} \\ 65^{\circ} 32^{\prime} \mathrm{N} & 52^{\circ} 15^{\prime} \mathrm{V} & \mathrm{E}\end{array}$

$65^{\circ} 33^{\prime} \mathrm{N} \quad 52^{\circ} 14^{\prime} \mathrm{W}$ SE

$65^{\circ} 34^{\prime} \mathrm{N} \quad 52^{\circ} 09^{\prime} \mathrm{V}$ SE

$\begin{array}{lll}65^{\circ} 35^{\prime} \mathrm{N} & 52^{\circ} 07^{\prime} \mathrm{W} & \mathrm{E} \\ 65^{\circ} 36^{\prime} \mathrm{N} & 52^{\circ} 05^{\prime} \mathrm{W} & \mathrm{S}\end{array}$

$65^{\circ} 37^{\prime} \mathrm{N} \quad 5^{\circ} 04^{\prime} \mathrm{V}$ SW

$65^{\circ} 35^{\prime} \mathrm{N} \quad 52^{\circ} 00^{\prime} \mathrm{V}$ NE

$65^{\circ} 36^{\prime} \mathrm{N} \quad 52^{\circ} 02^{\prime} \mathrm{V} \quad \mathrm{NE}$

$65^{\circ} 38^{\prime} \mathrm{N} \quad 52^{\circ} 011^{\prime} \mathrm{V}$

$\begin{array}{lll}65^{\circ} 38^{\prime} \mathrm{N} & 52^{\circ} 03^{\prime} \mathrm{W} & \mathrm{NE} \\ 65^{\circ} 37^{\prime} \mathrm{N} & 52^{\circ} 06^{\prime} \mathrm{W} & \mathrm{NW}\end{array}$

$65^{\circ} 36^{\prime} \mathrm{N} \quad 52^{\circ} 08^{\prime} \mathrm{W} \mathrm{W}$

$770012 \quad 6400-02$

640112 6400-02

$700010 \quad 6430-02$

$643112 \quad 6400-01$

$640112 \quad 6400-01$

$670010 \quad 6400-01$

$\begin{array}{ll}780010 & 6400-02 \\ 780010 & 6430-02\end{array}$

$770010 \quad 6400-01$

$\begin{array}{ll}613512 & 6400-01 \\ 640110 & 6400-01\end{array}$

$\begin{array}{ll}780010 & 6400-01 \\ 780010 & 6400-01\end{array}$
$1 \mathrm{DB10011}$

1DB10012

1DB10014

IDB10016

1DB10017
1DB10018 $65^{\circ} 34^{\prime} \mathrm{N} \quad 52^{\circ} 13^{\prime} \mathrm{N}$ N

$65^{\circ} 39^{\prime} \mathrm{N} \quad 52^{\circ} 13, \mathrm{~W}$ SE

$65^{\circ} 42$ 'N $52^{\circ} 07$ ' W SE

$\begin{array}{lll}65^{\circ} 43^{\prime} \mathrm{N} & 52^{\circ} 00^{\prime} \mathrm{W} & \mathrm{SE} \\ 65^{\circ} 46^{\prime} \mathrm{N} & 52^{\circ} 06^{\prime} \mathrm{V} & \mathrm{SE}\end{array}$

$65^{\circ} 45^{\prime} \mathrm{N} \quad 51^{\circ} 59^{\prime} \mathrm{W} S$

$65^{\circ} 50^{\prime} \mathrm{N} \quad 52^{\circ} 00^{\prime} \mathrm{W}$ SE

$65^{\circ} 50^{\prime} \mathrm{N} \quad 51^{\circ} 49^{\prime} \mathrm{W}$ SE

$\begin{array}{lll}65^{\circ} 51^{\prime} \mathrm{N} & 51^{\circ} 45^{\prime} \mathrm{W} & \mathrm{SE} \\ 65^{\circ} 41^{\prime} \mathrm{N} & 51^{\circ} 37^{\prime} \mathrm{W} & \mathrm{SW}\end{array}$

$\begin{array}{lll}65^{\circ} 44^{\prime} \mathrm{N} & 52^{\circ} 00^{\prime} \mathrm{W} & \mathrm{N} \\ 65^{\circ} 40^{\prime} \mathrm{N} & 51^{\circ} 56^{\prime} \mathrm{W} & \mathrm{NW}\end{array}$

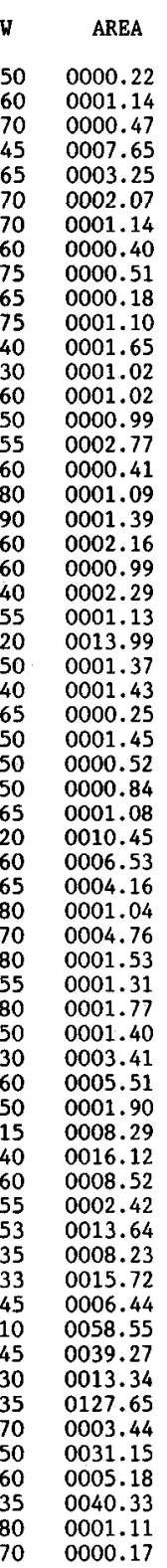

$\stackrel{M O}{\mathrm{CODE}}$

$780010 \quad 6400-01$

$643112 \quad 6400-01$

$373112 \quad 6500-02$

$300010 \quad 6500-02$

$300010 \quad 6500-02$

$750012 \quad 6500-03$

$750010 \quad 6500-03$

$\begin{array}{ll}780010 & 6530-02 \\ 370212 & 6530-02\end{array}$

$300512 \quad 6530-02$

$370212 \quad 6530-02$

$633212 \quad 6530-02$

$\begin{array}{ll}641111 & 6530-02 \\ 771010 & 6530-02\end{array}$

$780012 \quad 6530-02$

$780010 \quad 6530-02$

6530-02

$300112 \quad 6530-02$

$300110 \quad 6530-02$

$306112 \quad 6500-02$

$750010 \quad 6500-02$

$300110 \quad 6530-02$

$303010 \quad 6530-02$

$300010 \quad 6530-02$

$300010 \quad 6530-02$

$303112 \quad 6530-02$

$780010 \quad 6530-02$

$333112 \quad 6530-02$

$300112 \quad 6530-02$

303112 6530-02

$300112 \quad 6530-02$

$336112 \quad 6530-02$

$300112 \quad 6530-02$

$336112 \quad 6530-02$

$306311 \quad 6530-02$

$303212 \quad 6530-02$

$303112 \quad 6530-02$

$300012 \quad 6530-02$

$306212 \quad 6530-02$

$300112 \quad 6530-02$

$306112 \quad 6530-03$

$\begin{array}{ll}700010 & 6530-02 \\ 700010 & 6530-02\end{array}$ 


\begin{tabular}{|c|c|c|c|c|}
\hline CODE & LAT & LONG & ORI & HIGH \\
\hline 1DB11001 & $65^{\circ} 40^{\prime} \mathrm{N}$ & $51^{\circ} 55^{\prime} \mathrm{W}$ & $\mathbf{E}$ & 74 \\
\hline 1DB11002 & $65^{\circ} 43^{\prime} \mathrm{N}$ & $51^{\circ} 44^{\prime} \mathrm{W}$ & $\mathrm{SE}$ & 97 \\
\hline 1DB11003 & $65^{\circ} 50^{\prime} \mathrm{N}$ & $51^{\circ} 28^{\prime} \mathrm{W}$ & SW & 120 \\
\hline 1DB11004 & $65^{\circ} 51^{\prime} \mathrm{N}$ & $51^{\circ} 25^{\prime} \mathrm{W}$ & $\mathrm{s}$ & 110 \\
\hline 1DB11005 & $65^{\circ} 52^{\prime} \mathrm{N}$ & $51^{\circ} 24^{\prime} \mathrm{W}$ & NE & 107 \\
\hline 1DB11006 & $65^{\circ} 54^{\prime} \mathrm{N}$ & $51^{\circ} 20^{\circ} \mathrm{W}$ & $\mathbf{s}$ & 190 \\
\hline 1DB11007 & $65^{\circ} 54^{\prime} \mathrm{N}$ & $51^{\circ} 15^{\prime} \mathrm{W}$ & $\mathbf{S}$ & 190 \\
\hline 1DB11008 & $65^{\circ} 54^{\prime} \mathrm{N}$ & $51^{\circ} 13^{\prime} \mathrm{W}$ & $\mathrm{s}$ & 190 \\
\hline 1DB11009 & $65^{\circ} 54^{\prime} \mathrm{N}$ & $51^{\circ} 11^{\prime} \mathrm{W}$ & $\mathrm{s}$ & 190 \\
\hline 1DB11010 & $65^{\circ} 54^{\prime} \mathrm{N}$ & $51^{\circ} 06^{\prime} \mathrm{W}$ & $\mathrm{s}$ & 190 \\
\hline 1DB11011 & $65^{\circ} 52^{\prime} \mathrm{N}$ & $51^{\circ} 04^{\prime} \mathrm{W}$ & $\mathrm{s}$ & 153 \\
\hline 1DB11012 & $65^{\circ} 52^{\prime} \mathrm{N}$ & $51^{\circ} 01^{\prime} \mathrm{W}$ & $\mathbf{s}$ & 153 \\
\hline 1DB11013 & $65^{\circ} 54^{\prime} \mathrm{N}$ & $50^{\circ} 57^{\prime} \mathrm{W}$ & SW & 200 \\
\hline 1DB11014 & $65^{\circ} 55^{\prime} \mathrm{N}$ & $50^{\circ} 48^{\prime} \mathrm{W}$ & SE & 200 \\
\hline 1DB11015 & $65^{\circ} 55^{\prime} \mathrm{N}$ & $50^{\circ} 40^{\prime} \mathrm{W}$ & SE & 190 \\
\hline 1DB11016 & $65^{\circ} 55^{\prime} \mathrm{N}$ & $50^{\circ} 34^{\prime} \mathrm{W}$ & SE & 200 \\
\hline 1DB11017 & $65^{\circ} 55^{\prime} \mathrm{N}$ & $50^{\circ} 30^{\prime} \mathrm{W}$ & SE & 180 \\
\hline 1DB11018 & $65^{\circ} 55^{\prime} \mathrm{N}$ & $50^{\circ} 25^{\prime} \mathrm{W}$ & SE & 160 \\
\hline 1DB1 & $65^{\circ} 54^{\prime} \mathrm{N}$ & $50^{\circ} 15^{\prime} \mathrm{W}$ & $\mathrm{s}$ & 112 \\
\hline 1DB11020 & $65^{\circ} 54^{\prime} \mathrm{N}$ & $50^{\circ} 08^{\prime} \mathrm{W}$ & $\mathrm{W}$ & 180 \\
\hline 1DB11021 & $65^{\circ} 47^{\prime} \mathrm{N}$ & $50^{\circ} 25^{\prime} \mathrm{W}$ & W & 180 \\
\hline 1DB11022 & $65^{\circ} 43^{\prime} \mathrm{N}$ & $50^{\circ} 12^{\prime} \mathrm{W}$ & $\mathrm{W}$ & 180 \\
\hline 1DB1 & $65^{\circ} 41^{\prime} \mathrm{N}$ & $50^{\circ} 11^{\prime} \mathrm{W}$ & W & 180 \\
\hline 1DB & 65 & $51^{\circ} 05^{\prime} \mathrm{W}$ & NE & 95 \\
\hline 1DB11025 & $65^{\circ} 42^{\prime} \mathrm{N}$ & $51^{\circ} 08^{\prime} \mathrm{W}$ & NE & 106 \\
\hline 1DB11026 & $65^{\circ} 42^{\prime} \mathrm{N}$ & $51^{\circ} 10^{\prime} \mathrm{W}$ & $\mathbf{N}$ & 106 \\
\hline 1DB11027 & $65^{\circ} 42^{\prime} \mathrm{N}$ & $51^{\circ} 19^{\prime} \mathrm{W}$ & $\mathrm{NE}$ & 110 \\
\hline 1DB1 & $65^{\circ}$ & $51^{\circ} 24^{\prime} \mathrm{W}$ & $\mathbf{N}$ & 110 \\
\hline 1DB & $65^{\circ} 42^{\prime} \mathrm{N}$ & $51^{\circ} 34^{\prime} \mathrm{W}$ & $\mathbf{N}$ & 70 \\
\hline 1DB11030 & $65^{\circ} 42^{\prime} \mathrm{N}$ & $51^{\circ} 37^{\prime} \mathrm{W}$ & NW & 80 \\
\hline 1DB11031 & $65^{\circ} 38^{\prime} \mathrm{N}$ & $51^{\circ} 50^{\prime} \mathrm{W}$ & NW & 85 \\
\hline 1DB12001 & $65^{\circ} 38^{\prime} \mathrm{N}$ & $51^{\circ} 48^{\prime} \mathrm{W}$ & E & 85 \\
\hline 1DB & $65^{\circ} 42^{\prime} \mathrm{N}$ & $51^{\circ} 27^{\prime} \mathrm{W}$ & $s$ & 85 \\
\hline $10 B$ & $65^{\circ} 42^{\prime} \mathrm{N}$ & $51^{\circ} 25^{\prime} \mathrm{W}$ & W & 90 \\
\hline 1DB12004 & $65^{\circ} 40^{\prime} \mathrm{N}$ & $51^{\circ} 33^{\prime} \mathrm{W}$ & NW & 93 \\
\hline 1DB13001 & $65^{\circ} 40^{\prime} \mathrm{N}$ & $51^{\circ} 32^{\prime} \mathrm{W}$ & SE & 93 \\
\hline 1DB13002 & $65^{\circ}$ & $51^{\circ} 20^{\prime} \mathrm{W}$ & W & 110 \\
\hline 1DB13 & $65^{\circ} 41^{\prime} \mathrm{N}$ & $51^{\circ} 10^{\prime} \mathrm{W}$ & - & 106 \\
\hline 1DB13004 & $65^{\circ} 40^{\prime} \mathrm{N}$ & $51^{\circ} 05^{\prime} \mathrm{W}$ & NW & 95 \\
\hline 1DB13005 & $65^{\circ} 39^{\prime} \mathrm{N}$ & $51^{\circ} 17^{\prime} \mathrm{W}$ & & 96 \\
\hline $1 \mathrm{DB} 13$ & $65^{\circ} 38^{\prime} \mathrm{N}$ & $50^{\circ} 06^{\prime} \mathrm{W}$ & W & 180 \\
\hline 1DB13007 & $65^{\circ} 36^{\prime} \mathrm{N}$ & $49^{\circ} 59^{\prime} \mathrm{W}$ & W & 180 \\
\hline & & $49^{\circ} 58^{\prime} \mathrm{W}$ & W & 180 \\
\hline 1DB13009 & $65^{\circ} 28^{\prime} \mathrm{N}$ & $49^{\circ} 58^{\prime} \mathrm{W}$ & $\mathrm{NW}$ & 180 \\
\hline 1DB13010 & $65^{\circ} 27^{\prime} \mathrm{N}$ & $50^{\circ} 06^{\prime} \mathrm{Y}$ & W & 180 \\
\hline 1DB13 & 6 & $51^{\circ} 47^{\prime} \mathrm{W}$ & & 75 \\
\hline $10 B 130$ & $65^{\circ} 30^{\prime} \mathrm{N}$ & $51^{\circ} 50^{\prime} \mathrm{W}$ & SE & 5 \\
\hline & & $51^{\circ} 55^{\prime} \mathrm{V}$ & $w$ & \\
\hline 1DB14002 & $65^{\circ} 25^{\prime} \mathrm{N}$ & $51^{\circ} 49, \mathrm{~V}$ & $\mathrm{NE}$ & 80 \\
\hline 1DB14003 & & $51^{\circ} 54^{\prime} \mathrm{V}$ & $\mathbf{N}$ & 75 \\
\hline & $65^{\circ} 2$ & $52^{\circ} 00^{\circ} \mathrm{W}$ & NE & 60 \\
\hline 1DB1.4005 & $65^{\circ} 26^{\prime} \mathrm{N}$ & $52^{\circ} 03^{\prime} \mathrm{W}$ & $\mathbf{E}$ & 126 \\
\hline 1DB & $65^{\circ} 28^{\prime} \mathrm{N}$ & $52^{\circ} 02^{\prime} \mathrm{V}$ & $\mathbf{N}$ & 85 \\
\hline $1 \mathrm{DE}$ & 6. & $52^{\circ} 04^{\prime} \mathrm{V}$ & $\mathrm{NW}$ & 110 \\
\hline 1DB14C & $65^{\circ} 26^{\prime} \mathrm{N}$ & $52^{\circ} 06^{\prime} \mathrm{W}$ & $\mathrm{W}$ & 100 \\
\hline 1DB14009 & $65^{\circ} 25^{\prime} \mathrm{N}$ & $52^{\circ} 10^{\prime} \mathrm{V}$ & $\mathrm{NW}$ & 105 \\
\hline 1DB & & $52^{\circ} 13^{\prime} \mathrm{W}$ & SW & \\
\hline 1DB15001 & $65^{\circ} 24^{\prime} \mathrm{N}$ & $52^{\circ} 08^{\prime} \mathrm{W}$ & SN & 110 \\
\hline 1DB15C & $65^{\circ} 24^{\prime} \mathrm{N}$ & $52^{\circ} 06^{\prime} \mathrm{W}$ & E & 100 \\
\hline 1DB15C & $65^{\circ} 21^{\prime} \mathrm{N}$ & $52^{\circ} 01^{\prime} \mathrm{V}$ & $\mathrm{N}$ & 75 \\
\hline 1DB150 & $5^{\circ} 21^{\prime} \mathrm{N}$ & $52^{\circ} 05^{\prime}$ & $\mathbf{E}$ & 75 \\
\hline
\end{tabular}

$\begin{array}{cccc} & & \text { MO } & \\ \text { LOW } & \text { AREA } & \text { CODE } & \text { MAP } \\ 70 & 0000.07 & 700010 & 6530-02 \\ 90 & 0000.85 & 700010 & 6530-03 \\ 45 & 0015.64 & 306012 & 6530-03 \\ 55 & 0013.44 & 300012 & 6530-03 \\ 90 & 0003.84 & 350010 & 6530-03 \\ 40 & 0056.81 & 303012 & 6530-03 \\ 40 & 0051.66 & 303012 & 6530-03 \\ 90 & 0016.00 & 300010 & 6530-03 \\ 40 & 0035.87 & 303011 & 6530-03 \\ 5 & 0057.58 & 306311 & 6550-03 \\ 65 & 0016.87 & 303312 & 6530-03 \\ 90 & 0006.01 & 303312 & 6530-03 \\ 50 & 0185.06 & 303212 & 6530-03 \\ 30 & 0128.04 & 303112 & 6530-04 \\ 35 & 0092.53 & 303012 & 6530-04 \\ 50 & 0055.38 & 303212 & 6530-04 \\ 55 & 0042.12 & 300113 & 6530-04 \\ 85 & 0086.67 & 300113 & 6530-04 \\ 80 & 0018.22 & 300110 & 6530-04 \\ 85 & 0101.40 & 167113 & 6530-04 \\ 10 & 1413.39 & 163113 & 6530-04 \\ 10 & 0284.10 & 163113 & 6530-04 \\ 40 & 0158.64 & 163113 & 6550-04 \\ 85 & 0000.50 & 270110 & 6530-03 \\ 70 & 0002.98 & 270112 & 6530-03 \\ 70 & 0002.30 & 270112 & 6530-03 \\ 65 & 0006.16 & 300112 & 6530-03 \\ 60 & 0006.59 & 300112 & 65300-03 \\ 70 & 0000.43 & 780010 & 6530-03 \\ 70 & 0000.30 & 780010 & 6530-03 \\ 70 & 0001.19 & 300010 & 6530-02 \\ 70 & 0001.12 & 300010 & 6530-02 \\ 80 & 0000.36 & 780010 & 6530-03 \\ 80 & 0000.55 & 780010 & 6530-03 \\ 70 & 0005.89 & 300112 & 6530-03 \\ 80 & 0001.55 & 300110 & 6530-03 \\ 90 & 0002.07 & 300010 & 6530-03 \\ 95 & 0001.81 & 270010 & 6530-03 \\ 90 & 0000.56 & 776010 & 6530-03 \\ 90 & 0000.51 & 780010 & 6530-03 \\ 50 & 0284.24 & 163113 & 6550-04 \\ 50 & 0941.93 & 166113 & 6530-05 \\ 50 & 0798.19 & 163114 & 6530-05 \\ 55 & 0392.00 & 166113 & 6500-05 \\ 58 & 0195.64 & 163113 & 6500-04 \\ 65 & 0000.37 & 770010 & 6530-02 \\ 85 & 0000.53 & 670010 & 6530-02 \\ 80 & 0000.13 & 770010 & 6530-02 \\ 80 & 0000.22 & 780010 & 6500-02 \\ 70 & 0000.23 & 780010 & 6500-02 \\ 60 & 0000.13 & 780010 & 6500-02 \\ 35 & 0011.99 & 303112 & 6500-02 \\ 60 & 0002.21 & 270112 & 6500-02 \\ 50 & 0002.95 & 270112 & 6500-02 \\ 20 & 0004.20 & 333212 & 6500-02 \\ 60 & 0002.84 & 300010 & 6500-02 \\ 55 & 0000.34 & 780010 & 6500-02 \\ 45 & 0008.14 & 303212 & 6500-02 \\ 85 & 0000.67 & 750010 & 6500-02 \\ 50 & 0000.65 & 670012 & 6500-02 \\ 55 & 0000.59 & 200010 & 6500-02 \\ & & & \end{array}$

\begin{tabular}{|c|c|c|c|}
\hline CODE & LAT & LONG & ORI \\
\hline 1DB15005 & $65^{\circ} 22^{\prime} \mathrm{N}$ & $6 . \mathrm{W}$ & $\mathrm{N}$ \\
\hline $\begin{array}{l}\text { 1DB16001 } \\
\text { 1DB16002 }\end{array}$ & $65^{\circ} 17$ 'N & $52^{\circ} 06^{\prime} \mathrm{W}$ & NW \\
\hline $\begin{array}{l}\text { 1DB16002 } \\
\text { 1DC01001 }\end{array}$ & $\begin{array}{l}65^{\circ} 16 \% \mathrm{~N} \\
65^{\circ} 35^{\prime} \mathrm{N}\end{array}$ & $\begin{array}{l}52^{\circ} 08^{\prime} \mathrm{V} \\
53^{\circ} 08^{\prime} \mathrm{V}\end{array}$ & \\
\hline $\begin{array}{l}\text { 1DC01001 } \\
\text { 1DC01002 }\end{array}$ & $\begin{array}{l}65^{\circ} 55^{\prime} \mathrm{N} \\
65^{\circ} 36^{\prime} \mathrm{N}\end{array}$ & & $\mathrm{N}$ \\
\hline $\begin{array}{l}\text { 1DC01002 } \\
\text { 1DC01003 }\end{array}$ & 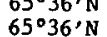 & $\begin{array}{l}53^{\circ} 05^{\prime} \mathrm{W} \\
53^{\circ} 03^{\prime} \mathrm{W}\end{array}$ & . \\
\hline $\begin{array}{l}1 \mathrm{DC} 01003 \\
1 \mathrm{DC} 01004\end{array}$ & & & $N$ \\
\hline 1DC01004 & $655^{3} 37 / \mathrm{N}$ & $53^{\circ} 00^{\prime} \mathrm{W}$ & SW \\
\hline 1DC01005 & $65^{\circ} 37 / \mathrm{N}$ & $52^{\circ} 55^{\prime} \mathrm{W}$ & \\
\hline $1 D C 01006$ & $65^{\circ} 37^{\prime} \mathrm{N}$ & $52^{\circ} 54^{\prime} \mathrm{W}$ & \\
\hline 1DC01007 & $65^{\circ} 36^{\prime} \mathrm{N}$ & $52^{\circ} 55^{\prime} \mathrm{W}$ & SW \\
\hline $1 \mathrm{DC} 01008$ & $65^{\circ} 36^{\prime} \mathrm{N}$ & $52^{\circ} 57^{\prime} \mathrm{w}$ & $c$ \\
\hline 1DC01009 & $65^{\circ} 35^{\prime} \mathrm{N}$ & 52057,16 & $\mathrm{NW}$ \\
\hline 1DC01010 & $65^{\circ} 35^{\prime} \mathrm{N}$ & $52^{\circ} 59^{\prime} \mathrm{W}$ & \\
\hline 1DC01011 & $65^{\circ} 34^{\prime} \mathrm{N}$ & $52^{\circ} 59^{\prime} \mathrm{W}$ & SW \\
\hline 1DC01012 & $65^{\circ} 34^{\prime} \mathrm{N}$ & $52 \circ 57 \cdot 6$ & \\
\hline 1DC01013 & $65^{\circ} 34^{\prime} \mathrm{N}$ & $52^{\circ} 56^{\prime} \mathrm{W}$ & $\mathrm{N}$ \\
\hline 1DC01014 & $65^{\circ} 36^{\prime} \mathrm{N}$ & $52^{\circ} 54^{\prime \prime ~}$ & E \\
\hline 1DC01015 & $65^{\circ} 36^{\prime} \mathrm{N}$ & $52^{\circ} 54^{\prime} \mathrm{W}$ & E \\
\hline 1DC01016 & $65^{\circ} 37^{\prime} \mathrm{N}$ & $52^{\circ} 54^{\prime} \mathrm{W}$ & SE \\
\hline 1DC01017 & $65^{\circ} 37^{\prime} \mathrm{N}$ & $52 \circ 54, \mathrm{~W}$ & $\mathrm{SE}$ \\
\hline 1DC02001 & $65^{\circ} 40^{\prime} \mathrm{N}$ & $52^{\circ} 40^{\prime} \mathrm{W}$ & \\
\hline $1 \mathrm{DCO} 2002$ & $65^{\circ} 42^{\prime} \mathrm{N}$ & $52^{\circ} 33^{\prime} \mathrm{W}$ & SW \\
\hline $10 \mathrm{CO} 2003$ & $65^{\circ} 42^{\prime} \mathrm{N}$ & $52^{\circ} 32^{\prime} \mathrm{W}$ & SW \\
\hline 1DC02004 & $65^{\circ} 42^{\prime} \mathrm{N}$ & $52^{\circ} 28^{\prime} \mathrm{W}$ & SW \\
\hline $1 \mathrm{DCO} 2005$ & $65^{\circ} 41^{\prime} \mathrm{N}$ & $52^{\circ} 28^{\prime} \mathrm{W}$ & SW \\
\hline 1DC02006 & $65^{\circ} 40^{\prime} \mathrm{N}$ & $52^{\circ} 30^{\prime} \mathrm{W}$ & \\
\hline 1DCC & $65^{\circ} 40^{\prime} \mathrm{N}$ & $52^{\circ} 32^{\prime} \mathrm{V}$ & N \\
\hline 1DC02008 & $65^{\circ} 39^{\prime} \mathrm{N}$ & $52^{\circ} 35^{\prime} \mathrm{V}$ & $\mathrm{NW}$ \\
\hline 1DC02009 & $65^{\circ} 38^{\prime} \mathrm{N}$ & $52^{\circ} 35^{\prime} \mathrm{W}$ & $\mathrm{NW}$ \\
\hline 1DC02010 & $65^{\circ} 38^{\prime} \mathrm{N}$ & $52^{\circ} 39^{\prime} \mathrm{W}$ & NU \\
\hline 1DC02011 & $65^{\circ} 38^{\prime} \mathrm{N}$ & $52^{\circ} 40^{\prime} \mathrm{W}$ & NW \\
\hline $1 \mathrm{DCC}$ & $65^{\circ} 37 \cdot \mathrm{N}$ & $52^{\circ} 41^{\prime} \mathrm{W}$ & $\mathrm{NW}$ \\
\hline 1DC02013 & $65^{\circ} 37^{\prime} \mathrm{N}$ & $52^{\circ} 43^{\prime} \mathrm{W}$ & NW \\
\hline $1 \mathrm{DCO} 2014$ & $65^{\circ} 37^{\prime} \mathrm{N}$ & $52^{\circ} 44^{\prime} \mathrm{W}$ & NW \\
\hline $1 D C 03001$ & $65^{\circ} 38^{\prime} \mathrm{N}$ & $52^{\circ} 35^{\prime} \mathrm{W}$ & $\mathrm{Y}$ \\
\hline $1 \mathrm{DCO}$ & $65^{\circ} 37^{\prime} \mathrm{N}$ & $52^{\circ} 38^{\prime} \mathrm{W}$ & NW \\
\hline & $65^{\circ} 36^{\prime} \mathrm{N}$ & $52^{\circ} 38^{\prime} \mathrm{W}$ & $\mathrm{NW}$ \\
\hline 1DC03004 & $65^{\circ} 36^{\prime} \mathrm{N}$ & $52^{\circ} 39^{\prime} \mathrm{W}$ & $\mathrm{NW}$ \\
\hline 1DC03005 & $65^{\circ} 36^{\prime} \mathrm{N}$ & $52^{\circ} 40^{\prime} \mathrm{W}$ & NW \\
\hline 1DC03006 & $65^{\circ} 35^{\prime} \mathrm{N}$ & $52^{\circ} 41 \times \mathrm{W}$ & NW \\
\hline 1DC0 & $65^{\circ} 35^{\prime} \mathrm{N}$ & $52^{\circ} 42^{\prime} \mathrm{W}$ & NW \\
\hline 1DC & $65^{\circ} 35^{\prime} \mathrm{N}$ & $52^{\circ} 41^{\prime} \mathrm{W}$ & sw \\
\hline 1DC04002 & $65^{\circ} 34^{\prime} \mathrm{N}$ & $52^{\circ} 41^{\prime} \mathrm{W}$ & SW \\
\hline 1DC04003 & $65^{\circ} 32^{\prime} \mathrm{N}$ & $52^{\circ} 45^{\prime} \mathrm{V}$ & NE \\
\hline 1DC05001 & $65^{\circ} 33^{\prime} \mathrm{N}$ & $52^{\circ} 41^{\prime} \mathrm{W}$ & \\
\hline $1 D C 05002$ & $65^{\circ} 34^{\prime} \mathrm{N}$ & $52^{\circ} 35^{\prime} \mathrm{W}$ & SE \\
\hline 1DC & $65^{\circ} 35^{\prime} \mathrm{N}$ & $52^{\circ} 35^{\prime} \mathrm{W}$ & SE \\
\hline 1DC06001 & $65^{\circ} 35^{\prime} \mathrm{N}$ & $52^{\circ} 34^{\prime} \mathrm{W}$ & SE \\
\hline 1DC06002 & $65^{\circ} 36^{\prime} \mathrm{N}$ & $52^{\circ} 33^{\prime} \mathrm{W}$ & $\mathrm{E}$ \\
\hline $1 D C 06003$ & $65^{\circ} 37^{\prime} \mathrm{N}$ & $52^{\circ} 34^{\prime} \mathrm{W}$ & $\mathrm{NE}$ \\
\hline & $65^{\circ} 39^{\prime} \mathrm{N}$ & $2^{\circ} 33^{\prime}$ & E \\
\hline 1DC06005 & $65^{\circ} 39^{\prime} \mathrm{N}$ & $52^{\circ} 31^{\prime} \mathrm{W}$ & E \\
\hline 1DC06006 & $65^{\circ} 39^{\prime} \mathrm{N}$ & $52^{\circ} 30^{\prime} \mathrm{W}$ & \\
\hline 1DC07001 & $65^{\circ} 41^{\prime} \mathrm{N}$ & $52^{\circ} 27 \cdot \mathrm{W}$ & sw \\
\hline 1DC07002 & $65^{\circ} 40^{\prime} \mathrm{N}$ & $52^{\circ} 23^{\prime} \mathrm{W}$ & 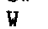 \\
\hline & $65^{\circ} 39^{\prime} \mathrm{N}$ & $023, \mathrm{~W}$ & NW \\
\hline 1DC07004 & $65^{\circ} 38^{\prime} \mathrm{N}$ & $52^{\circ} 25^{\prime} \mathrm{W}$ & NW \\
\hline 1DC07005 & $65^{\circ} 37^{\prime} \mathrm{N}$ & $52^{\circ} 26^{\prime} \mathrm{W}$ & NW \\
\hline 1DC07006 & $65^{\circ} 36^{\prime} \mathrm{N}$ & $52^{\circ} 27^{\prime} \mathrm{W}$ & NW \\
\hline & & & \\
\hline $1 \mathrm{DCC}$ & $34^{\prime} \mathrm{N}$ & $1027 \cdot \mathrm{W}$ & \\
\hline
\end{tabular}

HIGH
80
85
100
55
65
65
70
70
75
70
70
35
35
65
55
45
70
75
80
70
100
130
90
135
50
80
80
90
50
50
50
70
70
40
45
90
85
110
75
95
80
100
100
85
60
80
75
85
100
90

80
120
120
135
135
110
110
95
90

MAP

200012 6500-02

$213312 \quad 6500-02$

750010 6500-02

$750010 \quad 6530-01$

$780010 \quad 6530-01$

$750010 \quad 6530-01$

$700010 \quad 6530-01$

$670012 \quad 6530-01$

$640010 \quad 6530-01$

$790010 \quad 6530-01$

643112 6530-01

$613112 \quad 6530-01$

780010 6530-01

$750010 \quad 6530-01$

$750010 \quad 6530-01$

780010 6530-01

$600010 \quad 6530-01$

$780010 \quad 6530-01$

$780010 \quad 6530-01$

$750010 \quad 6530-02$

750010 6530-02

313112 6530-02

6530-02

$213010 \quad 6530-02$

$213312 \quad 6530-02$

$213312 \quad 6530-02$

610312 6530-01

$670010 \quad 6530-01$

643210 6530-01

6530-01

533112 6530-01

780010 6530-01

$670010 \quad 6530-02$

$780010 \quad 6530-02$

$670010 \quad 6530-02$

700010 6530-02

$523112 \quad 6530-02$

$750010 \quad 6530-02$

$700010 \quad 6530-02$

365111 6530-02

$363111 \quad 6530-02$

305312 6530-02

$300110 \quad 6530-02$

303312 6530-02

$300010 \quad 6530-02$

$\begin{array}{ll}300110 & 6530-02 \\ 700010 & 6530-02\end{array}$ 


\begin{tabular}{|c|c|c|c|c|c|c|c|c|c|c|c|c|c|c|c|c|c|}
\hline CODE & LAT & LONG & ORI & HIGH & LOW & AREA & $\begin{array}{c}\text { MO } \\
\text { CODE }\end{array}$ & MAP & CODE & LAT & LONG & ORI & HIGH & LOW & AREA & $\begin{array}{c}\text { MO } \\
\text { CODE }\end{array}$ & MAP \\
\hline $1 \mathrm{DC08003}$ & $65^{\circ} 33^{\prime} \mathrm{N}$ & $52^{\circ} 28^{\prime} \mathrm{W}$ & NW & 60 & 15 & 0000.18 & 200110 & $6530-02$ & 1DD07011 & $65^{\circ} 37^{\prime} \mathrm{N}$ & $53^{\circ} 03^{\prime} \mathrm{W}$ & NW & 60 & 10 & 0002.38 & 643112 & $6530-01$ \\
\hline $1 D C 08004$ & $65^{\circ} 33^{\prime} \mathrm{N}$ & $52^{\circ} 29^{\prime} \mathrm{W}$ & N & 45 & 15 & 0000.12 & 780010 & $6530-02$ & $1 \mathrm{DDD} 07012$ & $65^{\circ} 37^{\prime} \mathrm{N}$ & $53^{\circ} 04^{\prime} \mathrm{W}$ & $\mathbf{N}$ & 25 & 15 & 0000.04 & 780010 & $6530-01$ \\
\hline $1 \mathrm{DC} 08005$ & $65^{\circ} 32^{\prime} \mathrm{N}$ & $52^{\circ} 30^{\prime} \mathrm{W}$ & 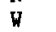 & 85 & 70 & 0000.10 & 780010 & $6530-02$ & $10 D 07013$ & $65^{\circ} 36^{\prime} \mathrm{N}$ & $53^{\circ} 03^{\prime} \mathrm{W}$ & NW & 55 & 45 & 0000.08 & 750010 & $6530-01$ \\
\hline 1DD01001 & $65^{\circ} 41$, N & $53^{\circ} 06, \mathrm{v}$ & SE & 60 & 60 & 0000.07 & 780010 & $6530-01$ & $10 D 07014$ & $65^{\circ} 36^{\prime} \mathrm{N}$ & $53^{\circ} 03 \cdot \mathrm{w}$ & $\mathrm{NV}$ & 60 & 30 & 0000.61 & 640110 & $6530-01$ \\
\hline 1DD01002 & $65^{\circ} 42$, N & $53^{\circ} 00^{\prime} \mathrm{v}$ & NW & 65 & 40 & 0000.78 & 643112 & $6530-01$ & 1DD07015 & $65^{\circ} 37^{\prime} \mathrm{N}$ & $53^{\circ} 04, \mathrm{~W}$ & $\mathrm{NV}$ & 50 & 25 & 0000.09 & 700010 & $6530-01$ \\
\hline 1DD01003 & $65^{\circ} 42, \mathrm{~N}$ & $52^{\circ} 57 \cdot \mathrm{W}$ & SW & 70 & 40 & 0002.42 & 643112 & 6530-01 & 1DD07016 & $65^{\circ} 36^{\prime} \mathrm{N}$ & $53^{\circ} 07 \cdot \mathrm{w}$ & $\mathrm{NW}$ & 35 & 15 & 0000.32 & 643112 & $6530-01$ \\
\hline 1DD01004 & $65^{\circ} 41^{\prime} \mathrm{N}$ & $52^{\circ} 58^{\prime} \mathrm{W}$ & $\mathrm{W}$ & & 25 & 0000.51 & 643112 & $6530-01$ & $1 \mathrm{DD} 07017$ & $65^{\circ} 36^{\prime} \mathrm{N}$ & $53^{\circ} 08^{\prime} \mathrm{W}$ & W & 45 & 20 & 0000.17 & 643112 & $6530-01$ \\
\hline 1DD01005 & $65^{\circ} 41^{\prime} \mathrm{N}$ & $52^{\circ} 58^{\prime} \mathrm{W}$ & sw & 70 & 50 & 0000.48 & 643112 & $6530-01$ & 1DD07018 & $65^{\circ} 35^{\prime} \mathrm{N}$ & $53^{\circ} 10^{\prime} \mathrm{W}$ & W & 50 & 35 & 0000.03 & 790010 & $6530-01$ \\
\hline 1DD02001 & $65^{\circ} 41^{\prime} \mathrm{N}$ & $52^{\circ} 56^{\prime} \mathrm{W}$ & SE & 65 & 40 & 0000.21 & 780010 & $6530-01$ & $1 \mathrm{DD07019}$ & $65^{\circ} 35^{\prime} \mathrm{N}$ & $53^{\circ} 11, \mathrm{~W}$ & $\mathrm{~N}$ & 35 & 20 & 0000.13 & 790010 & $6530-01$ \\
\hline 1DD02002 & $65^{\circ} 42, \mathrm{~N}$ & $52^{\circ} 54^{\prime \prime} \mathrm{W}$ & $\mathrm{NE}$ & 90 & 50 & 0000.33 & 643112 & $6530-01$ & 1DE02001 & $65^{\circ} 49^{\prime} \mathrm{N}$ & $52^{\circ} 56^{\prime} \mathrm{W}$ & $\mathrm{N}$ & 80 & 65 & 0000.16 & 750010 & $6530-01$ \\
\hline $1 \mathrm{DD02003}$ & $65^{\circ} 44^{\prime} \mathrm{N}$ & $52 \circ 52, \mathrm{~W}$ & $\mathrm{SE}$ & 80 & 30 & 0000.15 & 780010 & $6530-01$ & 1DE02002 & $65^{\circ} 50^{\prime} \mathrm{N}$ & $52^{\circ} 50^{\prime} \mathrm{W}$ & NW & & & 0000.07 & 790010 & $6530-01$ \\
\hline 1DD02004 & $65^{\circ} 43^{\prime} \mathrm{N}$ & $52 \circ 50 \cdot \mathrm{W}$ & $\mathrm{NW}$ & 110 & 55 & 0000.12 & 670010 & $6530-01$ & $1 \mathrm{DE} 02003$ & $65^{\circ} 50^{\prime} \mathrm{N}$ & $52^{\circ} 51 \cdot \mathrm{k}$ & NW & 90 & 60 & 0000.22 & 673112 & $6530-01$ \\
\hline $1 \mathrm{DD0} 2005$ & $65^{\circ} 42 \cdot \mathrm{N}$ & $52^{\circ} 49 \cdot \mathrm{V}$ & NW & & 20 & 0000.70 & 643112 & $6530-01$ & $1 \mathrm{DE} 02004$ & $65^{\circ} 50^{\prime} \mathrm{N}$ & $52^{\circ} 52 \cdot \mathrm{W}$ & NW & 85 & 65 & 0000.29 & 750010 & $6530-01$ \\
\hline $10 D 02006$ & $65^{\circ} 42 \cdot \mathrm{N}$ & $52^{\circ} 50^{\prime} \mathrm{W}$ & SW & 75 & 55 & 0000.51 & 750010 & $6530-01$ & 1DE03001 & $65^{\circ} 49^{\prime} \mathrm{N}$ & $52^{\circ} 48, \mathrm{~W}$ & $\mathrm{~W}$ & 85 & 60 & 0000.15 & 750110 & $6530-01$ \\
\hline 1DD02007 & $65^{\circ} 42$ ' N & $52^{\circ} 50^{\prime} \mathrm{W}$ & $\mathrm{NW}$ & 90 & 30 & 0000.61 & 643212 & $6530-01$ & IDE04001 & $65^{\circ} 49^{\prime} \mathrm{N}$ & $52^{\circ} 45, \mathrm{~W}$ & NV & 115 & 50 & 0001.18 & 303112 & $6530-01$ \\
\hline $1 \mathrm{DD} 02008$ & $65^{\circ} 41, \mathrm{~N}$ & $52^{\circ} 50^{\prime} \mathrm{W}$ & w & 70 & 60 & 0000.10 & 780010 & $6530-01$ & 1DE04002 & $65^{\circ} 48^{\prime} \mathrm{N}$ & $52^{\circ} 46^{\prime} \mathrm{W}$ & W & 120 & 60 & 0000.27 & 750112 & $\begin{array}{l}6530-01 \\
6530-01\end{array}$ \\
\hline 1DD03001 & $65^{\circ} 42 \cdot \mathrm{N}$ & $52^{\circ} 48^{\prime} \mathrm{W}$ & $\mathbf{E}$ & 65 & 40 & 0000.63 & 643112 & $6530-01$ & $1 \mathrm{DE} 04003$ & $65^{\circ} 48^{\prime} \mathrm{N}$ & $52^{\circ} 45^{\prime} \mathrm{W}$ & NE & 120 & 90 & 0000.20 & 780010 & $6530-01$ \\
\hline $1 D D 03002$ & $65^{\circ} 43^{\prime} \mathrm{N}$ & $52^{\circ} 47 \mathrm{~W}$ & NE & 90 & 50 & 0000.58 & 643112 & $6530-01$ & 1DE04004 & $65^{\circ} 46^{\prime} \mathrm{N}$ & $52^{\circ} 48^{\prime} \mathrm{W}$ & $\mathbf{N}$ & 90 & 15 & 0001.54 & 643212 & $6530-01$ \\
\hline $1 \mathrm{DD} 03003$ & $65^{\circ} 43^{\prime} \mathrm{N}$ & $52^{\circ} 48^{\prime} \mathrm{W}$ & $\mathrm{NE}$ & 90 & 35 & 0000.46 & 643112 & $6530-01$ & 1DE04005 & $65^{\circ} 46^{\prime} \mathrm{N}$ & $52^{\circ} 50^{\prime} \mathrm{W}$ & NW & 100 & 25 & 0001.04 & $\begin{array}{l}643212 \\
643112\end{array}$ & $\begin{array}{l}6330-01 \\
6530-01\end{array}$ \\
\hline 1DD03004 & $65^{\circ} 45^{\prime} \mathrm{N}$ & $52^{\circ} 51^{\prime} \mathrm{W}$ & $\mathrm{SE}$ & 100 & 50 & 0001.54 & 613212 & $6530-01$ & 1DE04006 & $65^{\circ} 46^{\prime} \mathrm{N}$ & $52 \circ 53, \mathrm{w}$ & NW & 100 & 10 & 0000.13 & 643112 & $6530-01$ \\
\hline 1DD03005 & $65^{\circ} 45^{\prime} \mathrm{N}$ & $52^{\circ} 49, \mathrm{~W}$ & E & & 45 & 0001.40 & 643212 & $6530-01$ & 1DE04007 & $65^{\circ} 47^{\prime} \mathrm{N}$ & $52^{\circ} 52^{\prime} \mathrm{W}$ & NW & 60 & 30 & 0001.54 & 780010 & $6530-01$ \\
\hline 1DD03006 & $65^{\circ} 46^{\prime} \mathrm{N}$ & $52^{\circ} 48^{\prime} \mathrm{W}$ & $\mathrm{s}$ & 95 & 70 & 0000.17 & 780010 & $6530-01$ & 1DE04008 & $65^{\circ} 46^{\prime} \mathrm{N}$ & $52^{\circ} 51, \mathrm{~W}$ & $\mathrm{v}$ & 100 & 50 & 0000.70 & 770010 & $6530-01$ \\
\hline $1 D D 03007$ & $65^{\circ} 45^{\prime} \mathrm{N}$ & $52^{\circ} 47 \cdot \mathrm{W}$ & E & 90 & 75 & 0000.44 & 670010 & $6530-01$ & 1DE04009 & $65^{\circ} 46^{\prime} \mathrm{N}$ & $52 \circ 53, \mathrm{v}$ & $\mathrm{NW}$ & 90 & 10 & 0002.26 & 616112 & $6530-01$ \\
\hline 10003008 & $65045 . \mathrm{N}$ & $52^{\circ} 44^{\prime} \mathrm{V}$ & v & 88 & 75 & 0000.14 & 780010 & $6530-01$ & 1DE05001 & $65^{\circ} 45, \mathrm{~N}$ & $52054^{\prime} \mathrm{w}$ & $\mathrm{U}$ & 70 & 40 & 0000.27 & 613212 & $6530-01$ \\
\hline 1DD04001 & $65^{\circ} 48^{\prime} \mathrm{N}$ & $52^{\circ} 42^{\prime} \mathrm{W}$ & $\mathbf{E}$ & 115 & 60 & 0003.13 & 303012 & $6530-01$ & 1DE05002 & $65^{\circ} 44^{\prime} \mathrm{N}$ & $52^{\circ} 54^{\prime} \mathrm{W}$ & NW & 95 & 30 & 0000.84 & 643112 & $6530-01$ \\
\hline $1 \mathrm{DD} 04002$ & $65^{\circ} 49^{\prime} \mathrm{N}$ & $52^{\circ} 42^{\prime} \mathrm{W}$ & $\mathrm{NE}$ & 100 & 90 & 0000.72 & 300012 & $6530-01$ & 1DE05003 & $65^{\circ} 44^{\prime} \mathrm{N}$ & $52 \circ 56, \mathrm{~W}$ & $\mathbf{N}$ & 65 & 35 & 0000.19 & 770012 & $6530-01$ \\
\hline $1 D D 04003$ & $65^{\circ} 51^{\prime} \mathrm{N}$ & $52^{\circ} 36^{\prime} \mathrm{W}$ & SW & 120 & 30 & 0004.46 & 513312 & $6530-02$ & 1DE06001 & $65^{\circ} 43^{\prime} \mathrm{N}$ & $52 \circ 55, \mathrm{~W}$ & N & 75 & 25 & 0000.38 & 643312 & $6530-01$ \\
\hline $10 D 04004$ & $65^{\circ} 50^{\prime} \mathrm{N}$ & $52^{\circ} 36^{\prime} \mathrm{W}$ & $\mathrm{w}$ & 150 & 25 & 0002.96 & 513412 & $6530-02$ & 1DE06002 & $65^{\circ} 43^{\prime} \mathrm{N}$ & $52 \circ 57 \cdot \mathrm{W}$ & $\mathrm{N}$ & 90 & 15 & 0000.84 & 643412 & $6530-01$ \\
\hline $10 D 04005$ & $65^{\circ} 49^{\prime} \mathrm{N}$ & $52^{\circ} 39^{\prime} \mathrm{W}$ & NW & 120 & 50 & 0000.22 & 643412 & $6530-01$ & 1DE06003 & $65^{\circ} 43^{\prime} \mathrm{N}$ & $52^{\circ} 58^{\prime} \mathrm{W}$ & $\mathrm{N}$ & 50 & 20 & 0000.18 & 640212 & $6530-01$ \\
\hline 1DD04006 & $65^{\circ} 48^{\prime} \mathrm{N}$ & $52^{\circ} 40^{\prime} \mathrm{W}$ & $\mathrm{NW}$ & 100 & 65 & 0000.18 & 643212 & $6530-01$ & 1DE06004 & $65^{\circ} 43^{\prime} \mathrm{N}$ & $52^{\circ} 59, \mathrm{~W}$ & $\mathbf{N}$ & 65 & 20 & 0001.25 & 643112 & $6530-01$ \\
\hline 1DD05001 & $65^{\circ} 49^{\prime} \mathrm{N}$ & $52^{\circ} 34^{\prime} \mathrm{W}$ & $\mathrm{SW}$ & 115 & 30 & 0006.03 & 513012 & $6530-02$ & 1DE06005 & $65^{\circ} 43^{\prime} \mathrm{N}$ & $53^{\circ} 00, \mathrm{w}$ & $\mathbf{N}$ & 70 & 50 & 0000.56 & 610112 & $6530-01$ \\
\hline 1DD05002 & $65^{\circ} 48^{\prime} \mathrm{N}$ & $52^{\circ} 31^{\prime} \mathrm{W}$ & s & 130 & 50 & 0001.69 & 643212 & $6530-02$ & 1DE07001 & $65^{\circ} 44^{\prime} \mathrm{N}$ & $53^{\circ} 02 \cdot \mathrm{W}$ & N & 55 & 15 & 0000.75 & 643412 & $6530-01$ \\
\hline 1DD05003 & $65^{\circ} 47 \cdot \mathrm{N}$ & $52^{\circ} 30^{\prime}$ & SW & 90 & 50 & 0002.22 & 533112 & $6530-02$ & 1DE07002 & $65^{\circ} 43^{\prime} \mathrm{N}$ & $53^{\circ} 03^{\circ} \mathrm{w}$ & NW & 60 & 50 & 0000.11 & 750010 & $6530-01$ \\
\hline $10 D 05004$ & $65^{\circ} 46^{\prime} \mathrm{N}$ & $52^{\circ} 31^{\prime} \mathrm{W}$ & W & 40 & 20 & 0000.71 & 643312 & $6530-02$ & 1DE07003 & $65^{\circ} 42^{\prime} \mathrm{N}$ & $53^{\circ} 03 \cdot \mathrm{W}$ & $\mathrm{N}$ & 60 & 45 & 0000.16 & 640112 & $6530-01$ \\
\hline $1 \mathrm{DD0} 05005$ & $65^{\circ} 46^{\prime} \mathrm{N}$ & $52^{\circ} 34^{\prime} \mathrm{W}$ & $\mathrm{NE}$ & 80 & 20 & 0001.02 & 643312 & $6530-02$ & 1DE07004 & $65^{\circ} 42^{\prime} \mathrm{N}$ & $53^{\circ} 05, \mathrm{~W}$ & $\mathrm{NW}$ & 60 & 20 & 0000.10 & 643112 & $6530-01$ \\
\hline $1 D D 05006$ & $65^{\circ} 47 \cdot \mathrm{N}$ & $52^{\circ} 35^{\prime} \mathrm{w}$ & $\mathrm{N}$ & 110 & 5 & 0000.57 & 643412 & $6530-02$ & 1DE07005 & $65^{\circ} 42^{\prime} \mathrm{N}$ & $53^{\circ} 06^{\prime} \mathrm{W}$ & $\mathrm{NU}$ & 70 & 50 & 0000.20 & 643112 & $6530-01$ \\
\hline $1 \mathrm{DD} 05007$ & $65^{\circ} 471 \mathrm{~N}$ & $52^{\circ} 38^{\prime} \mathrm{W}$ & NW & 110 & 5 & 0000.56 & 643312 & $6530-01$ & $1 \mathrm{DE} 07006$ & $65^{\circ} 42^{\prime} \mathrm{N}$ & $53^{\circ} 06^{\prime} \mathrm{W}$ & NW & 70 & 45 & 0000.42 & 643110 & $6530-01$ \\
\hline $1 D D 05008$ & $65^{\circ} 46^{\prime} \mathrm{N}$ & $52^{\circ} 39^{\prime} \mathrm{W}$ & NW & 100 & 30 & 0000.39 & 643112 & $6530-01$ & 1DF01001 & $65^{\circ} 58^{\prime} \mathrm{N}$ & $53^{\circ} 27 \cdot \mathrm{W}$ & $\mathrm{SW}$ & 55 & 45 & 0000.05 & 770110 & $6530-01$ \\
\hline 1DD05009 & $65^{\circ} 45^{\prime} \mathrm{N}$ & $52^{\circ} 39^{\prime \prime} \mathrm{W}$ & $\mathrm{W}$ & 150 & 35 & 0000.61 & 613112 & $6530-01$ & 1DF01002 & $65^{\circ} 57^{\prime} \mathrm{N}$ & $53^{\circ} 24^{\prime} \mathrm{W}$ & $\mathrm{N}$ & & & 0000.10 & 780010 & $6530-01$ \\
\hline $1 \mathrm{DD} 05010$ & $65^{\circ} 45^{\prime} \mathrm{N}$ & $52^{\circ} 39^{\prime \prime} \mathrm{V}$ & $\mathrm{H}$ & 110 & 50 & 0000.27 & 633112 & $6530-01$ & 1DF01003 & $65^{\circ} 57^{\prime} \mathrm{N}$ & $53^{\circ} 23^{\prime} \mathrm{W}$ & E & 50 & 30 & 0000.11 & 750010 & $6530-01$ \\
\hline 1DD05011 & $65^{\circ} 44^{\prime} \mathrm{N}$ & $52^{\circ} 40^{\prime} \mathrm{W}$ & sW & 95 & 60 & 0000.10 & 780010 & $6530-01$ & 1DF01004 & $65^{\circ} 56^{\prime} \mathrm{N}$ & $53^{\circ} 22, \mathrm{~W}$ & N & 50 & 30 & 0000.11 & 750010 & $6530-01$ \\
\hline $10 D 06001$ & $65^{\circ} 43^{\prime} \mathrm{N}$ & $52^{\circ} 38^{\prime} \mathrm{V}$ & w & 105 & 60 & 0000.31 & 790010 & $6530-01$ & 1DF01005 & $65^{\circ} 56^{\prime} \mathrm{N}$ & $53^{\circ} 23^{\prime} \mathrm{W}$ & $\mathrm{V}$ & & 50 & 0000.16 & 770010 & $6530-01$ \\
\hline 10006002 & $65^{\circ} 45, \mathrm{~N}$ & $52 \circ 33^{\prime} \mathrm{V}$ & $\mathrm{SW}$ & 90 & 50 & 0000.46 & 673212 & $6530-02$ & 1DF01006 & $65^{\circ} 57, \mathrm{~N}$ & $53^{\circ} 15, \mathrm{~W}$ & $\mathrm{~N}$ & & & 0000.10 & 780010 & $6530-01$ \\
\hline $1 D D 06$ & $65^{\circ} 44^{\prime} \mathrm{N}$ & $52^{\circ} 34^{\prime} \mathrm{W}$ & $\mathrm{SW}$ & 120 & 25 & 0002.37 & 533112 & 6530-02 & 1DF01007 & $65^{\circ} 57^{\prime} \mathrm{N}$ & $53^{\circ} 14, \mathrm{~W}$ & s & 81 & 80 & 0000.11 & 780010 & $6530-01$ \\
\hline $10 D 06$ & $65^{\circ} 43^{\prime} \mathrm{N}$ & $52^{\circ} 35^{\prime} \mathrm{V}$ & W & 125 & 25 & 0007.54 & 513112 & 6530-02 & 1DF02001 & $66^{\circ} 02^{\prime} \mathrm{N}$ & $53^{\circ} 01$, w & s & 75 & 75 & 0000.23 & 363112 & $6600-01$ \\
\hline 1DDO & $65^{\circ} 41^{\prime} \mathrm{N}$ & $52^{\circ} 36^{\prime} \mathrm{W}$ & $\mathrm{NW}$ & 85 & 45 & 0000.28 & 643212 & 65 & 1DF02 & $66^{\circ} 02^{\prime} \mathrm{N}$ & $53^{\circ} 02, \mathrm{w}$ & SE & 95 & 85 & 0000.26 & 300110 & $6600-01$ \\
\hline $1 \mathrm{DDO} 6$ & $65^{\circ} 41, \mathrm{~N}$ & $52^{\circ} 39^{\prime} \mathrm{W}$ & $\mathrm{N}$ & 120 & 15 & 0001.71 & 643112 & $6530-01$ & $1 \mathrm{DF} 02003$ & $66^{\circ} 04^{\prime} \mathrm{N}$ & $52^{\circ} 57 \cdot \mathrm{W}$ & SW & 110 & & 0049.24 & 360112 & $6600-01$ \\
\hline $10 D 06007$ & $65^{\circ} 41, N$ & $52^{\circ} 42$ & $\mathrm{y}$ & 45 & 15 & 0000.62 & 643112 & $6530-01$ & 1DF03001 & $66^{\circ} 03, \mathrm{~N}$ & $52054^{\prime} \mathrm{W}$ & $\mathrm{SE}$ & 120 & 105 & 0000.06 & 300110 & $6600-02$ \\
\hline 1DD06008 & $65^{\circ} 40^{\prime} \mathrm{N}$ & $52^{\circ} 42^{\prime} \mathrm{V}$ & $\mathrm{SW}$ & & & 0000.62 & 780010 & 6530-01 & 1DF03002 & $66^{\circ} 04^{\prime} \mathrm{N}$ & $52^{\circ} 50^{\prime} \mathrm{w}$ & SW & 100 & 40 & 0003.64 & 363112 & $6600-02$ \\
\hline 1DD06009 & $65^{\circ} 40^{\prime} \mathrm{N}$ & $52^{\circ} 43^{\prime} \mathrm{V}$ & NW & 50 & 35 & & 750 & & 10 & ${ }^{\prime} \mathrm{N}$ & $52^{\circ} 49, \mathrm{~W}$ & NW & 110 & 80 & 0000.45 & 270010 & $6600-02$ \\
\hline $10 D 06010$ & $65^{\circ} 39^{\prime} \mathrm{N}$ & $52^{\circ} 44^{\prime} \mathrm{V}$ & W & 90 & 70 & 0000.15 & 790010 & $6530-01$ & $1 \mathrm{DF} 03004$ & $66^{\circ} 02^{\prime} \mathrm{N}$ & $52^{\circ} 48, \mathrm{~W}$ & $\mathrm{SW}$ & 174 & & 0240.00 & 305111 & $6000-02$ \\
\hline $1 D D 07001$ & $65^{\circ} 38^{\prime} \mathrm{N}$ & $52053 \%$ & $\mathrm{~N}$ & & 20 & 0000.22 & 653212 & $6530-01$ & $10 F 03005$ & $66^{\circ} 01, \mathrm{~N}$ & $52^{\circ} 44^{\prime} \mathrm{W}$ & SW & 110 & 80 & 0003.48 & 513112 & $6600-02$ \\
\hline 1DD07002 & $65^{\circ} 38^{\prime} \mathrm{N}$ & $52^{\circ} 54^{\prime} \mathrm{W}$ & $\mathrm{N}$ & 55 & 15 & 0000.26 & 643312 & 6530-01 & 1DF03006 & $65^{\circ} 58^{\prime} \mathrm{N}$ & $52^{\circ} 50^{\circ} \mathrm{W}$ & $\mathrm{NE}$ & 75 & 55 & 0000.31 & 750010 & $6530-01$ \\
\hline & $65^{\circ} 39^{\prime} \mathrm{N}$ & $52 \circ 55$ & $N$ & & 35 & 00 & 670 & & $1 \mathrm{DF} 03007$ & $65^{\circ} 58^{\prime} \mathrm{N}$ & $52051, \mathrm{~W}$ & $\mathrm{NE}$ & 80 & 50 & 0000.55 & 750010 & $6530-01$ \\
\hline 100 & $65^{\circ} 38^{\prime} \mathrm{N}$ & $52^{\circ} 56^{\prime} \mathrm{V}$ & NW & 55 & 10 & 0001.26 & 533112 & $6530-01$ & 1DF03008 & $65^{\circ} 58^{\prime} \mathrm{N}$ & $52 \circ 52, w$ & $\mathrm{NE}$ & & 70 & 0000.09 & 643310 & $6530-01$ \\
\hline $1 D D 07005$ & $65^{\circ} 38^{\prime} \mathrm{N}$ & $52 \circ 56^{\prime} \mathrm{V}$ & $\mathrm{W}$ & & 10 & 0000.88 & 513112 & $6530-01$ & 1DF03009 & $65^{\circ} 58^{\prime} \mathrm{N}$ & $52053, \mathrm{w}$ & $\mathrm{NE}$ & & & 0000.39 & 643210 & $6530-01$ \\
\hline 1DD07006 & $65^{\circ} 37^{\prime N} \mathrm{~N}$ & $52057 \% \mathrm{~W}$ & N & 45 & 10 & 0001.97 & 643112 & $6530-01$ & 1DF03010 & $65^{\circ} 58^{\prime} \mathrm{N}$ & $52 \circ 54, \mathrm{~W}$ & $\mathrm{NE}$ & 115 & 35 & 0004.23 & 513212 & $6530-01$ \\
\hline 1D & $65^{\circ} 32^{\prime} \mathrm{N}$ & $52^{\circ} 59^{\prime} \mathrm{V}$ & $\mathrm{N}$ & 40 & 25 & 00 & 640110 & & & $65^{\circ} 59^{\prime} \mathrm{N}$ & $6, \mathrm{~W}$ & $\mathrm{NE}$ & 80 & 40 & 0001.58 & 513312 & $6530-01$ \\
\hline 1DD07008 & $65^{\circ} 38^{\prime} \mathrm{N}$ & $53^{\circ} 00^{\prime} \mathrm{V}$ & NW & 50 & 10 & 0000.65 & 643412 & 6530-01 & 1DF03012 & $66^{\circ} 00^{\circ} \mathrm{N}$ & $52^{\circ} 56^{\prime} \mathrm{w}$ & $\mathrm{NE}$ & 70 & 60 & 0000.19 & 643112 & $6530-01$ \\
\hline 1DD07009 & $65^{\circ} 37^{\prime} \mathrm{N}$ & $53^{\circ} 01$ ' & $\mathrm{NW}$ & 60 & 10 & 0002.37 & 513112 & 6530-01 & 1DF04001 & $65^{\circ} 59^{\prime} \mathrm{N}$ & $52058, \mathrm{~W}$ & NW & 70 & 55 & 0000.29 & 640112 & $6530-01$ \\
\hline 1DD07010 & $65^{\circ} 38^{\prime} \mathrm{N}$ & $53^{\circ} 02, \mathrm{w}$ & $\mathrm{NW}$ & 25 & 15 & 0000.11 & 780010 & $6530-01$ & 1DF04002 & $65^{\circ} 59^{\prime} \mathrm{N}$ & $52^{\circ} 57 \cdot \mathrm{W}$ & $\mathrm{NW}$ & 70 & 70 & 0000.08 & 640112 & $6530-01$ \\
\hline
\end{tabular}




\begin{tabular}{|c|c|c|c|c|c|c|c|c|c|c|c|c|c|}
\hline CODE & LAT & LONG & ORI & HIGH & LOW & AREA & $\begin{array}{l}\text { MO } \\
\text { CODE }\end{array}$ & MAP & CODE & LAT & LONG & ORI & HIGH \\
\hline 1DF04003 & $65^{\circ} 59^{\prime} \mathrm{N}$ & $52^{\circ} 58^{\prime} \mathrm{W}$ & NW & & 25 & 0000.32 & 643212 & $6530-01$ & 1DF19001 & $66^{\circ} 02^{\prime} \mathrm{N}$ & $52^{\circ} 00^{\prime} \mathrm{W}$ & $\mathbf{s}$ & 200 \\
\hline 1DF04004 & $65^{\circ} 58^{\prime} \mathrm{N}$ & $52^{\circ} 58^{\prime} \mathrm{W}$ & NW & 110 & 15 & 0002.80 & 643312 & $6530-01$ & 1DF19002 & $66^{\circ} 03^{\prime} \mathrm{N}$ & $51^{\circ} 59^{\prime \prime}$ & SW & 180 \\
\hline 1DF04005 & $65^{\circ} 58^{\prime} \mathrm{N}$ & $53^{\circ} 00^{\prime}$ & NW & 95 & 25 & 0001.95 & 613212 & $6530-01$ & 1DF19003 & $66^{\circ} 03^{\prime} \mathrm{N}$ & $51^{\circ} 58^{\prime} \mathrm{W}$ & SW & 190 \\
\hline 1DF04006 & $65^{\circ} 58^{\prime} \mathrm{N}$ & $53^{\circ} 02^{\prime} \mathrm{W}$ & NW & 80 & 70 & 0000.25 & 780010 & $6530-01$ & 1DF19004 & $66^{\circ} 03^{\prime} \mathrm{N}$ & $51^{\circ} 56^{\prime} \mathrm{V}$ & $\mathrm{s}$ & 190 \\
\hline 1DF04007 & $65^{\circ} 57^{\prime} \mathrm{N}$ & $53^{\circ} 02^{\prime} \mathrm{V}$ & W & 95 & 50 & 0000.54 & 643112 & $6530-01$ & 1DF19005 & $66^{\circ} 03^{\prime} \mathrm{N}$ & $51^{\circ} 54^{\prime} \mathrm{W}$ & $\mathrm{s}$ & 150 \\
\hline 1DF04008 & $65^{\circ} 57^{\circ} \mathrm{N}$ & $53^{\circ} 03^{\prime} \mathrm{w}$ & SW & 95 & 75 & 0000.39 & 643112 & $6530-01$ & 1DF19006 & $66^{\circ} 03^{\prime} \mathrm{N}$ & $51.53, \mathrm{~W}$ & s & 110 \\
\hline 1DF04009 & $65^{\circ} 56^{\prime} \mathrm{N}$ & $53^{\circ} 01^{\prime} \mathrm{W}$ & SW & 110 & 55 & 0002.84 & 513112 & $6530-01$ & 1DF19007 & $66^{\circ} 05^{\prime} \mathrm{N}$ & $51^{\circ} 52^{\prime} \mathrm{V}$ & $\mathrm{s}$ & 197 \\
\hline 1DF04010 & $65^{\circ} 55^{\prime} \mathrm{N}$ & $53^{\circ} 04^{\prime} \mathrm{W}$ & $\mathrm{N}$ & 70 & 45 & 0000.34 & 643112 & $6530-01$ & 1DF19008 & $66^{\circ} 05^{\circ} \mathrm{N}$ & $51^{\circ} 49 \cdot \mathrm{V}$ & $\mathrm{SE}$ & 200 \\
\hline 1DF04011 & $65^{\circ} 55^{\prime} \mathrm{N}$ & $53^{\circ} 05^{\prime} \mathrm{W}$ & E & 75 & 60 & 0001.01 & 670112 & $6530-01$ & 1DF20001 & $66^{\circ} 06^{\prime} \mathrm{N}$ & $51^{\circ} 50^{\prime} \mathrm{V}$ & $\mathrm{SE}$ & 180 \\
\hline 1DF04012 & $65^{\circ} 55^{\prime} \mathrm{N}$ & $53^{\circ} 07^{\prime} \mathrm{W}$ & $\mathrm{N}$ & 90 & 45 & 0000.24 & 646112 & $6530-01$ & 1DF20002 & $66^{\circ} 08^{\prime} \mathrm{N}$ & $51^{\circ} 45^{\prime} \mathrm{W}$ & $\mathrm{s}$ & 188 \\
\hline 1DF04013 & $65^{\circ} 54^{\prime} \mathrm{N}$ & $53^{\circ} 12^{\prime \prime}$ & NW & 70 & & 0000.06 & 780010 & $6530-01$ & 1DF20003 & $66^{\circ} 10^{\circ} \mathrm{N}$ & $51^{\circ} 37 \% \mathrm{~W}$ & $\mathrm{SE}$ & 170 \\
\hline 1DF0S001 & $65^{\circ} 55^{\prime} \mathrm{N}$ & $53^{\circ} 05^{\prime} \mathrm{W}$ & E & & & 0000.47 & 753112 & $6530-01$ & 1DF20004 & $66^{\circ} 12 \cdot \mathrm{N}$ & $51^{\circ} 35^{\prime} \mathrm{w}$ & $\mathrm{SE}$ & 170 \\
\hline 1DF05002 & $65^{\circ} 55^{\prime} \mathrm{N}$ & $53^{\circ} 02^{\prime} \mathrm{W}$ & $\overline{\mathrm{W}}$ & 80 & 70 & 0000.11 & 753112 & $6530-01$ & 1DF20005 & $66^{\circ} 12^{\prime} \mathrm{N}$ & $51^{\circ} 31^{\prime} \mathrm{W}$ & SW & 165 \\
\hline 1DF06001 & $65^{\circ} 56^{\prime} \mathrm{N}$ & $52^{\circ} 59^{\prime} \mathrm{W}$ & SE & 100 & 50 & 0003.40 & 643112 & $6530-01$ & 1DF20006 & $66^{\circ} 13^{\prime} \mathrm{N}$ & $51^{\circ} 29^{\prime} \mathrm{W}$ & $\mathrm{SE}$ & 160 \\
\hline $1 \mathrm{DF} 07001$ & $65^{\circ} 57^{\prime} \mathrm{N}$ & $52^{\circ} 57 \cdot \mathrm{V}$ & s & 105 & 50 & 0002.21 & 613112 & $6530-01$ & 1DF20007 & $66^{\circ} 14^{\prime} \mathrm{N}$ & $51^{\circ} 28^{\prime} \mathrm{W}$ & $\mathrm{E}$ & 160 \\
\hline 1DF07002 & $65 \circ 57 \cdot \mathrm{N}$ & $52.54 \cdot 6$ & $\mathrm{~s}$ & 110 & 100 & 0000.48 & 780010 & $6530-01$ & 1DF20008 & $66^{\circ} 11 / \mathrm{N}$ & $51^{\circ} 13^{\circ} \mathrm{v}$ & N & 195 \\
\hline 1DF08001 & $66^{\circ} 01$ ' N & $52^{\circ} 42^{\prime} \mathrm{V}$ & s & 130 & 130 & 0000.11 & 780010 & $6600-02$ & 1DF20009 & $66^{\circ} 09^{\prime} \mathrm{N}$ & $51^{\circ} 15^{\prime} \mathrm{W}$ & $\mathrm{N}$ & 195 \\
\hline 1DFO8002 & $66^{\circ} 00^{\prime} \mathrm{N}$ & $52^{\circ} 42^{\prime} \mathrm{V}$ & s & 110 & 30 & 0005.99 & 513112 & $6600-02$ & 1DF20010 & $66^{\circ} 09^{\prime} \mathrm{N}$ & $51^{\circ} 19$ ' & N & 155 \\
\hline 1DF09001 & $65^{\circ} 59^{\prime} \mathrm{N}$ & $52^{\circ} 39^{\prime} \mathrm{V}$ & SE & 110 & 70 & 0000.11 & 780010 & $6530-01$ & 1DF20011 & $66^{\circ} 08^{\prime} \mathrm{N}$ & $51^{\circ} 21^{\prime} \mathrm{V}$ & N & 195 \\
\hline 1DF09002 & $66^{\circ} 00^{\prime} \mathrm{N}$ & $52^{\circ} 40^{\prime} \mathrm{W}$ & $\mathrm{E}$ & 115 & 90 & 0000.12 & 643212 & $6600-02$ & 1DF20012 & $66^{\circ} 08^{\prime} \mathrm{N}$ & $51^{\circ} 24^{\prime} \mathrm{V}$ & N & 195 \\
\hline 1DF09003 & $66^{\circ} 01$ 'N & $52^{\circ} 39^{\prime \prime}$ & SE & 110 & 40 & 0002.52 & 643312 & $6600-02$ & 1DF20013 & $66^{\circ} 08^{\prime} \mathrm{N}$ & $51^{\circ} 27, \mathrm{v}$ & NW & 160 \\
\hline 1DF09004 & $66^{\circ} 02, \mathrm{~N}$ & $52^{\circ} 39^{\prime} \mathrm{W}$ & $\mathbf{s}$ & 115 & 70 & 0000.86 & 643112 & $6600-02$ & $1 D F 20014$ & $66^{\circ} 07 \cdot \mathrm{N}$ & $51^{\circ} 28^{\prime} \mathrm{V}$ & $\mathrm{NW}$ & 195 \\
\hline 1DF09005 & $66^{\circ} 02, \mathrm{~N}$ & $52.37, \mathrm{~W}$ & SW & 140 & 80 & 0000.68 & 643112 & $6600-02$ & 1DF20015 & $66^{\circ} 07^{\circ} \mathrm{N}$ & $51^{\circ} 32^{\circ} \mathrm{k}$ & NW & 150 \\
\hline 1DF 10001 & $66^{\circ} 02^{\prime} \mathrm{N}$ & $52^{\circ} 35^{\prime} \mathrm{W}$ & $\mathrm{E}$ & 130 & 60 & 0000.72 & 643212 & $6600-02$ & 1DF20016 & $66^{\circ} 05^{\prime} \mathrm{N}$ & $51^{\circ} 32, \mathrm{v}$ & $w$ & 195 \\
\hline 1DF10002 & $66^{\circ} 03^{\prime} \mathrm{N}$ & $52^{\circ} 35^{\prime} \mathrm{W}$ & $\bar{E}$ & 90 & 70 & 0000.09 & 750110 & $6600-02$ & 1DF20017 & $66^{\circ} 05^{\prime} \mathrm{N}$ & $51^{\circ} 34^{\prime} \mathrm{V}$ & NW & 150 \\
\hline 1DF10003 & $66^{\circ} 04 \cdot \mathrm{N}$ & $52^{\circ} 33^{\prime \prime}$ & SW & 140 & 50 & 0006.73 & 533112 & $6600-02$ & IDF20018 & $66^{\circ} 03^{\prime} \mathrm{N}$ & $51^{\circ} 36,6$ & NW & 190 \\
\hline 1DF10004 & $66^{\circ} 05^{\circ} \mathrm{N}$ & $52^{\circ} 30^{\prime} \mathrm{W}$ & SW & 105 & 75 & 0002.55 & 533112 & 6600 & 1DF20019 & $66^{\circ} 05^{\prime} \mathrm{N}$ & $51.39 \cdot v$ & N & 120 \\
\hline IDF10005 & $66^{\circ} 03 \cdot \mathrm{N}$ & $52^{\circ} 31^{\prime \prime}$ & SW & 160 & & 0315.83 & 353112 & $6600-02$ & 1DF20020 & $66^{\circ} 04^{\prime} \mathrm{N}$ & $51.41, v$ & N & 142 \\
\hline IDF10006 & $66^{\circ} 01, \mathrm{~N}$ & $52^{\circ} 32^{\prime} \mathrm{W}$ & $\mathrm{W}$ & 110 & 90 & 0000.07 & 643112 & $6600-02$ & 1DF20021 & $66^{\circ} 02^{\prime} \mathrm{N}$ & $511^{\circ} 1 \mathrm{v}$ & NW & 150 \\
\hline 1DF11001 & $66^{\circ} 01^{\prime} \mathrm{N}$ & $52^{\circ} 30^{\prime} \mathrm{w}$ & E & 100 & 65 & 0000.95 & 643212 & $6600-02$ & 1DF20022 & $66^{\circ} 02^{\prime} \mathrm{N}$ & $51^{\circ} 46^{\prime} \mathrm{W}$ & NW & 137 \\
\hline 1DF11002 & $66^{\circ} 02$ 'N & $52^{\circ} 29^{\prime} \mathrm{W}$ & SE & 95 & 80 & 0000.09 & 750010 & 6600 & 1DF20023 & $66^{\circ} 01{ }^{\prime} \mathrm{N}$ & $51^{\circ} 47,6$ & W & 137 \\
\hline 1DF 11003 & $66^{\circ} 02, \mathrm{~N}$ & $52^{\circ} 28^{\prime} \mathrm{W}$ & $\mathrm{s}$ & 80 & 60 & 0000.16 & 780010 & $6600-02$ & $1 \mathrm{DF} 20024$ & $66^{\circ} 01 \cdot \mathrm{N}$ & $51^{\circ} 47^{\prime} \mathrm{k}$ & W & 137 \\
\hline 1DF11004 & $66^{\circ} 07 \cdot \mathrm{N}$ & $52^{\circ} 20^{\prime \prime} \mathrm{V}$ & SW & 150 & 60 & 0015.98 & 363112 & $6600-02$ & 1DF20025 & $66^{\circ} 00^{\prime} \mathrm{N}$ & $51.45, \mathrm{~W}$ & SW & 137 \\
\hline 1DF11005 & $66^{\circ} 04^{\prime} \mathrm{N}$ & $52^{\circ} 27^{\prime} \mathrm{W}$ & SW & 160 & & 0211.11 & 364117 & 6600 & 1DF20026 & $66^{\circ} 01^{\prime} \mathrm{N}$ & $51039 \cdot 6$ & SW & 137 \\
\hline 1DF11006 & $66^{\circ} 00^{\prime} \mathrm{N}$ & $52^{\circ} 24^{\prime} \mathrm{V}$ & NW & 130 & 50 & 0000.20 & 643012 & 6600 & $1 \mathrm{DF} 21001$ & $65^{\circ} 58^{\prime} \mathrm{N}$ & $51^{\circ} 42,6$ & SW & 119 \\
\hline 1DF12001 & $65^{\circ} 58^{\prime} \mathrm{N}$ & $52^{\circ} 30^{\circ} \mathrm{v}$ & NW & 120 & 20 & 0004.00 & 643212 & 6530 & 1DF21002 & $65^{\circ} 57 / \mathrm{N}$ & $51^{\circ} 31,6$ & SW & 195 \\
\hline 1DF 12002 & $65^{\circ} 58^{\circ} \mathrm{N}$ & $52^{\circ} 32, \mathrm{w}$ & NW & 125 & 20 & 0001.04 & 643312 & 653 & $10 F 21003$ & $65^{\circ} 52^{\prime} \mathrm{N}$ & $51^{\circ} 45,6$ & $\mathrm{E}$ & 120 \\
\hline 1DF12003 & $65^{\circ} 57^{\prime} \mathrm{N}$ & $52 \cdot 34 . \mathrm{W}$ & NW & 130 & 20 & 0001.13 & 643212 & $6530-02$ & 1DF21004 & $65^{\circ} 54^{\prime} \mathrm{N}$ & $51^{\circ} 46^{\prime}$ & NE & 120 \\
\hline IDF12004 & $65^{\circ} 57^{\circ} \mathrm{N}$ & $52^{\circ} 35^{\prime} \mathrm{W}$ & NW & 80 & 50 & 0000.11 & 750010 & 6530 & 1DF21005 & $65^{\circ} 55^{\prime} \mathrm{N}$ & $51^{\circ} 49,6$ & $\mathrm{NE}$ & 120 \\
\hline 1DF12005 & $65^{\circ} 57^{\prime} \mathrm{N}$ & $52^{\circ} 35^{\prime} \mathrm{w}$ & NW & 100 & 50 & 0000.11 & 750010 & $6530-02$ & 1DF21006 & $65^{\circ} 57^{\prime} \mathrm{N}$ & $51 \circ 51,6$ & E & 142 \\
\hline 1DF12006 & $65^{\circ} 57^{\prime} \mathrm{N}$ & $52^{\circ} 32^{\prime} \mathrm{w}$ & N & 166 & 130 & 0000.34 & 700010 & $6530-02$ & 1DF22001 & $65^{\circ} 59^{\prime} \mathrm{N}$ & $51.53, \mathrm{~V}$ & N & 110 \\
\hline 1DF13001 & $65^{\circ} 57^{\circ} \mathrm{N}$ & $52^{\circ} 32^{\prime} \mathrm{W}$ & $\mathrm{s}$ & 150 & 100 & 0001.36 & 513112 & $6530-02$ & $10 F 22002$ & $65^{\circ} 54^{\prime} \mathrm{N}$ & $51.55 \% \mathrm{~W}$ & $\mathrm{~N}$ & 154 \\
\hline 1DF13002 & $65^{\circ} 57 \cdot \mathrm{N}$ & $52^{\circ} 30^{\prime \prime}$ & SW & 150 & 100 & 0000.52 & 643212 & 6530 & 1DF22003 & $66^{\circ} 00^{\prime} \mathrm{N}$ & $51^{\circ} 56^{\prime \prime}$ & SE & 150 \\
\hline 1DF13003 & $65^{\circ} 56^{\prime} \mathrm{N}$ & $52^{\circ} 30^{\prime} \mathrm{W}$ & $\mathrm{sW}$ & 160 & 30 & 0003.24 & 513112 & 65 & 1DF22004 & N & $51^{\circ} 58^{\prime} \mathrm{W}$ & $\mathrm{NE}$ & 158 \\
\hline 1DF 13004 & $65^{\circ} 55^{\prime} \mathrm{N}$ & $52^{\circ} 29, \mathrm{~W}$ & $\mathrm{sW}$ & 100 & 65 & 0001.06 & 533112 & 6530 & $1 D F 23001$ & $65^{\circ} 59, \mathrm{~N}$ & $52^{\circ} 00^{\prime} \mathrm{V}$ & $\mathbf{N}$ & 140 \\
\hline 1DF14001 & $65^{\circ} 56^{\prime} \mathrm{N}$ & $52^{\circ} 25, \mathrm{~V}$ & $\mathrm{~s}$ & 160 & 10 & 0008.63 & 533122 & $6530-02$ & 1DF23002 & $65^{\circ} 58^{\prime} \mathrm{N}$ & $52^{\circ} 03^{\prime} \mathrm{V}$ & $\mathrm{SE}$ & $\begin{array}{l}169 \\
169\end{array}$ \\
\hline & $65^{\circ} 56^{\prime} \mathrm{N}$ & $52^{\circ} 23^{\prime} \mathrm{W}$ & SW & 135 & 70 & 000 & 613212 & 6530 & IDF23003 & $65^{\circ} 58^{\prime} \mathrm{N}$ & $52^{\circ} 02^{\prime \prime}$ & $\mathrm{SE}$ & 150 \\
\hline $1 \mathrm{DF}$ & $65^{\circ} 55^{\prime} \mathrm{N}$ & $52^{\circ} 22^{\prime} \mathrm{V}$ & SW & 180 & 30 & 0005.65 & 513112 & & IDF23004 & $65^{\circ} 57^{\prime} \mathrm{N}$ & $52 \circ 04,6$ & NW & 160 \\
\hline 1DF16001 & $65^{\circ} 56^{\prime} \mathrm{N}$ & $52^{\circ} 18^{\prime} \mathrm{W}$ & $\mathrm{s}$ & 180 & 80 & 0001.78 & 533112 & $6530-02$ & $1 D F 23005$ & $65^{\circ} 57^{\prime} \mathrm{N}$ & $52^{\circ} 07$ W & $\mathrm{N}$ & 195 \\
\hline 1DF16002 & $65^{\circ} 56^{\prime} \mathrm{N}$ & $52^{\circ} 17, \mathrm{w}$ & E & 120 & 55 & 0000.73 & 640112 & $6530-02$ & 1DF23006 & $65^{\circ} 58^{\prime} \mathrm{N}$ & $52^{\circ} 07,6$ & $\mathrm{~N}$ & 95 \\
\hline IDF16003 & $65^{\circ} 57^{\circ} \mathrm{N}$ & $52^{\circ} 20^{\prime} \mathrm{W}$ & NE & 160 & & 0008.61 & 515112 & $6530-02$ & 1DF23007 & $65^{\circ} 58^{\prime} \mathrm{N}$ & $52^{\circ} 08^{\prime} \mathrm{w}$ & NW & 60 \\
\hline IDF & $65^{\circ} 58^{\prime} \cdot \mathrm{N}$ & $52^{\circ} 18^{\prime} \mathrm{W}$ & $\mathrm{s}$ & 210 & 105 & 000 & & & $10 \mathrm{~F}$ & $65^{\circ} 57 / \mathrm{N}$ & 0 & NW & 60 \\
\hline 1DF16005 & $65^{\circ} 59^{\prime} \mathrm{N}$ & $52^{\circ} 16^{\prime} \mathrm{W}$ & $\mathrm{SE}$ & 210 & 50 & 0004.29 & 513212 & 6530 & IDF24002 & $65^{\circ} 57 \cdot \mathrm{N}$ & $52^{\circ} 08^{\prime}$ & SW & \\
\hline IDF17001 & $66^{\circ} 01^{\prime} \mathrm{N}$ & $52^{\circ} 12^{\prime} \mathrm{W}$ & $\mathrm{SE}$ & 195 & & 0027.86 & 365312 . & $6600-02$ & 1DF24003 & $65^{\circ} 56^{\prime} \mathrm{N}$ & $52^{\circ} 10^{\prime} \mathrm{W}$ & $\mathbf{S E}$ & 130 \\
\hline 1DF17002 & $66^{\circ} 01^{\prime} \mathrm{N}$ & $52^{\circ} 10^{\prime} \mathrm{w}$ & $\mathrm{SE}$ & 115 & 100 & 0000.29 & 700010 & $6600-02$ & 1DF24004 & $65^{\circ} 55^{\prime} \mathrm{N}$ & $52^{\circ} 09^{\prime} \mathrm{W}$ & $\mathrm{s}$ & 180 \\
\hline IDF17003 & $66^{\circ} 01^{\prime} \mathrm{N}$ & $52^{\circ} 09^{\prime} \mathrm{W}$ & $\mathrm{s}$ & 13 & 80 & 0000 & & & 1DF2 & N & שי & Sw & 160 \\
\hline 1DF17004 & $66^{\circ} 02^{\prime} \mathrm{N}$ & $52^{\circ} 08^{\prime} \mathrm{w}$ & $\mathbf{s}$ & 200 & 160 & 0000.24 & 700010 & $6600-02$ & 1DF24006 & $65^{\circ} 54^{\prime} \mathrm{N}$ & $52^{\circ} 10^{\prime \prime}$ & SW & 150 \\
\hline 1DF18001 & $66^{\circ} 02^{\prime} \mathrm{N}$ & $52^{\circ} 07^{\circ} \mathrm{W}$ & SE & 210 & 180 & 0003.24 & 300010 & $6600-02$ & 1DF25001 & $65^{\circ} 53^{\prime} \mathrm{N}$ & $52^{\circ} 08^{\prime}$ & $\mathrm{W}$ & 210 \\
\hline 1DF18002 & $66^{\circ} 01^{\prime} \mathrm{N}$ & $52^{\circ} 06^{\prime} \mathrm{W}$ & $\mathrm{s}$ & 180 & 70 & 0001.78 & 373212 & $600-02$ & 1DF25002 & $65^{\circ} 52^{\prime} \mathrm{N}$ & $52^{\circ} 07 \cdot \mathrm{W}$ & SW & \\
\hline IDF18003 & $66^{\circ} 03^{\prime} \mathrm{N}$ & $52^{\circ} 04^{\prime} \mathrm{W}$ & $\mathrm{SE}$ & 180 & 20 & 0002 & 363412 & 660 & 1DF25003 & $65^{\circ} 51^{\prime} \mathrm{N}$ & $52^{\circ} 09^{\prime}$ & $\mathrm{NW}$ & 160 \\
\hline 1DF18004 & $66^{\circ} 03^{\prime} \mathrm{N}$ & $52^{\circ} 02^{\prime} \mathrm{w}$ & $\mathbf{s}$ & 160 & 130 & 0000.32 & 700010 & $6600-02$ & $1 D F 25004$ & $65^{\circ} 51^{\prime} \mathrm{N}$ & $52^{\circ} 12^{\prime} \mathrm{W}$ & Y & \\
\hline
\end{tabular}

$\begin{array}{rccc}\text { LOW } & \text { AREA } & \text { MODE } & \text { MAP } \\ & 0013.71 & 365412 & 6600-03 \\ 100 & 0000.63 & 780010 & 6600-03 \\ 80 & 0001.08 & 303312 & 6600-03 \\ 90 & 0001.51 & 300010 & 6600-03 \\ 30 & 0001.24 & 303312 & 6600-03 \\ 60 & 0000.74 & 303312 & 6600-03 \\ & 0023.86 & 363412 & 6600-03 \\ 60 & 0005.04 & 363312 & 6600-03 \\ 30 & 0005.03 & 363412 & 6600-03 \\ & 0093.93 & 362311 & 6600-03 \\ 85 & 0009.97 & 300210 & 6600-03 \\ 20 & 0038.44 & 362412 & 6600-03 \\ 135 & 0001.91 & 300010 & 6600-03 \\ 120 & 0002.67 & 300010 & 6600-03 \\ 95 & 0015.14 & 363112 & 6600-03 \\ 75 & 0043.74 & 363112 & 6600-03 \\ 40 & 0054.34 & 363212 & 6600-03 \\ 130 & 0004.82 & 300112 & 6600-03 \\ 20 & 0023.26 & 363312 & 6600-03 \\ 20 & 0013.93 & 366312 & 6600-03 \\ 125 & 0003.43 & 300010 & 6600-03 \\ 10 & 0099.76 & 362412 & 6600-03 \\ 110 & 0003.87 & 300010 & 6600-03 \\ 10 & 0040.86 & 362412 & 6600-03 \\ 120 & 0002.46 & 300010 & 6600-03 \\ 15 & 0054.25 & 362312 & 6600-03 \\ 85 & 0000.53 & 300012 & 6600-03 \\ 60 & 0004.96 & 363412 & 6600-03 \\ & 0021.00 & 365141 & 6600-03 \\ 60 & 0002.24 & 363412 & 6600-03 \\ 80 & 0001.46 & 300012 & 6600-03 \\ 80 & 0001.17 & 300012 & 6600-03 \\ 90 & 0005.99 & 303012 & 6600-03 \\ & 0004.71 & 365412 & 6600-03 \\ 90 & 0002.11 & 30012 & 6530-03 \\ 55 & 0248.21 & 364111 & 6530-03 \\ 6010.03 & 300012 & 6530-03 \\ 55 & 0005.54 & 300011 & 6530-02 \\ 40 & 0013.65 & 363112 & 6530-02 \\ 80 & 0007.94 & 200012 & 6530-02 \\ 80 & 0000.90 & 303012 & 6530-02 \\ & 0069.38 & 365112 & 6530-02 \\ 150 & 0000.26 & 700010 & 6600-03 \\ 10 & 0002.92 & 363512 & 6600-03 \\ 140 & 0002.00 & 360412 & 6530-02 \\ 90 & 0000.74 & 700010 & 6530-02 \\ & 001.35 & 643212 & 6530-02 \\ 100 & 0001.44 & 365412 & 6530-02 \\ 55 & 0000.84 & 633212 & 6530-02 \\ 25 & 0000.43 & 700010 & 6530-02 \\ 50 & 0000.40 & 700010 & 6530-02 \\ 100 & 0000.11 & 7800010 & 6530-02 \\ 100 & 0000.09 & 750010 & 6530-02 \\ 140 & 0000.18 & 7530-02 \\ 15 & 0018.81 & 313111 & 6530-02 \\ 60 & 0004.51 & 643112 & 6530-02 \\ 200 & 0000.08 & 700010 & 6530-02 \\ 150 & 0000.16 & 780010 & 6530-02 \\ 20 & 0018.36 & 363412 & 6530-02 \\ 50 & 0000.90 & 203312 & 6530-02 \\ & & & \end{array}$




\begin{tabular}{|c|c|c|c|c|c|c|c|c|c|c|c|c|c|c|c|c|c|}
\hline CODE & LAT & LONG & ORI & HIGH & LOW & AREA & $\begin{array}{l}\text { MO } \\
\text { CODE }\end{array}$ & MAP & CODE & LAT & LONG & ORI & HIGH & LOW & AREA & $\begin{array}{c}\text { MO } \\
\text { CODE }\end{array}$ & MAP \\
\hline F26001 & $65^{\circ} 51^{\prime} \mathrm{N}$ & $52^{\circ} 13^{\prime} \mathrm{V}$ & $\mathrm{s}$ & 120 & 110 & 0000.22 & 700010 & $6530-02$ & 1DG01019 & $66^{\circ} 24^{\prime} \mathrm{N}$ & $52^{\circ} 36^{\prime} \mathrm{W}$ & - & 140 & 100 & 0000.99 & 700010 & $6600-02$ \\
\hline DF26002 & $65^{\circ} 50^{\prime} \mathrm{N}$ & $52^{\circ} 12 \cdot \mathrm{W}$ & $\mathrm{W}$ & 170 & & 0005.77 & 535312 & $6530-02$ & $1 D G 01020$ & $66^{\circ} 24^{\prime} \mathrm{N}$ & $52 \circ 37 \cdot \mathrm{W}$ & $\bar{s}$ & $\begin{array}{l}140 \\
140\end{array}$ & 140 & 0000.13 & 700010 & $6600-02$ \\
\hline 1DF26003 & $65^{\circ} 50^{\prime} \mathrm{N}$ & $52^{\circ} 12^{\prime \prime}$ & $\tilde{\mathrm{v}}$ & 160 & 140 & 0000.30 & 203212 & $6530-02$ & 1DG01021 & $66^{\circ} 25^{\prime} \mathrm{N}$ & $52 \circ 37 \cdot \mathrm{W}$ & s & 150 & 140 & 0000.34 & 700010 & $6600-02$ \\
\hline 1DF27001 & $65^{\circ} 50^{\prime} \mathrm{N}$ & $52^{\circ} 15^{\prime} \mathrm{V}$ & SW & 150 & 135 & 0000.08 & 700010 & $6530-02$ & $1 \mathrm{DG} 01022$ & $66^{\circ} 25^{\prime} \mathrm{N}$ & $52 \cdot 35 \cdot \mathrm{V}$ & $\mathrm{E}$ & 150 & 125 & 0002.95 & 780010 & $6600-02$ \\
\hline 1DF27002 & $65^{\circ} 49^{\prime} \mathrm{N}$ & $52^{\circ} 13^{\prime} \mathrm{V}$ & $\mathrm{SW}$ & 160 & 135 & 0001.74 & 640112 & $6530-02$ & 1DG01023 & $66^{\circ} 26^{\prime} \mathrm{N}$ & $52055 \cdot \mathrm{v}$ & SE & 135 & 130 & 0002.95 & 750010 & $6600-02$ \\
\hline 1DF27003 & $65^{\circ} 49^{\prime} \mathrm{N}$ & $52^{\circ} 12^{\prime} \mathrm{W}$ & W & 135 & 50 & 0005.72 & 363312 & $6530-02$ & 1DG02001 & $67^{\circ} 10^{\prime} \mathrm{N}$ & $49^{\circ} 60^{\prime} \mathrm{v}$ & $\mathrm{W}$ & 180 & 50 & 0167.34 & 160112 & $6700-05$ \\
\hline 1DF27004 & $65^{\circ} 47, \mathrm{~N}$ & $52^{\circ} 14^{\prime} \mathrm{W}$ & $\mathrm{NW}$ & 135 & 10 & 0018.24 & 363312 & $6530-02$ & 1DG02002 & $67^{\circ} 07^{\circ} \mathrm{N}$ & $50^{\circ} 04^{\prime} \mathrm{W}$ & $\mathrm{W}$ & 180 & 25 & 0701.72 & 163112 & $6700-05$ \\
\hline 1DF27005 & $65^{\circ} 48^{\prime} \mathrm{N}$ & $52^{\circ} 17 \cdot 6$ & $\mathrm{~N}$ & 100 & 50 & 0001.46 & 643212 & $6530-02$ & 1DG03001 & $67^{\circ} 05^{\prime} \mathrm{N}$ & $50^{\circ} 07, \mathrm{v}$ & W & 180 & 25 & 0175.45 & 163112 & $6700-05$ \\
\hline 1DF27006 & $65^{\circ} 48^{\prime} \mathrm{N}$ & $52^{\circ} 18^{\prime} \mathrm{W}$ & $\mathrm{N}$ & 135 & 60 & 0002.12 & 643112 & $6530-02$ & $1 \mathrm{DG} 03002$ & $67^{\circ} 05^{\circ} \mathrm{N}$ & $50^{\circ} 00^{\circ} \mathrm{v}$ & W & 180 & 30 & 0161.04 & 163112 & $6700-05$ \\
\hline 1DF27007 & $65^{\circ} 49^{\prime} \mathrm{N}$ & $52^{\circ} 19^{\prime} \mathrm{W}$ & NE & 130 & 30 & 0001.14 & 363312 & $6530-02$ & $1 D G 03003$ & $67^{\circ} 04^{\prime} \mathrm{N}$ & $49^{\circ} 59^{\prime} \mathrm{V}$ & $\mathrm{W}$ & 180 & 55 & 0162.94 & 166112 & $6700-05$ \\
\hline 1DF28001 & $65^{\circ} 49 \cdot \mathrm{N}$ & $52^{\circ} 22^{\prime} \mathrm{W}$ & $\mathrm{NE}$ & 125 & & 0007.63 & 513312 & $6530-02$ & $1 \mathrm{DG} 03004$ & $67^{\circ} 01$, N & $49^{\circ} 54, \mathrm{~W}$ & $\hat{w}$ & 180 & 15 & 0379.80 & 163112 & $6700-05$ \\
\hline 1DF28002 & $65^{\circ} 50^{\prime} \mathrm{N}$ & $52^{\circ} 22^{\prime} \mathrm{W}$ & $\mathrm{s}$ & 90 & 80 & 0000.15 & 780010 & $6530-02$ & 1DG03005 & $67^{\circ} 00^{\prime} \mathrm{N}$ & $49^{\circ} 48^{\prime} \mathrm{W}$ & $\mathrm{w}$ & 180 & 20 & 0382.86 & 163112 & $6700-05$ \\
\hline 1DF29001 & $65^{\circ} 51^{\prime} \mathrm{N}$ & $52^{\circ} 21^{\prime} \mathrm{W}$ & $\mathrm{NE}$ & 130 & & 0004.64 & 513312 & $6530-02$ & 1DG03006 & $66^{\circ} 58^{\prime} \mathrm{N}$ & $49^{\circ} 44^{\prime} \mathrm{W}$ & V & 180 & 20 & 0306.21 & 163113 & $6630-05$ \\
\hline 1DF 29002 & $65^{\circ} 51 \cdot \mathrm{N}$ & $52^{\circ} 23^{\prime} \mathrm{V}$ & $\mathrm{NE}$ & 145 & 10 & 0003.35 & 533412 & $6530-02$ & $10 G 03007$ & $66^{\circ} 56^{\prime} \mathrm{N}$ & $49^{\circ} 50^{\circ} \mathrm{W}$ & NW & 180 & 15 & 1122.26 & 163113 & $6630-05$ \\
\hline IDF29003 & $65^{\circ} 52^{\prime} \mathrm{N}$ & $52^{\circ} 24^{\prime} \mathrm{W}$ & NE & 145 & & 0000.54 & 533412 & $6530-02$ & 1DG08001 & $66^{\circ} 53^{\prime} \mathrm{N}$ & $49^{\circ} 48^{\circ} \mathrm{W}$ & $\mathrm{W}$ & 180 & 20 & 0641.18 & 166113 & $6630-05$ \\
\hline IDF29004 & $65^{\circ} 52^{\prime} \mathrm{N}$ & $52^{\circ} 25^{\prime} \mathrm{V}$ & $\mathrm{N}$ & & & 0000.32 & 643212 & $6530-02$ & 1DG08002 & $66^{\circ} 49 \cdot \mathrm{N}$ & $49^{\circ} 40^{\circ} \mathrm{W}$ & V & 180 & 40 & 0287.76 & 163113 & $6630-05$ \\
\hline 1DF30001 & $65^{\circ} 49 \cdot \mathrm{N}$ & $52^{\circ} 28^{\prime} \mathrm{W}$ & N & 110 & & 0019.66 & 513312 & $6530-02$ & IDG08003 & $66^{\circ} 471 \mathrm{~N}$ & $49^{\circ} 40^{\prime} \mathrm{W}$ & $\hat{\mathrm{V}}$ & 180 & 30 & 0300.34 & 163113 & $6630-05$ \\
\hline 1DF31001 & $65^{\circ} 52, \mathrm{~N}$ & $52^{\circ} 30^{\prime} \mathrm{V}$ & $\mathbf{N}$ & 120 & 70 & 0000.45 & 700010 & $6530-02$ & 1DG08004 & $66^{\circ} 45, \mathrm{~N}$ & $49^{\circ} 45^{\prime} \mathrm{W}$ & V & 180 & 20 & 0199.98 & 163113 & $6630-05$ \\
\hline $1 \mathrm{DF} 31002$ & $65^{\circ} 51 \cdot \mathrm{N}$ & $52^{\circ} 32^{\prime} \mathrm{W}$ & NE & 160 & & 0005.36 & 645312 & $6530-02$ & 1DG08005 & $66^{\circ} 44^{\prime} \mathrm{N}$ & $49^{\circ} 47^{\prime} \mathrm{W}$ & W & 180 & 20 & 0354.46 & 163114 & $6630-05$ \\
\hline 1DF 32001 & $65^{\circ} 53^{\prime} \mathrm{N}$ & $52^{\circ} 33^{\prime} \mathrm{W}$ & NE & 100 & 20 & 0000.59 & 643412 & $6530-02$ & 1DG08006 & $66^{\circ} 41 \cdot \mathrm{N}$ & $49^{\circ} 32^{\prime} \mathrm{w}$ & W & 180 & 20 & 0465.46 & 163114 & $6630-05$ \\
\hline 1DF 32002 & $65^{\circ} 52 \cdot \mathrm{N}$ & $52^{\circ} 37^{\prime} \mathrm{W}$ & NE & & 15 & 0004.84 & 513112 & $6530-02$ & 1DG08007 & $66^{\circ} 39^{\prime} \mathrm{N}$ & $49^{\circ} 34^{\prime} \mathrm{V}$ & W & 180 & 45 & 0528.80 & 166114 & $6630-05$ \\
\hline 1DF32003 & $65^{\circ} 53^{\prime} \mathrm{N}$ & $52^{\circ} 38^{\prime} \mathrm{W}$ & SE & 115 & 50 & 0000.82 & 643112 & $6530-01$ & 1DG08008 & $66^{\circ} 37 \cdot N$ & $49^{\circ} 32^{\prime} \mathrm{W}$ & W & 180 & 45 & 0207.38 & 166113 & $6630-05$ \\
\hline 1DF32004 & $65^{\circ} 54^{\prime} \mathrm{N}$ & $52^{\circ} 38^{\prime} \mathrm{W}$ & SE & & 55 & 0000.44 & 643312 & $6530-01$ & 1DG08009 & $66^{\circ} 35^{\prime} \mathrm{N}$ & $49^{\circ} 30^{\prime} \mathrm{W}$ & W & $\begin{array}{l}180 \\
180\end{array}$ & 48 & 0851.36 & $\begin{array}{l}166113 \\
163113\end{array}$ & $6630-05$ \\
\hline 1DF32005 & $65^{\circ} 55^{\prime} \mathrm{N}$ & $52^{\circ} 39^{\prime} \mathrm{W}$ & $\mathrm{NE}$ & 140 & 20 & 0000.92 & 643312 & $6530-01$ & 1DG08010 & $66^{\circ} 31$ ! N & $49^{\circ} 32^{\prime} \mathrm{W}$ & W & 180 & 50 & 0776.82 & 163113 & $6630-05$ \\
\hline 1DF32006 & $65^{\circ} 54 \cdot \mathrm{N}$ & $52^{\circ} 41, \xi$ & $\mathrm{N}$ & 90 & 35 & 0003.22 & 533211 & $6530-01$ & $1 D G 08011$ & $66^{\circ} 25^{\prime} \mathrm{N}$ & $49^{\circ} 30^{\prime} \mathrm{W}$ & NW & 180 & 80 & 0299.58 & 266113 & $6600-05$ \\
\hline 1DF32007 & $65^{\circ} 54^{\prime} \mathrm{N}$ & $52^{\circ} 43^{\prime} \mathrm{V}$ & NW & 90 & 30 & 0001.23 & 643212 & $6530-01$ & 1DG12001 & $66^{\circ} 29 \cdot \mathrm{N}$ & $49^{\circ} 46^{\prime} \mathrm{W}$ & $\mathrm{N}$ & 95 & 90 & 0000.14 & 780010 & $6600-05$ \\
\hline 1DF 33001 & $65^{\circ} 53^{\prime} \mathrm{N}$ & $52^{\circ} 43^{\prime} \mathrm{V}$ & v & 100 & 50 & 0000.37 & 640112 & 6530-01 & 1DG12002 & $66^{\circ} 28^{\circ} \mathrm{N}$ & $49^{\circ} 45 \cdot \mathrm{W}$ & W & 105 & 100 & 0000.10 & 780010 & $6600-05$ \\
\hline 1DF33002 & $65^{\circ} 53^{\prime} \mathrm{N}$ & $52^{\circ} 40^{\prime} \mathrm{W}$ & sw & 95 & 55 & 0000.82 & 640112 & $6530-01$ & 1DG12003 & $66^{\circ} 28^{\prime} \mathrm{N}$ & $49^{\circ} 49 \cdot \mathrm{V}$ & $\mathrm{N}$ & 110 & 100 & 0000.11 & 750010 & $6600-05$ \\
\hline $1 D F 33003$ & $65^{\circ} 50^{\prime} \mathrm{N}$ & $52^{\circ} 44^{\prime} \mathrm{W}$ & $\mathrm{N}$ & 115 & 50 & 0000.84 & 200110 & $6530-01$ & 1DG12004 & $66^{\circ} 28^{\prime} \mathrm{N}$ & $49^{\circ} 50^{\prime} \mathrm{W}$ & - & 110 & 100 & 0000.19 & 780010 & $6600-05$ \\
\hline 1DF33004 & $65^{\circ} 50^{\prime} \mathrm{N}$ & $52^{\circ} 45, \mathrm{~V}$ & Nw & 115 & 40 & 0001.42 & 643312 & $6530-01$ & 1DG12005 & $66^{\circ} 25^{\prime} \mathrm{N}$ & $49^{\circ} 44^{\prime \prime} \mathrm{W}$ & N & 100 & 100 & 0000.16 & 780010 & $6600-05$ \\
\hline 1DF33005 & $65^{\circ} 50^{\prime} \mathrm{N}$ & $52^{\circ} 46^{\prime} \mathrm{V}$ & NW & 105 & 75 & 0000.33 & 750010 & $6530-01$ & 1DG12006 & $66^{\circ} 26^{\prime} \mathrm{N}$ & $49^{\circ} 50^{\prime} \mathrm{W}$ & $\mathrm{N}$ & 115 & 105 & 0000.34 & 780010 & $6600-05$ \\
\hline 1DF33006 & $65^{\circ} 50^{\prime} \mathrm{N}$ & $52^{\circ} 47^{\prime}$ & N & & 50 & 0000.40 & 643112 & $6530-01$ & 1DG12007 & $66^{\circ} 26^{\prime} \mathrm{N}$ & $49 \cdot 53 \cdot \mathrm{W}$ & s & 110 & 100 & 0000.52 & 780010 & $6600-05$ \\
\hline 1DF33007 & $65^{\circ} 51^{\prime} \mathrm{N}$ & $52^{\circ} 48^{\prime} \mathrm{W}$ & N & 55 & 40 & 0000.12 & 780010 & 6530 & 1DG12008 & $66^{\circ} 27 \cdot \mathrm{N}$ & $49 \circ 55, v$ & N & 115 & 110 & 0000.06 & 700010 & $6600-05$ \\
\hline 1DF33008 & $65^{\circ} 51^{\prime} \mathrm{N}$ & $52^{\circ} 50^{\prime \prime} \mathrm{W}$ & NW & 80 & 45 & 0000.19 & 750010 & $6530-01$ & 1DG12009 & $66^{\circ} 26^{\prime} \mathrm{N}$ & $49^{\circ} 50^{\prime} \mathrm{v}$ & s & 120 & 110 & 0000.50 & 780010 & $6600-05$ \\
\hline 1DF34001 & $65^{\circ} 49^{\prime} \mathrm{N}$ & $52^{\circ} 57 \cdot v$ & NW & 80 & 50 & 0000.13 & 750010 & $6530-01$ & 1DG12010 & $66^{\circ} 23^{\prime} \mathrm{N}$ & $49^{\circ} 30^{\prime} \mathrm{W}$ & $\mathrm{NW}$ & 180 & 83 & 0656.07 & 166112 & $6600-05$ \\
\hline 1DF34002 & $65^{\circ} 44^{\prime} \mathrm{N}$ & $52 \circ 57 \cdot \mathrm{W}$ & $\mathrm{W}$ & & & 0000.12 & 680010 & $6530-01$ & 1DG12011 & $66^{\circ} 22^{\prime} \mathrm{N}$ & $49.038, \mathrm{~W}$ & N & 100 & 100 & 0000.11 & 750010 & $6600-05$ \\
\hline 1DF34003 & $65^{\circ} 48^{\prime} \mathrm{N}$ & $52^{\circ} 58^{\prime}$, & $\mathrm{NW}$ & 75 & 55 & 0000.09 & 780010 & $6530-01$ & 1DG12012 & $66^{\circ} 23^{\prime} \mathrm{N}$ & $49^{\circ} 44^{\prime} \mathrm{k}$ & $\mathrm{NE}$ & 100 & 100 & 0000.10 & 700010 & $6600-05$ \\
\hline 1DF34004 & $65^{\circ} 48^{\prime} \mathrm{N}$ & $52^{\circ} 59, \bar{w}$ & $\mathrm{~N}$ & 65 & 45 & 0000.15 & 780010 & 6530 & 1DG12013 & $66^{\circ} 23^{\prime} \mathrm{N}$ & $49^{\circ} 45^{\prime \prime} \mathrm{V}$ & N & 100 & 100 & & 780010 & 05 \\
\hline 1DF34005 & $65^{\circ} 48^{\prime} \mathrm{N}$ & $53^{\circ} 00^{\prime} \mathrm{W}$ & NW & & 40 & 0000.15 & 640112 & $6530-01$ & 1DG12014 & $66^{\circ} 20^{\prime} \mathrm{N}$ & $49^{\circ} 36^{\prime} \mathrm{v}$ & NW & 180 & 85 & 0261.51 & 1600112 & $6600-05$ \\
\hline 1DF34006 & $65^{\circ} 47^{\prime} \mathrm{N}$ & $53^{\circ} 02^{\prime}$ W & N & 88 & 45 & 0000.24 & 640210 & $6530-01$ & 1DG12015 & $66^{\circ} 19^{\prime} \mathrm{N}$ & $49^{\circ} 38^{\prime} \mathrm{W}$ & NW & 180 & 81 & 0277.37 & 166113 & $6600-05$ \\
\hline 1DF34007 & $65^{\circ} 47^{\prime} \mathrm{N}$ & $53^{\circ} 02^{\prime} \mathrm{W}$ & $\mathrm{N}$ & 60 & 40 & 0000.24 & 790112 & $6530-01$ & 1DG12016 & $66^{\circ} 30^{\prime} \mathrm{N}$ & $49^{\circ} 50^{\prime} \mathrm{W}$ & B & 100 & 95 & 0000.16 & 780010 & $6600-05$ \\
\hline 1DF34008 & $65^{\circ} 46^{\prime} \mathrm{N}$ & $53^{\circ} 06^{\prime}$ & $\mathrm{N}$ & 70 & 55 & 0000.15 & 630110 & $6530-01$ & 1DG12017 & $66^{\circ} 21^{\prime} \mathrm{N}$ & $49^{\circ} 45^{\circ} \mathrm{W}$ & $\mathrm{NE}$ & 105 & 100 & 00 & 780010 & -05 \\
\hline 1DF34009 & $65^{\circ} 46^{\prime} \mathrm{N}$ & $53^{\circ} 07, \mathrm{~W}$ & $\mathrm{~N}$ & 75 & 65 & 0000.11 & 770012 & $6530-01$ & 1DG12018 & $66^{\circ} 21^{\prime} \mathrm{N}$ & $49^{\circ} 49^{\prime} \mathrm{W}$ & $\mathrm{NE}$ & 115 & 100 & 00 & 10 & (5) \\
\hline 1DG01001 & $66^{\circ} 03^{\prime} \mathrm{N}$ & $53^{\circ} 35, k$ & W & 70 & 55 & 0000.08 & 750010 & $6600-01$ & 1DG12019 & $66^{\circ} 222^{\prime} \mathrm{N}$ & $49^{\circ} 47, \mathrm{~V}$ & $\mathrm{NE}$ & 95 & 90 & 0000.08 & 700010 & $6000-05$ \\
\hline 1DG01002 & $66^{\circ} 12^{\prime} \mathrm{N}$ & $53^{\circ} 07^{\prime} \mathrm{W}$ & $\mathrm{E}$ & 95 & 80 & 0000.31 & 780010 & 6600 & 1DG12020 & $66^{\circ} 23 \cdot \mathrm{N}$ & $49^{\circ} 51 \cdot \mathrm{W}$ & $\mathrm{NE}$ & 110 & 100 & 0000.50 & 780010 & $6600-05$ \\
\hline 1DG01003 & $66^{\circ} 12^{\prime} \mathrm{N}$ & $53^{\circ} 06^{\prime}$ & SW & 100 & 90 & 0001.54 & 770010 & $6600-01$ & 1DG12021 & $66^{\circ} 23, \mathrm{~N}$ & 49053,6 & $\mathrm{~N}$ & 100 & 90 & 0000.33 & 780010 & 660 \\
\hline 1DG01004 & $66^{\circ} 11^{\prime} \mathrm{N}$ & $53^{\circ} 04^{\prime \prime} \mathrm{v}$ & $\mathrm{s}$ & 100 & 100 & 0000.44 & 770010 & $6600-01$ & 1DG12022 & $66^{\circ} 233^{\prime} \mathrm{N}$ & $49^{\circ} 56^{\prime} \mathrm{v}$ & NW & 120 & 100 & 0000.22 & 780010 & 66 \\
\hline 1DG01005 & $66^{\circ} 12^{\prime}, \mathrm{N}$ & $53^{\circ} 02^{\prime} \mathrm{W}$ & $\mathrm{SE}$ & 110 & 115 & 0000.43 & 700010 & $6600-01$ & 1DG12023 & $66^{\circ} 22^{\prime} \mathrm{N}$ & $49 \circ 58 \cdot \mathrm{W}$ & NW & $\begin{array}{l}100 \\
105\end{array}$ & 105 & 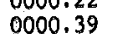 & 78 & $\begin{array}{l}-05 \\
-05\end{array}$ \\
\hline 1DG01006 & $66^{\circ} 13^{\prime} \mathrm{N}$ & $53^{\circ} 00^{\prime} \mathrm{W}$ & $\mathrm{SE}$ & 120 & & 0000.69 & 700010 & $6600-01$ & 1DG12024 & $66^{\circ} 24 . \mathrm{N}$ & $50^{\circ} 03, \mathrm{~W}$ & $\mathrm{E}$ & 105 & 105 & 0000.21 & 750010 & $6600-05$ \\
\hline 1DG01007 & $66^{\circ} 14^{\prime} \mathrm{N}$ & $52^{\circ} 58^{\prime} \mathrm{W}$ & $\mathrm{SE}$ & 140 & 100 & 0002.01 & 700110 & $6600-01$ & $1 D G 12025$ & $66^{\circ} 25^{\prime} \mathrm{N}$ & $49^{\circ} 55, \mathrm{~W}$ & N & 100 & 100 & 0000.06 & 700010 & $6600-05$ \\
\hline 1DG01008 & $66^{\circ} 15^{\prime} \mathrm{N}$ & $52^{\circ} 55^{\prime} \mathrm{W}$ & s & 130 & 100 & 0001.23 & 700110 & $6600=02$ & DG12026 & $66^{\circ} 251 \mathrm{~N}$ & $49^{\circ} 58, \mathrm{~W}$ & $\mathrm{~N}$ & 120 & 90 & 0000,75 & 700010 & $6600-05$ \\
\hline 1DG01009 & $66^{\circ} 15^{\prime N}$ & $52^{\circ} 55^{\prime} \mathrm{W}$ & $E$ & 115 & 90 & 0000.29 & 750010 & $6600-02$ & 1DG12027 & $66^{\circ} 26^{\prime} \mathrm{N}$ & $50^{\circ} 01, \mathrm{~W}$ & N & 120 & 120 & 0000.19 & 700010 & $6600-05$ \\
\hline 1DG01010 & $66^{\circ} 20^{\prime} \mathrm{N}$ & $52^{\circ} 50^{\prime} \mathrm{w}$ & $\mathrm{NE}$ & 130 & 110 & 0000.24 & 700010 & $6600-02$ & 1DG12028 & $\begin{array}{l}806^{\circ} 26^{\prime} \mathrm{N} \\
\end{array}$ & $50^{\circ} 00^{\prime} \mathrm{V}$ & N & 120 & $\begin{array}{l}120 \\
120\end{array}$ & 0000 & 700010 & $\begin{array}{l}60000-05 \\
6600-05\end{array}$ \\
\hline 1DG01011 & $66^{\circ} 20^{\prime} \mathrm{N}$ & $52^{\circ} 50^{\prime} \mathrm{W}$ & $\mathrm{E}$ & 105 & 95 & 0000.12 & 700010 & $6600-02$ & $10 G 16001$ & $66^{\circ} 26^{\prime} \mathrm{N} N$ & $50^{\circ} 02, \mathrm{v}$ & $\mathrm{N}$ & 115 & 1120 & 0000.12 & 700010 & $6600-05$ \\
\hline 1DG01012 & $66^{\circ} 20^{\prime} \mathrm{N}$ & $52^{\circ} 48 \cdot \mathrm{W}$ & 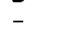 & 130 & 120 & 0000.31 & 700010 & $6600-02$ & $1 D G 16002$ & $668251 \mathrm{~N}$ & $50^{\circ} 00^{\prime} \mathrm{V}$ & $\mathrm{N}$ & 120 & 115 & 000011 & 700010 & $6600-05$ \\
\hline 1DG01013 & $66^{\circ} 21 \cdot \mathrm{N}$ & $52^{\circ} 46^{\prime} \mathrm{V}$ & $\mathrm{SE}$ & 120 & 100 & 0000.24 & 750010 & $6600-02$ & 1DG16003 & $66^{\circ} 25, \mathrm{~N}$ & $50^{\circ} 01{ }^{\prime} \mathrm{W}$ & N & 110 & 110 & 0000.22 & 700010 & $6600-05$ \\
\hline 1DG01014 & $66^{\circ} 21 \cdot \mathrm{N}$ & $52^{\circ} 47 \cdot \mathrm{W}$ & $\mathbf{E}$ & 130 & 100 & 0001.58 & 700010 & $6600=02$ & IDG16004 & $66025, \mathrm{~N}$ & $50^{\circ} 03, \mathrm{~W}$ & N & 110 & 110 & 0000.16 & 700010 & $6600-05$ \\
\hline 1DG01015 & $66^{\circ} 22, \mathrm{~N}$ & $52^{\circ} 46^{\prime} \mathrm{W}$ & s & 150 & 130 & 0000.46 & 700010 & $6600-02$ & 1DG16005 & $66^{\circ} 26, \mathrm{~N}$ & $50^{\circ} 04$ ? & $N^{n}$ & 115 & 1105 & 0000.17 & 780010 & $\begin{array}{l}6600-05 \\
6600-05\end{array}$ \\
\hline 1DG01016 & $66^{\circ} 22^{\prime} \mathrm{N}$ & $52^{\circ} 45^{\prime} \mathrm{W}$ & SW & 150 & 125 & 0001.92 & 750010 & 6600 & IDG16006 & $66^{\circ} 25^{\prime N}$ & $50^{\circ} 12 \cdot \mathrm{W}$ & E & 115 & 115 & 0000.08 & 770010 & $6600-04$ \\
\hline 1DG01017 & $66^{\circ} 23^{\prime} \mathrm{N}$ & $52^{\circ} 43^{\prime} \mathrm{W}$ & $\mathrm{s}$ & 150 & 120 & 0000.54 & 700010 & $6600-02$ & 1DG16007 & $66^{\circ} 26^{\prime} \mathrm{N}$ & $50^{\circ} 12^{\prime} \mathrm{W}$ & E & 115 & 110 & 0000.07 & 770010 & $6600-04$ \\
\hline 1DG01018 & $66^{\circ} 24^{\prime} \mathrm{N}$ & $52^{\circ} 40^{\prime} \mathrm{w}$ & - & 145 & 130 & 0000.88 & 780010 & $6600-02$ & 1DG16008 & $66^{\circ} 26^{\prime} \mathrm{N}$ & $50^{\circ} 15^{\prime} \mathrm{v}$ & N & 120 & 120 & 0000.35 & 770010 & $6600-04$ \\
\hline
\end{tabular}




\begin{tabular}{|c|c|c|c|}
\hline CODE & LAT & LONG & ORI \\
\hline 1DG16009 & $66^{\circ} 27^{\prime} \mathrm{N}$ & $50^{\circ} 15^{\prime} \mathrm{W}$ & $\mathrm{NE}$ \\
\hline 1DG16010 & $66^{\circ} 27^{\prime} \mathrm{N}$ & $50^{\circ} 18^{\prime \mathrm{W}}$ & $\mathrm{N}$ \\
\hline 1DG16011 & $66^{\circ} 27^{\prime} \mathrm{N}$ & $50^{\circ} 19^{\prime} \mathrm{W}$ & W \\
\hline IDG16012 & $66^{\circ} 27^{\circ} \mathrm{N}$ & $50^{\circ} 19^{\prime} \mathrm{W}$ & $\hat{\mathrm{w}}$ \\
\hline 1DG16013 & $66^{\circ} 25^{\prime} \mathrm{N}$ & $50^{\circ} 15^{\prime} \mathrm{W}$ & W \\
\hline 1DG16014 & $66^{\circ} 24^{\prime} \mathrm{N}$ & $50^{\circ} 12^{\prime} \mathrm{v}$ & $\mathrm{v}$ \\
\hline 1DG16015 & $66^{\circ} 23^{\prime} \mathrm{N}$ & $50^{\circ} 13^{\prime} \mathrm{v}$ & \\
\hline IDG16016 & $66^{\circ} 22^{\prime} \mathrm{N}$ & $50^{\circ} 20^{\prime} \mathrm{W}$ & $\mathrm{NE}$ \\
\hline 1DG16017 & $66^{\circ} 22, \mathrm{~N}$ & $50^{\circ} 25^{\prime} \mathrm{w}$ & $\mathrm{N}$ \\
\hline 1DG16018 & $66^{\circ} 23^{\prime} \mathrm{N}$ & $50^{\circ} 38^{\prime} \mathrm{W}$ & N \\
\hline 1DG16019 & $66^{\circ} 23^{\prime} \mathrm{N}$ & $50^{\circ} 40^{\prime} \mathrm{W}$ & NE \\
\hline 1DG16020 & $66^{\circ} 21^{\prime} \mathrm{N}$ & $50^{\circ} 41^{\prime} \mathrm{W}$ & N \\
\hline 1DG16021 & $66^{\circ} 21, \mathrm{~N}$ & $50^{\circ} 46^{\prime} \mathrm{W}$ & $\mathrm{N}$ \\
\hline 1DG16022 & $66^{\circ} 20^{\circ} \mathrm{N}$ & $50^{\circ} 44 \cdot \mathrm{W}$ & $\mathrm{NE}$ \\
\hline $1 D G 16023$ & $66^{\circ} 19^{\prime} \mathrm{N}$ & $50^{\circ} 45^{\prime} \mathrm{W}$ & $\mathrm{N}$ \\
\hline 1DG16024 & $66^{\circ} 20^{\prime} \mathrm{N}$ & $50^{\circ} 51^{\prime} \mathrm{W}$ & $\mathrm{N}$ \\
\hline IDG16025 & $66^{\circ} 19^{\prime} \mathrm{N}$ & $50^{\circ} 50^{\prime} \mathrm{w}$ & NE \\
\hline IDG16026 & $66^{\circ} 20^{\prime} \mathrm{N}$ & $50^{\circ} 54^{\prime}$ & $\mathrm{N}$ \\
\hline 1DG16027 & $66^{\circ} 22, \mathrm{~N}$ & $50^{\circ} 58^{\prime} \mathrm{W}$ & N \\
\hline 1DG16028 & $66^{\circ} 24^{\prime} \mathrm{N}$ & $51^{\circ} 06^{\prime} \mathrm{W}$ & $\mathrm{NW}$ \\
\hline 1DG16029 & $66^{\circ} 22^{\prime} \mathrm{N}$ & $51^{\circ} 06^{\prime} \mathrm{W}$ & $\mathrm{N}$ \\
\hline $1 D G 16030$ & $66^{\circ} 21, \mathrm{~N}$ & $51^{\circ} 04^{\prime} \mathrm{W}$ & \\
\hline 1DG16031 & $66^{\circ} 19, \mathrm{~N}$ & $50^{\circ} 58^{\prime} \mathrm{W}$ & N \\
\hline 1DG16032 & $66^{\circ} 18^{\prime} \mathrm{N}$ & $50^{\circ} 55^{\prime} \mathrm{W}$ & N \\
\hline $1 D G 16033$ & $66^{\circ} 18^{\prime} \mathrm{N}$ & $51^{\circ} 05 \cdot \mathrm{W}$ & $\mathrm{N}$ \\
\hline 1DG16034 & $66^{\circ} 19^{\prime} \mathrm{N}$ & $51^{\circ} 10^{\prime} \mathrm{V}$ & NE \\
\hline 1DG16035 & $66^{\circ} 17^{\prime} \mathrm{N}$ & $51^{\circ} 10^{\prime} \mathrm{W}$ & $\mathrm{NE}$ \\
\hline IDG16036 & $66^{\circ} 19^{\prime} \mathrm{N}$ & $51^{\circ} 13^{\prime} \mathrm{y}$ & $\mathrm{NE}$ \\
\hline 1DG16037 & $66^{\circ} 18^{\prime} \mathrm{N}$ & $51 \circ 15^{\prime} \mathrm{W}$ & $\mathrm{N}$ \\
\hline 1DG16038 & $66^{\circ} 20^{\circ} \mathrm{N}$ & $51^{\circ} 16^{\prime} \mathrm{W}$ & N \\
\hline 1DG16039 & $66^{\circ} 15^{\prime} \mathrm{N}$ & $50^{\circ} 57^{\prime} \mathrm{W}$ & $\mathbf{N}$ \\
\hline 1DG16040 & $66^{\circ} 15^{\prime} \mathrm{N}$ & $50^{\circ} 55^{\prime} \mathrm{W}$ & $\mathrm{s}$ \\
\hline IDG16041 & $66^{\circ} 15^{\prime} \mathrm{N}$ & $50^{\circ} 53^{\prime} \mathrm{W}$ & N \\
\hline 1DG16042 & $66^{\circ} 14^{\prime} \mathrm{N}$ & $50^{\circ} 47 \cdot \mathrm{W}$ & N \\
\hline 1DG16043 & $66^{\circ} 15^{\prime N}$ & $50^{\circ} 49 \cdot \mathrm{W}$ & $\mathrm{SE}$ \\
\hline IDG16044 & $66^{\circ} 15^{\prime} \mathrm{N}$ & $50^{\circ} 44^{\prime} \mathrm{V}$ & $\mathrm{N}$ \\
\hline 1DG16045 & $66^{\circ} 15^{\prime} \mathrm{N}$ & $50^{\circ} 42, \mathrm{~W}$ & $\mathrm{~N}$ \\
\hline 1DG16046 & $66^{\circ} 15^{\prime} \mathrm{N}$ & $50^{\circ} 35, \mathrm{~W}$ & $\mathrm{NE}$ \\
\hline 1DG16047 & $66^{\circ} 15^{\prime N}$ & $50^{\circ} 36^{\prime} \mathrm{W}$ & $\mathrm{NE}$ \\
\hline 1DG16048 & $66^{\circ} 14^{\prime} \mathrm{N}$ & $50^{\circ} 05^{\circ} \mathrm{w}$ & N \\
\hline 1DG16049 & $66^{\circ} 14^{\prime} \mathrm{N}$ & $50^{\circ} 37^{\prime} \mathrm{V}$ & N \\
\hline & $66^{\circ} 14 \cdot \mathrm{N}$ & $50^{\circ} 33^{\prime} \mathrm{W}$ & $\mathrm{N}$ \\
\hline 1DG16051 & $66^{\circ} 16^{\prime N}$ & $50^{\circ} 26^{\prime} \mathrm{W}$ & $\mathrm{NE}$ \\
\hline 1DG16052 & $66^{\circ} 16^{\prime} \mathrm{N}$ & $50 \circ 24^{\prime} \mathrm{W}$ & $\mathrm{NE}$ \\
\hline 1DG16053 & $66^{\circ} 14^{\prime} \mathrm{N}$ & $50^{\circ} 15^{\prime} \mathrm{V}$ & $\mathrm{sw}$ \\
\hline 1DG16054 & $66^{\circ} 14^{\prime} \mathrm{N}$ & $50^{\circ} 14^{\prime} \mathrm{W}$ & $\mathrm{SE}$ \\
\hline 1DG16055 & $66^{\circ} 14^{\prime} \mathrm{N}$ & $50^{\circ} 09^{\prime} \mathrm{W}$ & $\mathrm{N}$ \\
\hline 1DG16056 & $66^{\circ} 15^{\prime}, \mathrm{N}$ & $50^{\circ} 06^{\prime}$, w & N \\
\hline 1DG16057 & $66^{\circ} 15^{\prime N} \mathrm{~N}$ & $50^{\circ} 00^{\prime} \mathrm{W}$ & $\mathrm{N}$ \\
\hline 1DG16058 & $66^{\circ} 14^{\prime} \mathrm{N}$ & $50^{\circ} 08^{\prime} \mathrm{W}$ & $\mathrm{N}$ \\
\hline 1DG16059 & $66^{\circ} 14^{\prime} \mathrm{N}$ & $50^{\circ} 11^{\prime} \mathrm{W}$ & N \\
\hline 1DG16060 & $66^{\circ} 14^{\prime} \mathrm{N}$ & $50^{\circ} 12^{\prime} \mathrm{W}$ & $\mathrm{N}$ \\
\hline 1DG16061 & $66^{\circ} 15^{\prime} \mathrm{N}$ & $50^{\circ} 13^{\prime} \mathrm{W}$ & $\mathrm{N}$ \\
\hline 1DG16062 & $66^{\circ} 18^{\prime} \mathrm{N}$ & $50^{\circ} 16^{\prime} \mathrm{W}$ & $\mathrm{SE}$ \\
\hline 1DG16063 & $66^{\circ} 17 \cdot \mathrm{N}$ & $50^{\circ} 09^{\prime} \mathrm{W}$ & $\mathrm{N}$ \\
\hline 1DG16064 & $66^{\circ} 17 \cdot \mathrm{N}$ & $50^{\circ} 05 \cdot \mathrm{W}$ & $\mathrm{N}$ \\
\hline 1DG & $66^{\circ} 18^{\prime} \mathrm{N}$ & $50^{\circ} 08^{\prime} \mathrm{W}$ & $\mathrm{N}$ \\
\hline 1DG16066 & $66^{\circ} 19^{\prime} \mathrm{N}$ & $49^{\circ} 59^{\prime} \mathrm{W}$ & NE \\
\hline 1DG16067 & $66^{\circ} 18^{\prime} \mathrm{N}$ & $50^{\circ} 00^{\prime} \mathrm{W}$ & $\mathrm{NE}$ \\
\hline 1DG16068 & $66^{\circ} 18^{\prime} \mathrm{N}$ & $50^{\circ} 02, \mathrm{~W}$ & $\mathrm{NE}$ \\
\hline 1DG16069 & $66^{\circ} 18^{\prime} \mathrm{N}$ & $50^{\circ} 03^{\prime} \mathrm{V}$ & $\mathrm{NE}$ \\
\hline
\end{tabular}

$\begin{array}{rrc}\text { HIGH } & \text { LOW } & \text { AREA } \\ & & \\ 120 & 120 & 0000.18 \\ 115 & 115 & 0000.21 \\ 115 & 115 & 0000.06 \\ 115 & 115 & 0000.05 \\ 115 & 115 & 0000.14 \\ 115 & 110 & 0000.20 \\ 110 & 105 & 0000.48 \\ 120 & 115 & 0000.07 \\ 110 & 110 & 0000.09 \\ 120 & 115 & 0000.13 \\ 120 & 115 & 0000.06 \\ 120 & 120 & 0000.04 \\ 125 & 125 & 0000.05 \\ 125 & 125 & 0000.12 \\ 120 & 120 & 0000.09 \\ 120 & 120 & 0000.08 \\ 120 & 120 & 0000.07 \\ 120 & 120 & 0000.07 \\ 120 & 120 & 0000.06 \\ 120 & 120 & 0000.07 \\ 130 & 125 & 0000.25 \\ 135 & 135 & 0000.07 \\ 125 & 120 & 0000.12 \\ 130 & 130 & 0000.10 \\ 110 & 110 & 0000.06 \\ 110 & 110 & 0000.08 \\ 115 & 115 & 0000.07 \\ 115 & 115 & 0000.11 \\ 120 & 120 & 0000.05 \\ 120 & 115 & 0000.04 \\ 135 & 125 & 0000.10 \\ 145 & 140 & 0000.13 \\ 135 & 135 & 0000.08 \\ 125 & 120 & 0000.15 \\ 125 & 125 & 0000.18 \\ 135 & 130 & 0000.29 \\ 135 & 130 & 0000.19 \\ 105 & 105 & 0000.05 \\ 105 & 105 & 0000.06 \\ 105 & 100 & 0000.28 \\ 105 & 100 & 0000.11 \\ 110 & 110 & 0000.06 \\ 110 & 105 & 0000.13 \\ 115 & 110 & 0000.13 \\ 1215 & 115 & 0000.09 \\ 125 & 110 & 0000.18 \\ 125 & 120 & 0000.20 \\ 125 & 95 & 0001.25 \\ 105 & 90 & 0000.12 \\ 115 & 105 & 0000.08 \\ 120 & 115 & 0000.07 \\ 125 & 120 & 0000.10 \\ 115 & 110 & 0000.11 \\ 105 & 100 & 0000.16 \\ 100 & 95 & 0000.24 \\ 110 & 95 & 0000.26 \\ 115 & 110 & 0000.27 \\ 95 & 95 & 0000.15 \\ 105 & 105 & 0000.34 \\ 120 & 110 & 0000.59 \\ 125 & 120 & 0000.42 \\ & & \end{array}$

\begin{tabular}{cc|} 
MO & \\
CODE & MAP \\
770010 & $6600-04$ \\
780010 & $6600-04$ \\
700010 & $6600-04$ \\
700010 & $6600-04$ \\
770010 & $6600-04$ \\
770010 & $6600-04$ \\
780010 & $6600-04$ \\
700010 & $6600-04$ \\
700010 & $6600-04$ \\
700010 & $6600-04$ \\
700010 & $6600-04$ \\
750010 & $6600-04$ \\
750010 & $6600-04$ \\
750010 & $6600-04$ \\
700010 & $6600-04$ \\
750010 & $6600-04$ \\
700010 & $6600-04$ \\
700010 & $6600-04$ \\
700010 & $6600-04$ \\
750010 & $6600-03$ \\
780010 & $6600-03$ \\
750010 & $6600-04$ \\
750010 & $6600-04$ \\
750010 & $6600-04$ \\
700010 & $6600-04$ \\
700010 & $6600-03$ \\
700010 & $6600-03$ \\
750010 & $6600-03$ \\
750010 & $6600-03$ \\
750010 & $6600-03$ \\
770010 & $6600-04$ \\
700010 & $6600-04$ \\
750010 & $6600-04$ \\
750010 & $6600-04$ \\
750010 & $6600-04$ \\
750010 & $6600-04$ \\
780010 & $6600-04$ \\
750010 & $6600-04$ \\
750010 & $6600-04$ \\
700010 & $6600-05$ \\
780010 & $6600-04$ \\
780010 & $6600-04$ \\
770010 & $6600-04$ \\
750010 & $6600-04$ \\
700010 & $6600-04$ \\
700010 & $6600-04$ \\
700010 & $6600-05$ \\
780010 & $6600-05$ \\
780010 & $6600-05$ \\
780010 & $6600-05$ \\
750010 & $6600-04$ \\
750010 & $6600-04$ \\
780010 & $6600-04$ \\
700010 & $6600-04$ \\
780010 & $6600-05$ \\
780010 & $6600-05$ \\
780010 & $6600-05$ \\
700010 & $6600-05$ \\
700010 & $6600-05$ \\
780010 & $6600-05$ \\
780010 & $6600-05$ \\
&
\end{tabular}

CODE

LAT

LONG

ORI

1DG16070

1DG16071

$66^{\circ} 19^{\prime} \mathrm{N}$

$50^{\circ} 01^{\prime} \mathrm{W} \quad \mathrm{NE}$

1DG16072

$66^{\circ} 20^{\prime} \mathrm{N} \quad 50^{\circ} 09^{\prime} \mathrm{W}$ NE

DG16074 $66^{\circ} 21$ 'N

$50^{\circ} 06^{\prime} \mathrm{W}$ E

1DG16075

$66^{\circ} 21^{\prime}, \mathrm{N}$

$50^{\circ} 07 / \mathrm{W}$

16077

$66^{\circ} 20^{\prime} \mathrm{N}$

$50^{\circ} 13^{\prime} \mathrm{W} N$

1DG16079

$66^{\circ} 20^{\prime} \mathrm{N} \quad 50^{\circ} 14^{\prime} \mathrm{W}$

IDG16081

1DG1608

IDG16084

$66^{\circ} 17^{\prime} \mathrm{N}$

$66^{\circ} 16^{\prime} \mathrm{N}$

$50^{\circ} 30^{\prime} \mathrm{W}$ NW

$66^{\circ} 14^{\prime} \mathrm{N} 50^{\circ} 31^{\prime} \mathrm{W}$ N

1DG16086

1DG16087

(6.

$66^{\circ} 16^{\prime} \mathrm{N} 50^{\circ} 35^{\prime} \mathrm{W}$

1DG16089

$66^{\circ} 16^{\prime} \mathrm{N} 50^{\circ} 33^{\prime} \mathrm{W} \mathrm{S}$

1DG16091

$\begin{array}{lll}66^{\circ} 16^{\prime} \mathrm{N} & 50^{\circ} 35^{\prime} \mathrm{W} & \mathrm{SW} \\ 66^{\circ} 17^{\prime} \mathrm{N} & 50^{\circ} 34^{\prime} \mathrm{W} & \mathrm{NE}\end{array}$

1DG16092

IDG16094

1DG16095
1DG16096

$66^{\circ} 177^{-N}$

$50^{\circ} 33, \mathrm{~W} \mathrm{E}$

$\begin{array}{lll}66^{\circ} 78^{\prime}, \mathrm{N} & 50^{\circ} 29^{\prime} \mathrm{W} & \text { SE } \\ 50^{\circ} 32^{\prime} \mathrm{W} & \text { NE }\end{array}$

$66^{\circ} 17^{\prime} \mathrm{N}$

$66^{\circ} 18^{\prime} \mathrm{N}$

$50^{\circ} 34^{\prime} \mathrm{W} N$

1DG16098

$50^{\circ} 38^{\prime} \mathrm{W}$

1DG16100

1DG16101

1DG16103

$66^{\circ} 17^{\prime}$

$66^{\circ} 18^{\prime}$

$50^{\circ} 40^{\prime} \mathrm{W} \mathrm{N}$

1DG16104

$66^{\circ} 17^{\prime}$

$\begin{array}{lll} & \\ 50^{\circ} 38^{\prime}, \mathrm{W} & \mathrm{NE} & \mathrm{NE}\end{array}$

$50^{\circ} 42^{\prime} \mathrm{W} \mathrm{N}$

$66^{\circ} 16^{\prime} \mathrm{N}$

$50^{\circ} 46^{\prime} \mathrm{W}$

IDG1610

1DG16107

IDG16109

$66^{\circ} 16^{\prime} \mathrm{N}$

$66^{\circ} 17^{\prime}$

$50^{\circ} 48^{\prime} \mathrm{W} N \mathrm{~N}$

$66^{\circ} 16^{\prime} \mathrm{N} 50^{\circ} 54^{\circ} \mathrm{W}$ N

1DG16111

$66^{\circ} 16^{\prime} \mathrm{N}$

$50^{\circ} 57 \cdot \mathrm{W} N \mathrm{~N}$

1DG16112

1DG16114

$66^{\circ} 19^{\prime} \mathrm{N}$

$66^{\circ} 21^{\prime} \mathrm{N}$

$0^{\circ} 50^{\prime} \mathrm{W} N$

(2)

$66^{\circ} 22^{\prime} \mathrm{N}$

$\begin{array}{lll}50^{\circ} 34^{\prime} \mathrm{W} & \mathrm{NE} \\ 49^{\circ} 57^{\prime} \mathrm{W} & \mathrm{S}\end{array}$

1DG16117

1DG16118

1DG16120

1DG16121

$66^{\circ} 20^{\prime}$

$49^{\circ} 47, \mathrm{~W}$ NW

. $19^{\circ} \mathrm{N} \quad 49^{\circ} 47^{\prime} \mathrm{W}$ N

$66^{\circ} 17^{\prime} \mathrm{N}$

$49^{\circ} 40^{\circ} \mathrm{W}$ NW

$66^{\circ} 11$ ' N

$49^{\circ} 42^{\prime} \mathrm{V}$ NW

$1 D G 16123$

$66^{\circ} 12^{\prime} \mathrm{N} \quad 49^{\circ} 43^{\prime} \mathrm{W} N$

66.13'N $49^{\circ} 47^{\circ} \mathrm{N} N \mathrm{~N}$

$1 D G 16126 \quad 66^{\circ} 12$ 'N $49^{\circ} 45{ }^{\prime} \mathrm{N} N$

1DG16127 66 $66^{\circ} 11^{\prime} \mathrm{N} \quad 49^{\circ} 46^{\prime} \mathrm{W}$ NE

1DG16128

$66^{\circ} 11^{\prime} \mathrm{N} \quad 49^{\circ} 48^{\prime} \mathrm{W}$ NE

$\begin{array}{lll}66^{\circ} 11^{\prime} \mathrm{N} & 49^{\circ} 49^{\prime} \mathrm{W} & \mathrm{NE} \\ 66^{\circ} 11^{\prime} \mathrm{N} & 49^{\circ} 50^{\prime} \mathrm{W} & \mathrm{N}\end{array}$

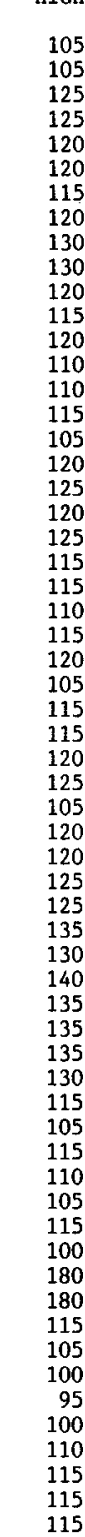

MAP N

$750010 \quad 6600-05$

$\begin{array}{llll}100 & 0000.14 & 750010 & 6600-05 \\ 100 & 0000.12 & 780010 & 6600-05 \\ 105 & 0001.03 & 780010 & 6600-05\end{array}$

$\begin{array}{llll}105 & 0001.03 & 780010 & 6600-05 \\ 115 & 0000.34 & 770010 & 6600-05\end{array}$

$\begin{array}{llll}110 & 0000.08 & 700010 & 6600-05\end{array}$

$\begin{array}{llll}110 & 0000.08 & 707010 & 6600-05 \\ 110 & 0000.37 & 770010 & 6600-04\end{array}$

$\begin{array}{lllll}110 & 0000.07 & 770010 & 6600-04\end{array}$

$\begin{array}{llll}130 & 0000.14 & 700010 & 6600-04 \\ 110 & 0000.25 & 780010 & 6600-04\end{array}$

$\begin{array}{llll}120 & 0000.08 & 700010 & 6600-04\end{array}$

$\begin{array}{llll}115 & 0000.15 & 700010 & 6600-04\end{array}$

$\begin{array}{llll}110 & 0000.10 & 750010 & 6600-04\end{array}$

$\begin{array}{llll}110 & 0000.14 & 780010 & 6600-04 \\ 110 & 0000.03 & 780010 & 6600-04\end{array}$

$\begin{array}{lllll}110 & 0000.24 & 780010 & 6600-04\end{array}$

$\begin{array}{llll}105 & 0000.09 & 750010 & 6600-04 \\ 115 & 0000.21 & 780010 & 6600-04\end{array}$

$\begin{array}{llll}120 & 0000.11 & 780010 & 6600-04\end{array}$

$\begin{array}{llll}120 & 0000.08 & 750010 & 6600-04\end{array}$

$125 \quad 0000.11 \quad 750010 \quad 6600-04$

$\begin{array}{llll}115 & 0000.08 & 780010 & 6600-04 \\ 115 & 0000.06 & 700010 & 6600-04\end{array}$

$\begin{array}{llll}110 & 0000.12 & 700010 & 6600-04\end{array}$

$\begin{array}{llll}115 & 0000.14 & 750010 & 6600-04\end{array}$

$\begin{array}{llll}120 & 0000.14 & 750010 & 6600-04\end{array}$

$\begin{array}{llll}105 & 0000.19 & 780010 & 6600-04 \\ 110 & 0000.16 & 780010 & 6600-04\end{array}$

$\begin{array}{llll}110 & 0000.34 & 780010 & 6600-04 \\ 110 & 0000.25 & 750010 & 6600-04\end{array}$

$\begin{array}{llll}112 & 000.25 & 750010 & 6600-04 \\ 120 & 0000.17 & 750010 & 6600-04\end{array}$

$\begin{array}{llll}105 & 0000.07 & 770010 & 6600-04\end{array}$

$\begin{array}{llll}120 & 0000.19 & 770010 & 6600-04 \\ 12000-04\end{array}$

$\begin{array}{llll}120 & 0000.12 & 700010 & 6600-04 \\ 125 & 0000.27 & 700010 & 6600-04\end{array}$

$\begin{array}{llll}125 & 0000.27 & 700010 & 6600-04 \\ 125 & 0000.15 & 780010 & 6600-04\end{array}$

$\begin{array}{llll}125 & 0000.15 & 780010 & 6600-04 \\ 130 & 0000.37 & 780010 & 6600-04\end{array}$

$\begin{array}{llll}125 & 0000.12 & 770010 & 6600-04\end{array}$

$\begin{array}{llll}130 & 0000.25 & 750010 & 6600-04\end{array}$

$\begin{array}{llll}135 & 0000.30 & 750010 & 6600-04 \\ 135 & 0000.09 & 750010 & 6600-04\end{array}$

$\begin{array}{llll}130 & 0000.25 & 750010 & 6600-04\end{array}$

$\begin{array}{llll}130 & 0000.09 & 750010 & 6600-04 \\ 115 & 0000.11 & 770010 & 6600-04\end{array}$

$\begin{array}{llll}115 & 0000.11 & 770010 & 6600-04 \\ 105 & 0000.08 & 700010 & 6600-05\end{array}$

$\begin{array}{llll}105 & 0000.08 & 700010 & 6600-05 \\ 100 & 0000.21 & 750010 & 6600-05\end{array}$

$\begin{array}{llll}100 & 0000.12 & 750010 & 6600-05 \\ 100 & 0000.07 & 750010 & 6600-05 \\ 115 & 0000.19 & 780010 & 6600-05\end{array}$

$\begin{array}{llll}100 & 0000.07 & 750010 & 6600-05 \\ 115 & 0000.19 & 780010 & 6600-05\end{array}$

$\begin{array}{llll}100 & 0000.27 & 780010 & 6600-05\end{array}$

\begin{tabular}{llll}
70 & 0606.34 & 166113 & $6600-05$ \\
\hline 8 & 0408.34 & 163112 & $6600-05$
\end{tabular}

$\begin{array}{rrrr}80 & 0408.34 & 163112 & 6600-05 \\ 105 & 0000.14 & 750010 & 6600-05\end{array}$

$\begin{array}{llll}105 & 0000.14 & 750010 & 6600-05 \\ 105 & 0000.07 & 750010 & 6600-05\end{array}$

$\begin{array}{llll}90 & 0000.19 & 750010 & 6600-05 \\ 95 & 0000.34 & 780010 & 6600-05\end{array}$

$\begin{array}{rrrr}95 & 0000.34 & 780010 & 6600-05 \\ 100 & 0000.22 & 780010 & 6600-05\end{array}$

$\begin{array}{llll}100 & 0000.22 & 780010 & 6600-05 \\ 100 & 0000.28 & 780010 & 6600-05\end{array}$

$\begin{array}{llll}115 & 0000.08 & 780010 & 6600-05 \\ 100 & 0000.44 & 780010 & 6000-05\end{array}$

$\begin{array}{llll}100 & 0000.44 & 780010 & 6600-05 \\ 105 & 0000.16 & 780010 & 6600-05\end{array}$ 


\begin{tabular}{|c|c|c|c|c|}
\hline CODE & LAT & LONG & ORI & \\
\hline 6131 & $66^{\circ} 11^{\prime} \mathrm{N}$ & $49^{\circ} 52^{\prime} \mathrm{W}$ & . & \\
\hline & $12, \mathrm{~N}$ & $49^{\circ} 56^{\prime} \mathrm{V}$ & N & \\
\hline 1DG16133 & $66^{\circ} 11^{\prime} \mathrm{N}$ & $49^{\circ} 55^{\prime} \mathrm{W}$ & $\mathrm{NE}$ & \\
\hline 1DG16134 & $66^{\circ} 11^{\prime} \mathrm{N}$ & $49^{\circ} 58^{\prime} \mathrm{W}$ & $\mathrm{N}$ & \\
\hline 1DG16135 & $66^{\circ} 12^{\prime} \mathrm{N}$ & $49^{\circ} 58^{\prime} \mathrm{W}$ & NE & \\
\hline 1DG16136 & $66^{\circ} 10^{\prime} \mathrm{N}$ & $50^{\circ} 00^{\prime} \mathrm{W}$ & $\mathrm{N}$ & \\
\hline 1DG16137 & $66^{\circ} 11^{\prime} \mathrm{N}$ & $49^{\circ} 57^{\circ} \mathrm{W}$ & NW & \\
\hline 1DG16138 & $66^{\circ} 11$, N & $49^{\circ} 54^{\prime \prime} \mathrm{W}$ & $\mathrm{s}$ & \\
\hline 1DG16139 & $66^{\circ} 10^{\prime} \mathrm{N}$ & $49^{\circ} 53^{\prime} \mathrm{W}$ & & \\
\hline 1DG16140 & $66^{\circ} 09^{\prime} \mathrm{N}$ & $49^{\circ} 43^{\prime} \mathrm{W}$ & $\mathrm{NW}$ & \\
\hline 1DG16141 & $66^{\circ} 08^{\prime} \mathrm{N}$ & $49^{\circ} 47^{\prime} \mathrm{W}$ & $\mathrm{NW}$ & \\
\hline 1DG16142 & $66^{\circ} 07^{\prime N N}$ & $49^{\circ} 54^{\prime \prime} \mathrm{W}$ & $\mathrm{NW}$ & \\
\hline 1DG16143 & $66^{\circ} 09^{\prime} \mathrm{N}$ & $50^{\circ} 03^{\prime} \mathrm{W}$ & N & \\
\hline 1DG16144 & $66^{\circ} 09^{\prime} \mathrm{N}$ & $50^{\circ} 04^{\prime} \mathrm{W}$ & $\mathrm{N}$ & \\
\hline 1DG16145 & $66^{\circ} 10^{\prime} \mathrm{N}$ & $50^{\circ} 05^{\prime} \mathrm{W}$ & $\mathrm{E}$ & \\
\hline 1DG16146 & $66^{\circ} 11^{\prime} \mathrm{N}$ & $50^{\circ} 03^{\prime}$ & $\mathrm{NE}$ & \\
\hline 1DG16147 & $66^{\circ} 11^{\prime} \mathrm{N}$ & $50^{\circ} 05^{\circ} \mathrm{W}$ & NE & \\
\hline 1DG1 & $66^{\circ} 07, \mathrm{~N}$ & $49^{\circ} 57^{\prime \prime} \mathrm{W}$ & $\mathrm{NE}$ & \\
\hline 1DG16149 & $66^{\circ} 07^{\prime} \mathrm{N}$ & $49^{\circ} 58^{\prime} \mathrm{W}$ & $\mathrm{sW}$ & \\
\hline 1DG16150 & $66^{\circ} 07^{\prime} \mathrm{N}$ & $49^{\circ} 58^{\prime} \mathrm{W}$ & $\mathrm{SE}$ & \\
\hline 1DG16151 & $66^{\circ} 06^{\prime} \mathrm{N}$ & $49 \circ 57, \mathrm{~W}$ & NE & \\
\hline 1DG1 & $66^{\circ} 06^{\prime N} \mathrm{~N}$ & $49057, \mathrm{~W}$ & $E$ & \\
\hline & $66^{\circ} 06^{\prime} \mathrm{N}$ & $49 \circ 59 \cdot \mathrm{W}$ & $\mathrm{NE}$ & \\
\hline 1DG1 & $66^{\circ} 07^{\prime} \mathrm{N}$ & $50^{\circ} 02 \cdot \mathrm{W}$ & $\mathbf{N}$ & \\
\hline 1DG16155 & $66^{\circ} 06^{\prime} \mathrm{N}$ & $50^{\circ} 00^{\prime} \mathrm{W}$ & $\mathrm{N}$ & \\
\hline 1DG16156 & $66^{\circ} 05^{\prime} \mathrm{N}$ & $50^{\circ} 01^{\prime} \mathrm{V}$ & NW & \\
\hline IDG16157 & $66^{\circ} 04^{\prime} \mathrm{N}$ & $50^{\circ} 04^{\prime} \mathrm{y}$ & $\mathrm{NE}$ & \\
\hline 1DG1 & & $50^{\circ} 05, v$ & $\mathrm{NE}$ & \\
\hline 1DG16159 & $66^{\circ} 06^{\prime} \mathrm{N}$ & $50^{\circ} 06^{\circ}$ ' & $\mathrm{E}$ & \\
\hline IDG16160 & $66^{\circ} 06^{\prime} \mathrm{N}$ & $50^{\circ} 06^{\prime} \mathrm{W}$ & N & \\
\hline 1DG16161 & $66^{\circ} 07 \cdot \mathrm{N}$ & $50^{\circ} 06^{\prime}$ & $\mathrm{N}$ & \\
\hline 1DG16162 & $66^{\circ} 05, \mathrm{~N}$ & $50^{\circ} 05^{\prime} \mathrm{V}$ & NE & \\
\hline 1DG1 & & $50^{\circ} 08^{\prime} \mathrm{W}$ & NE & \\
\hline $1 \mathrm{DG}$ & $6 e$ & $50^{\circ} 01^{\circ} \mathrm{W}$ & SE & \\
\hline 1DG16165 & $66^{\circ} 06^{\prime} \mathrm{N}$ & $50^{\circ} 08^{\prime}$ & $\mathrm{SE}$ & \\
\hline 1DG16166 & $66^{\circ} 06^{\prime} \mathrm{N}$ & $50^{\circ} 13^{\prime} \mathrm{V}$ & $\mathrm{SE}$ & \\
\hline 1DG? & $66^{\circ} 09^{\prime} \mathrm{N}$ & $50^{\circ} 12^{\prime}$ & $\mathrm{E}$ & \\
\hline IDG1 & $66^{\circ} 10^{\prime} \mathrm{N}$ & $50^{\circ} 14^{\prime \prime}$ & $\mathrm{NE}$ & \\
\hline 1DG16169 & $66^{\circ} 10^{\prime} \mathrm{N}$ & $50^{\circ} 16^{\prime} \mathrm{W}$ & $\mathrm{NE}$ & \\
\hline IDG16170 & $66^{\circ} 10^{\prime} \mathrm{N}$ & $50^{\circ} 18^{\prime} \mathrm{W}$ & $\mathrm{NE}$ & \\
\hline 1DG1 & $66^{\circ} 11^{\prime} \mathrm{N}$ & $50^{\circ} 21, \mathrm{~W}$ & NE & \\
\hline 1DG & $66^{\circ} 12^{\prime} \mathrm{N}$ & $50^{\circ} 24^{\prime} \mathrm{W}$ & $\mathrm{NE}$ & \\
\hline 1DG16173 & & $50^{\circ} 25^{\prime} \mathrm{W}$ & $\mathrm{N}$ & \\
\hline 1DG16174 & $66^{\circ} 12, \mathrm{~N}$ & $50^{\circ} 27, \mathrm{~V}$ & $\mathrm{~N}$ & \\
\hline & $66^{\circ} 12, \mathrm{~N}$ & $50^{\circ} 28^{\prime \prime} \mathrm{W}$ & NW & \\
\hline & $66^{\circ} 10^{\prime} \mathrm{N}$ & $50^{\circ} 27^{\prime} \mathrm{W}$ & NW & \\
\hline & 6 & $50^{\circ} 28^{\prime} \mathrm{V}$ & $\mathrm{W}$ & \\
\hline $1 \mathrm{DG}$ & $66^{\circ} 1$ & $50^{\circ} 33^{\prime} \mathrm{W}$ & $\mathrm{N}$ & \\
\hline 1DG16179 & $66^{\circ} 09^{\prime} \mathrm{N}$ & $50^{\circ} 33^{\prime \prime} \mathrm{W}$ & $\mathrm{N}$ & \\
\hline & $66^{\circ} 09^{\prime} \mathrm{N}$ & $50^{\circ} 33^{\prime} \mathrm{W}$ & SE & \\
\hline & $66^{\circ} 10^{\prime} \mathrm{N}$ & $50^{\circ} 34^{\prime}$ & NE & \\
\hline IDG & $66^{\circ} 12^{\prime} \mathrm{N}$ & $50^{\circ} 35, \mathrm{~V}$ & $\mathrm{NE}$ & \\
\hline 1DG161 & & $50^{\circ} 36^{\prime}$ W & $\mathrm{NE}$ & \\
\hline IDG & 6 & $50^{\circ} 40^{\prime} \mathrm{W}$ & $\mathrm{W}$ & \\
\hline 1DG16 & $66^{\circ} 12^{\prime} \mathrm{N}$ & $50^{\circ} 43^{\prime} \mathrm{W}$ & V & \\
\hline 1DG16186 & $66^{\circ} 11^{\prime} \mathrm{N}$ & $50^{\circ} 42, \mathrm{~V}$ & N & \\
\hline 1DG16187 & $66^{\circ} 11^{\prime} \mathrm{N}$ & $50^{\circ} 40^{\prime} \mathrm{W}$ & $\mathrm{NE}$ & \\
\hline 1DG16 & $66^{\circ} 10^{\prime} \mathrm{N}$ & $50^{\circ} 40^{\prime} \mathrm{V}$ & $\mathrm{NE}$ & \\
\hline 1DG16189 & $66^{\circ} 10^{\prime} \mathrm{N}$ & $50^{\circ} 38^{\prime} \mathrm{W}$ & N & \\
\hline 1DG16190 & $66^{\circ} 09^{\prime} \mathrm{N}$ & $50^{\circ} 36^{\prime} \mathrm{W}$ & N & \\
\hline 1DG16191 & $66^{\circ} 10^{\prime} \mathrm{N}$ & $50^{\circ} 35^{\prime} \mathrm{W}$ & N & \\
\hline
\end{tabular}

$\begin{array}{cc}\text { MO } & \\ \text { CODE } & \text { MAP } \\ 780010 & 6600-05 \\ 780010 & 6600-05 \\ 77010 & 6600-05 \\ 770010 & 6600-05 \\ 780010 & 6600-05 \\ 700010 & 6600-05 \\ 700010 & 6600-05 \\ 780010 & 6600-05 \\ 780010 & 6600-05 \\ 163112 & 6600-05 \\ 163113 & 6600-05 \\ 163112 & 6600-05 \\ 780010 & 6600-05 \\ 770010 & 6600-05 \\ 750010 & 6600-05 \\ 770010 & 6600-05 \\ 780010 & 6600-05 \\ 780010 & 6600-05 \\ 700010 & 6600-05 \\ 700010 & 6600-05 \\ 770010 & 6600-05 \\ 780010 & 6600-05 \\ 750010 & 6600-05 \\ 780010 & 6600-05 \\ 700010 & 6600-05 \\ 163113 & 6600-05 \\ 770010 & 6600-05 \\ 770010 & 6600-05 \\ 700010 & 6600-05 \\ 700010 & 6600-05 \\ 780010 & 6600-05 \\ 770010 & 6600-05 \\ 700010 & 6600-05 \\ 750010 & 6600-05 \\ 700010 & 6600-05 \\ 363114 & 6600-04 \\ 366112 & 6600-04 \\ 363212 & 6600-04 \\ 366212 & 6600-04 \\ 366310 & 6600-04 \\ 366212 & 6600-04 \\ 203212 & 6600-04 \\ 366312 & 6600-04 \\ 643212 & 6600-04 \\ 750010 & 6600-04 \\ 363112 & 6600-04 \\ 363112 & 6600-04 \\ 750010 & 6600-04 \\ 770010 & 6600-04 \\ 700010 & 6600-04 \\ 700010 & 6600-04 \\ 770010 & 6600-04 \\ 780010 & 6600-04 \\ 780010 & 6600-04 \\ 750010 & 6600-04 \\ 750010 & 6600-04 \\ 770010 & 6600-04 \\ 770010 & 6600-04 \\ 770010 & 6600-04 \\ 770010 & 6600-04 \\ 770010 & 6600-04 \\ & \end{array}$

\begin{tabular}{|c|c|c|c|}
\hline ODE & LAT & LONG & $\mathrm{OR}$ \\
\hline & $66^{\circ} 09^{\prime} \mathrm{N}$ & $50^{\circ} 36^{\prime} \mathrm{W}$ & \\
\hline & $66^{\circ} 09^{\prime} \mathrm{N}$ & $50^{\circ} 35^{\prime}$ & \\
\hline & $66^{\circ} 09^{\prime} \mathrm{N}$ & $50^{\circ} 35^{\prime}$ & \\
\hline 195 & $66^{\circ} 09^{\prime} \mathrm{N}$ & $50^{\circ} 34^{\prime} \mathrm{V}$ & \\
\hline 196 & $66^{\circ} 08^{\prime} \mathrm{N}$ & $50^{\circ} 28^{\prime} \mathrm{V}$ & \\
\hline 6197 & $66^{\circ} 08^{\prime} \mathrm{N}$ & $50^{\circ} 33^{\prime} \mathrm{V}$ & IE \\
\hline 6198 & $66^{\circ} 07^{\prime} \mathrm{N}$ & $50^{\circ} 31^{\prime} \mathrm{V}$ & \\
\hline 6700 & $66^{\circ} 07^{\prime} \mathrm{N}$ & $50^{\circ} 32^{\prime} \mathrm{V}$ & \\
\hline 200 & $66^{\circ} 06^{\prime} \mathrm{N}$ & $50^{\circ} 28^{\prime} \mathrm{V}$ & sw \\
\hline 5201 & $66^{\circ} 06^{\prime} \mathrm{N}$ & $50^{\circ} 29^{\prime}$ ' & su \\
\hline 6202 & $66^{\circ} 06^{\prime} \mathrm{N}$ & $50^{\circ} 30^{\prime} \mathrm{W}$ & SW \\
\hline 6002 & $66^{\circ} 06^{\prime} \mathrm{N}$ & $50^{\circ} 30^{\prime} \mathrm{W}$ & \\
\hline & & $50^{\circ} 29^{\prime} \mathrm{W}$ & \\
\hline & $66^{\circ} 05^{\prime} \mathrm{N}$ & $50^{\circ} 28^{\prime} \mathrm{W}$ & SW \\
\hline & $66^{\circ} 06^{\prime} \mathrm{N}$ & $50^{\circ} 27^{\prime} \mathrm{W}$ & \\
\hline & $66^{\circ} 0$ & $50^{\circ} 23^{\prime \prime}$ & \\
\hline & $66^{\circ} 06^{\prime} \mathrm{N}$ & $50^{\circ} 25^{\prime} \mathrm{W}$ & \\
\hline & $\mathrm{N}$ & $022^{\prime \prime} \mathrm{W}$ & \\
\hline & & $50^{\circ} 19^{\prime} \mathrm{W}$ & \\
\hline & $66^{\circ} 03^{\prime} \mathrm{N}$ & $50^{\circ} 18^{\prime} \mathrm{W}$ & \\
\hline 621 & $66^{\circ} 03^{\prime} \mathrm{N}$ & $50^{\circ} 21^{\prime} \mathrm{W}$ & NW \\
\hline$a_{1}$ & 'N & $50^{\circ} 23^{\prime} \mathrm{W}$ & \\
\hline & & $50^{\circ} 16^{\prime} \mathrm{W}$ & $\mathrm{NE}$ \\
\hline & & $50^{\circ} 19^{\prime} \mathrm{W}$ & \\
\hline & $66^{\circ} 03^{\prime} \mathrm{N}$ & $50^{\circ} 13^{\prime} \mathrm{W}$ & \\
\hline 6217 & $66^{\circ} \mathrm{C}$ & $50^{\circ} 12^{\prime} \mathrm{W}$ & \\
\hline & $\mathrm{N}$ & $50^{\circ} 13^{\prime} \mathrm{W}$ & \\
\hline & & $50^{\circ} 11, \mathrm{~W}$ & L \\
\hline & & $50^{\circ} 08^{\prime}, \mathrm{W}$ & $\mathrm{NE}$ \\
\hline & 66 & $50^{\circ} 06^{\prime} \mathrm{V}$ & \\
\hline 622 & $\mathbf{N}$ & $50^{\circ} 08^{\prime} \mathrm{W}$ & \\
\hline & N & $50^{\circ} 10^{\prime} \mathrm{W}$ & $\mathrm{NE}$ \\
\hline & & $50^{\circ} 15^{\prime} \mathrm{W}$ & \\
\hline & & $50^{\circ} 08^{\prime} \mathrm{W}$ & \\
\hline & $\mathbf{N}$ & $50^{\circ} 06^{\prime \prime} \mathrm{W}$ & \\
\hline & & $07 . n$ & \\
\hline & & $06, \mathrm{~W}$ & \\
\hline & & & \\
\hline & & $50^{\circ} \mathrm{C}$ & \\
\hline & N & $50^{\circ} 05^{\prime} \mathrm{W}$ & \\
\hline & & & \\
\hline & & $1 \mathrm{~W}$ & \\
\hline & & $49^{\circ} 58^{\prime} \mathrm{W}$ & $\mathrm{NW}$ \\
\hline & & $50^{\circ} 03^{\prime} \mathrm{W}$ & \\
\hline & $\mathbf{N}$ & i & \\
\hline & & & \\
\hline & & $25, \mathrm{~W}$ & \\
\hline & & $50^{\circ} 35^{\prime} \mathrm{W}$ & NE \\
\hline & & $50^{\circ} 38^{\prime} \mathrm{W}$ & \\
\hline & & $50^{\circ} 42^{\prime} \mathrm{V}$ & \\
\hline & & $50^{\circ} 44^{\prime} \mathrm{W}$ & \\
\hline & $66^{\circ} 0$ & $50^{\circ} 45^{\prime} \mathrm{W}$ & \\
\hline & $66^{\circ} 09$ & $50^{\circ} 46^{\prime} \mathrm{W}$ & NE \\
\hline & $66^{\circ} 05^{\circ} \mathrm{N}$ & $50^{\circ} 48^{\prime} \mathrm{W}$ & \\
\hline & & $50^{\circ} 57 \cdot \mathrm{W}$ & \\
\hline & $66^{\circ} 08$ & $51^{\circ} 02^{\prime} \mathrm{W}$ & \\
\hline & $66^{\circ} 08$ & $51^{\circ} 13^{\prime} \mathrm{W}$ & \\
\hline & $66^{\circ}$ & $51^{\circ} 21^{\prime} \mathrm{W}$ & \\
\hline & $66^{\circ} 16$ & $51^{\circ} 25^{\prime} \mathrm{W}$ & \\
\hline & 11 & $31-21 \mathrm{~W}$ & \\
\hline & & & \\
\hline
\end{tabular}

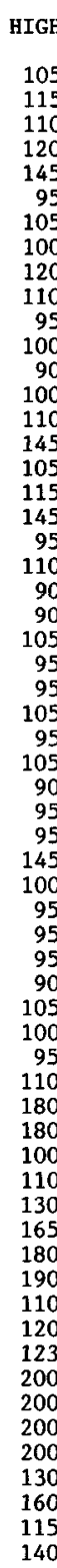




\begin{tabular}{|c|c|c|c|c|c|c|c|c|c|c|c|c|c|}
\hline CODE & LAT & LONG & ORI & HIGH & LOW & AREA & $\begin{array}{c}\text { MO } \\
\text { CODE }\end{array}$ & MAP & CODE & LAT & LONG & ORI & HIGH \\
\hline 253 & 66 & $51^{\circ} 26^{\prime} \mathrm{W}$ & NE & 150 & 105 & 0006.79 & 300010 & $6600-03$ & 1D & 6 & 5 & $\mathbf{N}$ & 140 \\
\hline 1DG16254 & $66^{\circ} 18^{\prime} \mathrm{N}$ & $51^{\circ} 23^{\prime} \mathrm{W}$ & $\mathrm{NE}$ & 105 & 80 & 0000.18 & 750010 & $6600-03$ & 1DG21013 & $66^{\circ} 15^{\prime} \mathrm{N}$ & $52^{\circ} 38^{\prime}$ W & sw & 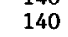 \\
\hline 1DG16255 & $66^{\circ} 18^{\prime} \mathrm{N}$ & $51^{\circ} 24^{\prime} \mathrm{W}$ & $\mathrm{N}$ & 100 & 80 & 0000.17 & 700010 & $6600-03$ & 1DG21014 & $66^{\circ} 15^{\prime} \mathrm{N}$ & $52^{\circ} 39^{\prime} \mathrm{W}$ & N & 6 \\
\hline $1 D G 16256$ & $66^{\circ} 18^{\prime} \mathrm{N}$ & $51^{\circ} 35 \% \mathrm{~W}$ & $N E$ & 180 & 70 & 0128.55 & 363012 & $6600-03$ & 1DG21015 & $66^{\circ} 15, \mathrm{~N}$ & $52^{\circ} 40^{\circ} \mathrm{W}$ & E & \\
\hline 1DG16257 & $66^{\circ} 21^{\prime} \mathrm{N}$ & $51^{\circ} 33^{\prime} \mathrm{W}$ & $\mathrm{NE}$ & 137 & 130 & 0000.36 & 780010 & $6600-03$ & 1DG21016 & $66^{\circ} 14^{\prime} \mathrm{N}$ & $52^{\circ} 41, \mathrm{~W}$ & E & 1 \\
\hline 1DG16258 & $66^{\circ} 20^{\prime} \mathrm{N}$ & $51^{\circ} 31^{\prime} \mathrm{v}$ & E & 125 & 110 & 0003.57 & 300010 & $6600-03$ & 1DG21017 & $66^{\circ} 15^{\prime} \mathrm{N}$ & $52 \circ 42, v$ & E & 1 \\
\hline 1DG16259 & $66^{\circ} 20^{\prime} \mathrm{N}$ & $51^{\circ} 34^{\prime} \mathrm{W}$ & $\mathrm{NE}$ & 125 & 115 & 0004.84 & 300010 & $6600-03$ & 1DG22001 & $66^{\circ} 15^{\prime} \mathrm{N}$ & $52^{\circ} 42 \cdot \mathrm{W}$ & NW & \\
\hline 1DG16260 & $66^{\circ} 21^{\prime} \mathrm{N}$ & $51^{\circ} 39^{\prime} \mathrm{W}$ & $\mathrm{N}$ & 120 & 120 & 0000.11 & 780010 & $6600-03$ & 1DG22002 & $66^{\circ} 14^{\prime} \mathrm{N}$ & $52^{\circ} 42 \cdot \mathrm{W}$ & $\mathrm{v}$ & 130 \\
\hline 1DG16261 & $66^{\circ} 20^{\prime} \mathrm{N}$ & $51^{\circ} 40^{\circ} \mathrm{W}$ & $\mathrm{NW}$ & 140 & 125 & 0007.04 & 363112 & $6600-03$ & 1DG22003 & $66^{\circ} 14^{\prime} \mathrm{N}$ & $52^{\circ} 41$ ' & $\mathrm{v}$ & \\
\hline $1 D G 16262$ & $66^{\circ} 21 \cdot \mathrm{N}$ & $51^{\circ} 40^{\circ} \mathrm{W}$ & NW & 125 & 125 & 0000.22 & 750010 & $6600-03$ & IDG22004 & $66^{\circ} 13^{\prime} \mathrm{N}$ & $52^{\circ} 40^{\prime \prime}$ & NW & \\
\hline 1DG16263 & $66^{\circ} 21 \cdot \mathrm{N}$ & $51^{\circ} 40^{\circ} \mathrm{W}$ & $\mathrm{NW}$ & 135 & 135 & 0000.25 & 780010 & $6600-03$ & 1DG22005 & $66^{\circ} 12^{\prime} \mathrm{N}$ & $52^{\circ} 40^{\circ} \mathrm{W}$ & NV & 175 \\
\hline 1DG16264 & $66^{\circ} 21^{\prime} \mathrm{N}$ & $51^{\circ} 43, \mathrm{~W}$ & $\mathrm{NE}$ & 130 & 120 & 0001.22 & 300012 & $6600-03$ & 1DG22006 & $66^{\circ} 12^{\prime} \mathrm{N}$ & $52^{\circ} 40^{\prime} \mathrm{W}$ & NW & 1 \\
\hline 1DG16265 & $66^{\circ} 21^{\prime} \mathrm{N}$ & $51^{\circ} 48^{\prime} \mathrm{W}$ & $\mathrm{N}$ & 170 & 80 & 0168.30 & 363112 & $6600-03$ & 1DG22007 & $66^{\circ} 11 \cdot \mathrm{N}$ & $52^{\circ} 41^{\prime \prime}$ & N & 1 \\
\hline 1DG16266 & $66^{\circ} 21^{\prime} \mathrm{N}$ & $51^{\circ} 53^{\prime} \mathrm{W}$ & $\mathrm{N}$ & 150 & 100 & 0027.96 & 300112 & $6600-03$ & 1DG22008 & $66^{\circ} 12^{\prime} \mathrm{N}$ & $52^{\circ} 44^{\prime} \mathrm{W}$ & E & \\
\hline 1DG16267 & $66^{\circ} 22^{\prime} \mathrm{N}$ & $51^{\circ} 58^{\prime} \mathrm{W}$ & $\mathrm{N}$ & 130 & 98 & 0011.55 & 366112 & $6600-03$ & 1DG22009 & $66^{\circ} 12^{\prime} \mathrm{N}$ & $52^{\circ} 46^{\prime} \mathrm{V}$ & $\mathrm{NE}$ & \\
\hline 1DG18001 & $66^{\circ} 24^{\prime} \mathrm{N}$ & $51.57 \%$ & $\mathrm{~N}$ & 115 & 100 & 0003.70 & 363112 & $6600-$ & 1DG22010 & $66^{\circ} 12^{\prime} \times \mathrm{N}$ & $52^{\circ} 46^{\prime} \mathrm{V}$ & SE & 145 \\
\hline 1DG18002 & $66^{\circ} 19^{\prime} \mathrm{N}$ & $52^{\circ} 02 \cdot \mathrm{v}$ & $\mathrm{NE}$ & 170 & 70 & 0189.52 & 363112 & $6600-1$ & 1DG23001 & $66^{\circ} 13^{\prime} \mathrm{N}$ & $52^{\circ} 47 \cdot \mathrm{v}$ & $\begin{array}{l}\mathrm{SE} \\
\mathrm{NW}\end{array}$ & \\
\hline 1DG18003 & $66^{\circ} 22^{\prime} \mathrm{N}$ & $52^{\circ} 07 \cdot \mathrm{W}$ & E & 160 & 85 & 0030.80 & 363112 & $6600-02$ & 1DG23002 & $66^{\circ} 13^{\prime} \mathrm{N}$ & $52^{\circ} 4 \mathrm{~B}^{\prime} \mathrm{W}$ & $\mathrm{N}$ & $\begin{array}{l}140 \\
110\end{array}$ \\
\hline 1DG18004 & $66^{\circ} 24^{\prime} \mathrm{N}$ & $52^{\circ} 05^{\prime} \mathrm{W}$ & $\bar{E}$ & 125 & 120 & 0000.04 & 770010 & $6600-02$ & 1DG23003 & $66^{\circ} 12^{\prime} \mathrm{N}$ & $52^{\circ} 48^{\prime} \mathrm{W}$ & $\begin{array}{l}\mathrm{N} \\
\mathrm{NW}\end{array}$ & \\
\hline 1DG18005 & $66^{\circ} 24^{\prime} \mathrm{N}$ & $52^{\circ} 06^{\prime} \mathrm{W}$ & $\mathrm{NE}$ & 126 & 125 & 0000.03 & 770010 & $6600-02$ & 1DG23004 & $66^{\circ} 12^{\prime} \mathrm{N}$ & $52^{\circ} 49 \cdot \mathrm{W}$ & N & \\
\hline 1DG18006 & $66^{\circ} 25^{\prime} \mathrm{N}$ & $52^{\circ} 07 \cdot \mathrm{V}$ & NE & 110 & 105 & 0000.05 & 770010 & 6600 & 1DG23005 & $66^{\circ} 12^{\prime} \mathrm{N}$ & $52^{\circ} 50^{\prime} \mathrm{V}$ & $\mathrm{NE}$ & 1 \\
\hline 1DG18007 & $66^{\circ} 24^{\prime} \mathrm{N}$ & $52^{\circ} 07.6$ & N & 125 & 120 & 0000.03 & 770010 & $6600-02$ & 1DG23006 & $66^{\circ} 11^{\prime} \mathrm{N}$ & $52^{\circ} 49^{\circ} \mathrm{W}$ & NU & \\
\hline 1DG18008 & $66^{\circ} 23^{\prime} \mathrm{N}$ & $52^{\circ} 09^{\prime} \mathrm{W}$ & NE & 160 & 75 & 0013.43 & 363122 & $6600-02$ & 1DG23007 & $66^{\circ} 10^{\prime} \mathrm{N}$ & $52^{\circ} 49^{\prime} \mathrm{W}$ & $\mathrm{N}$ & \\
\hline 1DG18009 & $66^{\circ} 23^{\prime} \mathrm{N}$ & $52^{\circ} 12^{\prime} \mathrm{W}$ & $\mathrm{NE}$ & 160 & 100 & 0012.57 & 300010 & $6600-02$ & 1DG23008 & $66^{\circ} 10^{\prime} \mathrm{N}$ & $52^{\circ} 52^{\prime} \mathrm{W}$ & $\mathrm{NE}$ & \\
\hline 1DG18010 & $66^{\circ} 23^{\prime} \mathrm{N}$ & $51^{\circ} 15^{\prime} \mathrm{W}$ & N & 160 & 70 & 0012.56 & 363312 & $6600-03$ & 1DG23009 & $66^{\circ} 11^{\prime} \mathrm{N}$ & $52^{\circ} 53^{\prime} \mathrm{W}$ & N & \\
\hline 1DG18011 & $66^{\circ} 23^{\prime} \mathrm{N}$ & $52^{\circ} 18^{\prime}$ ฟ & $\mathrm{N}$ & 160 & 90 & 0012.41 & 363012 & 660 & 1DG23010 & $66^{\circ} 11^{\prime} \mathrm{N}$ & $52^{\circ} 54^{\prime} \mathrm{W}$ & $\mathrm{NE}$ & 1 \\
\hline 1DG18012 & $66^{\circ} 23^{\prime} \mathrm{N}$ & $52^{\circ} 19^{\prime} \mathrm{V}$ & $\mathrm{N}$ & 160 & 115 & 0003.61 & 300010 & $6600-$ & 1DG23011 & $66^{\circ} 11$ 'N & $52 \circ 54, \mathrm{~W}$ & W & \\
\hline 1DG18013 & $66^{\circ} 23^{\prime} \mathrm{N}$ & $52^{\circ} 20^{\prime} \mathrm{W}$ & $\mathrm{N}$ & 160 & 90 & 0003.35 & 363312 & 6600 & 1DG24001 & $66^{\circ} 10^{\prime}, \mathrm{N}$ & $52 \circ 53, \mathrm{v}$ & SW & \\
\hline 1DG18014 & $66^{\circ} 24^{\prime} \mathrm{N}$ & $52^{\circ} 24^{\prime} \mathrm{W}$ & $\mathrm{NW}$ & 140 & 135 & 0000.09 & 780010 & 660 & 1DG24002 & & $52 \circ 52^{\prime} \mathrm{v}$ & WW & \\
\hline 1DG19001 & $66^{\circ} 23^{\prime} \mathrm{N}$ & $52^{\circ} 21^{\prime} \mathrm{W}$ & $\mathrm{N}$ & 160 & 125 & 0006.30 & 300112 & 6600 & 1DG24003 & $66^{\circ}$ & $52^{\circ} 55 \mathrm{~W}$ & N & 1 \\
\hline 1DG19002 & $66^{\circ} 22^{\prime} \mathrm{N}$ & $52023 \cdot \mathrm{W}$ & NW & 160 & 50 & 0008.65 & 363312 & 6600 & 1DG24004 & $66^{\circ} 08^{\prime} \mathrm{N}$ & $52^{\circ} 56^{\prime} \mathrm{W}$ & $\mathrm{N}$ & $\begin{array}{l}100 \\
125\end{array}$ \\
\hline 1DG19003 & $66^{\circ} 22, \mathrm{~N}$ & $52^{\circ} 23,6$ & NW & 160 & 120 & 0003.94 & 300010 & $6600-02$ & 1DG24005 & 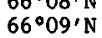 & $52^{\circ} 57 \cdot \mathrm{V}$ & $\begin{array}{l}\mathrm{N} \\
\mathrm{N}\end{array}$ & \\
\hline 1DG19004 & $66^{\circ} 21 \cdot \mathrm{N}$ & $52^{\circ} 24, y$ & NW & 160 & 140 & 0001.59 & 300012 & & $1 D G 24006$ & $66^{\circ} 09^{\circ} N$ & $52^{\circ} 58^{\prime} \mathrm{W}$ & N & 100 \\
\hline 1DG19005 & $66^{\circ} 22 \cdot \mathrm{N}$ & $52^{\circ} 27 i \mathrm{~W}$ & v & 14 ? & 105 & 0000.91 & 770010 & & 1DG24007 & & $52^{\circ} 58^{\prime}$ & $\begin{array}{l}N \\
N\end{array}$ & \\
\hline 1DG19006 & $66^{\circ} 21^{\prime} \mathrm{N}$ & $52^{\circ} 26^{\prime} \mathrm{W}$ & $\mathrm{v}$ & 145 & 145 & 0000.21 & 750010 & & 1DG25001 & $66^{\circ} 09^{\prime} \mathrm{N}$ & $53^{\circ} 00^{\circ} \mathrm{W}$ & NW & 140 \\
\hline 1DG20001 & $66^{\circ} 21 \cdot \mathrm{N}$ & $52^{\circ} 24^{\prime \prime W}$ & $\mathrm{v}$ & 160 & 145 & 0001.26 & 300010 & & $10 G 25$ & $66^{\circ} 08^{\prime}, \mathrm{N}$ & $52^{\circ} 57 \mathrm{~V}$ & s & \\
\hline 1DG20002 & $66^{\circ} 20^{\prime} \mathrm{N}$ & $52^{\circ} 23^{\prime \prime W}$ & $\mathrm{~V}$ & 16 & 80 & 0007.7 & 363312 & 66 & 1DG & 3 & 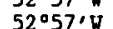 & y & $\begin{array}{l}140 \\
120\end{array}$ \\
\hline 1DG20003 & $66^{\circ} 20^{\prime} \mathrm{N}$ & $52^{\circ} 25, \mathrm{~W}$ & $\mathrm{H}$ & 16 & 135 & 0000 . & 300 & & $1 D G 25004$ & & $53^{\circ} 00^{\circ} \mathrm{V}$ & NQ & 140 \\
\hline 1DG20004 & $66^{\circ} 20^{\prime} \mathrm{N}$ & $52^{\circ} 27, \mathrm{~W}$ & NW & 16 & 90 & 000 & 643312 & & 1DG25005 & , N & $53^{\circ} 02^{\circ} \mathrm{v}$ & $N$ & $\begin{array}{l}140 \\
112\end{array}$ \\
\hline 1DG20005 & $66^{\circ} 20^{\prime} \mathrm{N}$ & $52^{\circ} 28^{\prime} \mathrm{W}$ & NW & 16. & 100 & 0000.84 & 670010 & 660 & IDG & $66^{\circ}$ & $53^{\circ} 03^{\prime} \mathrm{V}$ & NE & 12 \\
\hline 1DG20006 & $66^{\circ} 19^{\prime} \mathrm{N}$ & $52^{\circ} 24^{\prime} \mathrm{V}$ & $\mathrm{NW}$ & 160 & 100 & 0014.43 & 363312 & & 1DG26001 & $8^{\prime} \mathrm{N}$ & $53^{\circ} 05^{\circ}$ & NW & 100 \\
\hline 1DG20007 & $66^{\circ} 17 / \mathrm{N}$ & $52^{\circ} 24^{\prime} \mathrm{V}$ & $\mathrm{N}$ & 16 & 70 & 0049.54 & 363312 & $6600-02$ & 1DG26002 & $\mathbf{n}^{\mathrm{N}}$ & $53^{\circ} 04^{\prime} \mathrm{V}$ & w & 100 \\
\hline 1DG20008 & $66^{\circ} 17^{\prime} \mathrm{N}$ & $52^{\circ} 32^{\prime} \mathrm{V}$ & $\mathrm{N}$ & 13 & 130 & & 700010 & & 1DG26003 & , & $53^{\circ} 03^{\prime} \mathrm{W}$ & W & 110 \\
\hline 1DG20009 & $66^{\circ} 17 / \mathrm{N}$ & 52.33 .6 & NE & 130 & 130 & 0000.17 & 780010 & 6600 & 1DG26 & 66 & $53^{\circ} 07 \cdot \mathrm{W}$ & $\mathrm{N}$ & 140 \\
\hline 1DG20010 & $66^{\circ} 18^{\prime} \mathrm{N}$ & $52^{\circ} 36^{\prime} \mathrm{W}$ & B & 135 & 85 & 0000.90 & 643112 & 6600 & 1DG27001 & $\begin{array}{l}800 \\
66\end{array}$ & 9 & W & $\begin{array}{l}140 \\
100\end{array}$ \\
\hline 1DG20011 & $66^{\circ} 18^{\prime} N$ & $52^{\circ} 35, \mathrm{~V}$ & NE & 13 & 60 & 0002 & 643112 & & 1DG2 & $6 \cdot \mathrm{N}$ & $53^{\circ} 10^{\circ} \mathrm{W}$ & NW & \\
\hline 1DG20012 & $66^{\circ} 20^{\prime} \mathrm{N}$ & $52^{\circ} 34^{\prime} \mathrm{W}$ & NW & 11 & 100 & & 780010 & & 1DG27003 & & $10 . \mathrm{W}$ & NW & 110 \\
\hline 1DG20013 & $66^{\circ} 19 \cdot \mathrm{N}$ & $52^{\circ} 36^{\prime} \mathrm{V}$ & N & 135 & 110 & & 700010 & & 1DG27004 & 66 & $53^{\circ} 13^{\prime} \mathrm{W}$ & NW & 130 \\
\hline 1DG20014 & $66^{\circ} 19^{\prime} \mathrm{N}$ & $52^{\circ} 37 \mathrm{~V}$ & N & 135 & 110 & 0000.32 & 643112 & 6600 & 1DG27 & & $53^{\circ} 13^{\prime} \mathrm{V}$ & NV & 70 \\
\hline 1DG20015 & $66^{\circ} 19^{\prime} \mathrm{N}$ & $52^{\circ} 37 \% \mathrm{~V}$ & NW & 135 & 110 & 0000.75 & 643112 & 6600 & $1 D G 27006$ & $66^{\circ}$ & $53^{\circ} 13^{\prime} \mathrm{W}$ & SW & 100 \\
\hline 001 & $66^{\circ} 18^{\prime} N$ & $52^{\circ} 38^{\prime} \mathrm{V}$ & W & 130 & 85 & 0000.85 & 643112 & 6600 & 1DG27007 & $\begin{array}{l}60 \\
66\end{array}$ & $53^{\circ} 12^{\prime} \mathrm{V}$ & $\mathrm{W}$ & 100 \\
\hline 1DG & $66^{\circ} 18^{\prime} \mathrm{N}$ & $52^{\circ} 39^{\circ} \mathrm{W}$ & SH & 13 & 120 & & 700 & & 1DG27008 & $05, \mathrm{~N}$ & $53^{\circ} 14^{\prime} \mathrm{W}$ & $\mathrm{NH}$ & \\
\hline 1DG21003 & $66^{\circ} 18^{\prime} \mathrm{N}$ & $52^{\circ} 38^{\prime} \mathrm{V}$ & SW & 130 & 120 & 0000.31 & 700010 & $6600-02$ & 1DG27009 & $66^{\circ} 05^{\prime} \mathrm{N}$ & $53^{\circ} 15^{\prime} \mathrm{W}$ & $\mathrm{NW}$ & $\begin{array}{l}90 \\
100\end{array}$ \\
\hline 1DG21004 & $66^{\circ} 18^{\prime} \mathrm{N}$ & $52^{\circ} 37, \mathrm{~V}$ & $\mathrm{v}$ & 140 & 90 & 0001.33 & 643512 & $6600-02$ & 1DG27 & & $53^{\circ} 18^{\prime}$ & N & 100 \\
\hline 1DG21005 & $66^{\circ} 17^{\prime} \mathrm{N}$ & $52^{\circ} 37$, V & $\mathrm{v}$ & 14 & 130 & 0001.49 & 700012 & 660 & 1DG27011 & $5, \mathrm{~N}$ & $53^{\circ} 18^{\prime} \mathrm{V}$ & N & 95 \\
\hline 1DG21006 & $66^{\circ} 17 \cdot \mathrm{N}$ & $52^{\circ} 34^{\prime} \mathrm{W}$ & s & 13 & 130 & & 780010 & & 1DG27012 & $66^{\circ} 04^{\prime} \mathrm{N}$ & $53^{\circ} 18^{\prime \prime}$ & NV & \\
\hline 1DG21007 & $66^{\circ} 16^{\prime} \mathrm{N}$ & $52^{\circ} 29^{\prime} \mathrm{V}$ & Nh & 170 & 125 & 0006.49 & 363212 & $6600-02$ & 1DG27013 & $\begin{array}{l}66^{\circ} 03^{\prime} \mathrm{N} \\
\text { ton }\end{array}$ & $53^{\circ} 21^{\prime} \mathrm{V}$ & NE & 65 \\
\hline 1DG21008 & $66^{\circ} 15^{\prime} \mathrm{N}$ & $52^{\circ} 29^{\prime} \mathrm{V}$ & NW & 170 & 90 & 0004.92 & 363412 & $6600-02$ & 1 DG27014 & $66^{\circ} 03^{\prime} \mathrm{N}$ & $53^{\circ} 23^{\prime} \mathrm{W}$ & $\mathrm{N}$ & 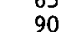 \\
\hline IDG21009 & & $52^{\circ} 33^{\prime \prime V}$ & - & 140 & 140 & 0000.21 & 780010 & $6600-02$ & 1DG27015 & $66^{\circ}$ & $53^{\circ} 24^{\prime} \mathrm{W}$ & N & \\
\hline IDG21010 & $66^{\circ} 16^{\prime} \mathrm{N}$ & $52^{\circ} 34^{\prime} \mathrm{V}$ & $\mathrm{SE}$ & 146 & 130 & 0000 & 780010 & 660 & 1DG28013 & $\begin{array}{l}66^{\circ} 03^{\prime} \mathrm{N} \\
n^{2} \\
6\end{array}$ & $53^{\circ} 22,0$ & su & \\
\hline 1DG21011 & $66^{\circ} 15^{\prime} \mathrm{N}$ & $52^{\circ} 35^{\prime} \mathrm{V}$ & $\mathrm{NV}$ & 170 & 15 & 0031.29 & 363411 & $6600-02$ & 1DG28002 & $66^{\circ} 03^{\prime} \mathrm{N}$ & $53^{\circ} 20^{\prime} \mathrm{W}$ & $\mathrm{W}$ & 90 \\
\hline
\end{tabular}

$\begin{array}{rccc}\text { LOW } & \text { AREA } & \text { MODE } & \text { MAP } \\ & & & \\ 70 & 0001.14 & 643212 & 6600-02 \\ 125 & 0000.30 & 780010 & 6600-02 \\ 20 & 0013.65 & 363311 & 6600-02 \\ 150 & 0000.50 & 200010 & 6600-02 \\ 130 & 0000.09 & 780010 & 6600-02 \\ 115 & 0000.05 & 780010 & 6600-02 \\ 125 & 0000.15 & 780010 & 6600-02 \\ 130 & 0000.10 & 700010 & 6600-02 \\ 130 & 0000.14 & 700010 & 6600-02 \\ 140 & 0000.65 & 200010 & 6600-02 \\ 160 & 0001.16 & 363112 & 6600-02 \\ 85 & 0001.10 & 363112 & 6600-02 \\ 10 & 0025.93 & 363312 & 6600-02 \\ 50 & 0000.35 & 643112 & 6600-02 \\ 40 & 0000.74 & 643312 & 6600-02 \\ 120 & 0000.49 & 700010 & 6600-02 \\ 130 & 0000.17 & 700010 & 6600-02 \\ 40 & 0000.78 & 643112 & 6600-02 \\ 135 & 0000.32 & 700010 & 6600-02 \\ 40 & 0001.81 & 643112 & 6600-02 \\ 120 & 0000.39 & 200010 & 6600-02 \\ 75 & 0002.95 & 363312 & 6600-02 \\ 25 & 0006.73 & 363312 & 6600-02 \\ 125 & 0000.34 & 750010 & 6600-02 \\ 50 & 0000.43 & 640010 & 6600-02 \\ 50 & 0000.27 & 750010 & 6600-02 \\ 70 & 0000.85 & 643212 & 6600-02 \\ 115 & 0000.67 & 780010 & 6600-02 \\ 90 & 0011.51 & 363312 & 6600-02 \\ 50 & 0000.51 & 643212 & 6600-02 \\ 50 & 0000.24 & 643212 & 6600-01 \\ 50 & 0000.54 & 643212 & 6600-01 \\ 65 & 0000.16 & 643212 & 6600-01 \\ 30 & 0000.33 & 643312 & 6600-01 \\ 125 & 0000.74 & 643312 & 6600-01 \\ 125 & 0000.08 & 780010 & 6600-01 \\ 110 & 0000.09 & 780010 & 6600-01 \\ 15 & 0013.42 & 363312 & 6600-01 \\ 95 & 0000.70 & 650510 & 6600-01 \\ 70 & 0000.36 & 780010 & 6600-01 \\ 25 & 0001.48 & 363112 & 6600-01 \\ 60 & 0002.34 & 363112 & 6600-01 \\ 90 & 0000.61 & 360010 & 6600-01 \\ 95 & 0000.56 & 770010 & 6600-01 \\ 65 & 0000.11 & 750010 & 6600-01 \\ 35 & 0001.55 & 643112 & 6600-01 \\ 105 & 0000.13 & 780010 & 6600-01 \\ 15 & 0001.17 & 643112 & 6600-01 \\ 40 & 0000.70 & 750010 & 6600-01 \\ 85 & 0000.07 & 780010 & 6600-01 \\ 70 & 0000.27 & 640010 & 6600-01 \\ 35 & 0001.17 & 673112 & 6600-01 \\ 15 & 0002.96 & 613312 & 6600-01 \\ 50 & 0000.12 & 750010 & 6600-01 \\ 70 & 0000.31 & 700010 & 6600-01 \\ 15 & 0002.88 & 643212 & 6600-01 \\ 40 & 0000.37 & 750010 & 6600-01 \\ 40 & 0000.30 & 750010 & 6600-01 \\ 20 & 0000.17 & 780010 & 6600-01 \\ 50 & 0000.40 & 780010 & 6600-01 \\ 60 & 0000.10 & 780010 & 6600-01 \\ & & & \end{array}$




\begin{tabular}{|c|c|c|c|c|c|c|c|c|c|c|c|c|c|c|c|c|c|}
\hline CODE & LAT & LONG & ORI & HIGH & LoW & AREA & $\begin{array}{c}\text { MO } \\
\text { CODE }\end{array}$ & MAP & CODE & LAT & LONG & ORI & HIGH & LOW & AREA & $\begin{array}{c}\text { MO } \\
\text { CODE }\end{array}$ & MAP \\
\hline 1DG28003 & $66^{\circ} 03^{\prime} \mathrm{N}$ & $53^{\circ} 18^{\prime} \mathrm{H}$ & $s$ & 85 & 60 & 0000.12 & 780010 & $6600-01$ & 1EA04013 & $66^{\circ} 21^{\prime} \mathrm{N}$ & $53^{\circ} 07^{\prime} \mathrm{W}$ & $\mathbf{N}$ & 130 & 75 & 0000.16 & 780010 & $6600-01$ \\
\hline 1DG28004 & $66^{\circ} 03^{\prime} \mathrm{N}$ & $53^{\circ} 17^{\prime} \mathrm{W}$ & SE & 100 & 70 & 0000.28 & 750010 & $6600-01$ & 1EA04014 & $66^{\circ} 20^{\prime} \mathrm{N}$ & $53^{\circ} 07^{\prime} \mathrm{W}$ & SW & 120 & 100 & 0000.09 & 700010 & $6600-01$ \\
\hline 1DG28005 & $66^{\circ} 04^{\prime} \mathrm{N}$ & $53^{\circ} 16^{\prime} \mathrm{W}$ & $\mathrm{W}$ & 100 & 80 & 0000.07 & 750010 & $6600-01$ & 1EA04015 & $66^{\circ} 20^{\prime} \mathrm{N}$ & $53^{\circ} 08^{\prime} \mathrm{W}$ & NW & 100 & 85 & 0000.05 & 750010 & $6600-01$ \\
\hline 1DG28006 & $66^{\circ} 03^{\prime} \mathrm{N}$ & $53^{\circ} 15^{\prime} \mathrm{W}$ & $\mathbf{s}$ & 90 & 65 & 0000.23 & 750010 & $6600-01$ & 1EA04016 & $66^{\circ} 19^{\prime} \mathrm{N}$ & $53^{\circ} 10^{\prime} \mathrm{W}$ & N & 90 & 75 & 0000.09 & 780010 & $6600-01$ \\
\hline 1DG28007 & $66^{\circ} 05^{\prime} \mathrm{N}$ & $53^{\circ} 07^{\prime} \mathrm{W}$ & W & 100 & 80 & 0000.27 & 700010 & $6600-01$ & 1EA04017 & $66^{\circ} 19^{\prime} \mathrm{N}$ & $53^{\circ} 11^{\prime} \mathrm{W}$ & NV & 95 & 70 & 0000.26 & 643112 & $6600-01$ \\
\hline 1DG28008 & $66^{\circ} 06^{\prime} \mathrm{N}$ & $53^{\circ} 04^{\prime} \mathrm{W}$ & w & 110 & 50 & 0004.35 & 363112 & $6600-01$ & 1EA04018 & $66^{\circ} 18^{\prime} \mathrm{N}$ & $53^{\circ} 13^{\prime} \mathrm{W}$ & NW & 75 & 55 & 0000.10 & 780010 & $6600-01$ \\
\hline 1DG28009 & $66^{\circ} 05^{\prime} \mathrm{N}$ & $53^{\circ} 05^{\prime} \mathrm{W}$ & NW & 110 & 40 & 0002.05 & 513112 & $6600-01$ & 1EA04019 & $66^{\circ} 18^{\prime} \mathrm{N}$ & $53^{\circ} 13^{\prime} \mathrm{W}$ & $\mathrm{w}$ & 70 & 50 & 0000.06 & 780010 & $6600-01$ \\
\hline 1DG28010 & $66^{\circ} 05^{\circ} \mathrm{N}$ & $53^{\circ} 07 . \mathrm{W}$ & $\mathbf{N}$ & 90 & 60 & 0000.07 & 750010 & $6600-01$ & 1EA04020 & $66^{\circ} 18^{\prime} \mathrm{N}$ & $53^{\circ} 14^{\prime} \mathrm{W}$ & w & 80 & 60 & 0000.07 & 780010 & $6600-01$ \\
\hline 1DG28011 & $66^{\circ} 05^{\prime} \mathrm{N}$ & $53^{\circ} 06^{\prime 6}$ & $\mathrm{NE}$ & 100 & 60 & 0000.11 & 750010 & $6600-01$ & 1EA05001 & $66^{\circ} 22^{\prime} \mathrm{N}$ & $52^{\circ} 58^{\prime} \mathrm{W}$ & sw & 130 & 110 & 0000.82 & 600010 & $6600-01$ \\
\hline 1DG28012 & $66^{\circ} 04^{\prime} \mathrm{N}$ & $53^{\circ} 07^{\prime} \mathrm{W}$ & NW & 95 & 45 & 0000.67 & 643112 & $6600-01$ & 1EA05002 & $66^{\circ} 21^{\prime} \mathrm{N}$ & $53^{\circ} 00^{\prime} \mathrm{V}$ & $\mathrm{W}$ & 150 & 65 & 0003.01 & 513112 & $6600-01$ \\
\hline 1DG28013 & $66^{\circ} 04^{\prime} \mathrm{N}$ & $53^{\circ} 07^{\prime} \mathrm{W}$ & $\mathrm{N}$ & 110 & 85 & 0000.22 & 750010 & $6600-01$ & 1EA05003 & $66^{\circ} 21^{\prime} \mathrm{N}$ & $53^{\circ} \mathrm{O} 2^{\prime} \mathrm{W}$ & $\mathrm{W}$ & 120 & 60 & 0001.17 & 643112 & $6600-01$ \\
\hline $1 D G 28014$ & $66^{\circ} 04^{\prime} \mathrm{N}$ & $53^{\circ} 09^{\prime} \mathrm{W}$ & $\mathrm{NW}$ & 90 & 45 & 0000.39 & 643112 & $6600-01$ & 1EA05004 & $66^{\circ} 20^{\circ} \mathrm{N}$ & $53^{\circ} 04^{\prime} \mathrm{W}$ & NW & 100 & 70 & 0000.32 & 643112 & $6600-01$ \\
\hline 1DG28015 & $66^{\circ} 03^{\prime} \mathrm{N}$ & $53^{\circ} 06^{\prime} \mathrm{W}$ & SW & 110 & 30 & 0006.42 & 363112 & $6600-01$ & 1EA05005 & $66^{\circ} 19^{\prime} \mathrm{N}$ & $53^{\circ} 04^{\prime} \mathrm{W}$ & SW & 1225 & 110 & 0000.27 & 700010 & $6600-01$ \\
\hline 1DG28016 & $66^{\circ} 02, \mathrm{~N}$ & $53^{\circ} 06^{\prime} \mathrm{V}$ & $\mathrm{N}$ & 90 & 70 & 0000.47 & 643112 & $6600-01$ & 1EA06001 & $66^{\circ} 20^{\prime} \mathrm{N}$ & $52^{\circ} 58^{\prime} \mathrm{W}$ & W & 150 & 70 & 0003.33 & 533012 & $6600-01$ \\
\hline 1DG28017 & $66^{\circ} 02^{\prime} \mathrm{N}$ & $53^{\circ} 08^{\prime} \mathrm{W}$ & $\mathrm{N}$ & 90 & 35 & 0000.81 & 643112 & $6600-01$ & 1EA06002 & $66^{\circ} 20^{\prime} \mathrm{N}$ & $52^{\circ} 54^{\prime} \mathrm{W}$ & E & 155 & 120 & 0000.25 & 643112 & $6600-02$ \\
\hline 1DG28018 & $66^{\circ} 02 \cdot \mathrm{N}$ & $53^{\circ} 10^{\prime} \mathrm{W}$ & $\mathrm{N}$ & 100 & 70 & 0000.23 & 750010 & $6600-01$ & 1EA06003 & $66^{\circ} 20^{\prime} \mathrm{N}$ & $52^{\circ} 53^{\prime} \mathrm{W}$ & SE & 150 & 140 & 0000.20 & 700010 & $6600-02$ \\
\hline 1DG28019 & $66^{\circ} 02^{\prime} \mathrm{N}$ & $53^{\circ} 11, \mathrm{~W}$ & NW & 75 & 50 & 0000.14 & 750010 & $6600-01$ & $1 \mathrm{EA06004}$ & $66^{\circ} 21 \cdot \mathrm{N}$ & $52^{\circ} 51^{\prime} \mathrm{W}$ & $\mathrm{SE}$ & 130 & 120 & 0000.32 & 700010 & $6600-02$ \\
\hline IDG28020 & $66^{\circ} 02, \mathrm{~N}$ & $53^{\circ} 11, \mathrm{~W}$ & $\mathrm{~N}$ & 100 & 80 & 0000.12 & 750110 & $6600-01$ & 1EA06005 & $66^{\circ} 21 \cdot \mathrm{N}$ & $52^{\circ} 50^{\prime} \mathrm{w}$ & $\mathrm{S}$ & 140 & 125 & 0000.07 & 770010 & $6600-02$ \\
\hline 1DG28021 & $66^{\circ} 02, \mathrm{~N}$ & $53^{\circ} 12, \mathrm{~W}$ & NW & 75 & 60 & 0000.14 & 780010 & $6600-01$ & 1EA06006 & $66^{\circ} 22 \cdot \mathrm{N}$ & $52^{\circ} 48^{\prime} \mathrm{W}$ & sw & 150 & 130 & 0002.02 & 700010 & $6600-02$ \\
\hline 1DG28022 & $66^{\circ} 02^{\prime} \mathrm{N}$ & $53^{\circ} 12^{\prime} \mathrm{V}$ & $\mathrm{N}$ & 115 & 70 & 0000.05 & 780010 & $6600-01$ & 1EA06007 & $66^{\circ} 21, \mathrm{~N}$ & $52^{\circ} 48^{\prime} \mathrm{W}$ & NW & 90 & 65 & 0001.20 & 513112 & $6600-02$ \\
\hline 1DG29001 & $66^{\circ} 03^{\prime} \mathrm{N}$ & $53^{\circ} 02^{\prime} \mathrm{W}$ & SW & 110 & 65 & 0009.12 & 300010 & $6600-01$ & 1EA06008 & $66^{\circ} 20^{\prime} \mathrm{N}$ & $52^{\circ} 50^{\prime} \mathrm{W}$ & NE & 130 & 110 & 0000.24 & 600010 & $6600-02$ \\
\hline 1DG29002 & $66^{\circ} 02^{\prime} \mathrm{N}$ & $53^{\circ} 03^{\prime} \mathrm{W}$ & s & 80 & 75 & 0000.50 & 300010 & $6600-01$ & 1EA06009 & $66^{\circ} 20^{\prime} \mathrm{N}$ & $52^{\circ} 51^{\prime} \mathrm{W}$ & NW & 150 & 90 & 0000.18 & 700010 & $6600-02$ \\
\hline 1DG29003 & $66^{\circ} 01, \mathrm{~N}$ & $53^{\circ} 06^{\prime} \mathrm{W}$ & $\mathrm{N}$ & 50 & 40 & 0000.09 & 770010 & $6600-01$ & 1EA06010 & $66^{\circ} 19^{\prime} \mathrm{N}$ & $52^{\circ} 51^{\prime} \mathrm{W}$ & $\mathrm{S}$ & 150 & 130 & 0000.70 & 700010 & $6600-02$ \\
\hline 1DG29004 & $66^{\circ} 00^{\prime} \mathrm{N}$ & $53^{\circ} 07^{\prime} \mathrm{W}$ & $\mathrm{N}$ & 90 & 60 & 0000.10 & 770010 & $6600-01$ & 1EA06011 & $66^{\circ} 17^{\prime} \mathrm{N}$ & $52^{\circ} 50^{\prime} \mathrm{W}$ & NW & 100 & 75 & 0000.15 & 643112 & $6600-02$ \\
\hline 1DG29005 & $66^{\circ} 00^{\circ} \mathrm{N}$ & $53^{\circ} 08^{\prime} \mathrm{W}$ & NW & 110 & 80 & 0000.14 & 780010 & $6600-01$ & $1 \mathrm{EA06012}$ & $66^{\circ} 17^{\prime} \mathrm{N}$ & $52^{\circ} 50^{\prime} \mathrm{W}$ & NV & 110 & 80 & 0000.16 & 643112 & $6600-02$ \\
\hline 1DG29006 & $65^{\circ} 59^{\prime} \mathrm{N}$ & $53^{\circ} 08^{\prime} \mathrm{K}$ & N & 100 & 50 & 0000.13 & 780010 & $6530-01$ & 1EA06013 & $66^{\circ} 17^{\prime} \mathrm{N}$ & $52^{\circ} 50^{\prime} \mathrm{W}$ & NW & 140 & 130 & 0000.08 & 700010 & $6600-02$ \\
\hline $1 D G 29007$ & $65^{\circ} 59 \cdot \mathrm{N}$ & $53^{\circ} 10 ! \mathrm{W}$ & $\mathrm{N}$ & & 15 & 0000.16 & 640110 & $6530-01$ & 1EA06014 & $66^{\circ} 17^{\prime} \mathrm{N}$ & $52^{\circ} 52^{\prime} \mathrm{W}$ & $\mathrm{NE}$ & 95 & 65 & 0001.89 & 643112 & $6600-02$ \\
\hline 1DG29008 & $65^{\circ} 59^{\circ} \mathrm{N}$ & $53^{\circ} 12, \mathrm{~W}$ & $\mathrm{NE}$ & 50 & & 0000.48 & 643212 & $6530-01$ & 1EA06015 & $66^{\circ} 18^{\prime} \mathrm{N}$ & $52^{\circ} 55^{\prime} \mathrm{W}$ & N & 120 & 60 & 0000.97 & 643112 & $6600-02$ \\
\hline IDG & $65^{\circ} 58^{\prime} \mathrm{N}$ & $53^{\circ} 13^{\prime} \mathrm{w}$ & NW & 100 & 40 & 0001.71 & 643112 & $6530-01$ & 1EA06016 & $66^{\circ} 17^{\circ} \mathrm{N}$ & $52^{\circ} 58^{\prime} \mathrm{W}$ & $\mathrm{W}$ & 65 & 50 & 0000.11 & 643112 & $6600-01$ \\
\hline 1DG29010 & $65^{\circ} 59^{\prime} \mathrm{N}$ & $53^{\circ} 16^{\prime} \mathrm{W}$ & $\mathrm{N}$ & & 30 & 0000.39 & 643212 & $6530-01$ & 1EA06017 & $66^{\circ} 16^{\prime} \mathrm{N}$ & $52^{\circ} 55^{\prime} \mathrm{W}$ & $\mathrm{NE}$ & 110 & 90 & 0000.57 & 670112 & $6600-02$ \\
\hline 1DG29011 & $65^{\circ} 59^{\prime} \mathrm{N}$ & $53^{\circ} 17^{\prime} \mathrm{V}$ & $\mathbf{N}$ & 65 & 40 & 0000.48 & 643212 & $6530-01$ & 1EA07001 & $66^{\circ} 16^{\prime} \mathrm{N}$ & $52^{\circ} 58^{\prime} \mathrm{W}$ & $\mathrm{N}$ & 125 & 100 & 0000.36 & 643112 & $6600-01$ \\
\hline 1DG29012 & $65^{\circ} 59^{\prime} \mathrm{N}$ & $53^{\circ} 18^{\prime} \mathrm{W}$ & $\mathbf{N}$ & 60 & 50 & 0000.18 & 750112 & $6530-01$ & 1EA07002 & $66^{\circ} 15^{\prime} \mathrm{N}$ & $52^{\circ} 56^{\prime} \mathrm{W}$ & NW & 120 & 90 & 0000.17 & 780010 & $6600-01$ \\
\hline 1DG29013 & $65^{\circ} 59^{\prime} \mathrm{N}$ & $53^{\circ} 19^{\prime} \mathrm{W}$ & N & 80 & 60 & 0000.44 & 600010 & $6530-01$ & 1EA07003 & $66^{\circ} 15^{\prime} \mathrm{N}$ & $52^{\circ} 57 \cdot \mathrm{W}$ & $\mathrm{NE}$ & 100 & 80 & 0000.26 & 770010 & $6600-01$ \\
\hline 1DG2 & $65^{\circ} 58^{\prime} \mathrm{N}$ & $53^{\circ} 21^{\prime} \mathrm{W}$ & N & & 25 & 0000.20 & 643010 & $6530-01$ & 1EA07004 & $66^{\circ} 15^{\prime} \mathrm{N}$ & $52^{\circ} 58^{\prime} \mathrm{W}$ & $\mathrm{NE}$ & 140 & 110 & 0000.23 & 700010 & $6600-01$ \\
\hline 1DG29015 & $65^{\circ} 58^{\prime} \mathrm{N}$ & $53^{\circ} 72 \mathrm{~W}$ & $\mathrm{~N}$ & 55 & 35 & 0000.18 & 643010 & $6530-01$ & 1EA07005 & $66^{\circ} 15^{\prime} \mathrm{N}$ & $52^{\circ} 59^{\prime} \mathrm{W}$ & $\mathrm{NE}$ & 130 & 70 & 0000.57 & 643512 & $6600-01$ \\
\hline 1DG29016 & $65^{\circ} 58^{\prime} \mathrm{N}$ & $53^{\circ} 23^{\prime} \mathrm{W}$ & NW & 50 & 25 & 0000.31 & 643012 & 653 & 1EA07006 & $66^{\circ} 16^{\prime} \mathrm{N}$ & $53^{\circ} 04^{\prime} \mathrm{W}$ & NE & 120 & 110 & 0000.13 & 780010 & $6600-01$ \\
\hline 1DG29017 & $65^{\circ} 58^{\prime} \mathrm{N}$ & $53^{\circ} 25^{\prime} \mathrm{W}$ & NW & & 15 & 0000.19 & 740012 & $6530-01$ & 1BA07007 & $66^{\circ} 16^{\prime} \mathrm{N}$ & $53^{\circ} 06^{\prime} \mathrm{W}$ & $\mathrm{NE}$ & 100 & 100 & 0000.03 & 780010 & $6600-01$ \\
\hline 1DG29018 & $65^{\circ} 58^{\prime} \mathrm{N}$ & $53^{\circ} 26^{\prime} \mathrm{W}$ & NW & 65 & 30 & 0000.30 & 643112 & $6530-01$ & 1EA08001 & $66^{\circ} 16^{\prime} \mathrm{N}$ & $53^{\circ} 07, \mathrm{~W}$ & $\mathrm{~W}$ & 105 & 90 & 0000.20 & 643010 & $6600-01$ \\
\hline 1DG29019 & $65^{\circ} 58^{\prime} \mathrm{N}$ & $53^{\circ} 27 \cdot \mathrm{W}$ & $\mathrm{w}$ & 45 & 20 & 0000.07 & 780010 & $6530-01$ & 1EA08002 & $66^{\circ} 15^{\prime} \mathrm{N}$ & $53^{\circ} 01^{\prime} \mathrm{w}$ & $\mathrm{w}$ & 140 & 70 & 0002.00 & 513312 & $6600-01$ \\
\hline 1EA01001 & $66^{\circ} 18^{\prime} \mathrm{N}$ & $53^{\circ} 32^{\prime} \mathrm{W}$ & $\mathrm{w}$ & 125 & 60 & 0003.54 & 200110 & $6600-01$ & 1EA08003 & $66^{\circ} 15^{\prime} \mathrm{N}$ & $53^{\circ} 03^{\prime} \mathrm{W}$ & NW & 130 & 90 & 0000.29 & 643012 & $6600-01$ \\
\hline 1 EA01002 & $66^{\circ} 17^{\prime} \mathrm{N}$ & $53^{\circ} 25^{\prime} \mathrm{W}$ & W & 90 & 50 & 0000.66 & 643012 & $6600-01$ & 1EA08004 & $66^{\circ} 14^{\prime} \mathrm{N}$ & $53^{\circ} 02^{\prime} \mathrm{W}$ & NW & 130 & 80 & 0003.21 & 513312 & $6600-01$ \\
\hline 1EA01003 & $66^{\circ} 18^{\prime} \mathrm{N}$ & $53^{\circ} 26^{\prime} \mathrm{W}$ & NW & 70 & 45 & 0000.14 & 643112 & $6600-01$ & 1EA08005 & $66^{\circ} 13^{\prime} \mathrm{N}$ & $53^{\circ} 04^{\prime} \mathrm{W}$ & NW & 110 & 70 & 0000.93 & 643212 & $6600-01$ \\
\hline 1EA01004 & $66^{\circ} 16^{\prime} \mathrm{N}$ & $53^{\circ} 31^{\prime} \mathrm{W}$ & $\mathrm{N}$ & 65 & 40 & 0000.70 & 643112 & $6600-01$ & 1EA08006 & $66^{\circ} 12^{\prime} \mathrm{N}$ & $53^{\circ} 05^{\prime} \mathrm{W}$ & $\mathrm{N}$ & 100 & 55 & 0001.22 & 673112 & $0-01$ \\
\hline 1EA01005 & $66^{\circ} 06^{\prime} \mathrm{N}$ & $53^{\circ} 32^{\prime} \mathrm{W}$ & NW & 55 & 40 & 0000.50 & 643112 & $6600-01$ & 1EA08007 & $66^{\circ} 13^{\prime} \mathrm{N}$ & $53^{\circ} 06^{\prime} \mathrm{W}$ & NE & 100 & 85 & 0000.28 & 750010 & $6600-01$ \\
\hline 1EA01006 & $66^{\circ} 16^{\prime} \mathrm{N}$ & $53^{\circ} 35^{\prime} \mathrm{W}$ & w & 70 & 50 & 0000.16 & 750010 & & 1EA08008 & $66^{\circ} 14^{\prime} \mathrm{N}$ & $53^{\circ} 09^{\prime} \mathrm{W}$ & E & 105 & 85 & 0000.30 & 643112 & $6600-01$ \\
\hline 1EA02001 & $66^{\circ} 17^{\prime} \mathrm{N}$ & $53^{\circ} 25^{\prime} \mathrm{W}$ & E & 95 & 80 & 000 & 643112 & & 1EA08009 & $66^{\circ} 15^{\prime N}$ & $53^{\circ} 10^{\prime} \mathrm{W}$ & E & 110 & 95 & 0000.11 & 643112 & $6600-01$ \\
\hline 1EA02002 & $66^{\circ} 18^{\prime} \mathrm{N}$ & $53^{\circ} 24^{\prime} \mathrm{W}$ & SE & 100 & 80 & 0000.32 & 700010 & $6600-01$ & 1EA08010 & $66^{\circ} 15^{\prime} \mathrm{N}$ & $53^{\circ} 11^{\prime} \mathrm{W}$ & E & 115 & 90 & 0000.09 & 780010 & $6600-01$ \\
\hline 1EA03001 & $66^{\circ} 18^{\prime} \mathrm{N}$ & $53^{\circ} 22^{\prime} \mathrm{W}$ & $\mathrm{SE}$ & 100 & 75 & 0000.59 & 750010 & $6600-01$ & 1EA08011 & $66^{\circ} 16^{\prime} \mathrm{N}$ & $53^{\circ} 11^{\prime} \mathrm{W}$ & N & 70 & 40 & 0000.07 & 780010 & $6600-01$ \\
\hline $1 \mathrm{EA0} 3002$ & $66^{\circ} 19^{\prime} \mathrm{N}$ & $53^{\circ} 21^{\prime} \mathrm{W}$ & $\mathrm{SE}$ & 100 & 85 & 0000.12 & 700010 & $6600-01$ & 1EA09001 & $66^{\circ} 15^{\prime} \mathrm{N}$ & $53^{\circ} 13^{\prime} \mathrm{W}$ & NW & 105 & 90 & 0000.28 & 750010 & $6600-01$ \\
\hline 1EA04001 & $66^{\circ} 20^{\prime} \mathrm{N}$ & $53^{\circ} 15^{\prime} \mathrm{W}$ & E & 100 & 70 & 0000.16 & 750010 & $6600-01$ & 1EA09002 & $66^{\circ} 15^{\prime} \mathrm{N}$ & $53^{\circ} 12^{\prime} \mathrm{W}$ & NW & 80 & 30 & 0001.21 & 533012 & $6600-01$ \\
\hline 1EA04002 & $66^{\circ} 211^{\prime} \mathrm{N}$ & $53^{\circ} 15^{\prime} \mathrm{W}$ & $\mathrm{SE}$ & 100 & 80 & 0000.13 & 700010 & $6600-01$ & 1EA09003 & $66^{\circ} 14^{\prime} \mathrm{N}$ & $53^{\circ} 13^{\prime} \mathrm{W}$ & NV & 120 & 90 & 0000.50 & 700010 & 6600-01 \\
\hline 1EA04003 & $66^{\circ} 21 \cdot \mathrm{N}$ & $53^{\circ} 13^{\prime} \mathrm{V}$ & NE & 120 & 80 & 0000.14 & 700010 & $6600-01$ & $1 \mathrm{EA} 09$ & $66^{\circ} 14^{\prime} \mathrm{N}$ & $53^{\circ} 15^{\prime} \mathrm{W}$ & NV & 90 & 40 & 0001.48 & 533112 & $6600-01$ \\
\hline 1 EA04004 & $66^{\circ} 22 \cdot \mathrm{N}$ & $53^{\circ} 13^{\prime} \mathrm{W}$ & $\mathrm{NE}$ & 120 & 70 & 0000.65 & 643112 & $6600-01$ & 1EA09005 & $66^{\circ} 14^{\prime} \mathrm{N}$ & $53^{\circ} 15^{\prime} \mathrm{W}$ & NW & 130 & 95 & 0000.36 & 513112 & $6600-01$ \\
\hline 1EA04005 & $66^{\circ} 21^{\prime} \mathrm{N}$ & $53^{\circ} 14^{\prime} \mathrm{W}$ & E & 140 & 125 & & 70 & & $1 \mathrm{EAO}$ & $66^{\circ} 13^{\prime} \mathrm{N}$ & $53^{\circ} 16^{\prime} \mathrm{W}$ & N. & 105 & 50 & 0002.63 & 643112 & $6600-01$ \\
\hline 1EA04006 & $66^{\circ} 24^{\prime} \mathrm{N}$ & $53^{\circ} 09^{\prime} \mathrm{W}$ & SE & 150 & 105 & 0000.36 & 643112 & $6600-01$ & 1EA09007 & $66^{\circ} 14^{\prime} \mathrm{N}$ & $53^{\circ} 19^{\prime} \mathrm{W}$ & W & 85 & 65 & 0000.16 & 643112 & $6600-01$ \\
\hline 1EA04007 & $66^{\circ} 24^{\prime} \mathrm{N}$ & $53^{\circ} 02^{\prime} \mathrm{W}$ & $\mathrm{NW}$ & 120 & 80 & 0000.47 & 750010 & $6600-01$ & 1EA09008 & $66^{\circ} 13^{\prime} \mathrm{N}$ & $53^{\circ} 19^{\prime} \mathrm{w}$ & NW & 100 & 55 & 0000.53 & 643112 & 6600-01 \\
\hline 1EA04008 & $66^{\circ} 23^{\prime} \mathrm{N}$ & $53^{\circ} 03^{\prime} \mathrm{W}$ & NW & 100 & 45 & 0001.02 & 533112 & $6600-01$ & 1EA09009 & $66^{\circ} 13^{\prime} \mathrm{N}$ & $53^{\circ} 21^{\prime} \mathrm{w}$ & NV & 100 & 60 & 0001.09 & 643112 & $6600-01$ \\
\hline 1EA04009 & $66^{\circ} 23^{\prime} \mathrm{N}$ & $53^{\circ} 04^{\prime} \mathrm{W}$ & $\mathbf{N}$ & 130 & 90 & 0000 & 780010 & $6600-01$ & 1EA10001 & $66^{\circ} 13^{\prime} \mathrm{N}$ & $52^{\circ} 23^{\prime} \mathrm{W}$ & NW & 85 & 40 & .0001 .13 & 643112 & $6600-02$ \\
\hline 1EA04010 & $66^{\circ} 22^{\prime} \mathrm{N}$ & $53^{\circ} 05^{\prime} \mathrm{W}$ & NW & 120 & 105 & 0000.30 & 643112 & $6600-01$ & 1EA10002 & $66^{\circ} 13^{\prime} \mathrm{N}$ & $53^{\circ} 23^{\prime} \mathrm{W}$ & $\mathrm{W}$ & 70 & 30 & 0000.11 & 750010 & $6600-01$ \\
\hline 1EA04011 & $66^{\circ} 21^{\prime} \mathrm{N}$ & $53^{\circ} 06^{\prime} \mathrm{W}$ & MV & 90 & 65 & 0000.05 & 780010 & $6600-01$ & 1EA10003 & $66^{\circ} 13^{\prime} \mathrm{N}$ & $53^{\circ} 25^{\prime} \mathrm{W}$ & $\hat{\mathrm{w}}$ & 75 & 30 & 0000.17 & 643112 & $6600-01$ \\
\hline 1EA04012 & $66^{\circ} 21^{\prime} \mathrm{N}$ & $53^{\circ} 07^{\prime} \mathrm{W}$ & $\mathbf{N}$ & 100 & 75 & 0000.17 & 780010 & $6600-01$ & 1EA10004 & $66^{\circ} 13^{\prime} \mathrm{N}$ & $53^{\circ} 25, \mathrm{~W}$ & $\mathrm{w}$ & 90 & 75 & 0000.06 & 700010 & $6600-01$ \\
\hline
\end{tabular}




\begin{tabular}{|c|c|c|c|c|c|c|c|c|c|c|c|c|c|c|c|c|c|}
\hline CODE & LAT & LONG & ORI & HIGH & LOW & AREA & $\begin{array}{c}\text { MO } \\
\text { CODE }\end{array}$ & MAP & CODE & LAT & LONG & ORI & HIGH & LOH & AREA & $\begin{array}{c}\text { MO } \\
\text { CODE }\end{array}$ & MAP \\
\hline 1EA11001 & $66^{\circ} 14^{\prime} \mathrm{N}$ & $53^{\circ} 12^{\prime} \mathrm{W}$ & NW & 85 & 75 & 0000.41 & 75 & $6600-01$ & 1EB10005 & $66^{\circ} 27 \cdot N$ & $53^{\circ} 00^{\prime} \mathrm{W}$ & NW & 110 & 80 & 78 & 750010 & $6600-01$ \\
\hline 1EA11002 & $66^{\circ} 13^{\prime} \mathrm{N}$ & $53^{\circ} 111^{\prime} \mathrm{W}$ & SW & 95 & 75 & 0000.24 & 780010 & $6600-01$ & 1EB10006 & $66^{\circ} 26^{\prime} \mathrm{N}$ & $53^{\circ} 00^{\prime} \mathrm{W}$ & $\mathrm{W}$ & 105 & 60 & 0000.36 & 643112 & $6600-01$ \\
\hline 1EA11003 & $66^{\circ} 13^{\prime} \mathrm{N}$ & $53^{\circ} 07$ ' W & $\mathbf{s}$ & 100 & 90 & 0000.40 & 780010 & $6600-01$ & $1 \mathrm{~EB} 10007$ & $66^{\circ} 26^{\prime} \mathrm{N}$ & $53^{\circ} 01^{\prime} \mathrm{V}$ & NW & 140 & 80 & 0001.84 & 543112 & $6600-01$ \\
\hline 1EA11004 & $66^{\circ} 12^{\prime} \mathrm{N}$ & $53^{\circ} 08^{\prime} \mathrm{W}$ & W & 100 & 70 & 0000.30 & 780010 & $6600-01$ & 1EB10008 & $66^{\circ} 26^{\prime} \mathrm{N}$ & $52^{\circ} 58^{\prime}$ & W & 130 & 110 & 0000.23 & 700010 & 6600-01 \\
\hline 1EA11005 & $66^{\circ} 10^{\prime} \mathrm{N}$ & $53^{\circ} 11^{\prime} \mathrm{W}$ & $\mathrm{NW}$ & 105 & 80 & 0000.18 & 640112 & $6600-01$ & 1EB10009 & $66^{\circ} 25 \cdot \mathrm{N}$ & $52 \circ 54^{\prime} \mathrm{W}$ & $\hat{W}$ & 115 & 100 & 0007.47 & 513112 & $6600-02$ \\
\hline 1EA11006 & $66^{\circ} 09^{\prime} \mathrm{N}$ & $53^{\circ} 13^{\prime} \mathrm{W}$ & $\mathrm{NE}$ & 70 & 50 & 0000.20 & 750010 & $6600-01$ & 1EB10010 & $66^{\circ} 25$, N & $52^{\circ} 56^{\prime} \mathrm{W}$ & s & 150 & 120 & 0000.36 & 640112 & $6600-01$ \\
\hline 1EA11007 & $66^{\circ} 09^{\prime} \mathrm{N}$ & $53^{\circ} 14^{\prime} \mathrm{W}$ & $\mathrm{NW}$ & 90 & 80 & 0000.11 & 700010 & $6600-01$ & 1EB10011 & $66^{\circ} 24^{\prime} \mathrm{N}$ & $52^{\circ} 55^{\prime}$ & W & 145 & 135 & 0000.27 & 643112 & $6600-02$ \\
\hline 1EA11008 & $66^{\circ} 08^{\prime} \mathrm{N}$ & $53^{\circ} 16^{\prime} \mathrm{W}$ & $\mathrm{N}$ & 80 & 55 & 0000.26 & 640010 & $6600-01$ & $1 \mathrm{~EB} 10012$ & $66^{\circ} 24^{\prime} \mathrm{N}$ & $52^{\circ} 54^{\prime} \mathrm{W}$ & E & 135 & 115 & 0000.23 & 750010 & $6600-02$ \\
\hline 1EA11009 & $66^{\circ} 08^{\prime} \mathrm{N}$ & $53^{\circ} 18^{\prime} \mathrm{W}$ & $\mathrm{N}$ & 90 & 45 & 0000.85 & 643112 & $6600-01$ & 1EB10013 & $66^{\circ} 25^{\prime} \mathrm{N}$ & $52^{\circ} 51^{\prime} \mathrm{W}$ & SE & 160 & 155 & 0000.33 & 700010 & $6600-02$ \\
\hline 1EA11010 & $66^{\circ} 09^{\prime} \mathrm{N}$ & $53^{\circ} 20^{\prime} \mathrm{W}$ & $\mathrm{NW}$ & 85 & 50 & 0000.27 & 643112 & $6600-01$ & 1 EB10014 & $66^{\circ} 25, \mathrm{~N}$ & $52^{\circ} 49^{\prime} \mathrm{V}$ & $\mathrm{SE}$ & 155 & 135 & 0000.48 & 700010 & $6600-02$ \\
\hline 1EA11011 & $66^{\circ} 09^{\prime} \mathrm{N}$ & $53^{\circ} 21^{\prime} \mathrm{W}$ & $\mathrm{NV}$ & 70 & 40 & 0000.21 & 640110 & $6600-01$ & 1EB10015 & $66^{\circ} 26^{\prime} \mathrm{N}$ & $52^{\circ} 49^{\prime} \mathrm{W}$ & $\mathrm{NE}$ & 110 & 100 & 0007.14 & 533112 & $6600-02$ \\
\hline 1EA11012 & $66^{\circ} 08^{\prime} \mathrm{N}$ & $53^{\circ} 22, \mathrm{w}$ & $\mathrm{W}$ & 80 & 50 & 0000.10 & 780010 & $6600-01$ & 1EB10016 & $66^{\circ} 27 \times \mathrm{N}$ & $52^{\circ} 50^{\prime} \mathrm{V}$ & $\mathrm{SE}$ & 140 & 110 & 0000.46 & 700010 & $6600-02$ \\
\hline 1EA11013 & $66^{\circ} 08^{\prime} \mathrm{N}$ & $53^{\circ} 24^{\prime} \mathrm{W}$ & E & 85 & 70 & 0000.15 & 780010 & $6600-01$ & 1EB10017 & $66^{\circ} 27^{\prime} \mathrm{N}$ & $52^{\circ} 48^{\prime} \mathrm{v}$ & SE & 135 & 120 & 0000.54 & 780010 & $6600-02$ \\
\hline 1EA12001 & $66^{\circ} 08^{\prime} \mathrm{N}$ & $53^{\circ} 25^{\prime} \mathrm{W}$ & $\mathrm{Nb}$ & 80 & 65 & 0000.08 & 780010 & $6600-01$ & 1EB10018 & $66^{\circ} 27 \cdot N$ & $52^{\circ} 48^{\prime} \mathrm{W}$ & $\mathrm{NE}$ & 135 & 110 & 0000.84 & 203112 & $6600-02$ \\
\hline 1EA12002 & $66^{\circ} 03^{\prime} \mathrm{N}$ & $53^{\circ} 33^{\prime} \mathrm{W}$ & $\mathrm{N}$ & 70 & 45 & 0000.17 & 643112 & $6600-01$ & 1EB10019 & $66^{\circ} 28^{\prime} \mathrm{N}$ & $52^{\circ} 50^{\prime} \mathrm{V}$ & $\mathrm{E}$ & 180 & 175 & 0000.11 & 700010 & $6600-02$ \\
\hline 1EA12003 & $66^{\circ} 03, N$ & $53^{\circ} 34^{\prime} \mathrm{V}$ & NW & 55 & 30 & 0000.15 & 750010 & $6600-01$ & IEB10020 & $66^{\circ} 28^{\prime} \mathrm{N}$ & $52^{\circ} 45 \cdot \mathrm{W}$ & s & 175 & 155 & 0000.70 & 700010 & $6600-02$ \\
\hline 1EB06001 & $66^{\circ} 27 \cdot \mathrm{N}$ & $52^{\circ} 32, \mathrm{k}$ & NE & 130 & 125 & 0000.15 & 750010 & $6600-02$ & $1 \mathrm{~EB} 10021$ & $66^{\circ} 28^{\prime} \mathrm{N}$ & $52^{\circ} 46^{\prime} \mathrm{V}$ & SW & $\begin{array}{l}173 \\
160\end{array}$ & 145 & 0000.13 & 780010 & $\begin{array}{l}66000-02 \\
6600-02\end{array}$ \\
\hline 1EB06002 & $66^{\circ} 26^{\prime} \mathrm{N}$ & $52^{\circ} 34^{\prime}$ & $\mathrm{NW}$ & 145 & 110 & 0004.04 & 203112 & $6600-02$ & IEB10022 & $66^{\circ} 25^{\prime} \mathrm{N}$ & $52^{\circ} 40^{\prime} \mathrm{V}$ & $\mathrm{W}$ & 110 & 100 & 0000.30 & 600010 & $6600-02$ \\
\hline 1EB06003 & $66^{\circ} 25^{\prime} \mathrm{N}$ & $52^{\circ} 36^{\prime} \mathrm{W}$ & $\mathrm{NW}$ & 150 & 100 & 0004.50 & 513112 & $6600-02$ & $1 \mathrm{~EB} 10023$ & $66^{\circ} 24^{\prime} \mathrm{N}$ & $52^{\circ} 41^{\prime} \mathrm{W}$ & W & 155 & 135 & 0000.89 & 200010 & $6600-02$ \\
\hline 1EB06004 & $66^{\circ} 25^{\prime} \mathrm{N}$ & $52^{\circ} 38^{\prime} \mathrm{W}$ & w & 150 & 120 & 0000.69 & 750010 & $6600-02$ & $1 \mathrm{~EB} 10024$ & $66^{\circ} 23, \mathrm{~N}$ & $52^{\circ} 41, \mathrm{~V}$ & $\mathrm{~W}$ & 150 & 150 & 0000,44 & 200010 & $6600-02$ \\
\hline 1EB06005 & $66^{\circ} 25^{\prime} N$ & $52^{\circ} 36^{\prime} \mathrm{W}$ & $\mathrm{NW}$ & 155 & 145 & 0000.09 & 643112 & $6600-02$ & 1EB10025 & $66^{\circ} 23^{\prime} \mathrm{N}$ & $52^{\circ} 44^{\prime} \mathrm{V}$ & $\mathrm{NE}$ & 150 & 80 & 0010.75 & 513112 & $6600-02$ \\
\hline 1EB06006 & $66^{\circ} 24^{\prime} \mathrm{N}$ & $52^{\circ} 37^{\prime \prime} \mathrm{W}$ & $\mathrm{NW}$ & 145 & 135 & 0000.38 & 643112 & $6600-02$ & 1EB10026 & $66^{\circ} 24^{\prime} \mathrm{N}$ & $52^{\circ} 45, \mathrm{~V}$ & $\mathrm{NW}$ & 150 & 115 & 0000.94 & 643112 & $6600-02$ \\
\hline 1EB06007 & $66^{\circ} 24^{\prime} \mathrm{N}$ & $52^{\circ} 39 \cdot \mathrm{W}$ & $\mathrm{N}$ & 135 & 105 & 0007.43 & 513112 & $6600-02$ & 1EB10027 & $66^{\circ} 24^{\prime} \mathrm{N}$ & $52^{\circ} 46^{\prime} \mathrm{W}$ & $\mathrm{NW}$ & 145 & 110 & 0000.39 & 643112 & $6600-02$ \\
\hline $1 \mathrm{~EB} 06008$ & $66^{\circ} 28^{\prime} \mathrm{N}$ & $52 \circ 43 \cdot 6$ & $\mathrm{SE}$ & 110 & 95 & 0001.04 & 700010 & $6600-02$ & 1EB10028 & $66^{\circ} 23^{\prime} \mathrm{N}$ & $52^{\circ} 46^{\prime} \mathrm{W}$ & $\mathrm{S}$ & 145 & 130 & 0000.50 & 700010 & $6600-02$ \\
\hline 1EB06009 & $66^{\circ} 29^{\prime} \mathrm{N}$ & $52^{\circ} 42, \mathrm{~W}$ & E & 105 & 90 & 0000.60 & 700010 & $6600-02$ & 1EB10029 & $66^{\circ} 24^{\prime} \mathrm{N}$ & $52^{\circ} 47, \mathrm{~W}$ & NW & $\begin{array}{l}145 \\
135\end{array}$ & $\begin{array}{r}130 \\
95\end{array}$ & 0000.38 & 643112 & $\begin{array}{l}60000-02 \\
6600-02\end{array}$ \\
\hline 1EB06010 & $66^{\circ} 29^{\prime} \mathrm{N}$ & $52^{\circ} 42^{\prime} \mathrm{W}$ & $\mathrm{s}$ & 105 & 105 & 0000.08 & 700010 & $6600-02$ & 1EB10030 & $66^{\circ} 23^{\prime} \mathrm{N}$ & $52^{\circ} 47, \mathrm{~W}$ & NV & 135 & 80 & 0000.55 & 533112 & $\begin{array}{l}00000-02 \\
6600-02\end{array}$ \\
\hline 1EB07001 & $66^{\circ} 30^{\prime} \mathrm{N}$ & $52^{\circ} 42, \mathrm{~W}$ & N & 155 & 45 & 0002.84 & 513312 & $6600-02$ & 1EB10031 & $66^{\circ} 23 \cdot \mathrm{N}$ & $52^{\circ} 48^{\prime} \mathrm{W}$ & NW & 140 & 90 & 0000.65 & 700010 & $\begin{array}{l}60000-02 \\
6600\end{array}$ \\
\hline $1 \mathrm{BB} 07002$ & $66^{\circ} 30^{\prime} \mathrm{N}$ & $52^{\circ} 43^{\prime} \mathrm{W}$ & $\mathrm{N}$ & 125 & 115 & 0000.11 & 750010 & $6600-02$ & 1EB10032 & $66^{\circ} 23^{\prime} \mathrm{N}$ & $52^{\circ} 50^{\prime} \mathrm{W}$ & $\mathrm{NW}$ & 140 & 105 & 0000.49 & 700010 & $6600-02$ \\
\hline 1EB07003 & $66^{\circ} 30^{\prime} \mathrm{N}$ & $52^{\circ} 44^{\prime} \mathrm{W}$ & NW & 130 & 100 & 0000.30 & 643112 & $6600-02$ & $1 \mathrm{~EB} 10033$ & $66^{\circ} 23^{\prime} N$ & $52^{\circ} 48^{\prime} \mathrm{W}$ & W & 140 & 120 & 0000.38 & 780010 & $6600-02$ \\
\hline 1EB07004 & $66^{\circ} 29^{\prime} \mathrm{N}$ & $52^{\circ} 45, \mathrm{~W}$ & $\mathrm{~N}$ & 160 & 65 & 0005.43 & 513312 & $6600-02$ & 1EB10034 & $66^{\circ} 23, \mathrm{~N}$ & $52^{\circ} 50^{\prime} \mathrm{W}$ & W & $\begin{array}{l}140 \\
105\end{array}$ & $\begin{array}{r}120 \\
85\end{array}$ & 0000.18 & 750010 & $\begin{array}{l}66000-02 \\
6600-02\end{array}$ \\
\hline 1EB07005 & $66^{\circ} 30^{\prime} \mathrm{N}$ & $52^{\circ} 47^{\prime} \mathrm{W}$ & N & 135 & 120 & 0000.06 & 780010 & $6600-c$ & 1EB10035 & $66^{\circ} 22 \cdot \mathrm{N}$ & $52 \circ 49, \mathrm{~W}$ & W & 150 & 90 & 0002.49 & 513112 & $6600-02$ \\
\hline 1EB07006 & $66^{\circ} 30^{\prime} \mathrm{N}$ & $52^{\circ} 47, \mathrm{~W}$ & N & 150 & 100 & 0001.46 & 600012 & $6600-02$ & 1EB10036 & $66^{\circ} 22^{\prime} \mathrm{N}$ & $52^{\circ} 50^{\prime} \mathrm{W}$ & W & $\begin{array}{l}130 \\
145\end{array}$ & $\begin{array}{r}90 \\
130\end{array}$ & 0001.01 & $\begin{array}{l}313112 \\
700010\end{array}$ & $\begin{array}{l}6600002 \\
6600-02\end{array}$ \\
\hline 1EB07007 & $66^{\circ} 30^{\prime} \mathrm{N}$ & $52^{\circ} 48^{\prime} \mathrm{W}$ & NW & 150 & 120 & 0000.78 & 700010 & $6600-02$ & $1 E B 10037$ & $66^{\circ} 21^{\prime} \mathrm{N}$ & $52 \circ 52, \mathrm{~W}$ & NW & 140 & 85 & 0004.70 & 513112 & $6600-02$ \\
\hline 1ЕВ08001 & $66^{\circ} 30^{\prime} \mathrm{N}$ & $52^{\circ} 50^{\prime} \mathrm{W}$ & NW & 120 & 30 & 0003.61 & 513112 & $6600-02$ & 1EB10038 & $66^{\circ} 21, \mathrm{~N}$ & $52^{\circ} 54^{\prime} \mathrm{W}$ & $\mathrm{N}$ & 125 & 110 & 0000.65 & 700010 & $6600-02$ \\
\hline 1EB08002 & $66^{\circ} 30^{\prime} \mathrm{N}$ & 52051 , & $\mathrm{N}$ & 100 & 30 & 0001.53 & 533112 & $6600-02$ & $1 \mathrm{~EB} 10039$ & $66^{\circ} 21, N$ & $52055, \mathrm{~W}$ & $\mathrm{~N}$ & 140 & 100 & 0004.10 & 513412 & $6600-02$ \\
\hline 1EB08003 & $66^{\circ} 29^{\prime} \mathrm{N}$ & $52 \circ 51,6$ & N & 130 & 115 & 0000.63 & 613212 & $6600-02$ & 1EB10040 & $66^{\circ} 22, \mathrm{~N}$ & $52 \circ 57, \mathrm{~V}$ & NE & $\begin{array}{l}140 \\
130\end{array}$ & 90 & 0000.61 & $\begin{array}{l}313412 \\
643112\end{array}$ & $\begin{array}{l}6000002 \\
6600-01\end{array}$ \\
\hline 1EB08004 & $66^{\circ} 30^{\prime} \mathrm{N}$ & $52^{\circ} 53^{\prime \prime}$ & $\mathbf{N}$ & 130 & 40 & 0002.47 & 643112 & $6600-02$ & 1EB10041 & $66^{\circ} 22, \mathrm{~N}$ & $52 \circ 57,6$ & NE & 130 & 105 & 0000.91 & 700010 & $6600-01$ \\
\hline $1 \mathrm{~EB} 08005$ & $66^{\circ} 30^{\prime} \mathrm{N}$ & $52^{\circ} 54^{\prime} \mathrm{W}$ & $\mathrm{N}$ & 85 & 50 & 0000.39 & 643112 & $6600-0$ & 1EB10042 & $66^{\circ} 22{ }^{\prime} \mathrm{N}$ & $52 \circ 57, \mathrm{v}$ & $\mathrm{N}$ & 130 & 55 & 0000.74 & 643312 & $6600-01$ \\
\hline 1EB08006 & $66^{\circ} 30^{\prime} \mathrm{N}$ & $52 \circ 55^{\prime \prime}$ & NE & 90 & 55 & 0000.33 & 640112 & $6600-02$ & 1EB10043 & $66^{\circ} 22^{\prime} \mathrm{N}$ & $52^{\circ} 59^{\prime} \mathrm{W}$ & $\mathrm{NE}$ & 120 & 70 & 0000.37 & 753210 & $6600-01$ \\
\hline 1EB08007 & $66^{\circ} 31^{\prime} \mathrm{N}$ & $52^{\circ} 56$, W & $\mathrm{N}$ & 65 & 30 & 0000.18 & 750112 & $6630-02$ & $1 \mathrm{BB} 10044$ & $66^{\circ} 23^{\prime} \mathrm{N}$ & $53^{\circ} 01, \mathrm{~W}$ & $\mathrm{SE}$ & 140 & 125 & 0000.26 & 750010 & $\begin{array}{l}6000001 \\
6600-01\end{array}$ \\
\hline 1EB08008 & $66^{\circ} 30^{\prime} \mathrm{N}$ & $52^{\circ} 56^{\prime} \mathrm{W}$ & N & 148 & 135 & 0000.14 & 700010 & $6600-0$ & 1EB10045 & $66^{\circ} 23^{\prime N} \mathrm{~N}$ & $53^{\circ} 02, \mathrm{~W}$ & B & $\begin{array}{l}140 \\
150\end{array}$ & 140 & $\begin{array}{l}0.20 \\
0.01\end{array}$ & 700010 & 01 \\
\hline $1 \mathrm{BB} 08009$ & $66^{\circ} 31^{\prime} \mathrm{N}$ & $52^{\circ} 58^{\prime}, \mathrm{W}$ & NW & 125 & 110 & 0000.32 & 700010 & $6630-0$ & 1EB10046 & $66^{\circ} 25^{\prime N} \mathrm{~N}$ & $53^{\circ} 05^{\prime}$ & NE & 90 & $\begin{array}{r}140 \\
65\end{array}$ & 0000.11 & 750010 & $6600-01$ \\
\hline 1EB09001 & $66^{\circ} 30^{\prime} \mathrm{N}$ & 52057 , W & SW & 145 & 135 & 0000.10 & 780010 & 663 & 1EB10047 & $66^{\circ} 25, \mathrm{~N}$ & $53^{\circ} 07, \mathrm{~V}$ & $\mathrm{NE}$ & 140 & 60 & 0004.26 & 613212 & $6600-01$ \\
\hline 1EB09002 & $66^{\circ} 29^{\prime} \mathrm{N}$ & $52^{\circ} 53^{\prime} \mathrm{W}$ & W & 150 & 140 & 0000.13 & 780010 & 6600 & 1EB10048 & $66^{\circ} 25^{\prime} \mathrm{N}$ & $53^{\circ} 08^{\prime} \mathrm{W}$ & $\mathrm{E}$ & 145 & 100 & 0001.45 & 600010 & $6600-01$ \\
\hline $1 \mathrm{BB} 09003$ & $66^{\circ} 29^{\prime} \mathrm{N}$ & $52^{\circ} 50, \mathrm{v}$ & $\mathrm{SW}$ & 184 & 180 & 0000.33 & 700010 & $6600-02$ & 1EB10049 & $66^{\circ} 26^{\prime} \mathrm{N}$ & $53^{\circ} 08^{\circ} \mathrm{y}$ & $\mathrm{N}$ & 125 & 50 & 0000.84 & 643112 & $6600-01$ \\
\hline 1EB09004 & $66^{\circ} 28^{\prime} \mathrm{N}$ & $52^{\circ} 48^{\prime} \mathrm{W}$ & W & 125 & 120 & 0000.20 & 700010 & 6600 & $1 \mathrm{~EB} 10050$ & $66^{\circ} 26^{\prime} \mathrm{N}$ & $53^{\circ} 10^{\prime} \mathrm{W}$ & N & 135 & 75 & 0000.65 & $\begin{array}{l}643112 \\
643112\end{array}$ & $\begin{array}{l}66000-01 \\
6600-01\end{array}$ \\
\hline 1EB09005 & $66^{\circ} 28^{\prime} \mathrm{N}$ & $52^{\circ} 49^{\prime} \mathrm{W}$ & NW & 120 & 115 & 0000.10 & 643112 & 6600 & 1EB10051 & $66^{\circ} 26^{\prime} \mathrm{N}$ & $53^{\circ} 09^{\prime}$ & NW & 145 & 105 & 0000.90 & $\begin{array}{l}043112 \\
533112\end{array}$ & $6600-01$ \\
\hline 1EB09006 & $66^{\circ} 28^{\prime} \mathrm{N}$ & $52^{\circ} 50^{\prime} \mathrm{W}$ & N & 85 & 75 & 0000.15 & 700010 & 6600-02 & 1EB10052 & $66^{\circ} 26^{\prime} \mathrm{N}$ & $53^{\circ} 12^{\prime} \mathrm{V}$ & N & 140 & 65 & 0005.27 & 533112 & $6600-01$ \\
\hline 1EB09007 & $66^{\circ} 27^{\prime} \mathrm{N}$ & $52^{\circ} 51, \mathrm{~W}$ & N & 115 & 60 & 0001.21 & 643110 & 6600-02 & 1EB11001 & $66^{\circ} 28^{\circ} \mathrm{N}$ & $53 \circ 11, \mathrm{y}$ & $\mathrm{N}$ & $\begin{array}{l}140 \\
105\end{array}$ & 55 & 0000.33 & 643112 & $6600-01$ \\
\hline 1EB09008 & $66^{\circ} 27 \cdot \mathrm{N}$ & $52 \circ 53, \mathrm{~W}$ & NW & 105 & 30 & 0001.14 & 513312 & 6600 & 1 EB11002 & $66^{\circ} 27 \cdot \mathrm{N}$ & $53^{\circ} 12, \bar{v}$ & NW & 110 & 65 & .39 & 643112 & $6600-01$ \\
\hline 1EB09009 & $66^{\circ} 29^{\prime} \mathrm{N}$ & $52^{\circ} 27^{\prime \prime} \mathrm{V}$ & $\mathbf{N}$ & 170 & 60 & 0003.83 & 513412 & 6600 & 1EB11003 & $66^{\circ} 27 \cdot \mathrm{N}$ & $53^{\circ} 13, \mathrm{~W}$ & $\mathrm{~N}$ & 90 & 70 & 0000.35 & 700010 & $6600-01$ \\
\hline 1EB09010 & $66^{\circ} 28^{\prime} \mathrm{N}$ & $52^{\circ} 58^{\prime}$ & $\mathrm{NE}$ & 155 & 140 & 0001.01 & 700010 & 660 & 1 BB1 1004 & $66^{\circ} 27 \cdot \mathrm{N}$ & $53^{\circ} 14^{\prime} \mathrm{V}$ & NE & 110 & 80 & 0000.42 & 643112 & $6600-01$ \\
\hline 1EB09011 & $66^{\circ} 28^{\prime} \mathrm{N}$ & $52^{\circ} 58^{\prime} \mathrm{W}$ & N & 140 & 80 & 0000.17 & 643112 & $6600-01$ & 1 EB 11005 & $66^{\circ} 27 \cdot N$ & $53^{\circ} 14 ! \mathrm{W}$ & B & 110 & 100 & 000007 & 700010 & $6600-01$ \\
\hline 1EB09012 & $66^{\circ} 29^{\prime} \mathrm{N}$ & $52^{\circ} 59^{\prime} \mathrm{V}$ & N & 110 & 90 & 0000.09 & 643212 & 6600-01 & $1 \mathrm{~EB} 11006$ & $66^{\circ} 29^{\prime} \mathrm{N}$ & $53 \circ 15, y$ & NE & 100 & 55 & 0000.45 & 643112 & $6600-01$ \\
\hline 1EB09013 & $66^{\circ} 29 \cdot \mathrm{N}$ & $52^{\circ} 59^{\prime} \mathrm{W}$ & N & 120 & 85 & 0000.22 & 643112 & $6600-01$ & 1EB11007 & $66^{\circ} 28^{\prime} \mathrm{N}$ & $53^{\circ} 14^{\prime} \mathrm{V}$ & $\mathbf{N}$ & 115 & 45 & 0000.90 & $\begin{array}{l}643112 \\
533112\end{array}$ & $\begin{array}{l}6600-01 \\
6600-01\end{array}$ \\
\hline 1EB09014 & $66^{\circ} 29^{\prime} \mathrm{N}$ & $53^{\circ} 05^{\prime} \mathrm{V}$ & NE & 125 & 110 & 0000.21 & 700010 & $6600-01$ & 1EB12001 & $66^{\circ} 28^{\prime} \mathrm{N}$ & $53^{\circ} 15^{\prime} \mathrm{v}$ & NW & $\begin{array}{l}113 \\
110\end{array}$ & $\begin{array}{l}45 \\
95\end{array}$ & 0000.12 & 643112 & $6600-01$ \\
\hline 1EB09015 & $66^{\circ} 30^{\prime} \mathrm{N}$ & $53^{\circ} 06^{\circ} \mathrm{W}$ & $\mathrm{N}$ & 90 & 60 & 0000.11 & 643112 & $6600-01$ & 1EB12002 & $66^{\circ} 28^{\prime} \mathrm{N}$ & $53^{\circ} 17^{\circ}$ & $\mathrm{N}$ & 115 & 50 & 0003.64 & 513112 & $6600-01$ \\
\hline 1EB10001 & $66^{\circ} 29^{\prime} \mathrm{N}$ & $53^{\circ} 06^{\prime} \mathrm{V}$ & Nh & 130 & 95 & 0000.53 & 533112 & $6600-01$ & 1EB13001 & $66^{\circ} 29^{\prime} \mathrm{N}$ & $53^{\circ} 20^{\circ}$ & NW & 90 & 60 & 0000.51 & 643112 & $6600-01$ \\
\hline 1EB10002 & $66^{\circ} 28^{\prime} \mathrm{N}$ & $52^{\circ} 59 \cdot \mathrm{V}$ & SW & 145 & 120 & 0000.14 & 750010 & $6600-01$ & 1EB13002 & $66^{\circ} 29^{\prime} \mathrm{N}$ & $53^{\circ} 21^{\prime}$ & $\mathrm{N}$ & $\begin{array}{l}90 \\
100\end{array}$ & $\begin{array}{l}50 \\
50\end{array}$ & 0000.58 & $\begin{array}{l}043112 \\
643112\end{array}$ & $6600-01$ \\
\hline 1EB10003 & $66^{\circ} 27 \cdot \mathrm{N}$ & $53^{\circ} 00^{\prime} \mathrm{W}$ & $\mathrm{v}$ & 140 & 75 & 0002.68 & 533112 & $6600-01$ & 1EB13003 & $66^{\circ} 29^{\circ} \mathrm{N}$ & $53^{\circ} 22^{\prime} \mathrm{W}$ & $\mathrm{N}$ & $\begin{array}{r}100 \\
90\end{array}$ & 55 & 0000.26 & 640112 & $6600-01$ \\
\hline 1 EB10004 & $66^{\circ} 26^{\prime} \mathrm{N}$ & $52^{\circ} 58^{\prime} \mathrm{W}$ & $\mathrm{NW}$ & 130 & 120 & 0000.49 & 700010 & $6600-01$ & 1EB13004 & $66^{\circ} 29^{\prime} \mathrm{N}$ & $53^{\circ} 24^{\prime} \mathrm{V}$ & N & 100 & 55 & 0000.52 & 643112 & $6600-01$ \\
\hline
\end{tabular}




\begin{tabular}{|c|c|c|c|c|c|c|c|c|c|c|c|c|c|c|c|c|c|}
\hline CODE & LAT & LONG & ORI & HIGH & LOW & AREA & $\begin{array}{c}\text { MO } \\
\text { CODE }\end{array}$ & MAP & CODE & LAT & LONG & ORI & HIGH & LOW & AREA & $\begin{array}{c}\text { MO } \\
\text { CODE }\end{array}$ & MAP \\
\hline $1 \mathrm{~EB} 13$ & $66^{\circ} 28^{\prime} \mathrm{N}$ & $53^{\circ} 25^{\prime} \mathrm{W}$ & $\mathrm{N}$ & 70 & 60 & 0000.10 & 700010 & $6600-01$ & 1EE08002 & $67^{\circ} 05^{\prime} \mathrm{N}$ & $53^{\circ}$ & SW & & & 00.13 & 750010 & $6700-01$ \\
\hline 1EB13006 & $66^{\circ} 28^{\prime} \mathrm{N}$ & $53^{\circ} 26^{\prime} \mathrm{W}$ & $\mathrm{N}$ & 60 & 50 & 0000.13 & 750010 & $6600-01$ & 1EE08003 & $67^{\circ} 05^{\prime} \mathrm{N}$ & $53^{\circ} 08^{\prime} \mathrm{W}$ & 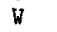 & 100 & 90 & 0000.78 & 643212 & $6700-02$ \\
\hline 1EB14001 & $66^{\circ} 28^{\prime} \mathrm{N}$ & $53^{\circ} 19^{\prime} \mathrm{W}$ & W & 85 & 70 & 0000.71 & 643112 & $6600-01$ & 1 EE08004 & $67^{\circ} 05^{\prime} \mathrm{N}$ & $53^{\circ} 07^{\prime} \mathrm{W}$ & $\mathrm{S}$ & 105 & 105 & 0000.23 & 750010 & $6700-02$ \\
\hline 1EB14002 & $66^{\circ} 27^{\prime} \mathrm{N}$ & $53^{\circ} 18^{\prime} \mathrm{W}$ & $v$ & 90 & 70 & 0000.18 & 643112 & $6600-01$ & 1EE08005 & $67^{\circ} 05^{\prime} \mathrm{N}$ & $53^{\circ} 06^{\prime} \mathrm{W}$ & $\mathrm{s}$ & 105 & 105 & 0000.06 & 780010 & $6700-02$ \\
\hline 1EB14003 & $66^{\circ} 27^{\circ} \mathrm{N}$ & $53^{\circ} 13^{\prime} \mathrm{W}$ & V & 100 & 80 & 0000.12 & 700010 & $6600-01$ & 1EF01001 & $67^{\circ} 05^{\prime} \mathrm{N}$ & $53^{\circ} 20^{\prime} \mathrm{W}$ & NE & 90 & 70 & 0000.22 & 770010 & $6700-01$ \\
\hline 1EB1 4004 & $66^{\circ} 26^{\prime} \mathrm{N}$ & $53^{\circ} 13^{\prime} \mathrm{W}$ & $\mathrm{NW}$ & 110 & 90 & 0000.39 & 643112 & $6600-01$ & 1EF05001 & $67^{\circ} 21^{\prime} \mathrm{N}$ & $52^{\circ} 52^{\prime} \mathrm{W}$ & E & 149 & 120 & 0000.33 & 700010 & $6700-02$ \\
\hline 1EB14005 & $66^{\circ} 26^{\prime} \mathrm{N}$ & $53^{\circ} 14^{\prime} \mathrm{W}$ & $\mathrm{N}$ & 130 & 80 & 0000.56 & 643112 & $6600-01$ & 1EF05002 & $67^{\circ} 21^{\prime} \mathrm{N}$ & $52^{\circ} 53^{\prime} \mathrm{W}$ & NE & 149 & 105 & 0000.46 & 750010 & $6700-02$ \\
\hline 1 EB14006 & $66^{\circ} 25^{\prime} \mathrm{N}$ & $53^{\circ} 14^{\prime} \mathrm{W}$ & W & 110 & 60 & 0000.45 & 643112 & $6600-01$ & 1EF05003 & $67^{\circ} 21^{\prime} \mathrm{N}$ & $52^{\circ} 54^{\prime} \mathrm{W}$ & NE & 125 & 100 & 0000.42 & 643112 & $6700-02$ \\
\hline 1EB14007 & $66^{\circ} 25^{\prime} \mathrm{N}$ & $53^{\circ} 14^{\prime} \mathrm{W}$ & NW & 120 & 65 & 0000.79 & 750112 & $6600-01$ & 1EF05004 & $67^{\circ} 21^{\prime} \mathrm{N}$ & $52^{\circ} 56^{\prime} \mathrm{W}$ & NE & 137 & 100 & 0000.43 & 670110 & $6700-02$ \\
\hline 1EB14008 & $66^{\circ} 24^{\prime} \mathrm{N}$ & $53^{\circ} 14^{\prime} \mathrm{W}$ & NW & 120 & 60 & 0000.20 & 773112 & $6600-01$ & $1 \mathrm{EF} 05005$ & $67^{\circ} 21^{\prime} \mathrm{N}$ & $52^{\circ} 58^{\prime} \mathrm{W}$ & $\mathrm{NE}$ & 150 & 90 & 0000.42 & 643112 & $6700-02$ \\
\hline 1EB14009 & $66^{\circ} 24^{\prime} \mathrm{N}$ & $53^{\circ} 16^{\prime} \mathrm{W}$ & $\mathrm{N}$ & 120 & 75 & 0000.34 & 643112 & $6600-01$ & 1EF05006 & $67^{\circ} 21^{\prime} \mathrm{N}$ & $53^{\circ} 00^{\prime} \mathrm{W}$ & $\mathbf{N}$ & 80 & 75 & 0000.25 & 750010 & $6700-02$ \\
\hline 1EB14010 & $66^{\circ} 24^{\prime} \mathrm{N}$ & $53^{\circ} 18^{\circ}$ W & $\mathrm{N}$ & 85 & 55 & 0000.47 & 750010 & $6600-01$ & 1 EF05007 & $67^{\circ} 21^{\prime} \mathrm{N}$ & $53^{\circ} 00^{\circ} \mathrm{W}$ & $\mathrm{N}$ & 150 & 130 & 0000.28 & 750010 & 6700-02 \\
\hline 1EB14011 & $66^{\circ} 24^{\prime} \mathrm{N}$ & $53^{\circ} 20^{\prime} \mathrm{W}$ & $\mathbf{N}$ & 80 & 60 & 0000.16 & 640112 & $6600-01$ & 1EF05008 & $67^{\circ} 20^{\prime} \mathrm{N}$ & $53^{\circ} 01^{\prime} \mathrm{w}$ & $\mathrm{N}$ & 130 & 120 & 0000.40 & 750010 & $6700-02$ \\
\hline 1EB14012 & $66^{\circ} 24^{\prime} \mathrm{N}$ & $53^{\circ} 21^{\prime} \mathrm{W}$ & N & 110 & 80 & 0000.24 & 643112 & $6600-01$ & 1EF05009 & $67^{\circ} 19^{\prime} \mathrm{N}$ & $53^{\circ} 04^{\prime} \mathrm{W}$ & NW & 1258 & 110 & 0000.36 & 750010 & $6700-02$ \\
\hline 1EB14013 & $66^{\circ} 25^{\prime} \mathrm{N}$ & $53^{\circ} 22 \cdot \mathrm{W}$ & $\mathrm{NE}$ & 85 & 60 & 0000.25 & 643112 & $6600-01$ & 1EF05010 & $67^{\circ} 18^{\prime} \mathrm{N}$ & $53^{\circ} 07^{\prime} \mathrm{v}$ & $\mathrm{N}$ & 128 & 115 & 0000.29 & 750010 & $6700-02$ \\
\hline 1EB14014 & $66^{\circ} 26^{\prime} \mathrm{N}$ & $53^{\circ} 22^{\prime} \mathrm{W}$ & $\mathrm{N}$ & 85 & 55 & 0000.23 & 640112 & $6600-01$ & 1EF15001 & $67^{\circ} 23^{\prime} \mathrm{N}$ & $49^{\circ} 43^{\prime} \mathrm{W}$ & W & 180 & 60 & 0614.02 & 166113 & $6700-05$ \\
\hline 1EB14015 & $66^{\circ} 26^{\prime} \mathrm{N}$ & $53^{\circ} 23^{\prime} \mathrm{W}$ & NW & 80 & 75 & 0000.08 & 640112 & $6600-01$ & $1 E F 15002$ & $67^{\circ} 20^{\prime} \mathrm{N}$ & $49^{\circ} 47 \mathrm{~W}$ & $\mathrm{~V}$ & 180 & 50 & 1565.67 & 163114 & $6700-05$ \\
\hline 1 EB14016 & $66^{\circ} 26^{\prime} \mathrm{N}$ & $53^{\circ} 24^{\prime} \mathrm{W}$ & $\mathbf{N}$ & 100 & 45 & 0001.05 & 643112 & $6600-01$ & 1EF15003 & $67^{\circ} 16^{\prime} \mathrm{N}$ & $49^{\circ} 48^{\prime} \mathrm{k}$ & $\ddot{u}$ & 180 & 40 & 1413.83 & 163113 & 6700-05 \\
\hline 1EB14017 & $66^{\circ} 26^{\prime} \mathrm{N}$ & $53^{\circ} 27^{\prime} \mathrm{W}$ & $\mathrm{N}$ & 60 & 50 & 0000.27 & 640112 & $6600-01$ & $1 \mathrm{EF} 16001$ & $67^{\circ} 11^{\prime} \mathrm{N}$ & $50^{\circ} 13^{\prime} \mathrm{W}$ & V & 180 & 10 & 1501.26 & 163113 & $6700-05$ \\
\hline 1EB14018 & $66^{\circ} 26^{\prime} \mathrm{N}$ & $53^{\circ} 28^{\prime} \mathrm{W}$ & N & 60 & 45 & 0000.30 & 700010 & $6600-01$ & $1 \mathrm{EF} 29001$ & $67^{\circ} 12^{\prime} \mathrm{N}$ & $52^{\circ} 27^{\prime} \mathrm{W}$ & $\mathrm{N}$ & 125 & 125 & 0000.11 & 700010 & $6700-02$ \\
\hline 1EB15001 & $66^{\circ} 25^{\prime} \mathrm{N}$ & $53^{\circ} 25^{\circ} \mathrm{W}$ & NW & 90 & 60 & 0000.65 & 643112 & $6600-01$ & 1EF29002 & $67^{\circ} 12^{\prime} \mathrm{N}$ & $52^{\circ} 28^{\prime \prime 6}$ & NW & 125 & 120 & 0000.08 & 700010 & $6700-02$ \\
\hline 1EB15002 & $66^{\circ} 25^{\prime} \mathrm{N}$ & $53^{\circ} 26^{\prime} \mathrm{W}$ & NW & 80 & 60 & 0000.16 & 700010 & $6600-01$ & 1EF32001 & $67^{\circ} 16^{\prime} \mathrm{N}$ & $52^{\circ} 23^{\prime} \mathrm{w}$ & $\mathrm{NE}$ & 120 & 105 & 0000.08 & 750010 & $6700-02$ \\
\hline 1EB15003 & $66^{\circ} 25^{\prime} \mathrm{N}$ & $53^{\circ} 28^{\circ} \mathrm{W}$ & $\mathrm{N}$ & 90 & 60 & 0000.31 & 643112 & $6600-01$ & 1EF32002 & $67^{\circ} 16^{\prime} \mathrm{N}$ & $52^{\circ} 24^{\prime}, y$ & $\mathrm{~N}$ & 115 & 105 & 0000.08 & 770010 & $6700-02$ \\
\hline 1EB15004 & $66^{\circ} 25^{\prime} \mathrm{N}$ & $53^{\circ} 29^{\prime} \mathrm{W}$ & $\mathrm{N}$ & 65 & 55 & 0000.11 & 750010 & $6600-01$ & 1EF32003 & $67^{\circ} 16^{\prime} \mathrm{N}$ & $52^{\circ} 25 \% \mathrm{~W}$ & NW & 130 & 115 & 0000.10 & 750010 & $6700-02$ \\
\hline 1EB15005 & $66^{\circ} 25^{\prime} \mathrm{N}$ & $53^{\circ} 30^{\prime} \mathrm{W}$ & N & 60 & 45 & 0000.14 & 643112 & $6600-01$ & 1EF32004 & $67^{\circ} 15^{\prime} \mathrm{N}$ & $52^{\circ} 32^{\prime} \mathrm{W}$ & NW & 120 & 110 & 0000.17 & 750010 & $6700-02$ \\
\hline 1EB16001 & $66^{\circ} 24^{\prime} \mathrm{N}$ & $53^{\circ} 21^{\prime} \mathrm{W}$ & $\mathrm{N}$ & 100 & 70 & 0000.11 & 643112 & $6600-01$ & 1EF32005 & $67^{\circ} 14^{\prime} \mathrm{N}$ & $52^{\circ} 34^{\prime} \mathrm{W}$ & NW & 120 & 110 & 0000.06 & 770010 & $6700-02$ \\
\hline 1EB16002 & $66^{\circ} 24^{\prime} \mathrm{N}$ & $53^{\circ} 12^{\prime} \mathrm{W}$ & NW & 115 & 90 & 0000.15 & 643112 & $6600-01$ & 1EF32006 & $67^{\circ} 13^{\prime} \mathrm{N}$ & $52^{\circ} 38^{\prime} \mathrm{W}$ & NW & 120 & 110 & 0000.06 & 770010 & $6700-02$ \\
\hline 1 EB16003 & $66^{\circ} 22^{\prime} \mathrm{N}$ & $53^{\circ} 11^{\prime \prime} \mathrm{W}$ & $\mathrm{NW}$ & 95 & 60 & 0000.46 & 643112 & $6600-01$ & 1EF32007 & $67^{\circ} 16^{\prime} \mathrm{N}$ & $52^{\circ} 43^{\prime} \mathrm{W}$ & $E$ & 150 & 120 & 0001.09 & 200010 & $6700-02$ \\
\hline 1EB16004 & $66^{\circ} 22^{\prime} \mathrm{N}$ & $53^{\circ} 12^{\prime} \mathrm{W}$ & $\mathrm{NW}$ & 90 & 50 & 0000.43 & 6.53112 & $6600-01$ & 1EF 33001 & $67^{\circ} 17^{\prime} \mathrm{N}$ & $52^{\circ} 39^{\prime} \mathrm{W}$ & $\mathrm{N}$ & 125 & 110 & 0000.21 & 750112 & $6700-02$ \\
\hline 1EB16005 & $66^{\circ} 22^{\prime} \mathrm{N}$ & $53^{\circ} 14^{\prime} \mathrm{W}$ & $\mathrm{NW}$ & 100 & 55 & 0000.30 & 643112 & $6600-01$ & $1 E F 33002$ & $67^{\circ} 17^{\prime} \mathrm{N}$ & $52^{\circ} 40 \cdot \mathrm{W}$ & N & 130 & 115 & 0000.12 & 700112 & $6700-02$ \\
\hline 1EB16006 & $66^{\circ} 22^{\prime} \mathrm{N}$ & $53^{\circ} 16^{\prime} \mathrm{W}$ & NV & 110 & 65 & 0001.17 & 643112 & $6600-01$ & 1EF 33003 & $67^{\circ} 16^{\prime} \mathrm{N}$ & $52 \circ 45$, w & N & 150 & 110 & 0000.83 & 200010 & $6700-02$ \\
\hline 1EB16007 & $66^{\circ} 22^{\prime} \mathrm{N}$ & $53^{\circ} 18^{\prime} \mathrm{W}$ & $\mathrm{N}$ & 85 & 70 & 0000.42 & 643112 & $6600-01$ & 1EF34001 & $67^{\circ} 16^{\prime} \mathrm{N}$ & $52^{\circ} 45, \mathrm{w}$ & SW & 150 & 120 & 0000.15 & 200010 & $6700-02$ \\
\hline 1EB16008 & $66^{\circ} 22$ ' N & $53^{\circ} 20^{\prime} \mathrm{W}$ & NW & 90 & 65 & 0000.29 & 740010 & $6600-01$ & 1EF34002 & $67^{\circ} 14^{\prime} \mathrm{N}$ & $52^{\circ} 47 . \mathrm{W}$ & $\mathrm{NW}$ & 115 & 95 & 0000.10 & 750010 & $6700-02$ \\
\hline 1EB16009 & $66^{\circ} 22^{\prime} \mathrm{N}$ & $53^{\circ} 21^{\prime} \mathrm{W}$ & $\mathrm{N}$ & 100 & 60 & 0000.36 & 670112 & $6600-01$ & 1EF34003 & $67^{\circ} 16^{\prime} \mathrm{N}$ & $52 \circ 51$ ' & E & 100 & 100 & 0000.03 & 700010 & $6700-02$ \\
\hline 1EB16010 & $66^{\circ} 22^{\prime} \mathrm{N}$ & $53^{\circ} 23^{\prime} \mathrm{W}$ & N & 80 & 65 & 0000.18 & 670112 & $6600-01$ & 1EF35001 & $67^{\circ} 16^{\prime} \mathrm{N}$ & 52052 W & W & 100 & 100 & 0000.06 & 700010 & $6700-02$ \\
\hline 1EB16011 & $66^{\circ} 22^{\prime} \mathrm{N}$ & $53^{\circ} 25^{\prime} \mathrm{W}$ & N & 85 & 60 & 0000.17 & 643112 & $6600-01$ & 1EF 35002 & $67^{\circ} 11^{\prime} \mathrm{N}$ & $52^{\circ} 31^{\prime} \mathrm{W}$ & NE & 125 & 115 & 0000.51 & 750010 & $6700-02$ \\
\hline 1EB16012 & $66^{\circ} 22^{\prime} \mathrm{N}$ & $53^{\circ} 28^{\prime} \mathrm{W}$ & N & 60 & 45 & 0000.05 & 700010 & $6600-01$ & 1EF35003 & $67^{\circ} 10^{\prime} \mathrm{N}$ & $52^{\circ} 35, \mathrm{w}$ & $\mathrm{N}$ & 136 & 105 & 0003.10 & 200010 & $6700-02$ \\
\hline 1EB16013 & $66^{\circ} 22^{\prime} \mathrm{N}$ & $53^{\circ} 30^{\prime} \mathrm{W}$ & $\mathbf{N}$ & 55 & 45 & 0000.11 & 640112 & $6600-01$ & 1EF35004 & $67^{\circ} 10^{\prime} \mathrm{N}$ & $52037 \% \mathrm{~W}$ & $\mathrm{~N}$ & 13 & 105 & 0002.53 & 200010 & $6700-02$ \\
\hline 1EB16014 & $66^{\circ} 22^{\prime} \mathrm{N}$ & $53^{\circ} 31^{\prime} \mathrm{W}$ & NW & 65 & 35 & 0000.16 & 640110 & $6600-01$ & 1EF 35005 & $67^{\circ} 11, \mathrm{~N}$ & $52^{\circ} 39^{\prime} \mathrm{W}$ & $\mathrm{N}$ & 115 & 90 & 0001.19 & 670012 & $6700-02$ \\
\hline 1EB17001 & $66^{\circ} 22^{\prime} \mathrm{N}$ & $53^{\circ} 25^{\prime} \mathrm{W}$ & W & 100 & 55 & 0000.48 & 643112 & $6600-01$ & 1EF 35006 & $67^{\circ} 11^{\prime} \mathrm{N}$ & $52^{\circ} 43,6$ & $\mathrm{E}$ & 120 & 100 & 0002.55 & 600010 & $6700-02$ \\
\hline 1EB17002 & $66^{\circ} 21 \cdot \mathrm{N}$ & $53^{\circ} 17^{\prime} \mathrm{W}$ & NW & 130 & 85 & 0000.44 & 643212 & $6600-01$ & 1EF 35007 & $67^{\circ} 12^{\prime} \mathrm{N}$ & $52^{\circ} 45 \cdot \mathrm{W}$ & N & 125 & 90 & 0003.08 & 670012 & $6700-02$ \\
\hline $1 \mathrm{~EB}$ & $66^{\circ} 19^{\prime} \mathrm{N}$ & $53^{\circ} 18^{\prime} \mathrm{V}$ & $\mathrm{N}$ & 100 & 60 & 0000.64 & 643112 & $6600-01$ & 1EF 35008 & $67^{\circ} 12^{\prime} \mathrm{N}$ & $52^{\circ} 48^{\prime} \mathrm{W}$ & NW & 125 & 115 & 0000.49 & 600010 & $6700-02$ \\
\hline 1EB17004 & $66^{\circ} 19^{\prime} \mathrm{N}$ & $53^{\circ} 19^{\circ} \mathrm{V}$ & $\mathrm{NE}$ & 105 & 55 & 0000.36 & 643112 & $6600-01$ & 1EF35009 & $67^{\circ} 12^{\prime} \mathrm{N}$ & $52^{\circ} 50^{\circ} \mathrm{k}$ & $\mathrm{N}$ & 105 & 105 & 0000.11 & 700010 & $6700-02$ \\
\hline 1EB17005 & $66^{\circ} 19^{\prime} \mathrm{N}$ & $53^{\circ} 21^{\prime} \mathrm{W}$ & $\mathrm{NW}$ & 90 & 45 & 0000.49 & 640010 & $6600-01$ & 1EF35010 & $67^{\circ} 12^{\prime} \mathrm{N}$ & $52^{\circ} 50^{\prime} \mathrm{w}$ & $\mathrm{NE}$ & 11 & 100 & 0000.49 & 700010 & $6700-02$ \\
\hline 1EB17006 & $66^{\circ} 19^{\prime} \mathrm{N}$ & $53^{\circ} 23^{\prime} \mathrm{W}$ & $\mathrm{N}$ & 120 & 20 & 0002.42 & 643212 & $6600-01$ & 1EF 35011 & $67^{\circ} 12^{\prime} \mathrm{N}$ & $52^{\circ} 52^{\prime} \mathrm{W}$ & $\mathrm{N}$ & 120 & 95 & 0000.49 & 640010 & $6700-02$ \\
\hline $1 \mathrm{~EB} 17007$ & $66^{\circ} 19^{\prime} \mathrm{N}$ & $53^{\circ} 25^{\prime} \mathrm{W}$ & NW & 100 & 50 & 0000.91 & 630010 & $6600-01$ & 1EF35012 & $67^{\circ} 13^{\prime} \mathrm{N}$ & $52^{\circ} 53^{\prime} \mathrm{W}$ & $\mathrm{N}$ & 120 & 90 & 0000.39 & 640010 & $6700-02$ \\
\hline $1 \mathrm{EB1} 7008$ & $66^{\circ} 19^{\prime N}$ & $53^{\circ} 27 \cdot \mathrm{W}$ & $\mathrm{NW}$ & 80 & 45 & 0000.33 & 643112 & $6600-01$ & 1EF35013 & $67^{\circ} 12^{\prime} \mathrm{N}$ & $52^{\circ} 54^{\prime} \mathrm{W}$ & N & 120 & 120 & 0000.15 & 700010 & $6700-02$ \\
\hline $1 \mathrm{~EB} 17009$ & $66^{\circ} 19^{\prime} \mathrm{N}$ & $53^{\circ} 28^{\prime} \mathrm{W}$ & $\mathrm{N}$ & 90 & 65 & 0000.48 & 700010 & $6600-01$ & 1EF35014 & $67^{\circ} 12^{\prime} \mathrm{N}$ & $52^{\circ} 56^{\circ} \mathrm{W}$ & $\mathrm{NE}$ & 145 & 95 & 0001.15 & 643112 & $6700-02$ \\
\hline $1 \mathrm{BB} 17010$ & $66^{\circ} 19^{\prime} \mathrm{N}$ & $53^{\circ} 31^{\prime} \mathrm{W}$ & $\mathrm{NE}$ & 65 & 40 & 0000.27 & 750010 & $6600-01$ & 1EF35015 & $67^{\circ} 13^{\prime} \mathrm{N}$ & $52^{\circ} 56^{\prime} \mathrm{W}$ & $\mathrm{N}$ & 13 & 120 & 0000.91 & 600010 & $6700-02$ \\
\hline 1EB17011 & $66^{\circ} 19^{\prime} \mathrm{N}$ & $53^{\circ} 33^{\prime} \mathrm{W}$ & $\mathrm{N}$ & 65 & 45 & 0000.12 & 750010 & 66 & 1EF35016 & $67^{\circ} 14^{\prime} \mathrm{N}$ & $52^{\circ} 55^{\prime} \mathrm{W}$ & $\mathrm{N}$ & 140 & 110 & 0000.26 & 750010 & $6700-02$ \\
\hline $1 \mathrm{EC} 07001$ & $66^{\circ} 38^{\prime} \mathrm{N}$ & $52^{\circ} 51^{\prime} \mathrm{W}$ & $\mathrm{E}$ & 145 & 120 & 0000.61 & 780010 & $6630-02$ & 1EF35017 & $67^{\circ} 14^{\prime} \mathrm{N}$ & 52057 W & NW & 140 & 100 & 0000.80 & 750010 & $6700-02$ \\
\hline 1EC08001 & $66^{\circ} 38^{\prime} \mathrm{N}$ & $52^{\circ} 52^{\prime} \mathrm{W}$ & NW & 145 & 130 & 0000.34 & 780010 & $6630-02$ & 1EF 35018 & $67^{\circ} 13^{\prime} \mathrm{N}$ & $52^{\circ} 58^{\prime} \mathrm{W}$ & N & 140 & 100 & 0001.40 & 643112 & $6700-02$ \\
\hline 1EE01001 & $67^{\circ} 08^{\circ} \mathrm{N}$ & $53^{\circ} 13^{\prime} \mathrm{W}$ & $\mathrm{W}$ & 95 & 65 & 0000.31 & 643012 & $6700-01$ & 1EF35019 & $67^{\circ} 13^{\prime} \mathrm{N}$ & $53^{\circ} 00^{\prime} \mathrm{w}$ & $\mathrm{N}$ & 14 & 100 & 0001.39 & 643112 & $6700-02$ \\
\hline $1 \mathrm{EE} 01002$ & $67^{\circ} 07^{\prime} \mathrm{N}$ & $53^{\circ} 13^{\prime} \mathrm{W}$ & NW & 95 & 55 & 0001.44 & 623112 & 67 & $1 \mathrm{EF}$ & $67^{\circ} 13^{\prime N}$ & $53^{\circ} 01 \cdot \mathrm{w}$ & $\mathrm{N}$ & 120 & 105 & 0000.43 & 700010 & $6700-02$ \\
\hline IEE02001 & $67^{\circ} 05^{\prime} \mathrm{N}$ & $53^{\circ} 12^{\prime} \mathrm{W}$ & NW & 115 & 55 & 0002.81 & 533112 & $6700-01$ & 1EF35021 & $67^{\circ} 13^{\prime} \mathrm{N}$ & $53^{\circ} 01^{\prime}$ & $\mathrm{NE}$ & & & 0000.37 & 700010 & $6700-02$ \\
\hline 1 EE 03001 & $67^{\circ} 05^{\prime} \mathrm{N}$ & $53^{\circ} 17^{\prime \prime}$ & NW & 90 & 65 & 0000.40 & 643112 & $6700-01$ & 1EF 35022 & $67^{\circ} 13^{\prime} \mathrm{N}$ & $53^{\circ} 03^{\prime} \mathrm{b}$ & $\mathrm{N}$ & 60 & 50 & 0000.34 & 643112 & $6700-02$ \\
\hline 1EE03002 & $67^{\circ} 05^{\circ} \mathrm{N}$ & $53^{\circ} 18^{\prime} \mathrm{W}$ & NW & 100 & 85 & 0000.09 & 700010 & $6700-01$ & 1EF 35023 & $67^{\circ} 13^{\prime} \mathrm{N}$ & $53^{\circ} 07^{\prime} \mathrm{w}$ & $\mathrm{N}$ & 130 & 105 & 0001.22 & 700010 & $6700-02$ \\
\hline 1EE03003 & $67^{\circ} 05^{\prime} \mathrm{N}$ & $53^{\circ} 14^{\prime} \mathrm{W}$ & $\mathrm{W}$ & 120 & 90 & 0001.33 & 670110 & $6700-01$ & 1EF35024 & $67^{\circ} 14^{\prime} \mathrm{N}$ & $53^{\circ} 09^{\prime} \mathrm{W}$ & $\mathrm{N}$ & & & 0000.28 & 643112 & $6700-01$ \\
\hline $1 \mathrm{EE} 03004$ & $67^{\circ} 04^{\prime} \mathrm{N}$ & $53^{\circ} 13^{\prime} \mathrm{W}$ & $\hat{~}$ & 100 & 90 & 0000.14 & 770010 & $6700-01$ & 1EF 35025 & $67^{\circ} 13^{\prime} \mathrm{N}$ & $53^{\circ} 10^{\prime} \mathrm{k}$ & NW & 120 & 90 & 0001.09 & 603012 & $6700-01$ \\
\hline 1 RE0 3005 & $67^{\circ} 04^{\prime} \mathrm{N}$ & $53^{\circ} 24^{\prime} \mathrm{W}$ & $\hat{N}$ & 90 & 60 & 0000.43 & 643112 & $6700-01$ & 1EF 35026 & $67^{\circ} 13^{\prime} \mathrm{N}$ & $53^{\circ} 13^{\prime} \mathrm{W}$ & N & 120 & 60 & 0001.93 & 643012 & $6700-01$ \\
\hline 1EE08001 & $67^{\circ} 05^{\prime} \mathrm{N}$ & $53^{\circ} 11$, W & $\mathrm{s}$ & 110 & 100 & 0000.19 & 650012 & $6700-01$ & 1EF35027 & $67^{\circ} 14^{\prime} \mathrm{N}$ & $53^{\circ} 16^{\prime} \mathrm{W}$ & NW & 105 & 85 & 0000.39 & 700010 & $6700-01$ \\
\hline
\end{tabular}




\begin{tabular}{|c|c|c|c|c|c|c|c|c|}
\hline CODE & LAT & LONG & ORI & HIGH & LOW & AREA & $\begin{array}{c}\text { MO } \\
\text { CODE }\end{array}$ & MAP \\
\hline F35028 & $67^{\circ} 13^{\prime} \mathrm{N}$ & $53^{\circ} 17^{\prime} \mathrm{W}$ & NW & 80 & 65 & 0000.56 & 643012 & $6700-01$ \\
\hline $1 E F 35029$ & $67^{\circ} 13^{\prime} \mathrm{N}$ & $53^{\circ} 19^{\prime} \mathrm{W}$ & NW & 105 & 80 & 0000.36 & 700010 & $6700-01$ \\
\hline $1 \mathrm{EF} 35030$ & $67^{\circ} 13^{\prime} \mathrm{N}$ & $52^{\circ} 37^{\prime} \mathrm{W}$ & NW & 120 & 110 & 0000.19 & 750010 & $6700-02$ \\
\hline $1 \mathrm{EF} 36001$ & $67^{\circ} 13^{\prime} \mathrm{N}$ & $53^{\circ} 15^{\prime} \mathrm{W}$ & V & 85 & 81 & 0000.10 & 750010 & $6700-01$ \\
\hline 1EF36002 & $67^{\circ} 12^{\prime} \mathrm{N}$ & $53^{\circ} 15^{\prime} \mathrm{W}$ & SW & 110 & 80 & 0000.13 & 750010 & $6700-01$ \\
\hline 1EF36003 & $67^{\circ} 12^{\prime} \mathrm{N}$ & $53^{\circ} 10^{\prime} \mathrm{W}$ & $v$ & 155 & 75 & 0006.88 & 533111 & $6700-01$ \\
\hline 1 EF 36004 & $67^{\circ} 11 \cdot \mathrm{N}$ & $53^{\circ} 11$ ' & SW & 115 & 75 & 0000.23 & 750010 & $6700-01$ \\
\hline 1EF36005 & $67^{\circ} 11^{\prime} \mathrm{N}$ & $53^{\circ} 08^{\prime} \mathrm{W}$ & E & 105 & 95 & 0000.15 & 750010 & $6700-02$ \\
\hline 1EF36006 & $67^{\circ} 12^{\prime} \mathrm{N}$ & $53^{\circ} 07^{\prime} \mathrm{W}$ & $\mathbf{E}$ & 140 & 110 & 0000.56 & 750010 & $6700-02$ \\
\hline 1EF 36007 & $67^{\circ} 13^{\prime} \mathrm{N}$ & $53^{\circ} 06^{\prime} \mathrm{V}$ & $\mathrm{SE}$ & 130 & 90 & 0002.31 & 750010 & $6700-02$ \\
\hline 1EF36008 & $67^{\circ} 13^{\prime} \mathrm{N}$ & $53^{\circ} 05^{\prime} \mathrm{W}$ & s & 135 & 130 & 0000.11 & 750010 & $6700-02$ \\
\hline 1 EF 36009 & $67^{\circ} 13^{\prime} \mathrm{N}$ & $53^{\circ} 04^{\prime} \mathrm{W}$ & SW & 115 & 105 & 0000.29 & 600010 & $6700-02$ \\
\hline 1EF36010 & $67^{\circ} 12^{\prime} \mathrm{N}$ & $53^{\circ} 02^{\prime} \mathrm{W}$ & E & 140 & 100 & 0002.96 & 533111 & $6700-02$ \\
\hline 1EF36011 & $67^{\circ} 11^{\prime} \mathrm{N}$ & $52^{\circ} 54^{\prime} \mathrm{W}$ & $\mathbf{N}$ & 130 & 95 & 0005.05 & 270110 & $6700-02$ \\
\hline $1 \mathrm{EF} 36012$ & $67^{\circ} 12^{\prime}, \mathrm{N}$ & $52^{\circ} 50^{\prime} \mathrm{W}$ & s & 110 & 95 & 0000.85 & 600010 & $6700-02$ \\
\hline 1EF 36013 & $67^{\circ} 11^{\prime} \mathrm{N}$ & $52^{\circ} 46^{\prime} \mathrm{W}$ & SW & 125 & 100 & 0001.32 & 563111 & $6700-02$ \\
\hline 1EF36014 & $67^{\circ} 10^{\prime} \mathrm{N}$ & $52^{\circ} 44^{\prime} \mathrm{W}$ & $\mathrm{SE}$ & 125 & 110 & 0003.28 & 600010 & 6700-02 \\
\hline 1EF36015 & $67^{\circ} 11^{\prime} \mathrm{N}$ & $52^{\circ} 39^{\prime} \mathrm{W}$ & $\mathrm{s}$ & 115 & 113 & 0000.76 & 700010 & $6700-02$ \\
\hline 1EF36016 & $67^{\circ} 10^{\prime} \mathrm{N}$ & $52^{\circ} 37^{\prime} \mathrm{W}$ & $\mathrm{NW}$ & 135 & 120 & 0000.45 & 700010 & $6700-02$ \\
\hline 1EF36017 & $67^{\circ} 09^{\prime} \mathrm{N}$ & $52^{\circ} 36^{\prime} \mathrm{W}$ & s & 140 & 135 & 0000.25 & 750010 & $6700-02$ \\
\hline 1EF36018 & $67^{\circ} 09^{\prime} \mathrm{N}$ & $52^{\circ} 34 \cdot \mathrm{W}$ & SE & 140 & 135 & 0000.26 & 700010 & $6700-02$ \\
\hline 1EF 36019 & $67^{\circ} 10^{\prime} \mathrm{N}$ & $52^{\circ} 32^{\circ} \mathrm{W}$ & $\mathrm{NE}$ & 120 & 110 & 0000.60 & 770010 & $6700-02$ \\
\hline $1 E F 36020$ & $67^{\circ} 09^{\prime} \mathrm{N}$ & $52^{\circ} 25^{\prime} \mathrm{W}$ & NW & 139 & 116 & 0001.04 & 670010 & $6700-02$ \\
\hline 1EF36021 & $67^{\circ} 07^{\prime} \mathrm{N}$ & $52^{\circ} 18^{\prime} \mathrm{W}$ & $\mathrm{N}$ & 135 & 130 & 0000.12 & 750010 & $6700-02$ \\
\hline 1EF 36022 & $67^{\circ} 077^{\prime N}$ & $52^{\circ} 19^{\prime} \mathrm{W}$ & N & 135 & 130 & 0000.11 & 750010 & $6700-02$ \\
\hline 1EF 36023 & $67^{\circ} 06^{\prime} \mathrm{N}$ & $52^{\circ} 23^{\prime} \mathrm{W}$ & NW & 127 & 120 & 0000.09 & 750010 & $6700-02$ \\
\hline $1 \mathrm{EF} 36024$ & $67^{\circ} 00^{\prime} \mathrm{N}$ & $52^{\circ} 43^{\prime} \mathrm{W}$ & $\mathrm{N}$ & 110 & 105 & 0000.10 & 750010 & $6630-02$ \\
\hline 1EF36025 & $67^{\circ} 09^{\prime} \mathrm{N}$ & $52^{\circ} 39^{\prime} \mathrm{W}$ & N & 120 & 115 & 0000.04 & 750010 & $6700-02$ \\
\hline 1EF 36026 & $67^{\circ} 09^{\prime} \mathrm{N}$ & $52^{\circ} 42^{\prime} \mathrm{W}$ & $\mathbf{N}$ & 115 & 113 & 0000.12 & 750010 & $6700-02$ \\
\hline 1EF 36027 & $67^{\circ} 09^{\prime} \mathrm{N}$ & $52^{\circ} 43^{\prime} \mathrm{W}$ & $\mathrm{N}$ & 115 & 100 & 0000.08 & 750010 & $6700-02$ \\
\hline 1EF36028 & $67^{\circ} 04^{\prime} \mathrm{N}$ & $52^{\circ} 45^{\prime} \mathrm{W}$ & N & 107 & 107 & 0000.09 & 750010 & $6700-02$ \\
\hline 1EF36029 & $67^{\circ} 08^{\prime} \mathrm{N}$ & $52^{\circ} 59^{\prime} \mathrm{W}$ & N & 95 & 90 & 0000.08 & 750010 & $6700-02$ \\
\hline 1EF 36030 & $67^{\circ} 06^{\prime} \mathrm{N}$ & $53^{\circ} 04^{\circ} \mathrm{W}$ & E & 115 & 83 & 0003.86 & 603012 & $6700-02$ \\
\hline 1EF36031 & $67^{\circ} 07^{\prime} \mathrm{N}$ & $53^{\circ} 05^{\prime} \mathrm{W}$ & $\mathrm{NE}$ & 105 & 96 & 0000.13 & 643110 & $6700-02$ \\
\hline $1 \mathrm{EF} 36032$ & $67^{\circ} 07^{\prime} \mathrm{N}$ & $53^{\circ} 07 \cdot \mathrm{W}$ & $\mathrm{NE}$ & 125 & 55 & 0000.68 & 533111 & $6700-02$ \\
\hline $1 E F 36033$ & $67^{\circ} 07^{\prime} \mathrm{N}$ & $53^{\circ} 06^{\prime} \mathrm{W}$ & $\mathbf{E}$ & 130 & 70 & 0001.11 & 500110 & $6700-02$ \\
\hline 1EF 36034 & $67^{\circ} 08^{\prime} \mathrm{N}$ & $53^{\circ} 08^{\prime} \mathrm{W}$ & $\mathrm{NE}$ & 130 & 90 & 0000.28 & 523111 & $6700-02$ \\
\hline 1EF 36035 & $67^{\circ} 07 \times \mathrm{N}$ & $53^{\circ} 09^{\prime} \mathrm{W}$ & $\mathrm{N}$ & 120 & 40 & 0005.00 & 523111 & 6700-01 \\
\hline 1EF 36036 & $67^{\circ} 08^{\prime} \mathrm{N}$ & $53^{\circ} 11^{\prime} \mathrm{W}$ & $E$ & 95 & 50 & 0000.67 & 633012 & $6700-01$ \\
\hline 1EF 36037 & $67^{\circ} 08^{\prime} \mathrm{N}$ & $53^{\circ} 22$, W & $\mathrm{N}$ & & & 0000.12 & 643012 & 6700-01 \\
\hline 1EF 36038 & $67^{\circ} 08^{\prime} \mathrm{N}$ & $53^{\circ} 24^{\prime} \mathrm{W}$ & N & 70 & 35 & 0000.37 & 533112 & 6700-01 \\
\hline 1EF 36039 & $67^{\circ} 09^{\prime} \mathrm{N}$ & $53^{\circ} 25^{\prime} \mathrm{W}$ & NE & 60 & 35 & 0001.03 & 643212 & $6700-01$ \\
\hline 1EF 37001 & $67^{\circ} 08^{\prime} \mathrm{N}$ & $53^{\circ} 29^{\prime} \mathrm{W}$ & $\mathrm{v}$ & 105 & 60 & 0001.72 & 643312 & $6700-01$ \\
\hline $1 \mathrm{EF} 37002$ & $67^{\circ} 08^{\prime} \mathrm{N}$ & $53^{\circ} 33^{\prime} \mathrm{W}$ & $\mathbf{N}$ & 80 & 60 & 0000.27 & 673012 & $6700-01$ \\
\hline 1EF 77003 & $67^{\circ} 08^{\prime} \mathrm{N}$ & $53^{\circ} 34^{\prime} \mathrm{W}$ & NE & 65 & 45 & 0000.10 & 750012 & $6700-01$ \\
\hline 1EF 37004 & $67^{\circ} 09^{\prime} \mathrm{N}$ & $53^{\circ} 35^{\prime} \mathrm{W}$ & $\mathrm{N}$ & 65 & 55 & 0000.19 & 750010 & $6700-01$ \\
\hline 1EF 37005 & $67^{\circ} 09^{\prime} \mathrm{N}$ & $53^{\circ} 38^{\prime} \mathrm{W}$ & $\mathrm{N}$ & 75 & 50 & 0000.47 & 670112 & $6700-01$ \\
\hline 1EF37006 & $67^{\circ} 09^{\prime} \mathrm{N}$ & $53^{\circ} 39^{\prime} \mathrm{W}$ & N & 70 & 65 & 0000.24 & 700010 & $6700-01$ \\
\hline $1 E F 39001$ & $67^{\circ} 08^{\prime} \mathrm{N}$ & $53^{\circ} 35^{\prime} \mathrm{W}$ & $\mathrm{NW}$ & 75 & 65 & 0000.11 & 700010 & $6700-01$ \\
\hline 1EF39002 & $67^{\circ} 07^{\prime} \mathrm{N}$ & $53^{\circ} 30^{\prime} \mathrm{W}$ & $\mathrm{N}$ & 80 & 65 & 0001.33 & 700010 & $6700-01$ \\
\hline 1EF39003 & $67^{\circ} 07^{\prime} \mathrm{N}$ & $53^{\circ} 32^{\prime} \mathrm{W}$ & NW & 80 & 50 & 0000.68 & 643012 & $6700-01$ \\
\hline 1EF39004 & $67^{\circ} 06^{\prime} \mathrm{N}$ & $53^{\circ} 34^{\prime} \mathrm{W}$ & $\mathrm{N}$ & 80 & 65 & 0000.18 & 700010 & $6700-01$ \\
\hline 1EF39005 & $67^{\circ} 06^{\prime} \mathrm{N}$ & $53^{\circ} 36^{\prime} \mathrm{W}$ & N & 70 & 55 & 0000.44 & 750012 & $6700-01$ \\
\hline 1FA16001 & $67^{\circ} 47^{\prime} \mathrm{N}$ & $49^{\circ} 47 \cdot \mathrm{W}$ & V & 180 & 50 & 0962.21 & 160113 & $6730-05$ \\
\hline 1FA16002 & $67^{\circ} 43^{\prime} \mathrm{N}$ & $49^{\circ} 50^{\prime} \mathrm{W}$ & w & 180 & 35 & 0502.20 & 163112 & $6730-05$ \\
\hline 1FA21001 & $67^{\circ} 39^{\prime} \mathrm{N}$ & $49^{\circ} 46^{\prime} \mathrm{W}$ & W & 180 & 40 & 0718.28 & 163113 & $6730-05$ \\
\hline $1 \mathrm{FA} 21002$ & $67^{\circ} 36^{\prime} \mathrm{N}$ & $49^{\circ} 45^{\prime} \mathrm{W}$ & $\mathrm{W}$ & 180 & 30 & 0902.24 & 163112 & $6730-05$ \\
\hline 1FA22001 & $67^{\circ} 31^{\prime} \mathrm{N}$ & $49^{\circ} 55^{\prime} \mathrm{W}$ & $\mathrm{W}$ & 180 & 15 & 1225.68 & 163112 & $6730-05$ \\
\hline 1FA23001 & $67^{\circ} 28^{\prime} \mathrm{N}$ & $49^{\circ} 44^{\prime} \mathrm{W}$ & w & 180 & 40 & 0820.67 & 163113 & $6700-05$ \\
\hline $1 F A 23002$ & $67^{\circ} 25^{\prime} \mathrm{N}$ & $49^{\circ} 42^{\prime} \mathrm{W}$ & $\mathrm{W}$ & 180 & 50 & 0798.99 & 163113 & $6700-05$ \\
\hline 1FA40001 & $67^{\circ} 23^{\prime} \mathrm{N}$ & $53^{\circ} 12^{\prime} \mathrm{W}$ & $\mathrm{N}$ & 75 & 70 & 0000.08 & 700010 & 6700-01 \\
\hline
\end{tabular}

CODE

1FA40002 1FA40003

1FA40005

1 FB17001

1 FC1600

IFC17001

1 FC19001

1FC21001

1 FC21002

1FC29001

1FC29002

1 FC30001

1 FC3000

1GA05001

1GA06001

1 GA06003

1GA06004

1GB05001

$1 \mathrm{~GB} 07001$

1 GB07002

1 GB07004

1GC04001

$1 \mathrm{GCO} 4003$

$1 \mathrm{GCO6001}$

1GC06003

$1 \mathrm{GE0} 3001$

1 GE03003

1 GE03004

1GE04001

$1 \mathrm{GE07001}$

1 GE07002

1GE08001

$1 \mathrm{GFO} 4002$

$1 \mathrm{GF06001}$

1 GF07001

1 GF07002

1 GHO1002

1GH01003

1GH01005
LAT

Low

ARA

CODE

MAP

$\begin{array}{llllllll}67^{\circ} 22^{\prime} \mathrm{N} & 53^{\circ} 17 \cdot \mathrm{N} & \mathrm{NE} & 100 & 95 & 0000.11 & 750010 & 6700-01\end{array}$ $\begin{array}{lllllll}67^{\circ} 2, N-53^{\circ} 19^{\prime}, \mathrm{W} & \mathrm{NE} & 115 & 85 & 0000.69 & 543112 & 6700-01\end{array}$

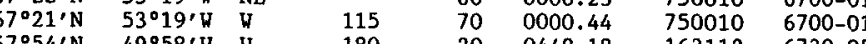
$\begin{array}{lllllll}67 \circ 52, \mathrm{~N} & 49^{\circ} 58^{\circ} \mathrm{V} & 180 & 20 & 0448.18 & 163112 & 6730-05 \\ 6 & 180 & 60 & 0802.38 & 163113 & 6730-05\end{array}$

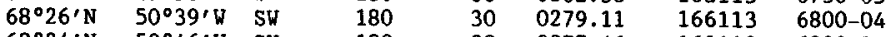
$50^{\circ} 51^{\prime} \mathrm{W}$ SH $68^{\circ} 15^{\prime} \mathrm{N} \quad 50^{\circ} 24^{\prime} \mathrm{W}$, $68^{\circ} 13^{\prime} \mathrm{N}$ $68^{\circ} 09^{\prime} \mathrm{N}$ $50^{\circ} 11^{\prime} \mathrm{W}$ W

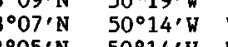
$68^{\circ} 03^{\prime} \mathrm{N}-50^{\circ} 11^{\prime}, \mathrm{H}$ $67^{\circ} 58^{\prime} \mathrm{N} \quad 50^{\circ} 18^{\prime} \mathrm{W} \quad \mathrm{SW}$ $67^{\circ} 58^{\prime} \mathrm{N} \quad 50^{\circ} 13^{\prime} \mathrm{V}$ Sh

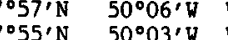
$68^{\circ} 41^{\prime} \mathrm{N} \quad 50^{\circ} 21^{\prime} \mathrm{W}$ $68^{\circ} 36^{\prime} \mathrm{N} \quad 50^{\circ} 24^{\prime} \mathrm{W}$ $68^{\circ} 34^{\prime} \mathrm{N}$ $68^{\circ} 31^{\prime} \mathrm{N}$
$68^{\circ} 28^{\prime} \mathrm{N}$

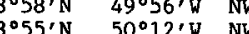
$68^{\circ} 54^{\prime} \mathrm{N} \quad 50^{\circ} 18^{\prime} \mathrm{W}$ NW $68^{\circ} 47^{\prime} \mathrm{N} \quad 50^{\circ} 12^{\prime} \mathrm{V} \quad \mathrm{NW}$ $68^{\circ} 43^{\prime} \mathrm{N} \quad 50^{\circ} 18^{\prime} \mathrm{W}$ W $\begin{array}{ll}69^{\circ} 22^{\prime} \mathrm{N} & 50^{\circ} 18^{\prime} \mathrm{W} \\ 69^{\circ} 22^{\prime} \mathrm{N} & 50^{\circ} 08^{\prime} \mathrm{W}\end{array}$ $69^{\circ} 20^{\prime} \mathrm{N} 50^{\circ} 13^{\prime} \mathrm{W}$ $69^{\circ} 11^{\prime} \mathrm{N} \quad 49^{\circ} 48^{\prime} \mathrm{W}$ W $69^{\circ} 06^{\prime} N \quad 49^{\circ} 45^{\prime} \mathrm{W}$ N $69^{\circ} 39^{\prime} \mathrm{N} \quad 50^{\circ} 00^{\circ} \mathrm{W}$ $69^{\circ} 36^{\prime} \mathrm{N} \quad 50^{\circ} 03^{\prime} \mathrm{W}$ $69^{\circ} 33^{\prime} \mathrm{N} \quad 50^{\circ} 02, \mathrm{~W}$

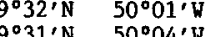
$69^{\circ} 29^{\prime} \mathrm{N} 50^{\circ} 07$ ' $\mathrm{W}$ $69^{\circ} 28^{\prime} \mathrm{N} \quad 50^{\circ} 12^{\prime} \mathrm{W}$ $50^{\circ} 11$ ' $\mathrm{W}$ (69. $50^{\circ} 18^{\prime} \mathrm{W}$ $69^{\circ} 52$ 'N $50^{\circ} 03$ ' W $69^{\circ} 50^{\prime} \mathrm{N} \quad 50^{\circ} 04^{\prime} \mathrm{W}$ $69^{\circ} 48^{\prime} \mathrm{N} \quad 50^{\circ} 13^{\prime} \mathrm{W}$ $69^{\circ} 42$ ' N $50^{\circ} 04^{\prime} \mathrm{W}$ $69^{\circ} 40^{\prime} \mathrm{N} \quad 50^{\circ} 02^{\prime} \mathrm{W}$ $70^{\circ} 22^{\prime} \mathrm{N} \quad 53^{\circ} 12^{\prime} \mathrm{W} \quad \mathrm{SW}$ $70^{\circ} 211^{\prime} \mathrm{N} \quad 52^{\circ} 57, \mathrm{~W}$ SE $70^{\circ} 20^{\prime} \mathrm{N} \quad 52^{\circ} 40^{\prime} \mathrm{W}$ W

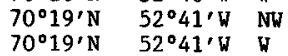

\begin{tabular}{ll}
20 & 0277.46 \\
& 3967.23 \\
\hline
\end{tabular}

$\begin{array}{ll}30 & 0167.68 \\ 10 & 0538.94\end{array}$

$\begin{array}{ll}10 & 0538.94 \\ 35 & 0622.83\end{array}$

$\begin{array}{ll}35 & 0622.83 \\ 10 & 0761.25\end{array}$

$15 \quad 0843.80$

$\begin{array}{ll}10 & 0200.48 \\ 30 & 0274.95\end{array}$ 0178.43 0395.07

$\begin{array}{ll}5 & 0620.39 \\ 35 & 0188.49\end{array}$ 19 0433.09

$\begin{array}{ll}30 & 0245.15 \\ 25 & 0474.62\end{array}$

$8 \quad 0184.86$ $\begin{array}{rr}8 & 0410.66 \\ 35 & 0198.93\end{array}$

$\begin{array}{ll}35 & 0198.93 \\ 35 & 0455.97\end{array}$ 1309.17 1428.01
0467.77 0182.28 0223.72

$25 \quad 0085.76$ 0145.03

$5 \quad 0132.92$ 8395.10

$\begin{array}{ll}25 & 0112.31 \\ 15 & 0329.01\end{array}$

$40 \quad 0144.23$

$\begin{array}{ll}30 & 0225.26 \\ 35 & 0155.86\end{array}$

$\begin{array}{ll}35 & 0155.86 \\ 55 & 0122.44\end{array}$

$35 \quad 0133.14$

\begin{tabular}{ll}
24 & 0316.56 \\
19 & 0131.24 \\
\hline & 027.49
\end{tabular}

$\begin{array}{ll}33 & 0207.49 \\ 10 & 0088.52\end{array}$

0997.59

$15 \quad 0236.22$ 0919.90

\begin{tabular}{ll}
0 & 0117.46 \\
\hline & 0127.64
\end{tabular}

$30 \quad 0139.49$ 0000.07

250000.08 $\begin{array}{rr}25 & 0008.36 \\ 85 & 0005.81\end{array}$

$\begin{array}{ll}100 & 0000.66 \\ 155 & 0000.31\end{array}$ $\begin{array}{ll}65112 & 6800-04 \\ 6800-04\end{array}$ 160113 6800-04 $163113 \quad 6800-04$ 163112 6800-04 $163112 \quad 6800-04$ 163112 6730-05 165112 6730-05 163112 6730-05 $1601126730-05$ $160113 \quad 6830-04$ $166112 \quad 6830-04$ $6830-04$
$6830-04$ $160113 \quad 6830-04$ $1601126830-04$ 164113 6830-04 166112 6830-04 $166112 \quad 6830-04$ $\begin{array}{ll}164112 & 6900-04 \\ 167112 & 6900-05\end{array}$ 163112 6900-04 16412 6900-05 $1631116900-05$ $166112 \quad 6900-05$ $163112 \quad 6930-05$ 166112 6930-05 163112 6930-05 166112 6930-05 $166112 \quad 6900-05$ 166112 6900-05 163112 6900-04 164112 6930-05 166112 6930-05 163112 6930-05 163112 6930-05 $700010 \quad 7000-02$ (7000-03 $260010 \quad 7000-03$ 


\begin{tabular}{|c|c|c|c|c|c|c|c|c|c|c|c|c|c|c|c|c|c|}
\hline CODE & LAT & LONG & ORI & HIGH & LOW & AREA & $\begin{array}{c}\text { MO } \\
\text { CODE }\end{array}$ & InAP & CODE & LAT & LONG & ORI & HIGH & LOW & AREA & $\begin{array}{c}\text { MO } \\
\text { CODE }\end{array}$ & MAP \\
\hline 1GH01007 & $70^{\circ} 19^{\prime} \mathrm{N}$ & $52^{\circ} 43^{\prime} \mathrm{W}$ & NW & 165 & 85 & 0 & 533012 & $7000-03$ & 6 & $\mathrm{~N}$ & $\mathbf{W}$ & NW & 105 & 85 & 0000.25 & 643112 & $7000-03$ \\
\hline 1GH01008 & $70^{\circ} 19^{\prime} \mathrm{N}$ & $52^{\circ} 45^{\prime} \mathrm{W}$ & $\mathbf{N}$ & 165 & 90 & 0002.15 & 530012 & $7000-03$ & 1GH02057 & $70^{\circ} 05^{\prime} \mathrm{N}$ & $51^{\circ} 51^{\prime} \mathrm{W}$ & W & 95 & 60 & 0000.43 & 643112 & $7000-03$ \\
\hline 1GH01009 & $70^{\circ} 19^{\prime} \mathrm{N}$ & $52^{\circ} 48^{\prime} \mathrm{W}$ & $\mathbf{N}$ & 170 & 85 & 0000.52 & 643010 & $7000-03$ & 1GH02058 & $70^{\circ} 04^{\prime} \mathrm{N}$ & $51^{\circ} 50^{\prime} \mathrm{W}$ & W & 95 & 90 & 0000.22 & 643112 & $7000-03$ \\
\hline 1GH01010 & $70^{\circ} 19^{\prime} \mathrm{N}$ & $52^{\circ} 49^{\prime} \mathrm{W}$ & $\mathbf{N}$ & 145 & & 0000.15 & 640010 & $7000-03$ & $1 \mathrm{GBO} 03001$ & $70^{\circ} 04^{\prime} \mathrm{N}$ & $51^{\circ} 50^{\circ} \mathrm{W}$ & NE & 100 & 95 & 0000.18 & 700010 & $7000-03$ \\
\hline 1GH01011 & $70^{\circ} 18^{\prime} \mathrm{N}$ & $52^{\circ} 45^{\prime} \mathrm{W}$ & NW & 110 & 80 & 0000.87 & 700010 & $7000-03$ & $1 \mathrm{GH} 03002$ & $70^{\circ} 05^{\prime} \mathrm{N}$ & $51^{\circ} 52^{\prime} \mathrm{H}$ & SE & 115 & 95 & 0000.28 & 670010 & $7000-03$ \\
\hline $1 \mathrm{GH} 01012$ & $70^{\circ} 17^{\prime} \mathrm{N}$ & $52^{\circ} 43^{\prime} \mathrm{W}$ & Sw & 200 & 145 & 0000.69 & 360010 & $7000-03$ & 1GH03003 & $70^{\circ} 06^{\prime} \mathrm{N}$ & $51^{\circ} 51 \% \mathrm{k}$ & s & 115 & 65 & 0002.88 & 600010 & $7000-03$ \\
\hline $1 \mathrm{GH} 02001$ & $70^{\circ} 17^{\prime} \cdot \mathrm{N}$ & $52^{\circ} 42^{\prime} \mathrm{W}$ & E & 150 & 115 & 0000.42 & 643012 & $7000-03$ & 1GH03004 & $70^{\circ} 07^{\prime} \mathrm{N}$ & $51^{\circ} 49 \%$ & SE & 110 & 85 & 0001.67 & 700010 & $7000-03$ \\
\hline $1 \mathrm{GH} 02002$ & $70^{\circ} 17^{\prime} \mathrm{N}$ & $52^{\circ} 42^{\prime} \mathrm{V}$ & $\mathrm{SE}$ & 200 & 150 & 0000.88 & 360010 & $7000-03$ & 1GH03005 & $70^{\circ} 06^{\prime} \mathrm{N}$ & $51^{\circ} 47^{\prime} \mathrm{W}$ & s & 125 & 60 & 0006.99 & 363012 & $7000-04$ \\
\hline $1 \mathrm{GH} 02003$ & $70^{\circ} 17^{\prime} \mathrm{N}$ & $52^{\circ} 39^{\prime} \mathrm{V}$ & - & 180 & 165 & 0000.33 & 300010 & $7000-03$ & $1 \mathrm{GH} 03006$ & $70^{\circ} 06^{\prime} \mathrm{N}$ & $51^{\circ} 46^{\prime} \mathrm{W}$ & SW & 120 & 80 & 0000.50 & 363012 & $7000-04$ \\
\hline $1 \mathrm{GH} 02004$ & $70^{\circ} 17^{\prime} \mathrm{N}$ & $52^{\circ} 37^{\prime} \mathrm{W}$ & - & 160 & 150 & 0000.16 & 700012 & $7000-03$ & $1 \mathrm{GH} 03007$ & $70^{\circ} 06^{\prime} \mathrm{N}$ & $51^{\circ} 44^{\prime} \mathrm{W}$ & SU & 120 & 105 & 0002.43 & 360010 & $7000-04$ \\
\hline $1 \mathrm{GH} 02005$ & $70^{\circ} 15^{\prime} \mathrm{N}$ & $52^{\circ} 34^{\prime} \mathrm{V}$ & SW & 176 & 145 & 0001.53 & 700010 & $7000-03$ & $1 \mathrm{GHO} 3008$ & $70^{\circ} 05^{\prime} \mathrm{N}$ & $51^{\circ} 42^{\prime} \mathrm{W}$ & SW & 110 & 25 & 0006.08 & 363312 & $7000-04$ \\
\hline 1GH02006 & $70^{\circ} 12$ ! N & $52^{\circ} 27^{\prime} \mathrm{W}$ & SE & 125 & 105 & 0000.69 & 260010 & 7000-03 & 1GH03009 & $70^{\circ} 04^{\prime} \mathrm{N}$ & $51^{\circ} 42^{\prime} \mathrm{W}$ & $\mathrm{W}$ & 95 & 60 & 0001.10 & 360010 & $7000-04$ \\
\hline 1GH02007 & $70^{\circ} 13^{\prime} \mathrm{N}$ & $52^{\circ} 26^{\prime} \mathrm{W}$ & SE & 135 & 105 & 0000.65 & 260010 & 7000-03 & 1GH03010 & $70^{\circ} 03^{\prime} \mathrm{N}$ & $51^{\circ} 43^{\prime} \mathrm{V}$ & sw & 85 & 85 & 0000.19 & 780010 & $7000-04$ \\
\hline $1 \mathrm{GH} 02008$ & $70^{\circ} 12^{\prime} \mathrm{N}$ & $52^{\circ} 19^{\prime} \mathrm{W}$ & - & 90 & 80 & 0000.28 & 700010 & 7000-03 & 1GH03011 & $70^{\circ} 03^{\prime} \mathrm{N}$ & $51^{\circ} 43^{\prime} \mathrm{W}$ & $w$ & 100 & 85 & 0000.17 & 780010 & $7000-04$ \\
\hline $1 \mathrm{GH} 02009$ & $70^{\circ} 13^{\prime} \mathrm{N}$ & $52^{\circ} 21^{\prime} \mathrm{W}$ & E & & 85 & 0001.64 & 263012 & 7000-03 & $1 \mathrm{GH} 04001$ & $70^{\circ} 03^{\prime} \mathrm{N}$ & $51^{\circ} 43^{\prime} \mathrm{W}$ & E & 100 & 100 & 0000.12 & 780010 & $7000-04$ \\
\hline $1 \mathrm{GH} 02010$ & $70^{\circ} 14^{\prime} \mathrm{N}$ & $52^{\circ} 23^{\prime} \mathrm{W}$ & $\mathrm{s}$ & 120 & 105 & 0000.46 & 770010 & $7000-03$ & 1GH04002 & $70^{\circ} 04^{\prime} \mathrm{N}$ & $51^{\circ} 43^{\prime}$ & SE & 100 & 90 & 0000.45 & 780010 & $7000-04$ \\
\hline $1 \mathrm{GH} 02011$ & $70^{\circ} 14^{\prime} \mathrm{N}$ & $52^{\circ} 22^{\prime} \mathrm{W}$ & E & 120 & 80 & 0001.16 & 643012 & $7000-03$ & $1 \mathrm{GH} 04003$ & $70^{\circ} 04^{\prime} \mathrm{N}$ & $51^{\circ} 42^{\prime} \mathrm{V}$ & $\mathrm{sW}$ & 90 & 85 & 0000.22 & 700010 & $7000-04$ \\
\hline 1GH02012 & $70^{\circ} 15^{\prime} \mathrm{N}$ & $52^{\circ} 22^{\prime} \mathrm{W}$ & E & 115 & 100 & 0000.32 & 670010 & $7000-03$ & 1GH05001 & $70^{\circ} 04^{\prime} \mathrm{N}$ & $51^{\circ} 40^{\prime} \mathrm{W}$ & SE & 95 & 50 & 0001.54 & 363112 & $7000-04$ \\
\hline $1 \mathrm{GH} 02013$ & $70^{\circ} 16^{\prime} \mathrm{N}$ & $52^{\circ} 21^{\prime} \mathrm{W}$ & $\mathbf{N}$ & 110 & 95 & 0001.02 & 643012 & $7000-03$ & $1 \mathrm{GH} 05002$ & $70^{\circ} 05^{\prime} \mathrm{N}$ & $51^{\circ} 40^{\prime} \mathrm{W}$ & SE & 100 & 55 & 0002.56 & 363112 & $7000-04$ \\
\hline $1 \mathrm{GH} 02014$ & $70^{\circ} 16^{\prime} \mathrm{N}$ & $52^{\circ} 23^{\prime} \mathrm{V}$ & E & 105 & 95 & 0000.30 & 640012 & $7000-03$ & $1 \mathrm{GH} 05003$ & $70^{\circ} 05^{\prime} \mathrm{N}$ & $51^{\circ} 38^{\prime} \mathrm{W}$ & B & 105 & 90 & 0000.42 & 360010 & $7000-04$ \\
\hline $1 \mathrm{GH} 02015$ & $70^{\circ} 15^{\prime} \mathrm{N}$ & $52^{\circ} 25^{\prime} \mathrm{W}$ & $\mathbf{N}$ & 158 & 65 & 0016.80 & 513316 & $7000-03$ & $1 \mathrm{GH} 05004$ & $70^{\circ} 06^{\prime} \mathrm{N}$ & $510^{\circ} 40, \mathrm{~V}$ & $\mathrm{E}$ & 110 & 14 & 0006.01 & 363312 & $7000-04$ \\
\hline $1 \mathrm{GH} 02016$ & $70^{\circ} 16^{\prime} \mathrm{N}$ & $52^{\circ} 30^{\prime} \mathrm{W}$ & NE & 115 & 95 & 0000.86 & 643112 & $7000-03$ & $1 \mathrm{GH} 05005$ & $70^{\circ} 06^{\prime} \mathrm{N}$ & $51^{\circ} 36^{\prime} \mathrm{W}$ & SE & 95 & 70 & 0000.73 & 363412 & $7000-04$ \\
\hline 1GH02017 & $70^{\circ} 16^{\prime} \mathrm{N}$ & $52^{\circ} 34^{\prime} \mathrm{W}$ & NE & 160 & 70 & 0004.83 & 513112 & 7000-03 & 1GH05006 & $70^{\circ} 07 \cdot \mathrm{N}$ & $51^{\circ} 40^{\prime} \mathrm{k}$ & E & 110 & 14 & 0008.58 & 363312 & $7000-04$ \\
\hline $1 \mathrm{GH} 02018$ & $70^{\circ} 17^{\prime N} \mathrm{~N}$ & $52^{\circ} 33^{\prime} \mathrm{W}$ & $\mathrm{SE}$ & 130 & 115 & 0000.11 & 700010 & $7000-03$ & $1 \mathrm{GH} 05007$ & $70^{\circ} 07^{\prime} \mathrm{N}$ & $51^{\circ} 40^{\prime} \mathrm{w}$ & SW & 110 & 105 & 0000.11 & 360012 & $7000-04$ \\
\hline $1 \mathrm{GHO} 2019$ & $70^{\circ} 17 \cdot \mathrm{N}$ & $52^{\circ} 36^{\prime} \mathrm{W}$ & $\mathbf{S}$ & 190 & 150 & 0001.40 & 260010 & $7000-03$ & $1 \mathrm{GH} 05008$ & $70^{\circ} 07 / \mathrm{N}$ & $51 \cdot 36, \mathrm{~V}$ & $\mathrm{E}$ & 110 & 85 & 0000.65 & 360012 & $7000-04$ \\
\hline $1 \mathrm{GHO} 2020$ & $70^{\circ} 17^{\prime} \mathrm{N}$ & $52^{\circ} 34^{\prime} \mathrm{W}$ & E & 190 & 105 & 0001.84 & 263112 & $7000-03$ & 1GH05009 & $70^{\circ} 08^{\prime} \mathrm{N}$ & $51^{\circ} 37 \cdot \mathrm{W}$ & $\bar{E}$ & 125 & 50 & 0004.42 & 363112 & $7000-04$ \\
\hline $1 \mathrm{GHO} 2021$ & $70^{\circ} 18^{\prime} \mathrm{N}$ & $52^{\circ} 31^{\prime} \mathrm{W}$ & $\mathrm{NE}$ & 130 & 105 & 0000.10 & 700010 & $7000-03$ & $1 \mathrm{GH} 05010$ & $70^{\circ} 09^{\prime} \mathrm{N}$ & $51^{\circ} 38^{\prime} \mathrm{W}$ & $\mathrm{E}$ & 125 & 65 & 0001.49 & 363112 & $7000-04$ \\
\hline $1 \mathrm{GH} 02022$ & $70^{\circ} 18^{\prime} \mathrm{N}$ & $52^{\circ} 32^{\prime} \mathrm{W}$ & E & 140 & 75 & 0001.79 & 643112 & $7000-03$ & 1GH05011 & $70^{\circ} 09^{\prime} \mathrm{N}$ & $51^{\circ} 38^{\prime} \mathrm{W}$ & E & 125 & 60 & 0003.85 & 360112 & $7000-04$ \\
\hline $1 \mathrm{GH} 02023$ & $70^{\circ} 19^{\prime} \mathrm{N}$ & $52^{\circ} 41^{\prime}$ & NE & & 125 & 0000.89 & 260010 & $7000-03$ & $1 \mathrm{GH} 05012$ & $70^{\circ} 09^{\prime} \mathrm{N}$ & $51^{\circ} 42^{\prime} \mathrm{W}$ & $\mathrm{NE}$ & 125 & 65 & 0014.38 & 366112 & $7000-04$ \\
\hline $1 \mathrm{GHO} 2024$ & $20^{\circ} 19^{\prime} \mathrm{N}$ & $52^{\circ} 36^{\prime} \mathrm{W}$ & $\mathbf{s}$ & 160 & 100 & 0026.77 & 263116 & $7000-03$ & $1 \mathrm{GH} 05013$ & $70^{\circ} 08^{\prime} \mathrm{N}$ & $51^{\circ} 46^{\prime} \mathrm{W}$ & $\mathbf{N}$ & 120 & 70 & 0005.36 & 360112 & $7000-04$ \\
\hline $1 \mathrm{GHO} 2025$ & $70^{\circ} 21^{\prime} \mathrm{N}$ & $52^{\circ} 35^{\prime} \mathrm{W}$ & $\mathrm{s}$ & 150 & 115 & 0000.85 & 260010 & $7000-03$ & $1 \mathrm{GH} 05014$ & $70^{\circ} 11 \cdot \mathrm{N}$ & $51^{\circ} 42^{\prime} \mathrm{V}$ & $\mathrm{s}$ & 125 & 115 & 0000.81 & 360010 & $7000-04$ \\
\hline 1 GH02026 & $70^{\circ} 22^{\prime} \mathrm{N}$ & $52^{\circ} 34^{\prime} \mathrm{W}$ & SW & 170 & 155 & 0000.56 & 260110 & $7000-03$ & 1GH05015 & $70^{\circ} 12^{\prime} \mathrm{N}$ & $510^{\circ}, \mathrm{v}$ & $\mathrm{s}$ & 125 & 100 & 0000.56 & 360010 & $7000-04$ \\
\hline 1GH02027 & $70^{\circ} 21^{\prime} \mathrm{N}$ & $52^{\circ} 31^{\prime} \mathrm{W}$ & SW & 172 & 150 & 0000.95 & 260110 & $7000-03$ & 1GH05016 & $70^{\circ} 11^{\prime} \mathrm{N}$ & $51^{\circ} 38^{\prime}$ & SW & 100 & 100 & 0000.58 & 360010 & $7000-04$ \\
\hline $1 \mathrm{GHO} 2028$ & $70^{\circ} 21^{\prime} \mathrm{N}$ & $52^{\circ} 29^{\prime} \mathrm{W}$ & $\mathbf{s}$ & 160 & 135 & 0001.50 & 263312 & $7000-03$ & 1GH05017 & $70^{\circ} 11^{\prime} \mathrm{N}$ & $51^{\circ} 36^{\prime} \mathrm{V}$ & SW & 85 & 85 & 0000.04 & 700010 & $7000-04$ \\
\hline $1 \mathrm{GH} 02029$ & $70^{\circ} 20^{\prime} \mathrm{N}$ & $52^{\circ} 27 ! \mathrm{W}$ & SE & 120 & 110 & 0000.45 & 260010 & $7000-03$ & 1GH05018 & $70^{\circ} 10^{\prime} \mathrm{N}$ & $51^{\circ} 36^{\prime \prime \prime}$ & $\mathrm{v}$ & 85 & 85 & 0000.09 & 700010 & $7000-04$ \\
\hline $1 \mathrm{GHO} 2030$ & $70^{\circ} 17^{\prime} \mathrm{N}$ & $52^{\circ} 09^{\prime} \mathrm{W}$ & NW & 121 & 90 & 0000.90 & 360012 & $7000-03$ & 1GH05019 & $70^{\circ} 09^{\prime} \mathrm{N}$ & $51^{\circ} 27^{\prime} \mathrm{W}$ & V & 110 & 75 & 000 & 363012 & $7000-04$ \\
\hline $1 \mathrm{GHO} 2031$ & $70^{\circ} \cdot 16^{\prime} \mathrm{N}$ & $52^{\circ} 09^{\prime} \mathrm{W}$ & NW & 121 & 70 & 0000.57 & 363012 & $7000-03$ & $1 \mathrm{GH} 05020$ & $70^{\circ} 09^{\prime} \mathrm{N}$ & $51^{\circ} 27 \mathrm{~W}$ & 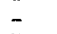 & 110 & 110 & 0000.27 & 360010 & $7000-04$ \\
\hline $1 \mathrm{GHO} 2032$ & $70^{\circ} 16^{\prime} \mathrm{N}$ & $52^{\circ} 08^{\prime} \mathrm{W}$ & SW & 121 & 105 & 0001.21 & 360010 & $7000-03$ & $16 \mathrm{H} 05021$ & $70^{\circ} 08^{\prime} \mathrm{N}$ & $51029 \% \mathrm{~V}$ & W & 115 & 90 & 0000.73 & 363112 & $7000-04$ \\
\hline 1GHO2033 & $70^{\circ} 14^{\prime} \mathrm{N}$ & $52^{\circ} 06^{\prime} \mathrm{W}$ & v & 125 & 95 & 0000.78 & 360012 & 700 & $1 \mathrm{GH} 05022$ & $70^{\circ} 07^{\prime} \mathrm{N}$ & $51^{\circ} 30^{\prime} \mathrm{V}$ & NW & 105 & 60 & 0000.85 & 643112 & $7000-04$ \\
\hline $1 \mathrm{GHO} 2034$ & $70^{\circ} 14^{\prime} \mathrm{N}$ & $52^{\circ} 05^{\prime} \mathrm{W}$ & v & 125 & 120 & 0000.42 & 360010 & 700 & $1 \mathrm{GH} 05023$ & $70^{\circ} 07 \cdot \mathrm{N}$ & $51^{\circ} 30^{\prime} \mathrm{W}$ & W & 110 & 55 & 00 & 600112 & $7000-04$ \\
\hline $1 \mathrm{GH} 02035$ & $70^{\circ} 13^{\prime} \mathrm{N}$ & $52^{\circ} 01^{\prime} \mathrm{W}$ & NW & 105 & 90 & 0000.19 & 260010 & $7000-03$ & $1 \mathrm{GH} 05024$ & $70^{\circ} 06^{\prime} \mathrm{N}$ & $51^{\circ} 30^{\prime} \mathrm{V}$ & W & 110 & 95 & 0000.41 & 700010 & $1-04$ \\
\hline 1GH02036 & $70^{\circ} 12^{\prime} \mathrm{N}$ & $51^{\circ} 59^{\prime} \mathrm{W}$ & NW & 140 & 110 & 0000.53 & 260010 & $7000-$ & $1 \mathrm{GHO} 05025$ & $70^{\circ} 06^{\prime N}$ & $51^{\circ} 29, \mathrm{~V}$ & SW & 115 & 100 & 0000.64 & 700010 & $7000-04$ \\
\hline 1GHO2037 & $70^{\circ} 12^{\prime} \mathrm{N}$ & $52^{\circ} 02^{\prime} \mathrm{W}$ & $\mathrm{N}$ & 130 & 85 & 0004.40 & 266112 & 700 & 1GH05026 & $70^{\circ} 06^{\prime} \mathrm{N}$ & $51^{\circ} 28, \mathrm{~W}$ & $\mathrm{~W}$ & 110 & 65 & 0001.12 & 643012 & $7000-04$ \\
\hline $1 \mathrm{GHO} 2038$ & $70^{\circ} 12^{\prime} \mathrm{N}$ & $52^{\circ} 05^{\prime} \mathrm{W}$ & NE & 110 & 90 & 0001.15 & 260112 & 700 & 1GH05027 & $70^{\circ} 05^{\prime} \mathrm{N}$ & $51^{\circ} 28^{\prime} \mathrm{W}$ & NV & 105 & 65 & 0001.29 & 643112 & $7000-04$ \\
\hline $1 \mathrm{GHO} 2039$ & $70^{\circ} 11^{\prime} \mathrm{N}$ & $52^{\circ} 05, \mathrm{~W}$ & $\mathrm{~W}$ & 110 & 100 & 0000.56 & 290010 & $7000-03$ & $1 \mathrm{GH} 05028$ & $70^{\circ} 05^{\prime} \mathrm{N}$ & $51030 \%$ & $\mathrm{~W}$ & 100 & 70 & 0000 & 600 & -04 \\
\hline 1GHO2040 & $70^{\circ} 12^{\prime} \mathrm{N}$ & $52^{\circ} 01^{\prime} \mathrm{W}$ & SE & 125 & 105 & 0001.25 & 260010 & $7000-03$ & 1GH05029 & $70^{\circ} 04^{\prime} \mathrm{N}$ & $51^{\circ} 30^{\prime} \mathrm{W}$ & sw & 100 & 95 & 0000.15 & 600010 & $7000-04$ \\
\hline $1 \mathrm{GHO} 2041$ & $70^{\circ} 12^{\prime} \mathrm{N}$ & $51^{\circ} 59^{\prime} \mathrm{F}$ & SW & 142 & 120 & 0000.70 & 260010 & $7000-03$ & & in & $51^{\circ} 29^{\prime} \mathrm{V}$ & w & 95 & 90 & 0000.16 & 700010 & $7000-04$ \\
\hline $1 \mathrm{GHO} 2042$ & $70^{\circ} 11^{\prime} \mathrm{N}$ & $51^{\circ} 57^{\prime} \mathrm{W}$ & SW & 125 & 120 & 0000.14 & 260010 & & $1 \mathrm{GH} 05031$ & & $51^{\circ} 29^{\prime} \mathrm{W}$ & NW & 85 & 65 & 0000.44 & 643112 & $7000-04$ \\
\hline $1 \mathrm{GHO} 2043$ & $70^{\circ} 10^{\prime} \mathrm{N}$ & $52^{\circ} 00^{\prime} \mathrm{W}$ & $\mathbf{N}$ & 105 & 70 & 0001.61 & 260112 & $7000-03$ & $1 \mathrm{GH} 08001$ & $70^{\circ} 03^{\prime} \mathrm{N}$ & $51^{\circ} 27^{\prime} \mathrm{V}$ & $\mathrm{E}$ & 95 & 55 & 0001.05 & 600010 & $7000-04$ \\
\hline $1 \mathrm{GH} 02044$ & $70^{\circ} 09^{\prime} \mathrm{N}$ & $52^{\circ} 01^{\prime} \mathrm{W}$ & w & 110 & 95 & 0000.27 & 263112 & $7000-03$ & $1 \mathrm{GHOBOO2}$ & $70^{\circ} 04^{\prime} \mathrm{N}$ & $51^{\circ} 28^{\prime \prime}$ & $\mathrm{SE}$ & 110 & 80 & 0001.51 & 300010 & $7000-04$ \\
\hline $1 \mathrm{GH} 02045$ & $70^{\circ} 09^{\prime} \mathrm{N}$ & $52^{\circ} 01^{\prime} \mathrm{W}$ & $\mathrm{W}$ & 110 & 70 & 0001.14 & 263112 & $7000-03$ & $1 \mathrm{GH} 08003$ & $70^{\circ} 05^{\prime N}$ & $51^{\circ} 26,6$ & E & 85 & 50 & 0000.67 & 643112 & $7000-04$ \\
\hline $1 \mathrm{GHO} 2046$ & $70^{\circ} 08^{\prime} \mathrm{N}$ & $52^{\circ} 01^{\prime} \mathrm{W}$ & NW & 115 & 115 & 0000.42 & 260010 & 7000 & $1 \mathrm{GHO}$ & & $51027 \%$ & $\mathrm{E}$ & 105 & 65 & 0000.43 & 643112 & $7000-04$ \\
\hline $1 \mathrm{GHO} 2047$ & $70^{\circ} 08^{\prime} \mathrm{N}$ & $52^{\circ} 00^{\prime} \mathrm{W}$ & SE & 115 & 100 & 0000.94 & 260010 & 7000 & 1GH08005 & $70^{\circ} 06^{\prime} \mathrm{N}$ & $51^{\circ} 28^{\prime}$ & E & 110 & 80 & 0000.48 & 643112 & $7000-04$ \\
\hline $1 \mathrm{GHO} 2048$ & $70^{\circ} 08^{\prime} \mathrm{N}$ & $51^{\circ} 57^{\prime} \mathrm{W}$ & SW & 119 & 100 & 0000.05 & 700010 & $7000-03$ & $1 \mathrm{GH} 08006$ & $70^{\circ} 06^{\prime} \mathrm{N}$ & $51^{\circ} 58^{\prime} \mathrm{W}$ & E & 110 & 90 & 0000 & 600012 & $7000-04$ \\
\hline $1 \mathrm{GHO} 2049$ & $70^{\circ} 09^{\prime} \mathrm{N}$ & $52^{\circ} 00^{\prime} \mathrm{W}$ & SE & 110 & 90 & 0000.89 & 260010 & $7000-03$ & 1GH08007 & $70^{\circ} 06^{\prime} \mathrm{N}$ & $51^{\circ} 27 \mathrm{~V}$ & B & 110 & 65 & 000 & 600112 & $7000-04$ \\
\hline 1GH02050 & $70^{\circ} 09^{\prime} \mathrm{N}$ & $51^{\circ} 53^{\prime} \mathrm{W}$ & $\mathrm{NW}$ & 110 & 85 & 0000.73 & 260010 & $7000-03$ & 1GH09001 & $70^{\circ} 06^{\prime} \mathrm{N}$ & $51^{\circ} 21^{\prime} \mathrm{v}$ & - & 96 & 85 & 0000.24 & 300010 & $7000-04$ \\
\hline $1 \mathrm{GHO} 2051$ & $70^{\circ} 09^{\prime} \mathrm{N}$ & $51^{\circ} 51^{\prime} \mathrm{W}$ & sw & 115 & 110 & 0000.44 & 260010 & $7000-03$ & $1 \mathrm{GH} 09002$ & $70^{\circ} 06^{\prime} \mathrm{N}$ & $51^{\circ} 22, \mathrm{k}$ & $\mathbf{N}$ & 75 & 55 & 0000.57 & 643112 & $7000-04$ \\
\hline 1GH02052 & $70^{\circ} 08^{\prime} \mathrm{N}$ & $51^{\circ} 52^{\prime} \mathrm{W}$ & w & 100 & 60 & 0000.48 & 363112 & $7000-03$ & $1 \mathrm{GH} 09003$ & $70^{\circ} 07^{\prime} \mathrm{N}$ & $51^{\circ} 26^{\prime} \mathrm{W}$ & & 115 & 85 & 0000.57 & 360010 & $7000-04$ \\
\hline 1GH02053 & $70^{\circ} 07 / \mathrm{N}$ & $51^{\circ} 51^{\prime} \mathrm{W}$ & $\mathrm{NH}$ & 127 & 50 & 0007.07 & 363112 & $7000-03$ & 1GH09004 & $70^{\circ} 08^{\prime} \mathrm{N}$ & $51^{\circ} 26,6$ & SE & 110 & 50 & 0002.46 & 363312 & 7000-04 \\
\hline $1 \mathrm{GHO} 2054$ & $70^{\circ} 06^{\prime} \mathrm{N}$ & $51^{\circ} 55^{\prime} \mathrm{W}$ & $\mathbf{N}$ & 100 & 50 & 0000.43 & 643112 & $000-03$ & $1 \mathrm{GHO9005}$ & $70^{\circ} 08^{\prime} \mathrm{N}$ & $51^{\circ} 24^{\prime} \mathrm{V}$ & $\mathrm{s}$ & 100 & 70 & 0001.72 & 363312 & $7000-04$ \\
\hline $1 \mathrm{GH} 02055$ & $70^{\circ} 06^{\prime} \mathrm{N}$ & $1057 \mathrm{~W}$ & $\mathbf{N}$ & 105 & 65 & 0001.15 & 603112 & $7000-03$ & 1GH09006 & $70^{\circ} 08^{\prime} \mathrm{N}$ & $51^{\circ} 22, \mathrm{v}$ & $\mathbf{s}$ & 95 & 80 & 0000.73 & 360010 & $7000-04$ \\
\hline
\end{tabular}




\begin{tabular}{|c|c|c|c|c|c|c|c|c|c|c|c|c|c|c|c|c|c|}
\hline CODE & LAT & LONG & ORI & HIGH & LOW & AREA & CODE & MAP & CODE & LAT & LONG & ORI & HIGH & LOW & AREA & $\begin{array}{c}\text { MO } \\
\text { CODE }\end{array}$ & MAP \\
\hline 1GH09007 & $70^{\circ} 08^{\prime} \mathrm{N}$ & $51^{\circ} 20^{\prime} \mathrm{W}$ & $\mathrm{s}$ & 95 & 90 & 0000.58 & 360100 & $7000-04$ & $1 \mathrm{HA02002}$ & $69^{\circ} 21^{\prime} \mathrm{N}$ & $53^{\circ} 38^{\prime} \mathrm{W}$ & SW & 90 & 85 & 0000.37 & 360110 & $6900-01$ \\
\hline $1 \mathrm{GH} 09008$ & $70^{\circ} 08^{\prime} \mathrm{N}$ & $51^{\circ} 19^{\prime} \mathrm{W}$ & E & 95 & 50 & 0000.95 & 363312 & $7000-04$ & $1 \mathrm{BA02003}$ & $69^{\circ} 20^{\prime} \mathrm{N}$ & $53^{\circ} 38^{\prime} \mathrm{W}$ & NW & 90 & 50 & 0010.79 & 363111 & $6900-01$ \\
\hline 1GH09009 & $70^{\circ} 08^{\prime} \mathrm{N}$ & $51^{\circ} 08^{\prime} \mathrm{W}$ & $\mathbf{N}$ & 90 & 55 & 0001.20 & 363012 & $7000-04$ & 1月A02004 & $69^{\circ} 19^{\prime} \mathrm{N}$ & $53^{\circ} 41$ ' & $\mathrm{N}$ & 80 & 50 & 0001.47 & 363111 & $6900-01$ \\
\hline 1GH09010 & $70^{\circ} 08^{\prime} \mathrm{N}$ & $51^{\circ} 10^{\prime} \mathrm{W}$ & NV & 95 & 50 & 0001.69 & 363012 & $7000-04$ & 1月A02005 & $69^{\circ} 18^{\prime} \mathrm{N}$ & $53^{\circ} 42^{\prime} \mathrm{W}$ & $s$ & 80 & 75 & 0000.08 & 690012 & $6900-01$ \\
\hline 1GH09011 & $70^{\circ} 07 ' \mathrm{~N}$ & $51^{\circ} 11^{\prime} \mathrm{W}$ & $w$ & 95 & 65 & 0001.86 & 363012 & $7000-04$ & 1月A02006 & $69^{\circ} 18^{\prime} \mathrm{N}$ & $53^{\circ} 41 \cdot \mathrm{W}$ & $s$ & 80 & 75 & 0000.36 & 700010 & $6900-01$ \\
\hline $1 \mathrm{GH} 10001$ & $70^{\circ} 07^{\prime} \mathrm{N}$ & $51^{\circ} 11^{\prime} \mathrm{W}$ & $\mathrm{SE}$ & 90 & 80 & 0000.49 & 360010 & $7000-04$ & 1HA02007 & $69^{\circ} 17^{\prime} \mathrm{N}$ & $53^{\circ} 41^{\prime} \mathrm{W}$ & SW & 65 & 65 & 0000.07 & 700010 & $6900-01$ \\
\hline $1 \mathrm{GH} 10002$ & $70^{\circ} 07^{\prime} \mathrm{N}$ & $51^{\circ} 09^{\prime} \mathrm{W}$ & $\mathbf{S}$ & 95 & 85 & 0000.64 & 360010 & $7000-04$ & 1HA03001 & $69^{\circ} 17^{\prime} \mathrm{N}$ & $53^{\circ} 40^{\prime} \mathrm{W}$ & & 60 & 60 & 0000.10 & 700010 & $6900-01$ \\
\hline 1GH11001 & $70^{\circ} 07 \cdot \mathrm{N}$ & $51^{\circ} 08^{\prime} \mathrm{W}$ & SE & 90 & 65 & 0001.54 & 360010 & $7000-04$ & $1 \mathrm{HA03002}$ & $69^{\circ} 17^{\prime} \mathrm{N}$ & $53^{\circ} 40^{\prime} \mathrm{W}$ & E & 55 & 55 & 0000.04 & 700010 & $6900-01$ \\
\hline $1 \mathrm{GH} 12001$ & $70^{\circ} 07^{\prime} \mathrm{N}$ & $51^{\circ} 07^{\prime} \mathrm{V}$ & $\mathrm{SE}$ & 80 & 70 & 0000.32 & 360010 & $7000-04$ & $1 \mathrm{HA} 03003$ & $69^{\circ} 18^{\prime} \mathrm{N}$ & $53^{\circ} 40^{\prime} \mathrm{W}$ & $\mathbf{s}$ & 75 & 70 & 0000.08 & 750010 & $6900-01$ \\
\hline 1GH15001 & $70^{\circ} 07^{\prime} \mathrm{N}$ & $51^{\circ} 07^{\prime} \mathrm{W}$ & $N$ & 70 & 50 & 0000.14 & 770010 & $7000-04$ & $1 \mathrm{HAO} 3004$ & $69^{\circ} 19^{\prime} \mathrm{N}$ & $53^{\circ} 37^{\prime}$ ט & y & 95 & 66 & 0001.23 & 363110 & $6900-01$ \\
\hline 1GH15002 & $70^{\circ} 08^{\prime} \mathrm{N}$ & $51^{\circ} 08^{\prime} \mathrm{W}$ & E & 95 & 45 & 0002.43 & 363112 & $7000-04$ & $1 \mathrm{HA} 03005$ & $69^{\circ} 18^{\prime} \mathrm{N}$ & $53^{\circ} 36^{\prime} \mathrm{v}$ & $\hat{W}$ & 95 & 71 & 0001.10 & 363110 & $6900-01$ \\
\hline $1 \mathrm{GH} 15003$ & $70^{\circ} 10^{\prime} \mathrm{N}$ & $51^{\circ} 13^{\prime} \mathrm{W}$ & SE & 90 & 80 & 0000.66 & 360110 & $7000-04$ & $1 \mathrm{HAO} 3006$ & $69^{\circ} 17^{\prime} \mathrm{N}$ & $53^{\circ} 34^{\prime} \mathrm{W}$ & SW & 80 & 70 & 0000.16 & 700010 & $6900-01$ \\
\hline $1 \mathrm{GH} 15004$ & $70^{\circ} 11^{\prime} \mathrm{N}$ & $51^{\circ} 12^{\prime} \mathrm{W}$ & SE & 110 & 80 & 0000.80 & 360010 & $7000-04$ & $1 \mathrm{HA03007}$ & $69^{\circ} 16^{\prime} \mathrm{N}$ & $53^{\circ} 33^{\prime} \mathrm{W}$ & $\mathrm{sW}$ & 70 & 65 & 0000.04 & 700010 & $6900-01$ \\
\hline $1 \mathrm{GH} 15005$ & $71^{\circ} 11^{\prime} \mathrm{N}$ & $51^{\circ} 11^{\prime} \mathrm{W}$ & SE & 110 & 90 & 0000.65 & 360010 & $7000-04$ & 1HA04001 & $69^{\circ} 17^{\prime} \mathrm{N}$ & $53^{\circ} 33^{\prime} \mathrm{W}$ & $\mathrm{SE}$ & 100 & 60 & 0001.70 & 363111 & $6900-01$ \\
\hline 1GH15006 & $70^{\circ} 12^{\prime} \mathrm{N}$ & $51^{\circ} 12^{\prime} \mathrm{W}$ & E & 110 & 50 & 0003.17 & 363012 & $7000-04$ & 1日A04002 & $69^{\circ} 18^{\prime} \mathrm{N}$ & $53^{\circ} 33^{\prime} \mathrm{W}$ & $\mathrm{E}$ & 95 & 49 & 0001.17 & 343111 & $6900-01$ \\
\hline 1GH15007 & $70^{\circ} 13^{\prime} \mathrm{N}$ & $51^{\circ} 12^{\prime} \mathrm{V}$ & E & 110 & 70 & 0003.40 & 360010 & $7000-04$ & 1HA04003 & $69^{\circ} 19^{\prime} \mathrm{N}$ & $53^{\circ} 32^{\prime} \mathrm{V}$ & E & 93 & 37 & 0005.73 & 343111 & $6900-01$ \\
\hline 1GH15008 & $70^{\circ} 13^{\prime} \mathrm{N}$ & $51^{\circ} 06^{\prime} \mathrm{W}$ & w & 90 & 80 & 0001.12 & 360112 & $7000-04$ & 1月A04004 & $69^{\circ} 20^{\prime} \mathrm{N}$ & $53^{\circ} 33^{\prime} \mathrm{V}$ & SW & 93 & 63 & 0001.31 & 343111 & $6900-01$ \\
\hline $1 \mathrm{GH} 15009$ & $70^{\circ} 12^{\prime} \mathrm{N}$ & $51^{\circ} 06^{\prime} \mathrm{V}$ & N & 95 & 70 & 0000.85 & 670012 & $7000-04$ & 1HA04005 & $69^{\circ} 20^{\prime} \mathrm{N}$ & $53^{\circ} 31^{\prime} \mathrm{W}$ & $\mathrm{NE}$ & 73 & 57 & 0000.07 & 643112 & $6900-01$ \\
\hline 1GH15010 & $70^{\circ} 11^{\prime} \mathrm{N}$ & $50^{\circ} 59^{\prime} \mathrm{W}$ & NE & 90 & 75 & 0000.54 & 670012 & $7000-04$ & 1HA04006 & $69^{\circ} 21^{\prime} \mathrm{N}$ & $53^{\circ} 33^{\prime} \mathrm{W}$ & $\mathrm{NE}$ & 93 & 47 & 0001.70 & 340111 & $6900-01$ \\
\hline 1GH15011 & $70^{\circ} 13^{\prime} \mathrm{N}$ & $51^{\circ} 04^{\prime} \mathrm{W}$ & $\mathrm{s}$ & 90 & 80 & 0001.12 & 360112 & $7000-04$ & 1HA04007 & $69^{\circ} 22^{\prime} \mathrm{N}$ & $53^{\circ} 26^{\prime} \mathrm{W}$ & $\mathrm{W}$ & 92 & 50 & 0002.22 & 770110 & $6900-01$ \\
\hline 1GH15012 & $70^{\circ} 13^{\prime} \mathrm{N}$ & $51^{\circ} 04^{\prime} \mathrm{V}$ & E & 90 & 70 & 0001.53 & 360112 & $7000-04$ & 1HA04008 & $69^{\circ} 21^{\prime} \mathrm{N}$ & $53^{\circ} 23^{\prime} \mathrm{W}$ & SW & 92 & 85 & 0000.61 & 770110 & $6900-01$ \\
\hline $1 \mathrm{GH15013}$ & $70^{\circ} 14^{\prime} \mathrm{N}$ & $51^{\circ} 05^{\prime} \mathrm{W}$ & $\mathrm{N}$ & 90 & 55 & 0001.28 & 360112 & $7000-04$ & $1 \mathrm{HA04009}$ & $69^{\circ} 19^{\prime} \mathrm{N}$ & $53^{\circ} 22^{\prime} \mathrm{W}$ & N & 93 & 43 & 0003.08 & 273111 & $6900-01$ \\
\hline 1GH15014 & $70^{\circ} 14^{\prime} \mathrm{N}$ & $50^{\circ} 57^{\prime} \mathrm{W}$ & SW & 100 & 90 & 0000.69 & 360012 & $7000-04$ & $1 \mathrm{HAO} 4010$ & $69^{\circ} 19^{\prime} \mathrm{N}$ & $53^{\circ} 25^{\circ} \mathrm{W}$ & N & 89 & 48 & 0000.76 & 343111 & $6900-01$ \\
\hline 1GH15015 & $70^{\circ} 13^{\prime} \mathrm{N}$ & $51^{\circ} 54^{\prime} \mathrm{W}$ & $\mathrm{N}$ & 80 & 55 & 0000.75 & 670012 & $7000-04$ & 1HA04011 & $69^{\circ} 18^{\prime} \mathrm{N}$ & $53^{\circ} 25^{\prime} \mathrm{W}$ & $\mathrm{W}$ & 93 & 43 & 0001.46 & 643111 & $6900-01$ \\
\hline 1GH15016 & $70^{\circ} 11^{\prime} \mathrm{N}$ & $51^{\circ} 51^{\prime} \mathrm{W}$ & NE & 75 & 65 & 0000.11 & 700010 & $7000-04$ & 1HA05001 & $69^{\circ} 18^{\prime} \mathrm{N}$ & $53^{\circ} 22^{\prime} \mathrm{W}$ & E & 87 & 67 & 0000.31 & 643112 & $6900-01$ \\
\hline 1GH15017 & $70^{\circ} 11^{\prime} \mathrm{N}$ & $50^{\circ} 44^{\prime} \mathrm{V}$ & $\mathrm{N}$ & 60 & 60 & 0000.20 & 700010 & $7000-05$ & 1HA05002 & $69^{\circ} 18^{\prime} \mathrm{N}$ & $53^{\circ} 20^{\prime} \mathrm{V}$ & $S E$ & 93 & 67 & 0001.02 & 293112 & $6900-01$ \\
\hline 1GH15018 & $70^{\circ} 14^{\prime} \mathrm{N}$ & $51^{\circ} 50^{\prime} \mathrm{W}$ & E & 80 & 70 & 0000.39 & 360010 & $7000-04$ & 1HA05003 & $69^{\circ} 19^{\prime} \mathrm{N}$ & $53^{\circ} 18^{\prime} \mathrm{K}$ & $\mathrm{E}$ & 76 & 47 & 0000.91 & 293112 & $6900-02$ \\
\hline 1GH15019 & $70^{\circ} 15^{\prime} \mathrm{N}$ & $51^{\circ} 48^{\prime} \mathrm{W}$ & SE & 75 & 60 & 0001.38 & 360010 & $7000-04$ & $1 \mathrm{HA} 05004$ & $69^{\circ} 20^{\prime} \mathrm{N}$ & $53^{\circ} 18^{\prime} \mathrm{W}$ & NE & 73 & 48 & 0000.47 & 293111 & $6900-02$ \\
\hline $1 \mathrm{GH} 15020$ & $70^{\circ} 13^{\prime} \mathrm{N}$ & $50^{\circ} 20^{\prime} \mathrm{W}$ & SW & 180 & 35 & 0128.93 & 163112 & $7000-05$ & 1HA05005 & $69^{\circ} 20^{\prime} \mathrm{N}$ & $53^{\circ} 17^{\prime} \mathrm{W}$ & $\mathrm{NE}$ & 65 & 55 & 0000.05 & 740111 & $6900-02$ \\
\hline 1GH16001 & $70^{\circ} 12^{\prime} \mathrm{N}$ & $50^{\circ} 15^{\prime} \mathrm{W}$ & $\mathrm{sW}$ & 180 & 20 & 0124.12 & 163112 & $7000-05$ & 1HA05006 & $69^{\circ} 21^{\prime} \mathrm{N}$ & $53^{\circ} 19^{\prime} \mathrm{W}$ & $\mathrm{E}$ & 65 & 60 & 0000.01 & 750111 & $6900-02$ \\
\hline $1 \mathrm{GH1} 700 \mathrm{I}$ & $70^{\circ} 04^{\prime} \mathrm{N}$ & $50^{\circ} 19^{\prime} \mathrm{V}$ & SW & 180 & & 1594.45 & 164112 & $7000-05$ & 1HA05007 & $69^{\circ} 211^{\prime} \mathrm{N}$ & $53^{\circ} 20^{\prime}$ & E & 92 & 48 & 0001.67 & 243111 & -02 \\
\hline $1 \mathrm{GH} 17002$ & $70^{\circ} 04^{\prime} \mathrm{N}$ & $50^{\circ} 00^{\prime} \mathrm{W}$ & Sw & 180 & 35 & 0076.66 & 163112 & $7000-05$ & 1HA05008 & $69^{\circ} 24^{\prime} \mathrm{N}$ & $53^{\circ} 12^{\prime \prime}$ & $\mathrm{W}$ & 103 & 72 & 0002.67 & 360112 & $6900-02$ \\
\hline $1 \mathrm{GH} 18001$ & $70^{\circ} 00^{\prime} \mathrm{N}$ & $50^{\circ} 09^{\prime} \mathrm{W}$ & W & 180 & & 2268.53 & 164111 & $6930-05$ & $1 \mathrm{HA} 05009$ & $69^{\circ} 23^{\circ} \mathrm{N}$ & $53^{\circ} 11, \mathrm{~W}$ & $\hat{W}$ & 90 & 55 & 0004.00 & 363111 & $6900-02$ \\
\hline $1 \mathrm{GH} 19001$ & $69^{\circ} 57^{\prime} \mathrm{N}$ & $50^{\circ} 16^{\prime} \mathrm{W}$ & W & 180 & & 0072.80 & 166111 & $6930-05$ & $1 \mathrm{HAO} 05010$ & $69^{\circ} 22^{\prime} \mathrm{N}$ & $53^{\circ} 11^{\prime} \mathrm{W}$ & $\mathrm{NW}$ & 95 & 60 & 0000.69 & 243111 & $6900-02$ \\
\hline $1 \mathrm{HAQ} 1001$ & $69^{\circ} 22^{\prime} \mathrm{N}$ & $54^{\circ} 08^{\prime} \mathrm{W}$ & - & 50 & 45 & 0000.05 & 750010 & $6900-01$ & 1HA05011 & $69^{\circ} 21^{\prime} \mathrm{N}$ & $53^{\circ} 12^{\prime} \mathrm{W}$ & NW & 95 & 45 & 0000.37 & 643111 & $6900-02$ \\
\hline $1 \mathrm{HAO} 1002$ & $69^{\circ} 22^{\prime} \mathrm{N}$ & $54^{\circ} 021 \mathrm{y}$ & $\mathbf{N}$ & 55 & 45 & 0000.17 & 740111 & $6900-01$ & $1 \mathrm{HAOSO12}$ & $69^{\circ} 21^{\prime} \mathrm{N}$ & $53^{\circ} 09^{\circ} \mathrm{V}$ & SW & 95 & 70 & 0000.48 & 200110 & $6900-02$ \\
\hline 1HA01003 & $69^{\circ} 22^{\prime} \mathrm{N}$ & $54^{\circ} 06^{\prime} \mathrm{W}$ & $\mathbf{N}$ & 65 & 50 & 0000.09 & 740112 & $6900-01$ & 1HA06001 & $69^{\circ} 21 \cdot \mathrm{N}$ & $53^{\circ} 08^{\prime} \mathrm{V}$ & $\mathrm{E}$ & 95 & 75 & 0000.09 & 700110 & $6900-02$ \\
\hline 1HA01004 & $69^{\circ} 25^{\prime} \mathrm{N}$ & $54^{\circ} 02.4$ & W & 80 & 65 & 0000.75 & 260111 & $6900-01$ & 1HA06002 & $69^{\circ} 21^{\prime} \mathrm{N}$ & $53^{\circ} 08^{\prime} \mathrm{W}$ & SE & 100 & 75 & 0000.97 & 790010 & $6900-02$ \\
\hline 1HA01005 & $69^{\circ} 24^{\prime} \mathrm{N}$ & $54^{\circ} 01^{\prime} \mathrm{W}$ & $W$ & 80 & 80 & 0000.43 & 270110 & $6900-01$ & 1 HA06003 & $69^{\circ} 22^{\prime} \mathrm{N}$ & $53^{\circ} 08^{\prime} \mathrm{W}$ & $\mathrm{SE}$ & 105 & 45 & 0002.20 & 360010 & $6900-02$ \\
\hline 1HA01006 & $69^{\circ} 24^{\prime} \mathrm{N}$ & $53^{\circ} 58^{\prime} \mathrm{W}$ & $\mathrm{s}$ & 80 & 70 & 0000.73 & 270100 & 690 & 1HA06004 & $69^{\circ} 22^{\prime} \mathrm{N}$ & $53^{\circ} 07^{\prime} \mathrm{V}$ & $\mathrm{E}$ & 85 & 50 & 0000.63 & 360010 & $6900-02$ \\
\hline 1HA01007 & $69^{\circ} 23^{\prime} \mathrm{N}$ & $53^{\circ} 57^{\prime} \mathrm{W}$ & NW & 60 & 50 & 0000.04 & 770000 & $6900-01$ & 1HA06005 & $69^{\circ} 23^{\prime} \mathrm{N}$ & $53^{\circ} 07^{\prime} \mathrm{V}$ & $\mathrm{E}$ & 100 & 55 & 000 & 360 & -02 \\
\hline 1HA01008 & $69^{\circ} 23^{\prime} \mathrm{N}$ & $53^{\circ} 56^{\prime} \mathrm{V}$ & NW & 70 & 70 & 0000.15 & 700010 & $6900-01$ & 1HA06006 & $69^{\circ} 23^{\prime} \mathrm{N}$ & $53^{\circ} 06^{\prime} \mathrm{V}$ & $\mathrm{E}$ & 65 & 55 & 0000.05 & 200010 & $6900-02$ \\
\hline 1НA01009 & $69^{\circ} 23^{\prime} \mathrm{N}$ & $53^{\circ} 55^{\prime} \mathrm{W}$ & E & 70 & 70 & 0000.07 & 700010 & $6900-01$ & 1HA06007 & $69^{\circ} 24^{\prime} \mathrm{N}$ & $53^{\circ} 07$ V & $\mathrm{SE}$ & & 65 & 0003.42 & 360010 & $6900-02$ \\
\hline 1HA01010 & $69^{\circ} 24^{\prime} \mathrm{N}$ & $53^{\circ} 56^{\prime} \mathrm{W}$ & E & 80 & 65 & 0001.67 & 200010 & 69 & 1HA06008 & $69^{\circ} 24^{\prime} \mathrm{N}$ & $53^{\circ} 05^{\prime} \mathrm{W}$ & $\mathbf{S}$ & & 65 & 0004.54 & 360010 & $6900-02$ \\
\hline 1HA01011 & $69^{\circ} 24^{\prime} \mathrm{N}$ & $53^{\circ} 53^{\prime} \mathrm{W}$ & s & 60 & 60 & 0000.21 & 770010 & 6900 & 1HA06009 & $69^{\circ} 23^{\prime} \mathrm{N}$ & $53^{\circ} 03^{\prime} \mathrm{W}$ & SW & & 60 & 0000.87 & 360010 & $6900-02$ \\
\hline 1HA01012 & $69^{\circ} 24^{\prime} \mathrm{N}$ & $53^{\circ} 52^{\prime} \mathrm{W}$ & W & 70 & 60 & 0000.01 & 780010 & $6900-01$ & 1HA06010 & $69^{\circ} 23^{\prime} \mathrm{N}$ & $53^{\circ} 02^{\prime} \mathrm{V}$ & $s$ & & 55 & 0000.62 & 360010 & $6900-02$ \\
\hline 1 HA01013 & $69^{\circ} 23^{\prime} \mathrm{N}$ & $53^{\circ} 52^{\prime} \mathrm{W}$ & S & 60 & 45 & 0000.17 & 770010 & $6900-01$ & 1HA07001 & $69^{\circ} 21^{\prime} \mathrm{N}$ & $53^{\circ} 02^{\prime} \mathrm{V}$ & SE & & 65 & 0001.63 & 360010 & $6900-02$ \\
\hline 1HA01014 & $69^{\circ} 24^{\prime} \mathrm{N}$ & $53^{\circ} 42^{\prime} \mathrm{W}$ & $\mathrm{sw}$ & 88 & 80 & 0001.62 & 270010 & $6900-01$ & $1 \mathrm{HAO}$ & $69^{\circ} 22^{\prime} \mathrm{N}$ & $53^{\circ} 00^{\prime} \mathrm{W}$ & $\mathrm{E}$ & 65 & 50 & 0000.93 & 370010 & $6900-02$ \\
\hline $1 \mathrm{HA} 01015$ & $69^{\circ} 24^{\prime} \mathrm{N}$ & $53^{\circ} 40^{\prime} \mathrm{W}$ & $\mathrm{s}$ & 88 & 80 & 0001.59 & 270010 & & 1HA07003 & $69^{\circ} 22^{\prime} \mathrm{N}$ & $53^{\circ} 04^{\prime} \mathrm{W}$ & $\mathrm{SE}$ & & 60 & 0003.99 & 363311 & $6900-02$ \\
\hline 1HA01016 & $69^{\circ} 22^{\prime} \mathrm{N}$ & $53^{\circ} 40^{\prime} \mathrm{W}$ & $\mathbf{N}$ & 70 & 60 & 0000.18 & 770010 & $6900-01$ & 1HA07004 & $69^{\circ} 23^{\prime} \mathrm{N}$ & $53^{\circ} 03^{\prime} \mathrm{W}$ & $\mathrm{SE}$ & & 65 & 0001.79 & 360010 & $6900-02$ \\
\hline 1HA01017 & $69^{\circ} 22^{\prime} \mathrm{N}$ & $53^{\circ} 41^{\prime} \mathrm{W}$ & $\mathbf{N}$ & 75 & 55 & 0000.14 & 770010 & $6900-01$ & 1HA07005 & $69^{\circ} 23^{\prime} \mathrm{N}$ & $53^{\circ} 03^{\prime} \mathrm{W}$ & $\mathrm{E}$ & & 55 & 0003.28 & 360010 & $6900-02$ \\
\hline $1 \mathrm{HA01018}$ & $69^{\circ} 22^{\prime} \mathrm{N}$ & $53^{\circ} 42^{\prime} \mathrm{W}$ & N & 80 & 55 & 0002.86 & 333111 & $6900-01$ & 1HA07 & $69^{\circ} 23^{\prime} \mathrm{N}$ & $53^{\circ} 03^{\prime}$ & E & & 35 & 0006.88 & 363012 & $6900-02$ \\
\hline 1HA01019 & $69^{\circ} 22^{\prime} \mathrm{N}$ & $53^{\circ} 44^{\prime} \mathrm{W}$ & NW & 85 & 70 & 0001.03 & 740012 & & 1HA07007 & $69^{\circ} 24^{\prime} \mathrm{N}$ & $53^{\circ} 02^{\prime} \mathrm{W}$ & SE & & 55 & 0004.33 & 360010 & $6900-02$ \\
\hline 1HA01020 & $69^{\circ} 22^{\prime} \mathrm{N}$ & $53^{\circ} 44^{\prime} \mathrm{W}$ & W & 85 & 70 & 0000.24 & 200010 & $6900-01$ & 1HA07008 & $69^{\circ} 25^{\prime} \mathrm{N}$ & $52^{\circ} 59^{\prime} \mathrm{V}$ & $\mathrm{S}$ & & 20 & 0013.11 & 363211 & $6900-02$ \\
\hline $1 \mathrm{HA01021}$ & $69^{\circ} 21^{\prime} \mathrm{N}$ & $53^{\circ} 42^{\prime} \mathrm{W}$ & NW & 75 & 50 & 0000.64 & 710010 & $6900-01$ & 1HA07009 & $69^{\circ} 25^{\prime} \mathrm{N}$ & $52^{\circ} 55^{\prime} \mathrm{V}$ & SW & & 70 & 0006.50 & 363012 & $6900-02$ \\
\hline 1HA01022 & $69^{\circ} 21^{\prime} \mathrm{N}$ & $53^{\circ} 44^{\prime} \mathrm{W}$ & NW & 80 & 50 & 0000.52 & 743010 & & $1 \mathrm{HA} 07010$ & $69^{\circ} 24^{\prime} \mathrm{N}$ & $52^{\circ} 53^{\prime} \mathrm{V}$ & u & & 80 & 0002.43 & 360010 & $6900-02$ \\
\hline $1 \mathrm{HAO} 1023$ & $69^{\circ} 22, \mathrm{~N}$ & $53^{\circ} 45 . \mathrm{W}$ & N & 80 & 45 & 0000.76 & 743112 & $6900-01$ & 1HAOTO11 & $69^{\circ} 28^{\prime} \mathrm{N}$ & $52^{\circ} 54^{\prime} \mathrm{W}$ & ฟ & 70 & 60 & 0000.15 & 630012 & $6900-02$ \\
\hline 1НA01024 & $69^{\circ} 21^{\prime} \mathrm{N}$ & $53^{\circ} 46^{\prime} \mathrm{W}$ & $\mathbf{N}$ & 70 & 50 & 0000.03 & 740010 & $6900-01$ & 1HA07012 & $69^{\circ} 24^{\prime} \mathrm{N}$ & $52^{\circ} 50^{\prime} \mathrm{W}$ & $\mathrm{N}$ & 70 & 60 & 0000.08 & 700010 & $6900-02$ \\
\hline 1HA01025 & $69^{\circ} 21^{\prime} \mathrm{N}$ & $53^{\circ} 47^{\prime} \mathrm{W}$ & $\mathrm{N}$ & 70 & 40 & 0000.35 & 740011 & 6900-01 & 1HA08001 & $69^{\circ} 24^{\prime} \mathrm{N}$ & $52^{\circ} 50^{\prime} \mathrm{W}$ & E & 70 & 60 & 0000.09 & 790010 & 6900-02 \\
\hline $1 \mathrm{HA} 01026$ & $69^{\circ} 21^{\prime} \mathrm{N}$ & $3^{\circ} 48^{\prime} \mathrm{V}$ & NW & 60 & 40 & 0000.06 & 740112 & $6900-01$ & 1HA08002 & $69^{\circ} 25^{\prime N} \mathrm{~N}$ & $52^{\circ} 48^{\prime} \mathrm{W}$ & $\mathrm{NE}$ & 80 & 65 & .0000 .54 & 673012 & $6900-02$ \\
\hline $1 \mathrm{HA} 02001$ & $69^{\circ} 20^{\prime} \mathrm{N}$ & $53^{\circ} 43^{\prime} \mathrm{V}$ & $\mathrm{s}$ & 75 & 75 & 0000.11 & 750010 & $6900-01$ & 1HA08003 & $69^{\circ} 26^{\prime} \mathrm{N}$ & $52 \circ 50^{\prime} \mathrm{V}$ & $\mathrm{NE}$ & 65 & 50 & 0000.24 & 770010 & $6900-02$ \\
\hline
\end{tabular}


LAT LONG ORI HIGH LOW AREA CODE MAP

$\begin{array}{llllllll}69^{\circ} 27^{\prime} \mathrm{N} & 52^{\circ} 47^{\prime} \mathrm{W} & \text { SW } & 80 & 70 & 0000.05 & 790010 & 6900-02\end{array}$

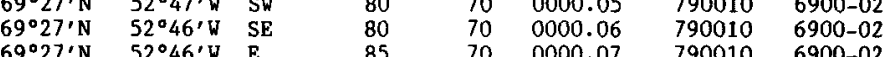

$\begin{array}{lll}69^{\circ} 27 \cdot \mathrm{N} & 52^{\circ} 46^{\prime} \mathrm{W} & \mathrm{E} \\ 69^{\circ} 27^{\prime} \mathrm{N} & 52^{\circ} 48^{\prime} \mathrm{W} & \mathrm{N}\end{array}$

$69^{\circ} 28^{\prime} \mathrm{N} \quad 52^{\circ} 48^{\prime} \mathrm{W} \quad \mathrm{N}$

$69^{\circ} 28^{\prime} \mathrm{N} \quad 52^{\circ} 52^{\prime} \mathrm{W}$ E

$69^{\circ} 29^{\prime} \mathrm{N} \quad 52^{\circ} 53^{\prime} \mathrm{W}$ SE

$69^{\circ} 30^{\prime} \mathrm{N} \quad 52^{\circ} 46^{\prime} \mathrm{W}$ SE

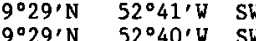

$69^{\circ} 28^{\prime} \mathrm{N} \quad 52^{\circ} 35^{\prime} \mathrm{W} \quad \mathrm{E}$

$69^{\circ} 28^{\prime} \mathrm{N} \quad 52^{\circ} 38^{\prime} \mathrm{W} \quad \mathrm{NE}$

$69^{\circ} 29^{\prime} \mathrm{N} \quad 52^{\circ} 39^{\prime} \mathrm{N}$

$69^{\circ} 30^{\prime} \mathrm{N} \quad 52^{\circ} 40^{\prime} \mathrm{W}$ E

$69^{\circ} 30^{\prime} \mathrm{N} \quad 52^{\circ} 41^{\prime} \mathrm{W}$

$69^{\circ} 31^{\prime} \mathrm{N} \quad 52^{\circ} 45, \mathrm{~W}$

$69^{\circ} 32$ 'N $52^{\circ} 50^{\prime} \mathrm{W}$ E

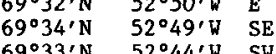

$69^{\circ} 33^{\prime} N 52^{\circ} 44^{\prime} \mathrm{W}$ S

$69^{\circ} 34^{\prime} \mathrm{N} \quad 52^{\circ} 45^{\prime} \mathrm{W}$ N

$69^{\circ} 34^{\prime} \mathrm{N} 52^{\circ} 45^{\prime} \mathrm{W}$ NE

$\begin{array}{lll}69^{\circ} 35^{\prime} \mathrm{N} & 52^{\circ} 45^{\prime} \mathrm{W} & \mathrm{E} \\ 69^{\circ} 37^{\prime} \mathrm{N} & 52^{\circ} 477^{\prime} \mathrm{W} & \mathrm{E}\end{array}$

$\begin{array}{lll}69^{\circ} 37^{\prime} \mathrm{N} & 52^{\circ} 47^{\prime} \mathrm{W} & \mathrm{E} \\ 69^{\circ} 37^{\prime} \mathrm{N} & 52^{\circ} 45^{\prime} \mathrm{W} & \mathrm{NE}\end{array}$

$52^{\circ} 00^{\prime} \mathrm{W} S$

$69^{\circ} 38^{\prime} \mathrm{N} 52^{\circ} 48^{\prime} \mathrm{N}$

$69^{\circ} 38^{\prime} \mathrm{N} \quad 52^{\circ} 48^{\prime} \mathrm{W}$

$69^{\circ} 33^{\prime} \mathrm{N} \quad 52^{\circ} 29^{\prime} \mathrm{N} N$

$69^{\circ} 33^{\prime} \mathrm{N} \quad 52^{\circ} 32^{\prime} \mathrm{N}$ NE

$69^{\circ} 34^{\prime} \mathrm{N} \quad 52^{\circ} 34^{\prime} \mathrm{W}$

$69^{\circ} 35^{\prime} \mathrm{N} 52^{\circ} 31^{\prime} \mathrm{W}$ NE

$\begin{array}{lll}69^{\circ} 35^{\prime} \mathrm{N} & 52^{\circ} 33^{\prime} \mathrm{W} & \mathrm{N} \\ 69^{\circ} 35^{\prime} \mathrm{N} & 52^{\circ} 34^{\prime} \mathrm{W} & \mathrm{NB}\end{array}$

$\begin{array}{lll}69^{\circ} 35^{\prime} \mathrm{N} & 52^{\circ} 34^{\prime}, \mathrm{W} & \mathrm{NE} \\ 69^{\circ} 35^{\prime} \mathrm{N} & 52^{\circ} 35^{\prime} \mathrm{W} & \mathrm{SW}\end{array}$

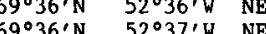

$69^{\circ} 37^{\prime} \mathrm{N} 52^{\circ} 38^{\prime} \mathrm{W} \quad \mathrm{NE}$

$69^{\circ} 38^{\prime} \mathrm{N} \quad 52^{\circ} 34^{\prime} \mathrm{W} \quad \mathrm{NE}$

$69^{\circ} 39^{\prime} \mathrm{N} \quad 52^{\circ} 40^{\prime} \mathrm{W}$ SW

$69^{\circ} 39^{\prime} \mathrm{N} \quad 52^{\circ} 36^{\prime} \mathrm{W}$ S

$69^{\circ} 37^{\prime} \mathrm{N} \quad 52^{\circ} 38^{\prime} \mathrm{W}$

$69^{\circ} 37^{\prime} \mathrm{N} \quad 52^{\circ} 29^{\prime} \mathrm{W}$ NE

$\begin{array}{lll}69^{\circ} 38^{\prime} \mathrm{N} & 52^{\circ} 30^{\prime} \mathrm{W} & \mathrm{S} \\ 69^{\circ} 38^{\prime} \mathrm{N} & 52^{\circ} 28^{\prime} \mathrm{V} & \mathrm{S}\end{array}$

$69^{\circ} 38^{\prime} \mathrm{N} \quad 52^{\circ} 23^{\prime} \mathrm{W}$

$69^{\circ} 42^{\prime} \mathrm{N} \quad 54^{\circ} 52^{\prime} \mathrm{V}$

$69^{\circ} 39^{\prime} \mathrm{N} \quad 54^{\circ} 41^{\prime} \mathrm{W}$ W

$69^{\circ} 39^{\prime} \mathrm{N} \quad 54^{\circ} 42^{\prime} \mathrm{W}$

$69^{\circ} 39^{\prime} \mathrm{N} \quad 54^{\circ} 31^{\circ} \mathrm{W}$

$\begin{array}{rrrr}69^{\circ} 38^{\prime} \mathrm{N} & 54^{\circ} 32^{\prime} \mathrm{N} & \mathrm{NW} & 105 \\ 69^{\circ} 38^{\prime} \mathrm{N} & 52^{\circ} 33^{\prime} \mathrm{N} & 85\end{array}$

0000.07
0000.46

60010
360010
$6900-02$

$670010 \quad 6900-02$

$0004.63 \quad 360010 \quad 6900-02$

$0012.91 \quad 363311 \quad 6930-03$

$0004.61 \quad 363312 \quad 6930-03$

$300010 \quad 6900-02$

$\begin{array}{rllll}105 & 95 & 0000.19 & 700010 & 6900-02 \\ 65 & 60 & 0000.06 & 790012 & 6900-02 \\ 65 & 50 & 0000.14 & 640012 & 6900-02 \\ 80 & 60 & 0000.21 & 643112 & 6900-02\end{array}$

$\begin{array}{lllll}80 & 60 & 0000.21 & 643112 & 6900-02 \\ 70 & 55 & 0000.17 & 790010 & 6900-02\end{array}$

70

80

0001.17

$\begin{array}{rr}790010 & 6900-02 \\ 360010 & 6900-02\end{array}$

$\begin{array}{ll}360010 & 6900-02 \\ 670112 & 6930-03\end{array}$

$363411 \quad 6930-03$

$\begin{array}{lll}0000.39 & 750010 \quad 6930-03\end{array}$

$\begin{array}{lllll} & 55 & 0007.67 & 363411 & 6930-03 \\ 80 \quad 50 & 0000.06 & 780010 & 6930-03\end{array}$

$\begin{array}{rrrrr}95 & 100 & 0000.16 & 300110 & 6930-03 \\ & 75 & 0000.92 & 770110 & 6930-03\end{array}$

$\begin{array}{llll}55 & 0000.61 & 360110 & 6930-03 \\ 0000.61 & 363411 & 6930-03 \\ & 0001.32 & 360110 & 6930-03\end{array}$

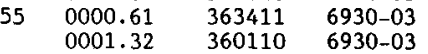

$\begin{array}{lll}0001.32 & 360110 & 6930-03 \\ 0000.22 & 770110 & 6930-03\end{array}$

$\begin{array}{lll}0000.22 & 360110 \quad 6930-03\end{array}$

$\begin{array}{lll}0004.17 & 363111 & 6930-03\end{array}$

$0004.17 \quad 360000 \quad 6930-03$

$\begin{array}{rllll}70 & 50 & 0000.33 & 730010 & 6930-03 \\ 70 & 45 & 0000.33 & 790012 & 6930-03 \\ 80 & 60 & 0000.16 & 790012 & 6930-03 \\ 105 & 90 & 0000.45 & 300010 & 6930-03\end{array}$

$\begin{array}{llll}90 & 0000.45 & 300010 & 6930-03 \\ 65 & 0000.07 & 790010 & 6930-03\end{array}$

$\begin{array}{lll}0000.07 & 790010 & 6930-03 \\ 0000.72 & 633112 & 6930-03\end{array}$

$\begin{array}{llll}70 & 0000.92 & 633112 & 6930-03 \\ 85 & 0000.17 & 770010 & 6930-03\end{array}$

0001.02

$\begin{array}{ll}770010 & 6930-03 \\ 603212 & 6930-03\end{array}$

$0000.54 \quad 600210 \quad 6930-03$

$0000.88 \quad 600210 \quad 6930-03$

$\begin{array}{lll}0003.34 & 363411 & 6930-03 \\ & 6930-03\end{array}$

$0002.18 \quad 360110 \quad 6930-03$

$0002.86 \quad 363110 \quad 6930-03$

$0000.25 \quad 700010 \quad 6930-03$

$0000.81 \quad 360110 \quad 6930-03$

$\begin{array}{lll}0002.34 & 300012 & 6930-03\end{array}$

$\begin{array}{ll}640112 & 6930-01 \\ 363412 & 6930=01\end{array}$

$740010 \quad 6930-01$

$740010 \quad 6930-01$

$300010 \quad 6930-01$

0000.07

0000.24

$50 \quad 0000.38$

$\begin{array}{ll}643112 & 6930-01 \\ 643112 & 6930-01\end{array}$

N $52^{\circ} 34^{\prime} \mathrm{WN} \quad \mathrm{NW}$

69.37'N 52.35' W -

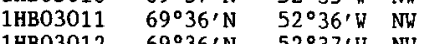

$\begin{array}{llll}1 \mathrm{HB} 03013 & 69^{\circ} 36^{\prime} \mathrm{N} & 52^{\circ} 38^{\prime} \mathrm{W} & \mathrm{N}\end{array}$

1 HB03014 $69^{\circ} 36^{\prime} \mathrm{N} \quad 52^{\circ} 49^{\prime} \mathrm{W}$

HB03015 69०36'N $52^{\circ} 46^{\prime} \mathrm{W} N$

$\begin{array}{lllll}1 \mathrm{HB} 03016 & 69^{\circ} 36 / \mathrm{N} & 52^{\circ} 41^{\prime} \mathrm{W} & \mathrm{N}\end{array}$

$1 \mathrm{HB03018} 69^{\circ} 36^{\prime} \mathrm{N} \quad 54^{\circ} 43^{\prime} \mathrm{W} N$

$\begin{array}{llll}\text { HB03019 } & 69^{\circ} 36^{\prime} \mathrm{N} & 54^{\circ} 45^{\prime} \mathrm{W} & \mathrm{N} \\ \mathrm{HB} 03020 & 69^{\circ} 36^{\prime} \mathrm{N} & 54^{\circ} 46^{\prime} \mathrm{W} & \mathrm{NW}\end{array}$

1 HB04001 $69^{\circ} 36^{\prime} \mathrm{N} \quad 54^{\circ} 40^{\prime} \mathrm{W} \quad \mathrm{NW}$

$\begin{array}{llll}1 \mathrm{BB} 04002 & 69^{\circ} 35^{\prime} \mathrm{N} & 54^{\circ} 37^{\prime} \mathrm{V} & \mathrm{SW} \\ 1 \mathrm{HB} 04003 & 69^{\circ} 36^{\prime} \mathrm{N} & 54^{\circ} 33^{\prime} \mathrm{W} & \mathrm{NW}\end{array}$

190403

HBO5002

$1 \mathrm{H} 05003$

1 HB05005

1HB05006

1 HB05008

$1 \mathrm{HB} 05009$

$1 \mathrm{HB} 05010$

$1 \mathrm{HB} 06002$

$1 \mathrm{HB} 07001$

$1 \mathrm{HB} 07002$

$1 \mathrm{HB} 07004$

1HB07005

1 HB08001

$1 \mathrm{HB} 08002$

$1 \mathrm{HB} 08003$

$1 \mathrm{HB} 08004$
$1 \mathrm{HB} 08005$

$1 \mathrm{HB} 08006$

1 HB08007

$1 \mathrm{HB} 09002$

$1 \mathrm{~B} 09004$

$1 \mathrm{HB} 09005$

$1 \mathrm{HB} 09006$

$1 \mathrm{HB} 09007$

$1 \mathrm{HB} 09008$

$1 \mathrm{HB} 10002$

1HB 10003
$1 \mathrm{HB} 10004$

1HB10004

$1 \mathrm{HB} 10006$

1 HBB 10007

$1 \mathrm{HB} 10009$

1HB10010

1HB10012 . $37^{\prime} \mathrm{N} 54^{\circ} 30^{\prime} \mathrm{N}$

$69^{\circ} 38^{\prime} \mathrm{N}$

$54^{\circ} 24^{\prime} \mathrm{W} S$

$69^{\circ} 36^{\prime} \mathrm{N} \quad 54^{\circ} 28^{\prime} \mathrm{W}$ SW

$69^{\circ} 36^{\prime} \mathrm{N} 54^{\circ} 27^{\prime} \mathrm{W}$

6.35'N $54^{\circ} 27 \mathrm{~W}$ W

$69^{\circ} 34^{\prime} \mathrm{N} \quad 54^{\circ} 28^{\prime} \mathrm{W}$ N

$69^{\circ} 34^{\prime} \mathrm{N} \quad 54^{\circ} 28^{\prime} \mathrm{W}$ NW

$69^{\circ} 36^{\prime} \mathrm{N} \quad 54^{\circ} 24^{\prime} \mathrm{W} \mathrm{S}$

69036 'N $54^{\circ} 233^{\prime} \mathrm{W}$ E

$69^{\circ} 36^{\prime} \mathrm{N} \quad 54^{\circ} 23^{\prime} \mathrm{W}$ E

$69^{\circ} 36^{\prime} \mathrm{N} \quad 54^{\circ} 16^{\circ} \mathrm{W}$ E

$69^{\circ} 35^{\prime} \mathrm{N} \quad 54^{\circ} 16^{\prime} \mathrm{W}$ W

$69^{\circ} 36^{\prime} \mathrm{N}$

$54^{\circ} 16^{\prime} \mathrm{W}$ W

$69^{\circ} 35{ }^{\prime} \mathrm{N}$

$54^{\circ} 11^{\prime} \mathrm{W}$

69.34' $54^{\circ} 13^{\prime} \mathrm{W} \mathrm{W}$

$69^{\circ} 35^{\prime} \mathrm{N} \quad 54^{\circ} 08^{\prime} \mathrm{W}$ SW

$69^{\circ} 34^{\prime} \mathrm{N} \quad 54^{\circ} 06^{\prime} \mathrm{W}$ W

$69^{\circ} 34^{\prime} \mathrm{N} \quad 54^{\circ} 03^{\prime} \mathrm{W} \quad \mathrm{N}$

$69^{\circ} 34^{\prime} \mathrm{N} \quad 54^{\circ} 04^{\prime} \mathrm{W}$.

$69^{\circ} 35^{\prime} \mathrm{N} \quad 54^{\circ} 04^{\prime} \mathrm{W} \quad \mathrm{N}$

$69^{\circ} 35^{\prime} \mathrm{N} \quad 54^{\circ} 06^{\prime} \mathrm{W} N \mathrm{~N}$

$69^{\circ} 36^{\prime} \mathrm{N} \quad 54^{\circ} 08^{\prime} \mathrm{W} \quad \mathrm{NE}$

$69^{\circ} 36^{\prime} \mathrm{N} \quad 54^{\circ} 09^{\prime} \mathrm{W} \quad \mathrm{N}$

$\begin{array}{llll}69^{\circ} 41^{\prime} N & 54^{\circ} 08^{\prime}, \mathrm{N} & \mathrm{NE} \\ 69^{\circ} 41^{\prime} \mathrm{N} & 54^{\circ} 09^{\prime} \mathrm{W} & \mathrm{B}\end{array}$

$69^{\circ} 42^{\prime} N \quad 54^{\circ} 08^{\prime} N$ SE

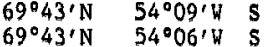

$69^{\circ} 42^{\prime} \mathrm{N} \quad 54^{\circ} 05^{\circ} \mathrm{N}$ N

$69^{\circ} 42^{\prime} \mathrm{N}$
$69^{\circ} 43 \prime \mathrm{N}$

$54^{\circ} 05^{\circ} \mathrm{W}$ N

$54^{\circ} 06^{\prime} \mathrm{W}$

$69^{\circ} 46^{\prime} \mathrm{N} \quad 54^{\circ} 06^{\prime} \mathrm{W}$ E

$\begin{array}{ll}69^{\circ} 48^{\prime} \mathrm{N} & 54^{\circ} 02^{\prime} \mathrm{W} \\ 69^{\circ} 47^{\prime} \mathrm{N} & 54^{\circ} 00^{\prime} \mathrm{W}\end{array}$

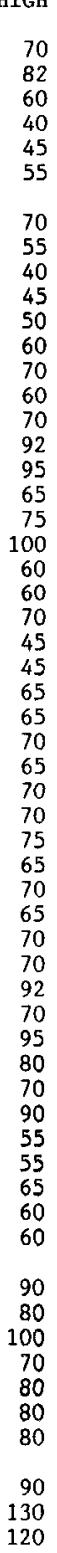

$643112 \quad 6930-01$

$790010 \quad 6930-01$

$790010 \quad 6930-01$

$790010 \quad 6930-01$

$790010 \quad 6930-01$

640112 6930-0

$640112 \quad 6930-01$

640112 6930-01

790010 6930-01

690010 630-01

$643112 \quad 6930-01$

$7000106930-01$

$6431126930-01$

640010 6930-01

$770012 \quad 6930-01$

$780012 \quad 6930-01$

770012 6930-01

$790010 \quad 6930-01$

$643112 \quad 6930-01$

$6431126930-01$

600010 6930-01

643112 6930-01

643112 6930-01

$300010 \quad 6930-01$

640010 6930-01

300010 6930-01

$643112 \quad 6930-01$

$643112 \quad 6930-01$

643112 6930-01

643112 6930-01

$700010 \quad 6930-01$

643112 6930-01

$\begin{array}{ll}780010 & 6930-01 \\ 300010 & 6930-01\end{array}$

$643112 \quad 6930-01$

223112 6930-01

$\begin{array}{ll}260010 & 6930-01 \\ 260010 & 6930-01\end{array}$

$\begin{array}{lll}300010 & 6930-01 & \infty\end{array} 643111 \quad 6930-01 \stackrel{\infty}{ }$ 


\begin{tabular}{|c|c|c|c|c|c|c|c|c|c|c|c|c|}
\hline CODE & LAT & LONG & ORI & $\mathrm{HIGH}$ & LOW & AREA & $\begin{array}{c}\text { Mo } \\
\text { CODE }\end{array}$ & MAP & CODE & LAT & LONG & ORI \\
\hline $1 \mathrm{HB} 10013$ & $69^{\circ} 46^{\prime} \mathrm{N}$ & $54^{\circ} 00^{\prime} \mathrm{W}$ & W & 120 & 65 & 0007.42 & 643017 & $6930-01$ & 1HB11027 & $69^{\circ} 42^{\prime} \mathrm{N}$ & $53^{\circ} 35^{\prime} \mathrm{H}$ & SE \\
\hline $1 \mathrm{HB1} 0014$ & $69^{\circ} 45^{\prime} \mathrm{N}$ & $53^{\circ} 58^{\prime} \mathrm{W}$ & W & 90 & 60 & 0002.35 & 643112 & $6930-01$ & $1 \mathrm{HB} 11028$ & $69^{\circ} 43^{\prime} \mathrm{N}$ & $53^{\circ} 36^{\prime} \mathrm{v}$ & $S E$ \\
\hline 1 HB10015 & $69^{\circ} 44^{\prime} \mathrm{N}$ & $53^{\circ} 59^{\prime} \mathrm{W}$ & NW & 80 & 60 & 0000.11 & 300010 & 6930-01 & 1HB11029 & $69^{\circ} 45^{\prime} \mathrm{N}$ & $53^{\circ} 36^{\prime} \mathrm{W}$ & $\mathrm{s}$ \\
\hline $1 \mathrm{HB} 10016$ & $69^{\circ} 44^{\prime} \mathrm{N}$ & $53^{\circ} 59^{\prime} \mathrm{W}$ & $\mathrm{sW}$ & 80 & 60 & 0000.23 & 600010 & $6930-01$ & $1 \mathrm{HB} 11030$ & $69^{\circ} 46^{\prime} \mathrm{N}$ & $53^{\circ} 31^{\prime} \mathrm{W}$ & SW \\
\hline $1 \mathrm{HB10017}$ & $59^{\circ} 43^{\prime} \mathrm{N}$ & $53^{\circ} 59^{\prime} \mathrm{W}$ & $v$ & 70 & 50 & 0001.06 & 600010 & $6930-01$ & $1 \mathrm{HB} 11031$ & $69^{\circ} 45^{\prime} \mathrm{N}$ & $53^{\circ} 31 \cdot \mathrm{w}$ & SW \\
\hline 1 HB10018 & $69^{\circ} 43^{\prime} \mathrm{N}$ & $53^{\circ} 58^{\prime} \mathrm{W}$ & NW & 70 & 50 & 0000.42 & 643112 & 6930-01 & 1HB11032 & $69^{\circ} 45^{\prime} \mathrm{N}$ & $53^{\circ} 30^{\circ} \mathrm{W}$ & SW \\
\hline 1 HB10019 & $69^{\circ} 42^{\prime} \mathrm{N}$ & $53^{\circ} 58^{\prime} \mathrm{W}$ & V & 70 & 55 & 0000.21 & 643112 & $6930-01$ & 1HB11033 & $69^{\circ} 45^{\prime} \mathrm{N}$ & $53^{\circ} 28^{\prime} \mathrm{W}$ & s \\
\hline $1 \mathrm{HB} 10020$ & $69^{\circ} 42^{\prime} \mathrm{N}$ & $53^{\circ} 58^{\prime} \mathrm{V}$ & & & & 0000.65 & 643112 & $6930-01$ & 1HB11034 & $69^{\circ} 43^{\prime} \mathrm{N}$ & $53^{\circ} 28^{\prime} \mathrm{W}$ & NW \\
\hline $1 \mathrm{HB} 10021$ & $69^{\circ} 42^{\prime} \mathrm{N}$ & $53^{\circ} 58^{\prime} \mathrm{V}$ & NW & & 40 & 0000.55 & 643112 & $6930-01$ & 1HB11035 & $69^{\circ} 43^{\prime} \mathrm{N}$ & $53^{\circ} 28^{\prime} \mathrm{W}$ & \\
\hline $1 \mathrm{HB} 10022$ & $69^{\circ} 43^{\prime} \mathrm{N}$ & $53^{\circ} 56^{\prime} \mathrm{W}$ & $\mathrm{E}$ & 75 & 50 & 0000.13 & 790112 & $6930-02$ & $1 \mathrm{HB} 11036$ & $69^{\circ} 42^{\prime} \mathrm{N}$ & $53^{\circ} 28^{\prime} \mathrm{W}$ & SW \\
\hline $1 \mathrm{HB} 10023$ & $69^{\circ} 44^{\prime} \mathrm{N}$ & $53^{\circ} 56^{\prime} \mathrm{V}$ & $\mathrm{E}$ & 85 & 50 & 0000.19 & 643112 & $6930-02$ & $1 \mathrm{HB} 11037$ & $69^{\circ} 42^{\prime} \mathrm{N}$ & $53^{\circ} 26^{\circ} \mathrm{V}$ & W \\
\hline $1 \mathrm{HB} 10024$ & $69^{\circ} 24^{\prime} \mathrm{N}$ & $53^{\circ} 55^{\prime} \mathrm{W}$ & E & 96 & 60 & 0000.15 & 643112 & $6930-02$ & 1HB11038 & $69^{\circ} 41^{\prime} \mathrm{N}$ & $53^{\circ} 26^{\prime} \mathrm{W}$ & SW \\
\hline $1 \mathrm{HB} 10025$ & $69^{\circ} 45^{\prime} \mathrm{N}$ & $53^{\circ} 56^{\prime} \mathrm{W}$ & SE & 110 & 70 & 0000.81 & 600112 & $6930-02$ & 1HB11039 & $69^{\circ} 38^{\prime} \mathrm{N}$ & $53^{\circ} 37^{\circ} \mathrm{W}$ & $\mathrm{NE}$ \\
\hline $1 \mathrm{HB} 1$ & $69^{\circ} 46^{\prime} \mathrm{N}$ & $53^{\circ} 56^{\prime} \mathrm{v}$ & 5 & 110 & 70 & 0001.42 & 600110 & 93 & $1 \mathrm{HB} 11040$ & $69^{\circ} 39^{\prime} \mathrm{N}$ & $53^{\circ} 38^{\prime} \mathrm{W}$ & $\mathrm{N}$ \\
\hline $1 \mathrm{HB} 10027$ & $69^{\circ} 48^{\prime} \mathrm{N}$ & $53^{\circ} 49 \cdot \mathrm{W}$ & $\mathrm{SW}$ & 120 & 115 & 0014.30 & 263111 & $6930-02$ & 1HB11041 & $69^{\circ} 38^{\prime} \mathrm{N}$ & $53^{\circ} 39^{\prime} \mathrm{w}$ & $\mathrm{N}$ \\
\hline $1 \mathrm{HB} 10028$ & $69^{\circ} 46^{\prime} \mathrm{N}$ & $53^{\circ} 52^{\prime} \mathrm{v}$ & - & 100 & 60 & 0000.06 & 700010 & $6930-02$ & 1HB11042 & $69^{\circ} 38^{\prime} \mathrm{N}$ & $53^{\circ} 40^{\prime} \mathrm{W}$ & $\mathrm{N}$ \\
\hline $1 \mathrm{HB} 10030$ & $69^{\circ} 45^{\prime} \mathrm{N}$ & $53^{\circ} 52^{\prime} \mathrm{V}$ & $\mathrm{SE}$ & 80 & 60 & 0000.08 & 700010 & $6930-02$ & $1 \mathrm{HB} 11043$ & $69^{\circ} 38^{\prime} \mathrm{N}$ & $53^{\circ} 40^{\prime} \mathrm{w}$ & $\mathrm{NW}$ \\
\hline $1 \mathrm{HB} 10031$ & $69^{\circ} 46^{\prime} \mathrm{N}$ & $53^{\circ} 50^{\prime} \mathrm{v}$ & $\mathrm{s}$ & 80 & 50 & 0000.52 & 643010 & $6930-02$ & $1 \mathrm{HB} 11044$ & $69^{\circ} 38^{\prime} \mathrm{N}$ & $53^{\circ} 42^{\prime} \mathrm{W}$ & V \\
\hline $1 \mathrm{HB} 10032$ & $69^{\circ} 47 \cdot \mathrm{N}$ & $53^{\circ} 50^{\prime} \mathrm{v}$ & $\mathrm{s}$ & 100 & 80 & 0000.18 & 643010 & 693 & $1 \mathrm{HB} 11045$ & $69^{\circ} 377^{\prime} \mathrm{N}$ & $53^{\circ} 43^{\prime} \mathrm{W}$ & $\hat{\mathrm{W}}$ \\
\hline $1 \mathrm{HB} 10033$ & $69^{\circ} 46^{\prime} \mathrm{N}$ & $53^{\circ} 48^{\prime} \mathrm{W}$ & $s$ & 60 & 40 & 0001.93 & 260010 & $6930-02$ & $1 \mathrm{HB} 11046$ & $69^{\circ} 37^{\prime} \mathrm{N}$ & $53^{\circ} 44^{\prime} \mathrm{W}$ & NW \\
\hline $1 \mathrm{HB} 10034$ & $69^{\circ} 45^{\prime} \mathrm{N}$ & $53^{\circ} 47^{\prime} \mathrm{W}$ & $\mathrm{s}$ & 70 & 60 & 0000.06 & 260010 & $6930-02$ & 1HB11047 & $69^{\circ} 37 \cdot \mathrm{N}$ & $53^{\circ} 45 \%$ & N \\
\hline $1 \mathrm{HB} 10035$ & $69^{\circ} 47^{\prime} \mathrm{N}$ & $53^{\circ} 48^{\prime} \mathrm{W}$ & $\mathrm{s}$ & 85 & 50 & 0005.08 & 263010 & $6930-02$ & 1HB11048 & $69^{\circ} 377^{\prime N}$ & $53^{\circ} 46^{\prime}$ & $\mathrm{NE}$ \\
\hline $1 \mathrm{HB} 1$ & $69^{\circ} 47 \times \mathrm{N}$ & $53^{\circ} 40^{\prime} \mathrm{v}$ & $\mathrm{sW}$ & 90 & 35 & 0030.07 & 263016 & 6930 & 1HB11049 & $69^{\circ} 36^{\prime}, \mathrm{N}$ & $53^{\circ} 48^{\prime} \mathrm{W}$ & N \\
\hline $1 \mathrm{HB1}$ & $69^{\circ} 45^{\prime} \mathrm{N}$ & $53^{\circ} 42^{\prime} \mathrm{v}$ & $\mathrm{NW}$ & 75 & 45 & 0003.48 & 200010 & 6930-02 & $1 \mathrm{HB} 12001$ & $69^{\circ} 36^{\prime} \mathrm{N}$ & $53^{\circ} 45, \mathrm{~W}$ & $\mathrm{SW}$ \\
\hline 1 HB10038 & $69^{\circ} 44^{\prime} \mathrm{N}$ & $53^{\circ} 44^{\prime} \mathrm{V}$ & $\mathrm{N}$ & & 50 & 0000.39 & 700010 & $6930-02$ & 1 HB12002 & $69^{\circ} 33^{\prime} \mathrm{N}$ & $53^{\circ} 42, \mathrm{~V}$ & $\mathrm{~N}$ \\
\hline $1 \mathrm{HB} 10039$ & $69^{\circ} 44^{\prime} \mathrm{N}$ & $53^{\circ} 44^{\prime} \mathrm{W}$ & $\mathrm{NW}$ & & 50 & 0000.20 & 700010 & $6930-02$ & $1 \mathrm{HB} 12003$ & $69^{\circ} 29^{\prime N}$ & $53^{\circ} 44^{\prime}$ & $\mathrm{NW}$ \\
\hline $1 \mathrm{HB} 10040$ & $69^{\circ} 43^{\prime} \mathrm{N}$ & $53^{\circ} 47^{\prime} \mathrm{W}$ & $\mathbf{N}$ & 60 & 35 & 0000.86 & 263112 & $6930-02$ & 1HB12004 & $69^{\circ} 30^{\prime} \mathrm{N}$ & $53^{\circ} 42^{\prime} \mathrm{W}$ & $\mathrm{SE}$ \\
\hline $1 \mathrm{HB} 10041$ & $69^{\circ} 43^{\prime} \mathrm{N}$ & $53^{\circ} 48, \mathrm{~W}$ & $\mathrm{~N}$ & 65 & 35 & 0001.22 & 643112 & & $1 \mathrm{HB} 13001$ & $69^{\circ} 32^{\prime} \mathrm{N}$ & $53^{\circ} 38^{\prime} \mathrm{W}$ & $\mathrm{NW}$ \\
\hline $1 \mathrm{HB} 10042$ & $69^{\circ} 44^{\prime} \mathrm{N}$ & $53^{\circ} 50^{\prime} \mathrm{W}$ & $\mathrm{N}$ & 60 & 50 & 0000.09 & 780010 & 693 & $1 \mathrm{HB} 13002$ & $69^{\circ} 30^{\prime} \mathrm{N}$ & $53^{\circ} 40^{\prime} \mathrm{V}$ & m \\
\hline $1 \mathrm{HB} 10043$ & $69^{\circ} 43^{\prime} \mathrm{N}$ & $53^{\circ} 49^{\prime} \mathrm{W}$ & - & 70 & 60 & 0000.08 & 700010 & $6930-02$ & 1HB13003 & $69^{\circ} 33^{\prime} \mathrm{N}$ & $53^{\circ} 41^{\prime \prime} \mathrm{W}$ & $\mathrm{SE}$ \\
\hline $1 \mathrm{HB} 10044$ & $69^{\circ} 42^{\prime} \mathrm{N}$ & $53^{\circ} 48^{\prime} \mathrm{W}$ & $\mathrm{NW}$ & 70 & 40 & 0001.95 & 643111 & 6930 & $1 \mathrm{HB} 13004$ & $69^{\circ} 36^{\prime} \mathrm{N}$ & $53^{\circ} 45$ & $E$ \\
\hline $1 \mathrm{HB} 10045$ & $69^{\circ} 42^{\prime} \mathrm{N}$ & $53^{\circ} 50^{\prime} \mathrm{W}$ & $\mathrm{N}$ & 70 & 50 & 0001.10 & 643112 & 693 & 1HB13005 & $69^{\circ} 37^{\prime} \mathrm{N}$ & $53^{\circ} 43^{\prime} \mathrm{V}$ & - \\
\hline & $69^{\circ} 41^{\prime} \mathrm{N}$ & $53^{\circ} 52^{\prime} \mathrm{W}$ & $\mathrm{NW}$ & 60 & 35 & 0000.1 & 700010 & 693 & 1HB13006 & $69^{\circ} 37^{\prime N} \mathrm{~N}$ & $53^{\circ} 43^{\prime} \mathrm{W}$ & $E$ \\
\hline 1HB10047 & $69^{\circ} 39^{\prime} \mathrm{N}$ & $53^{\circ} 53^{\prime} \mathrm{W}$ & NW & 75 & 50 & 0000.2 & 790010 & 693 & 1HB13007 & $69^{\circ} 37^{\prime} \mathrm{N}$ & $53^{\circ} 40^{\prime} \mathrm{W}$ & 5 \\
\hline $1 \mathrm{HB} 10048$ & $69^{\circ} 38^{\prime} \mathrm{N}$ & $53^{\circ} 55^{\prime} \mathrm{W}$ & $w$ & 65 & 45 & 0000.16 & 790010 & $6930-02$ & 1HB13008 & $69^{\circ} 36^{\prime} \mathrm{N}$ & $53^{\circ} 40^{\prime}$ & $\mathrm{SW}$ \\
\hline $1 \mathrm{HB} 11001$ & $69^{\circ} 38^{\prime} \mathrm{N}$ & $53^{\circ} 54^{\prime} \mathrm{W}$ & s & 80 & 70 & 0000.10 & 790010 & 693 & 1HB13009 & $69^{\circ} 36^{\prime N} \mathrm{~N}$ & $53^{\circ} 39^{\prime} \mathrm{y}$ & - \\
\hline 1HB11002 & $69^{\circ} 39^{\prime} \mathrm{N}$ & $53^{\circ} 54^{\prime} \mathrm{W}$ & NE & 85 & 75 & 0000.08 & 700010 & 6930 & $1 \mathrm{HB} 13010$ & $69^{\circ} 36^{\prime} \mathrm{N}$ & $53^{\circ} 38^{\prime} \mathrm{W}$ & $=$ \\
\hline $1 \mathrm{HB} 11003$ & $69^{\circ} 30^{\prime} \mathrm{N}$ & $53^{\circ} 43^{\prime} \mathrm{W}$ & $\mathbf{S}$ & & 70 & & 600012 & & $1 \mathrm{HB} 13011$ & $69^{\circ} 36^{\prime} \mathrm{N}$ & $53^{\circ} 37^{\prime} \mathrm{W}$ & $\overline{\mathrm{SW}}$ \\
\hline $1 \mathrm{HB} 11004$ & $69^{\circ} 41 \cdot \mathrm{N}$ & $53^{\circ} 48^{\prime} \mathrm{W}$ & SW & & 70 & 0001.14 & 200012 & 693 & 1HB14001 & $69^{\circ} 36^{\prime} \mathrm{N}$ & $53^{\circ} 37^{\prime} \mathrm{W}$ & $\mathrm{N}$ \\
\hline 1HB11005 & $69^{\circ} 41^{\prime} \mathrm{N}$ & $53^{\circ} 47 \cdot \mathrm{W}$ & $\mathrm{s}$ & & 10 & 0000.17 & 300010 & 693 & 1HB14002 & $69^{\circ} 36^{\prime} \mathrm{N}$ & $53^{\circ} 38^{\prime} \mathrm{W}$ & $\mathrm{NE}$ \\
\hline $1 \mathrm{HB} 11006$ & $69^{\circ} 40^{\prime} \mathrm{N}$ & $53^{\circ} 47^{\prime} \mathrm{W}$ & NW & 85 & 70 & 00 & 700010 & & 1HB14003 & $69^{\circ} 37^{\prime} \mathrm{N}$ & $53^{\circ} 38^{\prime} \mathrm{W}$ & E \\
\hline $1 \mathrm{HB} 11007$ & $69^{\circ} 39^{\prime} \mathrm{N}$ & $53^{\circ} 48^{\prime} \mathrm{W}$ & NW & 80 & 65 & 0000.11 & 640112 & 6930 & 1HB14004 & $69^{\circ} 37^{\prime} \mathrm{N}$ & $53^{\circ} 37^{\prime \prime} \mathrm{W}$ & $\mathrm{E}$ \\
\hline $1 \mathrm{HB} 11008$ & $69^{\circ} 39^{\prime} \mathrm{N}$ & $53^{\circ} 46^{\prime} \mathrm{W}$ & $\mathrm{NV}$ & 95 & 95 & 0000.11 & 640112 & & 1HB14005 & $69^{\circ} 37 \times \mathrm{N}$ & $53^{\circ} 38^{\prime} \mathrm{W}$ & \\
\hline $1 \mathrm{HB} 11009$ & $69^{\circ} 36^{\prime} \mathrm{N}$ & $53^{\circ} 39^{\prime} \mathrm{v}$ & - & 105 & 100 & 00 & 700010 & & 1HB14006 & $69^{\circ} 40^{\prime} \mathrm{N}$ & $53^{\circ} 25^{\prime} \mathrm{W}$ & $\mathrm{s}$ \\
\hline $1 \mathrm{HB} 1$ & $69^{\circ} 36^{\prime} \mathrm{N}$ & $53^{\circ} 38^{\prime} \mathrm{W}$ & SE & 100 & 60 & & 20 & & $1 \mathrm{HB1} 4007$ & $69^{\circ} 39^{\prime} \mathrm{N}$ & $53^{\circ} 22, \mathrm{~V}$ & $\overrightarrow{\mathrm{W}}$ \\
\hline 1HB11011 & $69^{\circ} 36^{\prime} \mathrm{N}$ & $53^{\circ} 37 / \mathrm{W}$ & $\mathrm{E}$ & & 90 & 0000.09 & 700010 & 693 & 1HB15001 & $69^{\circ} 38^{\prime} \mathrm{N}$ & $53^{\circ} 211 \mathrm{~V}$ & $\mathrm{~B}$ \\
\hline $1 \mathrm{HB} 11012$ & $69^{\circ} 42^{\prime} \mathrm{N}$ & $53^{\circ} 45^{\prime} \mathrm{W}$ & E & 110 & 90 & 0000.17 & 640012 & $6930-02$ & 1HB15002 & $69^{\circ} 39^{\prime} \mathrm{N}$ & $53^{\circ} 22, \mathrm{~W}$ & E \\
\hline $1 \mathrm{HB} 11013$ & $69^{\circ} 42^{\prime} \mathrm{N}$ & $53^{\circ} 46^{\prime} \mathrm{W}$ & E & 100 & 80 & 0001.24 & 263012 & & $1 \mathrm{HB} 1$ & $69^{\circ} 40^{\prime} \mathrm{N}$ & $53^{\circ} 22^{\prime} \mathrm{W}$ & NE \\
\hline $1 \mathrm{HB} 11014$ & $69^{\circ} 42^{\prime} \mathrm{N}$ & $53^{\circ} 47 \cdot \mathrm{W}$ & NE & & 80 & 00 & 263012 & & 1HB15004 & $69^{\circ} 41^{\prime} \mathrm{N}$ & $53^{\circ} 23^{\prime} \mathrm{W}$ & $\mathrm{E}$ \\
\hline 1HB11015 & $69^{\circ} 43^{\prime} \mathrm{N}$ & $53^{\circ} 44^{\prime} \mathrm{W}$ & $\mathrm{NE}$ & & 80 & 0001.76 & 263012 & 6930 & $1 \mathrm{HB} 15005$ & $69^{\circ} 41, \mathrm{~N}$ & $53^{\circ} 25^{\prime \prime}$ & $\mathrm{NE}$ \\
\hline $1 \mathrm{HB} 11016$ & $69^{\circ} 44^{\prime} \mathrm{N}$ & $53^{\circ} 41^{\prime} \mathrm{W}$ & SE & 110 & 90 & 0000.73 & 200010 & $6930-02$ & 1HB15006 & $69^{\circ} 42, \mathrm{~N}$ & 53025, & ND \\
\hline 1HB11017 & $69^{\circ} 44^{\prime} \mathrm{N}$ & $53^{\circ} 41^{\prime} \mathrm{W}$ & SW & 120 & 90 & & 643112 & 6930 & 1HB15007 & $69^{\circ} 42, \mathrm{~N}$ & $53^{\circ} 26^{\prime} \mathrm{W}$ & ${ }_{\mathrm{NE}}^{\mathrm{N}}$ \\
\hline $1 \mathrm{HB}$ & $69^{\circ} 43^{\prime} \mathrm{N}$ & $53^{\circ} 41^{\prime} \mathrm{W}$ & SW & & 90 & 000 & 643 & 69 & 1HB15008 & $69^{\circ} 43^{\prime} \mathrm{N}$ & $53^{\circ} 28^{\prime} \mathrm{W}$ & $\mathrm{NE}$ \\
\hline $1 \mathrm{HB} 11019$ & $69^{\circ} 43^{\prime} \mathrm{N}$ & $53^{\circ} 37^{\prime} \mathrm{W}$ & SW & & 90 & 000 & 643112 & 693 & 1HB15009 & $69^{\circ} 45 \cdot \mathrm{N}$ & $53^{\circ} 28^{\prime} \mathrm{W}$ & $\mathrm{SW}$ \\
\hline $1 \mathrm{HB} 11020$ & $69^{\circ} 43^{\prime} \mathrm{N}$ & $53^{\circ} 38^{\prime} \mathrm{W}$ & $\mathrm{SW}$ & & 90 & 0003.56 & 260010 & $6930-02$ & 1HB15010 & $69^{\circ} 45, \mathrm{~N}$ & $53^{\circ} 27^{\prime}$ & $S E$ \\
\hline $1 \mathrm{HB} 11021$ & $69^{\circ} 42^{\prime} \mathrm{N}$ & $53^{\circ} 38^{\prime} \mathrm{W}$ & W & & 90 & 0001.64 & 260010 & 6930 & 1HB15011 & $69^{\circ} 46^{\prime} \mathrm{N}$ & $53^{\circ} 27, \mathrm{~W}$ & $\begin{array}{l}S \mathrm{~S} \\
\mathrm{NE}\end{array}$ \\
\hline $1 \mathrm{HI}$ & $69^{\circ} 42, \mathrm{~N}$ & $53^{\circ} 38^{\prime} \mathrm{W}$ & $\eta$ & & 100 & & 260010 & & 1HB15012 & $69^{\circ} 46^{\prime N}$ & $53^{\circ} 25^{\prime} \mathrm{W}$ & E. \\
\hline $1 \mathrm{HB} 11023$ & $69^{\circ} 42^{\prime} \mathrm{N}$ & $8 . \mathrm{W}$ & SW & & 90 & & 260010 & 69 & 1HB15013 & $69^{\circ} 48^{\prime} \mathrm{N}$ & $53^{\circ} 28^{\prime} \mathrm{V}$ & $\mathrm{s}$ \\
\hline $1 \mathrm{HB} 11024$ & $69^{\circ} 41^{\prime} \mathrm{N}$ & $53^{\circ} 37^{\prime} \mathrm{W}$ & И & & 80 & 0000.36 & 260010 & $6930-02$ & 1HB15014 & $69^{\circ} 49^{\prime} \mathrm{N}$ & $53^{\circ} 23^{\prime} \mathrm{W}$ & W \\
\hline $1 \mathrm{HB} 11025$ & $69^{\circ} 41^{\prime} \mathrm{N}$ & $53^{\circ} 38^{\prime} \mathrm{W}$ & $\mathrm{N}$ & 90 & 80 & 0000.09 & 790012 & $6930-02$ & 1HB15015 & $69^{\circ} 49, \mathrm{~N}$ & $53^{\circ} 21^{\prime} \mathrm{W}$ & \\
\hline $1 \mathrm{HB} 11026$ & $69^{\circ} 41^{\prime} \mathrm{N}$ & $53^{\circ} 37^{\circ}$ & $\mathrm{SW}$ & 90 & 80 & 0000.31 & 260010 & $6930-02$ & 1 HB15016 & $69^{\circ} 50^{\prime} \mathrm{N}$ & $53^{\circ} 20^{\prime} \mathrm{W}$ & \\
\hline
\end{tabular}

$\begin{array}{rrccc}\text { HIGH } & \text { LOW } & \text { AREA } & \text { CODE } & \text { MAP } \\ & & & & \\ & 95 & 0002.07 & 260010 & 6930-02 \\ & 80 & 0001.48 & 263112 & 6930-02 \\ & 21 & 0038.49 & 263116 & 6930-02 \\ & 110 & 0000.75 & 260010 & 6930-02 \\ & 90 & 0001.28 & 263412 & 6930-02 \\ & 90 & 0001.15 & 260010 & 6930-02 \\ & 90 & 0000.64 & 263012 & 6930-02 \\ & 60 & 0001.47 & 363112 & 6930-02 \\ & 70 & 0001.13 & 363010 & 6930-02 \\ & 70 & 0000.74 & 360010 & 6930-02 \\ 115 & 80 & 0002.59 & 360010 & 6930-02 \\ 65 & 110 & 0000.40 & 360010 & 6930-02 \\ 65 & 45 & 0000.54 & 643112 & 6930-02 \\ 75 & 50 & 0000.11 & 640110 & 6930-02 \\ 60 & 55 & 0000.55 & 643112 & 6930-02 \\ 85 & 65 & 0000.09 & 643112 & 6930-02 \\ 80 & 45 & 0000.38 & 643112 & 6930-02 \\ 70 & 45 & 0000.89 & 643112 & 6930-02 \\ 75 & 35 & 0000.41 & 643112 & 6930-02 \\ 60 & 35 & 0000.35 & 643112 & 6930-02 \\ 55 & 35 & 0000.19 & 640112 & 6990-02 \\ 70 & 45 & 0000.25 & 640112 & 6930-02 \\ 105 & 80 & 0000.54 & 640010 & 6930-02 \\ 80 & 60 & 0000.44 & 363512 & 6930-02 \\ 87 & 65 & 0001.67 & 790010 & 6900-02 \\ 85 & 65 & 0001.74 & 790010 & 6900-02 \\ 83 & 75 & 0000.24 & 790010 & 6930-02 \\ 80 & 75 & 0000.19 & 790010 & 6930-02 \\ 90 & 80 & 0000.06 & 300010 & 6930-02 \\ 115 & 100 & 0000.08 & 640010 & 6930-02 \\ 125 & 120 & 0000.14 & 300010 & 6930-02 \\ 115 & 100 & 0000.05 & 790010 & 6930-02 \\ 118 & 100 & 0000.51 & 300010 & 6930-02 \\ 110 & 75 & 0001.37 & 643112 & 6930-02 \\ 110 & 95 & 0001.35 & 300010 & 6930-02 \\ 110 & 95 & 0000.23 & 700010 & 6930-02 \\ 90 & 75 & 0000.08 & 700010 & 6930-02 \\ 105 & 55 & 0001.67 & 643112 & 6930-02 \\ 90 & 80 & 0000.16 & 643112 & 6930-02 \\ 110 & 80 & 0000.15 & 643112 & 6930-02 \\ 85 & 70 & 0000.12 & 643112 & 6930-02 \\ 100 & 70 & 0001.39 & 643112 & 6930-02 \\ 105 & 85 & 0001.66 & 360010 & 6930-02 \\ 105 & 90 & 0000.83 & 360010 & 6930-02 \\ 80 & 70 & 0000.12 & 700010 & 6930-02 \\ 96 & 80 & 0000.75 & 363512 & 6930-02 \\ 108 & 75 & 0000.64 & 363412 & 6930-02 \\ 90 & 75 & 0000.65 & 363412 & 6930-02 \\ 100 & 65 & 0004.40 & 363312 & 6930-02 \\ 109 & 95 & 0000.17 & 640010 & 6930-02 \\ 100 & 75 & 0000.56 & 363012 & 6930-02 \\ 100 & 700 & 0001.23 & 360012 & 6930-02 \\ 120 & 85 & 0002.28 & 263112 & 6930-02 \\ 120 & 85 & 0000.89 & 263112 & 6930-02 \\ 120 & 80 & 0000.74 & 263112 & 6930-02 \\ & 55 & 0009.89 & 263117 & 6930-02 \\ & 55 & 0035.23 & 263116 & 6930-02 \\ & & 0000.45 & 643010 & 6930-02 \\ & & 0001.28 & 300010 & 6930-02 \\ & & 0002.48 & 360010 & 6930-02 \\ & & & & \end{array}$


LONG ORI

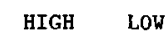

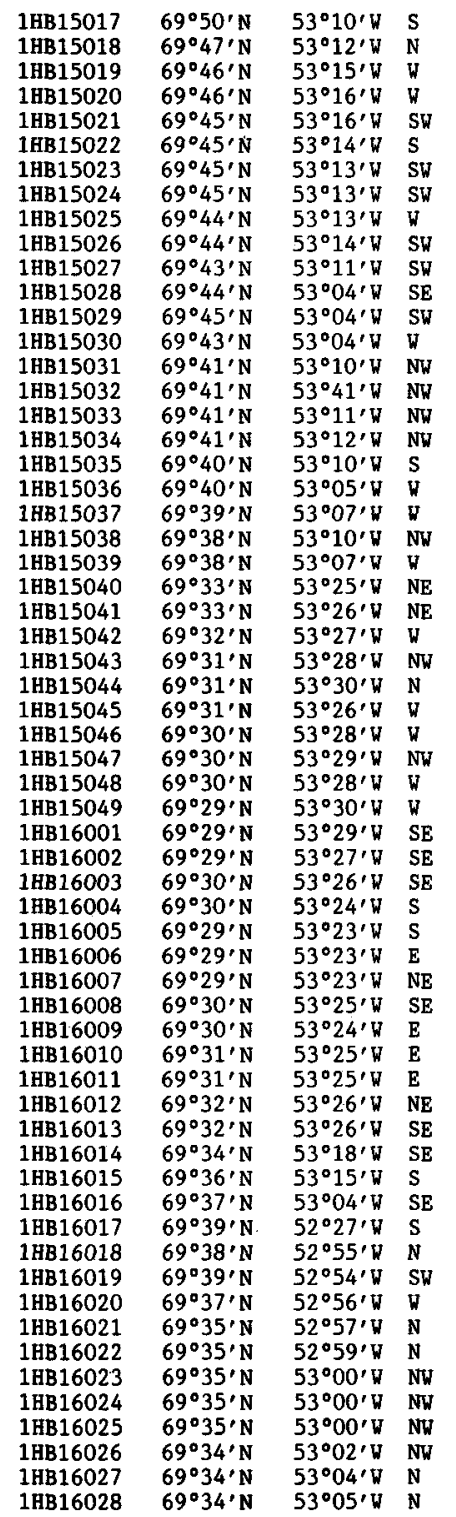
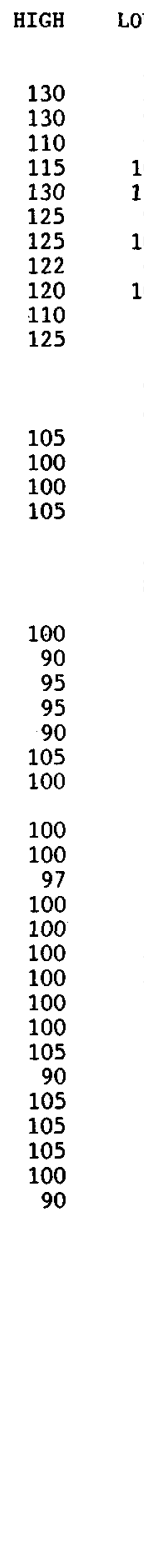

$\begin{array}{ccc} & & \\ \text { AREA } & \text { CODE } & \text { MAP } \\ \text { C0096.21 } & 363111 & 6930-02 \\ 0009.33 & 363016 & 6930-02 \\ 0003.03 & 360010 & 6930-02 \\ 0000.06 & 790010 & 6930-02 \\ 0000.07 & 790010 & 6930-02 \\ 0001.05 & 360010 & 6930-02 \\ 0000.59 & 363012 & 6930-02 \\ 0000.23 & 363010 & 6930-02 \\ 0001.86 & 363212 & 6930-02 \\ 0000.98 & 360010 & 6930-02 \\ 0000.69 & 360010 & 6930-02 \\ 0002.67 & 360010 & 6930-02 \\ 0091.55 & 363116 & 6930-02 \\ 0007.70 & 363112 & 6930-02 \\ 0027.62 & 363116 & 6930-02 \\ 0001.02 & 363312 & 6930-02 \\ 0000.47 & 363312 & 6930-02 \\ 0000.50 & 363412 & 6930-02 \\ 0001.76 & 360010 & 6930-02 \\ 0009.38 & 363111 & 6930-02 \\ 0003.18 & 363111 & 6930-02 \\ 0000.85 & 363212 & 6930-02 \\ 0007.98 & 363216 & 6930-02 \\ 0001.60 & 300010 & 6930-02 \\ 0000.25 & 643112 & 6930-02 \\ 0000.43 & 643112 & 6930-02 \\ 0001.41 & 363512 & 6930-02 \\ 0000.07 & 643112 & 6930-02 \\ 0000.94 & 363212 & 6930-02 \\ 0000.44 & 363212 & 6930-02 \\ 0000.59 & 360010 & 6930-02 \\ 0000.69 & 360010 & 6900-01 \\ 0001.23 & 360010 & 6900-01 \\ 0001.57 & 360010 & 6900-01 \\ 0001.85 & 360010 & 6900-01 \\ 0001.70 & 360010 & 6900-01 \\ 0001.95 & 360010 & 6900-01 \\ 0000.69 & 360010 & 6900-01 \\ 0000.96 & 643112 & 6900-01 \\ 0000.47 & 700010 & 6930-02 \\ 0001.26 & 640111 & 6930-02 \\ 0000.09 & 700010 & 6930-02 \\ 0001.23 & 740010 & 6930-02 \\ 0000.44 & 300010 & 6930-02 \\ 0003.89 & 360011 & 6930-02 \\ 0000.48 & 300010 & 6930-02 \\ 0000.04 & 780010 & 6930-02 \\ 0003.24 & 360110 & 6930-02 \\ 0004.43 & 360110 & 6930-02 \\ 0034.38 & 363111 & 6930-02 \\ 0000.16 & 770010 & 6930-02 \\ 0016.65 & 360112 & 6930-03 \\ 0045.15 & 363111 & 6930-03 \\ 0004.40 & 360110 & 6930-02 \\ 0003.76 & 363112 & 6930-02 \\ 0000.29 & 330110 & 6930-02 \\ 0000.26 & 360110 & 6930-02 \\ 0003.10 & 363112 & 6930-02 \\ 0004.69 & 363111 & 6930-02 \\ 0000.46 & 360110 & 6930-02 \\ 0000.74 & 363011 & 6930-02 \\ & & \\ 0\end{array}$

$\operatorname{CODE}$

LAT

LONG

ORI

$69^{\circ} 34^{\prime} \mathrm{N} \quad 53^{\circ} 06^{\prime} \mathrm{W} \quad \mathrm{N}$

$\begin{array}{llll}1 \mathrm{HB16030} & 69^{\circ} 34^{\prime} \mathrm{N} & 53^{\circ} 06^{\prime} \mathrm{V} & \mathrm{NW} \\ 1 \mathrm{HB} 16031 & 69^{\circ} 34^{\prime} \mathrm{N} & 53^{\circ} 07^{\prime} \mathrm{V} & \mathrm{N}\end{array}$

$\begin{array}{llll}1 \mathrm{HB} 16032 & 69^{\circ} 33^{\prime} \mathrm{N} & 53^{\circ} 10^{\prime} \mathrm{W} & \mathrm{NW}\end{array}$

1 HB16033

$69^{\circ} 33^{\prime} \mathrm{N} \quad 53^{\circ} 10^{\prime} \mathrm{W} \mathrm{NW}$

1 HB16035

$69^{\circ} 33^{\prime} \mathrm{N} 53^{\circ} 10^{\prime} \mathrm{W} \mathrm{W}$

1HB16037

1HB16038

$69^{\circ} 32^{\prime} \mathrm{N} \quad 53^{\circ} 11^{\prime} \mathrm{W}$ SE

1HB16039

$\begin{array}{lll}69^{\circ} 32^{\prime} \mathrm{N} & 53^{\circ} 13^{\prime} \mathrm{W} & \mathrm{NW} \\ 69^{\circ} \mathrm{N} & 53^{\circ} 14^{\prime} \mathrm{W} & \mathrm{NW}\end{array}$

$69^{\circ} 31^{\prime} \mathrm{N} \quad 53^{\circ} 15^{\prime} \mathrm{W} \quad \mathrm{N}$

$1 \mathrm{HB} 16041$

1HB16042

$1 \mathrm{HB} 16044$

$69^{\circ} 30^{\prime} \mathrm{N} 53^{\circ} 16^{\prime} \mathrm{W}$

$69^{\circ} 29^{\prime} \mathrm{N} \quad 53^{\circ} 16^{\prime} \mathrm{W}$ W

1 HB16045

$69^{\circ} 31^{\prime} \mathrm{N} \quad 53^{\circ} 12^{\prime} \mathrm{W}$ E

1AB16046

1 HB16048

1HB16049

$\begin{array}{lll}69^{\circ} 32^{\prime} \mathrm{N} & 53^{\circ} 07^{\prime} \mathrm{W} & \mathrm{SE} \\ 69^{\circ} 33^{\prime} \mathrm{N} & 53^{\circ} 04^{\prime} \mathrm{W} & \mathrm{SV}\end{array}$

$69^{\circ} 32^{\prime} \mathrm{N} \quad 53^{\circ} 05^{\prime} \mathrm{W}$ W

$69^{\circ} 31^{\prime} \mathrm{N} \quad 53^{\circ} 03^{\prime} \mathrm{W} N$

1 月B16051

$69^{\circ} 30^{\prime} \mathrm{N} \quad 53^{\circ} 06^{\prime} \mathrm{W} N$

1 HB16052

$1 \mathrm{HB16053}$

1 1月16054

$\begin{array}{lll}69^{\circ} 29^{\prime} \mathrm{N} & 53^{\circ} 05^{\prime} \mathrm{W} & \mathrm{NW} \\ & \mathrm{NW}\end{array}$

$69^{\circ} 29^{\prime} \mathrm{N} \quad 53^{\circ} 05^{\prime} \mathrm{W} \quad \mathrm{NW}$

$69^{\circ} 29^{\prime} \mathrm{N} \quad 53^{\circ} 07^{\prime} \mathrm{W} \quad \mathrm{N}$

AB16056

$69^{\circ} 28^{\prime} \mathrm{N} \quad 53^{\circ} 07^{\circ} \mathrm{W}$ NW

$60^{\circ} 27 \mathrm{~N} 53^{\circ} 12 . \mathrm{N}$

1 HB16059 $69^{\circ} 27^{\prime} \mathrm{N} \quad 53^{\circ} 12$ ' $\mathrm{V} N$

$1 \mathrm{HB} 1606069^{\circ} 27^{\prime} \mathrm{N} \quad 53^{\circ} 14^{\prime} \mathrm{W} N$

1 BB16061 $69^{\circ} 27^{\prime} \mathrm{N} \quad 53^{\circ} 15^{\prime} \mathrm{W} \quad \mathrm{NW}$

1 BB16062

1HB16064

$1 \mathrm{BB} 16065$

$1 \mathrm{HB16066}$

$69^{\circ} 27^{\prime} \mathrm{N} \quad 53^{\circ} 13^{\prime} \mathrm{W}$
$69^{\circ} 27^{\prime} \mathrm{N}$
$63^{\circ} 12^{\prime} \mathrm{W}$

$69^{\circ} 26^{\prime} \mathrm{N} \quad 53^{\circ} 11^{\prime} \mathrm{W}$

$69^{\circ} 26^{\prime} \mathrm{N} \quad 53^{\circ} 10^{\prime} \mathrm{W}$ SE

$60^{\circ} \mathrm{N}$ 53

69.25'N $53^{\circ} 12, \mathrm{~N} N$

1 HB16070

1 HB16071

$\begin{array}{llll}69^{\circ} 25^{\prime} \mathrm{N} & 53^{\circ} 12^{\prime} \mathrm{W} & \mathrm{N} \\ 69^{\circ} 22^{\prime} \mathrm{N} & 53^{\circ} 21^{\prime} \mathrm{W} & \mathrm{NE}\end{array}$

$1 \mathrm{HB} 16073 \quad 69^{\circ} 24^{\prime} \mathrm{N} \quad 53^{\circ} 22^{\prime} \mathrm{W} \quad \mathrm{SE}$

1 BB16074 $69^{\circ} 25^{\prime} \mathrm{N} \quad 53^{\circ} 22^{\prime} \mathrm{W}$ E

1 HB16075 $69^{\circ} 25^{\prime} \mathrm{N} \quad 53^{\circ} 20^{\prime} \mathrm{V}$ NE

1 BB16076 $69^{\circ} 25^{\prime} \mathrm{N} \quad 532^{\circ} 22^{\prime} \mathrm{W} N$

1HB16077 69.25'N $53^{\circ} 24^{\prime} \mathrm{W}$ N

$\begin{array}{llll}\text { 1AB16078 } & 69^{\circ} 25^{\prime} \mathrm{N} & 53^{\circ} 24^{\prime} \mathrm{W} & \mathrm{NW} \\ \text { 1月B17001 } & 69^{\circ} 26^{\prime} \mathrm{N} & 53^{\circ} 29^{\prime} \mathrm{W} & -\end{array}$

$1 \mathrm{HB} 17002 \quad 69^{\circ} 24^{\prime} \mathrm{N} \quad 53^{\circ} 23^{\prime} \mathrm{W}$ SW

$1 \mathrm{HB} 17003 \quad 69^{\circ} 23^{\prime} \mathrm{N} \quad 53^{\circ} 23^{\prime} \mathrm{V}$ N

$1 \mathrm{HB} 17004 \quad 69^{\circ} 23^{\prime} \mathrm{N} \quad 53^{\circ} 25^{\prime} \mathrm{W}$ NH

1HB17005 $69^{\circ} 23^{\prime} \mathrm{N} \quad 53^{\circ} 27^{\prime}, \mathrm{W} N$

1 BB17006 $69^{\circ} 21^{\prime} \mathrm{N} \quad 53^{\circ} 35^{\prime} \mathrm{W} N$

$1 \mathrm{HB} 17007 \quad 69^{\circ} 24^{\prime} \mathrm{N} \quad 53^{\circ} 38^{\prime} \mathrm{W}$ SE

$\begin{array}{llll}1 \text { HB17008 } & 69^{\circ} 25^{\prime} \mathrm{N} & 53^{\circ} 38^{\prime} \mathrm{W} & \mathrm{NE} \\ 1 \mathrm{HB} 17009 & 69^{\circ} 25^{\prime} \mathrm{N} & 53^{\circ} 39^{\prime} \mathrm{W} & \mathrm{N}\end{array}$

$\begin{array}{llll}1 \text { HB17010 } & 69^{\circ} 25^{\prime} \mathrm{N} & 53^{\circ} 40^{\prime} \mathrm{W} & \mathrm{N} \\ 1 \mathrm{HB} 17011 & 69^{\circ} 25^{\prime} \mathrm{N} & 53^{\circ} 41, \mathrm{~W} & \mathrm{~N}\end{array}$

AREA

$\begin{array}{llll}55 & 0000.09 & 740010 & 6930-02 \\ 85 & 0000.53 & 360110 & 6930-02\end{array}$

$\begin{array}{llll}50 & 0000.73 & 363011 & 6930-02\end{array}$ $0000.83 \quad 360110 \quad 6930-02$ $0001.06 \quad 363012 \quad 6930-02$ $0000.08 \quad 770010 \quad 6930-02$ $0000.60 \quad 300110 \quad 6930-02$ $0000.80 \quad 300110 \quad 6930-02$

$\begin{array}{llll}90 & 0000.76 & 363111 & 6930-02 \\ 95 & 0000.73 & 363111 & 6930-02\end{array}$

$\begin{array}{rrrr}75 & 0000.46 & 300110 & 6930-02 \\ 85 & 0000.69 & 363111 & 6930-02\end{array}$ 110

0000.63
0000.85

0001.1

0001.88

0040.63

0004.36

0005.19

0003.1

0001.82

0002.83

0000.15

000.75

0000.97

0000.55

0000.48

0000.82

$90 \quad 0000.54$

$\begin{array}{ll}85 & 0003.78 \\ 45 & 0009.00\end{array}$

$\begin{array}{ll}45 & 0009.00 \\ 60 & 0000.93\end{array}$

$55 \quad 0001.83$

650000.13

$\begin{array}{ll}80 & 0001.55 \\ 70 & 0000.44\end{array}$

$\begin{array}{ll}55 & 0000.05\end{array}$

$\begin{array}{ll}55 & 0000.11 \\ 55 & 0000.07\end{array}$

$60 \quad 0001.53$

$80 \quad 0000.09$

$50 \quad 0000.12$

$50 \quad 0000.20$

$50 \quad 0002.34$

$\begin{array}{ll}65 & 0001.30 \\ 65 & 0000.86\end{array}$

650000.57

$40 \quad 0000.11$

36311

6930-02 6900-02 $370110 \quad 6930-02$

$370110 \quad 6930-02$

$370110 \quad 6930-02$

333112 6900-02

300010 6900-02

$300010 \quad 6900-02$

640012 6900-02

$370010 \quad 6900-02$

$333111 \quad 6900-02$

$300110 \quad 6900-02$

6900-02

$370010 \quad 6900-02$

$370010 \quad 6900-02$

370010 6900-02

$300010 \quad 6900-02$

$333111 \quad 6900-02$

$300010 \quad 6900-01$

$200010 \quad 6900-01$

$780010 \quad 6900-01$

740010 6900-01

780010 6900-01

363112 6900-01

$280010 \quad 6900-01$

$790012 \quad 6900-01 \quad \infty$ 
$54^{\circ} 41^{\prime} \mathrm{W}$

$54^{\circ} 44^{\prime}, \mathrm{W}$

$69^{\circ} 24^{\prime} \mathrm{N} \quad 53^{\circ} 52^{\prime} \mathrm{W}$ NE

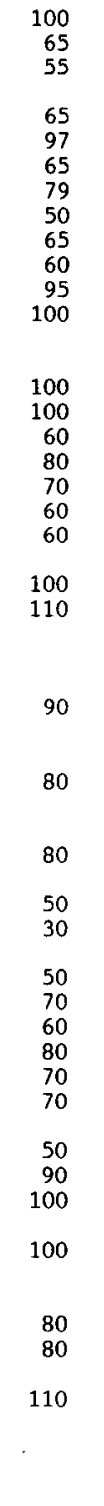

$\operatorname{MAP} \infty$
$360010 \quad 6930-01$

$643112 \quad 6930-01$

$640112 \quad 6930-01$

$643112 \quad 6930-01$

$300010 \quad 6930-01$ $643112 \quad 6930-01$ $6930-01$ $790010 \quad 6930-01$ $360010 \quad 6930-01$ $3600106930-01$ $6930-01$ $780010 \quad 6930-01$ $780010 \quad 6930-01$ $6431126930-01$ $790012-6930-01$ $640112 \quad 6930-01$ $300010 \quad 6930-01$ $300010 \quad 6930-01$ $360010 \quad 6930-01$ $360010 \quad 6930-01$ $780010 \quad 6930-01$ 263112 6930-01 790012 6930-01 263111 6930-01 $643112 \quad 6930-01$ 263112 6930-01 643112 6930-01 643112 6930-01 $\begin{array}{ll}613112 & 6930-01 \\ 750010 & 6930-01\end{array}$ 790012 6930-01 780010 6930-01 643112 6930-01 $\begin{array}{ll}643112 & 6930-01 \\ 790012 & 6930-01\end{array}$ $\begin{array}{ll}600010 & 6930-01 \\ 700010 & 6930-01\end{array}$ $\begin{array}{ll}700010 & 6930-01 \\ 600010 & 6930-01\end{array}$ $200010 \quad 6930-01$ 263011 6930-01 $\begin{array}{ll}200010 & 6930-01 \\ 260012 & 6930-01\end{array}$ $\begin{array}{ll}260011 & 6930-01 \\ 600010 & 6930-01\end{array}$ 600010 6930-01 700010 6930-01 260012 6930-01 $\begin{array}{ll}200010 & 6930-01 \\ 200010 & 6930-01\end{array}$ $\begin{aligned} & 200010 \\ & 260010\end{aligned} 6930-01$ $200010 \quad 6930-01$ 


\begin{tabular}{|c|c|c|c|}
\hline CODE & LAT & LONG & ORI \\
\hline C04017 & $69^{\circ} 44^{\prime} \mathrm{N}$ & $54^{\circ} 07^{\prime} \mathrm{V}$ & \\
\hline CO4018 & $69^{\circ} 44^{\prime} \mathrm{N}$ & $54^{\circ} 07^{\prime} \mathrm{W}$ & \\
\hline 1 HC04019 & $69^{\circ} 43^{\prime} \mathrm{N}$ & $54^{\circ} 10^{\prime} \mathrm{W}$ & W \\
\hline 1 HCO4020 & $69^{\circ} 42^{\prime} \mathrm{N}$ & $54^{\circ} 12^{\prime} \mathrm{W}$ & NW \\
\hline $1 \mathrm{HC} 04021$ & $69^{\circ} 42^{\prime} \mathrm{N}$ & $54^{\circ} 12^{\prime} \mathrm{W}$ & \\
\hline $1 \mathrm{HCO} 4022$ & $69^{\circ} 41^{\prime} \mathrm{N}$ & $54^{\circ} 13^{\prime} \mathrm{W}$ & \\
\hline $1 \mathrm{HCO} 4023$ & $69^{\circ} 42^{\prime} \mathrm{N}$ & $54^{\circ} 10^{\prime} \mathrm{W}$ & $\mathrm{SW}$ \\
\hline $1 \mathrm{HCO} 024$ & $69^{\circ} 41^{\prime} \mathrm{N}$ & $54^{\circ} 10^{\prime} \mathrm{V}$ & \\
\hline $1 \mathrm{HCO} 4025$ & $69^{\circ} 41^{\prime} \mathrm{N}$ & $54^{\circ} 08^{\prime} W$ & $\mathrm{~s}$ \\
\hline $1 \mathrm{HCO} 4026$ & $69^{\circ} 41^{\prime} \mathrm{N}$ & $54^{\circ} 08^{\prime} \mathrm{W}$ & $\mathrm{sw}$ \\
\hline $1 \mathrm{HCO} 04027$ & $69^{\circ} 36^{\prime} \mathrm{N}$ & $54^{\circ} 10^{\prime} \mathrm{W}$ & 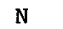 \\
\hline $1 \mathrm{HCO} 04028$ & $69^{\circ} 36^{\prime} \mathrm{N}$ & $54^{\circ} 12^{\prime} \mathrm{W}$ & $\mathrm{N}$ \\
\hline 1 HCO4029 & $69^{\circ} 37^{\prime N}$ & $54^{\circ} 13^{\prime} \mathrm{w}$ & Iv \\
\hline $1 \mathrm{HCO} 4030$ & $69^{\circ} 37^{\prime} \mathrm{N}$ & $54^{\circ} 15^{\prime}$ & \\
\hline $1 \mathrm{HCO} 4031$ & $69^{\circ} 37^{\prime} \mathrm{N}$ & $54^{\circ} 16^{\prime} \mathrm{W}$ & N \\
\hline $1 \mathrm{HC} 04032$ & $69^{\circ} 39^{\prime} \mathrm{N}$ & $54^{\circ} 18^{\prime} \mathrm{W}$ & N \\
\hline $1 \mathrm{HCO} 04033$ & $69^{\circ} 39^{\prime} \mathrm{N}$ & $54^{\circ} 20^{\prime} \mathrm{W}$ & N \\
\hline $1 \mathrm{HC} 04034$ & $69^{\circ} 39^{\prime} \mathrm{N}$ & $54^{\circ} 22, \mathrm{~V}$ & $\mathrm{NE}$ \\
\hline $1 \mathrm{HCO} 4035$ & $69^{\circ} 39^{\prime} \mathrm{N}$ & $54^{\circ} 23^{\prime} \mathrm{W}$ & - \\
\hline $1 \mathrm{HC} 05001$ & $69^{\circ} 39^{\prime} \mathrm{N}$ & $54^{\circ} 24^{\prime} \mathrm{W}$ & NW \\
\hline $1 \mathrm{HCO} 0002$ & $69^{\circ} 39^{\prime} \mathrm{N}$ & $54^{\circ} 23^{\prime} \mathrm{W}$ & sw \\
\hline $1 \mathrm{HC} 05003$ & $69^{\circ} 38^{\prime} \mathrm{N}$ & $54^{\circ} 23^{\prime} \mathrm{W}$ & \\
\hline $1 \mathrm{HCO5004}$ & $69^{\circ} 38^{\prime} \mathrm{N}$ & $54^{\circ} 26^{\prime \prime} \mathrm{W}$ & $\mathrm{N}$ \\
\hline $1 \mathrm{HC} 05005$ & $69^{\circ} 37^{\prime} \mathrm{N}$ & $54^{\circ} 27^{\prime} \mathrm{W}$ & \\
\hline $1 \mathrm{HC} 05006$ & $69^{\circ} 38^{\prime} \mathrm{N}$ & $54^{\circ} 28^{\prime} \mathrm{W}$ & \\
\hline $1 \mathrm{HC} 05007$ & $69^{\circ} 38^{\prime} \mathrm{N}$ & $54^{\circ} 30^{\prime \prime} \mathrm{W}$ & $\mathrm{NE}$ \\
\hline $1 \mathrm{HCO5008}$ & $69^{\circ} 39^{\prime} \mathrm{N}$ & $54^{\circ} 32^{\prime} \mathrm{W}$ & \\
\hline $1 \mathrm{HCO6001}$ & $69^{\circ} 40^{\prime} \mathrm{N}$ & $54^{\circ} 30^{\circ} \mathrm{W}$ & N \\
\hline $1 \mathrm{HCO6002}$ & $69^{\circ} 40^{\prime} \mathrm{N}$ & $54^{\circ} 32^{\prime} \mathrm{W}$ & N \\
\hline $1 \mathrm{HC} 06003$ & $69^{\circ} 40^{\prime} \mathrm{N}$ & $54^{\circ} 35^{\prime} \mathrm{W}$ & \\
\hline $1 \mathrm{HC} 06004$ & $69^{\circ} 41^{\prime} \mathrm{N}$ & $54^{\circ} 34^{\prime} \mathrm{W}$ & $\mathrm{NE}$ \\
\hline $1 \mathrm{HCO} 001$ & $69^{\circ} 41^{\prime} \mathrm{N}$ & $54^{\circ} 35^{\prime} \mathrm{w}$ & $\mathrm{NW}$ \\
\hline $1 \mathrm{HC} 07002$ & $69^{\circ} 40^{\prime} \mathrm{N}$ & $54^{\circ} 37^{\prime} \mathrm{W}$ & $\mathrm{N}$ \\
\hline $1 \mathrm{HC} 07003$ & $69^{\circ} 41, \mathrm{~N}$ & $54^{\circ} 38^{\prime} \mathrm{W}$ & N \\
\hline $1 \mathrm{HCO} 07004$ & $69^{\circ} 42^{\prime} \mathrm{N}$ & $54^{\circ} 40^{\prime} \mathrm{W}$ & $\mathrm{NE}$ \\
\hline $1 \mathrm{HCO}$ & $69^{\circ} 43^{\prime} \mathrm{N}$ & $54^{\circ} 42^{\prime} \mathrm{W}$ & $\mathrm{E}$ \\
\hline $1 \mathrm{HCOBO01}$ & $69^{\circ} 44^{\prime} \mathrm{N}$ & $54^{\circ} 43^{\prime} \mathrm{W}$ & N \\
\hline $1 \mathrm{HCOBOO2}$ & $69^{\circ} 48^{\prime} \mathrm{N}$ & $54^{\circ} 43^{\prime} \mathrm{W}$ & $\mathrm{y}-2>$ \\
\hline $1 \mathrm{HCO8003}$ & $69^{\circ} 43^{\prime} \mathrm{N}$ & $54^{\circ} 43^{\prime} \mathrm{W}$ & \\
\hline $1 \mathrm{HC} 08004$ & $69^{\circ} 41^{\prime} \mathrm{N}$ & $54^{\circ} 40^{\prime} \mathrm{W}$ & \\
\hline $\begin{array}{l}1 \mathrm{HCC} \\
\text { lat }\end{array}$ & $69^{\circ} 41^{\prime} \mathrm{N}$ & $54^{\circ} 44^{\prime} \mathrm{V}$ & NW \\
\hline $1 \mathrm{HC} 08006$ & $69^{\circ} 40^{\prime} \mathrm{N}$ & $54^{\circ} 45^{\prime} \mathrm{W}$ & 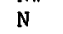 \\
\hline $1 \mathrm{HCOB007}$ & $69^{\circ} 41^{\prime} \mathrm{N}$ & $54^{\circ} 47^{\prime} \mathrm{W}$ & \\
\hline $1 \mathrm{HC} 08008$ & $69^{\circ} 42^{\prime} \mathrm{N}$ & $54^{\circ} 46^{\prime} \mathrm{W}$ & \\
\hline $1 \mathrm{HC} 08009$ & $69^{\circ} 42^{\prime} \mathrm{N}$ & $54^{\circ} 50^{\prime} \mathrm{W}$ & $\mathrm{NE}$ \\
\hline $1 \mathrm{HC} 08010$ & $69^{\circ} 42^{\prime} \mathrm{N}$ & $54^{\circ} 52^{\prime} \mathrm{W}$ & $\mathrm{NE}$ \\
\hline $1 \mathrm{HC} 08011$ & $69^{\circ} 43 \cdot \mathrm{N}$ & $54^{\circ} 53^{\circ}, \mathrm{W}$ & $\mathrm{NE}$ \\
\hline $1 \mathrm{HCO} 0001$ & $69^{\circ} 42^{\prime} \mathrm{N}$ & $54^{\circ} 55^{\prime} \mathrm{W}$ & \\
\hline $1 \mathrm{HCO} 0002$ & $69^{\circ} 41 \cdot \mathrm{N}$ & $54^{\circ} 56^{\prime} \mathrm{V}$ & \\
\hline $1 \mathrm{HD} 02001$ & $70^{\circ} 08^{\prime \prime N}$ & $54^{\circ} 21^{\prime} \mathrm{W}$ & SW \\
\hline $1 \mathrm{HDO} 2002$ & $70^{\circ} 07 \cdot \mathrm{N}$ & $54^{\circ} 17^{\prime} \mathrm{v}$ & SW \\
\hline $1 \mathrm{HD}$ & $70^{\circ} 06^{\prime} \mathrm{N}$ & $54^{\circ} 13^{\prime} \mathrm{w}$ & $\pi$ \\
\hline $1 \mathrm{HDO} 2004$ & $70^{\circ} 06^{\prime} \mathrm{N}$ & $54^{\circ} 15^{\prime} \mathrm{W}$ & v \\
\hline 1 HD02005 & $70^{\circ} 05^{\prime} \mathrm{N}$ & $54^{\circ} 18^{\prime} \mathrm{W}$ & $\mathrm{N}$ \\
\hline $1 \mathrm{HDO} 2006$ & $70^{\circ} 06^{\prime} \mathrm{N}$ & $54^{\circ} 22^{\prime} \mathrm{W}$ & \\
\hline $1 \mathrm{HDO} 2007$ & $70^{\circ}$ & $54^{\circ} 23^{\prime} \mathrm{W}$ & $\mathrm{N}$ \\
\hline 1HD02008 & $70^{\circ} 05^{\prime} \mathrm{N}$ & $54^{\circ} 24^{\prime} \mathrm{W}$ & \\
\hline IDO2009 & $70^{\circ} 07^{\prime} \mathrm{N}$ & $54^{\circ} 26^{\prime} \mathrm{W}$ & \\
\hline $14 \mathrm{M} 2010$ & $70^{\circ} 07^{\prime} \mathrm{N}$ & $54^{\circ} 76,6$ & \\
\hline & $70^{\circ} 06^{\prime} \mathrm{N}$ & $6^{\prime}$ & \\
\hline 1 HDO2012 & $70^{\circ} 06^{\prime} \mathrm{N}$ & $54^{\circ} 29^{\prime} \mathrm{W}$ & \\
\hline
\end{tabular}

HIGH



70
70
90

80
80
80
80
75
75
80
85
60
40
40
50
50
75
70
70
100
70
70
90
45
50
50
50
80
55
70
40
65
50
50
80
70
55
40
70
65
60
50
35
35
40
160
140
140
135
135
135
150
150
125
130
150
130

$\begin{array}{rc}\text { LOW } & \text { AREA } \\ 60 & 0001.40 \\ 70 & 0001.41 \\ 50 & 0002.56 \\ 50 & 0000.38 \\ 40 & 0000.53 \\ 40 & 0000.88 \\ 50 & 0000.96 \\ 80 & 0000.22 \\ 70 & 0000.17 \\ 50 & 0000.42 \\ 60 & 0001.43 \\ 50 & 0000.33 \\ 50 & 0001.26 \\ 55 & 0000.67 \\ 45 & 0000.61 \\ 45 & 0000.15 \\ 25 & 0000.37 \\ 35 & 0000.09 \\ 30 & 0000.07 \\ 40 & 0000.08 \\ 65 & 0000.16 \\ 48 & 0000.67 \\ 20 & 0004.17 \\ 90 & 0000.11 \\ 20 & 0001.65 \\ 20 & 0003.17 \\ 70 & 0000.12 \\ 35 & 0000.08 \\ 30 & 0000.64 \\ 35 & 0000.58 \\ 35 & 0000.06 \\ 55 & 0000.13 \\ 30 & 0000.58 \\ 35 & 0000.24 \\ 30 & 0000.09 \\ 35 & 0000.25 \\ 35 & 0000.04 \\ 40 & 0000.31 \\ 75 & 0000.22 \\ 40 & 0000.74 \\ 35 & 0000.24 \\ 35 & 0000.07 \\ 50 & 0000.07 \\ 45 & 0000.11 \\ 40 & 0000.10 \\ 40 & 0000.19 \\ 20 & 0000.18 \\ 30 & 0000.06 \\ 30 & 0000.09 \\ 115 & 0000.18 \\ 120 & 0000.21 \\ 105 & 0000.83 \\ 85 & 0003.35 \\ 65 & 0007.39 \\ 100 & 0000.26 \\ 80 & 0003.29 \\ 130 & 0001.11 \\ 100 & 0000.39 \\ 100 & 0000.24 \\ 70 & 0003.16 \\ 85 & 0001.18\end{array}$

MO
CODE MAP

CODE

LAT

LONG ORI

HIGH

AREA

MO

$260502 \quad 6930-01$

$\begin{array}{ll}260010 & 6930-01 \\ 623112 & 6930-01\end{array}$

643221 6930-01

643221
$69930-01$

$643221 \quad 6930-01$

700010 6930-01

$700010 \quad 6930-01$

$\begin{array}{ll}643112 & 6930-01 \\ 643112 & 6930-01\end{array}$

643112 6930-01

$\begin{array}{ll}643112 & 6930-01 \\ 643112 & 6930-01\end{array}$

$750012 \quad 6930-01$

$643112 \quad 6930-01$

$790010 \quad 6930-01$

$790010 \quad 6930-01$

$\begin{array}{ll}790010 & 6930-01 \\ 200010 & 6930-01\end{array}$

263116 6930-01

$300010 \quad 6930-01$

$\begin{array}{ll}263116 & 6930-01 \\ 643111 & 6930-01\end{array}$

$\begin{array}{ll}643111 & 6930-01 \\ 300010 & 6930-01\end{array}$

790012 6930-01

$643111 \quad 6930-01$

$790010 \quad 6930-01$

$\begin{array}{llll}1 \mathrm{HD} 02013 & 70^{\circ} 06^{\prime} \mathrm{N} & 54^{\circ} 32^{\prime} \mathrm{W} & \mathrm{E} \\ 1 \mathrm{HD} 04001 & 70^{\circ} 05^{\prime} \mathrm{N} & 54^{\circ} 29^{\prime} \mathrm{W} & \mathrm{SW}\end{array}$

$\begin{array}{lll}1 \mathrm{HD} 04001 & 70^{\circ} 05^{\prime} \mathrm{N} & 54^{\circ} 29^{\prime} \mathrm{W} \\ 1 \mathrm{HD} 04002 & 70^{\circ} 04^{\prime} \mathrm{N} & 54^{\circ} 27 \cdot \mathrm{W}\end{array}$

$\begin{array}{llll}1 \text { HD0 } 04003 & 70^{\circ} 04^{\prime} \mathrm{N} & 54^{\circ} 24^{\prime} \mathrm{W} & - \\ 1 \mathrm{HD} 04004 & 70^{\circ} 03^{\prime} \mathrm{N} & 54^{\circ} 20^{\prime} \mathrm{W} & \mathrm{W}\end{array}$

$\begin{array}{lll}1 \mathrm{HD} 04005 & 70^{\circ} 03 / \mathrm{N} & 54^{\circ} 17^{\prime} \mathrm{W} \\ 1 \mathrm{HD} 04006 & 70^{\circ} 03, \mathrm{~N} & 54^{\circ} 177^{\prime} \mathrm{W}\end{array}$

$1 \mathrm{HD} 04007 \quad 70^{\circ} 02{ }^{\prime} \mathrm{N} \quad 54^{\circ} 18^{\prime} \mathrm{W}$

$1 \mathrm{HD} 04008$

$70^{\circ} 01, \mathrm{~N} \quad 54^{\circ} 21, \mathrm{~W} \quad \mathrm{~N}$

$1 \mathrm{HD} 04010$

1 HDO4011

$\begin{array}{lll}70^{\circ} 02, \mathrm{~N} & 54^{\circ} 23^{\circ}, \mathrm{W} & \mathrm{NE} \\ 7 & \mathrm{~N}\end{array}$

1HD04012

$70^{\circ} 01, \mathrm{~N} \quad 54^{\circ} 255^{\prime} \mathrm{V}$ NW

$\begin{array}{lll}1 \text { HD04014 } & 70^{\circ} 00^{\prime} \mathrm{N} & 54^{\circ} 23^{\prime} \mathrm{W} \\ & 70^{\circ} 00^{\prime} \mathrm{N} & 54^{\circ} 25^{\prime} \mathrm{W}\end{array}$

$1 \mathrm{HD} 04015 \quad 69^{\circ} 59^{\prime} \mathrm{N} \quad 54^{\circ} 25^{\prime} \mathrm{W} \quad \mathrm{NW}$

$\begin{array}{llll}1 \mathrm{HD} 04016 & 70^{\circ} 00^{\prime} \mathrm{N} & 54^{\circ} 28^{\prime} \mathrm{W} & \mathrm{N}\end{array}$

1 HD04018

$70^{\circ} 00^{\prime} \mathrm{N} \quad 54^{\circ} 27^{\prime} \mathrm{W}$

$\begin{array}{llll}1 \mathrm{HD} 04020 & 70^{\circ} 03^{\prime} \mathrm{N} & 54^{\circ} 32^{\circ} \mathrm{W} & \mathrm{N} \\ \mathrm{H} & 54^{\circ} 3 \mathrm{w}^{\circ} \mathrm{N}\end{array}$

$\begin{array}{llll}1 \mathrm{HDO} 02021 & 70^{\circ} \mathrm{O} 2^{\prime} \mathrm{N} & 54^{\circ} 34, \mathrm{~W} & - \\ 1 \mathrm{HD} 04022 & 70^{\circ} \mathrm{OO} 0^{\prime} \mathrm{N} & 54^{\circ} 34^{\prime} \mathrm{W} & \mathrm{NW}\end{array}$

HD05002

$\begin{array}{ll}70^{\circ} 00^{\prime} \mathrm{N} & 54^{\circ} 34^{\prime} \mathrm{W} \\ 70^{\circ} 00^{\prime} \mathrm{N} & 54^{\circ} 34^{\prime} \mathrm{W}\end{array}$

$1 \mathrm{HD} 05004$

$69^{\circ} 59^{\prime} \mathrm{N} \quad 54^{\circ} 26^{\prime} \mathrm{W} \quad \mathrm{S}$

$\begin{array}{llll} & 70^{\circ} 01^{\prime} \mathrm{N} & 54^{\circ} 19^{\prime} \mathrm{W} & \mathrm{W} \\ \mathrm{HD} 05005 & 70^{\circ} 00^{\prime} \mathrm{N} & 54^{\circ} 17^{\prime} \mathrm{W} & \mathrm{NW}\end{array}$

$1 \mathrm{HD} 05006$

$70^{\circ} 00^{\prime} \mathrm{N} \quad 54^{\circ} 17, \mathrm{~W} \quad \mathrm{NW}$

$790010 \quad 6930-01$

$643112 \quad 6930-01$

$\begin{array}{ll}643112 & 6930-01 \\ 790012 & 6930-01\end{array}$

$790012 \quad 6930-01$

$790010 \quad 6930-01$

$\begin{aligned} & 643111 \\ & 700010\end{aligned} 6930-01$

$640112 \quad 6930-01$

$750112 \quad 6930-01$

$790010 \quad 6930-01$

$790010 \quad 6930-01$

$790010 \quad 6930-01$

$\begin{array}{ll}640112 & 6930-01 \\ 640112 & 6930-01\end{array}$

$790012 \quad 6930-01$

$\begin{array}{ll}790012 & 6930-01 \\ 750010 & 7000-01\end{array}$

$750010 \quad 7000-01$

$533112 \quad 7000-01$

533112
513112
$7000-01$

$700010 \quad 7000-01$

$5131127000-01$

$\begin{array}{ll}300010 & 7000-01\end{array}$

$770010 \quad 7000-01$

$\begin{array}{ll}533112 & 7000-01 \\ 600012 & 7000-01\end{array}$

$\begin{array}{llll} & 69^{\circ} 59^{\prime} \mathrm{N} & 54^{\circ} 15^{\prime} \mathrm{W} & \mathrm{N} \\ \mathrm{H} & 05008 & & \end{array}$

$1 \mathrm{HD} 05010 \quad 69^{\circ} 59^{\prime} \mathrm{N} \quad 54^{\circ} 20^{\prime} \mathrm{W} \quad \mathrm{W}$

$\begin{array}{llll}1 \mathrm{HD} 05011 & 69^{\circ} 58^{\prime} \mathrm{N} & 54^{\circ} 20^{\prime} \mathrm{W} & \mathrm{NW} \\ 1 \mathrm{HD} 06001 & 69^{\circ} 58^{\prime} \mathrm{N} & 54^{\circ} 18^{\prime} \mathrm{W} & \end{array}$

$1 \mathrm{HD} 06001$

$1 \mathrm{HD} 06002$

1 HDO6004

$\begin{array}{lll}69^{\circ} 58^{\prime} \mathrm{N} & 54^{\circ} 18^{\prime} \mathrm{W} & - \\ 70^{\circ} 01^{\prime} \mathrm{N} & 54^{\circ} 13^{\prime} \mathrm{W} & \mathrm{NE}\end{array}$

$\begin{array}{lll}70^{\circ} 01^{\prime} \mathrm{N} & 54^{\circ} 13^{\prime} \mathrm{W} & \mathrm{NE} \\ 70^{\circ} 03^{\prime} \mathrm{N} & 54^{\circ} 12^{\prime} \mathrm{W} & \mathrm{NE}\end{array}$

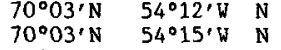

$1 \mathrm{HD} 06006$

1 HD06008

1 HD06009

$70^{\circ} 04^{\prime N} \quad 54^{\circ} 15^{\prime} \mathrm{W}$ NE

$70^{\circ} 04^{\prime} \mathrm{N} \quad 54^{\circ} 18^{\circ}, \mathrm{W}$ NE

1 HD06010

$70^{\circ} 05^{\prime} \mathrm{N} \quad 54^{\circ} 11^{\prime}, \mathrm{W}$ E

1HD06012

$\begin{array}{ll}70^{\circ} 06^{\prime} \mathrm{N} & 54^{\circ} 09^{\prime} \mathrm{W} \\ 70^{\circ} 05^{\prime} \mathrm{N} & 54^{\circ} 04^{\prime} \mathrm{W}\end{array}$

$1 \mathrm{HD} 06014$

1 HD06015

$\begin{array}{ll}70^{\circ} 05^{\prime} \mathrm{N} & 54^{\circ} 05^{\prime} \mathrm{W} \\ 70^{\circ} 04^{\prime} \mathrm{N} & 54^{\circ} 05^{\prime} \mathrm{W}\end{array}$

$\begin{array}{lll}70^{\circ} 04^{\prime} \mathrm{N} & 54^{\circ} 05^{\prime} \mathrm{W} & \mathrm{W} \\ 70^{\circ} 04^{\prime} \mathrm{N} & 54^{\circ} 02^{\prime} \mathrm{W} & \mathrm{W}\end{array}$

$70^{\circ} 03^{\prime} \mathrm{N} \quad 54^{\circ} 03^{\prime} \mathrm{W}$ NW

$70^{\circ} 03^{\prime} \mathrm{N} \quad 54^{\circ} 01^{\prime} \mathrm{W}$ W

1HD06019 70001'N $53^{\circ} 56, \mathrm{NW}$

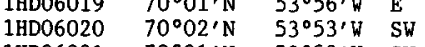

$1 \mathrm{HD} 0602170^{\circ} 01^{\prime} \mathrm{N} \quad 53^{\circ} 52^{\prime} \mathrm{W}$ SW

$1 \mathrm{HD06022}$

$70^{\circ} 00^{\prime} \mathrm{N} \quad 53^{\circ} 48^{\prime} \mathrm{W}$ SW

$\begin{array}{llll}1 \text { HD06023 } & 69^{\circ} 59^{\prime} \mathrm{N} & 53^{\circ} 44^{\prime} \mathrm{V} & \mathrm{E} \\ 1 \mathrm{HD} 06024 & 69^{\circ} 59^{\prime} \mathrm{N} & 53^{\circ} 41^{\prime} \mathrm{V} & \mathrm{SW}\end{array}$

$1 \mathrm{HD} 06025 \quad 69^{\circ} 58^{\prime} \mathrm{N} \quad 53^{\circ} 39^{\prime} \mathrm{W} \quad \mathrm{SW}$

$\begin{array}{llll}1 \mathrm{HD} 06026 & 69^{\circ} 57^{\prime} \mathrm{N} & 53^{\circ} 38^{\prime} \mathrm{W} & \mathrm{NW} \\ 1 \mathrm{HD} 06027 & 69^{\circ} 57^{\prime} \mathrm{N} & 53^{\circ} 40^{\prime} \mathrm{W} & \mathrm{NE}\end{array}$

90
100
130
157
120
115
135
115
110
100
90
110
120
110


75


70
105
90

120
100

151
151

$\begin{array}{rr}80 & 0000.16 \\ 80 & 0000.63\end{array}$

$700010 \quad 7000-01$

\begin{tabular}{llll}
20 & 0001.45 & 700010 & $7000-01$ \\
\hline & 0001.15 & 200010 & $7000-01$
\end{tabular}

$100 \quad 0000.11 \quad 790010 \quad 7000-01$

$\begin{array}{rrrr}90 & 0001.45 & 643112 & 7000-01 \\ 130 & 0000.12 & 300010 & 7000-01\end{array}$

$\begin{array}{llll}70 & 0005.82 & 513112 & 7000-01\end{array}$

$\begin{array}{llll}75 & 0000.79 & 643112 & 7000-01 \\ 75 & 0000.44 & 643112 & 7000-01\end{array}$

$\begin{array}{llll}75 & 0000.44 & 643112 & 7000-01 \\ 70 & 0000.11 & 643112 & 7000-01\end{array}$

$\begin{array}{llll}100 & 0000.07 & 790010 & 7000-01\end{array}$

$\begin{array}{llll}15 & 0000.50 & 300010 & 7000-01 \\ & 0000.59 & 700010 & 7000-01\end{array}$

$0003.57 \quad 603412 \quad 6930-01$

$\begin{array}{llll}100 & 0000.89 & 600012 & 6930-01\end{array}$

$\begin{array}{llll}60 & 0000.08 & 790010 & 7000-01\end{array}$

$\begin{array}{lll}0000.66 & 790010 \quad 6930-01\end{array}$

$\begin{array}{llll}65 & 0000.09 & 790112 & 7000-01\end{array}$

$\begin{array}{llll}80 & 0000.71 & 700010 & 7000-01\end{array}$ $\begin{array}{lll}0000.06 & 790112 & 7000-01\end{array}$ $0000.1470010 \quad 7000-01$

$\begin{array}{llll}100 & 0000.36 & 700010 & 6930-01\end{array}$

$\begin{array}{lll}0000.15 & 790010 & 7000-01\end{array}$

$\begin{array}{llll}30 & 0000.66 & 300010 & 7000-01\end{array}$

$0000.19 \quad 643112 \quad 6930-01$

$0000.30 \quad 643112 \quad 6930-01$

$0000.58 \quad 643112 \quad 6930-01$

$55 \quad \begin{array}{llll}0000.14 & 790012 & 6930-01 \\ & 0000.38 & 300010 & 6930-01\end{array}$

$\begin{array}{llll}80 & 0000.35 & 643112 & 7000-01 \\ 70 & 0000.65 & 673112 & 7000-01\end{array}$

$\begin{array}{llll}70 & 0000.65 & 673112 & 7000-01 \\ 65 & 0000.24 & 643112 & 7000-01\end{array}$

$\begin{array}{llll}65 & 0002.65 & 613116 & 7000-01 \\ 75 & 0000.34 & 643112 & 7000-01\end{array}$

$\begin{array}{llll}85 & 0000.73 & 200010 & 7000-01 \\ 95 & 0000.87 & 700010 & 7000-01\end{array}$

$\begin{array}{llll}95 & 0000.87 & 700010 & 7000-01\end{array}$

$\begin{array}{rrrr}65 & 0002.37 & 643017 & 7000-01\end{array}$

$\begin{array}{llll}110 & 0000.24 & 700010 & 7000-01 \\ 100 & 0000.72 & 643112 & 7000-01\end{array}$

$\begin{array}{llll}75 & 0001.48 & 643112 & 7000-01\end{array}$

$\begin{array}{llll}90 & 0000.25 & 643112 & 7000-01\end{array}$

$\begin{array}{llll}90 & 0002.43 & 643112 & 7000-01\end{array}$

$\begin{array}{llll}95 & 0002.18 & 613112 & 7000-01 \\ 95 & 0000.30 & 643112 & 7000-01\end{array}$

$\begin{array}{llll}125 & 0000.31 & 643012 & 7000-01 \\ & 0000.47 & 640312 & 7000-01\end{array}$

$100 \quad 0000.14 \quad 700010 \quad 7000-02$

$\begin{array}{rrrr}160 & 0002.01 & 543112 & 7000-02 \\ & 0000.13 & 300010 & 7000-02\end{array}$

$\begin{array}{llll}70 & 0000.07 & 700010 & 6930-02 \\ & 0000.79 & 700010 & 6930-02\end{array}$

$\begin{array}{lll}0000.05 & 700010 & 6930-02 \\ 0000.05 & 6930-02\end{array}$

$0000.28 \quad 643112 \quad 6930-02$

$\begin{array}{lll}0006.90 & 513016 & 6930-02 \\ 0000.25 & 640010 & 6930-02\end{array}$ 


\begin{tabular}{|c|c|c|c|c|c|c|c|c|c|c|c|c|c|c|c|c|c|}
\hline CODE & LAT & LONG & ORI & HIGH & LOW & AREA & $\begin{array}{c}\mathrm{MO} \\
\text { CODE }\end{array}$ & MAP & CODE & LAT & LONG & ORI & HIGH & LOW & AREA & $\begin{array}{c}\text { M0 } \\
\text { CODE }\end{array}$ & MAP \\
\hline 1HD06028 & $69^{\circ} 56^{\prime} \mathrm{N}$ & $53^{\circ} 37^{\prime} \mathrm{W}$ & - & & & 0001.01 & 700010 & $6930-02$ & 1HD07019 & $69^{\circ} 52^{\prime} \mathrm{N}$ & $53^{\circ} 54^{\prime} \mathrm{W}$ & $\mathrm{s}$ & 130 & & & & \\
\hline 1HD06029 & $69^{\circ} 55^{\prime} \mathrm{N}$ & $53^{\circ} 36^{\prime} \mathrm{W}$ & SE & & & 0000.60 & 300010 & $6930-02$ & $1 \mathrm{HD} 07020$ & $69^{\circ} 52^{\prime} \mathrm{N}$ & $53^{\circ} 54^{\prime} \mathrm{W}$ & $\mathrm{N}$ & 130 & 110 & 0000.57 & $\begin{array}{l}200010 \\
260010\end{array}$ & $\begin{array}{l}6930-02 \\
6930-02\end{array}$ \\
\hline 1HD06030 & $69^{\circ} 56^{\prime} \mathrm{N}$ & $53^{\circ} 35^{\prime} \mathrm{W}$ & SE & & & 0008.40 & 513016 & 6930-02 & $1 \mathrm{HD} 07021$ & $69^{\circ} 51^{\prime} \mathrm{N}$ & $53^{\circ} 48^{\prime} \mathrm{V}$ & $\mathrm{s}$ & & 130 & 0000.67 & 260010 & $\begin{array}{l}6930-02 \\
6930-02\end{array}$ \\
\hline 1HD06031 & $69^{\circ} 56^{\prime} \mathrm{N}$ & $53^{\circ} 32^{\prime} \mathrm{W}$ & - & & & 0001.60 & 300010 & 6930-02 & 1 HD07022 & $69^{\circ} 51^{\prime} \mathrm{N}$ & $53^{\circ} 50^{\prime} \mathrm{W}$ & $\mathrm{W}$ & 130 & 110 & 0001.51 & 643012 & $\begin{array}{l}6930-02 \\
6902\end{array}$ \\
\hline 1HD06032 & $69^{\circ} 56^{\prime} \mathrm{N}$ & $53^{\circ} 26^{\prime} \mathrm{y}$ & $w$ & & & 0000.56 & 300010 & $6930-02$ & 1HD07023 & $69^{\circ} 51^{\prime} \mathrm{N}$ & $53^{\circ} 51 \cdot \mathrm{W}$ & 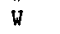 & 110 & 80 & 0000.22 & 780010 & $6930-02$ \\
\hline 1HD06033 & $69^{\circ} 55^{\prime} \mathrm{N}$ & $53^{\circ} 27 \cdot \mathrm{W}$ & - & & & 0006.68 & 363112 & 6930-02 & 1HD07024 & $69^{\circ} 51^{\prime} \mathrm{N}$ & $53^{\circ} 51^{\prime} \mathrm{W}$ & 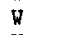 & 120 & 80 & 0000.54 & 640112 & $6930-02$ \\
\hline 1 HDO6034 & & $\begin{array}{l}53^{\circ} 27^{\prime} \mathrm{W} \\
53^{\circ} 25^{\prime} \mathrm{W}\end{array}$ & $\bar{w}$ & & & 0002.02 & 300010 & 6930-02 & $1 \mathrm{HD} 07025$ & $69^{\circ} 50^{\prime} \mathrm{N}$ & $53^{\circ} 50^{\circ} \mathrm{W}$ & W & 110 & 100 & 0000.04 & 780010 & $6930-02$ \\
\hline $\begin{array}{l}\text { 1HD06035 } \\
\text { 1HD06036 }\end{array}$ & $\begin{array}{l}69^{\circ} 53^{\prime} \mathrm{N} \\
69^{\circ} 53^{\prime} \mathrm{N}\end{array}$ & $\begin{array}{l}53^{\circ} 25^{\circ} \mathrm{W} \\
53^{\circ} 31^{\prime} \mathrm{W}\end{array}$ & $\begin{array}{l}\mathrm{N} \\
\mathrm{N}\end{array}$ & & & 0032.02 & 363111 & $6930-02$ & $1 \mathrm{HD} 07026$ & $69^{\circ} 50^{\prime} \mathrm{N}$ & $53^{\circ} 51^{\prime} \mathrm{W}$ & - & 90 & 90 & 0000.05 & 780010 & $6930-02$ \\
\hline $\begin{array}{l}\text { 1HD06036 } \\
\text { 1 HD06037 }\end{array}$ & & $\begin{array}{l}53^{\circ} 31 \% \mathrm{~W} \\
53^{\circ} 3 \mathrm{w}^{\prime}\end{array}$ & $\begin{array}{l}\mathrm{N} \\
\mathrm{N}\end{array}$ & & & 0001.28 & 643112 & $6930-02$ & 1HD07027 & $69^{\circ} 51^{\prime} \mathrm{N}$ & $53^{\circ} 49^{\prime} \mathrm{V}$ & $\mathrm{SE}$ & 130 & 130 & 0000.11 & 780010 & $6930-02$ \\
\hline & 69053 N & $53^{\circ} 33^{\prime \prime w}$ & & & & 0000.18 & 793012 & $6930-02$ & 1HD07028 & $69^{\circ} 49^{\prime} \mathrm{N}$ & $53^{\circ} 47^{\prime} \mathrm{V}$ & W & & 40 & 0028.97 & 263111 & $6930-02$ \\
\hline $\begin{array}{l}\text { 1HD06038 } \\
1 \text { HD06039 }\end{array}$ & $69^{\circ} 52^{\prime} \mathrm{N}$ & $53^{\circ} 30^{\circ} \mathrm{W}$ & $\begin{array}{l}N \\
-\end{array}$ & & & 0000.34 & 643112 & 6930-02 & $1 \mathrm{HD} 07029$ & $69^{\circ} 49^{\prime} \mathrm{N}$ & $53^{\circ} 52^{\prime \prime}$ & $\mathrm{N}$ & 90 & 60 & 0001.39 & 643112 & $6930-02$ \\
\hline $\begin{array}{l}\text { 1HD06039 } \\
1 \text { HD06040 }\end{array}$ & $69^{\circ} 52^{\prime} \mathrm{N}$ & $53^{\circ} 27^{\circ} \mathrm{W}$ & $\mathrm{NE}$ & & & 0000.15 & 700010 & $6930-02$ & 1 HD07030 & $69^{\circ} 49 \cdot \mathrm{N}$ & $53^{\circ} 54^{\prime} \mathrm{V}$ & $\mathbf{N}$ & 90 & 70 & 0000.15 & 770010 & $6930-02$ \\
\hline $\begin{array}{l}\text { 1日D06040 } \\
\text { 1日D06041 }\end{array}$ & $69^{\circ} 51^{\prime} \mathrm{N}$ & $53^{\circ} 30^{\circ} \mathrm{W}$ & NW & & & 0000.76 & 263112 & $6930-02$ & 1 HDO 7031 & $69^{\circ} 48^{\prime} \mathrm{N}$ & $53^{\circ} 53^{\prime} \mathrm{V}$ & . & 100 & 100 & 0000.19 & 300010 & $6930-02$ \\
\hline & $69^{\circ} 51 \cdot \mathrm{N}$ & $53^{\circ} 37 \cdot \mathrm{W}$ & $\mathrm{N}$ & & & 0033.68 & 263111 & 6930-02 & 1HD07032 & $69^{\circ} 48^{\prime} \mathrm{N}$ & $53^{\circ} 53^{\prime} \mathrm{W}$ & $\mathbf{N}$ & & 40 & 0003.82 & 203312 & 6930-02 \\
\hline $\begin{array}{l}1 \mathrm{HD} 06042 \\
1 \mathrm{HD} 06043\end{array}$ & $69^{\circ} 52 \cdot \mathrm{N}$ & $53^{\circ} 37^{\circ} \mathrm{W}$ & $\mathrm{NE}$ & & & 0005.39 & 263111 & 6930-02 & 1HD07033 & $69^{\circ} 48^{\prime} \mathrm{N}$ & $53^{\circ} 57$, & $\mathrm{N}$ & & 60 & 0000.68 & 643112 & $6930-01$ \\
\hline $\begin{array}{l}\text { 1HD06043 } \\
1 \text { HD06044 }\end{array}$ & $69^{\circ} 52^{\prime} \mathrm{N}$ & $53^{\circ} 38^{\prime} \mathrm{W}$ & NE & & & 0000.65 & 643112 & $6930-02$ & $1 \mathrm{HD} 07034$ & $69^{\circ} 49^{\prime} \mathrm{N}$ & $53^{\circ} 59^{\prime} \mathrm{W}$ & $\mathrm{NE}$ & 110 & 60 & 0001.01 & 643112 & $6930-01$ \\
\hline $\begin{array}{l}\text { 1HD06044 } \\
\text { 1HD06045 }\end{array}$ & $69^{\circ} 52^{\prime} \mathrm{N}$ & $53^{\circ} 39^{\prime} \mathrm{W}$ & $\mathrm{w}$ & & & 0000.15 & 200010 & $6930-02$ & 1 HD07035 & $69^{\circ} 49^{\prime} \mathrm{N}$ & $54^{\circ} 00^{\prime} \mathrm{V}$ & $\mathrm{N}$ & 120 & 70 & 0000.28 & 780010 & $6930-01$ \\
\hline $\begin{array}{l}\text { 1HD06045 } \\
\text { 1HD06046 }\end{array}$ & $69^{\circ} 52^{\prime} \mathrm{N}$ & $53^{\circ} 42 \cdot \mathrm{W}$ & $\mathbf{N}$ & & & 0000.13 & 700010 & $6930-02$ & $1 \mathrm{HD} 07036$ & $69^{\circ} 49^{\prime} \mathrm{N}$ & $54^{\circ} 01^{\prime} \mathrm{W}$ & $\mathbf{N}$ & & 70 & 0001.33 & 643112 & $6930-01$ \\
\hline & $69^{\circ} 52^{\prime} \mathrm{N}$ & $53^{\circ} 44^{\prime} \mathrm{W}$ & 10 & & & 0004.48 & 263111 & $6930-02$ & 1HD07037 & $69^{\circ} 49^{\prime} \mathrm{N}$ & $54^{\circ} 03^{\prime} \mathrm{V}$ & N & 100 & 70 & 0000.16 & 640112 & 6930-01 \\
\hline & $69^{\circ} 52^{\prime} \mathrm{N}$ & $53^{\circ} 46^{\prime} \mathrm{W}$ & $\mathrm{NE}$ & & & 0000.16 & 700010 & $6930-02$ & 1HD07038 & $69^{\circ} 48^{\prime} \mathrm{N}$ & $54^{\circ} 03^{\prime} \mathrm{W}$ & $\mathrm{W}$ & 20 & 60 & 0001.64 & 643112 & $6930-01$ \\
\hline $\begin{array}{l}\text { 1HD06048 } \\
\text { 1HD06049 }\end{array}$ & $69^{\circ} 54^{\prime} \mathrm{N}$ & $53^{\circ} 43^{\prime} \mathrm{W}$ & $\mathrm{NE}$ & & & 0006.15 & 263111 & 6930-02 & 1HD07039 & $69^{\circ} 47^{\circ} \mathrm{N}$ & $54^{\circ} 04^{\prime} \mathrm{W}$ & W & & 90 & 0000.64 & 643112 & 6930-01 \\
\hline & $69^{\circ} 54^{\prime} \mathrm{N}$ & $53^{\circ} 45^{\circ} \mathrm{W}$ & $\mathrm{N}$ & & & 0000.14 & 643112 & $6930-02$ & 1HD07040 & $69^{\circ} 47^{\prime} \mathrm{N}$ & $54^{\circ} 07^{\prime} \mathrm{W}$ & $\mathbf{N}$ & & 30 & 0008.16 & 263112 & 6930-01 \\
\hline & $69^{\circ} 53 \cdot \mathrm{N}$ & $53^{\circ} 48 \cdot \hat{W}$ & $\mathrm{NE}$ & & & 0000.76 & 643112 & $6930-02$ & 1 HD07041 & $69^{\circ} 49^{\prime} \mathrm{N}$ & $54^{\circ} 09^{\prime} \mathrm{W}$ & - & 90 & 90 & 0000.58 & 780010 & $6930-01$ \\
\hline & $69055, \mathrm{~N}$ & $53^{\circ} 49, \mathrm{U}$ & Nit & & & 0005.76 & 263111 & $6930-02$ & 1HD07042 & $69^{\circ} 50^{\prime} \mathrm{N}$ & $54^{\circ} 09^{\prime} \mathrm{W}$ & NE & 80 & 40 & 0000.12 & 790010 & 693 \\
\hline 1HD06052 & $69054, \mathrm{~N}$ & $\begin{array}{l}53^{\circ} 49 \% \mathrm{~W} \\
53^{\circ} 51^{\prime}, \mathrm{s}\end{array}$ & NW & & & 0000.13 & 700010 & 6930-02 & 1HD07043 & $69^{\circ} 50^{\prime} \mathrm{N}$ & $54^{\circ} 12^{\prime} \mathrm{W}$ & $\mathrm{NE}$ & 100 & 50 & 0000.78 & 640112 & $6930-01$ \\
\hline 1HD06053 & $\begin{array}{l}69^{\circ 55} 4^{\prime} \mathrm{N} \\
69^{\circ} 56^{\prime} \mathrm{N}\end{array}$ & $53^{\circ} 51 \cdot \mathrm{W}$ & NE & & & 0003.25 & 263441 & $6930-02$ & 1HD07044 & $69^{\circ} 51^{\prime} \mathrm{N}$ & $54^{\circ} 13^{\prime} \mathrm{W}$ & E & 180 & 60 & 0000.09 & 780010 & $6930-01$ \\
\hline 1HD06054 & $69^{\circ} 56^{\prime} \mathrm{N}$ & $53^{\circ} 51 \cdot \mathrm{W}$ & NW & & & 0000.19 & 790010 & $6930-02$ & 1нD07045 & $69^{\circ} 51 \cdot \mathrm{N}$ & $54^{\circ} 13^{\prime} \mathrm{W}$ & E & & 100 & 0000.39 & 360010 & $6930-01$ \\
\hline 1HD06055 & $69^{\circ} 55^{\prime} \mathrm{N}$ & $53^{\circ} 54^{\prime} \mathrm{W}$ & $\mathrm{NE}$ & & & 0001.50 & 513112 & $6930-02$ & $1 \mathrm{HD} 07046$ & $69^{\circ} 52^{\prime} \mathrm{N}$ & $54^{\circ} 14^{\prime} W$ & $N E$ & 70 & 50 & 0000.12 & 640112 & 6930 \\
\hline 1HD06056 & $69^{\circ} 55^{\prime} \mathrm{N}$ & $53^{\circ} 55 \cdot \mathrm{W}$ & $\mathrm{NE}$ & & & 0000.29 & 643112 & $6930-02$ & 1HD07047 & $69^{\circ} 52^{\prime} \mathrm{N}$ & $54^{\circ} 15^{\prime} \mathrm{W}$ & $\mathrm{NE}$ & 110 & 80 & 0000 & 780010 & $6930-01$ \\
\hline 1HD06057 & $69^{\circ} 55^{\prime} \mathrm{N}$ & $53^{\circ} 55^{\circ} \mathrm{W}$ & $\mathbf{E}$ & & & 0000.11 & 790010 & $6930-02$ & 1HD07048 & $69^{\circ} 53^{\prime} \mathrm{N}$ & $54^{\circ} 16^{\prime} \mathrm{W}$ & $\mathrm{NE}$ & & 110 & 0000.42 & 200010 & 6930-01 \\
\hline 1HD06058 & $69^{\circ} 56^{\prime} \mathrm{N}$ & $53^{\circ} 56^{\prime} \mathrm{W}$ & NE & & & 0001.71 & 513112 & $6930-02$ & 1 HD07049 & $69^{\circ} 53^{\prime} \mathrm{N}$ & $54^{\circ} 15^{\prime} \mathrm{W}$ & $\mathrm{NE}$ & 70 & 50 & 0000.09 & 780010 & 6930-01 \\
\hline 1HD06059 & $69^{\circ} 56^{\prime} \mathrm{N}$ & $53^{\circ} 57^{\prime} \mathrm{W}$ & $\mathrm{NE}$ & & & 0000.42 & 643112 & $6930-02$ & 1HD07050 & $69^{\circ} 54^{\prime} \mathrm{N}$ & $54^{\circ} 16^{\prime} \mathrm{W}$ & $\mathbf{N}$ & 40 & 30 & 0000.07 & 790010 & $6930-01$ \\
\hline 1HD06060 & $69^{\circ} 57^{\prime} \mathrm{N}$ & $53^{\circ} 55^{\prime} \mathrm{W}$ & N & & & 0000.10 & 643112 & $6930-02$ & IHD07051 & $69^{\circ} 54^{\prime} \mathrm{N}$ & $54^{\circ} 17, \mathrm{~W}$ & $\mathrm{~N}$ & 40 & 30 & 0000.07 & 790012 & $6930-01$ \\
\hline 1 HD06061 & $69^{\circ} 58^{\prime} \mathrm{N}$ & $53^{\circ} 58^{\circ} \mathrm{W}$ & NW & & & 0000.62 & 643112 & $6930-01$ & 1HD08001 & $69^{\circ} 53^{\prime} \mathrm{N}$ & $54^{\circ} 17^{\prime} \mathrm{W}$ & W & 90 & 70 & 0000.17 & 790010 & $6930-01$ \\
\hline 1HD06062 & $69^{\circ} 57 \cdot N$ & $53^{\circ} 59 \cdot \mathrm{W}$ & NW & 95 & 50 & 0000.74 & 643111 & $6930-01$ & 1HD08002 & $69^{\circ} 52^{\prime} \mathrm{N}$ & $54^{\circ} 15^{\prime} \mathrm{W}$ & NW & & 25 & 0001.90 & 363412 & $6930-01$ \\
\hline 1月D06063 & $69^{\circ} 57^{\prime} \mathrm{N}$ & $54^{\circ} 00^{\circ} \mathrm{W}$ & $\mathrm{N}$ & 90 & 50 & 0001.01 & 643111 & 6930-01 & 1HD08003 & $69^{\circ} 52^{\prime} \mathrm{N}$ & $54^{\circ} 20^{\prime} \mathrm{W}$ & - & & 90 & 0000.71 & 300010 & $6930-01$ \\
\hline 1HD06064 & $69^{\circ} 58^{\prime} \mathrm{N}$ & $54^{\circ} 03^{\prime} \mathrm{W}$ & $\mathbf{N}$ & 90 & 60 & 0001.11 & 643112 & $6930-01$ & $1 \mathrm{HD} 08004$ & $69^{\circ} 52^{\prime N} \mathrm{~N}$ & $54^{\circ} 21^{\prime} \mathrm{W}$ & - & & 100 & 0000.22 & 300010 & $6930-01$ \\
\hline 1HD06065 & $69^{\circ} 58^{\prime} \mathrm{N}$ & $54^{\circ} 02^{\prime} \mathrm{W}$ & $\mathbf{N}$ & 100 & 75 & 0000.33 & 643112 & $6930-01$ & 1HD08005 & $69^{\circ} 52^{\prime} \mathrm{N}$ & $54^{\circ} 21^{\prime} \mathrm{W}$ & NE & 80 & 60 & 0000.16 & 643112 & $6930-01$ \\
\hline 1HD06066 & $69^{\circ} 59^{\prime} \mathrm{N}$ & $54^{\circ} 03^{\prime} \mathrm{W}$ & $\mathbf{N}$ & 95 & 75 & 0000.07 & 790012 & 6930-01 & 1HD08006 & $69^{\circ} 53^{\prime} \mathrm{N}$ & $54^{\circ} 23^{\prime} \mathrm{W}$ & $\mathrm{N}$ & & 50 & 0000.70 & 643112 & $6930-01$ \\
\hline 1HD06067 & $\begin{array}{l}69^{\circ} 59^{\prime} \mathrm{N} \\
60^{\circ} 57^{\prime} \mathrm{N}\end{array}$ & $54^{\circ} 05^{\prime} \mathrm{W}$ & $\mathrm{N}_{\mathrm{CF}}$ & 80 & 60 & 0000.05 & 790012 & 6930-01 & 1HD08007 & $69^{\circ} 53^{\prime} \mathrm{N}$ & $54^{\circ} 24^{\prime} \mathrm{W}$ & $\mathrm{N}$ & & 50 & 0001.32 & 643112 & $6930-01$ \\
\hline 1HD06068 & $69^{\circ} 57^{\prime} \mathrm{N}$ & $54^{\circ} 05^{\prime} \mathrm{W}$ & SE & 75 & 60 & 0000.06 & 643112 & $6930-01$ & 1HD08008 & $69^{\circ} 53^{\prime} \mathrm{N}$ & $54^{\circ} 26^{\prime} \mathrm{W}$ & $\mathrm{E}$ & 110 & 80 & 0001.14 & 613112 & $6930-01$ \\
\hline 1HD06069 & $69^{\circ} 57^{\prime} \mathrm{N}$ & $54^{\circ} 07^{\prime} \mathrm{W}$ & $\mathbf{N}$ & 75 & 45 & 0001.04 & 643112 & $6930-01$ & 1HD08009 & $69^{\circ} 54, \mathrm{~N}$ & $54^{\circ} 25^{\circ} \mathrm{W}$ & $\bar{E}$ & 90 & 80 & 0000.06 & 790010 & $6930-01$ \\
\hline 1月D06070 & $69^{\circ} 56^{\prime} \mathrm{N}$ & $54^{\circ} 10^{\circ} \mathrm{W}$ & NW & 65 & 40 & 0000.17 & 790010 & 6930-01 & $1 \mathrm{HD} 08010$ & $69^{\circ} 54^{\prime} \mathrm{N}$ & $54^{\circ} 26^{\prime} \mathrm{W}$ & NE & 70 & 50 & 0000.21 & 640112 & $6930-01$ \\
\hline $\begin{array}{l}\text { 1日D07001 } \\
1 \mathrm{BD07002}\end{array}$ & $\begin{array}{l}69^{\circ} 577^{\prime N} \mathrm{~N} \\
69054 \cdot \mathrm{N}\end{array}$ & $\begin{array}{l}54^{\circ} 00^{\prime} \mathrm{W} \\
55053 \cdot \mathrm{V}\end{array}$ & $N$ & 150 & 100 & 0000.85 & 303012 & 6930- & 1HD08011 & $69^{\circ} 55^{\prime} \mathrm{N}$ & $54^{\circ} 28^{\prime} \mathrm{W}$ & E & & 110 & 0000.21 & 780010 & $6930-01$ \\
\hline 1日D07002 & $\begin{array}{l}69^{\circ} 54 \cdot \mathrm{N} \\
69^{\circ} 55^{\prime} \mathrm{N}\end{array}$ & $54^{\circ} 00 . \mathrm{W}$ & $\mathrm{N}$ & & $\begin{array}{l}60 \\
50\end{array}$ & 0007.29 & 513011 & 6930-02 & 1HD09001 & $69^{\circ} 55^{\prime} \mathrm{N}$ & $54^{\circ} 28^{\prime} W$ & $\mathrm{~N}$ & & & 0000.21 & 780010 & $6930-01$ \\
\hline 1月D07003 & $\begin{array}{l}69^{\circ} 53^{\circ} \mathrm{N} \\
69^{\circ} 53^{\prime} \mathrm{N}\end{array}$ & $54^{\circ} 02$ ' & N & 80 & $\begin{array}{l}50 \\
50\end{array}$ & 0000.23 & 640112 & 6930-01 & 1HD09002 & $69^{\circ} 55^{\prime} \mathrm{N}$ & $54^{\circ} 29^{\prime} \mathrm{V}$ & $\mathbf{N}$ & 500 & 30 & 0000.38 & 790012 & $6930-01$ \\
\hline 1HD07004 & $\begin{array}{l}69^{\circ} 53^{\prime} \mathrm{N} \\
69^{\circ} 54^{\prime} \mathrm{N}\end{array}$ & $54^{\circ} 03^{\prime} \mathrm{W}$ & $\mathrm{N}$ & 80 & $\begin{array}{l}50 \\
50\end{array}$ & 0001.88 & 643112 & $6930-01$ & 1HD09003 & $69^{\circ} 55^{\prime} \mathrm{N}$ & $54^{\circ} 30^{\prime} \mathrm{W}$ & $\mathbf{N}$ & 85 & 40 & 0000.15 & 780010 & $6930-01$ \\
\hline 1HD07005 & $\begin{array}{l}69^{\circ} 54^{\prime} \mathrm{N} \\
69^{\circ} 54^{\prime} \mathrm{N}\end{array}$ & $54^{\circ} 05, \mathrm{~W}$ & NW & 90 & $\begin{array}{l}50 \\
60\end{array}$ & 0000.72 & 643112 & 6930-01 & $1 \mathrm{HD} 09004$ & $69^{\circ} 55^{\prime} \mathrm{N}$ & $54^{\circ} 31^{\prime} \mathrm{W}$ & - & 1000 & 80 & 0000.13 & 780010 & $6930-01$ \\
\hline 1 HD07006 & & $54^{\circ} 03^{\prime} \mathrm{W}$ & $\mathrm{W}$ & 130 & $\begin{array}{l}60 \\
80\end{array}$ & 0000.53 & 643112 & 6930-01 & $1 \mathrm{HD} 09005$ & $69^{\circ} 56^{\prime} \mathrm{N}$ & $54^{\circ} 33^{\prime} \mathrm{W}$ & N & 90 & 70 & 0000.05 & 780010 & $6930-01$ \\
\hline 1HD07007 & $\begin{array}{l}69^{\circ} 53^{\prime} \mathrm{N} \\
69^{\circ} 52^{\prime} \mathrm{N}\end{array}$ & $54^{\circ} 02^{\prime} \mathrm{W}$ & $\mathbf{E}$ & 110 & $\begin{array}{r}80 \\
100\end{array}$ & 0001.06 & 643111 & 6930-01 & 1HD09006 & $69^{\circ} 55^{\prime} \mathrm{N}$ & $54^{\circ} 33^{\prime} \mathrm{W}$ & $\mathrm{NW}$ & 80 & 40 & 0000.76 & 643112 & 6930-01 \\
\hline 1HD07008 & $\begin{array}{l}69^{\circ} 52^{\prime} \mathrm{N} \\
69^{\circ} 52^{\prime} \mathrm{N}\end{array}$ & $54^{\circ} 00^{\prime} \mathrm{W}$ & E & 120 & $\begin{array}{r}100 \\
90\end{array}$ & 0000.09 & 790010 & $6930-01$ & 1HD09007 & $69^{\circ} 56^{\prime} \mathrm{N}$ & $54^{\circ} 36^{\prime} \mathrm{V}$ & - & 110 & 105 & 0000.16 & 780010 & 6930-01 \\
\hline $\begin{array}{l}\text { 1HD07009 } \\
1 \text { HDO7010 }\end{array}$ & $\begin{array}{l}69^{\circ} 52^{\prime} \mathrm{N} \\
69^{\circ} 54^{\prime} \mathrm{N}\end{array}$ & $53^{\circ} 59^{\circ} \mathrm{W}$ & $\mathbf{2}$ & 130 & $\begin{array}{r}90 \\
130\end{array}$ & 0000.28 & 640112 & $6930-01$ & 1HDO9008 & $69^{\circ} 56^{\prime} \mathrm{N}$ & $54^{\circ} 37^{\prime} \mathrm{V}$ & W & 60 & 40 & 0000.23 & 790010 & $6930-01$ \\
\hline 1ADDO7010 & $69^{\circ} 54^{\prime} \mathrm{N}$ & $53^{\circ} 58^{\circ} \mathrm{W}$ & $\mathbf{s}$ & 130 & $\begin{array}{l}130 \\
110\end{array}$ & & 780010 & $6930-01$ & & & $54^{\circ} 38^{\prime} \mathrm{W}$ & W & 90 & 50 & 0000.64 & 643112 & $6930-01$ \\
\hline $\begin{array}{l}\text { 1HD07011 } \\
\text { 1HD07012 }\end{array}$ & $\begin{array}{l}69^{\circ} 54^{\prime} \mathrm{N} \\
69^{\circ} 53^{\prime} \mathrm{N}\end{array}$ & $53^{\circ} 56^{\prime \prime} \mathrm{W}$ & $\mathrm{v}$ & 11 & $\begin{array}{r}110 \\
80\end{array}$ & $\begin{array}{l}0000.61 \\
0002.25\end{array}$ & $\begin{array}{l}300010 \\
643112\end{array}$ & $\begin{array}{l}6930-01 \\
6930-01\end{array}$ & 1HD09010 & $69^{\circ} 55^{\prime} \mathrm{N}$ & $54^{\circ} 40^{\prime} \mathrm{W}$ & W & 110 & 85 & 0000.42 & 780010 & $6930-01$ \\
\hline 1日D07013 & 69052 , N & $53^{\circ} 56^{\prime} \mathrm{W}$ & $\mathrm{W}$ & 110 & 80 & 0002.37 & $\begin{array}{l}643112 \\
643312\end{array}$ & $6930-01$ & 1HE01001 & $69^{\circ} 36^{\prime} \mathrm{N}$ & $52^{\circ} 15^{\prime} \mathrm{W}$ & NE & 65 & 60 & 0000.08 & 780010 & $6930-03$ \\
\hline 1HD07014 & $69^{\circ} 51^{\prime} \mathrm{N}$ & $53^{\circ} 57 \cdot \mathrm{W}$ & W & 120 & 70 & 0000.87 & 643112 & $6930-01$ & 1HE01002 & $69^{\circ} 36^{\prime} \mathrm{N}$ & $52^{\circ} 18^{\prime} \mathrm{W}$ & NE & 80 & 70 & 0000.12 & 793011 & $6930-03$ \\
\hline 1月007015 & $69^{\circ} 51^{\prime} \mathrm{N}$ & $53^{\circ} 56^{\prime} \mathrm{W}$ & $\mathbf{s}$ & 130 & 90 & 0000.97 & 780010 & $6930-02$ & 1HE01003 & $69^{\circ} 37^{\prime} \mathrm{N}$ & $52^{\circ} 20^{\prime} \mathrm{W}$ & NE & 80 & 65 & 0001.95 & 673112 & $6930-03$ \\
\hline 1月D07016 & $69^{\circ} 51^{\prime} \mathrm{N}$ & $53^{\circ} 55^{\prime} \mathrm{W}$ & $\mathrm{s}$ & 110 & 80 & 0000.29 & 790010 & $6930-02$ & $\begin{array}{l}\text { 1HE01004 } \\
\text { 1HE01005 }\end{array}$ & $\begin{array}{l}69^{\circ} 38^{\prime} \mathrm{N} \\
69^{\circ} 39^{\prime} \mathrm{N}\end{array}$ & $52^{\circ} 22^{\prime} \mathrm{W}$ & $\mathrm{E}$ & & & 0000.39 & 673112 & $6930-03$ \\
\hline 1HD07017 & $69^{\circ} 52^{\prime} \mathrm{N}$ & $53^{\circ} 55^{\prime} \mathrm{W}$ & SE & 120 & 80 & 0000.92 & 770010 & $6930-02$ & $\begin{array}{l}\text { 1HEU100 S } \\
1 \mathrm{HE} 01006\end{array}$ & $69^{\circ} 39^{\prime} \mathrm{N}$ & 52016,6 & $\mathrm{~N}$ & 90 & 65 & 0000.22 & 793112 & $6930-03$ \\
\hline 1HD07018 & $69^{\circ} 52^{\prime} \mathrm{N}$ & $53^{\circ} 54^{\prime} \mathrm{W}$ & $\mathbf{s}$ & & 60 & 0007.54 & 263112 & $6930-02$ & 1HE01007 & $69^{\circ} 39^{\prime} \mathrm{N}$ & $52^{\circ} 18^{\prime} \mathrm{W}$ & $\stackrel{N}{N}$ & $\begin{array}{l}95 \\
90\end{array}$ & $\begin{array}{r}700 \\
65\end{array}$ & $\begin{array}{l}0000.45 \\
0000.28\end{array}$ & $\begin{array}{l}643112 \\
643111\end{array}$ & $6930-03$ \\
\hline
\end{tabular}




\begin{tabular}{|c|c|c|c|}
\hline & LAT & LONG & \\
\hline & 6 & $52^{\circ} 20^{\prime} \mathrm{W}$ & \\
\hline 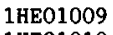 & 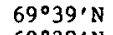 & ${ }^{\circ} 23 \cdot \mathrm{v}$ & \\
\hline IHE01010 & $69^{\circ} 38^{\prime} \mathrm{N}$ & $52^{\circ} 23^{\prime} \mathrm{V}$ & \\
\hline HEO1011 & $69^{\circ} 39^{\prime} \mathrm{N}$ & $52^{\circ} 30^{\circ} \mathrm{v}$ & \\
\hline HE01012 & $69^{\circ} 40^{\prime} \mathrm{N}$ & $33 . \mathrm{v}$ & \\
\hline 1HE01013 & $69^{\circ} 41^{\prime} \mathrm{N}$ & $2^{\circ} 28^{\prime} \mathrm{W}$ & \\
\hline 1 HE01014 & $69^{\circ} 41^{\prime} \mathrm{N}$ & ${ }^{\circ} 28^{\prime} \mathrm{W}$ & \\
\hline $1 \mathrm{HE} 01015$ & $69^{\circ} 40^{\prime} \mathrm{N}$ & $52^{\circ} 34^{\prime} \mathrm{W}$ & \\
\hline 1 HE01016 & $69^{\circ} 41^{\prime} \mathrm{N}$ & $52^{\circ} 34^{\prime} \mathrm{W}$ & \\
\hline 1HE01017 & $69^{\circ} 42^{\prime} \mathrm{N}$ & $34 . \mathrm{v}$ & \\
\hline 1HEO & $69^{\circ}$ & $\mathrm{W}$ & \\
\hline 1HEO & $69^{\circ} 4$ & $35 . \mathrm{k}$ & \\
\hline $1 \mathrm{HE} 01020$ & $69^{\circ} 40^{\prime} \mathrm{N}$ & $52^{\circ} 38^{\circ} \mathrm{W}$ & \\
\hline & & $41 \cdot \mathrm{w}$ & \\
\hline $1 \mathrm{HEO}$ & & $5, \mathrm{w}$ & \\
\hline $1 \mathrm{HEO}$ & & $47 \cdot \mathrm{W}$ & \\
\hline $1 \mathrm{HEO}$ & 69 & ${ }^{\circ} 55^{\prime} \mathrm{W}$ & \\
\hline $1 \mathrm{HEO}$ & ' & $52^{\circ} 46^{\prime} \mathrm{W}$ & SE \\
\hline & & $44 \cdot \mathrm{W}$ & \\
\hline & & $2, k$ & \\
\hline $1 \mathrm{HEO}$ & & & \\
\hline IEC & & $41, \mathrm{~V}$ & \\
\hline 00 & , N & $43 . \mathrm{V}$ & \\
\hline & & & \\
\hline & & $\mathrm{v}$ & \\
\hline $1 \mathrm{HE}$ & & & \\
\hline & & $66^{\prime} \mathrm{W}$ & \\
\hline & & & \\
\hline & & & \\
\hline & & & \\
\hline $1 \mathrm{HE}$ & & $2 \cdot \mathrm{W}$ & \\
\hline & & & \\
\hline & & & \\
\hline & & & \\
\hline & & & \\
\hline & & & \\
\hline & & & \\
\hline & & & \\
\hline & & & \\
\hline & & & \\
\hline & & & \\
\hline & & & \\
\hline & & & \\
\hline & & & \\
\hline & & & \\
\hline & & & \\
\hline & & & \\
\hline & & & \\
\hline & & & $\mathrm{N}$ \\
\hline & & & \\
\hline & & & \\
\hline & & & \\
\hline & & & \\
\hline & & & \\
\hline & & & \\
\hline & & & \\
\hline & & & \\
\hline & & & \\
\hline & & & \\
\hline & 3 & $2^{\circ} 39^{\prime} \mathrm{W}$ & $N$ \\
\hline & 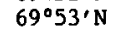 & 500 & \\
\hline
\end{tabular}

\begin{tabular}{|c|c|c|c|c|c|c|c|c|}
\hline \multirow[t]{42}{*}{ HIGH } & LOW & AREA & $\begin{array}{c}\text { MO } \\
\text { CODE }\end{array}$ & MAP & CODE & LAT & LONG & ORI \\
\hline & \multirow[t]{41}{*}{60} & 0000.21 & 643111 & $6930-03$ & 1HE02017 & $69^{\circ} 53^{\prime} \mathrm{N}$ & $52^{\circ} 46^{\prime} \mathrm{W}$ & $\mathbf{N}$ \\
\hline & & 0000.49 & 770112 & $6930-03$ & $1 \mathrm{HEO} 02018$ & $69^{\circ} 57 / \mathrm{N}$ & $52^{\circ} 55^{\prime} \mathrm{W}$ & $\mathrm{NW}$ \\
\hline & & 0001.50 & 363116 & $6930-03$ & 1HE02019 & $69^{\circ} 58^{\prime} \mathrm{N}$ & $52^{\circ} 58^{\prime} \mathrm{W}$ & $\mathrm{NE}$ \\
\hline & & 0002.46 & 363116 & $6930-03$ & 1HE02020 & $69^{\circ} 59^{\prime} \mathrm{N}$ & $53^{\circ} 01^{\prime} \mathrm{W}$ & 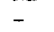 \\
\hline & & 0002.81 & 360010 & $6930-03$ & 1 HE02021 & $70^{\circ} 00^{\circ} \mathrm{N}$ & $53^{\circ} 02^{\prime} \mathrm{W}$ & - \\
\hline & & 0000.22 & 770010 & $6930-03$ & 1HE03001 & $70^{\circ} 00^{\prime} \mathrm{N}$ & $53^{\circ} 03^{\prime} \mathrm{W}$ & NW \\
\hline & & 0000.12 & 673112 & $6930-03$ & 1 HEO 3002 & $70^{\circ} 00^{\prime} \mathrm{N}$ & $53^{\circ} 06^{\circ}, \mathrm{W}$ & $\mathrm{N}$ \\
\hline & & 0004.07 & 363416 & $6930-03$ & 1 HE03003 & $70^{\circ} 01^{\prime} \mathrm{N}$ & $53^{\circ} 10^{\prime} \mathrm{W}$ & $\mathrm{NE}$ \\
\hline & & 0001.06 & 360010 & $6930-03$ & 1HE03004 & $70^{\circ} 01 ' \mathrm{~N}$ & $53^{\circ} 07^{\prime} \mathrm{W}$ & E \\
\hline & & 0000.54 & 363312 & $6930-03$ & $1 \mathrm{HE} 03005$ & $70^{\circ} 02^{\prime} \mathrm{N}$ & $53^{\circ} 06^{\prime W}$ & E \\
\hline & & 0001.12 & 363312 & $6930-03$ & 1HE04001 & $70^{\circ} 03^{\prime} \mathrm{N}$ & $53^{\circ} 05^{\prime} \mathrm{W}$ & $\mathbf{N}$ \\
\hline & & 0001.67 & 360012 & $6930-03$ & 1HE04002 & $70^{\circ} 04^{\prime} \mathrm{N}$ & $53^{\circ} 05 \cdot \mathrm{W}$ & $\mathrm{NE}$ \\
\hline & & 0010.40 & 363411 & $6930-03$ & $1 \mathrm{HE} 04003$ & $70^{\circ} 03^{\prime} \mathrm{N}$ & $53^{\circ} 07 \% \mathrm{~W}$ & $\mathrm{~N}$ \\
\hline & & 0000.27 & 643212 & $6930-03$ & 1HE04004 & $70^{\circ} 04^{\prime} \mathrm{N}$ & $53^{\circ} 09 \% \mathrm{~W}$ & $\mathrm{NE}$ \\
\hline & & 0010.21 & 363511 & $6930-03$ & $1 \mathrm{HE} 04005$ & $70^{\circ} 04^{\prime} \mathrm{N}$ & $53^{\circ} 10^{\prime} \mathrm{W}$ & $\mathrm{NE}$ \\
\hline & & 0000.28 & 670012 & $6930-03$ & 1HE04006 & $70^{\circ} 05^{\prime} \mathrm{N}$ & $53^{\circ} 10^{\circ} \mathrm{W}$ & $\mathrm{E}$ \\
\hline & & 0055.96 & 363416 & $6930-03$ & 1 HE0 0001 & $70^{\circ} 06^{\prime} \mathrm{N}$ & $53 \circ 11$. & $\mathrm{N}$ \\
\hline & & 0002.67 & 360110 & $6930-03$ & 1HE05002 & $70^{\circ} 05$, N & $53^{\circ} 13^{\prime} \mathrm{k}$ & NW \\
\hline & & 0002.87 & 360110 & $6930-03$ & 1HE05003 & $70^{\circ} 03^{\prime N} \mathrm{~N}$ & $53^{\circ} 14^{\prime} \mathrm{W}$ & $\mathrm{N}$ \\
\hline & & 0001.45 & 363412 & $6930-03$ & 1HE06001 & $70^{\circ} 03^{\prime} \mathrm{N}$ & $53^{\circ} 15^{\prime} \mathrm{W}$ & $\mathrm{N}$ \\
\hline & & 0000.22 & 750010 & $6930-03$ & 1 HE06002 & $70^{\circ} 05, \mathrm{~N}$ & $53^{\circ} 19, \mathrm{k}$ & E \\
\hline & & 0001.54 & 363412 & $6930-03$ & $1 \mathrm{HE} 06003$ & $70^{\circ} 05^{\prime N}$ & $53^{\circ} 21 \cdot \mathrm{y}$ & E \\
\hline & & 0003.58 & 360110 & $6930-03$ & 1FE06004 & $70^{\circ} 06^{\prime} \mathrm{N}$ & $53^{\circ} 21$, & $\mathbf{E}$ \\
\hline & & 0001.38 & 770010 & $6930-03$ & 1HE06005 & $70^{\circ} 07 \cdot \mathrm{N}$ & $53^{\circ} 20^{\prime} \mathrm{W}$ & NE \\
\hline & & 0003.08 & 363412 & $6930-03$ & 1HE06006 & $70^{\circ} 07 \cdot \mathrm{N}$ & $53^{\circ} 20^{\circ} \mathrm{W}$ & $\begin{array}{l}\mathrm{NE} \\
\mathrm{NE}\end{array}$ \\
\hline & & 0001.73 & 363412 & $6930-03$ & $1 \mathrm{HE} 07001$ & $70^{\circ} 07 \cdot \mathrm{N}$ & $53^{\circ} 22^{\prime} \mathrm{W}$ & $\mathrm{N}$ \\
\hline & & 0005.59 & 363411 & $6930-03$ & 1HE07002 & $70^{\circ} 08^{\prime} \mathrm{N}$ & $53^{\circ} 24^{\prime} \mathrm{W}$ & $\mathrm{NE}$ \\
\hline & & 0000.56 & 670110 & $6930-03$ & 1HE07003 & $70^{\circ} 09^{\prime} \mathrm{N}$ & $53^{\circ} 24^{\prime} \mathrm{W}$ & NE \\
\hline & & 0018.70 & 363311 & $6930-03$ & 1HE07004 & $70^{\circ} 09^{\circ} \mathrm{N}$ & $53^{\circ} 26^{\circ} \mathrm{W}$ & NE \\
\hline & & 0000.12 & 200010 & $6930-03$ & 1HE07005 & $70^{\circ} 09^{\prime} \mathrm{N}$ & $53^{\circ} 27 \% \mathrm{~W}$ & $\mathrm{NE}$ \\
\hline & & 0007.04 & 363012 & $6930-03$ & $1 \mathrm{HE} 08001$ & $70^{\circ} 11, \mathrm{~N}$ & $53^{\circ} 26^{\circ} \mathrm{W}$ & $\mathrm{NE}$ \\
\hline & & 0021.04 & 366211 & $6930-03$ & $1 \mathrm{HE} 08002$ & $70^{\circ} 11^{\prime N}$ & $53^{\circ} 28^{\prime} \mathrm{W}$ & $\mathrm{NE}$ \\
\hline & & 0013.49 & 366212 & $6930-03$ & 1 HE08003 & $70^{\circ} 11^{\prime} \mathrm{N}$ & $53^{\circ} 29 . \mathrm{W}$ & $\mathbf{N}$ \\
\hline & & 0001.04 & 770012 & $6930-03$ & 1 HE08004 & $70^{\circ} 10^{\prime} \mathrm{N}$ & $53^{\circ} 30^{\prime} \mathrm{W}$ & I \\
\hline & & 0009.75 & 363212 & $6930-02$ & 1HE08005 & $70^{\circ} 10^{\prime N} \mathrm{~N}$ & $53^{\circ} 32 \% \mathrm{k}$ & $\mathrm{N}$ \\
\hline & & 0008.58 & 363212 & $6930-02$ & 1HE08006 & $70^{\circ} 10^{\prime} \mathrm{N}$ & $53^{\circ} 34^{\prime} \mathrm{W}$ & $\mathrm{N}$ \\
\hline & & 0000.45 & 360010 & $6930-02$ & 1 HE08007 & $70^{\circ} 11^{\prime N}$ & $53^{\circ} 36^{\prime} \mathrm{V}$ & E \\
\hline & & 0000.22 & 360010 & $6930-02$ & $1 \mathrm{HE} 08008$ & $70^{\circ} 12^{\prime} \mathrm{N}$ & $53^{\circ} 38^{\circ} \mathrm{W}$ & $E$ \\
\hline & & 0006.41 & 360212 & $6930-02$ & 1HE08009 & $70^{\circ} 13^{\prime} \mathrm{N}$ & $53^{\circ} 36^{\prime} \mathrm{W}$ & N \\
\hline & & 0000.84 & 363312 & $6930-02$ & 1HE08010 & $70^{\circ} 13^{\prime} \mathrm{N}$ & $53^{\circ} 41^{\prime} \mathrm{W}$ & $\mathrm{N}$ \\
\hline & & 0000.11 & 780010 & $6930-03$ & $1 \mathrm{HE} 09001$ & $70^{\circ} 12, \mathrm{~N}$ & $53^{\circ} 40^{\prime} \mathrm{W}$ & n \\
\hline 130 & 110 & 0001.62 & 780010 & $6930-03$ & 1 HE 09002 & $70^{\circ} 10^{\prime} \mathrm{N}$ & $53^{\circ} 36^{\prime} \mathrm{W}$ & NW \\
\hline 93 & 95 & 0000.24 & 780010 & $6930-03$ & $1 \mathrm{HE} 09003$ & $70^{\circ} 10^{\prime} \mathrm{N}$ & $53^{\circ} 40^{\circ} \mathrm{W}$ & $\mathrm{N}$ \\
\hline 115 & 100 & 0000.24 & 700010 & $6930-03$ & 1HE09004 & $70^{\circ} 10^{\prime N}$ & $53^{\circ} 42^{\prime} \mathrm{W}$ & $\mathrm{N}$ \\
\hline \multirow{3}{*}{$\begin{array}{r}95 \\
100\end{array}$} & 90 & 0000.07 & 780010 & $6930-03$ & 1BE09005 & $70^{\circ} 10^{\prime} \mathrm{N}$ & $53^{\circ} 44^{\prime} \mathrm{W}$ & $\mathrm{N}$ \\
\hline & 75 & 0000.17 & 780010 & $6930-03$ & $1 \mathrm{HE} 09006$ & $70^{\circ} 11 \cdot \mathrm{N}$ & $53^{\circ} 47 \cdot \mathrm{W}$ & \\
\hline & 70 & 0000.43 & 790012 & $6930-03$ & 1 HE 090007 & $70^{\circ} 09^{\prime} \mathrm{N}$ & $53^{\circ} 43, \mathrm{~W}$ & SE \\
\hline 110 & 100 & 0000.42 & 700010 & $6930-03$ & 1HE09008 & $70^{\circ} 08^{\circ} \mathrm{N}$ & $53^{\circ} 36^{\prime} \mathrm{W}$ & \\
\hline 100 & 85 & 0000.30 & 790012 & $6930-03$ & 1HE09009 & $70^{\circ} 05^{\prime N}$ & $53^{\circ} 31, \mathrm{~W}$ & $\overline{\mathrm{NE}}$ \\
\hline 90 & 85 & 0000.15 & 790012 & $6930-03$ & $1 \mathrm{HE} 09010$ & $70^{\circ} 06^{\prime} \mathrm{N}$ & $53^{\circ} 32^{\prime} \mathrm{W}$ & \\
\hline 126 & 100 & 0000.69 & 780010 & $6930-03$ & $1 \mathrm{HE} 09011$ & $70^{\circ} 06^{\prime} \mathrm{N}$ & $53^{\circ} 32^{\prime} \mathrm{W}$ & $\vec{E}$ \\
\hline 100 & 90 & 0000.23 & 770010 & $6930-03$ & 1 HE09012 & $70^{\circ} 07 \cdot \mathrm{N}$ & $53^{\circ} 33^{\prime} \mathrm{W}$ & E \\
\hline 110 & 70 & 0000.98 & 643312 & $6930-03$ & 1 HE 09013 & $70^{\circ} 08^{\prime} \mathrm{N}$ & $53^{\circ} 35^{\prime} \mathrm{W}$ & E \\
\hline 90 & 80 & 0000.07 & 780012 & $6930-03$ & 1 HE0 09014 & $70^{\circ} 08^{\prime} \mathrm{N}$ & $53^{\circ} 33^{\circ}$, & $\mathrm{SE}$ \\
\hline 115 & 80 & 0001.79 & 673012 & $6930-03$ & 1HE09015 & $70^{\circ} 09^{\prime} \mathrm{N}$ & $53^{\circ} 29 \cdot \mathrm{W}$ & $\mathrm{SW}$ \\
\hline 115 & 90 & 0001.34 & 770010 & $6930-03$ & 1 HE 09016 & $70^{\circ} 08^{\circ} \mathrm{N}$ & $53^{\circ} 26^{\prime} \mathrm{k}$ & SE \\
\hline 75 & 70 & 0000.16 & 780010 & $6930-03$ & $1 \mathrm{HE} 09017$ & $70^{\circ} 06^{\circ} \mathrm{N}$ & $53025, y$ & $\mathrm{~B}$ \\
\hline & 80 & 0000.85 & 773010 & $6930-03$ & 1HE09018 & $70^{\circ} 05 \cdot \mathrm{N}$ & $53^{\circ} 24^{\prime} \mathrm{V}$ & $\ddot{v}$ \\
\hline \multirow{3}{*}{$\begin{array}{r}100 \\
75\end{array}$} & 65 & 0000.13 & 780010 & $6930-03$ & $1 \mathrm{HE} 09019$ & $70^{\circ} 04$, N & $53^{\circ} 23^{\prime} \mathrm{V}$ & W \\
\hline & & 0001.29 & 670112 & $6930-03$ & 1HE09020 & $70^{\circ} 03 \cdot \mathrm{N}$ & $53^{\circ} 23^{\prime} \mathrm{W}$ & $w$ \\
\hline & & 0000.30 & 773112 & $6930-03$ & 1HE09021 & $70^{\circ} 02$ ' N & $53^{\circ} 22^{\prime} \mathrm{W}$ & w \\
\hline
\end{tabular}




\begin{tabular}{|c|c|c|c|c|c|c|c|c|c|c|c|c|c|c|c|c|c|}
\hline CODE & LAT & LONG & ORI & HIGH & LOW & AREA & $\begin{array}{c}\text { MO } \\
\text { CODE }\end{array}$ & MAP & CODE & LAT & LONG & ORI & HIGH & LOW & AREA & $\begin{array}{c}\text { Mo } \\
\text { CODE }\end{array}$ & MAP \\
\hline E09022 & $70^{\circ} 00^{\prime} \mathrm{N}$ & $53^{\circ} 16^{\prime} \mathrm{W}$ & W & 166 & 135 & 0007.10 & 263111 & $7000-02$ & 1HE09083 & $70^{\circ} 01^{\prime} \mathrm{N}$ & $53^{\circ} 49^{\prime} \mathrm{W}$ & $\mathrm{N}$ & 150 & 75 & 0004.19 & 513112 & $7000-02$ \\
\hline 1 HE09023 & $70^{\circ} 00^{\prime} \mathrm{N}$ & $53^{\circ} 20^{\prime \prime}$ & W & 145 & 120 & 0000.51 & 643112 & $7000-02$ & 1HEO 09084 & $70^{\circ} 02, \mathrm{~N}$ & $53^{\circ} 50^{\prime} \mathrm{W}$ & $\mathrm{E}$ & 145 & 120 & 0000.37 & 780010 & $7000-02$ \\
\hline $1 \mathrm{HE} 09024$ & $69^{\circ} 59^{\prime} \mathrm{N}$ & $53^{\circ} 17^{\prime} \mathrm{K}$ & $\mathrm{SW}$ & & & 0001.30 & 260010 & $6930-02$ & 1HE09085 & $70^{\circ} 02 \cdot \mathrm{N}$ & $53^{\circ} 50^{\circ} \mathrm{W}$ & $\mathrm{NE}$ & 140 & 110 & 0000.46 & 643112 & $7000-02$ \\
\hline $1 \mathrm{HE0} 9025$ & $69^{\circ} 59^{\prime} \mathrm{N}$ & $53^{\circ} 14^{\prime} \mathrm{W}$ & $\mathrm{SW}$ & & & 0003.29 & 263112 & $6930-02$ & 1 HE09086 & $70^{\circ} 02^{\prime} \mathrm{N}$ & $53^{\circ} 50^{\prime} \mathrm{W}$ & - & 155 & 145 & 0000.17 & 700010 & $7000-02$ \\
\hline $1 \mathrm{HE} 09026$ & $69^{\circ} 58^{\prime} \mathrm{N}$ & $53^{\circ} 13^{\prime} \mathrm{W}$ & - & & & 0000.27 & 300010 & $6930-02$ & 1 HE09087 & $70^{\circ} 03 \cdot \mathrm{N}$ & $53050 \%$ & $\bar{N}$ & 110 & 95 & 0000.06 & 640112 & $7000-02$ \\
\hline 1HE09027 & $69^{\circ} 58^{\prime} \mathrm{N}$ & $53^{\circ} 12^{\prime} \mathrm{V}$ & $\mathrm{SE}$ & & & 0000.41 & 643112 & $6930-02$ & 1HE09088 & $70^{\circ} 03^{\prime} \mathrm{N}$ & $53^{\circ} 50 \% \mathrm{w}$ & $\mathrm{N}$ & 95 & 75 & 0000.38 & 643112 & $7000-02$ \\
\hline 1HE09028 & $69059 \cdot \mathrm{N}$ & $53^{\circ} 11^{\prime} \mathrm{W}$ & $\mathrm{SE}$ & & & 0002.57 & 260010 & $6930-02$ & 1HE09089 & $70^{\circ} 03^{\prime} \mathrm{N}$ & $53^{\circ} 54^{\prime} \mathrm{W}$ & $\mathrm{N}$ & 125 & 75 & 0003.22 & 613112 & $7000-02$ \\
\hline $1 \mathrm{HE} 09029$ & $69^{\circ} 59^{\prime} \mathrm{N}$ & $53^{\circ} 10^{\prime} \mathrm{W}$ & $\mathrm{SW}$ & & & 0000.08 & 770010 & $6930-02$ & 1 HE 09090 & $70^{\circ} 03^{\prime} \mathrm{N}$ & $53057 \% \mathrm{k}$ & NE & 125 & 75 & 0007.16 & 203116 & $7000-02$ \\
\hline 1HE09030 & $69^{\circ} 58^{\prime} \mathrm{N}$ & $53^{\circ} 09^{\prime} \mathrm{W}$ & $\mathrm{s}$ & & & 0000.37 & 260010 & $6930-02$ & 1HE09091 & $70^{\circ} 04^{\prime} \mathrm{N}$ & $53^{\circ} 56^{\prime} \mathrm{k}$ & $\mathrm{NE}$ & & 105 & 0000.59 & 643112 & $7000-02$ \\
\hline 1HE09031 & $69^{\circ} 59^{\prime} \mathrm{N}$ & $53^{\circ} 08^{\prime} \mathrm{W}$ & $\mathrm{SE}$ & & & 0000.86 & 263112 & $6930-02$ & $1 \mathrm{HE} 09092$ & $70^{\circ} 04^{\prime} \mathrm{N}$ & $53056, \mathrm{k}$ & $E$ & & 100 & 0000.19 & 643112 & $7000-02$ \\
\hline 1HE09032 & $69^{\circ} 59^{\prime} \mathrm{N}$ & $53^{\circ} 03^{\prime} \mathrm{W}$ & - & & & 0000.77 & 260010 & $6930-02$ & 1HE09093 & $70^{\circ} 05^{\prime} \mathrm{N}$ & $53^{\circ} 55, \mathrm{k}$ & $\mathrm{E}$ & 1.25 & 90 & 0000.74 & 643112 & $7000-02$ \\
\hline 1HE09033 & $6905 \mathrm{~B}^{\prime} \mathrm{N}$ & $53^{\circ} 00^{\prime \prime} \mathrm{W}$ & $\mathrm{SW}$ & & & 0001.54 & 263112 & $6930-02$ & 1HE09094 & $70^{\circ} 05^{\prime} \mathrm{N}$ & $53057 \%$ & $\mathrm{~N}$ & 110 & 90 & 0000.33 & 643116 & $7000-01$ \\
\hline 1HE09034 & $69^{\circ} 55^{\prime} \mathrm{N}$ & $53^{\circ} 02^{\prime \prime \mathrm{W}}$ & $\mathrm{N}$ & & & 0011.34 & 363012 & $6930-02$ & 1HE09095 & $70^{\circ} 05 \cdot \mathrm{N}$ & $54^{\circ} 00^{\circ}$ & $\mathrm{E}$ & 135 & 80 & 0003.82 & 513116 & $7000-01$ \\
\hline 1HE09035 & $69^{\circ} 55^{\prime} \mathrm{N}$ & $53^{\circ} 03^{\prime \prime}$ & - & & & 0000.47 & 780010 & 6930-02 & 1HE09096 & $70^{\circ} 08^{\prime} \mathrm{N}$ & $53^{\circ} 53^{\prime}, \mathrm{V}$ & $\mathrm{N}$ & 105 & 90 & 0000.06 & 513112 & $7000-02$ \\
\hline $1 \mathrm{HE} 09036$ & $69^{\circ} 55^{\prime} \mathrm{N}$ & $53^{\circ} 05^{\circ}, \mathrm{k}$ & $\vec{N}$ & & & 0005.15 & 363112 & $6930-02$ & $1 \mathrm{HE} 09097$ & $70^{\circ} 07^{\prime N}$ & 53053.4 & $\mathrm{Ny}$ & 110 & 90 & 0000.00 & 640112 & $7000-02$ \\
\hline 1HE09037 & $69^{\circ} 55^{\prime} \mathrm{N}$ & $53^{\circ} 06^{\prime} \mathrm{K}$ & $\mathrm{NE}$ & & & 0000.32 & 770010 & 6930-02 & 1HE09098 & $70^{\circ} 07 \cdot \mathrm{N}$ & $53^{\circ} 55 \% \mathrm{~W}$ & $\mathrm{~N}$ & $\begin{array}{l}120 \\
120\end{array}$ & 90 & 0000.50 & 670112 & $7000-02$ \\
\hline 1HE09038 & $69 \circ 56 \cdot \mathrm{N}$ & $53^{\circ} 07 \cdot n$ & $\mathrm{~N}$ & & & 0000.13 & 770010 & $\begin{array}{l}6730-02 \\
69302\end{array}$ & 1HE09099 & $70^{\circ} 06^{\prime} \mathrm{N}$ & $53^{\circ} 57 . \mathrm{K}$ & $\mathrm{NE}$ & 150 & 90 & 0001.11 & 670112 & $7000-01$ \\
\hline 1HE09039 & $69^{\circ} 56^{\prime} \mathrm{N}$ & $53^{\circ} 08^{\prime} \mathrm{V}$ & $\mathrm{NW}$ & & & 0001.88 & 643112 & $6930-02$ & 1HE09100 & $70^{\circ} 07{ }^{\circ} \mathrm{N}$ & $53^{\circ} 58^{\prime} \mathrm{k}$ & $\mathrm{E}$ & 150 & 110 & 0000.79 & 640110 & $7000-01$ \\
\hline $1 \mathrm{HE} 09040$ & $69^{\circ} 56^{\prime} \mathrm{N}$ & $53^{\circ} 10^{\prime} \mathrm{W}$ & NW & & & 0000.32 & 643112 & $6930-02$ & 1HE09101 & $70^{\circ} 08^{\prime} \mathrm{N}$ & $53^{\circ} 58, \mathrm{k}$ & $\mathrm{NE}$ & 145 & 105 & 0000.75 & 643112 & $7000-01$ \\
\hline $1 \mathrm{HE} 09041$ & $69^{\circ} 56^{\prime} \mathrm{N}$ & $53^{\circ} 11^{\prime} \mathrm{V}$ & $\mathrm{NW}$ & & & 0000.16 & 643112 & $\begin{array}{l}0930-02 \\
6930-02\end{array}$ & 1HE09101 & $70^{\circ} 09^{\prime} \mathrm{N}$ & $53059 \% \mathrm{v}$ & NE & $\begin{array}{l}145 \\
130\end{array}$ & 105 & 0000.42 & $\begin{array}{l}643112 \\
300010\end{array}$ & $7000-01$ \\
\hline 1HE09042 & $69^{\circ} 55^{\prime} \mathrm{N}$ & $53^{\circ} 10^{\prime \prime} \mathrm{W}$ & $\mathrm{NW}$ & & & 0002.50 & 363142 & 6930-02 & 1HE09103 & $70^{\circ} 08^{\prime} \mathrm{N}$ & $54^{\circ} 00^{\circ} \mathrm{v}$ & $\overrightarrow{\mathrm{N}}$ & 120 & 90 & 0000.91 & 643112 & $7000-01$ \\
\hline $1 \mathrm{HE} 09043$ & $69^{\circ} 54^{\prime} \mathrm{N}$ & $53^{\circ} 10^{\prime} \mathrm{W}$ & SW & & & 0004.78 & 360010 & $6930-02$ & 1HE09104 & $70^{\circ} 08^{\prime} \mathrm{N}$ & $54^{\circ} 01^{\prime} \mathrm{v}$ & NW & 115 & 95 & 0000.28 & $\begin{array}{l}643112 \\
643112\end{array}$ & $7000-01$ \\
\hline $1 \mathrm{HE} 09044$ & $69^{\circ} 53^{\prime} \mathrm{N}$ & $53^{\circ} 15^{\prime} \mathrm{W}$ & $\mathrm{N}$ & & & 0016.56 & 363112 & $6930-02$ & 1HE09105 & $70^{\circ} 07^{\prime} \mathbf{N}$ & $54^{\circ} 00^{\prime} \mathrm{w}$ & NW & 130 & 90 & 0001.35 & 643112 & $7000-01$ \\
\hline 1HE09045 & $69^{\circ} 55^{\prime} \mathrm{N}$ & $53^{\circ} 19^{\prime \prime}$ & $\mathrm{E}$ & & & 0002.34 & 643112 & $6930-02$ & 1 HE09106 & $70^{\circ} 071 \mathrm{~N}$ & $54^{\circ} 03^{\prime \prime} \mathrm{V}$ & $\mathrm{N}$ & 100 & 75 & 0000.56 & 643112 & $7000-01$ \\
\hline 1HE09046 & $69^{\circ} 56^{\prime} \mathrm{N}$ & $53^{\circ} 19^{\prime} \mathrm{W}$ & $\mathrm{NE}$ & & & 0001.19 & 640112 & $\begin{array}{l}0930-02 \\
6930-02\end{array}$ & 1HE09106 & $70^{\circ} 06^{\prime} \mathrm{N}$ & $54^{\circ} 04^{\prime} \mathrm{W}$ & $\mathrm{N}$ & 100 & 10 & 0000.21 & $\begin{array}{l}643112 \\
700010\end{array}$ & $7000-01$ \\
\hline 1 HE 09047 & $69^{\circ} 55^{\prime} \mathrm{N}$ & $53^{\circ} 21^{\prime} \mathrm{k}$ & $\mathrm{N}$ & & & 0000.26 & 780010 & $6930-02$ & $1 \mathrm{HE} 09108$ & $70^{\circ} 06^{\prime} \mathrm{N}$ & $54^{\circ} 066^{\prime} \mathrm{W}$ & $\bar{N}$ & 140 & 70 & 0003.51 & 513111 & $7000-01$ \\
\hline 1HE09048 & $69^{\circ} 56^{\prime N}$ & $53^{\circ} 18^{\prime} \mathrm{W}$ & $\mathbf{N}$ & & & 0000.23 & 780010 & $6930-02$ & 1HE09109 & $70^{\circ} 07 \cdot \mathrm{N}$ & $54^{\circ} 08^{\circ} \mathrm{W}$ & $\mathrm{NE}$ & 120 & 90 & 0000.48 & 643112 & $7000-01$ \\
\hline 1HE09049 & $69^{\circ} 56^{\prime} \mathrm{N}$ & $53^{\circ} 20^{\prime} \mathrm{k}$ & N & & & 0000.43 & 643112 & $6930-02$ & 1HE09110 & $70^{\circ} 09^{\prime} \mathrm{N}$ & $54^{\circ} 06^{\circ} \mathrm{W}$ & $\mathrm{NE}$ & 120 & 80 & 0000.61 & 643112 & $7000-01$ \\
\hline 1HE09050 & $69^{\circ} 56^{\prime} \mathrm{N}$ & $53^{\circ} 22^{\prime} \mathrm{k}$ & $\mathrm{NE}$ & & & 0003.40 & 363112 & $6930-02$ & $1 \mathrm{HEO} 0111$ & $70^{\circ} 10^{\prime} \mathrm{N}$ & $54^{\circ} 08^{\prime} \mathrm{w}$ & NE & 95 & 90 & 0000.18 & 643112 & $7000-01$ \\
\hline 1HE09051 & $69057, \mathrm{~N}$ & $53^{\circ} 28^{\prime} \mathrm{W}$ & E & & & 0010.51 & 213116 & $\begin{array}{l}0930-02 \\
6930-02\end{array}$ & 1HEO9111 & $70^{\circ} 10^{\circ} \mathrm{N}$ & $54^{\circ} 09^{\prime} \mathrm{v}$ & $\begin{array}{l}\mathrm{NE} \\
\mathrm{E}\end{array}$ & $\begin{array}{r}95 \\
120\end{array}$ & $\begin{array}{r}90 \\
105\end{array}$ & 0000.18 & $\begin{array}{l}643112 \\
643112\end{array}$ & $7000-01$ \\
\hline 1HE09052 & $69^{\circ} 58^{\prime} \mathrm{N}$ & $53^{\circ} 24^{\prime \prime} \mathrm{h}$ & $\mathrm{NE}$ & & & 0000.30 & 780010 & 6930-02 & $1 \mathrm{HE} 09113$ & $70^{\circ} 10^{\prime} \mathrm{N}$ & $54^{\circ} 09^{\circ}$ ' & $\mathrm{E}$ & 125 & 105 & 0000.44 & 643112 & $7000-01$ \\
\hline $1 \mathrm{HE} 09053$ & $69^{\circ} 58^{\prime} \mathrm{N}$ & $53^{\circ} 25^{\prime \prime}$ & $\mathrm{NE}$ & & & 0000.84 & 643112 & $\begin{array}{l}9730-02 \\
6930-02\end{array}$ & 1HE09114 & $70^{\circ} 11 \cdot \mathrm{N}$ & $54^{\circ} 06^{\prime} \mathrm{W}$ & $\mathrm{SE}$ & 145 & 110 & 0000.24 & 643112 & $7000-01$ \\
\hline $1 \mathrm{HE} 09054$ & $69^{\circ} 58^{\prime} \mathrm{N}$ & $53^{\circ} 25^{\prime \prime}$ & $\mathrm{NE}$ & & & 0000.38 & 300010 & 6930-02 & 1HE09115 & $70^{\circ} 11 \cdot \mathrm{N}$ & $54^{\circ} 06^{\circ} \mathrm{W}$ & $\mathrm{E}$ & 110 & 85 & 0000.34 & 670112 & $7000-01$ \\
\hline $1 \mathrm{HE} 09055$ & $69 \circ 57 \cdot \mathrm{N}$ & $53^{\circ} 27, \bar{v}$ & - & & & 0000.12 & 260010 & $6930-02$ & $1 \mathrm{HE} 09116$ & $70^{\circ} 11$, N & $54^{\circ} 07^{\circ} \mathrm{W}$ & $\mathrm{E}$ & 140 & 80 & 0000.97 & 643112 & $7000-01$ \\
\hline 1HE09056 & $69^{\circ} 58^{\prime} \mathrm{N}$ & $53^{\circ} 25^{\prime} \mathrm{V}$ & $\overline{\mathrm{N}}$ & & & 0001.23 & 543112 & 6930 & $\begin{array}{l}\text { 1HEO9116 } \\
\text { 1HE09117 }\end{array}$ & $70^{\circ} 11$, N & $54^{\circ} 077 \mathrm{~W}$ & ${ }_{\mathrm{E}}^{\mathrm{E}}$ & $\begin{array}{l}140 \\
140\end{array}$ & $\begin{array}{l}80 \\
95\end{array}$ & $\begin{array}{l}000.91 \\
0000.82\end{array}$ & $\begin{array}{l}643112 \\
643112\end{array}$ & $7000-01$ \\
\hline $1 \mathrm{HE} 09057$ & $69^{\circ} 59^{\prime} \mathrm{N}$ & $53^{\circ} 26^{\prime \prime}$ & $\mathrm{NE}$ & & & 0000.29 & 640110 & $6930-02$ & 1 1 & $70^{\circ} 12^{\prime} \mathrm{N}$ & $54^{\circ} 07 \mathrm{7}$ & 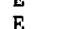 & 140 & 95 & 0001.02 & 643112 & $7000-01$ \\
\hline 1 HE0 09058 & $69^{\circ} 59^{\prime} \mathrm{N}$ & $53^{\circ} 27^{\prime} \mathrm{W}$ & $\mathrm{NE}$ & & & 0000.76 & 260010 & $6930-02$ & 1HEOY118 & $70^{\circ} 14^{\prime} \mathrm{N}$ & $54^{\circ} 03^{\prime} \mathrm{W}$ & N & 170 & 70 & 0001.52 & $\begin{array}{l}043112 \\
513112\end{array}$ & $7000-01$ \\
\hline 1HE09059 & $69^{\circ} 58^{\prime} \mathrm{N}$ & $53^{\circ} 29^{\prime}, \mathrm{W}$ & $\mathrm{N}$ & & & 0006.63 & 263111 & $6930-02$ & $1 \mathrm{HE} 09120$ & $70^{\circ} 17 \cdot \mathrm{N}$ & $54^{\circ} 04^{\prime} \mathrm{V}$ & NW & 150 & 100 & 0000.27 & 780010 & $7000-01$ \\
\hline $1 \mathrm{HE} 09060$ & $70^{\circ} 00^{\prime} \mathrm{N}$ & $53^{\circ} 26^{\prime} \mathrm{W}$ & $\mathrm{NE}$ & & 100 & 0000.25 & 260010 & $7000-02$ & $1 \mathrm{HEO} 0121$ & $70^{\circ} 16^{\prime} \mathrm{N}$ & $54^{\circ} 06^{\prime \prime}$ & $\mathrm{N}$ & 168 & 110 & 0002.22 & 200010 & $7000-01$ \\
\hline 1HE09061 & $70^{\circ} 00^{\prime} \mathrm{N}$ & $53^{\circ} 31^{\prime} \mathrm{W}$ & NW & & & 0002.97 & 263112 & $6930-02$ & $\begin{array}{l}\text { 1HEO9121 } \\
\text { 1HE09122 }\end{array}$ & $70^{\circ} 16^{\circ} \mathrm{N}$ & $54^{\circ} 08^{\prime}, \mathrm{k}$ & NW & $\begin{array}{l}168 \\
110\end{array}$ & $\begin{array}{r}110 \\
80\end{array}$ & 0000.34 & $\begin{array}{l}200010 \\
770010\end{array}$ & $7000-01$ \\
\hline $1 \mathrm{HE} 09062$ & $69^{\circ} 59^{\prime} \mathrm{N}$ & $53^{\circ} 36^{\prime} \mathrm{W}$ & $\mathbf{N}$ & & & 0011.89 & 513111 & 6930-02 & 1HE10001 & $70^{\circ} 15^{\prime} \mathrm{N}$ & $54^{\circ} 08^{\prime}, 6$ & $\mathrm{NW}$ & 140 & 70 & 0006.71 & 513111 & $7000-01$ \\
\hline 1HE09063 & $70^{\circ} 01 \cdot \mathrm{N}$ & $53^{\circ} 35^{\prime \prime}$ & $\mathrm{SE}$ & 130 & 125 & 0000.15 & 770010 & $7000-02$ & 1HE10002 & $70^{\circ} 15^{\prime} \mathrm{N}$ & $54^{\circ} 11^{\prime}, \mathrm{v}$ & $\mathrm{NW}$ & $\begin{array}{l}140 \\
180\end{array}$ & 70 & 0005.38 & 533111 & $7000-01$ \\
\hline 1HE09064 & $70^{\circ} 01 \cdot \mathrm{N}$ & $53^{\circ} 34^{\prime} \mathrm{W}$ & $\mathrm{NE}$ & 140 & 140 & 0000.18 & 700010 & 7000 & 1HE10003 & $70^{\circ} 14^{\prime} \mathrm{N}$ & $54^{\circ} 11^{\prime}, \mathrm{k}$ & w & $\begin{array}{l}180 \\
180\end{array}$ & 130 & 0001.82 & 260010 & $7000-01$ \\
\hline 1HE09065 & $70^{\circ} 02^{\prime} \mathrm{N}$ & $53^{\circ} 35^{\prime \prime} \mathrm{W}$ & $\mathrm{N}$ & 160 & 90 & 0001.72 & 643112 & $7000-02$ & 1HE10004 & $70^{\circ} 13, \mathrm{~N}$ & $54^{\circ} 10^{\prime}$ & 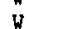 & 180 & 135 & 000180 & 260010 & $7000-01$ \\
\hline 1HE09066 & $70^{\circ} 02^{\prime} \mathrm{N}$ & $53^{\circ} 37, \mathrm{v}$ & $\mathrm{NE}$ & 145 & 95 & 0000.98 & 643112 & $7000-02$ & 1HE10005 & $70^{\circ} 13^{\prime} \mathrm{N}$ & $54^{\circ} 12^{\circ} \mathrm{k}$ & NW & 150 & 90 & 0003.24 & 533112 & $7000-01$ \\
\hline 1HE09067 & $70^{\circ} 03, \mathrm{~N}$ & $53^{\circ} 38^{\prime \prime}$ & $\mathrm{NE}$ & 135 & 90 & 0000.69 & 643112 & $7000-02$ & $1 \mathrm{HE} 10006$ & $70^{\circ} 12^{\prime} \mathrm{N}$ & $54^{\circ} 12^{\prime} \mathrm{v}$ & W & 135 & 90 & 0000.47 & 780010 & $7000-01$ \\
\hline 1 HE09068 & $70^{\circ} 03^{\prime} \mathrm{N}$ & $53^{\circ} 39^{\prime}, 0$ & $\begin{array}{l}\text { NSE } \\
\text { NE }\end{array}$ & $\begin{array}{l}130 \\
130\end{array}$ & 90 & 0000.07 & 643112 & $7000-02$ & 1HE10007 & $70^{\circ} 12^{\prime} \mathrm{N}$ & $54^{\circ} 15^{\prime \prime}$ & $\mathrm{N}$ & 190 & 35 & 0005.19 & 533112 & $7000-01$ \\
\hline 1 HE09069 & $70^{\circ} 04^{\prime} \mathrm{N}$ & $53^{\circ} 41^{\prime} \mathrm{w}$ & $\mathrm{N}$ & 130 & 90 & 0000.85 & 643112 & $7000-02$ & 1HE11001 & $70^{\circ} 13^{\prime} \mathrm{N}$ & $54^{\circ} 17 \% \mathrm{~W}$ & $\mathrm{NW}$ & 140 & 100 & 0001.36 & 200012 & $7000-01$ \\
\hline IHE09070 & $70^{\circ} 04^{\prime} \mathrm{N}$ & $53^{\circ} 42^{\prime} \mathrm{W}$ & $\mathrm{N}$ & 150 & 130 & 0000.43 & 360010 & $7000-02$ & 1HE11002 & $70^{\circ} 12^{\prime} \mathrm{N}$ & $54^{\circ} 17^{\prime} \mathrm{W}$ & NW & $\begin{array}{l}140 \\
150\end{array}$ & $\begin{array}{r}100 \\
95\end{array}$ & $\begin{array}{l}0001.36 \\
0001.85\end{array}$ & $\begin{array}{l}200012 \\
700010\end{array}$ & $\begin{array}{l}7000-01 \\
7000-01\end{array}$ \\
\hline 1HE09071 & $70^{\circ} 04^{\prime} \mathrm{N}$ & $53^{\circ} 44^{\prime} \mathrm{W}$ & $\mathrm{N}$ & 130 & 100 & 0000.53 & 643112 & 7000 & $1 \mathrm{HE}$ & $70^{\circ} 11$ ' N & $54^{\circ} 14^{\prime}$ & SW & 150 & 135 & 0000.43 & 700010 & $7000-01$ \\
\hline 1HE09072 & $70^{\circ} 05^{\prime} \mathrm{N}$ & $53^{\circ} 46^{\prime \prime} \mathrm{W}$ & $\mathrm{N}$ & 110 & $\begin{array}{r}100 \\
90\end{array}$ & 0000.15 & 643112 & 7000 & IHE11004 & $70^{\circ} 11$, N & $54^{\circ} 14^{\prime} \mathrm{W}$ & - & $\begin{array}{l}120 \\
163\end{array}$ & $\begin{array}{l}133 \\
135\end{array}$ & 0000.87 & 700010 & $7000-01$ \\
\hline 1HE09073 & $70^{\circ} 04^{\prime} \mathrm{N}$ & $53^{\circ} 44^{\prime} \mathrm{W}$ & $\mathrm{W}$ & 150 & 100 & 0000.43 & 360010 & $7000-02$ & 1HE11005 & $70^{\circ} 11 \cdot \mathrm{N}$ & $54^{\circ} 11$, w & - & 180 & 155 & 0000.89 & 300010 & $7000-01$ \\
\hline $1 \mathrm{HE} 09074$ & $70^{\circ} 03^{\prime} \mathrm{N}$ & $53^{\circ} 43^{\prime \prime}$ & $\mathrm{SE}$ & 140 & 110 & 0000.45 & 360010 & $7000-02$ & 1HE11006 & $70^{\circ} 11 \cdot \mathrm{N}$ & $54 \circ 12,8$ & $\bar{s}$ & 150 & 140 & 0000.10 & 700010 & $7000-01$ \\
\hline 1HE09075 & $70^{\circ} 03^{\prime} \mathrm{N}$ & $53^{\circ} 40^{\prime} \mathrm{W}$ & SW & 171 & 140 & 0000.34 & 700010 & $7000-02$ & 1HE11007 & $70^{\circ} 10^{\prime} \mathrm{N}$ & 54011,6 & $\vec{v}$ & 180 & 65 & 00 & 643112 & $7000-01$ \\
\hline $1 \mathrm{HE} 09076$ & $70^{\circ} 02, \mathrm{~N}$ & $53^{\circ} 38^{\prime} \mathrm{V}$ & $\mathrm{SW}$ & $\begin{array}{l}171 \\
150\end{array}$ & 115 & 0000.54 & 643112 & $7000-02$ & 1HE11008 & $70^{\circ} 09^{\prime} \mathrm{N}$ & $54^{\circ} 10^{\prime} \mathrm{v}$ & v & $\begin{array}{l}180 \\
140\end{array}$ & 110 & 0000.75 & 643112 & $7000-01$ \\
\hline 1HE09077 & $70^{\circ} 01 \cdot \mathrm{N}$ & $53^{\circ} 38^{\prime \prime} \mathrm{k}$ & $\mathrm{SW}$ & 145 & 135 & 0000.14 & 780010 & $7000-02$ & 1HE11009 & $70^{\circ} 09^{\prime} \mathrm{N}$ & $54^{\circ} 09^{\prime}$, & H & 145 & 90 & 0002.38 & 613112 & $7000-01$ \\
\hline $1 \mathrm{HE} 09078$ & $70^{\circ} 00^{\prime} \mathrm{N}$ & $53^{\circ} 40^{\prime \prime} \mathrm{W}$ & $\mathrm{NW}$ & & 145 & 0016.88 & 213111 & $7000-02$ & 1HE11010 & $70^{\circ} 09^{\prime} \mathrm{N}$ & $54^{\circ} 10^{\prime}, \mathrm{v}$ & $\mathrm{SW}$ & 120 & 100 & 0000.29 & 700010 & $7000-01$ \\
\hline 1HE09079 & $70^{\circ} 00^{\prime} \mathrm{N}$ & $53^{\circ} 39^{\prime \prime} \mathrm{V}$ & - & & 145 & 0001.12 & 200010 & $6930-02$ & 1HE11011 & $70^{\circ} 08^{\prime} \mathrm{N}$ & $54^{\circ} 10^{\prime} \mathrm{k}$ & NW & $\begin{array}{l}120 \\
140\end{array}$ & 100 & 0000.53 & 643112 & $7000-01$ \\
\hline 1HE09080 & $70^{\circ} 01$ ' N & $53^{\circ} 43^{\prime} \mathrm{V}$ & - & 155 & 145 & 0000.32 & 780010 & $7000-02$ & 1HE11012 & $70^{\circ} 07 \times \mathrm{N}$ & $54^{\circ} 09^{\prime} \mathrm{v}$ & NW & $\begin{array}{l}140 \\
130\end{array}$ & 110 & 0000.26 & 643112 & $7000-01$ \\
\hline 1HE09081 & $70^{\circ} 01 \cdot \mathrm{N}$ & $53^{\circ} 45,6$ & $\overline{\mathrm{NE}}$ & 150 & 85 & 0002.56 & 643112 & 7000 & 1HE11013 & $70^{\circ} 07 \cdot \mathrm{N}$ & $54^{\circ} 12^{\prime} \mathrm{k}$ & $\mathrm{NV}$ & $\begin{array}{l}130 \\
125\end{array}$ & $\begin{array}{l}1100 \\
70\end{array}$ & 0007.265 & $\begin{array}{l}043112 \\
513212\end{array}$ & $7000-01$ \\
\hline 1HE09082 & $70^{\circ} 02 \cdot \mathrm{N}$ & $53^{\circ} 46^{\prime} \mathrm{V}$ & $\mathrm{N}$ & 145 & 105 & 0000.41 & 200010 & $7000-02$ & 1HE11014 & איריס & $54^{\circ} 13^{\prime} \mathrm{k}$ & NWW & 150 & 140 & 0000.18 & 700010 & $7000-01$ \\
\hline
\end{tabular}


$\stackrel{\text { MO }}{\text { CODE }}$ MAP

CODE

LAT

LONG

ORI

1IA02051

$70^{\circ} 42$ ' N

$53^{\circ} 12^{\prime} \mathrm{W}$

1 HE11016

$\begin{array}{lll}70^{\circ} 10^{\prime} \mathrm{N} & 54^{\circ} 21^{\prime} \mathrm{W} & \mathrm{NE} \\ 70^{\circ} 09^{\prime} \mathrm{N} & 54^{\circ} 23^{\prime} \mathrm{W} & \mathrm{NW}\end{array}$

1 HE11018

$54^{\circ} 30^{\prime} \mathrm{W} N$

1 IA01002

$70^{\circ} 36^{\prime} \mathrm{N} 53^{\circ} 47^{\circ} \mathrm{W}$

1IA01003

$70^{\circ} 36^{\prime} \mathrm{N} \quad 53^{\circ} 49^{\prime} \mathrm{W}$ NW

$53^{\circ} 49^{\prime} \mathrm{W} \mathrm{NW}$

1 IA01006

$70^{\circ} 34^{\prime} \mathrm{N} \quad 53^{\circ} 47^{\prime} \mathrm{W}$ W

IIA01007

$70^{\circ} 33^{\prime} \mathrm{N} \quad 53^{\circ} 49^{\prime} \mathrm{W}$ NW

IIA02002

IIA02003

1IA02004

$70^{\circ} 32^{\prime} \mathrm{N} \quad 53^{\circ} 46^{\prime} \mathrm{W}$ SW

$53^{\circ} 46^{\prime} \mathrm{W} \mathrm{NW}$

$70^{\circ} 32 \mathrm{~N}$

11A02006

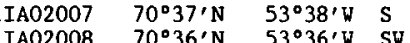

IIA02009

$70^{\circ} 35^{\prime} \mathrm{N} 53^{\circ} 36^{\prime}, \mathrm{W}$ SW

1IA02010

1 IA02012

$\begin{array}{lll} & -1 \\ & \end{array}$

1IA02013

$70^{\circ} 34^{\prime} \mathrm{N}$

$53^{\circ} 39^{\prime} \mathrm{V}$ E

1 IA02015

1 IA02017

湤 $34^{\prime} \mathrm{W}$

$\begin{array}{ll}70^{\circ} 32^{\prime} \mathrm{N} & 53^{\circ} 32^{\circ} \mathrm{V} \\ 75^{\circ} \mathrm{V}\end{array}$

1IA02018

$70^{\circ} 31^{\prime} \mathrm{N}$

$53^{\circ} 35^{\prime} \mathrm{W}$

1IAO2020

70. $31^{\prime} \mathrm{N} \quad 53^{\circ} 37^{\prime} \mathrm{W}$ W

1IA02021

IIA02023

$53032, \mathrm{~W} S$

1 IA02024

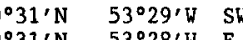

1IA02025

1 IA02027

1 IA02029

IIA0203

1I02031

$53^{\circ} 28^{\prime} \mathrm{W}$

$70^{\circ} 30^{\prime} \mathrm{N} \quad 53^{\circ} 23^{\prime} \mathrm{W}$ SW

$\begin{array}{lll}70^{\circ} 30^{\prime} \mathrm{N} & 53^{\circ} 11^{\prime} \mathrm{W} & \mathrm{N} \\ 70^{\circ} 30^{\prime} \mathrm{N} & 53^{\circ} 13^{\prime} \mathrm{W} & \mathrm{N}\end{array}$

$70^{\circ} 30^{\prime} \mathrm{N}$

$53^{\circ} 14^{\prime}, \mathrm{N}$

$70^{\circ} 30^{\prime} \mathrm{N}$

53019 , N 90

1 IA02033

$70^{\circ} 31, \mathrm{~N}$

政

1IA02034

IIA02035

$1 \mathrm{IA02037}$

1IA02038

$70^{\circ} 34^{\prime} \mathrm{N} \quad 53^{\circ} 27^{\prime} \mathrm{V}$

$70^{\circ} 35^{\prime} \mathrm{N}$
$70^{\circ} 33^{\prime} \mathrm{N}$

1 IA02040

(

$53^{\circ} 14^{\prime}, \mathrm{CE}$

1 IA02041

1 IA02043

11A02044

$70^{\circ} 34 \mathrm{~N}$

$53^{\circ} 11$ ' $N$

$70^{\circ} 32^{\prime} \mathrm{N}$

$53^{\circ} 15^{\prime} \mathrm{W}$ N

1 IA02045

(

$53^{\circ} 19^{\prime} \mathrm{W}$ E

$70^{\circ} 36^{\prime} \mathrm{N} \quad 53^{\circ} 20^{\prime} \mathrm{V}$ SE

IIA02047 70037'N $53^{\circ} 15^{\prime} \mathrm{V}$ E

$\begin{array}{llll}\text { IIA02049 } & 70^{\circ} 38^{\prime} \mathrm{N} & 53^{\circ} 14^{\prime} \mathrm{W} & \mathrm{SE} \\ \text { IIA02050 } & 70^{\circ} 40^{\prime} \mathrm{N} & 53^{\circ} 14^{\prime} \mathrm{W} & \mathrm{SE}\end{array}$

1 IA02050

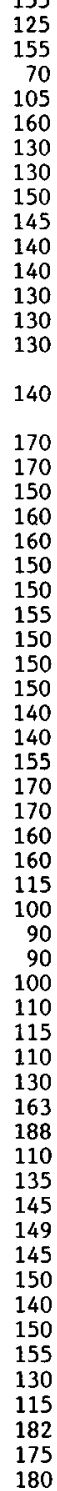

\begin{tabular}{rlll}
115 & 0000.34 & 700010 & $7000-01$ \\
65 & 0003.05 & 533112 & $7000-01$ \\
65 & 0000.17 & 700010 & $7000-01$ \\
105 & 0000.08 & 700010 & $7030-01$ \\
90 & 0007.60 & 513112 & $7030-01$ \\
110 & 0000.16 & 750110 & $7030-01$ \\
110 & 0000.43 & 640110 & $7030-01$ \\
105 & 0003.01 & 533112 & $7030-01$ \\
95 & 0002.47 & 533112 & $7030-01$ \\
90 & 0003.49 & 513112 & $7030-01$ \\
110 & 0001.05 & 700010 & $7030-01$ \\
110 & 0000.34 & 750010 & $7030-01$ \\
110 & 0000.50 & 750010 & $7030-01$ \\
100 & 0000.29 & 700010 & $7030-01$ \\
120 & 0000.48 & 700010 & $7030-01$ \\
140 & 0000.14 & 700010 & $7030-01$ \\
110 & 0000.14 & 700010 & $7030-02$ \\
85 & 0010.11 & 513012 & $7030-02$ \\
120 & 0000.47 & 700010 & $7030-02$ \\
120 & 0000.49 & 643212 & $7030-02$ \\
135 & 0000.39 & 700010 & $7030-02$ \\
85 & 00010.48 & 513012 & $7030-02$ \\
95 & 0001.28 & 643012 & $7030-02$ \\
95 & 0000.74 & 643012 & $7030-02$ \\
120 & 0000.74 & 643012 & $7030-02$ \\
110 & 0000.27 & 600012 & $7030-02$ \\
80 & 0001.17 & 643112 & $7030-02$ \\
130 & 0000.18 & 600010 & $7030-02$ \\
120 & 0000.08 & 700010 & $7030-02$ \\
100 & 0000.63 & 640010 & $7030-02$ \\
105 & 0002.34 & 613012 & $7030-02$ \\
150 & 0000.38 & 700010 & $7030-02$ \\
160 & 0000.31 & 600010 & $7030-02$ \\
150 & 0000.08 & 700010 & $7030-02$ \\
135 & 0000.20 & 700010 & $7030-02$ \\
100 & 0000.09 & 640112 & $7030-02$ \\
80 & 0000.22 & 640112 & $7030-02$ \\
80 & 0000.15 & 643112 & $7030-02$ \\
85 & 0000.35 & 643212 & $7030-02$ \\
80 & 0002.05 & 643212 & $7030-02$ \\
85 & 0000.85 & 643112 & $7030-02$ \\
90 & 0000.40 & 643212 & $7030-02$ \\
90 & 0000.72 & 643212 & $7030-02$ \\
75 & 0007.96 & 513216 & $7030-02$ \\
95 & 0001.08 & 643312 & $7030-02$ \\
75 & 0018.11 & 513112 & $7030-02$ \\
90 & 0001.16 & 643112 & $7030-02$ \\
75 & 0001.04 & 533112 & $7030-02$ \\
75 & 0003.07 & 503012 & $7030-02$ \\
90 & 0001.17 & 503012 & $7030-02$ \\
75 & 0001.34 & 513111 & $7030-02$ \\
70 & 0003.15 & 513112 & $7030-02$ \\
110 & 0000.27 & 643112 & $7030-02$ \\
85 & 0002.16 & 533112 & $7030-02$ \\
85 & 0008.39 & 513132 & $7030-02$ \\
90 & 0000.74 & 643112 & $7030-02$ \\
90 & 0000.97 & 643012 & $7030-02$ \\
80 & 0009.39 & 513111 & $7030-02$ \\
140 & 0000.28 & 600012 & $7030-02$ \\
80 & 0014.41 & 513112 & $7030-02$ \\
\hline
\end{tabular}

1IA02053

$70^{\circ} 41^{\prime} \mathrm{N} \quad 53^{\circ} 08^{\prime} \mathrm{W}$ S $\mathrm{S} \quad 13$

(1)

1IA02057

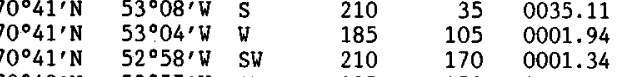

$\begin{array}{llll}70^{\circ} 40^{\prime} \mathrm{N} & 52 \circ 57 \cdot \mathrm{W} & \mathrm{SW} & 195 \\ 70^{\circ} 38^{\prime} \mathrm{N} & 53^{\circ} 00^{\prime} \mathrm{W} & \mathrm{W} & 170\end{array}$

IA02058

1 IA02060

$70^{\circ} 37^{\prime} \mathrm{N} 53^{\circ} 01^{\prime} \mathrm{N}$

$70^{\circ} 36^{\prime} \mathrm{N} \quad 53^{\circ} 01^{\prime} \mathrm{W}$

IA02061

$70^{\circ} 35^{\prime} \mathrm{N} 53^{\circ} 01, \mathrm{~W}$

IIA02063

$70^{\circ} 37^{\prime} \mathrm{N} \quad 52^{\circ} 53^{\prime} \mathrm{W} \mathrm{S}$

1IA02064

1 IA02066

$70^{\circ} 36^{\prime} \mathrm{N} 52^{\circ} 51^{\prime} \mathrm{W}$ SW

$70^{\circ} 34^{\prime} \mathrm{N} \quad 52^{\circ} 46^{\circ} \mathrm{W}$

1IA02067

1 IA02069

$70^{\circ} 32^{\prime} \mathrm{N} 52^{\circ} 42^{\prime} \mathrm{W} \mathrm{W}$

$70^{\circ} 32^{\prime} \mathrm{N} \quad 52^{\circ} 46^{\prime} \mathrm{W} \quad \mathrm{NW}$

1 IA02071

IA02072

$\begin{array}{ll}70^{\circ} 32^{\prime} \mathrm{N} & 52^{\circ} 44^{\prime} \mathrm{W} \\ 70^{\circ} 31^{\prime} \mathrm{N} & 52^{\circ} 49^{\prime} \mathrm{V}\end{array}$

$70^{\circ} 30^{\prime} \mathrm{N} \quad 52^{\circ} 47^{\prime} \mathrm{V}$ W

$70^{\circ} 30^{\prime} \mathrm{N} \quad 52^{\circ} 42, \mathrm{~W} \mathrm{~W}$

1 IA02074

$70^{\circ} 30^{\prime} \mathrm{N}$

$52^{\circ} 40^{\prime} \mathrm{W}$ SV

1 IAO2076

$70^{\circ} 30^{\prime} \mathrm{N} \quad 52^{\circ} 38^{\prime} \mathrm{W}$ S

IA02078

1IA02079

$70^{\circ} 30^{\prime} \mathrm{N} \quad 52^{\circ} 36^{\circ} \mathrm{W}$

$70^{\circ} 31, \mathrm{~N} \quad 52^{\circ} 36^{\prime} \mathrm{W}$ SW

$70^{\circ} 31^{\prime} \mathrm{N} \quad 52^{\circ} 37 \cdot \mathrm{W} S$

$70^{\circ} 30^{\prime} \mathrm{N} \quad 52^{\circ} 27^{\prime} \mathrm{W}$ S

IAO2083

$70^{\circ} 28^{\prime} \mathrm{N} \quad 52^{\circ} 31^{\prime} \mathrm{W}$ S

IA02084

IIA02086

$70^{\circ} 29^{\prime} \mathrm{N} \quad 52^{\circ} 27^{\prime} \mathrm{W}$ -

1IA02087

$70^{\circ} 29^{\prime} \mathrm{N} \quad 52^{\circ} 24^{\prime} \mathrm{W} \quad \mathrm{S}$

$\begin{array}{lll}70^{\circ} 29^{\prime} \mathrm{N} & 52^{\circ} 21^{\prime} \mathrm{W} & \mathrm{SE} \\ 70^{\circ} 29^{\prime} \mathrm{N} & 52^{\circ} 20^{\prime} \mathrm{W} & \mathrm{SW}\end{array}$

1 IA02089

1 IAO2099

1IA02091

$70^{\circ} 28^{\prime} \mathrm{N}$

$52^{\circ} 18, W \quad \mathrm{~W}$

$\begin{array}{llll}\text { IA02093 } & 70^{\circ} 25^{\prime} \mathrm{N} & 52^{\circ} 17, \mathrm{~W} & \mathrm{SW} \\ \text { IA02094 } & 70^{\circ} 25^{\prime} \mathrm{N} & 52^{\circ} 16^{\prime} \mathrm{W} & \mathrm{S}\end{array}$

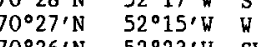

$70^{\circ} 25^{\prime} \mathrm{N} \quad 52^{\circ} 20^{\circ} \mathrm{W}$ S

IIA02096 70024'N 52014'W S

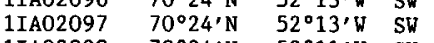

1 IA02098

1 IA02099

$70^{\circ} 24^{\prime} \mathrm{N} \quad 52^{\circ} 11^{\prime} \mathrm{W}$ SW

$\begin{array}{llll}1 \text { IA02100 } 70^{\circ} 22^{\prime} \mathrm{N} & 52^{\circ} 00^{\circ} \mathrm{W} & \mathrm{S} \\ \mathrm{I} & \mathrm{S}\end{array}$

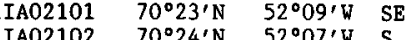

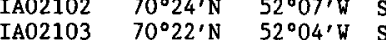

IA02104 $70^{\circ} 22^{\prime} \mathrm{N} \quad 52^{\circ} 03^{\prime} \mathrm{W}$

$\begin{array}{llll}\text { IIA02105 } & 7^{\circ} 24^{\prime} \mathrm{N} & 52^{\circ} 00^{\prime} \mathrm{W} & \mathrm{S} \\ \text { 1IA02106 } & 70^{\circ} 22^{\prime} \mathrm{N} & 51^{\circ} 57^{\prime} \mathrm{W} & \mathrm{SW}\end{array}$

1IA02107 $70^{\circ} 22^{\prime} \mathrm{N} \quad 51^{\circ} 56^{\prime} \mathrm{W}$ S

IA02108 $70^{\circ} 23^{\prime} \mathrm{N} \quad 51^{\circ} 53^{\prime} \mathrm{W}$ SW

$\begin{array}{llll}\text { IIA02109 } & 70^{\circ} 22^{\prime} \mathrm{N} & 51^{\circ} 51^{\prime} \mathrm{W} & \mathrm{SE} \\ \text { 1IA02110 } & 70^{\circ} 23^{\prime} \mathrm{N} & 51^{\circ} 49, \mathrm{~W} & \mathrm{SW}\end{array}$

1IA02111

$70^{\circ} 21^{\prime} \mathrm{N} \quad 51^{\circ} 49, \mathrm{~V}$

140
145
170
205
205
195
207
17
130
17
18
17
165
165
16
155
155
18
160
209
16
135
160
140
20
150
170
165
200
200
155
120
120
145
125
125
105
17
8
8
135
168
125
159
168
150
150
156
145
156
137

$125 \quad 0000.35$

$100 \quad 0000.61$

1450001.02

$130 \quad 0002.54$

$\begin{array}{ll}110 & 0003.45 \\ 140 & 0000.96\end{array}$

$80 \quad 0007.74$

$100 \quad 0000.45$

000.08

$\begin{array}{ll}100 & 0001.92 \\ 110 & 0000.67\end{array}$

$\begin{array}{ll}130 & 0000.17 \\ 120 & 0001.30\end{array}$

$\begin{array}{rr}130 & 0000.49 \\ 95 & 0000.76\end{array}$

$120 \quad 0000.86$

0000.72
$\quad 0025.89$

$\begin{array}{rr}135 & 0000.24\end{array}$

1350001.21

0000.47

1300000.61

$105 \quad 0002.40$

1050000.74

$\begin{array}{ll}140 & 0000.36\end{array}$

$120 \quad 0002.75$

$140 \quad 0001.64$

$45 \quad 0001.18$

0001.45

$\begin{array}{ll}100 & 0000.59\end{array}$

$\begin{array}{ll}5 & 0001.70 \\ 6 & 0000.93\end{array}$

$70 \quad 0006.04$

$\begin{array}{ll}60 & 0001.06\end{array}$

$\begin{array}{rr}35 & 0001.44 \\ 115 & 0000.47\end{array}$

$55 \quad 0013.25$

110000.67

$50 \quad 0012.66$

$90 \quad 0000.92$

$\begin{array}{lll}156 & 55 & 0015.07 \\ 137 & 65 & 0002.06\end{array}$

CODE

$700010 \quad 7030-02$

$50131117030-02$

$600012 \quad 7030-02$

$700010 \quad 7030-02$

$5131127030-02$

$643112 \quad 7030-02$

$643112 \quad 7030-02$

$2631117030-02$

$263112 \quad 7030-02$

$513012 \quad 7030-02$
640010

$640010 \quad 7030-02$

$700010 \quad 7030-02$

$2600127030-02$

7000-03

263112 7000-03

$2601127000-03$

$700010 \quad 7030-02$
263112

$2631127030-03$

$700010 \quad 7000-03$

600010
790012
643132

$643132 \quad 7000-03$

$643012 \quad 7000-03$

$643010 \quad 7000-03$

$790010 \quad 7000-03$

$363011 \quad 7000-03$

$360010 \quad 7000-03$

$360010 \quad 7000-03$

$363012 \quad 7000-03$

$363010 \quad 7000-03$

$\begin{aligned} & 363010 \\ & 363012\end{aligned} 7000-03$

$3630117000-03$

$3630127000-03$

360010 7000-03

7000-03

$363111 \quad 7000-03$

$360010 \quad 7000-03$

$363117000-03$

$360010 \quad 7000-03$

$3633127000-03$

$3633127000-03 \quad 0$ 


\begin{tabular}{|c|c|c|c|c|c|c|c|c|c|c|c|c|c|c|c|c|c|}
\hline CODE & LAT & LONG & ORI & HIGH & LOW & AREA & $\begin{array}{c}\text { MO } \\
\text { CODE }\end{array}$ & MAP & CODE & LAT & LONG & ORI & HIGH & LOW & AREA & $\begin{array}{c}\text { MO } \\
\text { CODE }\end{array}$ & MAP \\
\hline 1IA02112 & $70^{\circ} 21^{\prime} \mathrm{N}$ & $51^{\circ} 48^{\prime} \mathrm{W}$ & $\mathrm{SE}$ & 137 & & 0.67 & 36001 & $7000-04$ & 1I & ' N & ' $W$ & NE & 100 & 85 & 0000.37 & 700010 & $7000-04$ \\
\hline 1IA02113 & $70^{\circ} 211^{\prime} \mathrm{N}$ & $51^{\circ} 46^{\prime} \mathrm{W}$ & $\mathrm{s}$ & 130 & 70 & 0003.73 & 363312 & $7000-04$ & $1 \mathrm{IA0} 2174$ & $70^{\circ} 12^{\prime} \mathrm{N}$ & $51^{\circ} 36^{\prime} \mathrm{W}$ & $\mathrm{N}$ & 90 & 70 & 0000.12 & 600012 & $7000-04$ \\
\hline $1 I A 02114$ & $70^{\circ} 21^{\prime} \mathrm{N}$ & $51^{\circ} 4^{4}$ W & E & 125 & 95 & 0001.75 & 360012 & $7000-04$ & 1 IA02175 & $70^{\circ} 12^{\prime} \mathrm{N}$ & $51^{\circ} 38^{\prime} \mathrm{W}$ & NE & 100 & 70 & 0000.61 & 363412 & $7000-04$ \\
\hline 1IA02115 & $70^{\circ} 22^{\prime} \mathrm{N}$ & $51^{\circ} 42^{\prime}$ & $\mathrm{s}$ & 151 & 55 & 0015.16 & 363111 & $7000-04$ & 1IA02176 & $70^{\circ} 12^{\prime} \mathrm{N}$ & $51^{\circ} 43^{\prime} \mathrm{W}$ & E & 125 & 50 & 0005.93 & 363412 & $7000-04$ \\
\hline 1 IA02116 & $70^{\circ} 21^{\prime} \mathrm{N}$ & $51^{\circ} 39^{\prime} \mathrm{W}$ & SE & 130 & 115 & 0001.35 & 360010 & $7000-04$ & 1IA02177 & $70^{\circ} 13^{\prime} \mathrm{N}$ & $51^{\circ} 41^{\prime} \mathrm{W}$ & $\mathrm{s}$ & 120 & 110 & 0000.54 & 360010 & $7000-04$ \\
\hline 1IA02117 & $70^{\circ} 22^{\prime} \mathrm{N}$ & $51^{\circ} 37 \%$ & $\mathrm{SE}$ & 135 & 50 & 0005.36 & 363311 & $7000-04$ & 1 IA02178 & $70^{\circ} 13^{\prime} \mathrm{N}$ & $511^{\circ} 39 \cdot \mathrm{W}$ & NE & 115 & 85 & 0000.64 & 363412 & $7000-04$ \\
\hline 1IA02118 & $70^{\circ} 22^{\prime} \mathrm{N}$ & $51^{\circ} 33^{\prime} \mathrm{W}$ & SW & 150 & 55 & 0011.00 & 363311 & $7000-04$ & 1 IAO2179 & $70^{\circ} 13^{\prime} \mathrm{N}$ & $51^{\circ} 41^{\prime} \mathrm{W}$ & $\mathrm{NE}$ & 130 & 50 & 0001.29 & 363312 & $7000-04$ \\
\hline 1 IA02119 & $70^{\circ} 21 \cdot \mathrm{N}$ & $51^{\circ} 28^{\prime} \mathrm{W}$ & $\mathrm{s}$ & & 120 & 0003.00 & 360111 & $7000-04$ & $1 \mathrm{IA} 02180$ & $70^{\circ} 14^{\prime} \mathrm{N}$ & $51^{\circ} 43^{\prime} \mathrm{W}$ & $\mathrm{NE}$ & 134 & 27 & 0003.95 & 363312 & $7000-04$ \\
\hline 1 IA02120 & $70^{\circ} 21^{\prime} \mathrm{N}$ & $51^{\circ} 24^{\prime \prime}$ & sw & 155 & 110 & 0004.07 & 363112 & $7000-04$ & IIA02181 & $70^{\circ} 14^{\prime} \mathrm{N}$ & $51^{\circ} 44^{\prime} \mathrm{W}$ & NE & 130 & 40 & 0001.39 & 363412 & $7000-04$ \\
\hline $1 \mathrm{IA0} 2121$ & $70^{\circ} 20^{\prime} \mathrm{N}$ & $51^{\circ} 28^{\prime} \mathrm{W}$ & $\mathrm{N}$ & 110 & 90 & 0000.84 & 670112 & $7000-04$ & 1IA02182 & $70^{\circ} 14^{\prime} \mathrm{N}$ & $51^{\circ} 45^{\prime} \mathrm{W}$ & $\mathrm{NE}$ & 100 & 50 & 0001.03 & 363512 & $7000-04$ \\
\hline IIA02122 & $70^{\circ} 20^{\prime} \mathrm{N}$ & $51021, \mathrm{w}$ & $\mathrm{s}$ & 155 & 110 & 0006.17 & 363112 & $7000-04$ & 1IA02183 & $70^{\circ} 14^{\prime} \mathrm{N}$ & $51^{\circ} 47, \mathrm{~W}$ & $\mathrm{NE}$ & 130 & 30 & 0004.43 & 363412 & $7000-04$ \\
\hline 1IA02123 & $70^{\circ} 20^{\prime} \mathrm{N}$ & $51^{\circ} 00^{\prime} \mathrm{W}$ & SW & 150 & 110 & 0004.80 & 363112 & $7000-04$ & 1IA02184 & $70^{\circ} 16^{\prime} \mathrm{N}$ & $51^{\circ} 46^{\prime} \mathrm{W}$ & $\mathrm{NE}$ & 95 & 55 & 0000.09 & 750010 & $7000-04$ \\
\hline 1 IA02124 & $70^{\circ} 18^{\prime} \mathrm{N}$ & $51^{\circ} 15^{\prime} \mathrm{W}$ & $\mathrm{s}$ & 110 & 110 & 0000.16 & 363112 & $7000-04$ & 1IA02185 & $70^{\circ} 16^{\prime} \mathrm{N}$ & $51^{\circ} 48^{\prime} \mathrm{W}$ & $\mathrm{NE}$ & 140 & 60 & 0001.07 & 363412 & $7000-04$ \\
\hline 1IA02125 & $70^{\circ} 17 \cdot \mathrm{N}$ & $51^{\circ} 13^{\prime} \mathrm{W}$ & $\mathrm{N}$ & 90 & 85 & 0000.27 & 670012 & $7000-04$ & 1IA02186 & $70^{\circ} 17 \cdot \mathrm{N}$ & $51^{\circ} 00^{\prime} \hat{\mathrm{W}}$ & $\mathrm{NE}$ & & 45 & 0000.44 & 360512 & $7000-04$ \\
\hline 1IA02126 & $70^{\circ} 17 \cdot \mathrm{N}$ & $51^{\circ} 10^{\prime} \mathrm{W}$ & $\mathrm{V}$ & 115 & 90 & 0001.03 & 360012 & $7000-04$ & 1IA02187 & $70^{\circ} 16^{\prime} \mathrm{N}$ & $51^{\circ} 49, \mathrm{~W}$ & $\mathrm{NE}$ & 140 & 100 & 0000.96 & 360010 & $7000-03$ \\
\hline 1IA02127 & $70^{\circ} 17^{\prime} \mathrm{N}$ & $51^{\circ} 10^{\prime \prime} \mathrm{V}$ & u & & 100 & 0000.82 & 360012 & $7000-04$ & 1IA02188 & $70^{\circ} 17^{\circ} \mathrm{N}$ & $51^{\circ} 50^{\prime} \mathrm{W}$ & N & 130 & 85 & 0000.21 & 363010 & $7000-03$ \\
\hline 1IA02128 & $70^{\circ} 16^{\prime} \mathrm{N}$ & $51^{\circ} 10^{\prime} \mathrm{W}$ & $\mathrm{s}$ & & 110 & 0000.02 & 360012 & $7000-04$ & 1IA02189 & $70^{\circ} 16^{\prime} \mathrm{N}$ & $51^{\circ} 51^{\prime} \mathrm{W}$ & $\mathrm{NE}$ & 140 & 35 & 0002.01 & 363412 & $7000-03$ \\
\hline 1 IA02129 & $70^{\circ} 16^{\prime} \mathrm{N}$ & $51^{\circ} 11^{\prime} \mathrm{V}$ & SW & 105 & 95 & 0000.41 & 670112 & $7000-04$ & 1IA02190 & $70^{\circ} 17 \cdot \mathrm{N}$ & $51^{\circ} 51^{\prime} \mathrm{W}$ & $\mathrm{N}$ & 125 & 85 & 0000.25 & 363010 & $7000-03$ \\
\hline 1IA02130 & $70^{\circ} 16^{\prime} \mathrm{N}$ & $51^{\circ} 09^{\prime} \mathrm{W}$ & $\mathrm{SE}$ & 100 & 95 & 0000.11 & 750110 & $7000-04$ & 1 IA02191 & $70^{\circ} 17 \cdot \mathrm{N}$ & $51^{\circ} 52^{\prime} \mathrm{W}$ & $\mathrm{NE}$ & 140 & 35 & 0001.87 & 363312 & $7000-03$ \\
\hline IIAO2131 & $70^{\circ} 15^{\prime} \mathrm{N}$ & $51^{\circ} 07^{\prime} \mathrm{w}$ & $\mathrm{SW}$ & & & 0000.24 & 360010 & $7000-04$ & 1IA02192 & $70^{\circ} 17 \cdot \mathrm{N}$ & $51^{\circ} 54^{\prime} \mathrm{W}$ & NE & 140 & 35 & 0003.11 & 363312 & $7000-03$ \\
\hline $1 \mathrm{IAO} 2132$ & $70^{\circ} 14^{\prime} \mathrm{N}$ & $51^{\circ} 06^{\prime} \mathrm{W}$ & $\mathrm{NW}$ & 80 & 75 & 0000.18 & 360112 & $7000-04$ & 1IA02193 & $70^{\circ} 18^{\prime} \mathrm{N}$ & $51^{\circ} 56, \mathrm{~W}$ & NE & 120 & 70 & 0000.85 & 363412 & $7000-03$ \\
\hline IIA02133 & $70^{\circ} 14^{\prime} \mathrm{N}$ & $51^{\circ} 12^{\prime} \mathrm{V}$ & NE & 115 & 50 & 0001.46 & 363312 & $7000-04$ & 1 IAO2194 & $70^{\circ} 18^{\prime} \mathrm{N}$ & $51055 \% \mathrm{~W}$ & $N E$ & 130 & 50 & 0000.33 & 363412 & $7000-03$ \\
\hline 1 IA02134 & $70^{\circ} 14^{\prime} \mathrm{N}$ & $51^{\circ} 13^{\prime} \mathrm{W}$ & $\mathrm{NE}$ & 115 & 50 & 0001.04 & 363312 & $7000-04$ & 1IA02195 & $70^{\circ} 19^{\prime} \mathrm{N}$ & $51.56, \mathrm{~W}$ & $\mathrm{~N}$ & & 50 & 0001.18 & 363412 & $7000-03$ \\
\hline 1IA02135 & $70^{\circ} 14^{\prime} \mathrm{N}$ & $51^{\circ} 16^{\prime} \mathrm{W}$ & $\mathrm{N}$ & 128 & 32 & 0005.87 & 363312 & $7000-04$ & 1IA02196 & $70^{\circ} 18^{\prime} \mathrm{N}$ & $51^{\circ} 58, \mathrm{~W}$ & NW & 130 & 50 & 0003.91 & 363212 & $7000-03$ \\
\hline 1IA02136 & $70^{\circ} 14^{\prime} \mathrm{N}$ & $51^{\circ} 18^{\prime} \mathrm{W}$ & E & 120 & 100 & 0000.51 & 360112 & $7000-04$ & $11 A 02197$ & $70^{\circ} 18^{\prime} \mathrm{N}$ & $52^{\circ} 02^{\prime} \mathrm{W}$ & $\mathrm{W}$ & 145 & 85 & 0000.25 & 363512 & $7000-03$ \\
\hline 1IA02137 & $70^{\circ} 14^{\prime} \mathrm{N}$ & $51^{\circ} 19^{\prime} \mathrm{k}$ & $\mathrm{NE}$ & 120 & 40 & 0001.64 & 363312 & $7000-04$ & 1IA02198 & $70^{\circ} 18^{\prime} \mathrm{N}$ & $52^{\circ} 011^{\prime} \mathrm{W}$ & $\mathrm{W}$ & 145 & 120 & 0000.47 & 363012 & $7000-03$ \\
\hline 1IA02138 & $70^{\circ} 15^{\prime} \mathrm{N}$ & $51^{\circ} 21^{\prime} \mathrm{W}$ & $\mathrm{NE}$ & 120 & 55 & 0002.74 & 363312 & $7000-04$ & 1IA02199 & $70^{\circ} 17^{\prime} \mathrm{N}$ & $52^{\circ} 00^{\prime} \mathrm{W}$ & $\mathrm{W}$ & 135 & 105 & 0001.33 & 363112 & $7000-03$ \\
\hline $1 \mathrm{IAO} 2139$ & $70^{\circ} 15^{\prime} \mathrm{N}$ & $51^{\circ} 22^{\prime} \mathrm{V}$ & NE & 125 & 45 & 0001.65 & 363312 & $7000-04$ & $1 \mathrm{IA02200}$ & $70^{\circ} 17^{\prime} \mathrm{N}$ & $51^{\circ} 57 \cdot \mathrm{W}$ & sw & 135 & 110 & 0002.39 & 363112 & $7000-03$ \\
\hline 1 IA02140 & $70^{\circ} 15^{\prime} \mathrm{N}$ & $51^{\circ} 24^{\prime} \mathrm{W}$ & $\mathbf{N}$ & 125 & 40 & 0003.14 & 363312 & $7000-04$ & 1IA02201 & $70^{\circ} 17^{\circ} \mathrm{N}$ & $51^{\circ} 56^{\prime} \mathrm{W}$ & $\mathrm{W}$ & 140 & 80 & 0001.61 & 363112 & $7000-03$ \\
\hline $1 \mathrm{IA02141}$ & $70^{\circ} 16^{\prime} \mathrm{N}$ & $51^{\circ} 25^{\prime} \mathrm{W}$ & NE & 135 & 45 & 0002.67 & 363312 & $7000-04$ & 1IA02202 & $70^{\circ} 16^{\prime} \mathrm{N}$ & $51 \circ 55, \mathrm{~W}$ & $\mathrm{v}$ & 140 & 135 & 0000.39 & 360012 & $7000-03$ \\
\hline 1IA02142 & איר17 & $51^{\circ} 26^{\prime}, \mathrm{W}$ & $\mathrm{NE}$ & 125 & 100 & 0001.48 & 360012 & $7000-04$ & 1IA02203 & $70^{\circ} 16^{\prime}, \mathrm{N}$ & $51^{\circ} 53^{\prime} \mathrm{W}$ & s & 140 & 125 & 0000.83 & 360012 & $7000-03$ \\
\hline 1IA02143 & $70^{\circ} 18^{\prime} \mathrm{N}$ & $51^{\circ} 32 \% \mathrm{~W}$ & $\mathrm{~N}$ & 115 & 100 & 0000.13 & 363512 & $7000-04$ & 1IA02204 & $70^{\circ} 16^{\prime} \mathrm{N}$ & $51^{\circ} 52^{\prime} \mathrm{W}$ & SW & 130 & 75 & 0002.07 & 363112 & $7000-03$ \\
\hline 1 IA02144 & $70^{\circ} 18^{\prime} \mathrm{N}$ & $51^{\circ} 33^{\prime} \mathrm{W}$ & $\mathrm{NE}$ & 120 & 100 & 0000.42 & 363412 & $7000-04$ & $1 \mathrm{IA02205}$ & $70^{\circ} 15^{\prime} \mathrm{N}$ & $51^{\circ} 52^{\prime} \mathrm{W}$ & SW & 140 & 120 & 0000.94 & 360110 & $7000-03$ \\
\hline 1IA02145 & $70^{\circ} 18^{\prime} \mathrm{N}$ & $51^{\circ} 34^{\prime} \mathrm{W}$ & $\mathrm{NE}$ & 115 & 80 & 0000.70 & 363312 & $7000-04$ & 1IA02206 & $70^{\circ} 14^{\prime} \mathrm{N}$ & $51^{\circ} 49, \mathrm{~W}$ & SW & 140 & 80 & 0004.79 & 363112 & $7000-03$ \\
\hline 1IA02146 & $70^{\circ} 18^{\prime} \mathrm{N}$ & $51^{\circ} 35^{\prime} \mathrm{W}$ & $\mathrm{NE}$ & 115 & 100 & 0000.09 & 360010 & $7000-04$ & 1IA02207 & $70^{\circ} 14^{\prime} \mathrm{N}$ & $51^{\circ} 48^{\prime} \mathrm{W}$ & s & 130 & 115 & 0001.08 & 360112 & $7000-03$ \\
\hline 1IA02147 & $70^{\circ} 18^{\prime} \mathrm{N}$ & $51^{\circ} 35^{\prime} \mathrm{W}$ & $\mathrm{NW}$ & 115 & 55 & 0001.39 & 363312 & $7000-04$ & 1IA02208 & $70^{\circ} 14^{\prime} \mathrm{N}$ & $51^{\circ} 46^{\prime} \mathrm{W}$ & SW & 130 & 120 & 0002.16 & 360112 & $7000-04$ \\
\hline 1IA02148 & $70^{\circ} 18^{\prime} \mathrm{N}$ & $51^{\circ} 35^{\prime} \mathrm{W}$ & $\mathrm{s}$ & 120 & 105 & 0000.90 & 360010 & $7000-04$ & 1 IA02209 & $70^{\circ} 13^{\prime} \mathrm{N}$ & $51^{\circ} 43^{\prime} \mathrm{W}$ & SW & 130 & 130 & 0000.58 & 360112 & $7000-04$ \\
\hline 1IA02149 & $70^{\circ} 18^{\prime} \mathrm{N}$ & $51^{\circ} 32^{\prime} \mathrm{W}$ & $\mathrm{s}$ & 120 & 105 & 0000.32 & 360010 & $7000-04$ & $11 \mathrm{A0} 2210$ & $70^{\circ} 13^{\prime} \mathrm{N}$ & $51^{\circ} 46^{\prime} \mathrm{W}$ & $\mathrm{v}$ & 115 & 70 & 0003.79 & 363112 & $7000-04$ \\
\hline 1 IA02150 & $70^{\circ} 16^{\prime} \mathrm{N}$ & $51^{\circ} 27^{\prime} \mathrm{W}$ & $\mathrm{NW}$ & 130 & 100 & 0004.65 & 363112 & $7000-04$ & $1 I A 02211$ & $70^{\circ} 12^{\prime} \mathrm{N}$ & $51^{\circ} 46^{\prime} \mathrm{k}$ & NE & 143 & 130 & 000 & 360 & 70 \\
\hline 1IA02151 & $70^{\circ} 16^{\prime} \mathrm{N}$ & $51^{\circ} 27 \cdot \mathrm{v}$ & $\nabla$ & 130 & 115 & 0000.58 & 360112 & $7000-04$ & 1IA02212 & $70^{\circ} 12^{\prime} \mathrm{N}$ & $51^{\circ} 46, \mathrm{~V}$ & SW & 143 & 125 & 0000.85 & 360010 & $7000-04$ \\
\hline 1IA02152 & $70^{\circ} 15^{\prime} \mathrm{N}$ & $51^{\circ} 26^{\prime} \mathrm{w}$ & sW & 130 & 85 & 0001.95 & 363312 & $7000-04$ & 1IA02213 & $70^{\circ} 12^{\prime} \mathrm{N}$ & $51.44 \cdot \mathrm{W}$ & $\mathrm{V}$ & 125 & 100 & 0000.99 & 363112 & $7000-04$ \\
\hline 1 IA02153 & $70^{\circ} 15^{\prime} \mathrm{N}$ & $51^{\circ} 26^{\prime} \mathrm{W}$ & sW & 130 & 120 & 0000.78 & 360012 & 700 & 1IA02214 & $70^{\circ} 09^{\prime} \mathrm{N}$ & $51^{\circ} 47 \cdot \mathrm{W}$ & $\mathrm{N}$ & 90 & 65 & 0001.34 & 366112 & $7000-04$ \\
\hline 1IA02154 & $70^{\circ} 15^{\prime N} \mathrm{~N}$ & $51^{\circ} 24^{\prime} \mathrm{W}$ & sW & 130 & 110 & 0001.26 & 360012 & $7000-04$ & 1IA02215 & $70^{\circ} 09^{\prime} \mathrm{N}$ & $51^{\circ} 49 \cdot \mathrm{W}$ & $\mathrm{N}$ & 115 & 65 & 0004.64 & 363112 & $7000-03$ \\
\hline 1IA02155 & $70^{\circ} 14^{\prime} \mathrm{N}$ & $51^{\circ} 23^{\prime} \mathrm{W}$ & $\mathrm{s}$ & 130 & 110 & 0000.87 & 363112 & $7000-04$ & 1 IA02216 & $70^{\circ} 09^{\prime} \mathrm{N}$ & $51^{\circ} 50^{\prime} \mathrm{W}$ & $\mathrm{N}$ & 115 & 80 & 00 & 263012 & $0-03$ \\
\hline 1IA02156 & $70^{\circ} 14^{\prime} \mathrm{N}$ & $51^{\circ} 20^{\prime} \mathrm{K}$ & $\mathrm{SW}$ & 125 & 80 & 0009.23 & 362312 & $7000-04$ & 1IA02217 & $70^{\circ} 09^{\prime} \mathrm{N}$ & $51^{\circ} 52^{\prime} \mathrm{W}$ & $\mathrm{N}$ & 115 & 75 & 0001.83 & 263012 & $7000-03$ \\
\hline 1IA02157 & $70^{\circ} 13^{\prime} \mathrm{N}$ & $51^{\circ} 21^{\prime} \mathrm{W}$ & SW & 125 & 115 & 0000.50 & 360010 & 700 & $1 \mathrm{IAC}$ & $70^{\circ} 11^{\prime} \mathrm{N}$ & $51^{\circ} 57^{\prime} \mathrm{W}$ & $\mathrm{SE}$ & 125 & 110 & 0000.54 & 260012 & $7000-03$ \\
\hline 1IA02158 & $70^{\circ} 13^{\prime} \mathrm{N}$ & $51^{\circ} 20^{\prime} \mathrm{W}$ & $\mathrm{SE}$ & 125 & 115 & 0000.51 & 360010 & 700 & 1IA02219 & $70^{\circ} 11^{\prime} \mathrm{N}$ & $51^{\circ} 56^{\prime} \mathrm{W}$ & $\mathrm{NE}$ & 125 & 75 & 0001.63 & 263012 & $7000-03$ \\
\hline 1IA02159 & $70^{\circ} 13^{\prime} \mathrm{N}$ & $51^{\circ} 18^{\prime} \mathrm{W}$ & SW & 128 & 70 & 0001.53 & 363312 & $7000-04$ & 1 IA02220 & $70^{\circ} 12^{\prime} \mathrm{N}$ & $51^{\circ} 57^{\prime} \mathrm{v}$ & $\mathrm{NE}$ & 142 & 80 & 0001.08 & 26 & $7000-03$ \\
\hline 1IA02160 & $70^{\circ} 12^{\prime} \mathrm{N}$ & $51^{\circ} 15^{\prime} \mathrm{W}$ & $\mathrm{s}$ & 125 & 80 & 0007.64 & 363311 & $7000-04$ & $11 A 02221$ & $70^{\circ} 12^{\prime} \mathrm{N}$ & $51^{\circ} 58, \mathrm{~W}$ & NE & 142 & 75 & 00 & 26 & $7000-03$ \\
\hline 1IA02161 & $70^{\circ} 11^{\prime} \mathrm{N}$ & $51^{\circ} 14^{\prime} \mathrm{w}$ & - & 110 & 100 & 0000.29 & 363310 & $7000-04$ & 1IA02222 & $70^{\circ} 13^{\prime} \mathrm{N}$ & $51.59, \mathrm{~V}$ & $\mathrm{NE}$ & 115 & 90 & 0000.50 & 260010 & $7000-03$ \\
\hline 1IA02162 & $70^{\circ} 10^{\prime} \mathrm{N}$ & $51^{\circ} 14^{\prime} \mathrm{W}$ & NW & 90 & 85 & 0000.21 & 360110 & $7000-04$ & $1 \mathrm{IA02223}$ & $70^{\circ} 14^{\prime} \mathrm{N}$ & $52^{\circ} 03^{\prime} \mathrm{k}$ & $\mathrm{E}$ & 128 & 110 & 0000.86 & 360010 & $7000-03$ \\
\hline 1IA02163 & $70^{\circ} 12^{\prime} \mathrm{N}$ & $51^{\circ} 25^{\prime} \mathrm{W}$ & NE & 100 & 90 & 0000.51 & 370112 & $7000-04$ & $11 A 02224$ & $70^{\circ} 14^{\prime} \mathrm{N}$ & $52^{\circ} 03^{\prime} \mathrm{W}$ & $\mathrm{N}$ & 110 & 100 & 0000.19 & 360010 & $7000-03$ \\
\hline 1IA02164 & $70^{\circ} 06^{\prime} \mathrm{N}$ & $51^{\circ} 32^{\prime} \mathrm{W}$ & $\mathrm{NE}$ & 110 & 70 & 0004.09 & 370112 & $7000-04$ & 1IA02225 & $70^{\circ} 14^{\prime} \mathrm{N}$ & $52^{\circ} 04^{\prime} \mathrm{W}$ & $\mathrm{NE}$ & 128 & 75 & 000 & 36 & 70 \\
\hline 1IA02165 & $70^{\circ} 09^{\prime} \mathrm{N}$ & $51^{\circ} 22^{\prime} \mathrm{W}$ & $\mathrm{NE}$ & 100 & 50 & 0004.52 & 363312 & 7000 & 1IA02226 & $70^{\circ} 15^{\prime} \mathrm{N}$ & $52^{\circ} 05^{\circ} \mathrm{W}$ & $\mathrm{NE}$ & 10 & 80 & 0000.64 & 643112 & $7000-03$ \\
\hline 1IA02166 & $70^{\circ} 09^{\prime} \mathrm{N}$ & $51^{\circ} 25^{\prime} \mathrm{W}$ & $\mathbf{N}$ & 105 & 60 & 0005.41 & & 70 & 1IA02227 & $70^{\circ} 16^{\prime} \mathrm{N}$ & $52^{\circ} 07 \cdot \mathrm{W}$ & E & 121 & 105 & 0000.96 & 360112 & $7000-03$ \\
\hline 1IA02167 & $70^{\circ} 10^{\prime} \mathrm{N}$ & $51^{\circ} 28^{\prime} \mathrm{w}$ & $\mathrm{NW}$ & 90 & 65 & 0001.92 & 360112 & $7000-04$ & 1 IA02228 & $70^{\circ} 17 \cdot \mathrm{N}$ & $52^{\circ} 07^{\prime} \mathrm{W}$ & NE & 120 & 90 & 0000.50 & 363112 & $7000-03$ \\
\hline 1IA02168 & $70^{\circ} 10^{\prime} \mathrm{N}$ & $51^{\circ} 34^{\prime} \mathrm{W}$ & $\mathrm{N}$ & 80 & 65 & 0000.56 & 700010 & $7000-04$ & 1IA02229 & $70^{\circ} 17 \cdot \mathrm{N}$ & $52^{\circ} 07^{\circ} \mathrm{W}$ & $\mathrm{N}$ & 121 & 80 & 0000.62 & 363112 & $7000-03$ \\
\hline 1IA02169 & $70^{\circ} 10^{\prime} \mathrm{N}$ & $51^{\circ} 35^{\prime} \mathrm{W}$ & s & 80 & 75 & 0000.06 & 700010 & $7000-04$ & 1IA02230 & $70^{\circ} 21 \cdot \mathrm{N}$ & $52^{\circ} 25,6$ & $\mathrm{E}$ & 115 & 85 & 0002.54 & 260112 & $7000-03$ \\
\hline 1IA02170 & $70^{\circ} 11^{\prime} \mathrm{N}$ & $51^{\circ} 35^{\prime} \mathrm{W}$ & $\mathrm{s}$ & 80 & 75 & 0000.21 & 700 & 7000 & IIA02231 & $70^{\circ} 21$ ' N & $52^{\circ} 27^{\circ} \mathrm{v}$ & 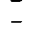 & 160 & 110 & 0000.66 & 260112 & $7000-03$ \\
\hline 1IA02171 & $70^{\circ} 11^{\prime} \mathrm{N}$ & $51^{\circ} 35^{\prime} \mathrm{W}$ & NE & 75 & 65 & 0000.11 & 700010 & $7000-04$ & 1IA02232 & $70^{\circ} 22^{\prime} \mathrm{N}$ & $52^{\circ} 25^{\prime} \mathrm{W}$ & $\mathbf{E}$ & 100 & 95 & 0000.60 & 260112 & $7000-03$ \\
\hline 1IA02172 & $70^{\circ} 11^{\prime} \mathrm{N}$ & $51^{\circ} 35^{\prime} \mathrm{W}$ & $\mathbf{N}$ & 75 & 70 & 0000.13 & 700010 & $7000-04$ & 1 IA02233 & $70^{\circ} 20^{\prime} \mathrm{N}$ & $52^{\circ} 29^{\prime} \mathrm{W}$ & $\mathrm{NE}$ & 170 & 90 & 0004.77 & 263111 & $7000-03$ \\
\hline
\end{tabular}




\begin{tabular}{|c|c|c|c|c|c|c|c|c|c|c|c|c|c|c|c|c|c|}
\hline CODE & LAT & LONG & ORI & HIGH & LOW & AREA & $\begin{array}{c}\text { MO } \\
\text { CODE }\end{array}$ & MAP & CODE & LAT & LONG & ORI & HIGH & LOW & AREA & $\begin{array}{c}\mathrm{MO} \\
\mathrm{CODE}\end{array}$ & MAP \\
\hline 1IA02234 & $70^{\circ} 22{ }^{\prime} \mathrm{N}$ & $52^{\circ} 31^{\prime} \mathrm{W}$ & $\mathrm{NE}$ & 170 & $>0$ & 000 & 2 & $7000-03$ & 1IB17006 & $70^{\circ}$ & $50^{\circ} 57 \cdot 6$ & v & 90 & 85 & 0001.73 & & \\
\hline 1IA02235 & $70^{\circ} 22 \mathrm{~N}$ & $52^{\circ} 32^{\prime} \mathrm{W}$ & $\mathrm{NE}$ & 170 & 105 & 0000.44 & 260112 & $7000-03$ & IIB17007 & $70^{\circ} 17 \times \mathrm{N}$ & $50^{\circ} 56^{\prime} \mathrm{V}$ & s & $\begin{array}{r}90 \\
100\end{array}$ & 95 & 0000.69 & 360010 & $7000-04$ \\
\hline 1IA02236 & $70^{\circ} 22^{\prime} \mathrm{N}$ & $52^{\circ} 34^{\prime} \mathrm{W}$ & $\mathrm{N}$ & 170 & 100 & 0002.15 & 260112 & $7000-03$ & 1IB17008 & $70^{\circ} 18^{\prime} \mathrm{N}$ & $50^{\circ} 55 \mathrm{~V}$ & $\mathrm{E}$ & 100 & 90 & 0000.83 & 360010 & $7000-04$ \\
\hline 11A02237 & $70^{\circ} 22^{\prime} \mathrm{N}$ & $52^{\circ} 35^{\prime} \mathrm{W}$ & $\mathrm{N}$ & 160 & 110 & 0000.56 & 263112 & $7000-03$ & 1IB17009 & $70^{\circ} 19^{\prime} \mathrm{N}$ & $50^{\circ} 55^{\circ} \mathrm{W}$ & SE & 100 & 90 & 0000.70 & 700010 & $7000-04$ \\
\hline 1IA02238 & $70^{\circ} 22$ ' N & $52 \circ 37 \% \mathrm{~W}$ & NW & 160 & 95 & 0000.81 & 643111 & $7000-03$ & $1 \mathrm{IB} 17010$ & $70^{\circ} 19^{\prime} \mathrm{N}$ & $50^{\circ} 53^{\prime} \mathrm{w}$ & $\mathrm{W}$ & 100 & 95 & 0000.17 & 700010 & $7000-04$ \\
\hline 1IA02239 & $70^{\circ} 23^{\prime} \mathrm{N}$ & $52 \circ 39 . \mathrm{W}$ & N & 110 & 100 & 0000.11 & 643112 & $7000-03$ & 1IB17011 & $70^{\circ} 16^{\prime} \mathrm{N}$ & $50^{\circ} 50^{\prime} \mathrm{W}$ & W & & 63 & 0000.56 & 360012 & $7000-04$ \\
\hline $1 \mathrm{IA02240}$ & $70^{\circ} 22, \mathrm{~N}$ & $52^{\circ} 39^{\circ} \mathrm{W}$ & w & 164 & 140 & 0000.97 & 260110 & $7000-03$ & 1IB17012 & $70^{\circ} 16^{\prime} \mathrm{N}$ & $50^{\circ} 52^{\prime \prime}$ & $\mathrm{NE}$ & 90 & 63 & 0000.99 & 670012 & $7000-04$ \\
\hline 1IA02241 & $70^{\circ} 22 \cdot \mathrm{N}$ & $52^{\circ} 41^{\prime} \mathrm{W}$ & NW & 130 & 100 & 0000.46 & 600012 & $7000-03$ & IIB17013 & $70^{\circ} 15^{\prime} \mathrm{N}$ & $50^{\circ} 50^{\prime} \mathrm{W}$ & $\begin{array}{l}\text { NE } \\
\mathrm{W}\end{array}$ & & & 0000.55 & 360010 & $7000-04$ \\
\hline 1IA02242 & $70^{\circ} 23^{\prime} \mathrm{N}$ & $52^{\circ} 46^{\prime} \mathrm{W}$ & $\mathrm{NE}$ & 170 & 105 & 0001.18 & 640112 & $7000-03$ & 1IB17014 & $70^{\circ} 15, \mathrm{~N}$ & $50^{\circ} 51^{\prime} \mathrm{W}$ & W & 85 & 70 & 0000.33 & 360010 & $7000-04$ \\
\hline 1IA02243 & $70^{\circ} 23^{\prime} \mathrm{N}$ & $52^{\circ} 48^{\prime} \mathrm{W}$ & $\mathbf{N}$ & 170 & 100 & 0001.45 & 643112 & $7000-03$ & 1IB17015 & $70^{\circ} 14^{\prime} \mathrm{N}$ & $50.57 \cdot \mathrm{W}$ & $\mathrm{NE}$ & 100 & & 0000.46 & 360010 & $7000-04$ \\
\hline 1IA02244 & $70^{\circ} 23^{\prime} \mathrm{N}$ & $52^{\circ} 49^{\prime} W$ & $\mathrm{~N}$ & 170 & 110 & 0000.60 & 600010 & $7000-03$ & $1 I B 17016$ & $70^{\circ} 15^{\prime} \mathrm{N}$ & $51^{\circ} 06^{\prime} \mathrm{W}$ & $\mathrm{NE}$ & 100 & 95 & 0000.45 & 360010 & $7000-04$ \\
\hline 1IA02245 & $70^{\circ} 23^{\prime} \mathrm{N}$ & $52^{\circ} 50^{\circ} \mathrm{k}$ & $\mathrm{N}$ & 170 & 95 & 0000.78 & 643112 & $7000-03$ & 1IB17017 & $70^{\circ} 16^{\prime} \mathrm{N}$ & $51^{\circ} 08^{\prime \prime}$ & $\mathrm{NE}$ & 145 & 145 & 0000.09 & 770012 & $7000-04$ \\
\hline $1 \mathrm{IAO} 2246$ & $70^{\circ} 23^{\prime} \mathrm{N}$ & $52^{\circ} 50^{\circ} \mathrm{W}$ & $\mathrm{N}$ & & & 0000.05 & 600010 & $7000-03$ & IIB17018 & $70^{\circ} 17^{\prime} \mathrm{N}$ & $51^{\circ} 09^{\prime}$ W & $\mathrm{E}$ & 117 & 105 & 0000.31 & 360112 & $7000-04$ \\
\hline 1IA02247 & $70^{\circ} 23^{\prime} \mathrm{N}$ & $52^{\circ} 51^{\prime} \mathrm{W}$ & $\mathrm{N}$ & 150 & 95 & 0001.25 & 643112 & $7000-03$ & 1IB17019 & $70^{\circ} 17 \cdot \mathrm{N}$ & $51^{\circ} 08^{\prime} \mathrm{W}$ & $\mathbf{E}$ & 115 & 90 & 0000.92 & 360112 & $7000-04$ \\
\hline $1 \mathrm{IA02248}$ & $70^{\circ} 23^{\prime} \mathrm{N}$ & $52^{\circ} 53^{\prime} \mathrm{W}$ & $\mathrm{N}$ & 140 & 95 & 0002.11 & 670012 & $7000-02$ & IIB18001 & $70^{\circ} 18^{\prime} \mathrm{N}$ & $51^{\circ} 08^{\prime}$ & $\mathbf{N}$ & 110 & 85 & 0001.41 & 360012 & $7000-04$ \\
\hline 1IA02249 & $70^{\circ} 24^{\prime} \mathrm{N}$ & $52^{\circ} 56^{\prime} \mathrm{W}$ & N & 150 & 95 & 0001.16 & 643112 & $7000-02$ & 1IB18002 & $70^{\circ} 18^{\prime} \mathrm{N}$ & $51^{\circ} 13^{\prime} \mathrm{V}$ & - & 100 & 95 & 0000.25 & 770012 & $7000-04$ \\
\hline 1IA02250 & $70^{\circ} 23^{\prime} \mathrm{N}$ & $52^{\circ} 57^{\prime} \mathrm{W}$ & NW & 110 & 90 & 0001.65 & 643112 & $7000-02$ & 1IB18003 & $70^{\circ} 19^{\prime} \mathrm{N}$ & $51 \circ 15, v$ & E & & 100 & 0001.87 & 360112 & $7000-04$ \\
\hline 1IA02251 & $70^{\circ} 22^{\prime} \mathrm{N}$ & $53^{\circ} 00^{\prime} \mathrm{W}$ & $\mathrm{N}$ & & 85 & 0008.23 & 513112 & $7000-02$ & 1 IB18004 & $70^{\circ} 20^{\prime} \mathrm{N}$ & $51^{\circ} 16^{\prime} \mathrm{V}$ & E & & 105 & 0002.39 & 360112 & $7000-04$ \\
\hline 1IA02252 & $70^{\circ} 24^{\prime} \mathrm{N}$ & $53^{\circ} 04^{\prime} \mathrm{W}$ & $\mathrm{N}$ & 167 & 105 & 0001.77 & 643132 & $7000-02$ & 1IB18005 & $70^{\circ} 21^{\prime} \mathrm{N}$ & $51^{\circ} 14^{\prime} \mathrm{W}$ & - & 105 & 85 & 0000.14 & 770010 & $7000-04$ \\
\hline 1IA02253 & $70^{\circ} 23^{\prime} \mathrm{N}$ & $53^{\circ} 06^{\prime} \mathrm{W}$ & NW & 140 & 120 & 0000.15 & 700010 & $7000-02$ & 1IB18006 & $70^{\circ} 21 \cdot \mathrm{N}$ & $51^{\circ} 17^{\prime} \mathrm{W}$ & NE & 150 & 60 & 0003.93 & 363212 & $7000-04$ \\
\hline 1IA02254 & $70^{\circ} 22^{\prime} \mathrm{N}$ & $53^{\circ} 06^{\prime} \mathrm{W}$ & $\mathrm{NW}$ & 155 & 110 & 0000.85 & 643112 & $7000-02$ & 1IB18007 & $70^{\circ} 22^{\prime} \mathrm{N}$ & $510^{\circ} 17^{\prime} \mathrm{W}$ & NE & 105 & 60 & 0000.25 & 543212 & $7000-04$ \\
\hline 1IA02255 & $70^{\circ} 22^{\prime} \mathrm{N}$ & $53^{\circ} 07^{\prime} \mathrm{W}$ & $\mathrm{N}$ & 150 & 75 & 0002.73 & 643112 & $7000-02$ & 1IB18008 & $70^{\circ} 22^{\prime} \mathrm{N}$ & $51^{\circ} 19^{\prime} \mathrm{W}$ & $\mathbf{N}$ & 155 & 55 & 0004.15 & 36 & 700 \\
\hline 1IA02256 & $70^{\circ} 22$, N & $53^{\circ} 10^{\prime} \mathrm{W}$ & $\mathrm{N}$ & 140 & 90 & 0000.87 & 643112 & $7000-02$ & 1IB18009 & $70^{\circ} 23 \cdot \mathrm{N}$ & $51^{\circ} 19^{\prime}$ W & NE & 60 & 45 & 0000.20 & 773212 & $7000-04$ \\
\hline 1IA02257 & $70^{\circ} 24^{\prime} \mathrm{N}$ & $53^{\circ} 12^{\prime} \mathrm{W}$ & NW & 150 & 120 & 0000.13 & 700010 & $7000-02$ & IIB19001 & $70^{\circ} 23^{\prime} \mathrm{N}$ & $51^{\circ} 20^{\prime} \mathrm{W}$ & $\mathrm{NE}$ & 115 & 90 & 0000.42 & 770112 & $7000-04$ \\
\hline 1IA02258 & $70^{\circ} 23 \cdot \mathrm{N}$ & $53^{\circ} 15^{\prime} \mathrm{W}$ & $\mathrm{N}$ & 140 & 80 & 0002.86 & 643112 & $7000-02$ & 1IB19002 & $70^{\circ} 22, \mathrm{~N}$ & $51^{\circ} 22^{\prime} \mathrm{W}$ & $\mathrm{N}$ & 155 & 11 & 0001.80 & 360010 & $7000-04$ \\
\hline 1IA02259 & $70^{\circ} 24^{\prime} \mathrm{N}$ & $53^{\circ} 17 \cdot \mathrm{W}$ & $\mathrm{N}$ & 130 & 95 & 0000.48 & 643112 & $7000-02$ & 1IB19003 & $70^{\circ} 22^{\prime} \mathrm{N}$ & $51^{\circ} 25^{\prime} \mathrm{W}$ & $\mathbf{N}$ & 155 & 60 & 0010.35 & 363312 & $7000-04$ \\
\hline 1IA02260 & $70^{\circ} 24^{\prime} \mathrm{N}$ & $53^{\circ} 18^{\prime} \mathrm{W}$ & $\mathrm{N}$ & 145 & 100 & 0000.96 & 643112 & $7000-02$ & 1IB19004 & $70^{\circ} 23 \cdot \mathrm{N}$ & $51 \circ 27 \cdot \mathrm{V}$ & E & 125 & 120 & 0000.14 & 770010 & 70 \\
\hline 1IA02261 & $70^{\circ} 23^{\prime} \mathrm{N}$ & $53^{\circ} 19^{\prime} \mathrm{W}$ & $\mathrm{N}$ & 150 & 95 & 0002.04 & 643112 & $7000-02$ & 1IB19005 & $70^{\circ} 23^{\prime} \mathrm{N}$ & $51^{\circ} 25, \mathrm{~W}$ & $\mathrm{SE}$ & 115 & 110 & 000 & 770010 & \\
\hline 1IA02262 & $70^{\circ} 24^{\prime} \mathrm{N}$ & $53^{\circ} 23^{\prime} \mathrm{W}$ & $\mathrm{N}$ & 145 & 95 & 0000.85 & 643112 & $7000-02$ & 1IB20001 & $70^{\circ} 23^{\prime} \mathrm{N}$ & $51^{\circ} 30^{\prime} \mathrm{W}$ & $\mathbf{N}$ & 125 & 125 & 0008.45 & 363311 & $7000-04$ \\
\hline 1IA02263 & $70^{\circ} 24^{\prime} \mathrm{N}$ & $53^{\circ} 24^{\prime} \mathrm{W}$ & NW & 105 & 95 & 0000.27 & 640012 & $7000-02$ & IIB20002 & $70^{\circ} 23^{\prime} \mathrm{N}$ & $51^{\circ} 27^{\prime} \mathrm{W}$ & $\mathbf{N}$ & 138 & 55 & 0000.08 & 780010 & $7000-04$ \\
\hline 1IA02264 & $70^{\circ} 24^{\prime} \mathrm{N}$ & $53^{\circ} 25, \mathrm{~W}$ & $\mathrm{~N}$ & 105 & 95 & 0000.27 & 790012 & $7000-02$ & 1IB20003 & $70^{\circ} 24^{\prime} \mathrm{N}$ & $51^{\circ} 30^{\prime} \mathrm{W}$ & E & 125 & 115 & 0000.19 & 770010 & $7000-04$ \\
\hline 1IA02265 & $70^{\circ} 24^{\prime} \mathrm{N}$ & $53^{\circ} 33^{\prime} \mathrm{v}$ & E & 100 & 90 & 0000.75 & 640012 & $7000-02$ & IIB20004 & $70^{\circ} 24^{\prime} \mathrm{N}$ & $51^{\circ} 30^{\prime} \mathrm{W}$ & NE & 125 & 85 & 0000.57 & 643112 & 70 \\
\hline 1IA02266 & $70^{\circ} 25^{\prime} \mathrm{N}$ & $53^{\circ} 34^{\prime} \mathrm{W}$ & NW & 95 & 70 & 0000.22 & 640012 & $7000-02$ & 1IB20005 & $70^{\circ} 24^{\prime} \mathrm{N}$ & $51^{\circ} 33^{\prime} \mathrm{W}$ & NE & 130 & 75 & 000 & 363012 & $00-04$ \\
\hline $11 A 02267$ & $70^{\circ} 24^{\prime} \mathrm{N}$ & $53^{\circ} 35^{\prime} \mathrm{W}$ & NW & & 85 & 0000.94 & 643012 & $7000-02$ & IIB21001 & $70^{\circ} 24^{\prime} \mathrm{N}$ & $51^{\circ} 40^{\prime} \mathrm{W}$ & NE & 130 & 20 & 0016.87 & $\begin{array}{l}363012 \\
363412\end{array}$ & $7000-04$ \\
\hline 1IB01001 & $70^{\circ} 42, \mathrm{~N}$ & $51^{\circ} 52^{\prime} \mathrm{W}$ & $\mathrm{W}$ & 120 & 105 & 0000.53 & 700010 & $7030-03$ & 1IB21002 & $70^{\circ} 25, \mathrm{~N}$ & $51^{\circ} 37 \mathrm{~W}$ & $\mathrm{NE}$ & 110 & 65 & 0000.38 & 650010 & $7000-04$ \\
\hline $1 \mathrm{IB} 01002$ & $70^{\circ} 42, \mathrm{~N}$ & $51^{\circ} 50^{\circ} \mathrm{W}$ & v & 125 & 120 & 0000.29 & 360010 & $7030-03$ & 1IB21003 & $70^{\circ} 26^{\prime N} \mathrm{~N}$ & $51^{\circ} 38^{\prime} \mathrm{W}$ & $\mathrm{SE}$ & 135 & 115 & 0001.12 & 260112 & $7000-04$ \\
\hline 1IB01003 & $70^{\circ} 41$, N & $51^{\circ} 49^{\prime} \mathrm{W}$ & SW & 125 & 120 & 0001.45 & 360010 & $7030-03$ & IIB22001 & $70^{\circ} 27, \mathrm{~N}$ & $51^{\circ} 40^{\prime} \mathrm{W}$ & $\mathrm{W}$ & 130 & 100 & 0000.26 & 263112 & 70 \\
\hline 1 IBO1004 & $70^{\circ} 40^{\prime} \mathrm{N}$ & $51^{\circ} 48^{\prime} \mathrm{W}$ & $s$ & 120 & 115 & 0000.89 & 360010 & $7030-03$ & 1IB22002 & $70^{\circ} 25^{\prime} \mathrm{N}$ & $51^{\circ} 42 \cdot \mathrm{W}$ & $\mathrm{N}$ & 135 & 70 & 000 & 363312 & $7000-04$ \\
\hline 1IB01005 & $70^{\circ} 41^{\prime} \mathrm{N}$ & $51^{\circ} 46^{\prime} \mathrm{W}$ & & 120 & 85 & 0002.39 & 362112 & $7030-03$ & 1IB22003 & $70^{\circ} 26^{\prime} \mathrm{N}$ & $51^{\circ} 44 \cdot \mathrm{W}$ & $\mathbf{N}$ & 135 & 95 & 0000.29 & 770010 & $7000-04$ \\
\hline $1 \mathrm{IB} 01006$ & $70^{\circ} 41, \mathrm{~N}$ & $51^{\circ} 42^{\prime} \mathrm{W}$ & $\mathrm{SE}$ & 100 & 95 & 0001.06 & 690112 & $7030-03$ & 1IB22004 & $70^{\circ} 26^{\prime} \mathrm{N}$ & $51^{\circ} 45, \mathrm{~W}$ & $\mathrm{~N}$ & 140 & 85 & 0002.28 & 513012 & $7000-04$ \\
\hline 1IB03001 & $70^{\circ} 39^{\prime} \mathrm{N}$ & $50^{\circ} 33^{\prime} \mathrm{W}$ & $\mathrm{SW}$ & 180 & & 0305.96 & 164112 & $7030-04$ & 1IB22005 & $70^{\circ} 26^{\prime} \mathrm{N}$ & $510^{\circ} 46 \mathrm{~W}$ & E & & 125 & 0000.39 & 700010 & $7000-04$ \\
\hline 1 IBO3002 & $70^{\circ} 37, \mathrm{~N}$ & $50^{\circ} 28^{\prime} \mathrm{W}$ & SW & 180 & 55 & 0057.20 & 166112 & $7030-04$ & 1IB22006 & $70^{\circ} 27^{\circ} \mathrm{N}$ & $51^{\circ} 46^{\prime} \mathrm{W}$ & $\mathrm{E}$ & & 105 & 0000.54 & 700010 & $7000-04$ \\
\hline 1IB11001 & $70^{\circ} 35^{\prime} \mathrm{N}$ & $50^{\circ} 28^{\prime} \mathrm{W}$ & SW & 180 & & 0288.27 & 164112 & $7030-04$ & 1IB23001 & $70^{\circ} 23^{\prime} \mathrm{N}$ & $51^{\circ} 47^{\prime} \mathrm{W}$ & NW & 125 & 110 & 0000.38 & 700010 & 700 \\
\hline 1IB11002 & $70^{\circ} 34^{\prime} \mathrm{N}$ & $50^{\circ} 25^{\prime} \mathrm{W}$ & $\mathrm{SW}$ & 180 & 60 & 0056.26 & 163112 & $7030-05$ & 1IB23002 & $70^{\circ} 25 \cdot \mathrm{N}$ & $51^{\circ} 45, \mathrm{~W}$ & SW & 130 & 120 & 0000.40 & 700010 & $7000-04$ \\
\hline 1IB12001 & $70^{\circ} 24^{\prime} \mathrm{N}$ & $50^{\circ} 32^{\prime} \mathrm{W}$ & $\mathrm{SW}$ & 180 & & 2796.39 & 164113 & $7000-05$ & 1IB23003 & $70^{\circ} 24^{\prime} \mathrm{N}$ & $51^{\circ} 45, \mathrm{~W}$ & $N$ & 150 & 20 & 0019.60 & 363311 & $7000-04$ \\
\hline IIB 13001 & $70^{\circ} 21^{\prime} \mathrm{N}$ & $50^{\circ} 24^{\prime} \mathrm{W}$ & $\mathrm{v}$ & 180 & 40 & 0073.89 & 163113 & $7000-0$ & 1IB23004 & $70^{\circ} 26^{\prime} \mathrm{N}$ & $51^{\circ} 50^{\circ} \mathrm{W}$ & $\mathrm{E}$ & 140 & 135 & 000 & 700010 & $7000-03$ \\
\hline IIB 14001 & $70^{\circ} 16^{\prime} \mathrm{N}$ & $50^{\circ} 20^{\prime} \mathrm{W}$ & SW & 180 & 50 & 0137.35 & 163112 & $7000-05$ & IIB2 & $70^{\circ}$ & $51^{\circ} 51^{\prime} \mathrm{V}$ & $\mathrm{E}$ & 150 & 135 & 0000 & 700010 & $7000-03$ \\
\hline 1IB15001 & $70^{\circ} 15, \mathrm{~N}$ & $50^{\circ} 47 \cdot \mathrm{V}$ & $N E$ & 8 & 70 & 0000.09 & 750010 & 7000 & 1IB23006 & $70^{\circ} 28^{\prime} \mathrm{N}$ & $51^{\circ} 50, \mathrm{~W}$ & E & 125 & 100 & 0000.13 & 70 & $7000-03$ \\
\hline 1IB15002 & $70^{\circ} 16^{\prime} \mathrm{N}$ & $50^{\circ} 49^{\prime} \mathrm{W}$ & $\mathrm{N}$ & 90 & 65 & 0002.51 & 360012 & $7000-$ & 1IB24001 & $70^{\circ} 28^{\prime} \mathrm{N}$ & $51^{\circ} 50^{\prime \prime} \mathrm{W}$ & $\mathrm{N}$ & 125 & 105 & 0000.39 & 700010 & $7000-03$ \\
\hline 1IB16001 & $70^{\circ} 19^{\prime} \mathrm{N}$ & $50^{\circ} 52^{\prime} \mathrm{W}$ & E & 100 & 95 & 0000.08 & 700010 & $7000-04$ & 1IB24 & $70^{\circ} 27^{\prime} \mathrm{N}$ & $51^{\circ} 51, \mathrm{~V}$ & NW & 150 & 125 & 0000.21 & 700010 & $7000-03$ \\
\hline 1IB16002 & $70^{\circ} 19^{\prime} \mathrm{N}$ & $50^{\circ} 52^{\prime} \mathrm{W}$ & NE & 100 & 90 & 0000.62 & 750010 & $7000-04$ & 1IB24003 & $70^{\circ} 26^{\prime} \mathrm{N}$ & $51^{\circ} 55^{\prime} \mathrm{W}$ & $\mathrm{N}$ & 150 & 25 & 0013.23 & 363311 & $7000-03$ \\
\hline 1 IB 16003 & $70^{\circ} 19^{\prime} \mathrm{N}$ & $50^{\circ} 54^{\prime} \mathrm{w}$ & $\mathbf{N}$ & 100 & 90 & 0001.01 & 700010 & $7000-$ & 1IB24004 & $70^{\circ} 27^{\prime} \mathrm{N}$ & $51^{\circ} 55^{\prime} \mathrm{W}$ & NE & 165 & 100 & 0002.46 & 360112 & $7000-03$ \\
\hline 1IB16004 & $70^{\circ} 20^{\prime} \mathrm{N}$ & $50^{\circ} 53^{\prime} \mathrm{W}$ & $\mathrm{N}$ & 8 & 80 & 0000.20 & 750010 & $7000-04$ & 1IB24005 & $70^{\circ} 28^{\prime} \mathrm{N}$ & $51057 \%$ & N & 155 & 60 & 0002.62 & 363112 & $7000-03$ \\
\hline 1IB16005 & $70^{\circ} 20^{\prime} \mathrm{N}$ & $50^{\circ} 55^{\prime} \mathrm{W}$ & $\mathrm{N}$ & 90 & 80 & 0000.79 & 700010 & $7000-04$ & 1IB25001 & $70^{\circ} 28^{\prime} \mathrm{N}$ & $51^{\circ} 58^{\prime} \mathrm{V}$ & NW & 170 & 60 & 0002.92 & 363312 & $7000-03$ \\
\hline 1IB16006 & $70^{\circ} 20^{\prime} \mathrm{N}$ & $50^{\circ} 56^{\circ} \mathrm{W}$ & $\mathrm{N}$ & 100 & 95 & 0000.35 & 700010 & $7000-04$ & 1IB25002 & $70^{\circ} 26^{\prime} \mathrm{N}$ & $52^{\circ} 07, \mathrm{~W}$ & $\mathbf{N}$ & 170 & & 0036.10 & 363112 & $7000-03$ \\
\hline 1 IB16007 & $70^{\circ} 20^{\prime} \mathrm{N}$ & $50^{\circ} 57 \cdot \mathrm{W}$ & $\mathrm{N}$ & 95 & 85 & 0000.51 & 700010 & $7000-04$ & 1IB25003 & $70^{\circ} 28^{\prime} \mathrm{N}$ & $52^{\circ} 01^{\prime} \mathrm{W}$ & $\mathrm{SE}$ & 145 & 120 & 0000.57 & 500010 & $7000-03$ \\
\hline 1IB17001 & $70^{\circ} 20^{\prime} \mathrm{N}$ & $51^{\circ} 00^{\prime} \mathrm{W}$ & $\mathbf{N}$ & 8 & 70 & 0000.56 & 700010 & $7000-04$ & 1IB25004 & $70^{\circ} 29^{\prime} \mathrm{N}$ & $52^{\circ} 04^{\prime} \mathrm{W}$ & $\mathrm{SE}$ & 170 & 120 & 0005.40 & 360010 & $7000-03$ \\
\hline 1IB17002 & $70^{\circ} 20^{\prime} \mathrm{N}$ & $50^{\circ} 59^{\prime} \mathrm{W}$ & NW & 100 & 90 & 0000.39 & 780010 & $7000-04$ & 1IB25005 & $70^{\circ} 29^{\prime} \mathrm{N}$ & $52^{\circ} 01 \cdot \mathrm{W}$ & $\mathrm{E}$ & 160 & 120 & 0001.09 & 360010 & $7000-03$ \\
\hline 1IB17003 & $70^{\circ} 20^{\prime} \mathrm{N}$ & $50^{\circ} 59^{\prime} \mathrm{W}$ & $\mathrm{W}$ & 100 & 100 & 0000.20 & 700010 & $7000-04$ & 1IB25006 & $70^{\circ} 30^{\prime} \mathrm{N}$ & $52^{\circ} 01, \mathrm{~W}$ & $\mathrm{NE}$ & 200 & 75 & 0002.09 & 363112 & $7030-03$ \\
\hline IIB 17004 & $70^{\circ} 19^{\prime} \mathrm{N}$ & $50^{\circ} 58^{\prime} \mathrm{W}$ & - & 104 & 104 & 0000.18 & 700010 & $7000-04$ & 1IB25007 & $70^{\circ} 31^{\prime} \mathrm{N}$ & $52^{\circ} 01, \mathrm{~V}$ & $\mathrm{~N}$ & 140 & 100 & 0001.07 & 360112 & $7030-03$ \\
\hline 1IB17005 & $70^{\circ} 19^{\prime} \mathrm{N}$ & $50^{\circ} 56^{\prime} \mathrm{W}$ & $\mathrm{v}$ & 105 & 100 & 0000.77 & 700010 & $7000-04$ & 1IB25008 & $70^{\circ} 31^{\prime} \mathrm{N}$ & $52^{\circ} 02^{\prime}, \mathrm{V}$ & NW & 130 & 115 & 0000.62 & 360112 & $7030-03$ \\
\hline
\end{tabular}




\begin{tabular}{|c|c|c|c|c|c|c|c|c|c|c|c|c|}
\hline CODE & LAT & LONG & ORI & HIGH & LOW & AREA & $\begin{array}{c}\text { MO } \\
\text { CODE }\end{array}$ & MAP & CODE & LAT & LONG & ORI \\
\hline 1 IB25009 & $70^{\circ} 32^{\prime} \mathrm{N}$ & $52^{\circ} 02^{\prime} \mathrm{W}$ & - & & & 0000.16 & 700010 & $7030-03$ & & $70^{\circ} 41^{\prime} \mathrm{N}$ & $53^{\circ} 24^{\prime} \mathrm{W}$ & \\
\hline 1IB26001 & $70^{\circ} 32^{\prime} \mathrm{N}$ & $52^{\circ} 02^{\prime} \mathrm{K}$ & $\mathrm{N}$ & 120 & 105 & 0000.15 & 770010 & $7030-03$ & 1IB40006 & $70^{\circ} 40^{\prime} \mathrm{N}$ & $53^{\circ} 23^{\prime} \mathrm{W}$ & W \\
\hline 1IB26002 & $70^{\circ} 31^{\prime} \mathrm{N}$ & $52^{\circ} 03^{\prime} \mathrm{W}$ & NW & 120 & 80 & 0000.18 & 553012 & $7030-03$ & 1IB40007 & $70^{\circ} 39^{\prime} \mathrm{N}$ & $53^{\circ} 23^{\prime} \mathrm{W}$ & $\hat{\mathrm{W}}$ \\
\hline 1 IB 26003 & $70^{\circ} 30^{\prime} \mathrm{N}$ & $52^{\circ} 05^{\prime} \mathrm{K}$ & $N$ & 185 & 50 & 0012.44 & 363016 & $7000-03$ & IIB40008 & $70^{\circ} 38^{\prime} \mathrm{N}$ & $53^{\circ} 25^{\prime} \mathrm{W}$ & $\mathrm{N}$ \\
\hline $1 \mathrm{IB} 26004$ & $70^{\circ} 30^{\prime} \mathrm{N}$ & $52^{\circ} 08^{\prime} \mathrm{W}$ & NE & 185 & 120 & 0001.21 & 360010 & $7030-03$ & 1IB40009 & $70^{\circ} 38^{\prime} \mathrm{N}$ & $53^{\circ} 30^{\prime} \mathrm{W}$ & Iv \\
\hline 1IB26005 & $70^{\circ} 31^{\prime} \mathrm{N}$ & $52^{\circ} 08^{\prime} \mathrm{W}$ & NE & 180 & 130 & 0002.00 & 360010 & 7030-03 & 1 IB40010 & $70^{\circ} 38^{\prime} \mathrm{N}$ & $53^{\circ} 28^{\prime} \mathrm{W}$ & $\mathrm{NE}$ \\
\hline 1IB26006 & $70^{\circ} 32^{\prime} \mathrm{N}$ & $52^{\circ} 07^{\prime} \mathrm{W}$ & E & 130 & 100 & 0000.24 & 700010 & $7030-03$ & 1IB40011 & $70^{\circ} 39 \cdot \mathrm{N}$ & $53^{\circ} 28^{\prime} \mathrm{W}$ & $\mathrm{NE}$ \\
\hline 1 IB 26007 & $70^{\circ} 32^{\prime} \mathrm{N}$ & $52^{\circ} 07^{\prime} \mathrm{W}$ & 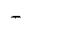 & 125 & 110 & 0000.18 & 700010 & $7030-03$ & 1 IB 40012 & $70^{\circ} 39^{\prime} \mathrm{N}$ & $53^{\circ} 30^{\prime} \mathrm{W}$ & $\mathrm{N}$ \\
\hline 1IB27001 & $70^{\circ} 32^{\prime} \mathrm{N}$ & $52^{\circ} 03^{\prime} \mathrm{W}$ & $\mathbf{N}$ & 120 & 75 & 0000.15 & 750010 & $7030-03$ & IIB40013 & $70^{\circ} 39 \cdot \mathrm{N}$ & $53^{\circ} 32^{\prime} \mathrm{W}$ & $\mathrm{N}$ \\
\hline 1IB27002 & $70^{\circ} 32^{\prime} \mathrm{N}$ & $52^{\circ} 08^{\prime} \mathrm{W}$ & $\mathrm{N}$ & 120 & 70 & 0000.15 & 640010 & $7030-03$ & 1 IB40014 & $70^{\circ} 38^{\prime} \mathrm{N}$ & $53^{\circ} 33^{\prime} \mathrm{W}$ & $\mathrm{NW}$ \\
\hline 1 IB27003 & $70^{\circ} 30^{\prime} \mathrm{N}$ & $52^{\circ} 13^{\prime} \mathrm{W}$ & NE & 200 & 15 & 0017.45 & 363312 & $7030-03$ & 1IB40015 & $70^{\circ} 38^{\prime} \mathrm{N}$ & $53^{\circ} 35^{\prime} \mathrm{W}$ & $\mathrm{N}$ \\
\hline 1 IB27004 & $70^{\circ} 32^{\prime} \mathrm{N}$ & $52^{\circ} 14^{\prime} \mathrm{W}$ & $\mathrm{E}$ & 168 & 130 & 0003.44 & 360010 & $7030-03$ & 1IB40016 & $70^{\circ} 38^{\prime} \mathrm{N}$ & $53^{\circ} 37^{\prime} \mathrm{W}$ & E \\
\hline 1IB27005 & $70^{\circ} 33^{\prime} \mathrm{N}$ & $52^{\circ} 11 \% \mathrm{~W}$ & $\mathrm{SE}$ & 135 & 130 & 0000.16 & 700010 & $7030-03$ & 1 IB 40017 & $70^{\circ} 38^{\prime} \mathrm{N}$ & $53^{\circ} 37^{\prime} \mathrm{V}$ & $\mathrm{E}$ \\
\hline 1IB28001 & $70^{\circ} 34 \cdot \mathrm{N}$ & $52^{\circ} 09^{\prime} \mathrm{V}$ & - & 130 & 120 & 0000.23 & 700010 & $7030-03$ & IIB40018 & $70^{\circ} 39^{\prime} \mathrm{N}$ & $53^{\circ} 38^{\prime}$ & NW \\
\hline $1 \mathrm{IB} 28002$ & $70^{\circ} 34^{\prime} \mathrm{N}$ & $52^{\circ} 09^{\prime}$ & - & 130 & 125 & 0000.18 & 700010 & $7030-03$ & IIB40019 & $70^{\circ} 38^{\prime} \mathrm{N}$ & $53^{\circ} 39^{\prime \prime}$ & $\mathrm{W}$ \\
\hline 1IB28003 & $70^{\circ} 33^{\prime} \mathrm{N}$ & $52^{\circ} 14^{\prime} \mathrm{V}$ & N & 168 & 60 & 0006.55 & 363312 & $7030-03$ & IIB40020 & $70^{\circ} 38^{\prime} \mathrm{N}$ & $53^{\circ} 40^{\prime} \mathrm{W}$ & W \\
\hline 1IB28004 & $70^{\circ} 34^{\prime} \mathrm{N}$ & $52^{\circ} 16^{\prime} \mathrm{V}$ & $\mathrm{N}$ & 155 & 95 & 0001.64 & 363312 & $7030-03$ & 1 IB 40021 & $70^{\circ} 38^{\prime} \mathrm{N}$ & $53^{\circ} 42^{\prime} \mathrm{V}$ & N \\
\hline 1IB29001 & $70^{\circ} 34^{\prime} \mathrm{N}$ & $52^{\circ} 17^{\prime} \mathrm{W}$ & NW & 145 & 130 & 0000.11 & 770010 & $7030-03$ & 1 IB 40022 & $70^{\circ} 40^{\prime} \mathrm{N}$ & $53^{\circ} 45^{\prime} \mathrm{W}$ & $\mathrm{NW}$ \\
\hline 1IB29002 & $70^{\circ} 34^{\prime} \mathrm{N}$ & $52^{\circ} 16^{\prime}$ w & $\mathrm{V}$ & 150 & 100 & 0000.92 & 363312 & $7030-03$ & 1IB40023 & $70^{\circ} 39 \cdot \mathrm{N}$ & $53^{\circ} 45^{\prime}$ & $\mathrm{W}$ \\
\hline 1IB29003 & $70^{\circ} 33^{\prime} \mathrm{N}$ & $52^{\circ} 16^{\prime} \mathrm{W}$ & SW & 168 & 95 & 0003.92 & 360112 & $7030-03$ & 1IB40024 & $70^{\circ} 38^{\prime} \mathrm{N}$ & $53^{\circ} 48^{\prime} \mathrm{W}$ & NW \\
\hline 1IB29004 & $70^{\circ} 33^{\prime} \mathrm{N}$ & $52^{\circ} 20^{\prime} \mathrm{W}$ & $\mathrm{N}$ & 200 & 50 & 0034.30 & 513016 & $7030-03$ & 1IB40025 & $70^{\circ} 37 \cdot \mathrm{N}$ & $53^{\circ} 50^{\prime} \mathrm{V}$ & NW \\
\hline 1IB29005 & $70^{\circ} 33^{\prime} \mathrm{N}$ & $52^{\circ} 27 \cdot \mathrm{W}$ & $\mathrm{E}$ & 195 & 150 & 0002.05 & 263010 & $7030-03$ & 1 IB40026 & $70^{\circ} 40^{\prime} \mathrm{N}$ & $54^{\circ} 13^{\prime} \mathrm{W}$ & $\mathrm{NE}$ \\
\hline 1IB29006 & $70^{\circ} 34^{\prime} \mathrm{N}$ & $52^{\circ} 24^{\prime} \mathrm{V}$ & $\mathrm{N}$ & 175 & 165 & 0000.10 & 700010 & $7030-03$ & 1 IB43001 & $70^{\circ} 40^{\prime} \mathrm{N}$ & $54^{\circ} 14^{\prime} \mathrm{W}$ & $\mathrm{W}$ \\
\hline 1IB29007 & $70^{\circ} 35^{\prime} \mathrm{N}$ & $52^{\circ} 27^{\prime} \mathrm{W}$ & NE & 165 & 105 & 0001.00 & 263010 & $7030-03$ & $1 \mathrm{IC} 01001$ & $70^{\circ} 55^{\prime} \mathrm{N}$ & $52^{\circ} 08^{\prime \prime ~}$ & W \\
\hline 1 IB 30001 & $70^{\circ} 35^{\prime} \mathrm{N}$ & $52^{\circ} 28^{\prime} \mathrm{W}$ & NW & 165 & 100 & 0001.61 & 263012 & $7030-03$ & $1 \mathrm{IC} 01002$ & $70^{\circ} 54^{\prime} \mathrm{N}$ & $52^{\circ} 07^{\prime} \mathrm{W}$ & $\mathrm{SW}$ \\
\hline 1 IB 30002 & $70^{\circ} 35^{\prime} \mathrm{N}$ & $52^{\circ} 30^{\prime} \mathrm{W}$ & $v$ & 145 & 115 & 0000.33 & 600010 & $7030-03$ & $1 \mathrm{IC} 01003$ & $70^{\circ} 54^{\prime} \mathrm{N}$ & $52^{\circ} 05^{\prime} \mathrm{W}$ & $\mathrm{SW}$ \\
\hline 1IB30003 & $70^{\circ} 35^{\prime} \mathrm{N}$ & $52^{\circ} 33^{\prime} \mathrm{W}$ & $\mathrm{N}$ & 200 & 40 & 0062.18 & 263012 & $7030-03$ & IIC01004 & $70^{\circ} 54^{\prime} \mathrm{N}$ & $52^{\circ} 03^{\prime} \mathrm{W}$ & $\mathrm{SE}$ \\
\hline 1 IB 30004 & $70^{\circ} 35^{\prime} \mathrm{N}$ & $52^{\circ} 35^{\prime} \mathrm{W}$ & $\mathrm{NE}$ & 150 & 80 & 0001.54 & 263012 & $7030-03$ & $1 \mathrm{ICO} 01005$ & $70^{\circ} 55^{\prime} \mathrm{N}$ & $52^{\circ} 00^{\prime} \mathrm{V}$ & $\mathrm{s}$ \\
\hline 1IB30005 & $70^{\circ} 34^{\prime} \mathrm{N}$ & $52^{\circ} 30^{\prime} \mathrm{W}$ & - & 170 & 140 & 0000.37 & 260010 & $7030-02$ & $1 \mathrm{IC01006}$ & $70^{\circ} 55^{\prime} \mathrm{N}$ & $51^{\circ} 58^{\prime} \mathrm{W}$ & NE \\
\hline 1IB30006 & $70^{\circ} 38^{\prime} \mathrm{N}$ & $52^{\circ} 40^{\prime} \mathrm{W}$ & s & 185 & 180 & 0000.25 & 700010 & $7030-02$ & $1 \mathrm{IC} 01007$ & $70^{\circ} 53^{\prime} \mathrm{N}$ & $52^{\circ} 02^{\prime} \mathrm{W}$ & $\mathrm{NW}$ \\
\hline 1IB31001 & $70^{\circ} 39^{\prime} \mathrm{N}$ & $52^{\circ} 38^{\prime} \mathrm{W}$ & $\mathrm{N}$ & 150 & 115 & 0000.84 & 640111 & $7030-02$ & $1 \mathrm{IC} 01008$ & $70^{\circ} 54^{\prime} \mathrm{N}$ & $52^{\circ} 00^{\prime} \mathrm{W}$ & $\mathrm{NW}$ \\
\hline 1IB 31002 & $70^{\circ} 39^{\prime} \mathrm{N}$ & $52^{\circ} 40^{\prime} \mathrm{W}$ & $\mathrm{NE}$ & 190 & 180 & 0000.15 & 700010 & $7030-02$ & $1 \mathrm{IC} 01009$ & $70^{\circ} 56^{\prime} \mathrm{N}$ & $51^{\circ} 54^{\prime} \mathrm{W}$ & $\mathrm{s}$ \\
\hline 1IB32001 & $70^{\circ} 39^{\prime} \mathrm{N}$ & $52^{\circ} 41^{\prime} \mathrm{W}$ & $\mathrm{N}$ & 160 & 120 & 0000.63 & 643111 & $7030-02$ & $1 \mathrm{IC} 01010$ & $70^{\circ} 53^{\prime} \mathrm{N}$ & $51^{\circ} 54^{\prime} \mathrm{W}$ & su \\
\hline 1IB32002 & $70^{\circ} 39^{\prime} \mathrm{N}$ & $52^{\circ} 41^{\prime} \mathrm{W}$ & $\mathrm{N}$ & 190 & 180 & 0000.16 & 700010 & $7030-02$ & $1 \mathrm{ICO} 01011$ & $70^{\circ} 53 \cdot \mathrm{N}$ & $51^{\circ} 50^{\prime} \mathrm{W}$ & $\mathrm{s}$ \\
\hline 1IB32003 & $70^{\circ} 39^{\prime} \mathrm{N}$ & $52^{\circ} 43^{\prime} \mathrm{W}$ & $\mathbf{N}$ & & 140 & 0000.60 & 600012 & $7030-02$ & $1 \mathrm{IC} 01012$ & $70^{\circ} 53^{\prime} \mathrm{N}$ & $51^{\circ} 48^{\prime} \mathrm{W}$ & E \\
\hline 1IB 33001 & $70^{\circ} 41^{\prime} \mathrm{N}$ & $52^{\circ} 49^{\prime} \mathrm{W}$ & NW & 170 & 125 & 0000.38 & 600010 & $7030-02$ & $1 \mathrm{ICO} 01013$ & $70^{\circ} 54^{\prime} \mathrm{N}$ & $51^{\circ} 47^{\prime} \mathrm{W}$ & $\mathrm{s}$ \\
\hline $1 \mathrm{IB} 33002$ & $70^{\circ} 40^{\prime} \mathrm{N}$ & $52^{\circ} 44^{\prime} \mathrm{W}$ & w & 170 & 145 & 0000.18 & 600010 & $7030-02$ & $1 \mathrm{IC} 01014$ & $70^{\circ} 53 \cdot \mathrm{N}$ & $51^{\circ} 46^{\prime} \mathrm{W}$ & $\mathrm{s}$ \\
\hline 1 IB 33003 & $70^{\circ} 40^{\prime} \mathrm{N}$ & $52^{\circ} 45^{\prime} \mathrm{W}$ & NW & 190 & 115 & 0000.49 & 260010 & 7030-02 & $1 \mathrm{ICO} 1015$ & $70^{\circ} 53^{\prime} \mathrm{N}$ & $51^{\circ} 45^{\prime} \mathrm{W}$ & W \\
\hline 1IB33004 & $70^{\circ} 41^{\prime} \mathrm{N}$ & $52^{\circ} 47 . \mathrm{W}$ & $\mathbf{N}$ & 206 & 55 & 0011.11 & 263312 & $7030-02$ & 1IC02001 & $70^{\circ} 58^{\prime} \mathrm{N}$ & $51^{\circ} 28^{\prime} \mathrm{W}$ & $\mathrm{SE}$ \\
\hline 1IB33005 & $70^{\circ} 41^{\prime} \mathrm{N}$ & $52^{\circ} 50^{\prime} \mathrm{W}$ & $\mathrm{N}$ & 200 & 40 & 0011.68 & 263312 & $7030-02$ & $1 \mathrm{ICO} 002$ & $70^{\circ} 58^{\prime} \mathrm{N}$ & $51^{\circ} 25^{\prime} \mathrm{W}$ & $\mathrm{SW}$ \\
\hline 1IB33006 & $70^{\circ} 41^{\prime} \mathrm{N}$ & $52^{\circ} 52^{\prime} \mathrm{W}$ & NE & 210 & 95 & 0004.86 & 643312 & $7030-02$ & $1 \mathrm{IC} 02003$ & $70^{\circ} 56^{\prime} \mathrm{N}$ & $51^{\circ} 23^{\prime} \mathrm{W}$ & $\mathrm{SW}$ \\
\hline $11 \mathrm{~B} 33007$ & $70^{\circ} 43^{\prime} \mathrm{N}$ & $52^{\circ} 52^{\prime} \mathrm{W}$ & N & 150 & 110 & 0000.23 & 600010 & $7030-02$ & $1 \mathrm{IC} 03001$ & $70^{\circ} 56^{\prime} \mathrm{N}$ & $51^{\circ} 20^{\prime} \mathrm{W}$ & SE \\
\hline 1IB34001 & $70^{\circ} 42^{\prime} \mathrm{N}$ & $52^{\circ} 55^{\prime} \mathrm{W}$ & $\mathbb{N}$ & 210 & 90 & 0004.07 & 513312 & 7030-02 & $1 \mathrm{ICO} 03002$ & $70^{\circ} 56^{\prime} \mathrm{N}$ & $51^{\circ} 18^{\prime} \mathrm{W}$ & $\mathrm{SE}$ \\
\hline 1IB 34002 & $70^{\circ} 42^{\prime} \mathrm{N}$ & $52^{\circ} 57 \cdot \mathrm{W}$ & NE & 210 & 120 & 0001.65 & 570012 & $7030-02$ & $1 \mathrm{IC} 03003$ & $70^{\circ} 56^{\prime} \mathrm{N}$ & $51^{\circ} 23^{\prime \prime}$ & $\mathrm{SE}$ \\
\hline 1 IB 34003 & $70^{\circ} \cdot 43^{\prime} \mathrm{N}$ & $52^{\circ} 58^{\prime} \mathrm{W}$ & $\mathbf{N}$ & 180 & 100 & 0001.11 & 263112 & $7030-02$ & $1 \mathrm{IC06001}$ & $70^{\circ} 57 \cdot \mathrm{N}$ & $50^{\circ} 47 \cdot \mathrm{W}$ & $\mathrm{SW}$ \\
\hline 1IB34004 & $70^{\circ} 43^{\prime} \mathrm{N}$ & $53^{\circ} 00^{\prime} \mathrm{W}$ & $\mathbf{N}$ & 210 & 80 & 0005.00 & 263112 & $7030-02$ & $1 \mathrm{IC} 06002$ & $70^{\circ} 56^{\prime} \mathrm{N}$ & $50^{\circ} 44^{\prime \prime}$ & $\mathrm{SW}$ \\
\hline 1 IB 35001 & $70^{\circ} 43^{\prime} \mathrm{N}$ & $53^{\circ} 02^{\prime} \mathrm{V}$ & NE & 185 & 60 & 0005.04 & 533012 & $7030-02$ & $1 \mathrm{IC} 07001$ & $70^{\circ} 51 \cdot \mathrm{N}$ & $50^{\circ} 44^{\prime} \mathrm{V}$ & SW \\
\hline $1 \mathrm{IB} 35002$ & $70^{\circ} 44^{\prime} \mathrm{N}$ & $53^{\circ} 05^{\prime} \mathrm{V}$ & $\mathrm{N}$ & 170 & 100 & 0001.67 & 643012 & $7030-02$ & $1 \mathrm{IC} 07002$ & $70^{\circ} 49^{\prime} \mathrm{N}$ & $50^{\circ} 27^{\prime} \mathrm{W}$ & SW \\
\hline 1 IB 36001 & $70^{\circ} 44^{\prime} \mathrm{N}$ & $53^{\circ} 08^{\prime} \mathrm{V}$ & $\mathrm{N}$ & 105 & 100 & 0000.06 & 790010 & 7030 & 1IC08001 & $70^{\circ} 47^{\prime} \mathrm{N}$ & $50^{\circ} 37^{\prime} \mathrm{W}$ & SW \\
\hline 1IB 36002 & $70^{\circ} 43^{\prime} \mathrm{N}$ & $53^{\circ} 10^{\circ} \mathrm{W}$ & $\mathbf{N}$ & 180 & 110 & 0000.52 & 770010 & $7030-02$ & 1 IC09001 & $70^{\circ} 45^{\prime} \mathrm{N}$ & $50^{\circ} 38^{\prime} \mathrm{W}$ & $\mathrm{SW}$ \\
\hline 1 IB 36003 & $70^{\circ} 43^{\prime} \mathrm{N}$ & $53^{\circ} 10^{\prime} \mathrm{W}$ & NW & 180 & 135 & 0000.17 & 770010 & $7030-02$ & 1IC09002 & $70^{\circ} 44^{\prime} \mathrm{N}$ & $50^{\circ} 35^{\prime} \mathrm{W}$ & $\mathrm{SW}$ \\
\hline 1IB36004 & $70^{\circ} 42^{\prime} \mathrm{N}$ & $53^{\circ} 10^{\prime} \mathrm{W}$ & W & 185 & 180 & 0000.08 & 700010 & $7030-02$ & 1IC09003 & $70^{\circ} 41 \cdot \mathrm{N}$ & $50^{\circ} 32^{\prime} \mathrm{W}$ & SW \\
\hline 1IB36005 & $70^{\circ} 42 \cdot \mathrm{N}$ & $53^{\circ} 12^{\prime} \mathrm{W}$ & N & 185 & 115 & 0003.64 & 513011 & $7030-02$ & $1 \mathrm{IC} 10001$ & $70^{\circ} 44^{\prime} \mathrm{N}$ & $50^{\circ} 57^{\prime} \mathrm{W}$ & $\mathrm{NE}$ \\
\hline 1 IB 36006 & $70^{\circ} 43, \mathrm{~N}$ & $53^{\circ} 14^{\prime}$ & N & 150 & 135 & 0000.19 & 513011 & $7030-02$ & 1 IC10002 & $70^{\circ} 44^{\prime} \mathrm{N}$ & $51^{\circ} 00^{\prime \prime} \mathrm{W}$ & $\mathrm{N}$ \\
\hline 1IB37001 & $70^{\circ} 42, \mathrm{~N}$ & $53^{\circ} 17^{\prime} \mathrm{W}$ & $\mathbf{N}$ & 125 & 25 & 0003.22 & 613312 & $7030-02$ & $1 \mathrm{IC} 10003$ & $70^{\circ} 45, \mathrm{~N}$ & $51^{\circ} 11^{\prime} \mathrm{W}$ & $\mathrm{N}$ \\
\hline & $70^{\circ} 42^{\prime} \mathrm{N}$ & $53^{\circ} 19^{\prime} \mathrm{W}$ & NE & & 120 & 0000.08 & 770010 & $7030-02$ & 1 IC10004 & $70^{\circ} 45, \mathrm{~N}$ & $51^{\circ} 13^{\prime} \mathrm{W}$ & $\mathrm{N}$ \\
\hline 1 IB 37003 & $70^{\circ} 42^{\prime} \mathrm{N}$ & $53^{\circ} 20^{\prime} \mathrm{W}$ & $\mathrm{NE}$ & & 130 & 0000.10 & 670010 & 7030 & $1 \mathrm{ICl} 10005$ & $70^{\circ} 44^{\prime} \mathrm{N}$ & $51^{\circ} 17^{\prime} \mathrm{W}$ & $\mathrm{NW}$ \\
\hline 1IB40001 & $70^{\circ} 41^{\prime} \mathrm{N}$ & $53^{\circ} 18^{\prime} \mathrm{W}$ & SW & 175 & 150 & 0000.16 & 700010 & $7030-02$ & $1 \mathrm{IC} 10006$ & $70^{\circ} 44^{\prime} \mathrm{N}$ & $51^{\circ} 20^{\prime} \mathrm{W}$ & $\mathrm{NW}$ \\
\hline $1 \mathrm{IB} 40002$ & $70^{\circ} 41 \cdot \mathrm{N}$ & $53^{\circ} 21^{\prime} \mathrm{V}$ & NW & 160 & 80 & 0010.90 & 513112 & $7030-02$ & $1 \mathrm{IC} 11001$ & $70^{\circ} 41^{\prime} \mathrm{N}$ & $51^{\circ} 39^{\prime} \mathrm{W}$ & N \\
\hline 1 IB 40003 & $70^{\circ} 39^{\prime} \mathrm{N}$ & $53^{\circ} 21^{\prime} \mathrm{W}$ & E & 190 & 130 & 0000.33 & 670110 & $7030-02$ & 1 IC11002 & $70^{\circ} 42 \cdot \mathrm{N}$ & $51^{\circ} 41^{\prime} \mathrm{W}$ & $\mathrm{N}$ \\
\hline 1IB40004 & $70^{\circ} 41 \cdot \mathrm{N}$ & $53^{\circ} 23^{\prime} \mathrm{W}$ & $\mathbf{N}$ & 130 & 105 & 0000.30 & 670010 & $7030-02$ & $1 \mathrm{IC} 11003$ & $70^{\circ} 43^{\prime} \mathrm{N}$ & $51^{\circ} 47^{\prime} \mathrm{W}$ & \\
\hline
\end{tabular}

\begin{tabular}{|c|c|c|c|c|}
\hline \multirow[b]{2}{*}{ IIGH } & \multirow[b]{2}{*}{ LOW } & \multicolumn{3}{|c|}{ Mo } \\
\hline & & AREA & CODE & MAP \\
\hline & 105 & 1.03 & 0112 & 70 \\
\hline 180 & 130 & 0000.34 & 650112 & $7030-02$ \\
\hline 190 & 125 & 0000.62 & 653112 & $7030-02$ \\
\hline 170 & 85 & 0012.57 & 513112 & $7030-02$ \\
\hline 185 & 150 & 0001.14 & 700010 & $7030-02$ \\
\hline 150 & 100 & 0001.42 & 670112 & $7030-02$ \\
\hline 140 & 110 & 0000.23 & 600010 & $7030-02$ \\
\hline 150 & 100 & 0002.13 & 270112 & $7030-02$ \\
\hline 175 & 100 & 0001.62 & 270112 & $7030-02$ \\
\hline 185 & 125 & 0000.38 & 750110 & $7030-02$ \\
\hline 190 & 170 & 0008. & 513112 & $7030-02$ \\
\hline 165 & 115 & 0000.35 & 770110 & $7030-02$ \\
\hline 155 & 140 & 0000.13 & 700010 & $7030-02$ \\
\hline 160 & 100 & 0000.93 & 653212 & $7030-02$ \\
\hline 190 & 95 & 0001.87 & 673312 & $7030-02$ \\
\hline 190 & 115 & 0000.48 & 670012 & $7030-02$ \\
\hline 170 & 70 & 0004.57 & 513212 & $7030-02$ \\
\hline 155 & 95 & 0002.70 & 260012 & $7030-01$ \\
\hline 155 & 145 & 0000.56 & 260010 & $7030-01$ \\
\hline 165 & 80 & 0008.30 & 263112 & $7030-01$ \\
\hline 150 & 115 & 0001 & 263112 & $7030-01$ \\
\hline 100 & 95 & 0000.10 & 700010 & $7030-01$ \\
\hline 105 & 95 & 0001.71 & 700010 & $7030-01$ \\
\hline 155 & 140 & 0000.20 & 700010 & $7030-03$ \\
\hline 140 & 130 & 0000.70 & 300112 & $7030-03$ \\
\hline 168 & 110 & & 360 & $7030-03$ \\
\hline 155 & 125 & 0000.43 & 360112 & $7030-03$ \\
\hline 140 & 75 & 0007.03 & 313111 & $7030-03$ \\
\hline 147 & 130 & 0000 . & 300112 & $7030-03$ \\
\hline 125 & 130 & & 770112 & \\
\hline 12 & 90 & & & \\
\hline 155 & 85 & 0003.22 & 360111 & $7030-03$ \\
\hline 145 & 105 & 0000. & 300112 & $7030-03$ \\
\hline 148 & 120 & & 300 & $7030-03$ \\
\hline 148 & 85 & & 363 & -03 \\
\hline 115 & 95 & & 300 & \\
\hline 11 & 10 & & 700 & $7030-03$ \\
\hline 115 & $+\infty$ & 000 & 600 & $7030-03$ \\
\hline 135 & 110 & & 363 & $0-04$ \\
\hline 145 & 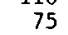 & & & 04 \\
\hline 13 & 90 & & 360 & -04 \\
\hline 13 & 95 & & 360 & $7030-04$ \\
\hline 11 & 90 & & 30 & $7030-04$ \\
\hline 125 & 110 & o & 60 & $7030-04$ \\
\hline 180 & & & 163 & $7030-04$ \\
\hline 100 & & & 163 & $7030-04$ \\
\hline 180 & & & 164 & $7030-04$ \\
\hline 180 & 55 & & 163 & \\
\hline 180 & & 0037 & 163 & $7030-04$ \\
\hline 18 & & 00 & 165 & $7030-04$ \\
\hline 180 & & & & \\
\hline 180 & 50 & o & 16 & 7 \\
\hline 0 & & & 700 & 70 \\
\hline 100 & 95 & 0000 . & 700010 & $7030-04$ \\
\hline 90 & 90 & 0000.11 & 700010 & $7030-04$ \\
\hline 95 & 0 & 0. & 700010 & $7030-04$ \\
\hline 105 & 100 & & 700 & 70 \\
\hline 100 & 95 & 0000.09 & 700010 & $7030-04$ \\
\hline 95 & 70 & 000 & 700010 & $7030-03$ \\
\hline 100 & 95 & 000 & 700010 & $7030-03$ \\
\hline 130 & 115 & 0000.30 & 780010 & $7030-03$ \\
\hline
\end{tabular}




\begin{tabular}{|c|c|c|c|c|c|c|c|c|}
\hline \multirow[b]{2}{*}{ CODE } & \multirow[b]{2}{*}{ LAT } & \multirow[b]{2}{*}{ LONG } & \multirow[b]{2}{*}{ ORI } & \multirow[b]{2}{*}{ HIGH } & \multirow[b]{2}{*}{ LOW } & \multicolumn{3}{|c|}{ м0 } \\
\hline & & & & & & AREA & CODE & MAP \\
\hline 11004 & $70^{\circ} 42^{\prime} \mathrm{N}$ & $51^{\circ} 43^{\prime} \mathrm{W}$ & NW & 100 & 95 & 0000.74 & 360010 & $7030-03$ \\
\hline c1100s & $70^{\circ} 42^{\prime} \mathrm{N}$ & $51^{\circ} 48^{\prime} \mathrm{W}$ & N & 120 & 85 & 0001.67 & 362112 & $7030-03$ \\
\hline $1 \mathrm{IC} 11006$ & $70^{\circ} 41^{\prime} \mathrm{N}$ & $51^{\circ} 49^{\prime} \mathrm{W}$ & $\mathrm{NE}$ & 115 & 95 & 0000.66 & 360010 & $7030-03$ \\
\hline 1IC11007 & $70^{\circ} 42^{\prime} \mathrm{N}$ & $51^{\circ} 51 / \mathrm{W}$ & $\mathrm{NE}$ & 115 & 100 & 0000.76 & 360010 & $7030-03$ \\
\hline 1IC1 1008 & $70^{\circ} 43^{\prime} \mathrm{N}$ & $51^{\circ} 52, \bar{w}$ & $\mathrm{NE}$ & 115 & 95 & 0000.41 & 780010 & $7030-03$ \\
\hline 1ID20001 & $70^{\circ} 59^{\prime} \mathrm{N}$ & $50^{\circ} 49^{\prime}$ & $\mathrm{NW}$ & 180 & & 0298.63 & 164111 & $7030-04$ \\
\hline 1ID22001 & $70^{\circ} 57^{\prime} \mathrm{N}$ & $51^{\circ} 17^{\prime} \mathrm{W}$ & $\mathrm{E}$ & 130 & 60 & 0003.43 & 360211 & $7030-04$ \\
\hline IID22002 & $70^{\circ} 57 \cdot \mathrm{N}$ & $51^{\circ} 19^{\prime} \mathrm{W}$ & $\begin{array}{l}\mathrm{E} \\
\mathrm{NE}\end{array}$ & 130 & 90 & 0002.27 & 303112 & $7030-04$ \\
\hline 1ID22003 & $70^{\circ} 57 \cdot \mathrm{N}$ & $51^{\circ} 19^{\prime} \mathrm{W}$ & $\mathrm{NE}$ & 130 & 100 & 0001.88 & 303111 & $7030-04$ \\
\hline 1ID22004 & $70^{\circ} 58^{\prime} \mathrm{N}$ & $51^{\circ} 22^{\prime} \mathrm{W}$ & N & 125 & 75 & 0003.27 & 360211 & $7030-04$ \\
\hline 1ID23001 & $70^{\circ} 58^{\prime} \mathrm{N}$ & $51^{\circ} 23^{\prime} \mathrm{W}$ & $\mathrm{NE}$ & 125 & 100 & 0001.70 & 300112 & $7030-04$ \\
\hline 1ID23002 & $70^{\circ} 58 \cdot \mathrm{N}$ & $51^{\circ} 24^{\prime} \mathrm{W}$ & $\mathrm{NE}$ & 125 & 100 & 0001.09 & 300112 & $7030-04$ \\
\hline 1TD23003 & $70^{\circ} 59 \cdot \mathrm{N}$ & $51^{\circ} 26^{\prime} \mathrm{W}$ & NE & 145 & 45 & 0002.46 & 360312 & $\begin{array}{r}7030-04 \\
7030\end{array}$ \\
\hline 1ID23004 & $70^{\circ} 59^{\prime} \mathrm{N}$ & $51^{\circ} 28^{\prime} \mathrm{W}$ & $\mathrm{NE}$ & 145 & 60 & 0002.09 & 360312 & $7030-04$ \\
\hline 1ID23005 & $70^{\circ} 59^{\prime N} \mathrm{~N}$ & $51^{\circ} 29^{\prime} \mathrm{W}$ & $\mathrm{NE}$ & 145 & 10 & 0002.00 & 360312 & $7030-04$ \\
\hline 1ID23006 & $70^{\circ} 59 \cdot \mathrm{N}$ & $51^{\circ} 31^{\prime} \mathrm{W}$ & N & 130 & 50 & 0003.10 & 360412 & $7030-04$ \\
\hline $11 D 23007$ & $71^{\circ} 00 \cdot \mathrm{N}$ & $51^{\circ} 33^{\prime \prime}$ & N & 145 & 70 & 0003.42 & 360312 & $7030-03$ \\
\hline 1ID23008 & $71^{\circ} 01^{\prime} \mathrm{N}$ & $51^{\circ} 37 \cdot \mathrm{W}$ & $\begin{array}{l}\text { N } \\
\text { N }\end{array}$ & 145 & & 0002.76 & 360412 & $7030-03$ \\
\hline 1ID23009 & $71^{\circ} 01$, & $51^{\circ} 42, \mathrm{~W}$ & $\mathrm{NE}$ & 120 & 50 & 0001.64 & 300010 & $7030-03$ \\
\hline 1ID23010 & $71^{\circ} 01{ }^{\prime} \mathrm{N}$ & $51^{\circ} 42, \mathrm{~W}$ & $\mathrm{NE}$ & 140 & 50 & 0002.25 & 360312 & $7030-03$ \\
\hline 1ID23011 & $71^{\circ} 01^{\prime} \mathrm{N}$ & $51^{\circ} 43, \mathrm{~W}$ & $\mathrm{NE}$ & 120 & 50 & 0001 & 360211 & $7030-03$ \\
\hline 1ID23012 & $71^{\circ} 02 \cdot \mathrm{N}$ & $51^{\circ} 46 \cdot \mathrm{W}$ & $\mathrm{N}$ & 140 & 130 & 0000.73 & 600010 & $7030-03$ \\
\hline 1ID23013 & $71^{\circ} 01^{\prime} \mathrm{N}$ & $51^{\circ} 46^{\prime} \mathrm{W}$ & $\mathrm{N}$ & $\begin{array}{l}140 \\
130\end{array}$ & 80 & 0004.25 & 360112 & $7030-03$ \\
\hline 1ID23014 & $71^{\circ} 01 \cdot \mathrm{N}$ & $51^{\circ} 50^{\prime} W$ & $\mathrm{NW}$ & & & 0000.54 & 300010 & $7030-03$ \\
\hline IID24001 & $71^{\circ} 01^{\prime N}$ & $51^{\circ} 43^{\prime} \mathrm{W}$ & SW & 140 & 120 & 0000.70 & 303112 & $7030-03$ \\
\hline 1ID24002 & $71^{\circ} 01^{\prime} \mathrm{N}$ & $51^{\circ} 42^{\prime} \mathrm{W}$ & $\mathrm{s}$ & 140 & 120 & 00 & 303 & -03 \\
\hline 1102 & $71^{\circ} 00^{\prime} \mathrm{N}$ & $51^{\circ} 40^{\prime} \mathrm{W}$ & sw & 145 & 110 & 0003.99 & 303112 & $7030-03$ \\
\hline IID24004 & $70^{\circ} 59 \cdot \mathrm{N}$ & $51^{\circ} 37^{\prime} \mathrm{W}$ & $\mathrm{su}$ & $\begin{array}{l}14 J \\
140\end{array}$ & 110 & 0003.86 & $\begin{array}{l}303112 \\
360112\end{array}$ & $7030-03$ \\
\hline 1ID24005 & $70^{\circ} 59 \cdot \mathrm{N}$ & $51^{\circ} 36^{\prime} \mathrm{W}$ & $\mathrm{s}$ & 140 & 130 & 0001.28 & 360112 & $7030-03$ \\
\hline 1ID24006 & $70^{\circ} 58^{\prime} \mathrm{N}$ & $51^{\circ} 33^{\prime} \mathrm{W}$ & SW & $\begin{array}{l}140 \\
145\end{array}$ & 50 & 0007.67 & 360311 & $7030-03$ \\
\hline $1 \mathrm{ID} 24$ & $70^{\circ} 57 \cdot \mathrm{N}$ & $51^{\circ} 31, \mathrm{~W}$ & S & 135 & 110 & 0001.79 & 300110 & $7030-03$ \\
\hline 1ID25001 & $70.53 \cdot \mathrm{N}$ & $51^{\circ} 45 \cdot \mathrm{W}$ & $\mathrm{NE}$ & 130 & 115 & $\begin{array}{l}3 \\
1\end{array}$ & 700112 & $7030-03$ \\
\hline 1ID25002 & $70^{\circ} 53$, N & $51^{\circ} 47 \cdot \mathrm{W}$ & NE & 135 & 105 & $\frac{1}{3}$ & 300110 & 0 \\
\hline 1ID25003 & $70^{\circ} 54^{\prime} \mathrm{N}$ & $51^{\circ} 47^{\prime} \mathrm{W}$ & $\mathrm{NE}$ & 130 & 115 & 00 & 300010 & $5-03$ \\
\hline 1ID25004 & $70^{\circ} 55^{\prime} \mathrm{N}$ & $51^{\circ} 48^{\prime} W$ & $\mathrm{~N}$ & 130 & 80 & 0000.71 & 360112 & $7030-03$ \\
\hline 1ID25005 & $70^{\circ} 55^{\prime N}$ & $51^{\circ} 49^{\prime} \mathrm{W}$ & $\mathrm{N}$ & 145 & 45 & 0002.51 & 360211 & $7030-03$ \\
\hline 1ID25006 & $70^{\circ} 54^{\prime} \mathrm{N}$ & $51^{\circ} 50^{\prime} \mathrm{W}$ & $\mathrm{N}$ & $\begin{array}{l}145 \\
135\end{array}$ & 85 & 00 & 643112 & $7030-03$ \\
\hline 1ID25007 & $70^{\circ} 54^{\prime} \mathrm{N}$ & $51^{\circ} 52, \mathrm{~W}$ & $\mathrm{~N}$ & $\begin{array}{l}133 \\
105\end{array}$ & $\begin{array}{l}83 \\
95\end{array}$ & 00 & $\begin{array}{l}643112 \\
640012\end{array}$ & $7030-03$ \\
\hline $1 \mathrm{ID} 250$ & 7005 & $51^{\circ} 51, \mathrm{~W}$ & $\mathrm{~W}$ & 146 & 60 & 0001.01 & 360010 & $7030-03$ \\
\hline 1ID25009 & $70.53 \cdot \mathrm{N}$ & $51052, \mathrm{~W}$ & NW & $\begin{array}{l}140 \\
148\end{array}$ & 100 & 0000.52 & 300010 & $7030-03$ \\
\hline 1ID25010 & $70^{\circ} 56^{\prime} \mathrm{N}$ & $51053, \mathrm{~W}$ & $\mathrm{SE}$ & 110 & 95 & 0 & 300010 & $7030-03$ \\
\hline 1ID25011 & $70056, \mathrm{~N}$ & $51052, \mathrm{~V}$ & $\mathrm{~N}$ & & & & 700010 & -03 \\
\hline 1ID25012 & $70^{\circ} 57 \cdot \mathrm{N}$ & $51053, \mathrm{~W}$ & $\mathbf{N}$ & 155 & 40 & & 300012 & $7030-03$ \\
\hline 1ID25013 & $70^{\circ} 56^{\prime} \mathrm{N}$ & $51^{\circ} 54^{\prime} \mathrm{W}$ & $\mathrm{NE}$ & 158 & 145 & 0000.30 & 300012 & $7030-03$ \\
\hline 1ID25014 & $70^{\circ} 56^{\prime} \mathrm{N}$ & $51.55 \% \mathrm{~W}$ & $\begin{array}{l}\mathrm{NE} \\
\mathrm{N}\end{array}$ & $\begin{array}{l}138 \\
158\end{array}$ & $\begin{array}{l}143 \\
135\end{array}$ & 0000.76 & $\begin{array}{l}300012 \\
300012\end{array}$ & $\begin{array}{l}-03 \\
-03 \\
-03\end{array}$ \\
\hline & $70^{\circ} 56^{\prime} \mathrm{N}$ & $51^{\circ} 58, \mathrm{~W}$ & $\mathrm{~N}$ & 150 & 60 & (1) & 360010 & \\
\hline & & W & $\mathrm{NE}$ & 1 & 145 & & & -03 \\
\hline & $70^{\circ} 57$ & $52^{\circ} 02^{\prime} \mathrm{W}$ & N & 160 & 135 & 0000.08 & 700010 & $7030-03$ \\
\hline 1ID25018 & $70^{\circ} 57 \times \mathrm{N}$ & $52^{\circ} 03, \mathrm{~W}$ & NW & 150 & 115 & 0000.07 & 640010 & $7030-03$ \\
\hline & $70^{\circ} 57 \cdot \mathrm{N}$ & $04 \%$ & $\mathrm{NW}$ & 14 & 60 & 000 & 640112 & $7030-03$ \\
\hline & 7 & $\mathrm{~W}$ & w & 16 & 55 & & 360 & $7030-03$ \\
\hline 1 ID2502 & 70055 & $52^{\circ} 06^{\prime} \mathrm{W}$ & W & 15 & 120 & 0000.72 & 300110 & $7030-03$ \\
\hline 1ID25022 & $70^{\circ} 55^{\prime N}$ & $52^{\circ} 07, \mathrm{~W}$ & NW & 125 & 50 & 0000.34 & 640012 & $7030-03$ \\
\hline
\end{tabular}




\section{ATLAS SHEETS}
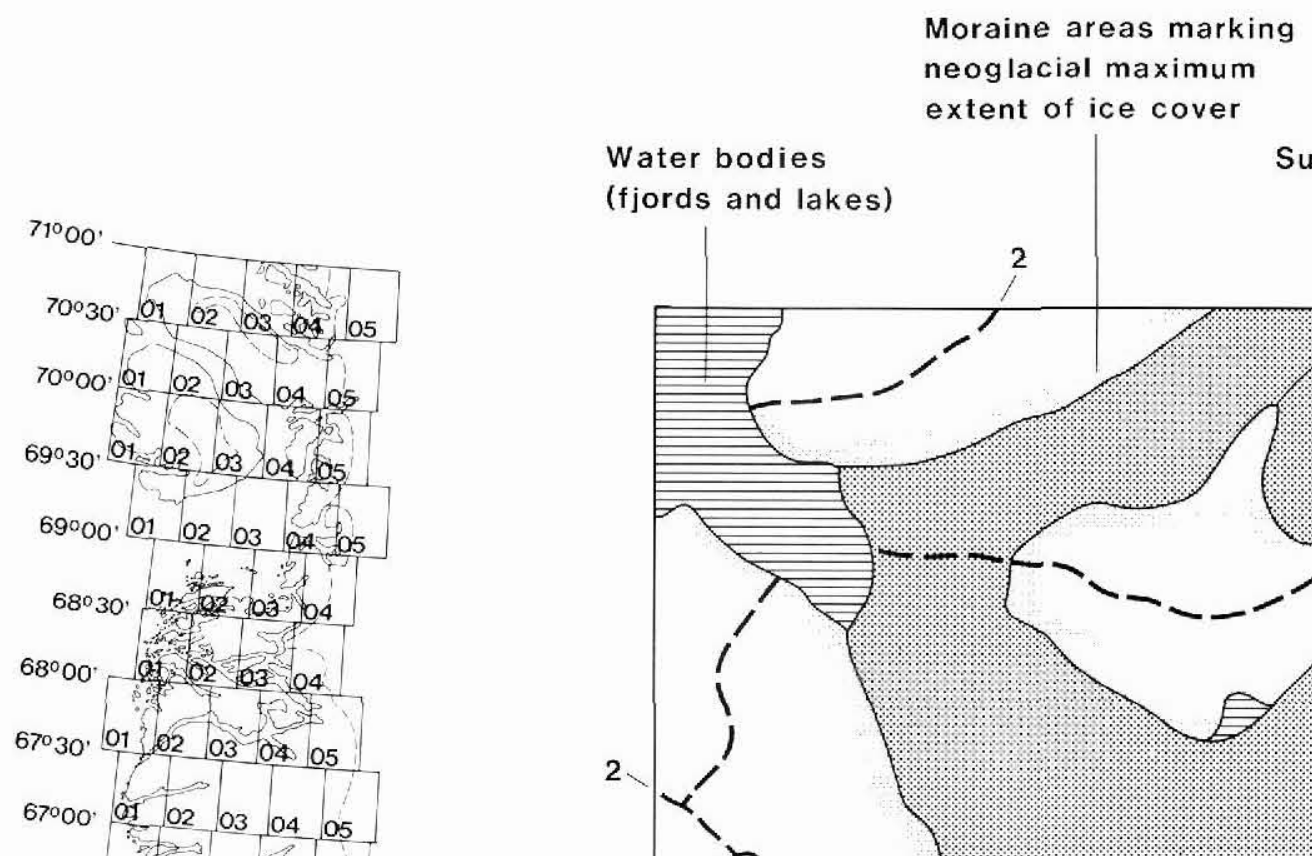

Water bodies

(fjords and lakes)

extent of ice cover

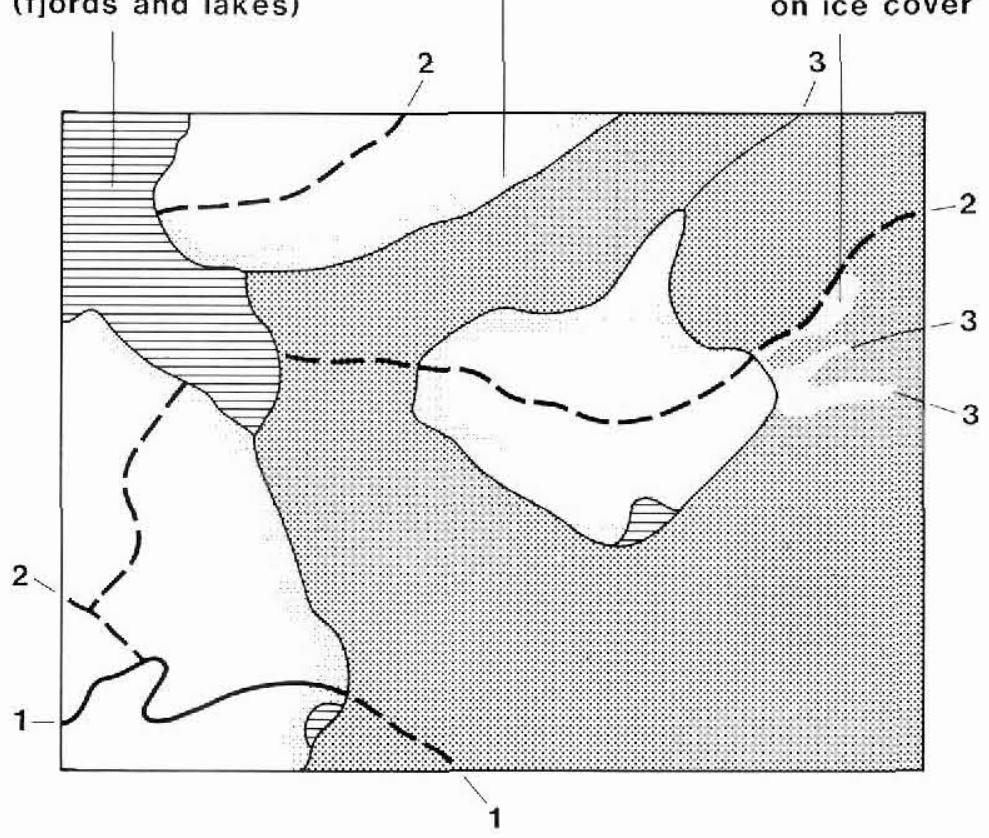

1. Limit of drainage areas between fjords; continuation on ice surface dashed.

2. Limit of hydrological basin areas and their continuation on ice surface.

3. Subdivision of ice cover into sectors.

Index of map sheets. 


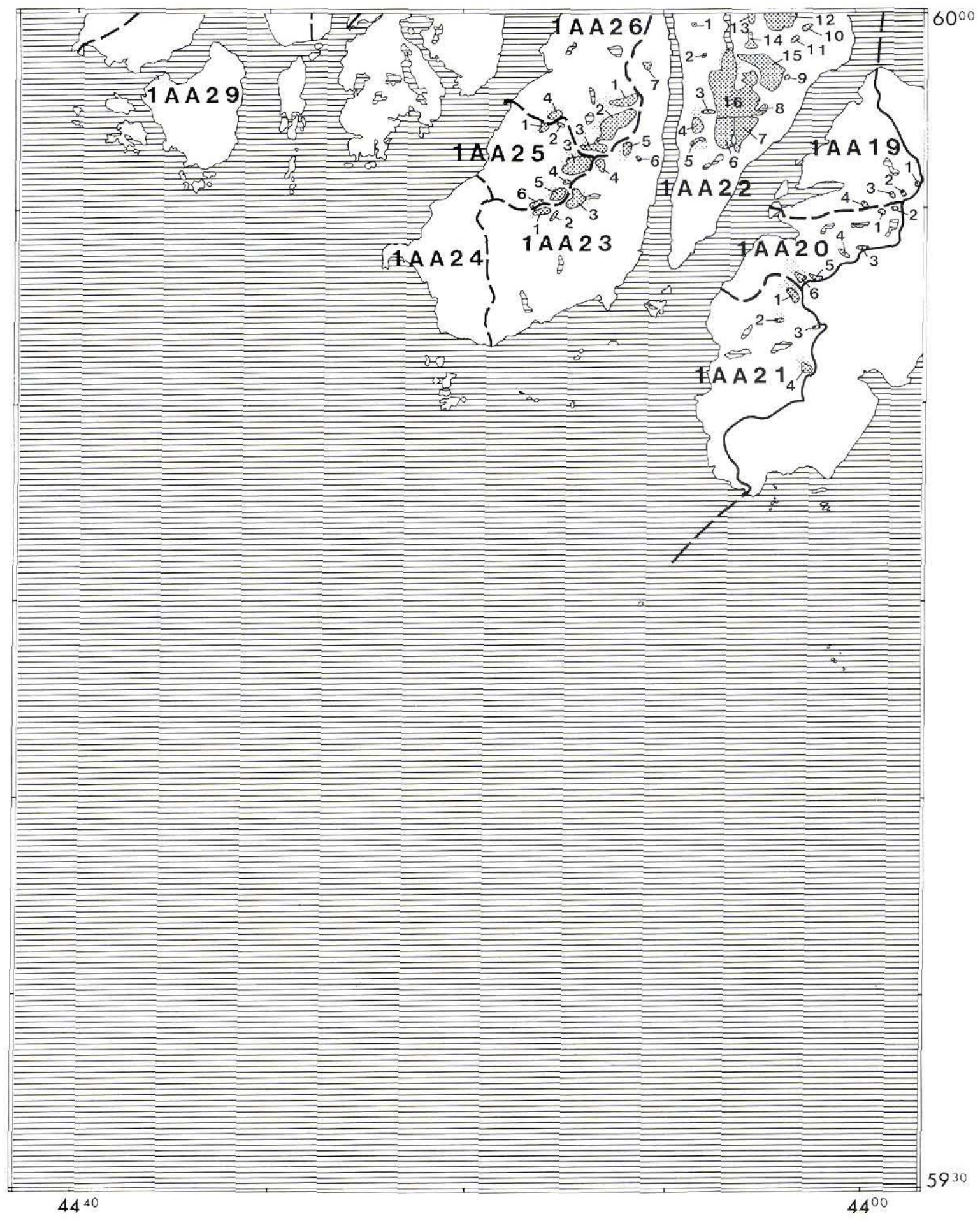

GGU GLACIER ATLAS

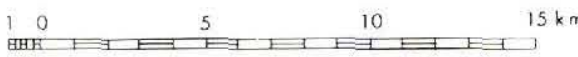

SHEET 5930-01 


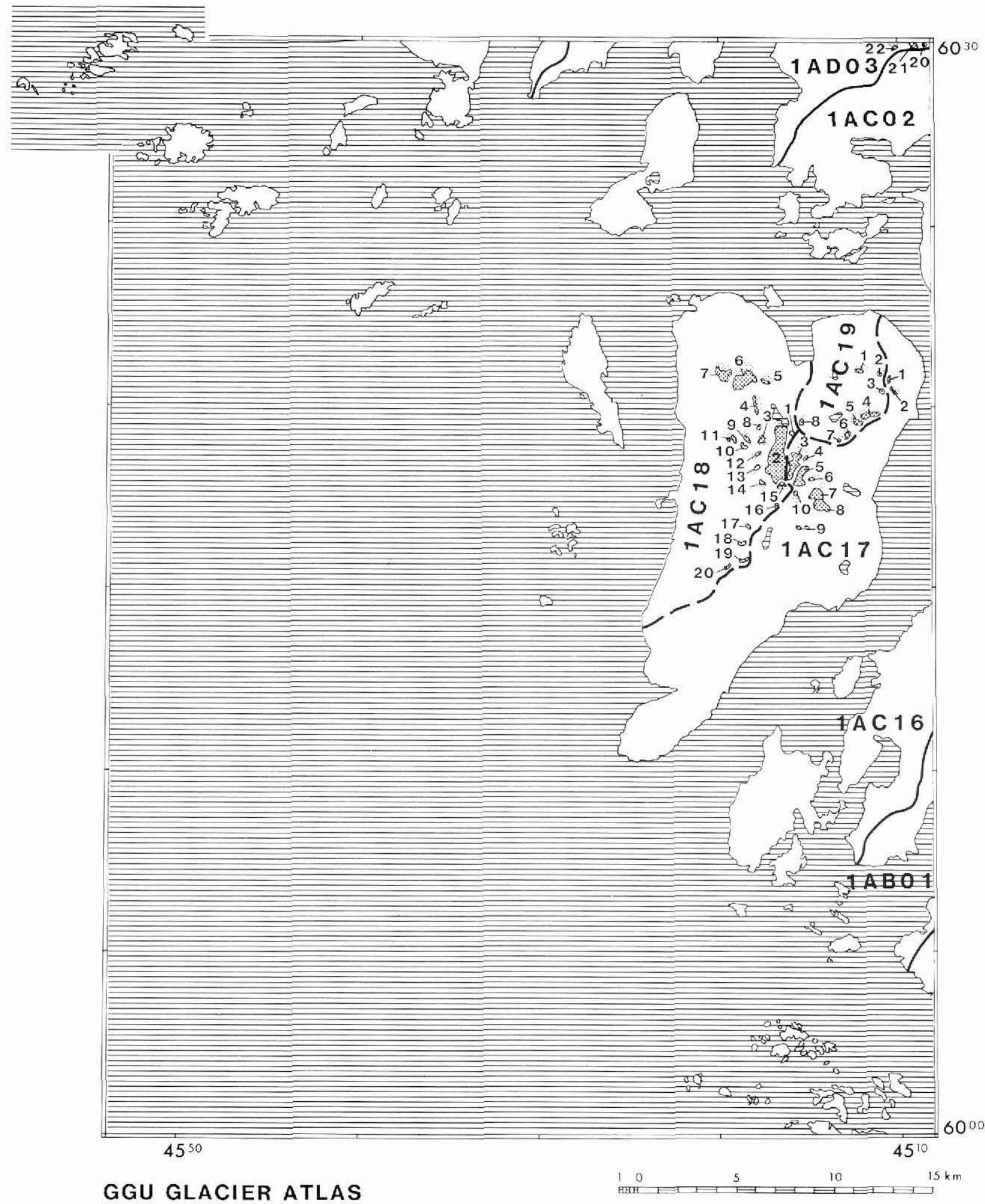

\section{SHEET 6000-01}




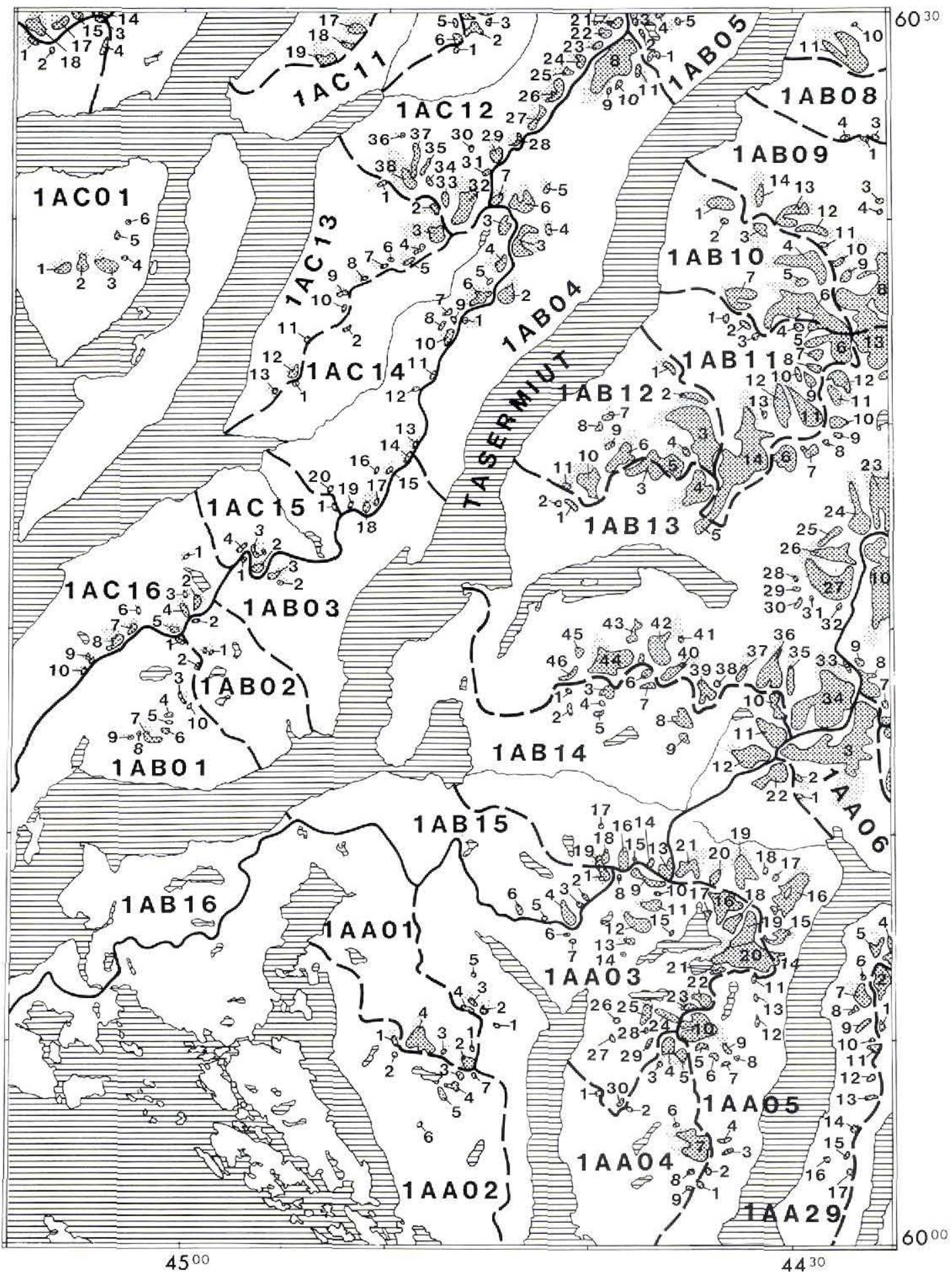

GGU GLACIER ATLAS

$\begin{array}{llll}10 & 5 & 10 & 15 \mathrm{~km}\end{array}$ SHEET 6000-02 


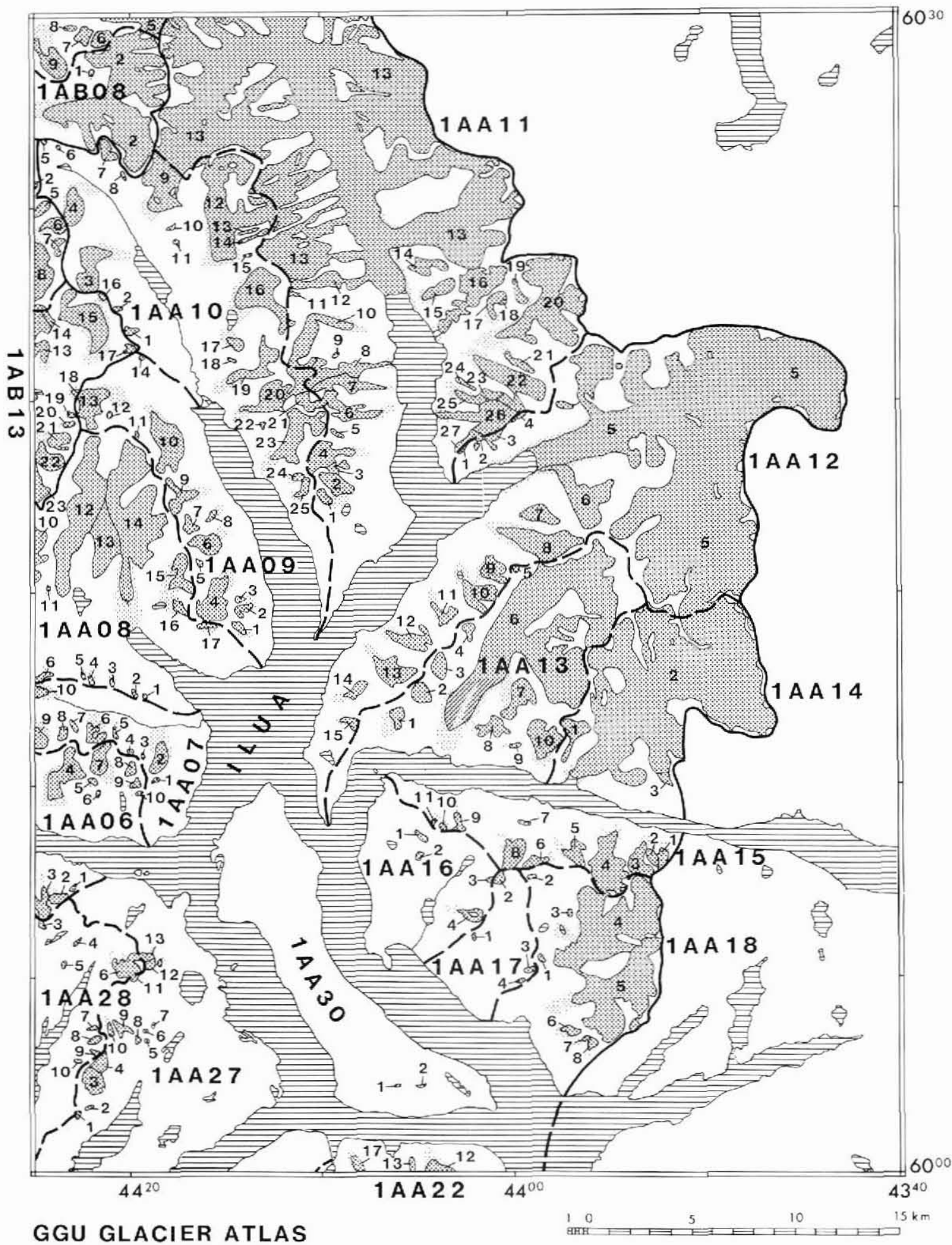

SHEET 6000-03 


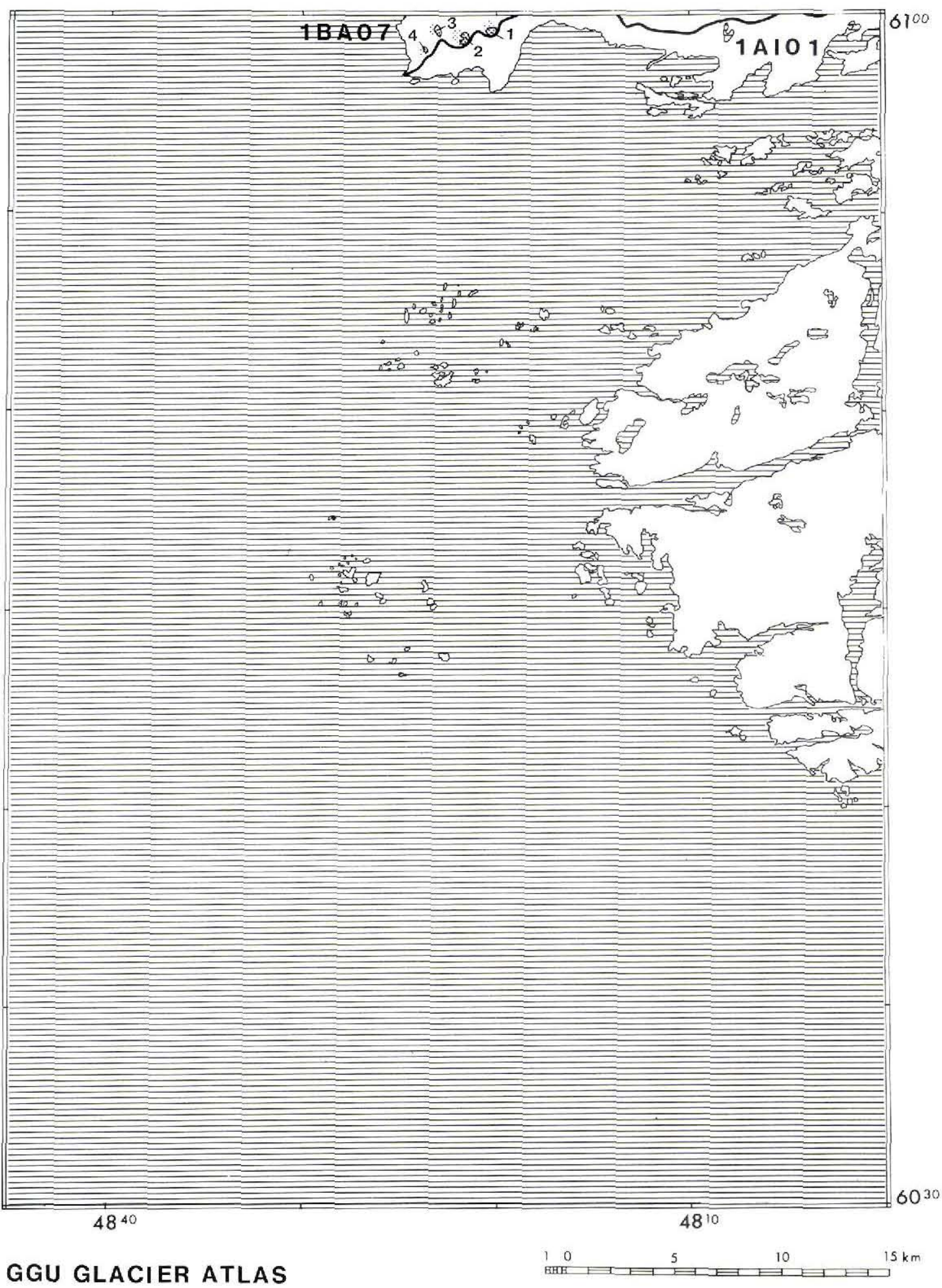
SHEET 6030-01 


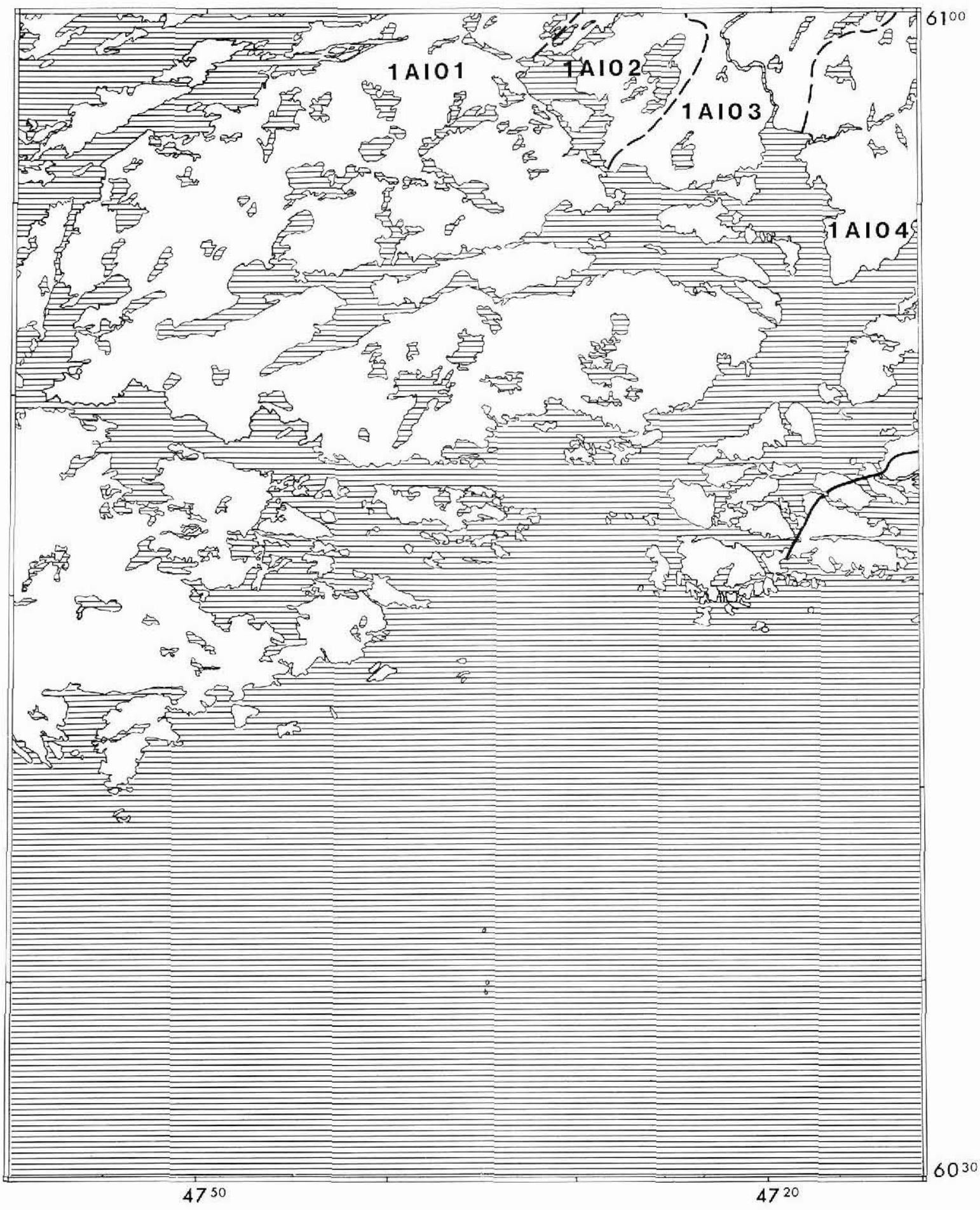

GGU GLACIER ATLAS

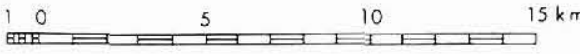




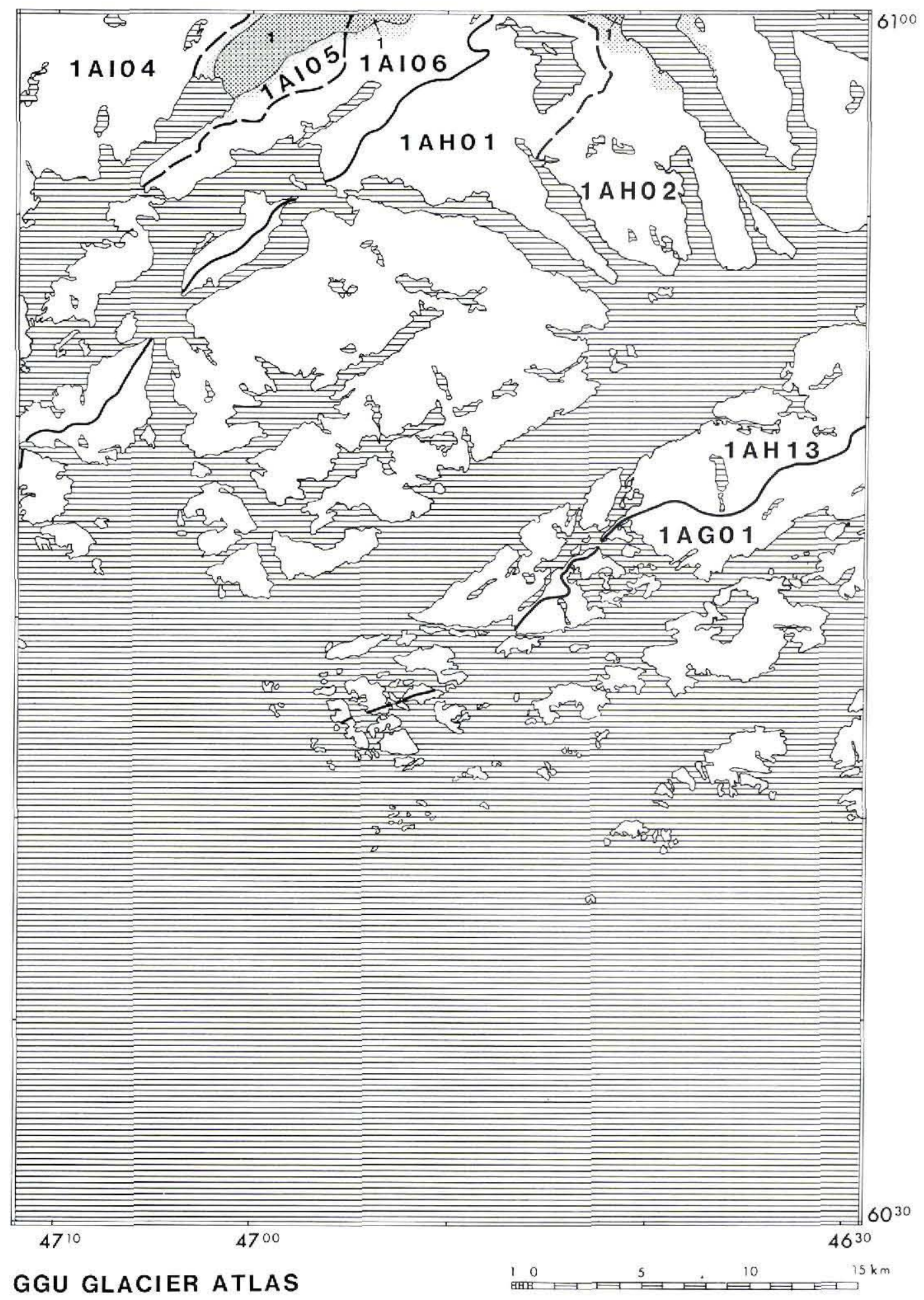




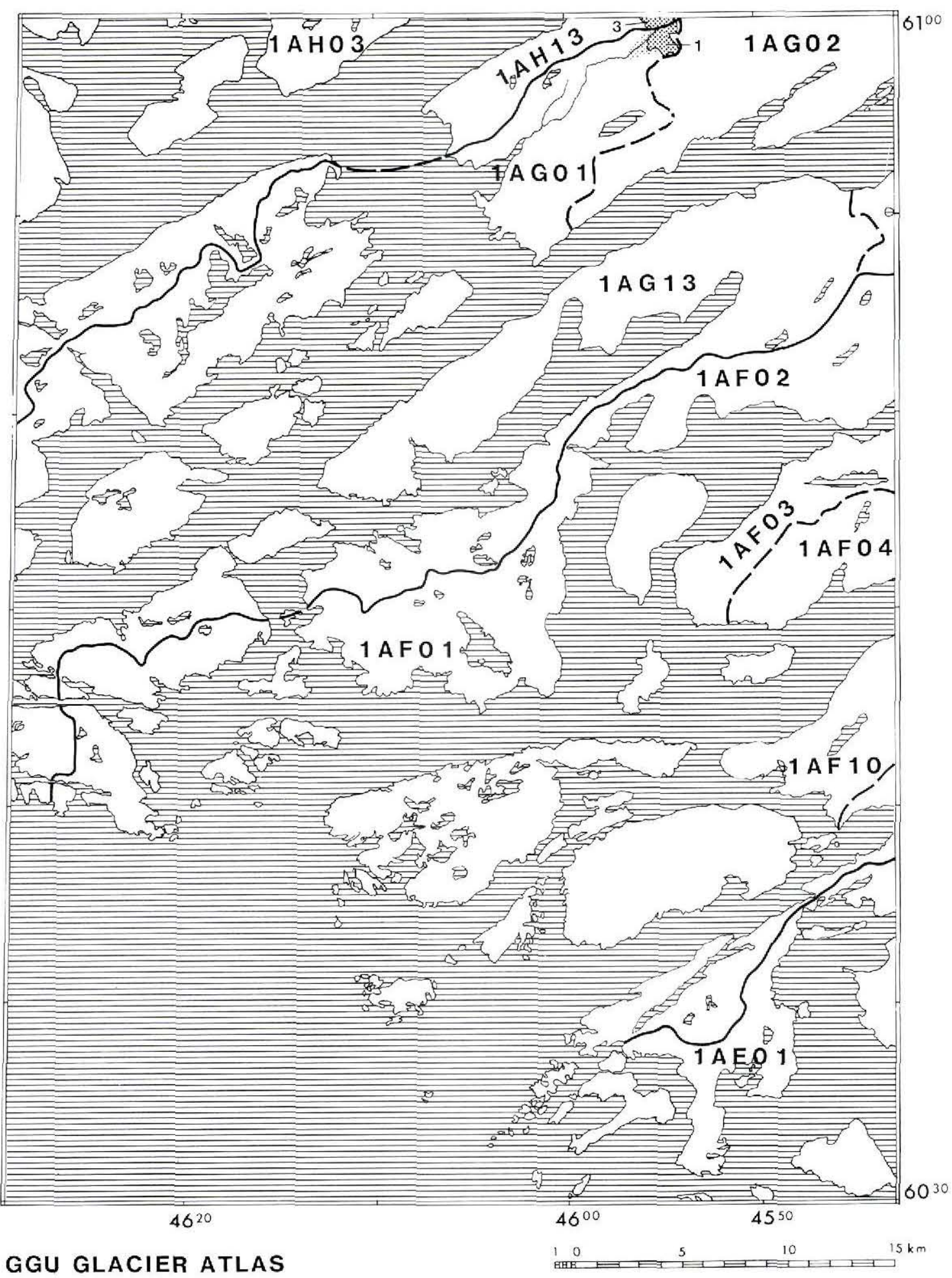

\section{SHEET 6030-04}




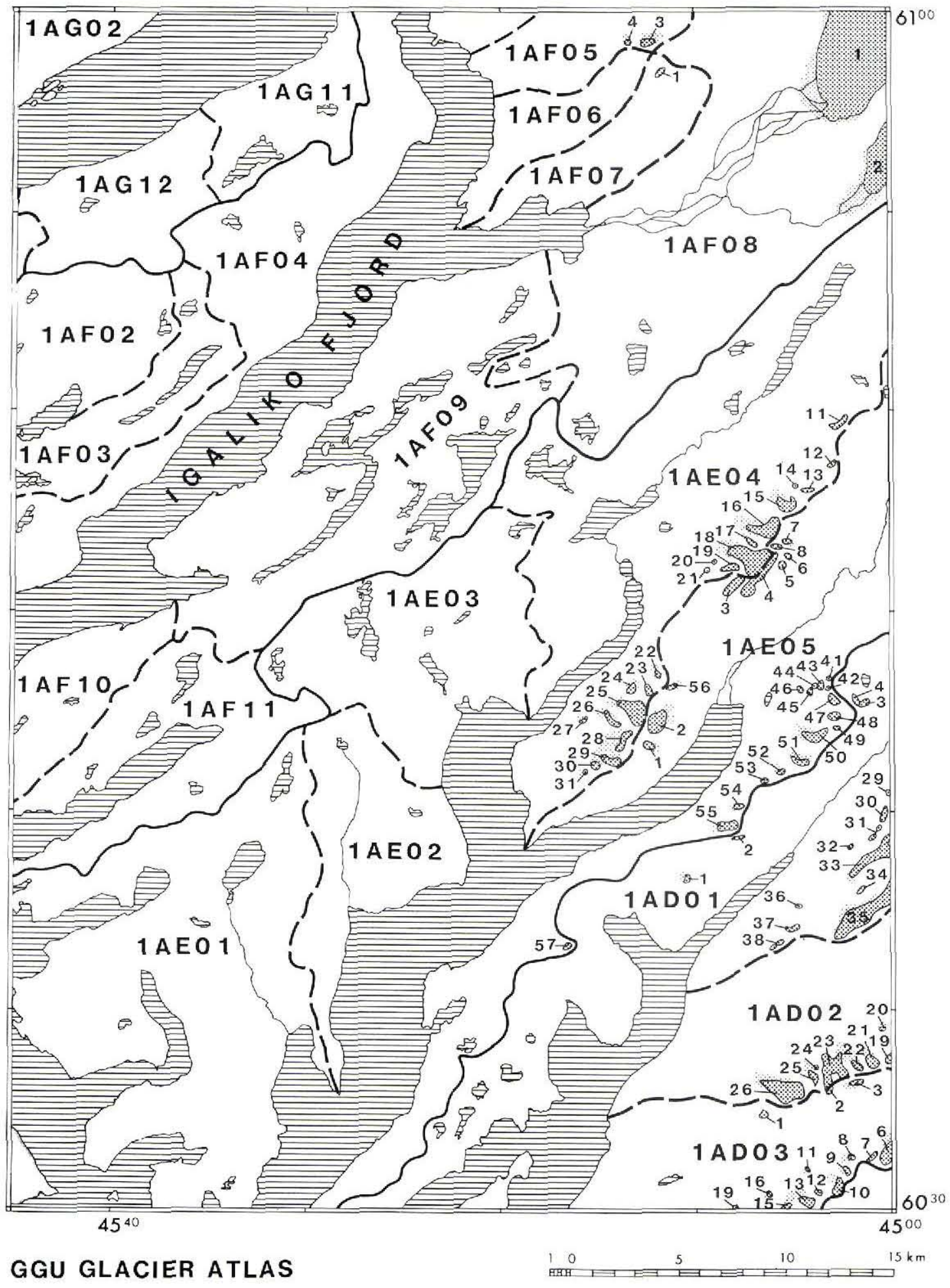

SHEET 6030-05 


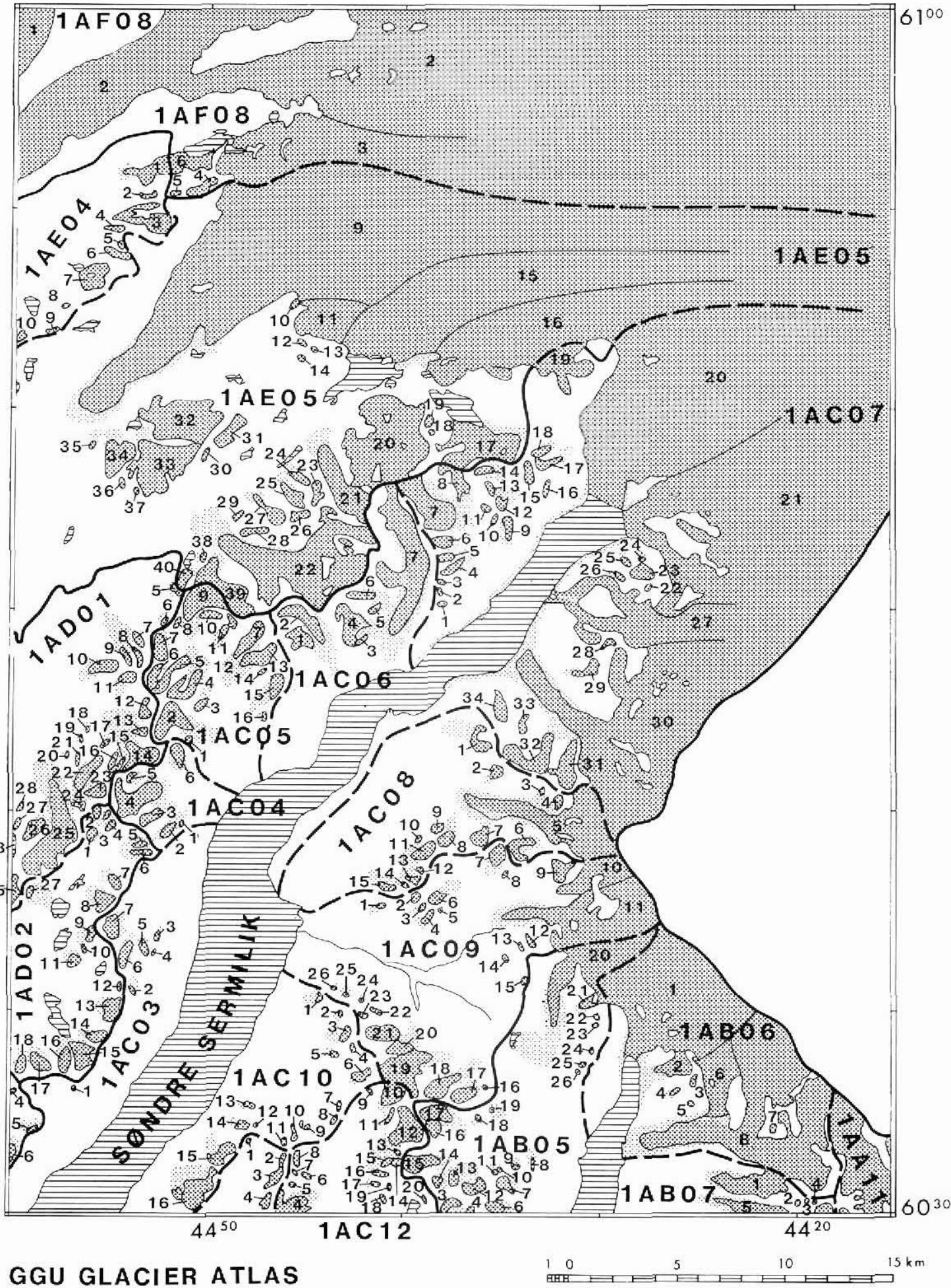

\section{SHEET 6030-06}




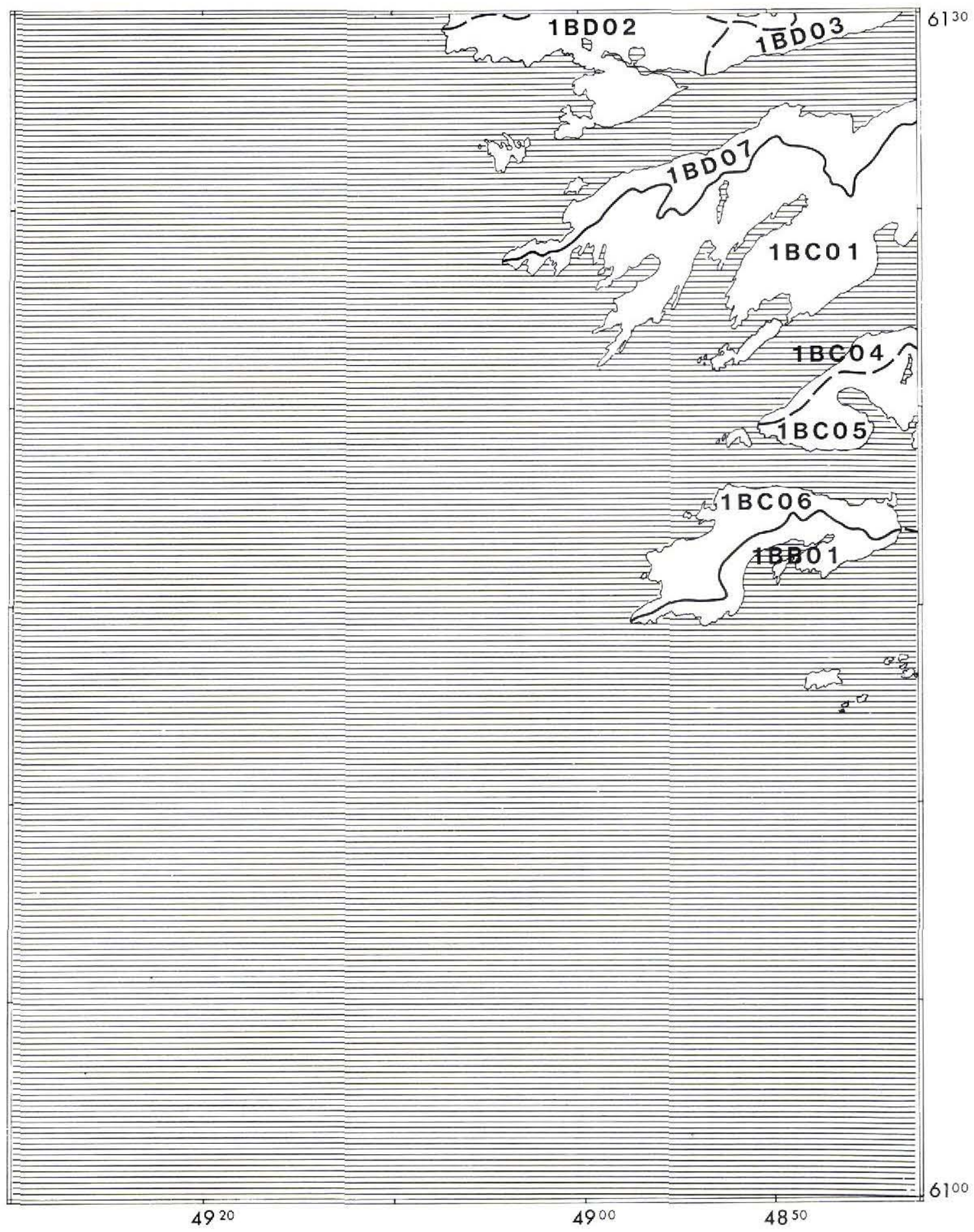

GGU GLACIER ATLAS

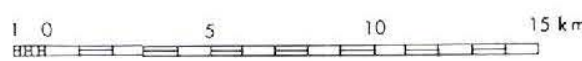

SHEET 6100-01 


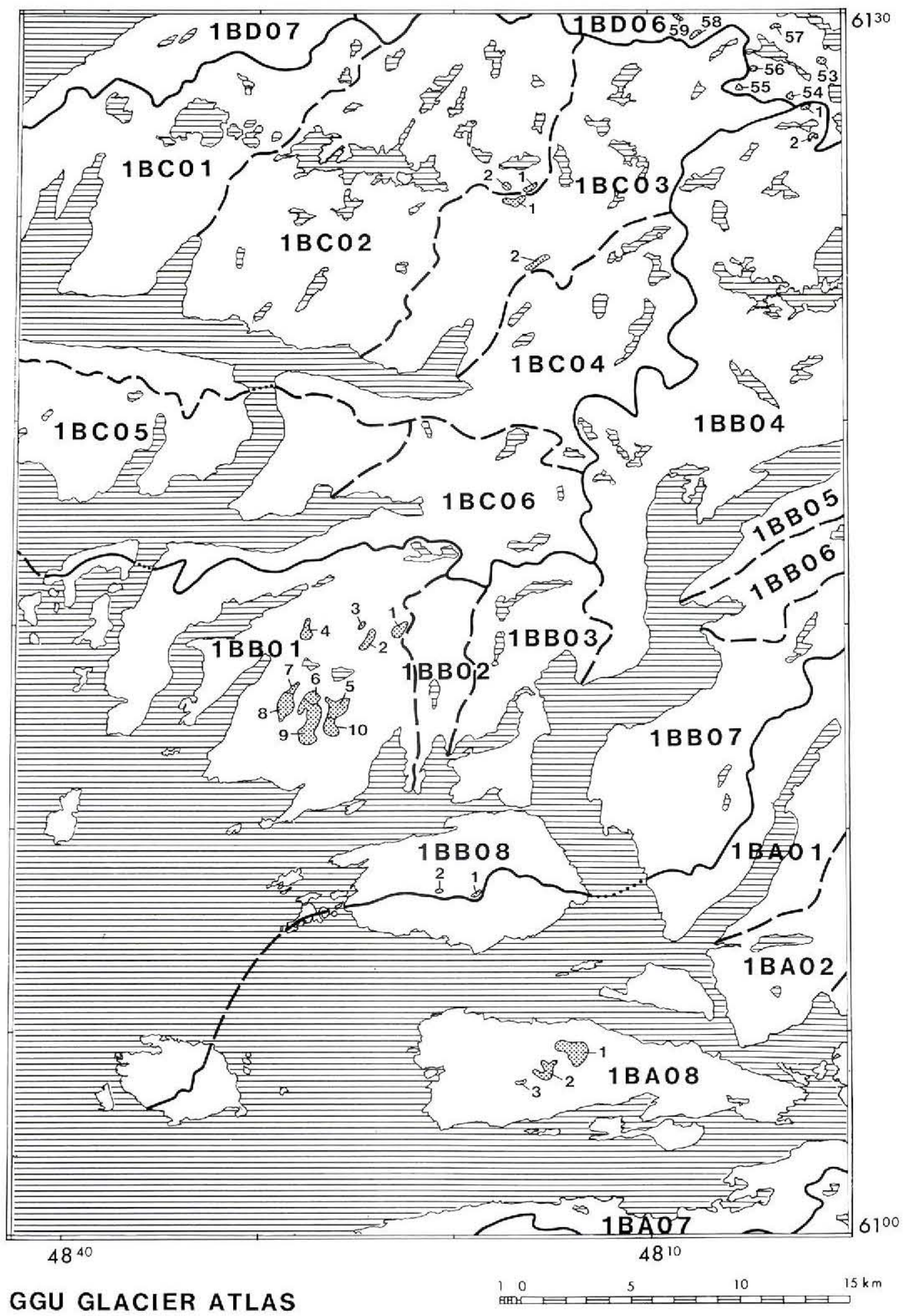

\section{SHEET 6100-02}




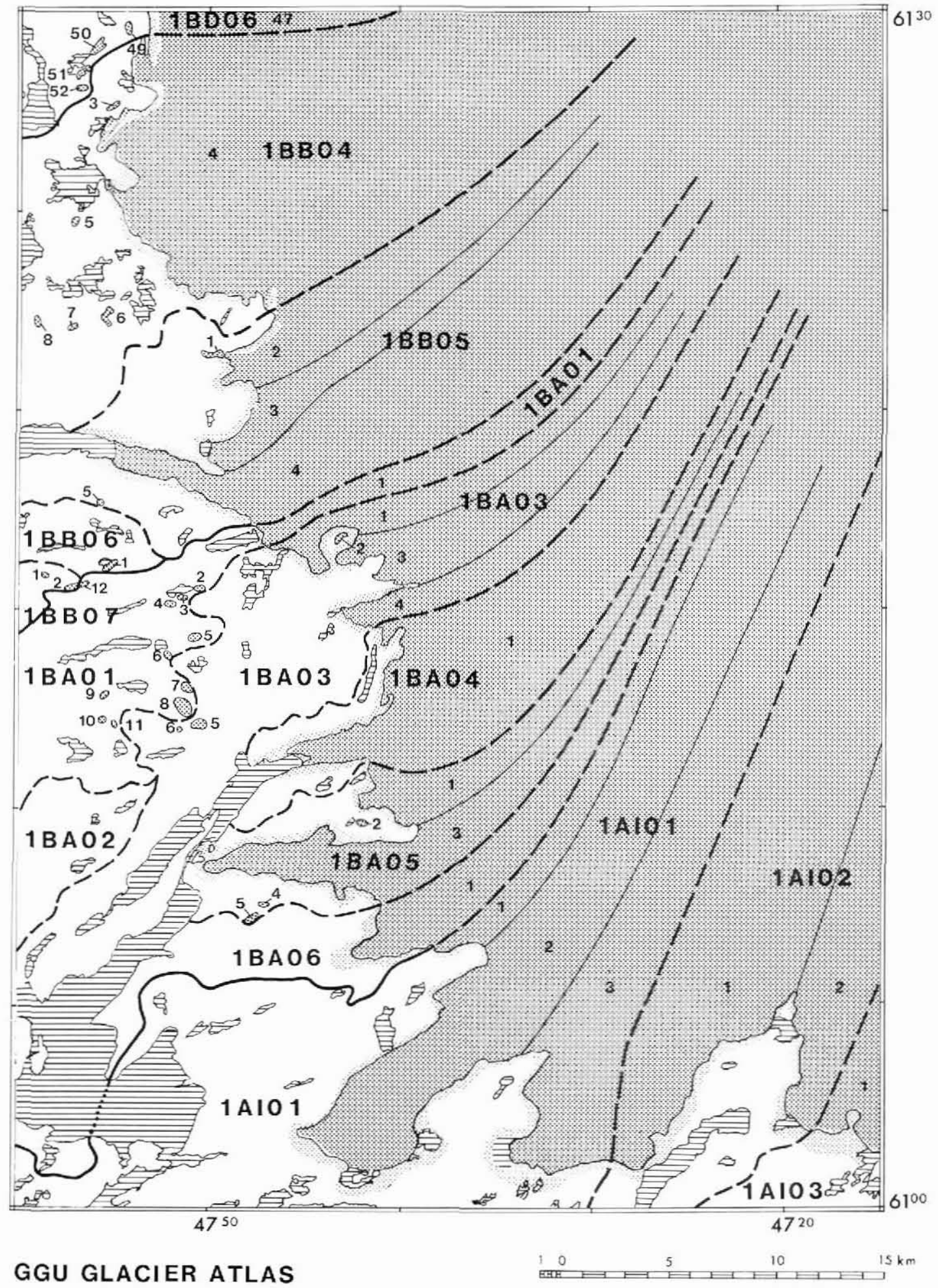

SHEET 6100-03 


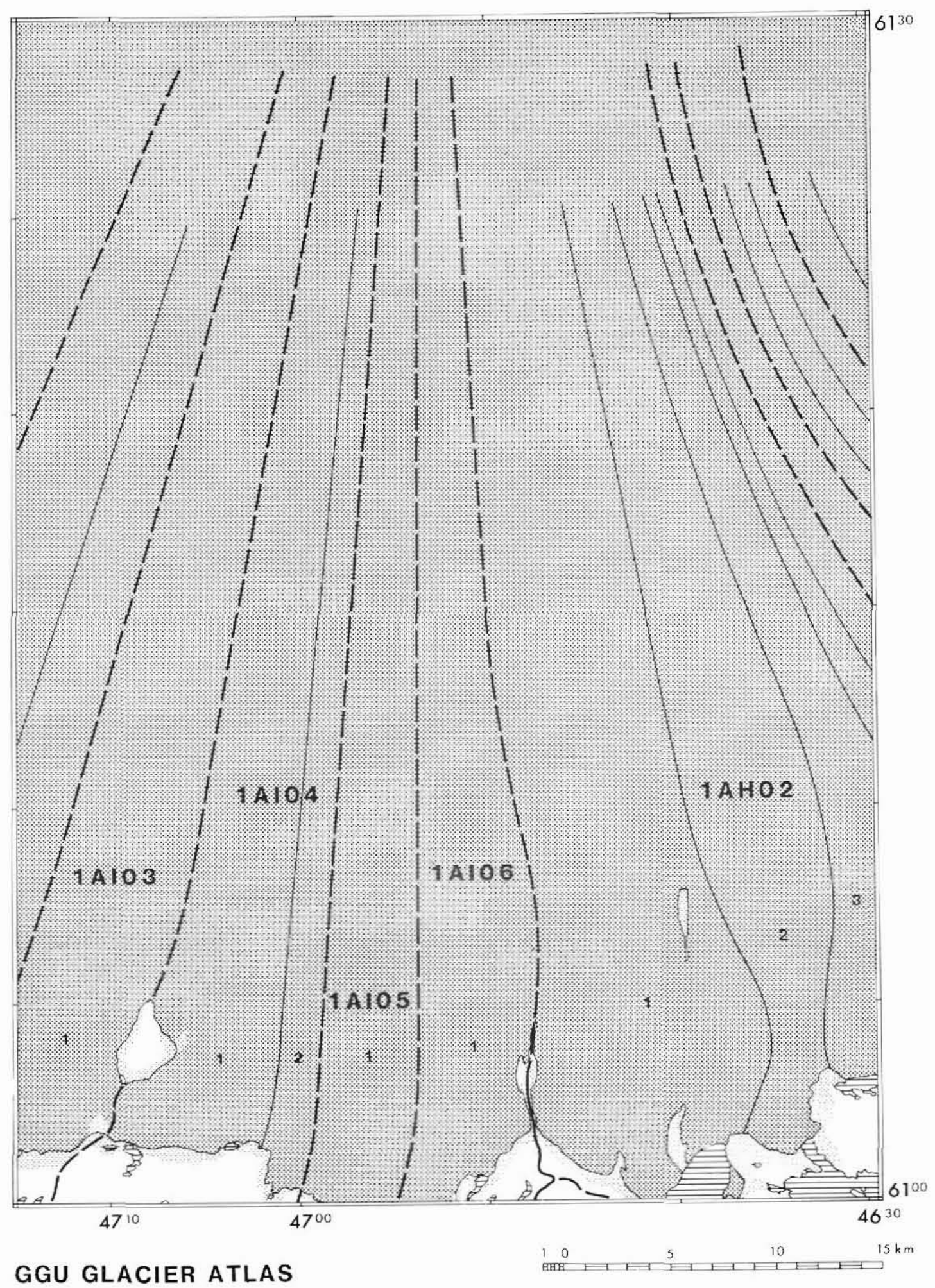

SHEET 6100-04 


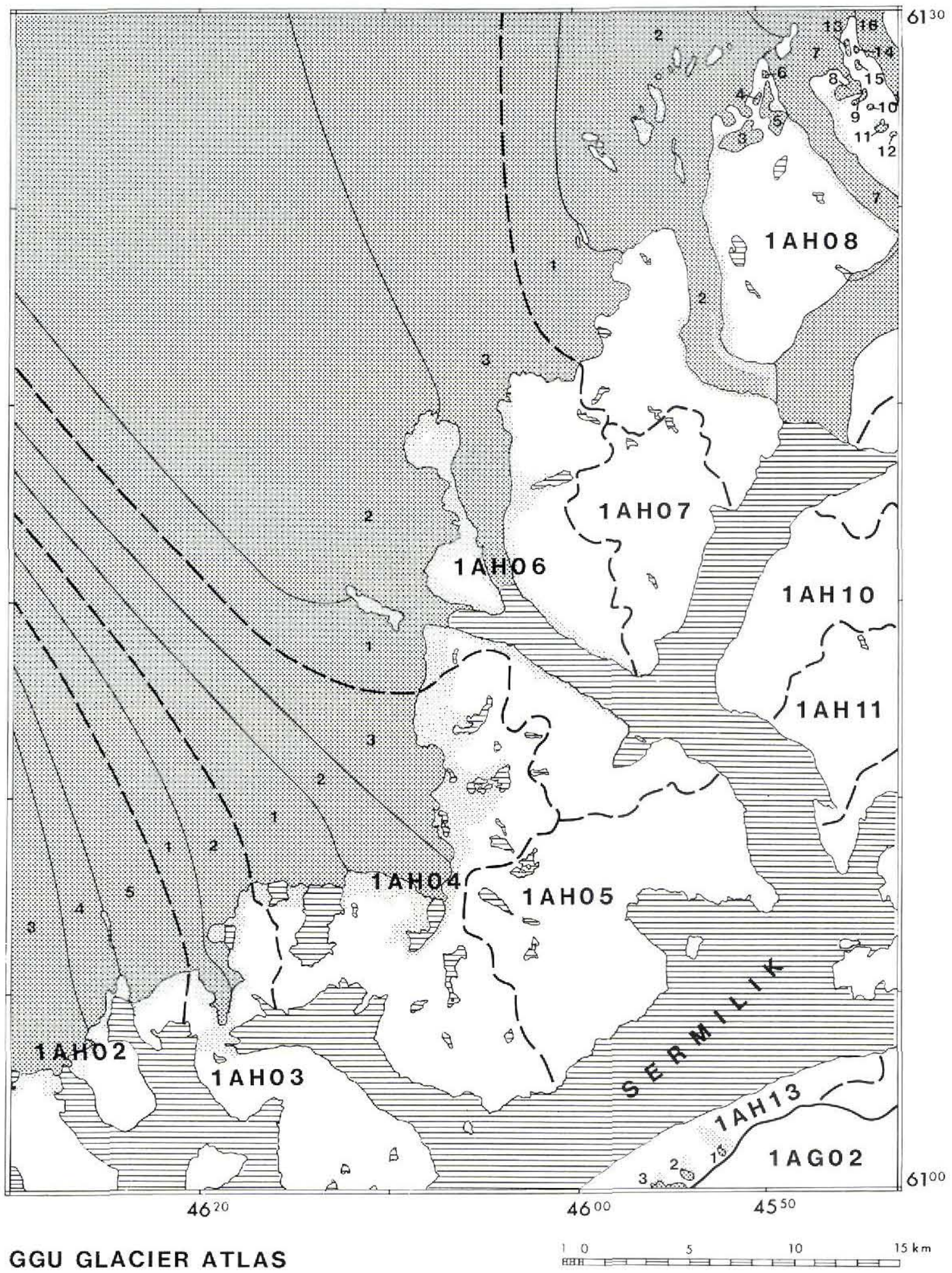

SHEET 6100-05 


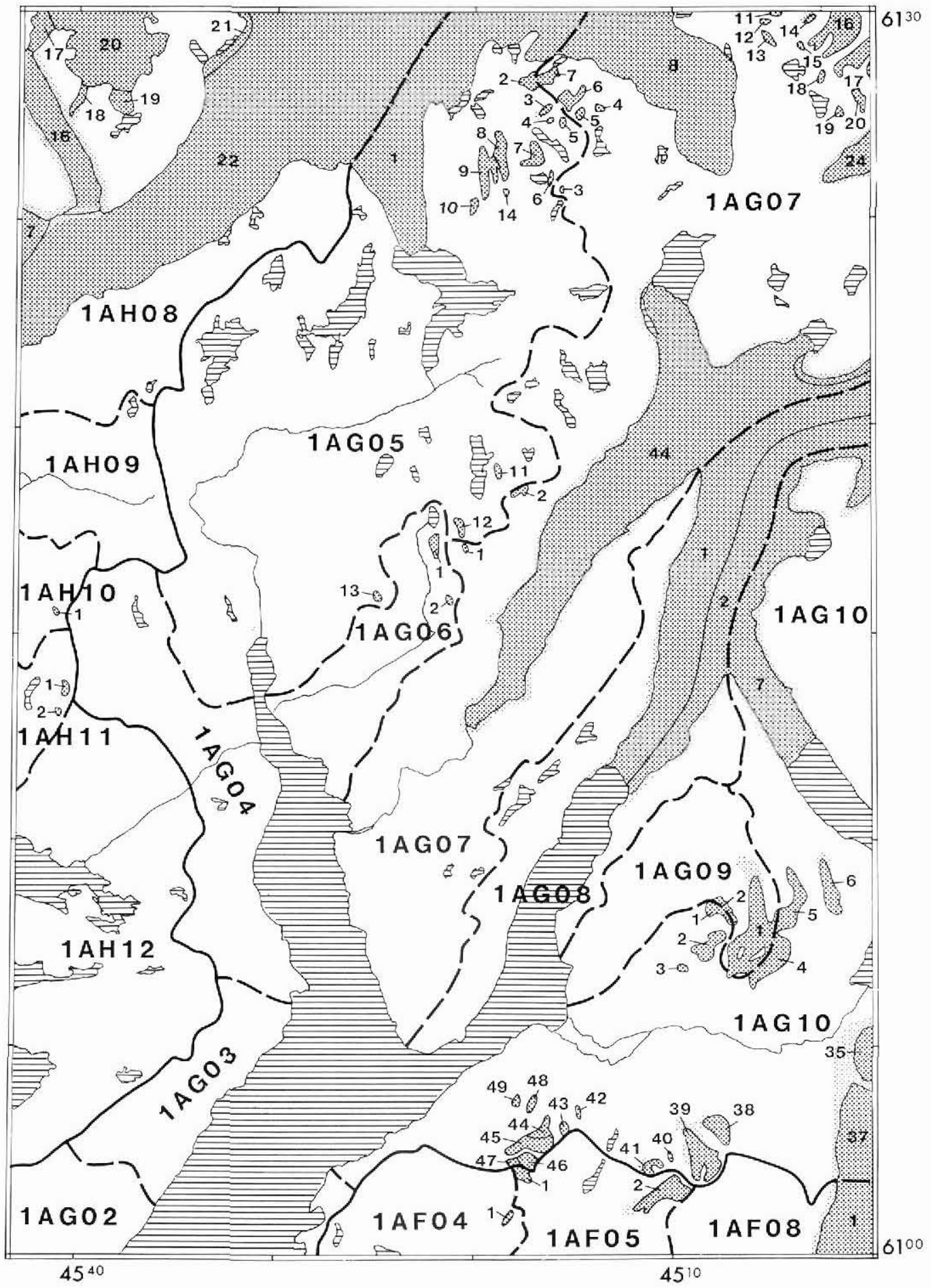

GGU GLACIER ATLAS

$10050102015 \mathrm{~km}$ 


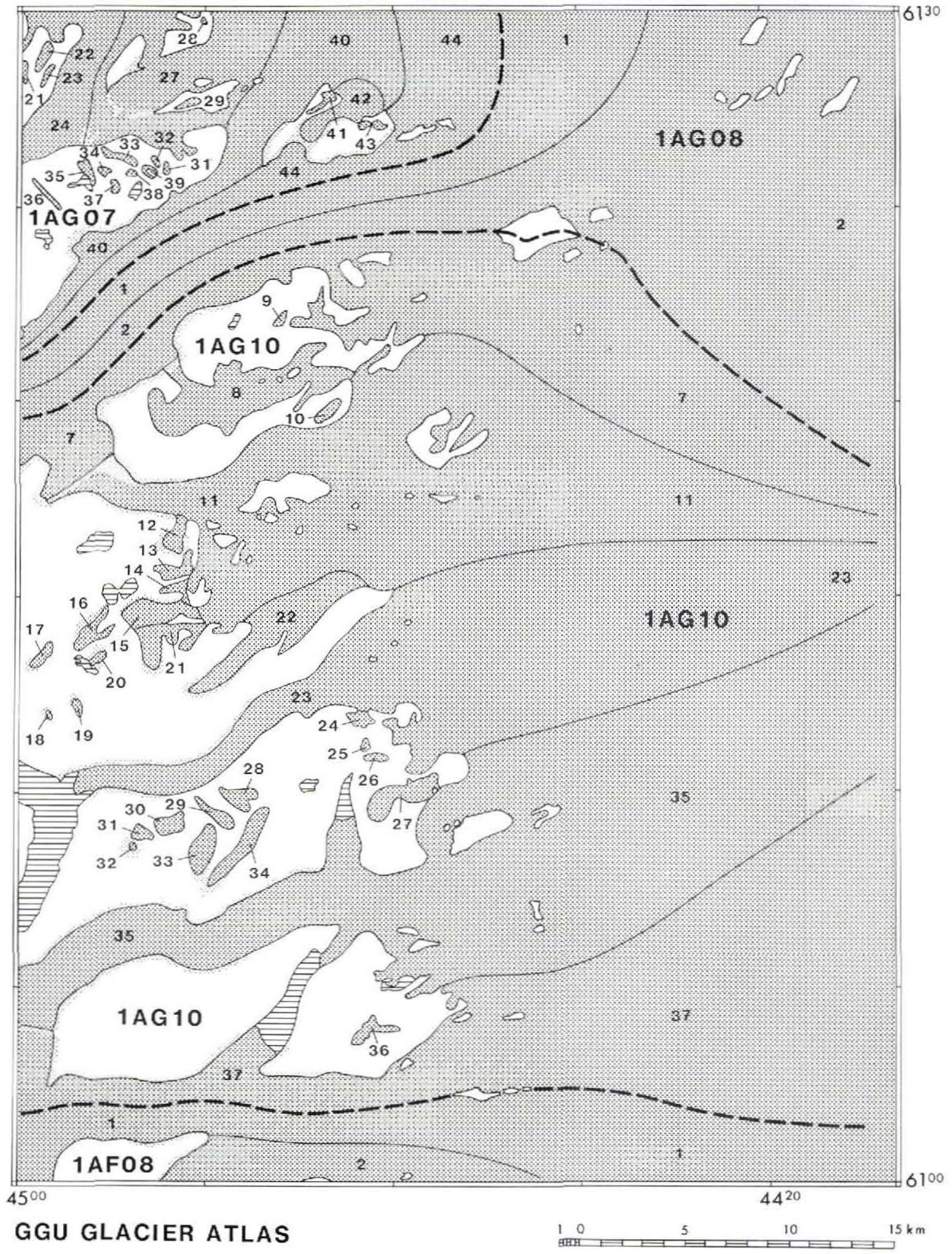

SHEET 6100-07 


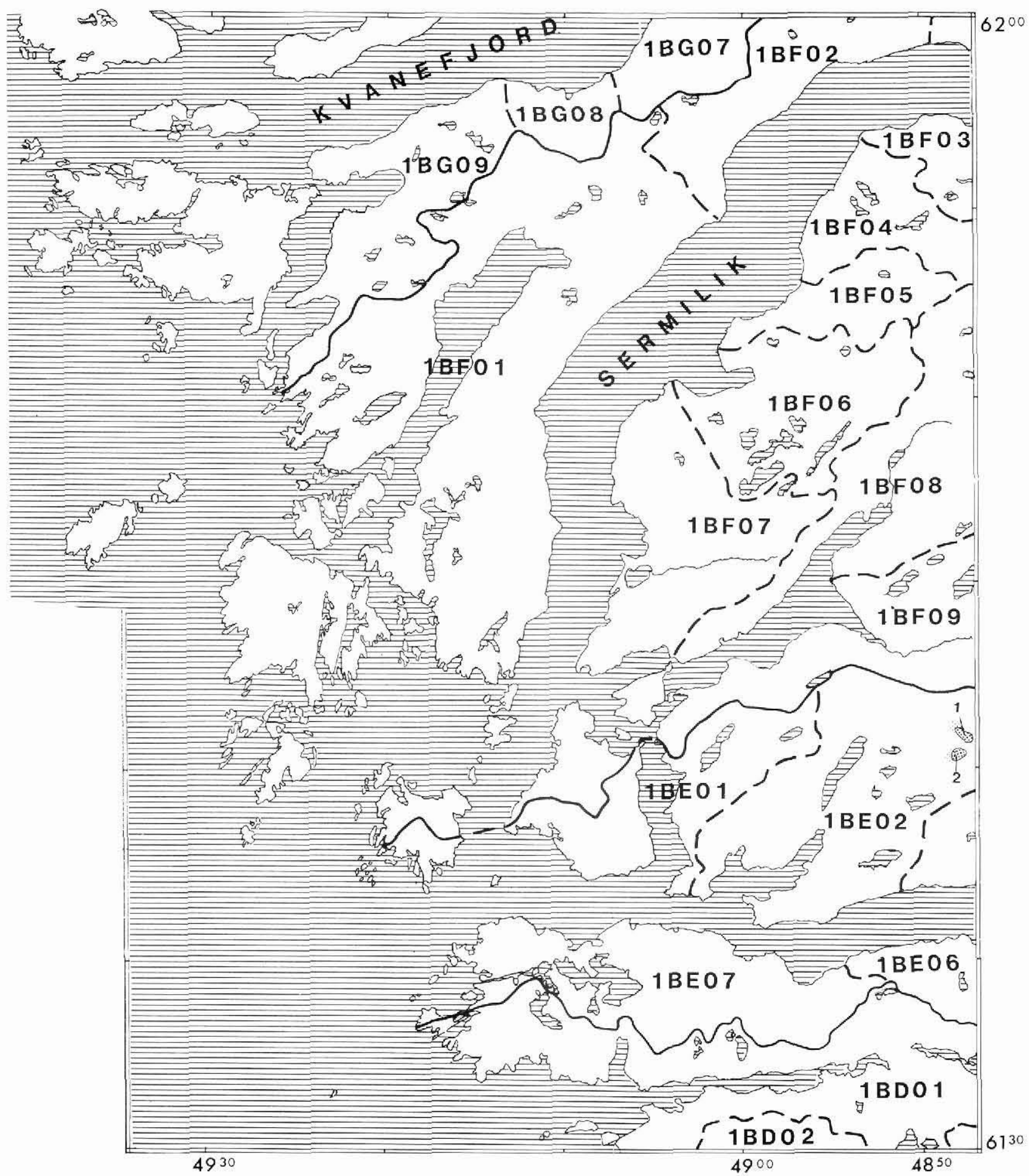

GGU GLACIER ATLAS 


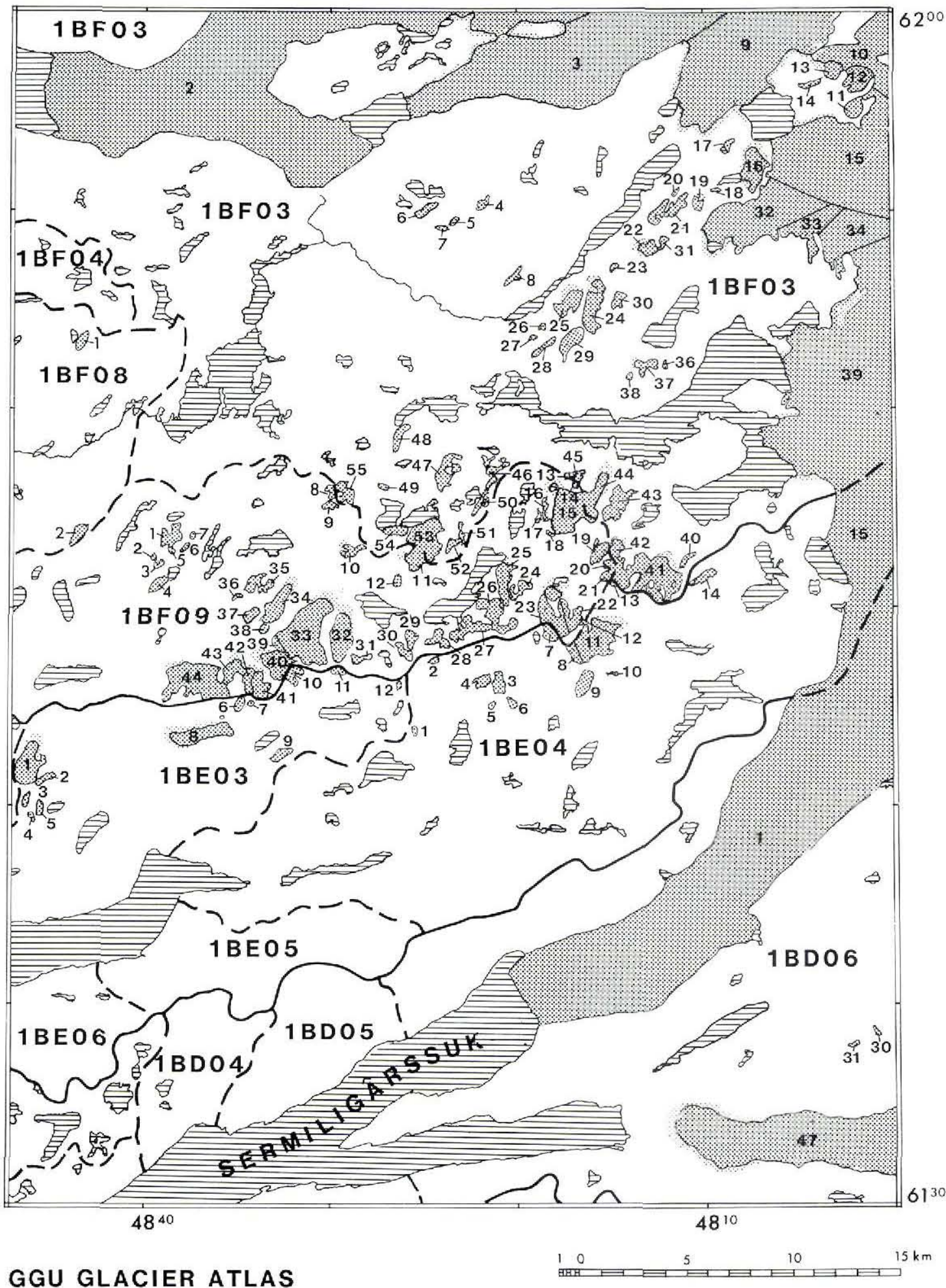




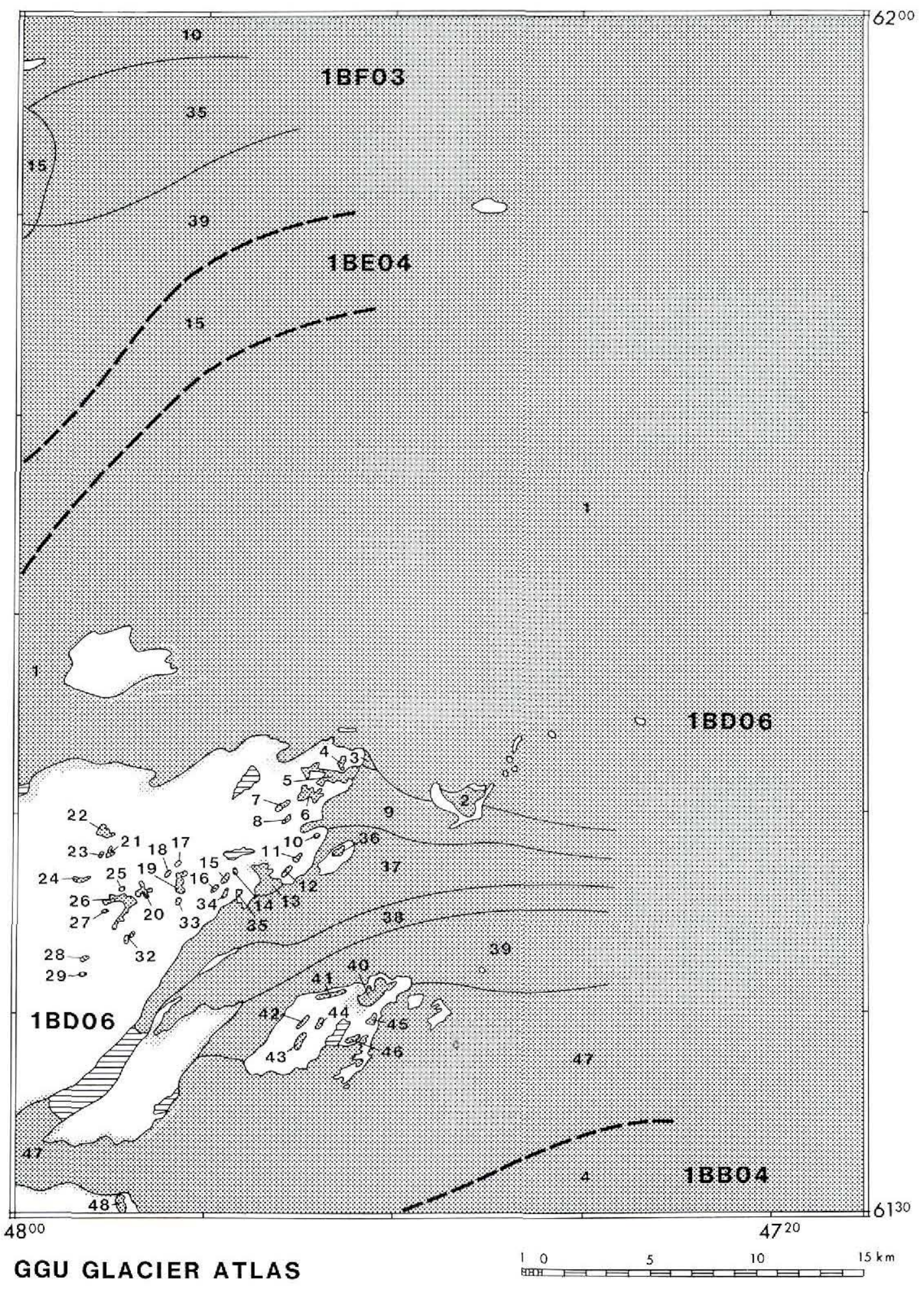

SHEET 6130-03 


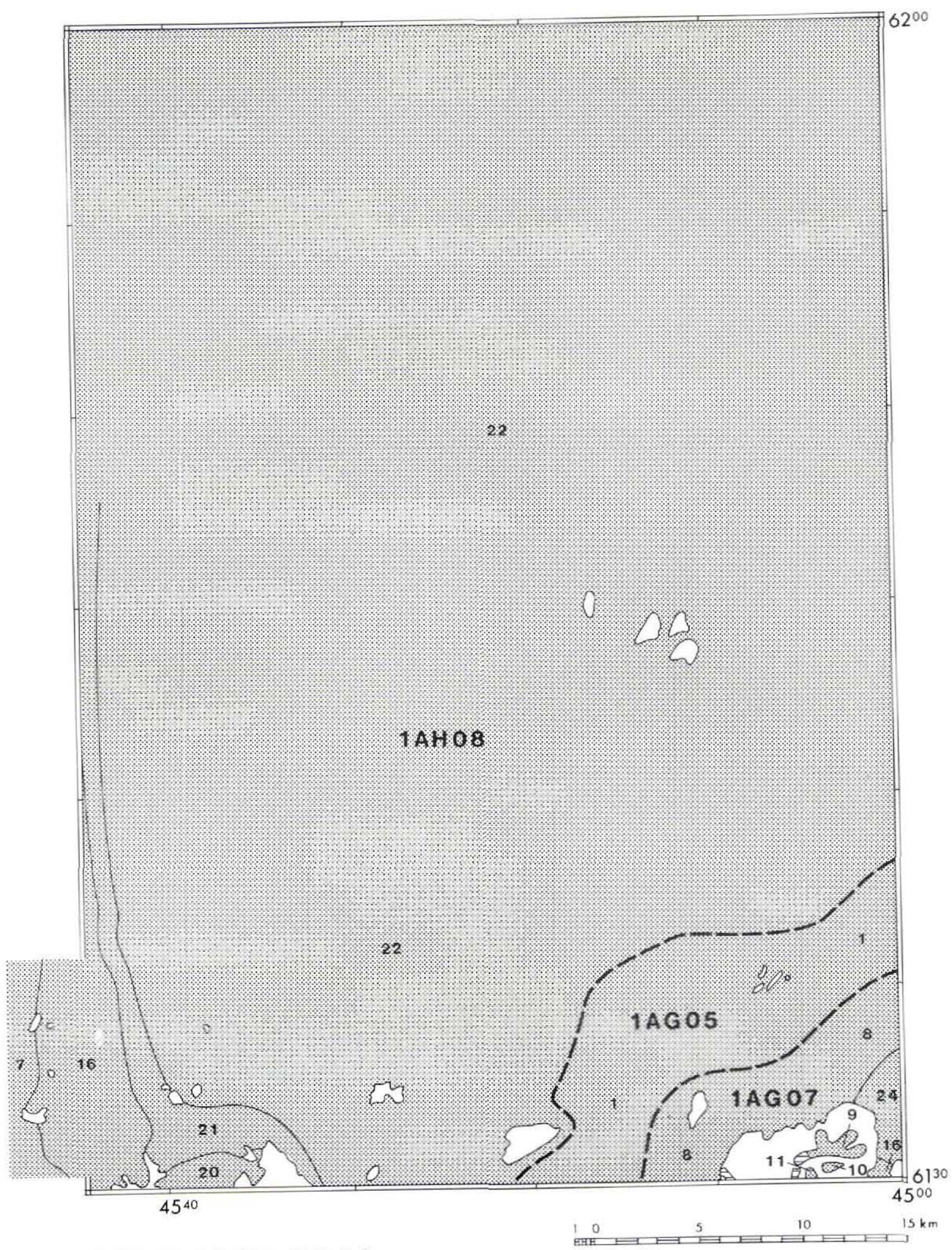

GGU GLACIER ATLAS

SHEET 6130-06 


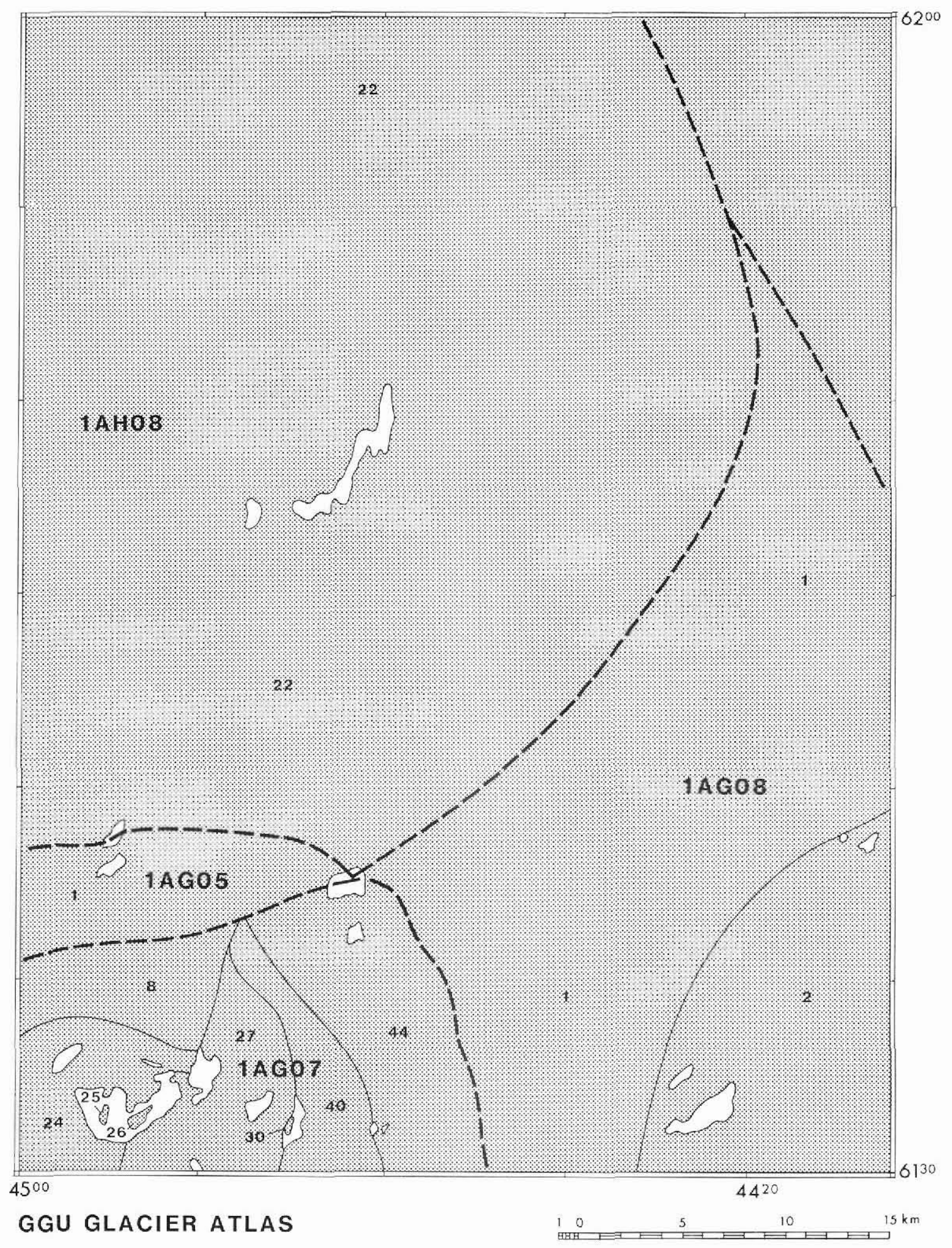

SHEET 6130-07 


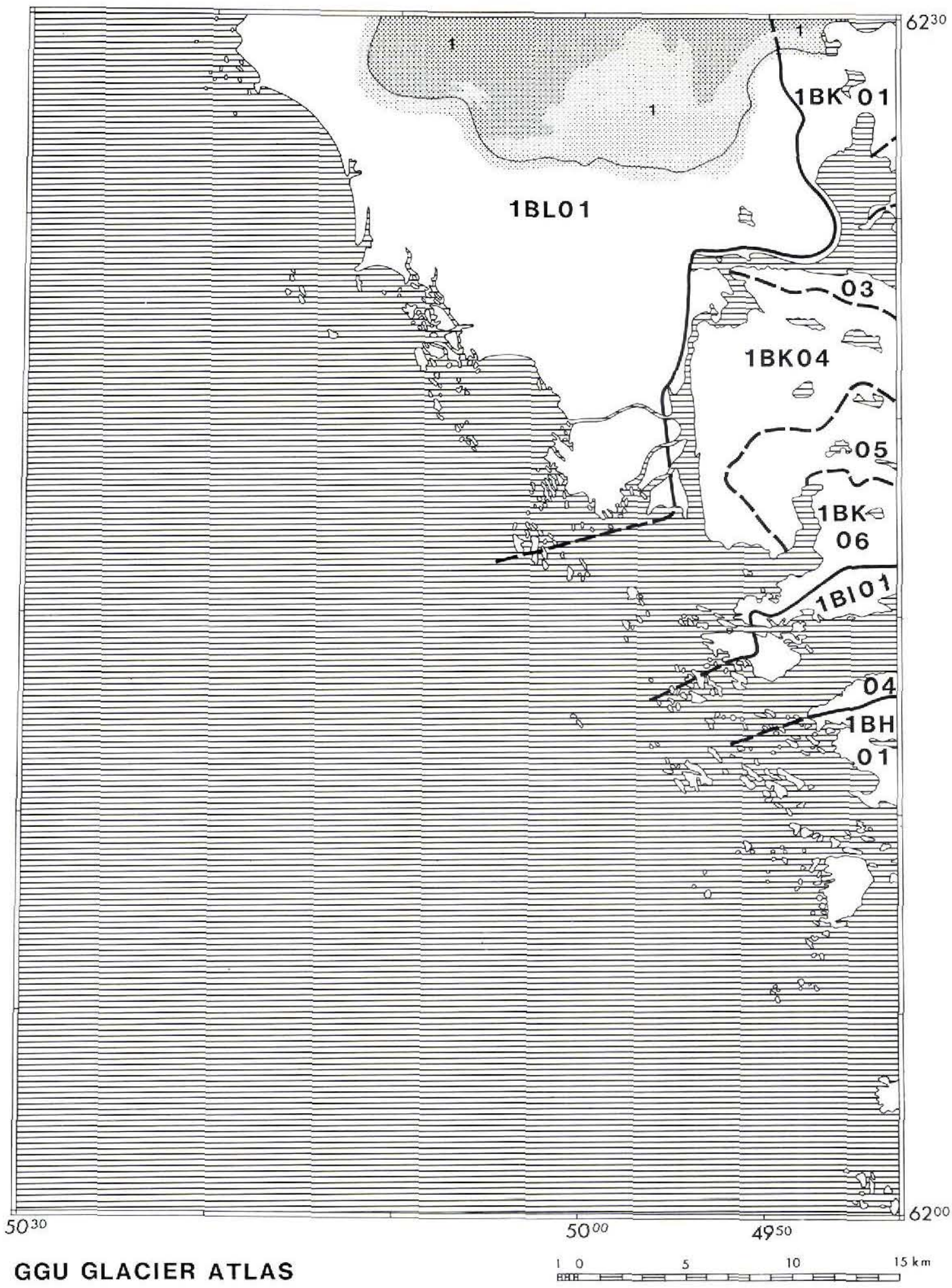

SHEET 6200-01 


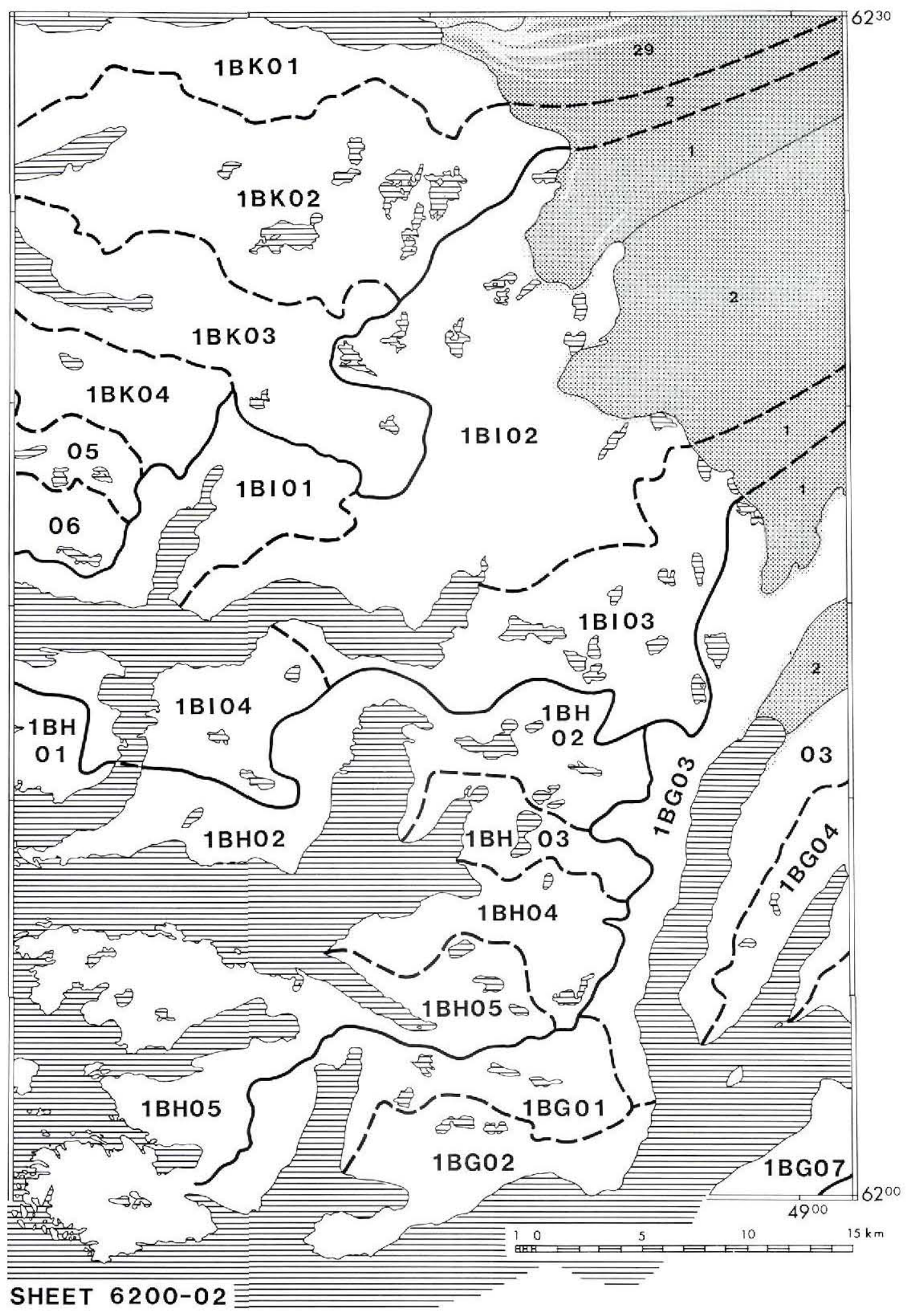




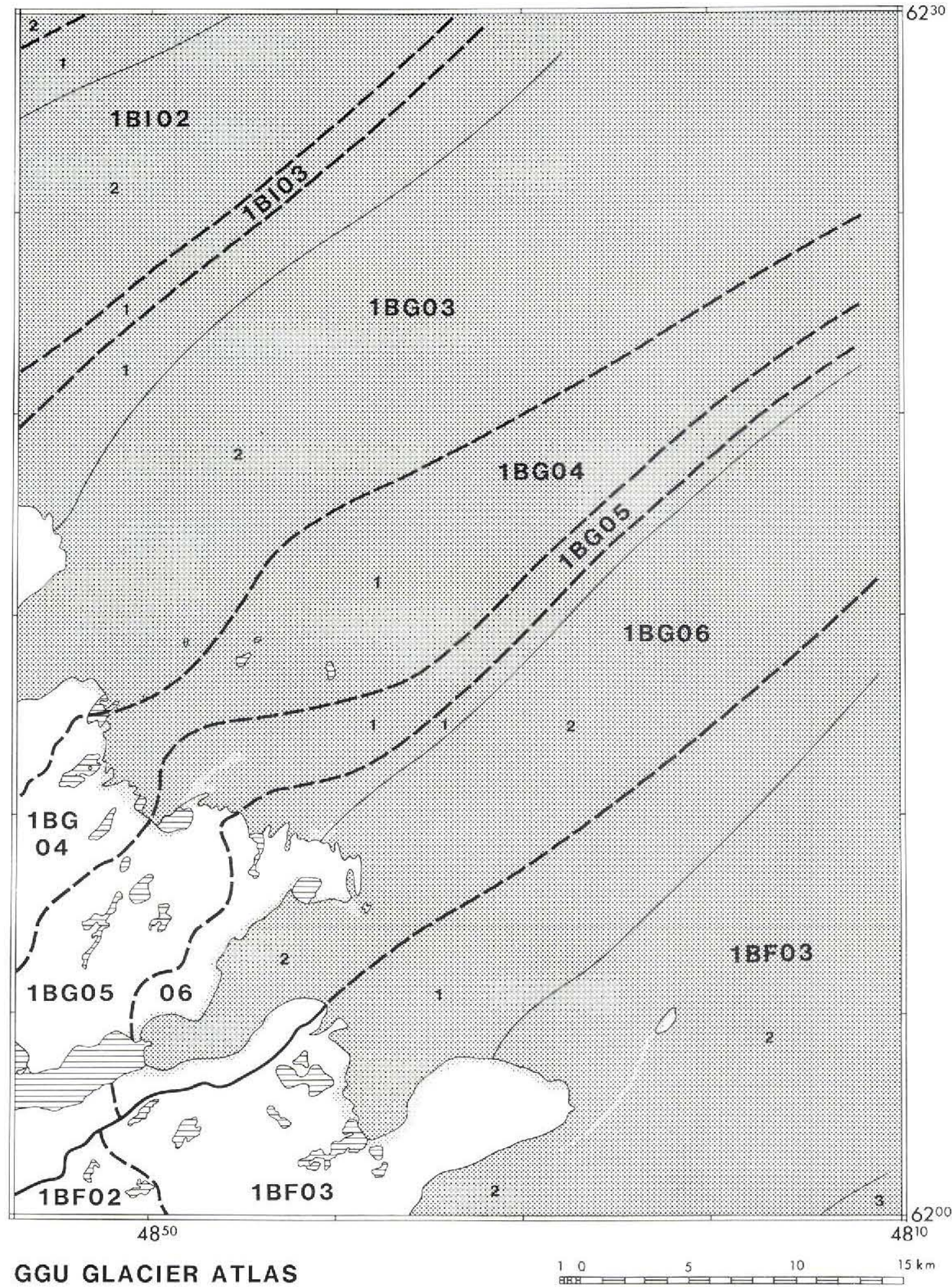

SHEET 6200-03 


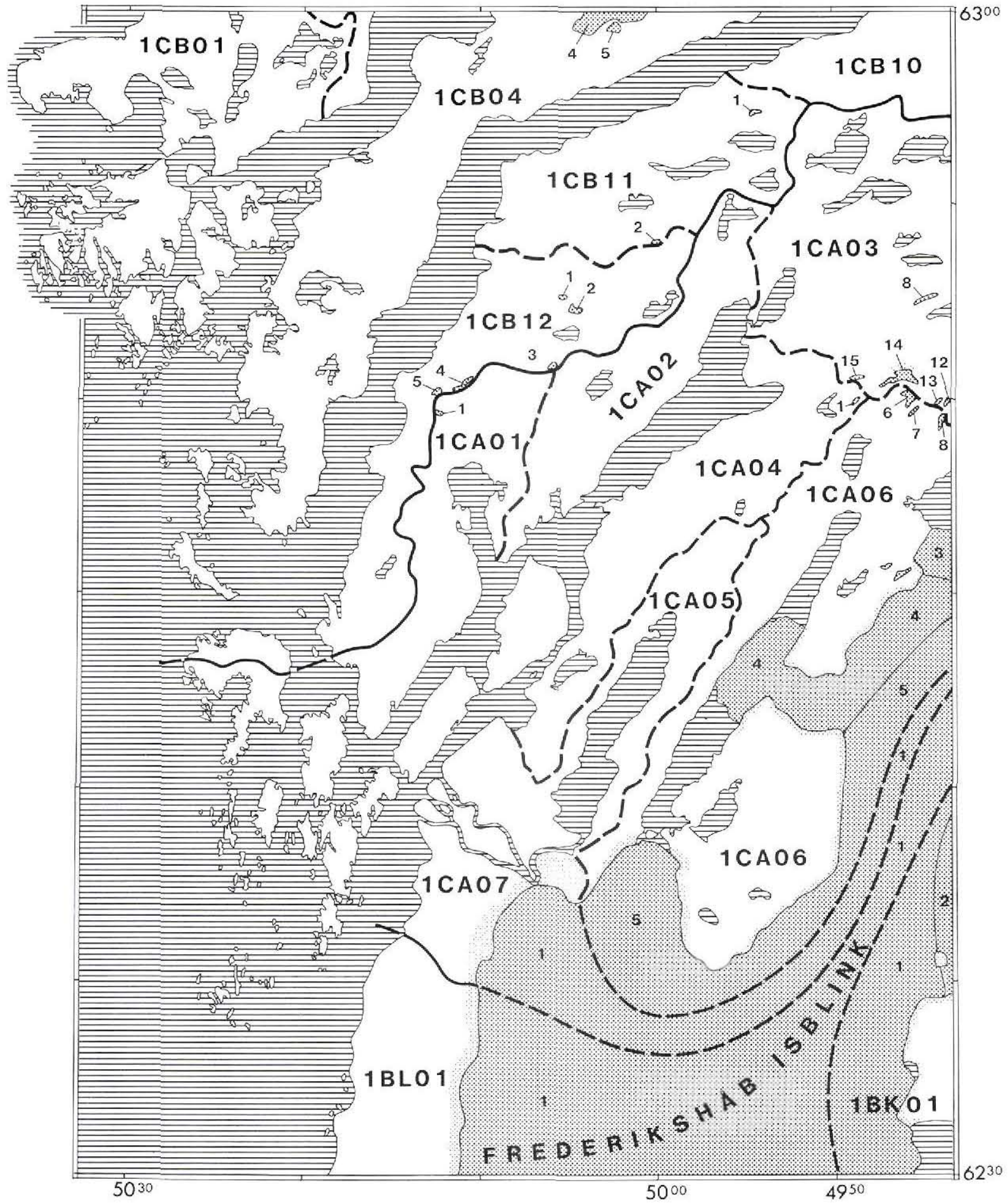

GGU GLACIER ATLAS

$10 \quad 5 \quad 10 \quad 15 \mathrm{~km}$

SHEET 6230-01 


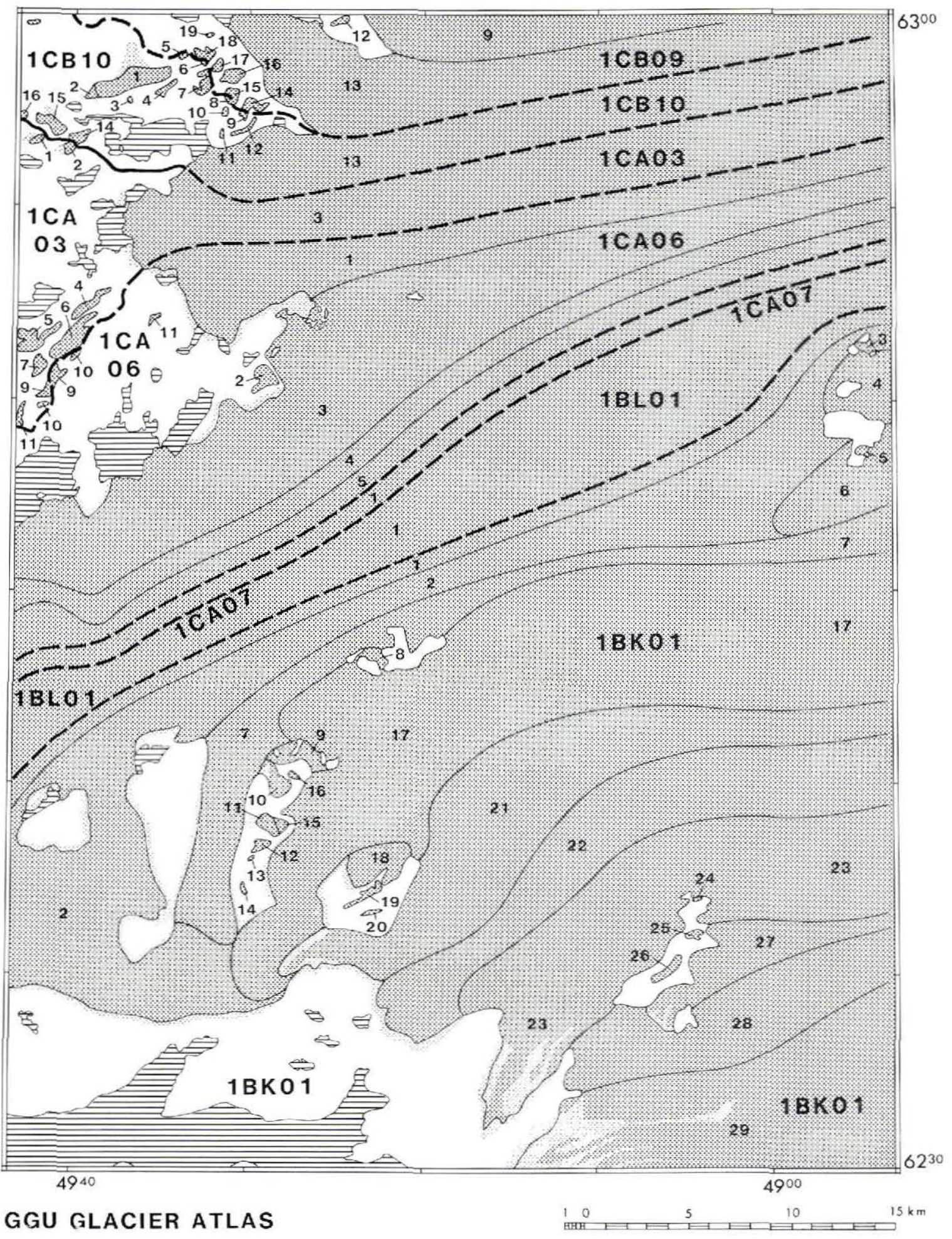

SHEET 6230-02 


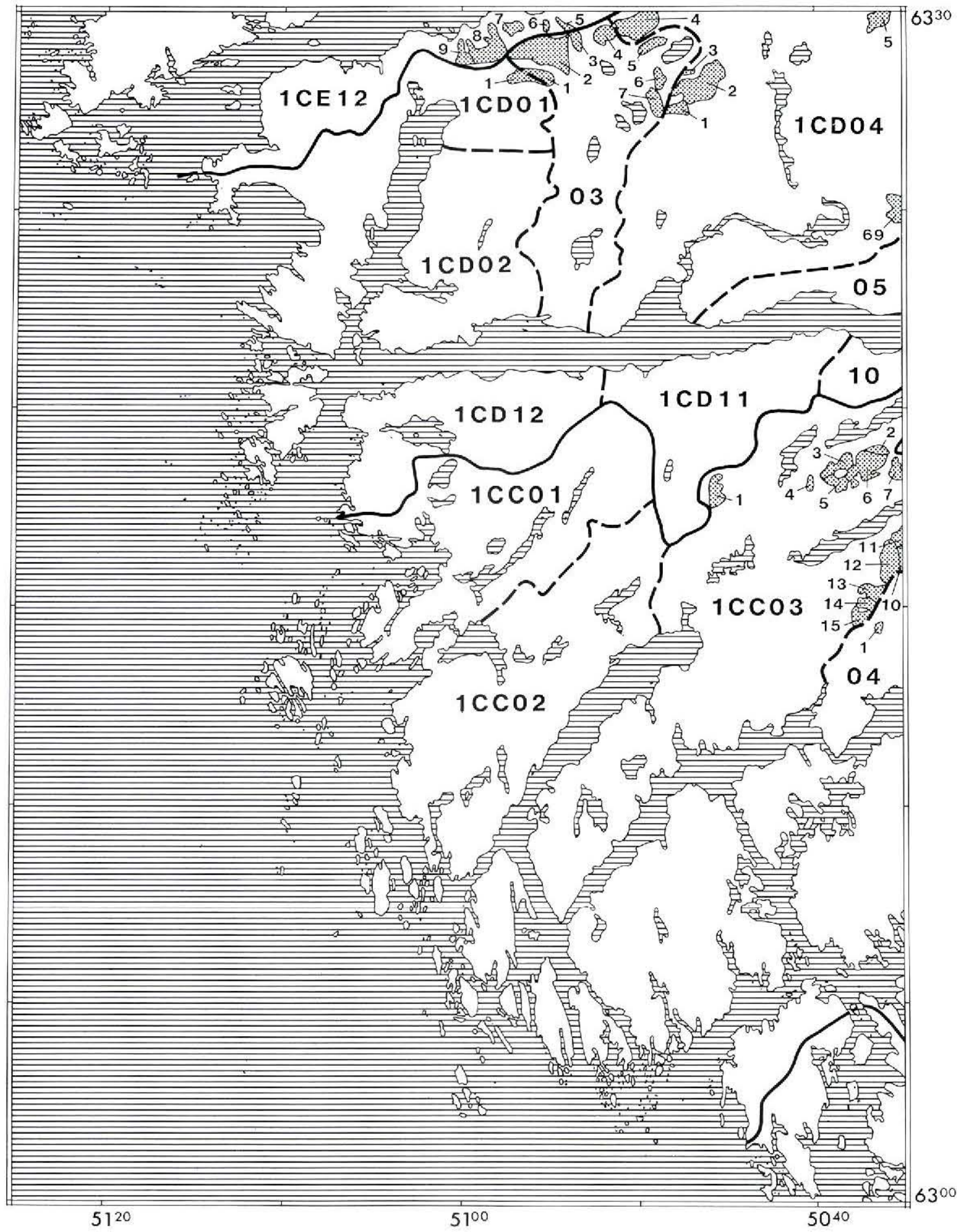




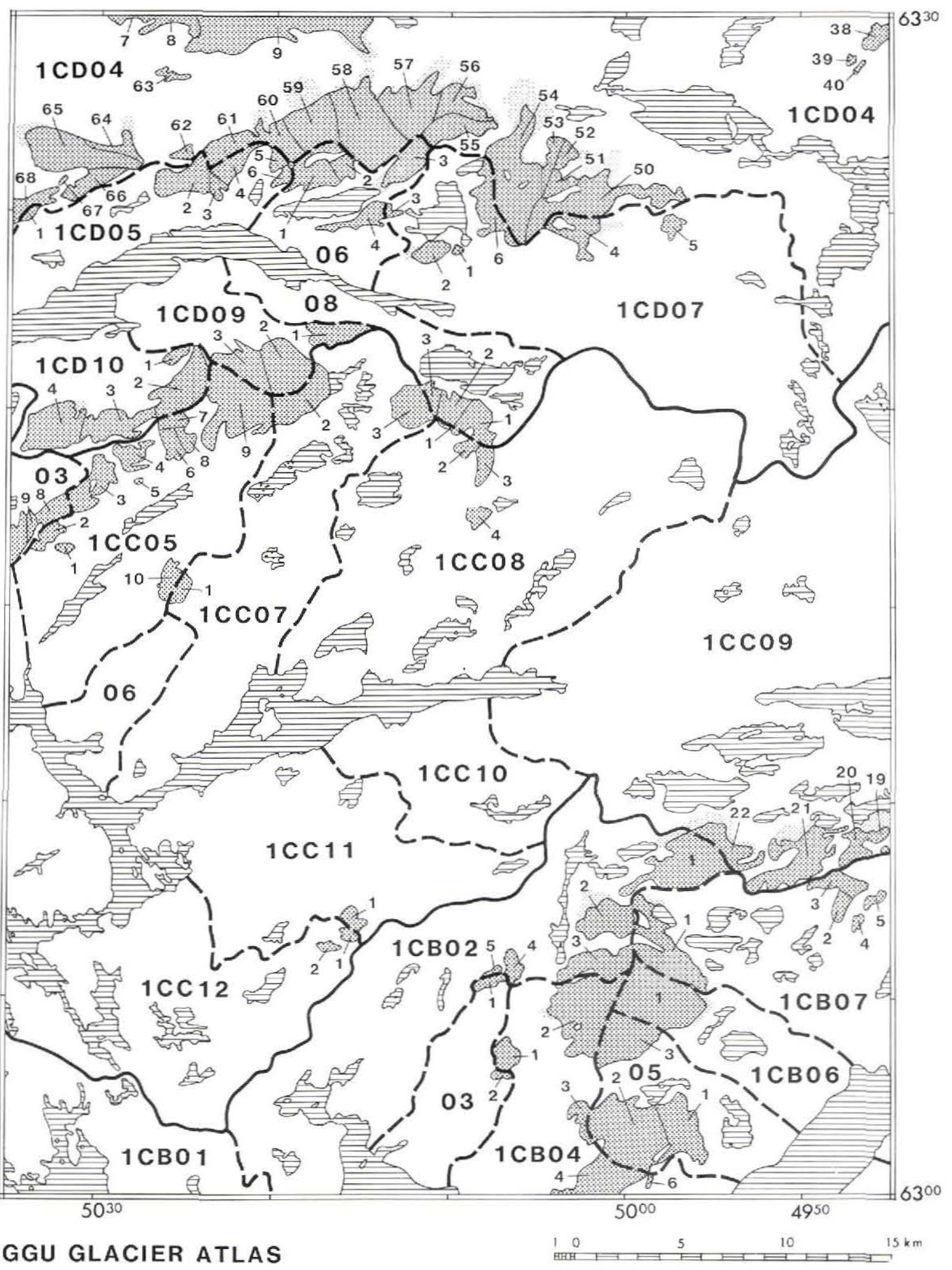

SHEET 6300-02 


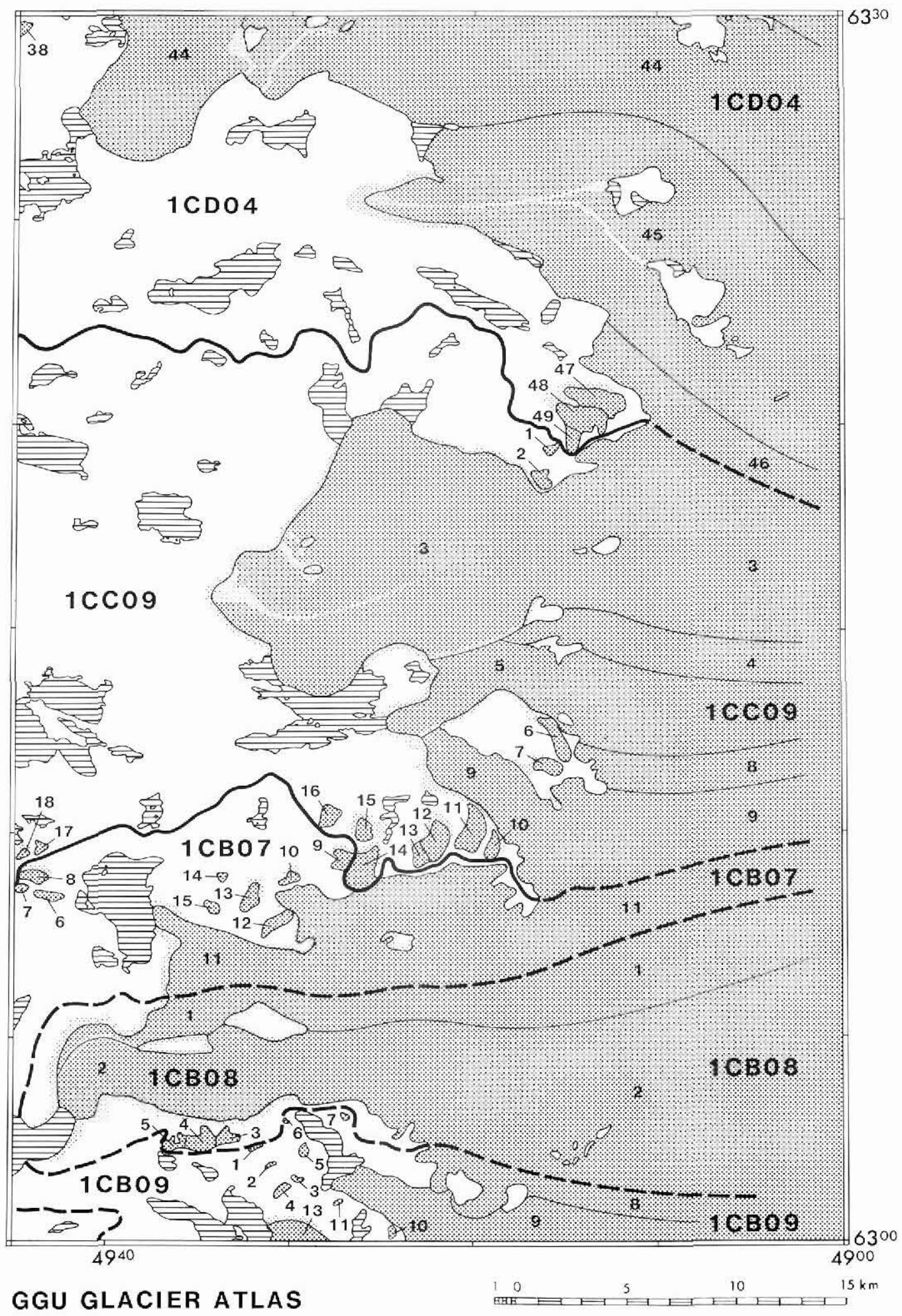

SHEET 6300-03 


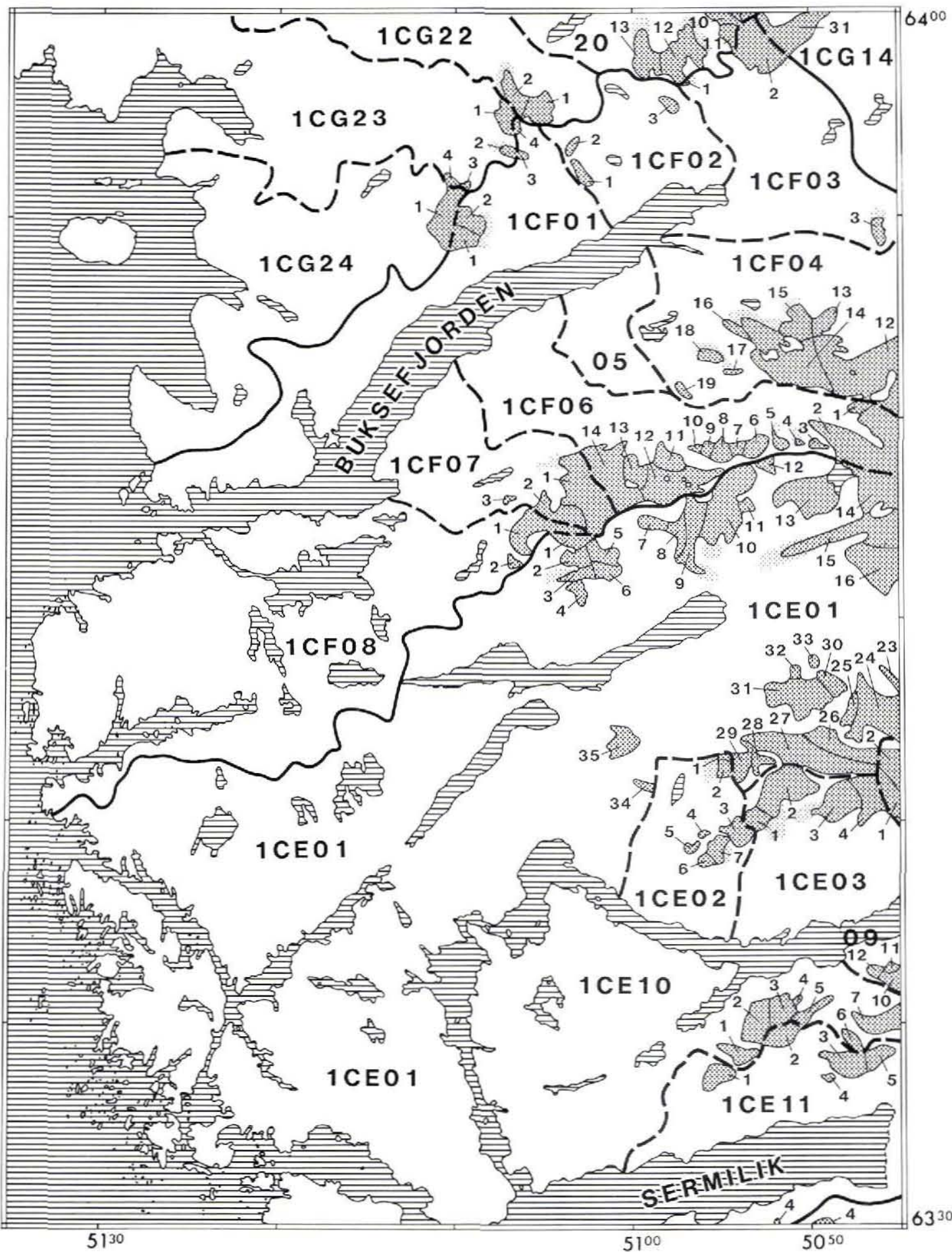

GGU GLACIER ATLAS

10

10 $15 \mathrm{~km}$ SHEET 6330-01 


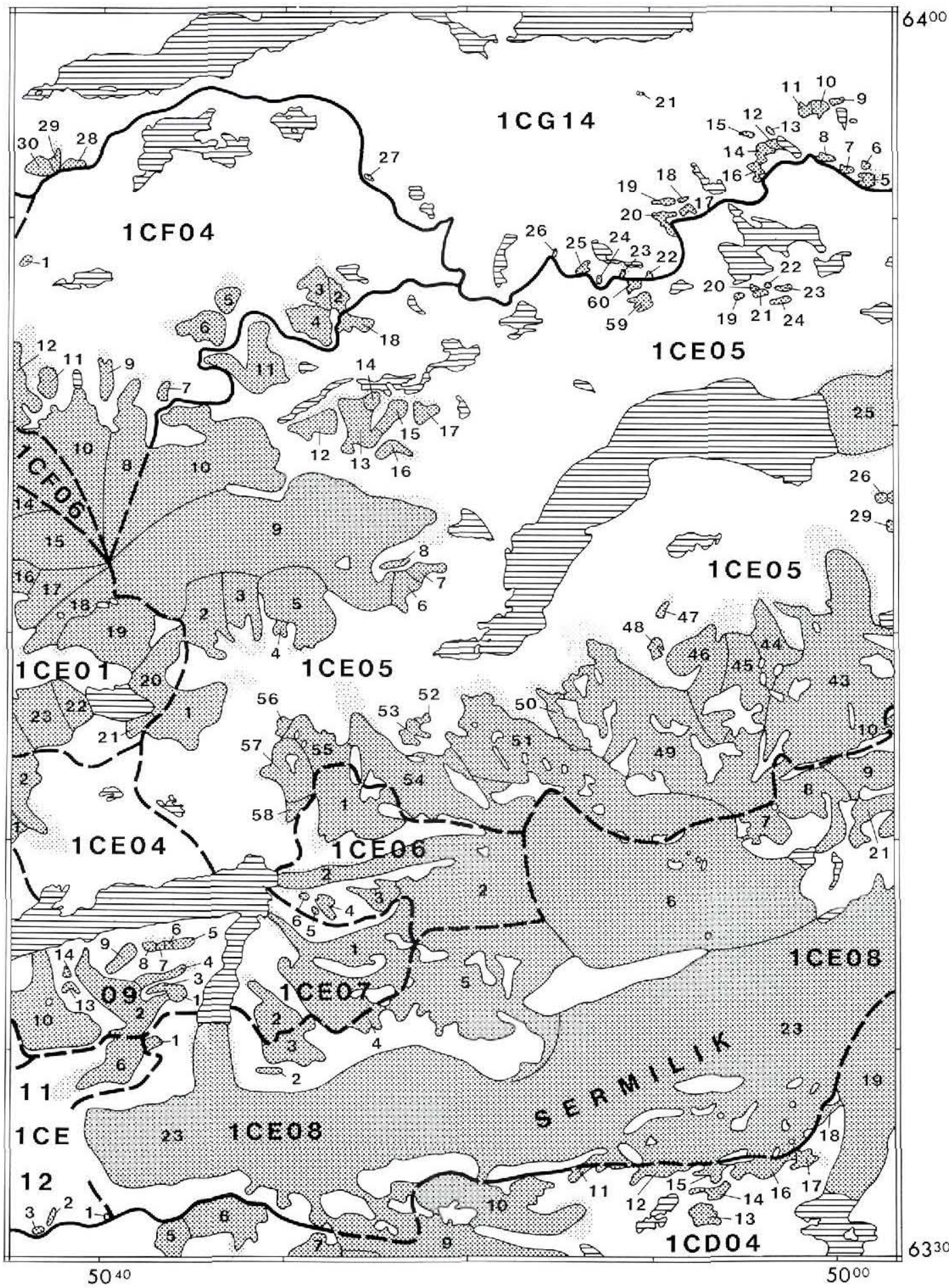

GGU GLACIER ATLAS

SHEET 6330-02 


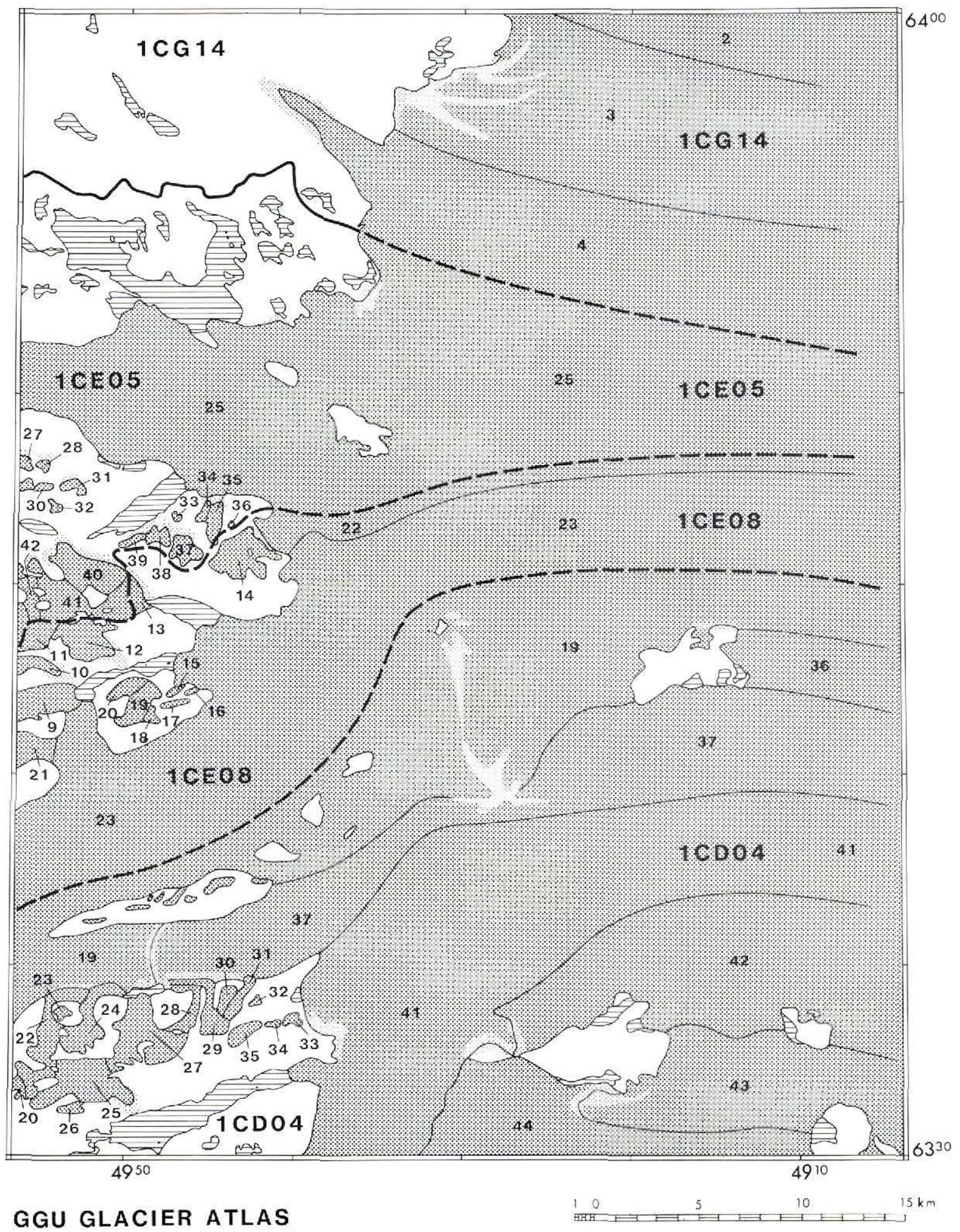

\section{SHEET 6330-03}




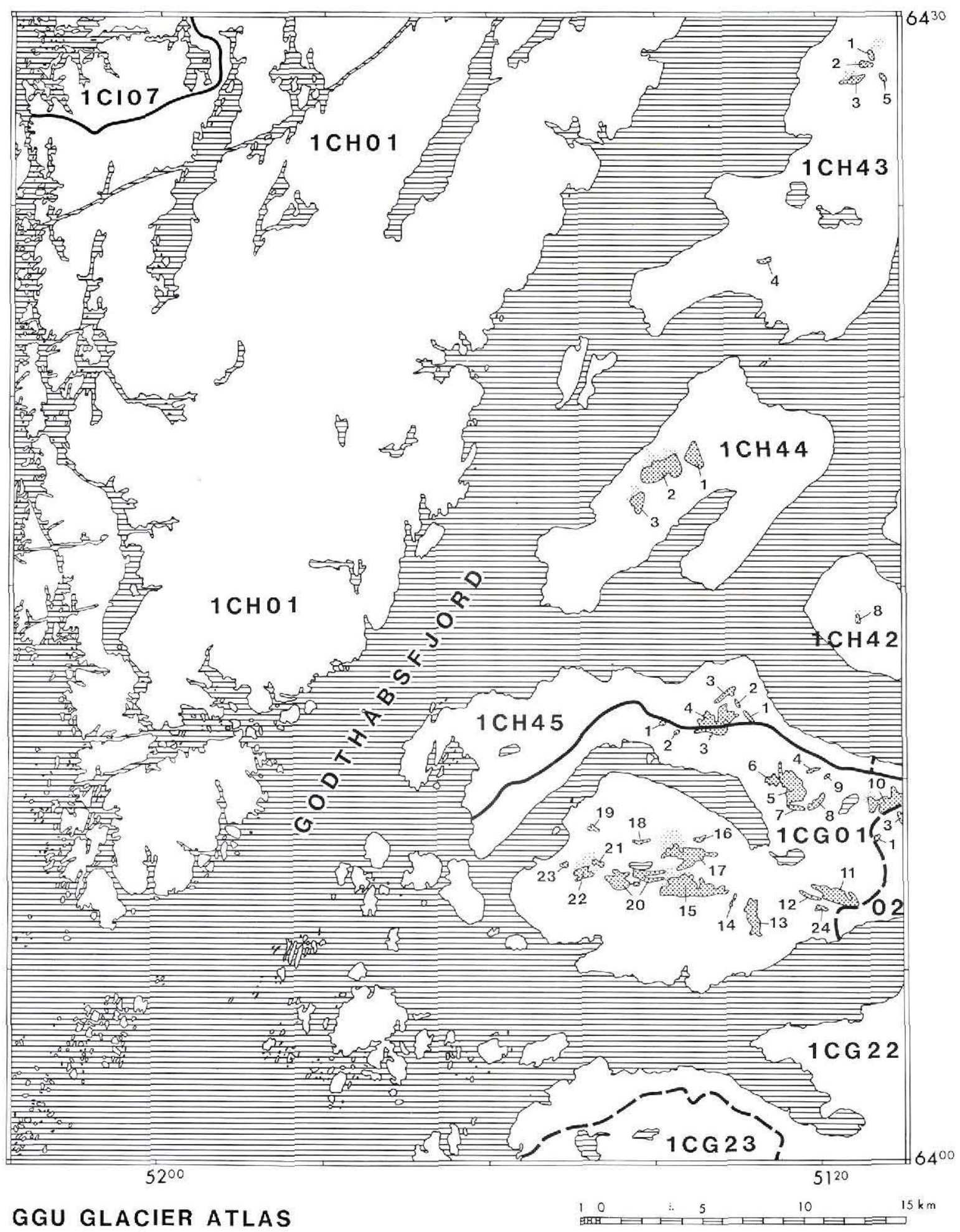

\section{SHEET 6400-01}




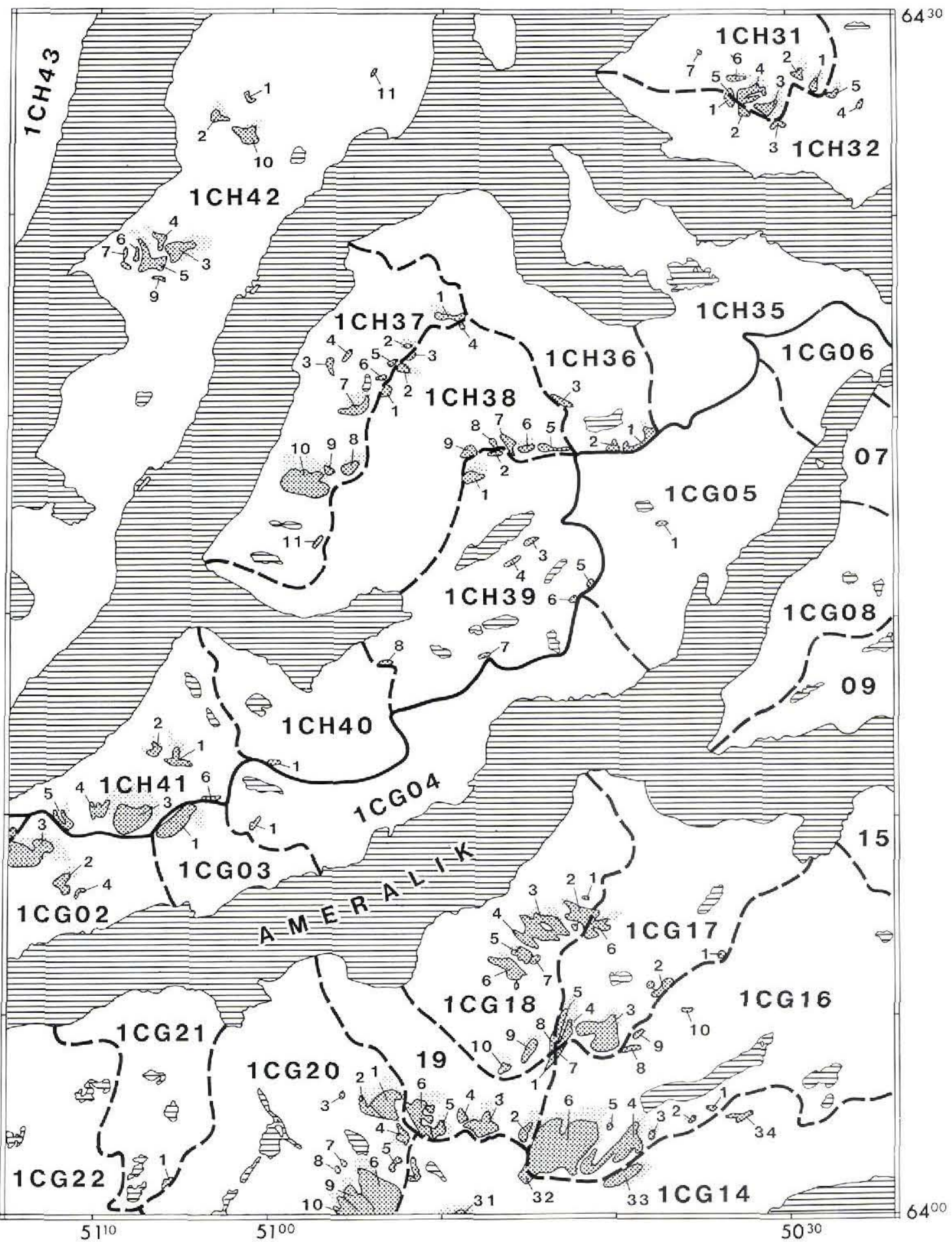

GGU GLACIER ATLAS

$\begin{array}{llll}10 & 5 & 10 & 15 k\end{array}$

SHEET 6400-02 


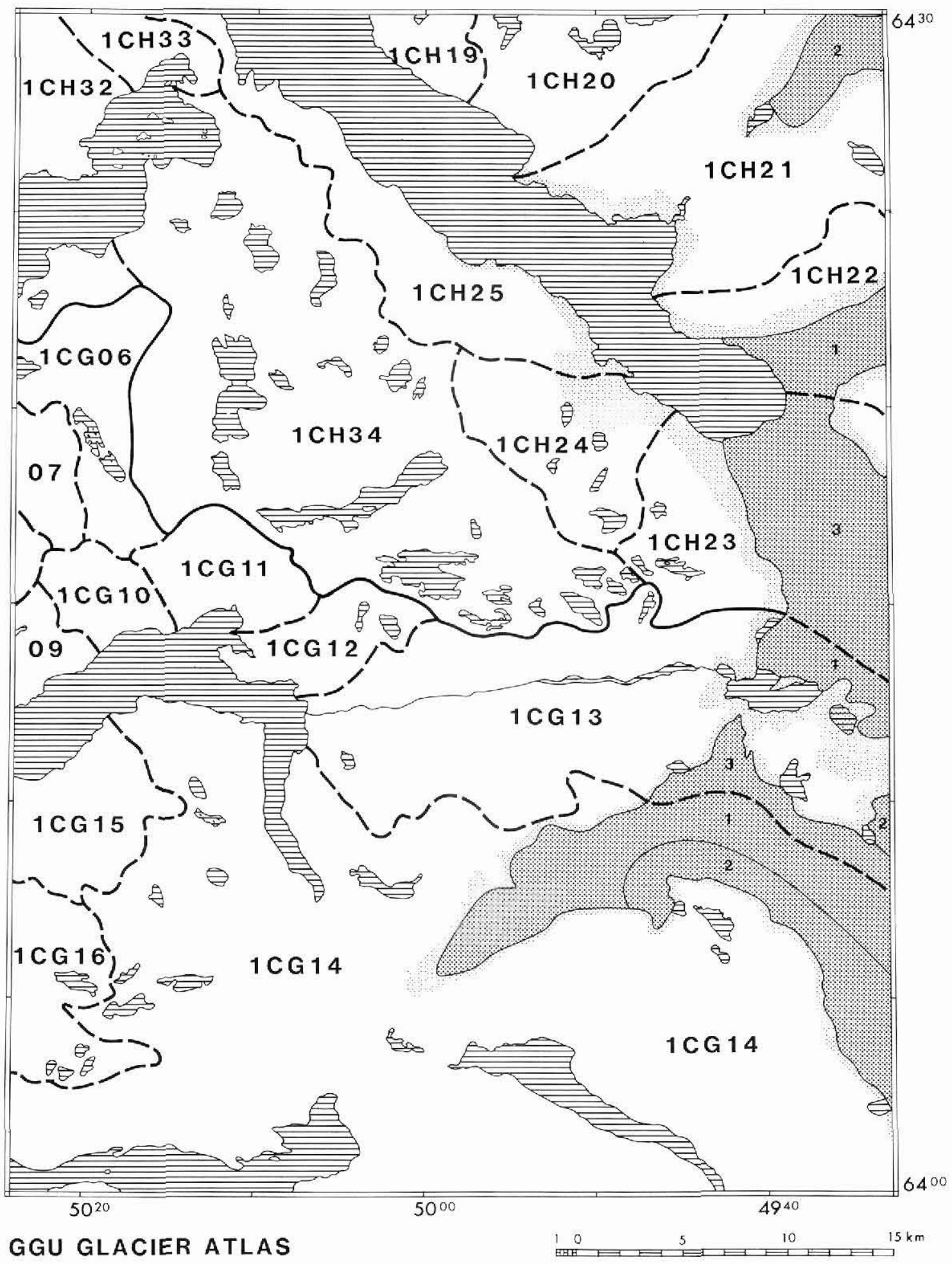

SHEET 6400-03 


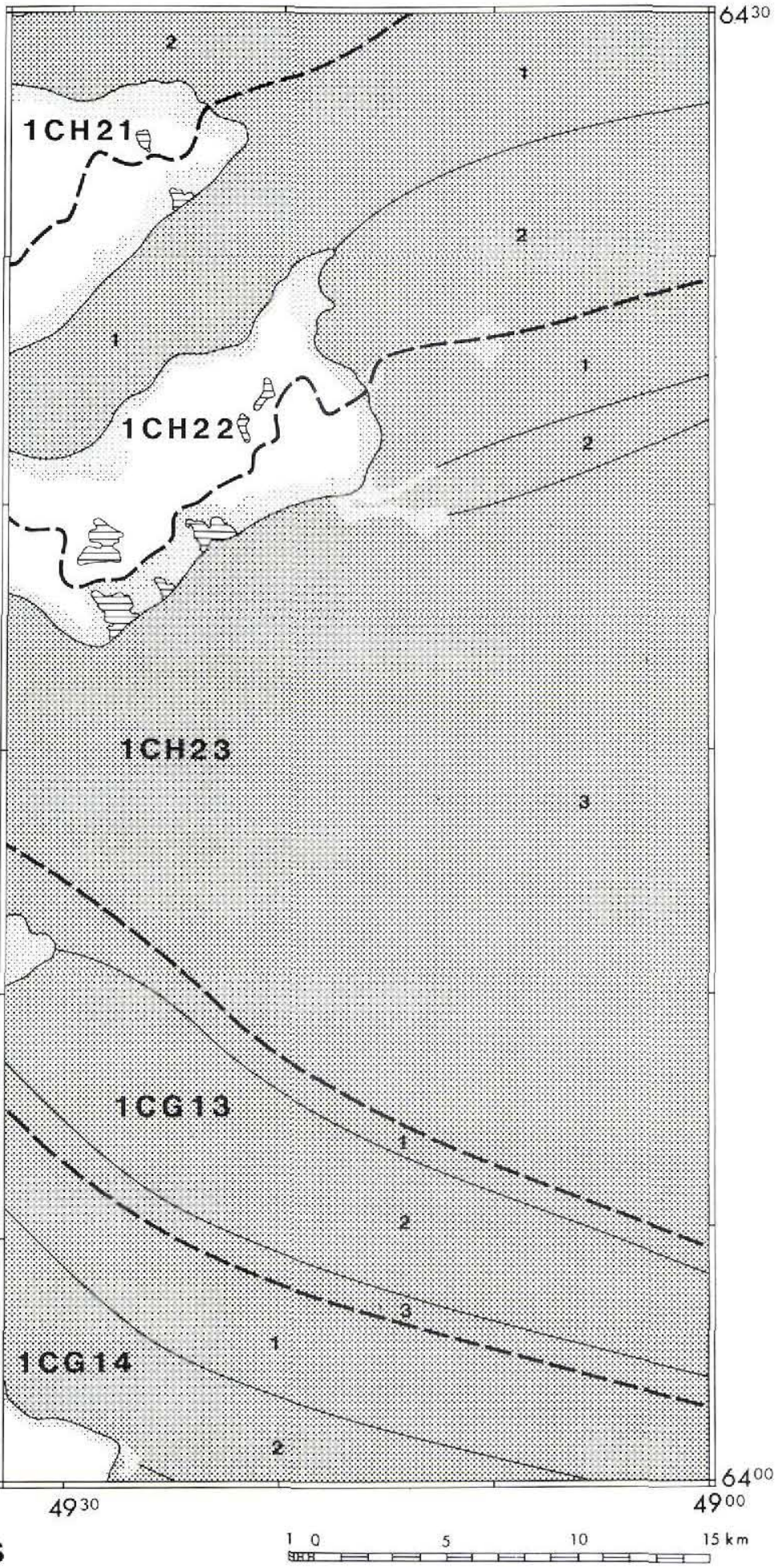

GGU GLACIER ATLAS

SHEET 6400-04 


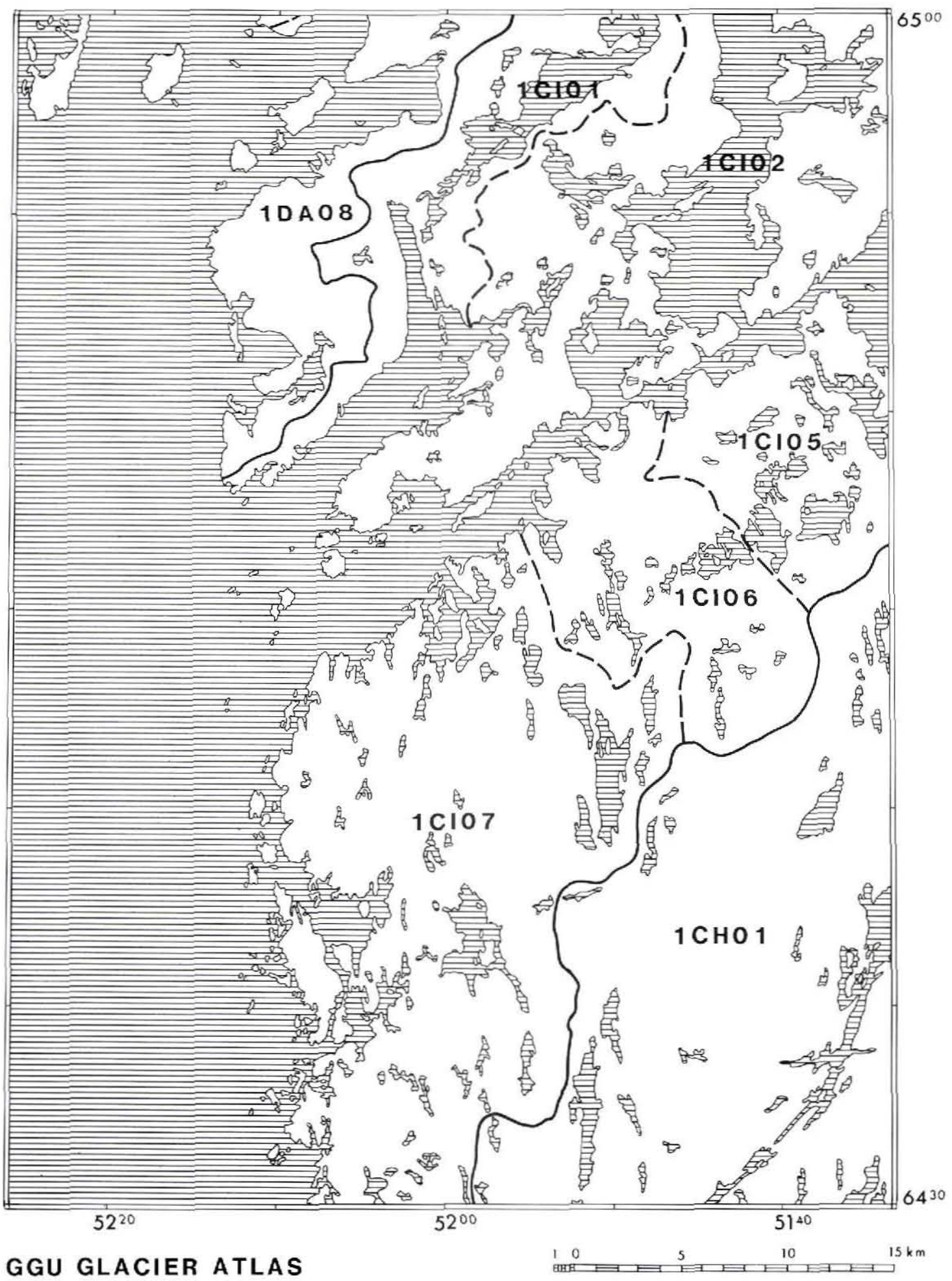

SHEET 6430-01 


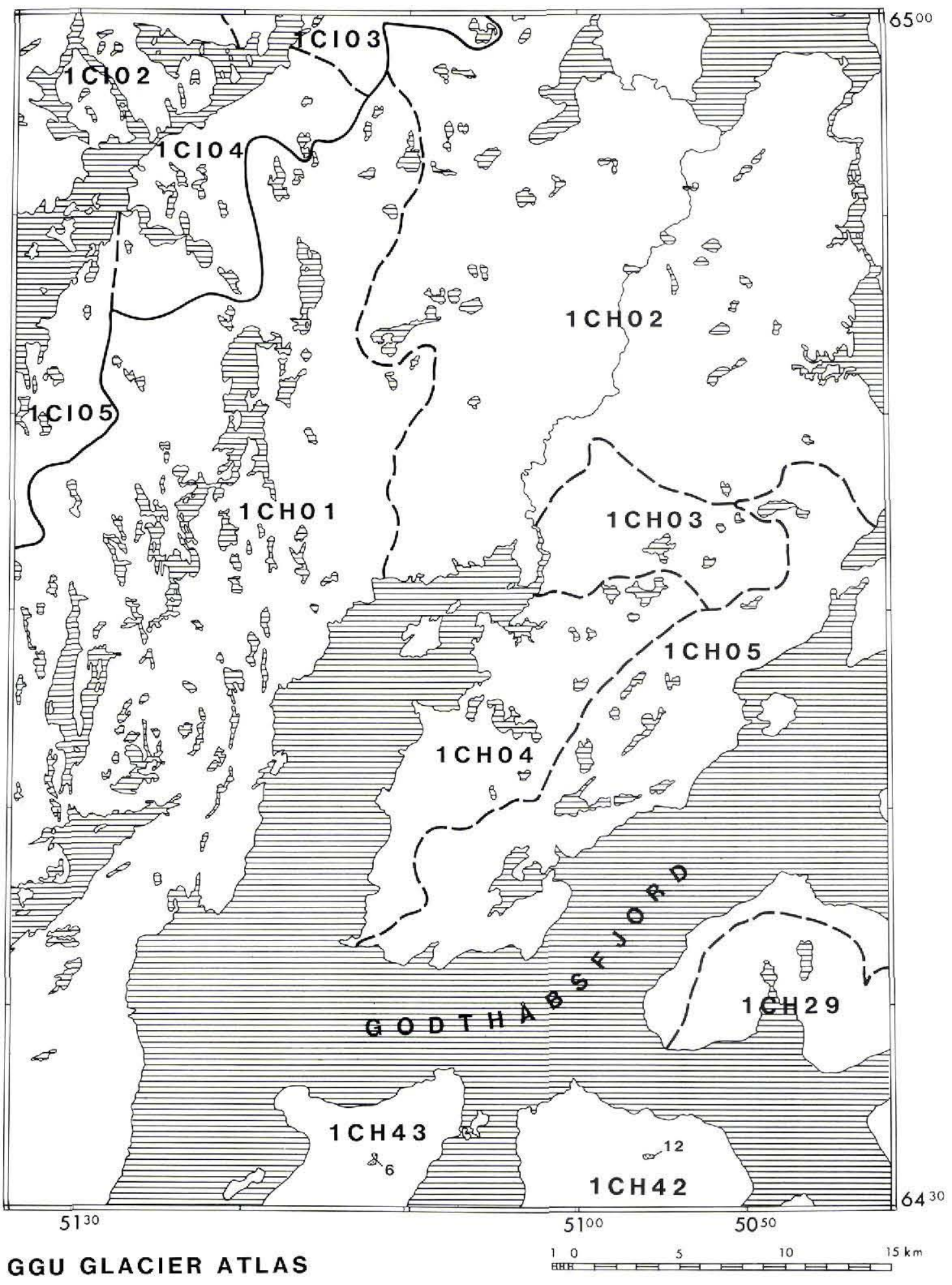

SHEET 6430-02 


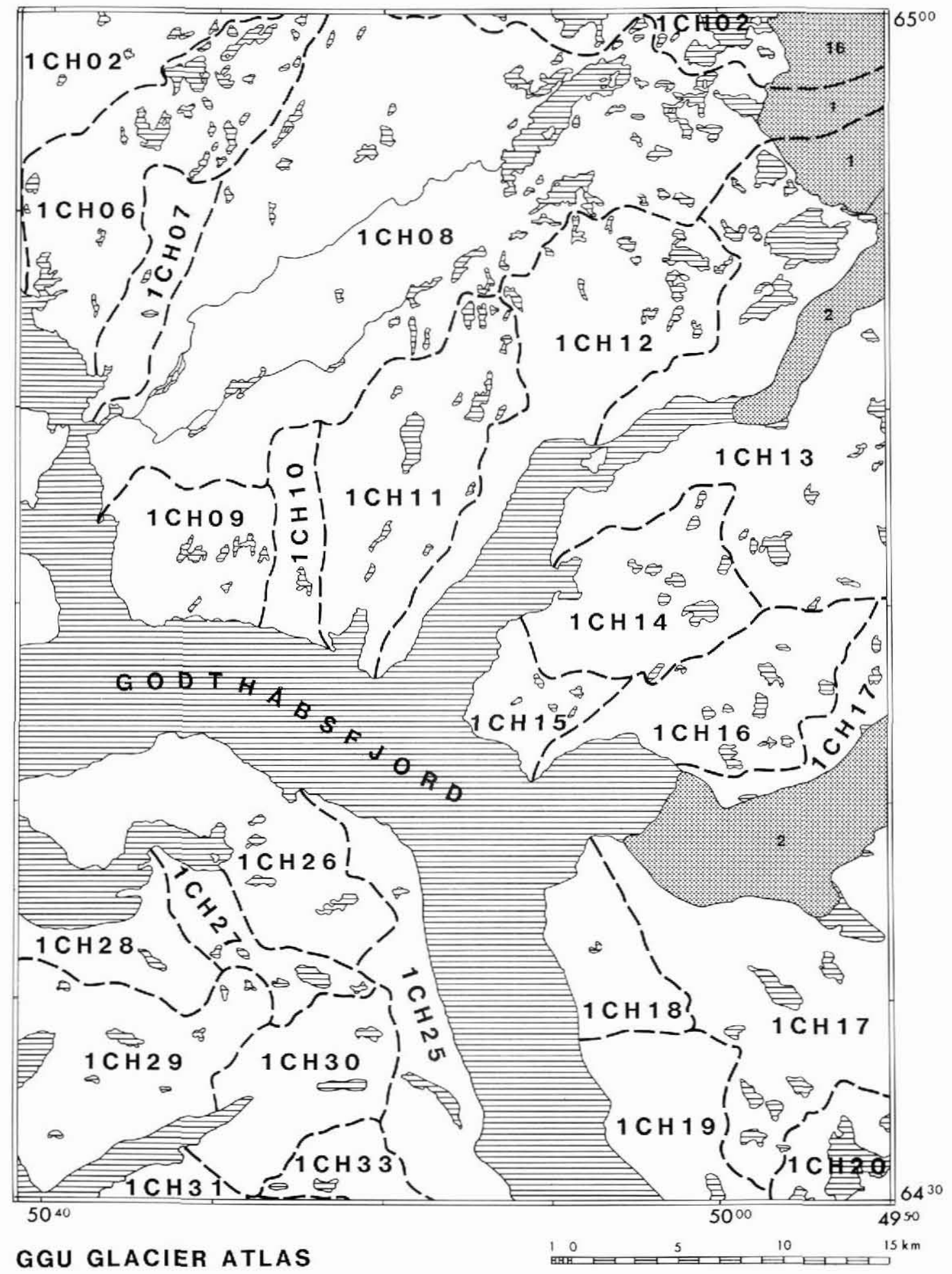

SHEET 6430-03 


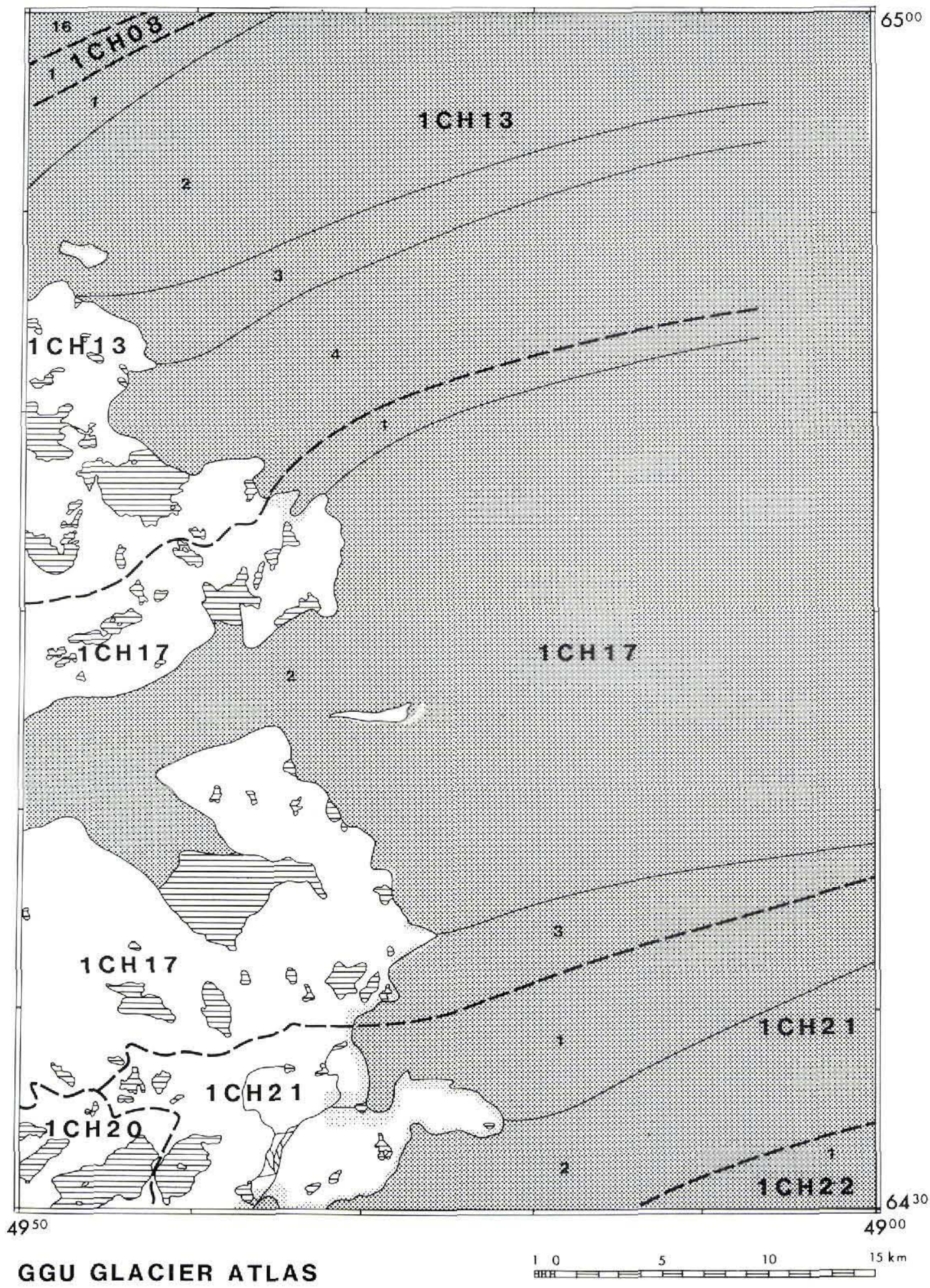

SHEET 6430-04 


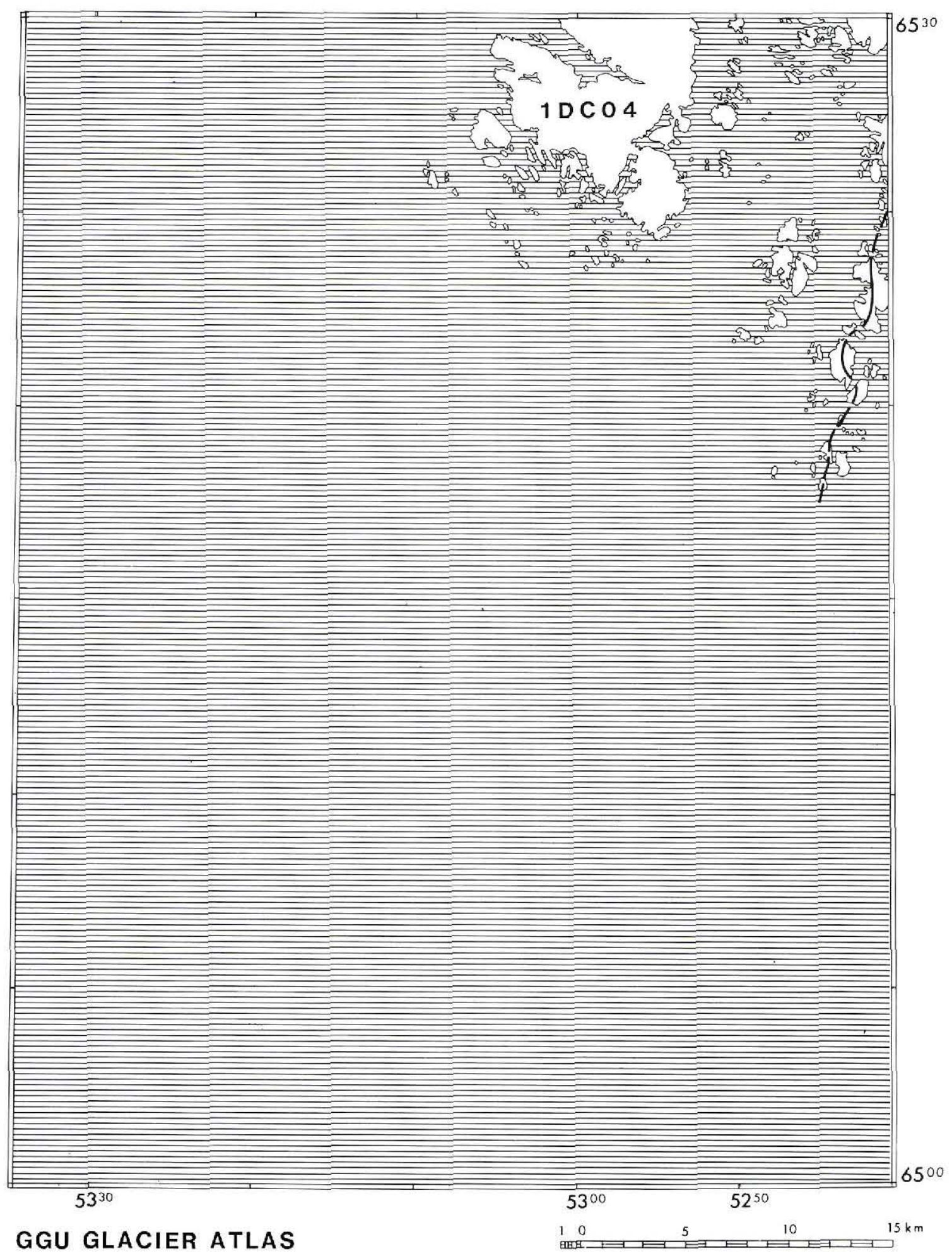

SHEET 6500-01 


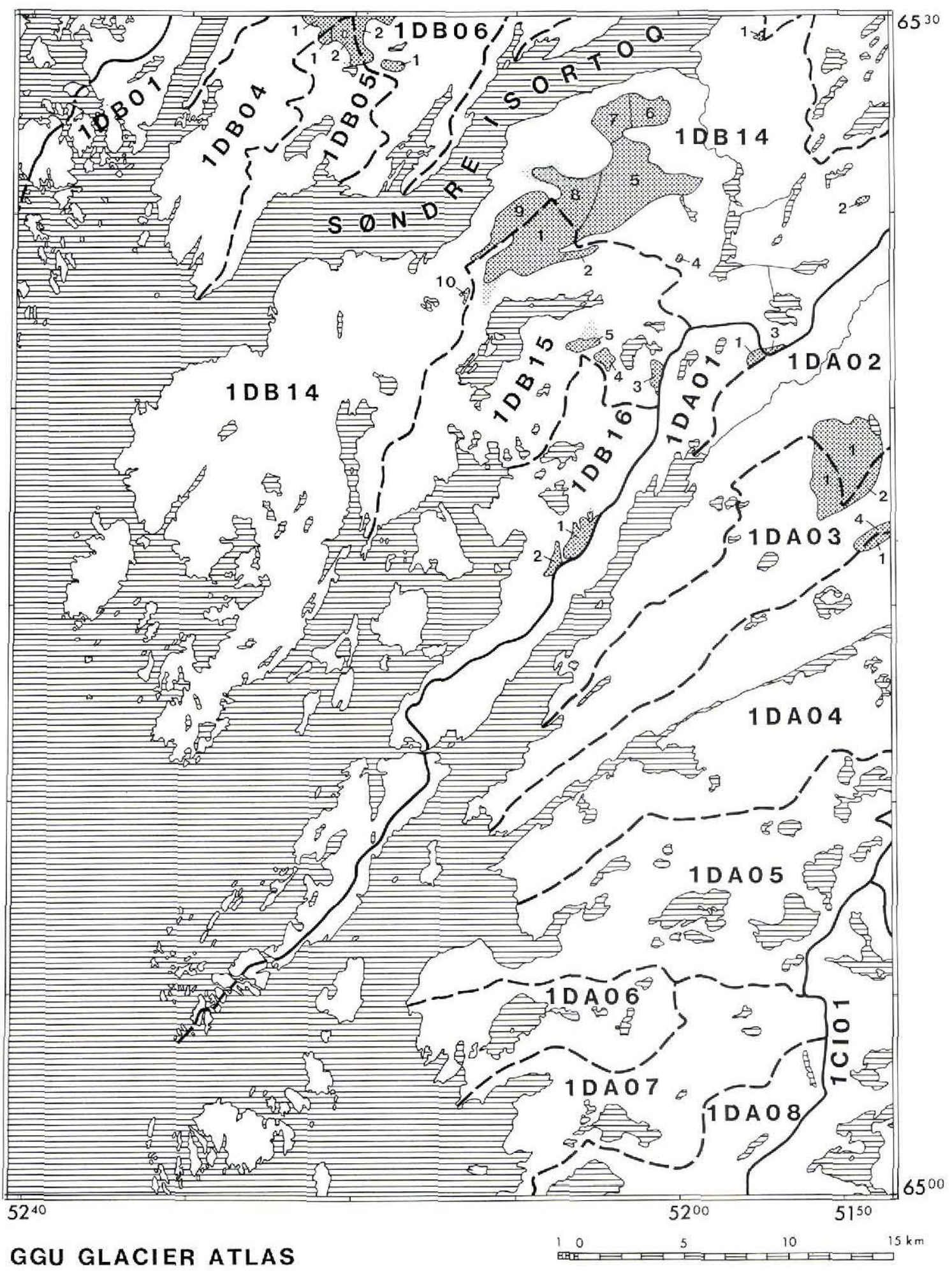

\section{SHEET 6500-02}




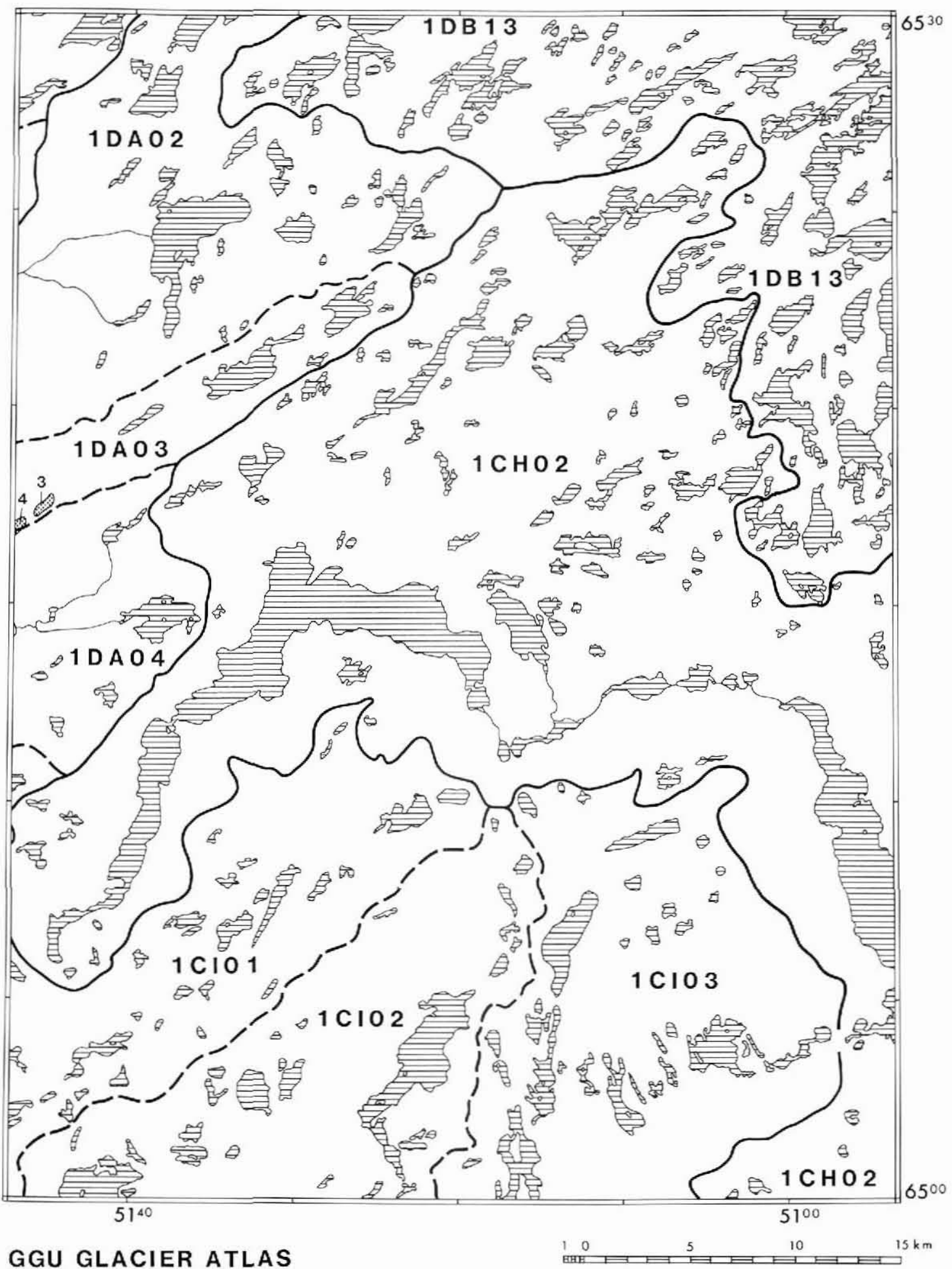

\section{SHEET 6500-03}




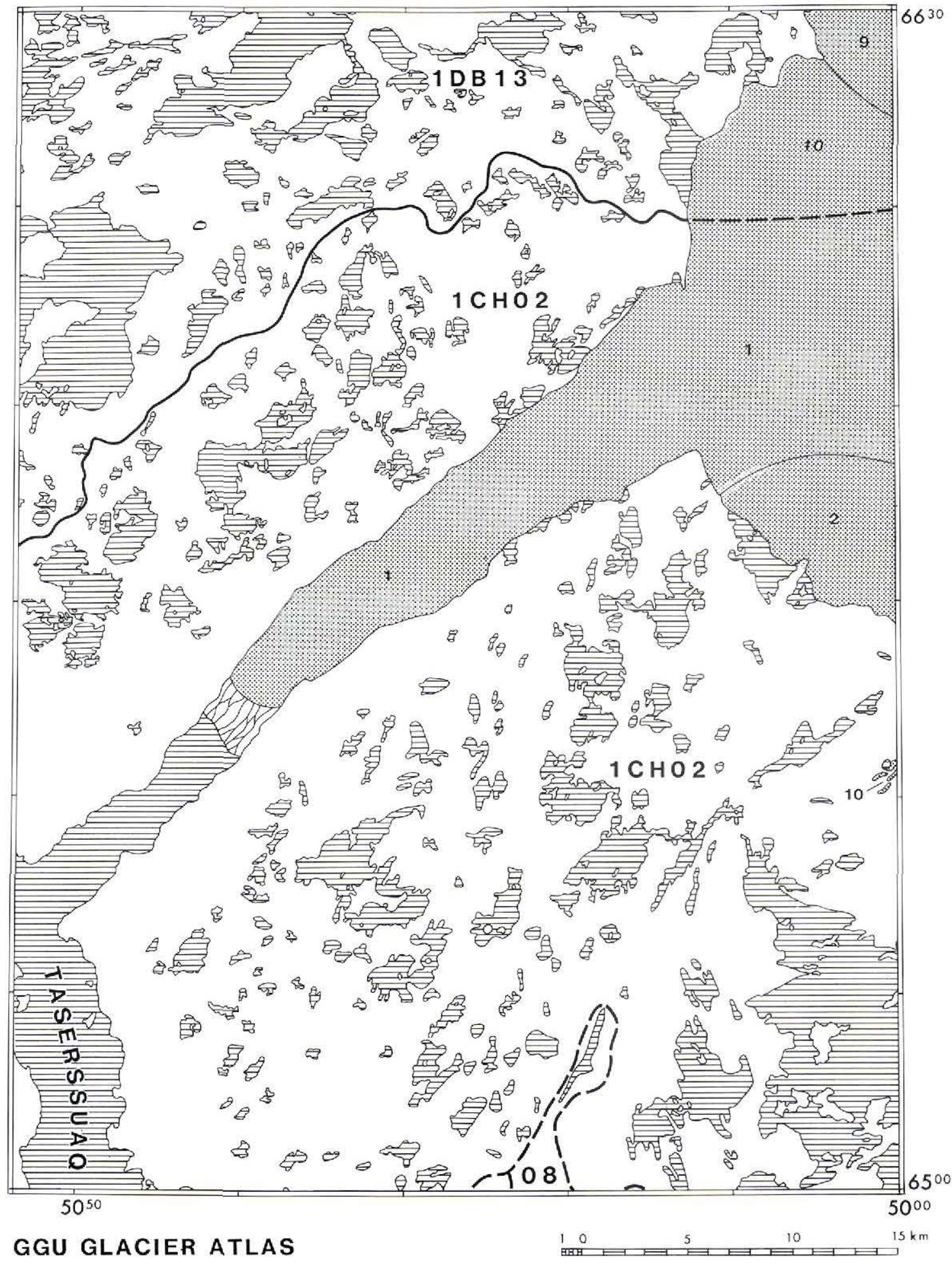

SHEET 6500-04 


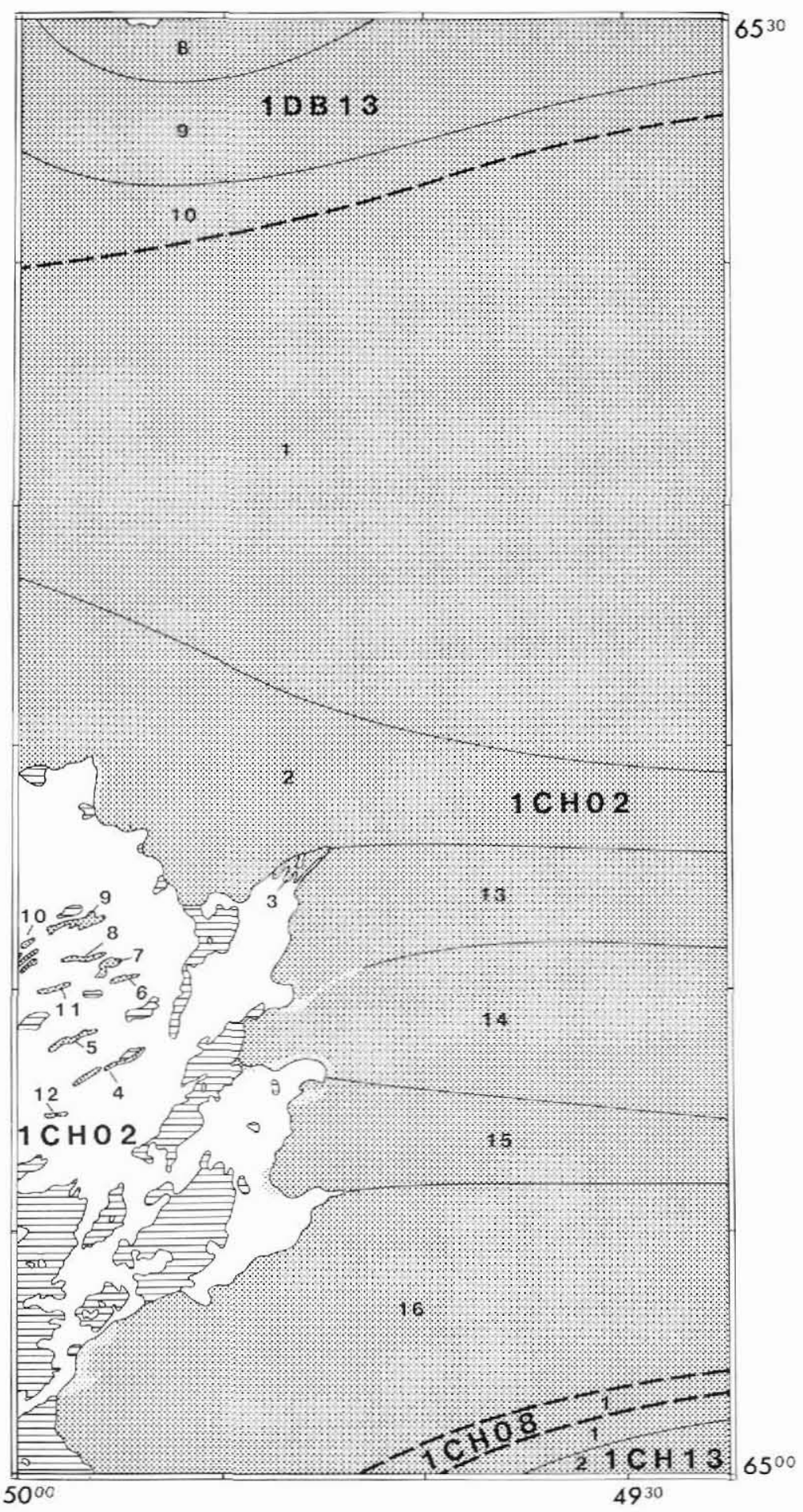

GGU GLACIER ATLAS

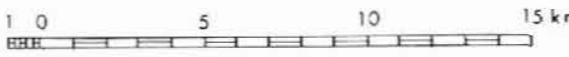
SHEET 6500-05 


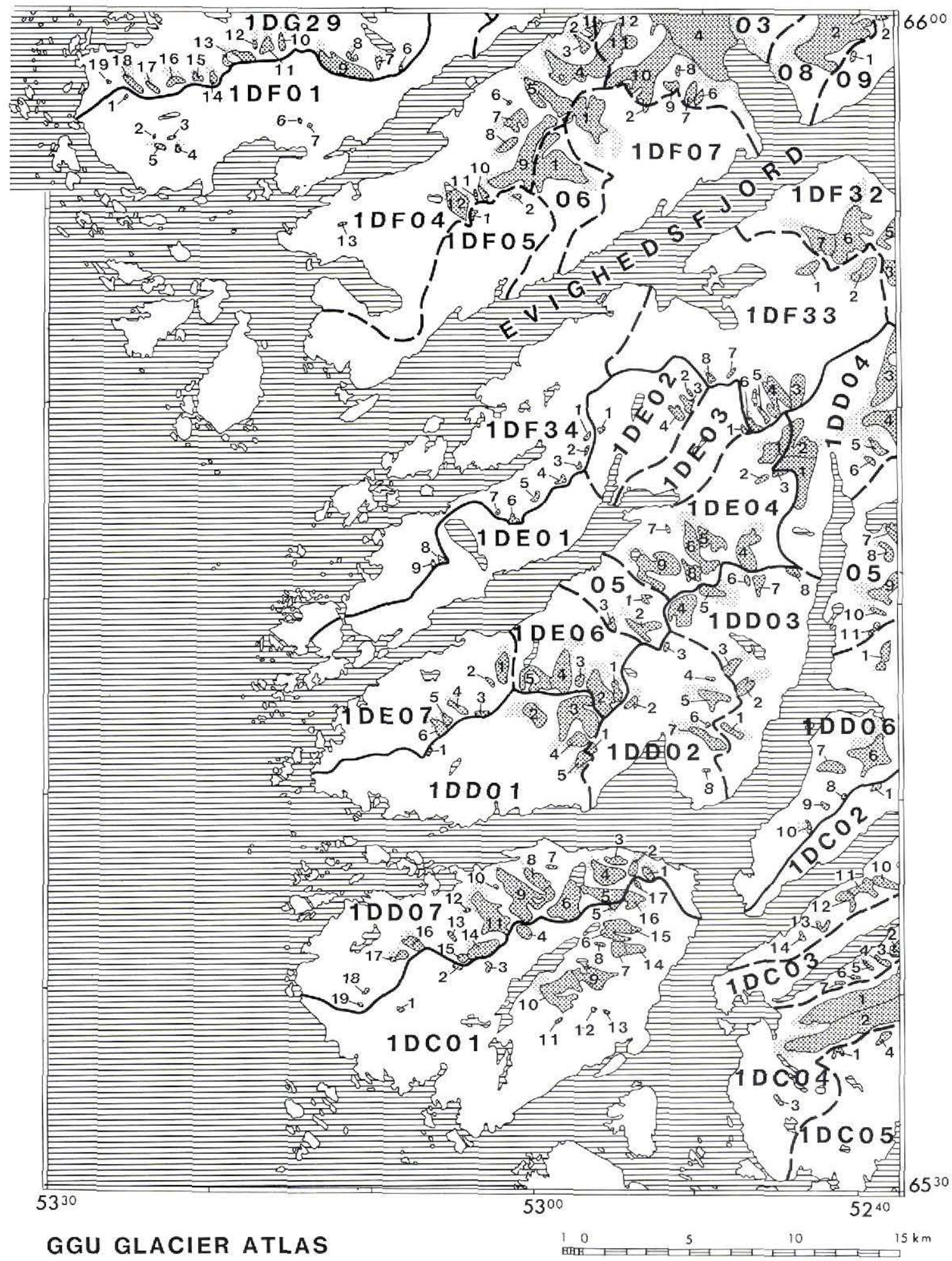

SHEET 6530-01 


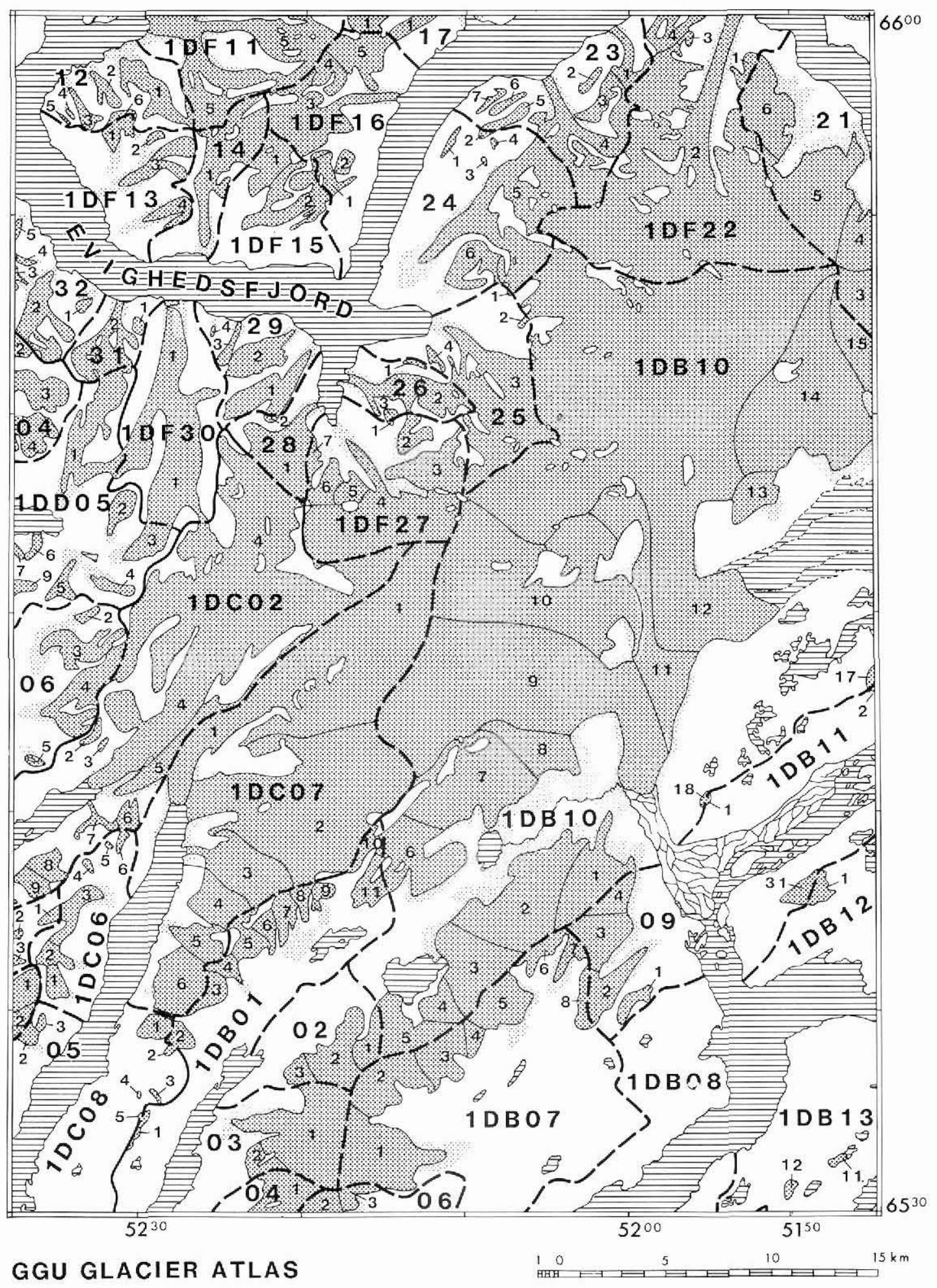

SHEET 6530-02 


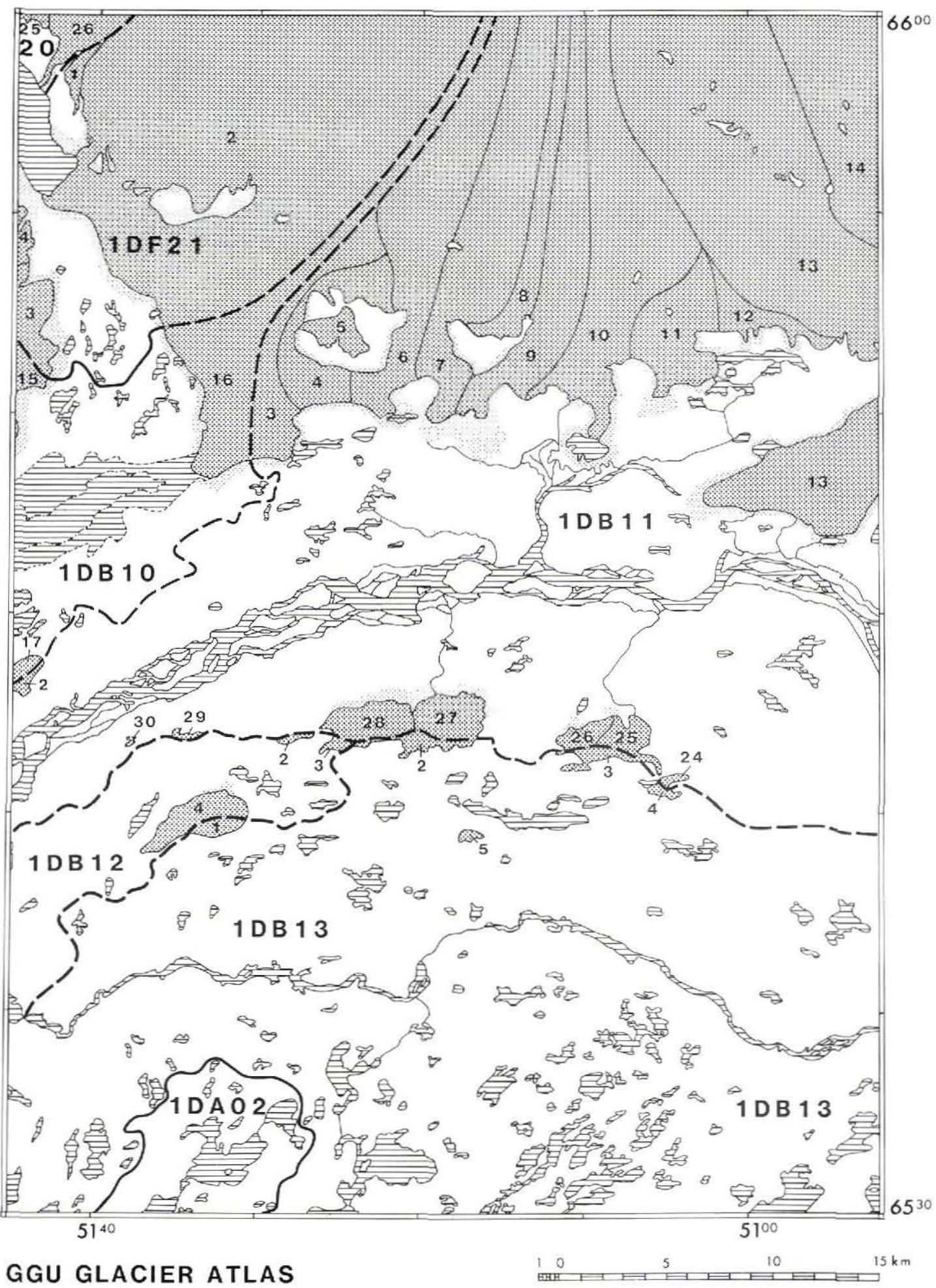

SHEET 6530-03 


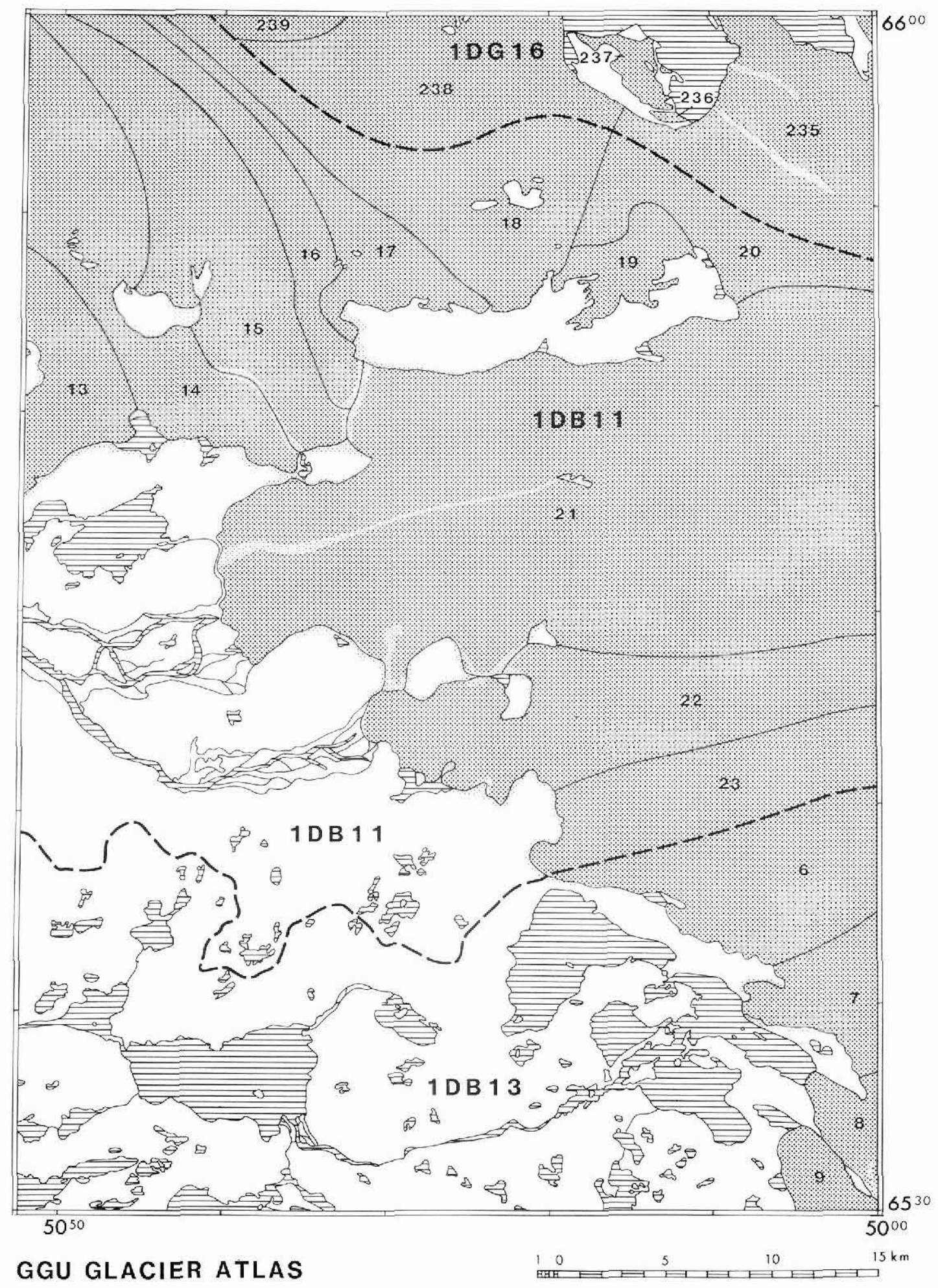

\section{SHEET 6530-04}




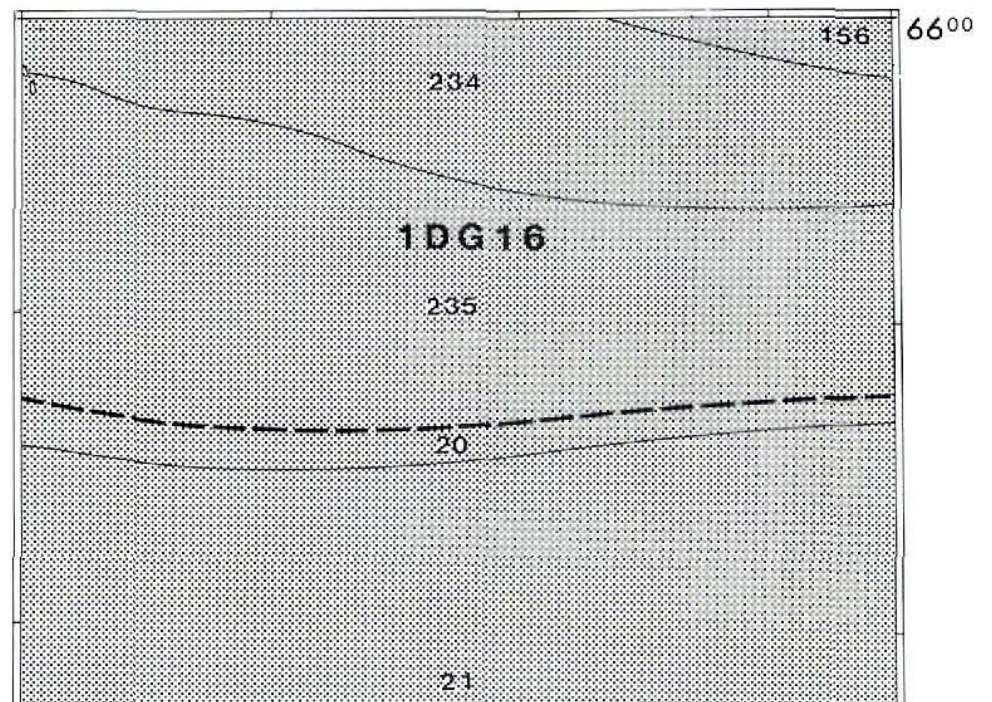

$108.1 \%$

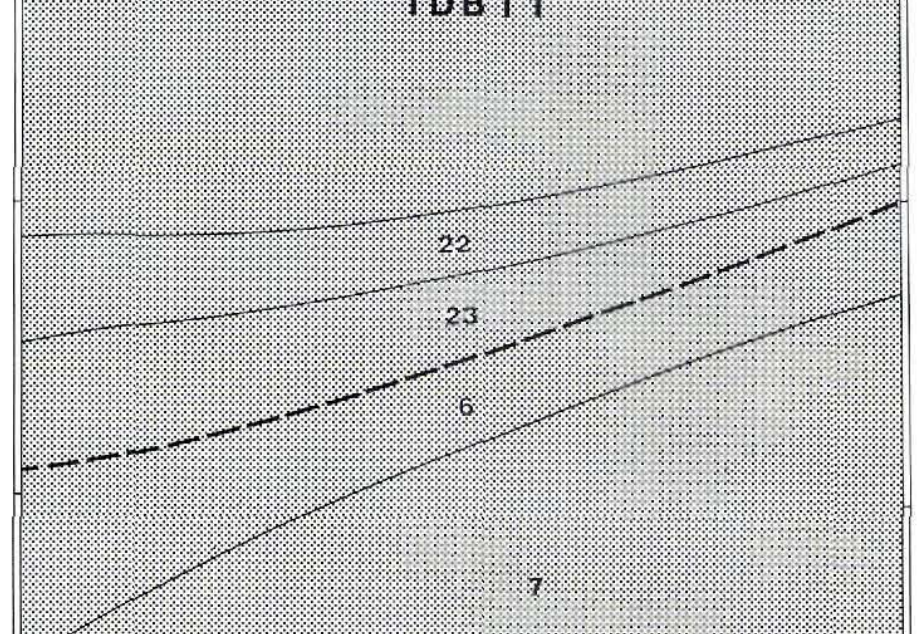

$1037: 2$

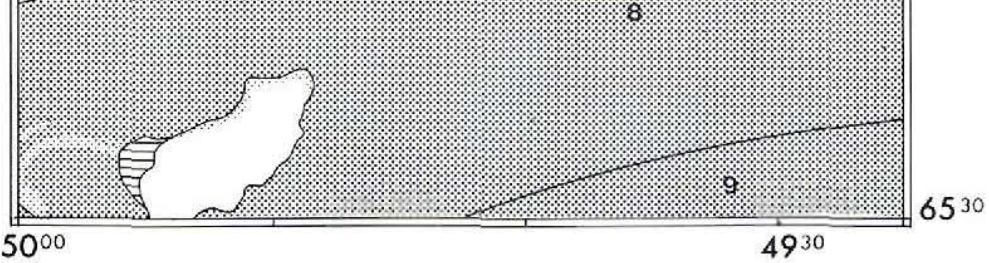

GGU GLACIER ATLAS

1

10 $15 \mathrm{~km}$

SHEET 6530-05 


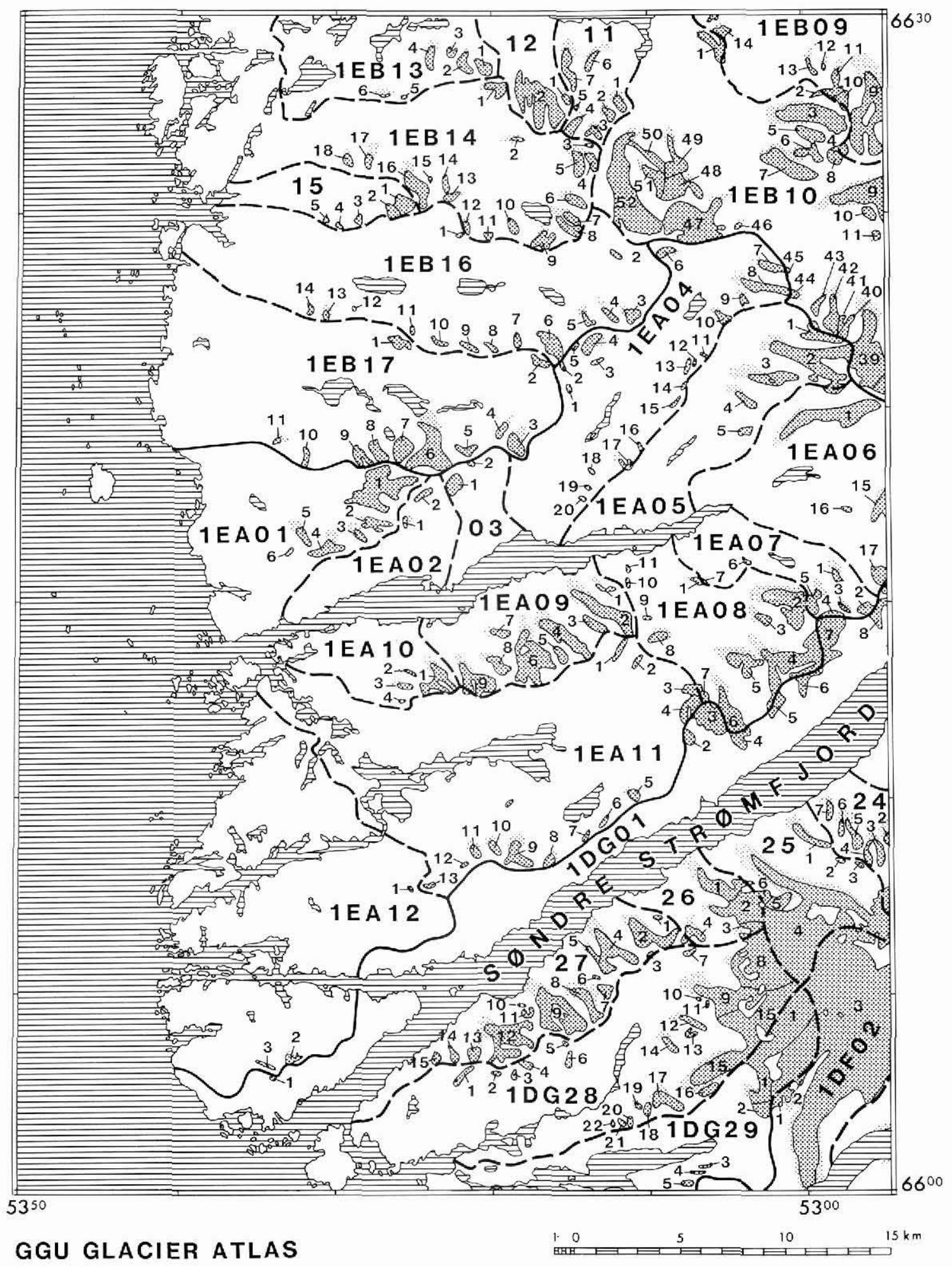

\section{SHEET 6600-01}




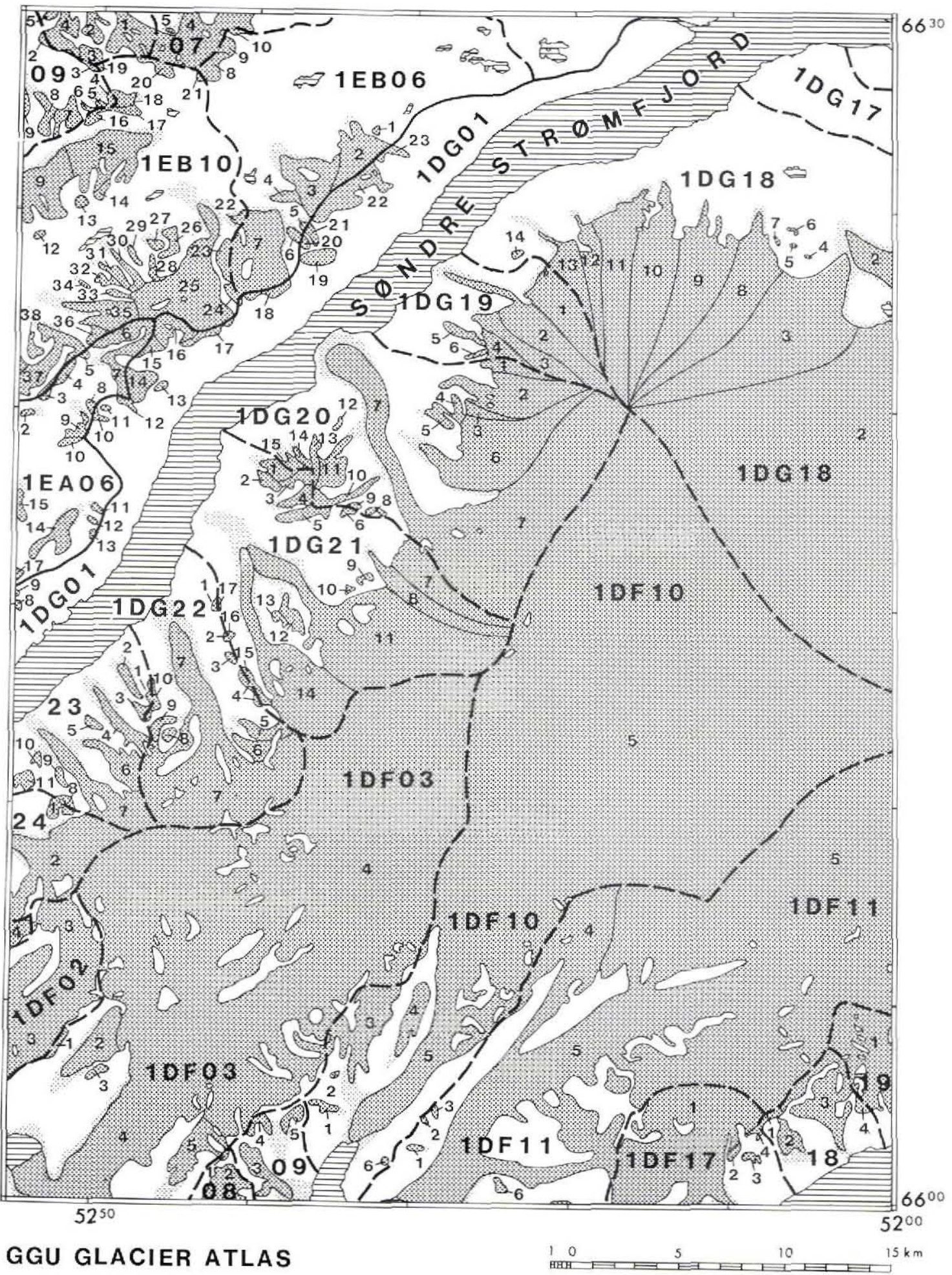

SHEET 6600-02 


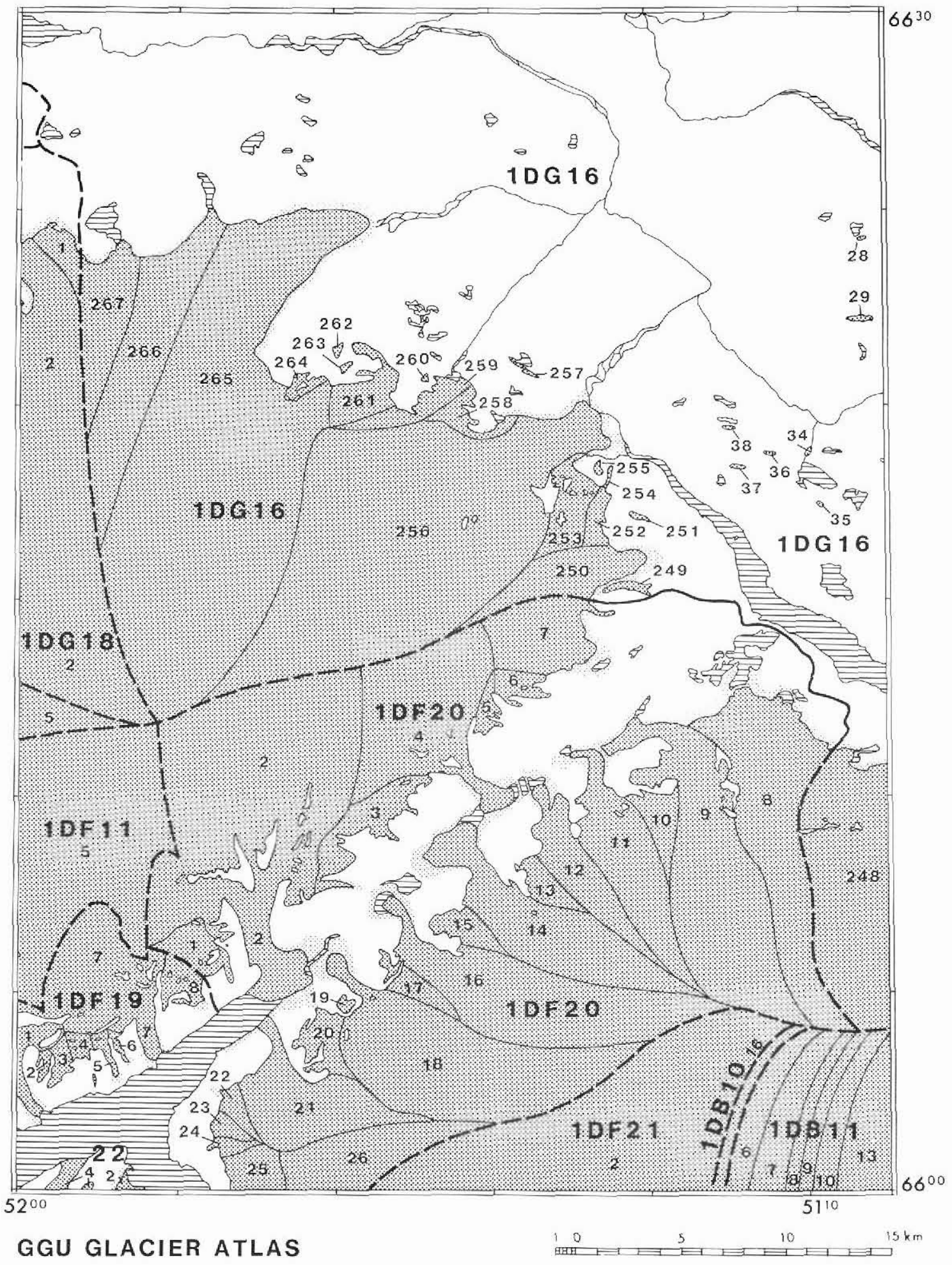

SHEET 6600-03 


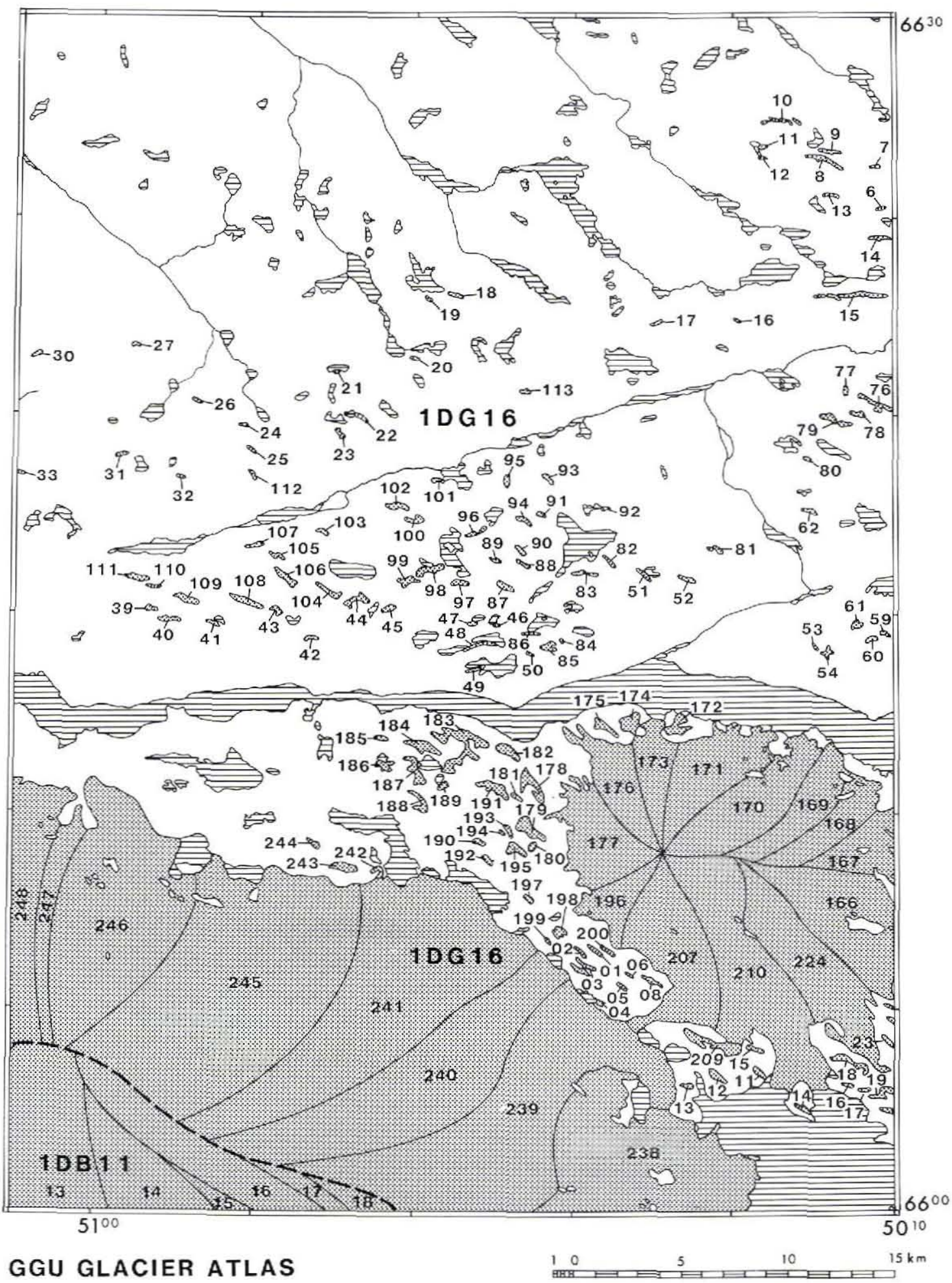

SHEET 6600-04 


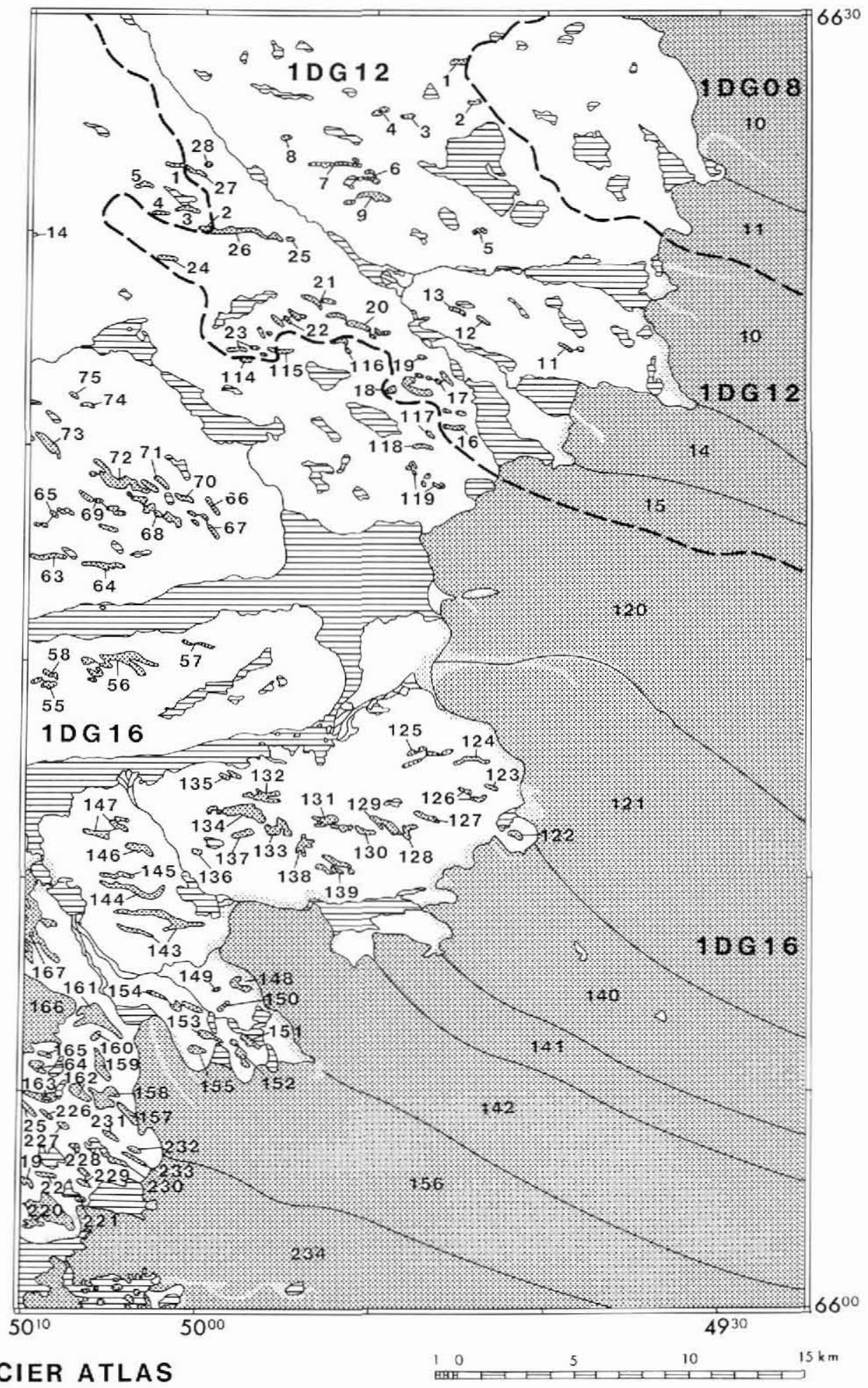

GGU GLACIER ATLAS

10

\section{SHEET 6600-05}




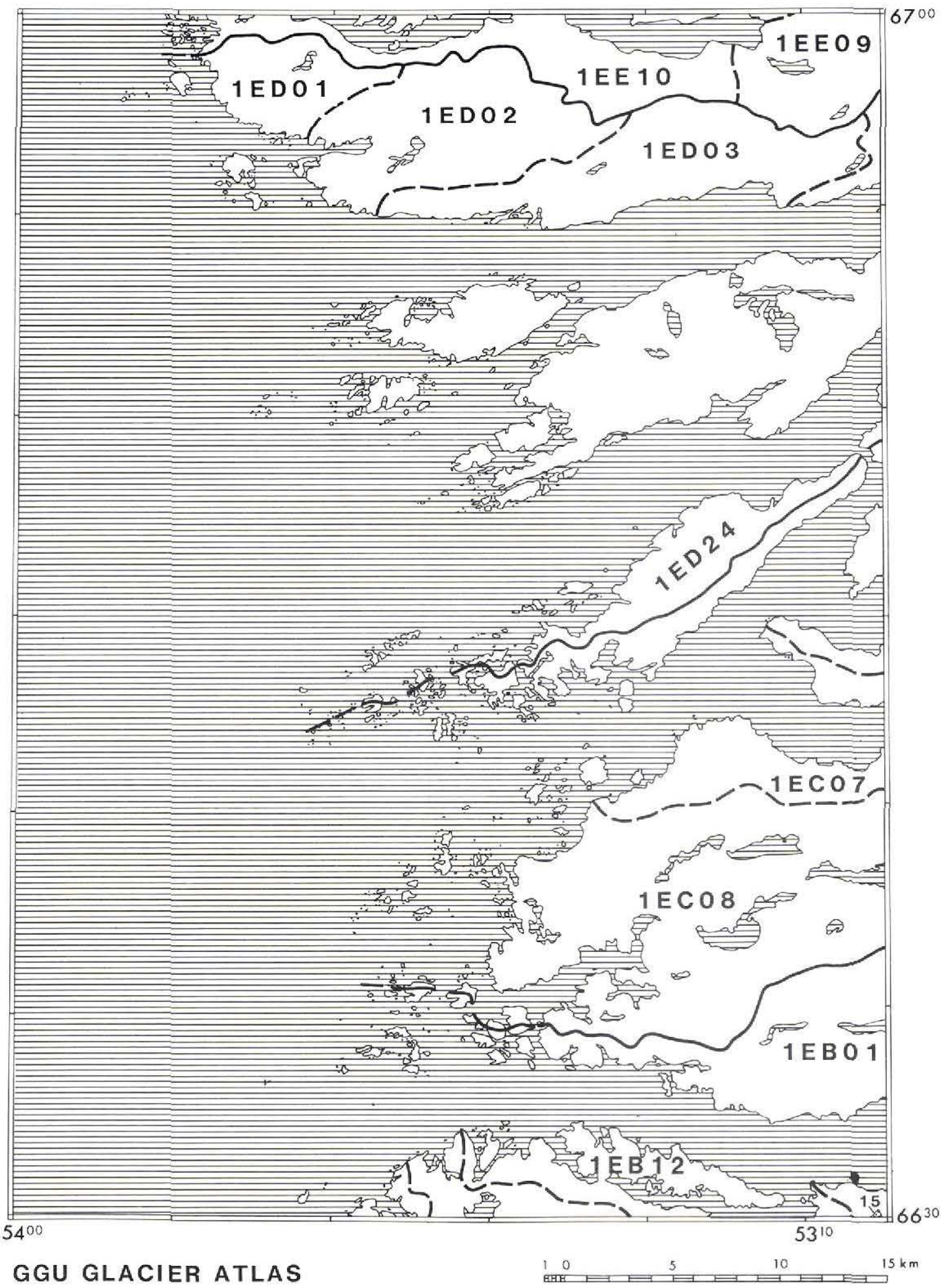




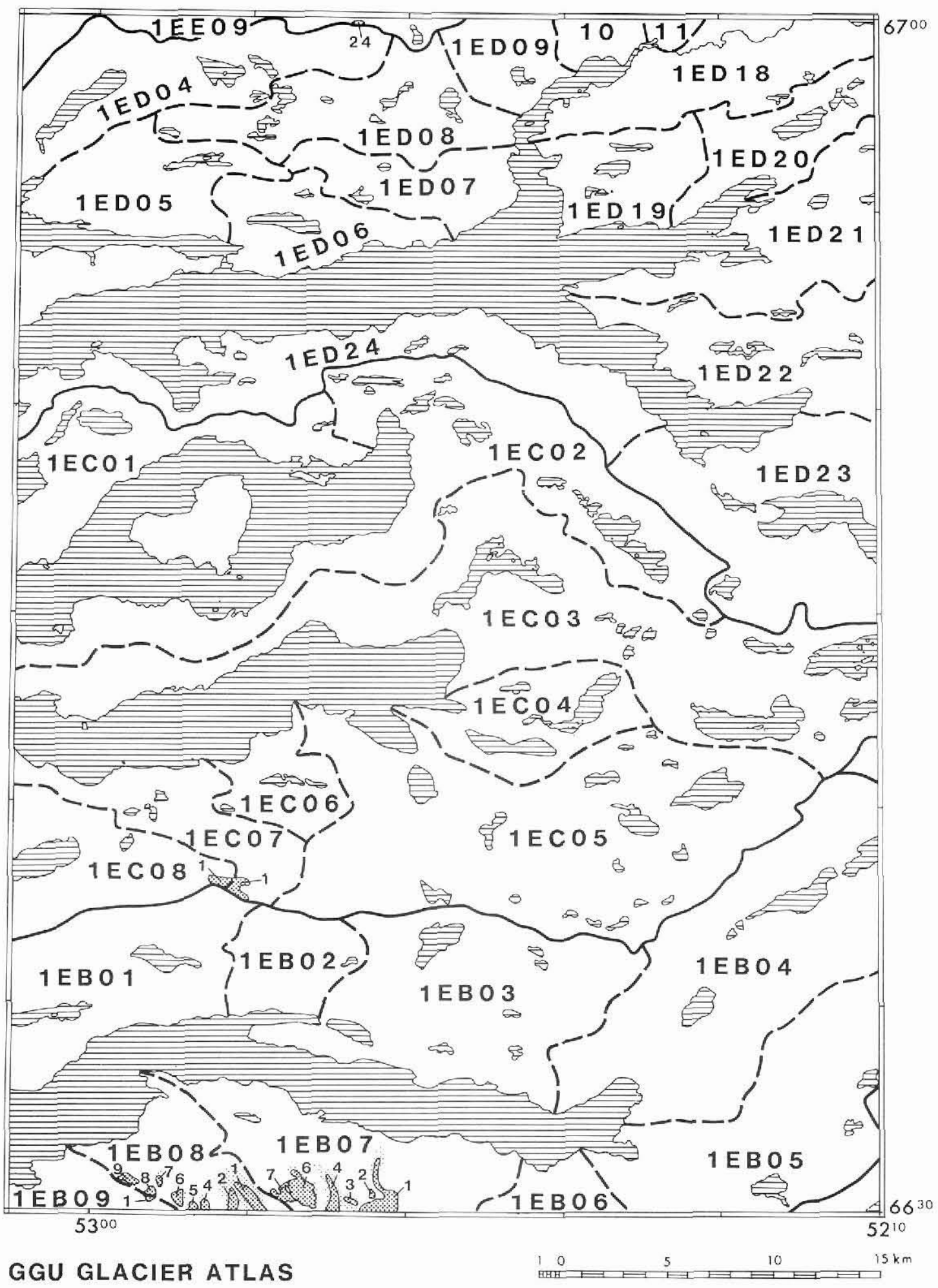

SHEET 6630-02 


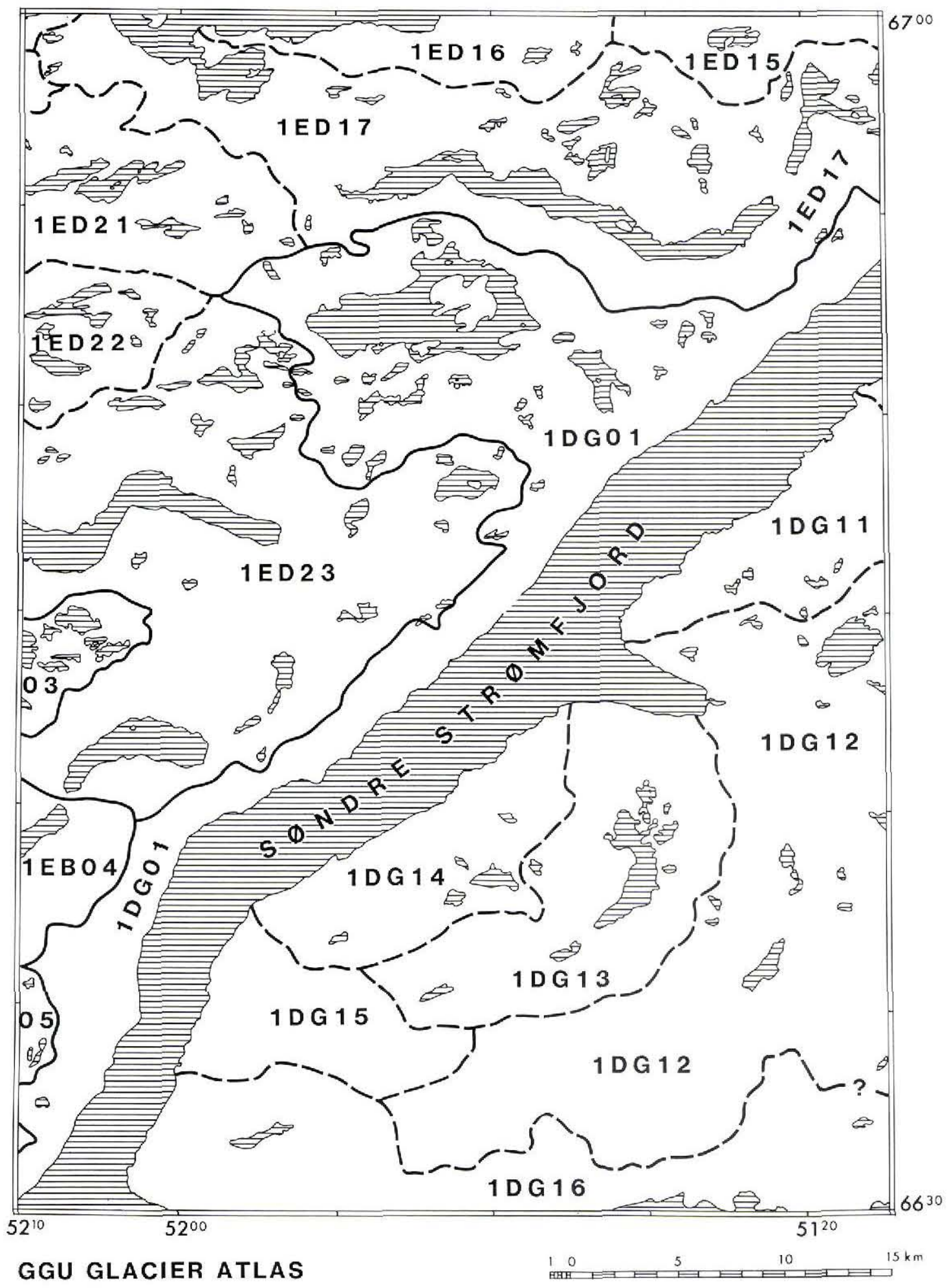

SHEET 6630-03 


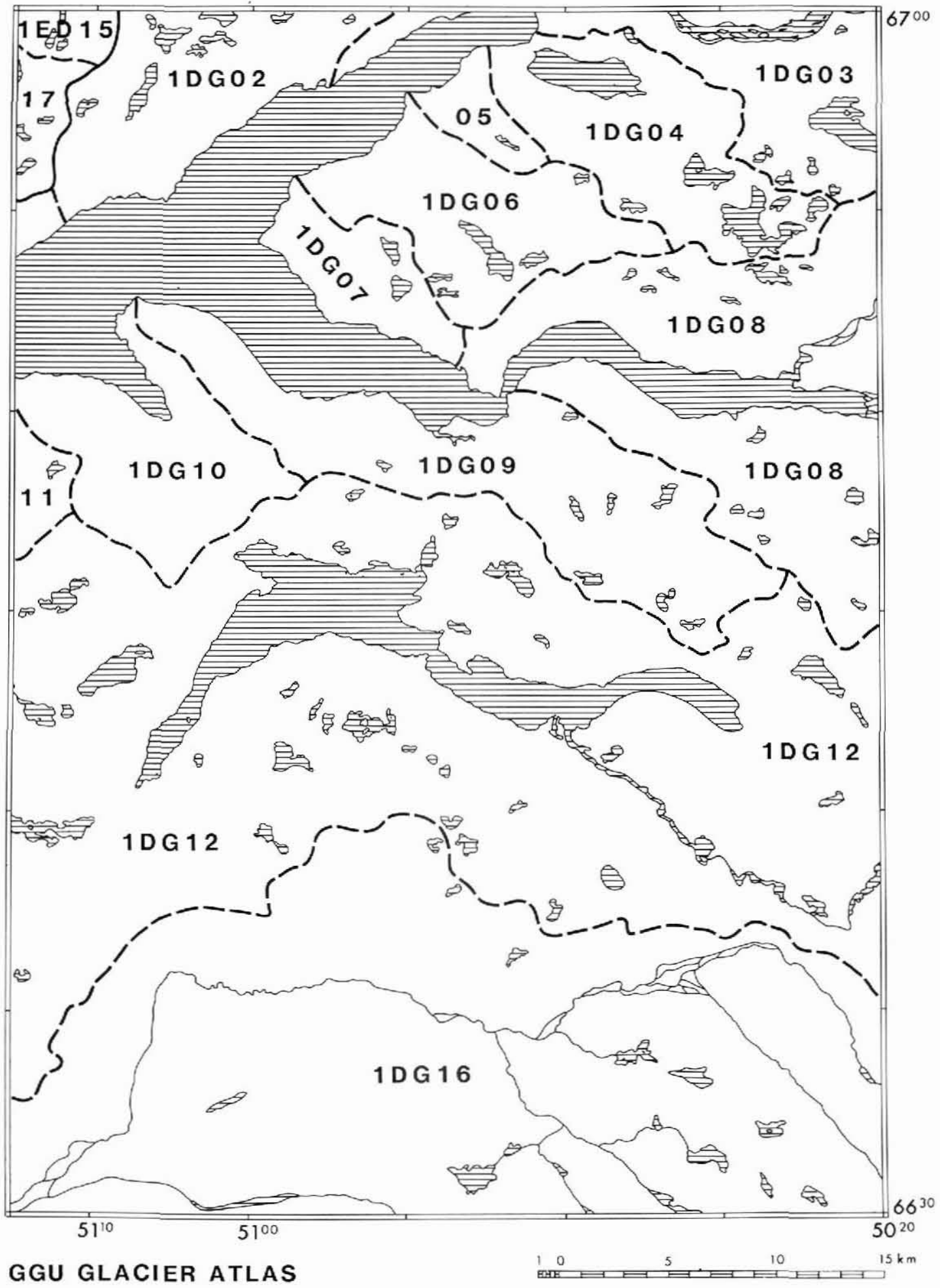

SHEET 6630-04 


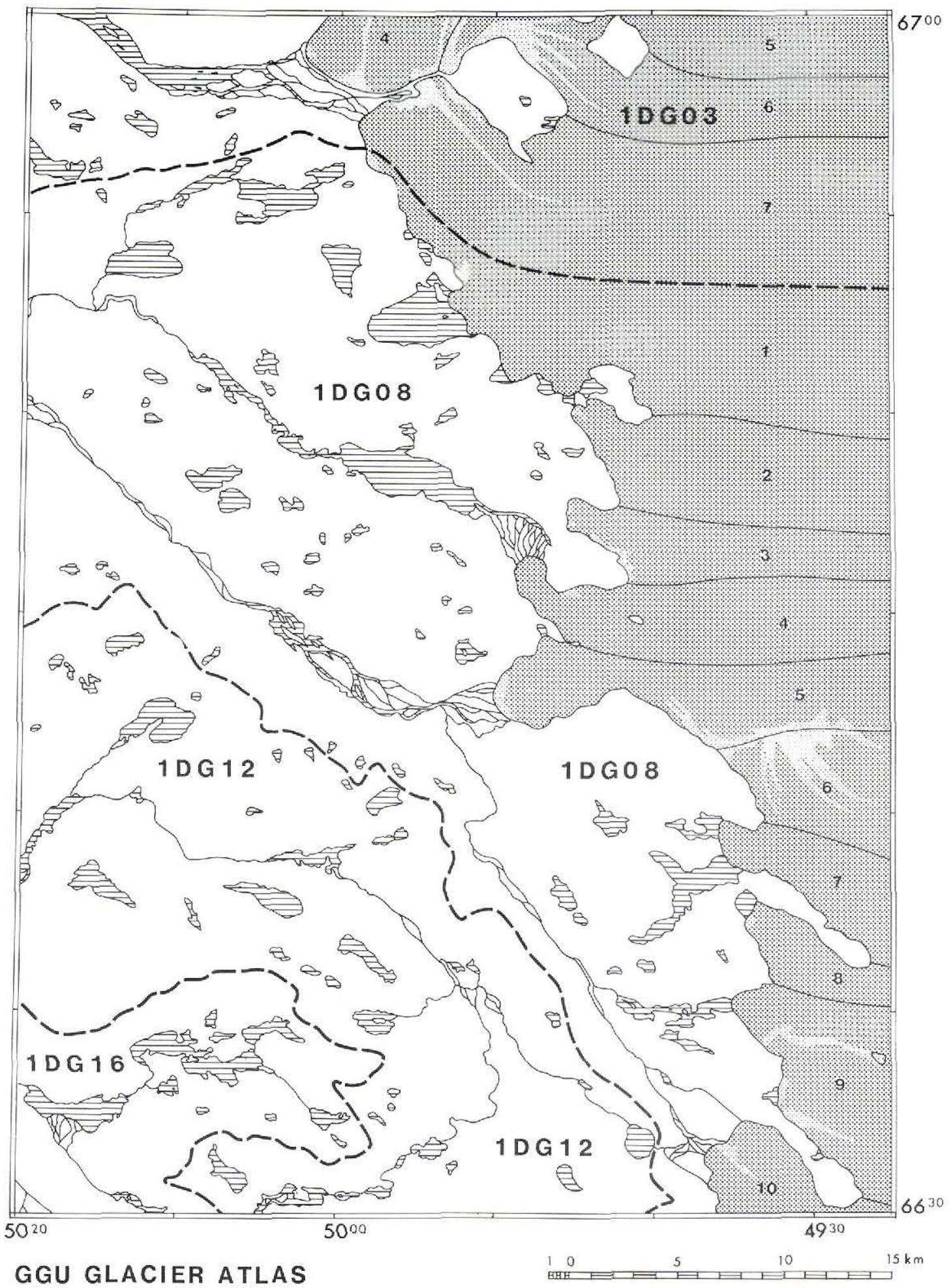

SHEET 6630-05 


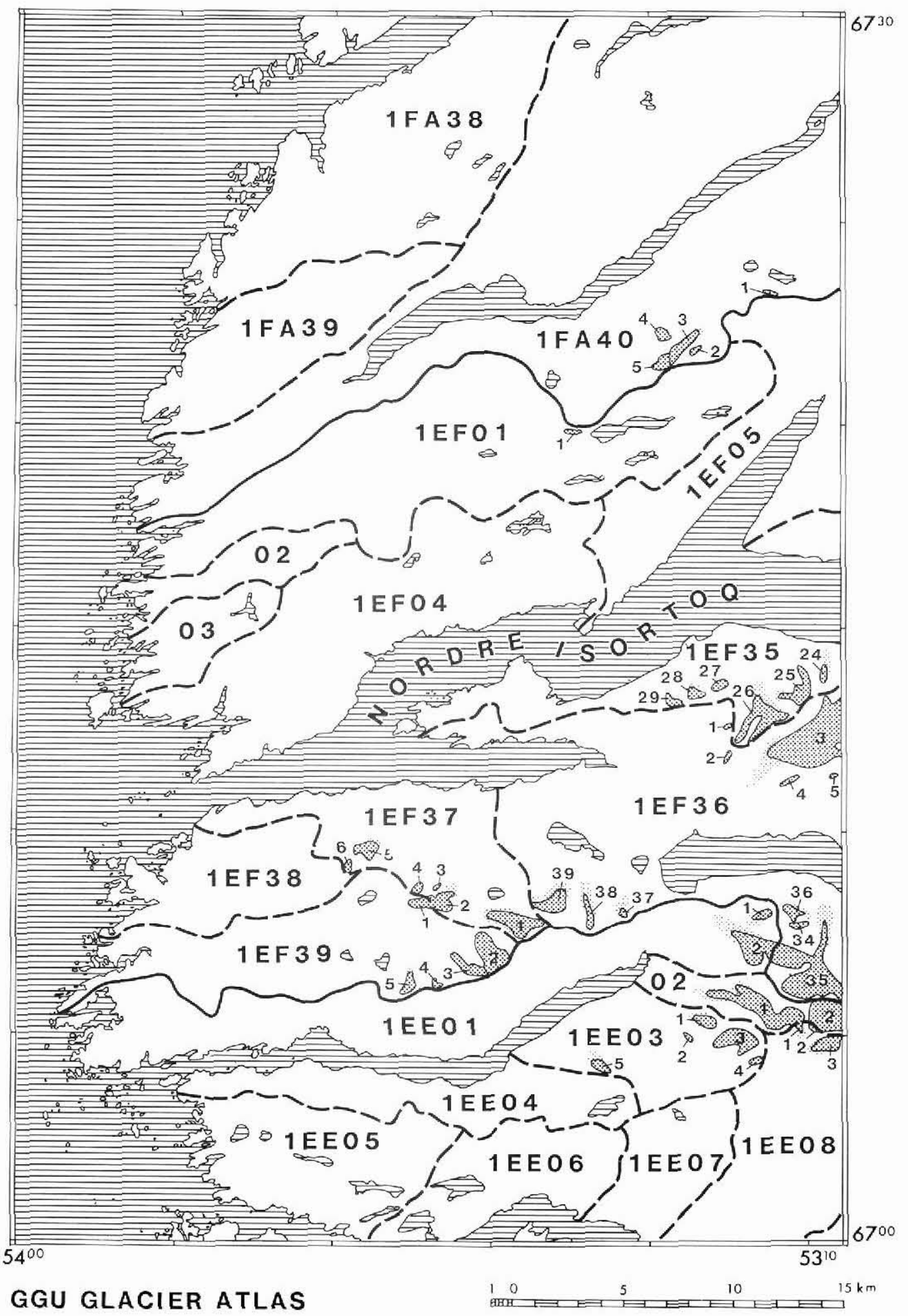
SHEET 6700-01 


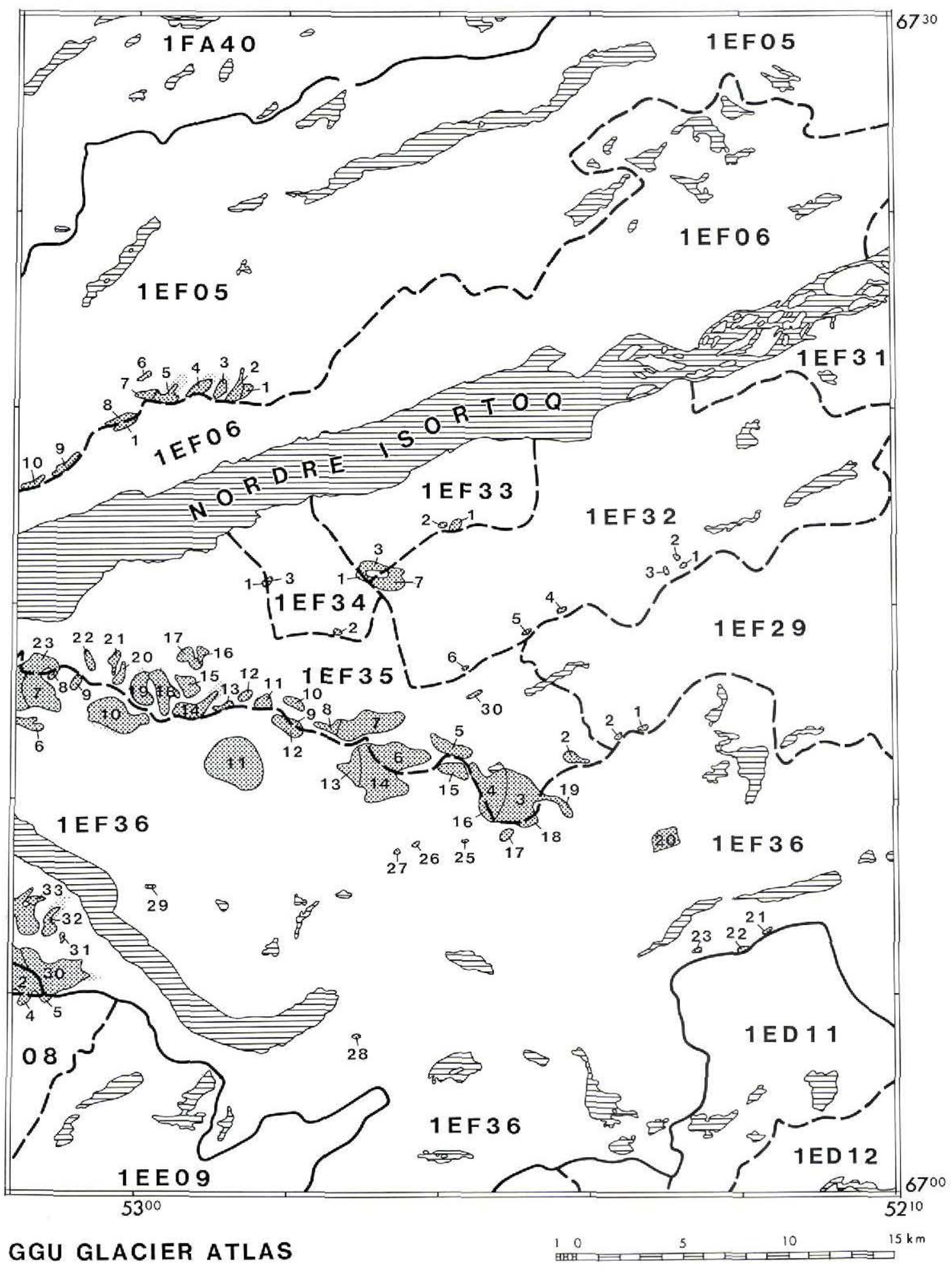

SHEET 6700-02 


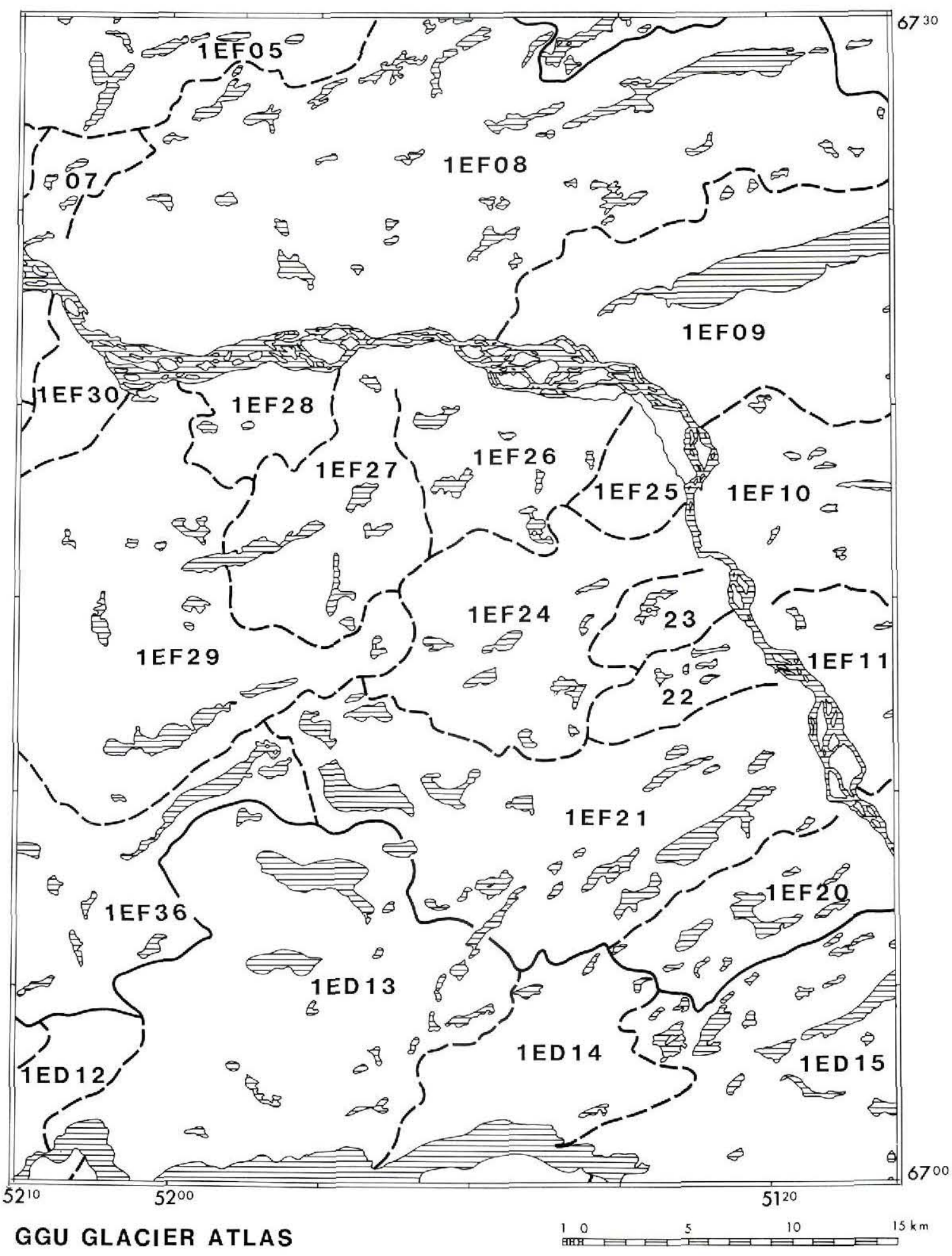

SHEET 6700-03 


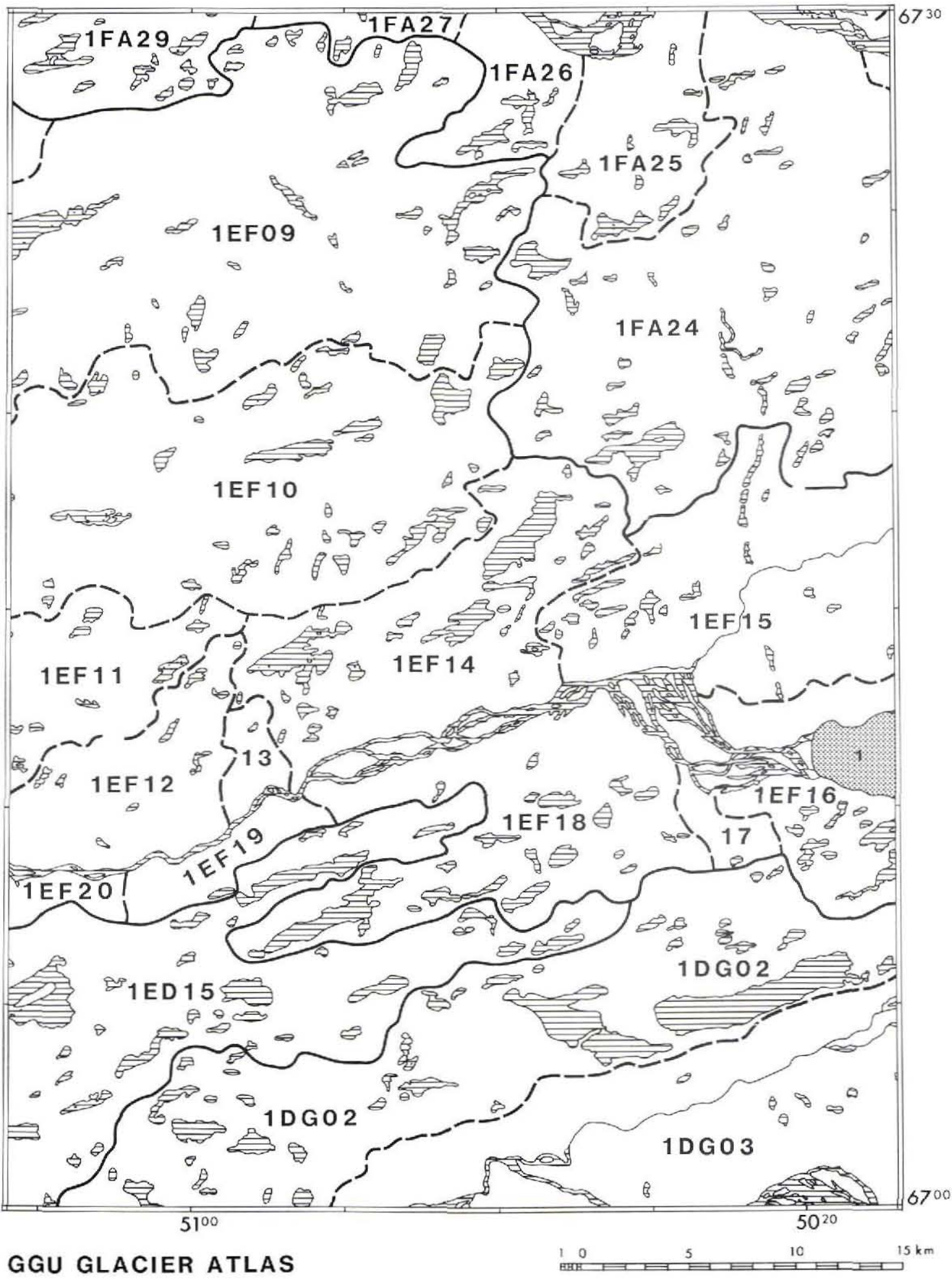

\section{SHEET 6700-04}




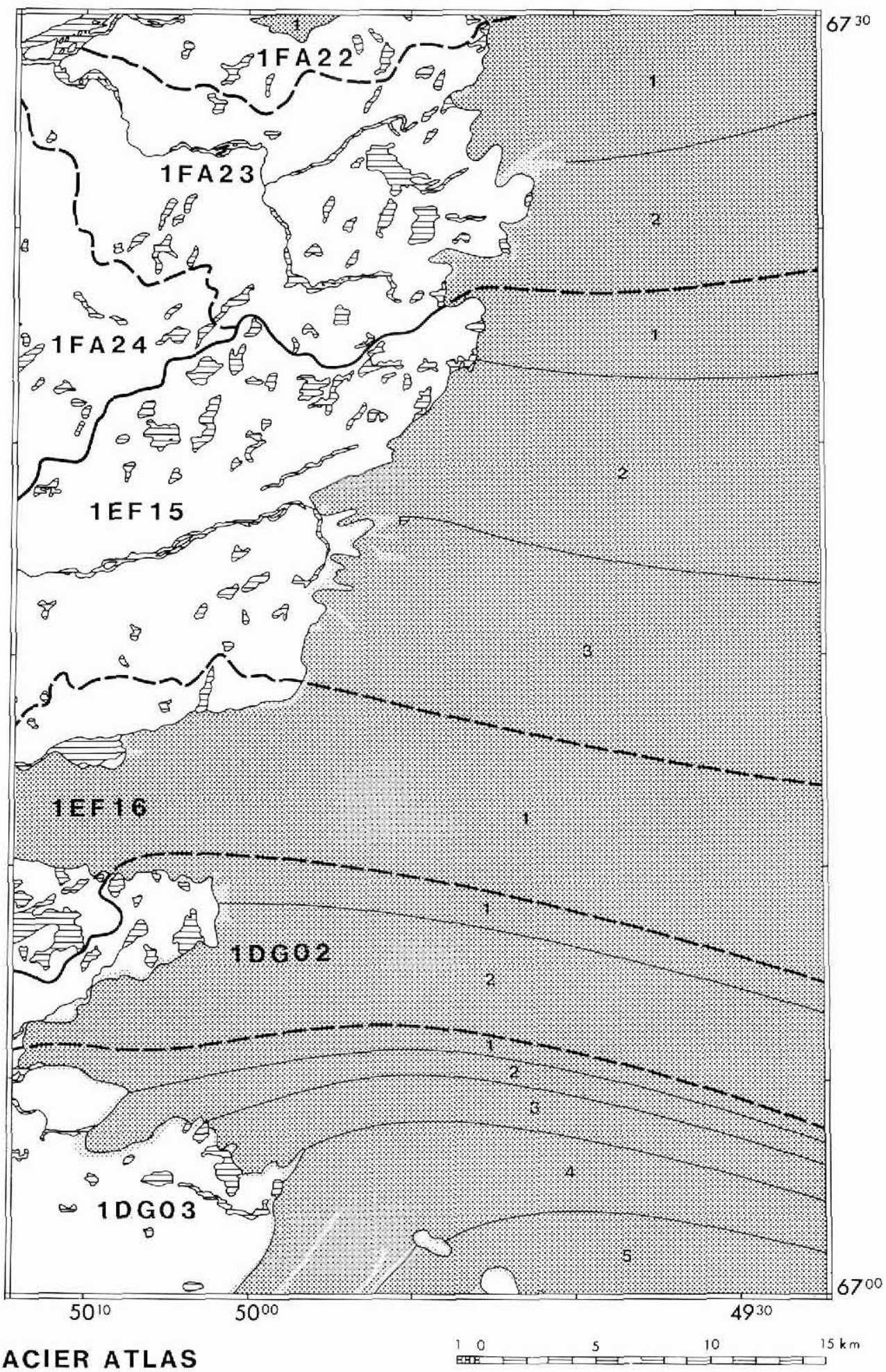

GGU GLACIER ATLAS

SHEET 6700-05 


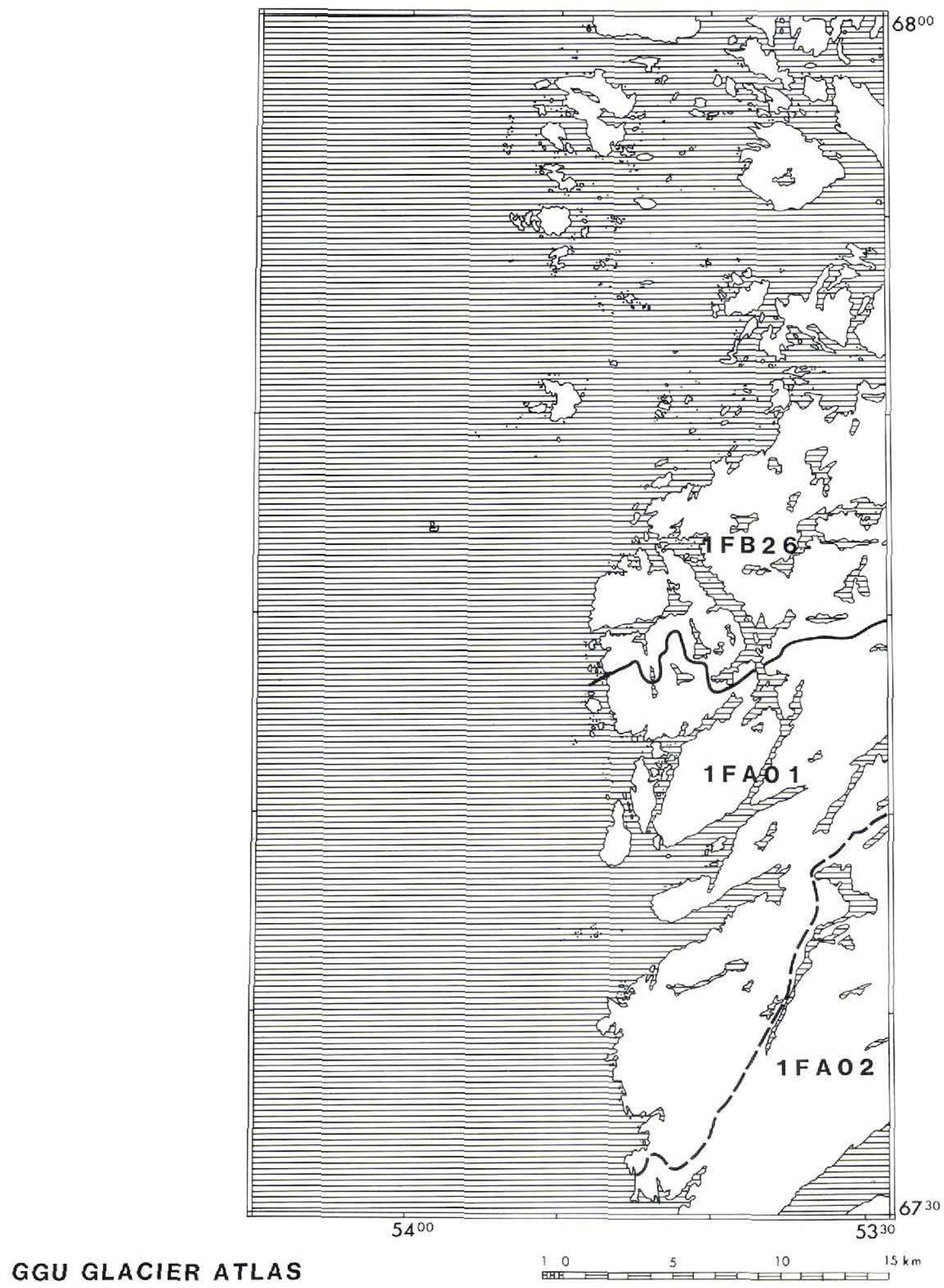




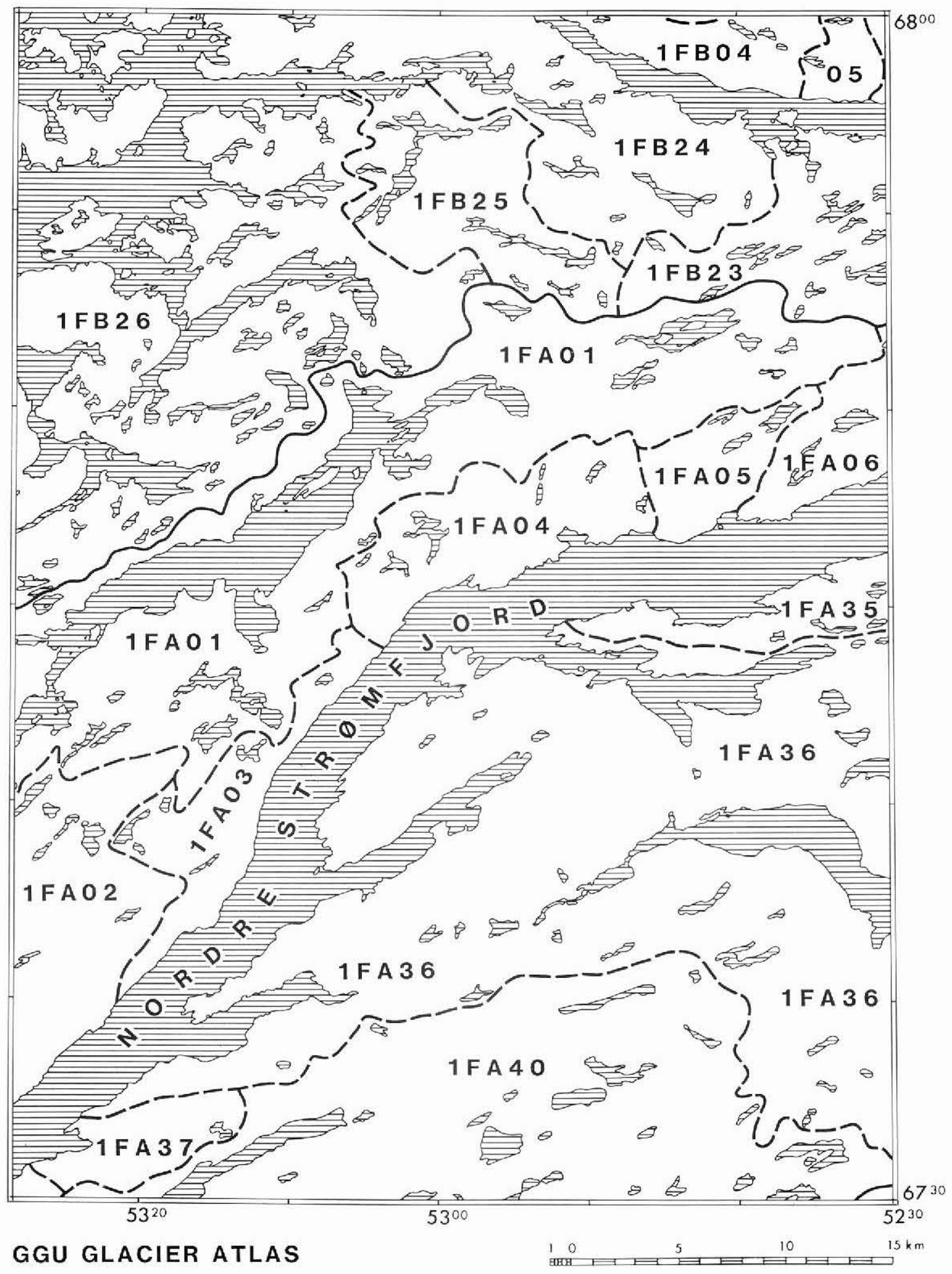

SHEET 6730-02 


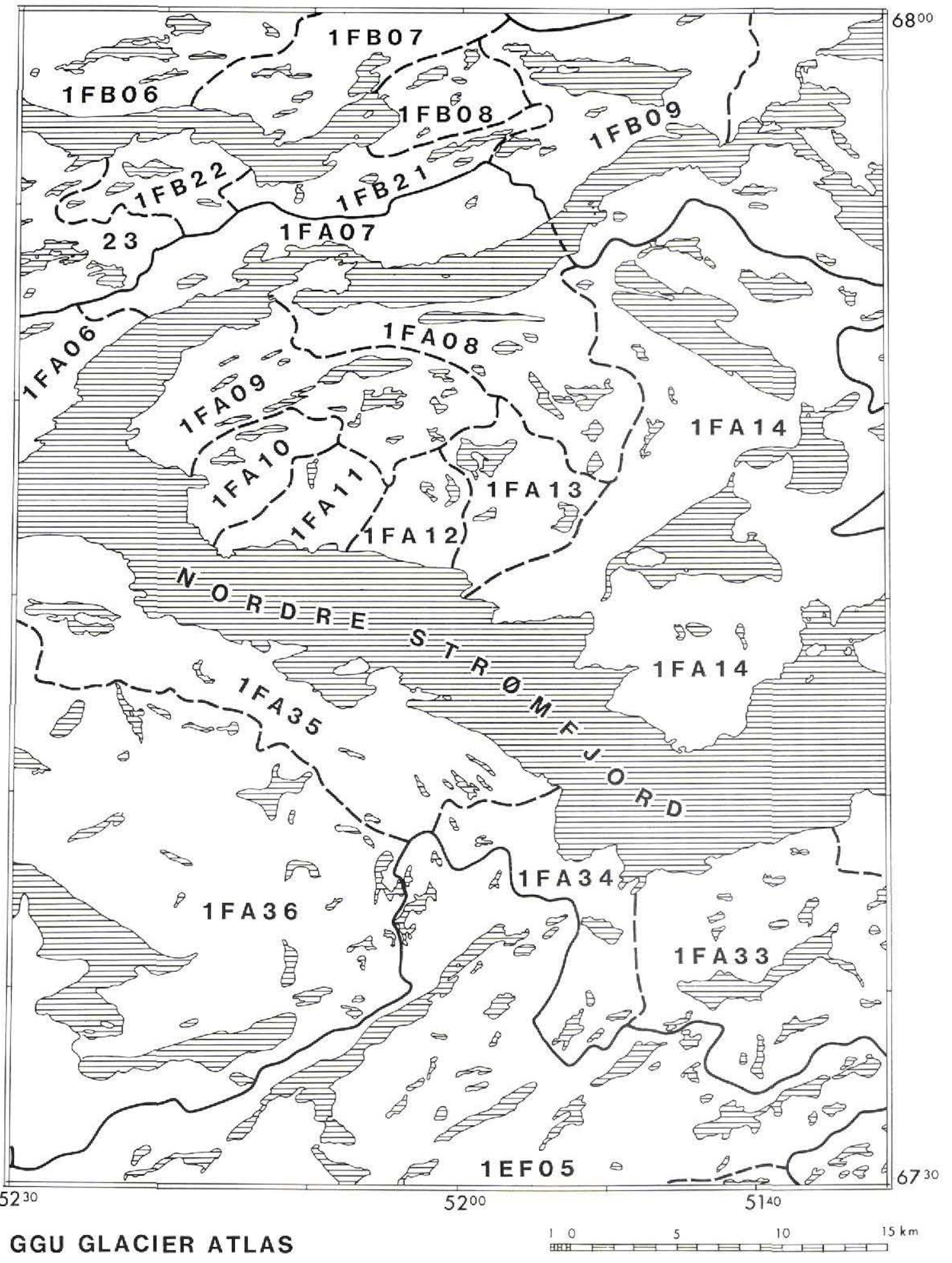

SHEET 6730-03 


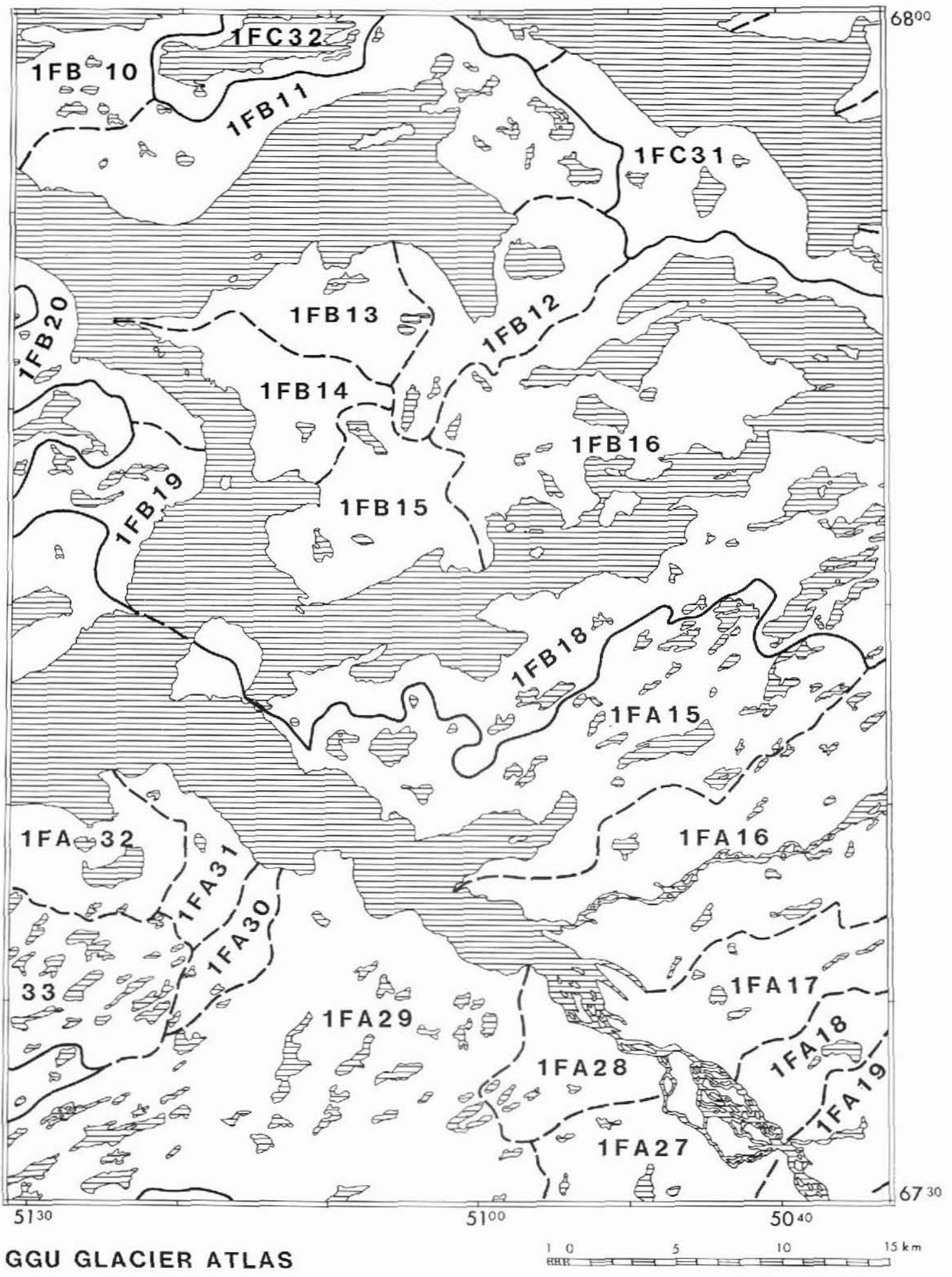

SHEET 6730-04 


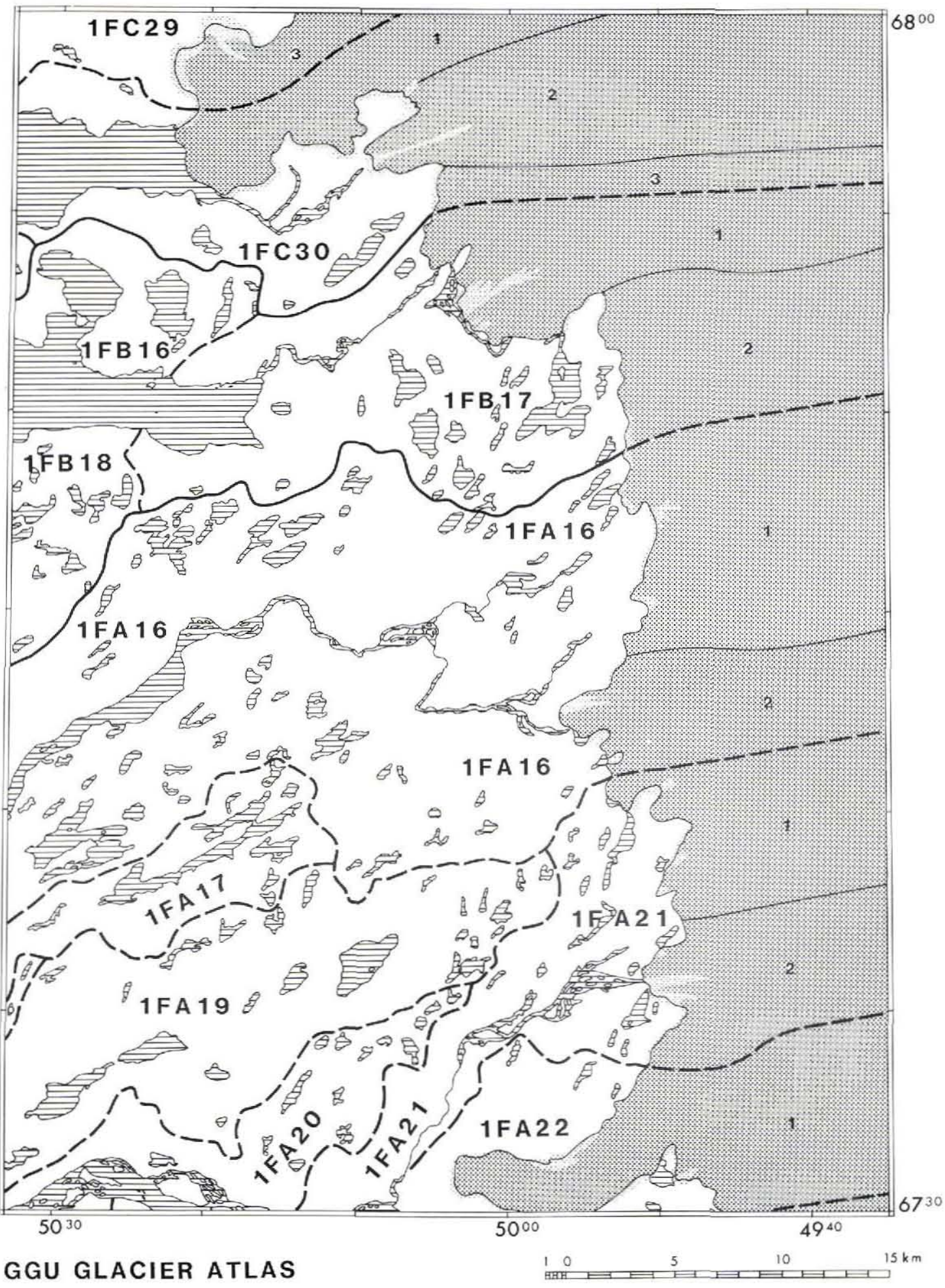

SHEET 6730-05 


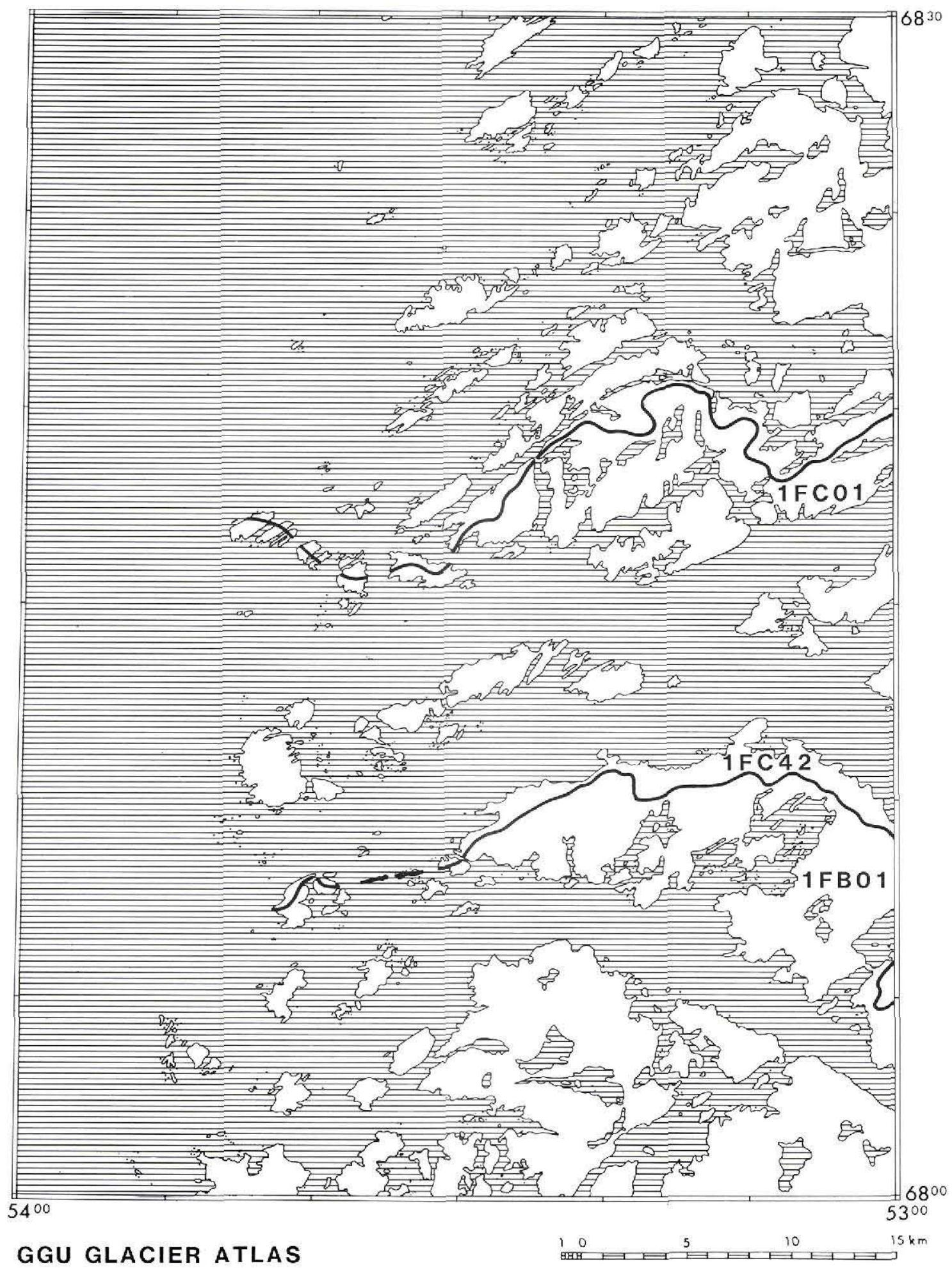




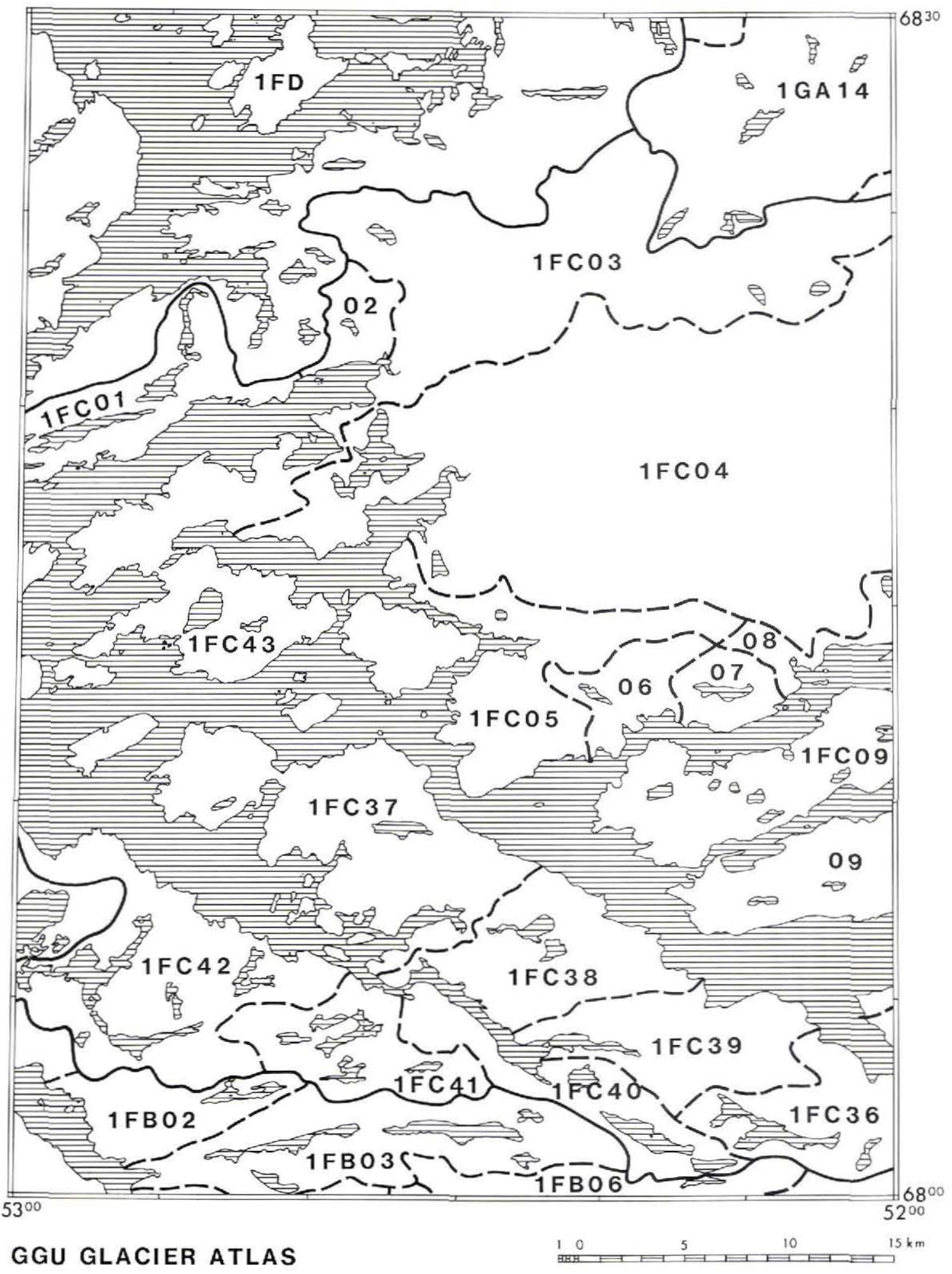

SHEET 6800-02 


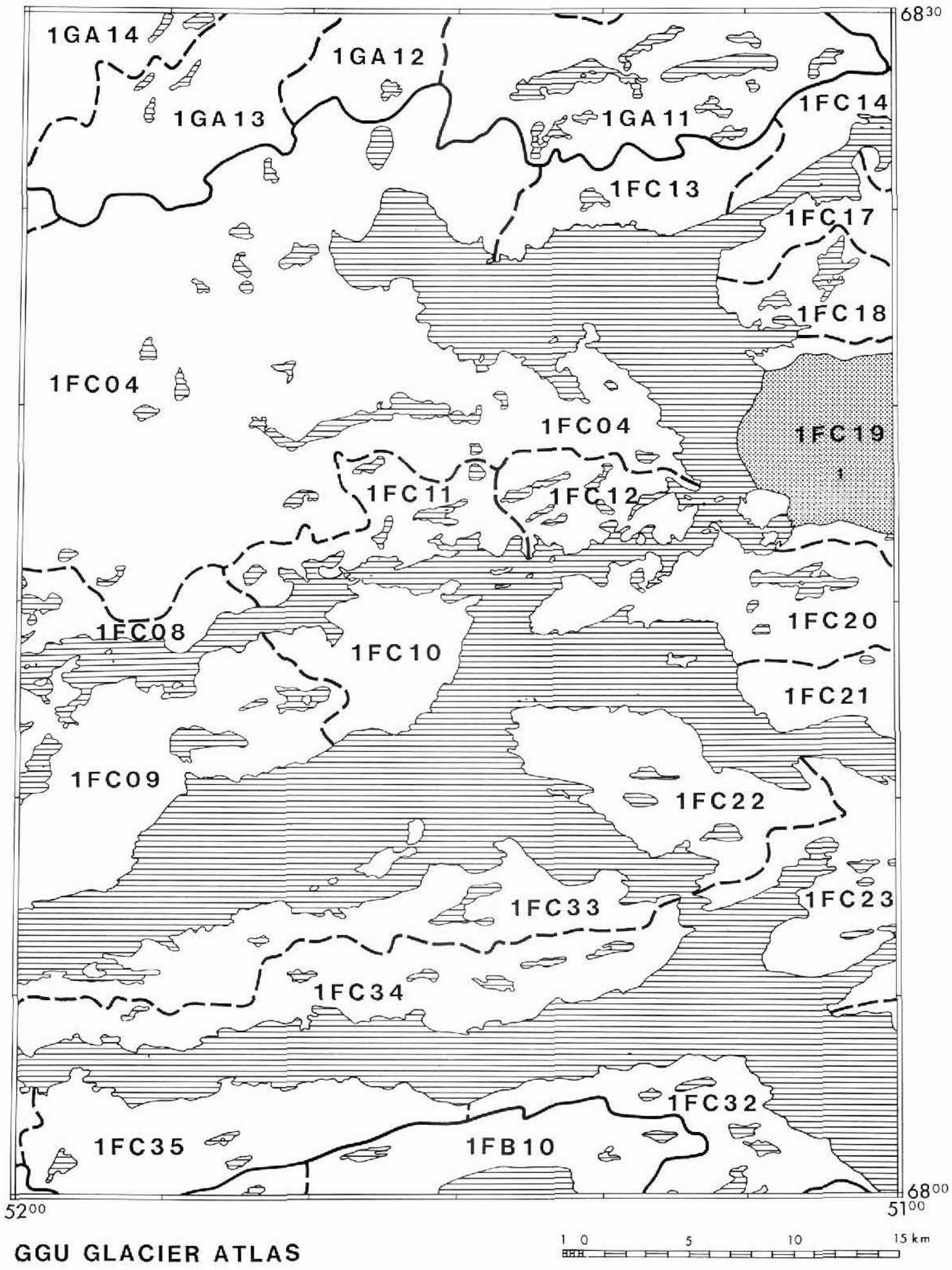

SHEET 6800-03 


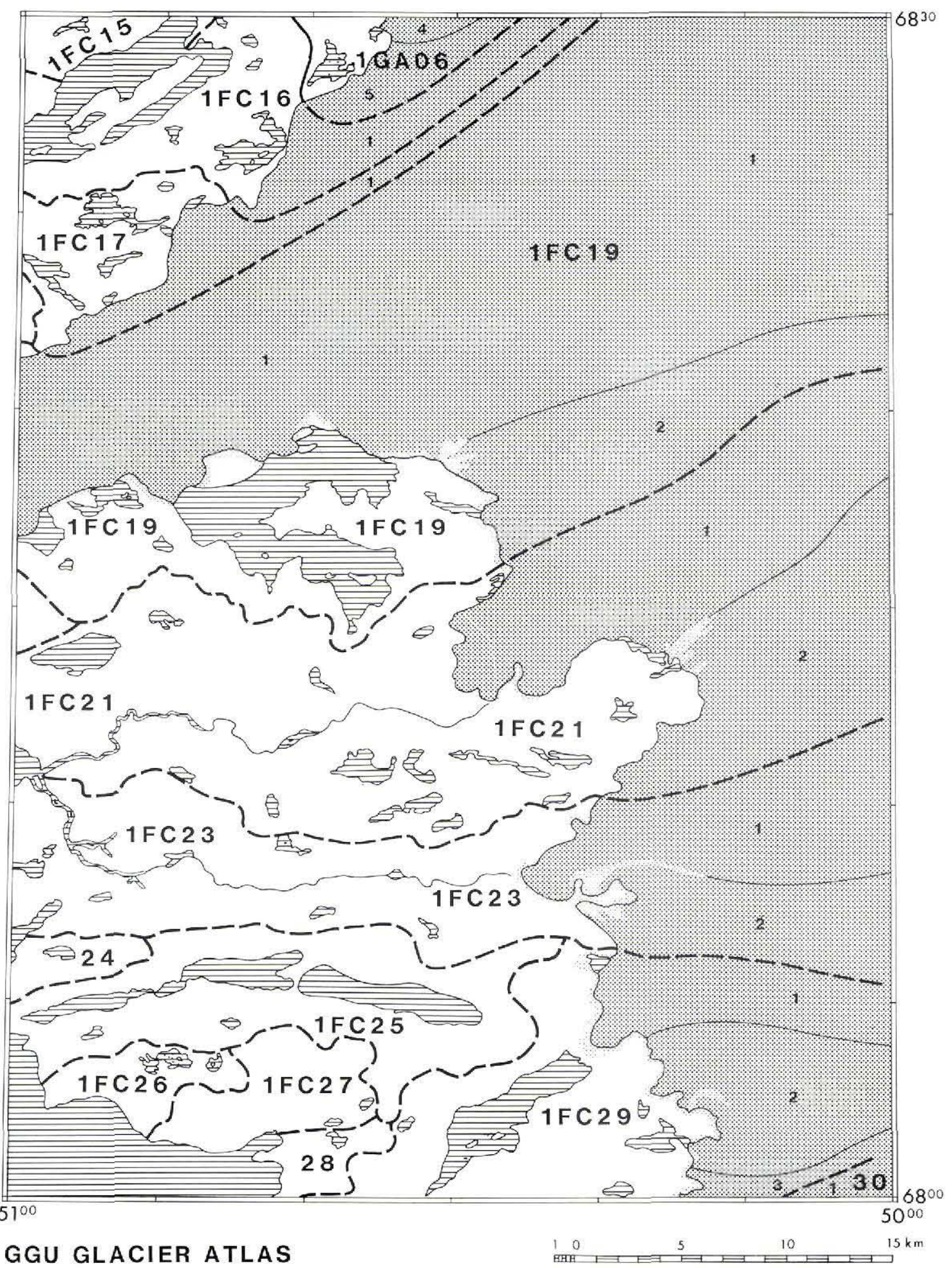

\section{SHEET 6800-04}




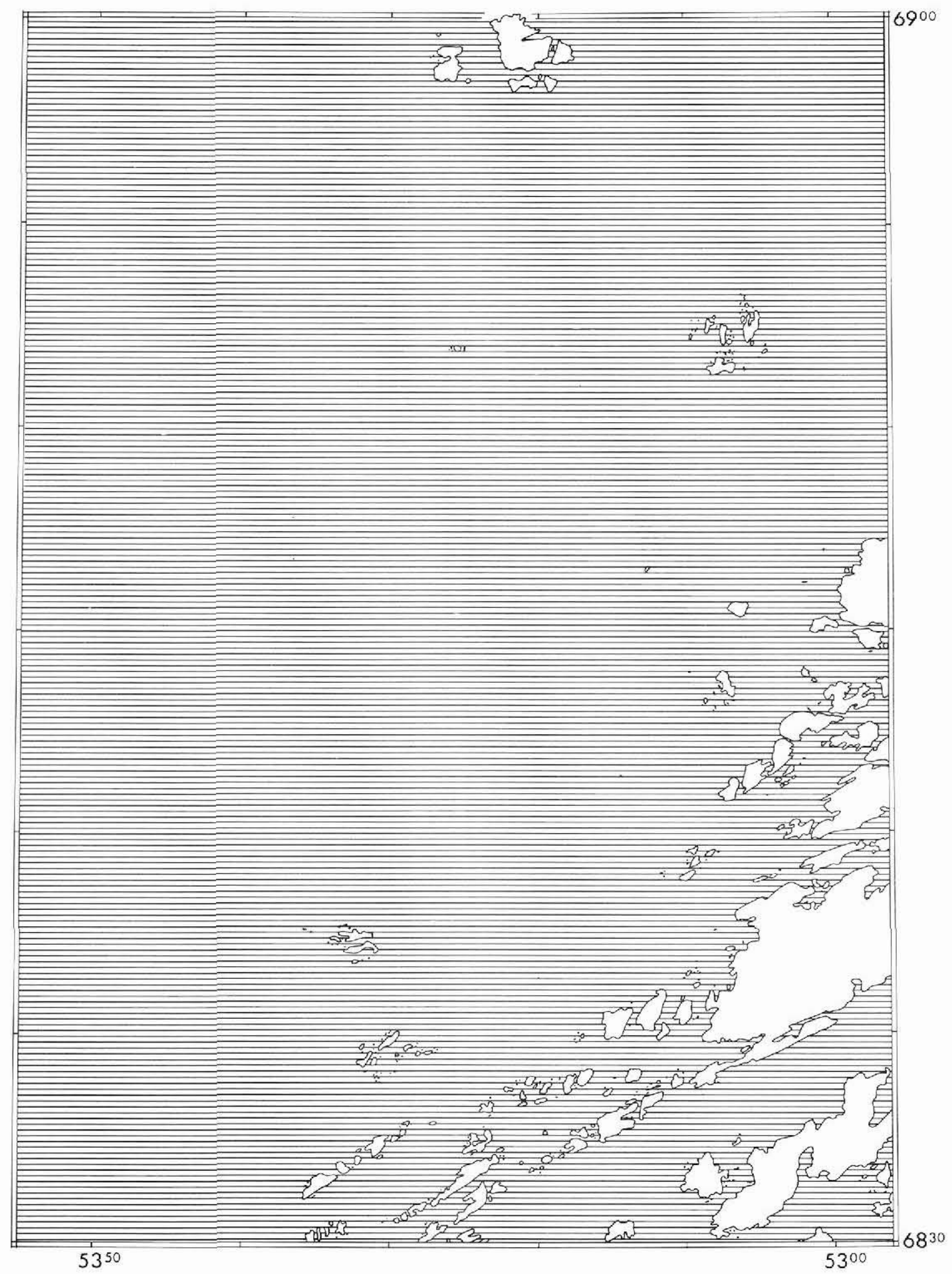

GGU GLACIER ATLAS

$1001015 \mathrm{~km}$

SHEET 6830-01 


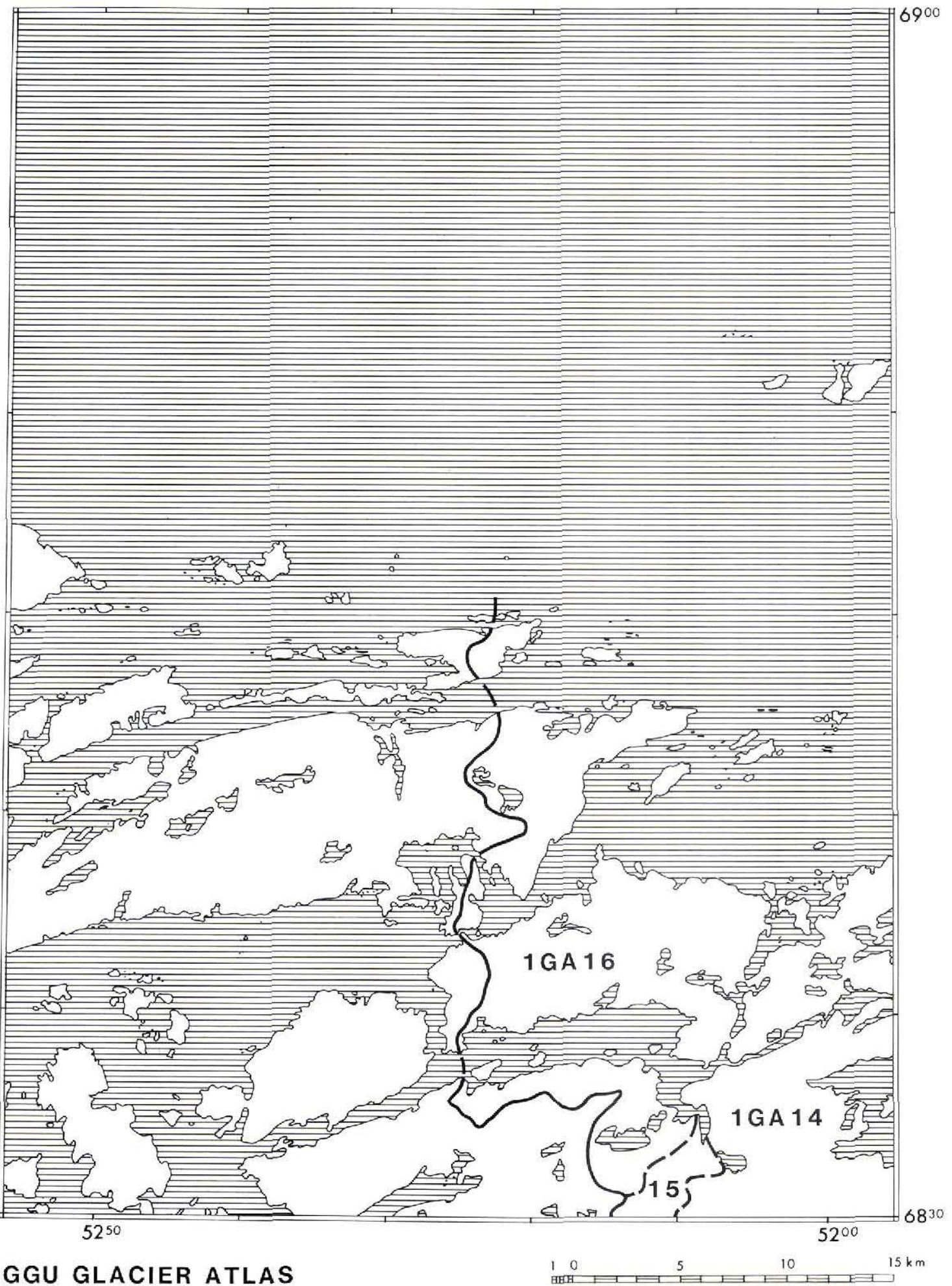

SHEET 6830-02 


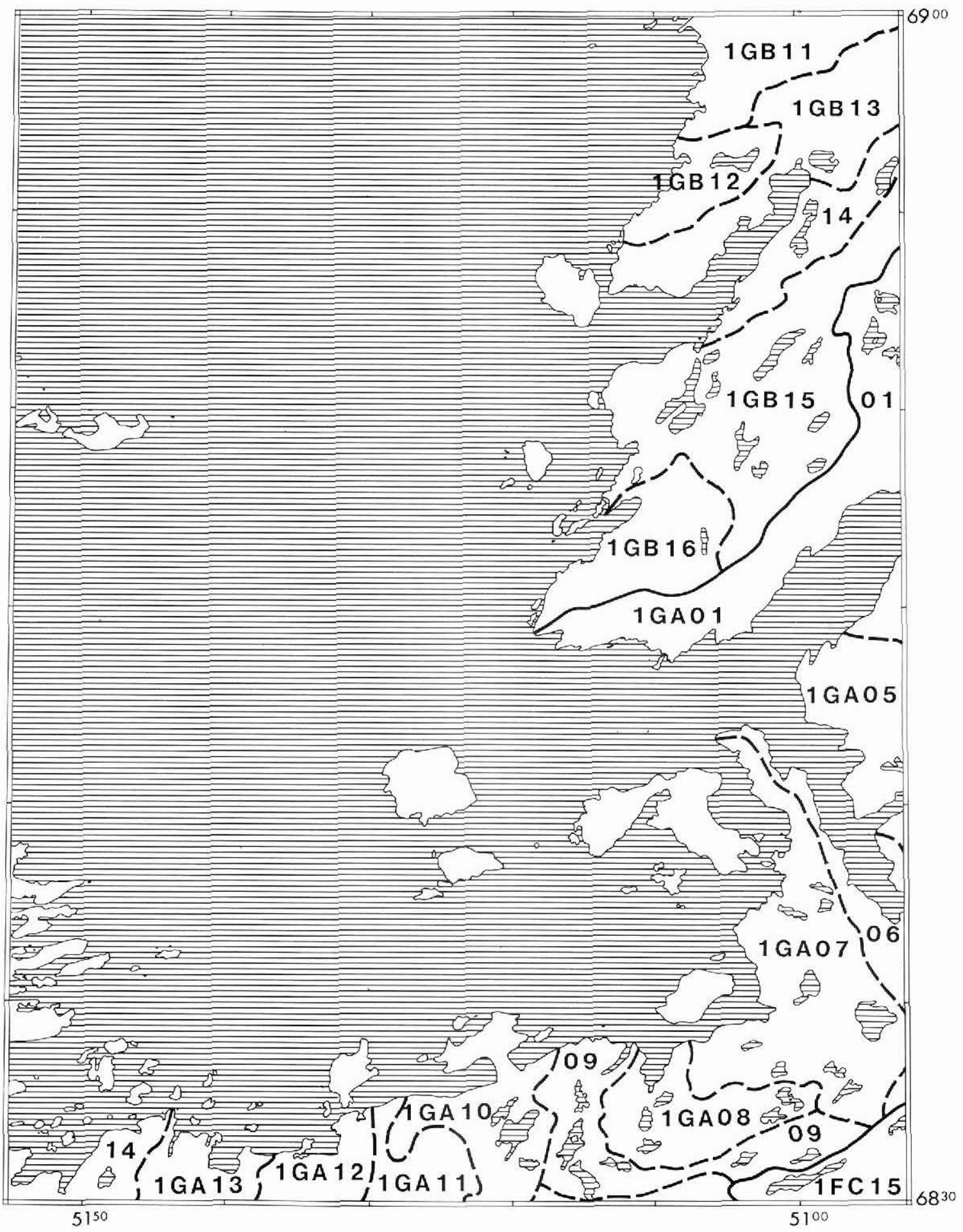




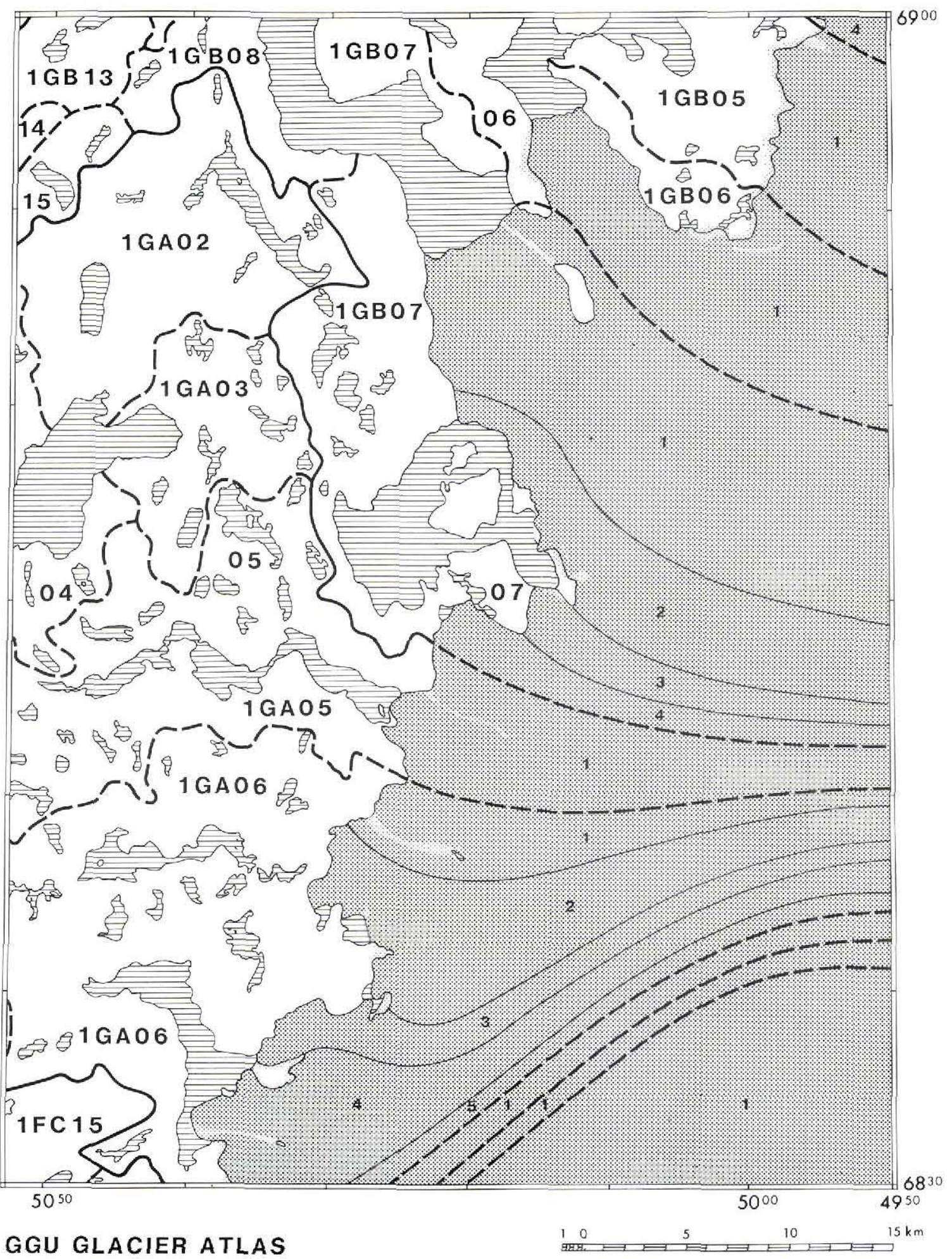

SHEET 6830-04 


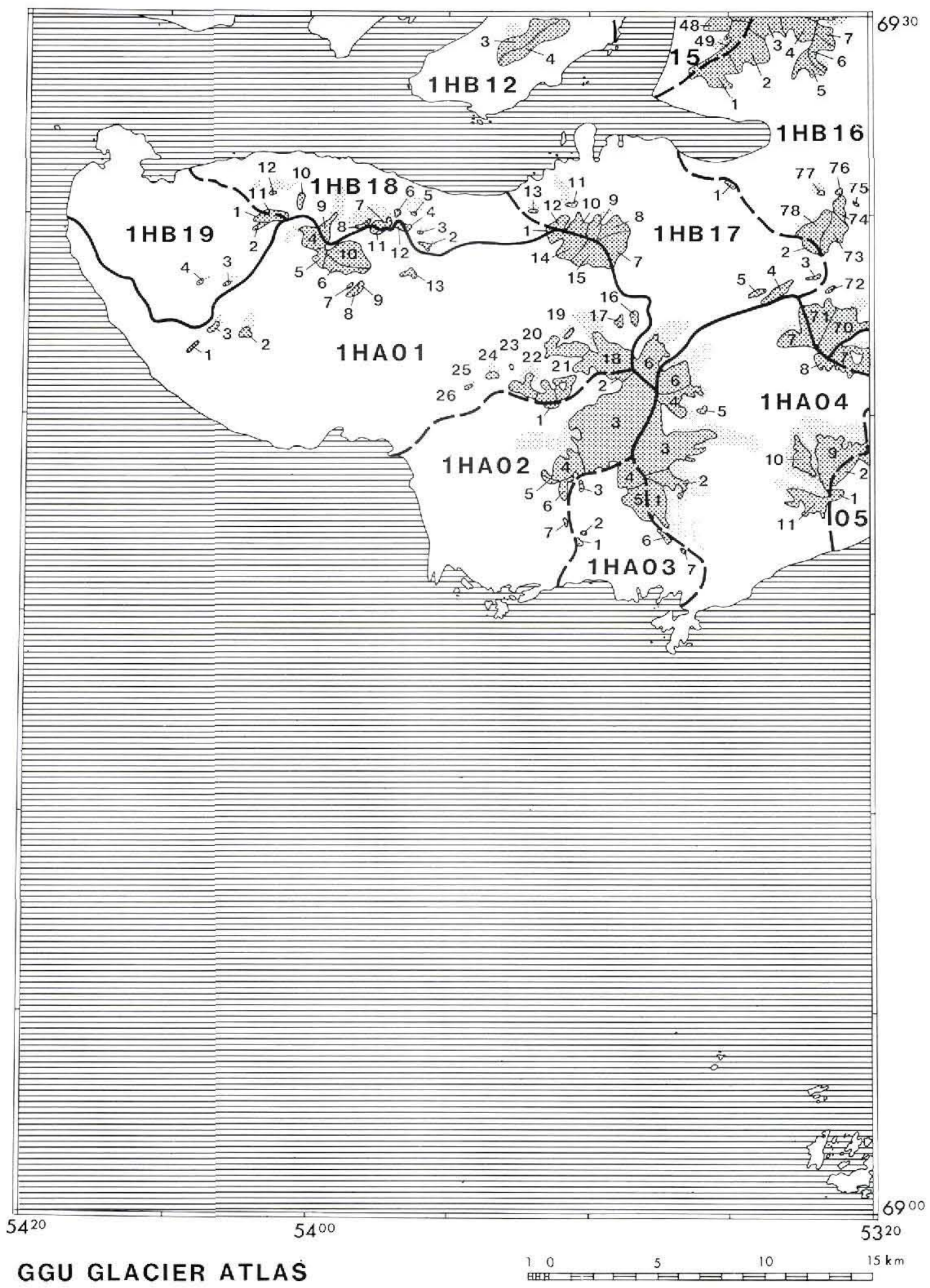




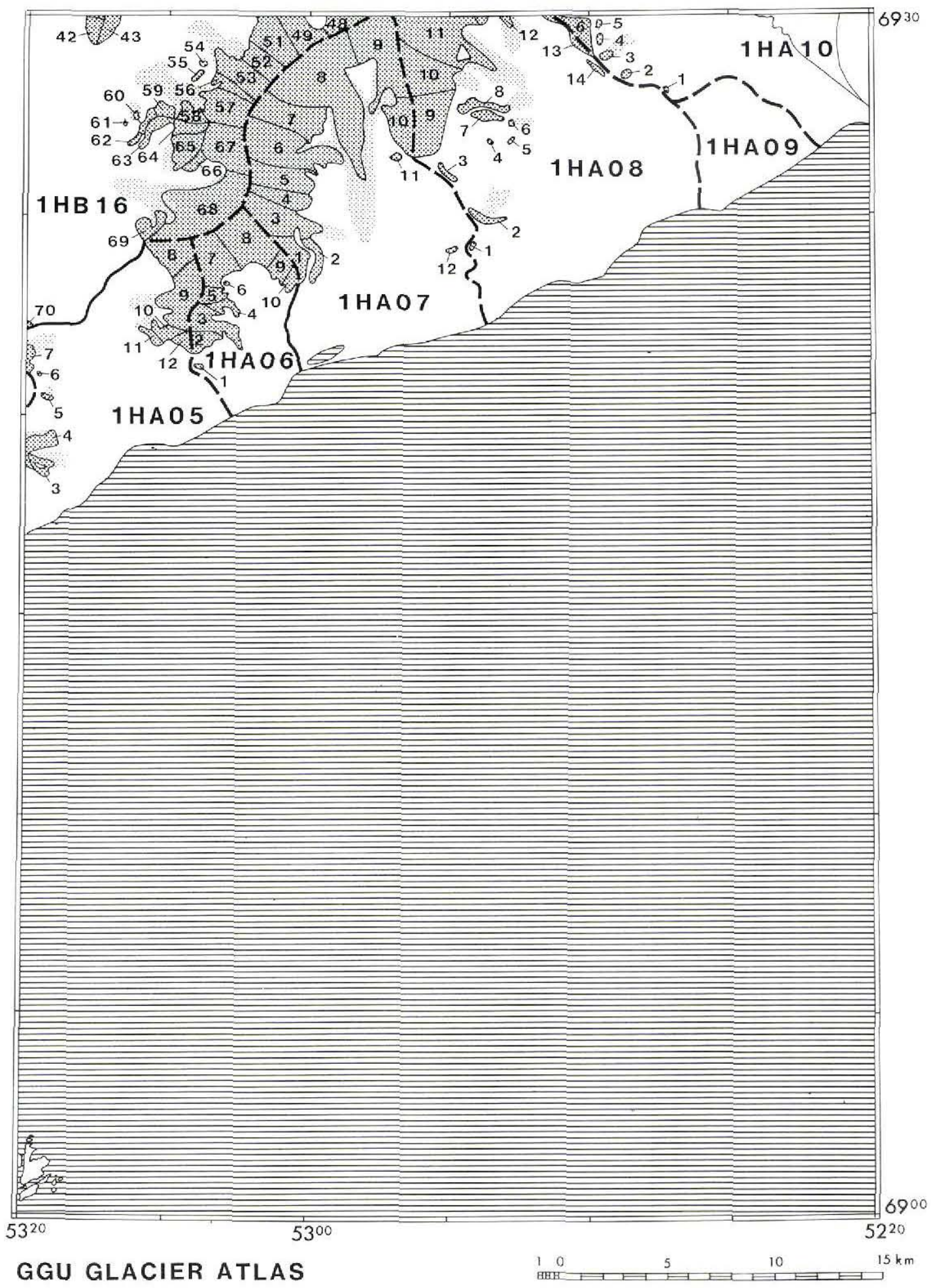

SHEET 6900-02 


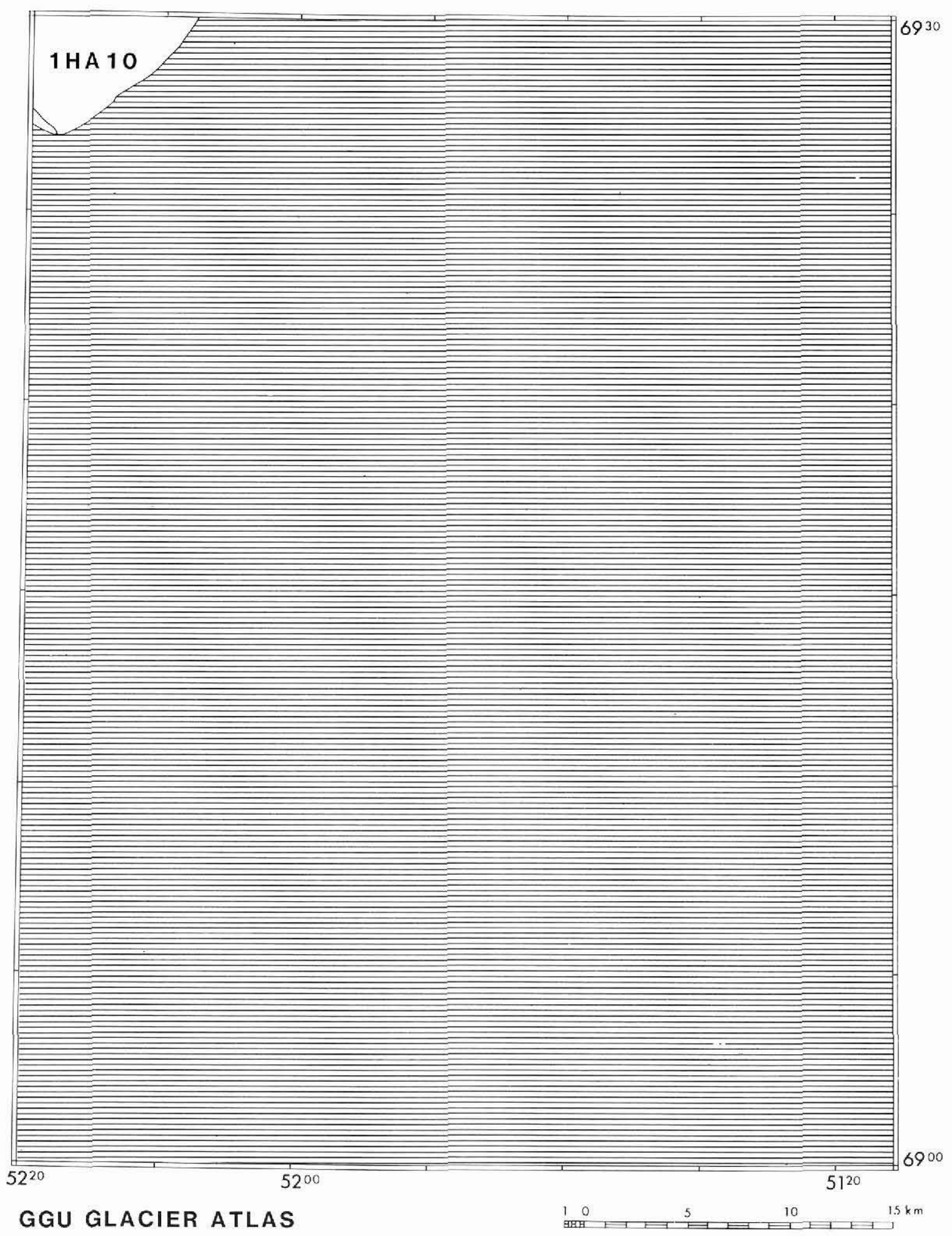

SHEET 6900-03 


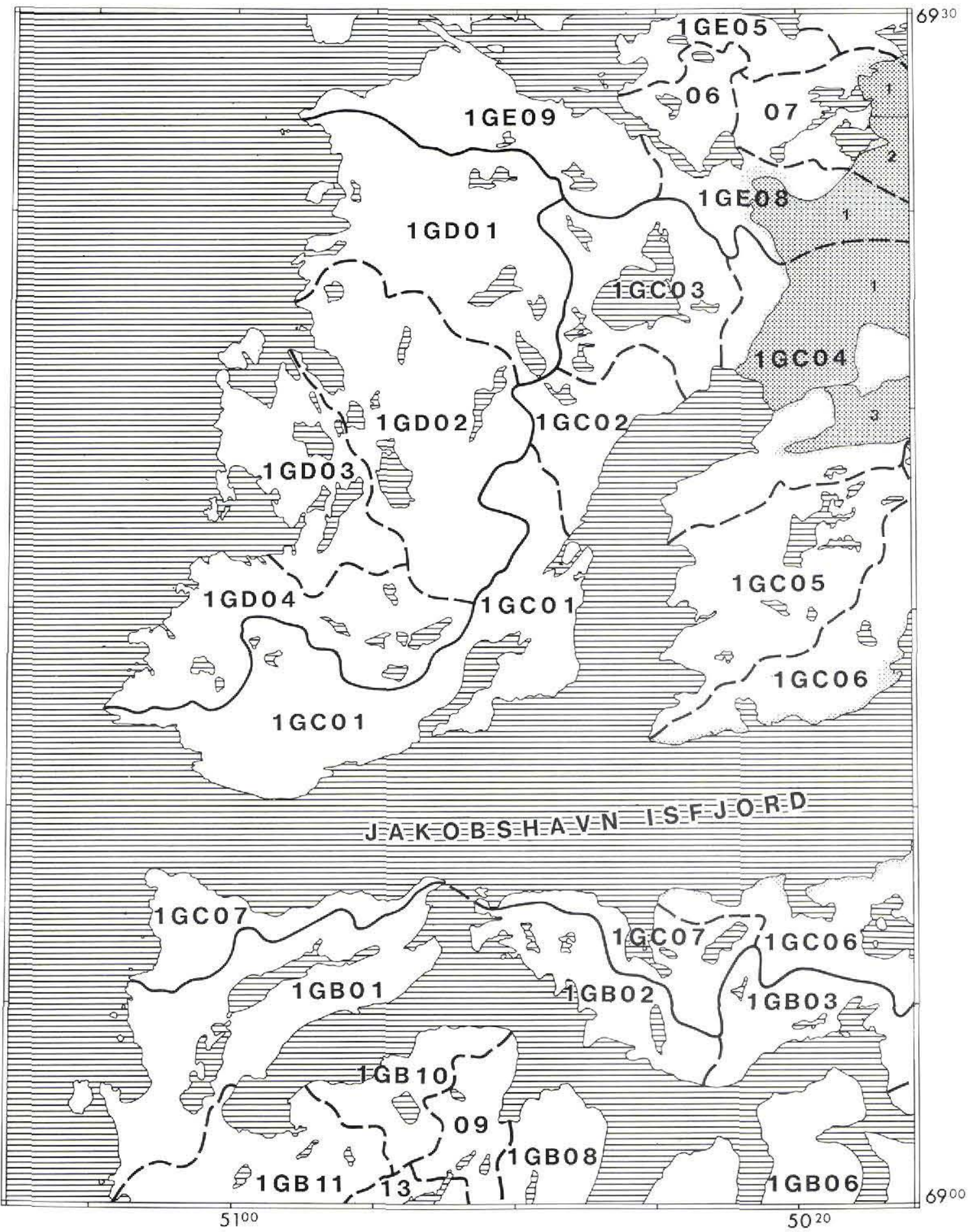




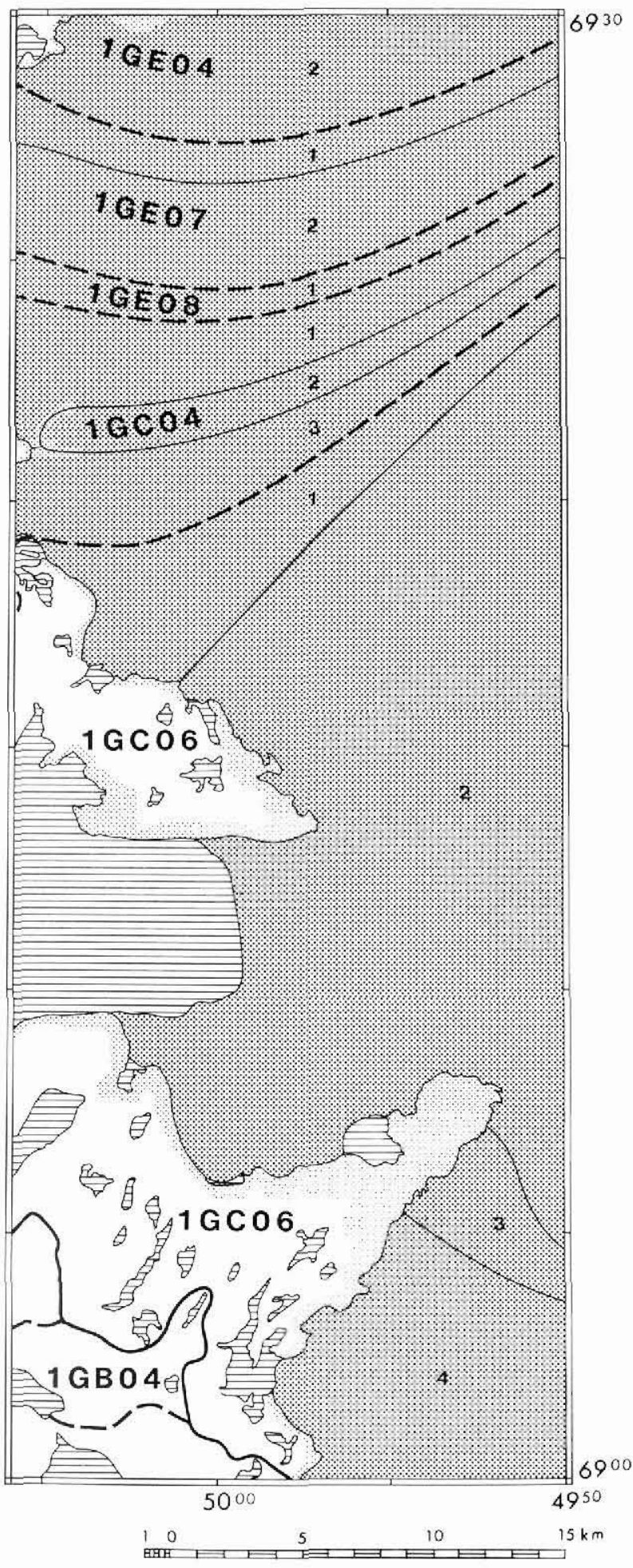




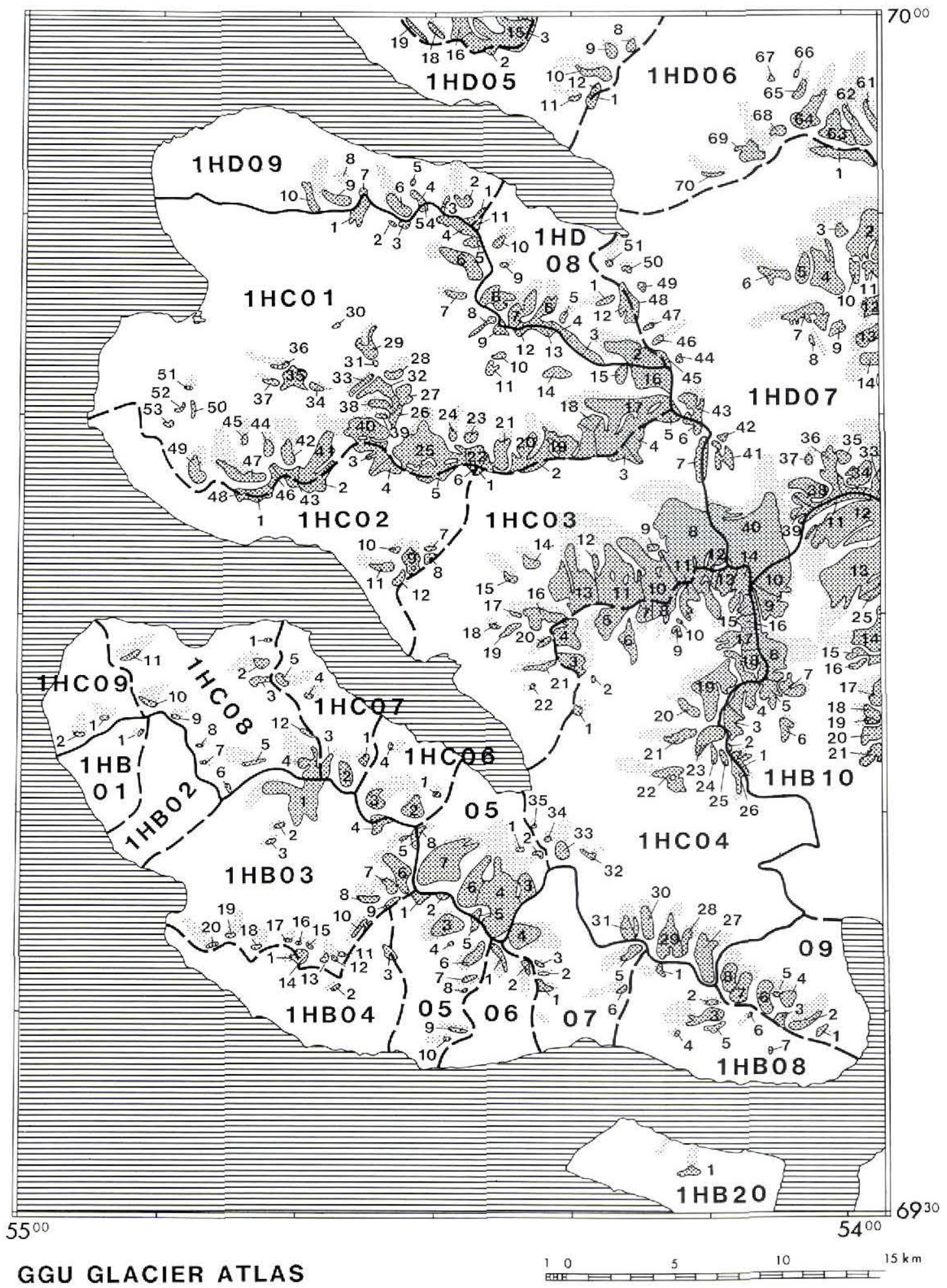

\section{SHEET 6930-01}




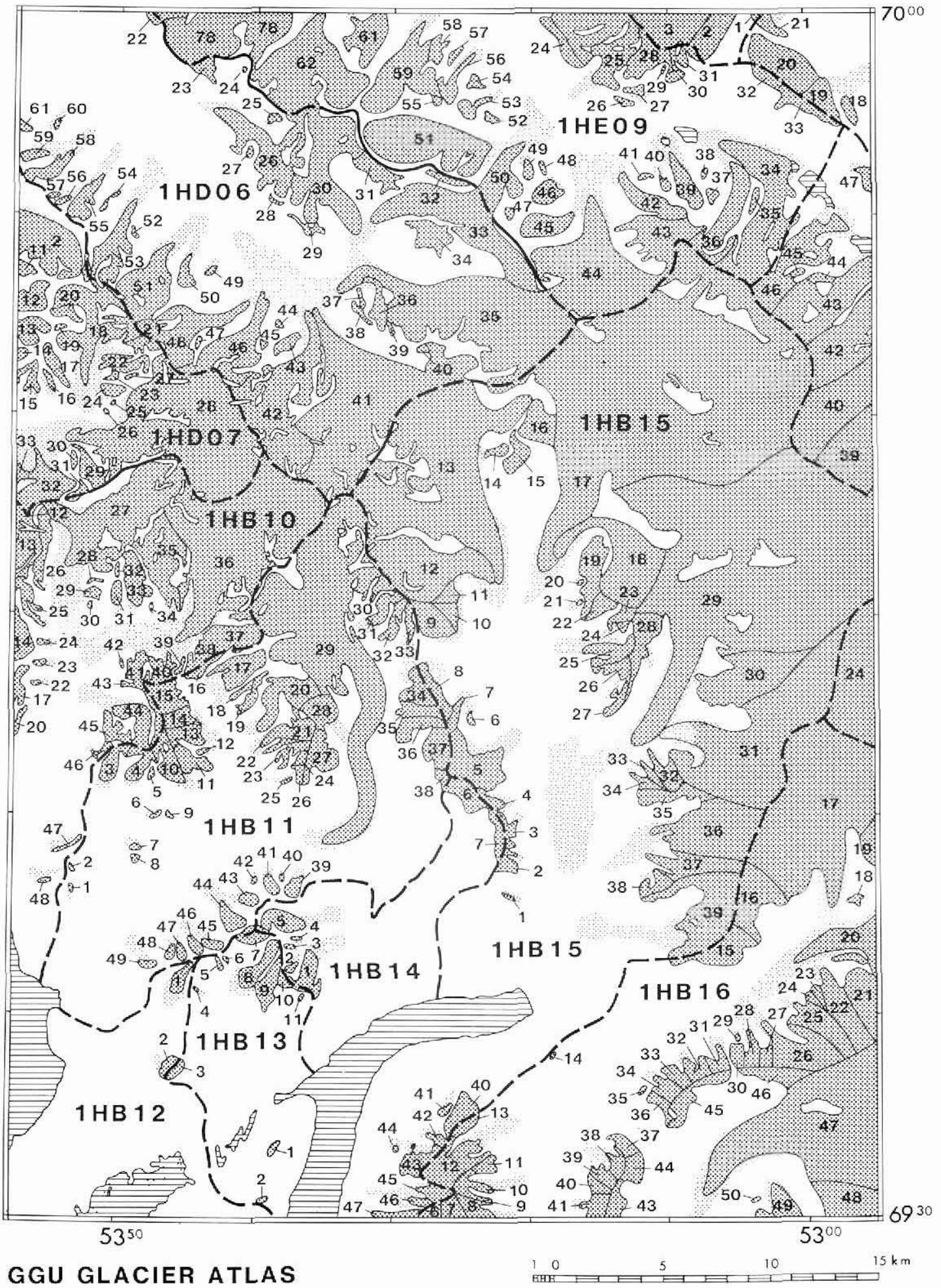

\section{SHEET 6930-02}




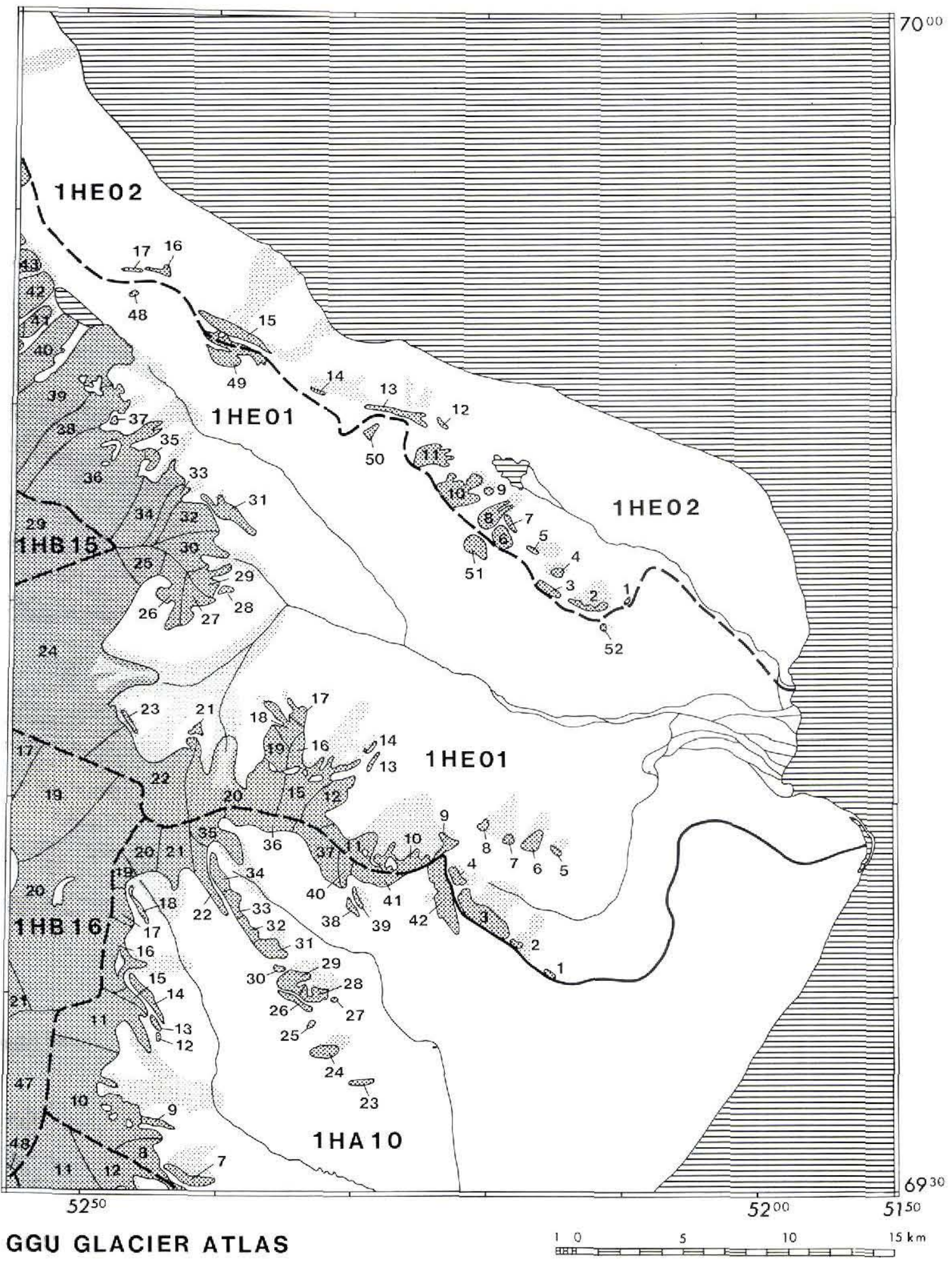

SHEET 6930-03 


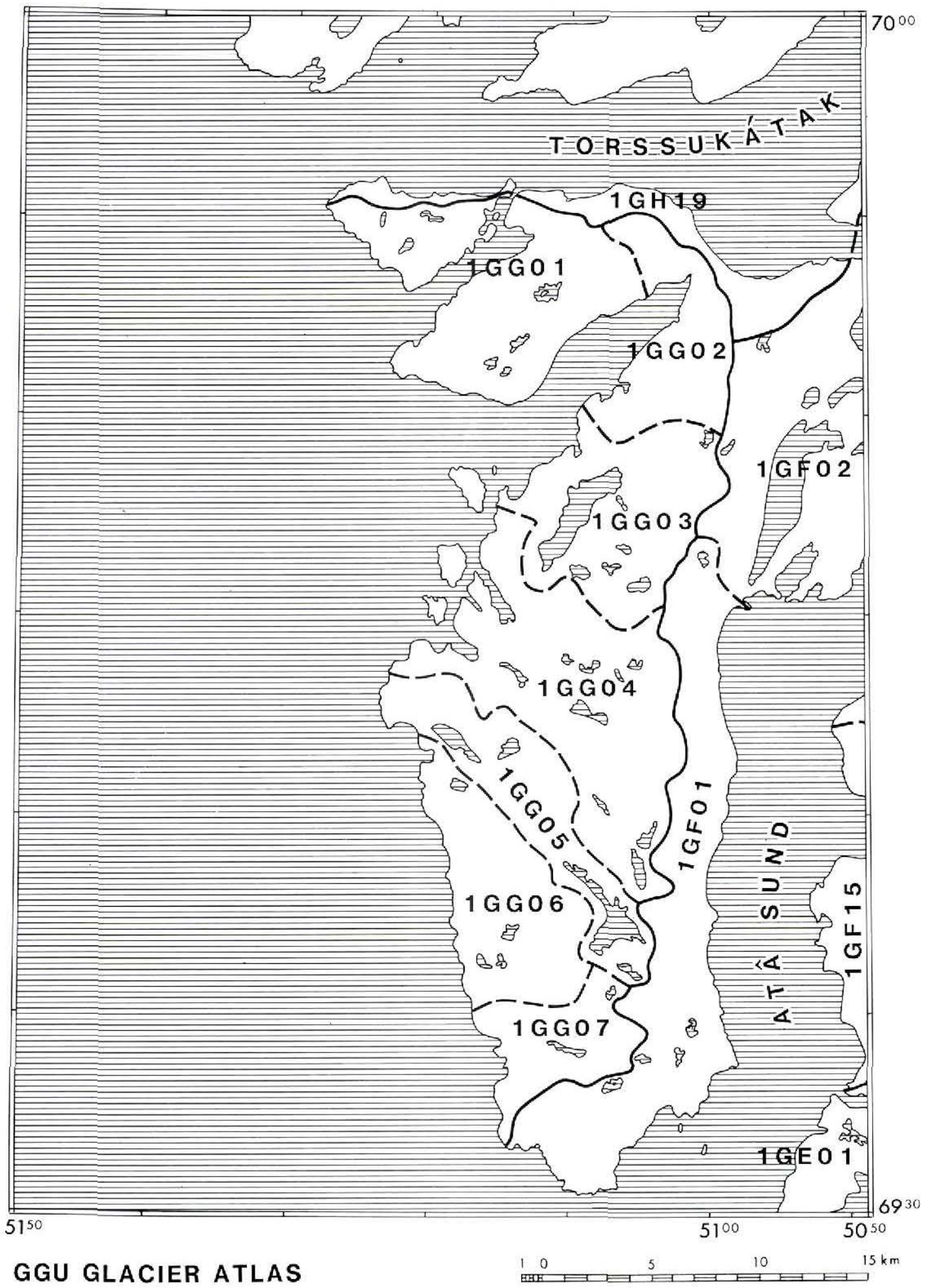

SHEET 6930-04 


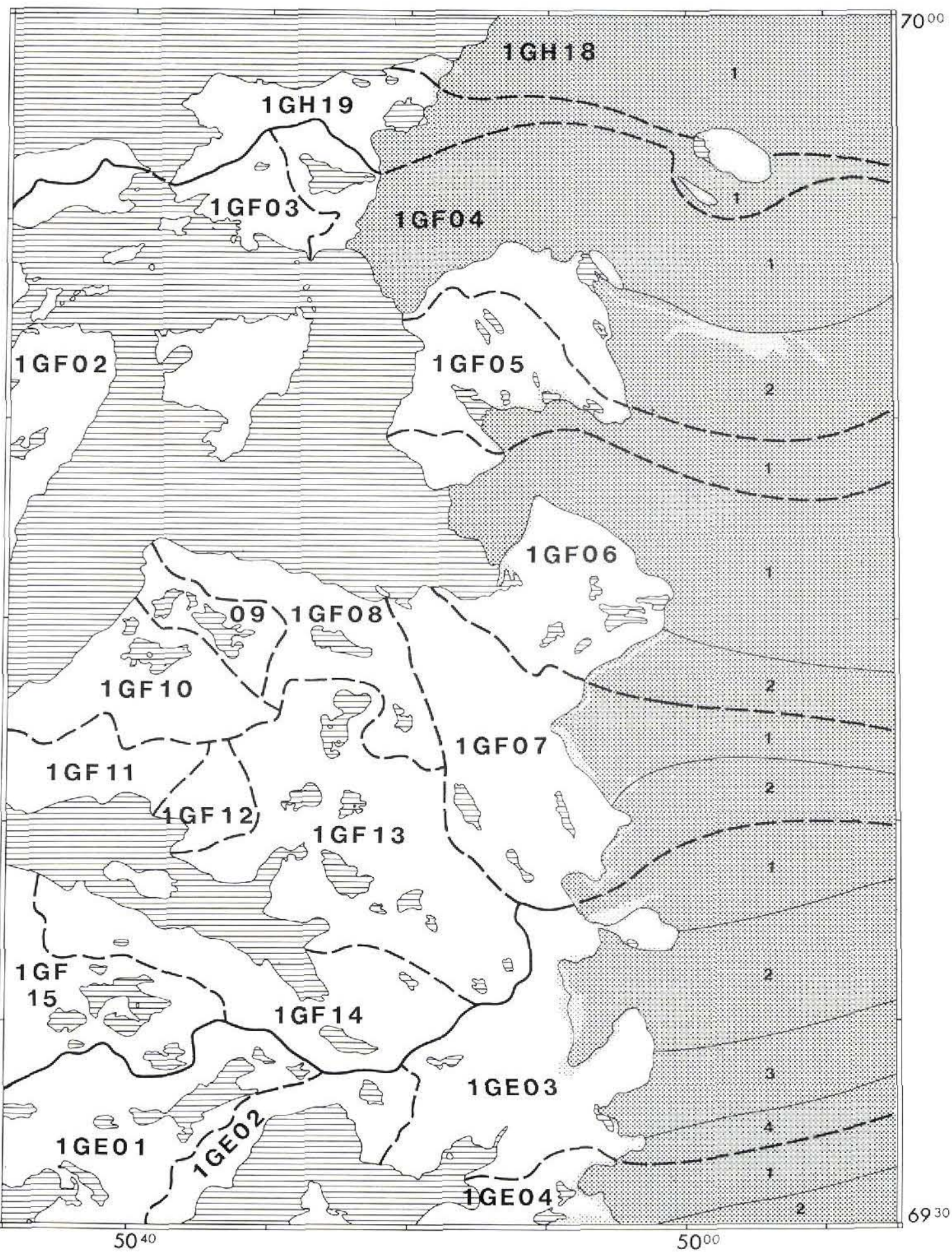

GGU GLACIER ATLAS

$10 \quad 5 \quad 10 \quad 15 \mathrm{~km}$

SHEET 6930-05 


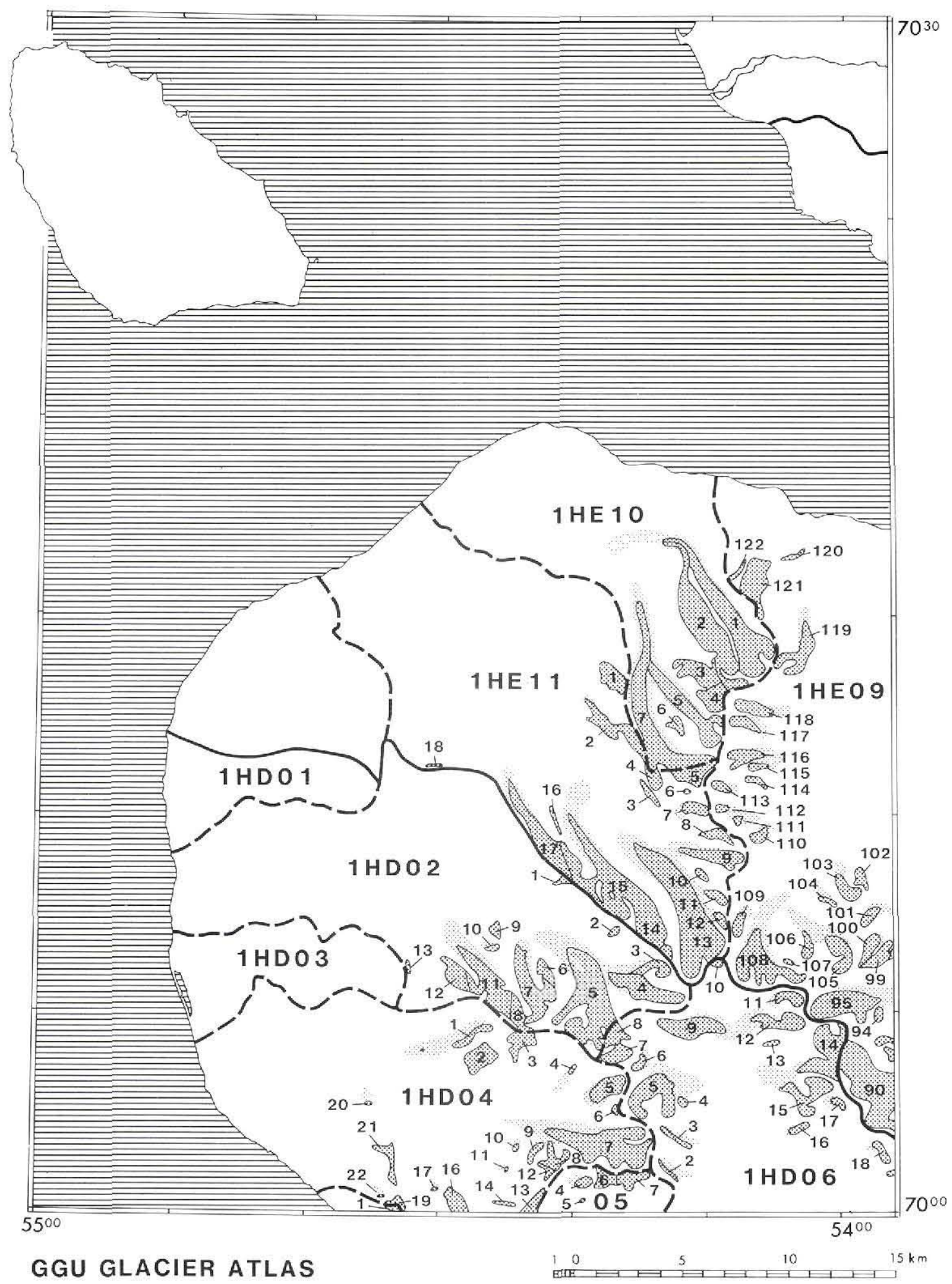

SHEET 7000-01 


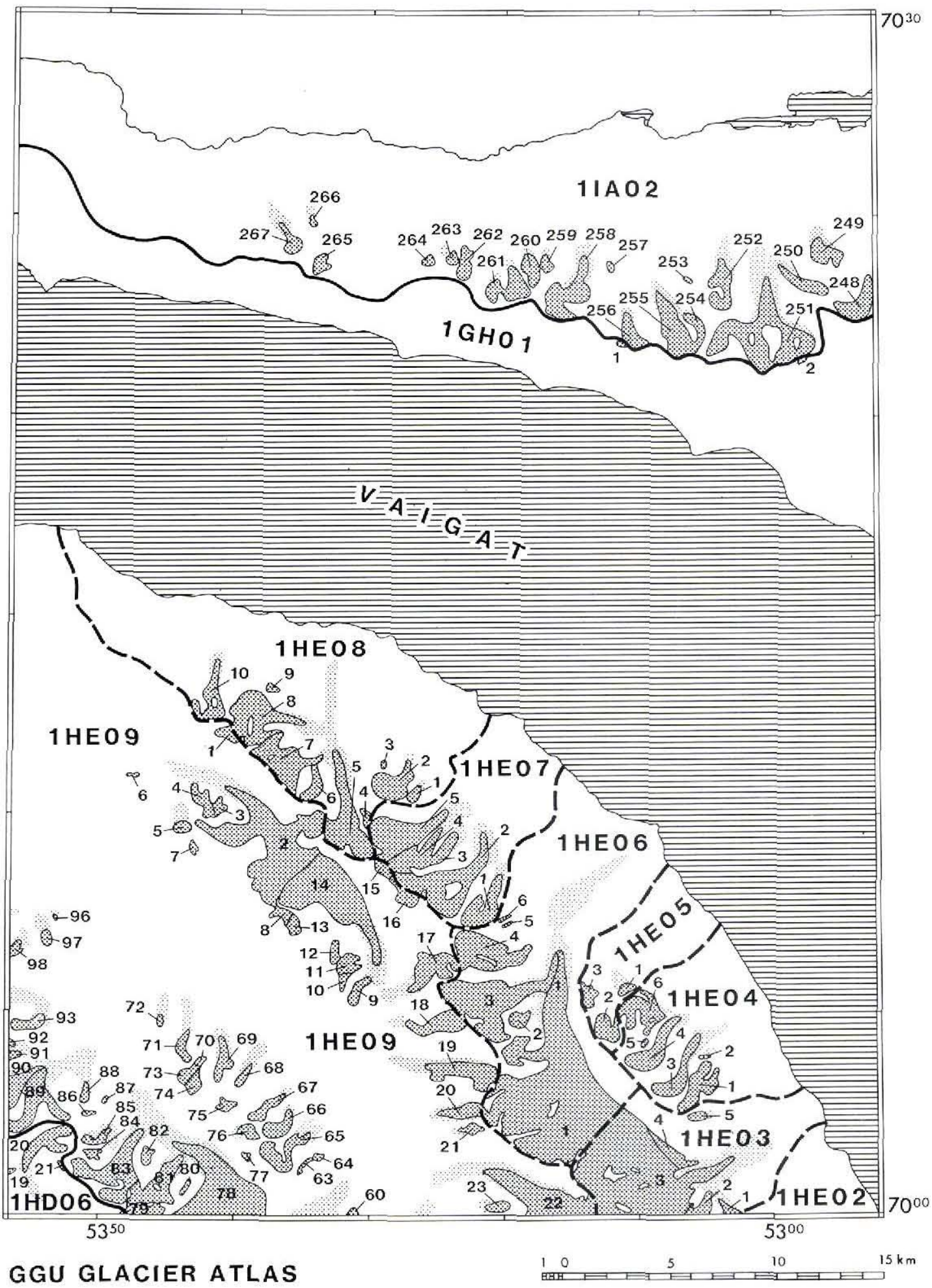

\section{SHEET 7000-02}




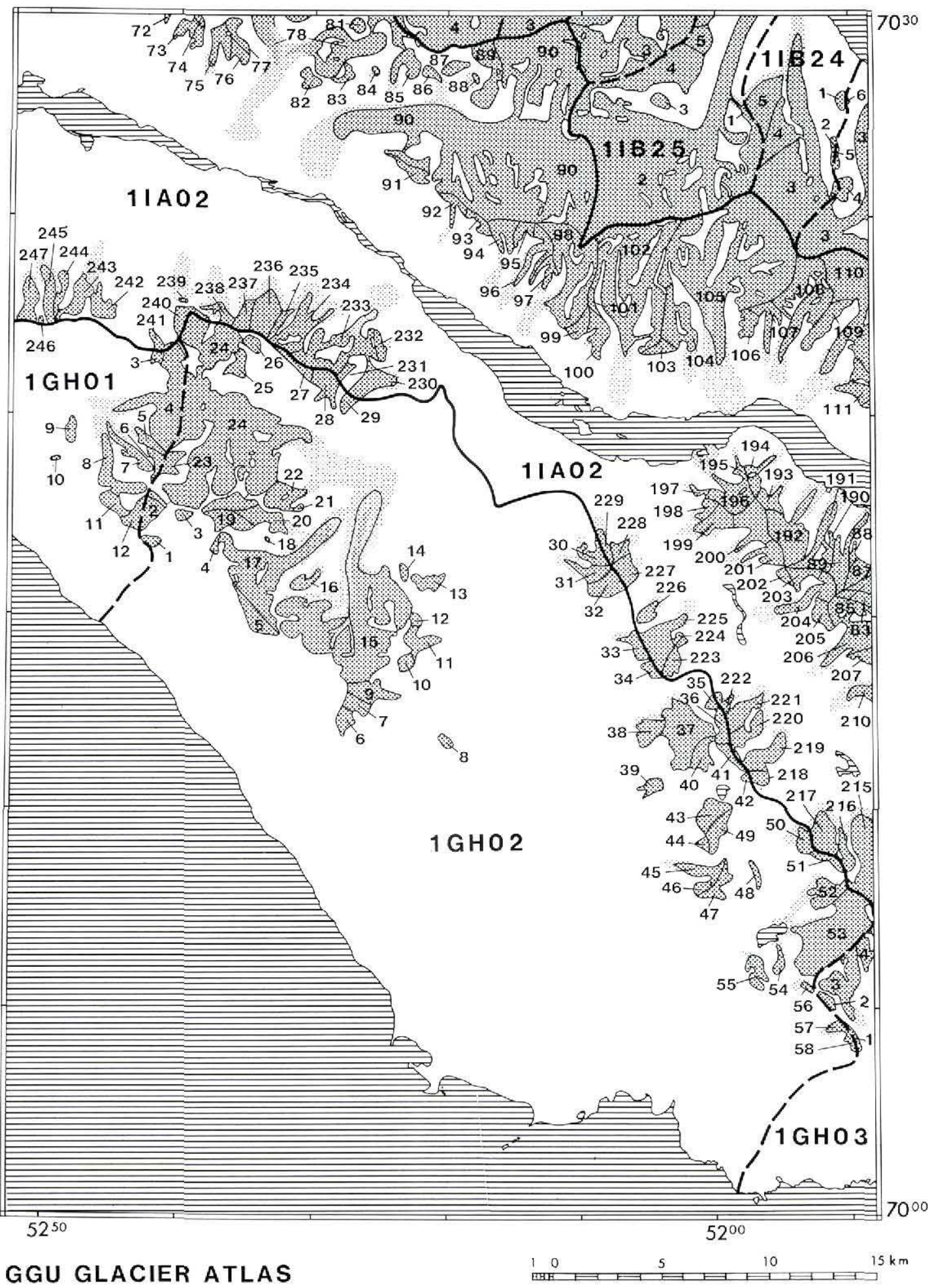




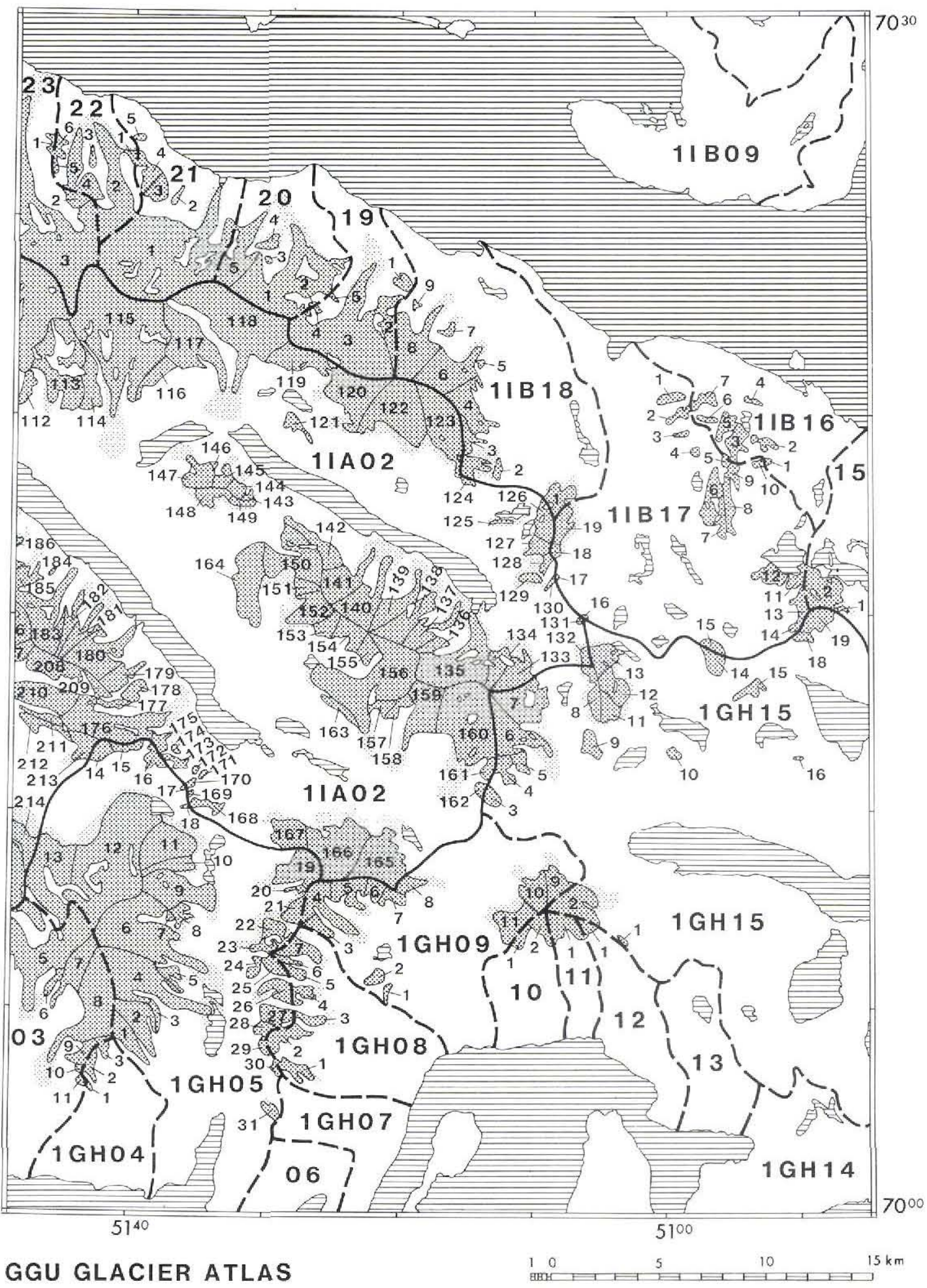

SHEET 7000-04 


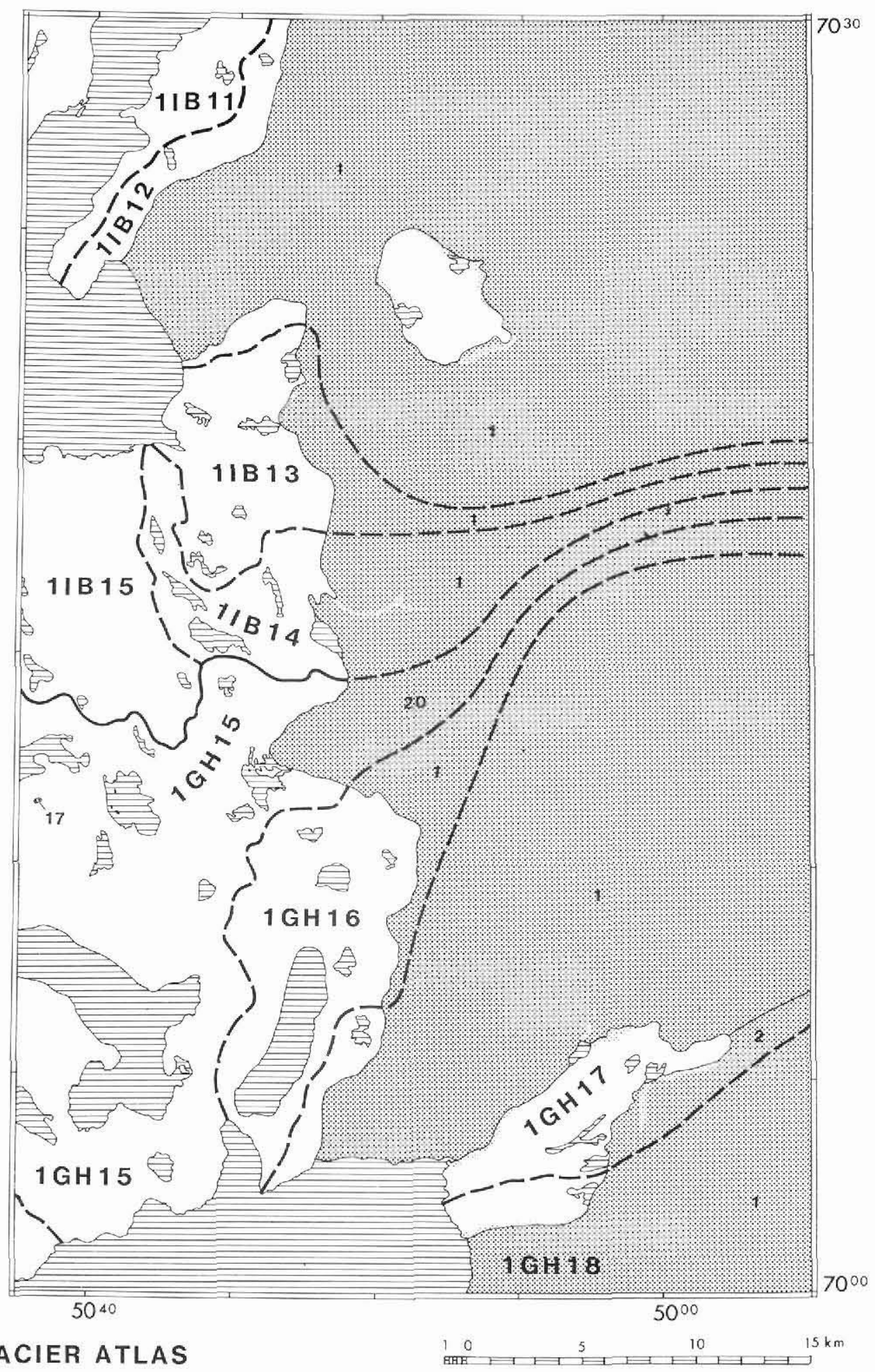

GGU GLACIER ATLAS

SHEET 7000-05 


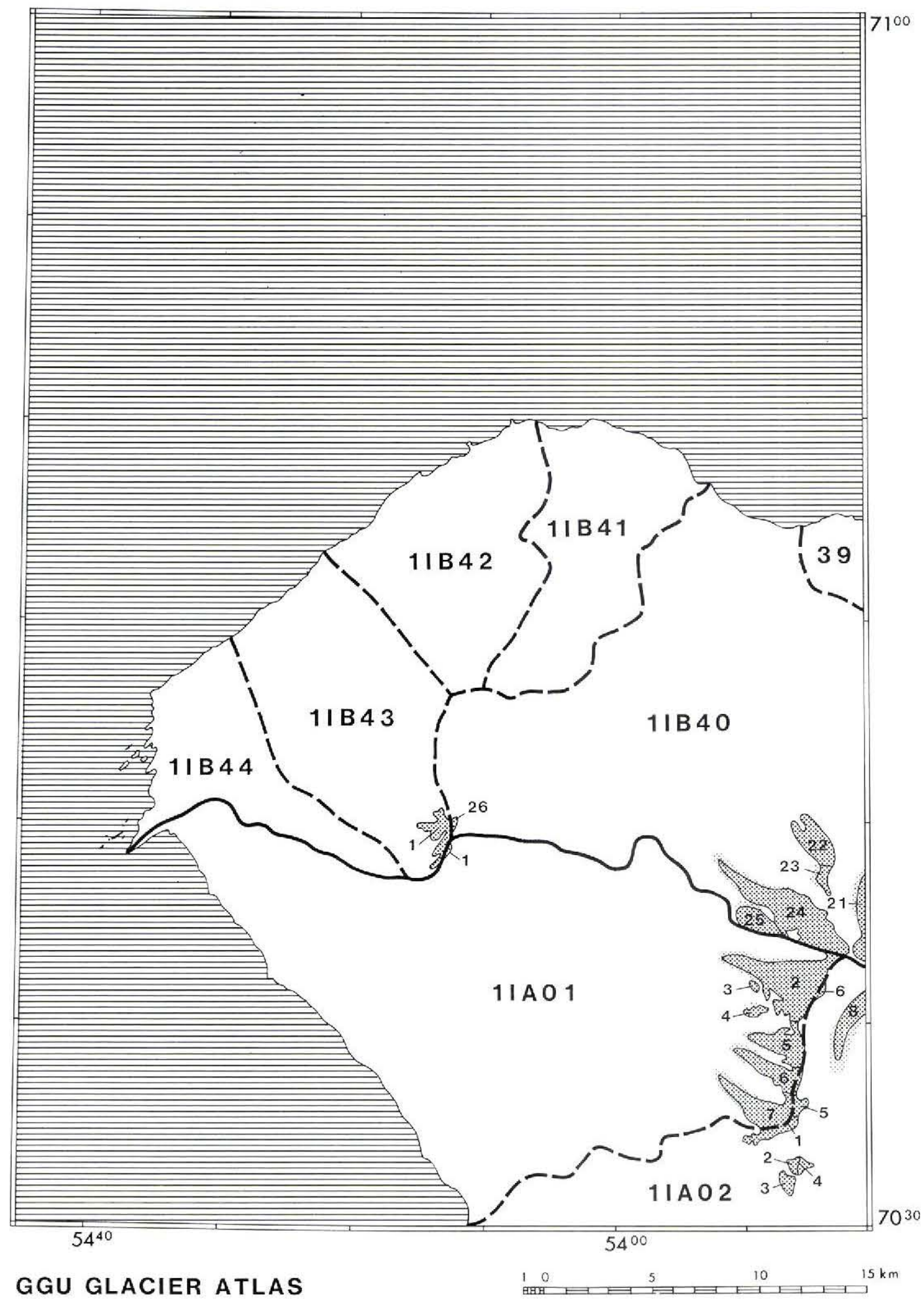

SHEET 7030-01 


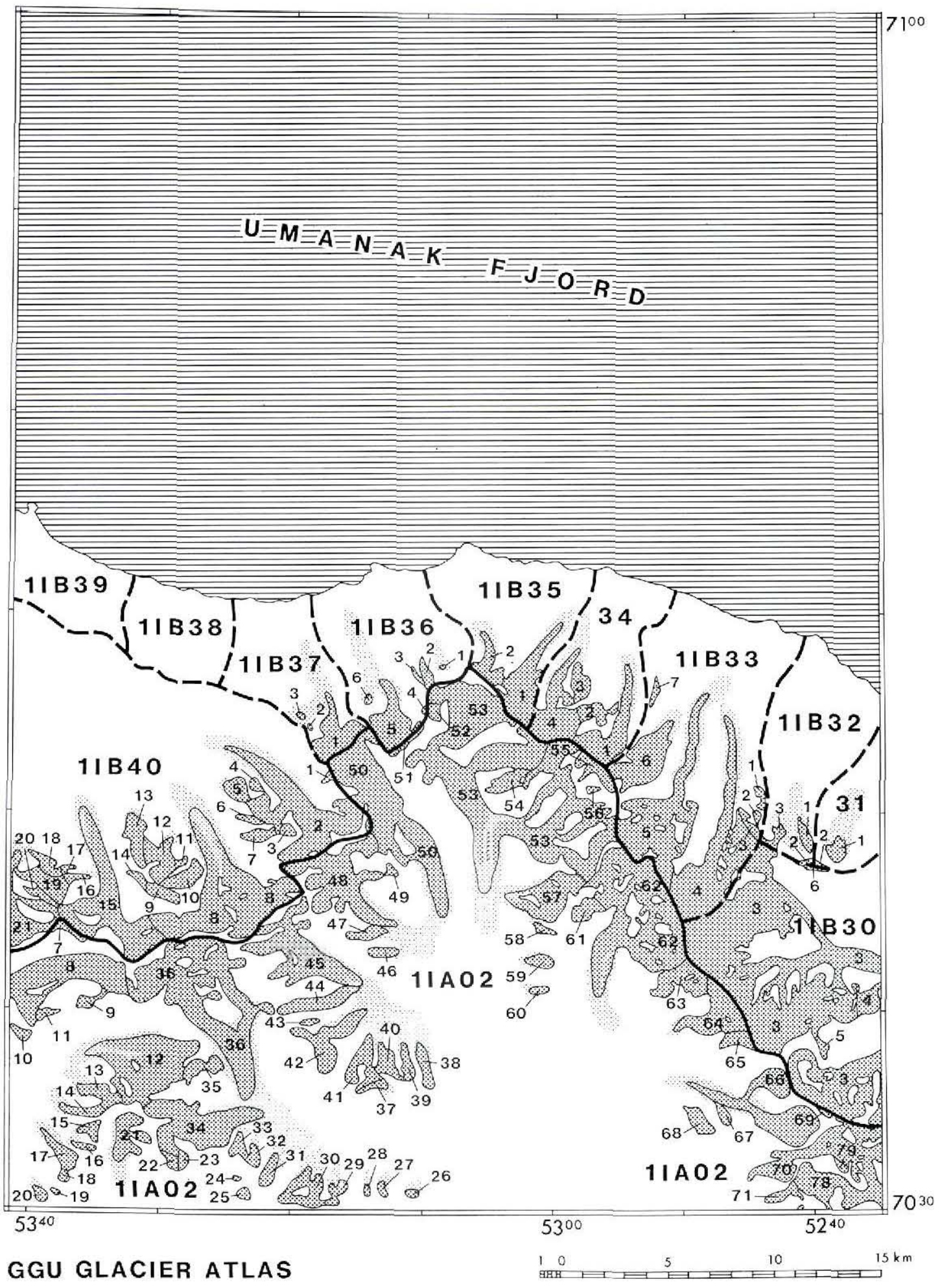

SHEET 7030-02 


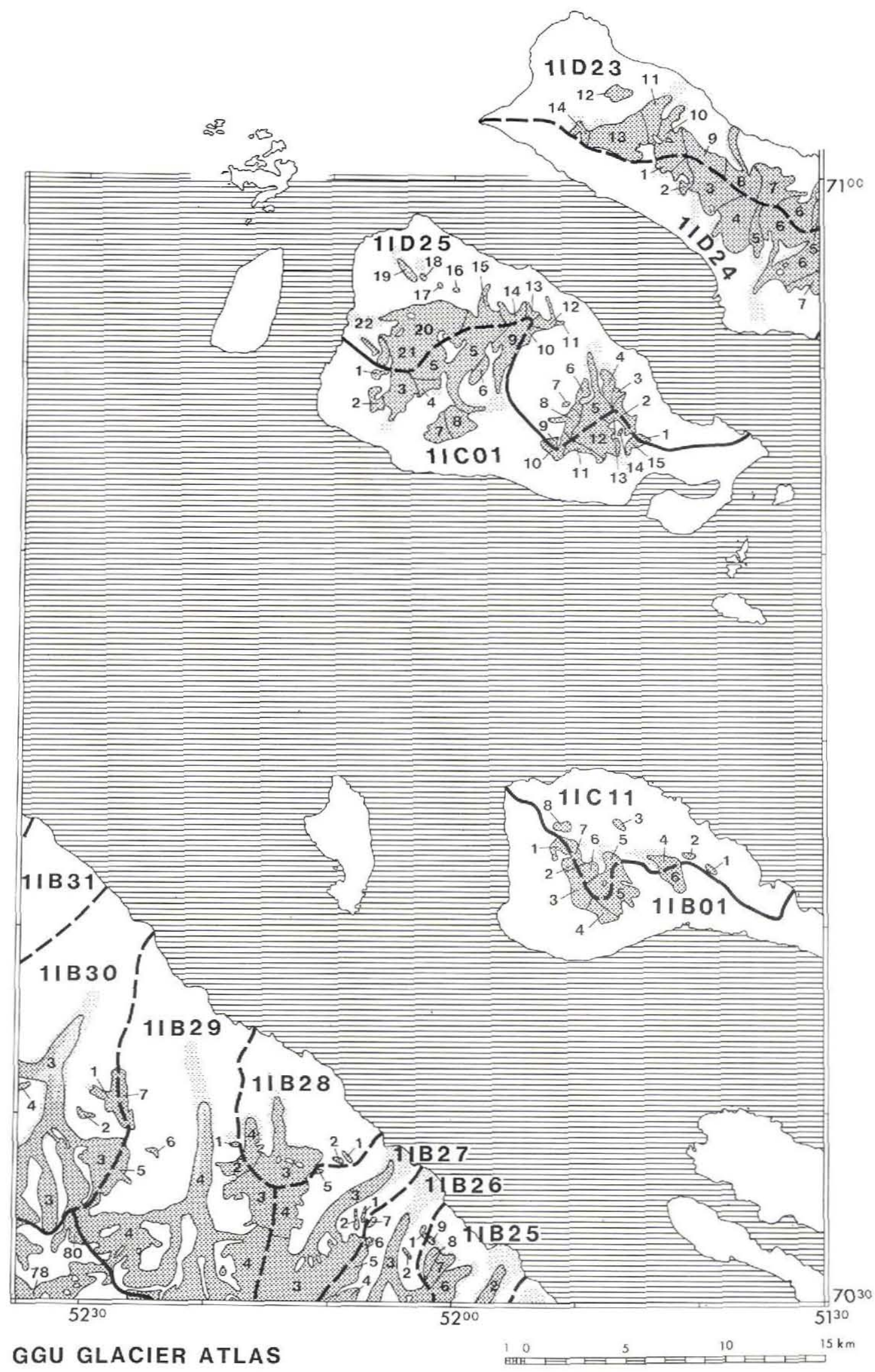

SHEET 7030-03 


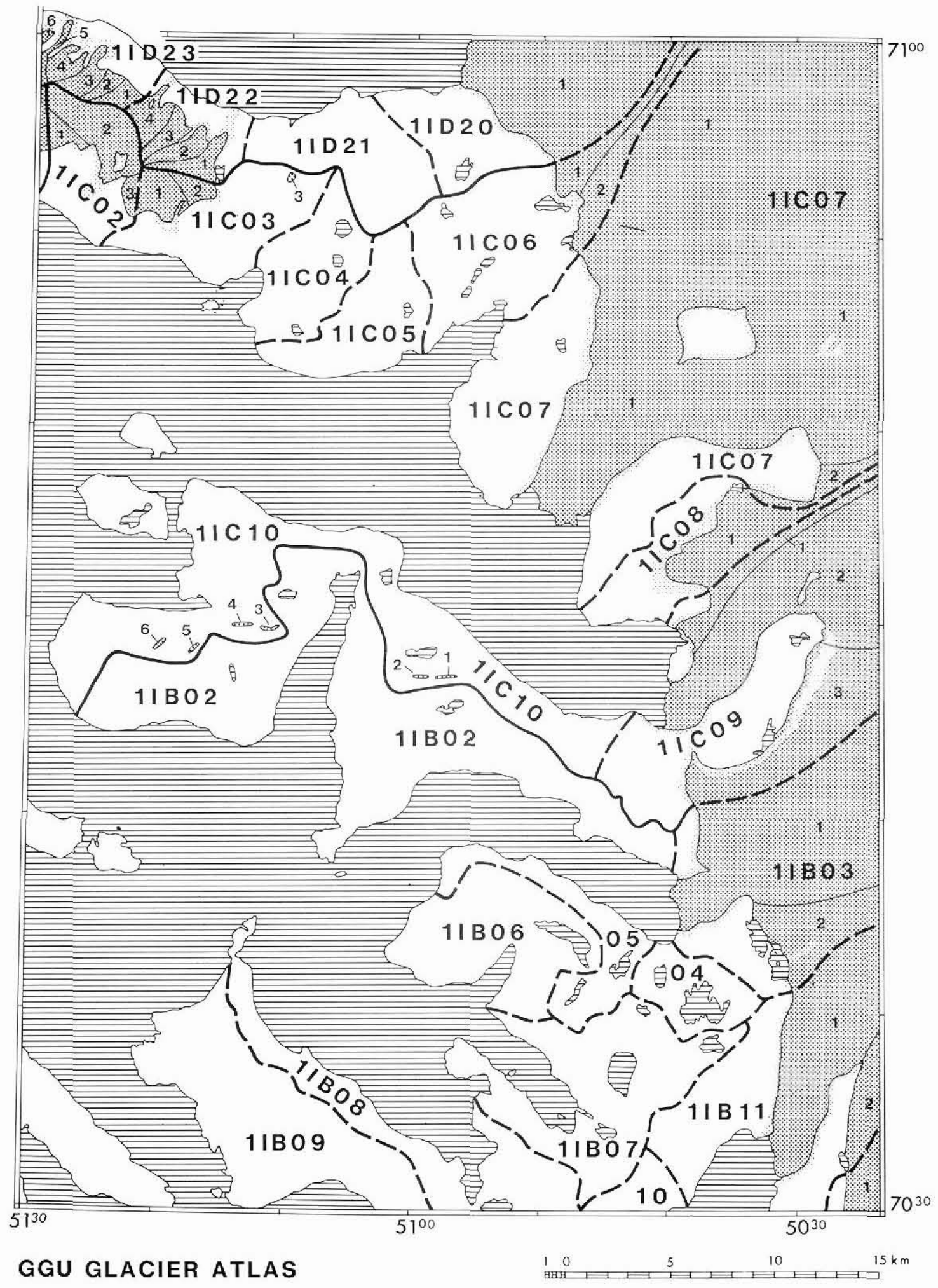

\section{SHEET 7030-04}




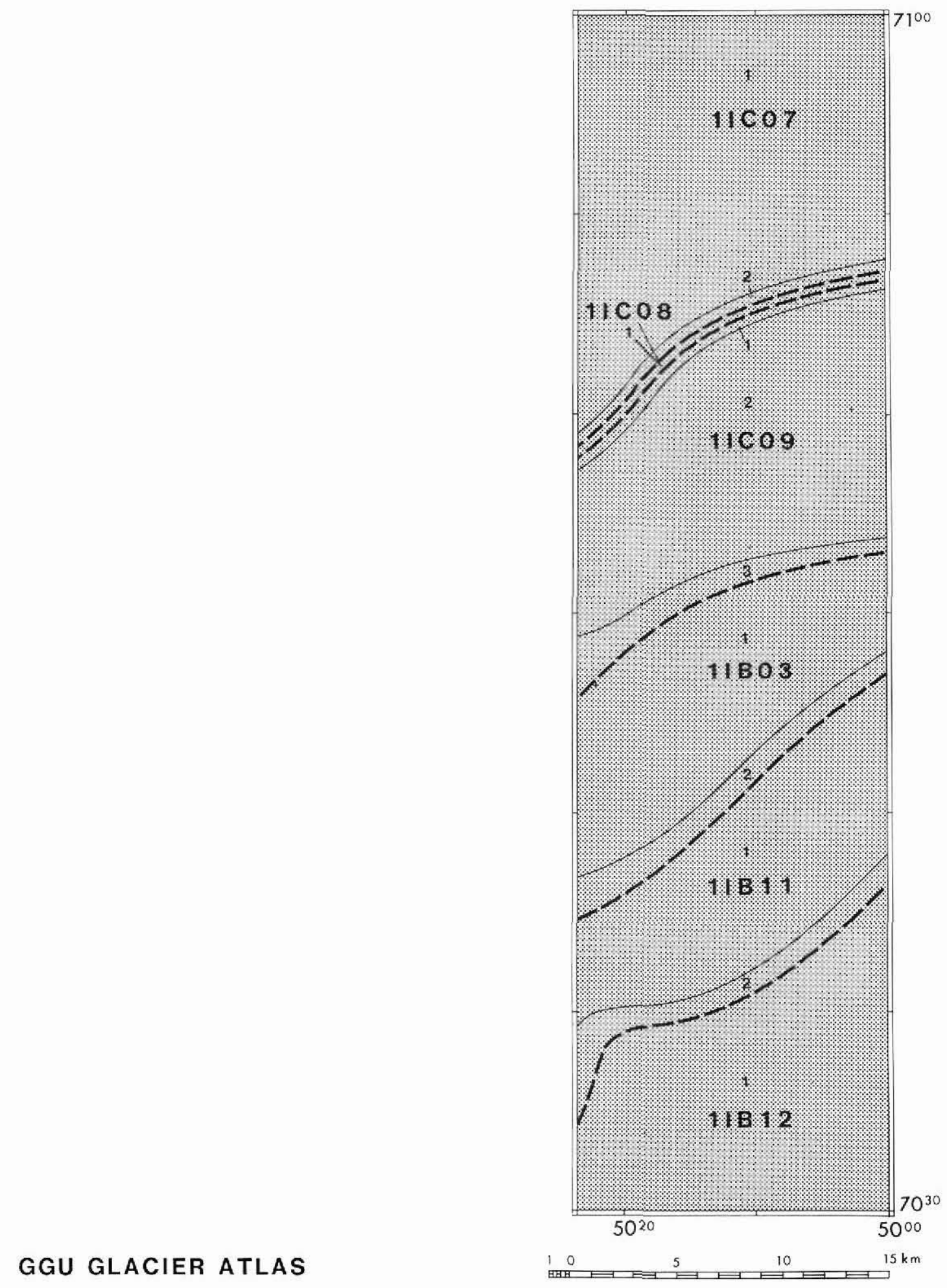


GRØNLANDS GEOLOGISKE UNDERSØGELSE

Geological Survey of Greenland

ISSN 0418-6559

Øster Voldgade 10, DK-1350 Copenhagen K

Denmark

AiO Print Ltd.

Odense, Denmark 